Supplement of Solid Earth, 11, 2425-2438, 2020

https://doi.org/10.5194/se-11-2425-2020-supplement

(C) Author(s) 2020. This work is distributed under

the Creative Commons Attribution 4.0 License.

(c) (1)

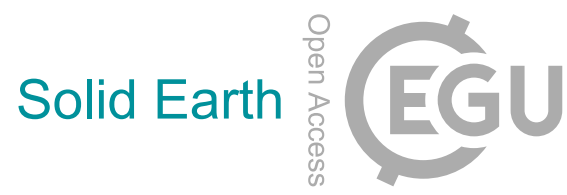

Supplement of

\title{
Micro- and nano-porosity of the active Alpine Fault zone, New Zealand
}

Martina Kirilova et al.

Correspondence to: Martina Kirilova (martina.kirilova@uni-mainz.de)

The copyright of individual parts of the supplement might differ from the CC BY 4.0 License. 


\section{Supplementary material 1}

Supplementary material for the methodologies implemented during thresholding and calculating total porosities in samples from rocks representing the Alpine Fault core.

\section{Content:}

- Figure 1: Avizo screenshots showing the threholded grey-scale values on (i) grey-scale images and (ii) grey-value histograms

- Table 1: Porosity estimates based on: 20, 50, 100, 200 connected voxels and polynomial fitting.

- Matlab script used for estimating total porosities by excluding cracks but predicting and including the volumes of large pores. The script allowed producing figure 3.

- A series of pore size data acquired from the segmentation of synchrotron X-ray microtomography for each sample.

Figure 1. For each sample are shown: (i) a representative grey-scale image, (ii) the same grey-sale image overlain by the selected threshold, and (iii) a grey-value histogram with the selected threshold range. The grey-value histograms were zoomed to the data histogram range for better visualization.

\section{(a) DFDP-1B 58-1.9}

(upper foliated $\mathrm{ccl}$ )

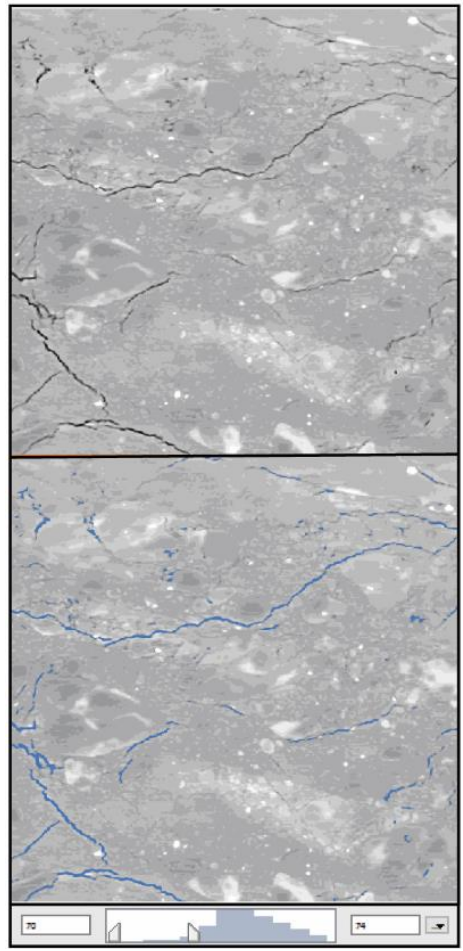

(b) DFDP-1B 69-2.48

(upper foliated $\mathrm{ccl}$ )

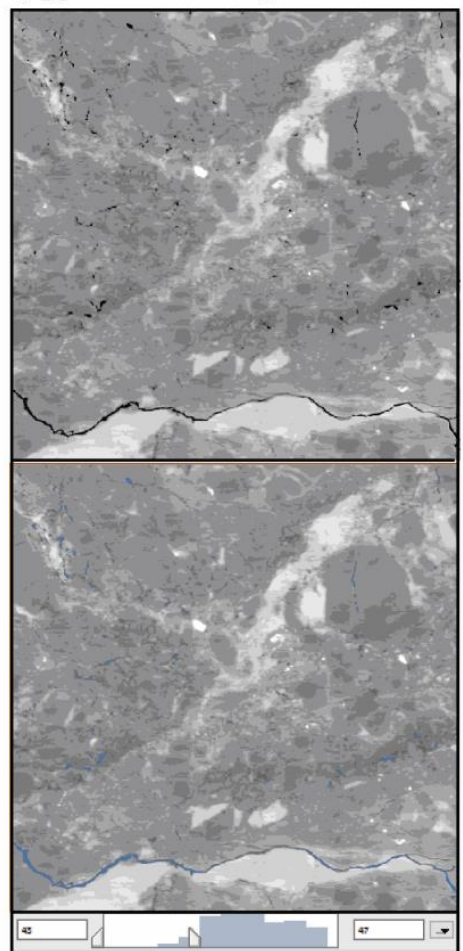

(c) DFDP-1B 69-2.54

(PSZ-2)

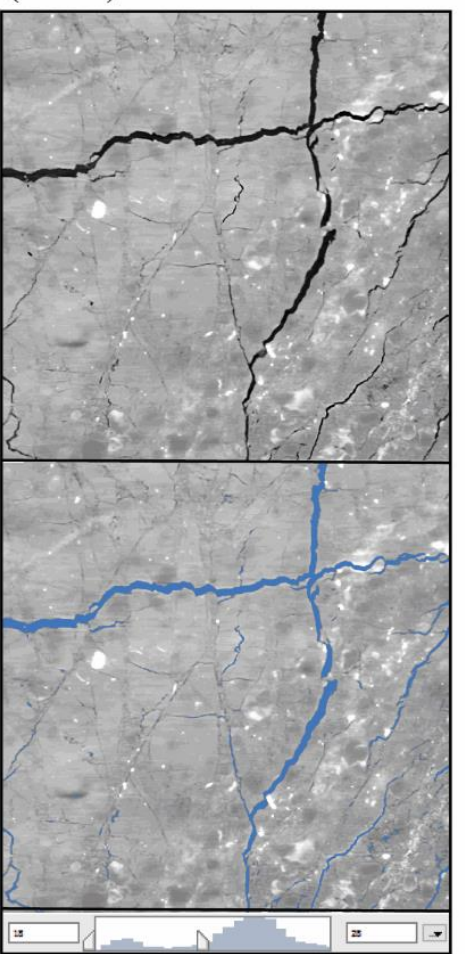

(d) DFDP-1B 69-2.57

(lower ccl)

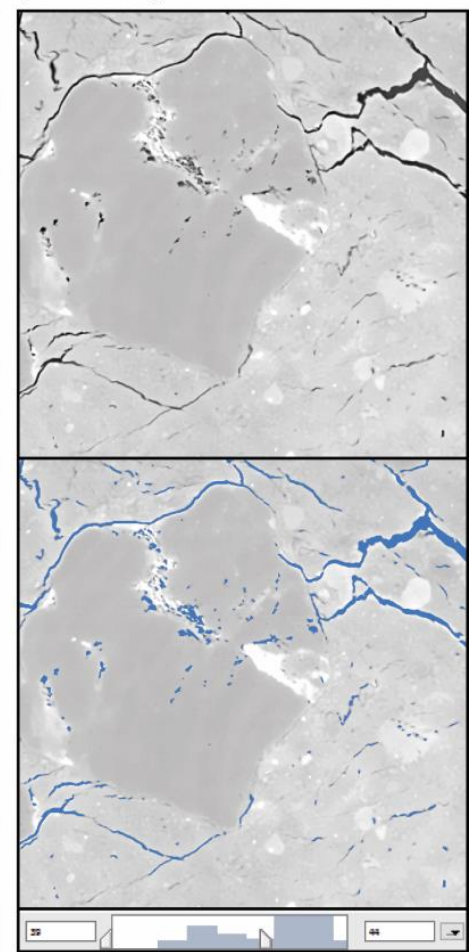

Table 1. Total porosity estimates

\begin{tabular}{|c|c|c|c|c|c|c|c|c|c|c|c|c|}
\hline Sample: & $\begin{array}{l}\text { Voxel } \\
\text { size }\end{array}$ & Lattice & $\begin{array}{l}\text { Sample } \\
\text { volume }\end{array}$ & $\begin{array}{c}\text { Porosity } \\
\text { volume at } 20 \\
\text { connected } \\
\text { voxels }\end{array}$ & $\begin{array}{l}\text { Total } \\
\text { porosity \% } \\
\text { at } 20 \\
\text { connected } \\
\text { voxels }\end{array}$ & $\begin{array}{c}\text { Porosity } \\
\text { volume at } 50 \\
\text { connected } \\
\text { voxels }\end{array}$ & $\begin{array}{l}\text { Total } \\
\text { porosity \% } \\
\text { at } 50 \\
\text { connected } \\
\text { voxels }\end{array}$ & $\begin{array}{l}\text { Porosity } \\
\text { volume at } \\
100 \\
\text { connected } \\
\text { voxels }\end{array}$ & $\begin{array}{l}\text { Total } \\
\text { porosity } \% \\
\text { at } 100 \\
\text { connected } \\
\text { voxels }\end{array}$ & $\begin{array}{l}\text { Porosity } \\
\text { volume at } \\
200 \\
\text { connected } \\
\text { voxels }\end{array}$ & $\begin{array}{c}\text { Total } \\
\text { porosity \% } \\
\text { at } 200 \\
\text { connected } \\
\text { voxels }\end{array}$ & $\begin{array}{c}\text { Total } \\
\text { porosity \% } \\
\text { based on } \\
\text { polynomial } \\
\text { regression }\end{array}$ \\
\hline DFDP-1B 58_1.9 & 1.3 & $601^{\wedge} 3$ & 476928716.8 & 129185.797 & 0.03 & 235351.428 & 0.05 & 337472.382 & 0.07 & 457749.34 & 0.10 & 0.10 \\
\hline DFDP-1B 69_2.48 & 1.3 & $701^{\wedge} 3$ & 756805205.9 & 222136.473 & 0.03 & 427294.53 & 0.06 & 625523.249 & 0.08 & 812323.17 & 0.11 & 0.12 \\
\hline DFDP-1B 69_2.54 & 1.3 & $801^{\wedge} 3$ & 1129087515 & 284722.412 & 0.03 & 506852.294 & 0.04 & & 0.06 & & 0.09 & 0.10 \\
\hline DFDP-1B 69_2.57 & 1.3 & $601^{\wedge} 3$ & 476928716.8 & 252736.289 & 0.05 & 504316.956 & 0.11 & 741067.873 & 0.16 & 796434.47 & 0.17 & 0.24 \\
\hline
\end{tabular}




\section{MATLAB CODE}

close all \% ignore this line

FILE = 'filename.csV';

HEADER_ROWS $=2$;

$f=$ importdata(FILE, ' , ', HEADER_ROWS);

data $=f$.data; headers $=f$. colheaders;

VOLUMES = "" "Volume3d"" ";

VOXEL_SIZE = 1.3;

FIT_LIMIT $=180 ; \%$ unique for each sample

FILTER = ' : '; \% no filter

INDICATE_EXTRAPOLATION = true;

data $=\operatorname{data}($ FILTER, headers $==$ VOLUMES $)$;

$\%$ create edges equidistant between possible volumes corresponding to actual

$\%$ volume of a voxel

$\mathrm{VV}=$ VOXEL_SIZE^3;

edges $=\min ($ data $)-(\mathrm{vv} / 2): \mathrm{vv}: 65535 * \mathrm{vv}+\mathrm{vv} / 2 ; \%$ limit*vv+vv/2;

Ys = histcounts (data, edges);

$\mathrm{Xs}=$ edges+vv/2;

$\mathrm{Xs}(\mathrm{end})=[]$;

$\%$ polyfit line, where counts are not zero and display the fit curve equation

fitYs = Ys (1:FIT_LIMIT);

fitXs = Xs (1:FIT LIMIT);

idx = fitYs $\sim=0$;

$c=\operatorname{polyfit}(\log (f i t X s(i d x)), \log (f i t Y s(i d x)), 2)$;

fit $=@(x) \exp \left(c(1) \cdot{ }^{*} \log (x) \cdot{ }^{\wedge} 2+c(2) \cdot * \log (x)+c(3)\right)$;

$\operatorname{disp}\left(\operatorname{strcat}\left(" y=", \operatorname{num} 2 \operatorname{str}(c(1)), " x^{\wedge} 2+", \operatorname{num} 2 \operatorname{str}(c(2)), " x+", \operatorname{num} 2 \operatorname{str}(c(3))\right)\right)$;

\% the least squares fit is calculated as described in "Computing R2 from Polynomial Fits"

\% https://wWw.mathworks.com/help/matlab/data analysis/linear-regression.html

$\operatorname{disp}\left(\operatorname{strcat}\left(" R^{\wedge} 2=", \operatorname{num} 2 \operatorname{str}\left(1-\operatorname{sum}\left((Y s(i d x)-f i \bar{t}(X s(i d x))) \cdot{ }^{\wedge} 2\right) /((\operatorname{length}(Y s(i d x))-1) * \operatorname{var}(Y s(i d x)))\right)\right)\right) ; \%$

least squares fit

$\%$ calculate the volume of the maximum pore size we expect to observe

syms $x$;

sol = double $\left(\operatorname{solve}\left(\log (1)==c(1)^{*} \log (x)^{\wedge} 2+c(2) * \log (x)+c(3), x\right) / v v\right)$;

maxPore $=\operatorname{sol}($ sol $>$ FIT LIMIT);

disp (strcat ("maxPore=", num2str (maxPore*vv)))

$\%$ show integration of volumes for different limits

func $=@(x) x . *$ fit $(x)$;

$\operatorname{disp}(\operatorname{strcat}("$ toOne $=$ ", num2str (integral (func, $\min (\mathrm{Xs}), \operatorname{maxPore} * v v) / v v))$ )

$\operatorname{disp}(\operatorname{strcat}("$ toInf $=$ ", num $2 \operatorname{str}($ integral (func, $\min (X s)$, inf $) / v v))$ )

$\operatorname{disp}(\operatorname{strcat}(" N a n o=", \operatorname{num} 2 \operatorname{str}($ integral $($ func, $\theta, \min (\mathrm{Xs})) / \mathrm{vv}))$ )

$\%$ Plot the data and best fit

$\log \log \left(\mathrm{Xs}, \mathrm{Ys}, \mathrm{o}^{\prime}\right)$

hold on

if(INDICATE_EXTRAPOLATION)

$\%$ plot the fit portion with a different color than the extrapolation

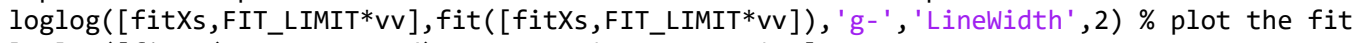

$\log \log ([$ fitXs(FIT_LIMIT:end),FIT_LIMIT*vv:maxPore*vV], . .

fit ([fitXs(FIT_LIMIT:end),FIT_LIMIT*vv:maxPore*vv]), 'r-', 'LineWidth',2) \% plot the extrapolation

else

$\%$ plot the entire fit as a single color end

$\log \log ([$ fitXs, maxPore*vv], fit ([fitXs, maxPore*vv]), 'r-', 'LineWidth',2) \% plot the fit

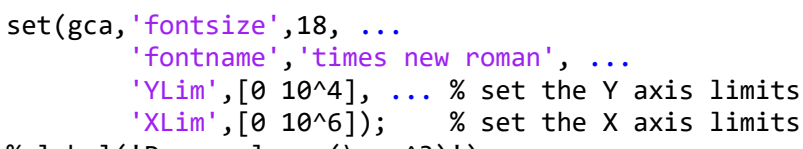

$\% x l a b e l\left(' P o r e ~ v o l u m e\left(\backslash m u m^{\wedge} 3\right)\right.$ ')

$\% y l a b e l$ ('Number of pores') 


\begin{tabular}{|c|c|c|c|}
\hline DFDP-1B 58-1.9 & DFDP-1B 69-2.48 & DFDP-1B 69-2.54 & DFDP-1B 69-2.57 \\
\hline Volume3d & Volume3d & Volume3d & Volume3d \\
\hline 361679 & 32.955 & 2287.08 & $4.78 \mathrm{E}+07$ \\
\hline 80807.8 & 15.379 & 6162.58 & 2421.09 \\
\hline 254.852 & 4.394 & $3.60 E+07$ & 34976.2 \\
\hline $2.10 \mathrm{E}+06$ & 94.471 & 24.167 & 24.167 \\
\hline 63.713 & 94.471 & 26.364 & 6.591 \\
\hline 15.379 & 24.167 & 2.197 & 2.197 \\
\hline 17.576 & 83.486 & 2.197 & 37.349 \\
\hline 2.197 & 61.516 & 885103 & 138.411 \\
\hline 24.167 & 2.197 & 13.182 & 36922.8 \\
\hline 982.059 & 21.97 & 2.197 & 254.852 \\
\hline 63.713 & 98.865 & 2.197 & 4.394 \\
\hline 145.002 & 6.591 & 3161.48 & 2.197 \\
\hline 112.047 & 4.394 & 4.394 & 1885.03 \\
\hline 7370.93 & 4666.43 & 35.152 & 2.197 \\
\hline 50.531 & 4.394 & 762.359 & 6.591 \\
\hline 2205.79 & $1.66 \mathrm{E}+06$ & 21.97 & 2.197 \\
\hline 8.788 & 2.197 & 296.595 & 230.685 \\
\hline 17.576 & 4.394 & 30.758 & 39.546 \\
\hline 79.092 & 10.985 & 4.394 & 2.197 \\
\hline 68.107 & 6.591 & 1575.25 & 19.773 \\
\hline 28.561 & 63.713 & 17543 & 101.062 \\
\hline 4646.65 & 2.197 & 9154.9 & 74.698 \\
\hline 6.591 & 4.394 & 8.788 & 21.97 \\
\hline 35.152 & 2.197 & 189217 & 17.576 \\
\hline 180.154 & 2.197 & 307.58 & 90.077 \\
\hline 35.152 & 2.197 & 2.197 & 10.985 \\
\hline 2.197 & 2.197 & 105.456 & 766.753 \\
\hline 134.017 & 2.197 & 261.443 & 41.743 \\
\hline 41.743 & 10.985 & 10.985 & 17.576 \\
\hline 6.591 & 10233.6 & 4.394 & 8.788 \\
\hline 61.516 & 10.985 & 13.182 & 4.394 \\
\hline 57.122 & 2.197 & 2.197 & 2.197 \\
\hline 417.43 & 8.788 & 2.197 & 2.197 \\
\hline 804.102 & 4.394 & 2.197 & 52.728 \\
\hline 6085.69 & 4.394 & 30.758 & 17.576 \\
\hline 98.865 & 4.394 & 2.197 & 1313.81 \\
\hline 2.197 & 43.94 & 2.197 & 2.197 \\
\hline 19.773 & 4.394 & 410.839 & 4.394 \\
\hline 2497.99 & 373.49 & 300.989 & 2.197 \\
\hline 37.349 & 4.394 & 133797 & 96.668 \\
\hline 26.364 & 355.914 & 10.985 & 12006.6 \\
\hline 37.349 & 39.546 & 30.758 & 228.488 \\
\hline 8.788 & 87.88 & 94.471 & 61.516 \\
\hline 10.985 & 59.319 & 4.394 & 19.773 \\
\hline 19.773 & 74.698 & 10.985 & 2.197 \\
\hline 2.197 & 4.394 & 2.197 & 8.788 \\
\hline 2.197 & 19.773 & 17.576 & 13173.2 \\
\hline 48.334 & 8.788 & 2.197 & 10.985 \\
\hline
\end{tabular}




\begin{tabular}{|c|c|c|c|}
\hline 555.841 & 26.364 & 2.197 & 17.576 \\
\hline 4.394 & 30.758 & 177.957 & 13.182 \\
\hline 63.713 & 1513.73 & 35.152 & 2.197 \\
\hline 116.441 & 46.137 & 344.929 & 2.197 \\
\hline 13.182 & 854.633 & 2.197 & 13.182 \\
\hline 2.197 & 123.032 & 4.394 & 2.197 \\
\hline 6511.91 & 155.987 & 13.182 & 4.394 \\
\hline 2.197 & 305.383 & 48.334 & 4.394 \\
\hline 6.591 & 17.576 & 276.822 & 26.364 \\
\hline 177.957 & 2.197 & 46.137 & 2.197 \\
\hline 2.197 & 52.728 & 13.182 & 2.197 \\
\hline 2.197 & 195.533 & 4.394 & 8.788 \\
\hline 2.197 & 15.379 & 1072.14 & 2952.77 \\
\hline 83.486 & 10.985 & 4.394 & 4.394 \\
\hline 421.824 & 10.985 & 2.197 & 21.97 \\
\hline 39.546 & 2.197 & 6.591 & 8.788 \\
\hline 10.985 & 6.591 & 13.182 & 8.788 \\
\hline 6.591 & 2.197 & 2.197 & 10.985 \\
\hline 2.197 & 13.182 & 8.788 & 13.182 \\
\hline 15.379 & 2.197 & 2.197 & 32.955 \\
\hline 19.773 & 2.197 & 2.197 & 2.197 \\
\hline 2.197 & 2.197 & 2.197 & 1915.78 \\
\hline 13.182 & 2.197 & 54.925 & 1116.08 \\
\hline 28.561 & 4.394 & 2.197 & 48.334 \\
\hline 15.379 & 10.985 & 4.394 & 87.88 \\
\hline 388.869 & 2.197 & 79.092 & 2.197 \\
\hline 806121 & 2.197 & 177.957 & 248.261 \\
\hline 19.773 & 4.394 & 2.197 & 2.197 \\
\hline 15.379 & 2.197 & 2.197 & 2.197 \\
\hline 2.197 & 46.137 & 2.197 & 2.197 \\
\hline 2.197 & 164.775 & 15.379 & 2.197 \\
\hline 26.364 & 4.394 & 153873 & 109.85 \\
\hline 85.683 & 6.591 & 162.578 & 2.197 \\
\hline 6.591 & 17.576 & 6.591 & 2.197 \\
\hline 26.364 & 2.197 & 24.167 & 164.775 \\
\hline 2.197 & 2.197 & 37.349 & 8.788 \\
\hline 558.038 & 15.379 & 158.184 & 6.591 \\
\hline 3712.93 & 2.197 & 24.167 & 4.394 \\
\hline 19.773 & 8.788 & 2.197 & 4.394 \\
\hline 4.394 & 43.94 & 105.456 & 2.197 \\
\hline 4.394 & 41.743 & 2.197 & 6.591 \\
\hline 10.985 & 2.197 & 6.591 & 2.197 \\
\hline 6.591 & 2.197 & 1004.03 & 2.197 \\
\hline 131.82 & 2.197 & 57.122 & 21.97 \\
\hline 43.94 & 41.743 & 74.698 & 2.197 \\
\hline 107.653 & 2.197 & 8.788 & 2.197 \\
\hline 129.623 & 2.197 & 13.182 & 2.197 \\
\hline 6.591 & 4.394 & 30.758 & 28.561 \\
\hline 24.167 & 2.197 & 6.591 & 2.197 \\
\hline 248.261 & 2.197 & 2.197 & 2.197 \\
\hline
\end{tabular}




\begin{tabular}{|c|c|c|c|}
\hline 2.197 & 213.109 & 2.197 & 125.229 \\
\hline 10.985 & 6.591 & 2.197 & 2.197 \\
\hline 10.985 & 2.197 & 37.349 & 10.985 \\
\hline 4.394 & 26.364 & 87078.1 & 2.197 \\
\hline 8.788 & 2.197 & 17.576 & 2.197 \\
\hline 65.91 & 2.197 & 8.788 & 2.197 \\
\hline 2.197 & 6.591 & 6456.98 & 2.197 \\
\hline 39.546 & 2.197 & 311.974 & 2.197 \\
\hline 2.197 & 2.197 & 2.197 & 2.197 \\
\hline 2.197 & 2.197 & 65.91 & 2.197 \\
\hline 15.379 & 2.197 & 41.743 & 4.394 \\
\hline 26.364 & 2.197 & 2.197 & 2.197 \\
\hline 6.591 & 4.394 & 3574.52 & 2.197 \\
\hline 129.623 & 2.197 & 17.576 & 72.501 \\
\hline 2.197 & 59.319 & 373.49 & 2.197 \\
\hline 17.576 & 2.197 & 2.197 & 6.591 \\
\hline 6.591 & 2.197 & 13.182 & 3723.91 \\
\hline 10.985 & 2.197 & 85.683 & 10.985 \\
\hline 37.349 & 283.413 & 114.244 & 2.197 \\
\hline 2.197 & 13.182 & 2445.26 & 2.197 \\
\hline 10.985 & 13.182 & 366.899 & 2.197 \\
\hline 103.259 & 8.788 & 177.957 & 32.955 \\
\hline 13.182 & 26.364 & 2.197 & 8.788 \\
\hline 13.182 & 10.985 & 4.394 & 2.197 \\
\hline 35.152 & 4.394 & 39.546 & 10.985 \\
\hline 6.591 & 8.788 & 10.985 & 2.197 \\
\hline 15.379 & 8.788 & 4.394 & 10.985 \\
\hline 2.197 & 19.773 & 30.758 & 10.985 \\
\hline 2.197 & 6.591 & 19.773 & 30.758 \\
\hline 4.394 & 13.182 & 4.394 & 28.561 \\
\hline 6.591 & 2.197 & 4.394 & 2.197 \\
\hline 10.985 & 2.197 & 35.152 & 21.97 \\
\hline 2.197 & 2.197 & 494.325 & 24.167 \\
\hline 13.182 & 2.197 & 2.197 & 13.182 \\
\hline 2.197 & 2.197 & 10.985 & 76.895 \\
\hline 87.88 & 4.394 & 19.773 & 118.638 \\
\hline 6.591 & 8.788 & 8.788 & 21.97 \\
\hline 83.486 & 2.197 & 54.925 & 292.201 \\
\hline 4.394 & 2.197 & 48.334 & 26.364 \\
\hline 85.683 & 782.132 & 109.85 & 13.182 \\
\hline 2.197 & 43.94 & 43.94 & 2.197 \\
\hline 4.394 & 6.591 & 553.644 & 2.197 \\
\hline 4.394 & 79.092 & 61.516 & 4.394 \\
\hline 2.197 & 2.197 & 2.197 & 13.182 \\
\hline 15.379 & 4.394 & 2.197 & 30.758 \\
\hline 6.591 & 2.197 & 16440.1 & 4.394 \\
\hline 6.591 & 70.304 & 8.788 & 13.182 \\
\hline 116.441 & 443.794 & 10.985 & 2.197 \\
\hline 2.197 & 2.197 & 2.197 & 2.197 \\
\hline 32.955 & 13.182 & 2.197 & 595.387 \\
\hline
\end{tabular}




\begin{tabular}{|c|c|c|c|}
\hline 81.289 & 6.591 & 5422.2 & 13.182 \\
\hline 2.197 & 2.197 & 621.751 & 2.197 \\
\hline 13.182 & 8.788 & 8.788 & 61.516 \\
\hline 30.758 & 4.394 & 2.197 & 2.197 \\
\hline 461.37 & 2.197 & 215.306 & 4.394 \\
\hline 4.394 & 2.197 & 2001.47 & 19.773 \\
\hline 2.197 & 2.197 & 2.197 & 628.342 \\
\hline 4.394 & 2.197 & 599234 & 4.394 \\
\hline 1748.81 & 2.197 & 4.394 & 2.197 \\
\hline 153.79 & 2.197 & 17.576 & 17.576 \\
\hline 4.394 & 2.197 & 188.942 & 6.591 \\
\hline 248.261 & 43.94 & 2.197 & 2.197 \\
\hline 2.197 & 17.576 & 123.032 & 2.197 \\
\hline 59.319 & 6.591 & 4.394 & 109.85 \\
\hline 2.197 & 1168.8 & 4.394 & 2.197 \\
\hline 158.184 & 217.503 & 2.197 & 114.244 \\
\hline 2.197 & 15.379 & 2.197 & 2.197 \\
\hline 4.394 & 101.062 & 2.197 & 4.394 \\
\hline 2.197 & 2.197 & 85.683 & 2.197 \\
\hline 8.788 & 6.591 & 151.593 & 2.197 \\
\hline 10.985 & 173.563 & 15.379 & 2.197 \\
\hline 13.182 & 6.591 & 35.152 & 138.411 \\
\hline 30.758 & 72.501 & 2.197 & 2.197 \\
\hline 10.985 & 4.394 & 8.788 & 2.197 \\
\hline 15.379 & 8.788 & 413.036 & 303.186 \\
\hline 2.197 & 50.531 & 19.773 & 2.197 \\
\hline 32.955 & 2.197 & 689.858 & 6.591 \\
\hline 2.197 & 4.394 & 1221.53 & 2.197 \\
\hline 2.197 & 8.788 & 4.394 & 2.197 \\
\hline 4.394 & 2.197 & 4.394 & 15.379 \\
\hline 2.197 & 2.197 & 2.197 & 2.197 \\
\hline 4.394 & 26.364 & 85.683 & 4.394 \\
\hline 10.985 & 21.97 & 12116.5 & 4.394 \\
\hline 2.197 & 8.788 & 162.578 & 32.955 \\
\hline 79.092 & 2.197 & 5068.48 & 164.775 \\
\hline 68.107 & 2.197 & 15.379 & 6.591 \\
\hline 127.426 & 2.197 & 116.441 & 8.788 \\
\hline 4.394 & 2.197 & 2.197 & 46.137 \\
\hline 10.985 & 182.351 & 46.137 & 54.925 \\
\hline 2.197 & 2.197 & 112.047 & 43.94 \\
\hline 2.197 & 4.394 & 4.394 & 2.197 \\
\hline 6.591 & 2.197 & 8.788 & 2.197 \\
\hline 13.182 & 2.197 & 8.788 & 4.394 \\
\hline 17.576 & 2.197 & 5611.14 & 19.773 \\
\hline 98.865 & 2.197 & 2.197 & 2.197 \\
\hline 2.197 & 8.788 & 41.743 & 59.319 \\
\hline 4.394 & 2.197 & 24.167 & 252.655 \\
\hline 239.473 & 2.197 & 731.601 & 2.197 \\
\hline 237.276 & 2.197 & 24.167 & 123.032 \\
\hline 208.715 & 2.197 & 790.92 & 2.197 \\
\hline
\end{tabular}




\begin{tabular}{|c|c|c|c|}
\hline 4.394 & 6.591 & 15.379 & 6.591 \\
\hline 41.743 & 4.394 & 2.197 & 2.197 \\
\hline 21.97 & 6.591 & 2.197 & 2.197 \\
\hline 8.788 & 6.591 & 131.82 & 2.197 \\
\hline 1986.09 & 2.197 & 8.788 & 2.197 \\
\hline 2.197 & 26.364 & 65.91 & 4.394 \\
\hline 182.351 & 6.591 & 1173.2 & 6.591 \\
\hline 2.197 & 6.591 & 18951.3 & 2.197 \\
\hline 2.197 & 197.73 & 103.259 & 4.394 \\
\hline 4.394 & 2.197 & 2.197 & 4.394 \\
\hline 2.197 & 43.94 & 15.379 & 4.394 \\
\hline 15.379 & 26.364 & 173.563 & 138.411 \\
\hline 17.576 & 48.334 & 15.379 & 2.197 \\
\hline 2.197 & 210.912 & 450.385 & 2.197 \\
\hline 2.197 & 4.394 & 41.743 & 2.197 \\
\hline 50.531 & 2.197 & 465.764 & 8.788 \\
\hline 65.91 & 19.773 & 347.126 & 4932.26 \\
\hline 4.394 & 2.197 & 938.119 & 8.788 \\
\hline 65.91 & 2.197 & 2.197 & 4.394 \\
\hline 24.167 & 4.394 & 263.64 & 204.321 \\
\hline 8.788 & 4.394 & 2.197 & 2.197 \\
\hline 2.197 & 79.092 & 4.394 & 2.197 \\
\hline 74.698 & 19.773 & 4.394 & 2.197 \\
\hline 28.561 & 8.788 & 518.492 & 2.197 \\
\hline 28.561 & 2.197 & 15.379 & 2.197 \\
\hline 2.197 & 2.197 & 2.197 & 2.197 \\
\hline 4.394 & 2.197 & 4.394 & 2.197 \\
\hline 145.002 & 8.788 & 224.094 & 4.394 \\
\hline 13.182 & 6.591 & 369.096 & 2.197 \\
\hline 2.197 & 2.197 & 2.197 & 2.197 \\
\hline 2.197 & 4.394 & 1291.84 & 37.349 \\
\hline 103.259 & 2.197 & 87.88 & 8.788 \\
\hline 32.955 & 35.152 & 2.197 & 2.197 \\
\hline 4.394 & 35.152 & 184.548 & 2.197 \\
\hline 24.167 & 10.985 & 199.927 & 2.197 \\
\hline 509.704 & 4.394 & 26.364 & 2.197 \\
\hline 4.394 & 2.197 & 8.788 & 2.197 \\
\hline 37.349 & 2.197 & 15.379 & 24.167 \\
\hline 4.394 & 1151.23 & 37.349 & 347.126 \\
\hline 2.197 & 254.852 & 4.394 & 2.197 \\
\hline 180.154 & 4.394 & 2.197 & 2.197 \\
\hline 169.169 & 6.591 & 2.197 & 2.197 \\
\hline 4.394 & 2.197 & 48.334 & 37.349 \\
\hline 134.017 & 2.197 & 2.197 & 6.591 \\
\hline 6.591 & 2.197 & 59.319 & 2.197 \\
\hline 6.591 & 81.289 & 4.394 & 8.788 \\
\hline 8.788 & 138.411 & 2.197 & 6.591 \\
\hline 2.197 & 8.788 & 2.197 & 2.197 \\
\hline 8.788 & 2.197 & 2.197 & 2.197 \\
\hline 2.197 & 4.394 & 4.394 & 8.788 \\
\hline
\end{tabular}




\begin{tabular}{|c|c|c|c|}
\hline 35.152 & 2.197 & 10.985 & 2.197 \\
\hline 10.985 & 2.197 & 57.122 & 2.197 \\
\hline 6.591 & 6.591 & 2.197 & 2.197 \\
\hline 90.077 & 2.197 & 4.394 & 2.197 \\
\hline 4.394 & 2.197 & 2.197 & 2.197 \\
\hline 4.394 & 13.182 & 4.394 & 2.197 \\
\hline 10.985 & 2.197 & 160.381 & 4.394 \\
\hline 8.788 & 2.197 & 4.394 & 13.182 \\
\hline 2.197 & 2.197 & 2.197 & 2.197 \\
\hline 13.182 & 4.394 & 2.197 & 32.955 \\
\hline 21.97 & 87.88 & 6.591 & 6.591 \\
\hline 28.561 & 2.197 & 32.955 & 4.394 \\
\hline 4.394 & 2.197 & 118.638 & 4.394 \\
\hline 6.591 & 15.379 & 2.197 & 26.364 \\
\hline 2.197 & 15.379 & 8.788 & 2.197 \\
\hline 2.197 & 863.421 & 2.197 & 2.197 \\
\hline 4.394 & 2.197 & 2.197 & 10.985 \\
\hline 2.197 & 2.197 & 37.349 & 30.758 \\
\hline 2.197 & 4.394 & 103.259 & 1065.54 \\
\hline 2.197 & 87.88 & 19.773 & 6.591 \\
\hline 6.591 & 15.379 & 153.79 & 41.743 \\
\hline 2.197 & 15.379 & 61.516 & 4.394 \\
\hline 8.788 & 15.379 & 169.169 & 2.197 \\
\hline 10.985 & 2.197 & 4.394 & 2.197 \\
\hline 109.85 & 2.197 & 76.895 & 2.197 \\
\hline 8.788 & 2.197 & 13.182 & 2.197 \\
\hline 2.197 & 2.197 & 4.394 & 4.394 \\
\hline 26.364 & 186.745 & 2.197 & 2.197 \\
\hline 4.394 & 10.985 & 4.394 & 52.728 \\
\hline 790.92 & 2.197 & 4.394 & 54.925 \\
\hline 4.394 & 2.197 & 786.526 & 2.197 \\
\hline 2.197 & 4.394 & 10.985 & 8.788 \\
\hline 13.182 & 4.394 & 164.775 & 2.197 \\
\hline 8.788 & 94.471 & 6.591 & 977.665 \\
\hline 4.394 & 2.197 & 2.197 & 2.197 \\
\hline 19.773 & 2.197 & 6.591 & 2.197 \\
\hline 17.576 & 2.197 & 3170.27 & 2.197 \\
\hline 2.197 & 2.197 & 50.531 & 2.197 \\
\hline 2.197 & 4.394 & 4.394 & 2.197 \\
\hline 26.364 & 4.394 & 2.197 & 2.197 \\
\hline 2.197 & 2.197 & 2.197 & 10.985 \\
\hline 2.197 & 6.591 & 81.289 & 2.197 \\
\hline 2.197 & 6.591 & 2.197 & 2.197 \\
\hline 2.197 & 10.985 & 2.197 & 4.394 \\
\hline 2.197 & 57.122 & 2.197 & 2.197 \\
\hline 4.394 & 43.94 & 92.274 & 4.394 \\
\hline 2.197 & 24.167 & 2.197 & 2.197 \\
\hline 13.182 & 17.576 & 46.137 & 2.197 \\
\hline 4.394 & 2.197 & 472.355 & 4.394 \\
\hline 4.394 & 8.788 & 4.394 & 2.197 \\
\hline
\end{tabular}




\begin{tabular}{|c|c|c|c|}
\hline 4.394 & 90.077 & 2.197 & 2.197 \\
\hline 188.942 & 138.411 & 2.197 & 4.394 \\
\hline 2.197 & 4.394 & 2.197 & 2.197 \\
\hline 2.197 & 65.91 & 101.062 & 17.576 \\
\hline 10.985 & 217.503 & 2.197 & 4.394 \\
\hline 2.197 & 2.197 & 8.788 & 2.197 \\
\hline 2.197 & 2.197 & 48.334 & 4.394 \\
\hline 72.501 & 2.197 & 2.197 & 6.591 \\
\hline 32.955 & 6.591 & 2.197 & 46.137 \\
\hline 2.197 & 2.197 & 2.197 & 2.197 \\
\hline 2.197 & 114.244 & 2.197 & 2.197 \\
\hline 35.152 & 4.394 & 2.197 & 2.197 \\
\hline 13.182 & 4.394 & 35.152 & 6.591 \\
\hline 2.197 & 2.197 & 2.197 & 2.197 \\
\hline 15.379 & 2.197 & 19.773 & 685.464 \\
\hline 30.758 & 4.394 & 149.396 & 2.197 \\
\hline 571.22 & 2.197 & 2.197 & 2.197 \\
\hline 6.591 & 2.197 & 4.394 & 2.197 \\
\hline 2.197 & 4.394 & 28.561 & 6.591 \\
\hline 109.85 & 6.591 & 2.197 & 2.197 \\
\hline 6.591 & 8.788 & 4.394 & 632.736 \\
\hline 257.049 & 2.197 & 24.167 & 2.197 \\
\hline 46.137 & 2.197 & 2.197 & 2.197 \\
\hline 15.379 & 2.197 & 4.394 & 2.197 \\
\hline 6.591 & 4.394 & 2.197 & 2.197 \\
\hline 4.394 & 2.197 & 8.788 & 8.788 \\
\hline 21.97 & 2.197 & 24.167 & 63.713 \\
\hline 13.182 & 2.197 & 2.197 & 83.486 \\
\hline 10.985 & 8.788 & 6.591 & 2.197 \\
\hline 2.197 & 4.394 & 2.197 & 2.197 \\
\hline 26.364 & 17.576 & 2.197 & 2.197 \\
\hline 105.456 & 4.394 & 4.394 & 10.985 \\
\hline 2.197 & 6.591 & 21.97 & 112.047 \\
\hline 2.197 & 52.728 & 2.197 & 15.379 \\
\hline 2.197 & 2.197 & 2.197 & 2.197 \\
\hline 2.197 & 2.197 & 4.394 & 2.197 \\
\hline 15.379 & 2.197 & 15.379 & 2.197 \\
\hline 2.197 & 2.197 & 2.197 & 2.197 \\
\hline 19.773 & 39.546 & 2.197 & 4.394 \\
\hline 90.077 & 2.197 & 2.197 & 26.364 \\
\hline 2.197 & 2.197 & 2.197 & 2.197 \\
\hline 2.197 & 43.94 & 8.788 & 2.197 \\
\hline 2.197 & 90.077 & 320.762 & 28.561 \\
\hline 15.379 & 2.197 & 2.197 & 8.788 \\
\hline 37.349 & 2.197 & 4.394 & 68.107 \\
\hline 2.197 & 13.182 & 19.773 & 2.197 \\
\hline 2.197 & 19.773 & 2.197 & 8.788 \\
\hline 19.773 & 81.289 & 2.197 & 2.197 \\
\hline 237.276 & 2.197 & 21.97 & 10.985 \\
\hline 13.182 & 8.788 & 2.197 & 17.576 \\
\hline
\end{tabular}




\begin{tabular}{|c|c|c|c|}
\hline 162.578 & 2.197 & 2.197 & 2.197 \\
\hline 21.97 & 2.197 & 8.788 & 35.152 \\
\hline 15.379 & 4.394 & 2.197 & 4.394 \\
\hline 4.394 & 10.985 & 3128.53 & 2.197 \\
\hline 74.698 & 4.394 & 2.197 & 2.197 \\
\hline 17.576 & 19.773 & 17.576 & 21.97 \\
\hline 15.379 & 15.379 & 2.197 & 35.152 \\
\hline 2.197 & 2.197 & 6.591 & 6.591 \\
\hline 4.394 & 2.197 & 2.197 & 8.788 \\
\hline 2.197 & 2.197 & 1410.47 & 2.197 \\
\hline 28.561 & 2.197 & 6.591 & 21.97 \\
\hline 48.334 & 4.394 & 61.516 & 112.047 \\
\hline 2.197 & 2.197 & 4.394 & 46.137 \\
\hline 105.456 & 4.394 & 17.576 & 70.304 \\
\hline 32.955 & 6.591 & 145.002 & 13.182 \\
\hline 13.182 & 2.197 & 6.591 & 68.107 \\
\hline 37.349 & 17.576 & 2.197 & 13.182 \\
\hline 21.97 & 21.97 & 158.184 & 4.394 \\
\hline 2.197 & 92.274 & 2.197 & 2.197 \\
\hline 32.955 & 4.394 & 8.788 & 17.576 \\
\hline 13.182 & 2.197 & 15.379 & 2.197 \\
\hline 79.092 & 2.197 & 2.197 & 8.788 \\
\hline 19.773 & 30.758 & 4.394 & 6.591 \\
\hline 1133.65 & 2.197 & 2.197 & 2.197 \\
\hline 6.591 & 2.197 & 2.197 & 8.788 \\
\hline 57.122 & 2.197 & 676.676 & 2.197 \\
\hline 171.366 & 2.197 & 369.096 & 2.197 \\
\hline 116.441 & 2.197 & 2.197 & 2.197 \\
\hline 59.319 & 2.197 & 2.197 & 4.394 \\
\hline 4.394 & 2.197 & 2.197 & 8.788 \\
\hline 6.591 & 35.152 & 2.197 & 94.471 \\
\hline 17.576 & 2.197 & 4.394 & 2.197 \\
\hline 13.182 & 6.591 & 2346.4 & 101.062 \\
\hline 2.197 & 39.546 & 10.985 & 2.197 \\
\hline 485.537 & 2.197 & 10.985 & 6.591 \\
\hline 15.379 & 8.788 & 2.197 & 639.327 \\
\hline 90.077 & 2.197 & 2.197 & 2.197 \\
\hline 10.985 & 2.197 & 259.246 & 2.197 \\
\hline 10.985 & 52.728 & 2.197 & 4.394 \\
\hline 2.197 & 2.197 & 2.197 & 26.364 \\
\hline 68.107 & 2.197 & 2.197 & 2.197 \\
\hline 2.197 & 138.411 & 21.97 & 8.788 \\
\hline 2.197 & 2.197 & 2.197 & 2.197 \\
\hline 2.197 & 17.576 & 2.197 & 2.197 \\
\hline 2.197 & 35.152 & 2.197 & 8.788 \\
\hline 4.394 & 8.788 & 6.591 & 8.788 \\
\hline 123.032 & 2.197 & 2.197 & 2.197 \\
\hline 41.743 & 68.107 & 2.197 & 6.591 \\
\hline 2.197 & 2.197 & 6.591 & 10.985 \\
\hline 15.379 & 6.591 & 8.788 & 43.94 \\
\hline
\end{tabular}




\begin{tabular}{|c|c|c|c|}
\hline 24.167 & 2.197 & 2.197 & 98.865 \\
\hline 2.197 & 4.394 & 2.197 & 19.773 \\
\hline 101.062 & 2.197 & 4.394 & 542.659 \\
\hline 4.394 & 2.197 & 325.156 & 8.788 \\
\hline 10.985 & 10.985 & 2.197 & 2.197 \\
\hline 21.97 & 2.197 & 6.591 & 8.788 \\
\hline 2.197 & 21.97 & 2.197 & 2.197 \\
\hline 13.182 & 8.788 & 4.394 & 13.182 \\
\hline 6.591 & 2.197 & 2.197 & 2.197 \\
\hline 2.197 & 2.197 & 2.197 & 2.197 \\
\hline 2.197 & 4.394 & 8.788 & 8.788 \\
\hline 2.197 & 193.336 & 8.788 & 6.591 \\
\hline 2.197 & 10.985 & 6.591 & 28.561 \\
\hline 125.229 & 4.394 & 10.985 & 2.197 \\
\hline 17.576 & 4.394 & 28.561 & 17.576 \\
\hline 2.197 & 2.197 & 6.591 & 2.197 \\
\hline 90.077 & 2.197 & 681.07 & 2.197 \\
\hline 43.94 & 2.197 & 4.394 & 19.773 \\
\hline 6.591 & 2.197 & 6.591 & 6.591 \\
\hline 8.788 & 6.591 & 2.197 & 24.167 \\
\hline 28.561 & 17.576 & 65.91 & 15.379 \\
\hline 2.197 & 6.591 & 2.197 & 6.591 \\
\hline 2.197 & 6.591 & 26.364 & 4.394 \\
\hline 2.197 & 2.197 & 2.197 & 232.882 \\
\hline 2.197 & 8.788 & 8.788 & 76.895 \\
\hline 28.561 & 63.713 & 6.591 & 6.591 \\
\hline 32.955 & 6.591 & 6.591 & 188.942 \\
\hline 2.197 & 21.97 & 1902.6 & 2.197 \\
\hline 28.561 & 6.591 & 2.197 & 2.197 \\
\hline 2.197 & 2.197 & 2.197 & 2.197 \\
\hline 2.197 & 37.349 & 2.197 & 2.197 \\
\hline 2.197 & 2.197 & 26.364 & 10.985 \\
\hline 2.197 & 2.197 & 4.394 & 2.197 \\
\hline 28.561 & 4.394 & 48.334 & 13.182 \\
\hline 2.197 & 19.773 & 4.394 & 2.197 \\
\hline 35.152 & 2.197 & 109.85 & 2.197 \\
\hline 43.94 & 2.197 & 70.304 & 2.197 \\
\hline 35.152 & 30.758 & 544.856 & 4.394 \\
\hline 2.197 & 8.788 & 35.152 & 13.182 \\
\hline 6.591 & 2.197 & 43.94 & 15.379 \\
\hline 2.197 & 59.319 & 15.379 & 2.197 \\
\hline 74.698 & 134.017 & 2.197 & 2.197 \\
\hline 6.591 & 30.758 & 4.394 & 30.758 \\
\hline 2.197 & 2.197 & 65.91 & 6.591 \\
\hline 2.197 & 13.182 & 6.591 & 2.197 \\
\hline 2.197 & 2.197 & 4.394 & 199.927 \\
\hline 8.788 & 28.561 & 2.197 & 4.394 \\
\hline 6.591 & 19.773 & 261.443 & 4.394 \\
\hline 125.229 & 17.576 & 43.94 & 81.289 \\
\hline 19.773 & 202.124 & 10.985 & 2.197 \\
\hline
\end{tabular}




\begin{tabular}{|c|c|c|c|}
\hline 13.182 & 2.197 & 2.197 & 8.788 \\
\hline 26.364 & 426.218 & 48.334 & 852.436 \\
\hline 17.576 & 10.985 & 408.642 & 2.197 \\
\hline 48.334 & 2.197 & 2.197 & 43.94 \\
\hline 21.97 & 4.394 & 2.197 & 2.197 \\
\hline 39.546 & 4.394 & 2.197 & 2.197 \\
\hline 215.306 & 54.925 & 2.197 & 2.197 \\
\hline 37.349 & 4.394 & 725.01 & 2.197 \\
\hline 4.394 & 164.775 & 2.197 & 166.972 \\
\hline 4.394 & 2.197 & 2.197 & 15.379 \\
\hline 2.197 & 46.137 & 2.197 & 17.576 \\
\hline 2.197 & 6.591 & 13.182 & 4.394 \\
\hline 2.197 & 197.73 & 2.197 & 2.197 \\
\hline 2.197 & 57.122 & 2.197 & 2.197 \\
\hline 2.197 & 4.394 & 2.197 & 797.511 \\
\hline 17.576 & 2.197 & 2.197 & 41.743 \\
\hline 2.197 & 10.985 & 2.197 & 30.758 \\
\hline 21.97 & 15.379 & 2.197 & 52.728 \\
\hline 4.394 & 2.197 & 2.197 & 2.197 \\
\hline 76.895 & 2.197 & 2.197 & 2.197 \\
\hline 8.788 & 2.197 & 2.197 & 2.197 \\
\hline 2.197 & 2.197 & 2.197 & 28.561 \\
\hline 2.197 & 15.379 & 2.197 & 35.152 \\
\hline 2.197 & 15.379 & 2.197 & 8.788 \\
\hline 17.576 & 4.394 & 2.197 & 4.394 \\
\hline 30.758 & 2.197 & 114.244 & 17.576 \\
\hline 4.394 & 4.394 & 8.788 & 4.394 \\
\hline 13.182 & 13.182 & 2.197 & 4.394 \\
\hline 6.591 & 2.197 & 6.591 & 13.182 \\
\hline 8.788 & 4.394 & 10.985 & 2.197 \\
\hline 2.197 & 2.197 & 2.197 & 4.394 \\
\hline 597.584 & 4.394 & 2.197 & 39.546 \\
\hline 8.788 & 2.197 & 2.197 & 4.394 \\
\hline 8.788 & 2.197 & 2.197 & 2.197 \\
\hline 6.591 & 81.289 & 2.197 & 4.394 \\
\hline 2.197 & 6.591 & 94.471 & 2.197 \\
\hline 2.197 & 114.244 & 2.197 & 2.197 \\
\hline 30.758 & 4.394 & 24.167 & 2.197 \\
\hline 2.197 & 2.197 & 6.591 & 2.197 \\
\hline 2.197 & 2.197 & 2.197 & 1366.53 \\
\hline 6.591 & 2.197 & 193.336 & 771.147 \\
\hline 131.82 & 8.788 & 79.092 & 8.788 \\
\hline 2.197 & 2.197 & 30.758 & 85.683 \\
\hline 307.58 & 15.379 & 2.197 & 158.184 \\
\hline 6.591 & 8.788 & 10.985 & 2.197 \\
\hline 4.394 & 4.394 & 180.154 & 2.197 \\
\hline 2.197 & 2.197 & 2.197 & 2.197 \\
\hline 17.576 & 72.501 & 2.197 & 2.197 \\
\hline 8911.03 & 6.591 & 180.154 & 2.197 \\
\hline 6.591 & 4.394 & 1067.74 & 2.197 \\
\hline
\end{tabular}




\begin{tabular}{|c|c|c|c|}
\hline 2.197 & 2.197 & 4.394 & 2021.24 \\
\hline 2.197 & 41.743 & 19.773 & 21.97 \\
\hline 2.197 & 6.591 & 8.788 & 2.197 \\
\hline 2.197 & 2.197 & 2.197 & 6.591 \\
\hline 2.197 & 6.591 & 8.788 & 426.218 \\
\hline 2.197 & 2.197 & 6.591 & 2.197 \\
\hline 6.591 & 13.182 & 13.182 & 2.197 \\
\hline 2.197 & 2.197 & 474.552 & 2.197 \\
\hline 2.197 & 2.197 & 6.591 & 35.152 \\
\hline 17.576 & 2.197 & 169.169 & 30.758 \\
\hline 2.197 & 4.394 & 2.197 & 54.925 \\
\hline 2.197 & 2.197 & 246.064 & 72.501 \\
\hline 125.229 & 2.197 & 41.743 & 4.394 \\
\hline 13.182 & 4.394 & 105.456 & 6.591 \\
\hline 13.182 & 659.1 & 10.985 & 2.197 \\
\hline 32.955 & 24.167 & 131.82 & 39.546 \\
\hline 2.197 & 103.259 & 4.394 & 54.925 \\
\hline 4.394 & 2.197 & 32.955 & 2.197 \\
\hline 8.788 & 8.788 & 2.197 & 2.197 \\
\hline 2.197 & 79.092 & 21.97 & 4.394 \\
\hline 2.197 & 10.985 & 2.197 & 2.197 \\
\hline 2.197 & 6.591 & 2.197 & 2.197 \\
\hline 2.197 & 17.576 & 8.788 & 2.197 \\
\hline 8.788 & 50.531 & 2.197 & 4.394 \\
\hline 4.394 & 112.047 & 2.197 & 4.394 \\
\hline 28.561 & 2.197 & 2.197 & 2.197 \\
\hline 2.197 & 8.788 & 2.197 & 2.197 \\
\hline 48.334 & 2.197 & 2.197 & 123.032 \\
\hline 999.635 & 2.197 & 2.197 & 10.985 \\
\hline 8.788 & 13.182 & 2.197 & 54.925 \\
\hline 4.394 & 4.394 & 2.197 & 65.91 \\
\hline 2.197 & 10.985 & 2.197 & 6.591 \\
\hline 21.97 & 4.394 & 2.197 & 2.197 \\
\hline 19.773 & 105.456 & 2.197 & 127.426 \\
\hline 4.394 & 4.394 & 2.197 & 103.259 \\
\hline 26.364 & 2.197 & 4.394 & 10.985 \\
\hline 2.197 & 15.379 & 4.394 & 19.773 \\
\hline 2.197 & 2.197 & 4.394 & 2.197 \\
\hline 10.985 & 4.394 & 450.385 & 2.197 \\
\hline 4.394 & 2.197 & 127.426 & 384.475 \\
\hline 2.197 & 2.197 & 8.788 & 4.394 \\
\hline 2.197 & 4.394 & 8.788 & 228.488 \\
\hline 6.591 & 2.197 & 4.394 & 39.546 \\
\hline 26.364 & 4.394 & 2.197 & 61.516 \\
\hline 21.97 & 2.197 & 4.394 & 8.788 \\
\hline 4.394 & 24.167 & 2.197 & 2.197 \\
\hline 8.788 & 13.182 & 4.394 & 32.955 \\
\hline 8.788 & 15.379 & 28.561 & 443.794 \\
\hline 54.925 & 2.197 & 8.788 & 2.197 \\
\hline 101.062 & 2.197 & 2.197 & 195.533 \\
\hline
\end{tabular}




\begin{tabular}{|c|c|c|c|}
\hline 24.167 & 2.197 & 2.197 & 2.197 \\
\hline 35.152 & 90.077 & 6.591 & 4.394 \\
\hline 2.197 & 8.788 & 8.788 & 2.197 \\
\hline 10.985 & 46.137 & 2.197 & 6.591 \\
\hline 4.394 & 2.197 & 4.394 & 98.865 \\
\hline 13.182 & 6.591 & 4.394 & 2.197 \\
\hline 2.197 & 4.394 & 8.788 & 2.197 \\
\hline 2.197 & 2.197 & 4.394 & 2.197 \\
\hline 15.379 & 2.197 & 4.394 & 2.197 \\
\hline 2.197 & 43.94 & 2.197 & 2.197 \\
\hline 21.97 & 2.197 & 2.197 & 17.576 \\
\hline 61.516 & 2.197 & 2.197 & 410.839 \\
\hline 2.197 & 6.591 & 70.304 & 74.698 \\
\hline 87.88 & 4.394 & 52.728 & 54.925 \\
\hline 8.788 & 24.167 & 2.197 & 32.955 \\
\hline 2.197 & 2.197 & 39.546 & 17.576 \\
\hline 57.122 & 2.197 & 2.197 & 2.197 \\
\hline 2.197 & 17.576 & 4.394 & 2.197 \\
\hline 2.197 & 94.471 & 4.394 & 2.197 \\
\hline 61.516 & 10.985 & 6.591 & 2.197 \\
\hline 155.987 & 2.197 & 2.197 & 4.394 \\
\hline 13.182 & 2.197 & 2.197 & 6.591 \\
\hline 140.608 & 15.379 & 2.197 & 2.197 \\
\hline 4.394 & 4.394 & 2.197 & 8.788 \\
\hline 112.047 & 10.985 & 2.197 & 4.394 \\
\hline 70.304 & 10.985 & 2.197 & 4.394 \\
\hline 2.197 & 30.758 & 2.197 & 204.321 \\
\hline 2.197 & 4.394 & 2.197 & 2.197 \\
\hline 48.334 & 2.197 & 4.394 & 94.471 \\
\hline 2.197 & 2.197 & 52.728 & 2.197 \\
\hline 2.197 & 72.501 & 2.197 & 4.394 \\
\hline 2.197 & 17.576 & 10.985 & 2.197 \\
\hline 2.197 & 2.197 & 70.304 & 2.197 \\
\hline 2.197 & 2.197 & 21.97 & 15.379 \\
\hline 48.334 & 87.88 & 50.531 & 6.591 \\
\hline 65.91 & 35.152 & 2.197 & 101.062 \\
\hline 4.394 & 10.985 & 2.197 & 6.591 \\
\hline 2.197 & 2.197 & 70.304 & 2.197 \\
\hline 2.197 & 2.197 & 6.591 & 2.197 \\
\hline 15.379 & 94.471 & 2.197 & 72.501 \\
\hline 6.591 & 17.576 & 2.197 & 6.591 \\
\hline 2.197 & 6.591 & 50.531 & 2.197 \\
\hline 2.197 & 2.197 & 8.788 & 37.349 \\
\hline 10.985 & 2.197 & 2.197 & 2.197 \\
\hline 2.197 & 101.062 & 8.788 & 2.197 \\
\hline 2.197 & 2.197 & 8.788 & 6.591 \\
\hline 2.197 & 2.197 & 2.197 & 10.985 \\
\hline 24.167 & 2.197 & 2.197 & 10.985 \\
\hline 24.167 & 76.895 & 2.197 & 2.197 \\
\hline 109.85 & 2.197 & 2.197 & 30.758 \\
\hline
\end{tabular}




\begin{tabular}{|c|c|c|c|}
\hline 4.394 & 2.197 & 13.182 & 957.892 \\
\hline 489.931 & 6.591 & 8.788 & 2.197 \\
\hline 4.394 & 2.197 & 4.394 & 2.197 \\
\hline 65.91 & 4.394 & 8.788 & 2.197 \\
\hline 2.197 & 6.591 & 10.985 & 2.197 \\
\hline 2.197 & 19.773 & 8.788 & 2.197 \\
\hline 26.364 & 2.197 & 19.773 & 2.197 \\
\hline 6.591 & 6.591 & 6.591 & 19.773 \\
\hline 30.758 & 26.364 & 57.122 & 358.111 \\
\hline 17.576 & 4.394 & 17.576 & 28.561 \\
\hline 226.291 & 2.197 & 2.197 & 2.197 \\
\hline 15.379 & 19.773 & 2.197 & 13.182 \\
\hline 4.394 & 2.197 & 2.197 & 19.773 \\
\hline 2.197 & 17.576 & 8.788 & 46.137 \\
\hline 83.486 & 4.394 & 2.197 & 2.197 \\
\hline 8.788 & 10.985 & 2.197 & 10.985 \\
\hline 2.197 & 2.197 & 2.197 & 4.394 \\
\hline 2.197 & 2.197 & 6.591 & 4.394 \\
\hline 10.985 & 2.197 & 2.197 & 2.197 \\
\hline 140.608 & 6.591 & 19.773 & 2.197 \\
\hline 191.139 & 2.197 & 2.197 & 19.773 \\
\hline 52.728 & 2.197 & 4.394 & 2.197 \\
\hline 2996.71 & 2.197 & 4.394 & 2.197 \\
\hline 6.591 & 2.197 & 4.394 & 4.394 \\
\hline 15.379 & 2.197 & 2.197 & 4.394 \\
\hline 4.394 & 2.197 & 2.197 & 39.546 \\
\hline 2.197 & 2.197 & 24.167 & 4310.51 \\
\hline 2.197 & 21.97 & 2.197 & 59.319 \\
\hline 21.97 & 2.197 & 17.576 & 13.182 \\
\hline 2.197 & 2.197 & 2.197 & 2.197 \\
\hline 2.197 & 2.197 & 2.197 & 2.197 \\
\hline 2.197 & 4.394 & 10.985 & 2.197 \\
\hline 2.197 & 2.197 & 2.197 & 39.546 \\
\hline 2.197 & 2.197 & 19.773 & 158.184 \\
\hline 2.197 & 17.576 & 2.197 & 2.197 \\
\hline 2.197 & 474.552 & 8.788 & 2.197 \\
\hline 2.197 & 43.94 & 15.379 & 76.895 \\
\hline 4.394 & 2.197 & 6.591 & 2.197 \\
\hline 10.985 & 2.197 & 279.019 & 4.394 \\
\hline 2.197 & 17.576 & 2.197 & 72.501 \\
\hline 28.561 & 21.97 & 147.199 & 2.197 \\
\hline 4.394 & 4.394 & 1643.36 & 617.357 \\
\hline 10.985 & 96.668 & 2.197 & 2.197 \\
\hline 8.788 & 17.576 & 123.032 & 13.182 \\
\hline 4.394 & 8.788 & 2.197 & 2.197 \\
\hline 15.379 & 158.184 & 4.394 & 19.773 \\
\hline 19.773 & 68.107 & 8.788 & 2.197 \\
\hline 21.97 & 2.197 & 2.197 & 8.788 \\
\hline 24.167 & 8.788 & 2.197 & 213.109 \\
\hline 28.561 & 2.197 & 2.197 & 6.591 \\
\hline
\end{tabular}




\begin{tabular}{|c|c|c|c|}
\hline 2.197 & 8.788 & 2.197 & 2.197 \\
\hline 2.197 & 2.197 & 2.197 & 15.379 \\
\hline 6.591 & 2.197 & 2.197 & 2.197 \\
\hline 26.364 & 4.394 & 2.197 & 76.895 \\
\hline 2.197 & 4.394 & 2.197 & 2.197 \\
\hline 8.788 & 2.197 & 2.197 & 24.167 \\
\hline 2.197 & 6.591 & 13.182 & 6.591 \\
\hline 21.97 & 6.591 & 2.197 & 8.788 \\
\hline 2.197 & 2.197 & 123.032 & 151.593 \\
\hline 4.394 & 4.394 & 13.182 & 43.94 \\
\hline 2.197 & 2.197 & 43.94 & 2.197 \\
\hline 2.197 & 2.197 & 186.745 & 10.985 \\
\hline 2.197 & 6.591 & 52.728 & 2.197 \\
\hline 2.197 & 2.197 & 2.197 & 26.364 \\
\hline 2.197 & 2.197 & 4.394 & 19.773 \\
\hline 10.985 & 2.197 & 2.197 & 79.092 \\
\hline 2.197 & 2.197 & 13.182 & 4.394 \\
\hline 2.197 & 4.394 & 2.197 & 57.122 \\
\hline 2.197 & 2.197 & 6.591 & 4.394 \\
\hline 43.94 & 10.985 & 2.197 & 2.197 \\
\hline 2.197 & 4.394 & 2.197 & 10.985 \\
\hline 4.394 & 28.561 & 2.197 & 2.197 \\
\hline 15.379 & 54.925 & 13.182 & 13.182 \\
\hline 39.546 & 4.394 & 4.394 & 2.197 \\
\hline 24.167 & 15.379 & 19.773 & 32.955 \\
\hline 6.591 & 15.379 & 6.591 & 96.668 \\
\hline 57.122 & 2.197 & 4.394 & 19.773 \\
\hline 4.394 & 8.788 & 689.858 & 32.955 \\
\hline 2.197 & 2.197 & 2.197 & 8.788 \\
\hline 57.122 & 2.197 & 2.197 & 2.197 \\
\hline 4.394 & 74.698 & 373.49 & 237.276 \\
\hline 2.197 & 184.548 & 153.79 & 13.182 \\
\hline 48.334 & 2.197 & 13.182 & 2.197 \\
\hline 15.379 & 1645.55 & 2.197 & 2.197 \\
\hline 28.561 & 2.197 & 2.197 & 4.394 \\
\hline 61.516 & 6.591 & 2.197 & 2.197 \\
\hline 13.182 & 6.591 & 13.182 & 2.197 \\
\hline 4.394 & 4.394 & 8.788 & 10.985 \\
\hline 2.197 & 6.591 & 268.034 & 2.197 \\
\hline 8.788 & 4.394 & 37.349 & 2.197 \\
\hline 6.591 & 17.576 & 26.364 & 19.773 \\
\hline 2.197 & 15.379 & 70.304 & 112.047 \\
\hline 2.197 & 4.394 & 2.197 & 6.591 \\
\hline 2.197 & 13.182 & 19.773 & 4.394 \\
\hline 6.591 & 13.182 & 10.985 & 142.805 \\
\hline 85.683 & 30.758 & 2.197 & 2.197 \\
\hline 439.4 & 2.197 & 2.197 & 28.561 \\
\hline 2.197 & 2.197 & 2.197 & 41.743 \\
\hline 2.197 & 21.97 & 10.985 & 239.473 \\
\hline 21.97 & 19.773 & 694.252 & 26.364 \\
\hline
\end{tabular}




\begin{tabular}{|c|c|c|c|}
\hline 4.394 & 2.197 & 4.394 & 4.394 \\
\hline 2.197 & 4.394 & 2.197 & 2.197 \\
\hline 52.728 & 4.394 & 2.197 & 59.319 \\
\hline 2.197 & 13.182 & 4.394 & 21.97 \\
\hline 2.197 & 2.197 & 2.197 & 2.197 \\
\hline 6.591 & 2.197 & 2.197 & 2.197 \\
\hline 4.394 & 2.197 & 2.197 & 2.197 \\
\hline 639.327 & 2.197 & 2.197 & 15.379 \\
\hline 6.591 & 2.197 & 2.197 & 2.197 \\
\hline 48.334 & 2.197 & 2.197 & 15.379 \\
\hline 232.882 & 2.197 & 2.197 & 2.197 \\
\hline 32.955 & 4.394 & 4.394 & 2.197 \\
\hline 35.152 & 26.364 & 13.182 & 4.394 \\
\hline 125.229 & 2.197 & 2.197 & 61.516 \\
\hline 13.182 & 274.625 & 8.788 & 443.794 \\
\hline 2.197 & 4.394 & 35.152 & 2.197 \\
\hline 2.197 & 4.394 & 2.197 & 70.304 \\
\hline 15.379 & 6.591 & 2.197 & 13.182 \\
\hline 6.591 & 8.788 & 2.197 & 2.197 \\
\hline 87.88 & 2.197 & 2.197 & 6.591 \\
\hline 8.788 & 52.728 & 13.182 & 2.197 \\
\hline 4.394 & 81.289 & 2.197 & 344.929 \\
\hline 4.394 & 2.197 & 2.197 & 85.683 \\
\hline 8.788 & 2.197 & 2.197 & 2.197 \\
\hline 2.197 & 2.197 & 2.197 & 4.394 \\
\hline 2880.27 & 2.197 & 8.788 & 2.197 \\
\hline 2.197 & 2.197 & 2.197 & 2.197 \\
\hline 2.197 & 4.394 & 2.197 & 2.197 \\
\hline 4.394 & 2.197 & 48.334 & 70.304 \\
\hline 6.591 & 4.394 & 4.394 & 83.486 \\
\hline 303.186 & 15.379 & 2.197 & 191.139 \\
\hline 57.122 & 2.197 & 2.197 & 2.197 \\
\hline 2.197 & 4.394 & 6.591 & 10.985 \\
\hline 2.197 & 8.788 & 2.197 & 4.394 \\
\hline 4.394 & 2.197 & 2.197 & 4.394 \\
\hline 6.591 & 13.182 & 2.197 & 4.394 \\
\hline 2.197 & 4.394 & 4.394 & 439.4 \\
\hline 2.197 & 2.197 & 2.197 & 4.394 \\
\hline 4.394 & 2.197 & 98.865 & 8.788 \\
\hline 4.394 & 21.97 & 4.394 & 2.197 \\
\hline 2.197 & 8.788 & 65.91 & 4.394 \\
\hline 4.394 & 4.394 & 8.788 & 4.394 \\
\hline 2.197 & 6.591 & 2.197 & 2.197 \\
\hline 21.97 & 32.955 & 2.197 & 8.788 \\
\hline 4.394 & 155.987 & 2.197 & 13.182 \\
\hline 2.197 & 173.563 & 8.788 & 10.985 \\
\hline 8.788 & 151.593 & 2.197 & 6.591 \\
\hline 2.197 & 65.91 & 46.137 & 2.197 \\
\hline 13.182 & 134.017 & 6.591 & 15.379 \\
\hline 2.197 & 30.758 & 2.197 & 4.394 \\
\hline
\end{tabular}




\begin{tabular}{|c|c|c|c|}
\hline 151.593 & 2.197 & 6.591 & 17.576 \\
\hline 2.197 & 4.394 & 15.379 & 2.197 \\
\hline 8.788 & 2.197 & 298.792 & 2.197 \\
\hline 4.394 & 26.364 & 2.197 & 2.197 \\
\hline 2.197 & 406.445 & 26.364 & 39.546 \\
\hline 4.394 & 2.197 & 166.972 & 15.379 \\
\hline 2.197 & 41.743 & 4.394 & 35.152 \\
\hline 24.167 & 2.197 & 4.394 & 307.58 \\
\hline 6.591 & 2.197 & 4.394 & 61.516 \\
\hline 61.516 & 4.394 & 4.394 & 35.152 \\
\hline 26.364 & 17.576 & 28.561 & 6.591 \\
\hline 8.788 & 4.394 & 15.379 & 2.197 \\
\hline 2.197 & 2.197 & 54.925 & 10.985 \\
\hline 2.197 & 24.167 & 6.591 & 41.743 \\
\hline 19.773 & 2.197 & 81.289 & 2.197 \\
\hline 2.197 & 2.197 & 2.197 & 2.197 \\
\hline 10.985 & 2.197 & 4.394 & 6.591 \\
\hline 2.197 & 2.197 & 2.197 & 6.591 \\
\hline 2.197 & 1098.5 & 101.062 & 2.197 \\
\hline 2.197 & 4.394 & 2.197 & 21.97 \\
\hline 2.197 & 65.91 & 1614.79 & 19.773 \\
\hline 46.137 & 2.197 & 79.092 & 2.197 \\
\hline 30.758 & 63.713 & 41.743 & 83.486 \\
\hline 2.197 & 4.394 & 8.788 & 52.728 \\
\hline 8.788 & 2.197 & 2.197 & 28.561 \\
\hline 10.985 & 2.197 & 2.197 & 2.197 \\
\hline 4.394 & 6.591 & 158.184 & 2.197 \\
\hline 32.955 & 4.394 & 10.985 & 13.182 \\
\hline 8.788 & 2.197 & 8.788 & 10.985 \\
\hline 2.197 & 83.486 & 2.197 & 46.137 \\
\hline 384.475 & 4.394 & 4350.06 & 4.394 \\
\hline 2.197 & 6.591 & 8.788 & 2.197 \\
\hline 2.197 & 4.394 & 2.197 & 4.394 \\
\hline 2.197 & 2.197 & 2.197 & 4.394 \\
\hline 17.576 & 8.788 & 2.197 & 4.394 \\
\hline 2.197 & 13.182 & 48.334 & 17.576 \\
\hline 2.197 & 54.925 & 123.032 & 30.758 \\
\hline 4.394 & 4.394 & 2.197 & 4.394 \\
\hline 2.197 & 4.394 & 2.197 & 41.743 \\
\hline 2.197 & 2.197 & 2.197 & 21.97 \\
\hline 4.394 & 15.379 & 2.197 & 136.214 \\
\hline 197.73 & 2.197 & 8.788 & 70.304 \\
\hline 35.152 & 2.197 & 2.197 & 162.578 \\
\hline 10.985 & 10.985 & 18015.4 & 2.197 \\
\hline 48.334 & 8.788 & 8.788 & 6.591 \\
\hline 320.762 & 10.985 & 19.773 & 13.182 \\
\hline 127.426 & 10.985 & 17.576 & 6.591 \\
\hline 10.985 & 136.214 & 2.197 & 24.167 \\
\hline 8.788 & 2.197 & 96.668 & 4.394 \\
\hline 2.197 & 21.97 & 2.197 & 8.788 \\
\hline
\end{tabular}




\begin{tabular}{|c|c|c|c|}
\hline 2.197 & 8.788 & 4.394 & 2.197 \\
\hline 2.197 & 74.698 & 4.394 & 2.197 \\
\hline 8.788 & 2.197 & 2.197 & 2.197 \\
\hline 421.824 & 2.197 & 2.197 & 35.152 \\
\hline 26.364 & 2.197 & 52.728 & 8.788 \\
\hline 8.788 & 2.197 & 2.197 & 85.683 \\
\hline 6.591 & 4.394 & 254.852 & 4.394 \\
\hline 2.197 & 2.197 & 353.717 & 246.064 \\
\hline 17.576 & 15.379 & 92.274 & 19.773 \\
\hline 6.591 & 4.394 & 2.197 & 30.758 \\
\hline 2.197 & 2.197 & 2.197 & 2.197 \\
\hline 26.364 & 2.197 & 2.197 & 2.197 \\
\hline 13.182 & 4.394 & 2.197 & 2.197 \\
\hline 4.394 & 2.197 & 2.197 & 41.743 \\
\hline 2.197 & 2.197 & 4.394 & 2.197 \\
\hline 2.197 & 4.394 & 6.591 & 2.197 \\
\hline 2.197 & 4.394 & 2.197 & 8.788 \\
\hline 6.591 & 2.197 & 6.591 & 83.486 \\
\hline 263.64 & 30.758 & 21.97 & 4.394 \\
\hline 2.197 & 6.591 & 6.591 & 2.197 \\
\hline 2.197 & 281.216 & 6.591 & 2.197 \\
\hline 2.197 & 6.591 & 6.591 & 10.985 \\
\hline 6.591 & 4.394 & 6.591 & 6.591 \\
\hline 949.104 & 87.88 & 4.394 & 6.591 \\
\hline 13.182 & 2.197 & 2.197 & 15.379 \\
\hline 107.653 & 2.197 & 2.197 & 2.197 \\
\hline 28.561 & 2.197 & 2.197 & 39.546 \\
\hline 2.197 & 2.197 & 17.576 & 129.623 \\
\hline 2.197 & 6.591 & 10.985 & 43.94 \\
\hline 2.197 & 13.182 & 4.394 & 2.197 \\
\hline 32.955 & 24.167 & 6.591 & 68.107 \\
\hline 17.576 & 2.197 & 10.985 & 4.394 \\
\hline 2.197 & 2.197 & 30.758 & 54.925 \\
\hline 13.182 & 2.197 & 134.017 & 4.394 \\
\hline 10.985 & 41.743 & 8.788 & 2.197 \\
\hline 24.167 & 2.197 & 8.788 & 30.758 \\
\hline 2.197 & 50.531 & 13.182 & 4.394 \\
\hline 2.197 & 2.197 & 6.591 & 4.394 \\
\hline 2.197 & 2.197 & 24.167 & 6.591 \\
\hline 2.197 & 10.985 & 57.122 & 8.788 \\
\hline 10.985 & 8.788 & 4.394 & 2.197 \\
\hline 2.197 & 6.591 & 94.471 & 2.197 \\
\hline 8.788 & 2.197 & 2.197 & 35.152 \\
\hline 131.82 & 107.653 & 8.788 & 232.882 \\
\hline 4.394 & 4.394 & 4.394 & 10.985 \\
\hline 4.394 & 41.743 & 92.274 & 175.76 \\
\hline 6.591 & 70.304 & 2.197 & 2.197 \\
\hline 4.394 & 26.364 & 10.985 & 2.197 \\
\hline 4.394 & 52.728 & 61.516 & 393.263 \\
\hline 2.197 & 2.197 & 2.197 & 384.475 \\
\hline
\end{tabular}




\begin{tabular}{|c|c|c|c|}
\hline 2.197 & 15.379 & 15.379 & 4.394 \\
\hline 1309.41 & 8.788 & 2.197 & 2.197 \\
\hline 180.154 & 2.197 & 2.197 & 41.743 \\
\hline 8.788 & 4.394 & 10.985 & 17.576 \\
\hline 6.591 & 2.197 & 24.167 & 8.788 \\
\hline 2.197 & 17.576 & 2.197 & 2.197 \\
\hline 6.591 & 6.591 & 2.197 & 2.197 \\
\hline 4.394 & 4.394 & 2.197 & 63.713 \\
\hline 2.197 & 2.197 & 2.197 & 6.591 \\
\hline 2.197 & 2.197 & 61.516 & 63.713 \\
\hline 26.364 & 4.394 & 2.197 & 6.591 \\
\hline 2.197 & 6.591 & 2.197 & 46.137 \\
\hline 10.985 & 2.197 & 1417.06 & 24.167 \\
\hline 314.171 & 28.561 & 6.591 & 2.197 \\
\hline 8.788 & 2.197 & 2.197 & 96.668 \\
\hline 15.379 & 2.197 & 2.197 & 2.197 \\
\hline 6.591 & 2.197 & 8.788 & 2.197 \\
\hline 2.197 & 2.197 & 10.985 & 4.394 \\
\hline 2.197 & 4.394 & 30.758 & 2.197 \\
\hline 2.197 & 437.203 & 4.394 & 2.197 \\
\hline 4.394 & 2.197 & 4.394 & 13.182 \\
\hline 8.788 & 15.379 & 2.197 & 6.591 \\
\hline 32.955 & 2.197 & 65.91 & 8.788 \\
\hline 13.182 & 2.197 & 8.788 & 441.597 \\
\hline 41.743 & 35.152 & 15.379 & 8.788 \\
\hline 19.773 & 43.94 & 235.079 & 35.152 \\
\hline 50.531 & 2.197 & 2.197 & 2.197 \\
\hline 883.194 & 21.97 & 8.788 & 2.197 \\
\hline 39.546 & 13.182 & 2.197 & 83.486 \\
\hline 1107.29 & 2.197 & 10.985 & 17.576 \\
\hline 4.394 & 2.197 & 147.199 & 2.197 \\
\hline 2476.02 & 4.394 & 4.394 & 4.394 \\
\hline 26.364 & 10.985 & 2.197 & 8.788 \\
\hline 2.197 & 2.197 & 30.758 & 17.576 \\
\hline 2.197 & 2.197 & 2.197 & 15.379 \\
\hline 2.197 & 26.364 & 2.197 & 2.197 \\
\hline 4.394 & 26.364 & 2.197 & 6.591 \\
\hline 4.394 & 4.394 & 6.591 & 4.394 \\
\hline 8.788 & 2.197 & 8.788 & 10.985 \\
\hline 2.197 & 2.197 & 17.576 & 388.869 \\
\hline 4.394 & 2.197 & 10.985 & 4.394 \\
\hline 54.925 & 138.411 & 28.561 & 4.394 \\
\hline 24.167 & 15.379 & 160.381 & 6.591 \\
\hline 4.394 & 2.197 & 2.197 & 79.092 \\
\hline 41.743 & 2.197 & 46.137 & 4.394 \\
\hline 2.197 & 4.394 & 37.349 & 19.773 \\
\hline 4.394 & 2.197 & 2.197 & 28.561 \\
\hline 2.197 & 13.182 & 52.728 & 8.788 \\
\hline 2.197 & 13.182 & 85.683 & 2.197 \\
\hline 15.379 & 6.591 & 6.591 & 2.197 \\
\hline
\end{tabular}




\begin{tabular}{|c|c|c|c|}
\hline 79.092 & 2.197 & 4.394 & 2.197 \\
\hline 283.413 & 4.394 & 2.197 & 8.788 \\
\hline 2.197 & 2.197 & 2.197 & 2.197 \\
\hline 2.197 & 28.561 & 2.197 & 39.546 \\
\hline 2.197 & 2.197 & 8.788 & 2.197 \\
\hline 4.394 & 8.788 & 39.546 & 83.486 \\
\hline 2.197 & 6.591 & 32.955 & 2.197 \\
\hline 63.713 & 17.576 & 32.955 & 2.197 \\
\hline 41.743 & 2.197 & 2.197 & 17.576 \\
\hline 54.925 & 35.152 & 4.394 & 13.182 \\
\hline 2.197 & 138.411 & 74.698 & 4.394 \\
\hline 2.197 & 2.197 & 28.561 & 6.591 \\
\hline 2.197 & 30.758 & 8.788 & 32.955 \\
\hline 10.985 & 41.743 & 43.94 & 2.197 \\
\hline 4.394 & 21.97 & 2.197 & 74.698 \\
\hline 2.197 & 6.591 & 28.561 & 17.576 \\
\hline 2.197 & 21.97 & 8.788 & 79.092 \\
\hline 2.197 & 6.591 & 2.197 & 2.197 \\
\hline 30.758 & 17.576 & 8.788 & 6.591 \\
\hline 13.182 & 17.576 & 2.197 & 2.197 \\
\hline 2.197 & 24.167 & 90.077 & 21.97 \\
\hline 37.349 & 2.197 & 37.349 & 21.97 \\
\hline 17.576 & 2.197 & 6.591 & 2.197 \\
\hline 6.591 & 21.97 & 8.788 & 10.985 \\
\hline 518.492 & 2.197 & 2.197 & 6.591 \\
\hline 2.197 & 2.197 & 2.197 & 6.591 \\
\hline 2.197 & 2.197 & 2.197 & 6.591 \\
\hline 4.394 & 8.788 & 4.394 & 4.394 \\
\hline 52.728 & 2.197 & 2.197 & 4.394 \\
\hline 4.394 & 2.197 & 4.394 & 2.197 \\
\hline 2.197 & 6.591 & 48.334 & 2.197 \\
\hline 2.197 & 4.394 & 43.94 & 2.197 \\
\hline 2.197 & 13.182 & 61.516 & 2.197 \\
\hline 202.124 & 15.379 & 4.394 & 41.743 \\
\hline 2.197 & 4.394 & 4.394 & 87.88 \\
\hline 2.197 & 4.394 & 6.591 & 2.197 \\
\hline 2.197 & 2.197 & 4.394 & 2.197 \\
\hline 2.197 & 24.167 & 48.334 & 2.197 \\
\hline 17.576 & 2.197 & 10.985 & 162.578 \\
\hline 4.394 & 2.197 & 8.788 & 28.561 \\
\hline 4.394 & 48.334 & 2.197 & 24.167 \\
\hline 17.576 & 2.197 & 2.197 & 2.197 \\
\hline 2.197 & 4.394 & 6.591 & 4.394 \\
\hline 6.591 & 4.394 & 101.062 & 6.591 \\
\hline 2.197 & 13.182 & 2.197 & 2.197 \\
\hline 2.197 & 37.349 & 81.289 & 2.197 \\
\hline 76.895 & 2.197 & 6.591 & 54.925 \\
\hline 2.197 & 8.788 & 13.182 & 17.576 \\
\hline 2.197 & 43.94 & 6.591 & 4.394 \\
\hline 10.985 & 65.91 & 4.394 & 2.197 \\
\hline
\end{tabular}




\begin{tabular}{|c|c|c|c|}
\hline 6.591 & 2.197 & 8.788 & 2.197 \\
\hline 8.788 & 2.197 & 2.197 & 2.197 \\
\hline 2.197 & 70.304 & 4.394 & 2.197 \\
\hline 4.394 & 2.197 & 2.197 & 2.197 \\
\hline 6.591 & 15.379 & 4.394 & 4.394 \\
\hline 2.197 & 8.788 & 4.394 & 10.985 \\
\hline 2.197 & 2.197 & 39.546 & 6.591 \\
\hline 874.406 & 32.955 & 65.91 & 6.591 \\
\hline 2.197 & 4.394 & 15.379 & 19.773 \\
\hline 26.364 & 2.197 & 15.379 & 2.197 \\
\hline 43.94 & 2.197 & 2.197 & 10.985 \\
\hline 2.197 & 4.394 & 2.197 & 155.987 \\
\hline 2.197 & 6.591 & 188.942 & 2.197 \\
\hline 2.197 & 21.97 & 8.788 & 6.591 \\
\hline 4.394 & 8.788 & 2.197 & 4.394 \\
\hline 8.788 & 8.788 & 2.197 & 21.97 \\
\hline 2.197 & 116.441 & 28.561 & 2.197 \\
\hline 2.197 & 19.773 & 6.591 & 8.788 \\
\hline 28.561 & 2.197 & 2.197 & 112.047 \\
\hline 28.561 & 30.758 & 2.197 & 281.216 \\
\hline 2.197 & 2.197 & 2.197 & 8.788 \\
\hline 2.197 & 13.182 & 13.182 & 2.197 \\
\hline 2.197 & 15.379 & 4.394 & 2.197 \\
\hline 28.561 & 2.197 & 2.197 & 6.591 \\
\hline 8.788 & 2.197 & 4.394 & 2.197 \\
\hline 2.197 & 8.788 & 4.394 & 2.197 \\
\hline 151.593 & 52.728 & 2.197 & 4.394 \\
\hline 2.197 & 10.985 & 19.773 & 4.394 \\
\hline 10.985 & 2.197 & 35.152 & 41.743 \\
\hline 285.61 & 2.197 & 98.865 & 4.394 \\
\hline 8.788 & 4.394 & 24.167 & 8.788 \\
\hline 2.197 & 52.728 & 17.576 & 92.274 \\
\hline 43.94 & 2.197 & 13.182 & 8.788 \\
\hline 13.182 & 2.197 & 4.394 & 30.758 \\
\hline 4.394 & 8.788 & 17.576 & 2.197 \\
\hline 2.197 & 2.197 & 2.197 & 2.197 \\
\hline 2.197 & 4.394 & 2.197 & 4.394 \\
\hline 105.456 & 28.561 & 6.591 & 120.835 \\
\hline 215.306 & 2.197 & 208.715 & 2.197 \\
\hline 1847.68 & 2.197 & 30.758 & 81.289 \\
\hline 32.955 & 2.197 & 13.182 & 39.546 \\
\hline 4.394 & 134.017 & 24.167 & 8.788 \\
\hline 4.394 & 46.137 & 17.576 & 8.788 \\
\hline 10.985 & 8.788 & 10.985 & 6.591 \\
\hline 2.197 & 2.197 & 2.197 & 2.197 \\
\hline 24.167 & 28.561 & 2.197 & 74.698 \\
\hline 2.197 & 4.394 & 4.394 & 57.122 \\
\hline 2.197 & 2.197 & 17.576 & 2.197 \\
\hline 2.197 & 4.394 & 6.591 & 24.167 \\
\hline 2.197 & 4.394 & 153.79 & 2.197 \\
\hline
\end{tabular}




\begin{tabular}{|c|c|c|c|}
\hline 6.591 & 101.062 & 2.197 & 2.197 \\
\hline 8.788 & 2.197 & 4.394 & 6.591 \\
\hline 6.591 & 2.197 & 10.985 & 2.197 \\
\hline 2.197 & 2.197 & 4.394 & 10.985 \\
\hline 2.197 & 2.197 & 2.197 & 46.137 \\
\hline 2.197 & 725.01 & 2.197 & 4.394 \\
\hline 6.591 & 2.197 & 2.197 & 2.197 \\
\hline 32.955 & 2.197 & 6.591 & 43.94 \\
\hline 2.197 & 2.197 & 2.197 & 4.394 \\
\hline 57.122 & 4.394 & 2.197 & 4.394 \\
\hline 2.197 & 6.591 & 2.197 & 10.985 \\
\hline 26.364 & 2.197 & 2.197 & 2.197 \\
\hline 10.985 & 2.197 & 32.955 & 13.182 \\
\hline 24.167 & 13.182 & 74.698 & 48.334 \\
\hline 2.197 & 26.364 & 4.394 & 4.394 \\
\hline 24002.2 & 129.623 & 2.197 & 10.985 \\
\hline 162.578 & 10.985 & 562.432 & 2.197 \\
\hline 177.957 & 4.394 & 6.591 & 28.561 \\
\hline 2.197 & 263.64 & 2.197 & 59.319 \\
\hline 15.379 & 140.608 & 4.394 & 2.197 \\
\hline 2.197 & 17.576 & 2.197 & 2.197 \\
\hline 6.591 & 6.591 & 2.197 & 8.788 \\
\hline 54.925 & 371.293 & 6.591 & 129.623 \\
\hline 4.394 & 2.197 & 4.394 & 21.97 \\
\hline 19.773 & 6.591 & 21.97 & 2.197 \\
\hline 2.197 & 2.197 & 46.137 & 19.773 \\
\hline 26.364 & 2.197 & 4.394 & 397.657 \\
\hline 6.591 & 6.591 & 2.197 & 13.182 \\
\hline 2.197 & 2.197 & 2.197 & 13.182 \\
\hline 520.689 & 2.197 & 2.197 & 2.197 \\
\hline 2.197 & 26.364 & 8.788 & 2.197 \\
\hline 2.197 & 2.197 & 19.773 & 151.593 \\
\hline 17.576 & 19.773 & 2.197 & 2.197 \\
\hline 522.886 & 6.591 & 4.394 & 92.274 \\
\hline 2.197 & 13.182 & 2.197 & 2.197 \\
\hline 2.197 & 2.197 & 10.985 & 83.486 \\
\hline 21.97 & 2.197 & 21.97 & 35.152 \\
\hline 4.394 & 4.394 & 2.197 & 2.197 \\
\hline 2.197 & 382.278 & 2.197 & 2.197 \\
\hline 10.985 & 28.561 & 2.197 & 2.197 \\
\hline 59.319 & 6.591 & 2.197 & 6.591 \\
\hline 4.394 & 2.197 & 2.197 & 68.107 \\
\hline 2.197 & 26.364 & 2.197 & 43.94 \\
\hline 2.197 & 8.788 & 8.788 & 237.276 \\
\hline 2.197 & 2.197 & 252.655 & 13.182 \\
\hline 2.197 & 52.728 & 37.349 & 35.152 \\
\hline 404.248 & 26.364 & 10.985 & 4.394 \\
\hline 2.197 & 46.137 & 59.319 & 2.197 \\
\hline 6.591 & 50.531 & 6.591 & 10.985 \\
\hline 74.698 & 6.591 & 79.092 & 76.895 \\
\hline
\end{tabular}




\begin{tabular}{|c|c|c|c|}
\hline 28.561 & 114.244 & 24.167 & 30.758 \\
\hline 173.563 & 21.97 & 2.197 & 15.379 \\
\hline 54.925 & 4.394 & 21.97 & 96.668 \\
\hline 331.747 & 700.843 & 2.197 & 76.895 \\
\hline 13.182 & 70.304 & 2.197 & 2.197 \\
\hline 2.197 & 21.97 & 48.334 & 4.394 \\
\hline 4.394 & 2.197 & 2.197 & 30.758 \\
\hline 6.591 & 41.743 & 72.501 & 37.349 \\
\hline 2.197 & 32.955 & 54.925 & 2.197 \\
\hline 4.394 & 4.394 & 15.379 & 94.471 \\
\hline 2.197 & 2.197 & 10.985 & 6.591 \\
\hline 28.561 & 6.591 & 8.788 & 32.955 \\
\hline 6.591 & 2.197 & 8.788 & 2.197 \\
\hline 4.394 & 2.197 & 1487.37 & 2.197 \\
\hline 2.197 & 2.197 & 232.882 & 6.591 \\
\hline 8.788 & 4.394 & 46.137 & 2.197 \\
\hline 2.197 & 50.531 & 2.197 & 2.197 \\
\hline 52.728 & 6.591 & 4.394 & 4.394 \\
\hline 50.531 & 2.197 & 4.394 & 4.394 \\
\hline 98.865 & 26.364 & 52.728 & 2.197 \\
\hline 2.197 & 2.197 & 6.591 & 4.394 \\
\hline 2.197 & 10.985 & 4.394 & 17.576 \\
\hline 8.788 & 6.591 & 2.197 & 2.197 \\
\hline 15.379 & 10.985 & 2.197 & 2.197 \\
\hline 52.728 & 188.942 & 2.197 & 4.394 \\
\hline 10.985 & 10.985 & 13.182 & 2.197 \\
\hline 2.197 & 43.94 & 2.197 & 2.197 \\
\hline 6.591 & 32.955 & 24.167 & 4.394 \\
\hline 2.197 & 2.197 & 2.197 & 2.197 \\
\hline 8.788 & 2.197 & 2.197 & 114.244 \\
\hline 2.197 & 2.197 & 15.379 & 8.788 \\
\hline 2.197 & 4.394 & 2.197 & 59.319 \\
\hline 17.576 & 28.561 & 920.543 & 355.914 \\
\hline 2.197 & 2.197 & 2.197 & 2.197 \\
\hline 6.591 & 2.197 & 2.197 & 2.197 \\
\hline 2.197 & 160.381 & 2.197 & 4.394 \\
\hline 248.261 & 15.379 & 8.788 & 21.97 \\
\hline 2.197 & 2.197 & 450.385 & 2.197 \\
\hline 98.865 & 48.334 & 2.197 & 6.591 \\
\hline 4.394 & 4.394 & 2.197 & 30.758 \\
\hline 21.97 & 2.197 & 2.197 & 13.182 \\
\hline 30.758 & 4.394 & 2.197 & 2.197 \\
\hline 8.788 & 305.383 & 2.197 & 8.788 \\
\hline 817.284 & 48.334 & 13.182 & 2.197 \\
\hline 4.394 & 26.364 & 109.85 & 2.197 \\
\hline 2.197 & 4.394 & 1157.82 & 24.167 \\
\hline 19.773 & 4.394 & 90.077 & 2.197 \\
\hline 13.182 & 2.197 & 15.379 & 2.197 \\
\hline 32.955 & 52.728 & 2.197 & 2.197 \\
\hline 8.788 & 2.197 & 6.591 & 4.394 \\
\hline
\end{tabular}




\begin{tabular}{|c|c|c|c|}
\hline 19.773 & 2.197 & 8.788 & 4.394 \\
\hline 2.197 & 2.197 & 6.591 & 2.197 \\
\hline 2.197 & 2.197 & 2.197 & 2.197 \\
\hline 2.197 & 2.197 & 2.197 & 10.985 \\
\hline 24.167 & 2.197 & 2.197 & 2.197 \\
\hline 28.561 & 2.197 & 2.197 & 2.197 \\
\hline 13.182 & 2.197 & 2.197 & 2.197 \\
\hline 4.394 & 54.925 & 305.383 & 2.197 \\
\hline 13.182 & 48.334 & 13.182 & 8.788 \\
\hline 87.88 & 26.364 & 2.197 & 2.197 \\
\hline 32.955 & 2.197 & 13.182 & 32.955 \\
\hline 2.197 & 2.197 & 2.197 & 6.591 \\
\hline 13.182 & 68.107 & 213.109 & 32.955 \\
\hline 21.97 & 459.173 & 81.289 & 2.197 \\
\hline 13.182 & 131.82 & 4.394 & 2.197 \\
\hline 4.394 & 2.197 & 2.197 & 2.197 \\
\hline 6.591 & 48.334 & 8.788 & 2.197 \\
\hline 2.197 & 8.788 & 2.197 & 8.788 \\
\hline 6.591 & 2.197 & 2.197 & 2.197 \\
\hline 355.914 & 4.394 & 292.201 & 4.394 \\
\hline 830.466 & 2.197 & 195.533 & 4.394 \\
\hline 6.591 & 4.394 & 125.229 & 13.182 \\
\hline 6.591 & 2.197 & 402.051 & 17.576 \\
\hline 6.591 & 35.152 & 6.591 & 4.394 \\
\hline 4.394 & 13.182 & 140.608 & 10.985 \\
\hline 15.379 & 10.985 & 333.944 & 2.197 \\
\hline 4.394 & 4.394 & 4.394 & 13.182 \\
\hline 15.379 & 2.197 & 8.788 & 96.668 \\
\hline 2.197 & 2.197 & 41.743 & 2.197 \\
\hline 2.197 & 188.942 & 2.197 & 6.591 \\
\hline 4.394 & 21.97 & 2.197 & 2.197 \\
\hline 2.197 & 2.197 & 94.471 & 17.576 \\
\hline 17.576 & 2.197 & 707.434 & 39.546 \\
\hline 4.394 & 4.394 & 2.197 & 6.591 \\
\hline 6.591 & 63.713 & 6.591 & 4.394 \\
\hline 28.561 & 50.531 & 43.94 & 4.394 \\
\hline 4.394 & 28.561 & 15.379 & 2.197 \\
\hline 4.394 & 39.546 & 4.394 & 4.394 \\
\hline 4.394 & 43.94 & 2.197 & 2.197 \\
\hline 39.546 & 2.197 & 15.379 & 10.985 \\
\hline 2.197 & 2.197 & 4.394 & 57.122 \\
\hline 4.394 & 10.985 & 2.197 & 6.591 \\
\hline 17.576 & 2.197 & 2.197 & 2.197 \\
\hline 10.985 & 4.394 & 21.97 & 24.167 \\
\hline 26.364 & 50.531 & 2.197 & 28.561 \\
\hline 2.197 & 13.182 & 2.197 & 96.668 \\
\hline 6.591 & 2.197 & 8.788 & 173.563 \\
\hline 59.319 & 21.97 & 140.608 & 8.788 \\
\hline 103.259 & 87.88 & 116.441 & 24.167 \\
\hline 2.197 & 215.306 & 413.036 & 8.788 \\
\hline
\end{tabular}




\begin{tabular}{|c|c|c|c|}
\hline 2.197 & 6.591 & 2.197 & 15.379 \\
\hline 13.182 & 2.197 & 4.394 & 8.788 \\
\hline 81.289 & 32.955 & 30.758 & 10.985 \\
\hline 6.591 & 1805.93 & 2.197 & 6.591 \\
\hline 8.788 & 2.197 & 8.788 & 4.394 \\
\hline 10.985 & 4.394 & 52.728 & 4.394 \\
\hline 19.773 & 2.197 & 2.197 & 118.638 \\
\hline 2.197 & 2.197 & 13.182 & 2.197 \\
\hline 10.985 & 2.197 & 2.197 & 6.591 \\
\hline 26.364 & 4.394 & 2.197 & 8.788 \\
\hline 4.394 & 2.197 & 228.488 & 2.197 \\
\hline 6.591 & 2.197 & 2.197 & 6.591 \\
\hline 4.394 & 15.379 & 41.743 & 65.91 \\
\hline 43.94 & 15.379 & 4.394 & 41.743 \\
\hline 246.064 & 2.197 & 105.456 & 402.051 \\
\hline 13.182 & 28.561 & 6322.97 & 41.743 \\
\hline 8.788 & 65.91 & 6.591 & 10.985 \\
\hline 30.758 & 57.122 & 2.197 & 250.458 \\
\hline 26.364 & 17.576 & 4.394 & 13.182 \\
\hline 15.379 & 4.394 & 2.197 & 2.197 \\
\hline 4.394 & 6.591 & 2.197 & 63.713 \\
\hline 6.591 & 85.683 & 2.197 & 39.546 \\
\hline 4.394 & 2.197 & 81.289 & 2.197 \\
\hline 8.788 & 21.97 & 50.531 & 2.197 \\
\hline 4.394 & 2.197 & 21.97 & 2.197 \\
\hline 17.576 & 2.197 & 32.955 & 2.197 \\
\hline 2.197 & 17.576 & 287.807 & 19.773 \\
\hline 61.516 & 101.062 & 15.379 & 21.97 \\
\hline 2.197 & 28.561 & 127.426 & 8.788 \\
\hline 2.197 & 2.197 & 26.364 & 4.394 \\
\hline 46.137 & 2.197 & 17.576 & 2.197 \\
\hline 59.319 & 10.985 & 10.985 & 57.122 \\
\hline 24.167 & 2.197 & 483.34 & 8.788 \\
\hline 2.197 & 6.591 & 8.788 & 6.591 \\
\hline 19.773 & 4.394 & 39.546 & 4.394 \\
\hline 65.91 & 2.197 & 4.394 & 6.591 \\
\hline 2.197 & 2.197 & 2.197 & 2.197 \\
\hline 4.394 & 4.394 & 68.107 & 6.591 \\
\hline 48.334 & 4.394 & 2.197 & 4.394 \\
\hline 2.197 & 15.379 & 76.895 & 6.591 \\
\hline 2.197 & 6.591 & 32.955 & 2.197 \\
\hline 28.561 & 8.788 & 2.197 & 2.197 \\
\hline 26.364 & 2.197 & 2.197 & 2.197 \\
\hline 98.865 & 6.591 & 2.197 & 2.197 \\
\hline 6.591 & 13.182 & 2.197 & 15.379 \\
\hline 4.394 & 13.182 & 4.394 & 4.394 \\
\hline 2.197 & 63.713 & 2.197 & 70.304 \\
\hline 2.197 & 6.591 & 4.394 & 2.197 \\
\hline 140.608 & 50.531 & 13.182 & 4.394 \\
\hline 358.111 & 72.501 & 2.197 & 4.394 \\
\hline
\end{tabular}




\begin{tabular}{|c|c|c|c|}
\hline 2.197 & 10.985 & 6.591 & 2.197 \\
\hline 15.379 & 21.97 & 4.394 & 54.925 \\
\hline 4.394 & 8.788 & 2.197 & 2.197 \\
\hline 37.349 & 2.197 & 2.197 & 127.426 \\
\hline 366.899 & 13.182 & 101.062 & 10.985 \\
\hline 4.394 & 8.788 & 2.197 & 10.985 \\
\hline 2.197 & 6.591 & 2.197 & 21.97 \\
\hline 2.197 & 4.394 & 2.197 & 4.394 \\
\hline 17.576 & 4.394 & 1351.15 & 63.713 \\
\hline 250.458 & 2.197 & 4.394 & 287.807 \\
\hline 10.985 & 2.197 & 24.167 & 65.91 \\
\hline 6.591 & 2.197 & 4.394 & 2.197 \\
\hline 4.394 & 4.394 & 2.197 & 4.394 \\
\hline 30.758 & 6.591 & 21.97 & 4.394 \\
\hline 2.197 & 2.197 & 13.182 & 265523 \\
\hline 153.79 & 74.698 & 2.197 & 2.197 \\
\hline 2.197 & 59.319 & 2.197 & 46.137 \\
\hline 2.197 & 4.394 & 4.394 & 32.955 \\
\hline 6.591 & 13.182 & 2.197 & 8.788 \\
\hline 6.591 & 6.591 & 2.197 & 6.591 \\
\hline 10.985 & 6.591 & 83.486 & 41.743 \\
\hline 2.197 & 4.394 & 19.773 & 6.591 \\
\hline 21.97 & 70.304 & 397.657 & 4.394 \\
\hline 4.394 & 30.758 & 4.394 & 57.122 \\
\hline 2.197 & 4.394 & 2.197 & 257.049 \\
\hline 4.394 & 24.167 & 2.197 & 15.379 \\
\hline 24.167 & 10.985 & 2.197 & 8.788 \\
\hline 2.197 & 101.062 & 4.394 & 24.167 \\
\hline 2.197 & 4.394 & 2.197 & 2.197 \\
\hline 54.925 & 160.381 & 2.197 & 2.197 \\
\hline 30.758 & 13.182 & 50.531 & 4.394 \\
\hline 19.773 & 2.197 & 32.955 & 2.197 \\
\hline 90.077 & 2.197 & 32.955 & 2.197 \\
\hline 19.773 & 19.773 & 4.394 & 13.182 \\
\hline 19.773 & 2.197 & 43.94 & 8.788 \\
\hline 2.197 & 2.197 & 2.197 & 17.576 \\
\hline 4.394 & 2.197 & 2.197 & 37.349 \\
\hline 4.394 & 2.197 & 28.561 & 8.788 \\
\hline 6.591 & 79.092 & 4.394 & 2.197 \\
\hline 199.927 & 4.394 & 30.758 & 15.379 \\
\hline 123.032 & 6.591 & 4.394 & 8.788 \\
\hline 2.197 & 4.394 & 4.394 & 4.394 \\
\hline 8.788 & 2.197 & 4.394 & 6.591 \\
\hline 26.364 & 4.394 & 2.197 & 15.379 \\
\hline 2.197 & 109.85 & 30.758 & 4.394 \\
\hline 125.229 & 2.197 & 4.394 & 2.197 \\
\hline 10.985 & 30.758 & 37.349 & 21.97 \\
\hline 68.107 & 2.197 & 2.197 & 2.197 \\
\hline 946.907 & 2.197 & 2.197 & 13.182 \\
\hline 2.197 & 8.788 & 4.394 & 2.197 \\
\hline
\end{tabular}




\begin{tabular}{|c|c|c|c|}
\hline 48.334 & 17.576 & 2.197 & 4.394 \\
\hline 2.197 & 2.197 & 2.197 & 2.197 \\
\hline 79.092 & 2.197 & 6.591 & 8.788 \\
\hline 13.182 & 2.197 & 2.197 & 52.728 \\
\hline 4.394 & 2.197 & 2.197 & 19.773 \\
\hline 4.394 & 2.197 & 6.591 & 362.505 \\
\hline 13.182 & 4.394 & 2.197 & 6.591 \\
\hline 96.668 & 2.197 & 4.394 & 4.394 \\
\hline 2.197 & 4.394 & 65.91 & 4.394 \\
\hline 4.394 & 2.197 & 2.197 & 74.698 \\
\hline 2.197 & 2.197 & 35.152 & 26.364 \\
\hline 15.379 & 19.773 & 1285.24 & 10.985 \\
\hline 109.85 & 4.394 & 2.197 & 2.197 \\
\hline 2.197 & 2.197 & 10.985 & 39.546 \\
\hline 2.197 & 8.788 & 41.743 & 4.394 \\
\hline 21.97 & 2.197 & 2.197 & 4.394 \\
\hline 285.61 & 2.197 & 76.895 & 118.638 \\
\hline 2.197 & 2.197 & 4.394 & 2.197 \\
\hline 2.197 & 4.394 & 2.197 & 2.197 \\
\hline 8.788 & 2.197 & 8.788 & 2.197 \\
\hline 4.394 & 2.197 & 2.197 & 2.197 \\
\hline 2.197 & 2.197 & 4.394 & 13.182 \\
\hline 2.197 & 2.197 & 6.591 & 2.197 \\
\hline 2.197 & 15.379 & 2.197 & 2.197 \\
\hline 1467.6 & 2.197 & 2.197 & 2.197 \\
\hline 6.591 & 4.394 & 2.197 & 2.197 \\
\hline 54.925 & 2.197 & 4.394 & 4.394 \\
\hline 118.638 & 10.985 & 408.642 & 61.516 \\
\hline 10.985 & 2.197 & 6.591 & 2.197 \\
\hline 2.197 & 2.197 & 6.591 & 279.019 \\
\hline 2.197 & 2.197 & 6.591 & 6.591 \\
\hline 2.197 & 6.591 & 2.197 & 76.895 \\
\hline 2.197 & 96.668 & 2.197 & 2.197 \\
\hline 2.197 & 2.197 & 8.788 & 2.197 \\
\hline 59.319 & 92.274 & 4.394 & 4.394 \\
\hline 2.197 & 2.197 & 4.394 & 35.152 \\
\hline 2.197 & 8.788 & 8.788 & 8.788 \\
\hline 48.334 & 4.394 & 94.471 & 4.394 \\
\hline 250.458 & 2.197 & 6.591 & 2.197 \\
\hline 8.788 & 2.197 & 65.91 & 4.394 \\
\hline 4.394 & 4.394 & 377.884 & 6.591 \\
\hline 6.591 & 4.394 & 175.76 & 63.713 \\
\hline 8.788 & 70.304 & 19.773 & 6.591 \\
\hline 10.985 & 4.394 & 17.576 & 4.394 \\
\hline 105.456 & 17.576 & 15.379 & 39.546 \\
\hline 90.077 & 10.985 & 13.182 & 489.931 \\
\hline 2.197 & 2.197 & 127.426 & 8.788 \\
\hline 15.379 & 2.197 & 2.197 & 2.197 \\
\hline 10.985 & 2.197 & 6.591 & 6.591 \\
\hline 4.394 & 2.197 & 4.394 & 2.197 \\
\hline
\end{tabular}




\begin{tabular}{|c|c|c|c|}
\hline 4.394 & 2.197 & 2.197 & 4.394 \\
\hline 6.591 & 2.197 & 10.985 & 10.985 \\
\hline 13.182 & 4.394 & 2.197 & 15.379 \\
\hline 4.394 & 8.788 & 2.197 & 138.411 \\
\hline 39.546 & 2.197 & 8.788 & 2.197 \\
\hline 10.985 & 6.591 & 140.608 & 147.199 \\
\hline 10.985 & 26.364 & 6.591 & 2.197 \\
\hline 81.289 & 2.197 & 68.107 & 13.182 \\
\hline 39.546 & 72.501 & 2.197 & 10.985 \\
\hline 118.638 & 2.197 & 2.197 & 6.591 \\
\hline 17.576 & 2.197 & 2.197 & 2.197 \\
\hline 13.182 & 26.364 & 2.197 & 2.197 \\
\hline 2.197 & 8.788 & 4.394 & 46.137 \\
\hline 35.152 & 138.411 & 10.985 & 2.197 \\
\hline 59.319 & 17.576 & 2.197 & 4.394 \\
\hline 4.394 & 54.925 & 35.152 & 19.773 \\
\hline 4.394 & 13.182 & 123.032 & 2.197 \\
\hline 10.985 & 4.394 & 2.197 & 17.576 \\
\hline 2.197 & 50.531 & 10.985 & 19.773 \\
\hline 2.197 & 2.197 & 57.122 & 197.73 \\
\hline 2.197 & 96.668 & 4.394 & 19.773 \\
\hline 2.197 & 4.394 & 237.276 & 4.394 \\
\hline 2.197 & 4.394 & 26.364 & 4.394 \\
\hline 4.394 & 2.197 & 68.107 & 8.788 \\
\hline 4.394 & 4.394 & 8.788 & 169.169 \\
\hline 1403.88 & 19.773 & 59.319 & 24.167 \\
\hline 35.152 & 2.197 & 1535.7 & 2.197 \\
\hline 2.197 & 2.197 & 180.154 & 2.197 \\
\hline 10.985 & 2.197 & 15.379 & 2.197 \\
\hline 19.773 & 2.197 & 2.197 & 6.591 \\
\hline 2.197 & 2.197 & 2.197 & 2.197 \\
\hline 4.394 & 2.197 & 2.197 & 259.246 \\
\hline 13.182 & 2.197 & 4.394 & 26.364 \\
\hline 2.197 & 6.591 & 4.394 & 2.197 \\
\hline 10.985 & 388.869 & 2.197 & 92.274 \\
\hline 6.591 & 2.197 & 6.591 & 50.531 \\
\hline 4.394 & 2.197 & 4.394 & 2.197 \\
\hline 2.197 & 2.197 & 2.197 & 2.197 \\
\hline 2.197 & 2.197 & 35.152 & 10.985 \\
\hline 2.197 & 10.985 & 10.985 & 15.379 \\
\hline 2.197 & 26.364 & 2.197 & 10.985 \\
\hline 2.197 & 2.197 & 2.197 & 2.197 \\
\hline 6.591 & 4.394 & 4.394 & 4.394 \\
\hline 4.394 & 2.197 & 4.394 & 2.197 \\
\hline 204.321 & 4.394 & 4.394 & 2.197 \\
\hline 21.97 & 2.197 & 28.561 & 6.591 \\
\hline 2.197 & 24.167 & 2.197 & 19.773 \\
\hline 142.805 & 4.394 & 2.197 & 4.394 \\
\hline 109.85 & 2.197 & 4.394 & 2.197 \\
\hline 35.152 & 4.394 & 2.197 & 4.394 \\
\hline
\end{tabular}




\begin{tabular}{|c|c|c|c|}
\hline 52.728 & 13.182 & 2.197 & 4.394 \\
\hline 197.73 & 4.394 & 2.197 & 74.698 \\
\hline 6.591 & 17.576 & 164.775 & 13.182 \\
\hline 26.364 & 19.773 & 2.197 & 819.481 \\
\hline 2.197 & 10.985 & 79.092 & 6.591 \\
\hline 94.471 & 8.788 & 2.197 & 2.197 \\
\hline 19.773 & 2.197 & 8.788 & 4.394 \\
\hline 2.197 & 2.197 & 2.197 & 39.546 \\
\hline 35.152 & 2.197 & 2.197 & 2.197 \\
\hline 32.955 & 4.394 & 116.441 & 2.197 \\
\hline 4.394 & 2.197 & 63.713 & 4.394 \\
\hline 4.394 & 15.379 & 13.182 & 98.865 \\
\hline 13.182 & 316.368 & 4.394 & 4.394 \\
\hline 2.197 & 30.758 & 5108.02 & 1168.8 \\
\hline 2.197 & 171.366 & 2.197 & 19.773 \\
\hline 26.364 & 8.788 & 4.394 & 76.895 \\
\hline 6.591 & 6.591 & 2.197 & 2.197 \\
\hline 13.182 & 4.394 & 4.394 & 15.379 \\
\hline 2.197 & 6.591 & 4.394 & 2.197 \\
\hline 4.394 & 10.985 & 59.319 & 87.88 \\
\hline 2.197 & 2.197 & 2.197 & 8.788 \\
\hline 4.394 & 35.152 & 72.501 & 2.197 \\
\hline 2.197 & 131.82 & 4.394 & 8.788 \\
\hline 4.394 & 2.197 & 2.197 & 6.591 \\
\hline 1098.5 & 2.197 & 2.197 & 21.97 \\
\hline 4.394 & 4.394 & 68.107 & 24.167 \\
\hline 8.788 & 2.197 & 650.312 & 2.197 \\
\hline 2.197 & 2.197 & 54.925 & 4.394 \\
\hline 41.743 & 15.379 & 199.927 & 2.197 \\
\hline 10.985 & 15.379 & 6.591 & 2.197 \\
\hline 2.197 & 4.394 & 2.197 & 17.576 \\
\hline 2.197 & 421.824 & 1709.27 & 15.379 \\
\hline 2.197 & 2.197 & 2.197 & 13.182 \\
\hline 4.394 & 2.197 & 2.197 & 2.197 \\
\hline 2.197 & 2.197 & 2.197 & 2.197 \\
\hline 2.197 & 2.197 & 2.197 & 4.394 \\
\hline 4.394 & 8.788 & 35.152 & 17.576 \\
\hline 4.394 & 43.94 & 8.788 & 15.379 \\
\hline 2.197 & 2.197 & 204.321 & 10.985 \\
\hline 52.728 & 2.197 & 2.197 & 4.394 \\
\hline 10.985 & 4.394 & 10.985 & 129.623 \\
\hline 2.197 & 2.197 & 2.197 & 2.197 \\
\hline 2.197 & 2.197 & 536.068 & 41.743 \\
\hline 4.394 & 2.197 & 2.197 & 2.197 \\
\hline 2.197 & 4.394 & 4.394 & 2.197 \\
\hline 2.197 & 2.197 & 171.366 & 2.197 \\
\hline 2.197 & 4.394 & 2.197 & 32.955 \\
\hline 8.788 & 4.394 & 2.197 & 2.197 \\
\hline 2.197 & 4.394 & 2.197 & 2.197 \\
\hline 85.683 & 4.394 & 6.591 & 2.197 \\
\hline
\end{tabular}




\begin{tabular}{|c|c|c|c|}
\hline 48.334 & 2.197 & 21.97 & 4.394 \\
\hline 63.713 & 4.394 & 6.591 & 2.197 \\
\hline 2.197 & 4.394 & 2.197 & 68.107 \\
\hline 2.197 & 10.985 & 2.197 & 17.576 \\
\hline 210.912 & 2.197 & 6.591 & 2.197 \\
\hline 307.58 & 4.394 & 2.197 & 6.591 \\
\hline 127.426 & 2.197 & 107.653 & 2.197 \\
\hline 448.188 & 2.197 & 4.394 & 907.361 \\
\hline 2.197 & 430.612 & 2.197 & 4.394 \\
\hline 10.985 & 4.394 & 116.441 & 50.531 \\
\hline 6.591 & 6.591 & 4.394 & 17.576 \\
\hline 61.516 & 2.197 & 2.197 & 6.591 \\
\hline 307.58 & 4.394 & 2.197 & 2.197 \\
\hline 2.197 & 2.197 & 8.788 & 2.197 \\
\hline 2.197 & 8.788 & 2.197 & 4.394 \\
\hline 37.349 & 2.197 & 2.197 & 8.788 \\
\hline 17.576 & 6.591 & 4.394 & 4.394 \\
\hline 17.576 & 4.394 & 2.197 & 17.576 \\
\hline 19.773 & 2.197 & 2.197 & 659840 \\
\hline 54.925 & 2.197 & 4.394 & 19.773 \\
\hline 2.197 & 2.197 & 2.197 & 13.182 \\
\hline 30.758 & 65.91 & 158.184 & 15.379 \\
\hline 2.197 & 37.349 & 2750.64 & 28.561 \\
\hline 2.197 & 6.591 & 4.394 & 10.985 \\
\hline 21.97 & 327.353 & 2.197 & 514.098 \\
\hline 76.895 & 2.197 & 2.197 & 252.655 \\
\hline 6.591 & 2.197 & 2.197 & 4.394 \\
\hline 26.364 & 2.197 & 2.197 & 2.197 \\
\hline 21.97 & 4.394 & 2.197 & 2.197 \\
\hline 10.985 & 4.394 & 10.985 & 2.197 \\
\hline 2.197 & 2.197 & 2.197 & 107.653 \\
\hline 6.591 & 2.197 & 2.197 & 43.94 \\
\hline 2.197 & 2.197 & 70.304 & 2.197 \\
\hline 393.263 & 2.197 & 2.197 & 17.576 \\
\hline 2.197 & 4.394 & 2.197 & 10.985 \\
\hline 10.985 & 8.788 & 24.167 & 2.197 \\
\hline 15.379 & 13.182 & 15.379 & 17.576 \\
\hline 41751.8 & 2.197 & 6.591 & 28.561 \\
\hline 8.788 & 2.197 & 76.895 & 17.576 \\
\hline 59.319 & 2.197 & 15.379 & 65.91 \\
\hline 118.638 & 2.197 & 59.319 & 4.394 \\
\hline 2.197 & 2.197 & 2.197 & 90.077 \\
\hline 6.591 & 4.394 & 4.394 & 8.788 \\
\hline 79.092 & 2.197 & 2.197 & 15.379 \\
\hline 2.197 & 24.167 & 2.197 & 2.197 \\
\hline 63.713 & 15.379 & 4.394 & 4.394 \\
\hline 632.736 & 186.745 & 6.591 & 52.728 \\
\hline 2.197 & 4.394 & 15.379 & 13.182 \\
\hline 6.591 & 2.197 & 50.531 & 6.591 \\
\hline 2.197 & 10.985 & 2.197 & 639.327 \\
\hline
\end{tabular}




\begin{tabular}{|c|c|c|c|}
\hline 32.955 & 21.97 & 4.394 & 2.197 \\
\hline 2.197 & 61.516 & 2.197 & 2.197 \\
\hline 4.394 & 2.197 & 19.773 & 4.394 \\
\hline 1069.94 & 230.685 & 26.364 & 2.197 \\
\hline 17.576 & 81.289 & 2.197 & 19.773 \\
\hline 26.364 & 4.394 & 2.197 & 2.197 \\
\hline 2.197 & 13.182 & 8.788 & 105.456 \\
\hline 19.773 & 4.394 & 21.97 & 39.546 \\
\hline 4.394 & 2.197 & 2.197 & 2.197 \\
\hline 6.591 & 48.334 & 61.516 & 4.394 \\
\hline 2.197 & 2.197 & 2.197 & 30.758 \\
\hline 6.591 & 2.197 & 6.591 & 6.591 \\
\hline 2.197 & 4.394 & 37.349 & 13.182 \\
\hline 2.197 & 2.197 & 10.985 & 65.91 \\
\hline 87.88 & 107.653 & 4.394 & 90.077 \\
\hline 13.182 & 2.197 & 50.531 & 17.576 \\
\hline 4.394 & 2.197 & 2.197 & 32.955 \\
\hline 4.394 & 2.197 & 2.197 & 26.364 \\
\hline 138.411 & 2.197 & 4.394 & 4.394 \\
\hline 2.197 & 2.197 & 21.97 & 8.788 \\
\hline 6.591 & 81.289 & 2.197 & 2.197 \\
\hline 239.473 & 8.788 & 4.394 & 24.167 \\
\hline 81.289 & 2.197 & 2.197 & 4.394 \\
\hline 6.591 & 79.092 & 4.394 & 331.747 \\
\hline 2.197 & 388.869 & 4.394 & 21.97 \\
\hline 48.334 & 4.394 & 10.985 & 43.94 \\
\hline 19.773 & 2.197 & 2.197 & 79.092 \\
\hline 2.197 & 2.197 & 2.197 & 70.304 \\
\hline 2.197 & 6.591 & 8.788 & 6.591 \\
\hline 81.289 & 50.531 & 2.197 & 2.197 \\
\hline 105.456 & 6.591 & 2.197 & 28.561 \\
\hline 19.773 & 6.591 & 4.394 & 21.97 \\
\hline 2.197 & 6.591 & 307.58 & 83.486 \\
\hline 8.788 & 2.197 & 252.655 & 4.394 \\
\hline 2.197 & 2.197 & 2.197 & 2.197 \\
\hline 21.97 & 2.197 & 10.985 & 2.197 \\
\hline 13.182 & 2.197 & 4.394 & 2.197 \\
\hline 76.895 & 8.788 & 2.197 & 2.197 \\
\hline 8.788 & 6.591 & 63.713 & 37.349 \\
\hline 26.364 & 17.576 & 2.197 & 4.394 \\
\hline 2.197 & 8.788 & 2.197 & 2.197 \\
\hline 21.97 & 279.019 & 2.197 & 2.197 \\
\hline 6.591 & 17.576 & 2.197 & 2.197 \\
\hline 21.97 & 221.897 & 4506.05 & 54.925 \\
\hline 68.107 & 21.97 & 2.197 & 263.64 \\
\hline 261.443 & 2.197 & 2.197 & 10.985 \\
\hline 17.576 & 13.182 & 2.197 & 21.97 \\
\hline 2.197 & 145.002 & 35.152 & 4.394 \\
\hline 26.364 & 2.197 & 32.955 & 188.942 \\
\hline 61.516 & 39.546 & 2.197 & 127.426 \\
\hline
\end{tabular}




\begin{tabular}{|c|c|c|c|}
\hline 6.591 & 10.985 & 15.379 & 2.197 \\
\hline 2.197 & 92.274 & 8.788 & 2.197 \\
\hline 474.552 & 6.591 & 2.197 & 114.244 \\
\hline 2.197 & 4.394 & 2.197 & 10.985 \\
\hline 2.197 & 2.197 & 2.197 & 2.197 \\
\hline 4.394 & 2.197 & 28.561 & 2.197 \\
\hline 13.182 & 261.443 & 120.835 & 4.394 \\
\hline 6.591 & 6.591 & 10.985 & 2.197 \\
\hline 4.394 & 52.728 & 85.683 & 26.364 \\
\hline 8.788 & 35.152 & 2.197 & 39.546 \\
\hline 10.985 & 19.773 & 92.274 & 2.197 \\
\hline 6.591 & 2.197 & 4.394 & 4.394 \\
\hline 2.197 & 2.197 & 2.197 & 35.152 \\
\hline 10.985 & 4.394 & 24.167 & 2.197 \\
\hline 87.88 & 10.985 & 432.809 & 2.197 \\
\hline 250.458 & 26.364 & 4.394 & 8.788 \\
\hline 26.364 & 13.182 & 2.197 & 4.394 \\
\hline 17.576 & 2.197 & 2.197 & 4.394 \\
\hline 6.591 & 2.197 & 35.152 & 15.379 \\
\hline 2.197 & 2.197 & 123.032 & 43.94 \\
\hline 39.546 & 6.591 & 2.197 & 311.974 \\
\hline 6.591 & 2.197 & 6.591 & 4.394 \\
\hline 28.561 & 2.197 & 2.197 & 2.197 \\
\hline 4.394 & 2.197 & 6.591 & 2.197 \\
\hline 8.788 & 2.197 & 4.394 & 4.394 \\
\hline 13.182 & 2.197 & 74.698 & 10.985 \\
\hline 8.788 & 8.788 & 2.197 & 114.244 \\
\hline 4.394 & 4.394 & 43.94 & 13.182 \\
\hline 32.955 & 10.985 & 43.94 & 566.826 \\
\hline 6.591 & 2.197 & 2.197 & 65.91 \\
\hline 10.985 & 2.197 & 46.137 & 2.197 \\
\hline 2.197 & 8.788 & 8.788 & 2.197 \\
\hline 1452.22 & 92.274 & 136.214 & 2.197 \\
\hline 13.182 & 8.788 & 2.197 & 13.182 \\
\hline 2.197 & 35.152 & 8.788 & 2.197 \\
\hline 125.229 & 147.199 & 2.197 & 8.788 \\
\hline 2.197 & 2.197 & 37.349 & 15.379 \\
\hline 175.76 & 2.197 & 2.197 & 13.182 \\
\hline 6.591 & 8.788 & 4.394 & 2.197 \\
\hline 6.591 & 2.197 & 2.197 & 52.728 \\
\hline 13.182 & 2.197 & 35.152 & 2.197 \\
\hline 87.88 & 17.576 & 2.197 & 2.197 \\
\hline 2.197 & 17.576 & 2.197 & 101.062 \\
\hline 8.788 & 2.197 & 533.871 & 6.591 \\
\hline 2.197 & 2.197 & 41.743 & 28.561 \\
\hline 2.197 & 2.197 & 19.773 & 309.777 \\
\hline 2.197 & 2.197 & 2.197 & 26.364 \\
\hline 8.788 & 30.758 & 15.379 & 8.788 \\
\hline 52.728 & 2.197 & 2.197 & 393.263 \\
\hline 2.197 & 127.426 & 2.197 & 131.82 \\
\hline
\end{tabular}




\begin{tabular}{|c|c|c|c|}
\hline 2.197 & 8.788 & 2.197 & 17.576 \\
\hline 6.591 & 2.197 & 8.788 & 26.364 \\
\hline 6.591 & 2.197 & 79.092 & 59.319 \\
\hline 4.394 & 6.591 & 2.197 & 48.334 \\
\hline 6.591 & 2.197 & 6.591 & 6.591 \\
\hline 771.147 & 4.394 & 285.61 & 13.182 \\
\hline 41.743 & 2.197 & 6.591 & 26.364 \\
\hline 2.197 & 2.197 & 48.334 & 57.122 \\
\hline 2.197 & 17.576 & 164.775 & 2.197 \\
\hline 2.197 & 2.197 & 2.197 & 46.137 \\
\hline 2.197 & 2.197 & 2.197 & 87.88 \\
\hline 2.197 & 43.94 & 17.576 & 6.591 \\
\hline 2.197 & 2.197 & 4.394 & 32.955 \\
\hline 4.394 & 2.197 & 92.274 & 4.394 \\
\hline 26.364 & 2.197 & 4.394 & 2.197 \\
\hline 19.773 & 59.319 & 4.394 & 15.379 \\
\hline 8.788 & 4.394 & 2.197 & 70.304 \\
\hline 52.728 & 2.197 & 13.182 & 2.197 \\
\hline 6.591 & 13.182 & 41.743 & 114.244 \\
\hline 15.379 & 6.591 & 46.137 & 4.394 \\
\hline 6.591 & 90.077 & 219.7 & 2.197 \\
\hline 4.394 & 120.835 & 2.197 & 24.167 \\
\hline 2.197 & 8.788 & 2.197 & 8.788 \\
\hline 4.394 & 24.167 & 2.197 & 4.394 \\
\hline 2.197 & 72.501 & 15.379 & 1764.19 \\
\hline 2.197 & 2.197 & 24.167 & 4.394 \\
\hline 98.865 & 15.379 & 2.197 & 2.197 \\
\hline 13.182 & 2.197 & 2.197 & 2.197 \\
\hline 6.591 & 2.197 & 4.394 & 8.788 \\
\hline 4.394 & 15.379 & 4.394 & 2.197 \\
\hline 2.197 & 8.788 & 2.197 & 2.197 \\
\hline 79.092 & 2.197 & 2.197 & 2.197 \\
\hline 15.379 & 4.394 & 8.788 & 8.788 \\
\hline 13.182 & 2.197 & 4.394 & 19.773 \\
\hline 4.394 & 17.576 & 6.591 & 39.546 \\
\hline 79.092 & 2.197 & 270.231 & 544.856 \\
\hline 727.207 & 2.197 & 2.197 & 26.364 \\
\hline 467.961 & 4.394 & 4.394 & 4.394 \\
\hline 4.394 & 2.197 & 10.985 & 17.576 \\
\hline 2.197 & 158.184 & 2.197 & 2.197 \\
\hline 4.394 & 4.394 & 26.364 & 28.561 \\
\hline 15.379 & 4.394 & 4.394 & 237.276 \\
\hline 8.788 & 2.197 & 2.197 & 2.197 \\
\hline 4.394 & 4.394 & 4.394 & 4.394 \\
\hline 13.182 & 2.197 & 68.107 & 15.379 \\
\hline 70.304 & 2.197 & 30.758 & 21.97 \\
\hline 2.197 & 8.788 & 2.197 & 2.197 \\
\hline 2.197 & 4.394 & 2.197 & 26.364 \\
\hline 26.364 & 8.788 & 70.304 & 13.182 \\
\hline 46.137 & 2.197 & 621.751 & 4.394 \\
\hline
\end{tabular}




\begin{tabular}{|c|c|c|c|}
\hline 2504.58 & 2.197 & 2.197 & 94.471 \\
\hline 103.259 & 8.788 & 2.197 & 2.197 \\
\hline 2.197 & 2.197 & 2.197 & 8.788 \\
\hline 2.197 & 2.197 & 494.325 & 4.394 \\
\hline 4.394 & 2.197 & 2.197 & 2.197 \\
\hline 2.197 & 2.197 & 2.197 & 2.197 \\
\hline 2.197 & 114.244 & 2.197 & 2.197 \\
\hline 15.379 & 4.394 & 13.182 & 15.379 \\
\hline 2.197 & 4.394 & 2.197 & 50.531 \\
\hline 2.197 & 2.197 & 2.197 & 2.197 \\
\hline 2.197 & 2.197 & 2.197 & 199.927 \\
\hline 15.379 & 2.197 & 2.197 & 4.394 \\
\hline 10.985 & 4.394 & 2.197 & 37.349 \\
\hline 4.394 & 4.394 & 4.394 & 35.152 \\
\hline 2.197 & 2.197 & 74.698 & 28.561 \\
\hline 30.758 & 2.197 & 2.197 & 2.197 \\
\hline 138.411 & 2.197 & 15.379 & 2.197 \\
\hline 48.334 & 2.197 & 28.561 & 2.197 \\
\hline 21.97 & 4.394 & 103.259 & 30.758 \\
\hline 10.985 & 6.591 & 87.88 & 6.591 \\
\hline 21.97 & 10.985 & 8.788 & 46.137 \\
\hline 6.591 & 4.394 & 90.077 & 2.197 \\
\hline 2.197 & 17.576 & 8.788 & 6.591 \\
\hline 10.985 & 61.516 & 2.197 & 19.773 \\
\hline 17.576 & 2.197 & 2.197 & 779.935 \\
\hline 41.743 & 2.197 & 771.147 & 2.197 \\
\hline 6.591 & 2.197 & 294.398 & 193.336 \\
\hline 2.197 & 2.197 & 92.274 & 24.167 \\
\hline 2.197 & 32.955 & 2.197 & 72.501 \\
\hline 254.852 & 2.197 & 2.197 & 30.758 \\
\hline 2.197 & 4.394 & 19.773 & 57.122 \\
\hline 4.394 & 4.394 & 2.197 & 2.197 \\
\hline 4.394 & 2.197 & 2.197 & 4.394 \\
\hline 10.985 & 2.197 & 536.068 & 63.713 \\
\hline 2.197 & 21.97 & 333.944 & 112.047 \\
\hline 2.197 & 4.394 & 2.197 & 8.788 \\
\hline 6.591 & 19.773 & 232.882 & 15.379 \\
\hline 10.985 & 13.182 & 6.591 & 2.197 \\
\hline 2.197 & 4.394 & 8.788 & 13.182 \\
\hline 2.197 & 6.591 & 28.561 & 2.197 \\
\hline 30.758 & 15.379 & 37.349 & 134.017 \\
\hline 397.657 & 43.94 & 2.197 & 81.289 \\
\hline 2.197 & 380.081 & 2.197 & 239.473 \\
\hline 8.788 & 2.197 & 2.197 & 6.591 \\
\hline 439.4 & 2.197 & 2.197 & 2.197 \\
\hline 4.394 & 8.788 & 74.698 & 1052.36 \\
\hline 158.184 & 2.197 & 2.197 & 81.289 \\
\hline 2625.41 & 986.453 & 2.197 & 21.97 \\
\hline 10.985 & 15.379 & 2.197 & 2.197 \\
\hline 50.531 & 4.394 & 2.197 & 2.197 \\
\hline
\end{tabular}




\begin{tabular}{|c|c|c|c|}
\hline 652.509 & 4.394 & 2.197 & 35.152 \\
\hline 15.379 & 2.197 & 2.197 & 92.274 \\
\hline 2.197 & 4.394 & 2.197 & 17.576 \\
\hline 21.97 & 52.728 & 6.591 & 2.197 \\
\hline 397.657 & 2.197 & 39.546 & 2.197 \\
\hline 2.197 & 6.591 & 2.197 & 10.985 \\
\hline 2.197 & 4.394 & 15.379 & 21.97 \\
\hline 101.062 & 2.197 & 2.197 & 377.884 \\
\hline 90.077 & 6.591 & 2.197 & 13.182 \\
\hline 4.394 & 2.197 & 254.852 & 10.985 \\
\hline 2.197 & 6.591 & 10.985 & 46.137 \\
\hline 15.379 & 10.985 & 37.349 & 15.379 \\
\hline 43.94 & 6.591 & 4.394 & 21.97 \\
\hline 2.197 & 13.182 & 2.197 & 2.197 \\
\hline 2.197 & 4.394 & 2.197 & 8.788 \\
\hline 325.156 & 2.197 & 2.197 & 2.197 \\
\hline 2.197 & 4.394 & 2.197 & 30.758 \\
\hline 50.531 & 103.259 & 28.561 & 32.955 \\
\hline 131.82 & 2.197 & 2.197 & 2.197 \\
\hline 2.197 & 4.394 & 2.197 & 6.591 \\
\hline 2.197 & 32.955 & 2.197 & 6.591 \\
\hline 35.152 & 52.728 & 2.197 & 138.411 \\
\hline 8.788 & 2.197 & 2.197 & 6.591 \\
\hline 15.379 & 19.773 & 2.197 & 2.197 \\
\hline 1478.58 & 2.197 & 6.591 & 2.197 \\
\hline 2.197 & 2.197 & 52.728 & 4.394 \\
\hline 2.197 & 2.197 & 2.197 & 2.197 \\
\hline 2.197 & 2.197 & 2.197 & 6.591 \\
\hline 17.576 & 2.197 & 26.364 & 1010.62 \\
\hline 2.197 & 2.197 & 2.197 & 46.137 \\
\hline 182.351 & 2.197 & 105.456 & 15.379 \\
\hline 252.655 & 4.394 & 24.167 & 21.97 \\
\hline 21.97 & 2.197 & 6.591 & 19.773 \\
\hline 654.706 & 4.394 & 83.486 & 13.182 \\
\hline 6.591 & 39.546 & 2.197 & 4.394 \\
\hline 8.788 & 6.591 & 2.197 & 806.299 \\
\hline 6.591 & 2.197 & 2.197 & 30.758 \\
\hline 6.591 & 4.394 & 2.197 & 2.197 \\
\hline 2.197 & 17.576 & 24.167 & 21.97 \\
\hline 6.591 & 8.788 & 4.394 & 6.591 \\
\hline 8.788 & 2.197 & 4.394 & 76.895 \\
\hline 10.985 & 8.788 & 2.197 & 2.197 \\
\hline 21.97 & 2.197 & 2.197 & 2.197 \\
\hline 191.139 & 2.197 & 2.197 & 19.773 \\
\hline 2.197 & 2.197 & 4.394 & 17.576 \\
\hline 2.197 & 123.032 & 2.197 & 193.336 \\
\hline 2.197 & 2.197 & 2.197 & 13.182 \\
\hline 162.578 & 2.197 & 2.197 & 6.591 \\
\hline 2.197 & 6.591 & 85.683 & 13.182 \\
\hline 2.197 & 883.194 & 2.197 & 2.197 \\
\hline
\end{tabular}




\begin{tabular}{|c|c|c|c|}
\hline 17.576 & 2.197 & 2.197 & 296.595 \\
\hline 4.394 & 2.197 & 2.197 & 2.197 \\
\hline 74.698 & 2.197 & 4.394 & 2.197 \\
\hline 12900.8 & 21.97 & 2.197 & 10.985 \\
\hline 2.197 & 2.197 & 2.197 & 4.394 \\
\hline 2.197 & 2.197 & 4.394 & 39.546 \\
\hline 17.576 & 13.182 & 2.197 & 17.576 \\
\hline 2.197 & 2.197 & 2.197 & 57.122 \\
\hline 2.197 & 4.394 & 2.197 & 404.248 \\
\hline 4.394 & 2.197 & 2.197 & 2.197 \\
\hline 2.197 & 13.182 & 1423.66 & 30.758 \\
\hline 2.197 & 8.788 & 2.197 & 48.334 \\
\hline 4.394 & 2.197 & 2.197 & 17.576 \\
\hline 2.197 & 2.197 & 19.773 & 4.394 \\
\hline 4.394 & 4.394 & 2.197 & 30.758 \\
\hline 2.197 & 2.197 & 2.197 & 6.591 \\
\hline 2.197 & 2.197 & 4.394 & 2.197 \\
\hline 155.987 & 2.197 & 2.197 & 2.197 \\
\hline 6.591 & 2.197 & 2.197 & 17.576 \\
\hline 2.197 & 32.955 & 2.197 & 10.985 \\
\hline 604.175 & 4.394 & 2.197 & 26.364 \\
\hline 2.197 & 2.197 & 2.197 & 35.152 \\
\hline 43.94 & 4.394 & 2.197 & 2.197 \\
\hline 30.758 & 4.394 & 6.591 & 230.685 \\
\hline 96.668 & 2.197 & 681.07 & 19.773 \\
\hline 4.394 & 17.576 & 2.197 & 35.152 \\
\hline 4.394 & 2.197 & 2.197 & 15.379 \\
\hline 17.576 & 21.97 & 35.152 & 13.182 \\
\hline 52.728 & 2.197 & 83.486 & 2.197 \\
\hline 2.197 & 4.394 & 15.379 & 32.955 \\
\hline 4.394 & 2.197 & 2.197 & 15.379 \\
\hline 2.197 & 2.197 & 6.591 & 26.364 \\
\hline 2.197 & 2.197 & 2.197 & 37.349 \\
\hline 4.394 & 37.349 & 26.364 & 48.334 \\
\hline 2.197 & 4.394 & 6.591 & 24.167 \\
\hline 2.197 & 28.561 & 41.743 & 52.728 \\
\hline 2.197 & 54.925 & 2.197 & 6.591 \\
\hline 54.925 & 4.394 & 714.025 & 2.197 \\
\hline 2.197 & 59.319 & 2.197 & 1067.74 \\
\hline 4.394 & 6.591 & 10.985 & 2.197 \\
\hline 103.259 & 63.713 & 19.773 & 4.394 \\
\hline 13.182 & 322.959 & 13.182 & 105.456 \\
\hline 171.366 & 37.349 & 4.394 & 10.985 \\
\hline 2.197 & 8.788 & 4.394 & 4.394 \\
\hline 1948.74 & 17.576 & 4.394 & 4.394 \\
\hline 30.758 & 2.197 & 2.197 & 2.197 \\
\hline 4.394 & 4.394 & 4.394 & 10.985 \\
\hline 59.319 & 4.394 & 10.985 & 4.394 \\
\hline 30.758 & 4.394 & 6.591 & 30.758 \\
\hline 8.788 & 8.788 & 2.197 & 13.182 \\
\hline
\end{tabular}




\begin{tabular}{|c|c|c|c|}
\hline 2.197 & 19.773 & 2.197 & 2.197 \\
\hline 30.758 & 2.197 & 195.533 & 8.788 \\
\hline 4.394 & 2.197 & 2.197 & 85.683 \\
\hline 8.788 & 6.591 & 13.182 & 48.334 \\
\hline 2.197 & 2.197 & 2.197 & 70.304 \\
\hline 46.137 & 6.591 & 2.197 & 6.591 \\
\hline 4.394 & 4.394 & 2.197 & 105.456 \\
\hline 2.197 & 4.394 & 2.197 & 2.197 \\
\hline 2.197 & 13.182 & 2.197 & 2.197 \\
\hline 4.394 & 19.773 & 17.576 & 4.394 \\
\hline 30.758 & 4.394 & 2.197 & 2.197 \\
\hline 10.985 & 35.152 & 17.576 & 2.197 \\
\hline 2.197 & 155.987 & 17.576 & 6.591 \\
\hline 2.197 & 8.788 & 2.197 & 19.773 \\
\hline 13.182 & 13.182 & 2.197 & 2.197 \\
\hline 127.426 & 2.197 & 8.788 & 4.394 \\
\hline 2.197 & 19.773 & 2.197 & 226.291 \\
\hline 4.394 & 21.97 & 85.683 & 6.591 \\
\hline 2.197 & 2.197 & 19.773 & 48.334 \\
\hline 2.197 & 8.788 & 2.197 & 2.197 \\
\hline 2.197 & 2.197 & 2.197 & 50.531 \\
\hline 32.955 & 13.182 & 50.531 & 8.788 \\
\hline 4.394 & 46.137 & 13.182 & 6.591 \\
\hline 6.591 & 13.182 & 2.197 & 41.743 \\
\hline 21.97 & 101.062 & 2.197 & 303.186 \\
\hline 2.197 & 79.092 & 2.197 & 17.576 \\
\hline 6.591 & 26.364 & 2.197 & 21.97 \\
\hline 35.152 & 8.788 & 2.197 & 103.259 \\
\hline 2.197 & 2.197 & 2.197 & 6.591 \\
\hline 6.591 & 2.197 & 2.197 & 41.743 \\
\hline 65.91 & 109.85 & 2.197 & 24.167 \\
\hline 13.182 & 4.394 & 2.197 & 4.394 \\
\hline 177.957 & 2.197 & 2.197 & 65.91 \\
\hline 2.197 & 2.197 & 287.807 & 2.197 \\
\hline 48.334 & 2.197 & 2.197 & 2.197 \\
\hline 2.197 & 2.197 & 4.394 & 2.197 \\
\hline 4.394 & 2.197 & 2.197 & 15.379 \\
\hline 2.197 & 2.197 & 2.197 & 10.985 \\
\hline 2.197 & 2.197 & 4.394 & 2.197 \\
\hline 322.959 & 28.561 & 2.197 & 4.394 \\
\hline 4.394 & 2.197 & 2.197 & 4.394 \\
\hline 2.197 & 4.394 & 2.197 & 2.197 \\
\hline 54.925 & 2.197 & 2.197 & 4.394 \\
\hline 6.591 & 2.197 & 2.197 & 13.182 \\
\hline 136.214 & 4.394 & 2.197 & 2.197 \\
\hline 2.197 & 2.197 & 2.197 & 4.394 \\
\hline 4.394 & 4.394 & 2.197 & 59.319 \\
\hline 6.591 & 8.788 & 2.197 & 63.713 \\
\hline 2.197 & 2.197 & 8.788 & 50.531 \\
\hline 485.537 & 2.197 & 2.197 & 147.199 \\
\hline
\end{tabular}




\begin{tabular}{|c|c|c|c|}
\hline 4.394 & 2.197 & 8.788 & 134.017 \\
\hline 50.531 & 571.22 & 8.788 & 2.197 \\
\hline 4.394 & 2.197 & 72.501 & 4.394 \\
\hline 2.197 & 26.364 & 2.197 & 50.531 \\
\hline 26.364 & 2.197 & 37.349 & 2.197 \\
\hline 32.955 & 2.197 & 2.197 & 2.197 \\
\hline 2.197 & 2.197 & 2.197 & 2.197 \\
\hline 2.197 & 2.197 & 2.197 & 21.97 \\
\hline 2.197 & 24.167 & 2.197 & 4.394 \\
\hline 4.394 & 28.561 & 2.197 & 300.989 \\
\hline 8.788 & 136.214 & 28.561 & 35.152 \\
\hline 2.197 & 2.197 & 30.758 & 413.036 \\
\hline 2.197 & 17.576 & 39.546 & 17.576 \\
\hline 13.182 & 2.197 & 2.197 & 2.197 \\
\hline 2.197 & 37.349 & 2.197 & 103.259 \\
\hline 2.197 & 2.197 & 6.591 & 566.826 \\
\hline 8.788 & 6.591 & 8.788 & 250.458 \\
\hline 4.394 & 6.591 & 4.394 & 8.788 \\
\hline 8.788 & 4.394 & 41.743 & 41.743 \\
\hline 46.137 & 35.152 & 4.394 & 2304.65 \\
\hline 2.197 & 2.197 & 134.017 & 4.394 \\
\hline 2.197 & 2.197 & 2.197 & 19.773 \\
\hline 10.985 & 65.91 & 13.182 & 2.197 \\
\hline 4.394 & 87.88 & 120.835 & 92.274 \\
\hline 2.197 & 2.197 & 641.524 & 2.197 \\
\hline 8.788 & 2.197 & 57.122 & 15.379 \\
\hline 8.788 & 52.728 & 316.368 & 17.576 \\
\hline 6.591 & 2.197 & 4.394 & 112.047 \\
\hline 70.304 & 15.379 & 2.197 & 8.788 \\
\hline 2.197 & 54.925 & 4.394 & 2.197 \\
\hline 643.721 & 2.197 & 2.197 & 4.394 \\
\hline 2.197 & 65.91 & 2.197 & 944.71 \\
\hline 10.985 & 2.197 & 105.456 & 13.182 \\
\hline 107.653 & 4.394 & 6.591 & 43.94 \\
\hline 2.197 & 8.788 & 4.394 & 19.773 \\
\hline 28.561 & 4.394 & 4.394 & 4.394 \\
\hline 13.182 & 2.197 & 4.394 & 4.394 \\
\hline 4.394 & 6.591 & 6.591 & 4.394 \\
\hline 48.334 & 4.394 & 57.122 & 6.591 \\
\hline 10.985 & 2.197 & 6.591 & 54.925 \\
\hline 8.788 & 2.197 & 4.394 & 136.214 \\
\hline 1131.45 & 4.394 & 164.775 & 2.197 \\
\hline 13.182 & 2.197 & 79.092 & 4.394 \\
\hline 4.394 & 8.788 & 21.97 & 46.137 \\
\hline 320.762 & 2.197 & 336.141 & 6.591 \\
\hline 6.591 & 8.788 & 6.591 & 2.197 \\
\hline 63.713 & 4.394 & 2.197 & 15.379 \\
\hline 24.167 & 6.591 & 2.197 & 166.972 \\
\hline 96.668 & 4.394 & 2.197 & 6.591 \\
\hline 129.623 & 48.334 & 30.758 & 15.379 \\
\hline
\end{tabular}




\begin{tabular}{|c|c|c|c|}
\hline 112.047 & 46.137 & 2.197 & 1432.44 \\
\hline 15.379 & 2.197 & 4.394 & 15.379 \\
\hline 101.062 & 2.197 & 4.394 & 226.291 \\
\hline 2.197 & 8.788 & 2.197 & 37.349 \\
\hline 2.197 & 19.773 & 2.197 & 35.152 \\
\hline 2.197 & 2.197 & 2.197 & 210.912 \\
\hline 2.197 & 30.758 & 2.197 & 24.167 \\
\hline 21.97 & 2.197 & 2.197 & 4.394 \\
\hline 4.394 & 2.197 & 2.197 & 2.197 \\
\hline 4.394 & 2.197 & 2.197 & 15.379 \\
\hline 43.94 & 2.197 & 2.197 & 8.788 \\
\hline 2.197 & 2.197 & 2.197 & 4.394 \\
\hline 2.197 & 37.349 & 116.441 & 21.97 \\
\hline 2.197 & 6.591 & 127.426 & 4.394 \\
\hline 134.017 & 4.394 & 2.197 & 2.197 \\
\hline 8.788 & 2.197 & 4.394 & 6.591 \\
\hline 32.955 & 2.197 & 6.591 & 2.197 \\
\hline 2.197 & 2.197 & 2.197 & 4.394 \\
\hline 2.197 & 4.394 & 35.152 & 81.289 \\
\hline 52.728 & 2.197 & 4.394 & 1592.82 \\
\hline 92.274 & 2.197 & 2.197 & 61.516 \\
\hline 2.197 & 32.955 & 2.197 & 41.743 \\
\hline 2.197 & 2.197 & 13.182 & 448.188 \\
\hline 109.85 & 208.715 & 2.197 & 2.197 \\
\hline 2.197 & 32.955 & 2.197 & 92.274 \\
\hline 131.82 & 2.197 & 2.197 & 8.788 \\
\hline 4.394 & 10.985 & 2.197 & 10.985 \\
\hline 15.379 & 2.197 & 4.394 & 21.97 \\
\hline 4.394 & 2.197 & 4.394 & 8.788 \\
\hline 2.197 & 4.394 & 46.137 & 17.576 \\
\hline 6.591 & 2.197 & 4.394 & 4.394 \\
\hline 4.394 & 4.394 & 1026 & 8.788 \\
\hline 90.077 & 2.197 & 6.591 & 107.653 \\
\hline 46.137 & 4.394 & 2.197 & 50.531 \\
\hline 4.394 & 19.773 & 15.379 & 4.394 \\
\hline 19.773 & 2.197 & 6.591 & 46.137 \\
\hline 2.197 & 2.197 & 2.197 & 4.394 \\
\hline 6.591 & 2.197 & 2.197 & 19.773 \\
\hline 6.591 & 2.197 & 2.197 & 2.197 \\
\hline 2.197 & 6.591 & 13.182 & 2.197 \\
\hline 35.152 & 4.394 & 6.591 & 2.197 \\
\hline 2.197 & 15.379 & 2.197 & 2.197 \\
\hline 4.394 & 4.394 & 120.835 & 2.197 \\
\hline 2.197 & 24.167 & 6.591 & 10.985 \\
\hline 2.197 & 2.197 & 2.197 & 2.197 \\
\hline 265.837 & 2.197 & 2.197 & 8.788 \\
\hline 10.985 & 13.182 & 48.334 & 6.591 \\
\hline 2.197 & 2.197 & 15.379 & 101.062 \\
\hline 2.197 & 2.197 & 4.394 & 2.197 \\
\hline 4.394 & 2.197 & 4.394 & 13.182 \\
\hline
\end{tabular}




\begin{tabular}{|c|c|c|c|}
\hline 15.379 & 2.197 & 2.197 & 41.743 \\
\hline 15.379 & 2.197 & 4.394 & 101.062 \\
\hline 2.197 & 4.394 & 2.197 & 10.985 \\
\hline 2.197 & 6.591 & 2.197 & 37.349 \\
\hline 8.788 & 2.197 & 2.197 & 39.546 \\
\hline 32.955 & 37.349 & 10.985 & 19.773 \\
\hline 2.197 & 4.394 & 4.394 & 2.197 \\
\hline 1726.84 & 41.743 & 2.197 & 177.957 \\
\hline 15.379 & 2.197 & 54.925 & 2.197 \\
\hline 107.653 & 8.788 & 2.197 & 21.97 \\
\hline 2.197 & 6.591 & 21.97 & 92.274 \\
\hline 28.561 & 28.561 & 28.561 & 155.987 \\
\hline 2.197 & 2.197 & 21.97 & 2.197 \\
\hline 4.394 & 72.501 & 41.743 & 10.985 \\
\hline 54.925 & 11321.1 & 2.197 & 215.306 \\
\hline 2.197 & 2.197 & 127.426 & 10.985 \\
\hline 2.197 & 13.182 & 2.197 & 30.758 \\
\hline 4.394 & 8.788 & 6.591 & 2.197 \\
\hline 4.394 & 2.197 & 2.197 & 2.197 \\
\hline 15.379 & 2.197 & 4.394 & 2.197 \\
\hline 129.623 & 2.197 & 41.743 & 2.197 \\
\hline 6.591 & 8.788 & 10.985 & 2.197 \\
\hline 4.394 & 2.197 & 8.788 & 39.546 \\
\hline 2.197 & 2.197 & 4.394 & 4.394 \\
\hline 8.788 & 2.197 & 2.197 & 35.152 \\
\hline 2.197 & 17.576 & 2.197 & 37.349 \\
\hline 2.197 & 2.197 & 4.394 & 8.788 \\
\hline 2.197 & 2.197 & 2.197 & 32.955 \\
\hline 30.758 & 37.349 & 1447.82 & 32.955 \\
\hline 54.925 & 26.364 & 2.197 & 2.197 \\
\hline 2.197 & 4.394 & 13.182 & 15.379 \\
\hline 76.895 & 2.197 & 2.197 & 15.379 \\
\hline 2.197 & 8.788 & 13.182 & 79.092 \\
\hline 2.197 & 2.197 & 4.394 & 2.197 \\
\hline 4.394 & 2.197 & 2.197 & 10.985 \\
\hline 28.561 & 2.197 & 7584.04 & 57.122 \\
\hline 164.775 & 2.197 & 2.197 & 13.182 \\
\hline 2.197 & 8.788 & 8.788 & 2.197 \\
\hline 74.698 & 2.197 & 2.197 & 2.197 \\
\hline 2.197 & 2.197 & 4.394 & 26.364 \\
\hline 8.788 & 4.394 & 2.197 & 145.002 \\
\hline 15.379 & 76.895 & 2.197 & 24.167 \\
\hline 2.197 & 2.197 & 2.197 & 15.379 \\
\hline 8.788 & 2.197 & 46.137 & 4.394 \\
\hline 17.576 & 2.197 & 90.077 & 92.274 \\
\hline 2.197 & 2.197 & 2.197 & 70.304 \\
\hline 32.955 & 19.773 & 6.591 & 2.197 \\
\hline 32.955 & 2.197 & 2.197 & 48.334 \\
\hline 6.591 & 6.591 & 15.379 & 19.773 \\
\hline 57.122 & 4.394 & 8.788 & 10.985 \\
\hline
\end{tabular}




\begin{tabular}{|c|c|c|c|}
\hline 13.182 & 4.394 & 2.197 & 514.098 \\
\hline 13.182 & 2.197 & 6.591 & 15.379 \\
\hline 8.788 & 2.197 & 21.97 & 59.319 \\
\hline 32.955 & 8.788 & 48.334 & 8.788 \\
\hline 2.197 & 6.591 & 2.197 & 2.197 \\
\hline 6.591 & 6.591 & 30.758 & 39.546 \\
\hline 4.394 & 50.531 & 2.197 & 2.197 \\
\hline 4.394 & 2.197 & 6.591 & 79.092 \\
\hline 15.379 & 2.197 & 2.197 & 6.591 \\
\hline 2.197 & 2.197 & 2.197 & 6.591 \\
\hline 2.197 & 2.197 & 2.197 & 2.197 \\
\hline 120.835 & 2.197 & 2.197 & 2.197 \\
\hline 8.788 & 2.197 & 2.197 & 6.591 \\
\hline 6.591 & 4.394 & 2.197 & 8.788 \\
\hline 243.867 & 2.197 & 2.197 & 10.985 \\
\hline 2.197 & 2.197 & 8.788 & 4.394 \\
\hline 158.184 & 19.773 & 24.167 & 26.364 \\
\hline 2.197 & 2.197 & 15.379 & 8.788 \\
\hline 41.743 & 4.394 & 2.197 & 2.197 \\
\hline 4.394 & 26.364 & 2.197 & 70.304 \\
\hline 125.229 & 4.394 & 19.773 & 41.743 \\
\hline 2.197 & 2.197 & 2.197 & 13.182 \\
\hline 81.289 & 221.897 & 2.197 & 19.773 \\
\hline 617.357 & 28.561 & 2.197 & 46.137 \\
\hline 6.591 & 2.197 & 2.197 & 2.197 \\
\hline 2.197 & 2.197 & 2.197 & 19.773 \\
\hline 65.91 & 4.394 & 48.334 & 76.895 \\
\hline 79.092 & 2.197 & 4.394 & 13.182 \\
\hline 595.387 & 4.394 & 2.197 & 4.394 \\
\hline 2.197 & 4.394 & 17.576 & 57.122 \\
\hline 8.788 & 2.197 & 2.197 & 6.591 \\
\hline 2.197 & 2.197 & 4.394 & 10.985 \\
\hline 41.743 & 2.197 & 4.394 & 924.937 \\
\hline 2.197 & 4.394 & 21.97 & 15.379 \\
\hline 4.394 & 2.197 & 145.002 & 13.182 \\
\hline 2.197 & 2.197 & 13.182 & 10.985 \\
\hline 10.985 & 2.197 & 8.788 & 10.985 \\
\hline 2.197 & 17.576 & 4.394 & 2.197 \\
\hline 17.576 & 2.197 & 46.137 & 6.591 \\
\hline 17.576 & 8.788 & 26.364 & 6.591 \\
\hline 2.197 & 2.197 & 87.88 & 8.788 \\
\hline 2.197 & 4.394 & 2.197 & 2.197 \\
\hline 4.394 & 2.197 & 10.985 & 10.985 \\
\hline 4372.03 & 4.394 & 103.259 & 2.197 \\
\hline 2.197 & 98.865 & 140.608 & 2.197 \\
\hline 21.97 & 8.788 & 2.197 & 2.197 \\
\hline 735.995 & 2.197 & 2.197 & 2.197 \\
\hline 2.197 & 2.197 & 2.197 & 13.182 \\
\hline 6.591 & 2.197 & 2.197 & 4.394 \\
\hline 8.788 & 2.197 & 21.97 & 68.107 \\
\hline
\end{tabular}




\begin{tabular}{|c|c|c|c|}
\hline 2.197 & 28.561 & 85.683 & 6.591 \\
\hline 2.197 & 2.197 & 4.394 & 32.955 \\
\hline 4.394 & 2.197 & 17.576 & 2.197 \\
\hline 2.197 & 2.197 & 2.197 & 4.394 \\
\hline 19.773 & 2.197 & 2.197 & 4.394 \\
\hline 8.788 & 2.197 & 4.394 & 28.561 \\
\hline 2.197 & 2.197 & 2.197 & 98.865 \\
\hline 2.197 & 4.394 & 2.197 & 46.137 \\
\hline 39.546 & 105.456 & 26.364 & 2.197 \\
\hline 21.97 & 2.197 & 2.197 & 13.182 \\
\hline 21.97 & 2.197 & 160.381 & 13.182 \\
\hline 4.394 & 17.576 & 2.197 & 2.197 \\
\hline 4.394 & 2.197 & 2.197 & 2.197 \\
\hline 8.788 & 63.713 & 171.366 & 21.97 \\
\hline 2.197 & 15.379 & 2.197 & 2.197 \\
\hline 2.197 & 4.394 & 2.197 & 2.197 \\
\hline 28.561 & 70.304 & 2.197 & 26.364 \\
\hline 6.591 & 2.197 & 2.197 & 43.94 \\
\hline 76.895 & 2.197 & 2.197 & 6.591 \\
\hline 21.97 & 4.394 & 15.379 & 15.379 \\
\hline 10.985 & 4.394 & 166.972 & 4.394 \\
\hline 39.546 & 2.197 & 6.591 & 54.925 \\
\hline 2.197 & 2.197 & 2.197 & 26.364 \\
\hline 28.561 & 4.394 & 2.197 & 202.124 \\
\hline 8.788 & 2.197 & 8.788 & 2.197 \\
\hline 2.197 & 8.788 & 4.394 & 17.576 \\
\hline 2.197 & 2.197 & 24.167 & 8.788 \\
\hline 2.197 & 2.197 & 2.197 & 24.167 \\
\hline 2.197 & 2.197 & 2.197 & 6.591 \\
\hline 8.788 & 4.394 & 2.197 & 180.154 \\
\hline 4.394 & 2.197 & 2.197 & 50.531 \\
\hline 6.591 & 213.109 & 281.216 & 2.197 \\
\hline 2.197 & 2.197 & 57.122 & 2.197 \\
\hline 2.197 & 2.197 & 2.197 & 2.197 \\
\hline 2.197 & 2.197 & 2.197 & 19.773 \\
\hline 21.97 & 2.197 & 85.683 & 8.788 \\
\hline 10.985 & 4.394 & 41.743 & 2.197 \\
\hline 2.197 & 2.197 & 59.319 & 6.591 \\
\hline 24.167 & 8.788 & 2062.98 & 2.197 \\
\hline 10.985 & 13.182 & 4.394 & 2.197 \\
\hline 2.197 & 6.591 & 257.049 & 2.197 \\
\hline 2.197 & 2.197 & 2.197 & 13.182 \\
\hline 2.197 & 6.591 & 2.197 & 13.182 \\
\hline 46.137 & 19.773 & 2.197 & 8.788 \\
\hline 252.655 & 35.152 & 37.349 & 15.379 \\
\hline 15.379 & 70.304 & 2.197 & 10.985 \\
\hline 85.683 & 2.197 & 61.516 & 74.698 \\
\hline 8.788 & 10.985 & 8.788 & 2.197 \\
\hline 17.576 & 30.758 & 2.197 & 2.197 \\
\hline 63.713 & 26.364 & 175.76 & 8.788 \\
\hline
\end{tabular}




\begin{tabular}{|c|c|c|c|}
\hline 6.591 & 2.197 & 10.985 & 6.591 \\
\hline 10.985 & 2.197 & 257.049 & 2.197 \\
\hline 15.379 & 2.197 & 2.197 & 17.576 \\
\hline 2.197 & 2.197 & 37.349 & 2223.36 \\
\hline 4.394 & 4.394 & 10.985 & 90.077 \\
\hline 19.773 & 2.197 & 4.394 & 2.197 \\
\hline 21.97 & 2.197 & 90.077 & 17.576 \\
\hline 61.516 & 2.197 & 2.197 & 123.032 \\
\hline 2.197 & 2.197 & 2.197 & 17.576 \\
\hline 30.758 & 2.197 & 13.182 & 8.788 \\
\hline 2.197 & 8.788 & 2.197 & 13.182 \\
\hline 13.182 & 2.197 & 155.987 & 101.062 \\
\hline 2.197 & 2.197 & 2.197 & 13.182 \\
\hline 2.197 & 2.197 & 2.197 & 6.591 \\
\hline 123.032 & 2.197 & 2.197 & 2.197 \\
\hline 10.985 & 2.197 & 2.197 & 2.197 \\
\hline 6.591 & 2.197 & 2.197 & 2.197 \\
\hline 2.197 & 4.394 & 2.197 & 6.591 \\
\hline 2.197 & 2.197 & 2.197 & 10.985 \\
\hline 2.197 & 2.197 & 17.576 & 26.364 \\
\hline 17.576 & 2.197 & 16121.6 & 35.152 \\
\hline 8.788 & 4.394 & 10.985 & 8.788 \\
\hline 19.773 & 2.197 & 2.197 & 37.349 \\
\hline 10.985 & 21.97 & 8.788 & 4.394 \\
\hline 26.364 & 2.197 & 4.394 & 6.591 \\
\hline 944.71 & 4.394 & 28.561 & 138.411 \\
\hline 4.394 & 2.197 & 1522.52 & 4.394 \\
\hline 46.137 & 2.197 & 8.788 & 6.591 \\
\hline 230.685 & 2.197 & 81.289 & 2.197 \\
\hline 8.788 & 2.197 & 4.394 & 191.139 \\
\hline 26.364 & 2.197 & 2.197 & 4.394 \\
\hline 10.985 & 2.197 & 2.197 & 2.197 \\
\hline 182.351 & 72.501 & 6.591 & 32.955 \\
\hline 17.576 & 21.97 & 2.197 & 8.788 \\
\hline 2.197 & 2.197 & 2.197 & 10.985 \\
\hline 2.197 & 2.197 & 4.394 & 30.758 \\
\hline 2.197 & 10.985 & 6.591 & 2.197 \\
\hline 2.197 & 6.591 & 48.334 & 4.394 \\
\hline 2.197 & 6.591 & 2.197 & 6.591 \\
\hline 2.197 & 4.394 & 2.197 & 17.576 \\
\hline 2.197 & 2.197 & 6.591 & 7586.24 \\
\hline 2.197 & 4.394 & 94.471 & 19.773 \\
\hline 10.985 & 2.197 & 4.394 & 17.576 \\
\hline 4.394 & 4.394 & 4.394 & 4.394 \\
\hline 2.197 & 2.197 & 10.985 & 2.197 \\
\hline 8.788 & 4.394 & 2.197 & 13.182 \\
\hline 112.047 & 8.788 & 2.197 & 15.379 \\
\hline 2.197 & 8.788 & 2.197 & 2.197 \\
\hline 2.197 & 4.394 & 2.197 & 19.773 \\
\hline 114.244 & 2.197 & 197.73 & 213.109 \\
\hline
\end{tabular}




\begin{tabular}{|c|c|c|c|}
\hline 2.197 & 2.197 & 17.576 & 6.591 \\
\hline 2.197 & 2.197 & 26.364 & 21.97 \\
\hline 48.334 & 2.197 & 206.518 & 21.97 \\
\hline 311.974 & 2.197 & 15.379 & 39.546 \\
\hline 2.197 & 3337.24 & 2.197 & 46.137 \\
\hline 6.591 & 13.182 & 32.955 & 162.578 \\
\hline 10.985 & 2.197 & 283.413 & 123.032 \\
\hline 26.364 & 26.364 & 28.561 & 103.259 \\
\hline 4.394 & 2.197 & 4.394 & 10.985 \\
\hline 265.837 & 2.197 & 35.152 & 15.379 \\
\hline 127.426 & 2.197 & 6.591 & 2.197 \\
\hline 8.788 & 2.197 & 19.773 & 10.985 \\
\hline 4.394 & 2.197 & 6.591 & 6.591 \\
\hline 43.94 & 2.197 & 2.197 & 103.259 \\
\hline 6.591 & 2.197 & 24.167 & 17.576 \\
\hline 2.197 & 2.197 & 10.985 & 19.773 \\
\hline 4.394 & 63.713 & 28.561 & 2.197 \\
\hline 2.197 & 2.197 & 17.576 & 8.788 \\
\hline 2.197 & 4.394 & 2.197 & 17.576 \\
\hline 2.197 & 2.197 & 4.394 & 2.197 \\
\hline 2.197 & 13.182 & 2.197 & 2.197 \\
\hline 4.394 & 15.379 & 6.591 & 2.197 \\
\hline 2.197 & 191.139 & 4.394 & 37.349 \\
\hline 15.379 & 15.379 & 6.591 & 46.137 \\
\hline 2.197 & 4.394 & 2.197 & 4.394 \\
\hline 4.394 & 2.197 & 13.182 & 6.591 \\
\hline 35.152 & 15.379 & 4.394 & 2.197 \\
\hline 2.197 & 52.728 & 487.734 & 112.047 \\
\hline 2.197 & 35.152 & 177.957 & 2.197 \\
\hline 61.516 & 2.197 & 13.182 & 4.394 \\
\hline 573.417 & 2.197 & 74.698 & 885.391 \\
\hline 2.197 & 2.197 & 76.895 & 4.394 \\
\hline 2.197 & 2.197 & 6.591 & 2.197 \\
\hline 65.91 & 2.197 & 2.197 & 15.379 \\
\hline 52.728 & 2.197 & 15.379 & 15.379 \\
\hline 8.788 & 2.197 & 2.197 & 2.197 \\
\hline 8.788 & 6.591 & 4.394 & 43.94 \\
\hline 6.591 & 2.197 & 2.197 & 4.394 \\
\hline 15.379 & 8.788 & 4.394 & 2.197 \\
\hline 2.197 & 6.591 & 2.197 & 19.773 \\
\hline 2.197 & 13.182 & 241.67 & 17.576 \\
\hline 2.197 & 2.197 & 48.334 & 2.197 \\
\hline 74.698 & 2.197 & 2.197 & 74.698 \\
\hline 2.197 & 2.197 & 2.197 & 4.394 \\
\hline 2.197 & 2.197 & 2.197 & 2.197 \\
\hline 2.197 & 2.197 & 2.197 & 4.394 \\
\hline 2.197 & 4.394 & 2.197 & 54.925 \\
\hline 13.182 & 2.197 & 2.197 & 2.197 \\
\hline 2.197 & 10.985 & 70.304 & 24.167 \\
\hline 125.229 & 2.197 & 4.394 & 32.955 \\
\hline
\end{tabular}




\begin{tabular}{|c|c|c|c|}
\hline 10.985 & 2.197 & 2.197 & 114.244 \\
\hline 4.394 & 4.394 & 24.167 & 2.197 \\
\hline 8.788 & 2.197 & 6.591 & 17.576 \\
\hline 2.197 & 123.032 & 61.516 & 13.182 \\
\hline 2.197 & 489.931 & 24.167 & 39.546 \\
\hline 17.576 & 6.591 & 2.197 & 21.97 \\
\hline 19.773 & 6.591 & 2.197 & 79.092 \\
\hline 2.197 & 6.591 & 21.97 & 6.591 \\
\hline 35.152 & 2.197 & 63.713 & 6.591 \\
\hline 35.152 & 2.197 & 2.197 & 4.394 \\
\hline 63.713 & 10.985 & 6.591 & 6.591 \\
\hline 10.985 & 6.591 & 2027.83 & 43.94 \\
\hline 21.97 & 4.394 & 2.197 & 28.561 \\
\hline 2.197 & 2.197 & 2.197 & 13.182 \\
\hline 6.591 & 13.182 & 17.576 & 15.379 \\
\hline 4.394 & 2.197 & 2.197 & 2.197 \\
\hline 6.591 & 2.197 & 2.197 & 2.197 \\
\hline 8.788 & 4.394 & 10.985 & 2.197 \\
\hline 4.394 & 8.788 & 32.955 & 4.394 \\
\hline 2.197 & 4.394 & 17.576 & 17.576 \\
\hline 10.985 & 2.197 & 2.197 & 13.182 \\
\hline 2.197 & 2.197 & 2.197 & 59.319 \\
\hline 17.576 & 21.97 & 4.394 & 118.638 \\
\hline 6.591 & 13.182 & 15.379 & 28.561 \\
\hline 2.197 & 2.197 & 10.985 & 10.985 \\
\hline 2.197 & 2.197 & 13.182 & 2.197 \\
\hline 6.591 & 19.773 & 6.591 & 123.032 \\
\hline 13.182 & 2.197 & 2.197 & 2.197 \\
\hline 21.97 & 2.197 & 2.197 & 6.591 \\
\hline 19.773 & 2.197 & 4.394 & 285.61 \\
\hline 2467.23 & 4110.59 & 2.197 & 17.576 \\
\hline 2.197 & 2.197 & 2.197 & 4.394 \\
\hline 2.197 & 2.197 & 90.077 & 151.593 \\
\hline 2.197 & 2.197 & 15.379 & 46.137 \\
\hline 4.394 & 17.576 & 2.197 & 430.612 \\
\hline 54.925 & 15.379 & 4.394 & 2.197 \\
\hline 6.591 & 52.728 & 105.456 & 8.788 \\
\hline 98.865 & 4.394 & 37.349 & 2.197 \\
\hline 366.899 & 6.591 & 2.197 & 2.197 \\
\hline 2.197 & 4.394 & 2.197 & 19.773 \\
\hline 59.319 & 2.197 & 10.985 & 6.591 \\
\hline 54.925 & 4.394 & 2.197 & 54.925 \\
\hline 623.948 & 2.197 & 246.064 & 8.788 \\
\hline 19.773 & 21.97 & 61.516 & 6.591 \\
\hline 10.985 & 4.394 & 2.197 & 2.197 \\
\hline 2.197 & 2.197 & 4.394 & 13.182 \\
\hline 2.197 & 4.394 & 4.394 & 74.698 \\
\hline 2.197 & 4.394 & 362.505 & 19.773 \\
\hline 68.107 & 4.394 & 2.197 & 21.97 \\
\hline 37.349 & 4.394 & 2.197 & 2.197 \\
\hline
\end{tabular}




\begin{tabular}{|c|c|c|c|}
\hline 15.379 & 4.394 & 48.334 & 4.394 \\
\hline 19.773 & 4.394 & 6.591 & 268.034 \\
\hline 2.197 & 2.197 & 4.394 & 4.394 \\
\hline 2.197 & 2.197 & 37.349 & 4.394 \\
\hline 72.501 & 338.338 & 2.197 & 2.197 \\
\hline 61.516 & 2.197 & 2.197 & 6.591 \\
\hline 8.788 & 2.197 & 24.167 & 272.428 \\
\hline 28.561 & 4.394 & 6.591 & 6.591 \\
\hline 4.394 & 2.197 & 4.394 & 8.788 \\
\hline 59.319 & 2.197 & 37.349 & 294.398 \\
\hline 15.379 & 1972.91 & 2.197 & 2.197 \\
\hline 2.197 & 2.197 & 2.197 & 4.394 \\
\hline 2.197 & 10.985 & 2.197 & 4.394 \\
\hline 6.591 & 2.197 & 2.197 & 83.486 \\
\hline 28.561 & 2.197 & 4.394 & 272.428 \\
\hline 19.773 & 2.197 & 2.197 & 37.349 \\
\hline 26.364 & 2.197 & 4.394 & 2.197 \\
\hline 30.758 & 15.379 & 2.197 & 4.394 \\
\hline 32.955 & 26.364 & 4.394 & 17.576 \\
\hline 15.379 & 4.394 & 2.197 & 17.576 \\
\hline 2.197 & 13.182 & 2.197 & 19.773 \\
\hline 43.94 & 2.197 & 2.197 & 63.713 \\
\hline 28.561 & 2.197 & 13.182 & 4.394 \\
\hline 6.591 & 2.197 & 8.788 & 21.97 \\
\hline 2.197 & 2.197 & 8.788 & 79.092 \\
\hline 46.137 & 2.197 & 2.197 & 17.576 \\
\hline 8.788 & 2.197 & 17.576 & 2.197 \\
\hline 2284.88 & 8.788 & 2.197 & 6.591 \\
\hline 2.197 & 4.394 & 2.197 & 101.062 \\
\hline 2.197 & 10.985 & 1080.92 & 8.788 \\
\hline 6.591 & 4.394 & 2.197 & 83.486 \\
\hline 21.97 & 193.336 & 4.394 & 2.197 \\
\hline 2.197 & 2.197 & 2.197 & 37.349 \\
\hline 2.197 & 4.394 & 120.835 & 8.788 \\
\hline 2.197 & 10.985 & 2.197 & 52.728 \\
\hline 2.197 & 2.197 & 15.379 & 116.441 \\
\hline 8.788 & 2.197 & 21.97 & 26.364 \\
\hline 2.197 & 287.807 & 4.394 & 2.197 \\
\hline 2.197 & 4.394 & 8.788 & 1135.85 \\
\hline 2.197 & 2.197 & 32.955 & 145.002 \\
\hline 199.927 & 2.197 & 26.364 & 39.546 \\
\hline 2.197 & 61.516 & 30.758 & 193.336 \\
\hline 384.475 & 2.197 & 15.379 & 470.158 \\
\hline 382.278 & 6.591 & 2.197 & 2.197 \\
\hline 4.394 & 2.197 & 6.591 & 26.364 \\
\hline 2.197 & 4.394 & 19.773 & 8.788 \\
\hline 2.197 & 4.394 & 8.788 & 6.591 \\
\hline 8.788 & 4.394 & 4.394 & 10.985 \\
\hline 2.197 & 26.364 & 68.107 & 15.379 \\
\hline 2.197 & 8.788 & 2.197 & 24.167 \\
\hline
\end{tabular}




\begin{tabular}{|c|c|c|c|}
\hline 2.197 & 8.788 & 2.197 & 2.197 \\
\hline 2.197 & 4.394 & 2.197 & 2.197 \\
\hline 10.985 & 61.516 & 4.394 & 8.788 \\
\hline 61.516 & 6.591 & 951.301 & 41.743 \\
\hline 2.197 & 46.137 & 2.197 & 30.758 \\
\hline 2.197 & 263.64 & 2.197 & 8.788 \\
\hline 2.197 & 6.591 & 4.394 & 19.773 \\
\hline 6.591 & 15.379 & 10.985 & 8.788 \\
\hline 4.394 & 30.758 & 32.955 & 4.394 \\
\hline 19.773 & 21.97 & 63.713 & 4.394 \\
\hline 39.546 & 6.591 & 10.985 & 76.895 \\
\hline 13.182 & 6.591 & 4.394 & 50.531 \\
\hline 19.773 & 2.197 & 2.197 & 74.698 \\
\hline 2.197 & 6.591 & 6.591 & 10.985 \\
\hline 2.197 & 2.197 & 2.197 & 2.197 \\
\hline 2.197 & 6.591 & 24.167 & 21.97 \\
\hline 261.443 & 2.197 & 6.591 & 4.394 \\
\hline 2.197 & 4.394 & 2.197 & 2.197 \\
\hline 15.379 & 4.394 & 2.197 & 8.788 \\
\hline 37.349 & 85.683 & 2.197 & 4.394 \\
\hline 19.773 & 2.197 & 2.197 & 975.468 \\
\hline 6.591 & 4.394 & 24.167 & 2.197 \\
\hline 92.274 & 2.197 & 2.197 & 2.197 \\
\hline 2.197 & 2.197 & 19.773 & 35.152 \\
\hline 2.197 & 2.197 & 19.773 & 10.985 \\
\hline 2.197 & 15.379 & 129.623 & 43.94 \\
\hline 2.197 & 17.576 & 2.197 & 246.064 \\
\hline 2.197 & 2.197 & 2.197 & 243.867 \\
\hline 127.426 & 2.197 & 101.062 & 2.197 \\
\hline 2.197 & 19.773 & 6.591 & 2.197 \\
\hline 4.394 & 8.788 & 8.788 & 54.925 \\
\hline 2.197 & 8.788 & 6.591 & 4.394 \\
\hline 2.197 & 2.197 & 2.197 & 134.017 \\
\hline 2.197 & 2.197 & 50.531 & 2.197 \\
\hline 193.336 & 4910.29 & 26.364 & 8.788 \\
\hline 52.728 & 10.985 & 2.197 & 15.379 \\
\hline 59.319 & 13.182 & 26.364 & 2.197 \\
\hline 28.561 & 15.379 & 6.591 & 2.197 \\
\hline 107.653 & 2.197 & 8.788 & 70.304 \\
\hline 24.167 & 2.197 & 2.197 & 2.197 \\
\hline 6.591 & 4.394 & 2.197 & 230.685 \\
\hline 17.576 & 2.197 & 2.197 & 21.97 \\
\hline 41.743 & 17.576 & 46.137 & 21.97 \\
\hline 15.379 & 2.197 & 21.97 & 4.394 \\
\hline 2.197 & 2.197 & 59.319 & 246.064 \\
\hline 2.197 & 4.394 & 15.379 & 10.985 \\
\hline 24.167 & 2.197 & 2.197 & 2.197 \\
\hline 72.501 & 4.394 & 15.379 & 2.197 \\
\hline 19.773 & 2.197 & 17.576 & 43.94 \\
\hline 2.197 & 4.394 & 4.394 & 2.197 \\
\hline
\end{tabular}




\begin{tabular}{|c|c|c|c|}
\hline 2.197 & 6.591 & 145.002 & 14085 \\
\hline 4.394 & 2.197 & 13.182 & 116.441 \\
\hline 2.197 & 2.197 & 8.788 & 8.788 \\
\hline 4.394 & 2.197 & 4.394 & 2.197 \\
\hline 24.167 & 48.334 & 2.197 & 4.394 \\
\hline 2.197 & 2.197 & 2.197 & 43.94 \\
\hline 52.728 & 4.394 & 2.197 & 4.394 \\
\hline 6.591 & 65.91 & 2.197 & 184.548 \\
\hline 13.182 & 2.197 & 2.197 & 54.925 \\
\hline 6.591 & 2.197 & 2.197 & 10.985 \\
\hline 2.197 & 2.197 & 8.788 & 8.788 \\
\hline 57.122 & 2.197 & 2.197 & 8.788 \\
\hline 17.576 & 2.197 & 2.197 & 153.79 \\
\hline 52.728 & 2.197 & 28.561 & 8.788 \\
\hline 485.537 & 2.197 & 4.394 & 32.955 \\
\hline 2.197 & 2.197 & 4.394 & 10.985 \\
\hline 35.152 & 2.197 & 13.182 & 8.788 \\
\hline 8.788 & 30.758 & 2.197 & 10.985 \\
\hline 6.591 & 37.349 & 2.197 & 24.167 \\
\hline 10.985 & 48.334 & 8.788 & 1102.89 \\
\hline 8.788 & 202.124 & 19.773 & 188.942 \\
\hline 6.591 & 17.576 & 6.591 & 4.394 \\
\hline 4.394 & 2.197 & 76.895 & 4.394 \\
\hline 19.773 & 2.197 & 2.197 & 6.591 \\
\hline 2.197 & 2.197 & 10.985 & 136.214 \\
\hline 2.197 & 4.394 & 8.788 & 37.349 \\
\hline 2.197 & 4.394 & 2.197 & 24.167 \\
\hline 2.197 & 28.561 & 2.197 & 6.591 \\
\hline 15.379 & 2.197 & 4.394 & 136.214 \\
\hline 4.394 & 4.394 & 2.197 & 2.197 \\
\hline 30.758 & 2.197 & 52.728 & 10.985 \\
\hline 30.758 & 4.394 & 13.182 & 10.985 \\
\hline 13.182 & 2.197 & 474.552 & 4.394 \\
\hline 32.955 & 2.197 & 17.576 & 54.925 \\
\hline 35.152 & 10.985 & 8.788 & 57.122 \\
\hline 5470.53 & 2.197 & 6.591 & 125.229 \\
\hline 19.773 & 2.197 & 250.458 & 2.197 \\
\hline 2.197 & 2.197 & 46.137 & 4.394 \\
\hline 2.197 & 21.97 & 2.197 & 6.591 \\
\hline 30795.3 & 2.197 & 2.197 & 54.925 \\
\hline 6.591 & 4.394 & 2.197 & 85.683 \\
\hline 21.97 & 4.394 & 2.197 & 2.197 \\
\hline 2.197 & 2.197 & 2.197 & 98.865 \\
\hline 2.197 & 4.394 & 8.788 & 4.394 \\
\hline 4.394 & 46.137 & 2.197 & 6.591 \\
\hline 39.546 & 10.985 & 24.167 & 15.379 \\
\hline 2.197 & 28.561 & 232.882 & 92.274 \\
\hline 2.197 & 2.197 & 2.197 & 4.394 \\
\hline 2.197 & 4.394 & 6.591 & 8.788 \\
\hline 6.591 & 4.394 & 2.197 & 4.394 \\
\hline
\end{tabular}




\begin{tabular}{|c|c|c|c|}
\hline 4.394 & 4.394 & 2.197 & 8.788 \\
\hline 2.197 & 2.197 & 19.773 & 15.379 \\
\hline 140.608 & 2.197 & 30.758 & 10.985 \\
\hline 4.394 & 2.197 & 2.197 & 8.788 \\
\hline 2.197 & 28.561 & 2.197 & 2.197 \\
\hline 2.197 & 8.788 & 41.743 & 2.197 \\
\hline 2.197 & 4.394 & 15.379 & 35.152 \\
\hline 2.197 & 6.591 & 26.364 & 4.394 \\
\hline 83.486 & 6.591 & 2.197 & 30.758 \\
\hline 35.152 & 61.516 & 26.364 & 52.728 \\
\hline 4.394 & 6.591 & 2.197 & 10.985 \\
\hline 2.197 & 19.773 & 2.197 & 2.197 \\
\hline 2.197 & 26.364 & 4.394 & 10.985 \\
\hline 17.576 & 2.197 & 24.167 & 120.835 \\
\hline 2.197 & 21.97 & 57.122 & 8.788 \\
\hline 2.197 & 2.197 & 2.197 & 2.197 \\
\hline 35.152 & 26.364 & 4.394 & 63.713 \\
\hline 2.197 & 6.591 & 2.197 & 10.985 \\
\hline 2.197 & 6.591 & 285.61 & 35.152 \\
\hline 2.197 & 4.394 & 37.349 & 2.197 \\
\hline 215.306 & 6.591 & 2.197 & 4.394 \\
\hline 72.501 & 2.197 & 61.516 & 24.167 \\
\hline 6.591 & 2.197 & 10.985 & 48.334 \\
\hline 8.788 & 4.394 & 2.197 & 15.379 \\
\hline 318.565 & 6.591 & 2.197 & 6.591 \\
\hline 2.197 & 276.822 & 59.319 & 19.773 \\
\hline 30.758 & 46.137 & 46.137 & 2.197 \\
\hline 8.788 & 13.182 & 15.379 & 2.197 \\
\hline 2.197 & 2.197 & 2.197 & 4.394 \\
\hline 2.197 & 8.788 & 2.197 & 6.591 \\
\hline 116.441 & 43.94 & 125.229 & 2.197 \\
\hline 2.197 & 2.197 & 2.197 & 461.37 \\
\hline 2.197 & 2.197 & 15.379 & 26.364 \\
\hline 2.197 & 2.197 & 35.152 & 10.985 \\
\hline 246.064 & 2.197 & 571.22 & 6.591 \\
\hline 2.197 & 140.608 & 2.197 & 8.788 \\
\hline 2.197 & 74.698 & 2.197 & 98.865 \\
\hline 4.394 & 2.197 & 4.394 & 2.197 \\
\hline 2.197 & 85.683 & 2.197 & 26.364 \\
\hline 17.576 & 2.197 & 2.197 & 2.197 \\
\hline 2.197 & 98.865 & 4.394 & 63.713 \\
\hline 4.394 & 4.394 & 4.394 & 2.197 \\
\hline 10.985 & 19.773 & 2.197 & 13.182 \\
\hline 13.182 & 6.591 & 2.197 & 15.379 \\
\hline 2.197 & 35.152 & 54.925 & 30.758 \\
\hline 6.591 & 81.289 & 285.61 & 35.152 \\
\hline 48.334 & 2.197 & 15.379 & 2.197 \\
\hline 498.719 & 2.197 & 24.167 & 10.985 \\
\hline 2.197 & 10.985 & 2.197 & 2.197 \\
\hline 21.97 & 2.197 & 123.032 & 32.955 \\
\hline
\end{tabular}




\begin{tabular}{|c|c|c|c|}
\hline 2.197 & 2.197 & 15.379 & 13.182 \\
\hline 164.775 & 6.591 & 4.394 & 13.182 \\
\hline 8.788 & 2.197 & 41.743 & 2.197 \\
\hline 4.394 & 4.394 & 2.197 & 10.985 \\
\hline 2.197 & 2.197 & 10.985 & 6.591 \\
\hline 32.955 & 2.197 & 70.304 & 219.7 \\
\hline 2.197 & 4.394 & 8.788 & 2.197 \\
\hline 21.97 & 4.394 & 10.985 & 4.394 \\
\hline 26.364 & 13.182 & 2.197 & 41.743 \\
\hline 21.97 & 2.197 & 28.561 & 10.985 \\
\hline 6.591 & 2.197 & 19.773 & 8.788 \\
\hline 61.516 & 6.591 & 2.197 & 30.758 \\
\hline 2.197 & 8.788 & 2.197 & 15.379 \\
\hline 2.197 & 28.561 & 63.713 & 6.591 \\
\hline 2.197 & 2.197 & 19.773 & 8.788 \\
\hline 2.197 & 8.788 & 17.576 & 68.107 \\
\hline 21.97 & 6.591 & 65.91 & 61.516 \\
\hline 10.985 & 2.197 & 17.576 & 4.394 \\
\hline 4.394 & 6.591 & 2.197 & 193.336 \\
\hline 63.713 & 4.394 & 2.197 & 6.591 \\
\hline 46.137 & 2.197 & 149.396 & 83.486 \\
\hline 2.197 & 4.394 & 10.985 & 2.197 \\
\hline 79.092 & 61.516 & 2.197 & 43.94 \\
\hline 54.925 & 2.197 & 4.394 & 8.788 \\
\hline 8.788 & 2.197 & 4.394 & 2.197 \\
\hline 5586.97 & 6.591 & 24.167 & 4.394 \\
\hline 2.197 & 2.197 & 4.394 & 2.197 \\
\hline 21.97 & 6.591 & 4.394 & 2.197 \\
\hline 2.197 & 2.197 & 2.197 & 2.197 \\
\hline 10.985 & 4.394 & 2.197 & 2.197 \\
\hline 2.197 & 10.985 & 2.197 & 538.265 \\
\hline 2.197 & 15.379 & 2.197 & 19.773 \\
\hline 2.197 & 13.182 & 2.197 & 8.788 \\
\hline 35.152 & 35.152 & 219.7 & 2.197 \\
\hline 4.394 & 4.394 & 43.94 & 2.197 \\
\hline 2.197 & 2.197 & 6.591 & 6.591 \\
\hline 184.548 & 2.197 & 6.591 & 2.197 \\
\hline 2.197 & 4.394 & 4.394 & 604.175 \\
\hline 2.197 & 4.394 & 2.197 & 6.591 \\
\hline 2.197 & 2.197 & 10.985 & 4.394 \\
\hline 2.197 & 52.728 & 21.97 & 2.197 \\
\hline 4.394 & 2.197 & 2.197 & 6.591 \\
\hline 4.394 & 2.197 & 10.985 & 8.788 \\
\hline 2.197 & 2.197 & 2.197 & 6.591 \\
\hline 2.197 & 116.441 & 90.077 & 4.394 \\
\hline 2.197 & 17.576 & 8.788 & 6.591 \\
\hline 8480.42 & 2.197 & 2.197 & 2.197 \\
\hline 21.97 & 2.197 & 2.197 & 6.591 \\
\hline 10.985 & 2.197 & 2.197 & 4.394 \\
\hline 10.985 & 6.591 & 2.197 & 39.546 \\
\hline
\end{tabular}




\begin{tabular}{|c|c|c|c|}
\hline 6.591 & 4.394 & 2.197 & 8.788 \\
\hline 30.758 & 6.591 & 2.197 & 68.107 \\
\hline 21.97 & 19.773 & 2.197 & 17.576 \\
\hline 15.379 & 6.591 & 2.197 & 10.985 \\
\hline 13.182 & 15.379 & 13.182 & 39.546 \\
\hline 1089.71 & 2.197 & 2.197 & 2.197 \\
\hline 166.972 & 4.394 & 2.197 & 2.197 \\
\hline 2.197 & 2.197 & 4.394 & 26.364 \\
\hline 2.197 & 298.792 & 188.942 & 2.197 \\
\hline 6.591 & 13.182 & 107.653 & 15.379 \\
\hline 155.987 & 24.167 & 2.197 & 21.97 \\
\hline 19.773 & 2.197 & 2.197 & 70.304 \\
\hline 74.698 & 24.167 & 4.394 & 4.394 \\
\hline 8.788 & 6.591 & 2.197 & 4.394 \\
\hline 134.017 & 131.82 & 4.394 & 6.591 \\
\hline 50.531 & 46.137 & 96.668 & 4.394 \\
\hline 4.394 & 17.576 & 26.364 & 173.563 \\
\hline 26.364 & 24.167 & 2.197 & 13.182 \\
\hline 105.456 & 2.197 & 2.197 & 43.94 \\
\hline 131.82 & 8.788 & 107.653 & 30.758 \\
\hline 13.182 & 32.955 & 6.591 & 2.197 \\
\hline 6.591 & 6.591 & 2.197 & 41.743 \\
\hline 435.006 & 2.197 & 2.197 & 70.304 \\
\hline 6.591 & 2.197 & 2.197 & 37.349 \\
\hline 10.985 & 4.394 & 142.805 & 13.182 \\
\hline 30.758 & 2.197 & 41.743 & 114.244 \\
\hline 8.788 & 2.197 & 2.197 & 257.049 \\
\hline 2.197 & 28.561 & 4.394 & 61.516 \\
\hline 246.064 & 8.788 & 2.197 & 30.758 \\
\hline 336.141 & 10.985 & 41.743 & 2.197 \\
\hline 13.182 & 4.394 & 2.197 & 318.565 \\
\hline 52.728 & 4.394 & 8.788 & 2.197 \\
\hline 2.197 & 4.394 & 2.197 & 21.97 \\
\hline 26.364 & 2.197 & 13.182 & 4.394 \\
\hline 4.394 & 2.197 & 2278.29 & 2.197 \\
\hline 2.197 & 4.394 & 13.182 & 386.672 \\
\hline 74.698 & 2.197 & 17.576 & 15.379 \\
\hline 2.197 & 6.591 & 243.867 & 382.278 \\
\hline 8.788 & 2.197 & 85.683 & 854.633 \\
\hline 2.197 & 4.394 & 182.351 & 2.197 \\
\hline 70.304 & 2.197 & 4.394 & 4.394 \\
\hline 6.591 & 2.197 & 2.197 & 4.394 \\
\hline 2.197 & 2.197 & 2.197 & 15.379 \\
\hline 57.122 & 2.197 & 184.548 & 57.122 \\
\hline 2.197 & 6.591 & 136.214 & 2.197 \\
\hline 6.591 & 2.197 & 2.197 & 10.985 \\
\hline 2.197 & 2.197 & 4.394 & 10.985 \\
\hline 6.591 & 2.197 & 28.561 & 2.197 \\
\hline 15.379 & 2.197 & 4.394 & 4.394 \\
\hline 2.197 & 2.197 & 2.197 & 4.394 \\
\hline
\end{tabular}




\begin{tabular}{|c|c|c|c|}
\hline 2.197 & 26.364 & 28.561 & 6.591 \\
\hline 2.197 & 2.197 & 19.773 & 10.985 \\
\hline 83.486 & 17.576 & 8.788 & 4.394 \\
\hline 8.788 & 4.394 & 2.197 & 4.394 \\
\hline 2.197 & 4.394 & 575.614 & 10.985 \\
\hline 85.683 & 10.985 & 54.925 & 48.334 \\
\hline 2.197 & 2.197 & 8.788 & 134.017 \\
\hline 2.197 & 344.929 & 2.197 & 2.197 \\
\hline 35.152 & 2.197 & 4.394 & 48.334 \\
\hline 2.197 & 6.591 & 129.623 & 2.197 \\
\hline 2.197 & 6.591 & 2.197 & 54.925 \\
\hline 10.985 & 6.591 & 4.394 & 182.351 \\
\hline 4.394 & 21.97 & 4.394 & 2.197 \\
\hline 28.561 & 2.197 & 2.197 & 4.394 \\
\hline 109.85 & 6.591 & 8.788 & 94.471 \\
\hline 48.334 & 2.197 & 4.394 & 63.713 \\
\hline 4.394 & 4.394 & 112.047 & 94.471 \\
\hline 13.182 & 13.182 & 2.197 & 4.394 \\
\hline 2.197 & 8.788 & 15.379 & 8.788 \\
\hline 17.576 & 2.197 & 4.394 & 32.955 \\
\hline 2.197 & 8.788 & 4.394 & 239.473 \\
\hline 4.394 & 8.788 & 2.197 & 26.364 \\
\hline 32.955 & 4.394 & 2.197 & 8.788 \\
\hline 13.182 & 1509.34 & 2.197 & 8.788 \\
\hline 145.002 & 19.773 & 6.591 & 590.993 \\
\hline 2.197 & 2.197 & 8.788 & 142.805 \\
\hline 2.197 & 6.591 & 2.197 & 2.197 \\
\hline 10.985 & 17.576 & 2.197 & 8.788 \\
\hline 10.985 & 2.197 & 2.197 & 13.182 \\
\hline 8.788 & 2.197 & 2.197 & 206.518 \\
\hline 35.152 & 90.077 & 39.546 & 19.773 \\
\hline 2.197 & 4.394 & 2.197 & 430.612 \\
\hline 6.591 & 6.591 & 8.788 & 2.197 \\
\hline 2.197 & 98.865 & 43.94 & 804.102 \\
\hline 35.152 & 17.576 & 2.197 & 2.197 \\
\hline 2.197 & 26.364 & 15.379 & 2.197 \\
\hline 50.531 & 37.349 & 2.197 & 142.805 \\
\hline 4.394 & 48.334 & 15.379 & 10.985 \\
\hline 2.197 & 4.394 & 1344.56 & 6.591 \\
\hline 1274.26 & 76.895 & 24.167 & 4.394 \\
\hline 87.88 & 41.743 & 4.394 & 8.788 \\
\hline 35.152 & 4.394 & 2.197 & 32.955 \\
\hline 2.197 & 4.394 & 2.197 & 2.197 \\
\hline 72.501 & 2.197 & 4.394 & 17.576 \\
\hline 19.773 & 8.788 & 103.259 & 63.713 \\
\hline 268.034 & 2.197 & 2.197 & 8.788 \\
\hline 90.077 & 2.197 & 8.788 & 1012.82 \\
\hline 880.997 & 4.394 & 2.197 & 6.591 \\
\hline 4.394 & 13.182 & 4.394 & 21.97 \\
\hline 2.197 & 6.591 & 43.94 & 125.229 \\
\hline
\end{tabular}




\begin{tabular}{|c|c|c|c|}
\hline 2.197 & 30.758 & 8.788 & 8.788 \\
\hline 2.197 & 2.197 & 10.985 & 2.197 \\
\hline 30.758 & 4.394 & 2.197 & 199.927 \\
\hline 186.745 & 13.182 & 2.197 & 452.582 \\
\hline 2.197 & 4.394 & 6.591 & 2.197 \\
\hline 10.985 & 2.197 & 30.758 & 142.805 \\
\hline 2.197 & 17.576 & 2.197 & 24.167 \\
\hline 13.182 & 4.394 & 10.985 & 2.197 \\
\hline 2.197 & 74.698 & 2.197 & 61.516 \\
\hline 303.186 & 13.182 & 2.197 & 2.197 \\
\hline 8.788 & 2.197 & 2.197 & 17.576 \\
\hline 2.197 & 2.197 & 2.197 & 41.743 \\
\hline 1975.1 & 46.137 & 19.773 & 24.167 \\
\hline 30.758 & 2.197 & 2.197 & 17.576 \\
\hline 2.197 & 2.197 & 2.197 & 430.612 \\
\hline 2.197 & 2.197 & 6.591 & 15.379 \\
\hline 57.122 & 4.394 & 13.182 & 10.985 \\
\hline 74.698 & 6.591 & 32.955 & 24.167 \\
\hline 26.364 & 24.167 & 4.394 & 2.197 \\
\hline 6.591 & 6.591 & 107.653 & 131.82 \\
\hline 101.062 & 257.049 & 188.942 & 2.197 \\
\hline 2.197 & 6.591 & 6.591 & 140.608 \\
\hline 2.197 & 10.985 & 57.122 & 26.364 \\
\hline 2.197 & 2.197 & 153.79 & 21.97 \\
\hline 2.197 & 2.197 & 880.997 & 8.788 \\
\hline 2.197 & 6.591 & 48.334 & 4.394 \\
\hline 37.349 & 2.197 & 6.591 & 24.167 \\
\hline 2.197 & 2.197 & 13.182 & 2.197 \\
\hline 3045.04 & 13.182 & 79.092 & 54.925 \\
\hline 15.379 & 6.591 & 4.394 & 292.201 \\
\hline 17.576 & 24.167 & 2.197 & 21.97 \\
\hline 2.197 & 2.197 & 6.591 & 68.107 \\
\hline 76.895 & 2.197 & 13.182 & 8.788 \\
\hline 8.788 & 4.394 & 2.197 & 103.259 \\
\hline 10.985 & 4.394 & 8.788 & 13.182 \\
\hline 2.197 & 2.197 & 4.394 & 19.773 \\
\hline 24.167 & 2.197 & 2.197 & 195.533 \\
\hline 2.197 & 2.197 & 2.197 & 6.591 \\
\hline 63.713 & 2.197 & 2.197 & 8.788 \\
\hline 3328.45 & 2.197 & 2.197 & 2.197 \\
\hline 8.788 & 10.985 & 2.197 & 230.685 \\
\hline 105.456 & 6.591 & 2.197 & 166.972 \\
\hline 547.053 & 217.503 & 2.197 & 43.94 \\
\hline 2.197 & 4.394 & 83.486 & 24.167 \\
\hline 65.91 & 4.394 & 19.773 & 707.434 \\
\hline 4.394 & 2.197 & 17.576 & 37.349 \\
\hline 2.197 & 2.197 & 213.109 & 2.197 \\
\hline 2.197 & 79.092 & 2.197 & 2.197 \\
\hline 19.773 & 4.394 & 4.394 & 2.197 \\
\hline 2.197 & 15.379 & 92.274 & 8.788 \\
\hline
\end{tabular}




\begin{tabular}{|c|c|c|c|}
\hline 4.394 & 2.197 & 2.197 & 2.197 \\
\hline 2.197 & 39.546 & 2.197 & 6.591 \\
\hline 2.197 & 15.379 & 2.197 & 2.197 \\
\hline 2.197 & 4.394 & 2.197 & 13.182 \\
\hline 604.175 & 4.394 & 10.985 & 142.805 \\
\hline 6.591 & 2.197 & 2.197 & 30.758 \\
\hline 4.394 & 24.167 & 4.394 & 21.97 \\
\hline 10.985 & 2.197 & 59.319 & 10.985 \\
\hline 2.197 & 19.773 & 2.197 & 10.985 \\
\hline 13.182 & 19.773 & 8.788 & 13.182 \\
\hline 50.531 & 2.197 & 2.197 & 2.197 \\
\hline 362.505 & 2.197 & 10.985 & 2.197 \\
\hline 13.182 & 2.197 & 349.323 & 2.197 \\
\hline 8.788 & 2.197 & 2.197 & 37.349 \\
\hline 2.197 & 8.788 & 2.197 & 2.197 \\
\hline 8.788 & 2.197 & 6.591 & 94.471 \\
\hline 2.197 & 2.197 & 4.394 & 2.197 \\
\hline 24.167 & 4.394 & 8.788 & 21.97 \\
\hline 2.197 & 4.394 & 8.788 & 8.788 \\
\hline 6.591 & 4.394 & 4.394 & 21.97 \\
\hline 2.197 & 65.91 & 342.732 & 32.955 \\
\hline 2.197 & 6.591 & 28.561 & 19.773 \\
\hline 24.167 & 10.985 & 30.758 & 24.167 \\
\hline 4.394 & 2.197 & 2.197 & 2.197 \\
\hline 32.955 & 2.197 & 15.379 & 6.591 \\
\hline 347.126 & 41.743 & 13.182 & 13.182 \\
\hline 87.88 & 2.197 & 2.197 & 10.985 \\
\hline 4.394 & 4.394 & 13.182 & 305.383 \\
\hline 2.197 & 2.197 & 39.546 & 17.576 \\
\hline 8.788 & 4.394 & 6.591 & 131.82 \\
\hline 19.773 & 8.788 & 2.197 & 50.531 \\
\hline 74.698 & 15.379 & 15.379 & 10.985 \\
\hline 2.197 & 94.471 & 2.197 & 41.743 \\
\hline 2.197 & 26.364 & 2.197 & 6.591 \\
\hline 8.788 & 10.985 & 2.197 & 2.197 \\
\hline 265.837 & 2.197 & 8.788 & 15.379 \\
\hline 2.197 & 8.788 & 4.394 & 120.835 \\
\hline 2.197 & 2.197 & 2.197 & 8.788 \\
\hline 8.788 & 2.197 & 101.062 & 19.773 \\
\hline 6.591 & 21.97 & 2.197 & 606.372 \\
\hline 6.591 & 2.197 & 13.182 & 2.197 \\
\hline 37.349 & 28.561 & 2.197 & 2.197 \\
\hline 123.032 & 4.394 & 2.197 & 2.197 \\
\hline 2.197 & 13.182 & 2.197 & 2.197 \\
\hline 2.197 & 4.394 & 158.184 & 2.197 \\
\hline 2.197 & 4.394 & 61.516 & 8.788 \\
\hline 2.197 & 4.394 & 2.197 & 224.094 \\
\hline 10.985 & 6.591 & 340.535 & 2.197 \\
\hline 24.167 & 2.197 & 2.197 & 43.94 \\
\hline 8.788 & 2.197 & 48.334 & 74.698 \\
\hline
\end{tabular}




\begin{tabular}{|c|c|c|c|}
\hline 2.197 & 4.394 & 15.379 & 2.197 \\
\hline 415.233 & 2.197 & 2.197 & 4.394 \\
\hline 2.197 & 4.394 & 2.197 & 15.379 \\
\hline 10.985 & 4.394 & 15.379 & 149.396 \\
\hline 257.049 & 4.394 & 50.531 & 2.197 \\
\hline 2.197 & 8.788 & 2.197 & 2416.7 \\
\hline 8.788 & 2.197 & 19.773 & 2.197 \\
\hline 2.197 & 2.197 & 2.197 & 2.197 \\
\hline 46.137 & 2.197 & 2.197 & 8.788 \\
\hline 13.182 & 37.349 & 2.197 & 4.394 \\
\hline 221.897 & 2.197 & 2.197 & 2.197 \\
\hline 13.182 & 2.197 & 821.678 & 21.97 \\
\hline 54.925 & 2.197 & 24.167 & 17.576 \\
\hline 8.788 & 2.197 & 15.379 & 2.197 \\
\hline 2.197 & 21.97 & 10.985 & 17.576 \\
\hline 32.955 & 2.197 & 61.516 & 21.97 \\
\hline 4.394 & 2.197 & 6.591 & 76.895 \\
\hline 15.379 & 13.182 & 6.591 & 48.334 \\
\hline 10.985 & 2.197 & 2.197 & 1535.7 \\
\hline 2.197 & 21.97 & 2.197 & 19.773 \\
\hline 6.591 & 6.591 & 2.197 & 6.591 \\
\hline 2.197 & 2.197 & 4.394 & 8.788 \\
\hline 2.197 & 2.197 & 2.197 & 81.289 \\
\hline 13.182 & 2.197 & 107.653 & 30.758 \\
\hline 15.379 & 105.456 & 2.197 & 2.197 \\
\hline 2.197 & 26.364 & 8.788 & 2.197 \\
\hline 2.197 & 26.364 & 4.394 & 6.591 \\
\hline 72.501 & 2.197 & 2.197 & 4.394 \\
\hline 2.197 & 15.379 & 24.167 & 37.349 \\
\hline 2.197 & 2.197 & 8.788 & 2.197 \\
\hline 2.197 & 4.394 & 6.591 & 13.182 \\
\hline 2.197 & 10.985 & 4.394 & 1432.44 \\
\hline 164.775 & 2.197 & 2.197 & 2.197 \\
\hline 2.197 & 2.197 & 46.137 & 197.73 \\
\hline 2.197 & 4.394 & 41.743 & 21.97 \\
\hline 32.955 & 8.788 & 11081.7 & 6.591 \\
\hline 2.197 & 2.197 & 2.197 & 39.546 \\
\hline 2.197 & 4.394 & 19.773 & 4.394 \\
\hline 2.197 & 13.182 & 2.197 & 3517.4 \\
\hline 175.76 & 19.773 & 843.648 & 6.591 \\
\hline 2.197 & 131.82 & 2.197 & 2.197 \\
\hline 4.394 & 2.197 & 8.788 & 15.379 \\
\hline 149.396 & 61.516 & 8.788 & 4.394 \\
\hline 15.379 & 158.184 & 6004.4 & 13.182 \\
\hline 6.591 & 41.743 & 94.471 & 2.197 \\
\hline 2.197 & 6.591 & 2.197 & 17.576 \\
\hline 2.197 & 2.197 & 13.182 & 72.501 \\
\hline 4.394 & 4.394 & 4.394 & 2.197 \\
\hline 19.773 & 2.197 & 52.728 & 2.197 \\
\hline 26.364 & 2.197 & 2.197 & 24.167 \\
\hline
\end{tabular}




\begin{tabular}{|c|c|c|c|}
\hline 2.197 & 6.591 & 52.728 & 2.197 \\
\hline 2.197 & 2.197 & 4.394 & 14010.3 \\
\hline 4.394 & 2.197 & 4.394 & 6.591 \\
\hline 4.394 & 57.122 & 4.394 & 39.546 \\
\hline 2.197 & 2.197 & 2.197 & 2.197 \\
\hline 8190.42 & 8.788 & 83.486 & 2.197 \\
\hline 2.197 & 13.182 & 2.197 & 24.167 \\
\hline 79.092 & 6.591 & 2.197 & 70.304 \\
\hline 8.788 & 2.197 & 2.197 & 2.197 \\
\hline 35.152 & 4.394 & 8.788 & 6.591 \\
\hline 2.197 & 26.364 & 2.197 & 35.152 \\
\hline 2.197 & 2.197 & 2.197 & 37.349 \\
\hline 232.882 & 2.197 & 4.394 & 2.197 \\
\hline 17.576 & 2.197 & 17.576 & 52.728 \\
\hline 70.304 & 10.985 & 10.985 & 6.591 \\
\hline 74.698 & 8.788 & 6.591 & 2.197 \\
\hline 24.167 & 63.713 & 4.394 & 8.788 \\
\hline 48.334 & 15.379 & 8.788 & 15.379 \\
\hline 4.394 & 2.197 & 4.394 & 4.394 \\
\hline 4.394 & 2.197 & 2.197 & 10.985 \\
\hline 4.394 & 2.197 & 6.591 & 83.486 \\
\hline 2.197 & 6.591 & 8.788 & 43.94 \\
\hline 2.197 & 17.576 & 13.182 & 26.364 \\
\hline 2.197 & 2.197 & 35.152 & 4.394 \\
\hline 109.85 & 17.576 & 15.379 & 617.357 \\
\hline 2.197 & 6.591 & 2.197 & 6.591 \\
\hline 2.197 & 2.197 & 2.197 & 6.591 \\
\hline 32.955 & 2.197 & 4.394 & 19.773 \\
\hline 15.379 & 6.591 & 13.182 & 2.197 \\
\hline 26.364 & 4.394 & 2.197 & 21.97 \\
\hline 32.955 & 2.197 & 32.955 & 37.349 \\
\hline 2.197 & 650.312 & 10.985 & 13.182 \\
\hline 41.743 & 2.197 & 1219.33 & 6.591 \\
\hline 70.304 & 2.197 & 105.456 & 13.182 \\
\hline 41.743 & 4.394 & 37.349 & 105.456 \\
\hline 90.077 & 4.394 & 15.379 & 24.167 \\
\hline 2.197 & 4.394 & 155.987 & 4.394 \\
\hline 13.182 & 10.985 & 26.364 & 15.379 \\
\hline 463.567 & 4.394 & 2.197 & 136.214 \\
\hline 8.788 & 2.197 & 103.259 & 83.486 \\
\hline 2.197 & 6.591 & 2.197 & 4071.04 \\
\hline 2.197 & 6.591 & 30.758 & 6.591 \\
\hline 2.197 & 2.197 & 13.182 & 24.167 \\
\hline 2.197 & 10.985 & 2.197 & 30.758 \\
\hline 8.788 & 6.591 & 54.925 & 2.197 \\
\hline 2.197 & 2.197 & 19.773 & 8.788 \\
\hline 8.788 & 125.229 & 2.197 & 2.197 \\
\hline 13.182 & 2.197 & 19.773 & 68.107 \\
\hline 248.261 & 2.197 & 37.349 & 6.591 \\
\hline 2.197 & 380.081 & 63.713 & 2.197 \\
\hline
\end{tabular}




\begin{tabular}{|c|c|c|c|}
\hline 2.197 & 2.197 & 8.788 & 6.591 \\
\hline 54.925 & 2.197 & 2.197 & 140.608 \\
\hline 2.197 & 2.197 & 30.758 & 30.758 \\
\hline 8.788 & 2.197 & 2.197 & 39.546 \\
\hline 13.182 & 4.394 & 2.197 & 21.97 \\
\hline 4.394 & 2.197 & 4.394 & 59.319 \\
\hline 2.197 & 2.197 & 6.591 & 509.704 \\
\hline 6.591 & 63.713 & 6.591 & 2.197 \\
\hline 2.197 & 13.182 & 48.334 & 2.197 \\
\hline 2.197 & 2.197 & 6.591 & 50.531 \\
\hline 2.197 & 2.197 & 4.394 & 10.985 \\
\hline 2.197 & 2.197 & 2.197 & 2.197 \\
\hline 2.197 & 279.019 & 2.197 & 2.197 \\
\hline 2.197 & 2.197 & 531.674 & 6.591 \\
\hline 257.049 & 26.364 & 4.394 & 2.197 \\
\hline 2.197 & 2.197 & 2.197 & 2.197 \\
\hline 26.364 & 19.773 & 6.591 & 2.197 \\
\hline 17.576 & 140.608 & 4.394 & 13.182 \\
\hline 2.197 & 8.788 & 10.985 & 575.614 \\
\hline 28.561 & 13.182 & 2.197 & 2.197 \\
\hline 30.758 & 2.197 & 10.985 & 2.197 \\
\hline 224.094 & 2.197 & 2.197 & 2.197 \\
\hline 1091.91 & 503.113 & 4.394 & 452.582 \\
\hline 2.197 & 10.985 & 2.197 & 17.576 \\
\hline 19.773 & 2.197 & 2.197 & 235.079 \\
\hline 6.591 & 4.394 & 2.197 & 1869.65 \\
\hline 733.798 & 90.077 & 2.197 & 24.167 \\
\hline 15.379 & 2.197 & 2.197 & 4.394 \\
\hline 10.985 & 2.197 & 30.758 & 6.591 \\
\hline 15.379 & 8.788 & 2.197 & 4.394 \\
\hline 4.394 & 4.394 & 2.197 & 2.197 \\
\hline 8.788 & 2.197 & 8.788 & 6.591 \\
\hline 19.773 & 21.97 & 41.743 & 118.638 \\
\hline 2.197 & 2.197 & 17.576 & 63.713 \\
\hline 4.394 & 4.394 & 2.197 & 4.394 \\
\hline 79.092 & 2.197 & 2.197 & 2.197 \\
\hline 2.197 & 94.471 & 2.197 & 4.394 \\
\hline 4.394 & 2.197 & 4.394 & 85.683 \\
\hline 2.197 & 2.197 & 2.197 & 228.488 \\
\hline 8.788 & 8.788 & 2.197 & 2.197 \\
\hline 2.197 & 13.182 & 138.411 & 17.576 \\
\hline 10.985 & 19.773 & 116.441 & 2.197 \\
\hline 15.379 & 52.728 & 15.379 & 13.182 \\
\hline 2.197 & 1085.32 & 4.394 & 13.182 \\
\hline 4.394 & 4.394 & 1603.81 & 57.122 \\
\hline 4.394 & 30.758 & 2.197 & 46.137 \\
\hline 2.197 & 10.985 & 24.167 & 2.197 \\
\hline 2.197 & 68.107 & 4.394 & 114.244 \\
\hline 13.182 & 4.394 & 4.394 & 424.021 \\
\hline 4.394 & 28.561 & 2.197 & 2.197 \\
\hline
\end{tabular}




\begin{tabular}{|c|c|c|c|}
\hline 15.379 & 2.197 & 2.197 & 28.561 \\
\hline 10.985 & 90.077 & 2.197 & 6.591 \\
\hline 2.197 & 4.394 & 98.865 & 63.713 \\
\hline 2.197 & 4.394 & 59.319 & 50.531 \\
\hline 41.743 & 6.591 & 2.197 & 4.394 \\
\hline 46.137 & 32.955 & 2.197 & 43.94 \\
\hline 2.197 & 2.197 & 2.197 & 2.197 \\
\hline 1241.3 & 21.97 & 2.197 & 199.927 \\
\hline 4.394 & 2.197 & 79.092 & 2.197 \\
\hline 4.394 & 2.197 & 443.794 & 10.985 \\
\hline 10.985 & 2.197 & 2.197 & 2.197 \\
\hline 13.182 & 8.788 & 2.197 & 2.197 \\
\hline 10.985 & 2.197 & 2.197 & 26.364 \\
\hline 32.955 & 6.591 & 2.197 & 6.591 \\
\hline 2.197 & 10.985 & 2.197 & 10.985 \\
\hline 2.197 & 2.197 & 10.985 & 8.788 \\
\hline 32.955 & 24.167 & 13.182 & 13.182 \\
\hline 8.788 & 2.197 & 2.197 & 61.516 \\
\hline 24.167 & 98.865 & 6.591 & 26.364 \\
\hline 2.197 & 6.591 & 21.97 & 129.623 \\
\hline 2.197 & 4.394 & 41.743 & 2.197 \\
\hline 2.197 & 4.394 & 4.394 & 2.197 \\
\hline 2.197 & 98.865 & 6.591 & 28.561 \\
\hline 2.197 & 8.788 & 8.788 & 2.197 \\
\hline 59.319 & 2.197 & 322.959 & 2.197 \\
\hline 39.546 & 8.788 & 2.197 & 10.985 \\
\hline 4.394 & 4.394 & 21.97 & 2.197 \\
\hline 4.394 & 43.94 & 2.197 & 2.197 \\
\hline 2.197 & 4.394 & 8.788 & 8.788 \\
\hline 10.985 & 10.985 & 977.665 & 2.197 \\
\hline 2.197 & 2.197 & 2.197 & 76.895 \\
\hline 6.591 & 2.197 & 19.773 & 10.985 \\
\hline 57.122 & 2.197 & 292.201 & 13.182 \\
\hline 10.985 & 24.167 & 21.97 & 13.182 \\
\hline 13.182 & 6.591 & 6.591 & 35.152 \\
\hline 2.197 & 4.394 & 2.197 & 6.591 \\
\hline 21.97 & 2.197 & 4.394 & 4.394 \\
\hline 6.591 & 2.197 & 4.394 & 123.032 \\
\hline 2.197 & 134.017 & 39.546 & 6.591 \\
\hline 8.788 & 35.152 & 15.379 & 26.364 \\
\hline 287.807 & 8.788 & 77835.3 & 2.197 \\
\hline 15.379 & 2.197 & 2.197 & 10.985 \\
\hline 74.698 & 4.394 & 2.197 & 2.197 \\
\hline 2.197 & 43.94 & 8.788 & 79.092 \\
\hline 4.394 & 4.394 & 48.334 & 8.788 \\
\hline 6.591 & 4.394 & 4.394 & 2.197 \\
\hline 26.364 & 2.197 & 2.197 & 4.394 \\
\hline 2.197 & 6.591 & 2.197 & 21.97 \\
\hline 26.364 & 2.197 & 15.379 & 8.788 \\
\hline 35.152 & 2.197 & 24.167 & 2.197 \\
\hline
\end{tabular}




\begin{tabular}{|c|c|c|c|}
\hline 13.182 & 2.197 & 4.394 & 32.955 \\
\hline 2.197 & 35.152 & 4.394 & 219.7 \\
\hline 6.591 & 6.591 & 4.394 & 4.394 \\
\hline 2.197 & 2.197 & 182.351 & 6.591 \\
\hline 4.394 & 19.773 & 4.394 & 2.197 \\
\hline 2.197 & 10.985 & 2.197 & 57.122 \\
\hline 17.576 & 241.67 & 2.197 & 19.773 \\
\hline 2.197 & 162.578 & 2.197 & 10.985 \\
\hline 61.516 & 50.531 & 2.197 & 30.758 \\
\hline 13.182 & 2.197 & 2.197 & 37.349 \\
\hline 10.985 & 2.197 & 4.394 & 68.107 \\
\hline 13.182 & 8.788 & 83.486 & 300.989 \\
\hline 85.683 & 8.788 & 17.576 & 26.364 \\
\hline 8.788 & 2.197 & 2.197 & 2.197 \\
\hline 2.197 & 85.683 & 2.197 & 10.985 \\
\hline 6.591 & 2.197 & 48.334 & 10.985 \\
\hline 8.788 & 101.062 & 2.197 & 43.94 \\
\hline 4.394 & 145.002 & 6.591 & 15.379 \\
\hline 307.58 & 41.743 & 2.197 & 10.985 \\
\hline 43.94 & 68.107 & 4.394 & 2.197 \\
\hline 15.379 & 2.197 & 2.197 & 2.197 \\
\hline 2.197 & 2.197 & 6.591 & 333.944 \\
\hline 4.394 & 4.394 & 6.591 & 481.143 \\
\hline 193.336 & 2.197 & 483.34 & 2.197 \\
\hline 10.985 & 2.197 & 83.486 & 197.73 \\
\hline 63.713 & 21.97 & 59.319 & 90.077 \\
\hline 2.197 & 2.197 & 6.591 & 112.047 \\
\hline 2.197 & 2.197 & 4.394 & 4.394 \\
\hline 2.197 & 13.182 & 4.394 & 4.394 \\
\hline 4.394 & 2.197 & 2.197 & 2.197 \\
\hline 364.702 & 2.197 & 2.197 & 136.214 \\
\hline 2.197 & 4.394 & 4.394 & 8.788 \\
\hline 252.655 & 35.152 & 15.379 & 52.728 \\
\hline 2.197 & 4.394 & 24.167 & 61.516 \\
\hline 2.197 & 4.394 & 4.394 & 81.289 \\
\hline 2.197 & 4.394 & 2.197 & 26.364 \\
\hline 24.167 & 39.546 & 2.197 & 131.82 \\
\hline 10.985 & 8.788 & 6.591 & 3007.69 \\
\hline 4.394 & 2.197 & 8.788 & 6.591 \\
\hline 4.394 & 4.394 & 65.91 & 15.379 \\
\hline 21.97 & 12604.2 & 8.788 & 2.197 \\
\hline 151.593 & 76.895 & 2.197 & 70.304 \\
\hline 2.197 & 10.985 & 105.456 & 4.394 \\
\hline 76.895 & 13.182 & 2.197 & 21.97 \\
\hline 19.773 & 10.985 & 4.394 & 10.985 \\
\hline 72.501 & 8.788 & 8.788 & 54.925 \\
\hline 8.788 & 4.394 & 623.948 & 4.394 \\
\hline 48.334 & 19.773 & 35.152 & 4.394 \\
\hline 2.197 & 41.743 & 43.94 & 81.289 \\
\hline 61.516 & 28.561 & 2.197 & 2.197 \\
\hline
\end{tabular}




\begin{tabular}{|c|c|c|c|}
\hline 2.197 & 2.197 & 10.985 & 2.197 \\
\hline 2.197 & 85.683 & 28.561 & 2.197 \\
\hline 57.122 & 8.788 & 24.167 & 35.152 \\
\hline 2.197 & 65.91 & 2.197 & 28.561 \\
\hline 2.197 & 8.788 & 43.94 & 46.137 \\
\hline 92.274 & 6.591 & 21.97 & 2.197 \\
\hline 48.334 & 2.197 & 263.64 & 74.698 \\
\hline 6.591 & 2.197 & 92.274 & 10.985 \\
\hline 4.394 & 4.394 & 2.197 & 2.197 \\
\hline 2.197 & 2.197 & 28.561 & 2.197 \\
\hline 2.197 & 2.197 & 94.471 & 4.394 \\
\hline 8.788 & 4.394 & 2.197 & 4.394 \\
\hline 8.788 & 2.197 & 2.197 & 28.561 \\
\hline 15.379 & 4.394 & 46.137 & 32.955 \\
\hline 2.197 & 4.394 & 2.197 & 15.379 \\
\hline 10.985 & 6.591 & 6.591 & 70.304 \\
\hline 24.167 & 32.955 & 2.197 & 10.985 \\
\hline 6.591 & 511.901 & 2.197 & 2.197 \\
\hline 17.576 & 37.349 & 2.197 & 2.197 \\
\hline 15.379 & 4.394 & 2.197 & 10.985 \\
\hline 19.773 & 4.394 & 4.394 & 272.428 \\
\hline 65.91 & 4.394 & 2.197 & 74.698 \\
\hline 2.197 & 6.591 & 2.197 & 28.561 \\
\hline 6.591 & 35.152 & 2.197 & 6.591 \\
\hline 182.351 & 2.197 & 2.197 & 21.97 \\
\hline 105.456 & 4.394 & 17.576 & 8.788 \\
\hline 4.394 & 4.394 & 254.852 & 8.788 \\
\hline 2.197 & 4.394 & 59.319 & 54.925 \\
\hline 2.197 & 2.197 & 2.197 & 235.079 \\
\hline 2.197 & 48.334 & 10.985 & 50.531 \\
\hline 2.197 & 2.197 & 28.561 & 57.122 \\
\hline 17.576 & 13.182 & 6.591 & 17.576 \\
\hline 498.719 & 68.107 & 2.197 & 10.985 \\
\hline 2.197 & 2.197 & 2.197 & 184.548 \\
\hline 4.394 & 2.197 & 57.122 & 19.773 \\
\hline 6.591 & 4.394 & 15.379 & 4.394 \\
\hline 6.591 & 46.137 & 63.713 & 164.775 \\
\hline 2.197 & 2.197 & 5617.73 & 39.546 \\
\hline 4.394 & 2.197 & 2.197 & 21.97 \\
\hline 4.394 & 32.955 & 2.197 & 13.182 \\
\hline 6.591 & 13.182 & 2.197 & 28.561 \\
\hline 2.197 & 4.394 & 2.197 & 30.758 \\
\hline 2.197 & 4.394 & 2.197 & 4.394 \\
\hline 8.788 & 4.394 & 79.092 & 50.531 \\
\hline 26.364 & 2.197 & 2.197 & 46.137 \\
\hline 6.591 & 13.182 & 2.197 & 54.925 \\
\hline 79.092 & 13.182 & 2.197 & 39.546 \\
\hline 2.197 & 4.394 & 540.462 & 6.591 \\
\hline 1243.5 & 8.788 & 17.576 & 2.197 \\
\hline 46.137 & 30.758 & 2.197 & 10.985 \\
\hline
\end{tabular}




\begin{tabular}{|c|c|c|c|}
\hline 2.197 & 4.394 & 13.182 & 68.107 \\
\hline 2.197 & 8.788 & 15.379 & 26.364 \\
\hline 2.197 & 8.788 & 37.349 & 85.683 \\
\hline 70.304 & 35.152 & 13.182 & 120.835 \\
\hline 10.985 & 10.985 & 887.588 & 2.197 \\
\hline 35.152 & 2.197 & 4.394 & 48.334 \\
\hline 13.182 & 2.197 & 48.334 & 2.197 \\
\hline 87.88 & 2.197 & 15.379 & 2.197 \\
\hline 6.591 & 48.334 & 4.394 & 4.394 \\
\hline 2.197 & 26.364 & 2.197 & 2.197 \\
\hline 13.182 & 2.197 & 2.197 & 4.394 \\
\hline 19.773 & 2.197 & 2.197 & 4.394 \\
\hline 13.182 & 2.197 & 2.197 & 90.077 \\
\hline 17.576 & 43.94 & 2.197 & 2.197 \\
\hline 155.987 & 4.394 & 2.197 & 96.668 \\
\hline 804.102 & 26.364 & 2.197 & 6.591 \\
\hline 8.788 & 2.197 & 2.197 & 142.805 \\
\hline 10.985 & 2.197 & 4.394 & 6.591 \\
\hline 2.197 & 2.197 & 4.394 & 61.516 \\
\hline 2.197 & 4.394 & 10.985 & 2.197 \\
\hline 2.197 & 6.591 & 13.182 & 4.394 \\
\hline 4.394 & 4.394 & 2.197 & 2.197 \\
\hline 4.394 & 2.197 & 134.017 & 250.458 \\
\hline 4.394 & 2.197 & 8.788 & 2.197 \\
\hline 393.263 & 2.197 & 17.576 & 13.182 \\
\hline 683.267 & 6.591 & 1181.99 & 43.94 \\
\hline 2.197 & 48.334 & 83.486 & 2.197 \\
\hline 8.788 & 13.182 & 2.197 & 4.394 \\
\hline 2.197 & 4.394 & 2.197 & 26.364 \\
\hline 2.197 & 13.182 & 4804.84 & 61.516 \\
\hline 4.394 & 6.591 & 10.985 & 8.788 \\
\hline 2.197 & 6.591 & 13.182 & 50.531 \\
\hline 373.49 & 4.394 & 2.197 & 4.394 \\
\hline 6.591 & 4.394 & 21.97 & 131.82 \\
\hline 76.895 & 21.97 & 4.394 & 35.152 \\
\hline 4.394 & 2.197 & 10.985 & 116.441 \\
\hline 35.152 & 4.394 & 8.788 & 2.197 \\
\hline 4.394 & 28.561 & 2.197 & 30.758 \\
\hline 2.197 & 30.758 & 39.546 & 2.197 \\
\hline 2.197 & 17.576 & 4.394 & 2.197 \\
\hline 127.426 & 57.122 & 4.394 & 2.197 \\
\hline 2.197 & 2.197 & 17.576 & 2.197 \\
\hline 2.197 & 48.334 & 2.197 & 30.758 \\
\hline 2.197 & 28.561 & 2.197 & 6.591 \\
\hline 4.394 & 8.788 & 39.546 & 37.349 \\
\hline 2.197 & 4.394 & 2.197 & 2.197 \\
\hline 30.758 & 149.396 & 2.197 & 8.788 \\
\hline 2.197 & 15.379 & 17.576 & 2.197 \\
\hline 13.182 & 4.394 & 10.985 & 4.394 \\
\hline 2.197 & 19.773 & 2.197 & 4.394 \\
\hline
\end{tabular}




\begin{tabular}{|c|c|c|c|}
\hline 2.197 & 6.591 & 28.561 & 13.182 \\
\hline 8.788 & 145.002 & 2.197 & 2.197 \\
\hline 8.788 & 2.197 & 6.591 & 19.773 \\
\hline 21.97 & 4.394 & 2.197 & 2.197 \\
\hline 17.576 & 2.197 & 2.197 & 894.179 \\
\hline 8.788 & 2.197 & 6.591 & 626.145 \\
\hline 10.985 & 2.197 & 252.655 & 2.197 \\
\hline 13.182 & 4.394 & 2.197 & 4.394 \\
\hline 4.394 & 17.576 & 2.197 & 94.471 \\
\hline 2.197 & 2.197 & 2.197 & 2.197 \\
\hline 2.197 & 2.197 & 2.197 & 2.197 \\
\hline 4.394 & 2.197 & 2.197 & 4.394 \\
\hline 4.394 & 2.197 & 13.182 & 2.197 \\
\hline 52.728 & 2.197 & 101.062 & 296.595 \\
\hline 21.97 & 21.97 & 2.197 & 8.788 \\
\hline 96.668 & 10.985 & 2.197 & 2.197 \\
\hline 63.713 & 6.591 & 2.197 & 30.758 \\
\hline 65.91 & 24.167 & 2.197 & 2.197 \\
\hline 2.197 & 2.197 & 8.788 & 393.263 \\
\hline 2.197 & 2.197 & 2.197 & 28.561 \\
\hline 85.683 & 2.197 & 8.788 & 2.197 \\
\hline 28.561 & 2.197 & 2.197 & 2.197 \\
\hline 2.197 & 4.394 & 4.394 & 4.394 \\
\hline 2.197 & 4.394 & 50.531 & 2.197 \\
\hline 2.197 & 2.197 & 6.591 & 6.591 \\
\hline 57.122 & 10.985 & 151.593 & 4.394 \\
\hline 2.197 & 2.197 & 4.394 & 6.591 \\
\hline 2.197 & 2.197 & 2.197 & 21.97 \\
\hline 2.197 & 4.394 & 2.197 & 8.788 \\
\hline 4.394 & 48.334 & 2.197 & 2.197 \\
\hline 2.197 & 213.109 & 2.197 & 2.197 \\
\hline 2.197 & 2.197 & 8.788 & 4.394 \\
\hline 6.591 & 32.955 & 26.364 & 13.182 \\
\hline 2.197 & 15.379 & 28.561 & 19.773 \\
\hline 2.197 & 2.197 & 2.197 & 8.788 \\
\hline 28.561 & 6.591 & 470.158 & 28.561 \\
\hline 2.197 & 4.394 & 2.197 & 30.758 \\
\hline 2.197 & 4.394 & 32.955 & 175.76 \\
\hline 2.197 & 2.197 & 2.197 & 6.591 \\
\hline 2.197 & 4.394 & 2.197 & 8.788 \\
\hline 4.394 & 4.394 & 292.201 & 32.955 \\
\hline 10.985 & 2.197 & 10.985 & 46.137 \\
\hline 2.197 & 4.394 & 2.197 & 15.379 \\
\hline 94.471 & 2.197 & 13.182 & 2.197 \\
\hline 2.197 & 2.197 & 230.685 & 2.197 \\
\hline 2.197 & 6.591 & 4.394 & 4.394 \\
\hline 2.197 & 6.591 & 2.197 & 19.773 \\
\hline 6.591 & 4.394 & 2.197 & 15.379 \\
\hline 2.197 & 8.788 & 2.197 & 19.773 \\
\hline 2.197 & 2.197 & 2.197 & 68.107 \\
\hline
\end{tabular}




\begin{tabular}{|c|c|c|c|}
\hline 4.394 & 2.197 & 924.937 & 39.546 \\
\hline 564.629 & 76.895 & 4.394 & 2.197 \\
\hline 2.197 & 2.197 & 8.788 & 2.197 \\
\hline 17.576 & 228.488 & 2.197 & 2.197 \\
\hline 10.985 & 4.394 & 2.197 & 10.985 \\
\hline 37.349 & 395.46 & 105.456 & 10.985 \\
\hline 6.591 & 103.259 & 2.197 & 19.773 \\
\hline 2.197 & 2.197 & 2.197 & 369.096 \\
\hline 8.788 & 2.197 & 4934.46 & 2.197 \\
\hline 19.773 & 377.884 & 19.773 & 2.197 \\
\hline 2.197 & 6.591 & 2.197 & 26.364 \\
\hline 2.197 & 46.137 & 2.197 & 2.197 \\
\hline 4.394 & 2.197 & 10.985 & 2.197 \\
\hline 4.394 & 41.743 & 4.394 & 10.985 \\
\hline 4.394 & 6.591 & 28.561 & 307.58 \\
\hline 2.197 & 6.591 & 43.94 & 2.197 \\
\hline 6.591 & 4.394 & 2.197 & 149.396 \\
\hline 2.197 & 4.394 & 4.394 & 2.197 \\
\hline 494.325 & 6.591 & 10.985 & 2.197 \\
\hline 8.788 & 4.394 & 2.197 & 10.985 \\
\hline 2.197 & 2.197 & 2.197 & 32.955 \\
\hline 17.576 & 2.197 & 2.197 & 463.567 \\
\hline 13.182 & 2.197 & 4.394 & 26.364 \\
\hline 2.197 & 2.197 & 2.197 & 733.798 \\
\hline 94.471 & 4.394 & 4.394 & 2.197 \\
\hline 17.576 & 2.197 & 41.743 & 4.394 \\
\hline 15.379 & 4.394 & 4.394 & 32.955 \\
\hline 2.197 & 2.197 & 2.197 & 2.197 \\
\hline 2.197 & 35.152 & 35.152 & 2.197 \\
\hline 26.364 & 2.197 & 26.364 & 24.167 \\
\hline 2.197 & 2.197 & 2.197 & 670.085 \\
\hline 4117.18 & 2.197 & 57.122 & 2.197 \\
\hline 28.561 & 2.197 & 2.197 & 83.486 \\
\hline 2.197 & 2.197 & 2.197 & 65.91 \\
\hline 8.788 & 2.197 & 96.668 & 6.591 \\
\hline 2.197 & 2.197 & 2.197 & 79.092 \\
\hline 2.197 & 2.197 & 2.197 & 4.394 \\
\hline 4.394 & 6.591 & 2.197 & 261.443 \\
\hline 2.197 & 2.197 & 2.197 & 81.289 \\
\hline 2.197 & 8.788 & 850.239 & 2.197 \\
\hline 2.197 & 2.197 & 37.349 & 59.319 \\
\hline 10.985 & 2.197 & 2.197 & 2.197 \\
\hline 6.591 & 2.197 & 2.197 & 4.394 \\
\hline 147.199 & 2.197 & 2.197 & 795.314 \\
\hline 129.623 & 4.394 & 2.197 & 19.773 \\
\hline 13.182 & 2.197 & 162.578 & 21.97 \\
\hline 2.197 & 4.394 & 2.197 & 2.197 \\
\hline 70.304 & 2.197 & 2.197 & 2.197 \\
\hline 6.591 & 309.777 & 19.773 & 10.985 \\
\hline 4.394 & 8.788 & 2.197 & 2.197 \\
\hline
\end{tabular}




\begin{tabular}{|c|c|c|c|}
\hline 2.197 & 2.197 & 2.197 & 6.591 \\
\hline 2.197 & 2.197 & 2.197 & 96.668 \\
\hline 2.197 & 2.197 & 2.197 & 2.197 \\
\hline 10.985 & 2.197 & 2.197 & 2.197 \\
\hline 2.197 & 2.197 & 30.758 & 2.197 \\
\hline 2.197 & 10.985 & 32.955 & 2.197 \\
\hline 35.152 & 13.182 & 4.394 & 2.197 \\
\hline 43.94 & 2.197 & 2.197 & 24.167 \\
\hline 2.197 & 2.197 & 2.197 & 2.197 \\
\hline 2.197 & 21.97 & 2.197 & 2.197 \\
\hline 4.394 & 4.394 & 63.713 & 634.933 \\
\hline 21.97 & 4.394 & 2.197 & 8.788 \\
\hline 2.197 & 4.394 & 2.197 & 10.985 \\
\hline 2.197 & 59.319 & 8.788 & 81.289 \\
\hline 2.197 & 6.591 & 50.531 & 169.169 \\
\hline 204.321 & 6.591 & 2.197 & 2.197 \\
\hline 4.394 & 8.788 & 2.197 & 136.214 \\
\hline 13.182 & 136.214 & 8.788 & 237.276 \\
\hline 92.274 & 15.379 & 2.197 & 232.882 \\
\hline 8.788 & 17.576 & 2.197 & 6.591 \\
\hline 487.734 & 6.591 & 2.197 & 13.182 \\
\hline 2.197 & 4.394 & 2.197 & 6.591 \\
\hline 43.94 & 2.197 & 8.788 & 112.047 \\
\hline 10.985 & 2.197 & 2.197 & 17.576 \\
\hline 125.229 & 2.197 & 4.394 & 15.379 \\
\hline 32.955 & 2.197 & 2.197 & 85.683 \\
\hline 4.394 & 2.197 & 15.379 & 2.197 \\
\hline 15.379 & 2.197 & 118.638 & 43.94 \\
\hline 4.394 & 4.394 & 10.985 & 81.289 \\
\hline 2.197 & 6.591 & 30.758 & 28.561 \\
\hline 2.197 & 59.319 & 21.97 & 2.197 \\
\hline 8.788 & 2.197 & 35747.4 & 15.379 \\
\hline 2.197 & 52.728 & 2.197 & 2.197 \\
\hline 2.197 & 48.334 & 2.197 & 2.197 \\
\hline 2.197 & 2.197 & 2.197 & 757.965 \\
\hline 10.985 & 6.591 & 2.197 & 2.197 \\
\hline 21.97 & 2.197 & 2.197 & 2.197 \\
\hline 2.197 & 4.394 & 2.197 & 2.197 \\
\hline 54.925 & 17.576 & 2.197 & 4.394 \\
\hline 15.379 & 2.197 & 6.591 & 8.788 \\
\hline 10.985 & 17.576 & 2.197 & 21.97 \\
\hline 79.092 & 4.394 & 773.344 & 252.655 \\
\hline 54.925 & 2.197 & 2.197 & 6.591 \\
\hline 2.197 & 4.394 & 4.394 & 8.788 \\
\hline 2.197 & 2.197 & 6.591 & 15.379 \\
\hline 8.788 & 19.773 & 166.972 & 65.91 \\
\hline 2.197 & 26.364 & 4.394 & 2.197 \\
\hline 3594.29 & 2.197 & 19.773 & 2.197 \\
\hline 2.197 & 2.197 & 8.788 & 2.197 \\
\hline 2.197 & 4.394 & 6.591 & 10.985 \\
\hline
\end{tabular}




\begin{tabular}{|c|c|c|c|}
\hline 10.985 & 2.197 & 17.576 & 61.516 \\
\hline 24.167 & 6.591 & 4.394 & 13.182 \\
\hline 4.394 & 155.987 & 2.197 & 90.077 \\
\hline 2.197 & 4.394 & 24.167 & 8.788 \\
\hline 1133.65 & 24.167 & 8.788 & 2.197 \\
\hline 2.197 & 96.668 & 2.197 & 149.396 \\
\hline 8.788 & 2.197 & 2.197 & 30.758 \\
\hline 6.591 & 4.394 & 2.197 & 19.773 \\
\hline 8.788 & 6.591 & 24.167 & 2.197 \\
\hline 2.197 & 4.394 & 6.591 & 2.197 \\
\hline 2.197 & 2.197 & 13.182 & 8.788 \\
\hline 2.197 & 2.197 & 4.394 & 30.758 \\
\hline 8.788 & 8.788 & 2.197 & 2.197 \\
\hline 6.591 & 6.591 & 2.197 & 13.182 \\
\hline 41.743 & 6.591 & 26.364 & 6.591 \\
\hline 2.197 & 15.379 & 2.197 & 24.167 \\
\hline 2.197 & 2.197 & 2.197 & 2.197 \\
\hline 2.197 & 35.152 & 4.394 & 2.197 \\
\hline 2.197 & 10.985 & 678.873 & 19.773 \\
\hline 96.668 & 2.197 & 2.197 & 46.137 \\
\hline 2.197 & 2.197 & 10.985 & 61.516 \\
\hline 32.955 & 4.394 & 2.197 & 79.092 \\
\hline 10.985 & 2.197 & 2.197 & 10.985 \\
\hline 6.591 & 4.394 & 54.925 & 19.773 \\
\hline 123.032 & 2.197 & 4.394 & 2.197 \\
\hline 360.308 & 2.197 & 2.197 & 46.137 \\
\hline 32.955 & 41.743 & 4.394 & 2.197 \\
\hline 2.197 & 2.197 & 2.197 & 173.563 \\
\hline 2.197 & 32.955 & 2.197 & 142.805 \\
\hline 2.197 & 19.773 & 235.079 & 39.546 \\
\hline 96.668 & 21.97 & 2.197 & 21.97 \\
\hline 13.182 & 2.197 & 2.197 & 21.97 \\
\hline 2.197 & 2.197 & 41.743 & 6.591 \\
\hline 8.788 & 13.182 & 19.773 & 103.259 \\
\hline 2.197 & 76.895 & 2.197 & 6.591 \\
\hline 4.394 & 15.379 & 2.197 & 2594.66 \\
\hline 2.197 & 13.182 & 2.197 & 43.94 \\
\hline 243.867 & 32.955 & 2.197 & 2.197 \\
\hline 186.745 & 8.788 & 2.197 & 13.182 \\
\hline 59.319 & 8.788 & 2.197 & 19.773 \\
\hline 2.197 & 2.197 & 96.668 & 2.197 \\
\hline 21.97 & 2.197 & 76.895 & 2.197 \\
\hline 2.197 & 309.777 & 213.109 & 173.563 \\
\hline 2.197 & 6.591 & 2.197 & 1825.71 \\
\hline 4.394 & 2.197 & 10.985 & 2.197 \\
\hline 6.591 & 2.197 & 6.591 & 347.126 \\
\hline 2.197 & 2.197 & 2.197 & 4.394 \\
\hline 162.578 & 6.591 & 17.576 & 39.546 \\
\hline 83.486 & 13.182 & 6.591 & 26.364 \\
\hline 10.985 & 13.182 & 2.197 & 2.197 \\
\hline
\end{tabular}




\begin{tabular}{|c|c|c|c|}
\hline 6.591 & 39.546 & 103.259 & 13.182 \\
\hline 6.591 & 6.591 & 2.197 & 8.788 \\
\hline 19.773 & 2.197 & 2.197 & 10.985 \\
\hline 2.197 & 8.788 & 37.349 & 4.394 \\
\hline 10.985 & 2.197 & 10.985 & 8.788 \\
\hline 4.394 & 2.197 & 2.197 & 15.379 \\
\hline 2.197 & 2.197 & 21.97 & 54.925 \\
\hline 2.197 & 2.197 & 30.758 & 175.76 \\
\hline 2.197 & 2.197 & 4.394 & 6.591 \\
\hline 127.426 & 2.197 & 2.197 & 224.094 \\
\hline 544.856 & 65.91 & 162.578 & 2.197 \\
\hline 14695.7 & 2.197 & 2.197 & 4.394 \\
\hline 8.788 & 2.197 & 6.591 & 369.096 \\
\hline 2.197 & 155.987 & 10.985 & 65.91 \\
\hline 43.94 & 35.152 & 10.985 & 17.576 \\
\hline 17.576 & 2.197 & 8.788 & 59.319 \\
\hline 2.197 & 6.591 & 2.197 & 13.182 \\
\hline 237.276 & 2.197 & 2.197 & 2.197 \\
\hline 21.97 & 2.197 & 136.214 & 2.197 \\
\hline 445.991 & 8.788 & 2.197 & 2.197 \\
\hline 52.728 & 85.683 & 41.743 & 2.197 \\
\hline 2.197 & 2.197 & 8.788 & 30.758 \\
\hline 4.394 & 26.364 & 4.394 & 6.591 \\
\hline 4.394 & 26.364 & 120.835 & 90.077 \\
\hline 2.197 & 54.925 & 10.985 & 2.197 \\
\hline 39.546 & 65.91 & 6.591 & 2.197 \\
\hline 2.197 & 6.591 & 2.197 & 6.591 \\
\hline 85.683 & 2.197 & 8.788 & 4.394 \\
\hline 2.197 & 2.197 & 2.197 & 290.004 \\
\hline 8.788 & 4.394 & 10.985 & 2.197 \\
\hline 2.197 & 8.788 & 2.197 & 2.197 \\
\hline 13.182 & 235.079 & 6.591 & 2.197 \\
\hline 2.197 & 76.895 & 26.364 & 4.394 \\
\hline 2.197 & 10.985 & 2.197 & 30.758 \\
\hline 2.197 & 4.394 & 48.334 & 2.197 \\
\hline 65.91 & 2.197 & 182.351 & 4.394 \\
\hline 4.394 & 2.197 & 2.197 & 41.743 \\
\hline 19.773 & 8.788 & 2.197 & 4.394 \\
\hline 2.197 & 10.985 & 2.197 & 28.561 \\
\hline 2.197 & 2.197 & 257.049 & 2.197 \\
\hline 24.167 & 37.349 & 2.197 & 162.578 \\
\hline 2.197 & 37.349 & 24.167 & 2.197 \\
\hline 41.743 & 37.349 & 2.197 & 24.167 \\
\hline 43.94 & 2.197 & 2.197 & 19.773 \\
\hline 105.456 & 2.197 & 2.197 & 2.197 \\
\hline 17.576 & 2.197 & 17.576 & 43.94 \\
\hline 28.561 & 2.197 & 8.788 & 2.197 \\
\hline 2.197 & 2.197 & 8.788 & 6.591 \\
\hline 2.197 & 17.576 & 10.985 & 2.197 \\
\hline 2.197 & 4.394 & 2.197 & 4.394 \\
\hline
\end{tabular}




\begin{tabular}{|c|c|c|c|}
\hline 2.197 & 6.591 & 2.197 & 10.985 \\
\hline 10.985 & 4139.15 & 4.394 & 39.546 \\
\hline 2.197 & 6.591 & 230.685 & 26.364 \\
\hline 8.788 & 2.197 & 28.561 & 2.197 \\
\hline 4.394 & 13.182 & 2.197 & 17.576 \\
\hline 2.197 & 215.306 & 26.364 & 10.985 \\
\hline 2.197 & 8.788 & 2.197 & 2.197 \\
\hline 4.394 & 2.197 & 4.394 & 2.197 \\
\hline 4.394 & 41.743 & 4.394 & 2.197 \\
\hline 6.591 & 296.595 & 138.411 & 41.743 \\
\hline 4.394 & 2.197 & 2.197 & 147.199 \\
\hline 2.197 & 32.955 & 182.351 & 6.591 \\
\hline 4.394 & 105.456 & 13.182 & 217.503 \\
\hline 2.197 & 15.379 & 2.197 & 4.394 \\
\hline 2.197 & 2.197 & 8.788 & 6.591 \\
\hline 2.197 & 48.334 & 2.197 & 2.197 \\
\hline 2.197 & 2.197 & 13.182 & 83.486 \\
\hline 19.773 & 4.394 & 59.319 & 1843.28 \\
\hline 2.197 & 2.197 & 15.379 & 2.197 \\
\hline 4.394 & 243.867 & 2.197 & 4.394 \\
\hline 2.197 & 2.197 & 2.197 & 10.985 \\
\hline 2.197 & 145.002 & 2.197 & 8.788 \\
\hline 6.591 & 4.394 & 2.197 & 17.576 \\
\hline 2.197 & 2.197 & 2.197 & 48.334 \\
\hline 43.94 & 2.197 & 166.972 & 6.591 \\
\hline 2.197 & 8.788 & 2.197 & 10.985 \\
\hline 2.197 & 2.197 & 2.197 & 6.591 \\
\hline 2.197 & 309.777 & 4.394 & 103.259 \\
\hline 975.468 & 10.985 & 2.197 & 2.197 \\
\hline 2.197 & 15.379 & 2.197 & 2.197 \\
\hline 2.197 & 4.394 & 13.182 & 6.591 \\
\hline 687.661 & 2.197 & 24.167 & 19.773 \\
\hline 30.758 & 2.197 & 94.471 & 41.743 \\
\hline 2.197 & 52.728 & 17.576 & 21.97 \\
\hline 54.925 & 32.955 & 2.197 & 2.197 \\
\hline 2.197 & 24.167 & 24.167 & 57.122 \\
\hline 1772.98 & 32.955 & 8.788 & 8.788 \\
\hline 26.364 & 6.591 & 21.97 & 2.197 \\
\hline 2.197 & 26.364 & 10.985 & 6.591 \\
\hline 37.349 & 13.182 & 6.591 & 166.972 \\
\hline 149.396 & 6.591 & 2.197 & 28.561 \\
\hline 6.591 & 6.591 & 46.137 & 4.394 \\
\hline 2.197 & 2.197 & 41.743 & 13.182 \\
\hline 303.186 & 37.349 & 2.197 & 32.955 \\
\hline 138.411 & 224.094 & 32.955 & 2.197 \\
\hline 19.773 & 2.197 & 4.394 & 4.394 \\
\hline 4.394 & 15.379 & 79.092 & 2.197 \\
\hline 4.394 & 8.788 & 90.077 & 8.788 \\
\hline 24.167 & 197.73 & 2.197 & 127.426 \\
\hline 2.197 & 17.576 & 2.197 & 123.032 \\
\hline
\end{tabular}




\begin{tabular}{|c|c|c|c|}
\hline 2.197 & 76.895 & 2.197 & 85.683 \\
\hline 2.197 & 15.379 & 2.197 & 6.591 \\
\hline 2.197 & 50.531 & 6.591 & 17.576 \\
\hline 8.788 & 26.364 & 2.197 & 30.758 \\
\hline 15.379 & 74.698 & 6.591 & 15.379 \\
\hline 2.197 & 65.91 & 4.394 & 6.591 \\
\hline 2.197 & 10.985 & 4.394 & 2.197 \\
\hline 2.197 & 353.717 & 43.94 & 131.82 \\
\hline 35.152 & 4.394 & 2.197 & 57.122 \\
\hline 283.413 & 10.985 & 13.182 & 2.197 \\
\hline 300.989 & 15.379 & 8.788 & 4.394 \\
\hline 21.97 & 2.197 & 2.197 & 21.97 \\
\hline 10.985 & 6.591 & 54.925 & 6.591 \\
\hline 630.539 & 4.394 & 2.197 & 98.865 \\
\hline 79.092 & 2.197 & 52.728 & 35.152 \\
\hline 424.021 & 2.197 & 43.94 & 2.197 \\
\hline 2.197 & 2.197 & 147.199 & 10.985 \\
\hline 2.197 & 2.197 & 4.394 & 19.773 \\
\hline 206.518 & 2.197 & 94.471 & 54.925 \\
\hline 72.501 & 46.137 & 63.713 & 199.927 \\
\hline 65.91 & 15.379 & 26.364 & 70.304 \\
\hline 2.197 & 32.955 & 2.197 & 61.516 \\
\hline 2.197 & 4.394 & 2.197 & 6.591 \\
\hline 2.197 & 4.394 & 2.197 & 4.394 \\
\hline 2.197 & 21.97 & 2.197 & 2.197 \\
\hline 4.394 & 74.698 & 2.197 & 6.591 \\
\hline 2.197 & 28.561 & 1181.99 & 4.394 \\
\hline 10.985 & 10.985 & 171.366 & 4.394 \\
\hline 2.197 & 2.197 & 4.394 & 19.773 \\
\hline 2.197 & 2.197 & 2.197 & 13.182 \\
\hline 21.97 & 4.394 & 4.394 & 10.985 \\
\hline 2.197 & 4.394 & 2.197 & 13.182 \\
\hline 76.895 & 2.197 & 2.197 & 2.197 \\
\hline 98.865 & 2.197 & 2.197 & 6.591 \\
\hline 8.788 & 4.394 & 39.546 & 2.197 \\
\hline 4.394 & 123.032 & 2.197 & 10.985 \\
\hline 2.197 & 13.182 & 118.638 & 26.364 \\
\hline 2.197 & 6.591 & 41.743 & 2.197 \\
\hline 2.197 & 4.394 & 2.197 & 320.762 \\
\hline 2.197 & 2.197 & 4.394 & 26.364 \\
\hline 4.394 & 92.274 & 57.122 & 50.531 \\
\hline 21.97 & 4.394 & 2.197 & 10.985 \\
\hline 8.788 & 2.197 & 2.197 & 26.364 \\
\hline 2.197 & 2.197 & 2.197 & 8.788 \\
\hline 2.197 & 2.197 & 4.394 & 41.743 \\
\hline 57.122 & 26.364 & 17.576 & 2.197 \\
\hline 8.788 & 8.788 & 17.576 & 4.394 \\
\hline 37.349 & 2.197 & 2.197 & 13.182 \\
\hline 2.197 & 448.188 & 17.576 & 19.773 \\
\hline 28185.3 & 30.758 & 10.985 & 6.591 \\
\hline
\end{tabular}




\begin{tabular}{|c|c|c|c|}
\hline 2.197 & 46.137 & 6.591 & 8.788 \\
\hline 2.197 & 4.394 & 37.349 & 94.471 \\
\hline 2.197 & 10.985 & 2.197 & 4.394 \\
\hline 2.197 & 35.152 & 4.394 & 65.91 \\
\hline 4.394 & 70.304 & 4.394 & 10.985 \\
\hline 46.137 & 4.394 & 4.394 & 37.349 \\
\hline 6.591 & 28.561 & 4.394 & 294.398 \\
\hline 2.197 & 2.197 & 707.434 & 2.197 \\
\hline 10.985 & 215.306 & 2.197 & 41.743 \\
\hline 24.167 & 21.97 & 193.336 & 2.197 \\
\hline 4.394 & 15.379 & 21.97 & 15.379 \\
\hline 3449.29 & 2.197 & 19.773 & 32.955 \\
\hline 2.197 & 2.197 & 2.197 & 224.094 \\
\hline 2.197 & 59.319 & 2.197 & 2.197 \\
\hline 2.197 & 30.758 & 2.197 & 48.334 \\
\hline 70.304 & 43.94 & 2.197 & 2.197 \\
\hline 41.743 & 28.561 & 2.197 & 2.197 \\
\hline 4.394 & 2.197 & 2.197 & 2.197 \\
\hline 127.426 & 2.197 & 85.683 & 459.173 \\
\hline 2.197 & 2.197 & 2.197 & 8.788 \\
\hline 2.197 & 8.788 & 54.925 & 28.561 \\
\hline 2.197 & 46.137 & 6.591 & 8.788 \\
\hline 2.197 & 6.591 & 17.576 & 50.531 \\
\hline 15.379 & 8.788 & 2.197 & 109.85 \\
\hline 2016.85 & 26.364 & 2.197 & 287.807 \\
\hline 279.019 & 4.394 & 6.591 & 61.516 \\
\hline 39.546 & 76.895 & 19.773 & 19.773 \\
\hline 4.394 & 6.591 & 6.591 & 103.259 \\
\hline 4.394 & 17.576 & 147.199 & 30.758 \\
\hline 2.197 & 13.182 & 2.197 & 43.94 \\
\hline 87.88 & 4.394 & 2.197 & 17.576 \\
\hline 2.197 & 6.591 & 2.197 & 26.364 \\
\hline 13.182 & 37.349 & 4.394 & 160.381 \\
\hline 199.927 & 2.197 & 4.394 & 2.197 \\
\hline 2.197 & 4.394 & 17.576 & 52.728 \\
\hline 2.197 & 21.97 & 854.633 & 17.576 \\
\hline 35.152 & 8.788 & 6.591 & 10.985 \\
\hline 21.97 & 6.591 & 2.197 & 6.591 \\
\hline 2.197 & 2.197 & 2.197 & 57.122 \\
\hline 2.197 & 105.456 & 2.197 & 28.561 \\
\hline 2.197 & 2.197 & 10.985 & 112.047 \\
\hline 2.197 & 2.197 & 17.576 & 125.229 \\
\hline 24.167 & 85.683 & 2.197 & 96.668 \\
\hline 30.758 & 4.394 & 4.394 & 13.182 \\
\hline 70.304 & 2.197 & 19.773 & 2.197 \\
\hline 21.97 & 2.197 & 50.531 & 35.152 \\
\hline 10.985 & 10.985 & 10.985 & 8.788 \\
\hline 10.985 & 2.197 & 226.291 & 2.197 \\
\hline 4.394 & 6.591 & 2.197 & 26.364 \\
\hline 2.197 & 39.546 & 6.591 & 92.274 \\
\hline
\end{tabular}




\begin{tabular}{|c|c|c|c|}
\hline 2.197 & 2.197 & 2.197 & 13.182 \\
\hline 6.591 & 24.167 & 2.197 & 19.773 \\
\hline 19.773 & 6.591 & 13.182 & 24.167 \\
\hline 52.728 & 2.197 & 19.773 & 6.591 \\
\hline 4.394 & 2.197 & 2.197 & 46.137 \\
\hline 4.394 & 57.122 & 10.985 & 8.788 \\
\hline 13.182 & 8.788 & 4.394 & 4.394 \\
\hline 2.197 & 4.394 & 24.167 & 6.591 \\
\hline 6.591 & 4.394 & 619.554 & 219.7 \\
\hline 61.516 & 2.197 & 59.319 & 26.364 \\
\hline 61.516 & 2.197 & 2.197 & 2.197 \\
\hline 46.137 & 2.197 & 2.197 & 2.197 \\
\hline 2.197 & 37.349 & 4.394 & 19.773 \\
\hline 393.263 & 2.197 & 2.197 & 68.107 \\
\hline 2.197 & 13.182 & 2.197 & 4.394 \\
\hline 4.394 & 4.394 & 487.734 & 6.591 \\
\hline 2.197 & 2.197 & 4.394 & 13.182 \\
\hline 21.97 & 79.092 & 4.394 & 4.394 \\
\hline 74.698 & 6.591 & 46.137 & 4.394 \\
\hline 17.576 & 10.985 & 4.394 & 79.092 \\
\hline 21.97 & 2.197 & 2.197 & 4.394 \\
\hline 4.394 & 68.107 & 2.197 & 35.152 \\
\hline 2.197 & 4.394 & 72.501 & 37.349 \\
\hline 2.197 & 2.197 & 2.197 & 24.167 \\
\hline 4.394 & 4.394 & 2.197 & 21.97 \\
\hline 2.197 & 6.591 & 2.197 & 2774.81 \\
\hline 2.197 & 2.197 & 35.152 & 4.394 \\
\hline 15.379 & 4.394 & 46.137 & 21.97 \\
\hline 103.259 & 140.608 & 10.985 & 50.531 \\
\hline 30.758 & 2.197 & 4.394 & 2.197 \\
\hline 15.379 & 2.197 & 43.94 & 24.167 \\
\hline 30.758 & 21.97 & 2.197 & 26.364 \\
\hline 8.788 & 6.591 & 35.152 & 4.394 \\
\hline 2.197 & 13.182 & 2.197 & 6.591 \\
\hline 28.561 & 6.591 & 2.197 & 10.985 \\
\hline 2.197 & 4.394 & 2.197 & 68.107 \\
\hline 2.197 & 2.197 & 43.94 & 8.788 \\
\hline 180.154 & 2.197 & 2.197 & 127.426 \\
\hline 2.197 & 2.197 & 2.197 & 19.773 \\
\hline 6.591 & 2.197 & 4.394 & 241.67 \\
\hline 4.394 & 6.591 & 74.698 & 15.379 \\
\hline 4.394 & 4.394 & 2.197 & 52.728 \\
\hline 35.152 & 2.197 & 237.276 & 198099 \\
\hline 2.197 & 2.197 & 2.197 & 8.788 \\
\hline 4.394 & 2.197 & 10.985 & 21.97 \\
\hline 39.546 & 2.197 & 4.394 & 2.197 \\
\hline 2.197 & 2.197 & 6.591 & 6.591 \\
\hline 8.788 & 2.197 & 2.197 & 4.394 \\
\hline 94.471 & 131.82 & 2.197 & 17.576 \\
\hline 2.197 & 48.334 & 4.394 & 4.394 \\
\hline
\end{tabular}




\begin{tabular}{|c|c|c|c|}
\hline 2.197 & 21.97 & 6.591 & 15.379 \\
\hline 98.865 & 4.394 & 28.561 & 32.955 \\
\hline 4.394 & 50.531 & 10.985 & 41.743 \\
\hline 2.197 & 6.591 & 30.758 & 2.197 \\
\hline 90.077 & 2.197 & 15.379 & 2.197 \\
\hline 369.096 & 6.591 & 118.638 & 83.486 \\
\hline 4.394 & 61.516 & 17.576 & 8.788 \\
\hline 6.591 & 24.167 & 2.197 & 309.777 \\
\hline 6.591 & 2.197 & 2.197 & 2.197 \\
\hline 6.591 & 13.182 & 2.197 & 46.137 \\
\hline 8.788 & 61.516 & 8.788 & 101.062 \\
\hline 145.002 & 61.516 & 2.197 & 4.394 \\
\hline 2.197 & 2.197 & 2.197 & 61.516 \\
\hline 4.394 & 17.576 & 2.197 & 10.985 \\
\hline 68.107 & 41.743 & 2.197 & 2.197 \\
\hline 2.197 & 2.197 & 6.591 & 4.394 \\
\hline 4.394 & 24.167 & 2.197 & 19.773 \\
\hline 10.985 & 4.394 & 2.197 & 43.94 \\
\hline 2.197 & 15.379 & 2.197 & 63.713 \\
\hline 19.773 & 8.788 & 2.197 & 6.591 \\
\hline 10.985 & 4.394 & 2.197 & 8.788 \\
\hline 2.197 & 8.788 & 2.197 & 105.456 \\
\hline 2.197 & 4.394 & 4.394 & 2.197 \\
\hline 2.197 & 2.197 & 6.591 & 50.531 \\
\hline 2.197 & 8.788 & 4.394 & 2.197 \\
\hline 76.895 & 2.197 & 4.394 & 2.197 \\
\hline 10.985 & 6.591 & 10.985 & 2.197 \\
\hline 17.576 & 6.591 & 2.197 & 26.364 \\
\hline 21.97 & 2.197 & 24.167 & 15.379 \\
\hline 10.985 & 2.197 & 63.713 & 17.576 \\
\hline 30.758 & 2.197 & 10.985 & 4.394 \\
\hline 19.773 & 2.197 & 8.788 & 17.576 \\
\hline 13.182 & 2.197 & 6.591 & 4.394 \\
\hline 26.364 & 2.197 & 2.197 & 2.197 \\
\hline 10.985 & 10.985 & 2.197 & 151.593 \\
\hline 13.182 & 28.561 & 2.197 & 19.773 \\
\hline 2.197 & 46.137 & 2.197 & 57.122 \\
\hline 2.197 & 10.985 & 4.394 & 6.591 \\
\hline 4.394 & 4.394 & 30.758 & 2.197 \\
\hline 2.197 & 4.394 & 35.152 & 318.565 \\
\hline 54.925 & 2.197 & 15.379 & 2.197 \\
\hline 4.394 & 59.319 & 125.229 & 24.167 \\
\hline 57.122 & 48.334 & 6.591 & 145.002 \\
\hline 24.167 & 57.122 & 4.394 & 210.912 \\
\hline 2.197 & 10.985 & 2.197 & 8.788 \\
\hline 2.197 & 2.197 & 2.197 & 17.576 \\
\hline 57.122 & 4.394 & 2.197 & 4.394 \\
\hline 70.304 & 243.867 & 2.197 & 15.379 \\
\hline 2.197 & 10.985 & 6.591 & 138.411 \\
\hline 6.591 & 2.197 & 4.394 & 17.576 \\
\hline
\end{tabular}




\begin{tabular}{|c|c|c|c|}
\hline 37.349 & 52.728 & 30.758 & 4.394 \\
\hline 48.334 & 17.576 & 2.197 & 39.546 \\
\hline 32.955 & 17.576 & 28.561 & 6.591 \\
\hline 8.788 & 17.576 & 2.197 & 94.471 \\
\hline 21.97 & 2.197 & 28.561 & 4.394 \\
\hline 2.197 & 125.229 & 2.197 & 4.394 \\
\hline 371.293 & 2.197 & 8.788 & 2.197 \\
\hline 2.197 & 4.394 & 2.197 & 120.835 \\
\hline 2.197 & 2.197 & 2.197 & 30.758 \\
\hline 79.092 & 2.197 & 2.197 & 8.788 \\
\hline 2.197 & 101.062 & 4.394 & 4.394 \\
\hline 30.758 & 2.197 & 4.394 & 4.394 \\
\hline 32.955 & 2.197 & 2.197 & 6.591 \\
\hline 2.197 & 2.197 & 2.197 & 32.955 \\
\hline 2.197 & 2.197 & 2.197 & 103.259 \\
\hline 2.197 & 2.197 & 72.501 & 46.137 \\
\hline 4.394 & 4.394 & 6.591 & 4.394 \\
\hline 10.985 & 4.394 & 2.197 & 30.758 \\
\hline 30.758 & 4.394 & 4.394 & 13.182 \\
\hline 2.197 & 2.197 & 30.758 & 2.197 \\
\hline 10.985 & 2.197 & 19.773 & 13.182 \\
\hline 2.197 & 4.394 & 24.167 & 41.743 \\
\hline 4.394 & 8.788 & 109.85 & 28.561 \\
\hline 138.411 & 2.197 & 2.197 & 46.137 \\
\hline 6.591 & 2.197 & 2.197 & 21.97 \\
\hline 32.955 & 48.334 & 10.985 & 81.289 \\
\hline 85.683 & 4.394 & 494.325 & 46.137 \\
\hline 4.394 & 4.394 & 2.197 & 162.578 \\
\hline 15.379 & 2.197 & 13.182 & 2.197 \\
\hline 276.822 & 105.456 & 96.668 & 4.394 \\
\hline 59.319 & 2.197 & 43.94 & 180.154 \\
\hline 19.773 & 15.379 & 2.197 & 4.394 \\
\hline 17.576 & 2.197 & 2.197 & 6.591 \\
\hline 450.385 & 8.788 & 2.197 & 4.394 \\
\hline 17.576 & 2.197 & 4.394 & 74.698 \\
\hline 4.394 & 52.728 & 208.715 & 2.197 \\
\hline 2126.7 & 2.197 & 2.197 & 98.865 \\
\hline 59.319 & 2.197 & 4.394 & 35.152 \\
\hline 17.576 & 21.97 & 2.197 & 50.531 \\
\hline 4.394 & 2.197 & 2.197 & 50.531 \\
\hline 10.985 & 10.985 & 4.394 & 314.171 \\
\hline 24.167 & 19.773 & 663.494 & 287.807 \\
\hline 2.197 & 70.304 & 4.394 & 10.985 \\
\hline 4.394 & 74.698 & 26.364 & 6.591 \\
\hline 4.394 & 2.197 & 4.394 & 17.576 \\
\hline 52.728 & 2.197 & 19.773 & 15.379 \\
\hline 2.197 & 4.394 & 4.394 & 10.985 \\
\hline 10.985 & 123.032 & 2.197 & 2.197 \\
\hline 19.773 & 2.197 & 191.139 & 2.197 \\
\hline 6.591 & 2.197 & 2.197 & 76.895 \\
\hline
\end{tabular}




\begin{tabular}{|c|c|c|c|}
\hline 2.197 & 4.394 & 4.394 & 72.501 \\
\hline 4.394 & 4.394 & 4.394 & 8.788 \\
\hline 6.591 & 2.197 & 2.197 & 13.182 \\
\hline 4.394 & 2.197 & 30.758 & 2.197 \\
\hline 2.197 & 2.197 & 2.197 & 63.713 \\
\hline 221.897 & 2.197 & 2.197 & 123.032 \\
\hline 24.167 & 4.394 & 134.017 & 4.394 \\
\hline 4.394 & 8.788 & 336.141 & 8.788 \\
\hline 2.197 & 4.394 & 2.197 & 169.169 \\
\hline 30.758 & 2.197 & 2.197 & 50.531 \\
\hline 13.182 & 4.394 & 2.197 & 4.394 \\
\hline 2.197 & 2.197 & 4.394 & 2.197 \\
\hline 50.531 & 4.394 & 26.364 & 2.197 \\
\hline 2.197 & 2.197 & 2.197 & 4.394 \\
\hline 6.591 & 2.197 & 17.576 & 79.092 \\
\hline 2.197 & 8.788 & 2.197 & 10.985 \\
\hline 10.985 & 2.197 & 2.197 & 17.576 \\
\hline 2.197 & 2.197 & 2.197 & 52.728 \\
\hline 151.593 & 65.91 & 10.985 & 153.79 \\
\hline 4.394 & 2.197 & 6.591 & 173.563 \\
\hline 2.197 & 4.394 & 2.197 & 4.394 \\
\hline 4.394 & 351.52 & 2.197 & 15.379 \\
\hline 13.182 & 17.576 & 2.197 & 210.912 \\
\hline 6.591 & 72.501 & 2.197 & 10.985 \\
\hline 4.394 & 15.379 & 2.197 & 6.591 \\
\hline 48.334 & 54.925 & 2.197 & 41.743 \\
\hline 8.788 & 795.314 & 28.561 & 4.394 \\
\hline 4.394 & 8.788 & 6.591 & 30.758 \\
\hline 4.394 & 4.394 & 43.94 & 30.758 \\
\hline 6.591 & 2.197 & 4.394 & 10.985 \\
\hline 2.197 & 2.197 & 2.197 & 81.289 \\
\hline 2.197 & 96.668 & 2.197 & 2.197 \\
\hline 2.197 & 4.394 & 2.197 & 169.169 \\
\hline 13.182 & 30.758 & 4.394 & 4.394 \\
\hline 8.788 & 6.591 & 13.182 & 6.591 \\
\hline 10.985 & 32.955 & 4.394 & 4.394 \\
\hline 2.197 & 149.396 & 28.561 & 2.197 \\
\hline 2.197 & 19.773 & 2.197 & 74.698 \\
\hline 2.197 & 2.197 & 4.394 & 13.182 \\
\hline 4.394 & 37.349 & 2.197 & 4.394 \\
\hline 10.985 & 2.197 & 202.124 & 13.182 \\
\hline 182.351 & 2.197 & 21.97 & 2.197 \\
\hline 2.197 & 2.197 & 8.788 & 2.197 \\
\hline 4.394 & 2.197 & 59.319 & 107.653 \\
\hline 13.182 & 2.197 & 2.197 & 52.728 \\
\hline 2.197 & 2.197 & 2.197 & 149.396 \\
\hline 90.077 & 2.197 & 2.197 & 180.154 \\
\hline 79.092 & 17.576 & 4.394 & 6.591 \\
\hline 402.051 & 2.197 & 155.987 & 131.82 \\
\hline 15.379 & 127.426 & 4.394 & 6.591 \\
\hline
\end{tabular}




\begin{tabular}{|c|c|c|c|}
\hline 2.197 & 8.788 & 2.197 & 6.591 \\
\hline 116.441 & 2.197 & 57.122 & 13.182 \\
\hline 46.137 & 2.197 & 2.197 & 37.349 \\
\hline 54.925 & 2.197 & 2.197 & 136.214 \\
\hline 13.182 & 2.197 & 2.197 & 177.957 \\
\hline 2.197 & 4.394 & 2.197 & 2.197 \\
\hline 92.274 & 6.591 & 2.197 & 13.182 \\
\hline 177.957 & 2.197 & 2.197 & 2.197 \\
\hline 2.197 & 2.197 & 2.197 & 4.394 \\
\hline 2.197 & 2.197 & 2.197 & 6.591 \\
\hline 2.197 & 28.561 & 4.394 & 362.505 \\
\hline 8.788 & 2.197 & 2.197 & 1452.22 \\
\hline 2.197 & 39.546 & 4.394 & 30.758 \\
\hline 17.576 & 17.576 & 4.394 & 32.955 \\
\hline 4.394 & 57.122 & 2.197 & 10.985 \\
\hline 2.197 & 2.197 & 8.788 & 13.182 \\
\hline 4.394 & 10.985 & 6.591 & 30.758 \\
\hline 2.197 & 2.197 & 10.985 & 8.788 \\
\hline 2.197 & 2.197 & 2.197 & 46.137 \\
\hline 2.197 & 2.197 & 2.197 & 4.394 \\
\hline 4.394 & 241.67 & 21.97 & 15.379 \\
\hline 8.788 & 28.561 & 6.591 & 2.197 \\
\hline 4.394 & 2.197 & 2.197 & 83.486 \\
\hline 6.591 & 2.197 & 2.197 & 4.394 \\
\hline 76.895 & 2.197 & 8.788 & 4.394 \\
\hline 2.197 & 4.394 & 125.229 & 50.531 \\
\hline 6.591 & 24.167 & 2.197 & 81.289 \\
\hline 2.197 & 2.197 & 59.319 & 50.531 \\
\hline 2.197 & 8.788 & 6.591 & 94.471 \\
\hline 2.197 & 61.516 & 8.788 & 2.197 \\
\hline 28.561 & 39.546 & 10.985 & 4.394 \\
\hline 6.591 & 2.197 & 2.197 & 30.758 \\
\hline 2.197 & 2.197 & 103.259 & 2.197 \\
\hline 92.274 & 2.197 & 28.561 & 17.576 \\
\hline 2.197 & 8.788 & 2.197 & 6.591 \\
\hline 41.743 & 2.197 & 4.394 & 2.197 \\
\hline 2.197 & 2.197 & 2.197 & 503.113 \\
\hline 21.97 & 2.197 & 2.197 & 26.364 \\
\hline 2.197 & 65.91 & 15.379 & 2.197 \\
\hline 2.197 & 8.788 & 87.88 & 2.197 \\
\hline 2.197 & 2.197 & 6.591 & 2.197 \\
\hline 2.197 & 2.197 & 8.788 & 4.394 \\
\hline 2.197 & 32.955 & 6.591 & 6.591 \\
\hline 2.197 & 4.394 & 2.197 & 6.591 \\
\hline 6.591 & 2.197 & 2.197 & 2.197 \\
\hline 2.197 & 6.591 & 10.985 & 2.197 \\
\hline 17.576 & 6.591 & 4.394 & 4.394 \\
\hline 2.197 & 37.349 & 4.394 & 2.197 \\
\hline 59.319 & 28.561 & 6.591 & 72.501 \\
\hline 4.394 & 2.197 & 2.197 & 2.197 \\
\hline
\end{tabular}




\begin{tabular}{|c|c|c|c|}
\hline 6.591 & 2.197 & 4.394 & 61.516 \\
\hline 26.364 & 4.394 & 35.152 & 6.591 \\
\hline 13.182 & 10.985 & 13.182 & 90.077 \\
\hline 2.197 & 8.788 & 775.541 & 10.985 \\
\hline 8.788 & 2.197 & 2.197 & 28.561 \\
\hline 50.531 & 4.394 & 2.197 & 32.955 \\
\hline 70.304 & 6.591 & 4.394 & 57.122 \\
\hline 2.197 & 6.591 & 2.197 & 48.334 \\
\hline 32.955 & 4.394 & 2.197 & 2.197 \\
\hline 2.197 & 15.379 & 2.197 & 6.591 \\
\hline 54.925 & 15.379 & 2.197 & 35.152 \\
\hline 2.197 & 6.591 & 2.197 & 2.197 \\
\hline 17.576 & 8.788 & 4.394 & 8.788 \\
\hline 6.591 & 90.077 & 8.788 & 138.411 \\
\hline 4.394 & 48.334 & 3128.53 & 6.591 \\
\hline 35.152 & 2.197 & 114.244 & 2.197 \\
\hline 2148.67 & 2.197 & 4.394 & 21.97 \\
\hline 2.197 & 2.197 & 8.788 & 15.379 \\
\hline 2.197 & 6.591 & 72.501 & 2.197 \\
\hline 373.49 & 4.394 & 120.835 & 68.107 \\
\hline 2.197 & 2.197 & 2.197 & 37.349 \\
\hline 59.319 & 2.197 & 30.758 & 43.94 \\
\hline 4.394 & 24.167 & 2.197 & 43.94 \\
\hline 1010.62 & 4.394 & 10.985 & 39.546 \\
\hline 2.197 & 2.197 & 2.197 & 19.773 \\
\hline 2.197 & 35.152 & 2.197 & 916.149 \\
\hline 4.394 & 2.197 & 41.743 & 10.985 \\
\hline 307.58 & 2.197 & 2.197 & 2.197 \\
\hline 54.925 & 2.197 & 2.197 & 2014.65 \\
\hline 4.394 & 98.865 & 2.197 & 17.576 \\
\hline 31647.8 & 8.788 & 4.394 & 10.985 \\
\hline 2.197 & 15.379 & 2.197 & 57.122 \\
\hline 199.927 & 2.197 & 8.788 & 2.197 \\
\hline 2.197 & 2.197 & 59.319 & 68.107 \\
\hline 4.394 & 138.411 & 4.394 & 13.182 \\
\hline 2.197 & 19.773 & 2.197 & 2.197 \\
\hline 2.197 & 68.107 & 10.985 & 4.394 \\
\hline 2.197 & 2.197 & 10.985 & 188.942 \\
\hline 4.394 & 8.788 & 4.394 & 177.957 \\
\hline 2.197 & 123.032 & 57.122 & 109.85 \\
\hline 6.591 & 19.773 & 59.319 & 4.394 \\
\hline 3020.87 & 17.576 & 2.197 & 6.591 \\
\hline 2.197 & 2.197 & 39.546 & 8.788 \\
\hline 26.364 & 2.197 & 2.197 & 2.197 \\
\hline 2.197 & 2.197 & 2.197 & 52.728 \\
\hline 2.197 & 15.379 & 2.197 & 2269.5 \\
\hline 2.197 & 4.394 & 6.591 & 10.985 \\
\hline 4.394 & 8.788 & 2.197 & 4.394 \\
\hline 50.531 & 4.394 & 43.94 & 17.576 \\
\hline 24.167 & 13.182 & 2.197 & 4.394 \\
\hline
\end{tabular}




\begin{tabular}{|c|c|c|c|}
\hline 57.122 & 2.197 & 2.197 & 180.154 \\
\hline 2.197 & 219.7 & 2.197 & 41.743 \\
\hline 166.972 & 8.788 & 8.788 & 2.197 \\
\hline 2.197 & 2.197 & 2.197 & 6.591 \\
\hline 4.394 & 8.788 & 2.197 & 19.773 \\
\hline 544.856 & 2.197 & 59.319 & 2.197 \\
\hline 15.379 & 4.394 & 2.197 & 28.561 \\
\hline 4.394 & 63.713 & 2.197 & 4.394 \\
\hline 35.152 & 10.985 & 8.788 & 15.379 \\
\hline 158.184 & 48.334 & 2.197 & 98.865 \\
\hline 2.197 & 114.244 & 103.259 & 24.167 \\
\hline 8.788 & 338.338 & 4.394 & 4.394 \\
\hline 2.197 & 17.576 & 2.197 & 4.394 \\
\hline 2.197 & 4.394 & 2.197 & 59.319 \\
\hline 2.197 & 4.394 & 2.197 & 46.137 \\
\hline 21.97 & 6.591 & 2.197 & 8.788 \\
\hline 2.197 & 2.197 & 2.197 & 87.88 \\
\hline 2.197 & 112.047 & 43.94 & 4.394 \\
\hline 2.197 & 24.167 & 21.97 & 26.364 \\
\hline 197.73 & 4.394 & 4.394 & 4.394 \\
\hline 2.197 & 2.197 & 13.182 & 32.955 \\
\hline 4.394 & 4.394 & 4.394 & 4.394 \\
\hline 15.379 & 4.394 & 2.197 & 2.197 \\
\hline 2.197 & 2.197 & 2.197 & 4.394 \\
\hline 2.197 & 4.394 & 2.197 & 4.394 \\
\hline 2.197 & 72.501 & 360.308 & 41.743 \\
\hline 2.197 & 2.197 & 6.591 & 2.197 \\
\hline 2.197 & 2.197 & 2.197 & 39.546 \\
\hline 4.394 & 52.728 & 2.197 & 68.107 \\
\hline 19.773 & 2.197 & 2.197 & 90.077 \\
\hline 2.197 & 19.773 & 4.394 & 8.788 \\
\hline 2.197 & 2.197 & 2.197 & 2.197 \\
\hline 21.97 & 2.197 & 2.197 & 2.197 \\
\hline 92.274 & 388.869 & 2.197 & 17.576 \\
\hline 2.197 & 2.197 & 2.197 & 4.394 \\
\hline 17.576 & 19.773 & 210.912 & 4.394 \\
\hline 2.197 & 6.591 & 30.758 & 19.773 \\
\hline 478.946 & 8.788 & 6.591 & 6.591 \\
\hline 2.197 & 4.394 & 2.197 & 96.668 \\
\hline 2.197 & 2.197 & 4.394 & 50.531 \\
\hline 2.197 & 4.394 & 2.197 & 90.077 \\
\hline 4.394 & 2.197 & 6.591 & 149.396 \\
\hline 50.531 & 2.197 & 4.394 & 8.788 \\
\hline 10.985 & 2.197 & 2.197 & 2.197 \\
\hline 2.197 & 2.197 & 72.501 & 188.942 \\
\hline 2.197 & 2.197 & 151.593 & 6.591 \\
\hline 2.197 & 2.197 & 19.773 & 164.775 \\
\hline 4.394 & 26.364 & 6.591 & 10.985 \\
\hline 19.773 & 6.591 & 2.197 & 193.336 \\
\hline 2.197 & 2.197 & 2.197 & 43.94 \\
\hline
\end{tabular}




\begin{tabular}{|c|c|c|c|}
\hline 2.197 & 2.197 & 15.379 & 2.197 \\
\hline 21.97 & 177.957 & 19.773 & 4404.98 \\
\hline 17.576 & 2.197 & 2.197 & 76.895 \\
\hline 131.82 & 544.856 & 39.546 & 4.394 \\
\hline 268.034 & 6.591 & 6.591 & 57.122 \\
\hline 8.788 & 17.576 & 2.197 & 1970.71 \\
\hline 4.394 & 13.182 & 24.167 & 6.591 \\
\hline 2.197 & 2.197 & 4.394 & 13.182 \\
\hline 8.788 & 8.788 & 63.713 & 10.985 \\
\hline 2.197 & 28.561 & 50.531 & 74.698 \\
\hline 4.394 & 2.197 & 8.788 & 10.985 \\
\hline 10.985 & 2.197 & 2.197 & 76.895 \\
\hline 2.197 & 6.591 & 6.591 & 2.197 \\
\hline 2.197 & 10.985 & 2.197 & 2.197 \\
\hline 2.197 & 2.197 & 70.304 & 10.985 \\
\hline 2.197 & 15.379 & 10.985 & 83.486 \\
\hline 52.728 & 6.591 & 2.197 & 28.561 \\
\hline 54.925 & 43.94 & 26.364 & 2.197 \\
\hline 15.379 & 2.197 & 2.197 & 6.591 \\
\hline 2.197 & 2.197 & 8.788 & 24.167 \\
\hline 4.394 & 2.197 & 2.197 & 6.591 \\
\hline 85.683 & 2.197 & 8.788 & 74.698 \\
\hline 4.394 & 2.197 & 10.985 & 21.97 \\
\hline 21.97 & 2.197 & 13.182 & 4.394 \\
\hline 4.394 & 2.197 & 2.197 & 8.788 \\
\hline 2.197 & 103.259 & 46.137 & 10.985 \\
\hline 8.788 & 2.197 & 10.985 & 10.985 \\
\hline 2.197 & 6.591 & 2.197 & 32.955 \\
\hline 6.591 & 2.197 & 65.91 & 79.092 \\
\hline 2.197 & 123.032 & 6.591 & 6.591 \\
\hline 470.158 & 2.197 & 2.197 & 2.197 \\
\hline 2.197 & 10.985 & 2.197 & 2.197 \\
\hline 819.481 & 2.197 & 2.197 & 2.197 \\
\hline 8.788 & 4.394 & 2.197 & 109.85 \\
\hline 4.394 & 2.197 & 2.197 & 6.591 \\
\hline 4.394 & 4.394 & 155.987 & 2.197 \\
\hline 8.788 & 17.576 & 2.197 & 17.576 \\
\hline 15.379 & 4.394 & 4.394 & 2.197 \\
\hline 50.531 & 10.985 & 2.197 & 4.394 \\
\hline 17.576 & 4.394 & 2.197 & 2.197 \\
\hline 8.788 & 15.379 & 81.289 & 8.788 \\
\hline 32.955 & 2.197 & 2.197 & 171.366 \\
\hline 4.394 & 2.197 & 2.197 & 39.546 \\
\hline 10.985 & 2.197 & 2.197 & 2.197 \\
\hline 2.197 & 2.197 & 2.197 & 48.334 \\
\hline 81.289 & 4.394 & 96.668 & 4.394 \\
\hline 2.197 & 70.304 & 15.379 & 2.197 \\
\hline 8.788 & 6.591 & 65.91 & 10.985 \\
\hline 4.394 & 15.379 & 2.197 & 2.197 \\
\hline 2.197 & 2.197 & 2.197 & 2.197 \\
\hline
\end{tabular}




\begin{tabular}{|c|c|c|c|}
\hline 4.394 & 52.728 & 4.394 & 2.197 \\
\hline 4.394 & 2.197 & 2.197 & 19.773 \\
\hline 2.197 & 8.788 & 4.394 & 2.197 \\
\hline 2.197 & 4.394 & 43.94 & 6.591 \\
\hline 2.197 & 35.152 & 15.379 & 28.561 \\
\hline 15034.1 & 4.394 & 17.576 & 6.591 \\
\hline 134.017 & 2.197 & 2.197 & 68.107 \\
\hline 4.394 & 2.197 & 13.182 & 17.576 \\
\hline 2.197 & 210.912 & 15.379 & 6.591 \\
\hline 2.197 & 2.197 & 2.197 & 8.788 \\
\hline 15.379 & 6.591 & 39.546 & 4.394 \\
\hline 2.197 & 13.182 & 2.197 & 41.743 \\
\hline 6.591 & 6.591 & 13.182 & 4.394 \\
\hline 13.182 & 8.788 & 15.379 & 582.205 \\
\hline 28.561 & 6.591 & 21.97 & 98.865 \\
\hline 4.394 & 2.197 & 4.394 & 821.678 \\
\hline 10.985 & 2.197 & 4.394 & 54.925 \\
\hline 2.197 & 2.197 & 2.197 & 57.122 \\
\hline 10.985 & 4.394 & 19.773 & 2.197 \\
\hline 195.533 & 2.197 & 4.394 & 19.773 \\
\hline 28.561 & 70.304 & 388.869 & 509.704 \\
\hline 2.197 & 2.197 & 2.197 & 10.985 \\
\hline 4.394 & 4.394 & 4.394 & 8.788 \\
\hline 2.197 & 2.197 & 2.197 & 21.97 \\
\hline 2.197 & 2.197 & 21.97 & 28.561 \\
\hline 2.197 & 2.197 & 2.197 & 32.955 \\
\hline 4.394 & 50.531 & 6.591 & 2.197 \\
\hline 72.501 & 2.197 & 2.197 & 2.197 \\
\hline 30.758 & 4.394 & 2.197 & 41.743 \\
\hline 2.197 & 8.788 & 6.591 & 13.182 \\
\hline 164.775 & 83.486 & 2.197 & 2.197 \\
\hline 166.972 & 54.925 & 32.955 & 290.004 \\
\hline 17.576 & 2.197 & 10.985 & 52.728 \\
\hline 2.197 & 10.985 & 2.197 & 101.062 \\
\hline 2.197 & 13.182 & 410.839 & 6.591 \\
\hline 61.516 & 2.197 & 13.182 & 19.773 \\
\hline 4.394 & 2.197 & 4.394 & 2.197 \\
\hline 6.591 & 6.591 & 2.197 & 24.167 \\
\hline 2.197 & 10.985 & 2.197 & 120.835 \\
\hline 2.197 & 19.773 & 2.197 & 8.788 \\
\hline 15.379 & 8.788 & 21.97 & 32.955 \\
\hline 158.184 & 2.197 & 2.197 & 2.197 \\
\hline 2.197 & 428.415 & 2.197 & 92.274 \\
\hline 10.985 & 2.197 & 2.197 & 6.591 \\
\hline 2.197 & 21.97 & 2.197 & 360.308 \\
\hline 2.197 & 2.197 & 13.182 & 232.882 \\
\hline 8.788 & 17.576 & 2.197 & 2.197 \\
\hline 15.379 & 13.182 & 2.197 & 41.743 \\
\hline 8.788 & 15.379 & 2.197 & 4.394 \\
\hline 32.955 & 694.252 & 6.591 & 347.126 \\
\hline
\end{tabular}




\begin{tabular}{|c|c|c|c|}
\hline 6.591 & 2.197 & 2.197 & 4.394 \\
\hline 13.182 & 35.152 & 13.182 & 2.197 \\
\hline 6.591 & 2.197 & 17.576 & 2.197 \\
\hline 2.197 & 2.197 & 6.591 & 2.197 \\
\hline 2.197 & 6.591 & 2.197 & 2.197 \\
\hline 2.197 & 2.197 & 2.197 & 41.743 \\
\hline 4.394 & 2.197 & 2.197 & 2.197 \\
\hline 2.197 & 6.591 & 2.197 & 15.379 \\
\hline 8.788 & 2.197 & 2.197 & 2.197 \\
\hline 2.197 & 2.197 & 2.197 & 129.623 \\
\hline 13.182 & 4.394 & 2.197 & 303.186 \\
\hline 6.591 & 10.985 & 17.576 & 28.561 \\
\hline 2.197 & 8.788 & 4.394 & 17.576 \\
\hline 2.197 & 6.591 & 2.197 & 6.591 \\
\hline 8.788 & 15.379 & 15.379 & 46.137 \\
\hline 2.197 & 2.197 & 4.394 & 59.319 \\
\hline 17.576 & 2.197 & 52.728 & 8.788 \\
\hline 94.471 & 2.197 & 2.197 & 13.182 \\
\hline 6.591 & 28.561 & 10.985 & 2.197 \\
\hline 2.197 & 4.394 & 13.182 & 15.379 \\
\hline 6.591 & 2.197 & 2.197 & 13.182 \\
\hline 4.394 & 118.638 & 4.394 & 79.092 \\
\hline 10.985 & 2.197 & 15.379 & 2.197 \\
\hline 63.713 & 32.955 & 70.304 & 2.197 \\
\hline 24.167 & 2.197 & 204.321 & 4.394 \\
\hline 173.563 & 988.65 & 50.531 & 32.955 \\
\hline 2.197 & 13.182 & 17.576 & 39.546 \\
\hline 13.182 & 10.985 & 28.561 & 6.591 \\
\hline 2.197 & 10.985 & 375.687 & 6.591 \\
\hline 2.197 & 2.197 & 6.591 & 15.379 \\
\hline 2.197 & 2.197 & 72.501 & 127.426 \\
\hline 112.047 & 2.197 & 2.197 & 2.197 \\
\hline 6.591 & 2.197 & 2.197 & 2.197 \\
\hline 57.122 & 6.591 & 63.713 & 61.516 \\
\hline 511.901 & 2.197 & 4.394 & 4.394 \\
\hline 28.561 & 2.197 & 2.197 & 2.197 \\
\hline 2.197 & 2.197 & 274.625 & 26.364 \\
\hline 10.985 & 2.197 & 112.047 & 2.197 \\
\hline 2.197 & 2.197 & 6.591 & 50.531 \\
\hline 4.394 & 6.591 & 6.591 & 4.394 \\
\hline 1463.2 & 19.773 & 4.394 & 10.985 \\
\hline 2.197 & 46.137 & 2.197 & 259.246 \\
\hline 2.197 & 10.985 & 327.353 & 310998 \\
\hline 63.713 & 6.591 & 28.561 & 2.197 \\
\hline 37.349 & 32.955 & 10.985 & 4.394 \\
\hline 28.561 & 2.197 & 2.197 & 10.985 \\
\hline 4.394 & 8.788 & 2.197 & 6.591 \\
\hline 2.197 & 28.561 & 107.653 & 32.955 \\
\hline 2.197 & 2.197 & 17.576 & 83.486 \\
\hline 4.394 & 2.197 & 10.985 & 2.197 \\
\hline
\end{tabular}




\begin{tabular}{|c|c|c|c|}
\hline 2.197 & 6.591 & 2.197 & 21.97 \\
\hline 2.197 & 2.197 & 4.394 & 54.925 \\
\hline 2.197 & 76.895 & 19.773 & 2.197 \\
\hline 19.773 & 544.856 & 35.152 & 43.94 \\
\hline 13.182 & 180.154 & 2.197 & 4.394 \\
\hline 2.197 & 10.985 & 4.394 & 26.364 \\
\hline 61.516 & 2.197 & 83.486 & 28.561 \\
\hline 2.197 & 4.394 & 2.197 & 4.394 \\
\hline 2.197 & 4.394 & 2.197 & 2.197 \\
\hline 2.197 & 4.394 & 2.197 & 2.197 \\
\hline 41.743 & 2.197 & 10.985 & 4.394 \\
\hline 6.591 & 2.197 & 257.049 & 4.394 \\
\hline 6.591 & 2.197 & 496.522 & 10.985 \\
\hline 580.008 & 2.197 & 2.197 & 28.561 \\
\hline 2.197 & 2.197 & 39.546 & 13.182 \\
\hline 2.197 & 2.197 & 70.304 & 19.773 \\
\hline 13.182 & 2.197 & 2.197 & 2.197 \\
\hline 24.167 & 2.197 & 2.197 & 6.591 \\
\hline 4.394 & 4.394 & 10.985 & 63.713 \\
\hline 17.576 & 13.182 & 2.197 & 13.182 \\
\hline 2.197 & 2366.17 & 232.882 & 2.197 \\
\hline 4.394 & 2.197 & 2.197 & 13.182 \\
\hline 4.394 & 15.379 & 21.97 & 8.788 \\
\hline 507.507 & 4.394 & 10.985 & 48.334 \\
\hline 2.197 & 2.197 & 2.197 & 24.167 \\
\hline 2.197 & 2.197 & 123.032 & 8.788 \\
\hline 2.197 & 2.197 & 2.197 & 15.379 \\
\hline 2.197 & 2.197 & 2.197 & 4.394 \\
\hline 50.531 & 10.985 & 2.197 & 32.955 \\
\hline 4.394 & 2.197 & 2.197 & 35.152 \\
\hline 2.197 & 4.394 & 2.197 & 19.773 \\
\hline 2.197 & 30.758 & 8.788 & 2394.73 \\
\hline 19.773 & 19.773 & 4.394 & 101.062 \\
\hline 65.91 & 2.197 & 8.788 & 2.197 \\
\hline 6.591 & 2.197 & 8.788 & 52.728 \\
\hline 2.197 & 4.394 & 32.955 & 37.349 \\
\hline 2.197 & 6.591 & 1423.66 & 15.379 \\
\hline 2.197 & 8.788 & 2.197 & 4.394 \\
\hline 10.985 & 24.167 & 76.895 & 2.197 \\
\hline 2.197 & 2.197 & 48.334 & 2.197 \\
\hline 8080.57 & 2.197 & 2.197 & 10.985 \\
\hline 8.788 & 4.394 & 8.788 & 109.85 \\
\hline 2.197 & 4.394 & 30.758 & 2.197 \\
\hline 4.394 & 2.197 & 2.197 & 4.394 \\
\hline 2.197 & 2.197 & 990.847 & 21.97 \\
\hline 63.713 & 2.197 & 6.591 & 4.394 \\
\hline 54.925 & 21.97 & 13.182 & 105.456 \\
\hline 52.728 & 2.197 & 83.486 & 10.985 \\
\hline 2.197 & 28.561 & 6.591 & 2.197 \\
\hline 2.197 & 2.197 & 6.591 & 8.788 \\
\hline
\end{tabular}




\begin{tabular}{|c|c|c|c|}
\hline 2.197 & 2.197 & 10.985 & 6.591 \\
\hline 4.394 & 2.197 & 118.638 & 4.394 \\
\hline 2.197 & 4.394 & 2.197 & 41.743 \\
\hline 2.197 & 2.197 & 2.197 & 103.259 \\
\hline 2.197 & 2.197 & 37.349 & 83.486 \\
\hline 2.197 & 2.197 & 2.197 & 4.394 \\
\hline 2.197 & 96.668 & 8.788 & 8.788 \\
\hline 2.197 & 2.197 & 173.563 & 4.394 \\
\hline 2.197 & 59.319 & 32.955 & 72.501 \\
\hline 2.197 & 41.743 & 15.379 & 4.394 \\
\hline 10.985 & 41.743 & 8.788 & 2.197 \\
\hline 10.985 & 61.516 & 8.788 & 24.167 \\
\hline 92.274 & 4.394 & 4.394 & 48.334 \\
\hline 4.394 & 15.379 & 4.394 & 37.349 \\
\hline 4.394 & 388.869 & 17.576 & 2.197 \\
\hline 13.182 & 17.576 & 6.591 & 10.985 \\
\hline 6.591 & 79.092 & 4.394 & 2.197 \\
\hline 2.197 & 13.182 & 1177.59 & 160.381 \\
\hline 4.394 & 2.197 & 2.197 & 13.182 \\
\hline 2.197 & 35.152 & 4.394 & 68.107 \\
\hline 2.197 & 2.197 & 10.985 & 2.197 \\
\hline 239.473 & 2.197 & 6.591 & 6.591 \\
\hline 4.394 & 2.197 & 2.197 & 21.97 \\
\hline 61.516 & 15.379 & 54.925 & 26.364 \\
\hline 6.591 & 4.394 & 2.197 & 2.197 \\
\hline 2.197 & 17.576 & 4.394 & 37.349 \\
\hline 2.197 & 2.197 & 2.197 & 72.501 \\
\hline 2.197 & 8.788 & 37.349 & 4.394 \\
\hline 2.197 & 4.394 & 2.197 & 136.214 \\
\hline 2.197 & 37.349 & 2.197 & 129.623 \\
\hline 13.182 & 87.88 & 2.197 & 2.197 \\
\hline 2.197 & 6.591 & 28.561 & 4.394 \\
\hline 2.197 & 2.197 & 2.197 & 28.561 \\
\hline 1652.14 & 17.576 & 6.591 & 10.985 \\
\hline 13.182 & 2.197 & 32.955 & 15.379 \\
\hline 63.713 & 2.197 & 4.394 & 164.775 \\
\hline 6.591 & 2.197 & 134.017 & 4.394 \\
\hline 26.364 & 2.197 & 2.197 & 6.591 \\
\hline 566.826 & 2.197 & 8.788 & 292.201 \\
\hline 162.578 & 15.379 & 4.394 & 21.97 \\
\hline 2.197 & 2.197 & 4.394 & 2.197 \\
\hline 2.197 & 19.773 & 2.197 & 92.274 \\
\hline 6.591 & 10.985 & 10.985 & 8.788 \\
\hline 19.773 & 41.743 & 10.985 & 30.758 \\
\hline 2.197 & 4.394 & 2.197 & 120.835 \\
\hline 2.197 & 2.197 & 4.394 & 2.197 \\
\hline 13.182 & 2.197 & 2.197 & 2.197 \\
\hline 2.197 & 2.197 & 28.561 & 661.297 \\
\hline 2.197 & 2.197 & 17.576 & 733.798 \\
\hline 2.197 & 28.561 & 10.985 & 48.334 \\
\hline
\end{tabular}




\begin{tabular}{|c|c|c|c|}
\hline 8.788 & 28.561 & 4.394 & 10.985 \\
\hline 2.197 & 2.197 & 74.698 & 17.576 \\
\hline 13.182 & 15.379 & 15.379 & 15.379 \\
\hline 6.591 & 4.394 & 2.197 & 57.122 \\
\hline 2.197 & 43.94 & 4.394 & 232.882 \\
\hline 10.985 & 2.197 & 4.394 & 10.985 \\
\hline 19.773 & 2.197 & 6.591 & 2.197 \\
\hline 13.182 & 4.394 & 8.788 & 19.773 \\
\hline 19.773 & 8.788 & 17.576 & 2.197 \\
\hline 6.591 & 2.197 & 26.364 & 2.197 \\
\hline 2.197 & 2.197 & 6.591 & 13.182 \\
\hline 4.394 & 50.531 & 8.788 & 1072.14 \\
\hline 2.197 & 2.197 & 39.546 & 48.334 \\
\hline 142.805 & 10.985 & 8.788 & 59.319 \\
\hline 2.197 & 21.97 & 90.077 & 43.94 \\
\hline 2.197 & 10.985 & 10.985 & 2.197 \\
\hline 2.197 & 105.456 & 2.197 & 8.788 \\
\hline 2.197 & 443.794 & 10.985 & 13.182 \\
\hline 13.182 & 454.779 & 39.546 & 10.985 \\
\hline 4.394 & 13.182 & 2.197 & 224.094 \\
\hline 2.197 & 2.197 & 4.394 & 142.805 \\
\hline 2.197 & 4.394 & 2.197 & 2.197 \\
\hline 6.591 & 2.197 & 17.576 & 32.955 \\
\hline 21.97 & 2.197 & 472.355 & 4.394 \\
\hline 74.698 & 6.591 & 6.591 & 235.079 \\
\hline 2.197 & 4.394 & 2.197 & 87.88 \\
\hline 21.97 & 4.394 & 2.197 & 6.591 \\
\hline 2.197 & 2.197 & 10.985 & 90.077 \\
\hline 2.197 & 2.197 & 15.379 & 8.788 \\
\hline 6.591 & 2.197 & 2.197 & 43.94 \\
\hline 8.788 & 8.788 & 6.591 & 2.197 \\
\hline 2.197 & 4.394 & 17.576 & 2.197 \\
\hline 13.182 & 4.394 & 6.591 & 4.394 \\
\hline 120.835 & 6.591 & 2.197 & 39.546 \\
\hline 13.182 & 2.197 & 13.182 & 4.394 \\
\hline 2.197 & 2.197 & 10.985 & 79.092 \\
\hline 48.334 & 2.197 & 2.197 & 1063.35 \\
\hline 4.394 & 35.152 & 4.394 & 72.501 \\
\hline 43.94 & 2.197 & 2.197 & 30.758 \\
\hline 2.197 & 21.97 & 48.334 & 10.985 \\
\hline 37.349 & 2.197 & 2.197 & 4.394 \\
\hline 114.244 & 2.197 & 19.773 & 46.137 \\
\hline 2.197 & 4.394 & 8.788 & 2.197 \\
\hline 2.197 & 4.394 & 626.145 & 134.017 \\
\hline 2.197 & 6.591 & 2.197 & 109.85 \\
\hline 2.197 & 50.531 & 2.197 & 59.319 \\
\hline 2.197 & 4.394 & 2.197 & 2.197 \\
\hline 17.576 & 19.773 & 2.197 & 105.456 \\
\hline 10.985 & 2.197 & 17.576 & 24.167 \\
\hline 4.394 & 2.197 & 2.197 & 92.274 \\
\hline
\end{tabular}




\begin{tabular}{|c|c|c|c|}
\hline 8.788 & 2.197 & 2.197 & 85.683 \\
\hline 13.182 & 2.197 & 2.197 & 2.197 \\
\hline 4.394 & 2.197 & 26.364 & 83.486 \\
\hline 35.152 & 2.197 & 2.197 & 6.591 \\
\hline 2.197 & 4.394 & 28.561 & 112.047 \\
\hline 13.182 & 2.197 & 4.394 & 2.197 \\
\hline 2.197 & 6.591 & 26.364 & 2.197 \\
\hline 2.197 & 2.197 & 2.197 & 4.394 \\
\hline 2.197 & 57.122 & 4.394 & 26.364 \\
\hline 2.197 & 8.788 & 2.197 & 109.85 \\
\hline 116.441 & 4.394 & 4.394 & 2.197 \\
\hline 2.197 & 300.989 & 2.197 & 2.197 \\
\hline 26.364 & 8.788 & 32.955 & 107.653 \\
\hline 2.197 & 35.152 & 118.638 & 116.441 \\
\hline 2.197 & 8.788 & 17.576 & 17.576 \\
\hline 2.197 & 6.591 & 4.394 & 43.94 \\
\hline 2.197 & 2.197 & 13.182 & 76.895 \\
\hline 2.197 & 101.062 & 2.197 & 26.364 \\
\hline 2.197 & 2.197 & 2.197 & 2.197 \\
\hline 96.668 & 90.077 & 6.591 & 15.379 \\
\hline 24.167 & 2.197 & 2.197 & 648.115 \\
\hline 2.197 & 2.197 & 2.197 & 2.197 \\
\hline 68.107 & 2.197 & 2.197 & 107.653 \\
\hline 37.349 & 4.394 & 2.197 & 2.197 \\
\hline 39.546 & 21.97 & 2.197 & 6.591 \\
\hline 35.152 & 2.197 & 2.197 & 15.379 \\
\hline 4.394 & 2.197 & 2.197 & 10.985 \\
\hline 4.394 & 24.167 & 634.933 & 21.97 \\
\hline 6.591 & 2.197 & 2.197 & 2.197 \\
\hline 2.197 & 6.591 & 8.788 & 2.197 \\
\hline 48.334 & 2.197 & 175.76 & 4.394 \\
\hline 182.351 & 2.197 & 456.976 & 2.197 \\
\hline 2.197 & 2.197 & 15.379 & 2.197 \\
\hline 2.197 & 4.394 & 2.197 & 125.229 \\
\hline 4.394 & 4.394 & 4.394 & 120.835 \\
\hline 2.197 & 19.773 & 79.092 & 2.197 \\
\hline 4.394 & 2.197 & 50.531 & 2.197 \\
\hline 2.197 & 4.394 & 17.576 & 2.197 \\
\hline 4.394 & 2.197 & 8.788 & 4.394 \\
\hline 4.394 & 2.197 & 4.394 & 2192.61 \\
\hline 2.197 & 4.394 & 2.197 & 10.985 \\
\hline 6.591 & 37.349 & 6.591 & 8.788 \\
\hline 4.394 & 43.94 & 94.471 & 54.925 \\
\hline 2.197 & 10.985 & 2.197 & 2.197 \\
\hline 2.197 & 2.197 & 8.788 & 2.197 \\
\hline 32.955 & 2.197 & 39.546 & 17.576 \\
\hline 2.197 & 2.197 & 4.394 & 10.985 \\
\hline 15.379 & 2.197 & 4.394 & 180.154 \\
\hline 4.394 & 2.197 & 8.788 & 2.197 \\
\hline 2.197 & 2.197 & 2.197 & 6.591 \\
\hline
\end{tabular}




\begin{tabular}{|c|c|c|c|}
\hline 26.364 & 10.985 & 4.394 & 8.788 \\
\hline 8.788 & 2.197 & 2.197 & 2.197 \\
\hline 4.394 & 15.379 & 2.197 & 2.197 \\
\hline 30.758 & 990.847 & 2.197 & 1414.87 \\
\hline 30.758 & 2.197 & 4.394 & 681.07 \\
\hline 4.394 & 2.197 & 265.837 & 169.169 \\
\hline 4.394 & 2.197 & 2.197 & 2.197 \\
\hline 41.743 & 2.197 & 2.197 & 63.713 \\
\hline 685.464 & 2.197 & 26.364 & 92.274 \\
\hline 21.97 & 2.197 & 2.197 & 6.591 \\
\hline 8.788 & 2.197 & 4.394 & 4.394 \\
\hline 10.985 & 2.197 & 4.394 & 39.546 \\
\hline 50.531 & 43.94 & 43.94 & 15.379 \\
\hline 24.167 & 4.394 & 2.197 & 2.197 \\
\hline 2.197 & 26.364 & 48.334 & 26.364 \\
\hline 2.197 & 2.197 & 61.516 & 2.197 \\
\hline 2.197 & 2.197 & 147.199 & 28.561 \\
\hline 37.349 & 13.182 & 2.197 & 21.97 \\
\hline 19.773 & 2.197 & 2.197 & 48.334 \\
\hline 17.576 & 10.985 & 2.197 & 46.137 \\
\hline 13.182 & 43.94 & 8.788 & 2.197 \\
\hline 8.788 & 41.743 & 2.197 & 5411.21 \\
\hline 15.379 & 4.394 & 2.197 & 2.197 \\
\hline 2.197 & 81.289 & 8.788 & 76.895 \\
\hline 54.925 & 35.152 & 4.394 & 2.197 \\
\hline 10.985 & 2.197 & 1399.49 & 2.197 \\
\hline 384.475 & 4.394 & 15.379 & 2.197 \\
\hline 2.197 & 2.197 & 2.197 & 10.985 \\
\hline 463.567 & 2.197 & 2.197 & 4.394 \\
\hline 61.516 & 2.197 & 661.297 & 15.379 \\
\hline 6.591 & 17.576 & 13.182 & 4.394 \\
\hline 2.197 & 2.197 & 2.197 & 28.561 \\
\hline 15.379 & 228.488 & 6.591 & 153.79 \\
\hline 19.773 & 8.788 & 2.197 & 1322.59 \\
\hline 2.197 & 2.197 & 2.197 & 32.955 \\
\hline 10.985 & 10.985 & 2.197 & 13.182 \\
\hline 4.394 & 15.379 & 6.591 & 26.364 \\
\hline 96.668 & 28.561 & 39.546 & 619.554 \\
\hline 19.773 & 4.394 & 8.788 & 2.197 \\
\hline 2.197 & 175.76 & 50.531 & 17.576 \\
\hline 2.197 & 19.773 & 6.591 & 485.537 \\
\hline 2.197 & 2.197 & 2.197 & 19.773 \\
\hline 395.46 & 4.394 & 26.364 & 2.197 \\
\hline 17.576 & 4.394 & 2.197 & 8.788 \\
\hline 35.152 & 2.197 & 2.197 & 30.758 \\
\hline 147.199 & 2.197 & 2.197 & 112.047 \\
\hline 15.379 & 2.197 & 2.197 & 2.197 \\
\hline 57.122 & 2.197 & 2.197 & 329.55 \\
\hline 123.032 & 2.197 & 26.364 & 63.713 \\
\hline 15.379 & 2.197 & 2.197 & 98.865 \\
\hline
\end{tabular}




\begin{tabular}{|c|c|c|c|}
\hline 8.788 & 2.197 & 2.197 & 21.97 \\
\hline 13.182 & 2.197 & 2.197 & 2.197 \\
\hline 24.167 & 4.394 & 2.197 & 28.561 \\
\hline 2.197 & 2.197 & 2.197 & 81.289 \\
\hline 8.788 & 4.394 & 4.394 & 90.077 \\
\hline 10.985 & 1432.44 & 303.186 & 2.197 \\
\hline 4.394 & 283.413 & 4.394 & 2.197 \\
\hline 13.182 & 13.182 & 483.34 & 2.197 \\
\hline 50.531 & 30.758 & 17.576 & 2.197 \\
\hline 2.197 & 15.379 & 13.182 & 39.546 \\
\hline 2.197 & 4.394 & 4.394 & 32.955 \\
\hline 2.197 & 6.591 & 2.197 & 37.349 \\
\hline 2.197 & 26.364 & 2.197 & 15.379 \\
\hline 2.197 & 10.985 & 2.197 & 10.985 \\
\hline 2.197 & 4.394 & 2.197 & 2.197 \\
\hline 2.197 & 2.197 & 6.591 & 26.364 \\
\hline 10.985 & 19.773 & 2.197 & 2.197 \\
\hline 2.197 & 24.167 & 2.197 & 68.107 \\
\hline 237.276 & 2647.38 & 2.197 & 2.197 \\
\hline 19.773 & 8.788 & 2.197 & 2.197 \\
\hline 2.197 & 2.197 & 4.394 & 2.197 \\
\hline 15.379 & 50.531 & 6.591 & 19.773 \\
\hline 109.85 & 96.668 & 13.182 & 8.788 \\
\hline 13.182 & 13.182 & 10.985 & 2.197 \\
\hline 19.773 & 2.197 & 2.197 & 2.197 \\
\hline 68.107 & 219.7 & 26.364 & 87.88 \\
\hline 6.591 & 2.197 & 26.364 & 6.591 \\
\hline 2.197 & 19.773 & 6.591 & 114.244 \\
\hline 17.576 & 2.197 & 4.394 & 8.788 \\
\hline 74.698 & 2.197 & 2.197 & 2.197 \\
\hline 2.197 & 2.197 & 2.197 & 2.197 \\
\hline 4.394 & 2.197 & 2.197 & 26.364 \\
\hline 8.788 & 2.197 & 4.394 & 155.987 \\
\hline 4.394 & 19.773 & 6.591 & 46.137 \\
\hline 10.985 & 2.197 & 26.364 & 30.758 \\
\hline 76.895 & 2.197 & 6.591 & 13.182 \\
\hline 2.197 & 2.197 & 2.197 & 15.379 \\
\hline 2.197 & 6.591 & 2.197 & 4.394 \\
\hline 360.308 & 248.261 & 540.462 & 57.122 \\
\hline 4.394 & 2.197 & 540.462 & 2.197 \\
\hline 4.394 & 4.394 & 13.182 & 2.197 \\
\hline 15.379 & 2.197 & 2.197 & 19.773 \\
\hline 4.394 & 2.197 & 2.197 & 2.197 \\
\hline 21.97 & 2.197 & 21.97 & 10.985 \\
\hline 2.197 & 2.197 & 4.394 & 2.197 \\
\hline 26.364 & 2.197 & 164.775 & 331.747 \\
\hline 28.561 & 2.197 & 63.713 & 2.197 \\
\hline 2.197 & 2.197 & 2.197 & 2.197 \\
\hline 2.197 & 2.197 & 4.394 & 87.88 \\
\hline 8.788 & 6.591 & 4.394 & 6.591 \\
\hline
\end{tabular}




\begin{tabular}{|c|c|c|c|}
\hline 13.182 & 2.197 & 4.394 & 2.197 \\
\hline 2.197 & 10.985 & 6.591 & 10.985 \\
\hline 2.197 & 2.197 & 15.379 & 41.743 \\
\hline 15.379 & 2.197 & 2.197 & 10.985 \\
\hline 2.197 & 2.197 & 4.394 & 24.167 \\
\hline 2.197 & 10.985 & 30.758 & 2.197 \\
\hline 24.167 & 32.955 & 874.406 & 28.561 \\
\hline 2.197 & 19.773 & 8.788 & 2.197 \\
\hline 2.197 & 4.394 & 2.197 & 74.698 \\
\hline 65.91 & 79.092 & 2.197 & 15.379 \\
\hline 41.743 & 2.197 & 32.955 & 37.349 \\
\hline 15.379 & 6.591 & 4.394 & 2.197 \\
\hline 4.394 & 15.379 & 4.394 & 2.197 \\
\hline 4.394 & 8.788 & 2.197 & 43.94 \\
\hline 4.394 & 2.197 & 2.197 & 2.197 \\
\hline 4.394 & 10.985 & 13.182 & 2.197 \\
\hline 2.197 & 70.304 & 2.197 & 2.197 \\
\hline 10.985 & 123.032 & 2.197 & 24.167 \\
\hline 2.197 & 6.591 & 4.394 & 8.788 \\
\hline 2.197 & 2.197 & 19.773 & 35.152 \\
\hline 37.349 & 8.788 & 123.032 & 8.788 \\
\hline 2.197 & 2.197 & 41.743 & 26.364 \\
\hline 13.182 & 61.516 & 24.167 & 87.88 \\
\hline 70.304 & 120.835 & 2.197 & 393.263 \\
\hline 46.137 & 10.985 & 4.394 & 2.197 \\
\hline 4.394 & 276.822 & 2.197 & 10.985 \\
\hline 4.394 & 15.379 & 2.197 & 17.576 \\
\hline 30.758 & 2.197 & 4.394 & 4.394 \\
\hline 116.441 & 2.197 & 2.197 & 43.94 \\
\hline 2.197 & 136.214 & 6.591 & 2.197 \\
\hline 261.443 & 6.591 & 15.379 & 2.197 \\
\hline 2.197 & 70.304 & 4.394 & 2.197 \\
\hline 65.91 & 32.955 & 13.182 & 6.591 \\
\hline 2.197 & 68.107 & 17.576 & 8.788 \\
\hline 577.811 & 4.394 & 15.379 & 2.197 \\
\hline 920.543 & 46.137 & 6.591 & 43.94 \\
\hline 6.591 & 74.698 & 21.97 & 32.955 \\
\hline 24.167 & 21.97 & 2.197 & 145.002 \\
\hline 2.197 & 21.97 & 4.394 & 101.062 \\
\hline 15.379 & 87.88 & 8.788 & 15.379 \\
\hline 6.591 & 37.349 & 15.379 & 6.591 \\
\hline 4.394 & 2.197 & 2.197 & 54.925 \\
\hline 4.394 & 2.197 & 48.334 & 481.143 \\
\hline 2.197 & 2.197 & 2.197 & 707.434 \\
\hline 6.591 & 2.197 & 2.197 & 2.197 \\
\hline 6.591 & 2.197 & 98.865 & 98.865 \\
\hline 26.364 & 2.197 & 2.197 & 2.197 \\
\hline 4.394 & 2.197 & 2.197 & 26.364 \\
\hline 2.197 & 4.394 & 19.773 & 13.182 \\
\hline 2.197 & 2.197 & 107.653 & 136.214 \\
\hline
\end{tabular}




\begin{tabular}{|c|c|c|c|}
\hline 2.197 & 2.197 & 28.561 & 2.197 \\
\hline 92.274 & 2.197 & 2.197 & 2.197 \\
\hline 10.985 & 2.197 & 226.291 & 2.197 \\
\hline 44030.1 & 2.197 & 8.788 & 186.745 \\
\hline 2.197 & 6.591 & 13.182 & 2.197 \\
\hline 10.985 & 6.591 & 2.197 & 2.197 \\
\hline 65.91 & 4.394 & 4.394 & 15.379 \\
\hline 2.197 & 2.197 & 17.576 & 50.531 \\
\hline 2.197 & 4.394 & 6.591 & 15.379 \\
\hline 2.197 & 19.773 & 2.197 & 2.197 \\
\hline 4.394 & 6.591 & 2.197 & 2.197 \\
\hline 26.364 & 942.513 & 2.197 & 15.379 \\
\hline 6.591 & 24.167 & 2.197 & 21.97 \\
\hline 4.394 & 8.788 & 15.379 & 8.788 \\
\hline 2.197 & 13.182 & 2.197 & 6.591 \\
\hline 24.167 & 6.591 & 2.197 & 4.394 \\
\hline 4.394 & 2.197 & 2.197 & 340.535 \\
\hline 2.197 & 2.197 & 2.197 & 2.197 \\
\hline 243.867 & 6.591 & 2.197 & 8.788 \\
\hline 5435.38 & 2.197 & 8.788 & 2.197 \\
\hline 10.985 & 6.591 & 2.197 & 2.197 \\
\hline 2.197 & 2.197 & 2.197 & 81.289 \\
\hline 2.197 & 2.197 & 63.713 & 202.124 \\
\hline 4.394 & 17.576 & 101.062 & 2.197 \\
\hline 8.788 & 15.379 & 2.197 & 2.197 \\
\hline 2.197 & 63.713 & 2.197 & 4.394 \\
\hline 4.394 & 10.985 & 2.197 & 125.229 \\
\hline 2.197 & 2.197 & 4.394 & 478.946 \\
\hline 6.591 & 37.349 & 4.394 & 19.773 \\
\hline 6.591 & 4.394 & 57.122 & 52.728 \\
\hline 65.91 & 15.379 & 21.97 & 4.394 \\
\hline 2.197 & 6.591 & 15.379 & 39.546 \\
\hline 2.197 & 2.197 & 2.197 & 4.394 \\
\hline 2.197 & 4.394 & 2.197 & 4.394 \\
\hline 92.274 & 2.197 & 17.576 & 8.788 \\
\hline 32.955 & 2.197 & 395.46 & 2.197 \\
\hline 4.394 & 24.167 & 2.197 & 4.394 \\
\hline 2.197 & 2.197 & 2.197 & 30.758 \\
\hline 2.197 & 2.197 & 2.197 & 2.197 \\
\hline 2.197 & 52.728 & 21.97 & 2.197 \\
\hline 4.394 & 2.197 & 26.364 & 2.197 \\
\hline 6.591 & 2.197 & 4.394 & 2.197 \\
\hline 2.197 & 48.334 & 4.394 & 2.197 \\
\hline 6.591 & 15.379 & 4.394 & 2.197 \\
\hline 2.197 & 2.197 & 2.197 & 10.985 \\
\hline 2.197 & 2.197 & 35.152 & 26.364 \\
\hline 30.758 & 35.152 & 107.653 & 41.743 \\
\hline 17.576 & 15.379 & 6.591 & 94.471 \\
\hline 606.372 & 4.394 & 2.197 & 43.94 \\
\hline 4.394 & 2.197 & 362.505 & 2.197 \\
\hline
\end{tabular}




\begin{tabular}{|c|c|c|c|}
\hline 4.394 & 15.379 & 2.197 & 230.685 \\
\hline 6.591 & 6.591 & 2.197 & 2.197 \\
\hline 8.788 & 10.985 & 2.197 & 2.197 \\
\hline 50.531 & 2.197 & 4.394 & 2.197 \\
\hline 10.985 & 54.925 & 6.591 & 2.197 \\
\hline 21.97 & 6.591 & 2.197 & 8.788 \\
\hline 2.197 & 2.197 & 2.197 & 105.456 \\
\hline 296.595 & 13.182 & 6.591 & 2.197 \\
\hline 2.197 & 26.364 & 4.394 & 2.197 \\
\hline 2.197 & 2.197 & 2.197 & 678.873 \\
\hline 2.197 & 4.394 & 2.197 & 41.743 \\
\hline 402.051 & 4.394 & 21.97 & 2.197 \\
\hline 4.394 & 2.197 & 2.197 & 2.197 \\
\hline 48.334 & 2.197 & 4.394 & 2.197 \\
\hline 24.167 & 2.197 & 2.197 & 85.683 \\
\hline 2.197 & 10.985 & 4.394 & 2.197 \\
\hline 15.379 & 43.94 & 2.197 & 2.197 \\
\hline 15.379 & 2.197 & 2.197 & 24.167 \\
\hline 2.197 & 4.394 & 2.197 & 19.773 \\
\hline 2.197 & 2.197 & 15.379 & 15.379 \\
\hline 2.197 & 21.97 & 24.167 & 136.214 \\
\hline 13.182 & 28.561 & 2.197 & 2.197 \\
\hline 2.197 & 2.197 & 19.773 & 13.182 \\
\hline 4.394 & 2.197 & 4.394 & 145.002 \\
\hline 2.197 & 32.955 & 35.152 & 6.591 \\
\hline 2.197 & 35.152 & 2.197 & 2.197 \\
\hline 2.197 & 17.576 & 2.197 & 2.197 \\
\hline 1513.73 & 32.955 & 4.394 & 13.182 \\
\hline 6.591 & 8.788 & 15.379 & 13.182 \\
\hline 2.197 & 57.122 & 13.182 & 103.259 \\
\hline 2.197 & 2.197 & 123.032 & 1746.61 \\
\hline 2.197 & 4.394 & 2.197 & 114.244 \\
\hline 2.197 & 37.349 & 2.197 & 2.197 \\
\hline 2.197 & 2.197 & 2.197 & 4.394 \\
\hline 6.591 & 2.197 & 4.394 & 13.182 \\
\hline 2.197 & 6.591 & 2.197 & 10.985 \\
\hline 2.197 & 2.197 & 2.197 & 142.805 \\
\hline 2.197 & 2.197 & 2.197 & 250.458 \\
\hline 2.197 & 2.197 & 2.197 & 37.349 \\
\hline 37.349 & 37.349 & 2.197 & 61.516 \\
\hline 41.743 & 2.197 & 2.197 & 74.698 \\
\hline 2.197 & 30.758 & 4.394 & 990.847 \\
\hline 2.197 & 2.197 & 4.394 & 19.773 \\
\hline 6.591 & 2.197 & 2.197 & 158.184 \\
\hline 2.197 & 2.197 & 2.197 & 43.94 \\
\hline 2.197 & 2.197 & 4.394 & 8.788 \\
\hline 2.197 & 2.197 & 41.743 & 2.197 \\
\hline 2.197 & 28.561 & 2.197 & 6.591 \\
\hline 4.394 & 8.788 & 2.197 & 4.394 \\
\hline 2.197 & 30.758 & 10.985 & 8.788 \\
\hline
\end{tabular}




\begin{tabular}{|c|c|c|c|}
\hline 4.394 & 2.197 & 2.197 & 4.394 \\
\hline 4.394 & 4.394 & 4.394 & 6.591 \\
\hline 13.182 & 6.591 & 8.788 & 17.576 \\
\hline 4.394 & 114.244 & 6.591 & 533.871 \\
\hline 6.591 & 125.229 & 2.197 & 2.197 \\
\hline 54.925 & 2.197 & 2.197 & 4.394 \\
\hline 48.334 & 98.865 & 2.197 & 120.835 \\
\hline 30.758 & 140.608 & 2.197 & 76.895 \\
\hline 15.379 & 2.197 & 355.914 & 21.97 \\
\hline 17.576 & 4.394 & 19.773 & 2.197 \\
\hline 2.197 & 15.379 & 39.546 & 59.319 \\
\hline 2.197 & 2.197 & 2.197 & 2.197 \\
\hline 2.197 & 2.197 & 2.197 & 2.197 \\
\hline 72.501 & 6.591 & 4.394 & 191.139 \\
\hline 4.394 & 2.197 & 547.053 & 2.197 \\
\hline 13.182 & 281.216 & 19.773 & 118.638 \\
\hline 2.197 & 6.591 & 4.394 & 4.394 \\
\hline 2.197 & 2.197 & 74.698 & 8.788 \\
\hline 2.197 & 2.197 & 10.985 & 2.197 \\
\hline 17.576 & 6.591 & 2.197 & 65.91 \\
\hline 13.182 & 4.394 & 8.788 & 6.591 \\
\hline 19.773 & 2.197 & 2.197 & 4.394 \\
\hline 4.394 & 2.197 & 19.773 & 68.107 \\
\hline 386.672 & 52.728 & 6.591 & 2.197 \\
\hline 2.197 & 4.394 & 13.182 & 322.959 \\
\hline 2.197 & 4.394 & 2.197 & 547.053 \\
\hline 6.591 & 8.788 & 2.197 & 8.788 \\
\hline 2.197 & 65.91 & 2.197 & 4.394 \\
\hline 151.593 & 8.788 & 21.97 & 17.576 \\
\hline 19.773 & 2.197 & 2.197 & 8.788 \\
\hline 6.591 & 2.197 & 13.182 & 2.197 \\
\hline 41.743 & 4.394 & 2.197 & 2.197 \\
\hline 28.561 & 4.394 & 2.197 & 24.167 \\
\hline 15.379 & 2.197 & 2.197 & 2.197 \\
\hline 2.197 & 41.743 & 2.197 & 4.394 \\
\hline 35.152 & 15.379 & 4.394 & 4.394 \\
\hline 10.985 & 8.788 & 2.197 & 32.955 \\
\hline 2.197 & 2.197 & 2.197 & 8.788 \\
\hline 6.591 & 2.197 & 2.197 & 32.955 \\
\hline 2.197 & 8.788 & 120.835 & 2.197 \\
\hline 8.788 & 8.788 & 2.197 & 6.591 \\
\hline 54.925 & 2.197 & 32.955 & 43.94 \\
\hline 4.394 & 2.197 & 8.788 & 2.197 \\
\hline 2.197 & 2.197 & 41.743 & 4.394 \\
\hline 4.394 & 4.394 & 314.171 & 32.955 \\
\hline 2.197 & 2.197 & 2948.37 & 6.591 \\
\hline 6.591 & 2.197 & 8.788 & 17.576 \\
\hline 2.197 & 76.895 & 4.394 & 221.897 \\
\hline 2.197 & 2.197 & 2.197 & 2.197 \\
\hline 17.576 & 2.197 & 8.788 & 52.728 \\
\hline
\end{tabular}




\begin{tabular}{|c|c|c|c|}
\hline 10.985 & 2.197 & 2.197 & 19.773 \\
\hline 2.197 & 8.788 & 30.758 & 4.394 \\
\hline 4.394 & 19.773 & 344.929 & 13.182 \\
\hline 2.197 & 35.152 & 4.394 & 1880.63 \\
\hline 10.985 & 6.591 & 21.97 & 8.788 \\
\hline 2.197 & 4.394 & 59.319 & 13.182 \\
\hline 94.471 & 2.197 & 2.197 & 43.94 \\
\hline 2.197 & 10.985 & 885.391 & 10.985 \\
\hline 2.197 & 4.394 & 13.182 & 19.773 \\
\hline 2.197 & 6.591 & 2.197 & 10.985 \\
\hline 2.197 & 35.152 & 21.97 & 8.788 \\
\hline 52.728 & 4.394 & 2.197 & 10.985 \\
\hline 314.171 & 2.197 & 6.591 & 13.182 \\
\hline 2.197 & 17.576 & 26.364 & 4.394 \\
\hline 19.773 & 395.46 & 2.197 & 30.758 \\
\hline 138.411 & 15.379 & 54.925 & 24.167 \\
\hline 4.394 & 15.379 & 2.197 & 19.773 \\
\hline 19.773 & 2.197 & 2.197 & 2.197 \\
\hline 6.591 & 24.167 & 2.197 & 2.197 \\
\hline 26.364 & 2.197 & 13.182 & 8.788 \\
\hline 4.394 & 13.182 & 6.591 & 37.349 \\
\hline 65.91 & 2.197 & 2.197 & 4.394 \\
\hline 4.394 & 2.197 & 2.197 & 17.576 \\
\hline 4.394 & 2.197 & 2.197 & 28.561 \\
\hline 2.197 & 13.182 & 2.197 & 2.197 \\
\hline 17.576 & 164.775 & 2.197 & 4.394 \\
\hline 6.591 & 6.591 & 39.546 & 2.197 \\
\hline 2.197 & 10.985 & 10.985 & 2.197 \\
\hline 2.197 & 2.197 & 4.394 & 65.91 \\
\hline 2.197 & 17.576 & 6.591 & 112.047 \\
\hline 2.197 & 15.379 & 2.197 & 30.758 \\
\hline 26.364 & 2.197 & 2.197 & 4.394 \\
\hline 2.197 & 4.394 & 287.807 & 4.394 \\
\hline 2.197 & 2.197 & 70.304 & 4.394 \\
\hline 2.197 & 4.394 & 2.197 & 109.85 \\
\hline 2.197 & 10.985 & 8.788 & 6.591 \\
\hline 74.698 & 37.349 & 6.591 & 10.985 \\
\hline 72.501 & 8.788 & 2.197 & 68.107 \\
\hline 4.394 & 4.394 & 415.233 & 52.728 \\
\hline 48.334 & 2.197 & 46.137 & 4.394 \\
\hline 6.591 & 15.379 & 2.197 & 4.394 \\
\hline 2.197 & 4.394 & 2.197 & 2.197 \\
\hline 41.743 & 2.197 & 2.197 & 6.591 \\
\hline 10.985 & 2.197 & 169.169 & 13.182 \\
\hline 2.197 & 2.197 & 2.197 & 24.167 \\
\hline 26.364 & 2.197 & 127.426 & 2.197 \\
\hline 2.197 & 593.19 & 2.197 & 191.139 \\
\hline 2.197 & 2.197 & 171.366 & 79.092 \\
\hline 2.197 & 2.197 & 2.197 & 2.197 \\
\hline 2.197 & 2.197 & 2.197 & 4.394 \\
\hline
\end{tabular}




\begin{tabular}{|c|c|c|c|}
\hline 2.197 & 2.197 & 4.394 & 28.561 \\
\hline 2.197 & 2.197 & 2.197 & 2.197 \\
\hline 21.97 & 4.394 & 8.788 & 46.137 \\
\hline 142.805 & 2.197 & 6.591 & 13.182 \\
\hline 8.788 & 2.197 & 74.698 & 10.985 \\
\hline 17.576 & 6.591 & 125.229 & 966.68 \\
\hline 158.184 & 2.197 & 6.591 & 292.201 \\
\hline 2.197 & 2.197 & 2.197 & 26.364 \\
\hline 2.197 & 2.197 & 4.394 & 4.394 \\
\hline 2.197 & 19.773 & 2.197 & 2.197 \\
\hline 4.394 & 8.788 & 2.197 & 17.576 \\
\hline 127.426 & 6.591 & 2.197 & 52.728 \\
\hline 6.591 & 1067.74 & 2.197 & 10.985 \\
\hline 6.591 & 8.788 & 1272.06 & 54.925 \\
\hline 13.182 & 13.182 & 8.788 & 10.985 \\
\hline 221.897 & 41.743 & 2.197 & 46.137 \\
\hline 19.773 & 2.197 & 2.197 & 65.91 \\
\hline 65.91 & 2.197 & 19.773 & 10.985 \\
\hline 4.394 & 41.743 & 2.197 & 32.955 \\
\hline 6.591 & 15.379 & 2.197 & 70.304 \\
\hline 2.197 & 8.788 & 2.197 & 21.97 \\
\hline 2.197 & 2.197 & 224.094 & 10.985 \\
\hline 2.197 & 279.019 & 4.394 & 43.94 \\
\hline 4.394 & 48.334 & 72.501 & 35.152 \\
\hline 2.197 & 98.865 & 6.591 & 263.64 \\
\hline 6.591 & 8.788 & 2.197 & 4.394 \\
\hline 6.591 & 131.82 & 4.394 & 4.394 \\
\hline 2.197 & 17.576 & 4.394 & 6.591 \\
\hline 17.576 & 10.985 & 2.197 & 2.197 \\
\hline 6.591 & 17.576 & 10.985 & 2.197 \\
\hline 30.758 & 204.321 & 13.182 & 2.197 \\
\hline 4.394 & 19.773 & 2.197 & 15.379 \\
\hline 2.197 & 2.197 & 6.591 & 2.197 \\
\hline 6.591 & 4.394 & 2.197 & 2.197 \\
\hline 180.154 & 120.835 & 4.394 & 39.546 \\
\hline 4.394 & 4.394 & 4.394 & 4.394 \\
\hline 81.289 & 8.788 & 13.182 & 2.197 \\
\hline 2.197 & 13.182 & 2.197 & 2.197 \\
\hline 2.197 & 2.197 & 13.182 & 2.197 \\
\hline 2.197 & 2.197 & 2.197 & 208.715 \\
\hline 2.197 & 4.394 & 2.197 & 142.805 \\
\hline 41.743 & 4.394 & 46.137 & 4.394 \\
\hline 2.197 & 13.182 & 2.197 & 4.394 \\
\hline 2.197 & 4.394 & 17.576 & 39.546 \\
\hline 2.197 & 10.985 & 2.197 & 2.197 \\
\hline 2.197 & 2.197 & 2.197 & 19.773 \\
\hline 4.394 & 2.197 & 2.197 & 24.167 \\
\hline 2.197 & 4.394 & 2.197 & 15.379 \\
\hline 2.197 & 4.394 & 166.972 & 6.591 \\
\hline 2.197 & 4.394 & 2.197 & 6.591 \\
\hline
\end{tabular}




\begin{tabular}{|c|c|c|c|}
\hline 2.197 & 30.758 & 2.197 & 8.788 \\
\hline 4.394 & 39.546 & 15.379 & 13.182 \\
\hline 2.197 & 10.985 & 4.394 & 8.788 \\
\hline 342.732 & 2.197 & 4.394 & 10.985 \\
\hline 4.394 & 2.197 & 2.197 & 21.97 \\
\hline 2.197 & 2.197 & 19.773 & 59.319 \\
\hline 63.713 & 2.197 & 171.366 & 2.197 \\
\hline 10.985 & 43.94 & 2.197 & 2.197 \\
\hline 153.79 & 2.197 & 2.197 & 2.197 \\
\hline 41.743 & 2.197 & 193.336 & 19.773 \\
\hline 4.394 & 21.97 & 4.394 & 24.167 \\
\hline 4.394 & 2.197 & 17.576 & 8.788 \\
\hline 2.197 & 15.379 & 6.591 & 6.591 \\
\hline 35.152 & 2.197 & 6.591 & 6.591 \\
\hline 2.197 & 2.197 & 268.034 & 4.394 \\
\hline 8.788 & 19.773 & 10.985 & 10.985 \\
\hline 2.197 & 15.379 & 2.197 & 4.394 \\
\hline 259.246 & 30.758 & 2.197 & 349.323 \\
\hline 4.394 & 125.229 & 2.197 & 32.955 \\
\hline 2.197 & 6.591 & 1548.88 & 101.062 \\
\hline 2.197 & 10.985 & 2.197 & 54.925 \\
\hline 2.197 & 8.788 & 2.197 & 37.349 \\
\hline 2.197 & 2.197 & 1526.91 & 52.728 \\
\hline 160.381 & 54.925 & 4.394 & 4.394 \\
\hline 4.394 & 19.773 & 8.788 & 54.925 \\
\hline 2.197 & 6.591 & 6.591 & 43.94 \\
\hline 10.985 & 2.197 & 2.197 & 4.394 \\
\hline 21.97 & 2.197 & 4.394 & 2.197 \\
\hline 2.197 & 2.197 & 2.197 & 10.985 \\
\hline 6.591 & 118.638 & 2471.62 & 24.167 \\
\hline 8.788 & 2.197 & 4.394 & 2.197 \\
\hline 85.683 & 13.182 & 118.638 & 2.197 \\
\hline 2.197 & 32.955 & 4.394 & 46.137 \\
\hline 2.197 & 10.985 & 2.197 & 17.576 \\
\hline 10.985 & 4.394 & 8.788 & 6.591 \\
\hline 28.561 & 2.197 & 129.623 & 2.197 \\
\hline 2.197 & 2.197 & 37.349 & 28.561 \\
\hline 2.197 & 48.334 & 2.197 & 63.713 \\
\hline 4.394 & 8.788 & 2.197 & 2.197 \\
\hline 13.182 & 2.197 & 2.197 & 4.394 \\
\hline 35.152 & 4.394 & 2.197 & 338.338 \\
\hline 2.197 & 4.394 & 2.197 & 4.394 \\
\hline 2.197 & 13.182 & 2.197 & 2.197 \\
\hline 210.912 & 13.182 & 2.197 & 114.244 \\
\hline 37.349 & 2.197 & 4.394 & 26.364 \\
\hline 32.955 & 13.182 & 15.379 & 118.638 \\
\hline 4.394 & 2.197 & 2.197 & 28.561 \\
\hline 19.773 & 2.197 & 28.561 & 6.591 \\
\hline 2.197 & 2.197 & 2.197 & 13.182 \\
\hline 19.773 & 2.197 & 2.197 & 2.197 \\
\hline
\end{tabular}




\begin{tabular}{|c|c|c|c|}
\hline 4.394 & 6.591 & 19.773 & 2.197 \\
\hline 2.197 & 4.394 & 39.546 & 39.546 \\
\hline 2.197 & 10.985 & 964.483 & 8.788 \\
\hline 35.152 & 10.985 & 2.197 & 26.364 \\
\hline 252.655 & 8.788 & 388.869 & 61.516 \\
\hline 10.985 & 96.668 & 2.197 & 54.925 \\
\hline 2.197 & 4.394 & 4.394 & 4.394 \\
\hline 2.197 & 2.197 & 63.713 & 19.773 \\
\hline 2.197 & 13.182 & 6.591 & 2.197 \\
\hline 2.197 & 6.591 & 2.197 & 6.591 \\
\hline 6.591 & 13.182 & 2.197 & 114.244 \\
\hline 19.773 & 373.49 & 10.985 & 8.788 \\
\hline 2.197 & 2.197 & 61.516 & 24.167 \\
\hline 4.394 & 2.197 & 2.197 & 17.576 \\
\hline 241.67 & 2.197 & 6.591 & 2.197 \\
\hline 2.197 & 8.788 & 65.91 & 59.319 \\
\hline 2.197 & 2.197 & 421.824 & 15.379 \\
\hline 8.788 & 4.394 & 2.197 & 131.82 \\
\hline 6.591 & 2.197 & 19.773 & 2.197 \\
\hline 15.379 & 2.197 & 35.152 & 2.197 \\
\hline 2.197 & 8.788 & 2.197 & 2.197 \\
\hline 54.925 & 8.788 & 2.197 & 4.394 \\
\hline 28.561 & 21.97 & 13.182 & 2.197 \\
\hline 41.743 & 24.167 & 542.659 & 21.97 \\
\hline 79.092 & 2.197 & 8.788 & 24.167 \\
\hline 2.197 & 2.197 & 2.197 & 24.167 \\
\hline 2.197 & 2.197 & 4.394 & 32.955 \\
\hline 2.197 & 4.394 & 4.394 & 15.379 \\
\hline 2.197 & 13.182 & 2.197 & 54.925 \\
\hline 6.591 & 2.197 & 74.698 & 65.91 \\
\hline 6.591 & 13.182 & 37.349 & 2.197 \\
\hline 6.591 & 24.167 & 2.197 & 10.985 \\
\hline 30.758 & 2.197 & 17.576 & 188.942 \\
\hline 2.197 & 2.197 & 13.182 & 39.546 \\
\hline 15.379 & 19.773 & 13.182 & 177.957 \\
\hline 1792.75 & 2.197 & 4.394 & 96.668 \\
\hline 6.591 & 4.394 & 85.683 & 94.471 \\
\hline 6.591 & 4.394 & 641.524 & 6.591 \\
\hline 92.274 & 6.591 & 17.576 & 4.394 \\
\hline 2.197 & 2.197 & 2.197 & 4.394 \\
\hline 2.197 & 6.591 & 13.182 & 10.985 \\
\hline 2.197 & 52.728 & 13.182 & 101.062 \\
\hline 2.197 & 54.925 & 2.197 & 8.788 \\
\hline 2.197 & 6.591 & 43.94 & 13.182 \\
\hline 6.591 & 24.167 & 6.591 & 3794.22 \\
\hline 2.197 & 2.197 & 4.394 & 2.197 \\
\hline 213.109 & 13.182 & 2.197 & 120.835 \\
\hline 4.394 & 13.182 & 147.199 & 6.591 \\
\hline 109.85 & 68.107 & 2.197 & 4.394 \\
\hline 2.197 & 13.182 & 2.197 & 13.182 \\
\hline
\end{tabular}




\begin{tabular}{|c|c|c|c|}
\hline 39.546 & 24.167 & 2.197 & 1177.59 \\
\hline 6.591 & 2.197 & 2.197 & 8.788 \\
\hline 476.749 & 2.197 & 2.197 & 540.462 \\
\hline 2.197 & 8.788 & 2.197 & 4.394 \\
\hline 72.501 & 4.394 & 6.591 & 2.197 \\
\hline 2.197 & 2.197 & 2.197 & 4.394 \\
\hline 28.561 & 10.985 & 2.197 & 13.182 \\
\hline 15.379 & 6.591 & 15.379 & 10.985 \\
\hline 6.591 & 2.197 & 2.197 & 2.197 \\
\hline 2.197 & 2.197 & 2.197 & 2.197 \\
\hline 142.805 & 4.394 & 107.653 & 24.167 \\
\hline 43.94 & 4.394 & 2.197 & 2.197 \\
\hline 4.394 & 4.394 & 30.758 & 52.728 \\
\hline 2.197 & 4.394 & 37.349 & 10.985 \\
\hline 6.591 & 13.182 & 6.591 & 24.167 \\
\hline 4.394 & 2.197 & 30.758 & 24.167 \\
\hline 2.197 & 4.394 & 32.955 & 15.379 \\
\hline 4.394 & 6.591 & 4.394 & 6.591 \\
\hline 4.394 & 10.985 & 30.758 & 325.156 \\
\hline 4.394 & 6.591 & 4.394 & 2.197 \\
\hline 26.364 & 2.197 & 4.394 & 960.089 \\
\hline 6.591 & 2.197 & 2.197 & 8.788 \\
\hline 2.197 & 28.561 & 6.591 & 2.197 \\
\hline 2.197 & 2.197 & 48.334 & 74.698 \\
\hline 2.197 & 41.743 & 2.197 & 57.122 \\
\hline 2.197 & 10.985 & 2.197 & 61.516 \\
\hline 28.561 & 2.197 & 2.197 & 72.501 \\
\hline 37.349 & 28.561 & 2.197 & 158.184 \\
\hline 8.788 & 94.471 & 92.274 & 94.471 \\
\hline 6.591 & 39.546 & 2.197 & 74.698 \\
\hline 46.137 & 2.197 & 39.546 & 2.197 \\
\hline 2.197 & 2.197 & 2.197 & 2.197 \\
\hline 2.197 & 17.576 & 10.985 & 4.394 \\
\hline 35.152 & 2.197 & 4.394 & 6.591 \\
\hline 68.107 & 2.197 & 4.394 & 6.591 \\
\hline 2.197 & 4.394 & 4.394 & 35.152 \\
\hline 13.182 & 43.94 & 8.788 & 483.34 \\
\hline 43.94 & 41.743 & 24.167 & 70.304 \\
\hline 4.394 & 83.486 & 2.197 & 4.394 \\
\hline 6.591 & 13.182 & 10.985 & 2.197 \\
\hline 2.197 & 2.197 & 4.394 & 74.698 \\
\hline 2.197 & 116.441 & 6.591 & 65.91 \\
\hline 2.197 & 2.197 & 4.394 & 4.394 \\
\hline 10.985 & 13.182 & 28.561 & 4.394 \\
\hline 2.197 & 2.197 & 65.91 & 4.394 \\
\hline 35.152 & 4.394 & 6.591 & 371.293 \\
\hline 2.197 & 2.197 & 4.394 & 2.197 \\
\hline 2.197 & 112.047 & 81.289 & 2.197 \\
\hline 4.394 & 15.379 & 74.698 & 4.394 \\
\hline 263.64 & 2.197 & 2.197 & 10.985 \\
\hline
\end{tabular}




\begin{tabular}{|c|c|c|c|}
\hline 2.197 & 2.197 & 2.197 & 46.137 \\
\hline 123.032 & 6.591 & 35.152 & 10.985 \\
\hline 4.394 & 4.394 & 19.773 & 8.788 \\
\hline 2.197 & 15.379 & 10.985 & 6.591 \\
\hline 52.728 & 2.197 & 2.197 & 13.182 \\
\hline 2.197 & 2.197 & 2.197 & 15.379 \\
\hline 17.576 & 6.591 & 2.197 & 2.197 \\
\hline 134.017 & 6.591 & 6.591 & 2.197 \\
\hline 221.897 & 4.394 & 13.182 & 171.366 \\
\hline 2.197 & 4.394 & 21.97 & 76.895 \\
\hline 6.591 & 13.182 & 68.107 & 19.773 \\
\hline 10.985 & 17.576 & 28.561 & 32.955 \\
\hline 39.546 & 24.167 & 2.197 & 4.394 \\
\hline 28.561 & 15.379 & 8.788 & 6129.63 \\
\hline 4.394 & 6.591 & 2.197 & 48.334 \\
\hline 13.182 & 4.394 & 26.364 & 19.773 \\
\hline 2.197 & 2.197 & 2.197 & 39.546 \\
\hline 6.591 & 4.394 & 2.197 & 2.197 \\
\hline 2.197 & 2.197 & 2.197 & 2.197 \\
\hline 2.197 & 2.197 & 2.197 & 6.591 \\
\hline 2.197 & 173.563 & 6.591 & 13.182 \\
\hline 6.591 & 41.743 & 2.197 & 2.197 \\
\hline 2.197 & 10.985 & 92.274 & 151.593 \\
\hline 2.197 & 4.394 & 35.152 & 2.197 \\
\hline 10.985 & 6.591 & 21.97 & 2.197 \\
\hline 8.788 & 10.985 & 4.394 & 37.349 \\
\hline 37.349 & 138.411 & 2.197 & 72.501 \\
\hline 2.197 & 8.788 & 15.379 & $8.7 \varepsilon$ \\
\hline 558.038 & 37.349 & 360.308 & 8.788 \\
\hline 35.152 & 28.561 & 2.197 & 391.066 \\
\hline 2.197 & 1467.6 & 2.197 & 59.319 \\
\hline 2.197 & 1129.26 & 32.955 & 2.197 \\
\hline 65.91 & 8.788 & 2.197 & 10.985 \\
\hline 35.152 & 6.591 & 2.197 & 698.646 \\
\hline 4.394 & 6.591 & 15.379 & 19.773 \\
\hline 52.728 & 4.394 & 2.197 & 2.197 \\
\hline 57.122 & 6.591 & 4.394 & 59.31 \\
\hline 2.197 & 4.394 & 105.456 & 268.03 \\
\hline 4.394 & 4.394 & 2.197 & 32.955 \\
\hline 21.97 & 15.379 & 2.197 & 41.743 \\
\hline 13.182 & 6.591 & 4.394 & 2.197 \\
\hline 13.182 & 782.132 & 2.197 & 15.379 \\
\hline 6.591 & 28.561 & 6.591 & 19.77 \\
\hline 43.94 & 13.182 & 2.197 & 142.805 \\
\hline 24.167 & 2.197 & 35.152 & 4.394 \\
\hline 2.197 & 2.197 & 2.197 & 8.78 \\
\hline 101.062 & 2.197 & 2.197 & 43.94 \\
\hline 13.182 & 46.137 & 4.394 & 96.668 \\
\hline 70.304 & 46.137 & 2.197 & 15.379 \\
\hline 10.985 & 2.197 & 6.591 & 4.394 \\
\hline
\end{tabular}




\begin{tabular}{|c|c|c|c|}
\hline 21.97 & 15.379 & 2.197 & 15.379 \\
\hline 6.591 & 2.197 & 8.788 & 8.788 \\
\hline 2.197 & 2.197 & 4.394 & 19.773 \\
\hline 2.197 & 15.379 & 2.197 & 2.197 \\
\hline 421.824 & 2.197 & 4.394 & 8.788 \\
\hline 4.394 & 8.788 & 4.394 & 13.182 \\
\hline 2.197 & 2.197 & 2.197 & 8.788 \\
\hline 2.197 & 2.197 & 17.576 & 10.985 \\
\hline 17.576 & 24.167 & 19.773 & 105.456 \\
\hline 228.488 & 6.591 & 6.591 & 10.985 \\
\hline 4.394 & 623.948 & 8.788 & 13.182 \\
\hline 2.197 & 13.182 & 4.394 & 10.985 \\
\hline 4.394 & 2.197 & 15.379 & 217.503 \\
\hline 4.394 & 37.349 & 21.97 & 30.758 \\
\hline 2.197 & 17.576 & 50.531 & 17.576 \\
\hline 2.197 & 48.334 & 4.394 & 26.364 \\
\hline 30.758 & 35.152 & 37.349 & 10.985 \\
\hline 4.394 & 2.197 & 13.182 & 2.197 \\
\hline 2.197 & 2.197 & 79.092 & 263.64 \\
\hline 98.865 & 2.197 & 13.182 & 10.985 \\
\hline 94.471 & 19.773 & 876.603 & 2.197 \\
\hline 6.591 & 4.394 & 59.319 & 2.197 \\
\hline 6.591 & 43.94 & 4.394 & 13.182 \\
\hline 41.743 & 6.591 & 8.788 & 228.488 \\
\hline 10.985 & 10.985 & 35.152 & 28.561 \\
\hline 2.197 & 6.591 & 2.197 & 26.364 \\
\hline 2.197 & 2.197 & 2.197 & 57.122 \\
\hline 6.591 & 2.197 & 41.743 & 21.97 \\
\hline 2.197 & 4.394 & 8.788 & 17.576 \\
\hline 2.197 & 2.197 & 2.197 & 8.788 \\
\hline 2.197 & 4.394 & 2.197 & 6.591 \\
\hline 10.985 & 2.197 & 2.197 & 24.167 \\
\hline 13.182 & 2.197 & 8.788 & 4.394 \\
\hline 6.591 & 21.97 & 140.608 & 2.197 \\
\hline 4.394 & 15.379 & 382.278 & 4.394 \\
\hline 340.535 & 4.394 & 17.576 & 94.471 \\
\hline 10.985 & 6.591 & 26.364 & 2.197 \\
\hline 4.394 & 30.758 & 76.895 & 2.197 \\
\hline 2.197 & 118.638 & 2.197 & 10.985 \\
\hline 52.728 & 32.955 & 4.394 & 17.576 \\
\hline 43.94 & 19.773 & 10.985 & 3534.97 \\
\hline 1647.75 & 2.197 & 2.197 & 360.308 \\
\hline 10.985 & 4.394 & 4.394 & 2.197 \\
\hline 6.591 & 4.394 & 2.197 & 2.197 \\
\hline 1566.46 & 43.94 & 2.197 & 4.394 \\
\hline 4.394 & 24.167 & 76.895 & 13.182 \\
\hline 68.107 & 6.591 & 4.394 & 13.182 \\
\hline 4.394 & 4.394 & 4.394 & 4.394 \\
\hline 6.591 & 4.394 & 2.197 & 2.197 \\
\hline 4.394 & 4.394 & 2.197 & 2.197 \\
\hline
\end{tabular}




\begin{tabular}{|c|c|c|c|}
\hline 10.985 & 2.197 & 2.197 & 35.152 \\
\hline 8.788 & 10.985 & 41.743 & 68.107 \\
\hline 116.441 & 2.197 & 2.197 & 164.775 \\
\hline 2.197 & 2.197 & 2.197 & 10.985 \\
\hline 2.197 & 4.394 & 2.197 & 226.291 \\
\hline 2.197 & 15.379 & 2.197 & 8.788 \\
\hline 74.698 & 2.197 & 2.197 & 175.76 \\
\hline 94.471 & 2.197 & 4.394 & 2.197 \\
\hline 142.805 & 2.197 & 4.394 & 94.471 \\
\hline 2.197 & 19.773 & 2.197 & 8.788 \\
\hline 35.152 & 2.197 & 2.197 & 2.197 \\
\hline 252.655 & 2.197 & 2.197 & 2.197 \\
\hline 2.197 & 6.591 & 43.94 & 2.197 \\
\hline 2.197 & 4.394 & 2.197 & 4.394 \\
\hline 6.591 & 37.349 & 2.197 & 10.985 \\
\hline 15.379 & 2.197 & 432.809 & 8.788 \\
\hline 2.197 & 173.563 & 8.788 & 61.516 \\
\hline 2.197 & 2.197 & 2.197 & 8.788 \\
\hline 4.394 & 8.788 & 13.182 & 26.364 \\
\hline 2.197 & 2.197 & 355.914 & 195.533 \\
\hline 48.334 & 30.758 & 2.197 & 2.197 \\
\hline 2.197 & 4.394 & 6.591 & 2.197 \\
\hline 19.773 & 13.182 & 39.546 & 2.197 \\
\hline 4.394 & 2.197 & 2.197 & 4.394 \\
\hline 2.197 & 2.197 & 19.773 & 2.197 \\
\hline 2.197 & 10.985 & 21.97 & 2.197 \\
\hline 2.197 & 28.561 & 19.773 & 2.197 \\
\hline 2.197 & 6.591 & 4.394 & 74.698 \\
\hline 2.197 & 2.197 & 2.197 & 21.97 \\
\hline 2.197 & 2.197 & 4.394 & 19.773 \\
\hline 2.197 & 4.394 & 24.167 & 15.379 \\
\hline 2.197 & 4.394 & 6.591 & 2.197 \\
\hline 4.394 & 2.197 & 2.197 & 19.773 \\
\hline 8.788 & 15.379 & 21.97 & 103.259 \\
\hline 2.197 & 2.197 & 72.501 & 169.169 \\
\hline 2.197 & 28.561 & 10.985 & 79.092 \\
\hline 13.182 & 13.182 & 24.167 & 10.985 \\
\hline 35.152 & 21.97 & 2.197 & 10.985 \\
\hline 2.197 & 17.576 & 13.182 & 19.773 \\
\hline 68.107 & 120.835 & 2.197 & 138.411 \\
\hline 2.197 & 1289.64 & 19.773 & 15.379 \\
\hline 17.576 & 114.244 & 2.197 & 149.396 \\
\hline 61.516 & 257.049 & 17.576 & 6.591 \\
\hline 4.394 & 4.394 & 2.197 & 2.197 \\
\hline 2.197 & 4.394 & 2.197 & 4.394 \\
\hline 2.197 & 2.197 & 2.197 & 17.576 \\
\hline 8.788 & 397.657 & 2.197 & 10.985 \\
\hline 4.394 & 226.291 & 65.91 & 6.591 \\
\hline 13.182 & 2.197 & 6.591 & 4.394 \\
\hline 2.197 & 6.591 & 43.94 & 24.167 \\
\hline
\end{tabular}




\begin{tabular}{|c|c|c|c|}
\hline 24.167 & 4.394 & 17.576 & 1149.03 \\
\hline 39.546 & 63.713 & 2.197 & 26.364 \\
\hline 2.197 & 76.895 & 4.394 & 6.591 \\
\hline 2.197 & 386.672 & 54.925 & 8.788 \\
\hline 92.274 & 50.531 & 2.197 & 2.197 \\
\hline 2.197 & 2.197 & 13.182 & 2.197 \\
\hline 2.197 & 24.167 & 116.441 & 4.394 \\
\hline 2282.68 & 101.062 & 2.197 & 4.394 \\
\hline 6.591 & 24.167 & 4.394 & 8.788 \\
\hline 2.197 & 2.197 & 8.788 & 2.197 \\
\hline 4.394 & 2.197 & 32146.5 & 2.197 \\
\hline 2.197 & 4.394 & 2.197 & 2.197 \\
\hline 2.197 & 4.394 & 2.197 & 10.985 \\
\hline 4.394 & 4.394 & 2.197 & 2.197 \\
\hline 2.197 & 24.167 & 2.197 & 270.231 \\
\hline 2.197 & 2.197 & 4.394 & 10.985 \\
\hline 8.788 & 2.197 & 8.788 & 880.997 \\
\hline 2.197 & 2.197 & 8.788 & 6.591 \\
\hline 39.546 & 4.394 & 4.394 & 2.197 \\
\hline 4.394 & 6.591 & 2.197 & 2.197 \\
\hline 6.591 & 30.758 & 2.197 & 24.167 \\
\hline 2.197 & 2.197 & 13.182 & 32.955 \\
\hline 13.182 & 13.182 & 2.197 & 6.591 \\
\hline 2.197 & 10.985 & 2.197 & 46.137 \\
\hline 6.591 & 2.197 & 2.197 & 10.985 \\
\hline 2.197 & 2.197 & 2.197 & 2.197 \\
\hline 2.197 & 6.591 & 2.197 & 142.805 \\
\hline 2.197 & 2.197 & 2.197 & 17.576 \\
\hline 61.516 & 4.394 & 2.197 & 8.788 \\
\hline 8.788 & 2.197 & 2.197 & 2.197 \\
\hline 15.379 & 10.985 & 2.197 & 19.773 \\
\hline 83.486 & 2.197 & 2.197 & 2.197 \\
\hline 1056.76 & 54.925 & 2.197 & 17.576 \\
\hline 6.591 & 6.591 & 2.197 & 30.758 \\
\hline 4.394 & 74.698 & 2.197 & 276.822 \\
\hline 61.516 & 6.591 & 2.197 & 8.788 \\
\hline 2.197 & 28.561 & 43.94 & 2.197 \\
\hline 2.197 & 19.773 & 19.773 & 2.197 \\
\hline 2.197 & 6.591 & 21.97 & 2.197 \\
\hline 8.788 & 2.197 & 8.788 & 4.394 \\
\hline 4.394 & 6.591 & 10.985 & 17.576 \\
\hline 26.364 & 4.394 & 142.805 & 4.394 \\
\hline 17.576 & 65.91 & 2.197 & 2.197 \\
\hline 2.197 & 52.728 & 19.773 & 4.394 \\
\hline 2.197 & 46.137 & 2.197 & 2.197 \\
\hline 4.394 & 90.077 & 19.773 & 2.197 \\
\hline 4.394 & 46.137 & 2.197 & 2.197 \\
\hline 2.197 & 6.591 & 68.107 & 2.197 \\
\hline 4.394 & 155.987 & 2.197 & 2.197 \\
\hline 6.591 & 2.197 & 46.137 & 4.394 \\
\hline
\end{tabular}




\begin{tabular}{|c|c|c|c|}
\hline 54.925 & 13.182 & 10.985 & 21.97 \\
\hline 19.773 & 2.197 & 177.957 & 125.229 \\
\hline 2.197 & 2.197 & 2.197 & 2.197 \\
\hline 4.394 & 21.97 & 2.197 & 2.197 \\
\hline 10.985 & 4.394 & 2.197 & 10.985 \\
\hline 8.788 & 2.197 & 28.561 & 28.561 \\
\hline 15.379 & 182.351 & 2.197 & 2.197 \\
\hline 4.394 & 2.197 & 2.197 & 4.394 \\
\hline 8.788 & 30.758 & 120.835 & 76.895 \\
\hline 8.788 & 6.591 & 4.394 & 13.182 \\
\hline 17.576 & 13.182 & 37.349 & 8.788 \\
\hline 6.591 & 13.182 & 2.197 & 13.182 \\
\hline 4.394 & 4.394 & 41.743 & 4.394 \\
\hline 2.197 & 206.518 & 94.471 & 6.591 \\
\hline 32.955 & 39.546 & 2.197 & 4.394 \\
\hline 32.955 & 17.576 & 35.152 & 19.773 \\
\hline 98.865 & 4.394 & 4.394 & 6.591 \\
\hline 2.197 & 4.394 & 6.591 & 4.394 \\
\hline 2.197 & 4.394 & 2.197 & 2.197 \\
\hline 2.197 & 15.379 & 32.955 & 6.591 \\
\hline 8.788 & 4.394 & 2.197 & 10.985 \\
\hline 6.591 & 4.394 & 4.394 & 4.394 \\
\hline 43.94 & 4.394 & 28.561 & 384.475 \\
\hline 6.591 & 2.197 & 10.985 & 2.197 \\
\hline 2.197 & 2.197 & 410.839 & 2.197 \\
\hline 4.394 & 28.561 & 2.197 & 32.955 \\
\hline 35.152 & 2.197 & 19.773 & 46.137 \\
\hline 2.197 & 10.985 & 4.394 & 2.197 \\
\hline 21.97 & 2.197 & 28.561 & 50.531 \\
\hline 43.94 & 15.379 & 46.137 & 65.91 \\
\hline 1072.14 & 206.518 & 54.925 & 8.788 \\
\hline 4.394 & 2.197 & 4.394 & 2.197 \\
\hline 2.197 & 2.197 & 2.197 & 30.758 \\
\hline 2.197 & 2.197 & 2.197 & 2.197 \\
\hline 74.698 & 98.865 & 13.182 & 6.591 \\
\hline 37.349 & 248.261 & 4.394 & 30.758 \\
\hline 2.197 & 2.197 & 2.197 & 4.394 \\
\hline 4.394 & 2.197 & 4.394 & 6.591 \\
\hline 4.394 & 2.197 & 2.197 & 13.182 \\
\hline 26.364 & 276.822 & 4.394 & 1379.72 \\
\hline 2.197 & 2.197 & 6.591 & 1050.17 \\
\hline 8.788 & 10.985 & 2.197 & 61.516 \\
\hline 2.197 & 4.394 & 2.197 & 15.379 \\
\hline 2.197 & 24.167 & 46.137 & 59.319 \\
\hline 26.364 & 10.985 & 15.379 & 2.197 \\
\hline 318.565 & 4.394 & 13.182 & 15.379 \\
\hline 10.985 & 48.334 & 50.531 & 32.955 \\
\hline 8.788 & 6.591 & 6.591 & 2.197 \\
\hline 2.197 & 4.394 & 19.773 & 37.349 \\
\hline 2.197 & 4.394 & 10.985 & 13.182 \\
\hline
\end{tabular}




\begin{tabular}{|c|c|c|c|}
\hline 17.576 & 2.197 & 61.516 & 61.516 \\
\hline 94.471 & 2.197 & 105.456 & 4.394 \\
\hline 2.197 & 2.197 & 149.396 & 96.668 \\
\hline 4.394 & 19.773 & 13.182 & 39.546 \\
\hline 2.197 & 26.364 & 2.197 & 17.576 \\
\hline 52.728 & 21.97 & 4.394 & 32.955 \\
\hline 24.167 & 2.197 & 8.788 & 6.591 \\
\hline 6.591 & 46.137 & 2.197 & 52.728 \\
\hline 2.197 & 8.788 & 2.197 & 41.743 \\
\hline 10.985 & 6.591 & 2.197 & 4.394 \\
\hline 41.743 & 15.379 & 4.394 & 4.394 \\
\hline 63.713 & 4.394 & 4.394 & 15.379 \\
\hline 2.197 & 2.197 & 8.788 & 10.985 \\
\hline 61.516 & 4.394 & 8.788 & 294.398 \\
\hline 30.758 & 2.197 & 2.197 & 226.291 \\
\hline 8.788 & 4.394 & 4.394 & 13.182 \\
\hline 2.197 & 2.197 & 2.197 & 173.563 \\
\hline 2.197 & 2.197 & 2.197 & 8.788 \\
\hline 2.197 & 24.167 & 2.197 & 28.561 \\
\hline 2.197 & 15.379 & 2.197 & 175.76 \\
\hline 6.591 & 2.197 & 68.107 & 17.576 \\
\hline 2.197 & 41.743 & 2.197 & 158.184 \\
\hline 59.319 & 2.197 & 2.197 & 290.004 \\
\hline 2.197 & 2.197 & 30.758 & 17.576 \\
\hline 4.394 & 2.197 & 2.197 & 101.062 \\
\hline 580.008 & 52.728 & 10.985 & 17.576 \\
\hline 4.394 & 107.653 & 30.758 & 50.531 \\
\hline 2.197 & 4.394 & 8.788 & 2.197 \\
\hline 268.034 & 2.197 & 2.197 & 1458.81 \\
\hline 90.077 & 19.773 & 57.122 & 6.591 \\
\hline 2.197 & 21.97 & 4.394 & 2.197 \\
\hline 134.017 & 21.97 & 2.197 & 24.167 \\
\hline 41.743 & 30.758 & 21.97 & 15.379 \\
\hline 2.197 & 109.85 & 2.197 & 8.788 \\
\hline 13.182 & 140.608 & 6.591 & 41.743 \\
\hline 153.79 & 13.182 & 4.394 & 17.576 \\
\hline 6.591 & 6.591 & 24.167 & 957.892 \\
\hline 21.97 & 2.197 & 26.364 & 2.197 \\
\hline 2.197 & 4.394 & 2.197 & 4.394 \\
\hline 129.623 & 19.773 & 406.445 & 37.349 \\
\hline 8.788 & 21.97 & 37.349 & 43.94 \\
\hline 2.197 & 2.197 & 4.394 & 2.197 \\
\hline 13.182 & 15.379 & 2.197 & 10.985 \\
\hline 10.985 & 17.576 & 30.758 & 2.197 \\
\hline 4.394 & 6.591 & 54.925 & 2.197 \\
\hline 26.364 & 41.743 & 37.349 & 6.591 \\
\hline 35.152 & 2.197 & 10.985 & 2.197 \\
\hline 2.197 & 4.394 & 2.197 & 13.182 \\
\hline 17.576 & 125.229 & 2.197 & 26.364 \\
\hline 6.591 & 2.197 & 10.985 & 8.788 \\
\hline
\end{tabular}




\begin{tabular}{|c|c|c|c|}
\hline 6.591 & 21.97 & 4.394 & 81.289 \\
\hline 8.788 & 2.197 & 17.576 & 81.289 \\
\hline 2.197 & 2.197 & 13.182 & 2.197 \\
\hline 2.197 & 2.197 & 2.197 & 13.182 \\
\hline 2.197 & 147.199 & 52.728 & 46.137 \\
\hline 2.197 & 2.197 & 6.591 & 37.349 \\
\hline 2.197 & 8.788 & 59.319 & 13.182 \\
\hline 2.197 & 272.428 & 2.197 & 2.197 \\
\hline 4.394 & 2.197 & 10.985 & 2.197 \\
\hline 6.591 & 4.394 & 2.197 & 8.788 \\
\hline 6.591 & 2.197 & 131.82 & 2.197 \\
\hline 61.516 & 4.394 & 8.788 & 10.985 \\
\hline 57.122 & 2.197 & 24.167 & 4.394 \\
\hline 4.394 & 2.197 & 21.97 & 2.197 \\
\hline 2.197 & 2.197 & 24.167 & 6.591 \\
\hline 50.531 & 8.788 & 140.608 & 90.077 \\
\hline 2.197 & 164.775 & 4.394 & 2.197 \\
\hline 21.97 & 2.197 & 2.197 & 2.197 \\
\hline 2.197 & 2.197 & 4.394 & 2.197 \\
\hline 2.197 & 4.394 & 2.197 & 19.773 \\
\hline 226.291 & 26.364 & 15.379 & 8.788 \\
\hline 4.394 & 8.788 & 2.197 & 24.167 \\
\hline 41.743 & 41.743 & 2.197 & 6.591 \\
\hline 2.197 & 2.197 & 19.773 & 2.197 \\
\hline 6.591 & 4.394 & 17.576 & 2.197 \\
\hline 48.334 & 13.182 & 6.591 & 4.394 \\
\hline 6.591 & 6.591 & 4.394 & 8.788 \\
\hline 10.985 & 2.197 & 4.394 & 2.197 \\
\hline 2.197 & 19.773 & 2.197 & 76.895 \\
\hline 17.576 & 48.334 & 2.197 & 2.197 \\
\hline 2.197 & 17.576 & 2.197 & 648.115 \\
\hline 2.197 & 52.728 & 4.394 & 10.985 \\
\hline 2.197 & 263.64 & 4.394 & 2.197 \\
\hline 2.197 & 10.985 & 57.122 & 863.421 \\
\hline 2.197 & 2.197 & 2.197 & 107.653 \\
\hline 39.546 & 24.167 & 6.591 & 2.197 \\
\hline 6.591 & 17.576 & 2.197 & 17.576 \\
\hline 2372.76 & 2.197 & 4.394 & 76.895 \\
\hline 4.394 & 6.591 & 6.591 & 2.197 \\
\hline 6.591 & 6.591 & 8.788 & 250.458 \\
\hline 4.394 & 39.546 & 4.394 & 230.685 \\
\hline 6.591 & 39.546 & 19.773 & 2.197 \\
\hline 15.379 & 2.197 & 37.349 & 10.985 \\
\hline 46.137 & 2.197 & 6.591 & 17.576 \\
\hline 8.788 & 28.561 & 2.197 & 2.197 \\
\hline 2.197 & 2.197 & 101.062 & 6.591 \\
\hline 6.591 & 15.379 & 96.668 & 19.773 \\
\hline 6.591 & 2.197 & 4.394 & 213.109 \\
\hline 2.197 & 2.197 & 2.197 & 283.413 \\
\hline 13.182 & 2.197 & 48.334 & 4.394 \\
\hline
\end{tabular}




\begin{tabular}{|c|c|c|c|}
\hline 68.107 & 4.394 & 8.788 & 6.591 \\
\hline 10.985 & 19.773 & 17.576 & 26.364 \\
\hline 2.197 & 2.197 & 10.985 & 13.182 \\
\hline 4.394 & 4.394 & 6.591 & 17.576 \\
\hline 4.394 & 2.197 & 13.182 & 17.576 \\
\hline 4.394 & 2.197 & 10.985 & 92.274 \\
\hline 2.197 & 13.182 & 2.197 & 13.182 \\
\hline 2.197 & 2.197 & 19.773 & 6.591 \\
\hline 2.197 & 2.197 & 46.137 & 2.197 \\
\hline 2.197 & 17.576 & 24.167 & 13.182 \\
\hline 2.197 & 2.197 & 4.394 & 2.197 \\
\hline 21.97 & 48.334 & 2.197 & 2.197 \\
\hline 4.394 & 553.644 & 2.197 & 10.985 \\
\hline 2.197 & 2.197 & 2.197 & 85.683 \\
\hline 2.197 & 2.197 & 8.788 & 2.197 \\
\hline 2.197 & 4.394 & 4.394 & 2.197 \\
\hline 2.197 & 28.561 & 2.197 & 54.925 \\
\hline 41.743 & 6.591 & 79.092 & 2.197 \\
\hline 2.197 & 2.197 & 2.197 & 10.985 \\
\hline 4.394 & 28.561 & 107.653 & 268.034 \\
\hline 37.349 & 2.197 & 2.197 & 615.16 \\
\hline 4.394 & 2.197 & 2.197 & 643.721 \\
\hline 2.197 & 2.197 & 2.197 & 59.319 \\
\hline 4.394 & 2.197 & 2.197 & 85.683 \\
\hline 13.182 & 2.197 & 4.394 & 4.394 \\
\hline 52.728 & 2.197 & 4.394 & 85.683 \\
\hline 2.197 & 30.758 & 2.197 & 2.197 \\
\hline 2.197 & 2.197 & 26.364 & 2.197 \\
\hline 2.197 & 10.985 & 8.788 & 4.394 \\
\hline 6.591 & 6.591 & 96.668 & 2.197 \\
\hline 24.167 & 24.167 & 6.591 & 19.773 \\
\hline 2.197 & 2.197 & 41.743 & 8.788 \\
\hline 4.394 & 26.364 & 2.197 & 59.319 \\
\hline 2.197 & 46.137 & 2.197 & 8.788 \\
\hline 15.379 & 21.97 & 2.197 & 28.561 \\
\hline 17.576 & 26.364 & 10.985 & 4.394 \\
\hline 2.197 & 28.561 & 138.411 & 8.788 \\
\hline 35.152 & 2.197 & 296.595 & 2.197 \\
\hline 2.197 & 10.985 & 24.167 & 2.197 \\
\hline 65.91 & 24.167 & 2.197 & 6.591 \\
\hline 30.758 & 6.591 & 4.394 & 24.167 \\
\hline 4.394 & 2.197 & 6.591 & 35.152 \\
\hline 2.197 & 4.394 & 2.197 & 2.197 \\
\hline 2.197 & 24.167 & 8.788 & 4.394 \\
\hline 6.591 & 2.197 & 15.379 & 8.788 \\
\hline 2.197 & 2.197 & 6.591 & 6.591 \\
\hline 8.788 & 2.197 & 13.182 & 32.955 \\
\hline 2.197 & 2.197 & 6.591 & 81.289 \\
\hline 10.985 & 10.985 & 6.591 & 173.563 \\
\hline 65.91 & 6.591 & 6.591 & 30.758 \\
\hline
\end{tabular}




\begin{tabular}{|c|c|c|c|}
\hline 2.197 & 2.197 & 13.182 & 4.394 \\
\hline 8.788 & 4.394 & 6.591 & 28.561 \\
\hline 2.197 & 2.197 & 2.197 & 2.197 \\
\hline 2.197 & 19.773 & 15.379 & 2.197 \\
\hline 30.758 & 13.182 & 2.197 & 2.197 \\
\hline 8.788 & 24.167 & 4.394 & 549.25 \\
\hline 21.97 & 2.197 & 10.985 & 17.576 \\
\hline 2326.62 & 2.197 & 2.197 & 6.591 \\
\hline 4.394 & 2.197 & 2.197 & 2.197 \\
\hline 28.561 & 2.197 & 1856.46 & 421.824 \\
\hline 4.394 & 2.197 & 2.197 & 8.788 \\
\hline 2.197 & 2.197 & 4.394 & 26.364 \\
\hline 10.985 & 2.197 & 10.985 & 2.197 \\
\hline 2.197 & 8.788 & 2.197 & 57.122 \\
\hline 2.197 & 2.197 & 2.197 & 37.349 \\
\hline 39.546 & 2.197 & 2.197 & 39.546 \\
\hline 2.197 & 2.197 & 8.788 & 2.197 \\
\hline 2.197 & 314.171 & 35.152 & 24.167 \\
\hline 2.197 & 2.197 & 15.379 & 432.809 \\
\hline 4.394 & 17.576 & 6.591 & 2.197 \\
\hline 4.394 & 2.197 & 2.197 & 155.987 \\
\hline 24.167 & 2.197 & 24.167 & 94.471 \\
\hline 388.869 & 2.197 & 2.197 & 13.182 \\
\hline 10.985 & 2.197 & 19.773 & 19.773 \\
\hline 2.197 & 4.394 & 24.167 & 4.394 \\
\hline 48.334 & 2.197 & 4.394 & 76.895 \\
\hline 2.197 & 41.743 & 2.197 & 6.591 \\
\hline 83.486 & 28.561 & 2.197 & 24.167 \\
\hline 15.379 & 2.197 & 2.197 & 6.591 \\
\hline 10.985 & 74.698 & 2.197 & 4.394 \\
\hline 2.197 & 10.985 & 8.788 & 173.563 \\
\hline 2.197 & 2.197 & 59.319 & 109.85 \\
\hline 8.788 & 28.561 & 8.788 & 569.023 \\
\hline 26.364 & 2.197 & 39.546 & 10.985 \\
\hline 2.197 & 37.349 & 105.456 & 39.546 \\
\hline 17.576 & 8.788 & 6.591 & 155.987 \\
\hline 13.182 & 4.394 & 6.591 & 24.167 \\
\hline 2.197 & 10.985 & 6.591 & 10.985 \\
\hline 202.124 & 2.197 & 4.394 & 397.657 \\
\hline 2.197 & 39.546 & 4.394 & 8.788 \\
\hline 35.152 & 2.197 & 6.591 & 2.197 \\
\hline 17.576 & 2.197 & 2.197 & 72.501 \\
\hline 2.197 & 10.985 & 2.197 & 4.394 \\
\hline 6.591 & 17.576 & 13.182 & 202.124 \\
\hline 2.197 & 13.182 & 2.197 & 52.728 \\
\hline 2.197 & 2.197 & 2.197 & 134.017 \\
\hline 2.197 & 2.197 & 2.197 & 2.197 \\
\hline 8.788 & 2.197 & 17.576 & 21.97 \\
\hline 147.199 & 10.985 & 4.394 & 2.197 \\
\hline 21.97 & 4.394 & 2.197 & 2.197 \\
\hline
\end{tabular}




\begin{tabular}{|c|c|c|c|}
\hline 39.546 & 2.197 & 2.197 & 2.197 \\
\hline 206.518 & 2.197 & 4.394 & 79.092 \\
\hline 186.745 & 15.379 & 61.516 & 2.197 \\
\hline 4.394 & 2.197 & 2.197 & 4.394 \\
\hline 10.985 & 6.591 & 2.197 & 13.182 \\
\hline 2.197 & 2.197 & 2.197 & 50.531 \\
\hline 2.197 & 8.788 & 2.197 & 61.516 \\
\hline 15.379 & 37.349 & 6.591 & 160.381 \\
\hline 13.182 & 26.364 & 2.197 & 19.773 \\
\hline 2.197 & 145.002 & 2.197 & 2.197 \\
\hline 90.077 & 28.561 & 13.182 & 19.773 \\
\hline 2.197 & 19.773 & 8.788 & 39.546 \\
\hline 470.158 & 2.197 & 46.137 & 6.591 \\
\hline 4.394 & 2.197 & 2.197 & 2.197 \\
\hline 15.379 & 2.197 & 2.197 & 2.197 \\
\hline 41.743 & 19.773 & 2.197 & 4.394 \\
\hline 21.97 & 2.197 & 8.788 & 4.394 \\
\hline 2.197 & 10.985 & 2.197 & 8.788 \\
\hline 8.788 & 2.197 & 2.197 & 105.456 \\
\hline 4.394 & 17.576 & 2.197 & 26.364 \\
\hline 2.197 & 2.197 & 17.576 & 21.97 \\
\hline 2.197 & 2.197 & 13.182 & 2.197 \\
\hline 57.122 & 10.985 & 186.745 & 19.773 \\
\hline 2.197 & 2.197 & 2.197 & 48.334 \\
\hline 2.197 & 96.668 & 2300.26 & 145.002 \\
\hline 2.197 & 2.197 & 2.197 & 4.394 \\
\hline 21.97 & 4.394 & 4.394 & 79.092 \\
\hline 731.601 & 26.364 & 6.591 & 59.319 \\
\hline 2.197 & 2.197 & 37.349 & 54.925 \\
\hline 85.683 & 2.197 & 2.197 & 4.394 \\
\hline 28.561 & 74.698 & 6.591 & 13.182 \\
\hline 4.394 & 4.394 & 2.197 & 61.516 \\
\hline 30.758 & 2.197 & 30.758 & 243.867 \\
\hline 30.758 & 2.197 & 2.197 & 2.197 \\
\hline 6.591 & 2.197 & 59.319 & 2.197 \\
\hline 2.197 & 2.197 & 2.197 & 2.197 \\
\hline 8.788 & 2.197 & 32.955 & 6.591 \\
\hline 2.197 & 2.197 & 2.197 & 10.985 \\
\hline 4.394 & 2.197 & 2.197 & 2.197 \\
\hline 2.197 & 6.591 & 2.197 & 70.304 \\
\hline 885.391 & 4.394 & 2.197 & 50.531 \\
\hline 118.638 & 4.394 & 28.561 & 21.97 \\
\hline 2.197 & 21.97 & 2106.92 & 2.197 \\
\hline 8.788 & 2.197 & 17.576 & 28.561 \\
\hline 52.728 & 2.197 & 13.182 & 26.364 \\
\hline 112.047 & 2.197 & 4.394 & 637.13 \\
\hline 2.197 & 2.197 & 2.197 & 4.394 \\
\hline 8.788 & 2.197 & 2.197 & 17.576 \\
\hline 2.197 & 2.197 & 2.197 & 415.233 \\
\hline 46.137 & 6.591 & 54.925 & 10.985 \\
\hline
\end{tabular}




\begin{tabular}{|c|c|c|c|}
\hline 61.516 & 4.394 & 2.197 & 6.591 \\
\hline 10.985 & 43.94 & 180.154 & 54.925 \\
\hline 2.197 & 4.394 & 2.197 & 37.349 \\
\hline 4.394 & 2.197 & 6.591 & 19.773 \\
\hline 2.197 & 2.197 & 26.364 & 2.197 \\
\hline 2.197 & 2.197 & 13.182 & 4.394 \\
\hline 2.197 & 4.394 & 8.788 & 8.788 \\
\hline 4.394 & 2.197 & 2.197 & 28.561 \\
\hline 4.394 & 4.394 & 2.197 & 4.394 \\
\hline 4.394 & 6.591 & 48.334 & 4.394 \\
\hline 17.576 & 8.788 & 6.591 & 531.674 \\
\hline 10.985 & 50.531 & 2.197 & 6.591 \\
\hline 10.985 & 2.197 & 2.197 & 8.788 \\
\hline 2.197 & 283.413 & 4.394 & 2.197 \\
\hline 2.197 & 2.197 & 4.394 & 2.197 \\
\hline 2.197 & 4.394 & 6.591 & 15.379 \\
\hline 4.394 & 76.895 & 2.197 & 6.591 \\
\hline 2.197 & 13.182 & 13.182 & 54.925 \\
\hline 13.182 & 30.758 & 13.182 & 2.197 \\
\hline 935.922 & 41.743 & 2.197 & 2.197 \\
\hline 153.79 & 28.561 & 525.083 & 2.197 \\
\hline 28.561 & 2.197 & 35.152 & 138.411 \\
\hline 30.758 & 2.197 & 2.197 & 2.197 \\
\hline 21.97 & 4.394 & 4.394 & 2.197 \\
\hline 54.925 & 2.197 & 6.591 & 43.94 \\
\hline 2.197 & 6.591 & 6.591 & 703.04 \\
\hline 2.197 & 2.197 & 2.197 & 8.788 \\
\hline 4.394 & 2.197 & 2.197 & 2.197 \\
\hline 63.713 & 4.394 & 2.197 & 8.788 \\
\hline 21.97 & 2.197 & 2.197 & 2.197 \\
\hline 50.531 & 2.197 & 2.197 & 24.167 \\
\hline 2.197 & 2.197 & 6.591 & 48.334 \\
\hline 2.197 & 2.197 & 2.197 & 74.698 \\
\hline 371.293 & 59.319 & 96.668 & 4.394 \\
\hline 2.197 & 17.576 & 2.197 & 10.985 \\
\hline 322.959 & 2.197 & 17.576 & 2.197 \\
\hline 2.197 & 2.197 & 153.79 & 24.167 \\
\hline 74.698 & 6.591 & 2394.73 & 59.319 \\
\hline 2.197 & 4.394 & 46.137 & 164.775 \\
\hline 2.197 & 4.394 & 2.197 & 46.137 \\
\hline 50.531 & 85.683 & 2.197 & 10.985 \\
\hline 4.394 & 4.394 & 2.197 & 6.591 \\
\hline 6.591 & 13.182 & 2.197 & 8.788 \\
\hline 120.835 & 37.349 & 2.197 & 30.758 \\
\hline 10.985 & 4.394 & 2.197 & 26.364 \\
\hline 76.895 & 2.197 & 10.985 & 2.197 \\
\hline 37.349 & 454.779 & 2.197 & 17.576 \\
\hline 160.381 & 2.197 & 26.364 & 2.197 \\
\hline 2.197 & 15.379 & 2.197 & 2.197 \\
\hline 4.394 & 542.659 & 2.197 & 4.394 \\
\hline
\end{tabular}




\begin{tabular}{|c|c|c|c|}
\hline 21.97 & 13.182 & 8.788 & 4.394 \\
\hline 8.788 & 2.197 & 790.92 & 10.985 \\
\hline 206.518 & 2.197 & 79.092 & 17.576 \\
\hline 10.985 & 2.197 & 2.197 & 15.379 \\
\hline 4.394 & 4.394 & 2.197 & 1078.73 \\
\hline 2.197 & 2.197 & 2.197 & 272.428 \\
\hline 46.137 & 2.197 & 2.197 & 98.865 \\
\hline 2.197 & 131.82 & 15.379 & 4.394 \\
\hline 2.197 & 2.197 & 6.591 & 17.576 \\
\hline 2.197 & 2.197 & 2.197 & 17.576 \\
\hline 2.197 & 4.394 & 199.927 & 6.591 \\
\hline 2.197 & 2.197 & 160.381 & 10.985 \\
\hline 10.985 & 2.197 & 2.197 & 2.197 \\
\hline 30.758 & 2.197 & 2.197 & 112.047 \\
\hline 2.197 & 410.839 & 4.394 & 46.137 \\
\hline 15.379 & 4.394 & 114.244 & 2.197 \\
\hline 4.394 & 6.591 & 6.591 & 2.197 \\
\hline 6.591 & 24.167 & 4.394 & 26.364 \\
\hline 21.97 & 10.985 & 2.197 & 4.394 \\
\hline 4.394 & 2.197 & 4.394 & 4.394 \\
\hline 2.197 & 2.197 & 24.167 & 32.955 \\
\hline 2.197 & 2.197 & 1228.12 & 19.773 \\
\hline 208.715 & 2.197 & 6.591 & 57.122 \\
\hline 2.197 & 2.197 & 2.197 & 6.591 \\
\hline 6.591 & 4.394 & 2.197 & 52.728 \\
\hline 112.047 & 32.955 & 4.394 & 134.017 \\
\hline 2.197 & 118.638 & 134.017 & 1162.21 \\
\hline 8.788 & 72.501 & 2.197 & 65.91 \\
\hline 26.364 & 19.773 & 338.338 & 21.97 \\
\hline 360.308 & 17.576 & 2.197 & 103.259 \\
\hline 4.394 & 169.169 & 72.501 & 2.197 \\
\hline 8.788 & 2.197 & 6.591 & 15.379 \\
\hline 30.758 & 24.167 & 2.197 & 21.97 \\
\hline 2.197 & 6.591 & 2.197 & 37.349 \\
\hline 2.197 & 21.97 & 13.182 & 6.591 \\
\hline 6.591 & 83.486 & 6.591 & 2.197 \\
\hline 2.197 & 8.788 & 2.197 & 30.758 \\
\hline 127.426 & 92.274 & 2.197 & 10.985 \\
\hline 6.591 & 2.197 & 2.197 & 8.788 \\
\hline 2.197 & 151.593 & 17.576 & 8.788 \\
\hline 4.394 & 41.743 & 43.94 & 65.91 \\
\hline 2.197 & 2.197 & 21.97 & 162.578 \\
\hline 30.758 & 4.394 & 2.197 & 17.576 \\
\hline 13.182 & 8.788 & 2.197 & 4.394 \\
\hline 10.985 & 2.197 & 10.985 & 59.319 \\
\hline 74.698 & 1968.51 & 2.197 & 1272.06 \\
\hline 4.394 & 26.364 & 2.197 & 134.017 \\
\hline 32.955 & 4.394 & 2.197 & 74.698 \\
\hline 26.364 & 2.197 & 2.197 & 21.97 \\
\hline 98.865 & 2.197 & 10.985 & 54.925 \\
\hline
\end{tabular}




\begin{tabular}{|c|c|c|c|}
\hline 944.71 & 2.197 & 8.788 & 2.197 \\
\hline 2.197 & 2.197 & 2.197 & 15.379 \\
\hline 68.107 & 4.394 & 2.197 & 527.28 \\
\hline 2.197 & 50.531 & 2.197 & 52.728 \\
\hline 8.788 & 2.197 & 4.394 & 259.246 \\
\hline 54.925 & 10.985 & 4.394 & 35.152 \\
\hline 8.788 & 24.167 & 2.197 & 142.805 \\
\hline 4.394 & 30.758 & 2.197 & 24.167 \\
\hline 2.197 & 2.197 & 1140.24 & 4.394 \\
\hline 2.197 & 2.197 & 72.501 & 17.576 \\
\hline 4.394 & 2.197 & 2.197 & 105.456 \\
\hline 2.197 & 6.591 & 94.471 & 2.197 \\
\hline 430.612 & 6.591 & 13.182 & 4.394 \\
\hline 2.197 & 4.394 & 57.122 & 10.985 \\
\hline 6.591 & 21.97 & 4.394 & 2.197 \\
\hline 2.197 & 2.197 & 19.773 & 81.289 \\
\hline 2.197 & 2.197 & 6.591 & 120.835 \\
\hline 46.137 & 15.379 & 2.197 & 10.985 \\
\hline 30.758 & 2.197 & 6.591 & 8.788 \\
\hline 4.394 & 2.197 & 10.985 & 24.167 \\
\hline 476.749 & 2.197 & 13.182 & 4.394 \\
\hline 6.591 & 8.788 & 24.167 & 6.591 \\
\hline 2.197 & 672.282 & 10.985 & 2.197 \\
\hline 61.516 & 15.379 & 2.197 & 4.394 \\
\hline 2.197 & 4.394 & 2.197 & 6.591 \\
\hline 2.197 & 37.349 & 164.775 & 531.674 \\
\hline 2.197 & 35.152 & 21.97 & 63.713 \\
\hline 2.197 & 268.034 & 19.773 & 87.88 \\
\hline 10.985 & 35.152 & 37.349 & 2.197 \\
\hline 4.394 & 50.531 & 54.925 & 10.985 \\
\hline 8.788 & 2.197 & 8.788 & 72.501 \\
\hline 123.032 & 6.591 & 2.197 & 8.788 \\
\hline 92.274 & 4.394 & 10.985 & 2322.23 \\
\hline 2.197 & 15.379 & 2.197 & 10.985 \\
\hline 2.197 & 2.197 & 2.197 & 41.743 \\
\hline 8.788 & 2.197 & 13.182 & 6.591 \\
\hline 85.683 & 4.394 & 2.197 & 10.985 \\
\hline 6.591 & 2.197 & 2.197 & 85.683 \\
\hline 4.394 & 2.197 & 2.197 & 4.394 \\
\hline 162.578 & 173.563 & 37.349 & 112.047 \\
\hline 4.394 & 19.773 & 2.197 & 24.167 \\
\hline 4.394 & 2.197 & 4.394 & 4.394 \\
\hline 2.197 & 2.197 & 2.197 & 329.55 \\
\hline 8.788 & 2.197 & 2.197 & 6.591 \\
\hline 43.94 & 2.197 & 59.319 & 116.441 \\
\hline 6.591 & 2.197 & 4.394 & 10.985 \\
\hline 13.182 & 52.728 & 6.591 & 90.077 \\
\hline 123.032 & 21.97 & 2.197 & 21.97 \\
\hline 4.394 & 2.197 & 2.197 & 2.197 \\
\hline 41.743 & 4.394 & 15.379 & 105.456 \\
\hline
\end{tabular}




\begin{tabular}{|c|c|c|c|}
\hline 13.182 & 4.394 & 2.197 & 32.955 \\
\hline 6.591 & 4.394 & 10.985 & 4.394 \\
\hline 15.379 & 21.97 & 6.591 & 2.197 \\
\hline 32.955 & 2.197 & 37.349 & 2.197 \\
\hline 6.591 & 2.197 & 2.197 & 26.364 \\
\hline 2.197 & 17.576 & 4.394 & 101.062 \\
\hline 2.197 & 8.788 & 6.591 & 10.985 \\
\hline 2.197 & 112.047 & 2.197 & 2.197 \\
\hline 2.197 & 2.197 & 13.182 & 74.698 \\
\hline 15.379 & 4.394 & 4.394 & 13.182 \\
\hline 2.197 & 2.197 & 8.788 & 10.985 \\
\hline 2.197 & 127.426 & 6.591 & 6.591 \\
\hline 76.895 & 90.077 & 4.394 & 6.591 \\
\hline 28.561 & 173.563 & 2.197 & 4.394 \\
\hline 6.591 & 4.394 & 8.788 & 70.304 \\
\hline 450.385 & 54.925 & 2.197 & 65.91 \\
\hline 26.364 & 39.546 & 2.197 & 17.576 \\
\hline 2.197 & 30.758 & 74.698 & 19.773 \\
\hline 15.379 & 10.985 & 118.638 & 180.154 \\
\hline 4.394 & 4.394 & 70.304 & 2.197 \\
\hline 90.077 & 2.197 & 4.394 & 2.197 \\
\hline 2.197 & 10.985 & 63.713 & 109.85 \\
\hline 2.197 & 15.379 & 2.197 & 8.788 \\
\hline 186.745 & 15.379 & 63.713 & 366.899 \\
\hline 2.197 & 2.197 & 35.152 & 2.197 \\
\hline 4.394 & 4.394 & 13.182 & 125.229 \\
\hline 19.773 & 2.197 & 8.788 & 4.394 \\
\hline 2.197 & 57.122 & 2.197 & 65.91 \\
\hline 123.032 & 43.94 & 2.197 & 37.349 \\
\hline 2.197 & 2.197 & 2.197 & 2.197 \\
\hline 8.788 & 8.788 & 2.197 & 2.197 \\
\hline 2.197 & 4.394 & 2.197 & 2.197 \\
\hline 2.197 & 48.334 & 2.197 & 55223.8 \\
\hline 2.197 & 15.379 & 2.197 & 2210.18 \\
\hline 4.394 & 2.197 & 21.97 & 8.788 \\
\hline 127.426 & 37.349 & 4.394 & 6.591 \\
\hline 2.197 & 6.591 & 2.197 & 21.97 \\
\hline 6.591 & 6.591 & 2.197 & 37.349 \\
\hline 41.743 & 2.197 & 6.591 & 2.197 \\
\hline 92.274 & 4.394 & 4.394 & 4.394 \\
\hline 63.713 & 4.394 & 2.197 & 109.85 \\
\hline 2.197 & 2.197 & 39.546 & 210.912 \\
\hline 2.197 & 2.197 & 8.788 & 41.743 \\
\hline 57.122 & 43.94 & 10.985 & 2.197 \\
\hline 4.394 & 2.197 & 13.182 & 2.197 \\
\hline 6.591 & 2.197 & 4.394 & 2.197 \\
\hline 85.683 & 2.197 & 4.394 & 2.197 \\
\hline 32.955 & 2.197 & 8.788 & 10.985 \\
\hline 2.197 & 6.591 & 2.197 & 4.394 \\
\hline 2.197 & 8.788 & 4963.02 & 4.394 \\
\hline
\end{tabular}




\begin{tabular}{|c|c|c|c|}
\hline 37.349 & 2.197 & 39.546 & 17.576 \\
\hline 386.672 & 2.197 & 4.394 & 6.591 \\
\hline 2.197 & 2.197 & 2.197 & 138.411 \\
\hline 63.713 & 2.197 & 180.154 & 10.985 \\
\hline 2.197 & 8.788 & 2.197 & 296.595 \\
\hline 127.426 & 8.788 & 2.197 & 2.197 \\
\hline 160.381 & 4.394 & 2.197 & 2.197 \\
\hline 4.394 & 6.591 & 4.394 & 2.197 \\
\hline 2.197 & 43.94 & 2.197 & 17.576 \\
\hline 8.788 & 2.197 & 2.197 & 19.773 \\
\hline 6.591 & 2.197 & 74.698 & 2.197 \\
\hline 151.593 & 2.197 & 28.561 & 32.955 \\
\hline 2.197 & 2.197 & 90.077 & 10.985 \\
\hline 35.152 & 2.197 & 4.394 & 19.773 \\
\hline 30.758 & 8.788 & 4.394 & 24.167 \\
\hline 6.591 & 2.197 & 2.197 & 30.758 \\
\hline 57.122 & 24.167 & 2.197 & 98.865 \\
\hline 85.683 & 248.261 & 2.197 & 2.197 \\
\hline 6.591 & 6.591 & 10.985 & 2.197 \\
\hline 4.394 & 138.411 & 2.197 & 2.197 \\
\hline 4.394 & 2.197 & 46.137 & 43.94 \\
\hline 4.394 & 30.758 & 2.197 & 68.107 \\
\hline 2.197 & 2.197 & 6.591 & 6.591 \\
\hline 74.698 & 2.197 & 2.197 & 8.788 \\
\hline 2.197 & 2.197 & 2.197 & 74.698 \\
\hline 2.197 & 4.394 & 300.989 & 59.319 \\
\hline 90.077 & 8.788 & 6.591 & 6.591 \\
\hline 6.591 & 131.82 & 252.655 & 10042.5 \\
\hline 8.788 & 4.394 & 10.985 & 72.501 \\
\hline 2.197 & 711.828 & 804.102 & 6.591 \\
\hline 2.197 & 17.576 & 81.289 & 10.985 \\
\hline 52.728 & 13.182 & 15.379 & 2.197 \\
\hline 15.379 & 15.379 & 2.197 & 896.376 \\
\hline 107.653 & 2.197 & 4.394 & 26.364 \\
\hline 4.394 & 2036.62 & 2.197 & 2.197 \\
\hline 28.561 & 43.94 & 57.122 & 235.079 \\
\hline 2.197 & 2.197 & 2.197 & 2.197 \\
\hline 2.197 & 2.197 & 15.379 & 2.197 \\
\hline 166.972 & 4.394 & 65.91 & 4.394 \\
\hline 6.591 & 2.197 & 6.591 & 4.394 \\
\hline 2.197 & 4.394 & 2.197 & 171.366 \\
\hline 406.445 & 2.197 & 2.197 & 4.394 \\
\hline 2.197 & 2.197 & 4.394 & 202.124 \\
\hline 4.394 & 2.197 & 2.197 & 39.546 \\
\hline 2.197 & 4.394 & 4.394 & 24.167 \\
\hline 13.182 & 2.197 & 197.73 & 4.394 \\
\hline 2.197 & 2.197 & 2.197 & 72.501 \\
\hline 801.905 & 6.591 & 2.197 & 2.197 \\
\hline 2.197 & 2.197 & 6.591 & 2.197 \\
\hline 2.197 & 2.197 & 21.97 & 15.379 \\
\hline
\end{tabular}




\begin{tabular}{|c|c|c|c|}
\hline 2.197 & 4.394 & 2.197 & 6.591 \\
\hline 2.197 & 2.197 & 6.591 & 2.197 \\
\hline 6.591 & 4.394 & 48.334 & 6.591 \\
\hline 2.197 & 8.788 & 46.137 & 2.197 \\
\hline 2.197 & 24.167 & 2.197 & 65.91 \\
\hline 2.197 & 4.394 & 13.182 & 39.546 \\
\hline 2.197 & 2.197 & 48.334 & 2.197 \\
\hline 2.197 & 6.591 & 85.683 & 32.955 \\
\hline 2.197 & 4.394 & 39.546 & 10.985 \\
\hline 2.197 & 10.985 & 2.197 & 2.197 \\
\hline 4.394 & 28.561 & 2.197 & 15.379 \\
\hline 4.394 & 8.788 & 4.394 & 2.197 \\
\hline 15.379 & 2.197 & 103.259 & 2.197 \\
\hline 366.899 & 24.167 & 17.576 & 2.197 \\
\hline 24.167 & 259.246 & 6.591 & 2.197 \\
\hline 54.925 & 2.197 & 2.197 & 13.182 \\
\hline 2.197 & 2.197 & 2.197 & 472.355 \\
\hline 4.394 & 166.972 & 920.543 & 92.274 \\
\hline 10.985 & 6.591 & 149.396 & 6.591 \\
\hline 74.698 & 2.197 & 2.197 & 6.591 \\
\hline 4.394 & 4.394 & 573.417 & 6.591 \\
\hline 2.197 & 2.197 & 2.197 & 8.788 \\
\hline 4.394 & 4.394 & 2.197 & 6.591 \\
\hline 32.955 & 2.197 & 4.394 & 10.985 \\
\hline 26.364 & 2.197 & 28.561 & 1276.46 \\
\hline 79.092 & 4.394 & 2.197 & 2.197 \\
\hline 4.394 & 4.394 & 2.197 & 52.728 \\
\hline 79.092 & 76.895 & 4.394 & 329.55 \\
\hline 4.394 & 2.197 & 81.289 & 964.483 \\
\hline 2.197 & 52.728 & 2.197 & 10.985 \\
\hline 149.396 & 72.501 & 6.591 & 8.788 \\
\hline 26.364 & 180.154 & 8.788 & 4.394 \\
\hline 2.197 & 13.182 & 13.182 & 8.788 \\
\hline 8.788 & 8.788 & 24.167 & 54.925 \\
\hline 2.197 & 538.265 & 2.197 & 74.698 \\
\hline 2.197 & 4.394 & 13.182 & 2.197 \\
\hline 19.773 & 2.197 & 8.788 & 4.394 \\
\hline 15.379 & 4.394 & 8.788 & 39.546 \\
\hline 8.788 & 2.197 & 2.197 & 41.743 \\
\hline 8.788 & 59.319 & 300.989 & 50.531 \\
\hline 2.197 & 43.94 & 4.394 & 509.704 \\
\hline 13.182 & 10.985 & 1698.28 & 39.546 \\
\hline 8.788 & 43.94 & 2.197 & 4.394 \\
\hline 17.576 & 2.197 & 145.002 & 8.788 \\
\hline 235.079 & 2.197 & 2.197 & 35.152 \\
\hline 2.197 & 2.197 & 41.743 & 39.546 \\
\hline 2.197 & 2.197 & 2.197 & 2.197 \\
\hline 2.197 & 4.394 & 4.394 & 2.197 \\
\hline 158.184 & 6.591 & 2.197 & 2.197 \\
\hline 2.197 & 4.394 & 2.197 & 17.576 \\
\hline
\end{tabular}




\begin{tabular}{|c|c|c|c|}
\hline 19.773 & 2.197 & 2.197 & 235.079 \\
\hline 35.152 & 2.197 & 13.182 & 191.139 \\
\hline 2436.47 & 4.394 & 15.379 & 37.349 \\
\hline 8.788 & 2.197 & 3592.09 & 21.97 \\
\hline 2.197 & 2.197 & 2.197 & 32.955 \\
\hline 1028.2 & 8.788 & 8.788 & 2.197 \\
\hline 2.197 & 8.788 & 162.578 & 2.197 \\
\hline 4.394 & 2.197 & 15.379 & 2.197 \\
\hline 6.591 & 2.197 & 254.852 & 48.334 \\
\hline 2.197 & 2.197 & 744.783 & 2.197 \\
\hline 689.858 & 2.197 & 342.732 & 617.357 \\
\hline 2.197 & 39.546 & 2.197 & 169.169 \\
\hline 2.197 & 65.91 & 74.698 & 4.394 \\
\hline 21.97 & 136.214 & 6.591 & 2.197 \\
\hline 4.394 & 6.591 & 79.092 & 6.591 \\
\hline 8.788 & 24.167 & 32.955 & 2.197 \\
\hline 184.548 & 41.743 & 13.182 & 2.197 \\
\hline 2.197 & 2.197 & 59.319 & 8.788 \\
\hline 26.364 & 2.197 & 2.197 & 21.97 \\
\hline 8.788 & 2.197 & 54.925 & 237.276 \\
\hline 476.749 & 2.197 & 37.349 & 85.683 \\
\hline 17.576 & 2.197 & 50.531 & 2.197 \\
\hline 2.197 & 2.197 & 8.788 & 15.379 \\
\hline 2.197 & 4.394 & 13.182 & 8.788 \\
\hline 4.394 & 2.197 & 2.197 & 2.197 \\
\hline 19.773 & 15.379 & 2.197 & 2.197 \\
\hline 4.394 & 6.591 & 13.182 & 4.394 \\
\hline 83.486 & 43.94 & 8.788 & 6.591 \\
\hline 353.717 & 2.197 & 8.788 & 103.259 \\
\hline 10.985 & 2.197 & 2.197 & 48.334 \\
\hline 4.394 & 2.197 & 2.197 & 8.788 \\
\hline 4.394 & 2.197 & 2.197 & 43.94 \\
\hline 4.394 & 2.197 & 2.197 & 751.374 \\
\hline 4.394 & 2.197 & 2.197 & 68.107 \\
\hline 2.197 & 2.197 & 2.197 & 57.122 \\
\hline 2.197 & 4.394 & 2.197 & 6.591 \\
\hline 4.394 & 8.788 & 8.788 & 2.197 \\
\hline 2.197 & 2.197 & 2.197 & 13.182 \\
\hline 4.394 & 52.728 & 2.197 & 322.959 \\
\hline 4.394 & 13.182 & 2.197 & 24.167 \\
\hline 2.197 & 2.197 & 8.788 & 37.349 \\
\hline 342.732 & 32.955 & 2.197 & 4.394 \\
\hline 4.394 & 19.773 & 8.788 & 50.531 \\
\hline 186.745 & 2.197 & 2.197 & 4.394 \\
\hline 2.197 & 4.394 & 2.197 & 281.216 \\
\hline 2.197 & 13.182 & 2.197 & 2.197 \\
\hline 10.985 & 4.394 & 2.197 & 28.561 \\
\hline 2.197 & 4.394 & 2.197 & 4.394 \\
\hline 472.355 & 136.214 & 13.182 & 2.197 \\
\hline 2.197 & 8.788 & 2.197 & 118.638 \\
\hline
\end{tabular}




\begin{tabular}{|c|c|c|c|}
\hline 2.197 & 19.773 & 2.197 & 4.394 \\
\hline 2.197 & 459.173 & 2.197 & 6.591 \\
\hline 2278.29 & 4.394 & 2.197 & 15.379 \\
\hline 2.197 & 105.456 & 4.394 & 2.197 \\
\hline 2.197 & 2.197 & 2.197 & 10.985 \\
\hline 70.304 & 116.441 & 21.97 & 41.743 \\
\hline 2.197 & 30.758 & 112.047 & 3778.84 \\
\hline 2.197 & 2.197 & 6.591 & 26.364 \\
\hline 2.197 & 2.197 & 131.82 & 10.985 \\
\hline 2.197 & 2.197 & 2.197 & 46.137 \\
\hline 2.197 & 2.197 & 2.197 & 4.394 \\
\hline 4.394 & 2.197 & 13.182 & 2.197 \\
\hline 4.394 & 4.394 & 2.197 & 6.591 \\
\hline 2.197 & 2.197 & 2.197 & 2.197 \\
\hline 2.197 & 15.379 & 2.197 & 32.955 \\
\hline 226.291 & 96.668 & 48.334 & 10.985 \\
\hline 39.546 & 8.788 & 17.576 & 2.197 \\
\hline 147.199 & 2.197 & 8.788 & 2.197 \\
\hline 41.743 & 2.197 & 79.092 & 2.197 \\
\hline 4.394 & 533.871 & 4.394 & 32.955 \\
\hline 261.443 & 2.197 & 37.349 & 13.182 \\
\hline 50.531 & 2.197 & 17.576 & 15.379 \\
\hline 138.411 & 24.167 & 54.925 & 2.197 \\
\hline 171.366 & 109.85 & 46.137 & 13.182 \\
\hline 4.394 & 2.197 & 32.955 & 65.91 \\
\hline 2.197 & 21.97 & 92.274 & 336.141 \\
\hline 6.591 & 2.197 & 2.197 & 83.486 \\
\hline 6.591 & 2.197 & 6.591 & 35.152 \\
\hline 54.925 & 32.955 & 8.788 & 8.788 \\
\hline 755.768 & 6.591 & 140.608 & 43.94 \\
\hline 4.394 & 2.197 & 2.197 & 10.985 \\
\hline 13.182 & 17.576 & 37.349 & 63.713 \\
\hline 2.197 & 2.197 & 17.576 & 2.197 \\
\hline 2.197 & 10.985 & 2.197 & 474.552 \\
\hline 13.182 & 15.379 & 4.394 & 162.578 \\
\hline 2.197 & 2.197 & 94.471 & 41.743 \\
\hline 4.394 & 2.197 & 4.394 & 8.788 \\
\hline 4.394 & 15.379 & 8.788 & 8.788 \\
\hline 2.197 & 54.925 & 1214.94 & 2.197 \\
\hline 6.591 & 2.197 & 15.379 & 2.197 \\
\hline 2.197 & 2.197 & 41.743 & 432.809 \\
\hline 15.379 & 13.182 & 37.349 & 52.728 \\
\hline 52.728 & 4.394 & 2.197 & 239.473 \\
\hline 4.394 & 140.608 & 2.197 & 2.197 \\
\hline 2.197 & 6.591 & 46.137 & 2.197 \\
\hline 10.985 & 50.531 & 2.197 & 147.199 \\
\hline 8.788 & 2.197 & 2.197 & 10.985 \\
\hline 2.197 & 76.895 & 2.197 & 21.97 \\
\hline 4.394 & 15.379 & 2.197 & 2.197 \\
\hline 4.394 & 37.349 & 2.197 & 8.788 \\
\hline
\end{tabular}




\begin{tabular}{|c|c|c|c|}
\hline 2.197 & 21.97 & 2.197 & 153.79 \\
\hline 2.197 & 13.182 & 2.197 & 35.152 \\
\hline 24.167 & 2.197 & 2.197 & 32.955 \\
\hline 52.728 & 17.576 & 123.032 & 136.214 \\
\hline 17.576 & 4.394 & 8.788 & 2.197 \\
\hline 2.197 & 2.197 & 30.758 & 193.336 \\
\hline 2.197 & 96.668 & 6.591 & 13.182 \\
\hline 26.364 & 4.394 & 2.197 & 15.379 \\
\hline 2.197 & 8.788 & 2.197 & 2.197 \\
\hline 6.591 & 6.591 & 2.197 & 2.197 \\
\hline 17.576 & 72.501 & 57.122 & 54.925 \\
\hline 4.394 & 4.394 & 118.638 & 2.197 \\
\hline 232.882 & 4.394 & 8.788 & 2.197 \\
\hline 17.576 & 2.197 & 6.591 & 4.394 \\
\hline 10.985 & 2.197 & 305.383 & 10.985 \\
\hline 10.985 & 2.197 & 4.394 & 24.167 \\
\hline 2.197 & 2.197 & 2.197 & 2.197 \\
\hline 37.349 & 2.197 & 2.197 & 35.152 \\
\hline 733.798 & 2.197 & 2.197 & 8.788 \\
\hline 94.471 & 2.197 & 13.182 & 2.197 \\
\hline 2.197 & 4.394 & 131.82 & 13.182 \\
\hline 2.197 & 476.749 & 15.379 & 17.576 \\
\hline 19.773 & 4.394 & 2.197 & 28.561 \\
\hline 2.197 & 4.394 & 39.546 & 2.197 \\
\hline 8.788 & 6.591 & 10.985 & 19.773 \\
\hline 46.137 & 4.394 & 8.788 & 37.349 \\
\hline 4.394 & 2.197 & 6.591 & 21.97 \\
\hline 2.197 & 2.197 & 6.591 & 90.077 \\
\hline 6.591 & 2.197 & 2.197 & 21.97 \\
\hline 17.576 & 2.197 & 2.197 & 32.955 \\
\hline 94.471 & 4.394 & 2.197 & 79.092 \\
\hline 2.197 & 4.394 & 2.197 & 15.379 \\
\hline 2.197 & 8.788 & 2.197 & 131.82 \\
\hline 2.197 & 8.788 & 17.576 & 2.197 \\
\hline 15.379 & 4.394 & 2.197 & 19.773 \\
\hline 70.304 & 105.456 & 2.197 & 4.394 \\
\hline 4.394 & 2.197 & 13.182 & 2.197 \\
\hline 6.591 & 6.591 & 2.197 & 2.197 \\
\hline 10.985 & 2.197 & 2.197 & 2.197 \\
\hline 10.985 & 37.349 & 21.97 & 4.394 \\
\hline 94.471 & 4.394 & 2.197 & 2.197 \\
\hline 15.379 & 15.379 & 2.197 & 13.182 \\
\hline 2.197 & 8.788 & 2.197 & 59.319 \\
\hline 2.197 & 496.522 & 2.197 & 604.175 \\
\hline 26.364 & 4.394 & 2.197 & 2.197 \\
\hline 54.925 & 2.197 & 4.394 & 17.576 \\
\hline 17.576 & 6.591 & 2.197 & 8.788 \\
\hline 105.456 & 10.985 & 4.394 & 17.576 \\
\hline 404.248 & 2.197 & 15.379 & 2.197 \\
\hline 2.197 & 10.985 & 2.197 & 4.394 \\
\hline
\end{tabular}




\begin{tabular}{|c|c|c|c|}
\hline 2.197 & 2.197 & 2.197 & 2.197 \\
\hline 4.394 & 6.591 & 4.394 & 43.94 \\
\hline 140.608 & 2.197 & 10.985 & 2.197 \\
\hline 87.88 & 13.182 & 2.197 & 4.394 \\
\hline 13.182 & 2.197 & 2.197 & 2.197 \\
\hline 4.394 & 4.394 & 2.197 & 2.197 \\
\hline 17.576 & 10.985 & 2.197 & 2.197 \\
\hline 35.152 & 2.197 & 2.197 & 30.758 \\
\hline 2.197 & 6.591 & 2.197 & 10.985 \\
\hline 2.197 & 4.394 & 2.197 & 76.895 \\
\hline 2.197 & 8.788 & 2.197 & 107.653 \\
\hline 424.021 & 4.394 & 2.197 & 2.197 \\
\hline 281.216 & 2.197 & 8.788 & 186.745 \\
\hline 6.591 & 15.379 & 8.788 & 6.591 \\
\hline 2.197 & 4.394 & 6.591 & 52.728 \\
\hline 96.668 & 4.394 & 2.197 & 305.383 \\
\hline 6.591 & 4.394 & 6.591 & 8.788 \\
\hline 2.197 & 46.137 & 8.788 & 2.197 \\
\hline 2.197 & 4.394 & 4.394 & 6.591 \\
\hline 2.197 & 4.394 & 2.197 & 19.773 \\
\hline 4.394 & 4.394 & 2.197 & 109.85 \\
\hline 2.197 & 2.197 & 175.76 & 37.349 \\
\hline 2.197 & 87.88 & 2.197 & 2.197 \\
\hline 17.576 & 114.244 & 2.197 & 57.122 \\
\hline 6.591 & 2.197 & 2.197 & 8.788 \\
\hline 2.197 & 4.394 & 395.46 & 54.925 \\
\hline 2.197 & 63.713 & 2.197 & 17.576 \\
\hline 4.394 & 68.107 & 2.197 & 13.182 \\
\hline 13.182 & 74.698 & 30.758 & 878.8 \\
\hline 35.152 & 566.826 & 2.197 & 8.788 \\
\hline 2.197 & 2.197 & 2.197 & 4.394 \\
\hline 2.197 & 2.197 & 4.394 & 81.289 \\
\hline 2.197 & 13.182 & 206.518 & 2.197 \\
\hline 39.546 & 52.728 & 35.152 & 2.197 \\
\hline 46.137 & 2.197 & 2.197 & 4.394 \\
\hline 4.394 & 76.895 & 10.985 & 6.591 \\
\hline 13.182 & 4.394 & 2.197 & 21.97 \\
\hline 17.576 & 2.197 & 120.835 & 37.349 \\
\hline 57.122 & 61.516 & 28.561 & 2.197 \\
\hline 2.197 & 13.182 & 2.197 & 136.214 \\
\hline 2.197 & 68.107 & 8.788 & 4.394 \\
\hline 2.197 & 2.197 & 32.955 & 2.197 \\
\hline 2.197 & 2.197 & 4.394 & 887.588 \\
\hline 2.197 & 8.788 & 28.561 & 1428.05 \\
\hline 6.591 & 4.394 & 4.394 & 6.591 \\
\hline 10.985 & 8.788 & 2.197 & 4.394 \\
\hline 4.394 & 4.394 & 2.197 & 142.805 \\
\hline 15.379 & 4.394 & 2.197 & 8.788 \\
\hline 2.197 & 2.197 & 70.304 & 13.182 \\
\hline 2.197 & 15.379 & 112.047 & 2.197 \\
\hline
\end{tabular}




\begin{tabular}{|c|c|c|c|}
\hline 63.713 & 2.197 & 4.394 & 15.379 \\
\hline 4.394 & 2.197 & 92.274 & 175.76 \\
\hline 4.394 & 2.197 & 6.591 & 8.788 \\
\hline 2.197 & 2.197 & 4.394 & 10.985 \\
\hline 2.197 & 2.197 & 6.591 & 355.914 \\
\hline 13.182 & 6.591 & 2.197 & 125.229 \\
\hline 314.171 & 2.197 & 4.394 & 4.394 \\
\hline 81.289 & 2.197 & 2.197 & 4.394 \\
\hline 8.788 & 2.197 & 2.197 & 2.197 \\
\hline 2.197 & 63.713 & 2.197 & 8.788 \\
\hline 4.394 & 2.197 & 2.197 & 2.197 \\
\hline 65.91 & 2.197 & 2.197 & 2.197 \\
\hline 2.197 & 2.197 & 283.413 & 2.197 \\
\hline 375.687 & 6.591 & 6.591 & 2.197 \\
\hline 4.394 & 2.197 & 6.591 & 4.394 \\
\hline 2.197 & 4.394 & 6.591 & 4.394 \\
\hline 2.197 & 2.197 & 17.576 & 4.394 \\
\hline 2.197 & 2.197 & 2.197 & 2.197 \\
\hline 2.197 & 17.576 & 2.197 & 24.167 \\
\hline 2.197 & 28.561 & 294.398 & 28.561 \\
\hline 4.394 & 4.394 & 2.197 & 2.197 \\
\hline 19.773 & 2.197 & 2.197 & 2.197 \\
\hline 6.591 & 2.197 & 2.197 & 48.334 \\
\hline 4.394 & 300.989 & 13.182 & 2.197 \\
\hline 70.304 & 2.197 & 4.394 & 2.197 \\
\hline 4.394 & 261.443 & 24.167 & 4.394 \\
\hline 8.788 & 2.197 & 4.394 & 10.985 \\
\hline 2.197 & 81.289 & 4.394 & 2.197 \\
\hline 26.364 & 147.199 & 2.197 & 4.394 \\
\hline 1298.43 & 4.394 & 92.274 & 114.244 \\
\hline 35.152 & 65.91 & 17.576 & 6.591 \\
\hline 35.152 & 6.591 & 2.197 & 21.97 \\
\hline 6.591 & 2.197 & 2.197 & 15.379 \\
\hline 6.591 & 2.197 & 4.394 & 2.197 \\
\hline 2.197 & 50.531 & 2.197 & 4.394 \\
\hline 6.591 & 8.788 & 2.197 & 4.394 \\
\hline 46.137 & 2.197 & 2.197 & 52.728 \\
\hline 4.394 & 21.97 & 2.197 & 2.197 \\
\hline 50.531 & 2.197 & 4.394 & 17.576 \\
\hline 2.197 & 2.197 & 2.197 & 39.546 \\
\hline 2.197 & 2.197 & 2.197 & 2.197 \\
\hline 2.197 & 2.197 & 2.197 & 13.182 \\
\hline 10.985 & 2.197 & 2.197 & 577.811 \\
\hline 2.197 & 4.394 & 2.197 & 199.927 \\
\hline 4.394 & 2.197 & 2.197 & 4.394 \\
\hline 10.985 & 8.788 & 2.197 & 15.379 \\
\hline 13.182 & 4.394 & 8.788 & 13.182 \\
\hline 2.197 & 59.319 & 2.197 & 443.794 \\
\hline 52.728 & 2.197 & 2.197 & 48.334 \\
\hline 2.197 & 8.788 & 4.394 & 10.985 \\
\hline
\end{tabular}




\begin{tabular}{|c|c|c|c|}
\hline 2.197 & 6.591 & 4.394 & 10.985 \\
\hline 2.197 & 4.394 & 109.85 & 8.788 \\
\hline 2.197 & 85.683 & 41.743 & 13.182 \\
\hline 6.591 & 2.197 & 2.197 & 8.788 \\
\hline 28.561 & 2.197 & 50.531 & 10.985 \\
\hline 13.182 & 21.97 & 2.197 & 21.97 \\
\hline 17.576 & 81.289 & 30.758 & 441.597 \\
\hline 13.182 & 180.154 & 2.197 & 21.97 \\
\hline 26.364 & 6.591 & 2.197 & 109.85 \\
\hline 1133.65 & 2.197 & 283.413 & 24.167 \\
\hline 10.985 & 32.955 & 8.788 & 8.788 \\
\hline 50.531 & 123.032 & 17.576 & 19.773 \\
\hline 105.456 & 32.955 & 2.197 & 2.197 \\
\hline 32.955 & 6.591 & 2.197 & 15.379 \\
\hline 6.591 & 2.197 & 2.197 & 2.197 \\
\hline 21.97 & 10.985 & 13.182 & 2.197 \\
\hline 2.197 & 2.197 & 8.788 & 2.197 \\
\hline 2.197 & 2.197 & 39.546 & 2.197 \\
\hline 2.197 & 30.758 & 2.197 & 2.197 \\
\hline 17.576 & 28.561 & 164.775 & 10.985 \\
\hline 224.094 & 2.197 & 15.379 & 2.197 \\
\hline 2.197 & 2.197 & 2.197 & 8.788 \\
\hline 2.197 & 2.197 & 184.548 & 13.182 \\
\hline 4.394 & 4.394 & 15.379 & 8.788 \\
\hline 2.197 & 39.546 & 17.576 & 28.561 \\
\hline 166.972 & 6.591 & 28.561 & 26.364 \\
\hline 2.197 & 4.394 & 10.985 & 21.97 \\
\hline 15.379 & 2.197 & 2.197 & 595.387 \\
\hline 2.197 & 83.486 & 494.325 & 13.182 \\
\hline 4.394 & 142.805 & 4.394 & 6.591 \\
\hline 2.197 & 17.576 & 10.985 & 353.717 \\
\hline 98.865 & 4.394 & 358.111 & 2.197 \\
\hline 4.394 & 41.743 & 3295.5 & 13.182 \\
\hline 39.546 & 37.349 & 4.394 & 4.394 \\
\hline 19.773 & 87.88 & 2.197 & 17.576 \\
\hline 2.197 & 63.713 & 96.668 & 15.379 \\
\hline 4.394 & 37.349 & 24.167 & 2.197 \\
\hline 2.197 & 8.788 & 2.197 & 21.97 \\
\hline 4.394 & 145.002 & 2.197 & 252.655 \\
\hline 79.092 & 2.197 & 2.197 & 1047.97 \\
\hline 265.837 & 8.788 & 2.197 & 15.379 \\
\hline 21.97 & 13.182 & 32.955 & 2.197 \\
\hline 15.379 & 26.364 & 4.394 & 15.379 \\
\hline 5103.63 & 2.197 & 380.081 & 21.97 \\
\hline 221.897 & 8.788 & 105.456 & 59.319 \\
\hline 4.394 & 30.758 & 4.394 & 4.394 \\
\hline 2.197 & 4.394 & 2.197 & 70.304 \\
\hline 41.743 & 79.092 & 2.197 & 61.516 \\
\hline 298.792 & 6.591 & 6.591 & 290.004 \\
\hline 2.197 & 204.321 & 2.197 & 250.458 \\
\hline
\end{tabular}




\begin{tabular}{|c|c|c|c|}
\hline 4.394 & 2.197 & 2.197 & 4.394 \\
\hline 2.197 & 136.214 & 2.197 & 54.925 \\
\hline 2.197 & 15.379 & 2.197 & 6.591 \\
\hline 2.197 & 4.394 & 2.197 & 35.152 \\
\hline 2.197 & 61.516 & 13.182 & 59.319 \\
\hline 2.197 & 8.788 & 2.197 & 6.591 \\
\hline 228.488 & 8.788 & 6.591 & 19.773 \\
\hline 4.394 & 10.985 & 2.197 & 13.182 \\
\hline 6.591 & 478.946 & 32.955 & 4.394 \\
\hline 2.197 & 2.197 & 39.546 & 13.182 \\
\hline 4.394 & 112.047 & 2.197 & 127.426 \\
\hline 6.591 & 8.788 & 6.591 & 149.396 \\
\hline 2.197 & 2.197 & 2.197 & 2.197 \\
\hline 112.047 & 2.197 & 6.591 & 118.638 \\
\hline 21.97 & 17.576 & 2.197 & 2.197 \\
\hline 81.289 & 2.197 & 4.394 & 333.944 \\
\hline 4.394 & 4.394 & 2.197 & 4.394 \\
\hline 24.167 & 4.394 & 2.197 & 6.591 \\
\hline 37.349 & 151.593 & 28.561 & 10.985 \\
\hline 134.017 & 2.197 & 2.197 & 6.591 \\
\hline 98.865 & 4.394 & 2.197 & 15.379 \\
\hline 21.97 & 2.197 & 39.546 & 8.788 \\
\hline 13854.3 & 2.197 & 32.955 & 2.197 \\
\hline 59.319 & 8.788 & 2.197 & 4.394 \\
\hline 2.197 & 4.394 & 32.955 & 2.197 \\
\hline 287.807 & 4.394 & 2.197 & 6.591 \\
\hline 37.349 & 2.197 & 19.773 & 4.394 \\
\hline 4.394 & 24.167 & 4.394 & 15.379 \\
\hline 13.182 & 2.197 & 2.197 & 13.182 \\
\hline 136.214 & 2.197 & 37.349 & 4.394 \\
\hline 32.955 & 37.349 & 2.197 & 37.349 \\
\hline 2.197 & 287.807 & 2.197 & 2.197 \\
\hline 2.197 & 13.182 & 61.516 & 2.197 \\
\hline 2.197 & 6.591 & 2.197 & 268.034 \\
\hline 13.182 & 8.788 & 4.394 & 10.985 \\
\hline 6.591 & 2.197 & 6.591 & 2.197 \\
\hline 4.394 & 6.591 & 2.197 & 2.197 \\
\hline 2.197 & 41.743 & 2.197 & 17.576 \\
\hline 2.197 & 2.197 & 10.985 & 28.561 \\
\hline 4.394 & 109.85 & 6.591 & 4.394 \\
\hline 19.773 & 2.197 & 316.368 & 68.107 \\
\hline 8.788 & 2.197 & 19.773 & 182.351 \\
\hline 6.591 & 65.91 & 19.773 & 21.97 \\
\hline 6.591 & 96.668 & 6.591 & 4.394 \\
\hline 6.591 & 6.591 & 2.197 & 4038.09 \\
\hline 41.743 & 4.394 & 2.197 & 140.608 \\
\hline 2.197 & 6.591 & 17.576 & 26.364 \\
\hline 28.561 & 13.182 & 6.591 & 4.394 \\
\hline 8.788 & 2.197 & 10.985 & 2.197 \\
\hline 2.197 & 6.591 & 10.985 & 2.197 \\
\hline
\end{tabular}




\begin{tabular}{|c|c|c|c|}
\hline 10.985 & 26.364 & 2.197 & 24.167 \\
\hline 8.788 & 17.576 & 2.197 & 290.004 \\
\hline 2.197 & 2.197 & 2.197 & 188.942 \\
\hline 13.182 & 8.788 & 2.197 & 19.773 \\
\hline 13.182 & 4.394 & 2.197 & 26.364 \\
\hline 2.197 & 2.197 & 4.394 & 24.167 \\
\hline 2.197 & 8.788 & 4.394 & 3462.47 \\
\hline 2.197 & 4.394 & 2.197 & 24.167 \\
\hline 4.394 & 13.182 & 13.182 & 2.197 \\
\hline 4.394 & 4.394 & 208.715 & 235.079 \\
\hline 2.197 & 13.182 & 85.683 & 2.197 \\
\hline 2.197 & 6.591 & 30.758 & 41.743 \\
\hline 21.97 & 2.197 & 2.197 & 15.379 \\
\hline 2.197 & 2.197 & 48.334 & 4.394 \\
\hline 2.197 & 2.197 & 19.773 & 2.197 \\
\hline 15.379 & 4.394 & 2.197 & 120.835 \\
\hline 4.394 & 2.197 & 2.197 & 52.728 \\
\hline 6.591 & 2.197 & 8.788 & 2.197 \\
\hline 13.182 & 4.394 & 2.197 & 10.985 \\
\hline 8.788 & 10.985 & 2.197 & 786.526 \\
\hline 8.788 & 10.985 & 2.197 & 4.394 \\
\hline 4.394 & 32.955 & 2.197 & 2.197 \\
\hline 2.197 & 2.197 & 26.364 & 6.591 \\
\hline 81.289 & 43.94 & 83.486 & 15.379 \\
\hline 98.865 & 2.197 & 6.591 & 2.197 \\
\hline 43.94 & 26.364 & 10.985 & 166.972 \\
\hline 2.197 & 2.197 & 2.197 & 188.942 \\
\hline 41.743 & 2.197 & 6.591 & 386.672 \\
\hline 24.167 & 21.97 & 2.197 & 43.94 \\
\hline 72.501 & 32.955 & 21.97 & 4.394 \\
\hline 2.197 & 4.394 & 6.591 & 39.546 \\
\hline 2.197 & 21.97 & 2.197 & 41.743 \\
\hline 140.608 & 52.728 & 2.197 & 30.758 \\
\hline 13.182 & 4.394 & 28.561 & 4.394 \\
\hline 92.274 & 6.591 & 2.197 & 2.197 \\
\hline 489.931 & 8.788 & 4.394 & 37.349 \\
\hline 2.197 & 61.516 & 2.197 & 109.85 \\
\hline 4.394 & 26.364 & 4.394 & 4.394 \\
\hline 6.591 & 4.394 & 10.985 & 2.197 \\
\hline 4.394 & 6.591 & 2.197 & 2.197 \\
\hline 2.197 & 79.092 & 17.576 & 50.531 \\
\hline 2.197 & 10.985 & 2.197 & 30.758 \\
\hline 2.197 & 2.197 & 2.197 & 659.1 \\
\hline 2.197 & 10.985 & 70.304 & 2.197 \\
\hline 10.985 & 4.394 & 4.394 & 90.077 \\
\hline 2.197 & 2.197 & 13.182 & 4.394 \\
\hline 2.197 & 2.197 & 65.91 & 2.197 \\
\hline 15.379 & 46.137 & 4.394 & 4.394 \\
\hline 19.773 & 4.394 & 4.394 & 116.441 \\
\hline 8.788 & 2.197 & 15.379 & 529.477 \\
\hline
\end{tabular}




\begin{tabular}{|c|c|c|c|}
\hline 2.197 & 2.197 & 17.576 & 32.955 \\
\hline 2.197 & 771.147 & 2.197 & 8.788 \\
\hline 61.516 & 2.197 & 13.182 & 50.531 \\
\hline 2.197 & 2.197 & 2.197 & 2.197 \\
\hline 6.591 & 26.364 & 2.197 & 2.197 \\
\hline 6.591 & 2.197 & 2.197 & 4.394 \\
\hline 26.364 & 13.182 & 2.197 & 6.591 \\
\hline 50.531 & 4.394 & 2.197 & 6.591 \\
\hline 6.591 & 6.591 & 2.197 & 2.197 \\
\hline 4.394 & 6.591 & 2.197 & 2.197 \\
\hline 2.197 & 72.501 & 2.197 & 57.122 \\
\hline 32.955 & 19.773 & 2.197 & 210.912 \\
\hline 2.197 & 17.576 & 2.197 & 2.197 \\
\hline 24.167 & 2.197 & 2.197 & 4.394 \\
\hline 6.591 & 129.623 & 10.985 & 19.773 \\
\hline 2.197 & 2.197 & 2.197 & 41.743 \\
\hline 2.197 & 325.156 & 19.773 & 74.698 \\
\hline 2.197 & 39.546 & 2.197 & 109.85 \\
\hline 2.197 & 2.197 & 4.394 & 46.137 \\
\hline 10.985 & 101.062 & 4.394 & 21.97 \\
\hline 43.94 & 2.197 & 4.394 & 2.197 \\
\hline 13.182 & 2.197 & 2.197 & 2.197 \\
\hline 2.197 & 232.882 & 15.379 & 107.653 \\
\hline 2.197 & 2.197 & 2.197 & 52.728 \\
\hline 70.304 & 4.394 & 2.197 & 43.94 \\
\hline 10.985 & 2.197 & 4.394 & 26.364 \\
\hline 35.152 & 10.985 & 2.197 & 32.955 \\
\hline 2.197 & 26.364 & 496.522 & 37.349 \\
\hline 2.197 & 2.197 & 2.197 & 4.394 \\
\hline 219.7 & 2.197 & 2.197 & 2.197 \\
\hline 6.591 & 6.591 & 2.197 & 24.167 \\
\hline 2.197 & 2.197 & 21.97 & 26.364 \\
\hline 52.728 & 43.94 & 13.182 & 24.167 \\
\hline 26.364 & 2.197 & 15.379 & 24.167 \\
\hline 8.788 & 15.379 & 166.972 & 2.197 \\
\hline 13.182 & 2.197 & 2.197 & 15.379 \\
\hline 2.197 & 4.394 & 2.197 & 15.379 \\
\hline 2.197 & 2.197 & 32.955 & 8.788 \\
\hline 13.182 & 4.394 & 2.197 & 2.197 \\
\hline 19.773 & 2.197 & 85.683 & 2.197 \\
\hline 50.531 & 2.197 & 6.591 & 13.182 \\
\hline 26.364 & 6.591 & 2.197 & 2.197 \\
\hline 2.197 & 2.197 & 6.591 & 2.197 \\
\hline 114.244 & 2.197 & 8.788 & 2.197 \\
\hline 28.561 & 8.788 & 2.197 & 155.987 \\
\hline 50.531 & 4.394 & 2.197 & 2.197 \\
\hline 63.713 & 2.197 & 2.197 & 39.546 \\
\hline 8.788 & 57.122 & 2.197 & 2.197 \\
\hline 2.197 & 2.197 & 215.306 & 32.955 \\
\hline 13.182 & 13.182 & 4.394 & 28.561 \\
\hline
\end{tabular}




\begin{tabular}{|c|c|c|c|}
\hline 24.167 & 13.182 & 2.197 & 2.197 \\
\hline 2.197 & 2.197 & 149.396 & 15.379 \\
\hline 120.835 & 2.197 & 6.591 & 68.107 \\
\hline 21.97 & 2.197 & 155.987 & 19.773 \\
\hline 4.394 & 2.197 & 145.002 & 6.591 \\
\hline 2.197 & 2.197 & 303.186 & 10.985 \\
\hline 4.394 & 2.197 & 17.576 & 10.985 \\
\hline 4.394 & 2.197 & 15.379 & 19.773 \\
\hline 17.576 & 2.197 & 8.788 & 2.197 \\
\hline 2.197 & 2.197 & 2.197 & 694.252 \\
\hline 2.197 & 17.576 & 19.773 & 4.394 \\
\hline 43.94 & 43.94 & 6.591 & 2.197 \\
\hline 15.379 & 2.197 & 2.197 & 2.197 \\
\hline 26.364 & 2.197 & 311.974 & 1680.7 \\
\hline 15.379 & 6.591 & 2.197 & 81.289 \\
\hline 2.197 & 2.197 & 2.197 & 2.197 \\
\hline 582.205 & 4.394 & 2.197 & 15.379 \\
\hline 10.985 & 13.182 & 2.197 & 105.456 \\
\hline 4.394 & 2.197 & 103.259 & 2.197 \\
\hline 59.319 & 2.197 & 2.197 & 35.152 \\
\hline 2.197 & 6.591 & 2.197 & 15.379 \\
\hline 2.197 & 4.394 & 2.197 & 226.291 \\
\hline 142.805 & 4.394 & 6.591 & 59.319 \\
\hline 19.773 & 2.197 & 17.576 & 175.76 \\
\hline 2.197 & 17.576 & 4.394 & 32.955 \\
\hline 26.364 & 2.197 & 2.197 & 32.955 \\
\hline 2.197 & 8.788 & 2.197 & 87.88 \\
\hline 4.394 & 2.197 & 2.197 & 4.394 \\
\hline 162.578 & 4.394 & 2.197 & 8.788 \\
\hline 2.197 & 2.197 & 2.197 & 160.381 \\
\hline 98.865 & 24.167 & 4.394 & 2.197 \\
\hline 21.97 & 8.788 & 2.197 & 6.591 \\
\hline 8.788 & 166.972 & 30.758 & 17.576 \\
\hline 87.88 & 10.985 & 19.773 & 230.685 \\
\hline 193.336 & 26.364 & 19.773 & 4.394 \\
\hline 65.91 & 2.197 & 2.197 & 50.531 \\
\hline 15.379 & 30.758 & 76.895 & 2.197 \\
\hline 4.394 & 4.394 & 4.394 & 4.394 \\
\hline 17.576 & 4.394 & 2.197 & 8.788 \\
\hline 54.925 & 35.152 & 2.197 & 2.197 \\
\hline 2.197 & 8.788 & 2.197 & 21.97 \\
\hline 2.197 & 2.197 & 355.914 & 41.743 \\
\hline 2.197 & 46.137 & 2.197 & 149.396 \\
\hline 17.576 & 4.394 & 72.501 & 4.394 \\
\hline 24.167 & 8.788 & 2.197 & 158.184 \\
\hline 6.591 & 4.394 & 160.381 & 46.137 \\
\hline 107.653 & 2.197 & 90.077 & 6.591 \\
\hline 85.683 & 4.394 & 30.758 & 6.591 \\
\hline 2.197 & 2.197 & 2.197 & 259.246 \\
\hline 96.668 & 101.062 & 10.985 & 2.197 \\
\hline
\end{tabular}




\begin{tabular}{|c|c|c|c|}
\hline 641.524 & 2.197 & 8.788 & 2.197 \\
\hline 87.88 & 2.197 & 4.394 & 50.531 \\
\hline 48.334 & 2.197 & 10.985 & 15.379 \\
\hline 2.197 & 21.97 & 46.137 & 46.137 \\
\hline 15.379 & 21.97 & 2.197 & 4.394 \\
\hline 43.94 & 4.394 & 2.197 & 4.394 \\
\hline 26.364 & 2.197 & 2.197 & 4.394 \\
\hline 43.94 & 2.197 & 2.197 & 19.773 \\
\hline 2.197 & 2.197 & 2.197 & 4.394 \\
\hline 4.394 & 2.197 & 2.197 & 70.304 \\
\hline 13.182 & 2.197 & 2.197 & 2.197 \\
\hline 2.197 & 4.394 & 10.985 & 59.319 \\
\hline 24.167 & 35.152 & 2.197 & 105.456 \\
\hline 2.197 & 2.197 & 204.321 & 24.167 \\
\hline 8.788 & 2.197 & 8.788 & 503.113 \\
\hline 2.197 & 10.985 & 4.394 & 39.546 \\
\hline 2.197 & 2.197 & 2.197 & 2.197 \\
\hline 2.197 & 2.197 & 2.197 & 6.591 \\
\hline 94.471 & 2.197 & 2.197 & 349.323 \\
\hline 4.394 & 2.197 & 54.925 & 2.197 \\
\hline 4.394 & 1107.29 & 637.13 & 10.985 \\
\hline 2.197 & 2.197 & 13.182 & 6.591 \\
\hline 294.398 & 2.197 & 2.197 & 6.591 \\
\hline 24.167 & 2.197 & 2.197 & 2.197 \\
\hline 4.394 & 2.197 & 6.591 & 1118.27 \\
\hline 17.576 & 2.197 & 83.486 & 24.167 \\
\hline 4.394 & 10.985 & 8.788 & 186.745 \\
\hline 2.197 & 4.394 & 4.394 & 17.576 \\
\hline 15.379 & 292.201 & 2.197 & 500.916 \\
\hline 4.394 & 13.182 & 4.394 & 2.197 \\
\hline 24.167 & 239.473 & 85.683 & 43.94 \\
\hline 2.197 & 2.197 & 10.985 & 2.197 \\
\hline 13.182 & 2.197 & 6.591 & 215.306 \\
\hline 4.394 & 6.591 & 2.197 & 553.644 \\
\hline 19.773 & 72.501 & 8.788 & 2.197 \\
\hline 8.788 & 8.788 & 155.987 & 13.182 \\
\hline 43.94 & 452.582 & 10.985 & 4.394 \\
\hline 2.197 & 1219.33 & 261.443 & 98.865 \\
\hline 2.197 & 2.197 & 2.197 & 2.197 \\
\hline 52.728 & 2.197 & 4.394 & 68.107 \\
\hline 2.197 & 2.197 & 4.394 & 210.912 \\
\hline 2.197 & 2.197 & 2.197 & 17.576 \\
\hline 4.394 & 6.591 & 37.349 & 30.758 \\
\hline 2.197 & 37.349 & 375.687 & 2.197 \\
\hline 2.197 & 131.82 & 114.244 & 13.182 \\
\hline 21.97 & 4.394 & 4.394 & 180.154 \\
\hline 13.182 & 2.197 & 21.97 & 24.167 \\
\hline 4.394 & 8.788 & 2.197 & 13.182 \\
\hline 4.394 & 4.394 & 52.728 & 4.394 \\
\hline 50.531 & 8.788 & 2.197 & 2.197 \\
\hline
\end{tabular}




\begin{tabular}{|c|c|c|c|}
\hline 6.591 & 21.97 & 13.182 & 2.197 \\
\hline 224.094 & 2.197 & 4.394 & 15.379 \\
\hline 10.985 & 4.394 & 2.197 & 98.865 \\
\hline 21.97 & 13.182 & 79.092 & 2.197 \\
\hline 2.197 & 13.182 & 2.197 & 85.683 \\
\hline 39.546 & 2.197 & 35.152 & 8.788 \\
\hline 2.197 & 2.197 & 8.788 & 125.229 \\
\hline 46.137 & 2.197 & 2.197 & 48.334 \\
\hline 17.576 & 6.591 & 2.197 & 21.97 \\
\hline 32.955 & 103.259 & 2.197 & 54.925 \\
\hline 136.214 & 15.379 & 2.197 & 6.591 \\
\hline 6.591 & 2.197 & 2.197 & 3365.8 \\
\hline 50.531 & 2.197 & 39.546 & 41.743 \\
\hline 6.591 & 155.987 & 4.394 & 70.304 \\
\hline 6.591 & 472.355 & 4.394 & 17.576 \\
\hline 15.379 & 6.591 & 2.197 & 4.394 \\
\hline 2.197 & 6.591 & 2.197 & 2.197 \\
\hline 6.591 & 2.197 & 28.561 & 37.349 \\
\hline 10.985 & 28.561 & 26.364 & 2.197 \\
\hline 10.985 & 2.197 & 131.82 & 131.82 \\
\hline 50.531 & 2.197 & 19.773 & 61.516 \\
\hline 2.197 & 2.197 & 2.197 & 2.197 \\
\hline 2.197 & 8.788 & 2.197 & 46.137 \\
\hline 4.394 & 6.591 & 2.197 & 8.788 \\
\hline 32.955 & 2.197 & 2.197 & 4.394 \\
\hline 344.929 & 2.197 & 32.955 & 6.591 \\
\hline 15.379 & 6.591 & 4.394 & 21.97 \\
\hline 13.182 & 2.197 & 191.139 & 522.886 \\
\hline 2.197 & 6.591 & 298.792 & 28.561 \\
\hline 13.182 & 2.197 & 15.379 & 2.197 \\
\hline 681.07 & 13.182 & 4.394 & 85.683 \\
\hline 2.197 & 21.97 & 15.379 & 10.985 \\
\hline 17.576 & 17.576 & 2.197 & 103.259 \\
\hline 10.985 & 2.197 & 21.97 & 17.576 \\
\hline 6.591 & 2.197 & 2.197 & 2.197 \\
\hline 4.394 & 19.773 & 2.197 & 2.197 \\
\hline 24.167 & 8.788 & 2.197 & 10.985 \\
\hline 2.197 & 4.394 & 2.197 & 2.197 \\
\hline 2.197 & 2.197 & 6.591 & 4.394 \\
\hline 2.197 & 19.773 & 4.394 & 2.197 \\
\hline 6.591 & 26.364 & 26.364 & 2.197 \\
\hline 114.244 & 13.182 & 43.94 & 195.533 \\
\hline 2.197 & 2.197 & 2.197 & 2.197 \\
\hline 4.394 & 114.244 & 166.972 & 8.788 \\
\hline 10.985 & 160.381 & 37.349 & 13.182 \\
\hline 2.197 & 180.154 & 2.197 & 21.97 \\
\hline 13.182 & 2.197 & 8.788 & 118.638 \\
\hline 2.197 & 4.394 & 2.197 & 2.197 \\
\hline 2.197 & 4.394 & 15.379 & 10.985 \\
\hline 4.394 & 13.182 & 41.743 & 4.394 \\
\hline
\end{tabular}




\begin{tabular}{|c|c|c|c|}
\hline 1298.43 & 105.456 & 15.379 & 2.197 \\
\hline 4.394 & 2.197 & 2.197 & 116.441 \\
\hline 2.197 & 2.197 & 35.152 & 26.364 \\
\hline 4.394 & 10.985 & 19.773 & 685.464 \\
\hline 4.394 & 2.197 & 2.197 & 10.985 \\
\hline 6.591 & 79.092 & 48.334 & 8.788 \\
\hline 76.895 & 19.773 & 19.773 & 6.591 \\
\hline 4.394 & 6.591 & 2.197 & 6.591 \\
\hline 61.516 & 2.197 & 2.197 & 17.576 \\
\hline 4.394 & 57.122 & 10.985 & 15.379 \\
\hline 28.561 & 448.188 & 4.394 & 8.788 \\
\hline 413.036 & 2.197 & 2.197 & 4.394 \\
\hline 32.955 & 21.97 & 2.197 & 28.561 \\
\hline 4.394 & 2.197 & 8.788 & 13.182 \\
\hline 2.197 & 2.197 & 213.109 & 13.182 \\
\hline 2.197 & 2.197 & 74.698 & 8.788 \\
\hline 6.591 & 2.197 & 2.197 & 6.591 \\
\hline 2.197 & 13.182 & 2.197 & 13.182 \\
\hline 2.197 & 2.197 & 2.197 & 6.591 \\
\hline 2.197 & 4.394 & 2.197 & 24.167 \\
\hline 2.197 & 30.758 & 2.197 & 2.197 \\
\hline 4.394 & 2.197 & 46.137 & 26.364 \\
\hline 2.197 & 6.591 & 61.516 & 10.985 \\
\hline 2.197 & 26.364 & 13.182 & 226.291 \\
\hline 8.788 & 17.576 & 2.197 & 2.197 \\
\hline 17.576 & 6.591 & 2.197 & 4.394 \\
\hline 6.591 & 2.197 & 4.394 & 8.788 \\
\hline 630.539 & 35.152 & 2.197 & 171.366 \\
\hline 4.394 & 2.197 & 2.197 & 384.475 \\
\hline 28.561 & 39.546 & 2.197 & 10.985 \\
\hline 177.957 & 120.835 & 2.197 & 21.97 \\
\hline 2.197 & 2.197 & 2.197 & 6.591 \\
\hline 24.167 & 2.197 & 2.197 & 13.182 \\
\hline 2.197 & 4.394 & 8.788 & 6.591 \\
\hline 281.216 & 2.197 & 2.197 & 2.197 \\
\hline 13.182 & 2.197 & 2.197 & 2.197 \\
\hline 8.788 & 112.047 & 2.197 & 2.197 \\
\hline 37.349 & 125.229 & 28.561 & 85.683 \\
\hline 2.197 & 2.197 & 15.379 & 10.985 \\
\hline 2.197 & 35.152 & 2.197 & 8.788 \\
\hline 2.197 & 4.394 & 6.591 & 37.349 \\
\hline 2.197 & 2.197 & 2.197 & 4.394 \\
\hline 4.394 & 2.197 & 2.197 & 2192.61 \\
\hline 2.197 & 4.394 & 4.394 & 6.591 \\
\hline 180.154 & 15.379 & 4.394 & 191.139 \\
\hline 6.591 & 2.197 & 19.773 & 2.197 \\
\hline 6.591 & 2.197 & 4.394 & 2.197 \\
\hline 105.456 & 6.591 & 2.197 & 2.197 \\
\hline 4.394 & 61.516 & 2.197 & 15.379 \\
\hline 265.837 & 10.985 & 478.946 & 17.576 \\
\hline
\end{tabular}




\begin{tabular}{|c|c|c|c|}
\hline 2.197 & 26.364 & 2.197 & 10.985 \\
\hline 2.197 & 10.985 & 2.197 & 10.985 \\
\hline 10.985 & 2.197 & 8.788 & 26.364 \\
\hline 8.788 & 15.379 & 2.197 & 408.642 \\
\hline 2.197 & 15.379 & 10.985 & 10.985 \\
\hline 76.895 & 4.394 & 21.97 & 140.608 \\
\hline 105.456 & 2.197 & 10.985 & 32.955 \\
\hline 8.788 & 2.197 & 4.394 & 6.591 \\
\hline 2.197 & 83.486 & 1324.79 & 19.773 \\
\hline 10.985 & 21.97 & 24.167 & 8.788 \\
\hline 2.197 & 4.394 & 2.197 & 52.728 \\
\hline 13.182 & 103.259 & 2.197 & 2.197 \\
\hline 54.925 & 17.576 & 2.197 & 4.394 \\
\hline 6.591 & 2.197 & 2383.74 & 4.394 \\
\hline 2.197 & 6.591 & 2.197 & 10.985 \\
\hline 8.788 & 15.379 & 24.167 & 8.788 \\
\hline 2.197 & 13.182 & 4.394 & 15.379 \\
\hline 46.137 & 4.394 & 4.394 & 8.788 \\
\hline 2.197 & 2.197 & 19.773 & 2.197 \\
\hline 17.576 & 19.773 & 2.197 & 6.591 \\
\hline 2.197 & 68.107 & 2.197 & 13.182 \\
\hline 2.197 & 4.394 & 2.197 & 402.051 \\
\hline 2.197 & 74.698 & 202.124 & 65.91 \\
\hline 4.394 & 199.927 & 2.197 & 32.955 \\
\hline 41.743 & 15.379 & 197.73 & 362.505 \\
\hline 17.576 & 2.197 & 2.197 & 2.197 \\
\hline 10.985 & 2.197 & 2.197 & 28.561 \\
\hline 461.37 & 2.197 & 10.985 & 2.197 \\
\hline 6.591 & 2.197 & 10.985 & 19.773 \\
\hline 50.531 & 2.197 & 2.197 & 2.197 \\
\hline 21.97 & 112.047 & 4.394 & 158.184 \\
\hline 2.197 & 6.591 & 2.197 & 4.394 \\
\hline 2.197 & 6.591 & 4.394 & 6.591 \\
\hline 2.197 & 6.591 & 17.576 & 4.394 \\
\hline 13.182 & 6.591 & 10.985 & 2.197 \\
\hline 454.779 & 26.364 & 2.197 & 2.197 \\
\hline 2.197 & 4.394 & 2.197 & 2.197 \\
\hline 8.788 & 13.182 & 4.394 & 2.197 \\
\hline 15.379 & 39.546 & 32.955 & 2.197 \\
\hline 134.017 & 8.788 & 92.274 & 68.107 \\
\hline 32.955 & 4.394 & 6.591 & 48.334 \\
\hline 2.197 & 2.197 & 4.394 & 441.597 \\
\hline 2.197 & 87.88 & 2.197 & 2.197 \\
\hline 2.197 & 2.197 & 2.197 & 6.591 \\
\hline 2.197 & 8.788 & 4.394 & 15.379 \\
\hline 13.182 & 43.94 & 2.197 & 13.182 \\
\hline 4.394 & 2.197 & 2.197 & 15.379 \\
\hline 24.167 & 39.546 & 2.197 & 13.182 \\
\hline 4.394 & 2.197 & 8.788 & 17.576 \\
\hline 2.197 & 13.182 & 26.364 & 2.197 \\
\hline
\end{tabular}




\begin{tabular}{|c|c|c|c|}
\hline 2.197 & 2.197 & 2.197 & 8.788 \\
\hline 32.955 & 24.167 & 30.758 & 15.379 \\
\hline 35.152 & 10.985 & 10.985 & 2.197 \\
\hline 2.197 & 2.197 & 2.197 & 13.182 \\
\hline 2.197 & 21.97 & 2.197 & 61.516 \\
\hline 2.197 & 4.394 & 2.197 & 6.591 \\
\hline 2.197 & 2.197 & 6.591 & 4.394 \\
\hline 6.591 & 87.88 & 21.97 & 134.017 \\
\hline 13.182 & 13.182 & 2.197 & 17.576 \\
\hline 10.985 & 2.197 & 2.197 & 4.394 \\
\hline 52.728 & 2.197 & 2.197 & 2.197 \\
\hline 2.197 & 2.197 & 10.985 & 59.319 \\
\hline 15.379 & 21.97 & 8.788 & 2.197 \\
\hline 15.379 & 17.576 & 2.197 & 4.394 \\
\hline 2.197 & 8.788 & 6.591 & 32.955 \\
\hline 6.591 & 2.197 & 114.244 & 50.531 \\
\hline 4.394 & 4.394 & 2.197 & 2.197 \\
\hline 68.107 & 2.197 & 21.97 & 2.197 \\
\hline 28.561 & 57.122 & 2.197 & 5268.41 \\
\hline 8.788 & 30.758 & 2.197 & 4.394 \\
\hline 287.807 & 2.197 & 63.713 & 2.197 \\
\hline 116.441 & 2.197 & 3137.32 & 676.676 \\
\hline 57.122 & 2.197 & 2.197 & 68.107 \\
\hline 2.197 & 68.107 & 2.197 & 32.955 \\
\hline 48.334 & 30.758 & 2.197 & 2.197 \\
\hline 96.668 & 2.197 & 2.197 & 6.591 \\
\hline 4.394 & 17.576 & 2.197 & 2.197 \\
\hline 6.591 & 10.985 & 2.197 & 2.197 \\
\hline 28.561 & 2.197 & 61.516 & 197.73 \\
\hline 21.97 & 19.773 & 516.295 & 112.047 \\
\hline 2.197 & 2.197 & 2.197 & 2.197 \\
\hline 17.576 & 105.456 & 6.591 & 46.137 \\
\hline 2225.56 & 19.773 & 2.197 & 13.182 \\
\hline 2.197 & 17.576 & 4.394 & 503.113 \\
\hline 21.97 & 2.197 & 4.394 & 2.197 \\
\hline 70.304 & 2.197 & 15.379 & 303.186 \\
\hline 2.197 & 4.394 & 4.394 & 15.379 \\
\hline 4.394 & 2.197 & 4.394 & 26.364 \\
\hline 6.591 & 15.379 & 2.197 & 2.197 \\
\hline 43.94 & 19.773 & 2.197 & 2.197 \\
\hline 2.197 & 39.546 & 2.197 & 13.182 \\
\hline 2.197 & 2.197 & 2.197 & 19.773 \\
\hline 2.197 & 2.197 & 2.197 & 94.471 \\
\hline 2.197 & 2.197 & 371.293 & 41.743 \\
\hline 2.197 & 2.197 & 437.203 & 4.394 \\
\hline 25940 & 2.197 & 2.197 & 13.182 \\
\hline 4.394 & 2.197 & 4.394 & 4.394 \\
\hline 116.441 & 2.197 & 4.394 & 15.379 \\
\hline 2.197 & 2.197 & 2.197 & 13.182 \\
\hline 41.743 & 2.197 & 30.758 & 2.197 \\
\hline
\end{tabular}




\begin{tabular}{|c|c|c|c|}
\hline 188.942 & 2.197 & 4.394 & 2.197 \\
\hline 65.91 & 4.394 & 15.379 & 6.591 \\
\hline 2.197 & 8.788 & 46.137 & 8.788 \\
\hline 43.94 & 2.197 & 8.788 & 2.197 \\
\hline 76.895 & 8.788 & 2.197 & 2.197 \\
\hline 13.182 & 2.197 & 10.985 & 19.773 \\
\hline 2.197 & 2.197 & 24.167 & 30.758 \\
\hline 2.197 & 4.394 & 6.591 & 2.197 \\
\hline 2.197 & 2.197 & 131.82 & 2.197 \\
\hline 4.394 & 8.788 & 8.788 & 43.94 \\
\hline 2.197 & 21.97 & 57.122 & 30.758 \\
\hline 96.668 & 6.591 & 30.758 & 26.364 \\
\hline 24.167 & 4.394 & 329.55 & 24.167 \\
\hline 2.197 & 15.379 & 15.379 & 2.197 \\
\hline 4.394 & 96.668 & 2.197 & 35.152 \\
\hline 21.97 & 2.197 & 10.985 & 39.546 \\
\hline 10.985 & 2.197 & 8.788 & 6.591 \\
\hline 24.167 & 83.486 & 13.182 & 2.197 \\
\hline 6.591 & 4.394 & 92.274 & 8.788 \\
\hline 4.394 & 48.334 & 2.197 & 2.197 \\
\hline 13.182 & 24.167 & 4.394 & 4.394 \\
\hline 15.379 & 21.97 & 13.182 & 2.197 \\
\hline 24.167 & 8.788 & 2.197 & 6.591 \\
\hline 15.379 & 4.394 & 28.561 & 24.167 \\
\hline 2.197 & 4.394 & 2.197 & 4.394 \\
\hline 4.394 & 8.788 & 2.197 & 2.197 \\
\hline 21.97 & 4.394 & 2.197 & 4.394 \\
\hline 13.182 & 35.152 & 28.561 & 10.985 \\
\hline 10.985 & 8.788 & 15.379 & 4.394 \\
\hline 8.788 & 52.728 & 35.152 & 2.197 \\
\hline 13.182 & 6.591 & 2.197 & 2.197 \\
\hline 380.081 & 107.653 & 4.394 & 2.197 \\
\hline 4.394 & 4.394 & 2.197 & 2.197 \\
\hline 213.109 & 15.379 & 2.197 & 26.364 \\
\hline 57.122 & 19.773 & 2.197 & 2.197 \\
\hline 2.197 & 2.197 & 2.197 & 68.107 \\
\hline 2.197 & 6.591 & 15.379 & 37.349 \\
\hline 6.591 & 32.955 & 2.197 & 24.167 \\
\hline 2.197 & 35.152 & 2.197 & 2.197 \\
\hline 15.379 & 26.364 & 8.788 & 2.197 \\
\hline 19210.6 & 2.197 & 15.379 & 26.364 \\
\hline 6.591 & 19.773 & 2.197 & 70.304 \\
\hline 41.743 & 2.197 & 6.591 & 2.197 \\
\hline 6.591 & 43.94 & 2.197 & 43.94 \\
\hline 4.394 & 48.334 & 6.591 & 4.394 \\
\hline 2.197 & 74.698 & 2.197 & 8.788 \\
\hline 2.197 & 2.197 & 8.788 & 26.364 \\
\hline 2.197 & 6.591 & 2.197 & 2.197 \\
\hline 8.788 & 2.197 & 24.167 & 4.394 \\
\hline 24.167 & 2.197 & 4.394 & 8.788 \\
\hline
\end{tabular}




\begin{tabular}{|c|c|c|c|}
\hline 4.394 & 4.394 & 2.197 & 4.394 \\
\hline 52.728 & 4.394 & 2.197 & 39.546 \\
\hline 2.197 & 2.197 & 2.197 & 63.713 \\
\hline 4.394 & 4.394 & 2.197 & 28.561 \\
\hline 2.197 & 13.182 & 2.197 & 6.591 \\
\hline 2.197 & 8.788 & 101.062 & 17.576 \\
\hline 35.152 & 4.394 & 2.197 & 15.379 \\
\hline 2.197 & 138.411 & 76.895 & 2.197 \\
\hline 8.788 & 30.758 & 8.788 & 87.88 \\
\hline 13.182 & 74.698 & 2.197 & 13.182 \\
\hline 35.152 & 46.137 & 2.197 & 2.197 \\
\hline 17.576 & 876.603 & 4.394 & 2.197 \\
\hline 24.167 & 283.413 & 15.379 & 19.773 \\
\hline 8.788 & 101.062 & 10.985 & 59.319 \\
\hline 76.895 & 6.591 & 87.88 & 26.364 \\
\hline 4.394 & 6.591 & 8.788 & 72.501 \\
\hline 4.394 & 17.576 & 6.591 & 19.773 \\
\hline 10.985 & 4.394 & 15.379 & 4.394 \\
\hline 2.197 & 26.364 & 15.379 & 30.758 \\
\hline 4.394 & 2.197 & 4.394 & 2.197 \\
\hline 28.561 & 4.394 & 2.197 & 173.563 \\
\hline 19.773 & 349.323 & 2.197 & 125.229 \\
\hline 72.501 & 48.334 & 28.561 & 2.197 \\
\hline 13.182 & 85.683 & 2.197 & 10.985 \\
\hline 4.394 & 68.107 & 2.197 & 4.394 \\
\hline 2.197 & 8.788 & 4.394 & 6.591 \\
\hline 76.895 & 2.197 & 2.197 & 24.167 \\
\hline 32.955 & 2.197 & 6.591 & 83.486 \\
\hline 48.334 & 4.394 & 15.379 & 24.167 \\
\hline 8.788 & 4.394 & 10.985 & 4.394 \\
\hline 72.501 & 4.394 & 2.197 & 2.197 \\
\hline 52.728 & 17793.5 & 10.985 & 15.379 \\
\hline 2.197 & 2.197 & 4.394 & 2.197 \\
\hline 2.197 & 10.985 & 28.561 & 8.788 \\
\hline 8.788 & 59.319 & 2.197 & 37.349 \\
\hline 4.394 & 153.79 & 40165.5 & 43.94 \\
\hline 2.197 & 2.197 & 24.167 & 19.773 \\
\hline 15.379 & 4.394 & 151.593 & 6.591 \\
\hline 57.122 & 2.197 & 2.197 & 2.197 \\
\hline 15.379 & 2.197 & 2985.72 & 19.773 \\
\hline 4.394 & 4.394 & 13.182 & 4.394 \\
\hline 160.381 & 2.197 & 6.591 & 131.82 \\
\hline 94.471 & 2.197 & 2.197 & 13.182 \\
\hline 4.394 & 2.197 & 92.274 & 24.167 \\
\hline 24.167 & 32.955 & 4.394 & 43.94 \\
\hline 4.394 & 32.955 & 13.182 & 59.319 \\
\hline 4.394 & 19.773 & 28.561 & 30.758 \\
\hline 2.197 & 4.394 & 6.591 & 4.394 \\
\hline 2.197 & 2.197 & 2.197 & 17.576 \\
\hline 2.197 & 2.197 & 19.773 & 8.788 \\
\hline
\end{tabular}




\begin{tabular}{|c|c|c|c|}
\hline 2.197 & 76.895 & 2.197 & 2.197 \\
\hline 2.197 & 8.788 & 28.561 & 6.591 \\
\hline 2.197 & 39.546 & 8.788 & 6.591 \\
\hline 2.197 & 15.379 & 2.197 & 13.182 \\
\hline 4.394 & 28.561 & 13.182 & 24.167 \\
\hline 6.591 & 37.349 & 2.197 & 2.197 \\
\hline 6.591 & 4.394 & 2.197 & 2.197 \\
\hline 2.197 & 8.788 & 4.394 & 272.428 \\
\hline 2.197 & 2.197 & 2.197 & 70.304 \\
\hline 2.197 & 2.197 & 290.004 & 8.788 \\
\hline 32.955 & 21.97 & 2.197 & 173.563 \\
\hline 13.182 & 4.394 & 17.576 & 70.304 \\
\hline 90.077 & 48.334 & 50.531 & 109.85 \\
\hline 4.394 & 2.197 & 2.197 & 10.985 \\
\hline 13.182 & 6916.16 & 120.835 & 48.334 \\
\hline 988.65 & 8.788 & 32.955 & 50.531 \\
\hline 4.394 & 2.197 & 2.197 & 4.394 \\
\hline 15.379 & 2.197 & 2.197 & 13.182 \\
\hline 2.197 & 2.197 & 4.394 & 13.182 \\
\hline 6.591 & 2.197 & 2.197 & 1050.17 \\
\hline 2.197 & 2.197 & 2.197 & 63.713 \\
\hline 4.394 & 2.197 & 2.197 & 2.197 \\
\hline 8.788 & 68.107 & 2.197 & 2.197 \\
\hline 15.379 & 6.591 & 2.197 & 8.788 \\
\hline 4.394 & 13.182 & 2.197 & 109.85 \\
\hline 2.197 & 24.167 & 2.197 & 2.197 \\
\hline 2.197 & 2.197 & 2.197 & 28.561 \\
\hline 6.591 & 2.197 & 4.394 & 2.197 \\
\hline 15.379 & 17.576 & 68.107 & 2.197 \\
\hline 2.197 & 4.394 & 2.197 & 2.197 \\
\hline 15.379 & 35.152 & 15.379 & 15.379 \\
\hline 2.197 & 8.788 & 2.197 & 57.122 \\
\hline 2.197 & 2.197 & 17.576 & 10.985 \\
\hline 13.182 & 68.107 & 43.94 & 8.788 \\
\hline 2.197 & 4.394 & 103.259 & 17.576 \\
\hline 2.197 & 26.364 & 15.379 & 70.304 \\
\hline 24.167 & 59.319 & 6.591 & 24.167 \\
\hline 4.394 & 52.728 & 4.394 & 4.394 \\
\hline 61.516 & 54.925 & 39.546 & 94.471 \\
\hline 2.197 & 6.591 & 2.197 & 2.197 \\
\hline 52.728 & 2.197 & 15.379 & 2.197 \\
\hline 2.197 & 2.197 & 19.773 & 4.394 \\
\hline 2.197 & 4.394 & 8.788 & 147.199 \\
\hline 2.197 & 10.985 & 2.197 & 4.394 \\
\hline 4.394 & 2.197 & 13.182 & 2.197 \\
\hline 17.576 & 30.758 & 15.379 & 4.394 \\
\hline 2.197 & 4.394 & 2.197 & 83.486 \\
\hline 13.182 & 6.591 & 6698.65 & 19.773 \\
\hline 2.197 & 8.788 & 15.379 & 4.394 \\
\hline 2.197 & 19.773 & 17.576 & 8.788 \\
\hline
\end{tabular}




\begin{tabular}{|c|c|c|c|}
\hline 8.788 & 4.394 & 10.985 & 219.7 \\
\hline 2.197 & 4.394 & 4.394 & 21.97 \\
\hline 15.379 & 4.394 & 41.743 & 134.017 \\
\hline 4.394 & 4.394 & 2.197 & 2.197 \\
\hline 2.197 & 13.182 & 8.788 & 8.788 \\
\hline 96.668 & 30.758 & 175.76 & 35.152 \\
\hline 15.379 & 2.197 & 2.197 & 1017.21 \\
\hline 6.591 & 4.394 & 57.122 & 2.197 \\
\hline 2.197 & 2.197 & 19.773 & 4.394 \\
\hline 2.197 & 4.394 & 43.94 & 6.591 \\
\hline 2.197 & 2.197 & 4.394 & 37.349 \\
\hline 13.182 & 13.182 & 10.985 & 2.197 \\
\hline 4.394 & 46.137 & 2.197 & 39.546 \\
\hline 2.197 & 4.394 & 199.927 & 21.97 \\
\hline 94.471 & 2.197 & 6.591 & 98.865 \\
\hline 6.591 & 4.394 & 4.394 & 30.758 \\
\hline 2.197 & 2.197 & 6.591 & 4.394 \\
\hline 8.788 & 134.017 & 2.197 & 134.017 \\
\hline 6.591 & 6.591 & 2.197 & 39.546 \\
\hline 19.773 & 13.182 & 125.229 & 1171 \\
\hline 32.955 & 2.197 & 2.197 & 2.197 \\
\hline 6.591 & 2.197 & 2.197 & 2.197 \\
\hline 2.197 & 2.197 & 61.516 & 79.092 \\
\hline 2.197 & 2.197 & 685.464 & 309.777 \\
\hline 2.197 & 2.197 & 13.182 & 4.394 \\
\hline 13.182 & 30.758 & 4.394 & 39.546 \\
\hline 2.197 & 4.394 & 32.955 & 15.379 \\
\hline 4.394 & 2.197 & 2.197 & 70.304 \\
\hline 142.805 & 13.182 & 2.197 & 1050.17 \\
\hline 83.486 & 4.394 & 98.865 & 17.576 \\
\hline 65.91 & 4.394 & 2.197 & 2.197 \\
\hline 10.985 & 4.394 & 2.197 & 4.394 \\
\hline 2.197 & 4.394 & 2.197 & 2.197 \\
\hline 10.985 & 24.167 & 4.394 & 2.197 \\
\hline 52.728 & 2.197 & 2.197 & 4.394 \\
\hline 2.197 & 17.576 & 4.394 & 2.197 \\
\hline 8.788 & 4.394 & 2.197 & 2.197 \\
\hline 2.197 & 2.197 & 2.197 & 6.591 \\
\hline 4.394 & 28.561 & 2.197 & 37.349 \\
\hline 2.197 & 4.394 & 2.197 & 1010.62 \\
\hline 39.546 & 125.229 & 2.197 & 39.546 \\
\hline 2.197 & 41.743 & 2.197 & 105.456 \\
\hline 6.591 & 4.394 & 2.197 & 6.591 \\
\hline 4.394 & 4.394 & 2.197 & 52.728 \\
\hline 10.985 & 2.197 & 19.773 & 13.182 \\
\hline 4.394 & 6.591 & 8.788 & 15.379 \\
\hline 6.591 & 10.985 & 2.197 & 151.593 \\
\hline 2.197 & 2.197 & 2.197 & 2.197 \\
\hline 2.197 & 2.197 & 2.197 & 2.197 \\
\hline 2.197 & 26.364 & 2.197 & 2.197 \\
\hline
\end{tabular}




\begin{tabular}{|c|c|c|c|}
\hline 2.197 & 28.561 & 2.197 & 15.379 \\
\hline 2.197 & 4.394 & 2.197 & 17.576 \\
\hline 2.197 & 2.197 & 2.197 & 4.394 \\
\hline 15.379 & 2.197 & 4.394 & 2.197 \\
\hline 2.197 & 26.364 & 30.758 & 8.788 \\
\hline 4.394 & 241.67 & 35.152 & 2.197 \\
\hline 6.591 & 52.728 & 2.197 & 6.591 \\
\hline 28.561 & 2.197 & 2.197 & 4.394 \\
\hline 6.591 & 2.197 & 13.182 & 2.197 \\
\hline 184.548 & 2.197 & 2.197 & 72.501 \\
\hline 15.379 & 2.197 & 2.197 & 8.788 \\
\hline 2.197 & 2.197 & 8.788 & 8.788 \\
\hline 6112.05 & 13.182 & 4.394 & 268.034 \\
\hline 4.394 & 4.394 & 6.591 & 13.182 \\
\hline 6.591 & 6.591 & 15.379 & 81.289 \\
\hline 6.591 & 15.379 & 2.197 & 54.925 \\
\hline 4.394 & 2.197 & 50.531 & 2.197 \\
\hline 2.197 & 2.197 & 2.197 & 2.197 \\
\hline 2.197 & 2.197 & 2.197 & 2.197 \\
\hline 19.773 & 8.788 & 2.197 & 30.758 \\
\hline 2.197 & 4.394 & 285.61 & 30.758 \\
\hline 131.82 & 2.197 & 2.197 & 2.197 \\
\hline 2.197 & 2.197 & 28.561 & 2.197 \\
\hline 76.895 & 2.197 & 2.197 & 4.394 \\
\hline 17.576 & 21.97 & 6.591 & 32.955 \\
\hline 98.865 & 2.197 & 4.394 & 8.788 \\
\hline 19.773 & 2.197 & 28.561 & 109.85 \\
\hline 10.985 & 2.197 & 10.985 & 8.788 \\
\hline 171.366 & 10.985 & 35.152 & 6.591 \\
\hline 35.152 & 59.319 & 30.758 & 21.97 \\
\hline 8.788 & 2.197 & 2.197 & 2.197 \\
\hline 19.773 & 4.394 & 2.197 & 50.531 \\
\hline 6.591 & 10.985 & 2.197 & 2.197 \\
\hline 191.139 & 4.394 & 8.788 & 37.349 \\
\hline 2.197 & 138.411 & 17.576 & 2.197 \\
\hline 4.394 & 2.197 & 2.197 & 2.197 \\
\hline 4.394 & 76.895 & 4.394 & 2.197 \\
\hline 68.107 & 2.197 & 2.197 & 61.516 \\
\hline 65.91 & 2.197 & 8.788 & 15.379 \\
\hline 76.895 & 32.955 & 2.197 & 2.197 \\
\hline 8.788 & 32.955 & 2.197 & 10.985 \\
\hline 17.576 & 15.379 & 6.591 & 17.576 \\
\hline 10.985 & 35.152 & 32.955 & 90.077 \\
\hline 10.985 & 13.182 & 13.182 & 2.197 \\
\hline 4.394 & 43.94 & 2.197 & 41.743 \\
\hline 243.867 & 2.197 & 30.758 & 2.197 \\
\hline 48.334 & 6.591 & 48.334 & 4.394 \\
\hline 6.591 & 39.546 & 2.197 & 6.591 \\
\hline 50.531 & 10.985 & 2.197 & 4.394 \\
\hline 17.576 & 2.197 & 2.197 & 2.197 \\
\hline
\end{tabular}




\begin{tabular}{|c|c|c|c|}
\hline 4.394 & 2.197 & 2.197 & 2.197 \\
\hline 2.197 & 2.197 & 30.758 & 6.591 \\
\hline 4.394 & 13.182 & 13.182 & 437.203 \\
\hline 549.25 & 2.197 & 2.197 & 19.773 \\
\hline 2.197 & 2.197 & 10.985 & 4.394 \\
\hline 118.638 & 2.197 & 164.775 & 4.394 \\
\hline 2.197 & 15.379 & 26.364 & 221.897 \\
\hline 2.197 & 61.516 & 13.182 & 57.122 \\
\hline 15.379 & 2.197 & 8.788 & 10.985 \\
\hline 17.576 & 4.394 & 2.197 & 109.85 \\
\hline 2.197 & 2.197 & 8.788 & 2.197 \\
\hline 4.394 & 43.94 & 79.092 & 21.97 \\
\hline 19.773 & 2.197 & 6.591 & 26.364 \\
\hline 52.728 & 17.576 & 151.593 & 19.773 \\
\hline 2.197 & 2.197 & 6.591 & 2.197 \\
\hline 43.94 & 2.197 & 2.197 & 19.773 \\
\hline 8.788 & 4.394 & 2.197 & 85.683 \\
\hline 173.563 & 8.788 & 35.152 & 30.758 \\
\hline 10.985 & 4.394 & 140.608 & 1680.7 \\
\hline 2.197 & 2.197 & 2.197 & 2.197 \\
\hline 15.379 & 2.197 & 2.197 & 2.197 \\
\hline 94.471 & 2.197 & 17.576 & 10.985 \\
\hline 2.197 & 4.394 & 68.107 & 8.788 \\
\hline 19.773 & 2.197 & 2.197 & 54.925 \\
\hline 2.197 & 8.788 & 65.91 & 4.394 \\
\hline 13.182 & 24.167 & 2.197 & 57.122 \\
\hline 2.197 & 79.092 & 13.182 & 26.364 \\
\hline 6.591 & 81.289 & 2.197 & 2.197 \\
\hline 35.152 & 2.197 & 2.197 & 39.546 \\
\hline 4.394 & 2.197 & 2.197 & 2.197 \\
\hline 2.197 & 2.197 & 2.197 & 107.653 \\
\hline 24.167 & 2.197 & 2.197 & 2.197 \\
\hline 85.683 & 24.167 & 2.197 & 4.394 \\
\hline 26.364 & 19.773 & 2.197 & 2.197 \\
\hline 2.197 & 6.591 & 13.182 & 2.197 \\
\hline 30.758 & 2.197 & 15.379 & 8.788 \\
\hline 6.591 & 4.394 & 2.197 & 4.394 \\
\hline 10.985 & 2.197 & 19.773 & 6.591 \\
\hline 2.197 & 2.197 & 19.773 & 2.197 \\
\hline 17.576 & 4.394 & 2.197 & 4.394 \\
\hline 28.561 & 2.197 & 15.379 & 21.97 \\
\hline 10.985 & 4.394 & 6.591 & 76.895 \\
\hline 13.182 & 17.576 & 28.561 & 79.092 \\
\hline 24.167 & 70.304 & 2.197 & 8.788 \\
\hline 13.182 & 101.062 & 2.197 & 4.394 \\
\hline 2.197 & 2.197 & 2.197 & 2.197 \\
\hline 4.394 & 83.486 & 2.197 & 94.471 \\
\hline 180.154 & 35.152 & 2.197 & 30.758 \\
\hline 4.394 & 57.122 & 2.197 & 76.895 \\
\hline 2.197 & 94.471 & 41.743 & 26.364 \\
\hline
\end{tabular}




\begin{tabular}{|c|c|c|c|}
\hline 1067.74 & 2.197 & 2.197 & 254.852 \\
\hline 46.137 & 2.197 & 2.197 & 2.197 \\
\hline 2.197 & 21.97 & 4.394 & 4.394 \\
\hline 2.197 & 17.576 & 2.197 & 8.788 \\
\hline 96.668 & 2.197 & 4.394 & 2.197 \\
\hline 74.698 & 2.197 & 2.197 & 10.985 \\
\hline 2.197 & 2.197 & 4.394 & 2.197 \\
\hline 2.197 & 13.182 & 10.985 & 2.197 \\
\hline 10.985 & 2.197 & 2.197 & 4.394 \\
\hline 2.197 & 2.197 & 4.394 & 6.591 \\
\hline 4.394 & 2.197 & 4.394 & 39.546 \\
\hline 2.197 & 4.394 & 13.182 & 4.394 \\
\hline 17.576 & 4.394 & 2.197 & 70.304 \\
\hline 4.394 & 2.197 & 46.137 & 2.197 \\
\hline 28.561 & 8.788 & 50.531 & 68.107 \\
\hline 2.197 & 4.394 & 32.955 & 107.653 \\
\hline 2.197 & 13.182 & 4.394 & 4.394 \\
\hline 59.319 & 2.197 & 6.591 & 4.394 \\
\hline 52.728 & 61.516 & 10.985 & 13.182 \\
\hline 35.152 & 2.197 & 8.788 & 2.197 \\
\hline 8.788 & 10.985 & 2.197 & 6.591 \\
\hline 10.985 & 2.197 & 19.773 & 61.516 \\
\hline 4.394 & 19.773 & 4.394 & 26.364 \\
\hline 17.576 & 2.197 & 160.381 & 72.501 \\
\hline 10.985 & 8.788 & 26.364 & 428.415 \\
\hline 2.197 & 4.394 & 52.728 & 2.197 \\
\hline 10.985 & 63.713 & 2.197 & 15.379 \\
\hline 2.197 & 2.197 & 2.197 & 8.788 \\
\hline 2.197 & 21.97 & 6.591 & 437.203 \\
\hline 4.394 & 2.197 & 10.985 & 13.182 \\
\hline 2.197 & 2.197 & 2.197 & 2.197 \\
\hline 2.197 & 1278.65 & 43.94 & 2.197 \\
\hline 2.197 & 4.394 & 2.197 & 6.591 \\
\hline 2.197 & 2.197 & 15.379 & 8.788 \\
\hline 8.788 & 784.329 & 15.379 & 2.197 \\
\hline 8.788 & 123.032 & 424.021 & 2.197 \\
\hline 617.357 & 6.591 & 2.197 & 13.182 \\
\hline 52.728 & 2.197 & 2.197 & 1465.4 \\
\hline 15.379 & 2.197 & 4.394 & 46.137 \\
\hline 2.197 & 6.591 & 2.197 & 158.184 \\
\hline 2.197 & 8.788 & 6.591 & 24.167 \\
\hline 6.591 & 4.394 & 17.576 & 2.197 \\
\hline 4.394 & 28.561 & 2.197 & 32.955 \\
\hline 21.97 & 153.79 & 186.745 & 2.197 \\
\hline 26.364 & 35.152 & 1210.55 & 17.576 \\
\hline 41.743 & 4.394 & 61.516 & 4.394 \\
\hline 2.197 & 2.197 & 2.197 & 4.394 \\
\hline 10.985 & 4.394 & 2.197 & 39.546 \\
\hline 186.745 & 2.197 & 2.197 & 10.985 \\
\hline 2.197 & 4.394 & 109.85 & 2.197 \\
\hline
\end{tabular}




\begin{tabular}{|c|c|c|c|}
\hline 13.182 & 13.182 & 109.85 & 8.788 \\
\hline 138.411 & 15.379 & 2.197 & 2.197 \\
\hline 2.197 & 4.394 & 15.379 & 13.182 \\
\hline 28.561 & 2.197 & 2.197 & 46.137 \\
\hline 10.985 & 2.197 & 4.394 & 35.152 \\
\hline 2.197 & 2.197 & 63.713 & 2.197 \\
\hline 2.197 & 2.197 & 2.197 & 4.394 \\
\hline 2.197 & 15.379 & 4.394 & 13.182 \\
\hline 21.97 & 15.379 & 2.197 & 2.197 \\
\hline 2.197 & 8.788 & 15.379 & 32.955 \\
\hline 4.394 & 6.591 & 2.197 & 21.97 \\
\hline 4.394 & 4.394 & 2.197 & 13.182 \\
\hline 4.394 & 4.394 & 2.197 & 13.182 \\
\hline 24.167 & 85.683 & 4.394 & 8.788 \\
\hline 4.394 & 13.182 & 10.985 & 41.743 \\
\hline 4.394 & 70.304 & 4.394 & 19.773 \\
\hline 17.576 & 15.379 & 298.792 & 61.516 \\
\hline 21.97 & 17.576 & 2.197 & 24.167 \\
\hline 15.379 & 4.394 & 2.197 & 1274.26 \\
\hline 28.561 & 48.334 & 101.062 & 61.516 \\
\hline 2.197 & 13.182 & 85.683 & 2.197 \\
\hline 26.364 & 2.197 & 2.197 & 2.197 \\
\hline 4.394 & 8.788 & 10.985 & 2.197 \\
\hline 105.456 & 140.608 & 17.576 & 57.122 \\
\hline 8.788 & 4.394 & 6.591 & 4.394 \\
\hline 52.728 & 6.591 & 4.394 & 2.197 \\
\hline 35.152 & 2.197 & 2.197 & 373.49 \\
\hline 2.197 & 8.788 & 2.197 & 28.561 \\
\hline 2.197 & 318.565 & 6.591 & 4.394 \\
\hline 188.942 & 751.374 & 10.985 & 19.773 \\
\hline 61.516 & 24.167 & 4.394 & 2.197 \\
\hline 57.122 & 10.985 & 300.989 & 2.197 \\
\hline 946.907 & 2.197 & 107.653 & 2.197 \\
\hline 35.152 & 145.002 & 6.591 & 41.743 \\
\hline 101.062 & 2.197 & 6.591 & 164.775 \\
\hline 2.197 & 2.197 & 2.197 & 6.591 \\
\hline 26.364 & 2.197 & 24.167 & 52.728 \\
\hline 13.182 & 2.197 & 4.394 & 8.788 \\
\hline 17.576 & 26.364 & 2.197 & 48.334 \\
\hline 2.197 & 32.955 & 17.576 & 940.316 \\
\hline 26.364 & 41.743 & 48.334 & 281.216 \\
\hline 2.197 & 17.576 & 2.197 & 2.197 \\
\hline 4.394 & 17.576 & 2.197 & 166.972 \\
\hline 4.394 & 24.167 & 2.197 & 2.197 \\
\hline 10.985 & 6.591 & 21.97 & 48.334 \\
\hline 2.197 & 4.394 & 8.788 & 6.591 \\
\hline 24.167 & 2.197 & 2.197 & 26.364 \\
\hline 8.788 & 2.197 & 32.955 & 13.182 \\
\hline 2.197 & 28.561 & 17.576 & 263.64 \\
\hline 2.197 & 2.197 & 430.612 & 98.865 \\
\hline
\end{tabular}




\begin{tabular}{|c|c|c|c|}
\hline 2.197 & 4.394 & 191.139 & 10.985 \\
\hline 142.805 & 2.197 & 19.773 & 48.334 \\
\hline 2.197 & 13.182 & 4.394 & 2.197 \\
\hline 2.197 & 4.394 & 2.197 & 24.167 \\
\hline 13.182 & 4.394 & 17.576 & 4.394 \\
\hline 32.955 & 15.379 & 2.197 & 10.985 \\
\hline 35.152 & 24.167 & 4.394 & 2.197 \\
\hline 76.895 & 2.197 & 8.788 & 46.137 \\
\hline 2.197 & 2.197 & 8.788 & 4.39 \\
\hline 30.758 & 4.394 & 13.182 & 6.591 \\
\hline 39.546 & 4.394 & 134.017 & 2.197 \\
\hline 496.522 & 8.788 & 8.788 & $404.24 \varepsilon$ \\
\hline 6.591 & 15.379 & 2.197 & 246.064 \\
\hline 15.379 & 109.85 & 10.985 & 19.773 \\
\hline 441.597 & 8.788 & 13.182 & 2.197 \\
\hline 24.167 & 4.394 & 21.97 & 65.91 \\
\hline 812.89 & 6.591 & 2.197 & 182.351 \\
\hline 17.576 & 2.197 & 15.379 & 54.92 \\
\hline 8.788 & 105.456 & 2.197 & 21.97 \\
\hline 2.197 & 2.197 & 2.197 & 138.411 \\
\hline 10.985 & 8.788 & 21.97 & 28.561 \\
\hline 549.25 & 8.788 & 621.751 & 4.394 \\
\hline 226.291 & 8.788 & 4.394 & 2.197 \\
\hline 17.576 & 8.788 & 2.197 & 4.394 \\
\hline 2.197 & 2.197 & 26.364 & 2.197 \\
\hline 21.97 & 2.197 & 2.197 & 48.334 \\
\hline 8.788 & 2.197 & 59.319 & 263.64 \\
\hline 13.182 & 2.197 & 2.197 & 4.39 \\
\hline 6.591 & 2.197 & 118.638 & 219 \\
\hline 26.364 & 4.394 & 10.985 & 13.182 \\
\hline 2.197 & 6.591 & 2.197 & 2.197 \\
\hline 2.197 & 50.531 & 2.197 & 2.19 \\
\hline 2.197 & 2.197 & 17.576 & 120.835 \\
\hline 21.97 & 2.197 & 6.591 & 4.394 \\
\hline 26.364 & 4.394 & 2.197 & 43.5 \\
\hline 2.197 & 2.197 & 112.047 & 26.36 \\
\hline 4.394 & 388.869 & 2.197 & 147.199 \\
\hline 2.197 & 57.122 & 2.197 & 46.137 \\
\hline 48.334 & 259.246 & 2.197 & 17.57 \\
\hline 43.94 & 2.197 & 6.591 & 2.19 \\
\hline 10.985 & 2.197 & 8.788 & 48.334 \\
\hline 4.394 & 2.197 & 149.396 & 4.35 \\
\hline 4.394 & 24.167 & 17.576 & 228.488 \\
\hline 2.197 & 61.516 & 147.199 & 26.36 \\
\hline 4.394 & 4.394 & 2.197 & 2.197 \\
\hline 2.197 & 2.197 & 2.197 & 2.197 \\
\hline 2.197 & 85.683 & 327.353 & 2.197 \\
\hline 2.197 & 6.591 & 2.197 & 4.394 \\
\hline 2.197 & 35.152 & 92.274 & 96.668 \\
\hline 4.394 & 19.773 & 2.197 & 6.591 \\
\hline
\end{tabular}




\begin{tabular}{|c|c|c|c|}
\hline 26.364 & 2.197 & 8.788 & 577.811 \\
\hline 2.197 & 2.197 & 10.985 & 2.197 \\
\hline 2.197 & 4.394 & 17.576 & 30.758 \\
\hline 30.758 & 2.197 & 112.047 & 21.97 \\
\hline 511.901 & 2.197 & 10.985 & 13.182 \\
\hline 87.88 & 10.985 & 85.683 & 6.591 \\
\hline 43.94 & 19.773 & 10.985 & 15.379 \\
\hline 26.364 & 13.182 & 2.197 & 70.304 \\
\hline 2.197 & 6.591 & 2.197 & 199.927 \\
\hline 2.197 & 4.394 & 6.591 & 21.97 \\
\hline 2.197 & 2.197 & 2.197 & 2.197 \\
\hline 15.379 & 4.394 & 6.591 & 57.122 \\
\hline 2.197 & 4.394 & 2.197 & 2.197 \\
\hline 63.713 & 24.167 & 2.197 & 8.788 \\
\hline 2.197 & 10.985 & 6.591 & 15.379 \\
\hline 6.591 & 6.591 & 2.197 & 6.591 \\
\hline 10.985 & 4.394 & 4.394 & 4.394 \\
\hline 6.591 & 2.197 & 8.788 & 2.197 \\
\hline 2.197 & 8.788 & 6.591 & 2.197 \\
\hline 61.516 & 4.394 & 10.985 & 193.336 \\
\hline 19.773 & 4.394 & 4.394 & 54.925 \\
\hline 21.97 & 10.985 & 8.788 & 107.653 \\
\hline 19.773 & 8.788 & 15.379 & 8.788 \\
\hline 107.653 & 4.394 & 8.788 & 2.197 \\
\hline 13.182 & 4.394 & 103.259 & 17.576 \\
\hline 2.197 & 109.85 & 35.152 & 2.197 \\
\hline 2.197 & 228.488 & 2.197 & 70.304 \\
\hline 13.182 & 1228.12 & 364.702 & 4.394 \\
\hline 8.788 & 46.137 & 2.197 & 145.002 \\
\hline 8.788 & 4.394 & 8.788 & 6.591 \\
\hline 68.107 & 6.591 & 28.561 & 94.471 \\
\hline 2.197 & 74.698 & 340.535 & 65.91 \\
\hline 4.394 & 116.441 & 35.152 & 21.97 \\
\hline 8.788 & 6.591 & 24.167 & 114.244 \\
\hline 195.533 & 6.591 & 28.561 & 3058.22 \\
\hline 8.788 & 8.788 & 6.591 & 6.591 \\
\hline 2.197 & 4.394 & 118.638 & 61.516 \\
\hline 6.591 & 43.94 & 8.788 & 72.501 \\
\hline 171.366 & 21.97 & 76.895 & 15.379 \\
\hline 955.695 & 145.002 & 2.197 & 8.788 \\
\hline 57.122 & 2.197 & 48.334 & 4.394 \\
\hline 2.197 & 32.955 & 2.197 & 15.379 \\
\hline 6.591 & 6.591 & 52.728 & 4.394 \\
\hline 6.591 & 8.788 & 4.394 & 10.985 \\
\hline 30.758 & 116.441 & 2.197 & 19.773 \\
\hline 17.576 & 2.197 & 19.773 & 663.494 \\
\hline 54.925 & 107.653 & 50.531 & 4.394 \\
\hline 6.591 & 13.182 & 2.197 & 4.394 \\
\hline 186.745 & 6.591 & 2.197 & 41.743 \\
\hline 2.197 & 2.197 & 2.197 & 10.985 \\
\hline
\end{tabular}




\begin{tabular}{|c|c|c|c|}
\hline 13.182 & 28.561 & 2.197 & 8.788 \\
\hline 13.182 & 4.394 & 41.743 & 48.334 \\
\hline 8.788 & 41.743 & 2.197 & 15.379 \\
\hline 2.197 & 8.788 & 10.985 & 4.394 \\
\hline 85.683 & 2.197 & 2.197 & 54.925 \\
\hline 2.197 & 4.394 & 590.993 & 24.167 \\
\hline 2.197 & 6.591 & 2.197 & 21.97 \\
\hline 21.97 & 2.197 & 10.985 & 2.197 \\
\hline 4.394 & 4.394 & 4.394 & 4.394 \\
\hline 2.197 & 4.394 & 2.197 & 28.561 \\
\hline 10960.8 & 4.394 & 2.197 & 13.182 \\
\hline 26.364 & 10.985 & 26.364 & 17.576 \\
\hline 26.364 & 6.591 & 17.576 & 2.197 \\
\hline 8.788 & 2.197 & 21.97 & 17.576 \\
\hline 48.334 & 13.182 & 19.773 & 487.734 \\
\hline 13.182 & 2.197 & 35.152 & 298.792 \\
\hline 228.488 & 4.394 & 4.394 & 65.91 \\
\hline 8.788 & 13.182 & 2.197 & 10.985 \\
\hline 2.197 & 127.426 & 2.197 & 4.394 \\
\hline 8.788 & 85.683 & 98.865 & 15.379 \\
\hline 13.182 & 46.137 & 2.197 & 4.394 \\
\hline 114.244 & 285.61 & 2.197 & 19.773 \\
\hline 37.349 & 2.197 & 2.197 & 54.925 \\
\hline 10.985 & 46.137 & 4.394 & 129.623 \\
\hline 72.501 & 4.394 & 43.94 & 2.197 \\
\hline 2.197 & 6.591 & 426.218 & 101.062 \\
\hline 2.197 & 2.197 & 2.197 & 54.925 \\
\hline 8680.35 & 28.561 & 188.942 & 2.197 \\
\hline 6.591 & 90.077 & 2.197 & 2.197 \\
\hline 4.394 & 2.197 & 6.591 & 65.91 \\
\hline 197.73 & 2.197 & 4.394 & 79.092 \\
\hline 10.985 & 8.788 & 6.591 & 4.394 \\
\hline 2.197 & 4.394 & 17.576 & 2.197 \\
\hline 2.197 & 10.985 & 153.79 & 15.379 \\
\hline 4.394 & 2.197 & 10.985 & 13.182 \\
\hline 8.788 & 2.197 & 2.197 & 10.985 \\
\hline 8.788 & 17.576 & 2.197 & 2.197 \\
\hline 13.182 & 4.394 & 30.758 & 24.167 \\
\hline 1151.23 & 4.394 & 94.471 & 43.94 \\
\hline 48.334 & 2.197 & 4.394 & 19.773 \\
\hline 2.197 & 24.167 & 2.197 & 8.788 \\
\hline 17.576 & 6.591 & 4.394 & 41.743 \\
\hline 8.788 & 4.394 & 2.197 & 103.259 \\
\hline 8.788 & 2.197 & 26.364 & 2.197 \\
\hline 90.077 & 2.197 & 6.591 & 46.137 \\
\hline 4.394 & 2.197 & 2.197 & 2.197 \\
\hline 1034.79 & 2.197 & 13.182 & 4.394 \\
\hline 2.197 & 2.197 & 41.743 & 26.364 \\
\hline 10.985 & 6.591 & 2.197 & 4.394 \\
\hline 2.197 & 2.197 & 2.197 & 10.985 \\
\hline
\end{tabular}




\begin{tabular}{|c|c|c|c|}
\hline 2.197 & 6.591 & 2.197 & 70.304 \\
\hline 2.197 & 6.591 & 4.394 & 2.197 \\
\hline 6.591 & 2.197 & 41.743 & 151.593 \\
\hline 2.197 & 2.197 & 2.197 & 147.199 \\
\hline 718.419 & 2.197 & 24.167 & 54.925 \\
\hline 4.394 & 2.197 & 2.197 & 87.88 \\
\hline 2.197 & 19.773 & 70.304 & 8.788 \\
\hline 2.197 & 15.379 & 19.773 & 21.97 \\
\hline 2.197 & 4.394 & 8.788 & 2.197 \\
\hline 8.788 & 8.788 & 65.91 & 10.985 \\
\hline 2.197 & 48.334 & 41.743 & 3135.12 \\
\hline 2.197 & 10.985 & 2.197 & 72.501 \\
\hline 4.394 & 2.197 & 4.394 & 2.197 \\
\hline 32.955 & 15.379 & 2.197 & 79.092 \\
\hline 6.591 & 6.591 & 2.197 & 2.197 \\
\hline 2.197 & 4.394 & 2.197 & 2.197 \\
\hline 8.788 & 2.197 & 24.167 & 2.197 \\
\hline 2.197 & 65.91 & 2.197 & 4.394 \\
\hline 21.97 & 46.137 & 21.97 & 268.034 \\
\hline 15.379 & 10.985 & 4.394 & 46.137 \\
\hline 305.383 & 2.197 & 6.591 & 2.197 \\
\hline 2.197 & 2.197 & 76.895 & 2.197 \\
\hline 2.197 & 8.788 & 2.197 & 180.154 \\
\hline 149.396 & 10.985 & 2.197 & 129.623 \\
\hline 221.897 & 2.197 & 8.788 & 19.773 \\
\hline 2.197 & 46.137 & 4.394 & 8.788 \\
\hline 2.197 & 48.334 & 21.97 & 173.563 \\
\hline 13.182 & 235.079 & 57.122 & 2.197 \\
\hline 98.865 & 2.197 & 37.349 & 30.758 \\
\hline 96.668 & 4.394 & 6.591 & 30.758 \\
\hline 96.668 & 2.197 & 6.591 & 2.197 \\
\hline 48.334 & 2.197 & 6.591 & 85.683 \\
\hline 46.137 & 4.394 & 2.197 & 153.79 \\
\hline 2.197 & 6.591 & 2.197 & 8.788 \\
\hline 6.591 & 46.137 & 4.394 & 322.959 \\
\hline 6.591 & 4.394 & 2.197 & 8.788 \\
\hline 26.364 & 4.394 & 2.197 & 15.379 \\
\hline 8.788 & 8.788 & 2.197 & 2.197 \\
\hline 4.394 & 127.426 & 21.97 & 140.608 \\
\hline 2.197 & 2.197 & 4.394 & 566.826 \\
\hline 2.197 & 2.197 & 74.698 & 283.413 \\
\hline 2.197 & 2.197 & 2.197 & 26.364 \\
\hline 2.197 & 4.394 & 2.197 & 307.58 \\
\hline 2.197 & 2.197 & 2.197 & 252.655 \\
\hline 2.197 & 2.197 & 6.591 & 30.758 \\
\hline 8.788 & 39.546 & 2.197 & 2.197 \\
\hline 4.394 & 21.97 & 841.451 & 2.197 \\
\hline 112.047 & 2.197 & 2.197 & 6.591 \\
\hline 6.591 & 4.394 & 24.167 & 6.591 \\
\hline 4.394 & 28.561 & 2.197 & 127.426 \\
\hline
\end{tabular}




\begin{tabular}{|c|c|c|c|}
\hline 2.197 & 39.546 & 4.394 & 26.364 \\
\hline 2.197 & 2.197 & 149.396 & 2.197 \\
\hline 2.197 & 52.728 & 8.788 & 496.522 \\
\hline 6.591 & 450.385 & 2.197 & 50.531 \\
\hline 24.167 & 2.197 & 2.197 & 2.197 \\
\hline 195.533 & 13.182 & 2.197 & 54.925 \\
\hline 2.197 & 70.304 & 2.197 & 4.394 \\
\hline 17.576 & 32.955 & 2.197 & 263.64 \\
\hline 6.591 & 92.274 & 24.167 & 32.955 \\
\hline 2.197 & 15.379 & 2.197 & 6.591 \\
\hline 4.394 & 2.197 & 2.197 & 61.516 \\
\hline 2.197 & 2.197 & 2.197 & 37.349 \\
\hline 4.394 & 6.591 & 13.182 & 63.713 \\
\hline 4.394 & 2.197 & 2.197 & 79.092 \\
\hline 120.835 & 2.197 & 79.092 & 41.743 \\
\hline 445.991 & 8.788 & 2.197 & 2.197 \\
\hline 2.197 & 2.197 & 2.197 & 2.197 \\
\hline 37.349 & 4.394 & 6.591 & 2.197 \\
\hline 15.379 & 101.062 & 2.197 & 4.394 \\
\hline 2.197 & 2.197 & 2.197 & 109.85 \\
\hline 338.338 & 2.197 & 6.591 & 87.88 \\
\hline 19.773 & 6.591 & 2.197 & 2.197 \\
\hline 8.788 & 2.197 & 2.197 & 10.985 \\
\hline 2.197 & 8.788 & 8.788 & 1898.21 \\
\hline 10.985 & 2.197 & 28.561 & 17.576 \\
\hline 63.713 & 2.197 & 46.137 & 2.197 \\
\hline 2.197 & 4.394 & 2.197 & 2.197 \\
\hline 2.197 & 4.394 & 6.591 & 2.197 \\
\hline 26.364 & 4.394 & 2.197 & 2.197 \\
\hline 28.561 & 362.505 & 263.64 & 13.182 \\
\hline 2.197 & 566.826 & 2.197 & 10.985 \\
\hline 10.985 & 2.197 & 4.394 & 92.274 \\
\hline 105.456 & 2.197 & 65.91 & 43.94 \\
\hline 4.394 & 4.394 & 661.297 & 6.591 \\
\hline 54.925 & 21.97 & 21.97 & 21.97 \\
\hline 4.394 & 2.197 & 2.197 & 105.456 \\
\hline 2.197 & 74.698 & 24.167 & 4.394 \\
\hline 10.985 & 4.394 & 2.197 & 8.788 \\
\hline 8.788 & 8.788 & 10.985 & 2.197 \\
\hline 57.122 & 155.987 & 46.137 & 4.394 \\
\hline 2.197 & 43.94 & 70.304 & 48.334 \\
\hline 59.319 & 134.017 & 4.394 & 15.379 \\
\hline 6.591 & 2.197 & 149.396 & 206.518 \\
\hline 303.186 & 2.197 & 140.608 & 4.394 \\
\hline 85.683 & 2.197 & 230.685 & 14423.3 \\
\hline 19.773 & 2.197 & 8.788 & 6.591 \\
\hline 4.394 & 50.531 & 4.394 & 46.137 \\
\hline 4.394 & 2.197 & 151.593 & 2.197 \\
\hline 177.957 & 4.394 & 2.197 & 19.773 \\
\hline 4.394 & 17.576 & 2.197 & 2.197 \\
\hline
\end{tabular}




\begin{tabular}{|c|c|c|c|}
\hline 15.379 & 2.197 & 2.197 & 2.197 \\
\hline 6.591 & 6.591 & 4.394 & 2.197 \\
\hline 39.546 & 15.379 & 4.394 & 87.88 \\
\hline 21.97 & 2.197 & 2.197 & 4.394 \\
\hline 2.197 & 15.379 & 180.154 & 2.197 \\
\hline 4.394 & 2.197 & 2.197 & 2.197 \\
\hline 2.197 & 2.197 & 4.394 & 2.197 \\
\hline 6.591 & 13.182 & 6.591 & 15.379 \\
\hline 54.925 & 4.394 & 2.197 & 24.167 \\
\hline 2.197 & 2.197 & 2.197 & 35.152 \\
\hline 2.197 & 4.394 & 2.197 & 10.985 \\
\hline 2.197 & 6.591 & 19.773 & 19.773 \\
\hline 2.197 & 2.197 & 2.197 & 15.379 \\
\hline 24.167 & 4.394 & 2.197 & 2.197 \\
\hline 2.197 & 72.501 & 2.197 & 68.107 \\
\hline 2.197 & 2.197 & 24.167 & 4.394 \\
\hline 2.197 & 43.94 & 2.197 & 10.985 \\
\hline 2.197 & 10.985 & 2.197 & 24.167 \\
\hline 2.197 & 2.197 & 2.197 & 35.152 \\
\hline 4.394 & 2.197 & 4.394 & 85.683 \\
\hline 74.698 & 10.985 & 2.197 & 57.122 \\
\hline 28.561 & 19.773 & 4.394 & 17.576 \\
\hline 4.394 & 4.394 & 2.197 & 17.576 \\
\hline 10.985 & 2.197 & 2.197 & 678.873 \\
\hline 2.197 & 4.394 & 2.197 & 13.182 \\
\hline 6.591 & 2.197 & 2.197 & 37.349 \\
\hline 28.561 & 2.197 & 2.197 & 10.985 \\
\hline 13.182 & 2.197 & 43.94 & 2.197 \\
\hline 131.82 & 4.394 & 13.182 & 250.458 \\
\hline 35.152 & 8.788 & 15.379 & 2.197 \\
\hline 10.985 & 2.197 & 2.197 & 8.788 \\
\hline 30.758 & 2.197 & 4.394 & 54.925 \\
\hline 2.197 & 2.197 & 2.197 & 3939.22 \\
\hline 17.576 & 287.807 & 21.97 & 46.137 \\
\hline 2.197 & 61.516 & 8.788 & 4.394 \\
\hline 336.141 & 854.633 & 2.197 & 43.94 \\
\hline 2.197 & 6.591 & 2.197 & 2.197 \\
\hline 4.394 & 2.197 & 2.197 & 2.197 \\
\hline 8.788 & 15.379 & 4.394 & 2.197 \\
\hline 739251 & 213.109 & 327.353 & 61.516 \\
\hline 39.546 & 50.531 & 4.394 & 28.561 \\
\hline 46.137 & 2.197 & 4.394 & 81.289 \\
\hline 24.167 & 2.197 & 10.985 & 10.985 \\
\hline 4.394 & 4.394 & 2.197 & 2.197 \\
\hline 37.349 & 2.197 & 15.379 & 17.576 \\
\hline 13.182 & 30.758 & 4.394 & 230.685 \\
\hline 6.591 & 28.561 & 2.197 & 13.182 \\
\hline 2.197 & 26.364 & 138.411 & 129.623 \\
\hline 2.197 & 4.394 & 2.197 & 52.728 \\
\hline 2.197 & 2.197 & 2.197 & 4.394 \\
\hline
\end{tabular}




\begin{tabular}{|c|c|c|c|}
\hline 21.97 & 2.197 & 10.985 & 32.955 \\
\hline 46.137 & 10.985 & 2.197 & 6.591 \\
\hline 10.985 & 2.197 & 10.985 & 2.197 \\
\hline 2.197 & 2.197 & 13.182 & 30.758 \\
\hline 2.197 & 2.197 & 72.501 & 2.197 \\
\hline 125.229 & 35.152 & 46.137 & 459.173 \\
\hline 32.955 & 2.197 & 2.197 & 285.61 \\
\hline 2.197 & 35.152 & 4.394 & 2.197 \\
\hline 2.197 & 2.197 & 35.152 & 57.122 \\
\hline 2.197 & 2.197 & 116.441 & 24.167 \\
\hline 142.805 & 2.197 & 2.197 & 94.471 \\
\hline 2.197 & 2.197 & 2.197 & 2.197 \\
\hline 527.28 & 2.197 & 2.197 & 4.394 \\
\hline 2.197 & 8.788 & 2.197 & 2.197 \\
\hline 2.197 & 2.197 & 2.197 & 2.197 \\
\hline 2.197 & 2.197 & 2.197 & 13.182 \\
\hline 2.197 & 8.788 & 8.788 & 8.788 \\
\hline 13.182 & 397.657 & 2.197 & 134.017 \\
\hline 2.197 & 13.182 & 6.591 & 2.197 \\
\hline 6.591 & 4.394 & 4.394 & 4.394 \\
\hline 30.758 & 2.197 & 6.591 & 4.394 \\
\hline 2.197 & 2.197 & 4.394 & 4.394 \\
\hline 2.197 & 2.197 & 4.394 & 74.698 \\
\hline 2.197 & 17.576 & 6.591 & 79.092 \\
\hline 10.985 & 2.197 & 10.985 & 2.197 \\
\hline 2.197 & 21.97 & 6.591 & 2.197 \\
\hline 1775.18 & 2.197 & 8.788 & 4.394 \\
\hline 4.394 & 48.334 & 2.197 & 79.092 \\
\hline 43.94 & 2.197 & 2.197 & 19.773 \\
\hline 15.379 & 17.576 & 46.137 & 70.304 \\
\hline 2.197 & 10.985 & 4.394 & 26.364 \\
\hline 1678.51 & 6.591 & 2.197 & $3.40 \mathrm{E}+06$ \\
\hline 10.985 & 4.394 & 2.197 & 15.379 \\
\hline 10.985 & 15.379 & 50.531 & 184.548 \\
\hline 2.197 & 421.824 & 10.985 & 4.394 \\
\hline 2.197 & 6.591 & 26.364 & 2.197 \\
\hline 6.591 & 74.698 & 2.197 & 2.197 \\
\hline 2.197 & 6.591 & 2.197 & 2.197 \\
\hline 21.97 & 13.182 & 59.319 & 4.394 \\
\hline 158.184 & 94.471 & 70.304 & 127.426 \\
\hline 4.394 & 43.94 & 28.561 & 37.349 \\
\hline 2.197 & 24.167 & 37.349 & 2.197 \\
\hline 8.788 & 177.957 & 199.927 & 120.835 \\
\hline 136.214 & 59.319 & 2.197 & 15.379 \\
\hline 4.394 & 2.197 & 4.394 & 8381.55 \\
\hline 2.197 & 8.788 & 4.394 & 2.197 \\
\hline 2.197 & 103.259 & 65.91 & 10.985 \\
\hline 2.197 & 2.197 & 2.197 & 2.197 \\
\hline 107.653 & 41.743 & 2.197 & 2.197 \\
\hline 21.97 & 72.501 & 2.197 & 8.788 \\
\hline
\end{tabular}




\begin{tabular}{|c|c|c|c|}
\hline 4.394 & 2.197 & 13.182 & 83.486 \\
\hline 4.394 & 4.394 & 4.394 & 4.394 \\
\hline 4.394 & 13.182 & 2.197 & 41.743 \\
\hline 951.301 & 4.394 & 2.197 & 10.985 \\
\hline 171.366 & 4.394 & 2.197 & 10.985 \\
\hline 2.197 & 2.197 & 2.197 & 32.955 \\
\hline 50.531 & 4.394 & 8.788 & 79.092 \\
\hline 1045.77 & 63.713 & 2.197 & 125.229 \\
\hline 2.197 & 26.364 & 8.788 & 2.197 \\
\hline 6.591 & 13.182 & 6.591 & 2.197 \\
\hline 48.334 & 2.197 & 2.197 & 112.047 \\
\hline 2.197 & 2.197 & 4.394 & 2.197 \\
\hline 2.197 & 4.394 & 10.985 & 103.259 \\
\hline 65.91 & 116.441 & 2.197 & 10.985 \\
\hline 32.955 & 4.394 & 92.274 & 131.82 \\
\hline 10.985 & 21.97 & 83.486 & 10.985 \\
\hline 4.394 & 2.197 & 103.259 & 193.336 \\
\hline 2.197 & 98.865 & 8.788 & 79.092 \\
\hline 8.788 & 30.758 & 50.531 & 395.46 \\
\hline 54.925 & 24.167 & 26.364 & 366.899 \\
\hline 57.122 & 46.137 & 19.773 & 114.244 \\
\hline 85.683 & 68.107 & 15.379 & 8.788 \\
\hline 2.197 & 4.394 & 158.184 & 2.197 \\
\hline 4.394 & 15.379 & 2.197 & 52.728 \\
\hline 2.197 & 6.591 & 2.197 & 4.394 \\
\hline 4.394 & 21.97 & 2.197 & 2.197 \\
\hline 6.591 & 6.591 & 4.394 & 19.773 \\
\hline 10.985 & 2.197 & 2.197 & 2.197 \\
\hline 129.623 & 2.197 & 4.394 & 2.197 \\
\hline 241.67 & 4.394 & 863.421 & 6.591 \\
\hline 4.394 & 4.394 & 17.576 & 21.97 \\
\hline 2.197 & 2.197 & 2.197 & 17.576 \\
\hline 13.182 & 28.561 & 4.394 & 6.591 \\
\hline 10.985 & 2.197 & 4.394 & 21.97 \\
\hline 10.985 & 2.197 & 360.308 & 107.653 \\
\hline 8.788 & 4.394 & 166.972 & 26.364 \\
\hline 2.197 & 8.788 & 2.197 & 2.197 \\
\hline 74.698 & 2.197 & 2.197 & 2.197 \\
\hline 35.152 & 10.985 & 2.197 & 26.364 \\
\hline 373.49 & 6.591 & 4.394 & 13.182 \\
\hline 24.167 & 694.252 & 2.197 & 83.486 \\
\hline 17.576 & 261.443 & 2.197 & 149.396 \\
\hline 13.182 & 6.591 & 2.197 & 28.561 \\
\hline 50.531 & 2.197 & 4.394 & 92.274 \\
\hline 4.394 & 4.394 & 39.546 & 6.591 \\
\hline 54.925 & 2.197 & 6.591 & 105.456 \\
\hline 19.773 & 46.137 & 2.197 & 15.379 \\
\hline 96.668 & 6.591 & 26.364 & 70.304 \\
\hline 2.197 & 4.394 & 4.394 & 2.197 \\
\hline 186.745 & 8.788 & 30.758 & 373.49 \\
\hline
\end{tabular}




\begin{tabular}{|c|c|c|c|}
\hline 703.04 & 15.379 & 10.985 & 8.788 \\
\hline 8.788 & 6.591 & 2.197 & 6.591 \\
\hline 50.531 & 2.197 & 17.576 & 4.394 \\
\hline 206.518 & 2.197 & 210.912 & 2.197 \\
\hline 4.394 & 125.229 & 2.197 & 257.049 \\
\hline 8.788 & 4.394 & 2.197 & 10.985 \\
\hline 2.197 & 2.197 & 6.591 & 4.394 \\
\hline 2.197 & 2.197 & 8.788 & 76.895 \\
\hline 19.773 & 8.788 & 2.197 & 41.743 \\
\hline 6.591 & 13.182 & 2.197 & 50.531 \\
\hline 8.788 & 4.394 & 54.925 & 2.197 \\
\hline 43.94 & 8.788 & 2.197 & 24.167 \\
\hline 21.97 & 6.591 & 65.91 & 59.319 \\
\hline 6.591 & 30.758 & 13.182 & 2.197 \\
\hline 2.197 & 4.394 & 24.167 & 2.197 \\
\hline 2.197 & 8.788 & 24.167 & 10.985 \\
\hline 2.197 & 2.197 & 4.394 & 57.122 \\
\hline 8.788 & 2.197 & 2.197 & 17.576 \\
\hline 8.788 & 2.197 & 2.197 & 32.955 \\
\hline 2.197 & 4.394 & 2.197 & 28.561 \\
\hline 2.197 & 17.576 & 2.197 & 17.576 \\
\hline 2.197 & 195.533 & 2.197 & 10.985 \\
\hline 6.591 & 6.591 & 37.349 & 46.137 \\
\hline 2.197 & 889.785 & 4.394 & 118.638 \\
\hline 4.394 & 4.394 & 8.788 & 10.985 \\
\hline 21.97 & 19.773 & 24.167 & 6.591 \\
\hline 8.788 & 6.591 & 13.182 & 48.334 \\
\hline 4.394 & 2.197 & 149.396 & 37.349 \\
\hline 320.762 & 19.773 & 2.197 & 68.107 \\
\hline 109.85 & 4.394 & 26.364 & 46.137 \\
\hline 85.683 & 21.97 & 83.486 & 2.197 \\
\hline 6.591 & 35.152 & 15.379 & 188.942 \\
\hline 125.229 & 4.394 & 4.394 & 2.197 \\
\hline 4.394 & 6.591 & 2.197 & 511.901 \\
\hline 248.261 & 8.788 & 8.788 & 180.154 \\
\hline 59.319 & 15.379 & 4.394 & 24.167 \\
\hline 4.394 & 15.379 & 19.773 & 6.591 \\
\hline 26.364 & 2.197 & 2.197 & 2.197 \\
\hline 2.197 & 2106.92 & 24.167 & 147.199 \\
\hline 19.773 & 4.394 & 81.289 & 4.394 \\
\hline 4.394 & 4.394 & 13.182 & 4.394 \\
\hline 10.985 & 2.197 & 180.154 & 8.788 \\
\hline 6.591 & 17.576 & 8.788 & 10.985 \\
\hline 125.229 & 2.197 & 6.591 & 2.197 \\
\hline 2.197 & 2.197 & 15.379 & 700.843 \\
\hline 2.197 & 2.197 & 13.182 & 8.788 \\
\hline 2.197 & 2.197 & 8.788 & 4.394 \\
\hline 2.197 & 2.197 & 2.197 & 98.865 \\
\hline 2.197 & 4.394 & 35.152 & 101.062 \\
\hline 43.94 & 2.197 & 2.197 & 74.698 \\
\hline
\end{tabular}




\begin{tabular}{|c|c|c|c|}
\hline 4.394 & 30.758 & 2.197 & 4.394 \\
\hline 8.788 & 2.197 & 160.381 & 41.743 \\
\hline 2.197 & 2.197 & 2.197 & 10.985 \\
\hline 4.394 & 2.197 & 37.349 & 2.197 \\
\hline 6.591 & 2.197 & 2.197 & 10.985 \\
\hline 13.182 & 195.533 & 2.197 & 151.593 \\
\hline 21.97 & 2.197 & 26.364 & 21.97 \\
\hline 10.985 & 351.52 & 435.006 & 2.197 \\
\hline 19.773 & 19.773 & 10.985 & 32.955 \\
\hline 8.788 & 72.501 & 6.591 & 13.182 \\
\hline 6.591 & 683.267 & 15.379 & 2.197 \\
\hline 2.197 & 30.758 & 2.197 & 2.197 \\
\hline 2.197 & 4.394 & 81.289 & 4.394 \\
\hline 50.531 & 32.955 & 13.182 & 24.167 \\
\hline 2.197 & 41.743 & 4.394 & 2.197 \\
\hline 6.591 & 8.788 & 6.591 & 2.197 \\
\hline 2.197 & 2.197 & 24.167 & 2.197 \\
\hline 2.197 & 2.197 & 2.197 & 39.546 \\
\hline 15.379 & 4.394 & 21.97 & 6.591 \\
\hline 2.197 & 219.7 & 487.734 & 4.394 \\
\hline 94.471 & 48.334 & 4.394 & 112.047 \\
\hline 21.97 & 138.411 & 2.197 & 4.394 \\
\hline 8.788 & 333.944 & 2.197 & 10.985 \\
\hline 8.788 & 2.197 & 2.197 & 2.197 \\
\hline 2.197 & 2.197 & 2.197 & 2.197 \\
\hline 441.597 & 35.152 & 2.197 & 24.167 \\
\hline 2.197 & 2.197 & 2.197 & 8.788 \\
\hline 8.788 & 2.197 & 19.773 & 46.137 \\
\hline 2.197 & 8.788 & 2.197 & 6.591 \\
\hline 2.197 & 2.197 & 6.591 & 41.743 \\
\hline 6.591 & 2.197 & 13.182 & 2.197 \\
\hline 15.379 & 2.197 & 2.197 & 2.197 \\
\hline 4.394 & 2.197 & 65.91 & 8.788 \\
\hline 32.955 & 4.394 & 2.197 & 21.97 \\
\hline 13.182 & 2.197 & 2.197 & 17.576 \\
\hline 28.561 & 2.197 & 43.94 & 4.394 \\
\hline 2.197 & 2.197 & 2.197 & 13.182 \\
\hline 2.197 & 2.197 & 2.197 & 32.955 \\
\hline 10.985 & 2.197 & 1746.61 & 30.758 \\
\hline 4.394 & 2.197 & 621.751 & 2.197 \\
\hline 13.182 & 46.137 & 94.471 & 61.516 \\
\hline 41.743 & 2.197 & 21.97 & 2.197 \\
\hline 46.137 & 2.197 & 6.591 & 202.124 \\
\hline 63.713 & 2.197 & 125.229 & 37.349 \\
\hline 2.197 & 2.197 & 17.576 & 10.985 \\
\hline 4.394 & 72.501 & 6.591 & 79.092 \\
\hline 259.246 & 2.197 & 61.516 & 17.576 \\
\hline 2.197 & 336.141 & 2.197 & 2.197 \\
\hline 2.197 & 4.394 & 4.394 & 4.394 \\
\hline 19.773 & 4.394 & 96.668 & 8.788 \\
\hline
\end{tabular}




\begin{tabular}{|c|c|c|c|}
\hline 2.197 & 2.197 & 2.197 & 72.501 \\
\hline 4.394 & 2.197 & 2.197 & 4.394 \\
\hline 19.773 & 2.197 & 2.197 & 26.364 \\
\hline 17.576 & 2.197 & 35.152 & 72.501 \\
\hline 87.88 & 63.713 & 10.985 & 43.94 \\
\hline 4.394 & 59.319 & 50.531 & 162.578 \\
\hline 21.97 & 6.591 & 8.788 & 8.788 \\
\hline 32.955 & 17.576 & 8.788 & 2.197 \\
\hline 21.97 & 48.334 & 134.017 & 2.197 \\
\hline 15.379 & 19.773 & 2.197 & 4.394 \\
\hline 21.97 & 68.107 & 2.197 & 406.445 \\
\hline 722.813 & 4.394 & 2.197 & 6.591 \\
\hline 103.259 & 90.077 & 2.197 & 52.728 \\
\hline 294.398 & 8.788 & 21.97 & 85.683 \\
\hline 162.578 & 41.743 & 2.197 & 28.561 \\
\hline 2.197 & 169.169 & 48.334 & 107.653 \\
\hline 6.591 & 2.197 & 2.197 & 13.182 \\
\hline 6.591 & 120.835 & 30.758 & 17.576 \\
\hline 4.394 & 8.788 & 2.197 & 2.197 \\
\hline 4.394 & 2.197 & 13.182 & 2.197 \\
\hline 6.591 & 129.623 & 2.197 & 72.501 \\
\hline 2.197 & 30.758 & 83.486 & 2.197 \\
\hline 4.394 & 15.379 & 21.97 & 61.516 \\
\hline 13.182 & 28.561 & 2.197 & 101.062 \\
\hline 13.182 & 2.197 & 2.197 & 329.55 \\
\hline 283.413 & 15.379 & 24.167 & 2.197 \\
\hline 65.91 & 4.394 & 2.197 & 2.197 \\
\hline 2.197 & 2.197 & 1948.74 & 612.963 \\
\hline 2.197 & 2.197 & 4.394 & 98.865 \\
\hline 15.379 & 32.955 & 30.758 & 15.379 \\
\hline 4.394 & 2.197 & 4.394 & 96.668 \\
\hline 4.394 & 2.197 & 4.394 & 250.458 \\
\hline 98.865 & 2.197 & 4.394 & 98.865 \\
\hline 21.97 & 2.197 & 8.788 & 263.64 \\
\hline 4.394 & 13.182 & 6.591 & 32.955 \\
\hline 158.184 & 2.197 & 2.197 & 8.788 \\
\hline 2.197 & 4.394 & 8.788 & 15.379 \\
\hline 15.379 & 6.591 & 19.773 & 32.955 \\
\hline 6.591 & 4.394 & 4.394 & 2.197 \\
\hline 10.985 & 43.94 & 2.197 & 2.197 \\
\hline 254.852 & 17.576 & 2.197 & 21.97 \\
\hline 26.364 & 147.199 & 2.197 & 15.379 \\
\hline 164.775 & 15.379 & 65.91 & 2.197 \\
\hline 90.077 & 2.197 & 2.197 & 155.987 \\
\hline 2.197 & 2.197 & 2.197 & 2.197 \\
\hline 8.788 & 2.197 & 2.197 & 382.278 \\
\hline 39.546 & 2.197 & 120.835 & 15.379 \\
\hline 2.197 & 2.197 & 2.197 & 2.197 \\
\hline 4.394 & 10.985 & 2.197 & 4.394 \\
\hline 628.342 & 391.066 & 2.197 & 4.394 \\
\hline
\end{tabular}




\begin{tabular}{|c|c|c|c|}
\hline 8.788 & 24.167 & 2.197 & 2.197 \\
\hline 2.197 & 4.394 & 2.197 & 2.197 \\
\hline 2.197 & 10.985 & 13.182 & 215.306 \\
\hline 8.788 & 81.289 & 2.197 & 10.985 \\
\hline 6.591 & 10.985 & 2.197 & 105.456 \\
\hline 360.308 & 2.197 & 2.197 & 65.91 \\
\hline 4.394 & 19.773 & 2.197 & 2.197 \\
\hline 153.79 & 2.197 & 2.197 & 151.593 \\
\hline 6.591 & 2.197 & 2.197 & 61.516 \\
\hline 24.167 & 15.379 & 6.591 & 454.779 \\
\hline 15.379 & 391.066 & 2.197 & 13.182 \\
\hline 2.197 & 2.197 & 2.197 & 2.197 \\
\hline 8.788 & 30.758 & 4.394 & 10.985 \\
\hline 155.987 & 136.214 & 158.184 & 30.758 \\
\hline 8.788 & 4.394 & 2.197 & 26.364 \\
\hline 8.788 & 2.197 & 2.197 & 6.591 \\
\hline 4.394 & 2.197 & 2.197 & 472.355 \\
\hline 2.197 & 4.394 & 4.394 & 6.591 \\
\hline 19.773 & 2.197 & 76.895 & 142.805 \\
\hline 2.197 & 2.197 & 2.197 & 2.197 \\
\hline 287.807 & 21.97 & 2.197 & 15.379 \\
\hline 8.788 & 13.182 & 2.197 & 13.182 \\
\hline 13.182 & 4.394 & 252.655 & 19.773 \\
\hline 4.394 & 6.591 & 2.197 & 2.197 \\
\hline 2.197 & 24.167 & 17.576 & 2.197 \\
\hline 10.985 & 2.197 & 98.865 & 2.197 \\
\hline 2.197 & 4.394 & 131.82 & 114.244 \\
\hline 98.865 & 2.197 & 2.197 & 52.728 \\
\hline 155.987 & 2.197 & 26.364 & 10.985 \\
\hline 72.501 & 8.788 & 2.197 & 103.259 \\
\hline 17.576 & 8.788 & 2.197 & 4.394 \\
\hline 2.197 & 4.394 & 2.197 & 2.197 \\
\hline 10.985 & 10.985 & 2.197 & 72.501 \\
\hline 2.197 & 70.304 & 4.394 & 4.394 \\
\hline 65.91 & 39.546 & 2.197 & 6.591 \\
\hline 8.788 & 46.137 & 2.197 & 26.364 \\
\hline 8.788 & 2.197 & 2.197 & 2.197 \\
\hline 8.788 & 8.788 & 287.807 & 116.441 \\
\hline 21.97 & 10.985 & 13.182 & 10.985 \\
\hline 2.197 & 2.197 & 8.788 & 10.985 \\
\hline 6.591 & 2.197 & 2.197 & 19.773 \\
\hline 26.364 & 57.122 & 10.985 & 26.364 \\
\hline 13.182 & 4.394 & 2.197 & 19.773 \\
\hline 6.591 & 17.576 & 4.394 & 13.182 \\
\hline 65.91 & 118.638 & 2.197 & 2.197 \\
\hline 4.394 & 10.985 & 287.807 & 114.244 \\
\hline 2.197 & 612.963 & 182.351 & 32.955 \\
\hline 2.197 & 54.925 & 2.197 & 39.546 \\
\hline 2.197 & 2.197 & 10.985 & 2853.9 \\
\hline 19.773 & 4.394 & 6.591 & 54.925 \\
\hline
\end{tabular}




\begin{tabular}{|c|c|c|c|}
\hline 4.394 & 2.197 & 15.379 & 35.152 \\
\hline 6.591 & 241.67 & 2.197 & 2.197 \\
\hline 8.788 & 4.394 & 714.025 & 6.591 \\
\hline 39.546 & 4.394 & 52.728 & 24.167 \\
\hline 2.197 & 2.197 & 4.394 & 2.197 \\
\hline 2.197 & 2.197 & 15.379 & 10.985 \\
\hline 2.197 & 2.197 & 307.58 & 114.244 \\
\hline 52.728 & 2.197 & 17.576 & 4.394 \\
\hline 15.379 & 13.182 & 2.197 & 4.394 \\
\hline 2.197 & 4.394 & 2.197 & 2.197 \\
\hline 287.807 & 2.197 & 4.394 & 37.349 \\
\hline 10.985 & 15.379 & 241.67 & 2.197 \\
\hline 4.394 & 2.197 & 4.394 & 6.591 \\
\hline 145.002 & 4.394 & 43.94 & 98.865 \\
\hline 35.152 & 8.788 & 2.197 & 2.197 \\
\hline 43.94 & 2.197 & 498.719 & 4.394 \\
\hline 76.895 & 2.197 & 6.591 & 4.394 \\
\hline 13.182 & 24.167 & 940.316 & 738.192 \\
\hline 6.591 & 6.591 & 2.197 & 63.713 \\
\hline 155.987 & 2.197 & 342.732 & 2.197 \\
\hline 4.394 & 15.379 & 2.197 & 2.197 \\
\hline 41.743 & 59.319 & 61.516 & 2.197 \\
\hline 4.394 & 21.97 & 1039.18 & 54.925 \\
\hline 13.182 & 26.364 & 28.561 & 76.895 \\
\hline 19.773 & 13.182 & 2.197 & 26.364 \\
\hline 4.394 & 116.441 & 79.092 & 2.197 \\
\hline 6.591 & 171.366 & 17.576 & 13.182 \\
\hline 555.841 & 87.88 & 13.182 & 158.184 \\
\hline 6.591 & 2.197 & 6.591 & 2.197 \\
\hline 186.745 & 4.394 & 2.197 & 4.394 \\
\hline 8.788 & 6.591 & 4.394 & 46.137 \\
\hline 6.591 & 2.197 & 4.394 & 15.379 \\
\hline 63.713 & 19.773 & 2.197 & 24.167 \\
\hline 26.364 & 96.668 & 2.197 & 41.743 \\
\hline 2.197 & 2.197 & 2.197 & 28.561 \\
\hline 17.576 & 2.197 & 19.773 & 4.394 \\
\hline 2.197 & 15.379 & 2.197 & 83.486 \\
\hline 2.197 & 4.394 & 2.197 & 8.788 \\
\hline 2.197 & 162.578 & 4.394 & 19.773 \\
\hline 8.788 & 2.197 & 2.197 & 2.197 \\
\hline 61.516 & 4.394 & 28.561 & 2.197 \\
\hline 68.107 & 2.197 & 2.197 & 37.349 \\
\hline 2.197 & 2.197 & 2.197 & 248.261 \\
\hline 6.591 & 8.788 & 74.698 & 2.197 \\
\hline 4.394 & 2.197 & 2.197 & 19.773 \\
\hline 2.197 & 2.197 & 2.197 & 2.197 \\
\hline 2.197 & 2.197 & 2.197 & 32.955 \\
\hline 21.97 & 2.197 & 2.197 & 52.728 \\
\hline 270.231 & 4.394 & 2.197 & 15.379 \\
\hline 30.758 & 2.197 & 2.197 & 2.197 \\
\hline
\end{tabular}




\begin{tabular}{|c|c|c|c|}
\hline 2.197 & 4.394 & 2.197 & 349.323 \\
\hline 39.546 & 147.199 & 2.197 & 2.197 \\
\hline 4.394 & 8.788 & 2.197 & 2.197 \\
\hline 2.197 & 6.591 & 8.788 & 10.985 \\
\hline 98.865 & 43.94 & 2.197 & 52.728 \\
\hline 2254.12 & 6.591 & 2.197 & 61.516 \\
\hline 8.788 & 2.197 & 2.197 & 2.197 \\
\hline 15.379 & 2.197 & 2.197 & 2.197 \\
\hline 2.197 & 2.197 & 4.394 & 6.591 \\
\hline 65.91 & 24.167 & 8.788 & 6.591 \\
\hline 70.304 & 21.97 & 2.197 & 17.576 \\
\hline 10.985 & 13.182 & 2.197 & 2.197 \\
\hline 17.576 & 32.955 & 19.773 & 173.563 \\
\hline 28.561 & 2.197 & 2.197 & 2.197 \\
\hline 6.591 & 26.364 & 4.394 & 2.197 \\
\hline 26.364 & 59.319 & 103.259 & 6.591 \\
\hline 6.591 & 41.743 & 2.197 & 894.179 \\
\hline 2.197 & 186.745 & 2.197 & 79.092 \\
\hline 15.379 & 4.394 & 6.591 & 4.394 \\
\hline 2071.77 & 2.197 & 4.394 & 2.197 \\
\hline 160.381 & 70.304 & 2.197 & 2.197 \\
\hline 28.561 & 340.535 & 30.758 & 1063.35 \\
\hline 7485.18 & 2.197 & 2.197 & 2.197 \\
\hline 10.985 & 145.002 & 2.197 & 2.197 \\
\hline 2.197 & 2.197 & 2.197 & 2.197 \\
\hline 6.591 & 17.576 & 39.546 & 290.004 \\
\hline 10.985 & 2.197 & 6.591 & 243.867 \\
\hline 13.182 & 4.394 & 6.591 & 30.758 \\
\hline 10.985 & 4.394 & 2.197 & 94.471 \\
\hline 21.97 & 4.394 & 2.197 & 24.167 \\
\hline 8.788 & 2.197 & 2.197 & 2.197 \\
\hline 2.197 & 2.197 & 2.197 & 17.576 \\
\hline 19.773 & 2.197 & 6.591 & 116.441 \\
\hline 2.197 & 4.394 & 4.394 & 2.197 \\
\hline 6.591 & 4.394 & 8.788 & 28.561 \\
\hline 2.197 & 2.197 & 2.197 & 65.91 \\
\hline 518.492 & 37.349 & 37.349 & 26.364 \\
\hline 74.698 & 6.591 & 2.197 & 24.167 \\
\hline 24.167 & 2.197 & 248.261 & 8.788 \\
\hline 2.197 & 30.758 & 6.591 & 52.728 \\
\hline 28.561 & 19.773 & 28.561 & 2.197 \\
\hline 6.591 & 13.182 & 164.775 & 232.882 \\
\hline 83.486 & 24.167 & 4.394 & 32.955 \\
\hline 127.426 & 8.788 & 4.394 & 41.743 \\
\hline 2.197 & 4.394 & 50.531 & 4.394 \\
\hline 4.394 & 298.792 & 112.047 & 10.985 \\
\hline 17.576 & 6.591 & 4.394 & 4.394 \\
\hline 101.062 & 39.546 & 6.591 & 2.197 \\
\hline 241.67 & 37.349 & 24.167 & 13.182 \\
\hline 28.561 & 4.394 & 2.197 & 628.342 \\
\hline
\end{tabular}




\begin{tabular}{|c|c|c|c|}
\hline 254.852 & 17.576 & 2.197 & 13.182 \\
\hline 52.728 & 28.561 & 2.197 & 24.167 \\
\hline 2451.85 & 215.306 & 2.197 & 30.758 \\
\hline 48.334 & 2.197 & 2.197 & 35.152 \\
\hline 46.137 & 2.197 & 4.394 & 2.197 \\
\hline 2.197 & 6.591 & 2.197 & 2.197 \\
\hline 2.197 & 4.394 & 2.197 & 28.561 \\
\hline 6.591 & 81.289 & 2.197 & 15.379 \\
\hline 2.197 & 8.788 & 35.152 & 8.788 \\
\hline 2.197 & 166.972 & 61.516 & 4.394 \\
\hline 6.591 & 169.169 & 2.197 & 41.743 \\
\hline 19.773 & 8.788 & 105.456 & 81.289 \\
\hline 50.531 & 2.197 & 2.197 & 4.394 \\
\hline 8.788 & 4.394 & 2293.67 & 24.167 \\
\hline 41.743 & 2.197 & 4.394 & 564.629 \\
\hline 4.394 & 74.698 & 8.788 & 344.929 \\
\hline 35.152 & 4.394 & 2.197 & 6.591 \\
\hline 37.349 & 2.197 & 2.197 & 8.788 \\
\hline 127.426 & 2.197 & 2.197 & 2.197 \\
\hline 2.197 & 4.394 & 2.197 & 2.197 \\
\hline 19.773 & 15.379 & 2.197 & 2.197 \\
\hline 6.591 & 4.394 & 8.788 & 193.336 \\
\hline 2.197 & 17.576 & 2.197 & 2.197 \\
\hline 151.593 & 2.197 & 4.394 & 2.197 \\
\hline 10.985 & 4.394 & 2.197 & 2.197 \\
\hline 4.394 & 6.591 & 2.197 & 4.394 \\
\hline 2.197 & 6.591 & 2.197 & 13.182 \\
\hline 8.788 & 2.197 & 2.197 & 6.591 \\
\hline 13.182 & 2.197 & 4.394 & 281.216 \\
\hline 24.167 & 2.197 & 2.197 & 10.985 \\
\hline 6.591 & 39.546 & 2.197 & 24.167 \\
\hline 4.394 & 2.197 & 2.197 & 2.197 \\
\hline 13.182 & 4.394 & 2.197 & 2.197 \\
\hline 21.97 & 4.394 & 2.197 & 10.985 \\
\hline 2.197 & 6.591 & 2.197 & 134.017 \\
\hline 13.182 & 4.394 & 2.197 & 6.591 \\
\hline 24.167 & 2.197 & 6.591 & 8.788 \\
\hline 13.182 & 2.197 & 15.379 & 10.985 \\
\hline 2.197 & 24.167 & 37.349 & 21.97 \\
\hline 19.773 & 10.985 & 6.591 & 2.197 \\
\hline 2.197 & 19.773 & 37.349 & 298.792 \\
\hline 268.034 & 821.678 & 2.197 & 85.683 \\
\hline 26.364 & 4.394 & 50.531 & 4.394 \\
\hline 2.197 & 4.394 & 107.653 & 15.379 \\
\hline 35.152 & 32.955 & 109.85 & 10.985 \\
\hline 8.788 & 10.985 & 26687 & 309.777 \\
\hline 41.743 & 30.758 & 6.591 & 103.259 \\
\hline 2.197 & 24.167 & 8.788 & 30.758 \\
\hline 4.394 & 21.97 & 2.197 & 50.531 \\
\hline 2.197 & 4.394 & 4.394 & 6.591 \\
\hline
\end{tabular}




\begin{tabular}{|c|c|c|c|}
\hline 2.197 & 26.364 & 619.554 & 8.788 \\
\hline 10.985 & 2.197 & 26.364 & 79.092 \\
\hline 153.79 & 6.591 & 57.122 & 63.713 \\
\hline 15.379 & 8.788 & 4.394 & 41.743 \\
\hline 19.773 & 4.394 & 8.788 & 2.197 \\
\hline 59.319 & 83.486 & 2.197 & 10.985 \\
\hline 10.985 & 2.197 & 21.97 & 6.591 \\
\hline 6.591 & 2.197 & 2.197 & 4.394 \\
\hline 2.197 & 2.197 & 259.246 & 17.576 \\
\hline 4.394 & 2.197 & 4.394 & 4.394 \\
\hline 24.167 & 17.576 & 17.576 & 103.259 \\
\hline 26.364 & 6.591 & 2.197 & 4.394 \\
\hline 2.197 & 6.591 & 37.349 & 13.182 \\
\hline 63.713 & 4.394 & 21.97 & 997.438 \\
\hline 19.773 & 15.379 & 21.97 & 17.576 \\
\hline 221.897 & 2.197 & 81.289 & 2.197 \\
\hline 2.197 & 8.788 & 2.197 & 4.394 \\
\hline 180.154 & 13.182 & 4.394 & 21.97 \\
\hline 2.197 & 4.394 & 15.379 & 2.197 \\
\hline 28.561 & 21.97 & 4.394 & 8.788 \\
\hline 2.197 & 6.591 & 2.197 & 2.197 \\
\hline 2.197 & 13.182 & 2.197 & 2.197 \\
\hline 26.364 & 30.758 & 112.047 & 4.394 \\
\hline 2.197 & 2.197 & 2.197 & 4.394 \\
\hline 17.576 & 4.394 & 50.531 & 250.458 \\
\hline 6.591 & 2.197 & 4.394 & 2.197 \\
\hline 2.197 & 2.197 & 6.591 & 2.197 \\
\hline 2.197 & 2.197 & 2.197 & 30.758 \\
\hline 35.152 & 2.197 & 15.379 & 2.197 \\
\hline 41.743 & 4.394 & 2.197 & 6.591 \\
\hline 2.197 & 6.591 & 2.197 & 2.197 \\
\hline 17.576 & 19.773 & 2.197 & 2.197 \\
\hline 191.139 & 63.713 & 63.713 & 4.394 \\
\hline 2.197 & 21.97 & 39.546 & 15.379 \\
\hline 2.197 & 369.096 & 2.197 & 2.197 \\
\hline 2.197 & 6.591 & 8.788 & 24.167 \\
\hline 48.334 & 13.182 & 2.197 & 6.591 \\
\hline 28.561 & 3396.56 & 2.197 & 10.985 \\
\hline 24.167 & 4.394 & 2.197 & 2533.14 \\
\hline 10.985 & 4.394 & 26.364 & 24.167 \\
\hline 6.591 & 32.955 & 2.197 & 2.197 \\
\hline 2.197 & 4.394 & 8.788 & 2.197 \\
\hline 4.394 & 8.788 & 2.197 & 63.713 \\
\hline 6.591 & 2.197 & 2.197 & 10.985 \\
\hline 52.728 & 19.773 & 2.197 & 19.773 \\
\hline 2.197 & 2.197 & 8.788 & 10.985 \\
\hline 8.788 & 2.197 & 2.197 & 6.591 \\
\hline 6.591 & 21.97 & 2.197 & 41.743 \\
\hline 13.182 & 6.591 & 4.394 & 15.379 \\
\hline 2.197 & 2.197 & 2.197 & 68.107 \\
\hline
\end{tabular}




\begin{tabular}{|c|c|c|c|}
\hline 2.197 & 2.197 & 2.197 & 17.576 \\
\hline 2.197 & 28.561 & 2.197 & 2.197 \\
\hline 6.591 & 13.182 & 2.197 & 2.197 \\
\hline 215.306 & 21.97 & 4.394 & 13.182 \\
\hline 74.698 & 2.197 & 2.197 & 274.625 \\
\hline 54.925 & 2.197 & 2.197 & 2.197 \\
\hline 4.394 & 2.197 & 17.576 & 2.197 \\
\hline 2.197 & 2.197 & 2.197 & 10.985 \\
\hline 4.394 & 263.64 & 2.197 & 8.788 \\
\hline 79.092 & 2.197 & 2.197 & 15.379 \\
\hline 2.197 & 19.773 & 68.107 & 98.865 \\
\hline 41.743 & 10.985 & 2.197 & 21.97 \\
\hline 2.197 & 4.394 & 2.197 & 2.197 \\
\hline 2.197 & 4.394 & 13.182 & 28.561 \\
\hline 2.197 & 4.394 & 2.197 & 4.394 \\
\hline 4.394 & 4.394 & 2.197 & 6.591 \\
\hline 4.394 & 2.197 & 68.107 & 63.713 \\
\hline 21.97 & 2.197 & 13.182 & 43.94 \\
\hline 13.182 & 2.197 & 46.137 & 39.546 \\
\hline 2.197 & 2.197 & 15.379 & 54.925 \\
\hline 2.197 & 4.394 & 57.122 & 307.58 \\
\hline 4.394 & 6.591 & 2.197 & 15.379 \\
\hline 81.289 & 2.197 & 6.591 & 2.197 \\
\hline 8.788 & 6.591 & 28.561 & 208.715 \\
\hline 2.197 & 2.197 & 90.077 & 4.394 \\
\hline 24.167 & 17.576 & 2.197 & 10.985 \\
\hline 17.576 & 4.394 & 2.197 & 2.197 \\
\hline 19.773 & 2.197 & 10.985 & 8.788 \\
\hline 2.197 & 15.379 & 8.788 & 8.788 \\
\hline 215.306 & 125.229 & 6.591 & 17.576 \\
\hline 160.381 & 39.546 & 2.197 & 8.788 \\
\hline 28.561 & 19.773 & 15.379 & 39.546 \\
\hline 4.394 & 6.591 & 17.576 & 13.182 \\
\hline 593.19 & 2.197 & 19.773 & 26.364 \\
\hline 2.197 & 2.197 & 2.197 & 13.182 \\
\hline 2.197 & 15.379 & 8.788 & 2.197 \\
\hline 2.197 & 65.91 & 2.197 & 17.576 \\
\hline 2.197 & 2.197 & 28.561 & 26.364 \\
\hline 26.364 & 13.182 & 2.197 & 59.319 \\
\hline 32.955 & 4.394 & 83.486 & 2.197 \\
\hline 8.788 & 41.743 & 43.94 & 6.591 \\
\hline 101.062 & 19.773 & 2.197 & 445.991 \\
\hline 10.985 & 2.197 & 8.788 & 2.197 \\
\hline 26.364 & 764.556 & 2.197 & 17.576 \\
\hline 8.788 & 6.591 & 4.394 & 83.486 \\
\hline 79.092 & 2.197 & 4.394 & 21.97 \\
\hline 8.788 & 48.334 & 6.591 & 116.441 \\
\hline 125.229 & 21.97 & 2.197 & 134.017 \\
\hline 283.413 & 2.197 & 21.97 & 48.334 \\
\hline 17.576 & 13.182 & 10.985 & 46.137 \\
\hline
\end{tabular}




\begin{tabular}{|c|c|c|c|}
\hline 41.743 & 15.379 & 2.197 & 2.197 \\
\hline 2.197 & 4.394 & 2.197 & 39.546 \\
\hline 2.197 & 54.925 & 2.197 & 4.394 \\
\hline 2.197 & 2.197 & 24.167 & 4.394 \\
\hline 2.197 & 4.394 & 10.985 & 2.197 \\
\hline 2.197 & 4.394 & 4.394 & 307.58 \\
\hline 90.077 & 26.364 & 4.394 & 1118.27 \\
\hline 10.985 & 2.197 & 4.394 & 424.021 \\
\hline 123.032 & 76.895 & 2.197 & 2.197 \\
\hline 6.591 & 4.394 & 21.97 & 39.546 \\
\hline 6.591 & 2.197 & 349.323 & 4.394 \\
\hline 2.197 & 2.197 & 2.197 & 1289.64 \\
\hline 4.394 & 4.394 & 2.197 & 2.197 \\
\hline 2.197 & 2.197 & 142.805 & 2.197 \\
\hline 65.91 & 76.895 & 2.197 & 41.743 \\
\hline 6.591 & 4.394 & 63.713 & 2.197 \\
\hline 2.197 & 4.394 & 6.591 & 2.197 \\
\hline 2.197 & 4.394 & 2.197 & 13.182 \\
\hline 2.197 & 4.394 & 2.197 & 2.197 \\
\hline 2.197 & 2.197 & 2.197 & 6.591 \\
\hline 8.788 & 2.197 & 4.394 & 35.152 \\
\hline 1454.41 & 4.394 & 129.623 & 32.955 \\
\hline 87.88 & 68.107 & 35.152 & 4.394 \\
\hline 2.197 & 2.197 & 8.788 & 8.788 \\
\hline 2.197 & 4.394 & 21.97 & 63.713 \\
\hline 4.394 & 4.394 & 2.197 & 8.788 \\
\hline 1535.7 & 4.394 & 8.788 & 8.788 \\
\hline 8.788 & 4.394 & 2.197 & 24.167 \\
\hline 10.985 & 2.197 & 2.197 & 26.364 \\
\hline 8.788 & 4.394 & 6.591 & 2.197 \\
\hline 32.955 & 4.394 & 8.788 & 19.773 \\
\hline 2.197 & 8.788 & 2.197 & 68.107 \\
\hline 2.197 & 8.788 & 37.349 & 986.453 \\
\hline 19.773 & 26.364 & 6.591 & 417.43 \\
\hline 2.197 & 8.788 & 90.077 & 68.107 \\
\hline 26.364 & 13.182 & 2.197 & 149.396 \\
\hline 2.197 & 30.758 & 24.167 & 8.788 \\
\hline 6.591 & 138.411 & 2.197 & 43.94 \\
\hline 129.623 & 2.197 & 2.197 & 6.591 \\
\hline 619.554 & 2.197 & 2.197 & 4.394 \\
\hline 650.312 & 2.197 & 10.985 & 2.197 \\
\hline 6.591 & 186.745 & 8.788 & 4.394 \\
\hline 118.638 & 32.955 & 2.197 & 2.197 \\
\hline 184.548 & 65.91 & 1722.45 & 74.698 \\
\hline 68.107 & 6.591 & 2.197 & 2.197 \\
\hline 2.197 & 2.197 & 2.197 & 6.591 \\
\hline 4.394 & 2.197 & 2.197 & 114.244 \\
\hline 2.197 & 2.197 & 2.197 & 2.197 \\
\hline 10.985 & 65.91 & 32.955 & 6.591 \\
\hline 2.197 & 4.394 & 4.394 & 17.576 \\
\hline
\end{tabular}




\begin{tabular}{|c|c|c|c|}
\hline 2.197 & 2.197 & 2.197 & 4.394 \\
\hline 307.58 & 101.062 & 2.197 & 30.758 \\
\hline 6.591 & 2.197 & 2.197 & 19.773 \\
\hline 46.137 & 4.394 & 2.197 & 10.985 \\
\hline 10.985 & 2.197 & 2.197 & 2.197 \\
\hline 8.788 & 6.591 & 15.379 & 395.46 \\
\hline 2.197 & 10.985 & 30.758 & 4.394 \\
\hline 4.394 & 13.182 & 17.576 & 2.197 \\
\hline 2.197 & 54.925 & 32.955 & 6.591 \\
\hline 13.182 & 26.364 & 2.197 & 2.197 \\
\hline 6.591 & 2.197 & 8.788 & 2.197 \\
\hline 24.167 & 2.197 & 4.394 & 4.394 \\
\hline 28.561 & 4.394 & 109.85 & 41.743 \\
\hline 6.591 & 2.197 & 4.394 & 8.788 \\
\hline 13.182 & 2.197 & 2.197 & 2.197 \\
\hline 112.047 & 4.394 & 26.364 & 2.197 \\
\hline 24.167 & 2.197 & 15.379 & 353.717 \\
\hline 24.167 & 2.197 & 61.516 & 2.197 \\
\hline 2.197 & 65.91 & 6.591 & 2.197 \\
\hline 10.985 & 4.394 & 4.394 & 68.107 \\
\hline 2.197 & 2.197 & 13.182 & 2.197 \\
\hline 13.182 & 4.394 & 10.985 & 15.379 \\
\hline 4.394 & 13.182 & 2.197 & 19.773 \\
\hline 4.394 & 8.788 & 2.197 & 41.743 \\
\hline 4.394 & 2.197 & 2.197 & 54.925 \\
\hline 2.197 & 4.394 & 4.394 & 2.197 \\
\hline 8.788 & 2.197 & 6.591 & 8.788 \\
\hline 39.546 & 186.745 & 907.361 & 13.182 \\
\hline 4.394 & 2.197 & 4.394 & 10.985 \\
\hline 10.985 & 21.97 & 21.97 & 2.197 \\
\hline 2.197 & 10.985 & 6.591 & 2.197 \\
\hline 4.394 & 43.94 & 65.91 & 4.394 \\
\hline 26.364 & 8.788 & 19.773 & 6.591 \\
\hline 68.107 & 2.197 & 4.394 & 19.773 \\
\hline 2.197 & 10.985 & 2.197 & 21.97 \\
\hline 2.197 & 17.576 & 2.197 & 6.591 \\
\hline 15.379 & 83.486 & 2.197 & 186.745 \\
\hline 2.197 & 48.334 & 252.655 & 2.197 \\
\hline 2.197 & 17.576 & 617.357 & 13.182 \\
\hline 13.182 & 6.591 & 2.197 & 98.865 \\
\hline 41.743 & 2.197 & 2.197 & 2.197 \\
\hline 2.197 & 601.978 & 10.985 & 2.197 \\
\hline 19.773 & 175.76 & 26.364 & 2.197 \\
\hline 2.197 & 2.197 & 2.197 & 81.289 \\
\hline 4.394 & 4.394 & 2.197 & 2.197 \\
\hline 4.394 & 19.773 & 4.394 & 2.197 \\
\hline 2.197 & 4.394 & 2.197 & 109.85 \\
\hline 2.197 & 2.197 & 2.197 & 2.197 \\
\hline 2.197 & 2.197 & 2.197 & 6.591 \\
\hline 26.364 & 30.758 & 37.349 & 4.394 \\
\hline
\end{tabular}




\begin{tabular}{|c|c|c|c|}
\hline 8.788 & 10.985 & 2.197 & 786.526 \\
\hline 6.591 & 2.197 & 4.394 & 17.576 \\
\hline 17.576 & 2.197 & 6.591 & 4.394 \\
\hline 4.394 & 2.197 & 2.197 & 21.97 \\
\hline 24.167 & 601.978 & 142.805 & 35.152 \\
\hline 10.985 & 26.364 & 2.197 & 4.394 \\
\hline 21.97 & 2.197 & 2.197 & 2.197 \\
\hline 2.197 & 15.379 & 8.788 & 8.788 \\
\hline 2.197 & 2.197 & 6.591 & 76.895 \\
\hline 4.394 & 43.94 & 17.576 & 35.152 \\
\hline 2.197 & 2.197 & 2.197 & 6.591 \\
\hline 2.197 & 15.379 & 2.197 & 8.788 \\
\hline 2.197 & 2.197 & 2.197 & 28.561 \\
\hline 4.394 & 4.394 & 26.364 & 4.394 \\
\hline 13.182 & 2.197 & 2.197 & 103.259 \\
\hline 28.561 & 2.197 & 2.197 & 322.959 \\
\hline 74.698 & 4.394 & 2.197 & 15.379 \\
\hline 19.773 & 4.394 & 4.394 & 2.197 \\
\hline 2.197 & 2.197 & 21.97 & 21.97 \\
\hline 39.546 & 4.394 & 8.788 & 2.197 \\
\hline 6.591 & 2.197 & 54.925 & 6.591 \\
\hline 2.197 & 2.197 & 2.197 & 4.394 \\
\hline 329.55 & 4.394 & 2.197 & 2.197 \\
\hline 21.97 & 10.985 & 2.197 & 32.955 \\
\hline 70.304 & 8.788 & 6.591 & 4.394 \\
\hline 24.167 & 164.775 & 2.197 & 2.197 \\
\hline 4.394 & 30.758 & 2.197 & 26.364 \\
\hline 21.97 & 17.576 & 8.788 & 4.394 \\
\hline 2.197 & 57.122 & 26.364 & 228.488 \\
\hline 57.122 & 4.394 & 571.22 & 4.394 \\
\hline 6.591 & 2.197 & 70.304 & 48.334 \\
\hline 362.505 & 2.197 & 32.955 & 138.411 \\
\hline 4.394 & 59.319 & 46.137 & 30.758 \\
\hline 4.394 & 2.197 & 2.197 & 4.394 \\
\hline 2.197 & 98.865 & 17.576 & 4.394 \\
\hline 147.199 & 8.788 & 2.197 & 26.364 \\
\hline 8.788 & 30.758 & 48.334 & 2.197 \\
\hline 112.047 & 2.197 & 2.197 & 4.394 \\
\hline 15.379 & 2.197 & 2.197 & 21.97 \\
\hline 2.197 & 24.167 & 4.394 & 219.7 \\
\hline 6.591 & 2.197 & 1118.27 & 8.788 \\
\hline 83.486 & 28.561 & 2.197 & 2.197 \\
\hline 13.182 & 4.394 & 2.197 & 17.576 \\
\hline 8.788 & 142.805 & 2.197 & 15.379 \\
\hline 21.97 & 6.591 & 2.197 & 15.379 \\
\hline 2.197 & 10.985 & 4.394 & 2.197 \\
\hline 6.591 & 10.985 & 186.745 & 6.591 \\
\hline 28.561 & 24.167 & 2.197 & 2.197 \\
\hline 2.197 & 13.182 & 2.197 & 2.197 \\
\hline 19.773 & 96.668 & 107.653 & 4.394 \\
\hline
\end{tabular}




\begin{tabular}{|c|c|c|c|}
\hline 4.394 & 65.91 & 2.197 & 4.394 \\
\hline 28.561 & 4.394 & 2.197 & 68.107 \\
\hline 81.289 & 2.197 & 17.576 & 15.379 \\
\hline 28.561 & 17.576 & 2.197 & 15.379 \\
\hline 2.197 & 2.197 & 2.197 & 17.576 \\
\hline 8.788 & 2.197 & 13.182 & 13.182 \\
\hline 4.394 & 4.394 & 153.79 & 2.197 \\
\hline 19.773 & 2.197 & 41.743 & 2.197 \\
\hline 2.197 & 2.197 & 17.576 & 2.197 \\
\hline 8.788 & 2.197 & 2.197 & 4.394 \\
\hline 26.364 & 2.197 & 4.394 & 19.773 \\
\hline 4.394 & 2.197 & 10.985 & 2.197 \\
\hline 59.319 & 21.97 & 4.394 & 15.379 \\
\hline 24.167 & 8.788 & 4.394 & 2.197 \\
\hline 32.955 & 2.197 & 120.835 & 28.561 \\
\hline 57.122 & 4.394 & 6.591 & 2.197 \\
\hline 4.394 & 2.197 & 46.137 & 70.304 \\
\hline 2.197 & 4.394 & 13.182 & 153.79 \\
\hline 17.576 & 2.197 & 41.743 & 24.167 \\
\hline 6.591 & 410.839 & 4.394 & 2.197 \\
\hline 2.197 & 83.486 & 2.197 & 2.197 \\
\hline 26.364 & 259.246 & 2.197 & 17.576 \\
\hline 6.591 & 4.394 & 2.197 & 127.426 \\
\hline 4.394 & 2.197 & 123.032 & 4.394 \\
\hline 4.394 & 54.925 & 2.197 & 46.137 \\
\hline 4.394 & 2.197 & 2.197 & 375.687 \\
\hline 2.197 & 26.364 & 2.197 & 4.394 \\
\hline 6.591 & 4.394 & 10.985 & 2.197 \\
\hline 2.197 & 639.327 & 2.197 & 6.591 \\
\hline 2.197 & 24.167 & 15.379 & 15.379 \\
\hline 10.985 & 15.379 & 2.197 & 17.576 \\
\hline 2.197 & 2.197 & 4.394 & 2.197 \\
\hline 2.197 & 2.197 & 10.985 & 2.197 \\
\hline 2.197 & 4.394 & 2.197 & 2.197 \\
\hline 2.197 & 445.991 & 19.773 & 2.197 \\
\hline 4.394 & 2.197 & 13.182 & 2.197 \\
\hline 2.197 & 2.197 & 2.197 & 15.379 \\
\hline 59.319 & 6.591 & 41.743 & 2.197 \\
\hline 15.379 & 158.184 & 17.576 & 2.197 \\
\hline 30.758 & 13.182 & 2.197 & 268.034 \\
\hline 21.97 & 21.97 & 2.197 & 41.743 \\
\hline 2.197 & 96.668 & 2.197 & 2.197 \\
\hline 2.197 & 206.518 & 2.197 & 2.197 \\
\hline 2.197 & 2.197 & 2.197 & 6.591 \\
\hline 6.591 & 103.259 & 2.197 & 320.762 \\
\hline 2320.03 & 2.197 & 2.197 & 8.788 \\
\hline 2.197 & 2.197 & 6.591 & 54.925 \\
\hline 4.394 & 2.197 & 2.197 & 129.623 \\
\hline 6.591 & 2.197 & 4.394 & 8.788 \\
\hline 4.394 & 8.788 & 2.197 & 2.197 \\
\hline
\end{tabular}




\begin{tabular}{|c|c|c|c|}
\hline 2.197 & 4.394 & 35.152 & 2.197 \\
\hline 4.394 & 4.394 & 10.985 & 41.743 \\
\hline 2.197 & 2.197 & 10.985 & 8.788 \\
\hline 4.394 & 10.985 & 2.197 & 2.197 \\
\hline 39.546 & 2.197 & 8.788 & 2.197 \\
\hline 13.182 & 2.197 & 353.717 & 70.304 \\
\hline 2.197 & 2.197 & 26.364 & 10.985 \\
\hline 112.047 & 2.197 & 39.546 & 46.137 \\
\hline 768.95 & 10662 & 39.546 & 125.229 \\
\hline 2.197 & 61.516 & 593.19 & 2.197 \\
\hline 2.197 & 4.394 & 6.591 & 604.175 \\
\hline 26.364 & 6.591 & 2.197 & 13.182 \\
\hline 48.334 & 4.394 & 4.394 & 103.259 \\
\hline 2.197 & 2.197 & 2.197 & 4.394 \\
\hline 10.985 & 41.743 & 4.394 & 37.349 \\
\hline 2.197 & 184.548 & 21.97 & 4.394 \\
\hline 17.576 & 19.773 & 2.197 & 2713.29 \\
\hline 2.197 & 13.182 & 2.197 & 10.985 \\
\hline 26.364 & 2.197 & 2.197 & 79.092 \\
\hline 4.394 & 118.638 & 2.197 & 13.182 \\
\hline 2.197 & 50.531 & 21.97 & 2.197 \\
\hline 2.197 & 2.197 & 8.788 & 103.259 \\
\hline 8.788 & 37.349 & 90.077 & 6.591 \\
\hline 50.531 & 2.197 & 6.591 & 26.364 \\
\hline 6.591 & 2.197 & 13.182 & 2.197 \\
\hline 4.394 & 52.728 & 21.97 & 2.197 \\
\hline 68.107 & 4.394 & 2.197 & 28.561 \\
\hline 59.319 & 2.197 & 2.197 & 136.214 \\
\hline 2.197 & 17.576 & 2.197 & 2.197 \\
\hline 8.788 & 57.122 & 54.925 & 2.197 \\
\hline 54.925 & 2.197 & 2.197 & 2.197 \\
\hline 21.97 & 2.197 & 2.197 & 2.197 \\
\hline 15.379 & 4.394 & 21.97 & 2.197 \\
\hline 32.955 & 2.197 & 8.788 & 2.197 \\
\hline 746.98 & 2.197 & 2.197 & 2.197 \\
\hline 2.197 & 10.985 & 2.197 & 19.773 \\
\hline 15.379 & 2.197 & 8.788 & 573.417 \\
\hline 6.591 & 4.394 & 6.591 & 2.197 \\
\hline 1034.79 & 2.197 & 46.137 & 2.197 \\
\hline 2.197 & 6.591 & 2.197 & 13.182 \\
\hline 4.394 & 26.364 & 19.773 & 8.788 \\
\hline 2.197 & 57.122 & 2.197 & 13.182 \\
\hline 2377.15 & 6.591 & 4.394 & 195.533 \\
\hline 2.197 & 2.197 & 15.379 & 2.197 \\
\hline 52.728 & 2.197 & 2.197 & 6.591 \\
\hline 4.394 & 2.197 & 10.985 & 30.758 \\
\hline 2.197 & 2.197 & 6.591 & 26.364 \\
\hline 19.773 & 8.788 & 4.394 & 6.591 \\
\hline 136.214 & 4.394 & 2.197 & 19.773 \\
\hline 83.486 & 2.197 & 1243.5 & 8.788 \\
\hline
\end{tabular}




\begin{tabular}{|c|c|c|c|}
\hline 2.197 & 30.758 & 65.91 & 65.91 \\
\hline 2.197 & 2.197 & 17.576 & 6716.23 \\
\hline 4.394 & 2.197 & 2.197 & 59.319 \\
\hline 21.97 & 13.182 & 4.394 & 6.591 \\
\hline 26.364 & 8.788 & 2.197 & 2.197 \\
\hline 2.197 & 2.197 & 2.197 & 8.788 \\
\hline 4.394 & 2.197 & 2.197 & 15.379 \\
\hline 10.985 & 4.394 & 6.591 & 2.197 \\
\hline 2.197 & 4.394 & 2.197 & 13.182 \\
\hline 2.197 & 6.591 & 10.985 & 37.349 \\
\hline 2.197 & 103.259 & 26.364 & 6.591 \\
\hline 2.197 & 8.788 & 10.985 & 10.985 \\
\hline 2.197 & 50.531 & 4.394 & 10.985 \\
\hline 2.197 & 6.591 & 2.197 & 2.197 \\
\hline 24.167 & 50.531 & 2.197 & 13.182 \\
\hline 90.077 & 10.985 & 2.197 & 19.773 \\
\hline 2.197 & 26.364 & 2.197 & 4.394 \\
\hline 1008.42 & 24.167 & 2.197 & 8.788 \\
\hline 13.182 & 87.88 & 2.197 & 2.197 \\
\hline 39.546 & 2.197 & 2.197 & 2.197 \\
\hline 246.064 & 2.197 & 6.591 & 10.985 \\
\hline 562.432 & 17.576 & 2.197 & 2.197 \\
\hline 24.167 & 2.197 & 8.788 & 17.576 \\
\hline 2.197 & 2.197 & 4.394 & 54.925 \\
\hline 45220.8 & 10.985 & 2.197 & 186.745 \\
\hline 2.197 & 37.349 & 2.197 & 26.364 \\
\hline 4.394 & 81.289 & 52.728 & 4.394 \\
\hline 2.197 & 2.197 & 24.167 & 32.955 \\
\hline 10.985 & 2.197 & 4.394 & 57.122 \\
\hline 6.591 & 2.197 & 2.197 & 145.002 \\
\hline 85.683 & 538.265 & 6.591 & 15.379 \\
\hline 228.488 & 2.197 & 2.197 & 13.182 \\
\hline 30.758 & 52.728 & 2.197 & 30.758 \\
\hline 2.197 & 65.91 & 2.197 & 13.182 \\
\hline 26.364 & 2.197 & 4.394 & 13.182 \\
\hline 6.591 & 2.197 & 2.197 & 2.197 \\
\hline 6.591 & 2.197 & 2.197 & 219.7 \\
\hline 4.394 & 2.197 & 15.379 & 59.319 \\
\hline 2.197 & 2.197 & 19.773 & 10.985 \\
\hline 41.743 & 4.394 & 199.927 & 2.197 \\
\hline 6.591 & 2.197 & 2.197 & 2.197 \\
\hline 2.197 & 4.394 & 2.197 & 6.591 \\
\hline 32.955 & 2.197 & 2.197 & 2.197 \\
\hline 6.591 & 2.197 & 32.955 & 2.197 \\
\hline 6.591 & 2.197 & 2.197 & 138.411 \\
\hline 24.167 & 6.591 & 2.197 & 90.077 \\
\hline 2.197 & 4.394 & 2.197 & 6.591 \\
\hline 2.197 & 177.957 & 6.591 & 54.925 \\
\hline 6.591 & 4.394 & 81.289 & 2.197 \\
\hline 6.591 & 13.182 & 15.379 & 2.197 \\
\hline
\end{tabular}




\begin{tabular}{|c|c|c|c|}
\hline 21.97 & 17.576 & 79.092 & 13.182 \\
\hline 28.561 & 61.516 & 1111.68 & 2.197 \\
\hline 2.197 & 2.197 & 129.623 & 59.319 \\
\hline 15.379 & 6.591 & 2.197 & 61.516 \\
\hline 32.955 & 32.955 & 83.486 & 13.182 \\
\hline 134.017 & 2.197 & 2.197 & 129.623 \\
\hline 2.197 & 76.895 & 48.334 & 4.394 \\
\hline 2.197 & 8.788 & 15.379 & 2.197 \\
\hline 13.182 & 4.394 & 35.152 & 6.591 \\
\hline 1030.39 & 54.925 & 112.047 & 19.773 \\
\hline 4.394 & 85.683 & 4.394 & 48.334 \\
\hline 4.394 & 39.546 & 32.955 & 6.591 \\
\hline 10.985 & 2.197 & 6.591 & 30.758 \\
\hline 2.197 & 70.304 & 10.985 & 35.152 \\
\hline 2.197 & 8.788 & 2.197 & 4.394 \\
\hline 2.197 & 50.531 & 10.985 & 2.197 \\
\hline 239.473 & 39.546 & 24.167 & 6.591 \\
\hline 158.184 & 2.197 & 2.197 & 35.152 \\
\hline 2.197 & 147.199 & 8.788 & 26.364 \\
\hline 74.698 & 2.197 & 421.824 & 10.985 \\
\hline 4.394 & 32.955 & 2.197 & 57.122 \\
\hline 2.197 & 452.582 & 2.197 & 50.531 \\
\hline 28.561 & 4.394 & 2.197 & 2.197 \\
\hline 4.394 & 114.244 & 92.274 & 2.197 \\
\hline 2.197 & 2.197 & 4.394 & 83.486 \\
\hline 2.197 & 2.197 & 2.197 & 1140.24 \\
\hline 10.985 & 43.94 & 2.197 & 19.773 \\
\hline 24.167 & 4.394 & 4.394 & 4.394 \\
\hline 46.137 & 2.197 & 2.197 & 6.591 \\
\hline 17.576 & 2.197 & 4.394 & 90.077 \\
\hline 24.167 & 2.197 & 4.394 & 4.394 \\
\hline 28.561 & 13.182 & 10.985 & 8.788 \\
\hline 2.197 & 2.197 & 4.394 & 48.334 \\
\hline 15.379 & 2.197 & 21.97 & 61.516 \\
\hline 92.274 & 8.788 & 2.197 & 8.788 \\
\hline 85.683 & 2.197 & 2.197 & 10.985 \\
\hline 15.379 & 2.197 & 8.788 & 6.591 \\
\hline 13.182 & 2.197 & 6.591 & 4.394 \\
\hline 26.364 & 2.197 & 54.925 & 10.985 \\
\hline 10.985 & 4.394 & 2.197 & 52.728 \\
\hline 39.546 & 28.561 & 2.197 & 39.546 \\
\hline 37.349 & 6.591 & 8.788 & 2.197 \\
\hline 17.576 & 2.197 & 10.985 & 41.743 \\
\hline 2.197 & 4.394 & 59.319 & 136.214 \\
\hline 2016.85 & 2.197 & 17.576 & 10.985 \\
\hline 26.364 & 6.591 & 2.197 & 24.167 \\
\hline 6105.46 & 35.152 & 2.197 & 43.94 \\
\hline 6.591 & 2.197 & 13.182 & 2.197 \\
\hline 2.197 & 2.197 & 2.197 & 43.94 \\
\hline 2.197 & 2.197 & 2.197 & 2.197 \\
\hline
\end{tabular}




\begin{tabular}{|c|c|c|c|}
\hline 37.349 & 120.835 & 8.788 & 2.197 \\
\hline 4.394 & 15.379 & 2.197 & 2.197 \\
\hline 26.364 & 28.561 & 2.197 & 26.364 \\
\hline 112.047 & 2.197 & 2.197 & 46.137 \\
\hline 2.197 & 32.955 & 2.197 & 28.561 \\
\hline 10.985 & 142.805 & 2.197 & 39.546 \\
\hline 8.788 & 641.524 & 2.197 & 19.773 \\
\hline 15.379 & 149.396 & 4.394 & 558.038 \\
\hline 68.107 & 859.027 & 2.197 & 595.387 \\
\hline 19.773 & 2.197 & 4.394 & 6.591 \\
\hline 2.197 & 241.67 & 2.197 & 19.773 \\
\hline 10.985 & 70.304 & 8.788 & 2.197 \\
\hline 6.591 & 19.773 & 30.758 & 13.182 \\
\hline 19.773 & 50.531 & 2.197 & 2.197 \\
\hline 4.394 & 17.576 & 2.197 & 2.197 \\
\hline 4.394 & 2.197 & 2.197 & 50.531 \\
\hline 21.97 & 41.743 & 2.197 & 8.788 \\
\hline 2.197 & 2.197 & 2.197 & 17.576 \\
\hline 2.197 & 261.443 & 2.197 & 10.985 \\
\hline 13.182 & 8.788 & 4.394 & 4.394 \\
\hline 1201.76 & 2.197 & 15.379 & 155.987 \\
\hline 17.576 & 2.197 & 13.182 & 15.379 \\
\hline 19.773 & 17.576 & 2.197 & 72.501 \\
\hline 2.197 & 30.758 & 2.197 & 155.987 \\
\hline 57.122 & 4.394 & 2.197 & 2.197 \\
\hline 129.623 & 6.591 & 2.197 & 2.197 \\
\hline 19.773 & 2.197 & 6.591 & 4.394 \\
\hline 6.591 & 2.197 & 6032.96 & 2.197 \\
\hline 2.197 & 32.955 & 72.501 & 2.197 \\
\hline 57.122 & 2.197 & 2.197 & 15.379 \\
\hline 4.394 & 2.197 & 37.349 & 54.925 \\
\hline 10.985 & 4.394 & 6.591 & 4.394 \\
\hline 2.197 & 68.107 & 138.411 & 2.197 \\
\hline 898.573 & 2.197 & 4.394 & 8.788 \\
\hline 606.372 & 13.182 & 2.197 & 28.561 \\
\hline 10.985 & 41.743 & 2.197 & 63.713 \\
\hline 709.631 & 65.91 & 68.107 & 17.576 \\
\hline 8.788 & 2.197 & 2.197 & 193.336 \\
\hline 182.351 & 10.985 & 30.758 & 4.394 \\
\hline 4.394 & 2.197 & 2.197 & 83.486 \\
\hline 4.394 & 4.394 & 37.349 & 17.576 \\
\hline 15.379 & 79.092 & 19.773 & 577.811 \\
\hline 6.591 & 4.394 & 147.199 & 4.394 \\
\hline 50.531 & 2.197 & 59.319 & 15.379 \\
\hline 6.591 & 43.94 & 79.092 & 2.197 \\
\hline 2.197 & 2.197 & 4.394 & 8.788 \\
\hline 4.394 & 2.197 & 4.394 & 10.985 \\
\hline 4.394 & 2.197 & 10.985 & 2.197 \\
\hline 2.197 & 272.428 & 169.169 & 10.985 \\
\hline 2.197 & 6.591 & 10.985 & 39.546 \\
\hline
\end{tabular}




\begin{tabular}{|c|c|c|c|}
\hline 2.197 & 2.197 & 26.364 & 129.623 \\
\hline 424.021 & 46.137 & 17.576 & 35.152 \\
\hline 6.591 & 2.197 & 2.197 & 2.197 \\
\hline 59.319 & 76.895 & 2.197 & 2.197 \\
\hline 2.197 & 2.197 & 2.197 & 46.137 \\
\hline 2.197 & 68.107 & 2.197 & 6.591 \\
\hline 2.197 & 8.788 & 518.492 & 2.197 \\
\hline 15.379 & 10.985 & 2.197 & 13.182 \\
\hline 162.578 & 52.728 & 2.197 & 142.805 \\
\hline 456.976 & 2.197 & 19.773 & 107.653 \\
\hline 309.777 & 2.197 & 4.394 & 4.394 \\
\hline 57.122 & 6.591 & 6.591 & 61.516 \\
\hline 2.197 & 10.985 & 2.197 & 28.561 \\
\hline 2.197 & 43.94 & 17.576 & 6.591 \\
\hline 50.531 & 4.394 & 6.591 & 10.985 \\
\hline 26.364 & 2.197 & 2.197 & 46.137 \\
\hline 4.394 & 2.197 & 424.021 & 4.394 \\
\hline 362.505 & 2.197 & 2.197 & 2.197 \\
\hline 4.394 & 6.591 & 2.197 & 4.394 \\
\hline 68.107 & 10.985 & 564.629 & 28.561 \\
\hline 21.97 & 2.197 & 2.197 & 43.94 \\
\hline 4.394 & 19.773 & 2.197 & 4.394 \\
\hline 2.197 & 13.182 & 6.591 & 46.137 \\
\hline 6.591 & 2.197 & 2.197 & 15.379 \\
\hline 360.308 & 15.379 & 2.197 & 81.289 \\
\hline 2.197 & 2.197 & 158.184 & 127.426 \\
\hline 6.591 & 32.955 & 6.591 & 10.985 \\
\hline 4.394 & 4.394 & 2.197 & 68.107 \\
\hline 4.394 & 35.152 & 26.364 & 2.197 \\
\hline 2.197 & 2.197 & 2.197 & 2.197 \\
\hline 4.394 & 50.531 & 2.197 & 41.743 \\
\hline 13.182 & 39.546 & 19.773 & 4.394 \\
\hline 21.97 & 4.394 & 8.788 & 43.94 \\
\hline 17.576 & 2.197 & 2.197 & 4.394 \\
\hline 6.591 & 2.197 & 2.197 & 6.591 \\
\hline 801.905 & 4.394 & 48.334 & 6.591 \\
\hline 8.788 & 96.668 & 2.197 & 61.516 \\
\hline 4.394 & 215.306 & 30.758 & 10.985 \\
\hline 43.94 & 217.503 & 4.394 & 4.394 \\
\hline 2.197 & 13.182 & 28.561 & 41.743 \\
\hline 10.985 & 270.231 & 2.197 & 149.396 \\
\hline 4198.47 & 15.379 & 2.197 & 4.394 \\
\hline 28.561 & 70.304 & 4.394 & 13.182 \\
\hline 270.231 & 24.167 & 87.88 & 13.182 \\
\hline 8.788 & 2.197 & 85.683 & 6.591 \\
\hline 2.197 & 21.97 & 4.394 & 4.394 \\
\hline 4.394 & 10.985 & 2.197 & 10.985 \\
\hline 2.197 & 257.049 & 2.197 & 13.182 \\
\hline 10.985 & 4.394 & 2.197 & 4.394 \\
\hline 2.197 & 4.394 & 2.197 & 15.379 \\
\hline
\end{tabular}




\begin{tabular}{|c|c|c|c|}
\hline 37.349 & 4.394 & 98.865 & 2.197 \\
\hline 19.773 & 28.561 & 142.805 & 21.97 \\
\hline 24.167 & 10.985 & 2.197 & 90.077 \\
\hline 186.745 & 4.394 & 8.788 & 30.758 \\
\hline 6.591 & 68.107 & 90.077 & 32.955 \\
\hline 2.197 & 30.758 & 2.197 & 4.394 \\
\hline 19.773 & 28.561 & 2.197 & 4.394 \\
\hline 2.197 & 35.152 & 17.576 & 272.428 \\
\hline 2.197 & 10.985 & 19.773 & 4.394 \\
\hline 68.107 & 2.197 & 2.197 & 8.788 \\
\hline 2.197 & 2.197 & 4.394 & 19.773 \\
\hline 4.394 & 10.985 & 6.591 & 127.426 \\
\hline 206.518 & 2.197 & 2.197 & 21.97 \\
\hline 13.182 & 4.394 & 2.197 & 2.197 \\
\hline 2.197 & 2.197 & 13.182 & 6.591 \\
\hline 10.985 & 2.197 & 10.985 & 114.244 \\
\hline 2.197 & 2.197 & 2.197 & 105.456 \\
\hline 21.97 & 8.788 & 2.197 & 6.591 \\
\hline 2.197 & 2.197 & 107.653 & 32.955 \\
\hline 48.334 & 2.197 & 2.197 & 46.137 \\
\hline 2.197 & 2.197 & 2.197 & 26.364 \\
\hline 2.197 & 13.182 & 50.531 & 2.197 \\
\hline 4.394 & 2.197 & 28.561 & 17.576 \\
\hline 8.788 & 2.197 & 26.364 & 57.122 \\
\hline 17.576 & 13.182 & 98.865 & 4.394 \\
\hline 2.197 & 28.561 & 4.394 & 2.197 \\
\hline 2.197 & 6.591 & 105.456 & 2.197 \\
\hline 2.197 & 4.394 & 2.197 & 8.788 \\
\hline 6.591 & 28.561 & 13.182 & 160.381 \\
\hline 2.197 & 2.197 & 2.197 & 15.379 \\
\hline 6.591 & 2.197 & 160.381 & 59892.4 \\
\hline 2.197 & 6.591 & 237.276 & 4.394 \\
\hline 4.394 & 142.805 & 2.197 & 54.925 \\
\hline 17.576 & 2.197 & 2.197 & 17.576 \\
\hline 6.591 & 28.561 & 26.364 & 2787.99 \\
\hline 46.137 & 13.182 & 4.394 & 52.728 \\
\hline 26.364 & 46.137 & 19.773 & 2.197 \\
\hline 8.788 & 2.197 & 72.501 & 4.394 \\
\hline 4.394 & 2.197 & 2.197 & 10.985 \\
\hline 4.394 & 2.197 & 2.197 & 2.197 \\
\hline 19.773 & 94.471 & 74.698 & 17.576 \\
\hline 17.576 & 2.197 & 4.394 & 57.122 \\
\hline 2.197 & 13.182 & 2.197 & 2.197 \\
\hline 107.653 & 19.773 & 4.394 & 2.197 \\
\hline 10.985 & 2.197 & 37.349 & 21.97 \\
\hline 1212.74 & 2.197 & 2.197 & 24.167 \\
\hline 4.394 & 6.591 & 2.197 & 8.788 \\
\hline 17.576 & 13.182 & 46.137 & 2.197 \\
\hline 10.985 & 2.197 & 2.197 & 43.94 \\
\hline 8.788 & 4.394 & 2.197 & 2.197 \\
\hline
\end{tabular}




\begin{tabular}{|c|c|c|c|}
\hline 63.713 & 2.197 & 15.379 & 15.379 \\
\hline 4.394 & 4.394 & 2.197 & 15.379 \\
\hline 2.197 & 2.197 & 87.88 & 2.197 \\
\hline 79.092 & 4.394 & 4.394 & 74.698 \\
\hline 24.167 & 2.197 & 4.394 & 4.394 \\
\hline 8.788 & 2.197 & 2.197 & 6.591 \\
\hline 8.788 & 481.143 & 4.394 & 2.197 \\
\hline 8.788 & 1239.11 & 2.197 & 26.364 \\
\hline 26.364 & 2.197 & 2.197 & 2.197 \\
\hline 15.379 & 24.167 & 32.955 & 8.788 \\
\hline 10.985 & 276.822 & 39.546 & 41.743 \\
\hline 4.394 & 4.394 & 2.197 & 17.576 \\
\hline 8.788 & 2.197 & 2.197 & 6.591 \\
\hline 2.197 & 8.788 & 8.788 & 265.837 \\
\hline 2.197 & 2.197 & 2.197 & 39.546 \\
\hline 268.034 & 162.578 & 2.197 & 26.364 \\
\hline 4.394 & 13.182 & 19.773 & 21.97 \\
\hline 2.197 & 2.197 & 17.576 & 1023.8 \\
\hline 2.197 & 105.456 & 13.182 & 107.653 \\
\hline 13.182 & 2.197 & 35.152 & 32.955 \\
\hline 4.394 & 4.394 & 8.788 & 1920.18 \\
\hline 2.197 & 17.576 & 4.394 & 114.244 \\
\hline 131.82 & 2.197 & 50.531 & 158.184 \\
\hline 944.71 & 4.394 & 4.394 & 37.349 \\
\hline 32.955 & 2.197 & 1357.75 & 2.197 \\
\hline 4.394 & 74.698 & 2.197 & 6.591 \\
\hline 8.788 & 2.197 & 4.394 & 28.561 \\
\hline 6.591 & 28.561 & 2.197 & 94.471 \\
\hline 10.985 & 2.197 & 6.591 & 65.91 \\
\hline 4.394 & 4.394 & 4.394 & 74.698 \\
\hline 10.985 & 6.591 & 2.197 & 43.94 \\
\hline 2.197 & 13.182 & 90.077 & 2.197 \\
\hline 2.197 & 2.197 & 2.197 & 2.197 \\
\hline 96.668 & 2.197 & 173.563 & 4.394 \\
\hline 92.274 & 6.591 & 2.197 & 2.197 \\
\hline 21.97 & 4.394 & 2.197 & 164.775 \\
\hline 21.97 & 2.197 & 2.197 & 10.985 \\
\hline 52.728 & 2.197 & 153.79 & 2.197 \\
\hline 2.197 & 2.197 & 2.197 & 6.591 \\
\hline 15.379 & 2.197 & 2.197 & 8.788 \\
\hline 46.137 & 57.122 & 4.394 & 2.197 \\
\hline 13.182 & 61.516 & 4.394 & 8.788 \\
\hline 197.73 & 4.394 & 2.197 & 46343.5 \\
\hline 8.788 & 83.486 & 2.197 & 8.788 \\
\hline 6.591 & 4.394 & 6.591 & 522.886 \\
\hline 15.379 & 659.1 & 4.394 & 2.197 \\
\hline 57.122 & 61.516 & 138.411 & 13.182 \\
\hline 2.197 & 19.773 & 2.197 & 2.197 \\
\hline 2.197 & 2.197 & 2.197 & 4.394 \\
\hline 2.197 & 8.788 & 2.197 & 15.379 \\
\hline
\end{tabular}




\begin{tabular}{|c|c|c|c|}
\hline 8.788 & 10.985 & 52.728 & 107.653 \\
\hline 17.576 & 13.182 & 6.591 & 8.788 \\
\hline 2.197 & 2.197 & 2.197 & 19.773 \\
\hline 28.561 & 2.197 & 2.197 & 17.576 \\
\hline 17.576 & 208.715 & 6.591 & 2.197 \\
\hline 213.109 & 17.576 & 2.197 & 57.122 \\
\hline 2.197 & 32.955 & 15.379 & 6.591 \\
\hline 4.394 & 2.197 & 6.591 & 90.077 \\
\hline 2.197 & 15.379 & 10.985 & 68.107 \\
\hline 186.745 & 13.182 & 28.561 & 6.591 \\
\hline 17.576 & 2.197 & 54.925 & 2.197 \\
\hline 700.843 & 2.197 & 2.197 & 4.394 \\
\hline 4.394 & 59.319 & 116.441 & 105.456 \\
\hline 4.394 & 72.501 & 15.379 & 72.501 \\
\hline 15.379 & 4.394 & 2.197 & 2.197 \\
\hline 2.197 & 2.197 & 2.197 & 2.197 \\
\hline 10.985 & 15.379 & 2.197 & 35.152 \\
\hline 2.197 & 4.394 & 2.197 & 2.197 \\
\hline 2.197 & 41.743 & 2.197 & 10.985 \\
\hline 50.531 & 4.394 & 15.379 & 6.591 \\
\hline 112.047 & 2.197 & 6.591 & 4.394 \\
\hline 2.197 & 2.197 & 10.985 & 35.152 \\
\hline 4.394 & 8.788 & 17.576 & 8.788 \\
\hline 2.197 & 2.197 & 4.394 & 81.289 \\
\hline 2.197 & 70.304 & 10.985 & 10.985 \\
\hline 28.561 & 6.591 & 54.925 & 26.364 \\
\hline 10.985 & 2.197 & 2.197 & 46.137 \\
\hline 4.394 & 26.364 & 2.197 & 2.197 \\
\hline 2.197 & 2.197 & 4.394 & 24.167 \\
\hline 2.197 & 2.197 & 4.394 & 15.379 \\
\hline 2.197 & 15.379 & 6.591 & 43.94 \\
\hline 13.182 & 4.394 & 2.197 & 24.167 \\
\hline 32.955 & 57.122 & 2.197 & 2.197 \\
\hline 41.743 & 98.865 & 2.197 & 24.167 \\
\hline 4.394 & 41.743 & 4.394 & 26.364 \\
\hline 2.197 & 4.394 & 2.197 & 2.197 \\
\hline 76.895 & 2.197 & 19.773 & 6.591 \\
\hline 4.394 & 2.197 & 2.197 & 2.197 \\
\hline 2.197 & 26.364 & 2.197 & 448.188 \\
\hline 2.197 & 2.197 & 2.197 & 8.788 \\
\hline 2.197 & 39.546 & 2.197 & 3170.27 \\
\hline 35.152 & 2.197 & 74.698 & 19.773 \\
\hline 81.289 & 2.197 & 2.197 & 8.788 \\
\hline 15.379 & 28.561 & 41.743 & 19.773 \\
\hline 2.197 & 94.471 & 32.955 & 10.985 \\
\hline 8.788 & 24.167 & 1168.8 & 17.576 \\
\hline 4.394 & 397.657 & 24.167 & 52.728 \\
\hline 15.379 & 24.167 & 2.197 & 28.561 \\
\hline 4.394 & 4.394 & 13.182 & 76.895 \\
\hline 4.394 & 10.985 & 1054.56 & 8.788 \\
\hline
\end{tabular}




\begin{tabular}{|c|c|c|c|}
\hline 2.197 & 6.591 & 4.394 & 30.758 \\
\hline 50.531 & 59.319 & 32.955 & 10.985 \\
\hline 4.394 & 2.197 & 2.197 & 15.379 \\
\hline 1102.89 & 2.197 & 2.197 & 42349.4 \\
\hline 6.591 & 57.122 & 2.197 & 2.197 \\
\hline 46.137 & 2.197 & 2.197 & 2.197 \\
\hline 2.197 & 6.591 & 17.576 & 4.394 \\
\hline 21.97 & 15.379 & 2.197 & 2.197 \\
\hline 6.591 & 109.85 & 6.591 & 4.394 \\
\hline 6.591 & 21.97 & 10.985 & 2.197 \\
\hline 2.197 & 8.788 & 2.197 & 24.167 \\
\hline 2.197 & 10.985 & 41.743 & 17.576 \\
\hline 2.197 & 28.561 & 254.852 & 30.758 \\
\hline 2.197 & 4.394 & 46.137 & 68.107 \\
\hline 26.364 & 2.197 & 1019.41 & 21.97 \\
\hline 83.486 & 8.788 & 19.773 & 492.128 \\
\hline 2.197 & 2.197 & 13.182 & 6.591 \\
\hline 2.197 & 2.197 & 90.077 & 6.591 \\
\hline 8.788 & 2.197 & 6.591 & 2.197 \\
\hline 2.197 & 24.167 & 6.591 & 10.985 \\
\hline 2.197 & 2.197 & 2.197 & 21.97 \\
\hline 32.955 & 2.197 & 24.167 & 2.197 \\
\hline 13.182 & 8.788 & 15.379 & 2.197 \\
\hline 4.394 & 6.591 & 2.197 & 10.985 \\
\hline 219.7 & 2.197 & 2.197 & 329.55 \\
\hline 2.197 & 2.197 & 4.394 & 6.591 \\
\hline 4.394 & 35.152 & 2.197 & 8.788 \\
\hline 17.576 & 59.319 & 2.197 & 17.576 \\
\hline 2.197 & 2.197 & 4.394 & 48.334 \\
\hline 35.152 & 4.394 & 37.349 & 4.394 \\
\hline 87.88 & 46.137 & 243.867 & 2.197 \\
\hline 10.985 & 6.591 & 4.394 & 2.197 \\
\hline 2.197 & 6.591 & 2.197 & 13.182 \\
\hline 2.197 & 17.576 & 2.197 & 15.379 \\
\hline 2.197 & 6.591 & 85.683 & 6.591 \\
\hline 10.985 & 2.197 & 114.244 & 21.97 \\
\hline 2.197 & 19.773 & 4.394 & 10.985 \\
\hline 10.985 & 105.456 & 153.79 & 17.576 \\
\hline 4.394 & 355.914 & 4.394 & 52.728 \\
\hline 2.197 & 2.197 & 10.985 & 10.985 \\
\hline 2.197 & 32.955 & 8.788 & 8.788 \\
\hline 6.591 & 2.197 & 2.197 & 24.167 \\
\hline 21.97 & 2.197 & 17.576 & 10.985 \\
\hline 8.788 & 140.608 & 2.197 & 8.788 \\
\hline 116.441 & 2.197 & 4.394 & 746.98 \\
\hline 19.773 & 2.197 & 2.197 & 4.394 \\
\hline 2.197 & 2.197 & 28.561 & 63.713 \\
\hline 17.576 & 4.394 & 2.197 & 13.182 \\
\hline 2.197 & 2.197 & 4.394 & 4.394 \\
\hline 2.197 & 2.197 & 13.182 & 2.197 \\
\hline
\end{tabular}




\begin{tabular}{|c|c|c|c|}
\hline 2.197 & 4.394 & 2.197 & 28.561 \\
\hline 21.97 & 8.788 & 2.197 & 13.182 \\
\hline 72.501 & 6.591 & 2.197 & 35.152 \\
\hline 19.773 & 2.197 & 2.197 & 10.985 \\
\hline 24.167 & 2.197 & 2.197 & 2.197 \\
\hline 2.197 & 6.591 & 2.197 & 8.788 \\
\hline 79.092 & 2.197 & 2.197 & 8.788 \\
\hline 10.985 & 30.758 & 10.985 & 2.197 \\
\hline 2.197 & 4.394 & 2.197 & 145.002 \\
\hline 24.167 & 2.197 & 2.197 & 263.64 \\
\hline 2.197 & 10.985 & 2.197 & 246.064 \\
\hline 17.576 & 26.364 & 17.576 & 10.985 \\
\hline 13.182 & 432.809 & 2.197 & 32.955 \\
\hline 24.167 & 28.561 & 2.197 & 2.197 \\
\hline 4.394 & 26.364 & 2.197 & 57.122 \\
\hline 74.698 & 755.768 & 114.244 & 52.728 \\
\hline 2.197 & 4.394 & 2.197 & 2.197 \\
\hline 4.394 & 2.197 & 21.97 & 2.197 \\
\hline 8.788 & 2.197 & 35.152 & 4.394 \\
\hline 4.394 & 57.122 & 2.197 & 2.197 \\
\hline 4.394 & 426.218 & 17.576 & 17.576 \\
\hline 2.197 & 48.334 & 118.638 & 2.197 \\
\hline 2.197 & 28.561 & 6.591 & 2.197 \\
\hline 2.197 & 6.591 & 37.349 & 2.197 \\
\hline 153.79 & 30.758 & 30.758 & 10.985 \\
\hline 241.67 & 50.531 & 19.773 & 2.197 \\
\hline 2.197 & 37.349 & 92.274 & 19.773 \\
\hline 17.576 & 6.591 & 4.394 & 2.197 \\
\hline 4.394 & 57.122 & 4.394 & 54.925 \\
\hline 1430.25 & 41.743 & 1830.1 & 105.456 \\
\hline 129.623 & 2.197 & 19.773 & 2.197 \\
\hline 2.197 & 10.985 & 17.576 & 2.197 \\
\hline 2.197 & 2.197 & 4.394 & 15.379 \\
\hline 4.394 & 13.182 & 8.788 & 4.394 \\
\hline 13.182 & 2.197 & 109.85 & 8.788 \\
\hline 4.394 & 2.197 & 6.591 & 2.197 \\
\hline 6.591 & 10.985 & 485.537 & 10.985 \\
\hline 28.561 & 43.94 & 4.394 & 17.576 \\
\hline 4.394 & 6.591 & 2.197 & 13.182 \\
\hline 28.561 & 2.197 & 8.788 & 8.788 \\
\hline 6.591 & 285.61 & 6.591 & 10.985 \\
\hline 2.197 & 4.394 & 4.394 & 4.394 \\
\hline 2.197 & 4.394 & 10.985 & 2.197 \\
\hline 410.839 & 6.591 & 2.197 & 65.91 \\
\hline 6.591 & 140.608 & 2.197 & 250.458 \\
\hline 10.985 & 8.788 & 6.591 & 50.531 \\
\hline 8.788 & 50.531 & 6.591 & 2.197 \\
\hline 2.197 & 83.486 & 48.334 & 4.394 \\
\hline 2.197 & 90.077 & 2.197 & 188.942 \\
\hline 6.591 & 149.396 & 2.197 & 2.197 \\
\hline
\end{tabular}




\begin{tabular}{|c|c|c|c|}
\hline 4.394 & 8.788 & 6.591 & 4.394 \\
\hline 52.728 & 4.394 & 6.591 & 13.182 \\
\hline 2.197 & 8.788 & 2.197 & 226.291 \\
\hline 2.197 & 24.167 & 2.197 & 4.394 \\
\hline 4.394 & 46.137 & 21.97 & 35.152 \\
\hline 37.349 & 4.394 & 230.685 & 37.349 \\
\hline 2.197 & 10.985 & 2.197 & 57.122 \\
\hline 19.773 & 8.788 & 241.67 & 8.788 \\
\hline 112.047 & 8.788 & 2.197 & 10.985 \\
\hline 2.197 & 8.788 & 4.394 & 10.985 \\
\hline 2.197 & 136.214 & 120.835 & 105.456 \\
\hline 243.867 & 6.591 & 2.197 & 6.591 \\
\hline 287.807 & 2.197 & 2.197 & 19.773 \\
\hline 15.379 & 4.394 & 136.214 & 68.107 \\
\hline 70.304 & 4.394 & 2.197 & 1742.22 \\
\hline 2.197 & 127.426 & 4.394 & 72.501 \\
\hline 28.561 & 48.334 & 10.985 & 2.197 \\
\hline 2.197 & 123.032 & 2.197 & 164.775 \\
\hline 4.394 & 101.062 & 2.197 & 2.197 \\
\hline 26.364 & 164.775 & 2.197 & 4.394 \\
\hline 26.364 & 4.394 & 953.498 & 171.366 \\
\hline 2.197 & 6.591 & 2.197 & 2.197 \\
\hline 19.773 & 4.394 & 2.197 & 2.197 \\
\hline 28.561 & 4.394 & 4.394 & 2.197 \\
\hline 2.197 & 2.197 & 2.197 & 43.94 \\
\hline 13.182 & 4.394 & 2.197 & 26.364 \\
\hline 28.561 & 4.394 & 2.197 & 70.304 \\
\hline 4.394 & 8.788 & 2.197 & 10.985 \\
\hline 19.773 & 17.576 & 2.197 & 59.319 \\
\hline 2.197 & 2.197 & 4.394 & 37.349 \\
\hline 6.591 & 19.773 & 2.197 & 19.773 \\
\hline 6.591 & 2.197 & 2.197 & 131.82 \\
\hline 2.197 & 13.182 & 2.197 & 59.319 \\
\hline 6.591 & 2.197 & 6.591 & 17.576 \\
\hline 6.591 & 2.197 & 4.394 & 2.197 \\
\hline 37.349 & 8.788 & 32.955 & 2.197 \\
\hline 79.092 & 8.788 & 4.394 & 17.576 \\
\hline 2.197 & 13.182 & 112.047 & 2.197 \\
\hline 2.197 & 15.379 & 4.394 & 17.576 \\
\hline 2.197 & 15.379 & 2.197 & 10.985 \\
\hline 61.516 & 10.985 & 2.197 & 10.985 \\
\hline 46.137 & 254.852 & 2.197 & 50.531 \\
\hline 6.591 & 63.713 & 30.758 & 24.167 \\
\hline 24.167 & 6.591 & 2.197 & 74.698 \\
\hline 615.16 & 4.394 & 6.591 & 2.197 \\
\hline 2.197 & 54.925 & 2.197 & 43.94 \\
\hline 32.955 & 338.338 & 2.197 & 6.591 \\
\hline 6.591 & 4.394 & 2.197 & 272.428 \\
\hline 32.955 & 26.364 & 2.197 & 6.591 \\
\hline 87.88 & 6.591 & 4.394 & 2.197 \\
\hline
\end{tabular}




\begin{tabular}{|c|c|c|c|}
\hline 2.197 & 39.546 & 2.197 & 70.304 \\
\hline 2.197 & 72.501 & 6.591 & 325.156 \\
\hline 39.546 & 39.546 & 8.788 & 2.197 \\
\hline 63.713 & 26.364 & 2.197 & 17.576 \\
\hline 171.366 & 2.197 & 61.516 & 32.955 \\
\hline 2.197 & 4.394 & 4.394 & 2.197 \\
\hline 21.97 & 2.197 & 6.591 & 2.197 \\
\hline 35.152 & 37.349 & 6.591 & 10.985 \\
\hline 2.197 & 788.723 & 2.197 & 26.364 \\
\hline 32.955 & 24.167 & 2.197 & 2.197 \\
\hline 21.97 & 43.94 & 79.092 & 2.197 \\
\hline 2.197 & 15.379 & 41.743 & 2.197 \\
\hline 6.591 & 6.591 & 155.987 & 19.773 \\
\hline 2.197 & 13.182 & 4.394 & 48.334 \\
\hline 2.197 & 26.364 & 2.197 & 81.289 \\
\hline 19.773 & 19.773 & 2.197 & 6.591 \\
\hline 2.197 & 57.122 & 4.394 & 48.334 \\
\hline 2.197 & 2.197 & 4.394 & 59.319 \\
\hline 2.197 & 17.576 & 15.379 & 107.653 \\
\hline 6.591 & 2.197 & 17.576 & 50.531 \\
\hline 2.197 & 210.912 & 2.197 & 149.396 \\
\hline 98.865 & 2.197 & 1713.66 & 8.788 \\
\hline 2.197 & 2.197 & 4.394 & 2.197 \\
\hline 2.197 & 6.591 & 17.576 & 15.379 \\
\hline 2.197 & 2.197 & 2.197 & 13.182 \\
\hline 2.197 & 4.394 & 2.197 & 4.394 \\
\hline 35.152 & 2.197 & 2.197 & 15.379 \\
\hline 2.197 & 2.197 & 28.561 & 4.394 \\
\hline 2.197 & 2.197 & 316.368 & 2.197 \\
\hline 4.394 & 2.197 & 8.788 & 72.501 \\
\hline 290.004 & 410.839 & 4.394 & 35.152 \\
\hline 6.591 & 68.107 & 2.197 & 2.197 \\
\hline 6.591 & 4.394 & 2.197 & 30.758 \\
\hline 4.394 & 57.122 & 2.197 & 63.713 \\
\hline 6.591 & 28.561 & 2.197 & 2.197 \\
\hline 2.197 & 2.197 & 2.197 & 6.591 \\
\hline 2.197 & 28.561 & 19.773 & 105.456 \\
\hline 8.788 & 4.394 & 13.182 & 2.197 \\
\hline 2.197 & 61.516 & 2.197 & 76.895 \\
\hline 8.788 & 4.394 & 4.394 & 13.182 \\
\hline 10.985 & 41.743 & 2.197 & 116.441 \\
\hline 2.197 & 8.788 & 10.985 & 6.591 \\
\hline 10.985 & 4.394 & 2.197 & 8.788 \\
\hline 24.167 & 8.788 & 2.197 & 21.97 \\
\hline 4.394 & 4.394 & 2.197 & 1935.56 \\
\hline 4.394 & 2.197 & 371.293 & 2.197 \\
\hline 32.955 & 186.745 & 116.441 & 6.591 \\
\hline 8.788 & 6.591 & 41.743 & 6.591 \\
\hline 2.197 & 2.197 & 639.327 & 4.394 \\
\hline 6.591 & 145.002 & 1232.52 & 26.364 \\
\hline
\end{tabular}




\begin{tabular}{|c|c|c|c|}
\hline 10.985 & 15.379 & 6.591 & 6.591 \\
\hline 15.379 & 2.197 & 4.394 & 2.197 \\
\hline 142.805 & 2.197 & 2.197 & 2.197 \\
\hline 21.97 & 4.394 & 2.197 & 4.394 \\
\hline 127.426 & 2.197 & 2.197 & 6.591 \\
\hline 15.379 & 6.591 & 131.82 & 8.788 \\
\hline 2.197 & 4.394 & 2.197 & 151.593 \\
\hline 26.364 & 17.576 & 10.985 & 46.137 \\
\hline 79.092 & 2.197 & 2.197 & 6.591 \\
\hline 26.364 & 15.379 & 2.197 & 8.788 \\
\hline 10.985 & 26.364 & 19.773 & 2.197 \\
\hline 4.394 & 2.197 & 2.197 & 63.713 \\
\hline 114.244 & 2.197 & 406.445 & 202.124 \\
\hline 21.97 & 4.394 & 213.109 & 32.955 \\
\hline 2.197 & 2.197 & 50.531 & 10.985 \\
\hline 2.197 & 2.197 & 8.788 & 2.197 \\
\hline 4.394 & 230.685 & 2.197 & 2.197 \\
\hline 39.546 & 4.394 & 2.197 & 2.197 \\
\hline 2.197 & 48.334 & 2.197 & 4.394 \\
\hline 17.576 & 151.593 & 2.197 & 322.959 \\
\hline 166.972 & 4.394 & 2.197 & 2.197 \\
\hline 4.394 & 2.197 & 2.197 & 2.197 \\
\hline 105.456 & 2.197 & 2.197 & 37.349 \\
\hline 8.788 & 41.743 & 2.197 & 17.576 \\
\hline 2.197 & 5512.27 & 4.394 & 8.788 \\
\hline 15.379 & 54.925 & 4.394 & 103.259 \\
\hline 21.97 & 10.985 & 2.197 & 24.167 \\
\hline 116.441 & 114.244 & 2.197 & 32.955 \\
\hline 26.364 & 382.278 & 2.197 & 2.197 \\
\hline 177.957 & 15.379 & 2.197 & 265.837 \\
\hline 8.788 & 15.379 & 2.197 & 8.788 \\
\hline 2.197 & 26.364 & 145.002 & 13.182 \\
\hline 2.197 & 21.97 & 105.456 & 2.197 \\
\hline 2.197 & 2.197 & 10.985 & 268.034 \\
\hline 2.197 & 19.773 & 15.379 & 13.182 \\
\hline 85.683 & 8.788 & 2.197 & 79.092 \\
\hline 153.79 & 8.788 & 2.197 & 393.263 \\
\hline 10.985 & 2.197 & 4.394 & 268.034 \\
\hline 320.762 & 199.927 & 2.197 & 19.773 \\
\hline 74.698 & 2.197 & 2.197 & 35.152 \\
\hline 28.561 & 6.591 & 2.197 & 4.394 \\
\hline 4.394 & 35.152 & 2.197 & 2.197 \\
\hline 4.394 & 2.197 & 193.336 & 24.167 \\
\hline 107.653 & 4.394 & 10.985 & 26.364 \\
\hline 2.197 & 2.197 & 2.197 & 8.788 \\
\hline 2.197 & 2.197 & 4.394 & 76.895 \\
\hline 2.197 & 2.197 & 2.197 & 17.576 \\
\hline 1915.78 & 4.394 & 2.197 & 125.229 \\
\hline 2.197 & 2.197 & 15.379 & 6.591 \\
\hline 6.591 & 4.394 & 2.197 & 4.394 \\
\hline
\end{tabular}




\begin{tabular}{|c|c|c|c|}
\hline 2.197 & 4.394 & 2.197 & 15.379 \\
\hline 2.197 & 26.364 & 4.394 & 6.591 \\
\hline 19.773 & 4.394 & 2.197 & 2.197 \\
\hline 28.561 & 8.788 & 2.197 & 2.197 \\
\hline 92.274 & 6.591 & 21.97 & 6.591 \\
\hline 4.394 & 19.773 & 123.032 & 2.197 \\
\hline 19.773 & 213.109 & 134.017 & 28.561 \\
\hline 4.394 & 2.197 & 6.591 & 70.304 \\
\hline 2.197 & 2.197 & 2.197 & 4.394 \\
\hline 4.394 & 6.591 & 81.289 & 52.728 \\
\hline 15.379 & 2.197 & 10.985 & 17.576 \\
\hline 15.379 & 2.197 & 4.394 & 4.394 \\
\hline 17.576 & 239.473 & 65.91 & 2.197 \\
\hline 4.394 & 46.137 & 24.167 & 13.182 \\
\hline 2.197 & 175.76 & 4.394 & 17.576 \\
\hline 8.788 & 6.591 & 2.197 & 8.788 \\
\hline 26.364 & 30.758 & 24.167 & 8.788 \\
\hline 26.364 & 2.197 & 2.197 & 4.394 \\
\hline 2.197 & 10.985 & 2.197 & 2.197 \\
\hline 4.394 & 48.334 & 13.182 & 257.049 \\
\hline 4.394 & 10.985 & 2.197 & 13.182 \\
\hline 2.197 & 63.713 & 10.985 & 13.182 \\
\hline 2.197 & 166.972 & 2.197 & 48.334 \\
\hline 4.394 & 19.773 & 2.197 & 2.197 \\
\hline 101.062 & 35.152 & 2.197 & 8.788 \\
\hline 6.591 & 2.197 & 303.186 & 21.97 \\
\hline 65.91 & 136.214 & 175.76 & 15.379 \\
\hline 103.259 & 13.182 & 59.319 & 98.865 \\
\hline 15.379 & 2.197 & 2.197 & 2.197 \\
\hline 2.197 & 2.197 & 2.197 & 2.197 \\
\hline 2.197 & 6.591 & 2.197 & 252.655 \\
\hline 2.197 & 24.167 & 2.197 & 2.197 \\
\hline 2.197 & 4.394 & 2.197 & 2.197 \\
\hline 2.197 & 2.197 & 4.394 & 65.91 \\
\hline 21.97 & 2.197 & 6.591 & 329.55 \\
\hline 2.197 & 4.394 & 13.182 & 2.197 \\
\hline 79.092 & 2.197 & 2.197 & 13.182 \\
\hline 30.758 & 13.182 & 35.152 & 68.107 \\
\hline 2.197 & 2.197 & 15.379 & 21.97 \\
\hline 2.197 & 191.139 & 2.197 & 2.197 \\
\hline 10.985 & 2.197 & 2.197 & 37.349 \\
\hline 74.698 & 2.197 & 2.197 & 19.773 \\
\hline 623.948 & 2.197 & 72.501 & 1461 \\
\hline 26.364 & 37.349 & 6.591 & 39.546 \\
\hline 2.197 & 4.394 & 2.197 & 76.895 \\
\hline 79.092 & 30.758 & 6.591 & 6.591 \\
\hline 2.197 & 2.197 & 48.334 & 13.182 \\
\hline 39.546 & 4.394 & 1195.17 & 48.334 \\
\hline 19.773 & 4.394 & 2.197 & 35.152 \\
\hline 2.197 & 15.379 & 2.197 & 241.67 \\
\hline
\end{tabular}




\begin{tabular}{|c|c|c|c|}
\hline 4.394 & 26.364 & 2.197 & 2.197 \\
\hline 4.394 & 6.591 & 6.591 & 8.788 \\
\hline 2.197 & 2.197 & 2.197 & 2.197 \\
\hline 81.289 & 2.197 & 2.197 & 406.445 \\
\hline 35.152 & 2.197 & 8.788 & 6.591 \\
\hline 2.197 & 2.197 & 4.394 & 2.197 \\
\hline 28.561 & 114.244 & 2.197 & 21.97 \\
\hline 32.955 & 2.197 & 2.197 & 136.214 \\
\hline 2.197 & 8.788 & 4.394 & 13.182 \\
\hline 15.379 & 30.758 & 21.97 & 320.762 \\
\hline 2.197 & 221.897 & 15.379 & 2.197 \\
\hline 4.394 & 15.379 & 10.985 & 257.049 \\
\hline 10.985 & 2.197 & 366.899 & 68.107 \\
\hline 37.349 & 13.182 & 2.197 & 2.197 \\
\hline 2.197 & 21.97 & 2.197 & 4.394 \\
\hline 65.91 & 13.182 & 2.197 & 6.591 \\
\hline 2.197 & 50.531 & 2.197 & 4.394 \\
\hline 2.197 & 4.394 & 28.561 & 2.197 \\
\hline 32.955 & 279.019 & 2.197 & 6.591 \\
\hline 2.197 & 83.486 & 50.531 & 50.531 \\
\hline 2.197 & 15.379 & 2.197 & 2.197 \\
\hline 21.97 & 164.775 & 2.197 & 10.985 \\
\hline 68.107 & 19.773 & 28.561 & 68.107 \\
\hline 4.394 & 257.049 & 114.244 & 2.197 \\
\hline 2.197 & 2.197 & 15.379 & 4.394 \\
\hline 19.773 & 13.182 & 4.394 & 39.546 \\
\hline 63.713 & 2.197 & 8.788 & 134.017 \\
\hline 65.91 & 24.167 & 2.197 & 2.197 \\
\hline 2.197 & 4.394 & 15.379 & 26.364 \\
\hline 13.182 & 169.169 & 2.197 & 4.394 \\
\hline 2.197 & 2.197 & 8.788 & 2.197 \\
\hline 8.788 & 2.197 & 4.394 & 21.97 \\
\hline 2.197 & 15.379 & 30.758 & 2.197 \\
\hline 6.591 & 4.394 & 2.197 & 6.591 \\
\hline 2.197 & 13.182 & 15.379 & 2.197 \\
\hline 177.957 & 4.394 & 6.591 & 8.788 \\
\hline 15.379 & 6.591 & 2.197 & 8.788 \\
\hline 39.546 & 4.394 & 4.394 & 26.364 \\
\hline 15.379 & 57.122 & 6.591 & 131.82 \\
\hline 2.197 & 15.379 & 114.244 & 4.394 \\
\hline 2.197 & 6.591 & 2.197 & 39.546 \\
\hline 213.109 & 2.197 & 6.591 & 15.379 \\
\hline 68.107 & 2.197 & 2.197 & 10.985 \\
\hline 2.197 & 2.197 & 2.197 & 720.616 \\
\hline 24.167 & 50.531 & 417.43 & 41.743 \\
\hline 19.773 & 2.197 & 290.004 & 2069.57 \\
\hline 4.394 & 17.576 & 2.197 & 83.486 \\
\hline 59.319 & 19.773 & 26.364 & 2.197 \\
\hline 659.1 & 57.122 & 24.167 & 54.925 \\
\hline 4.394 & 2.197 & 2.197 & 24.167 \\
\hline
\end{tabular}




\begin{tabular}{|c|c|c|c|}
\hline 48.334 & 2.197 & 39.546 & 54.925 \\
\hline 8.788 & 26.364 & 4.394 & 8.788 \\
\hline 39.546 & 19.773 & 2.197 & 6.591 \\
\hline 6.591 & 4.394 & 2.197 & 6.591 \\
\hline 96.668 & 10.985 & 845.845 & 169.169 \\
\hline 202.124 & 118.638 & 43.94 & 443.794 \\
\hline 2.197 & 166.972 & 6.591 & 2.197 \\
\hline 210.912 & 1445.63 & 294.398 & 4.394 \\
\hline 2.197 & 15.379 & 4.394 & 162.578 \\
\hline 15.379 & 13.182 & 8.788 & 37.349 \\
\hline 2.197 & 4.394 & 2.197 & 2.197 \\
\hline 2.197 & 2.197 & 8.788 & 2678.14 \\
\hline 6.591 & 26.364 & 2.197 & 17.576 \\
\hline 6.591 & 6.591 & 19.773 & 4.394 \\
\hline 6.591 & 2.197 & 2.197 & 2.197 \\
\hline 94.471 & 2.197 & 2.197 & 72.501 \\
\hline 2.197 & 10.985 & 6.591 & 24.167 \\
\hline 48.334 & 2.197 & 2.197 & 2.197 \\
\hline 4.394 & 2.197 & 2.197 & 8.788 \\
\hline 26.364 & 8.788 & 2.197 & 15.379 \\
\hline 2.197 & 142.805 & 2.197 & 19.773 \\
\hline 8.788 & 4.394 & 6.591 & 12353.7 \\
\hline 4.394 & 17.576 & 2.197 & 32.955 \\
\hline 2.197 & 61.516 & 17.576 & 76.895 \\
\hline 134.017 & 41.743 & 61.516 & 15.379 \\
\hline 2.197 & 43.94 & 6.591 & 43.94 \\
\hline 2.197 & 149.396 & 26.364 & 2.197 \\
\hline 4.394 & 2.197 & 2.197 & 197.73 \\
\hline 2.197 & 4.394 & 2.197 & 2.197 \\
\hline 6.591 & 485.537 & 8.788 & 2.197 \\
\hline 94.471 & 48.334 & 4.394 & 4.394 \\
\hline 2.197 & 74.698 & 127.426 & 2.197 \\
\hline 2.197 & 24.167 & 2.197 & 4.394 \\
\hline 8.788 & 2.197 & 2.197 & 2.197 \\
\hline 10.985 & 4.394 & 15.379 & 4.394 \\
\hline 57.122 & 4.394 & 2.197 & 2.197 \\
\hline 8.788 & 17.576 & 2.197 & 90.077 \\
\hline 6.591 & 2.197 & 15.379 & 2.197 \\
\hline 6.591 & 15.379 & 2.197 & 37.349 \\
\hline 2.197 & 2.197 & 83.486 & 6.591 \\
\hline 2.197 & 2.197 & 26.364 & 13.182 \\
\hline 2.197 & 2.197 & 21.97 & 54.925 \\
\hline 2.197 & 48.334 & 68.107 & 43.94 \\
\hline 2.197 & 4.394 & 39.546 & 4.394 \\
\hline 2.197 & 6.591 & 2.197 & 2.197 \\
\hline 4.394 & 15.379 & 2.197 & 13.182 \\
\hline 4.394 & 15.379 & 2.197 & 6.591 \\
\hline 2.197 & 2.197 & 92.274 & 41.743 \\
\hline 2.197 & 2.197 & 4.394 & 2.197 \\
\hline 2.197 & 41.743 & 2.197 & 2.197 \\
\hline
\end{tabular}




\begin{tabular}{|c|c|c|c|}
\hline 8.788 & 8.788 & 8.788 & 50.531 \\
\hline 96.668 & 4.394 & 10.985 & 10.985 \\
\hline 4.394 & 2.197 & 4.394 & 1950.94 \\
\hline 4.394 & 2.197 & 2.197 & 28.561 \\
\hline 311.974 & 4.394 & 19.773 & 2.197 \\
\hline 1825.71 & 2.197 & 107.653 & 10.985 \\
\hline 2.197 & 76.895 & 43.94 & 4.394 \\
\hline 10.985 & 2.197 & 4.394 & 15.379 \\
\hline 10.985 & 4.394 & 6.591 & 2.197 \\
\hline 808.496 & 4.394 & 81.289 & 32.955 \\
\hline 2.197 & 4.394 & 142.805 & 72.501 \\
\hline 15.379 & 2.197 & 79.092 & 6.591 \\
\hline 298.792 & 105.456 & 2.197 & 2.197 \\
\hline 164.775 & 155.987 & 8.788 & 8.788 \\
\hline 2.197 & 8.788 & 2.197 & 2.197 \\
\hline 8.788 & 2.197 & 2.197 & 8.788 \\
\hline 85.683 & 4.394 & 4.394 & 13.182 \\
\hline 6.591 & 2.197 & 10.985 & 15.379 \\
\hline 2.197 & 208.715 & 727.207 & 107.653 \\
\hline 257.049 & 2.197 & 1425.85 & 2.197 \\
\hline 17.576 & 4.394 & 2.197 & 2.197 \\
\hline 32.955 & 10.985 & 4.394 & 2.197 \\
\hline 1981.69 & 226.291 & 6.591 & 2.197 \\
\hline 2.197 & 13.182 & 2.197 & 57.122 \\
\hline 4.394 & 87.88 & 2.197 & 39.546 \\
\hline 21.97 & 8.788 & 48.334 & 2.197 \\
\hline 52.728 & 2.197 & 37.349 & 13.182 \\
\hline 4.394 & 17.576 & 2.197 & 41.743 \\
\hline 4.394 & 134.017 & 15.379 & 116.441 \\
\hline 2.197 & 72.501 & 2.197 & 2.197 \\
\hline 8.788 & 2.197 & 4.394 & 2.197 \\
\hline 4.394 & 2.197 & 4.394 & 2.197 \\
\hline 4.394 & 4.394 & 6.591 & 2.197 \\
\hline 2.197 & 4.394 & 81.289 & 2.197 \\
\hline 4.394 & 303.186 & 166.972 & 24.167 \\
\hline 525.083 & 4.394 & 125.229 & 4.394 \\
\hline 2.197 & 4.394 & 61.516 & 79.092 \\
\hline 2.197 & 81.289 & 15.379 & 17.576 \\
\hline 6.591 & 2.197 & 2.197 & 39.546 \\
\hline 243.867 & 15.379 & 15.379 & 24.167 \\
\hline 2.197 & 2.197 & 63.713 & 32.955 \\
\hline 2.197 & 4.394 & 274.625 & 2.197 \\
\hline 10.985 & 6.591 & 2.197 & 13.182 \\
\hline 6.591 & 2.197 & 2.197 & 70.304 \\
\hline 52.728 & 6.591 & 28.561 & 28.561 \\
\hline 6.591 & 2.197 & 2.197 & 15.379 \\
\hline 2.197 & 68.107 & 274.625 & 2.197 \\
\hline 3242.77 & 6.591 & 4.394 & 19.773 \\
\hline 8.788 & 6.591 & 2.197 & 46.137 \\
\hline 2.197 & 2.197 & 8.788 & 24.167 \\
\hline
\end{tabular}




\begin{tabular}{|c|c|c|c|}
\hline 10.985 & 2.197 & 2.197 & 2.197 \\
\hline 2.197 & 4.394 & 2.197 & 21.97 \\
\hline 4.394 & 114.244 & 2.197 & 6.591 \\
\hline 2.197 & 4.394 & 30.758 & 17.576 \\
\hline 8.788 & 15.379 & 10.985 & 2.197 \\
\hline 6.591 & 2.197 & 2.197 & 13.182 \\
\hline 13.182 & 4.394 & 4.394 & 4.394 \\
\hline 4.394 & 13.182 & 4.394 & 2.197 \\
\hline 13.182 & 4.394 & 2.197 & 147.199 \\
\hline 257.049 & 4.394 & 32.955 & 8.788 \\
\hline 32.955 & 24.167 & 15.379 & 17.576 \\
\hline 2.197 & 720.616 & 10.985 & 6.591 \\
\hline 2.197 & 116.441 & 2963.75 & 2.197 \\
\hline 61.516 & 2.197 & 26.364 & 2.197 \\
\hline 749.177 & 4.394 & 2.197 & 2.197 \\
\hline 2.197 & 6.591 & 140.608 & 4.394 \\
\hline 17.576 & 351.52 & 50.531 & 2.197 \\
\hline 2.197 & 41.743 & 10.985 & 2.197 \\
\hline 2.197 & 4.394 & 2.197 & 2.197 \\
\hline 946.907 & 2.197 & 24.167 & 10.985 \\
\hline 8.788 & 8.788 & 6.591 & 19.773 \\
\hline 13.182 & 2.197 & 8.788 & 2.197 \\
\hline 2.197 & 2.197 & 57.122 & 120.835 \\
\hline 105.456 & 4.394 & 4.394 & 2.197 \\
\hline 28.561 & 10.985 & 2.197 & 3269.14 \\
\hline 2.197 & 2.197 & 2.197 & 96.668 \\
\hline 2.197 & 2.197 & 8.788 & 32.955 \\
\hline 2.197 & 2.197 & 15.379 & 10.985 \\
\hline 4.394 & 2.197 & 37.349 & 344.929 \\
\hline 107.653 & 2.197 & 2.197 & 68.107 \\
\hline 19.773 & 2.197 & 2.197 & 145.002 \\
\hline 63.713 & 417.43 & 2.197 & 3950.21 \\
\hline 2.197 & 155.987 & 10.985 & 2.197 \\
\hline 118.638 & 4.394 & 2.197 & 2.197 \\
\hline 10.985 & 2.197 & 2.197 & 4.394 \\
\hline 24.167 & 54.925 & 37.349 & 8.788 \\
\hline 21.97 & 65.91 & 2.197 & 4.394 \\
\hline 74.698 & 24.167 & 10.985 & 4.394 \\
\hline 15.379 & 21.97 & 24.167 & 57.122 \\
\hline 30.758 & 2.197 & 2.197 & 4.394 \\
\hline 2.197 & 2.197 & 6.591 & 13.182 \\
\hline 2.197 & 4.394 & 46.137 & 2.197 \\
\hline 8.788 & 76.895 & 30.758 & 2.197 \\
\hline 19.773 & 2.197 & 61.516 & 15.379 \\
\hline 2.197 & 2.197 & 28.561 & 1781.77 \\
\hline 28.561 & 35.152 & 15.379 & 17.576 \\
\hline 4.394 & 2.197 & 2.197 & 2.197 \\
\hline 2.197 & 109.85 & 4.394 & 10.985 \\
\hline 28.561 & 6.591 & 145.002 & 10.985 \\
\hline 4.394 & 173.563 & 4.394 & 2.197 \\
\hline
\end{tabular}




\begin{tabular}{|c|c|c|c|}
\hline 19.773 & 2.197 & 46.137 & 4.394 \\
\hline 17.576 & 2.197 & 8.788 & 26.364 \\
\hline 6.591 & 248.261 & 2.197 & 15.379 \\
\hline 4.394 & 24.167 & 13.182 & 43.94 \\
\hline 274.625 & 24.167 & 4.394 & 417.43 \\
\hline 6.591 & 4.394 & 2.197 & 19.773 \\
\hline 17.576 & 320.762 & 15.379 & 2.197 \\
\hline 2.197 & 2.197 & 2.197 & 2.197 \\
\hline 10.985 & 2.197 & 4.394 & 4.394 \\
\hline 2.197 & 17.576 & 21.97 & 10.985 \\
\hline 2.197 & 206.518 & 8.788 & 2.197 \\
\hline 2.197 & 54.925 & 6.591 & 28.561 \\
\hline 6.591 & 8.788 & 4.394 & 15.379 \\
\hline 2.197 & 2.197 & 76.895 & 17.576 \\
\hline 2.197 & 4.394 & 270.231 & 24.167 \\
\hline 6.591 & 10.985 & 114.244 & 19.773 \\
\hline 90.077 & 46.137 & 19.773 & 41.743 \\
\hline 2.197 & 26.364 & 19.773 & 19.773 \\
\hline 2.197 & 4.394 & 90.077 & 4.394 \\
\hline 2.197 & 57.122 & 39.546 & 2.197 \\
\hline 2.197 & 228.488 & 4.394 & 2.197 \\
\hline 116.441 & 52.728 & 2.197 & 68.107 \\
\hline 2.197 & 109.85 & 2.197 & 155.987 \\
\hline 2.197 & 10.985 & 21.97 & 87.88 \\
\hline 13.182 & 166.972 & 4.394 & 26.364 \\
\hline 15.379 & 17.576 & 2.197 & 8.788 \\
\hline 2.197 & 2.197 & 2.197 & 6.591 \\
\hline 2.197 & 50.531 & 10.985 & 1015.01 \\
\hline 87.88 & 2.197 & 2.197 & 2.197 \\
\hline 4.394 & 15.379 & 2.197 & 4.394 \\
\hline 30.758 & 4.394 & 10.985 & 37.349 \\
\hline 1133.65 & 8.788 & 2.197 & 15.379 \\
\hline 4.394 & 8.788 & 21.97 & 43.94 \\
\hline 19.773 & 6.591 & 4.394 & 6.591 \\
\hline 8.788 & 32.955 & 2.197 & 4.394 \\
\hline 4.394 & 8.788 & 2.197 & 2.197 \\
\hline 6.591 & 35.152 & 4.394 & 261.443 \\
\hline 21.97 & 874.406 & 2.197 & 2.197 \\
\hline 2.197 & 2.197 & 52.728 & 4.394 \\
\hline 6.591 & 2.197 & 459.173 & 65.91 \\
\hline 4.394 & 2.197 & 6.591 & 2.197 \\
\hline 35.152 & 6.591 & 2.197 & 8.788 \\
\hline 50.531 & 8.788 & 2.197 & 4.394 \\
\hline 2.197 & 2.197 & 2.197 & 2.197 \\
\hline 32.955 & 15.379 & 2.197 & 61.516 \\
\hline 94.471 & 2.197 & 4.394 & 566.826 \\
\hline 4.394 & 68.107 & 37.349 & 8.788 \\
\hline 2.197 & 10.985 & 2.197 & 4.394 \\
\hline 15.379 & 2.197 & 2.197 & 13.182 \\
\hline 6.591 & 4.394 & 2.197 & 24.167 \\
\hline
\end{tabular}




\begin{tabular}{|c|c|c|c|}
\hline 4.394 & 8.788 & 2.197 & 54.925 \\
\hline 2.197 & 2.197 & 6.591 & 28.561 \\
\hline 2.197 & 24.167 & 2.197 & 2.197 \\
\hline 6.591 & 118.638 & 13.182 & 2.197 \\
\hline 13.182 & 103.259 & 81.289 & 4.394 \\
\hline 72.501 & 237.276 & 1087.51 & 10.985 \\
\hline 2.197 & 2.197 & 2.197 & 41.743 \\
\hline 24.167 & 2.197 & 17.576 & 10.985 \\
\hline 2.197 & 65.91 & 13.182 & 2.197 \\
\hline 4.394 & 2.197 & 30.758 & 2.197 \\
\hline 8.788 & 4.394 & 4.394 & 6.591 \\
\hline 13.182 & 28.561 & 2.197 & 6.591 \\
\hline 2.197 & 138.411 & 2.197 & 4.394 \\
\hline 32.955 & 2.197 & 2.197 & 2.197 \\
\hline 2.197 & 10.985 & 2.197 & 17.576 \\
\hline 19.773 & 2.197 & 2.197 & 19.773 \\
\hline 8.788 & 32.955 & 4.394 & 2.197 \\
\hline 4.394 & 4.394 & 2.197 & 17.576 \\
\hline 2.197 & 81.289 & 2.197 & 2.197 \\
\hline 17.576 & 19.773 & 4.394 & 13.182 \\
\hline 15.379 & 2.197 & 2.197 & 17.576 \\
\hline 235.079 & 2.197 & 68.107 & 10.985 \\
\hline 15.379 & 2.197 & 2.197 & 68.107 \\
\hline 4.394 & 2.197 & 30.758 & 30.758 \\
\hline 61.516 & 13.182 & 15.379 & 4.394 \\
\hline 10.985 & 13.182 & 6.591 & 68.107 \\
\hline 2.197 & 2.197 & 2.197 & 4.394 \\
\hline 2.197 & 4.394 & 43.94 & 2.197 \\
\hline 2.197 & 2.197 & 48.334 & 2.197 \\
\hline 59.319 & 2.197 & 17.576 & 2.197 \\
\hline 10.985 & 6.591 & 17.576 & 2.197 \\
\hline 2.197 & 92.274 & 8.788 & 15.379 \\
\hline 39.546 & 81.289 & 6.591 & 41.743 \\
\hline 2.197 & 2.197 & 13.182 & 166.972 \\
\hline 21.97 & 24.167 & 74.698 & 15.379 \\
\hline 2.197 & 2.197 & 85.683 & 123.032 \\
\hline 15.379 & 2.197 & 4.394 & 4.394 \\
\hline 2.197 & 2.197 & 17.576 & 39.546 \\
\hline 6.591 & 26.364 & 13.182 & 35.152 \\
\hline 4.394 & 2.197 & 26.364 & 4.394 \\
\hline 4.394 & 10.985 & 4.394 & 4.394 \\
\hline 8.788 & 35.152 & 2.197 & 2.197 \\
\hline 6.591 & 4.394 & 2.197 & 2.197 \\
\hline 2.197 & 30.758 & 2.197 & 2.197 \\
\hline 4.394 & 2.197 & 26.364 & 2.197 \\
\hline 2.197 & 39.546 & 39.546 & 2.197 \\
\hline 24.167 & 54.925 & 76.895 & 2.197 \\
\hline 2.197 & 6.591 & 30.758 & 1898.21 \\
\hline 2.197 & 6.591 & 2.197 & 2.197 \\
\hline 6.591 & 4.394 & 2.197 & 26.364 \\
\hline
\end{tabular}




\begin{tabular}{|c|c|c|c|}
\hline 4.394 & 26.364 & 2.197 & 4.394 \\
\hline 74.698 & 2.197 & 2.197 & 92.274 \\
\hline 2.197 & 2.197 & 2.197 & 2.197 \\
\hline 2.197 & 4.394 & 2.197 & 2.197 \\
\hline 4.394 & 626.145 & 2.197 & 125.229 \\
\hline 37.349 & 26.364 & 39.546 & 2.197 \\
\hline 26.364 & 2.197 & 17.576 & 17.576 \\
\hline 2.197 & 2.197 & 4.394 & 21.97 \\
\hline 48.334 & 37.349 & 2.197 & 2.197 \\
\hline 6.591 & 4.394 & 2.197 & 2.197 \\
\hline 4.394 & 6.591 & 70.304 & 2.197 \\
\hline 2.197 & 76.895 & 2.197 & 1915.78 \\
\hline 50.531 & 87.88 & 81.289 & 2.197 \\
\hline 2.197 & 4.394 & 2.197 & 10.985 \\
\hline 2.197 & 4.394 & 2.197 & 2.197 \\
\hline 26.364 & 15.379 & 26.364 & 8.788 \\
\hline 32.955 & 10.985 & 6.591 & 21.97 \\
\hline 6.591 & 4.394 & 2.197 & 6.591 \\
\hline 2.197 & 76.895 & 4.394 & 19.773 \\
\hline 70.304 & 2.197 & 2.197 & 2.197 \\
\hline 107.653 & 6.591 & 4.394 & 6.591 \\
\hline 2.197 & 2.197 & 2.197 & 43.94 \\
\hline 8.788 & 123.032 & 2.197 & 30.758 \\
\hline 518.492 & 2.197 & 10.985 & 35.152 \\
\hline 41.743 & 50.531 & 2.197 & 2.197 \\
\hline 4.394 & 21.97 & 52.728 & 68.107 \\
\hline 10.985 & 4.394 & 4.394 & 6.591 \\
\hline 48.334 & 46.137 & 2.197 & 186.745 \\
\hline 6.591 & 4.394 & 2.197 & 15.379 \\
\hline 43.94 & 586.599 & 10.985 & 41.743 \\
\hline 2.197 & 4.394 & 35.152 & 52.728 \\
\hline 4.394 & 2.197 & 79.092 & 2.197 \\
\hline 274.625 & 272.428 & 2.197 & 68.107 \\
\hline 4.394 & 2.197 & 213.109 & 83.486 \\
\hline 17.576 & 68.107 & 4.394 & 2.197 \\
\hline 2.197 & 114.244 & 2.197 & 6.591 \\
\hline 2.197 & 17.576 & 74.698 & 2.197 \\
\hline 63.713 & 17.576 & 46.137 & 54.925 \\
\hline 70.304 & 4.394 & 4.394 & 57.122 \\
\hline 87.88 & 28.561 & 10.985 & 4.394 \\
\hline 6.591 & 4.394 & 2.197 & 17.576 \\
\hline 147.199 & 6.591 & 15.379 & 92.274 \\
\hline 2.197 & 2.197 & 48.334 & 28.561 \\
\hline 2.197 & 28.561 & 13.182 & 8.788 \\
\hline 6.591 & 6.591 & 2.197 & 52.728 \\
\hline 2.197 & 2.197 & 65.91 & 2.197 \\
\hline 6.591 & 8.788 & 2.197 & 432.809 \\
\hline 24.167 & 46.137 & 2.197 & 19.773 \\
\hline 2871.48 & 72.501 & 6.591 & 2.197 \\
\hline 15.379 & 72.501 & 6.591 & 4.394 \\
\hline
\end{tabular}




\begin{tabular}{|c|c|c|c|}
\hline 4.394 & 52.728 & 6.591 & 13.182 \\
\hline 2.197 & 41.743 & 21.97 & 2.197 \\
\hline 2.197 & 6.591 & 37.349 & 15.379 \\
\hline 35.152 & 2.197 & 4.394 & 8.788 \\
\hline 6.591 & 2.197 & 17.576 & 4.394 \\
\hline 70.304 & 2.197 & 24.167 & 28.561 \\
\hline 32.955 & 4.394 & 2.197 & 478.946 \\
\hline 13.182 & 2.197 & 2.197 & 48.334 \\
\hline 61.516 & 13.182 & 48.334 & 6.591 \\
\hline 4.394 & 4.394 & 17.576 & 105.456 \\
\hline 19.773 & 239.473 & 83.486 & 19.773 \\
\hline 6.591 & 2.197 & 2.197 & 424.021 \\
\hline 96.668 & 2.197 & 2.197 & 15.379 \\
\hline 4.394 & 50.531 & 2.197 & 13.182 \\
\hline 43.94 & 2.197 & 2.197 & 10.985 \\
\hline 2.197 & 2.197 & 2.197 & 26.364 \\
\hline 46.137 & 4.394 & 8.788 & 2.197 \\
\hline 8.788 & 8.788 & 43.94 & 2.197 \\
\hline 4.394 & 2.197 & 2.197 & 63.713 \\
\hline 105.456 & 2.197 & 2.197 & 8.788 \\
\hline 4.394 & 2.197 & 2.197 & 8.788 \\
\hline 2.197 & 32.955 & 2.197 & 96.668 \\
\hline 4.394 & 2.197 & 2.197 & 26.364 \\
\hline 6.591 & 406.445 & 2.197 & 10.985 \\
\hline 17.576 & 24.167 & 8.788 & 4.394 \\
\hline 32.955 & 1693.89 & 10.985 & 4.394 \\
\hline 4.394 & 2.197 & 2.197 & 87.88 \\
\hline 6.591 & 39.546 & 35.152 & 6.591 \\
\hline 2.197 & 2.197 & 2.197 & 8.788 \\
\hline 4.394 & 2.197 & 4.394 & 10.985 \\
\hline 2.197 & 2.197 & 2.197 & 8.788 \\
\hline 2.197 & 6.591 & 2.197 & 48.334 \\
\hline 13.182 & 15.379 & 57.122 & 2.197 \\
\hline 4.394 & 2.197 & 232.882 & 7192.98 \\
\hline 6.591 & 61.516 & 221.897 & 2.197 \\
\hline 74.698 & 39.546 & 8.788 & 2.197 \\
\hline 3001.1 & 6.591 & 2.197 & 2.197 \\
\hline 10.985 & 26.364 & 74.698 & 26.364 \\
\hline 2.197 & 2.197 & 6.591 & 4.394 \\
\hline 6.591 & 10.985 & 193.336 & 2.197 \\
\hline 65.91 & 6.591 & 6.591 & 2.197 \\
\hline 30.758 & 50.531 & 4.394 & 2.197 \\
\hline 6.591 & 8.788 & 2.197 & 413.036 \\
\hline 2.197 & 24.167 & 10.985 & 79.092 \\
\hline 30.758 & 6.591 & 10.985 & 24.167 \\
\hline 24.167 & 10.985 & 15.379 & 26.364 \\
\hline 239.473 & 2.197 & 4.394 & 76.895 \\
\hline 217.503 & 19.773 & 6.591 & 2.197 \\
\hline 30.758 & 50.531 & 4.394 & 35.152 \\
\hline 140.608 & 6.591 & 10.985 & 6.591 \\
\hline
\end{tabular}




\begin{tabular}{|c|c|c|c|}
\hline 2.197 & 2.197 & 2.197 & 2.197 \\
\hline 19.773 & 6.591 & 2.197 & 2.197 \\
\hline 8.788 & 48.334 & 8.788 & 290.004 \\
\hline 2.197 & 2.197 & 2.197 & 13.182 \\
\hline 48.334 & 2.197 & 2359.58 & 17.576 \\
\hline 2.197 & 32.955 & 2.197 & 50.531 \\
\hline 2.197 & 2.197 & 13.182 & 4.394 \\
\hline 2.197 & 2.197 & 8.788 & 13.182 \\
\hline 26.364 & 2.197 & 19.773 & 2.197 \\
\hline 28.561 & 2.197 & 8.788 & 6.591 \\
\hline 48.334 & 2.197 & 2.197 & 28.561 \\
\hline 2.197 & 8.788 & 72.501 & 221.897 \\
\hline 2.197 & 13.182 & 2.197 & 13.182 \\
\hline 2.197 & 21.97 & 6.591 & 13.182 \\
\hline 2.197 & 107.653 & 4.394 & 2.197 \\
\hline 17.576 & 4.394 & 6.591 & 988.65 \\
\hline 112.047 & 2.197 & 2.197 & 15.379 \\
\hline 17.576 & 4.394 & 6.591 & 2.197 \\
\hline 8.788 & 15.379 & 2.197 & 24.167 \\
\hline 8.788 & 17.576 & 2.197 & 90.077 \\
\hline 65.91 & 15.379 & 2.197 & 4.394 \\
\hline 13.182 & 59.319 & 2.197 & 2.197 \\
\hline 4.394 & 15.379 & 35.152 & 206.518 \\
\hline 2.197 & 28.561 & 2.197 & 4.394 \\
\hline 15.379 & 2.197 & 2.197 & 2.197 \\
\hline 147.199 & 59.319 & 6.591 & 52.728 \\
\hline 191.139 & 2.197 & 2.197 & 15.379 \\
\hline 10.985 & 2.197 & 41.743 & 13.182 \\
\hline 4.394 & 2.197 & 26.364 & 2.197 \\
\hline 243.867 & 4.394 & 2.197 & 15.379 \\
\hline 2.197 & 1017.21 & 2.197 & 6.591 \\
\hline 24.167 & 32.955 & 2.197 & 2.197 \\
\hline 138.411 & 52.728 & 8.788 & 674.479 \\
\hline 48.334 & 2.197 & 456.976 & 6.591 \\
\hline 2.197 & 2.197 & 8.788 & 4.394 \\
\hline 2.197 & 65.91 & 4.394 & 2.197 \\
\hline 54.925 & 6.591 & 2.197 & 2.197 \\
\hline 6.591 & 4.394 & 10.985 & 540.462 \\
\hline 6.591 & 4.394 & 24.167 & 8.788 \\
\hline 2.197 & 13.182 & 2.197 & 103.259 \\
\hline 8.788 & 2.197 & 13.182 & 35.152 \\
\hline 19.773 & 65.91 & 21.97 & 4.394 \\
\hline 204.321 & 4.394 & 52.728 & 39.546 \\
\hline 32.955 & 15.379 & 98.865 & 6.591 \\
\hline 182.351 & 50.531 & 2.197 & 43.94 \\
\hline 2.197 & 4.394 & 8.788 & 8.788 \\
\hline 4.394 & 37.349 & 2.197 & 30.758 \\
\hline 8.788 & 2.197 & 8.788 & 2.197 \\
\hline 15.379 & 2.197 & 2.197 & 2.197 \\
\hline 2.197 & 2.197 & 21.97 & 74.698 \\
\hline
\end{tabular}




\begin{tabular}{|c|c|c|c|}
\hline 2.197 & 8.788 & 2.197 & 30.758 \\
\hline 4.394 & 13.182 & 2.197 & 6.591 \\
\hline 2.197 & 15.379 & 13.182 & 314.171 \\
\hline 59.319 & 37.349 & 6.591 & 4.394 \\
\hline 26.364 & 96.668 & 2.197 & 6.591 \\
\hline 597.584 & 2.197 & 2.197 & 4106.19 \\
\hline 17.576 & 8.788 & 2.197 & 2.197 \\
\hline 4.394 & 2.197 & 2.197 & 21.97 \\
\hline 19.773 & 72.501 & 848.042 & 2.197 \\
\hline 2.197 & 6.591 & 208.715 & 6.591 \\
\hline 2.197 & 2.197 & 6.591 & 2.197 \\
\hline 10.985 & 46.137 & 2.197 & 96.668 \\
\hline 8.788 & 2.197 & 2.197 & 6.591 \\
\hline 2.197 & 39.546 & 2.197 & 35.152 \\
\hline 4.394 & 2.197 & 2.197 & 98.865 \\
\hline 219.7 & 6.591 & 65.91 & 2.197 \\
\hline 15.379 & 2.197 & 2.197 & 105.456 \\
\hline 10.985 & 8.788 & 344.929 & 661.297 \\
\hline 8.788 & 6.591 & 4.394 & 2.197 \\
\hline 2.197 & 2.197 & 4.394 & 2.197 \\
\hline 4.394 & 21.97 & 4.394 & 2.197 \\
\hline 8.788 & 6.591 & 2.197 & 15.379 \\
\hline 83.486 & 6.591 & 2.197 & 4.394 \\
\hline 2.197 & 4.394 & 2.197 & 8.788 \\
\hline 30.758 & 30.758 & 10.985 & 4.394 \\
\hline 15.379 & 8.788 & 10.985 & 182.351 \\
\hline 4.394 & 2.197 & 57.122 & 151.593 \\
\hline 4.394 & 2.197 & 2.197 & 10.985 \\
\hline 4.394 & 4.394 & 4.394 & 10.985 \\
\hline 10.985 & 2.197 & 8.788 & 26.364 \\
\hline 2.197 & 59.319 & 8.788 & 2.197 \\
\hline 15.379 & 28.561 & 417.43 & 6.591 \\
\hline 10.985 & 65.91 & 2.197 & 221.897 \\
\hline 2.197 & 4.394 & 2.197 & 21.97 \\
\hline 17.576 & 2.197 & 15.379 & 76.895 \\
\hline 79.092 & 1316 & 30.758 & 13.182 \\
\hline 13.182 & 21.97 & 4.394 & 8.788 \\
\hline 6.591 & 140.608 & 41.743 & 2.197 \\
\hline 2.197 & 13.182 & 4.394 & 13.182 \\
\hline 39.546 & 13.182 & 13.182 & 2.197 \\
\hline 8.788 & 4.394 & 2.197 & 19.773 \\
\hline 57.122 & 10.985 & 4.394 & 24.167 \\
\hline 10.985 & 61.516 & 6.591 & 6.591 \\
\hline 81.289 & 32.955 & 1269.87 & 125.229 \\
\hline 2.197 & 24.167 & 250.458 & 28.561 \\
\hline 2.197 & 8.788 & 15.379 & 2.197 \\
\hline 10.985 & 2.197 & 2.197 & 10.985 \\
\hline 2.197 & 2.197 & 2.197 & 6.591 \\
\hline 30.758 & 2.197 & 4.394 & 90.077 \\
\hline 2.197 & 142.805 & 15.379 & 32.955 \\
\hline
\end{tabular}




\begin{tabular}{|c|c|c|c|}
\hline 2.197 & 17.576 & 41.743 & 19.773 \\
\hline 153.79 & 6.591 & 2.197 & 10.985 \\
\hline 6.591 & 4.394 & 6.591 & 10.985 \\
\hline 2.197 & 6.591 & 15.379 & 15.379 \\
\hline 15.379 & 4.394 & 2.197 & 13.182 \\
\hline 4.394 & 2.197 & 2.197 & 4.394 \\
\hline 2.197 & 8.788 & 2.197 & 13.182 \\
\hline 13.182 & 259.246 & 2.197 & 2.197 \\
\hline 2.197 & 213.109 & 2.197 & 21.97 \\
\hline 4.394 & 6.591 & 21.97 & 169.169 \\
\hline 68.107 & 226.291 & 2.197 & 26.364 \\
\hline 210.912 & 114.244 & 43.94 & 6.591 \\
\hline 173.563 & 4.394 & 21.97 & 2.197 \\
\hline 4.394 & 8.788 & 184.548 & 2.197 \\
\hline 13.182 & 8.788 & 188.942 & 2.197 \\
\hline 4.394 & 6.591 & 2.197 & 2.197 \\
\hline 6.591 & 4.394 & 2.197 & 28.561 \\
\hline 17.576 & 371.293 & 142.805 & 2.197 \\
\hline 19.773 & 8.788 & 6.591 & 15.379 \\
\hline 61.516 & 2.197 & 184.548 & 15.379 \\
\hline 19.773 & 10.985 & 2.197 & 96.668 \\
\hline 41.743 & 384.475 & 2.197 & 1298.43 \\
\hline 6.591 & 15.379 & 140.608 & 13.182 \\
\hline 15.379 & 2.197 & 2.197 & 2.197 \\
\hline 19.773 & 1601.61 & 2.197 & 59.319 \\
\hline 6.591 & 32.955 & 4.394 & 2.197 \\
\hline 402.051 & 15.379 & 32.955 & 79.092 \\
\hline 2.197 & 13.182 & 2.197 & 8.788 \\
\hline 4.394 & 2.197 & 2.197 & 6.591 \\
\hline 2.197 & 37.349 & 13.182 & 2.197 \\
\hline 41.743 & 4.394 & 4.394 & 270.231 \\
\hline 72.501 & 85.683 & 2.197 & 72.501 \\
\hline 4.394 & 39.546 & 118.638 & 87.88 \\
\hline 4.394 & 235.079 & 2.197 & 15.379 \\
\hline 81.289 & 15.379 & 2.197 & 50.531 \\
\hline 2.197 & 54.925 & 2.197 & 2.197 \\
\hline 2.197 & 2.197 & 2.197 & 10.985 \\
\hline 2.197 & 2.197 & 24.167 & 232.882 \\
\hline 65.91 & 10.985 & 8.788 & 202.124 \\
\hline 6.591 & 406.445 & 59.319 & 158.184 \\
\hline 4.394 & 32.955 & 10.985 & 41.743 \\
\hline 2.197 & 35.152 & 4.394 & 2.197 \\
\hline 26.364 & 41.743 & 2.197 & 8.788 \\
\hline 2.197 & 13.182 & 134.017 & 10.985 \\
\hline 2.197 & 2.197 & 2.197 & 19.773 \\
\hline 10.985 & 2.197 & 2.197 & 2.197 \\
\hline 4.394 & 4.394 & 4.394 & 19.773 \\
\hline 46.137 & 751.374 & 2.197 & 6.591 \\
\hline 155.987 & 239.473 & 2.197 & 54.925 \\
\hline 15.379 & 13.182 & 6.591 & 24.167 \\
\hline
\end{tabular}




\begin{tabular}{|c|c|c|c|}
\hline 76.895 & 246.064 & 2.197 & 8.788 \\
\hline 15.379 & 8.788 & 2.197 & 2.197 \\
\hline 32.955 & 10.985 & 2.197 & 21.97 \\
\hline 8.788 & 13.182 & 2.197 & 2.197 \\
\hline 4.394 & 35.152 & 24.167 & 13.182 \\
\hline 10.985 & 2.197 & 32.955 & 37.349 \\
\hline 4.394 & 8.788 & 4.394 & 57.122 \\
\hline 79.092 & 8.788 & 2.197 & 2.197 \\
\hline 2.197 & 57.122 & 136.214 & 21.97 \\
\hline 2.197 & 4.394 & 2.197 & 26.364 \\
\hline 4.394 & 50.531 & 2.197 & 19.773 \\
\hline 2.197 & 30.758 & 13.182 & 92.274 \\
\hline 26.364 & 17.576 & 4.394 & 2.197 \\
\hline 127.426 & 4.394 & 2.197 & 10.985 \\
\hline 41.743 & 4.394 & 2.197 & 2.197 \\
\hline 10.985 & 39.546 & 94.471 & 10.985 \\
\hline 2.197 & 206.518 & 155.987 & 4.394 \\
\hline 2.197 & 6.591 & 41.743 & 285.61 \\
\hline 8.788 & 153.79 & 2.197 & 10.985 \\
\hline 2.197 & 74.698 & 43.94 & 50.531 \\
\hline 4.394 & 2.197 & 4.394 & 10.985 \\
\hline 2.197 & 6.591 & 8.788 & 2.197 \\
\hline 13.182 & 2.197 & 2.197 & 17.576 \\
\hline 2.197 & 6.591 & 2.197 & 17.576 \\
\hline 2.197 & 2.197 & 2.197 & 1786.16 \\
\hline 4.394 & 413.036 & 10.985 & 2.197 \\
\hline 547.053 & 21.97 & 4.394 & 2.197 \\
\hline 2.197 & 74.698 & 2.197 & 10.985 \\
\hline 4.394 & 2.197 & 2.197 & 74.698 \\
\hline 30.758 & 2.197 & 2.197 & 13.182 \\
\hline 35.152 & 70.304 & 4.394 & 6.591 \\
\hline 54.925 & 2.197 & 2.197 & 70.304 \\
\hline 2.197 & 8.788 & 2.197 & 8.788 \\
\hline 35.152 & 28.561 & 68.107 & 52.728 \\
\hline 24.167 & 6.591 & 4.394 & 2.197 \\
\hline 8.788 & 158.184 & 17.576 & 21.97 \\
\hline 118.638 & 4.394 & 305.383 & 155.987 \\
\hline 2.197 & 17.576 & 2.197 & 101.062 \\
\hline 2.197 & 4.394 & 2.197 & 39.546 \\
\hline 2.197 & 17.576 & 35.152 & 41.743 \\
\hline 15.379 & 17.576 & 10.985 & 2.197 \\
\hline 10.985 & 17.576 & 2.197 & 6.591 \\
\hline 17.576 & 2.197 & 2.197 & 30.758 \\
\hline 24.167 & 59.319 & 2.197 & 151.593 \\
\hline 26.364 & 2.197 & 4.394 & 13.182 \\
\hline 4.394 & 4.394 & 15.379 & 4.394 \\
\hline 2.197 & 6.591 & 6.591 & 226.291 \\
\hline 4.394 & 8.788 & 2.197 & 43.94 \\
\hline 15.379 & 19.773 & 15.379 & 26.364 \\
\hline 2.197 & 94.471 & 4.394 & 24.167 \\
\hline
\end{tabular}




\begin{tabular}{|c|c|c|c|}
\hline 2.197 & 26.364 & 4.394 & 8.788 \\
\hline 2.197 & 6.591 & 98.865 & 259.246 \\
\hline 2.197 & 4.394 & 4.394 & 6.591 \\
\hline 19.773 & 2.197 & 10.985 & 2.197 \\
\hline 4.394 & 4.394 & 336.141 & 2.197 \\
\hline 281.216 & 2.197 & 580.008 & 30.758 \\
\hline 8.788 & 2.197 & 39.546 & 109.85 \\
\hline 15.379 & 2.197 & 2.197 & 2.197 \\
\hline 2.197 & 2.197 & 8.788 & 2.197 \\
\hline 65.91 & 4.394 & 2.197 & 2.197 \\
\hline 285.61 & 1520.32 & 454.779 & 6.591 \\
\hline 180.154 & 4.394 & 186.745 & 2.197 \\
\hline 2.197 & 4.394 & 4.394 & 54.925 \\
\hline 17.576 & 13.182 & 32.955 & 4.394 \\
\hline 2.197 & 13.182 & 2.197 & 208.715 \\
\hline 59.319 & 4.394 & 2.197 & 6.591 \\
\hline 4.394 & 4.394 & 114.244 & 2.197 \\
\hline 1243.5 & 2.197 & 13375.3 & 28.561 \\
\hline 2.197 & 2.197 & 8.788 & 4.394 \\
\hline 2.197 & 28.561 & 230.685 & 6.591 \\
\hline 329.55 & 2.197 & 2.197 & 2.197 \\
\hline 4.394 & 109.85 & 24.167 & 2.197 \\
\hline 8.788 & 166.972 & 127.426 & 399.854 \\
\hline 2.197 & 2.197 & 8.788 & 2.197 \\
\hline 6.591 & 15.379 & 54.925 & 883.194 \\
\hline 4.394 & 96.668 & 2.197 & 10.985 \\
\hline 4.394 & 87.88 & 4110.59 & 39.546 \\
\hline 6.591 & 531.674 & 2.197 & 2.197 \\
\hline 2.197 & 61.516 & 2.197 & 2.197 \\
\hline 10.985 & 13.182 & 2.197 & 30.758 \\
\hline 2.197 & 2.197 & 2.197 & 13.182 \\
\hline 4.394 & 68.107 & 68.107 & 279.019 \\
\hline 4.394 & 2.197 & 140.608 & 43.94 \\
\hline 2.197 & 85.683 & 2.197 & 6.591 \\
\hline 2.197 & 10.985 & 2.197 & 197.73 \\
\hline 21.97 & 151.593 & 2.197 & 46.137 \\
\hline 6.591 & 2.197 & 2.197 & 116.441 \\
\hline 17.576 & 2.197 & 79.092 & 2.197 \\
\hline 112.047 & 21.97 & 8.788 & 17.576 \\
\hline 125.229 & 153.79 & 8.788 & 8.788 \\
\hline 4.394 & 26.364 & 2.197 & 8.788 \\
\hline 6.591 & 4.394 & 257.049 & 96.668 \\
\hline 2.197 & 61.516 & 2.197 & 21.97 \\
\hline 2.197 & 8.788 & 2.197 & 10.985 \\
\hline 2.197 & 2.197 & 2.197 & 2.197 \\
\hline 2.197 & 46.137 & 4.394 & 2.197 \\
\hline 2.197 & 6.591 & 2.197 & 13.182 \\
\hline 8.788 & 2.197 & 4.394 & 8.788 \\
\hline 2.197 & 309.777 & 2.197 & 2115.71 \\
\hline 2.197 & 2.197 & 2.197 & 2177.23 \\
\hline
\end{tabular}




\begin{tabular}{|c|c|c|c|}
\hline 2.197 & 2.197 & 2.197 & 2.197 \\
\hline 28.561 & 2.197 & 37.349 & 2.197 \\
\hline 177.957 & 2.197 & 140.608 & 8.788 \\
\hline 2.197 & 2.197 & 2.197 & 4.394 \\
\hline 4.394 & 24.167 & 21.97 & 2.197 \\
\hline 13.182 & 2.197 & 5110.22 & 487.734 \\
\hline 8.788 & 10.985 & 2.197 & 6.591 \\
\hline 4.394 & 8.788 & 2.197 & 21.97 \\
\hline 54.925 & 37.349 & 30.758 & 149.396 \\
\hline 2.197 & 10.985 & 50.531 & 26.364 \\
\hline 8.788 & 155.987 & 15.379 & 351.52 \\
\hline 13.182 & 2.197 & 2.197 & 10.985 \\
\hline 2.197 & 4.394 & 2.197 & 2.197 \\
\hline 155.987 & 6.591 & 39.546 & 17.576 \\
\hline 10.985 & 10.985 & 2.197 & 2.197 \\
\hline 6.591 & 2.197 & 2262.91 & 24.167 \\
\hline 2.197 & 46.137 & 8.788 & 415.233 \\
\hline 2.197 & 61.516 & 2.197 & 2.197 \\
\hline 4.394 & 76.895 & 68.107 & 195.533 \\
\hline 6.591 & 10.985 & 13.182 & 384.475 \\
\hline 10.985 & 35.152 & 21.97 & 15.379 \\
\hline 96.668 & 28.561 & 2.197 & 4.394 \\
\hline 13.182 & 13.182 & 2.197 & 19.773 \\
\hline 2.197 & 4.394 & 2.197 & 10.985 \\
\hline 619.554 & 470.158 & 43.94 & 4.394 \\
\hline 10.985 & 37.349 & 19.773 & 169.169 \\
\hline 94.471 & 114.244 & 4.394 & 6.591 \\
\hline 13.182 & 2.197 & 8.788 & 74.698 \\
\hline 13.182 & 188.942 & 109.85 & 2.197 \\
\hline 2.197 & 188.942 & 2.197 & 2.197 \\
\hline 2.197 & 2.197 & 2.197 & 2.197 \\
\hline 4.394 & 2.197 & 2.197 & 6.591 \\
\hline 15.379 & 4.394 & 2.197 & 2.197 \\
\hline 2.197 & 2.197 & 2.197 & 186.745 \\
\hline 8.788 & 2.197 & 2.197 & 13.182 \\
\hline 118.638 & 2.197 & 2.197 & 2.197 \\
\hline 8.788 & 8.788 & 59.319 & 15.379 \\
\hline 2.197 & 2.197 & 2.197 & 4.394 \\
\hline 2.197 & 19.773 & 2.197 & 878.8 \\
\hline 15.379 & 4.394 & 4.394 & 2.197 \\
\hline 2.197 & 2.197 & 4.394 & 4.394 \\
\hline 2.197 & 8.788 & 2.197 & 153.79 \\
\hline 2.197 & 17.576 & 2.197 & 21.97 \\
\hline 2.197 & 4.394 & 2.197 & 6.591 \\
\hline 2.197 & 4.394 & 188.942 & 2.197 \\
\hline 2.197 & 13.182 & 24.167 & 26.364 \\
\hline 43.94 & 32.955 & 24.167 & 90.077 \\
\hline 2.197 & 2.197 & 30.758 & 24.167 \\
\hline 2.197 & 14473.8 & 8.788 & 2.197 \\
\hline 15.379 & 63.713 & 54.925 & 59.319 \\
\hline
\end{tabular}




\begin{tabular}{|c|c|c|c|}
\hline 180.154 & 6.591 & 112.047 & 2.197 \\
\hline 2.197 & 54.925 & 4.394 & 10.985 \\
\hline 6.591 & 6.591 & 2.197 & 8.788 \\
\hline 94.471 & 17.576 & 74.698 & 292.201 \\
\hline 87.88 & 41.743 & 17.576 & 8.788 \\
\hline 2.197 & 105.456 & 8.788 & 4.394 \\
\hline 4.394 & 2.197 & 2.197 & 26.364 \\
\hline 24.167 & 15.379 & 2.197 & 32.955 \\
\hline 2.197 & 151.593 & 2.197 & 8.788 \\
\hline 4.394 & 19.773 & 2.197 & 1386.31 \\
\hline 665.691 & 21.97 & 2.197 & 2.197 \\
\hline 21.97 & 2.197 & 24.167 & 8.788 \\
\hline 24.167 & 6.591 & 26.364 & 2.197 \\
\hline 2.197 & 17.576 & 2.197 & 17.576 \\
\hline 8.788 & 2.197 & 2.197 & 15.379 \\
\hline 17.576 & 2.197 & 2.197 & 4.394 \\
\hline 17.576 & 2.197 & 4.394 & 8.788 \\
\hline 4.394 & 17.576 & 2.197 & 8.788 \\
\hline 4.394 & 2.197 & 2.197 & 61.516 \\
\hline 6.591 & 369.096 & 2.197 & 28.561 \\
\hline 2.197 & 54.925 & 2.197 & 195.533 \\
\hline 4.394 & 2.197 & 6.591 & 2.197 \\
\hline 171.366 & 8.788 & 186.745 & 2.197 \\
\hline 2.197 & 83.486 & 2.197 & 2.197 \\
\hline 10.985 & 4.394 & 2.197 & 2.197 \\
\hline 10.985 & 68.107 & 15.379 & 98.865 \\
\hline 2.197 & 46.137 & 2.197 & 2.197 \\
\hline 2.197 & 13.182 & 2.197 & 166.972 \\
\hline 2.197 & 90.077 & 2.197 & 59.319 \\
\hline 8.788 & 4.394 & 2.197 & 15.379 \\
\hline 2.197 & 142.805 & 2.197 & 24.167 \\
\hline 4.394 & 101.062 & 92.274 & 2.197 \\
\hline 76.895 & 155.987 & 10.985 & 2.197 \\
\hline 2.197 & 4.394 & 4.394 & 6.591 \\
\hline 4.394 & 729.404 & 4.394 & 10.985 \\
\hline 4.394 & 54.925 & 4.394 & 8.788 \\
\hline 59.319 & 140.608 & 395.46 & 21.97 \\
\hline 2.197 & 2.197 & 37.349 & 96.668 \\
\hline 4.394 & 107.653 & 10.985 & 8.788 \\
\hline 2.197 & 13.182 & 4.394 & 450.385 \\
\hline 2.197 & 2.197 & 2.197 & 35.152 \\
\hline 85.683 & 6.591 & 35.152 & 4.394 \\
\hline 2.197 & 2.197 & 30.758 & 46.137 \\
\hline 2.197 & 204.321 & 13.182 & 87.88 \\
\hline 2.197 & 28.561 & 13.182 & 87.88 \\
\hline 19.773 & 533.871 & 2.197 & 2.197 \\
\hline 2.197 & 120.835 & 4.394 & 4.394 \\
\hline 2.197 & 35.152 & 17.576 & 2.197 \\
\hline 24.167 & 109.85 & 15.379 & 382.278 \\
\hline 696.449 & 15.379 & 35.152 & 6.591 \\
\hline
\end{tabular}




\begin{tabular}{|c|c|c|c|}
\hline 2.197 & 8.788 & 2.197 & 195.533 \\
\hline 4.394 & 8.788 & 8.788 & 30.758 \\
\hline 37.349 & 2.197 & 2.197 & 8.788 \\
\hline 94.471 & 2.197 & 70.304 & 70.304 \\
\hline 2.197 & 2.197 & 2.197 & 10.985 \\
\hline 2.197 & 6.591 & 8.788 & 241.67 \\
\hline 15.379 & 6.591 & 10.985 & 2.197 \\
\hline 2.197 & 30.758 & 2.197 & 2.197 \\
\hline 8.788 & 17.576 & 2.197 & 13.182 \\
\hline 26.364 & 355.914 & 8.788 & 4.394 \\
\hline 2.197 & 296.595 & 2.197 & 15.379 \\
\hline 2.197 & 186.745 & 15.379 & 41.743 \\
\hline 6.591 & 13.182 & 4.394 & 8.788 \\
\hline 92.274 & 6.591 & 2.197 & 4.394 \\
\hline 85.683 & 4.394 & 2.197 & 6.591 \\
\hline 4.394 & 10.985 & 134.017 & 4.394 \\
\hline 46.137 & 19.773 & 19.773 & 63.713 \\
\hline 2.197 & 2.197 & 15.379 & 162.578 \\
\hline 17.576 & 46.137 & 32.955 & 13.182 \\
\hline 2.197 & 48.334 & 4.394 & 59.319 \\
\hline 30.758 & 13.182 & 2.197 & 4.394 \\
\hline 2.197 & 2.197 & 79.092 & 6.591 \\
\hline 8.788 & 13.182 & 2.197 & 2.197 \\
\hline 2.197 & 35.152 & 2.197 & 145.002 \\
\hline 13.182 & 6.591 & 4.394 & 241.67 \\
\hline 151.593 & 2.197 & 2.197 & 65.91 \\
\hline 2.197 & 2.197 & 10.985 & 6.591 \\
\hline 35.152 & 8.788 & 2.197 & 6.591 \\
\hline 63.713 & 21.97 & 4.394 & 2.197 \\
\hline 79.092 & 13.182 & 2.197 & 4.394 \\
\hline 182.351 & 2.197 & 8.788 & 2.197 \\
\hline 450.385 & 2.197 & 2.197 & 2.197 \\
\hline 87.88 & 19.773 & 105.456 & 48.334 \\
\hline 46.137 & 54.925 & 43.94 & 13.182 \\
\hline 138.411 & 6.591 & 4.394 & 24.167 \\
\hline 70.304 & 13.182 & 10.985 & 177.957 \\
\hline 2.197 & 19.773 & 15.379 & 2.197 \\
\hline 37.349 & 4.394 & 57.122 & 2.197 \\
\hline 2.197 & 4.394 & 2.197 & 2.197 \\
\hline 19.773 & 2.197 & 10.985 & 6.591 \\
\hline 2.197 & 24.167 & 10.985 & 10.985 \\
\hline 59.319 & 2.197 & 2.197 & 2.197 \\
\hline 10.985 & 17.576 & 2.197 & 35.152 \\
\hline 2.197 & 142.805 & 6.591 & 54.925 \\
\hline 4.394 & 21.97 & 59.319 & 4.394 \\
\hline 2.197 & 8.788 & 10.985 & 32.955 \\
\hline 72.501 & 76.895 & 8.788 & 46.137 \\
\hline 4.394 & 17.576 & 4.394 & 24.167 \\
\hline 4.394 & 4.394 & 4.394 & 138.411 \\
\hline 8.788 & 94.471 & 4.394 & 17.576 \\
\hline
\end{tabular}




\begin{tabular}{|c|c|c|c|}
\hline 946.907 & 19.773 & 4.394 & 68.107 \\
\hline 169.169 & 43.94 & 2.197 & 283.413 \\
\hline 15.379 & 26.364 & 13.182 & 188.942 \\
\hline 6.591 & 2.197 & 46.137 & 8.788 \\
\hline 2.197 & 21.97 & 2.197 & 2.197 \\
\hline 37.349 & 15.379 & 4.394 & 171.366 \\
\hline 17.576 & 48.334 & 4.394 & 7678.51 \\
\hline 2.197 & 2.197 & 6.591 & 2.197 \\
\hline 4.394 & 17.576 & 92.274 & 2.197 \\
\hline 6.591 & 393.263 & 920.543 & 2.197 \\
\hline 17.576 & 4.394 & 28.561 & 2.197 \\
\hline 193.336 & 10.985 & 43.94 & 17.576 \\
\hline 2.197 & 4.394 & 2.197 & 2.197 \\
\hline 2.197 & 19.773 & 6.591 & 13.182 \\
\hline 92.274 & 2.197 & 2.197 & 2.197 \\
\hline 2.197 & 2.197 & 65.91 & 6.591 \\
\hline 2.197 & 26.364 & 2.197 & 15.379 \\
\hline 32.955 & 24.167 & 4.394 & 15.379 \\
\hline 57.122 & 4.394 & 21.97 & 6.591 \\
\hline 2.197 & 32.955 & 235.079 & 2.197 \\
\hline 39.546 & 268.034 & 4.394 & 48.334 \\
\hline 28.561 & 2.197 & 134.017 & 2.197 \\
\hline 19.773 & 2.197 & 294.398 & 2.197 \\
\hline 35.152 & 8.788 & 37.349 & 206.518 \\
\hline 2.197 & 2.197 & 28.561 & 131.82 \\
\hline 2.197 & 4.394 & 2.197 & 4.394 \\
\hline 10.985 & 4.394 & 8.788 & 8.788 \\
\hline 48.334 & 2.197 & 6.591 & 6.591 \\
\hline 2.197 & 2.197 & 50.531 & 15.379 \\
\hline 83.486 & 54.925 & 10.985 & 112.047 \\
\hline 2.197 & 478.946 & 10.985 & 10.985 \\
\hline 182.351 & 8.788 & 454.779 & 239.473 \\
\hline 4.394 & 2.197 & 13.182 & 4.394 \\
\hline 2.197 & 2.197 & 21.97 & 4.394 \\
\hline 2.197 & 4.394 & 131.82 & 2.197 \\
\hline 10.985 & 4.394 & 2.197 & 26.364 \\
\hline 2.197 & 17.576 & 2.197 & 861.224 \\
\hline 6.591 & 4549.99 & 2.197 & 4.394 \\
\hline 6.591 & 70.304 & 2.197 & 17.576 \\
\hline 50.531 & 10.985 & 2.197 & 19.773 \\
\hline 2.197 & 4.394 & 15.379 & 2.197 \\
\hline 13.182 & 17.576 & 2.197 & 4.394 \\
\hline 83.486 & 6.591 & 6.591 & 2.197 \\
\hline 4.394 & 79.092 & 2.197 & 4.394 \\
\hline 2.197 & 184.548 & 2.197 & 2.197 \\
\hline 19.773 & 8.788 & 13.182 & 3385.58 \\
\hline 10.985 & 17.576 & 2.197 & 162.578 \\
\hline 173.563 & 10.985 & 2.197 & 2.197 \\
\hline 79.092 & 32.955 & 2.197 & 2.197 \\
\hline 2.197 & 8.788 & 13.182 & 6.591 \\
\hline
\end{tabular}




\begin{tabular}{|c|c|c|c|}
\hline 37.349 & 2.197 & 21.97 & 32.955 \\
\hline 8.788 & 2.197 & 19.773 & 26.364 \\
\hline 2.197 & 2.197 & 28.561 & 6.591 \\
\hline 2.197 & 426.218 & 109.85 & 8.788 \\
\hline 228.488 & 17.576 & 10.985 & 32.955 \\
\hline 24.167 & 21.97 & 2.197 & 15.379 \\
\hline 2.197 & 17.576 & 149.396 & 318.565 \\
\hline 2.197 & 63.713 & 2.197 & 703.04 \\
\hline 2.197 & 6.591 & 2.197 & 50.531 \\
\hline 21.97 & 149.396 & 2.197 & 13.182 \\
\hline 2.197 & 8.788 & 15.379 & 2.197 \\
\hline 52.728 & 391.066 & 4.394 & 24.167 \\
\hline 30.758 & 41.743 & 2.197 & 13.182 \\
\hline 28.561 & 10.985 & 525.083 & 134.017 \\
\hline 4.394 & 147.199 & 2.197 & 2.197 \\
\hline 4.394 & 37.349 & 2.197 & 15.379 \\
\hline 2.197 & 17.576 & 43.94 & 4.394 \\
\hline 65.91 & 2.197 & 134.017 & 26.364 \\
\hline 8.788 & 4.394 & 2.197 & 2.197 \\
\hline 8.788 & 2.197 & 2.197 & 2.197 \\
\hline 35.152 & 13.182 & 35.152 & 2.197 \\
\hline 2.197 & 2.197 & 112.047 & 24.167 \\
\hline 2.197 & 10.985 & 2.197 & 2.197 \\
\hline 15.379 & 4.394 & 2.197 & 19.773 \\
\hline 1078.73 & 8.788 & 2.197 & 2.197 \\
\hline 30.758 & 2.197 & 30.758 & 4.394 \\
\hline 28.561 & 21.97 & 2.197 & 57.122 \\
\hline 169.169 & 120.835 & 4.394 & 52.728 \\
\hline 259.246 & 107.653 & 90.077 & 76.895 \\
\hline 10.985 & 2.197 & 2.197 & 2.197 \\
\hline 15.379 & 2.197 & 4.394 & 114.244 \\
\hline 2.197 & 147.199 & 2.197 & 72.501 \\
\hline 87.88 & 17.576 & 2.197 & 4.394 \\
\hline 6.591 & 6.591 & 4.394 & 10.985 \\
\hline 59.319 & 6.591 & 2.197 & 2.197 \\
\hline 21.97 & 2.197 & 2.197 & 21.97 \\
\hline 21.97 & 50.531 & 2.197 & 2.197 \\
\hline 13.182 & 30.758 & 15.379 & 10.985 \\
\hline 4.394 & 15.379 & 6.591 & 13.182 \\
\hline 28.561 & 17.576 & 2.197 & 4.394 \\
\hline 2.197 & 2.197 & 3398.76 & 35.152 \\
\hline 2.197 & 171.366 & 4.394 & 4.394 \\
\hline 28.561 & 2.197 & 13.182 & 4.394 \\
\hline 2.197 & 35.152 & 2.197 & 2.197 \\
\hline 21.97 & 6.591 & 15.379 & 35.152 \\
\hline 1026 & 2.197 & 52.728 & 2994.51 \\
\hline 2.197 & 4.394 & 2.197 & 30.758 \\
\hline 4.394 & 6.591 & 4.394 & 399.854 \\
\hline 10.985 & 6.591 & 17.576 & 6.591 \\
\hline 2.197 & 41.743 & 19.773 & 8.788 \\
\hline
\end{tabular}




\begin{tabular}{|c|c|c|c|}
\hline 13.182 & 4.394 & 4.394 & 2.197 \\
\hline 57.122 & 2.197 & 4.394 & 6.591 \\
\hline 2.197 & 2.197 & 37.349 & 15.379 \\
\hline 2.197 & 2.197 & 2.197 & 2.197 \\
\hline 2.197 & 2.197 & 2.197 & 10.985 \\
\hline 76.895 & 30.758 & 2.197 & 10.985 \\
\hline 24.167 & 48.334 & 13.182 & 57.122 \\
\hline 2.197 & 2.197 & 2.197 & 30.758 \\
\hline 186.745 & 13.182 & 123.032 & 8.788 \\
\hline 2.197 & 13.182 & 8.788 & 2.197 \\
\hline 307.58 & 43.94 & 2.197 & 2.197 \\
\hline 13.182 & 2.197 & 4.394 & 2.197 \\
\hline 2.197 & 13.182 & 6.591 & 28.561 \\
\hline 17.576 & 2.197 & 28.561 & 2.197 \\
\hline 54.925 & 32.955 & 48.334 & 136.214 \\
\hline 467.961 & 35.152 & 2.197 & 2.197 \\
\hline 46.137 & 2.197 & 61.516 & 2.197 \\
\hline 39.546 & 17.576 & 26.364 & 15.379 \\
\hline 2.197 & 10.985 & 90.077 & 6.591 \\
\hline 17.576 & 6.591 & 43.94 & 43.94 \\
\hline 6.591 & 314.171 & 2.197 & 590.993 \\
\hline 8.788 & 6.591 & 13.182 & 6.591 \\
\hline 4.394 & 81.289 & 4.394 & 2.197 \\
\hline 10.985 & 4.394 & 2.197 & 26.364 \\
\hline 48.334 & 35.152 & 2.197 & 10.985 \\
\hline 6.591 & 17.576 & 4.394 & 30.758 \\
\hline 4.394 & 153.79 & 4.394 & 8.788 \\
\hline 17.576 & 4.394 & 2.197 & 204.321 \\
\hline 2.197 & 2.197 & 2.197 & 32.955 \\
\hline 15.379 & 2.197 & 8.788 & 37.349 \\
\hline 4.394 & 4.394 & 2.197 & 28.561 \\
\hline 6.591 & 2.197 & 2.197 & 166.972 \\
\hline 4.394 & 2.197 & 4.394 & 90.077 \\
\hline 41.743 & 17.576 & 4.394 & 2.197 \\
\hline 6.591 & 2.197 & 39.546 & 4.394 \\
\hline 19.773 & 2.197 & 8.788 & 30.758 \\
\hline 2.197 & 6.591 & 4.394 & 39.546 \\
\hline 2.197 & 15.379 & 4.394 & 19.773 \\
\hline 43.94 & 8.788 & 2.197 & 2.197 \\
\hline 2.197 & 4.394 & 2.197 & 4.394 \\
\hline 4.394 & 2.197 & 39.546 & 2.197 \\
\hline 2.197 & 17.576 & 15.379 & 2.197 \\
\hline 4.394 & 8.788 & 4.394 & 4.394 \\
\hline 2.197 & 70.304 & 103.259 & 340.535 \\
\hline 13.182 & 74.698 & 2.197 & 2.197 \\
\hline 2.197 & 35.152 & 2.197 & 8.788 \\
\hline 24.167 & 43.94 & 2.197 & 2.197 \\
\hline 19.773 & 2.197 & 4.394 & 4.394 \\
\hline 4.394 & 2.197 & 2.197 & 24.167 \\
\hline 2.197 & 13.182 & 79092 & 10.985 \\
\hline
\end{tabular}




\begin{tabular}{|c|c|c|c|}
\hline 4.394 & 8.788 & 6.591 & 4.394 \\
\hline 2.197 & 17.576 & 6.591 & 21.97 \\
\hline 2.197 & 4.394 & 2.197 & 17.576 \\
\hline 24.167 & 2.197 & 4.394 & 35.152 \\
\hline 8.788 & 511.901 & 10.985 & 79.092 \\
\hline 8.788 & 26.364 & 417.43 & 140.608 \\
\hline 6.591 & 17.576 & 10.985 & 17.576 \\
\hline 2.197 & 8.788 & 59.319 & 8.788 \\
\hline 2.197 & 2.197 & 10.985 & 39.546 \\
\hline 15.379 & 6.591 & 17.576 & 6.591 \\
\hline 6.591 & 81.289 & 2.197 & 10.985 \\
\hline 2.197 & 8.788 & 2.197 & 28.561 \\
\hline 4.394 & 2.197 & 10.985 & 37.349 \\
\hline 4.394 & 6.591 & 2.197 & 13.182 \\
\hline 68.107 & 254.852 & 2.197 & 92.274 \\
\hline 72.501 & 19.773 & 2.197 & 90.077 \\
\hline 26.364 & 6.591 & 10.985 & 10.985 \\
\hline 4.394 & 37.349 & 2.197 & 2.197 \\
\hline 24.167 & 13.182 & 2.197 & 2.197 \\
\hline 2.197 & 4.394 & 2.197 & 2.197 \\
\hline 2.197 & 2.197 & 2.197 & 4.394 \\
\hline 2.197 & 10.985 & 2.197 & 4.394 \\
\hline 2.197 & 4.394 & 15.379 & 10.985 \\
\hline 2.197 & 2.197 & 1326.99 & 13.182 \\
\hline 13.182 & 21.97 & 2.197 & 2.197 \\
\hline 2.197 & 116.441 & 2.197 & 828.269 \\
\hline 57.122 & 32.955 & 10.985 & 2.197 \\
\hline 4.394 & 6.591 & 4.394 & 2.197 \\
\hline 4.394 & 19.773 & 2.197 & 8.788 \\
\hline 2.197 & 19.773 & 13.182 & 59.319 \\
\hline 2.197 & 15.379 & 8.788 & 46.137 \\
\hline 138.411 & 98.865 & 2.197 & 4.394 \\
\hline 28.561 & 2.197 & 26.364 & 2.197 \\
\hline 10.985 & 6.591 & 2.197 & 125.229 \\
\hline 4.394 & 118.638 & 2.197 & 10.985 \\
\hline 17.576 & 21.97 & 2.197 & 65.91 \\
\hline 4.394 & 8.788 & 2.197 & 2.197 \\
\hline 8.788 & 4.394 & 10.985 & 2.197 \\
\hline 48.334 & 21.97 & 2.197 & 8.788 \\
\hline 68.107 & 2.197 & 2.197 & 2.197 \\
\hline 83.486 & 2.197 & 72.501 & 15.379 \\
\hline 4.394 & 2.197 & 4.394 & 4.394 \\
\hline 32.955 & 2.197 & 2.197 & 6.591 \\
\hline 6.591 & 8.788 & 2.197 & 2.197 \\
\hline 17.576 & 4.394 & 2.197 & 6.591 \\
\hline 50.531 & 32.955 & 68.107 & 2.197 \\
\hline 2.197 & 2.197 & 4.394 & 19.773 \\
\hline 35.152 & 4.394 & 2.197 & 28.561 \\
\hline 81.289 & 2.197 & 4.394 & 8.788 \\
\hline 6.591 & 1390.7 & 5887.96 & 8.788 \\
\hline
\end{tabular}




\begin{tabular}{|c|c|c|c|}
\hline 2.197 & 15.379 & 4.394 & 54.925 \\
\hline 4.394 & 76.895 & 2.197 & 50.531 \\
\hline 112.047 & 2.197 & 4.394 & 8.788 \\
\hline 487.734 & 2.197 & 2.197 & 59.319 \\
\hline 2.197 & 164.775 & 2.197 & 13.182 \\
\hline 4.394 & 24.167 & 4.394 & 698.646 \\
\hline 4.394 & 17.576 & 2.197 & 6.591 \\
\hline 6.591 & 52.728 & 4.394 & 54.925 \\
\hline 2.197 & 2.197 & 2.197 & 8.788 \\
\hline 6.591 & 4.394 & 4.394 & 2.197 \\
\hline 21.97 & 4.394 & 2.197 & 2.197 \\
\hline 15.379 & 92.274 & 2.197 & 2.197 \\
\hline 2.197 & 224.094 & 2.197 & 10.985 \\
\hline 74.698 & 4.394 & 6.591 & 35.152 \\
\hline 2.197 & 59.319 & 3207.62 & 210.912 \\
\hline 2.197 & 57.122 & 2.197 & 96.668 \\
\hline 2.197 & 10.985 & 2.197 & 2.197 \\
\hline 2.197 & 101.062 & 6.591 & 2.197 \\
\hline 2.197 & 125.229 & 2.197 & 2.197 \\
\hline 4.394 & 4.394 & 4.394 & 129.623 \\
\hline 2.197 & 8.788 & 94.471 & 19.773 \\
\hline 13.182 & 10.985 & 2.197 & 10.985 \\
\hline 2.197 & 2.197 & 43.94 & 21.97 \\
\hline 2.197 & 2.197 & 46.137 & 4.394 \\
\hline 2.197 & 2.197 & 39.546 & 8.788 \\
\hline 54.925 & 4.394 & 169.169 & 2.197 \\
\hline 52.728 & 224.094 & 2.197 & 79.092 \\
\hline 37.349 & 217.503 & 2.197 & 6.591 \\
\hline 2.197 & 2.197 & 2.197 & 87.88 \\
\hline 21.97 & 2.197 & 2.197 & 8.788 \\
\hline 30.758 & 2.197 & 2.197 & 6.591 \\
\hline 4.394 & 48.334 & 24.167 & 13.182 \\
\hline 13.182 & 10.985 & 2.197 & 13.182 \\
\hline 6.591 & 107.653 & 61.516 & 17.576 \\
\hline 8.788 & 19.773 & 2.197 & 48.334 \\
\hline 2.197 & 140.608 & 2.197 & 2.197 \\
\hline 2.197 & 8.788 & 6.591 & 8.788 \\
\hline 2.197 & 13.182 & 160.381 & 4.394 \\
\hline 6.591 & 24.167 & 2.197 & 2.197 \\
\hline 17.576 & 922.74 & 2.197 & 65.91 \\
\hline 4.394 & 8.788 & 4.394 & 8.788 \\
\hline 8.788 & 10.985 & 15.379 & 6.591 \\
\hline 2.197 & 28.561 & 112.047 & 2.197 \\
\hline 2.197 & 160.381 & 28.561 & 17.576 \\
\hline 224.094 & 6.591 & 15.379 & 15.379 \\
\hline 6.591 & 6.591 & 2.197 & 2.197 \\
\hline 24.167 & 92.274 & 2.197 & 2.197 \\
\hline 2.197 & 6.591 & 10.985 & 4.394 \\
\hline 4.394 & 2.197 & 2.197 & 10.985 \\
\hline 2383.74 & 2.197 & 2.197 & 6.591 \\
\hline
\end{tabular}




\begin{tabular}{|c|c|c|c|}
\hline 120.835 & 2.197 & 103.259 & 6.591 \\
\hline 2.197 & 2.197 & 68.107 & 19.773 \\
\hline 1395.09 & 2.197 & 4.394 & 13.182 \\
\hline 4.394 & 2.197 & 57.122 & 13.182 \\
\hline 17.576 & 2.197 & 52.728 & 19.773 \\
\hline 2.197 & 2.197 & 2.197 & 2.197 \\
\hline 2.197 & 8.788 & 683.267 & 2.197 \\
\hline 2.197 & 4.394 & 2.197 & 6.591 \\
\hline 68.107 & 6.591 & 136.214 & 15.379 \\
\hline 2.197 & 4.394 & 2.197 & 19.773 \\
\hline 2.197 & 41.743 & 17.576 & 2.197 \\
\hline 2.197 & 2.197 & 10.985 & 2.197 \\
\hline 6.591 & 68.107 & 2.197 & 4104 \\
\hline 188.942 & 15.379 & 2.197 & 83.486 \\
\hline 46.137 & 43.94 & 32.955 & 10.985 \\
\hline 175.76 & 4.394 & 2.197 & 37.349 \\
\hline 39.546 & 10.985 & 6.591 & 2.197 \\
\hline 2.197 & 4.394 & 4.394 & 2.197 \\
\hline 26.364 & 2.197 & 2.197 & 2.197 \\
\hline 13.182 & 4.394 & 2.197 & 43.94 \\
\hline 2.197 & 4.394 & 15.379 & 43.94 \\
\hline 2025.63 & 15.379 & 2.197 & 15.379 \\
\hline 17.576 & 2.197 & 2.197 & 2.197 \\
\hline 17.576 & 26.364 & 2.197 & 2.197 \\
\hline 43.94 & 17.576 & 2.197 & 2.197 \\
\hline 39.546 & 46.137 & 371.293 & 37.349 \\
\hline 4.394 & 10.985 & 2.197 & 13.182 \\
\hline 28.561 & 35.152 & 41.743 & 4.394 \\
\hline 2.197 & 2.197 & 2.197 & 26.364 \\
\hline 10.985 & 134.017 & 2.197 & 46.137 \\
\hline 2.197 & 2.197 & 4.394 & 2.197 \\
\hline 28.561 & 2.197 & 6.591 & 70.304 \\
\hline 59.319 & 59.319 & 76.895 & 6.591 \\
\hline 2.197 & 2.197 & 2.197 & 30.758 \\
\hline 2.197 & 17.576 & 19.773 & 32.955 \\
\hline 24.167 & 32.955 & 13.182 & 2.197 \\
\hline 206.518 & 65.91 & 15.379 & 2.197 \\
\hline 188.942 & 10.985 & 26.364 & 19.773 \\
\hline 342.732 & 2.197 & 2.197 & 8.788 \\
\hline 2.197 & 8.788 & 2.197 & 19.773 \\
\hline 6.591 & 10.985 & 4.394 & 162.578 \\
\hline 2.197 & 72.501 & 6.591 & 2.197 \\
\hline 2.197 & 2.197 & 8.788 & 2.197 \\
\hline 2.197 & 74.698 & 13.182 & 340.535 \\
\hline 70.304 & 15.379 & 2.197 & 13.182 \\
\hline 17.576 & 13.182 & 6.591 & 24.167 \\
\hline 2.197 & 118.638 & 4.394 & 2.197 \\
\hline 114.244 & 15.379 & 2.197 & 169.169 \\
\hline 2.197 & 10.985 & 2.197 & 2.197 \\
\hline 13.182 & 2.197 & 2.197 & 6.591 \\
\hline
\end{tabular}




\begin{tabular}{|c|c|c|c|}
\hline 90.077 & 2.197 & 10.985 & 26.364 \\
\hline 13.182 & 2.197 & 2.197 & 4.394 \\
\hline 4.394 & 10.985 & 2.197 & 2.197 \\
\hline 76.895 & 6.591 & 4.394 & 2.197 \\
\hline 2.197 & 2.197 & 6.591 & 2.197 \\
\hline 83.486 & 2.197 & 13.182 & 2.197 \\
\hline 8.788 & 2.197 & 4.394 & 2.197 \\
\hline 10.985 & 2.197 & 2.197 & 2.197 \\
\hline 6.591 & 582.205 & 2.197 & 2.197 \\
\hline 2.197 & 87.88 & 21.97 & 94.471 \\
\hline 6.591 & 105.456 & 2.197 & 21.97 \\
\hline 13.182 & 4.394 & 35.152 & 30.758 \\
\hline 364.702 & 4.394 & 10.985 & 2.197 \\
\hline 8.788 & 35.152 & 6.591 & 2.197 \\
\hline 129.623 & 68.107 & 59.319 & 30.758 \\
\hline 2.197 & 120.835 & 6.591 & 10.985 \\
\hline 2.197 & 2.197 & 10.985 & 171.366 \\
\hline 2.197 & 4.394 & 8372.77 & 19.773 \\
\hline 4.394 & 2.197 & 4.394 & 2.197 \\
\hline 2.197 & 4.394 & 2.197 & 15.379 \\
\hline 68.107 & 24.167 & 4.394 & 2.197 \\
\hline 6.591 & 50.531 & 17.576 & 2.197 \\
\hline 2.197 & 48.334 & 37.349 & 17.576 \\
\hline 134.017 & 2.197 & 4.394 & 2.197 \\
\hline 6.591 & 98.865 & 101.062 & 2.197 \\
\hline 50.531 & 4.394 & 8.788 & 1034.79 \\
\hline 6.591 & 180.154 & 2.197 & 19.773 \\
\hline 26.364 & 28.561 & 4.394 & 2.197 \\
\hline 83.486 & 35.152 & 2.197 & 2.197 \\
\hline 19.773 & 39.546 & 43.94 & 8.788 \\
\hline 2.197 & 404.248 & 2.197 & 79.092 \\
\hline 52.728 & 8.788 & 4.394 & 4.394 \\
\hline 4.394 & 395.46 & 6.591 & 65.91 \\
\hline 10.985 & 76.895 & 2.197 & 26.364 \\
\hline 15.379 & 13.182 & 2.197 & 13.182 \\
\hline 6.591 & 2.197 & 2.197 & 6.591 \\
\hline 17.576 & 2.197 & 26.364 & 4.394 \\
\hline 8.788 & 6.591 & 265.837 & 193.336 \\
\hline 4.394 & 8.788 & 2.197 & 5896.75 \\
\hline 2.197 & 2.197 & 26.364 & 2.197 \\
\hline 15.379 & 10.985 & 243.867 & 197.73 \\
\hline 2.197 & 2.197 & 2.197 & 4.394 \\
\hline 2.197 & 10.985 & 72.501 & 13.182 \\
\hline 2.197 & 32.955 & 19.773 & 15.379 \\
\hline 2.197 & 4.394 & 10.985 & 26.364 \\
\hline 46.137 & 13.182 & 10.985 & 52.728 \\
\hline 377.884 & 142.805 & 10.985 & 2.197 \\
\hline 2.197 & 4.394 & 2.197 & 105.456 \\
\hline 15.379 & 74.698 & 2.197 & 4.394 \\
\hline 26.364 & 2.197 & 61.516 & 2.197 \\
\hline
\end{tabular}




\begin{tabular}{|c|c|c|c|}
\hline 2.197 & 2.197 & 2.197 & 2.197 \\
\hline 10.985 & 21.97 & 28.561 & 72.501 \\
\hline 6.591 & 2.197 & 2.197 & 13.182 \\
\hline 131.82 & 28.561 & 2.197 & 10.985 \\
\hline 39.546 & 4.394 & 2.197 & 70.304 \\
\hline 2.197 & 4.394 & 2.197 & 13.182 \\
\hline 30.758 & 15.379 & 2.197 & 265.837 \\
\hline 4.394 & 19.773 & 2.197 & 48.334 \\
\hline 331.747 & 6.591 & 2.197 & 24.167 \\
\hline 162.578 & 8.788 & 30.758 & 98.865 \\
\hline 2133.29 & 112.047 & 52.728 & 199.927 \\
\hline 2.197 & 4.394 & 2.197 & 4.394 \\
\hline 2.197 & 2.197 & 2.197 & 4.394 \\
\hline 2.197 & 4.394 & 2.197 & 35.152 \\
\hline 134.017 & 90.077 & 6.591 & 50.531 \\
\hline 2.197 & 8.788 & 2.197 & 4.394 \\
\hline 6.591 & 26.364 & 2.197 & 145.002 \\
\hline 2.197 & 13.182 & 61.516 & 2.197 \\
\hline 21.97 & 898.573 & 41.743 & 2.197 \\
\hline 4.394 & 26.364 & 4.394 & 2.197 \\
\hline 10.985 & 8.788 & 2.197 & 59.319 \\
\hline 63.713 & 2.197 & 2.197 & 4.394 \\
\hline 2.197 & 2.197 & 2.197 & 87.88 \\
\hline 4.394 & 19.773 & 2.197 & 2.197 \\
\hline 19.773 & 41.743 & 37.349 & 2.197 \\
\hline 2.197 & 2.197 & 4.394 & 8.788 \\
\hline 2.197 & 2.197 & 2.197 & 285.61 \\
\hline 2.197 & 2.197 & 2.197 & 2.197 \\
\hline 2.197 & 393.263 & 21.97 & 2.197 \\
\hline 2.197 & 15.379 & 2.197 & 2.197 \\
\hline 2.197 & 2.197 & 15.379 & 13.182 \\
\hline 2.197 & 10.985 & 24.167 & 8.788 \\
\hline 973.271 & 21.97 & 19.773 & 8.788 \\
\hline 4.394 & 116.441 & 6.591 & 17.576 \\
\hline 6.591 & 4.394 & 573.417 & 8.788 \\
\hline 4.394 & 41.743 & 17.576 & 2.197 \\
\hline 4.394 & 4.394 & 8.788 & 2.197 \\
\hline 8.788 & 19.773 & 94.471 & 24.167 \\
\hline 13.182 & 6.591 & 46.137 & 54.925 \\
\hline 57.122 & 173.563 & 2.197 & 8.788 \\
\hline 19.773 & 19.773 & 2.197 & 670.085 \\
\hline 26.364 & 6.591 & 2.197 & 17.576 \\
\hline 15.379 & 6.591 & 2.197 & 2.197 \\
\hline 2.197 & 10.985 & 24.167 & 52.728 \\
\hline 30.758 & 24.167 & 191.139 & 26.364 \\
\hline 19.773 & 19.773 & 2.197 & 4.394 \\
\hline 2.197 & 151.593 & 17.576 & 19.773 \\
\hline 13.182 & 48.334 & 2.197 & 2.197 \\
\hline 26.364 & 2.197 & 2.197 & 8.788 \\
\hline 2.197 & 30.758 & 13.182 & 8.788 \\
\hline
\end{tabular}




\begin{tabular}{|c|c|c|c|}
\hline 48.334 & 74.698 & 2.197 & 17.576 \\
\hline 2.197 & 4.394 & 2.197 & 2.197 \\
\hline 2.197 & 98.865 & 13.182 & 13.182 \\
\hline 39.546 & 24.167 & 15.379 & 2.197 \\
\hline 21.97 & 764.556 & 4.394 & 4.394 \\
\hline 28.561 & 39.546 & 2.197 & 107.653 \\
\hline 2.197 & 70.304 & 2.197 & 32.955 \\
\hline 2.197 & 186.745 & 2.197 & 3124.13 \\
\hline 2.197 & 28.561 & 2.197 & 19.773 \\
\hline 6.591 & 24.167 & 37.349 & 2.197 \\
\hline 4.394 & 63.713 & 2.197 & 15.379 \\
\hline 50.531 & 6.591 & 2.197 & 24.167 \\
\hline 85.683 & 50.531 & 2.197 & 503.113 \\
\hline 129.623 & 4.394 & 2.197 & 17.576 \\
\hline 2.197 & 46.137 & 13.182 & 72.501 \\
\hline 2.197 & 2.197 & 26.364 & 2.197 \\
\hline 4.394 & 10.985 & 13.182 & 15.379 \\
\hline 37.349 & 4.394 & 4.394 & 292.201 \\
\hline 28.561 & 142.805 & 2.197 & 8.788 \\
\hline 13.182 & 6.591 & 26.364 & 4.394 \\
\hline 2.197 & 2.197 & 8.788 & 59.319 \\
\hline 39.546 & 6.591 & 83.486 & 48.334 \\
\hline 10.985 & 2.197 & 2.197 & 8.788 \\
\hline 4.394 & 41.743 & 2.197 & 19.773 \\
\hline 15.379 & 13.182 & 2.197 & 30.758 \\
\hline 6.591 & 4.394 & 8.788 & 8.788 \\
\hline 15.379 & 6.591 & 6.591 & 294.398 \\
\hline 4.394 & 4.394 & 2.197 & 4.394 \\
\hline 101.062 & 2.197 & 43.94 & 421.824 \\
\hline 6.591 & 2.197 & 8.788 & 46.137 \\
\hline 250.458 & 4.394 & 30.758 & 109.85 \\
\hline 6.591 & 4.394 & 41.743 & 2.197 \\
\hline 2.197 & 2.197 & 2.197 & 10.985 \\
\hline 2.197 & 2.197 & 2.197 & 61.516 \\
\hline 4.394 & 4.394 & 2.197 & 10.985 \\
\hline 24.167 & 2.197 & 2.197 & 2.197 \\
\hline 10.985 & 4.394 & 4.394 & 4.394 \\
\hline 4.394 & 2.197 & 10.985 & 2.197 \\
\hline 8.788 & 8.788 & 6.591 & 2.197 \\
\hline 6.591 & 6.591 & 87.88 & 2.197 \\
\hline 6.591 & 4.394 & 2.197 & 265.837 \\
\hline 17.576 & 50.531 & 2.197 & 37.349 \\
\hline 746.98 & 13.182 & 2.197 & 2.197 \\
\hline 19.773 & 13.182 & 2.197 & 8.788 \\
\hline 2.197 & 4.394 & 6.591 & 54.925 \\
\hline 2.197 & 13.182 & 4.394 & 4.394 \\
\hline 2.197 & 26.364 & 2.197 & 76.895 \\
\hline 2.197 & 21.97 & 2.197 & 2.197 \\
\hline 15.379 & 57.122 & 2.197 & 2.197 \\
\hline 127.426 & 2.197 & 30.758 & 57.122 \\
\hline
\end{tabular}




\begin{tabular}{|c|c|c|c|}
\hline 136.214 & 10.985 & 2.197 & 120.835 \\
\hline 39.546 & 24.167 & 4.394 & 30.758 \\
\hline 123.032 & 2.197 & 2.197 & 2.197 \\
\hline 2.197 & 4.394 & 2.197 & 87.88 \\
\hline 10.985 & 8.788 & 2.197 & 68.107 \\
\hline 15.379 & 35.152 & 2.197 & 19.773 \\
\hline 2.197 & 6.591 & 2.197 & 2.197 \\
\hline 4.394 & 8.788 & 2.197 & 193.336 \\
\hline 10.985 & 193.336 & 2.197 & 4.394 \\
\hline 8.788 & 72.501 & 8.788 & 237.276 \\
\hline 6.591 & 2.197 & 2.197 & 41.743 \\
\hline 61.516 & 2.197 & 2.197 & 39.546 \\
\hline 2.197 & 2.197 & 24.167 & 30.758 \\
\hline 8.788 & 6.591 & 4.394 & 6.591 \\
\hline 8.788 & 26.364 & 24.167 & 4.394 \\
\hline 8.788 & 24.167 & 35.152 & 2.197 \\
\hline 26.364 & 4.394 & 6.591 & 2.197 \\
\hline 6.591 & 8.788 & 48.334 & 2.197 \\
\hline 17.576 & 4.394 & 4.394 & 39.546 \\
\hline 4.394 & 162.578 & 19.773 & 15.379 \\
\hline 28.561 & 50.531 & 2.197 & 92.274 \\
\hline 8.788 & 21.97 & 13.182 & 4.394 \\
\hline 4.394 & 2.197 & 116.441 & 8.788 \\
\hline 8.788 & 2.197 & 4.394 & 13.182 \\
\hline 2.197 & 140.608 & 96.668 & 2.197 \\
\hline 4.394 & 2.197 & 72.501 & 2.197 \\
\hline 10.985 & 2.197 & 4.394 & 24.167 \\
\hline 4.394 & 10.985 & 15.379 & 435.006 \\
\hline 263.64 & 2.197 & 1632.37 & 2.197 \\
\hline 8.788 & 6.591 & 39.546 & 6.591 \\
\hline 2.197 & 4.394 & 4.394 & 17.576 \\
\hline 2.197 & 72.501 & 6.591 & 92.274 \\
\hline 2.197 & 74.698 & 4.394 & 46.137 \\
\hline 2.197 & 2390.34 & 147.199 & 2.197 \\
\hline 6.591 & 13.182 & 2.197 & 90.077 \\
\hline 8.788 & 92.274 & 13.182 & 2.197 \\
\hline 92.274 & 26.364 & 162.578 & 4.394 \\
\hline 10.985 & 4.394 & 10.985 & 17.576 \\
\hline 17.576 & 4.394 & 2.197 & 4.394 \\
\hline 46.137 & 39.546 & 4.394 & 10.985 \\
\hline 2.197 & 145.002 & 6.591 & 8.788 \\
\hline 2.197 & 184.548 & 2.197 & 54.925 \\
\hline 2.197 & 6.591 & 4.394 & 43.94 \\
\hline 2.197 & 2.197 & 61.516 & 46.137 \\
\hline 186.745 & 2.197 & 2.197 & 15.379 \\
\hline 2.197 & 76.895 & 2.197 & 4.394 \\
\hline 2.197 & 59.319 & 2.197 & 24.167 \\
\hline 2.197 & 2.197 & 2.197 & 43.94 \\
\hline 2.197 & 6.591 & 8.788 & 250.458 \\
\hline 2.197 & 13.182 & 61.516 & 4.394 \\
\hline
\end{tabular}




\begin{tabular}{|c|c|c|c|}
\hline 2.197 & 6.591 & 61.516 & 169.169 \\
\hline 2.197 & 360.308 & 2.197 & 41.743 \\
\hline 13.182 & 6.591 & 145.002 & 2.197 \\
\hline 8.788 & 2.197 & 2.197 & 8.788 \\
\hline 142.805 & 63.713 & 4.394 & 2.197 \\
\hline 2.197 & 2.197 & 19.773 & 300.989 \\
\hline 26.364 & 8.788 & 35.152 & 2.197 \\
\hline 8.788 & 241.67 & 4.394 & 2.197 \\
\hline 24.167 & 13.182 & 4.394 & 2.197 \\
\hline 6.591 & 8.788 & 2.197 & 21.97 \\
\hline 8.788 & 24.167 & 2.197 & 2.197 \\
\hline 2.197 & 32.955 & 2.197 & 4.394 \\
\hline 4.394 & 26.364 & 19.773 & 28.561 \\
\hline 13.182 & 57.122 & 50.531 & 17.576 \\
\hline 4.394 & 52.728 & 2.197 & 2.197 \\
\hline 35.152 & 28.561 & 2.197 & 2.197 \\
\hline 32.955 & 24.167 & 4.394 & 4.394 \\
\hline 241.67 & 8.788 & 2.197 & 35.152 \\
\hline 2.197 & 37.349 & 2.197 & 26.364 \\
\hline 13.182 & 177.957 & 2.197 & 2.197 \\
\hline 6.591 & 4.394 & 2.197 & 35.152 \\
\hline 4.394 & 4.394 & 2.197 & 173.563 \\
\hline 15.379 & 2.197 & 24.167 & 4.394 \\
\hline 50.531 & 6.591 & 4.394 & 856.83 \\
\hline 6.591 & 2.197 & 2.197 & 76.895 \\
\hline 263.64 & 30.758 & 4.394 & 8.788 \\
\hline 2.197 & 263.64 & 4.394 & 4.394 \\
\hline 2.197 & 2.197 & 19.773 & 19.773 \\
\hline 2.197 & 6.591 & 2.197 & 2.197 \\
\hline 8.788 & 8.788 & 2.197 & 32.955 \\
\hline 83.486 & 654.706 & 17.576 & 2.197 \\
\hline 2.197 & 6.591 & 13.182 & 90.077 \\
\hline 2.197 & 6.591 & 2.197 & 24.167 \\
\hline 2.197 & 4.394 & 2.197 & 15.379 \\
\hline 2.197 & 4.394 & 2.197 & 2.197 \\
\hline 8.788 & 874.406 & 2.197 & 81.289 \\
\hline 4.394 & 2.197 & 2.197 & 2.197 \\
\hline 48.334 & 2.197 & 2.197 & 779.935 \\
\hline 26.364 & 2.197 & 2.197 & 79.092 \\
\hline 2.197 & 46.137 & 2.197 & 142.805 \\
\hline 6.591 & 21.97 & 2.197 & 2673.75 \\
\hline 3866.72 & 24.167 & 6.591 & 114.244 \\
\hline 2.197 & 65.91 & 2.197 & 17.576 \\
\hline 8.788 & 250.458 & 21.97 & 13.182 \\
\hline 6.591 & 43.94 & 6.591 & 2.197 \\
\hline 2.197 & 46.137 & 8.788 & 4.394 \\
\hline 37.349 & 4.394 & 10.985 & 46.137 \\
\hline 10.985 & 2.197 & 15.379 & 32.955 \\
\hline 4.394 & 15.379 & 342.732 & 1702.67 \\
\hline 4.394 & 6.591 & 4.394 & 2.197 \\
\hline
\end{tabular}




\begin{tabular}{|c|c|c|c|}
\hline 4.394 & 24.167 & 13.182 & 533.871 \\
\hline 4.394 & 2.197 & 2.197 & 8.788 \\
\hline 79.092 & 13.182 & 2.197 & 54.925 \\
\hline 13.182 & 79.092 & 2.197 & 2.197 \\
\hline 21.97 & 19.773 & 28.561 & 92.274 \\
\hline 19.773 & 39.546 & 4.394 & 13.182 \\
\hline 101.062 & 4.394 & 6.591 & 35.152 \\
\hline 32.955 & 92.274 & 6.591 & 26.364 \\
\hline 4.394 & 4.394 & 54.925 & 48.334 \\
\hline 17.576 & 17.576 & 2.197 & 8.788 \\
\hline 17.576 & 2.197 & 39.546 & 52.728 \\
\hline 2.197 & 2.197 & 2.197 & 30.758 \\
\hline 4.394 & 2.197 & 32.955 & 2.197 \\
\hline 2.197 & 6.591 & 2.197 & 2.197 \\
\hline 2.197 & 2.197 & 2.197 & 1155.62 \\
\hline 408.642 & 4.394 & 2.197 & 24.167 \\
\hline 160.381 & 6.591 & 19.773 & 2.197 \\
\hline 2.197 & 24.167 & 13.182 & 1981.69 \\
\hline 2.197 & 391.066 & 21.97 & 2.197 \\
\hline 2.197 & 6.591 & 4.394 & 2.197 \\
\hline 94.471 & 6.591 & 2.197 & 2.197 \\
\hline 39.546 & 8.788 & 38517.8 & 19.773 \\
\hline 8.788 & 762.359 & 4.394 & 24.167 \\
\hline 17.576 & 6.591 & 37.349 & 2.197 \\
\hline 2.197 & 8.788 & 2.197 & 2.197 \\
\hline 2.197 & 98.865 & 59.319 & 19.773 \\
\hline 8.788 & 65.91 & 2.197 & 2.197 \\
\hline 2.197 & 6.591 & 52.728 & 43.94 \\
\hline 26.364 & 17.576 & 2.197 & 13.182 \\
\hline 2.197 & 8.788 & 2.197 & 2.197 \\
\hline 1210.55 & 74.698 & 6.591 & 2.197 \\
\hline 39.546 & 2.197 & 4.394 & 21.97 \\
\hline 2.197 & 10.985 & 830.466 & 57.122 \\
\hline 112.047 & 24.167 & 50.531 & 2.197 \\
\hline 8.788 & 2.197 & 65.91 & 2.197 \\
\hline 17.576 & 8.788 & 134.017 & 37.349 \\
\hline 6.591 & 2.197 & 4.394 & 2.197 \\
\hline 24.167 & 41.743 & 428.415 & 30.758 \\
\hline 68.107 & 2.197 & 325.156 & 2.197 \\
\hline 17.576 & 32.955 & 2.197 & 15.379 \\
\hline 4.394 & 4.394 & 19.773 & 2.197 \\
\hline 4.394 & 4.394 & 39.546 & 21.97 \\
\hline 10.985 & 8.788 & 2.197 & 19.773 \\
\hline 1856.46 & 338.338 & 10.985 & 4.394 \\
\hline 6.591 & 30.758 & 2.197 & 21.97 \\
\hline 6.591 & 2.197 & 8.788 & 2.197 \\
\hline 6.591 & 8.788 & 2.197 & 2.197 \\
\hline 2.197 & 2.197 & 2.197 & 19.773 \\
\hline 160.381 & 2.197 & 61.516 & 24.167 \\
\hline 13.182 & 21.97 & 8.788 & 6.591 \\
\hline
\end{tabular}




\begin{tabular}{|c|c|c|c|}
\hline 2.197 & 13.182 & 2.197 & 10.985 \\
\hline 17.576 & 2.197 & 4.394 & 15.379 \\
\hline 274.625 & 61.516 & 2.197 & 54.925 \\
\hline 2.197 & 6.591 & 2.197 & 2.197 \\
\hline 32.955 & 6.591 & 2.197 & 2.197 \\
\hline 28.561 & 4.394 & 4.394 & 2.197 \\
\hline 2.197 & 2.197 & 2.197 & 48.334 \\
\hline 2.197 & 8.788 & 171.366 & 17.576 \\
\hline 6.591 & 17.576 & 2.197 & 28.561 \\
\hline 92.274 & 26.364 & 2.197 & 6.591 \\
\hline 57.122 & 21.97 & 832.663 & 48.334 \\
\hline 50.531 & 63.713 & 6.591 & 2.197 \\
\hline 2.197 & 4.394 & 2.197 & 35.152 \\
\hline 28.561 & 43.94 & 2.197 & 74.698 \\
\hline 26.364 & 2.197 & 4.394 & 10.985 \\
\hline 2.197 & 81.289 & 2.197 & 2.197 \\
\hline 52.728 & 2.197 & 2.197 & 2.197 \\
\hline 2.197 & 13.182 & 2.197 & 309.777 \\
\hline 2.197 & 6.591 & 2.197 & 68.107 \\
\hline 2.197 & 13.182 & 74.698 & 2.197 \\
\hline 2.197 & 257.049 & 26.364 & 2.197 \\
\hline 63.713 & 98.865 & 8.788 & 221.897 \\
\hline 2.197 & 13.182 & 2.197 & 39.546 \\
\hline 6.591 & 2.197 & 10.985 & 15.379 \\
\hline 2.197 & 2.197 & 2.197 & 2.197 \\
\hline 2.197 & 4.394 & 48.334 & 19.773 \\
\hline 2.197 & 13.182 & 6.591 & 81.289 \\
\hline 65.91 & 65.91 & 92.274 & 4.394 \\
\hline 28.561 & 10.985 & 103.259 & 43.94 \\
\hline 6.591 & 21.97 & 8.788 & 6.591 \\
\hline 2.197 & 2.197 & 98.865 & 48.334 \\
\hline 13.182 & 2.197 & 6.591 & 6.591 \\
\hline 61.516 & 30.758 & 4.394 & 43.94 \\
\hline 2.197 & 2.197 & 2.197 & 217.503 \\
\hline 85.683 & 2.197 & 2.197 & 13.182 \\
\hline 48.334 & 10.985 & 2.197 & 4.394 \\
\hline 4.394 & 30.758 & 10.985 & 13.182 \\
\hline 6.591 & 2.197 & 6.591 & 2.197 \\
\hline 8.788 & 39.546 & 10.985 & 4.394 \\
\hline 21.97 & 127.426 & 2.197 & 8.788 \\
\hline 2.197 & 2.197 & 4.394 & 10.985 \\
\hline 39.546 & 72.501 & 2.197 & 32.955 \\
\hline 2.197 & 28.561 & 4.394 & 28.561 \\
\hline 6.591 & 2.197 & 8.788 & 2.197 \\
\hline 24.167 & 2.197 & 4.394 & 920.543 \\
\hline 6.591 & 2.197 & 4.394 & 4.394 \\
\hline 2.197 & 24.167 & 4.394 & 112.047 \\
\hline 8.788 & 76.895 & 59.319 & 4.394 \\
\hline 4.394 & 2.197 & 2.197 & 4.394 \\
\hline 76.895 & 2.197 & 8.788 & 83.486 \\
\hline
\end{tabular}




\begin{tabular}{|c|c|c|c|}
\hline 48.334 & 65.91 & 2.197 & 4.394 \\
\hline 4.394 & 52.728 & 2.197 & 2.197 \\
\hline 4.394 & 10.985 & 50.531 & 4.394 \\
\hline 8.788 & 125.229 & 28.561 & 26.364 \\
\hline 2.197 & 226.291 & 4.394 & 2.197 \\
\hline 2.197 & 54.925 & 10.985 & 134.017 \\
\hline 6.591 & 2.197 & 2.197 & 19.773 \\
\hline 2.197 & 4.394 & 197.73 & 4.394 \\
\hline 2.197 & 10.985 & 4.394 & 43.94 \\
\hline 59.319 & 2.197 & 186.745 & 2.197 \\
\hline 2.197 & 32.955 & 37.349 & 2.197 \\
\hline 2.197 & 206.518 & 2.197 & 109.85 \\
\hline 2.197 & 4.394 & 2.197 & 2.197 \\
\hline 32.955 & 151.593 & 4.394 & 17.576 \\
\hline 2.197 & 2.197 & 226.291 & 2.197 \\
\hline 4.394 & 1302.82 & 2.197 & 96.668 \\
\hline 19.773 & 193.336 & 2.197 & 54.925 \\
\hline 35.152 & 2.197 & 17.576 & 6.591 \\
\hline 4.394 & 15.379 & 2.197 & 2592.46 \\
\hline 26.364 & 41.743 & 2.197 & 2.197 \\
\hline 15.379 & 15.379 & 21.97 & 1566.46 \\
\hline 24.167 & 61.516 & 2.197 & 13.182 \\
\hline 19.773 & 63.713 & 2.197 & 252.655 \\
\hline 2.197 & 2.197 & 2.197 & 8.788 \\
\hline 2.197 & 2.197 & 2.197 & 13.182 \\
\hline 10.985 & 2.197 & 2.197 & 2.197 \\
\hline 35.152 & 98.865 & 10.985 & 6.591 \\
\hline 37.349 & 2.197 & 54.925 & 19.773 \\
\hline 584.402 & 2.197 & 46.137 & 57.122 \\
\hline 13.182 & 6.591 & 6.591 & 2.197 \\
\hline 2.197 & 2.197 & 13.182 & 2.197 \\
\hline 8.788 & 2.197 & 2.197 & 13.182 \\
\hline 2.197 & 8.788 & 15.379 & 2.197 \\
\hline 2.197 & 48.334 & 13.182 & 43.94 \\
\hline 2.197 & 19.773 & 15.379 & 2.197 \\
\hline 101.062 & 518.492 & 4.394 & 2.197 \\
\hline 2.197 & 6.591 & 19.773 & 65.91 \\
\hline 136.214 & 4.394 & 2.197 & 39.546 \\
\hline 2.197 & 461.37 & 2.197 & 6.591 \\
\hline 2.197 & 2.197 & 2.197 & 4.394 \\
\hline 2.197 & 2.197 & 2.197 & 8.788 \\
\hline 4.394 & 24.167 & 61.516 & 2.197 \\
\hline 2.197 & 4.394 & 41.743 & 54.925 \\
\hline 2.197 & 4.394 & 65.91 & 19.773 \\
\hline 4.394 & 10.985 & 4.394 & 4.394 \\
\hline 2.197 & 10.985 & 456.976 & 72.501 \\
\hline 2.197 & 4.394 & 4.394 & 32.955 \\
\hline 2.197 & 2.197 & 2.197 & 177.957 \\
\hline 32.955 & 8.788 & 19.773 & 28.561 \\
\hline 109.85 & 4.394 & 6.591 & 118.638 \\
\hline
\end{tabular}




\begin{tabular}{|c|c|c|c|}
\hline 13.182 & 17.576 & 2.197 & 6.591 \\
\hline 145.002 & 57.122 & 2.197 & 4.394 \\
\hline 4.394 & 74.698 & 2.197 & 59.319 \\
\hline 10.985 & 6.591 & 2.197 & 2454.05 \\
\hline 2.197 & 17.576 & 2.197 & 4.394 \\
\hline 35.152 & 8.788 & 2.197 & 2.197 \\
\hline 131.82 & 19.773 & 17.576 & 6.591 \\
\hline 13.182 & 13.182 & 4.394 & 145.002 \\
\hline 2.197 & 59.319 & 2.197 & 65.91 \\
\hline 24.167 & 107.653 & 2.197 & 2.197 \\
\hline 26.364 & 410.839 & 2.197 & 2.197 \\
\hline 8.788 & 19.773 & 2.197 & 2.197 \\
\hline 8.788 & 6.591 & 2.197 & 28.561 \\
\hline 2.197 & 13.182 & 43.94 & 2.197 \\
\hline 19.773 & 8.788 & 2.197 & 2.197 \\
\hline 2.197 & 21.97 & 4.394 & 2.197 \\
\hline 24.167 & 85.683 & 2.197 & 2.197 \\
\hline 24.167 & 8.788 & 2.197 & 2.197 \\
\hline 2.197 & 4.394 & 2.197 & 15.379 \\
\hline 6.591 & 28.561 & 2.197 & 4.394 \\
\hline 10.985 & 6.591 & 4.394 & 2.197 \\
\hline 140.608 & 15.379 & 68.107 & 2.197 \\
\hline 41.743 & 13.182 & 232.882 & 94.471 \\
\hline 237.276 & 4.394 & 2.197 & 2.197 \\
\hline 6.591 & 26.364 & 118.638 & 26.364 \\
\hline 173.563 & 46.137 & 76.895 & 2.197 \\
\hline 2.197 & 10.985 & 290.004 & 160.381 \\
\hline 2.197 & 24.167 & 4.394 & 4.394 \\
\hline 2.197 & 8.788 & 2.197 & 28.561 \\
\hline 8.788 & 28.561 & 2.197 & 15.379 \\
\hline 2.197 & 4.394 & 2.197 & 37.349 \\
\hline 2.197 & 10.985 & 2.197 & 2.197 \\
\hline 10.985 & 59.319 & 147.199 & 2.197 \\
\hline 2.197 & 85.683 & 2.197 & 6.591 \\
\hline 15.379 & 13.182 & 19.773 & 2.197 \\
\hline 2.197 & 35.152 & 4.394 & 4.394 \\
\hline 39.546 & 17.576 & 2.197 & 6.591 \\
\hline 415.233 & 6.591 & 2.197 & 24.167 \\
\hline 4.394 & 2.197 & 85.683 & 13.182 \\
\hline 2.197 & 15.379 & 2.197 & 6.591 \\
\hline 2.197 & 24.167 & 6.591 & 2.197 \\
\hline 10.985 & 4.394 & 2.197 & 4.394 \\
\hline 2.197 & 4.394 & 48.334 & 2.197 \\
\hline 2.197 & 2016.85 & 6.591 & 83.486 \\
\hline 2.197 & 26.364 & 2.197 & 15.379 \\
\hline 85.683 & 17.576 & 4.394 & 2.197 \\
\hline 8.788 & 4.394 & 2.197 & 28.561 \\
\hline 2.197 & 505.31 & 15.379 & 2.197 \\
\hline 10.985 & 2.197 & 314.171 & 54.925 \\
\hline 83.486 & 2.197 & 2.197 & 17.576 \\
\hline
\end{tabular}




\begin{tabular}{|c|c|c|c|}
\hline 13.182 & 8.788 & 24.167 & 177.957 \\
\hline 4.394 & 17.576 & 19.773 & 4.394 \\
\hline 2.197 & 2.197 & 2.197 & 2.197 \\
\hline 19.773 & 41.743 & 4.394 & 4.394 \\
\hline 85.683 & 4.394 & 2.197 & 6.591 \\
\hline 72.501 & 2.197 & 2.197 & 4.394 \\
\hline 6.591 & 13.182 & 2.197 & 6.591 \\
\hline 2.197 & 2.197 & 15.379 & 8.788 \\
\hline 52.728 & 28.561 & 2.197 & 2.197 \\
\hline 4.394 & 37.349 & 10.985 & 6.591 \\
\hline 35.152 & 136.214 & 4.394 & 15.379 \\
\hline 8.788 & 4.394 & 142.805 & 6.591 \\
\hline 54.925 & 19.773 & 2.197 & 17.576 \\
\hline 79.092 & 6.591 & 2.197 & 2.197 \\
\hline 26.364 & 158.184 & 4.394 & 6.591 \\
\hline 2.197 & 2.197 & 757.965 & 103.259 \\
\hline 46.137 & 19.773 & 4.394 & 155.987 \\
\hline 2.197 & 10.985 & 10.985 & 4.394 \\
\hline 1010.62 & 2.197 & 15.379 & 15.379 \\
\hline 70.304 & 105.456 & 4.394 & 279.019 \\
\hline 57.122 & 2.197 & 2.197 & 2.197 \\
\hline 2.197 & 2.197 & 10.985 & 19.773 \\
\hline 2.197 & 2.197 & 4.394 & 19.773 \\
\hline 85.683 & 4.394 & 74.698 & 224.094 \\
\hline 2.197 & 6.591 & 520.689 & 2.197 \\
\hline 219.7 & 8.788 & 2.197 & 79.092 \\
\hline 4.394 & 2.197 & 2.197 & 911.755 \\
\hline 2.197 & 2.197 & 2.197 & 507.507 \\
\hline 8.788 & 2.197 & 13.182 & 2.197 \\
\hline 2.197 & 59.319 & 13.182 & 2.197 \\
\hline 21.97 & 8.788 & 105.456 & 11024.5 \\
\hline 558.038 & 54.925 & 6.591 & 43.94 \\
\hline 2.197 & 8.788 & 2.197 & 90.077 \\
\hline 2.197 & 180.154 & 4.394 & 2.197 \\
\hline 17.576 & 8.788 & 8.788 & 4.394 \\
\hline 8091.55 & 87.88 & 35.152 & 13.182 \\
\hline 4.394 & 6.591 & 2.197 & 619.554 \\
\hline 4.394 & 21.97 & 2.197 & 2.197 \\
\hline 2.197 & 13.182 & 4.394 & 448.188 \\
\hline 2.197 & 6.591 & 2.197 & 4.394 \\
\hline 15.379 & 2.197 & 2.197 & 2.197 \\
\hline 6.591 & 4.394 & 2.197 & 30.758 \\
\hline 28.561 & 2.197 & 2.197 & 443.794 \\
\hline 39.546 & 72.501 & 2.197 & 2.197 \\
\hline 46.137 & 8.788 & 2.197 & 19.773 \\
\hline 2.197 & 131.82 & 4.394 & 8.788 \\
\hline 2.197 & 39.546 & 2.197 & 37.349 \\
\hline 237.276 & 292.201 & 13.182 & 2.197 \\
\hline 24.167 & 21.97 & 2.197 & 2.197 \\
\hline 28.561 & 2.197 & 2.197 & 2.197 \\
\hline
\end{tabular}




\begin{tabular}{|c|c|c|c|}
\hline 6.591 & 4.394 & 2.197 & 70.304 \\
\hline 10.985 & 268.034 & 101.062 & 15.379 \\
\hline 10.985 & 195.533 & 103.259 & 2.197 \\
\hline 13.182 & 2.197 & 17.576 & 52.728 \\
\hline 237.276 & 4.394 & 4.394 & 4.394 \\
\hline 17.576 & 13.182 & 39.546 & 15.379 \\
\hline 6.591 & 2.197 & 2.197 & 2.197 \\
\hline 4.394 & 21.97 & 2.197 & 4.394 \\
\hline 2.197 & 4.394 & 2.197 & 41.743 \\
\hline 2.197 & 52.728 & 2.197 & 2.197 \\
\hline 2.197 & 28.561 & 2.197 & 478.946 \\
\hline 92.274 & 26.364 & 2.197 & 4.394 \\
\hline 4.394 & 50.531 & 4.394 & 4.394 \\
\hline 180.154 & 1535.7 & 26.364 & 4.394 \\
\hline 2.197 & 17.576 & 4.394 & 4.394 \\
\hline 2.197 & 17.576 & 2.197 & 15.379 \\
\hline 6.591 & 15.379 & 68.107 & 2.197 \\
\hline 48.334 & 123.032 & 2.197 & 32.955 \\
\hline 8.788 & 30.758 & 2.197 & 57.122 \\
\hline 13.182 & 4.394 & 4.394 & 793.117 \\
\hline 85.683 & 2.197 & 6.591 & 79.092 \\
\hline 1102.89 & 2.197 & 2.197 & 13.182 \\
\hline 2.197 & 10.985 & 2.197 & 2.197 \\
\hline 17.576 & 13.182 & 2.197 & 28.561 \\
\hline 8.788 & 98.865 & 8.788 & 32.955 \\
\hline 2946.18 & 76.895 & 21.97 & 4.394 \\
\hline 4.394 & 8.788 & 2.197 & 4.394 \\
\hline 8.788 & 2.197 & 2.197 & 4.394 \\
\hline 17.576 & 6.591 & 2.197 & 21.97 \\
\hline 8.788 & 6.591 & 4.394 & 2.197 \\
\hline 6.591 & 90.077 & 4.394 & 65.91 \\
\hline 210.912 & 24.167 & 116.441 & 2.197 \\
\hline 2.197 & 76.895 & 2.197 & 72.501 \\
\hline 2.197 & 15.379 & 2.197 & 28.561 \\
\hline 50.531 & 4.394 & 2.197 & 4.394 \\
\hline 13.182 & 2.197 & 4.394 & 2.197 \\
\hline 2.197 & 15.379 & 15.379 & 21.97 \\
\hline 2.197 & 90.077 & 4.394 & 8.788 \\
\hline 13.182 & 4.394 & 18217.5 & 24.167 \\
\hline 2.197 & 2.197 & 2.197 & 2.197 \\
\hline 2.197 & 2.197 & 116.441 & 59.319 \\
\hline 2.197 & 6.591 & 4.394 & 13.182 \\
\hline 4.394 & 13.182 & 6.591 & 487.734 \\
\hline 2.197 & 6.591 & 19.773 & 4.394 \\
\hline 4.394 & 6.591 & 15.379 & 2.197 \\
\hline 85.683 & 114.244 & 2.197 & 153.79 \\
\hline 138.411 & 10.985 & 6.591 & 2.197 \\
\hline 2.197 & 87.88 & 15.379 & 2.197 \\
\hline 8.788 & 8.788 & 54.925 & 10.985 \\
\hline 2.197 & 46.137 & 142.805 & 4.394 \\
\hline
\end{tabular}




\begin{tabular}{|c|c|c|c|}
\hline 2.197 & 2.197 & 2.197 & 48.334 \\
\hline 2.197 & 6.591 & 8.788 & 2.197 \\
\hline 6.591 & 6.591 & 48.334 & 68.107 \\
\hline 2.197 & 2.197 & 37.349 & 2.197 \\
\hline 224.094 & 8.788 & 4.394 & 15.379 \\
\hline 43.94 & 13.182 & 4.394 & 2.197 \\
\hline 4.394 & 2.197 & 4.394 & 81.289 \\
\hline 2.197 & 2.197 & 17.576 & 2.197 \\
\hline 138.411 & 4.394 & 13.182 & 2.197 \\
\hline 138.411 & 26.364 & 2.197 & 4.394 \\
\hline 2.197 & 2.197 & 32.955 & 63.713 \\
\hline 13.182 & 107.653 & 2.197 & 1261.08 \\
\hline 2.197 & 52.728 & 101.062 & 15.379 \\
\hline 435.006 & 2.197 & 65.91 & 2.197 \\
\hline 2.197 & 2.197 & 21.97 & 116.441 \\
\hline 10.985 & 6.591 & 2.197 & 2.197 \\
\hline 8.788 & 2.197 & 2.197 & 35.152 \\
\hline 8.788 & 206.518 & 2.197 & 2.197 \\
\hline 13.182 & 2.197 & 2.197 & 2.197 \\
\hline 24.167 & 2.197 & 15.379 & 199.927 \\
\hline 10.985 & 142.805 & 2.197 & 32.955 \\
\hline 2.197 & 13.182 & 2.197 & 184.548 \\
\hline 2.197 & 2.197 & 8.788 & 92.274 \\
\hline 2.197 & 28.561 & 2.197 & 50.531 \\
\hline 24.167 & 15.379 & 2.197 & 2.197 \\
\hline 2.197 & 21.97 & 2.197 & 2.197 \\
\hline 6.591 & 6.591 & 10.985 & 10.985 \\
\hline 8.788 & 46.137 & 2.197 & 21.97 \\
\hline 358.111 & 230.685 & 19.773 & 4.394 \\
\hline 28.561 & 4.394 & 2.197 & 4.394 \\
\hline 2.197 & 4.394 & 2.197 & 6.591 \\
\hline 59.319 & 17.576 & 2.197 & 2.197 \\
\hline 2.197 & 2.197 & 2.197 & 136.214 \\
\hline 2.197 & 2.197 & 2.197 & 2.197 \\
\hline 68.107 & 13.182 & 2.197 & 8.788 \\
\hline 2.197 & 4.394 & 4.394 & 17.576 \\
\hline 188.942 & 2.197 & 2.197 & 2.197 \\
\hline 2.197 & 4.394 & 4.394 & 24.167 \\
\hline 15.379 & 246.064 & 140.608 & 2.197 \\
\hline 6.591 & 2.197 & 2.197 & 19.773 \\
\hline 21.97 & 4.394 & 2.197 & 48.334 \\
\hline 173.563 & 204.321 & 2.197 & 10.985 \\
\hline 30.758 & 4.394 & 2.197 & 8.788 \\
\hline 6.591 & 6.591 & 4.394 & 6.591 \\
\hline 2.197 & 19.773 & 4.394 & 85.683 \\
\hline 61.516 & 8.788 & 8.788 & 35.152 \\
\hline 2.197 & 2.197 & 2.197 & 6.591 \\
\hline 109.85 & 2.197 & 2.197 & 2.197 \\
\hline 2.197 & 2.197 & 10.985 & 197.73 \\
\hline 19.773 & 8.788 & 4.394 & 76.895 \\
\hline
\end{tabular}




\begin{tabular}{|c|c|c|c|}
\hline 2.197 & 57.122 & 46.137 & 17.576 \\
\hline 10.985 & 2.197 & 2.197 & 4.394 \\
\hline 2.197 & 8.788 & 4.394 & 4.394 \\
\hline 2.197 & 2.197 & 224.094 & 15.379 \\
\hline 24.167 & 296.595 & 15.379 & 2.197 \\
\hline 2.197 & 210.912 & 8.788 & 4.394 \\
\hline 2.197 & 4.394 & 4.394 & 87.88 \\
\hline 2.197 & 4.394 & 81.289 & 2.197 \\
\hline 127.426 & 2.197 & 46.137 & 19.773 \\
\hline 61.516 & 2.197 & 2.197 & 107.653 \\
\hline 2.197 & 6.591 & 2.197 & 81.289 \\
\hline 8.788 & 2.197 & 68.107 & 4.394 \\
\hline 4.394 & 2.197 & 4.394 & 4.394 \\
\hline 81.289 & 2.197 & 2.197 & 28.561 \\
\hline 2.197 & 771.147 & 21.97 & 819.481 \\
\hline 142.805 & 54.925 & 17.576 & 4.394 \\
\hline 4.394 & 54.925 & 13.182 & 2.197 \\
\hline 15.379 & 68.107 & 2.197 & 52.728 \\
\hline 19.773 & 2.197 & 584.402 & 24.167 \\
\hline 281.216 & 4.394 & 94.471 & 4.394 \\
\hline 2.197 & 2.197 & 4.394 & 2.197 \\
\hline 259.246 & 8.788 & 4.394 & 4.394 \\
\hline 2.197 & 2.197 & 10.985 & 37.349 \\
\hline 2.197 & 2.197 & 2.197 & 2.197 \\
\hline 19.773 & 57.122 & 15.379 & 10.985 \\
\hline 2.197 & 10.985 & 21.97 & 2.197 \\
\hline 4.394 & 72.501 & 10.985 & 6.591 \\
\hline 6.591 & 24.167 & 125.229 & 30.758 \\
\hline 4.394 & 13.182 & 2.197 & 19.773 \\
\hline 4.394 & 98.865 & 35.152 & 6.591 \\
\hline 10.985 & 107.653 & 239.473 & 6.591 \\
\hline 26.364 & 2.197 & 6.591 & 4.394 \\
\hline 2.197 & 63.713 & 2.197 & 10.985 \\
\hline 21.97 & 4.394 & 24.167 & 24.167 \\
\hline 6.591 & 2.197 & 8.788 & 2.197 \\
\hline 3187.85 & 19.773 & 32.955 & 35.152 \\
\hline 2.197 & 76.895 & 2.197 & 28.561 \\
\hline 15.379 & 17.576 & 32.955 & 2.197 \\
\hline 2.197 & 162.578 & 74.698 & 2.197 \\
\hline 2.197 & 21.97 & 2.197 & 8.788 \\
\hline 2.197 & 2.197 & 2.197 & 2.197 \\
\hline 127.426 & 13.182 & 4.394 & 2.197 \\
\hline 109.85 & 224.094 & 2.197 & 13.182 \\
\hline 19.773 & 6.591 & 2.197 & 59.319 \\
\hline 2.197 & 2.197 & 2.197 & 10.985 \\
\hline 2122.3 & 2.197 & 4.394 & 347.126 \\
\hline 59.319 & 2.197 & 2.197 & 641.524 \\
\hline 285.61 & 8.788 & 2.197 & 109.85 \\
\hline 2.197 & 46.137 & 2.197 & 26.364 \\
\hline 1300.62 & 1355.55 & 2.197 & 6.591 \\
\hline
\end{tabular}




\begin{tabular}{|c|c|c|c|}
\hline 8.788 & 4.394 & 4.394 & 8.788 \\
\hline 4.394 & 2.197 & 2.197 & 4.394 \\
\hline 171.366 & 197.73 & 4.394 & 21.97 \\
\hline 263.64 & 6.591 & 4.394 & 2.197 \\
\hline 17.576 & 24.167 & 2.197 & 28.561 \\
\hline 83.486 & 13.182 & 2.197 & 2.197 \\
\hline 241.67 & 19.773 & 2.197 & 37.349 \\
\hline 17.576 & 8.788 & 2.197 & 109.85 \\
\hline 235.079 & 68.107 & 8.788 & 85.683 \\
\hline 714.025 & 114.244 & 59.319 & 105.456 \\
\hline 4.394 & 17.576 & 2.197 & 6.591 \\
\hline 527.28 & 2.197 & 90.077 & 8.788 \\
\hline 285.61 & 37.349 & 2.197 & 8.788 \\
\hline 6.591 & 76.895 & 2.197 & 10.985 \\
\hline 10.985 & 24.167 & 57.122 & 4.394 \\
\hline 4.394 & 2.197 & 6.591 & 4.394 \\
\hline 30.758 & 10.985 & 2.197 & 4.394 \\
\hline 2.197 & 41.743 & 2.197 & 21.97 \\
\hline 2.197 & 8.788 & 2.197 & 13.182 \\
\hline 4.394 & 30.758 & 2.197 & 2.197 \\
\hline 81.289 & 48.334 & 2.197 & 2.197 \\
\hline 2.197 & 2.197 & 15.379 & 4.394 \\
\hline 6.591 & 327.353 & 2.197 & 70.304 \\
\hline 43.94 & 101.062 & 2.197 & 2.197 \\
\hline 81.289 & 2.197 & 19.773 & 2.197 \\
\hline 2.197 & 2.197 & 4.394 & 57.122 \\
\hline 10.985 & 15.379 & 6.591 & 50.531 \\
\hline 8.788 & 17.576 & 2.197 & 4.394 \\
\hline 199.927 & 219.7 & 2.197 & 1395.09 \\
\hline 15.379 & 2.197 & 37.349 & 19.773 \\
\hline 186.745 & 2.197 & 8.788 & 35.152 \\
\hline 6.591 & 17.576 & 2.197 & 4.394 \\
\hline 13.182 & 116.441 & 13.182 & 19.773 \\
\hline 2.197 & 74.698 & 21.97 & 6.591 \\
\hline 32.955 & 2.197 & 35.152 & 10.985 \\
\hline 158.184 & 2.197 & 6.591 & 57.122 \\
\hline 2.197 & 4.394 & 81.289 & 19.773 \\
\hline 2.197 & 6.591 & 6.591 & 8.788 \\
\hline 63.713 & 2.197 & 661.297 & 10.985 \\
\hline 4.394 & 2.197 & 2.197 & 37.349 \\
\hline 8.788 & 2.197 & 4.394 & 4.394 \\
\hline 2.197 & 41.743 & 4.394 & 24.167 \\
\hline 2.197 & 26.364 & 2.197 & 153.79 \\
\hline 54.925 & 2.197 & 2.197 & 50.531 \\
\hline 2.197 & 35.152 & 63.713 & 112.047 \\
\hline 2.197 & 4.394 & 2.197 & 2.197 \\
\hline 140.608 & 2.197 & 2.197 & 6.591 \\
\hline 57.122 & 2.197 & 2.197 & 2.197 \\
\hline 2.197 & 98.865 & 43.94 & 59.319 \\
\hline 15.379 & 70.304 & 2.197 & 21.97 \\
\hline
\end{tabular}




\begin{tabular}{|c|c|c|c|}
\hline 2.197 & 279.019 & 10.985 & 6.591 \\
\hline 19.773 & 232.882 & 2.197 & 6.591 \\
\hline 6.591 & 28.561 & 4.394 & 2.197 \\
\hline 15.379 & 2.197 & 4.394 & 4.394 \\
\hline 50.531 & 19.773 & 2.197 & 4.394 \\
\hline 2.197 & 17.576 & 6.591 & 48.334 \\
\hline 2.197 & 6.591 & 28.561 & 2.197 \\
\hline 2.197 & 2.197 & 1034.79 & 175.76 \\
\hline 4.394 & 6.591 & 2.197 & 8.788 \\
\hline 2.197 & 2.197 & 2.197 & 10.985 \\
\hline 7067.75 & 50.531 & 8.788 & 35059.7 \\
\hline 6.591 & 35.152 & 8.788 & 28.561 \\
\hline 4.394 & 30.758 & 8.788 & 2.197 \\
\hline 204.321 & 28.561 & 4.394 & 2.197 \\
\hline 6.591 & 76.895 & 2.197 & 4.394 \\
\hline 4.394 & 13.182 & 2.197 & 4.394 \\
\hline 30.758 & 72.501 & 2.197 & 8.788 \\
\hline 2.197 & 2.197 & 32.955 & 4.394 \\
\hline 2.197 & 6.591 & 2.197 & 35.152 \\
\hline 2.197 & 2.197 & 2.197 & 2.197 \\
\hline 21.97 & 3840.36 & 19.773 & 19.773 \\
\hline 4.394 & 4.394 & 41.743 & 30.758 \\
\hline 2.197 & 2.197 & 2.197 & 118.638 \\
\hline 2546.32 & 2.197 & 2.197 & 6.591 \\
\hline 10.985 & 2.197 & 2.197 & 19.773 \\
\hline 8.788 & 28.561 & 4.394 & 5569.39 \\
\hline 123.032 & 2.197 & 2.197 & 254.852 \\
\hline 2.197 & 2.197 & 2.197 & 92.274 \\
\hline 549.25 & 8.788 & 2.197 & 10.985 \\
\hline 4.394 & 145.002 & 15.379 & 2.197 \\
\hline 2.197 & 92.274 & 6.591 & 2.197 \\
\hline 415.233 & 2.197 & 41.743 & 21.97 \\
\hline 2.197 & 8.788 & 158.184 & 39.546 \\
\hline 2.197 & 2.197 & 17.576 & 2.197 \\
\hline 771.147 & 10.985 & 2.197 & 2.197 \\
\hline 333.944 & 4.394 & 2.197 & 17.576 \\
\hline 13.182 & 15.379 & 4.394 & 32.955 \\
\hline 8.788 & 4.394 & 2.197 & 107.653 \\
\hline 929.331 & 338.338 & 2.197 & 10.985 \\
\hline 2.197 & 2.197 & 4.394 & 575.614 \\
\hline 1461 & 2.197 & 4.394 & 2.197 \\
\hline 751.374 & 118.638 & 4.394 & 2.197 \\
\hline 39.546 & 166.972 & 4.394 & 65.91 \\
\hline 2.197 & 8.788 & 2.197 & 13.182 \\
\hline 2.197 & 46.137 & 4.394 & 50.531 \\
\hline 6.591 & 19.773 & 2.197 & 30.758 \\
\hline 19.773 & 15.379 & 8.788 & 17.576 \\
\hline 6.591 & 2.197 & 4.394 & 6.591 \\
\hline 129.623 & 21.97 & 4.394 & 13.182 \\
\hline 6.591 & 21.97 & 2.197 & 160.381 \\
\hline
\end{tabular}




\begin{tabular}{|c|c|c|c|}
\hline 2.197 & 54.925 & 2.197 & 2.197 \\
\hline 6.591 & 19.773 & 2.197 & 2.197 \\
\hline 21.97 & 101.062 & 2.197 & 83.486 \\
\hline 39.546 & 6.591 & 2.197 & 2.197 \\
\hline 2.197 & 4.394 & 2.197 & 74.698 \\
\hline 6.591 & 83.486 & 2.197 & 2.197 \\
\hline 2.197 & 8.788 & 986.453 & 41.743 \\
\hline 2.197 & 127.426 & 2.197 & 21.97 \\
\hline 2.197 & 2.197 & 2.197 & 30.758 \\
\hline 6.591 & 2.197 & 2.197 & 8.788 \\
\hline 6.591 & 32.955 & 35.152 & 35.152 \\
\hline 2.197 & 96.668 & 2.197 & 15.379 \\
\hline 46.137 & 65.91 & 13.182 & 57.122 \\
\hline 8.788 & 2.197 & 4.394 & 2.197 \\
\hline 6.591 & 15.379 & 61.516 & 2.197 \\
\hline 344.929 & 2.197 & 8.788 & 2.197 \\
\hline 210.912 & 32.955 & 4.394 & 6.591 \\
\hline 63.713 & 441.597 & 2.197 & 54.925 \\
\hline 6.591 & 6.591 & 83.486 & 19.773 \\
\hline 2.197 & 13.182 & 6.591 & 63.713 \\
\hline 2.197 & 26.364 & 2.197 & 366.899 \\
\hline 2.197 & 39.546 & 24.167 & 2.197 \\
\hline 806.299 & 35.152 & 15.379 & 15.379 \\
\hline 21.97 & 8.788 & 4.394 & 4.394 \\
\hline 6.591 & 443.794 & 4.394 & 6.591 \\
\hline 2.197 & 13.182 & 8.788 & 2.197 \\
\hline 129.623 & 24.167 & 2.197 & 17.576 \\
\hline 10.985 & 4.394 & 2.197 & 37.349 \\
\hline 8.788 & 2.197 & 2.197 & 6.591 \\
\hline 4.394 & 2.197 & 2.197 & 2.197 \\
\hline 4.394 & 6.591 & 4.394 & 2.197 \\
\hline 10.985 & 30.758 & 2.197 & 4.394 \\
\hline 15.379 & 131.82 & 2.197 & 265.837 \\
\hline 4.394 & 6.591 & 2.197 & 1124.86 \\
\hline 61.516 & 8.788 & 2.197 & 2.197 \\
\hline 2.197 & 65.91 & 2.197 & 219.7 \\
\hline 10.985 & 2.197 & 219.7 & 2.197 \\
\hline 8.788 & 2.197 & 6.591 & 15.379 \\
\hline 2.197 & 2.197 & 13.182 & 2.197 \\
\hline 265.837 & 24.167 & 2.197 & 85.683 \\
\hline 8.788 & 57.122 & 17.576 & 35.152 \\
\hline 74.698 & 83.486 & 421.824 & 138.411 \\
\hline 531553 & 8.788 & 2.197 & 6.591 \\
\hline 28.561 & 4.394 & 6.591 & 2.197 \\
\hline 2.197 & 50.531 & 2.197 & 4.394 \\
\hline 30.758 & 37.349 & 35.152 & 2.197 \\
\hline 2.197 & 2.197 & 221.897 & 8.788 \\
\hline 15.379 & 68.107 & 2.197 & 2.197 \\
\hline 4.394 & 10.985 & 13.182 & 2.197 \\
\hline 17.576 & 26.364 & 2.197 & 8.788 \\
\hline
\end{tabular}




\begin{tabular}{|c|c|c|c|}
\hline 2.197 & 10.985 & 248.261 & 329.55 \\
\hline 8.788 & 542.659 & 2.197 & 2.197 \\
\hline 10.985 & 2.197 & 2.197 & 10.985 \\
\hline 6.591 & 2.197 & 2.197 & 10.985 \\
\hline 46.137 & 32.955 & 2.197 & 10.985 \\
\hline 2.197 & 26.364 & 8.788 & 2.197 \\
\hline 2.197 & 63.713 & 2.197 & 21.97 \\
\hline 2.197 & 35.152 & 2.197 & 149.396 \\
\hline 8.788 & 70.304 & 2.197 & 2.197 \\
\hline 2.197 & 30.758 & 2.197 & 19.773 \\
\hline 15.379 & 2.197 & 4.394 & 87.88 \\
\hline 10.985 & 120.835 & 2.197 & 180.154 \\
\hline 2.197 & 4.394 & 2324.43 & 2.197 \\
\hline 4.394 & 6.591 & 2.197 & 32.955 \\
\hline 4.394 & 10.985 & 2.197 & 15.379 \\
\hline 19.773 & 21.97 & 6.591 & 2.197 \\
\hline 43.94 & 24.167 & 2.197 & 2.197 \\
\hline 4.394 & 37.349 & 2.197 & 21.97 \\
\hline 15.379 & 2.197 & 13.182 & 2.197 \\
\hline 19.773 & 43.94 & 2.197 & 2.197 \\
\hline 2.197 & 2.197 & 2.197 & 6.591 \\
\hline 1412.67 & 6.591 & 98.865 & 4.394 \\
\hline 2.197 & 6.591 & 2.197 & 2.197 \\
\hline 4.394 & 54.925 & 2.197 & 35.152 \\
\hline 46.137 & 2.197 & 63.713 & 10.985 \\
\hline 17.576 & 54.925 & 4.394 & 2.197 \\
\hline 2.197 & 61.516 & 13.182 & 87.88 \\
\hline 10.985 & 153.79 & 15.379 & 94.471 \\
\hline 10.985 & 10.985 & 2.197 & 26.364 \\
\hline 2.197 & 26.364 & 46.137 & 2.197 \\
\hline 153.79 & 2.197 & 19.773 & 2.197 \\
\hline 2.197 & 175.76 & 2.197 & 116.441 \\
\hline 2.197 & 8.788 & 349.323 & 4.394 \\
\hline 2.197 & 2.197 & 2.197 & 2.197 \\
\hline 6.591 & 4.394 & 46.137 & 8.788 \\
\hline 272.428 & 13.182 & 2.197 & 2.197 \\
\hline 8.788 & 1882.83 & 8.788 & 149.396 \\
\hline 2.197 & 2.197 & 911.755 & 17.576 \\
\hline 24.167 & 101.062 & 8.788 & 2.197 \\
\hline 32.955 & 118.638 & 61.516 & 8.788 \\
\hline 2.197 & 2.197 & 2.197 & 2.197 \\
\hline 2.197 & 15.379 & 2.197 & 65.91 \\
\hline 2.197 & 17.576 & 177.957 & 4.394 \\
\hline 2.197 & 6.591 & 6.591 & 4.394 \\
\hline 15.379 & 6.591 & 483.34 & 2.197 \\
\hline 2.197 & 2.197 & 2.197 & 2.197 \\
\hline 46.137 & 2.197 & 2.197 & 8.788 \\
\hline 28.561 & 2.197 & 2.197 & 28.561 \\
\hline 6.591 & 4.394 & 79.092 & 8.788 \\
\hline 2.197 & 2.197 & 2.197 & 2.197 \\
\hline
\end{tabular}




\begin{tabular}{|c|c|c|c|}
\hline 2.197 & 28.561 & 37.349 & 21.97 \\
\hline 2.197 & 19.773 & 4.394 & 2.197 \\
\hline 2.197 & 63.713 & 4.394 & 2.197 \\
\hline 65.91 & 6.591 & 26.364 & 2.197 \\
\hline 57.122 & 382.278 & 2.197 & 4.394 \\
\hline 2.197 & 50.531 & 43.94 & 32.955 \\
\hline 2.197 & 2.197 & 13.182 & 50.531 \\
\hline 2.197 & 6.591 & 2.197 & 112.047 \\
\hline 2.197 & 28.561 & 65.91 & 276.822 \\
\hline 92.274 & 70.304 & 2.197 & 118.638 \\
\hline 8.788 & 2.197 & 120.835 & 21.97 \\
\hline 2.197 & 4.394 & 19.773 & 4.394 \\
\hline 327.353 & 2.197 & 6.591 & 8.788 \\
\hline 2.197 & 175.76 & 4.394 & 4.394 \\
\hline 2.197 & 2.197 & 2.197 & 2.197 \\
\hline 32.955 & 19.773 & 4.394 & 123.032 \\
\hline 70.304 & 340.535 & 2.197 & 4.394 \\
\hline 57.122 & 10.985 & 2.197 & 4.394 \\
\hline 4.394 & 43.94 & 2.197 & 8.788 \\
\hline 4.394 & 2.197 & 2.197 & 138.411 \\
\hline 2.197 & 162.578 & 13.182 & 2.197 \\
\hline 2.197 & 2.197 & 24.167 & 804.102 \\
\hline 2.197 & 4.394 & 2.197 & 4.394 \\
\hline 2.197 & 2.197 & 2.197 & 2.197 \\
\hline 2.197 & 2.197 & 2.197 & 2.197 \\
\hline 19.773 & 2.197 & 2.197 & 2.197 \\
\hline 101.062 & 4.394 & 2.197 & 2.197 \\
\hline 4.394 & 72.501 & 4.394 & 2.197 \\
\hline 13.182 & 13.182 & 2.197 & 15.379 \\
\hline 182.351 & 10.985 & 4.394 & 2.197 \\
\hline 48.334 & 127.426 & 2.197 & 4.394 \\
\hline 10.985 & 2.197 & 98.865 & 4.394 \\
\hline 2.197 & 2.197 & 2.197 & 24.167 \\
\hline 17.576 & 24.167 & 2.197 & 485.537 \\
\hline 15.379 & 2.197 & 2.197 & 26.364 \\
\hline 2.197 & 6.591 & 8.788 & 254.852 \\
\hline 54.925 & 2.197 & 2.197 & 2.197 \\
\hline 2.197 & 182.351 & 2.197 & 21.97 \\
\hline 2.197 & 6.591 & 2.197 & 6.591 \\
\hline 2.197 & 4.394 & 2.197 & 4.394 \\
\hline 4.394 & 30.758 & 13.182 & 46.137 \\
\hline 74.698 & 347.126 & 41.743 & 8.788 \\
\hline 17.576 & 973.271 & 17.576 & 6.591 \\
\hline 421.824 & 2.197 & 13.182 & 2.197 \\
\hline 37.349 & 10.985 & 15.379 & 105.456 \\
\hline 241.67 & 162.578 & 39.546 & 2.197 \\
\hline 61.516 & 2.197 & 17.576 & 2.197 \\
\hline 2.197 & 8.788 & 2.197 & 15.379 \\
\hline 37.349 & 10.985 & 4.394 & 81.289 \\
\hline 21.97 & 4.394 & 120.835 & 2.197 \\
\hline
\end{tabular}




\begin{tabular}{|c|c|c|c|}
\hline 2.197 & 2.197 & 2.197 & 2.197 \\
\hline 6.591 & 101.062 & 10.985 & 2.197 \\
\hline 10.985 & 10.985 & 2.197 & 2.197 \\
\hline 4.394 & 2.197 & 2.197 & 2.197 \\
\hline 2.197 & 2.197 & 109.85 & 226.291 \\
\hline 35.152 & 8.788 & 243.867 & 4.394 \\
\hline 2.197 & 2.197 & 15.379 & 2.197 \\
\hline 19.773 & 164.775 & 13.182 & 6.591 \\
\hline 4.394 & 184.548 & 2.197 & 30.758 \\
\hline 2.197 & 2.197 & 15.379 & 24.167 \\
\hline 2.197 & 15.379 & 250.458 & 2.197 \\
\hline 2.197 & 2.197 & 63.713 & 2.197 \\
\hline 4.394 & 2.197 & 8.788 & 2.197 \\
\hline 2.197 & 39.546 & 8.788 & 10.985 \\
\hline 2.197 & 81.289 & 19.773 & 17.576 \\
\hline 30.758 & 24.167 & 13.182 & 4.394 \\
\hline 6.591 & 186.745 & 10.985 & 2.197 \\
\hline 3378.99 & 26.364 & 2.197 & 4.394 \\
\hline 6.591 & 37.349 & 15.379 & 206.518 \\
\hline 10.985 & 19.773 & 15.379 & 184.548 \\
\hline 2.197 & 219.7 & 2.197 & 15.379 \\
\hline 63.713 & 169.169 & 90.077 & 32.955 \\
\hline 2.197 & 37.349 & 452.582 & 19.773 \\
\hline 10.985 & 2.197 & 105.456 & 10.985 \\
\hline 21.97 & 2.197 & 8.788 & 2.197 \\
\hline 2.197 & 39.546 & 2.197 & 15.379 \\
\hline 13.182 & 2.197 & 4.394 & 2.197 \\
\hline 2.197 & 48.334 & 2.197 & 8.788 \\
\hline 2.197 & 6.591 & 17.576 & 4.394 \\
\hline 2.197 & 6.591 & 2.197 & 17.576 \\
\hline 2.197 & 2.197 & 4.394 & 8.788 \\
\hline 107.653 & 15.379 & 123.032 & 35.152 \\
\hline 8.788 & 6.591 & 68.107 & 6.591 \\
\hline 2.197 & 2.197 & 2.197 & 21.97 \\
\hline 8.788 & 6.591 & 2.197 & 2.197 \\
\hline 2.197 & 8.788 & 2.197 & 112.047 \\
\hline 8.788 & 2.197 & 35.152 & 13.182 \\
\hline 2.197 & 2.197 & 6.591 & 2.197 \\
\hline 13.182 & 13.182 & 2.197 & 8.788 \\
\hline 8.788 & 4.394 & 2.197 & 24.167 \\
\hline 2.197 & 35.152 & 2.197 & 2.197 \\
\hline 52.728 & 859.027 & 10.985 & 237.276 \\
\hline 48.334 & 2.197 & 817.284 & 127.426 \\
\hline 4.394 & 4.394 & 2.197 & 2.197 \\
\hline 2.197 & 4.394 & 19.773 & 24.167 \\
\hline 2.197 & 2.197 & 17.576 & 2.197 \\
\hline 6.591 & 76.895 & 41.743 & 2.197 \\
\hline 2.197 & 41.743 & 4.394 & 10.985 \\
\hline 2.197 & 2.197 & 2.197 & 21.97 \\
\hline 19.773 & 72.501 & 4.394 & 4.394 \\
\hline
\end{tabular}




\begin{tabular}{|c|c|c|c|}
\hline 4.394 & 13.182 & 4.394 & 1772.98 \\
\hline 46.137 & 193.336 & 6.591 & 2.197 \\
\hline 2480.41 & 4.394 & 2.197 & 24.167 \\
\hline 2.197 & 2.197 & 74.698 & 2.197 \\
\hline 2.197 & 43.94 & 2.197 & 8.788 \\
\hline 41.743 & 35.152 & 2.197 & 3007.69 \\
\hline 15.379 & 2.197 & 21.97 & 48.334 \\
\hline 26.364 & 94.471 & 2.197 & 4.394 \\
\hline 2.197 & 105.456 & 2.197 & 6.591 \\
\hline 2.197 & 2.197 & 2.197 & 4.394 \\
\hline 24.167 & 10.985 & 2.197 & 63.713 \\
\hline 2.197 & 2.197 & 2.197 & 6.591 \\
\hline 140.608 & 6.591 & 2.197 & 30.758 \\
\hline 2.197 & 17.576 & 6.591 & 54.925 \\
\hline 889.785 & 2.197 & 2.197 & 4.394 \\
\hline 4.394 & 8.788 & 2.197 & 68.107 \\
\hline 129.623 & 4.394 & 4.394 & 81.289 \\
\hline 2.197 & 2.197 & 2.197 & 13.182 \\
\hline 41.743 & 76.895 & 2.197 & 24.167 \\
\hline 46.137 & 37.349 & 6.591 & 4.394 \\
\hline 4.394 & 2.197 & 2.197 & 6.591 \\
\hline 8.788 & 6.591 & 2.197 & 13.182 \\
\hline 2.197 & 6.591 & 6.591 & 59.319 \\
\hline 2.197 & 92.274 & 2.197 & 59.319 \\
\hline 17.576 & 19.773 & 180.154 & 59.319 \\
\hline 6.591 & 2.197 & 4.394 & 2.197 \\
\hline 2.197 & 2.197 & 2.197 & 19.773 \\
\hline 4.394 & 4.394 & 6.591 & 1390.7 \\
\hline 191.139 & 1094.11 & 129.623 & 202.124 \\
\hline 10.985 & 10.985 & 13.182 & 6.591 \\
\hline 17.576 & 2.197 & 2.197 & 2.197 \\
\hline 4.394 & 2629.81 & 4.394 & 41.743 \\
\hline 4.394 & 41.743 & 4.394 & 30.758 \\
\hline 8.788 & 2.197 & 52.728 & 17.576 \\
\hline 136.214 & 6.591 & 2.197 & 1366.53 \\
\hline 35.152 & 4.394 & 4.394 & 8.788 \\
\hline 17.576 & 72.501 & 8.788 & 10.985 \\
\hline 112.047 & 112.047 & 2.197 & 6.591 \\
\hline 4.394 & 15.379 & 21.97 & 8.788 \\
\hline 94.471 & 30.758 & 470.158 & 17.576 \\
\hline 2.197 & 2.197 & 10.985 & 2.197 \\
\hline 15.379 & 15.379 & 2.197 & 2.197 \\
\hline 6.591 & 116.441 & 8.788 & 2.197 \\
\hline 43.94 & 2.197 & 21.97 & 125.229 \\
\hline 8.788 & 2.197 & 19.773 & 65.91 \\
\hline 2.197 & 17.576 & 2.197 & 13.182 \\
\hline 8.788 & 17.576 & 6.591 & 4.394 \\
\hline 2750.64 & 2.197 & 21.97 & 10.985 \\
\hline 2.197 & 2.197 & 81.289 & 2.197 \\
\hline 2.197 & 72.501 & 2.197 & 32.955 \\
\hline
\end{tabular}




\begin{tabular}{|c|c|c|c|}
\hline 101.062 & 8.788 & 15.379 & 2.197 \\
\hline 2.197 & 15.379 & 35.152 & 540.462 \\
\hline 8.788 & 290.004 & 13.182 & 17.576 \\
\hline 32.955 & 10.985 & 2.197 & 19.773 \\
\hline 6.591 & 72.501 & 2.197 & 6.591 \\
\hline 13.182 & 801.905 & 8.788 & 2.197 \\
\hline 17.576 & 13.182 & 2.197 & 2.197 \\
\hline 48.334 & 19.773 & 61.516 & 2.197 \\
\hline 17.576 & 4.394 & 2.197 & 13.182 \\
\hline 158.184 & 19.773 & 2.197 & 32.955 \\
\hline 2157.45 & 48.334 & 10.985 & 6.591 \\
\hline 107.653 & 17.576 & 17.576 & 17.576 \\
\hline 17.576 & 17.576 & 2.197 & 2.197 \\
\hline 85.683 & 2.197 & 2.197 & 2.197 \\
\hline 2.197 & 2.197 & 2.197 & 4.394 \\
\hline 2.197 & 2.197 & 2.197 & 68.107 \\
\hline 2.197 & 4.394 & 8.788 & 19.773 \\
\hline 8.788 & 17.576 & 39.546 & 2.197 \\
\hline 57.122 & 2.197 & 6.591 & 17.576 \\
\hline 21.97 & 2.197 & 2.197 & 6.591 \\
\hline 2.197 & 39.546 & 2.197 & 2.197 \\
\hline 4.394 & 46.137 & 4.394 & 6.591 \\
\hline 1116.08 & 4.394 & 4.394 & 10.985 \\
\hline 4.394 & 2.197 & 2.197 & 2.197 \\
\hline 4.394 & 41.743 & 2.197 & 6.591 \\
\hline 4.394 & 39.546 & 2.197 & 4.394 \\
\hline 26.364 & 153.79 & 2.197 & 2.197 \\
\hline 8.788 & 298.792 & 2.197 & 6.591 \\
\hline 2.197 & 2.197 & 2.197 & 24.167 \\
\hline 35.152 & 314.171 & 6.591 & 24.167 \\
\hline 19.773 & 81.289 & 2.197 & 4.394 \\
\hline 863.421 & 10.985 & 4.394 & 2.197 \\
\hline 2.197 & 2.197 & 2.197 & 26.364 \\
\hline 19.773 & 2.197 & 2.197 & 98.865 \\
\hline 30.758 & 2.197 & 39.546 & 54.925 \\
\hline 4.394 & 2.197 & 2.197 & 13.182 \\
\hline 70.304 & 2.197 & 2.197 & 4.394 \\
\hline 4.394 & 4.394 & 21.97 & 2.197 \\
\hline 351.52 & 15.379 & 13.182 & 4.394 \\
\hline 6.591 & 43.94 & 87.88 & 4.394 \\
\hline 41.743 & 4.394 & 2.197 & 30.758 \\
\hline 2.197 & 87.88 & 41.743 & 10.985 \\
\hline 39.546 & 2.197 & 2.197 & 65.91 \\
\hline 2.197 & 2.197 & 2.197 & 166.972 \\
\hline 43.94 & 4.394 & 2.197 & 8.788 \\
\hline 2.197 & 2.197 & 35.152 & 990.847 \\
\hline 703.04 & 15.379 & 10.985 & 8.788 \\
\hline 4.394 & 8.788 & 109.85 & 13.182 \\
\hline 2.197 & 21.97 & 4.394 & 1515.93 \\
\hline 2.197 & 2.197 & 19.773 & 2.197 \\
\hline
\end{tabular}




\begin{tabular}{|c|c|c|c|}
\hline 83.486 & 4.394 & 46.137 & 19.773 \\
\hline 114.244 & 4.394 & 147.199 & 2.197 \\
\hline 4.394 & 4.394 & 2.197 & 4.394 \\
\hline 2.197 & 43.94 & 2.197 & 2.197 \\
\hline 2.197 & 4.394 & 199.927 & 2.197 \\
\hline 4.394 & 6.591 & 4.394 & 90.077 \\
\hline 2.197 & 2.197 & 2.197 & 2.197 \\
\hline 13.182 & 8.788 & 8.788 & 2.197 \\
\hline 15.379 & 2.197 & 109.85 & 303.186 \\
\hline 4.394 & 2.197 & 54.925 & 4.394 \\
\hline 50.531 & 6.591 & 2.197 & 6.591 \\
\hline 2.197 & 6.591 & 48.334 & 6.591 \\
\hline 4.394 & 65.91 & 15.379 & 2.197 \\
\hline 2.197 & 2.197 & 35.152 & 344.929 \\
\hline 21.97 & 2.197 & 4.394 & 2.197 \\
\hline 1768.58 & 2.197 & 35.152 & 2.197 \\
\hline 50.531 & 2.197 & 41.743 & 8.788 \\
\hline 76.895 & 2.197 & 2.197 & 2.197 \\
\hline 6.591 & 6.591 & 2.197 & 10.985 \\
\hline 13.182 & 4.394 & 2.197 & 333.944 \\
\hline 10.985 & 2.197 & 2.197 & 24.167 \\
\hline 8.788 & 2.197 & 2.197 & 8.788 \\
\hline 151.593 & 2.197 & 41.743 & 19.773 \\
\hline 2.197 & 4.394 & 2.197 & 17.576 \\
\hline 6.591 & 426.218 & 2.197 & 26.364 \\
\hline 48.334 & 6.591 & 4.394 & 2.197 \\
\hline 74.698 & 140.608 & 6.591 & 17.576 \\
\hline 19.773 & 413.036 & 85.683 & 10.985 \\
\hline 2.197 & 13.182 & 8.788 & 8.788 \\
\hline 4.394 & 39.546 & 61.516 & 2.197 \\
\hline 24.167 & 160.381 & 2.197 & 2.197 \\
\hline 8.788 & 26.364 & 2.197 & 41.743 \\
\hline 2.197 & 197.73 & 2.197 & 30.758 \\
\hline 2.197 & 8.788 & 2.197 & 2.197 \\
\hline 797.511 & 13.182 & 17.576 & 8.788 \\
\hline 2.197 & 81.289 & 32.955 & 13.182 \\
\hline 30.758 & 35.152 & 13.182 & 1713.66 \\
\hline 10.985 & 2.197 & 50.531 & 8.788 \\
\hline 4.394 & 6.591 & 4.394 & 17.576 \\
\hline 10.985 & 2.197 & 61.516 & 68.107 \\
\hline 15.379 & 8.788 & 39.546 & 8.788 \\
\hline 30.758 & 2.197 & 6.591 & 6.591 \\
\hline 2.197 & 2.197 & 2.197 & 4.394 \\
\hline 2.197 & 21.97 & 2.197 & 138.411 \\
\hline 2.197 & 2.197 & 2.197 & 2.197 \\
\hline 2.197 & 283.413 & 2.197 & 2.197 \\
\hline 2.197 & 2.197 & 2.197 & 39.546 \\
\hline 15.379 & 2.197 & 57.122 & 8.788 \\
\hline 8.788 & 2.197 & 4.394 & 2.197 \\
\hline 2.197 & 2.197 & 2.197 & 2.197 \\
\hline
\end{tabular}




\begin{tabular}{|c|c|c|c|}
\hline 30.758 & 8.788 & 10.985 & 2.197 \\
\hline 2.197 & 120.835 & 4.394 & 10.985 \\
\hline 4.394 & 17.576 & 39.546 & 68.107 \\
\hline 8.788 & 4.394 & 2.197 & 4.394 \\
\hline 2.197 & 19.773 & 6.591 & 21.97 \\
\hline 2.197 & 98.865 & 2.197 & 39.546 \\
\hline 15.379 & 90.077 & 10.985 & 2.197 \\
\hline 2.197 & 26.364 & 2.197 & 4.394 \\
\hline 6.591 & 10.985 & 4.394 & 4.394 \\
\hline 2.197 & 4.394 & 2.197 & 6.591 \\
\hline 2.197 & 2.197 & 2.197 & 28.561 \\
\hline 74.698 & 32.955 & 13.182 & 26.364 \\
\hline 180.154 & 213.109 & 180.154 & 13.182 \\
\hline 13.182 & 21.97 & 4.394 & 16189.7 \\
\hline 21.97 & 6.591 & 2.197 & 28.561 \\
\hline 6.591 & 10.985 & 2.197 & 2.197 \\
\hline 19.773 & 37.349 & 21.97 & 24.167 \\
\hline 6.591 & 48.334 & 41.743 & 13.182 \\
\hline 13.182 & 2.197 & 4.394 & 2.197 \\
\hline 63.713 & 6.591 & 6.591 & 6.591 \\
\hline 13.182 & 15.379 & 6.591 & 105.456 \\
\hline 26.364 & 35.152 & 6.591 & 13.182 \\
\hline 4.394 & 17.576 & 2.197 & 2.197 \\
\hline 2.197 & 2.197 & 2.197 & 6.591 \\
\hline 8.788 & 2.197 & 72.501 & 30.758 \\
\hline 6.591 & 4.394 & 4.394 & 17.576 \\
\hline 6.591 & 4.394 & 32.955 & 2.197 \\
\hline 57.122 & 832.663 & 219.7 & 46.137 \\
\hline 8.788 & 2.197 & 4.394 & 21.97 \\
\hline 6.591 & 2.197 & 2.197 & 13.182 \\
\hline 2.197 & 92.274 & 2.197 & 63.713 \\
\hline 2.197 & 10.985 & 2.197 & 103.259 \\
\hline 404.248 & 2.197 & 2.197 & 2.197 \\
\hline 2.197 & 17.576 & 2.197 & 10.985 \\
\hline 2.197 & 41.743 & 2.197 & 4.394 \\
\hline 41.743 & 4.394 & 2.197 & 39.546 \\
\hline 6.591 & 2.197 & 2.197 & 2.197 \\
\hline 17.576 & 2.197 & 2.197 & 4.394 \\
\hline 6.591 & 17.576 & 2.197 & 24.167 \\
\hline 6.591 & 8.788 & 68.107 & 4.394 \\
\hline 28.561 & 4.394 & 13.182 & 4.394 \\
\hline 4.394 & 46.137 & 4.394 & 2.197 \\
\hline 2.197 & 294.398 & 2.197 & 6.591 \\
\hline 2.197 & 10.985 & 2.197 & 2.197 \\
\hline 24.167 & 136.214 & 2.197 & 6.591 \\
\hline 4.394 & 2.197 & 8.788 & 13.182 \\
\hline 2.197 & 21.97 & 2.197 & 37.349 \\
\hline 2.197 & 4.394 & 2.197 & 48.334 \\
\hline 17.576 & 8.788 & 83.486 & 54.925 \\
\hline 4.394 & 24.167 & 2.197 & 94.471 \\
\hline
\end{tabular}




\begin{tabular}{|c|c|c|c|}
\hline 127.426 & 2.197 & 140.608 & 13.182 \\
\hline 2.197 & 494.325 & 2.197 & 4.394 \\
\hline 15.379 & 70.304 & 4.394 & 94.471 \\
\hline 2.197 & 39.546 & 15.379 & 2.197 \\
\hline 887.588 & 13.182 & 2.197 & 35.152 \\
\hline 2.197 & 2.197 & 2.197 & 4.394 \\
\hline 2.197 & 4.394 & 24.167 & 17.576 \\
\hline 2.197 & 41.743 & 6.591 & 13.182 \\
\hline 2.197 & 2.197 & 52.728 & 10.985 \\
\hline 305.383 & 2.197 & 4.394 & 10.985 \\
\hline 46.137 & 2.197 & 4.394 & 54.925 \\
\hline 2109.12 & 120.835 & 4.394 & 2.197 \\
\hline 4.394 & 8.788 & 6.591 & 17.576 \\
\hline 37.349 & 10.985 & 2.197 & 8.788 \\
\hline 2.197 & 43.94 & 2.197 & 2.197 \\
\hline 4.394 & 19.773 & 2.197 & 123.032 \\
\hline 2.197 & 2.197 & 2.197 & 3554.75 \\
\hline 2.197 & 26.364 & 2.197 & 2.197 \\
\hline 10.985 & 142.805 & 2.197 & 4.394 \\
\hline 2.197 & 2.197 & 2.197 & 4.394 \\
\hline 2.197 & 2.197 & 6.591 & 6.591 \\
\hline 87.88 & 6.591 & 2.197 & 17.576 \\
\hline 1146.83 & 4.394 & 2.197 & 221.897 \\
\hline 35.152 & 2.197 & 8.788 & 76.895 \\
\hline 2.197 & 4.394 & 17.576 & 2.197 \\
\hline 2.197 & 41.743 & 63.713 & 6.591 \\
\hline 2.197 & 4.394 & 17.576 & 26.364 \\
\hline 17.576 & 103.259 & 2.197 & 32.955 \\
\hline 2.197 & 292.201 & 2.197 & 30.758 \\
\hline 2.197 & 2.197 & 4.394 & 4.394 \\
\hline 2.197 & 2.197 & 4.394 & 4.394 \\
\hline 21.97 & 274.625 & 4.394 & 2.197 \\
\hline 4.394 & 2.197 & 1098.5 & 2.197 \\
\hline 6.591 & 10.985 & 19.773 & 4.394 \\
\hline 2.197 & 87.88 & 274.625 & 149.396 \\
\hline 2.197 & 15.379 & 1188.58 & 24.167 \\
\hline 2.197 & 10.985 & 8.788 & 35.152 \\
\hline 2.197 & 8.788 & 2.197 & 79.092 \\
\hline 17.576 & 13.182 & 17.576 & 61.516 \\
\hline 2.197 & 6.591 & 74.698 & 316.368 \\
\hline 6.591 & 24.167 & 2.197 & 2.197 \\
\hline 10.985 & 4.394 & 4.394 & 419.627 \\
\hline 37.349 & 6.591 & 8.788 & 1401.69 \\
\hline 2.197 & 46.137 & 2.197 & 8.788 \\
\hline 2.197 & 54.925 & 2.197 & 2.197 \\
\hline 478.946 & 24.167 & 2.197 & 21.97 \\
\hline 4.394 & 81.289 & 2.197 & 2.197 \\
\hline 349.323 & 37.349 & 96.668 & 2.197 \\
\hline 2.197 & 28.561 & 13.182 & 37.349 \\
\hline 2.197 & 21.97 & 369.096 & 6.591 \\
\hline
\end{tabular}




\begin{tabular}{|c|c|c|c|}
\hline 63.713 & 4.394 & 2.197 & 26.364 \\
\hline 35.152 & 221.897 & 2.197 & 19.773 \\
\hline 4.394 & 10.985 & 54.925 & 2.197 \\
\hline 6.591 & 48.334 & 2.197 & 1390.7 \\
\hline 2.197 & 10.985 & 6.591 & 2.197 \\
\hline 2.197 & 2.197 & 72.501 & 2.197 \\
\hline 8.788 & 2.197 & 2.197 & 17.576 \\
\hline 4.394 & 65.91 & 30.758 & 13.182 \\
\hline 4.394 & 467.961 & 13.182 & 10.985 \\
\hline 28.561 & 2.197 & 2.197 & 6.591 \\
\hline 476.749 & 15.379 & 8.788 & 2.197 \\
\hline 2.197 & 2.197 & 21.97 & 2.197 \\
\hline 4.394 & 28.561 & 107.653 & 10.985 \\
\hline 6.591 & 8.788 & 32.955 & 2.197 \\
\hline 17.576 & 2.197 & 4.394 & 2.197 \\
\hline 584.402 & 101.062 & 8.788 & 4.394 \\
\hline 206.518 & 6.591 & 32.955 & 28.561 \\
\hline 329.55 & 21.97 & 2.197 & 2.197 \\
\hline 112.047 & 79.092 & 2.197 & 2.197 \\
\hline 2.197 & 37.349 & 2.197 & 79.092 \\
\hline 4.394 & 10.985 & 15.379 & 2.197 \\
\hline 19.773 & 4.394 & 32.955 & 909.558 \\
\hline 8.788 & 8.788 & 2.197 & 94.471 \\
\hline 8.788 & 17.576 & 2.197 & 5140.98 \\
\hline 21.97 & 2.197 & 2.197 & 4.394 \\
\hline 8.788 & 15.379 & 2.197 & 10.985 \\
\hline 85.683 & 39.546 & 52.728 & 92.274 \\
\hline 4.394 & 24.167 & 2.197 & 90.077 \\
\hline 2.197 & 13.182 & 2.197 & 21.97 \\
\hline 2.197 & 6.591 & 4.394 & 4.394 \\
\hline 224.094 & 2.197 & 2.197 & 48.334 \\
\hline 138.411 & 10.985 & 2.197 & 35.152 \\
\hline 2.197 & 15.379 & 92.274 & 57.122 \\
\hline 17.576 & 2.197 & 2.197 & 28.561 \\
\hline 19.773 & 2.197 & 274.625 & 10.985 \\
\hline 4.394 & 2.197 & 127.426 & 48.334 \\
\hline 2.197 & 283.413 & 6.591 & 28.561 \\
\hline 2.197 & 2.197 & 6.591 & 8.788 \\
\hline 15.379 & 2.197 & 2.197 & 41.743 \\
\hline 2.197 & 103.259 & 57.122 & 17.576 \\
\hline 50.531 & 76.895 & 6.591 & 13.182 \\
\hline 26.364 & 6.591 & 2.197 & 101.062 \\
\hline 116.441 & 50.531 & 59.319 & 13.182 \\
\hline 76.895 & 8.788 & 2.197 & 10.985 \\
\hline 2.197 & 208.715 & 92.274 & 2.197 \\
\hline 4.394 & 50.531 & 2.197 & 37.349 \\
\hline 90.077 & 4.394 & 6.591 & 6.591 \\
\hline 10.985 & 4.394 & 2.197 & 48.334 \\
\hline 26.364 & 197.73 & 13.182 & 10.985 \\
\hline 142.805 & 15.379 & 2.197 & 41.743 \\
\hline
\end{tabular}




\begin{tabular}{|c|c|c|c|}
\hline 15.379 & 2.197 & 2.197 & 6.591 \\
\hline 8.788 & 10.985 & 2.197 & 15.379 \\
\hline 2.197 & 2.197 & 4.394 & 26.364 \\
\hline 8.788 & 35.152 & 2.197 & 28.561 \\
\hline 2.197 & 10.985 & 6.591 & 2.197 \\
\hline 28.561 & 619.554 & 4.394 & 72.501 \\
\hline 2.197 & 2.197 & 2.197 & 2.197 \\
\hline 2.197 & 2.197 & 2.197 & 2.197 \\
\hline 2.197 & 15.379 & 6.591 & 10.985 \\
\hline 2.197 & 6.591 & 6.591 & 2.197 \\
\hline 2.197 & 101.062 & 2.197 & 26.364 \\
\hline 19.773 & 19.773 & 2.197 & 48.334 \\
\hline 6.591 & 362.505 & 4.394 & 43.94 \\
\hline 4.394 & 136.214 & 2.197 & 175.76 \\
\hline 2.197 & 87.88 & 4.394 & 4.394 \\
\hline 79.092 & 2.197 & 41.743 & 4.394 \\
\hline 6.591 & 30.758 & 2.197 & 2.197 \\
\hline 4.394 & 173.563 & 4.394 & 2.197 \\
\hline 6.591 & 52.728 & 4.394 & 92.274 \\
\hline 2.197 & 19.773 & 2.197 & 48.334 \\
\hline 120.835 & 32.955 & 15.379 & 21.97 \\
\hline 15.379 & 213.109 & 1746.61 & 17.576 \\
\hline 24.167 & 103.259 & 41.743 & 138.411 \\
\hline 2.197 & 13.182 & 2.197 & 26.364 \\
\hline 2.197 & 8.788 & 2.197 & 2.197 \\
\hline 2.197 & 57.122 & 10.985 & 270.231 \\
\hline 2.197 & 57.122 & 2.197 & 4.394 \\
\hline 2.197 & 188.942 & 1208.35 & 4.394 \\
\hline 2.197 & 8.788 & 2.197 & 4.394 \\
\hline 2.197 & 13.182 & 2.197 & 2.197 \\
\hline 2.197 & 2.197 & 125.229 & 6.591 \\
\hline 8.788 & 37.349 & 70.304 & 160.381 \\
\hline 28.561 & 2.197 & 26.364 & 15.379 \\
\hline 8.788 & 10.985 & 162.578 & 101.062 \\
\hline 1100.7 & 6.591 & 8.788 & 2.197 \\
\hline 2.197 & 2.197 & 2.197 & 15.379 \\
\hline 525.083 & 2.197 & 2.197 & 2.197 \\
\hline 70.304 & 2.197 & 2.197 & 8.788 \\
\hline 4.394 & 10.985 & 15.379 & 2.197 \\
\hline 8.788 & 13.182 & 6.591 & 2.197 \\
\hline 2.197 & 2.197 & 105.456 & 2.197 \\
\hline 2.197 & 92.274 & 2.197 & 2.197 \\
\hline 2.197 & 43.94 & 2.197 & 65.91 \\
\hline 6.591 & 8.788 & 2.197 & 2.197 \\
\hline 145.002 & 4.394 & 8.788 & 4.394 \\
\hline 15.379 & 30.758 & 21.97 & 4.394 \\
\hline 30.758 & 8.788 & 2.197 & 2.197 \\
\hline 2.197 & 2.197 & 8.788 & 573.417 \\
\hline 8.788 & 8.788 & 2.197 & 2.197 \\
\hline 2.197 & 208.715 & 147.199 & 52.728 \\
\hline
\end{tabular}




\begin{tabular}{|c|c|c|c|}
\hline 2.197 & 28.561 & 2.197 & 52.728 \\
\hline 4.394 & 10.985 & 2.197 & 397.657 \\
\hline 2.197 & 2.197 & 26.364 & 39.546 \\
\hline 2.197 & 2.197 & 2.197 & 8.788 \\
\hline 2.197 & 118.638 & 24.167 & 37.349 \\
\hline 8.788 & 4.394 & 30.758 & 2.197 \\
\hline 24.167 & 19.773 & 4.394 & 2.197 \\
\hline 72.501 & 6.591 & 2.197 & 39.546 \\
\hline 4.394 & 10.985 & 1406.08 & 32.955 \\
\hline 8.788 & 219.7 & 2.197 & 4.394 \\
\hline 19.773 & 35.152 & 2.197 & 15.379 \\
\hline 13.182 & 6.591 & 2.197 & 26.364 \\
\hline 2.197 & 8.788 & 50.531 & 61.516 \\
\hline 2.197 & 136.214 & 83.486 & 13.182 \\
\hline 8.788 & 590.993 & 13.182 & 2.197 \\
\hline 10.985 & 13.182 & 4.394 & 10.985 \\
\hline 2.197 & 118.638 & 2.197 & 2.197 \\
\hline 2.197 & 2.197 & 65.91 & 8.788 \\
\hline 4.394 & 4.394 & 13.182 & 6.591 \\
\hline 10.985 & 26.364 & 116.441 & 2.197 \\
\hline 13.182 & 6.591 & 54.925 & 4.394 \\
\hline 2.197 & 2.197 & 2.197 & 4.394 \\
\hline 4.394 & 2.197 & 2.197 & 2.197 \\
\hline 19.773 & 4.394 & 24.167 & 2.197 \\
\hline 2.197 & 2.197 & 4.394 & 2.197 \\
\hline 32.955 & 4.394 & 17.576 & 2.197 \\
\hline 10.985 & 13.182 & 13.182 & 2.197 \\
\hline 4.394 & 10.985 & 6.591 & 4.394 \\
\hline 105.456 & 2.197 & 2.197 & 17.576 \\
\hline 2.197 & 2.197 & 48.334 & 19.773 \\
\hline 107.653 & 4.394 & 147.199 & 164.775 \\
\hline 10.985 & 28.561 & 2.197 & 129.623 \\
\hline 21.97 & 4.394 & 15.379 & 206.518 \\
\hline 123.032 & 4.394 & 2.197 & 4.394 \\
\hline 39.546 & 2.197 & 24.167 & 13.182 \\
\hline 8.788 & 30.758 & 147.199 & 4.394 \\
\hline 2.197 & 4.394 & 2.197 & 2.197 \\
\hline 90.077 & 2.197 & 13.182 & 19.773 \\
\hline 2.197 & 4.394 & 4.394 & 4.394 \\
\hline 8.788 & 2.197 & 6.591 & 2.197 \\
\hline 15.379 & 2.197 & 722.813 & 4.394 \\
\hline 2.197 & 601.978 & 1164.41 & 305.383 \\
\hline 2.197 & 61.516 & 19.773 & 4571.96 \\
\hline 10.985 & 13.182 & 15.379 & 4.394 \\
\hline 6.591 & 2.197 & 54.925 & 39.546 \\
\hline 4.394 & 4.394 & 4.394 & 237.276 \\
\hline 2.197 & 8.788 & 2.197 & 24.167 \\
\hline 177.957 & 17.576 & 4.394 & 17.576 \\
\hline 6.591 & 2.197 & 4.394 & 2.197 \\
\hline 35.152 & 6.591 & 2.197 & 6.591 \\
\hline
\end{tabular}




\begin{tabular}{|c|c|c|c|}
\hline 8.788 & 17.576 & 2.197 & 158.184 \\
\hline 6.591 & 6.591 & 4.394 & 4.394 \\
\hline 6.591 & 2.197 & 15.379 & 4.394 \\
\hline 2.197 & 2.197 & 6.591 & 2.197 \\
\hline 2.197 & 2.197 & 2.197 & 2.197 \\
\hline 4.394 & 3884.3 & 15.379 & 2.197 \\
\hline 6.591 & 4.394 & 30.758 & 87.88 \\
\hline 21.97 & 8.788 & 10.985 & 2.197 \\
\hline 4.394 & 2.197 & 2.197 & 134.017 \\
\hline 10.985 & 6.591 & 54.925 & 15.379 \\
\hline 2.197 & 529.477 & 24.167 & 210.912 \\
\hline 6.591 & 17.576 & 2.197 & 65.91 \\
\hline 2.197 & 35.152 & 327.353 & 2.197 \\
\hline 142.805 & 2.197 & 2.197 & 10.985 \\
\hline 2.197 & 10.985 & 2.197 & 2.197 \\
\hline 2.197 & 2.197 & 2.197 & 2.197 \\
\hline 2.197 & 6.591 & 2.197 & 10.985 \\
\hline 2.197 & 2.197 & 2.197 & 202.124 \\
\hline 2.197 & 6.591 & 2.197 & 180.154 \\
\hline 8.788 & 4.394 & 4.394 & 4.394 \\
\hline 2.197 & 2.197 & 4.394 & 13.182 \\
\hline 59.319 & 2.197 & 2.197 & 2.197 \\
\hline 426.218 & 15.379 & 2.197 & 63.713 \\
\hline 2.197 & 2.197 & 2.197 & 2.197 \\
\hline 2.197 & 2.197 & 2.197 & 2.197 \\
\hline 1245.7 & 2.197 & 50.531 & 6.591 \\
\hline 2.197 & 2.197 & 164.775 & 2.197 \\
\hline 2.197 & 2.197 & 10.985 & 2.197 \\
\hline 6.591 & 2.197 & 2.197 & 2.197 \\
\hline 4.394 & 28.561 & 2.197 & 10.985 \\
\hline 2.197 & 8.788 & 37.349 & 2.197 \\
\hline 46.137 & 2.197 & 2.197 & 4.394 \\
\hline 28.561 & 2.197 & 72.501 & 182.351 \\
\hline 2.197 & 2.197 & 10.985 & 13.182 \\
\hline 2.197 & 13.182 & 138.411 & 290.004 \\
\hline 52508.3 & 461.37 & 2.197 & 61.516 \\
\hline 48.334 & 2.197 & 6.591 & 2.197 \\
\hline 4.394 & 30.758 & 2.197 & 30.758 \\
\hline 292.201 & 15.379 & 2.197 & 2.197 \\
\hline 114.244 & 2.197 & 17.576 & 8.788 \\
\hline 2.197 & 10.985 & 37.349 & 4.394 \\
\hline 2.197 & 10.985 & 2.197 & 2.197 \\
\hline 15.379 & 2.197 & 4.394 & 2.197 \\
\hline 2.197 & 13.182 & 2.197 & 10.985 \\
\hline 15.379 & 21.97 & 8.788 & 2.197 \\
\hline 24.167 & 6.591 & 4.394 & 2.197 \\
\hline 4.394 & 2.197 & 4.394 & 4.394 \\
\hline 13.182 & 2.197 & 186.745 & 257.049 \\
\hline 26.364 & 2.197 & 28.561 & 13.182 \\
\hline 10.985 & 118.638 & 188.942 & 435.006 \\
\hline
\end{tabular}




\begin{tabular}{|c|c|c|c|}
\hline 13.182 & 2.197 & 4.394 & 2.197 \\
\hline 54.925 & 26.364 & 4.394 & 2.197 \\
\hline 6.591 & 37.349 & 4.394 & 2.197 \\
\hline 8.788 & 136.214 & 6.591 & 2.197 \\
\hline 2.197 & 2.197 & 57.122 & 10.985 \\
\hline 4.394 & 2.197 & 50.531 & 15.379 \\
\hline 54.925 & 2.197 & 114.244 & 2.197 \\
\hline 57.122 & 8.788 & 2.197 & 48.334 \\
\hline 1153.42 & 2.197 & 103.259 & 8.788 \\
\hline 2.197 & 808.496 & 4.394 & 17.576 \\
\hline 70.304 & 6.591 & 10.985 & 68.107 \\
\hline 8.788 & 112.047 & 2.197 & 2.197 \\
\hline 2.197 & 21.97 & 4.394 & 2.197 \\
\hline 10.985 & 360.308 & 21.97 & 4.394 \\
\hline 17.576 & 21.97 & 17.576 & 402.051 \\
\hline 2.197 & 8.788 & 79.092 & 2.197 \\
\hline 2.197 & 6.591 & 17.576 & 193.336 \\
\hline 2.197 & 10.985 & 2.197 & 8.788 \\
\hline 2.197 & 35.152 & 28.561 & 96.668 \\
\hline 43.94 & 2.197 & 4.394 & 8.788 \\
\hline 4.394 & 6.591 & 15.379 & 13.182 \\
\hline 26.364 & 2.197 & 2.197 & 2.197 \\
\hline 4.394 & 2.197 & 2.197 & 2.197 \\
\hline 4.394 & 10.985 & 4.394 & 2.197 \\
\hline 2.197 & 134.017 & 6.591 & 4.394 \\
\hline 2.197 & 2.197 & 2.197 & 4.394 \\
\hline 2.197 & 8.788 & 8.788 & 32.955 \\
\hline 4.394 & 2.197 & 2.197 & 6.591 \\
\hline 4.394 & 2.197 & 2.197 & 35.152 \\
\hline 28.561 & 2.197 & 2.197 & 134.017 \\
\hline 116.441 & 2.197 & 169.169 & 46.137 \\
\hline 8.788 & 13.182 & 2.197 & 2.197 \\
\hline 164.775 & 24.167 & 2.197 & 2.197 \\
\hline 116.441 & 191.139 & 2.197 & 46.137 \\
\hline 4.394 & 4.394 & 21.97 & 4.394 \\
\hline 81.289 & 74.698 & 13.182 & 48.334 \\
\hline 2.197 & 2.197 & 2.197 & 2.197 \\
\hline 4.394 & 13.182 & 26.364 & 15.379 \\
\hline 83.486 & 4.394 & 19.773 & 2.197 \\
\hline 127.426 & 41.743 & 2.197 & 2.197 \\
\hline 10.985 & 2.197 & 2.197 & 2.197 \\
\hline 2001.47 & 26.364 & 21.97 & 19.773 \\
\hline 19.773 & 17.576 & 4.394 & 65.91 \\
\hline 4.394 & 17.576 & 4.394 & 61.516 \\
\hline 21.97 & 2.197 & 8.788 & 329.55 \\
\hline 6454.79 & 4.394 & 2.197 & 2.197 \\
\hline 322.959 & 2.197 & 2.197 & 2.197 \\
\hline 21.97 & 204.321 & 2.197 & 15.379 \\
\hline 4.394 & 2.197 & 2.197 & 8.788 \\
\hline 4.394 & 8.788 & 2.197 & 2.197 \\
\hline
\end{tabular}




\begin{tabular}{|c|c|c|c|}
\hline 6.591 & 8.788 & 2.197 & 2.197 \\
\hline 39.546 & 4.394 & 4.394 & 32.955 \\
\hline 13.182 & 24.167 & 2.197 & 4.394 \\
\hline 2.197 & 2.197 & 2.197 & 3244.97 \\
\hline 342.732 & 6.591 & 2.197 & 1113.88 \\
\hline 2.197 & 13.182 & 2.197 & 2.197 \\
\hline 13.182 & 2.197 & 2.197 & 10.985 \\
\hline 2.197 & 4.394 & 2.197 & 13.182 \\
\hline 153.79 & 17.576 & 2.197 & 2.197 \\
\hline 2.197 & 10.985 & 13.182 & 6.591 \\
\hline 103.259 & 815.087 & 2.197 & 63.713 \\
\hline 2.197 & 41.743 & 2.197 & 6.591 \\
\hline 2.197 & 30.758 & 2.197 & 10.985 \\
\hline 103.259 & 4.394 & 2.197 & 4.394 \\
\hline 8.788 & 4.394 & 8.788 & 2.197 \\
\hline 21.97 & 63.713 & 54.925 & 30.758 \\
\hline 19.773 & 10.985 & 8.788 & 74.698 \\
\hline 13.182 & 2.197 & 8.788 & 72.501 \\
\hline 13.182 & 19.773 & 2.197 & 17.576 \\
\hline 2.197 & 19.773 & 13.182 & 2.197 \\
\hline 221.897 & 6.591 & 52.728 & 2.197 \\
\hline 118.638 & 15.379 & 17.576 & 39.546 \\
\hline 2.197 & 2.197 & 4.394 & 6.591 \\
\hline 4.394 & 54.925 & 2.197 & 21.97 \\
\hline 4.394 & 15.379 & 2.197 & 109.85 \\
\hline 41.743 & 2.197 & 2.197 & 52.728 \\
\hline 2.197 & 2.197 & 2.197 & 2.197 \\
\hline 65.91 & 355.914 & 2.197 & 6.591 \\
\hline 74.698 & 160.381 & 2.197 & 13.182 \\
\hline 2.197 & 2.197 & 4.394 & 2.197 \\
\hline 296.595 & 17.576 & 2.197 & 6.591 \\
\hline 46.137 & 2.197 & 15.379 & 54.925 \\
\hline 4.394 & 162.578 & 54.925 & 26.364 \\
\hline 90.077 & 96.668 & 4.394 & 109.85 \\
\hline 48.334 & 8.788 & 4.394 & 8.788 \\
\hline 4.394 & 4.394 & 48.334 & 4.394 \\
\hline 2.197 & 2.197 & 39.546 & 8.788 \\
\hline 6.591 & 2.197 & 39.546 & 6.591 \\
\hline 2.197 & 6.591 & 2.197 & 456.976 \\
\hline 4.394 & 52.728 & 2.197 & 4.394 \\
\hline 4.394 & 4.394 & 2.197 & 162.578 \\
\hline 4.394 & 4.394 & 19.773 & 35.152 \\
\hline 10.985 & 4.394 & 4.394 & 103.259 \\
\hline 41.743 & 39.546 & 52.728 & 17.576 \\
\hline 2.197 & 26.364 & 15.379 & 4.394 \\
\hline 191.139 & 2.197 & 8.788 & 65.91 \\
\hline 74.698 & 2.197 & 32.955 & 21.97 \\
\hline 2.197 & 6.591 & 340.535 & 52.728 \\
\hline 8.788 & 2.197 & 30.758 & 2.197 \\
\hline 4.394 & 4.394 & 24.167 & 41.743 \\
\hline
\end{tabular}




\begin{tabular}{|c|c|c|c|}
\hline 6.591 & 4.394 & 2.197 & 2.197 \\
\hline 2.197 & 180.154 & 2.197 & 2.197 \\
\hline 4.394 & 1885.03 & 10.985 & 6.591 \\
\hline 43.94 & 32.955 & 2.197 & 2.197 \\
\hline 19.773 & 112.047 & 2.197 & 2.197 \\
\hline 24.167 & 2.197 & 2.197 & 6.591 \\
\hline 30.758 & 2.197 & 2.197 & 202.124 \\
\hline 4.394 & 2.197 & 2.197 & 98.865 \\
\hline 2.197 & 13.182 & 2.197 & 2.197 \\
\hline 6.591 & 2.197 & 2.197 & 4.394 \\
\hline 46.137 & 13.182 & 4.394 & 8.788 \\
\hline 4.394 & 2.197 & 4.394 & 164.775 \\
\hline 2.197 & 46.137 & 2.197 & 2.197 \\
\hline 15.379 & 2.197 & 2.197 & 4.394 \\
\hline 2.197 & 94.471 & 2.197 & 4.394 \\
\hline 120.835 & 125.229 & 2.197 & 6.591 \\
\hline 10.985 & 2.197 & 2.197 & 2.197 \\
\hline 28.561 & 2.197 & 2.197 & 10.985 \\
\hline 8.788 & 15.379 & 2.197 & 2.197 \\
\hline 2.197 & 4.394 & 2.197 & 15.379 \\
\hline 2.197 & 8.788 & 547.053 & 83.486 \\
\hline 2.197 & 1790.55 & 2.197 & 8.788 \\
\hline 2.197 & 138.411 & 2.197 & 26.364 \\
\hline 4.394 & 10.985 & 2.197 & 4.394 \\
\hline 2.197 & 8.788 & 2.197 & 4.394 \\
\hline 2.197 & 15.379 & 61.516 & 63.713 \\
\hline 19.773 & 17.576 & 4.394 & 2.197 \\
\hline 8.788 & 6.591 & 21.97 & 4.394 \\
\hline 2.197 & 4.394 & 2.197 & 268.034 \\
\hline 103.259 & 10.985 & 4.394 & 76.895 \\
\hline 2.197 & 2.197 & 2.197 & 439.4 \\
\hline 2.197 & 4.394 & 8.788 & 17.576 \\
\hline 4.394 & 19.773 & 2.197 & 2.197 \\
\hline 46.137 & 2.197 & 4.394 & 32.955 \\
\hline 942.513 & 21.97 & 2.197 & 21.97 \\
\hline 2.197 & 6.591 & 136.214 & 2.197 \\
\hline 72.501 & 2.197 & 81.289 & 145.002 \\
\hline 2.197 & 24.167 & 2.197 & 41.743 \\
\hline 2.197 & 94.471 & 2.197 & 8.788 \\
\hline 103.259 & 8.788 & 4.394 & 19.773 \\
\hline 2.197 & 2.197 & 186.745 & 6.591 \\
\hline 10.985 & 2.197 & 21.97 & 10.985 \\
\hline 4.394 & 4.394 & 8.788 & 37.349 \\
\hline 10.985 & 2.197 & 4.394 & 13.182 \\
\hline 2.197 & 2.197 & 4.394 & 8.788 \\
\hline 39.546 & 2.197 & 4.394 & 4.394 \\
\hline 347.126 & 46.137 & 2.197 & 2.197 \\
\hline 2.197 & 2.197 & 4.394 & 6.591 \\
\hline 6.591 & 6.591 & 83.486 & 8.788 \\
\hline 15.379 & 4.394 & 21.97 & 2.197 \\
\hline
\end{tabular}




\begin{tabular}{|c|c|c|c|}
\hline 2.197 & 28.561 & 2.197 & 37.349 \\
\hline 2.197 & 4.394 & 2.197 & 270.231 \\
\hline 4.394 & 149.396 & 2.197 & 8.788 \\
\hline 241.67 & 35.152 & 2.197 & 41.743 \\
\hline 17.576 & 4.394 & 2.197 & 8.788 \\
\hline 155.987 & 6.591 & 2.197 & 2.197 \\
\hline 8.788 & 2.197 & 2.197 & 8.788 \\
\hline 28.561 & 8.788 & 2.197 & 79.092 \\
\hline 6.591 & 37.349 & 10.985 & 4.394 \\
\hline 2.197 & 4.394 & 2.197 & 13.182 \\
\hline 12615.2 & 2.197 & 2.197 & 8.788 \\
\hline 380.081 & 2.197 & 4.394 & 6.591 \\
\hline 105.456 & 4.394 & 4.394 & 2.197 \\
\hline 2.197 & 39.546 & 26.364 & 2.197 \\
\hline 6.591 & 19.773 & 8.788 & 147.199 \\
\hline 8.788 & 4.394 & 37.349 & 13.182 \\
\hline 2.197 & 74.698 & 72.501 & 4.394 \\
\hline 4.394 & 4.394 & 24.167 & 2.197 \\
\hline 2.197 & 19.773 & 2.197 & 68.107 \\
\hline 6.591 & 2.197 & 13.182 & 26.364 \\
\hline 15.379 & 32.955 & 21.97 & 8.788 \\
\hline 2.197 & 2.197 & 54.925 & 8.788 \\
\hline 37.349 & 39.546 & 6.591 & 2.197 \\
\hline 2.197 & 43.94 & 2.197 & 4.394 \\
\hline 2.197 & 2.197 & 8.788 & 35.152 \\
\hline 6.591 & 57.122 & 2.197 & 2.197 \\
\hline 17.576 & 4.394 & 166.972 & 2.197 \\
\hline 57.122 & 21.97 & 24.167 & 2.197 \\
\hline 2.197 & 2.197 & 4.394 & 10.985 \\
\hline 10.985 & 54.925 & 2.197 & 54.925 \\
\hline 10.985 & 8.788 & 39.546 & 2.197 \\
\hline 15.379 & 10.985 & 2.197 & 48.334 \\
\hline 2.197 & 2.197 & 4.394 & 35.152 \\
\hline 28.561 & 2.197 & 10.985 & 79.092 \\
\hline 26.364 & 28.561 & 2.197 & 276.822 \\
\hline 13.182 & 2.197 & 2.197 & 6.591 \\
\hline 21.97 & 32.955 & 2.197 & 2.197 \\
\hline 8.788 & 17.576 & 4.394 & 103.259 \\
\hline 2.197 & 19.773 & 6.591 & 32.955 \\
\hline 2.197 & 10.985 & 65.91 & 6.591 \\
\hline 279.019 & 1061.15 & 19.773 & 54.925 \\
\hline 35.152 & 87.88 & 2.197 & 17.576 \\
\hline 558.038 & 2.197 & 109.85 & 2.197 \\
\hline 4.394 & 6.591 & 2.197 & 19.773 \\
\hline 21.97 & 98.865 & 2.197 & 35.152 \\
\hline 17.576 & 2.197 & 4.394 & 8.788 \\
\hline 6.591 & 4.394 & 8.788 & 8.788 \\
\hline 142.805 & 19.773 & 2.197 & 28.561 \\
\hline 10.985 & 2.197 & 2.197 & 2.197 \\
\hline 4.394 & 6.591 & 2.197 & 4.394 \\
\hline
\end{tabular}




\begin{tabular}{|c|c|c|c|}
\hline 6.591 & 4.394 & 6.591 & 2.197 \\
\hline 8.788 & 4.394 & 13.182 & 4.394 \\
\hline 4.394 & 4.394 & 2.197 & 13.182 \\
\hline 21.97 & 2.197 & 17.576 & 8.788 \\
\hline 6.591 & 35.152 & 2.197 & 184.548 \\
\hline 2.197 & 2.197 & 2.197 & 467.961 \\
\hline 145.002 & 21.97 & 4.394 & 6.591 \\
\hline 2.197 & 2.197 & 2.197 & 634.933 \\
\hline 2.197 & 103.259 & 2.197 & 2.197 \\
\hline 2.197 & 300.989 & 4.394 & 4.394 \\
\hline 6.591 & 54.925 & 2.197 & 19.773 \\
\hline 70.304 & 2.197 & 4.394 & 4.394 \\
\hline 32.955 & 61.516 & 4.394 & 17.576 \\
\hline 4.394 & 2.197 & 2.197 & 252.655 \\
\hline 6.591 & 10.985 & 39.546 & 125.229 \\
\hline 6.591 & 4.394 & 2.197 & 15.379 \\
\hline 2.197 & 92.274 & 4.394 & 8.788 \\
\hline 24.167 & 511.901 & 2.197 & 17.576 \\
\hline 15.379 & 2.197 & 4.394 & 10.985 \\
\hline 2.197 & 2.197 & 2.197 & 6.591 \\
\hline 2.197 & 377.884 & 2.197 & 4.394 \\
\hline 2.197 & 2.197 & 32.955 & 290.004 \\
\hline 35.152 & 24.167 & 2.197 & 4.394 \\
\hline 28.561 & 50.531 & 70.304 & 13.182 \\
\hline 4.394 & 21.97 & 13.182 & 4.394 \\
\hline 94.471 & 43.94 & 2.197 & 208.715 \\
\hline 2.197 & 81.289 & 2.197 & 6.591 \\
\hline 8.788 & 4.394 & 2.197 & 2.197 \\
\hline 2041.01 & 10.985 & 4.394 & 2.197 \\
\hline 2.197 & 30.758 & 2.197 & 2.197 \\
\hline 2.197 & 21.97 & 4.394 & 2.197 \\
\hline 2.197 & 15.379 & 13.182 & 17.576 \\
\hline 2.197 & 6.591 & 4.394 & 19.773 \\
\hline 2.197 & 8.788 & 4.394 & 13.182 \\
\hline 8.788 & 19.773 & 4.394 & 2.197 \\
\hline 17.576 & 21.97 & 6.591 & 50.531 \\
\hline 72.501 & 6.591 & 4.394 & 2.197 \\
\hline 4.394 & 8.788 & 2.197 & 823.875 \\
\hline 2.197 & 6.591 & 2.197 & 2.197 \\
\hline 4.394 & 21.97 & 48.334 & 4.394 \\
\hline 2.197 & 2.197 & 2.197 & 26.364 \\
\hline 6.591 & 17.576 & 15.379 & 2.197 \\
\hline 2.197 & 13.182 & 19.773 & 65.91 \\
\hline 2.197 & 4.394 & 968.877 & 8.788 \\
\hline 26.364 & 59.319 & 2.197 & 2.197 \\
\hline 4.394 & 24.167 & 140.608 & 2.197 \\
\hline 120.835 & 2.197 & 6.591 & 15.379 \\
\hline 10.985 & 26.364 & 26.364 & 43.94 \\
\hline 61.516 & 24.167 & 6.591 & 10.985 \\
\hline 560.235 & 125.229 & 46.137 & 11215.7 \\
\hline
\end{tabular}




\begin{tabular}{|c|c|c|c|}
\hline 19.773 & 6.591 & 4.394 & 116.441 \\
\hline 2.197 & 2.197 & 30.758 & 61.516 \\
\hline 72.501 & 4.394 & 2.197 & 2.197 \\
\hline 4.394 & 4.394 & 6.591 & 2.197 \\
\hline 6.591 & 4.394 & 59.319 & 4.394 \\
\hline 4.394 & 2.197 & 2.197 & 39.546 \\
\hline 59.319 & 406.445 & 2.197 & 6.591 \\
\hline 2.197 & 2.197 & 4.394 & 2.197 \\
\hline 4.394 & 4.394 & 125.229 & 4.394 \\
\hline 19.773 & 2.197 & 19.773 & 2.197 \\
\hline 2.197 & 2.197 & 2.197 & 2.197 \\
\hline 4.394 & 19.773 & 37.349 & 6.591 \\
\hline 81.289 & 2.197 & 8.788 & 2.197 \\
\hline 4.394 & 2.197 & 2.197 & 79.092 \\
\hline 28.561 & 2.197 & 2.197 & 4.394 \\
\hline 24.167 & 8.788 & 2.197 & 4.394 \\
\hline 6.591 & 2.197 & 2.197 & 17.576 \\
\hline 30.758 & 2.197 & 4.394 & 21.97 \\
\hline 2.197 & 10.985 & 250.458 & 10.985 \\
\hline 74.698 & 6.591 & 10.985 & 37.349 \\
\hline 98.865 & 270.231 & 2.197 & 37.349 \\
\hline 2.197 & 6.591 & 4.394 & 2104.73 \\
\hline 2.197 & 21.97 & 10.985 & 2.197 \\
\hline 13.182 & 32.955 & 2.197 & 8.788 \\
\hline 257.049 & 15.379 & 4.394 & 6.591 \\
\hline 41.743 & 2.197 & 2.197 & 2895.65 \\
\hline 8.788 & 28.561 & 19.773 & 252.655 \\
\hline 2.197 & 8.788 & 2.197 & 10.985 \\
\hline 4.394 & 94.471 & 4.394 & 94.471 \\
\hline 2027.83 & 41.743 & 4.394 & 112.047 \\
\hline 4.394 & 2.197 & 2.197 & 107.653 \\
\hline 615.16 & 109.85 & 2.197 & 166.972 \\
\hline 2.197 & 4.394 & 4.394 & 48.334 \\
\hline 4.394 & 10.985 & 2.197 & 8.788 \\
\hline 10.985 & 41.743 & 2.197 & 50.531 \\
\hline 2.197 & 2.197 & 2.197 & 61.516 \\
\hline 2.197 & 21.97 & 2.197 & 28.561 \\
\hline 158.184 & 2.197 & 2.197 & 2.197 \\
\hline 2.197 & 37.349 & 4.394 & 2.197 \\
\hline 35.152 & 13.182 & 4.394 & 72.501 \\
\hline 2.197 & 50.531 & 2.197 & 2.197 \\
\hline 4.394 & 103.259 & 8.788 & 6.591 \\
\hline 2.197 & 37.349 & 2.197 & 6.591 \\
\hline 391.066 & 536.068 & 2.197 & 2.197 \\
\hline 35.152 & 21.97 & 4.394 & 2.197 \\
\hline 30.758 & 2.197 & 2.197 & 4.394 \\
\hline 26.364 & 2.197 & 2.197 & 46.137 \\
\hline 4286.35 & 2.197 & 32.955 & 6.591 \\
\hline 2.197 & 4.394 & 2.197 & 2.197 \\
\hline 2.197 & 39.546 & 2.197 & 74.698 \\
\hline
\end{tabular}




\begin{tabular}{|c|c|c|c|}
\hline 46.137 & 4.394 & 4.394 & 2.197 \\
\hline 68.107 & 2.197 & 32.955 & 2.197 \\
\hline 52.728 & 8.788 & 48.334 & 4.394 \\
\hline 76.895 & 24.167 & 19.773 & 2.197 \\
\hline 8.788 & 10.985 & 17.576 & 6.591 \\
\hline 24.167 & 6.591 & 6.591 & 28.561 \\
\hline 2.197 & 8.788 & 4.394 & 2.197 \\
\hline 50.531 & 4.394 & 26.364 & 2.197 \\
\hline 35.152 & 57.122 & 4.394 & 6.591 \\
\hline 6.591 & 21.97 & 4.394 & 4.394 \\
\hline 2.197 & 13.182 & 2.197 & 112.047 \\
\hline 2.197 & 59.319 & 2.197 & 28.561 \\
\hline 2.197 & 10.985 & 2.197 & 19.773 \\
\hline 2.197 & 35.152 & 6.591 & 6.591 \\
\hline 24.167 & 26.364 & 2.197 & 48.334 \\
\hline 2.197 & 19.773 & 2.197 & 10.985 \\
\hline 4.394 & 39.546 & 2.197 & 4.394 \\
\hline 2.197 & 2.197 & 8.788 & 204.321 \\
\hline 171.366 & 2.197 & 487.734 & 2.197 \\
\hline 6.591 & 70.304 & 10.985 & 19.773 \\
\hline 37.349 & 2.197 & 6.591 & 4.394 \\
\hline 41.743 & 239.473 & 4.394 & 13.182 \\
\hline 177.957 & 204.321 & 13.182 & 52.728 \\
\hline 112.047 & 2.197 & 2.197 & 2.197 \\
\hline 2.197 & 487.734 & 39.546 & 8.788 \\
\hline 10.985 & 6.591 & 21.97 & 2.197 \\
\hline 74.698 & 186.745 & 19.773 & 2.197 \\
\hline 2.197 & 4.394 & 4.394 & 114.244 \\
\hline 4.394 & 2.197 & 24.167 & 15.379 \\
\hline 2.197 & 4.394 & 2.197 & 28.561 \\
\hline 204.321 & 13.182 & 4.394 & 4.394 \\
\hline 63.713 & 10.985 & 2.197 & 703.04 \\
\hline 57.122 & 15.379 & 17.576 & 15.379 \\
\hline 2.197 & 92.274 & 2.197 & 4.394 \\
\hline 2.197 & 8.788 & 52.728 & 24.167 \\
\hline 2.197 & 4.394 & 2.197 & 6.591 \\
\hline 8.788 & 8.788 & 193.336 & 10.985 \\
\hline 2.197 & 4.394 & 2.197 & 127.426 \\
\hline 4.394 & 2.197 & 70.304 & 2.197 \\
\hline 35.152 & 15.379 & 123.032 & 366.899 \\
\hline 2.197 & 59.319 & 61.516 & 3372.39 \\
\hline 6.591 & 13.182 & 81.289 & 17.576 \\
\hline 10.985 & 6.591 & 8.788 & 10.985 \\
\hline 37.349 & 76.895 & 6.591 & 4.394 \\
\hline 4.394 & 2.197 & 2.197 & 26.364 \\
\hline 2.197 & 30.758 & 10.985 & 15.379 \\
\hline 48.334 & 2.197 & 2.197 & 65.91 \\
\hline 26.364 & 15.379 & 2.197 & 10.985 \\
\hline 3161.48 & 13.182 & 2.197 & 35.152 \\
\hline 112.047 & 4.394 & 10.985 & 2.197 \\
\hline
\end{tabular}




\begin{tabular}{|c|c|c|c|}
\hline 241.67 & 2.197 & 516.295 & 920.543 \\
\hline 2.197 & 2.197 & 4.394 & 2.197 \\
\hline 2.197 & 17.576 & 96.668 & 65.91 \\
\hline 2.197 & 8.788 & 2.197 & 26.364 \\
\hline 21.97 & 15.379 & 2.197 & 30.758 \\
\hline 2.197 & 2.197 & 32.955 & 2.197 \\
\hline 17.576 & 2.197 & 19.773 & 2.197 \\
\hline 4.394 & 210.912 & 4.394 & 13.182 \\
\hline 4.394 & 2.197 & 2.197 & 171.366 \\
\hline 2.197 & 2.197 & 8.788 & 39.546 \\
\hline 318.565 & 13.182 & 2.197 & 2.197 \\
\hline 2.197 & 17.576 & 2.197 & 13.182 \\
\hline 2.197 & 1634.57 & 37.349 & 1032.59 \\
\hline 24.167 & 2.197 & 4.394 & 8.788 \\
\hline 153.79 & 68.107 & 2.197 & 101.062 \\
\hline 430.612 & 4.394 & 2.197 & 37.349 \\
\hline 21.97 & 6.591 & 2.197 & 35.152 \\
\hline 2.197 & 2.197 & 2.197 & 6.591 \\
\hline 2.197 & 10.985 & 2.197 & 19.773 \\
\hline 8.788 & 4.394 & 2.197 & 4.394 \\
\hline 2.197 & 17.576 & 2.197 & 76.895 \\
\hline 2.197 & 2.197 & 41.743 & 8.788 \\
\hline 2.197 & 4.394 & 140.608 & 558.038 \\
\hline 2.197 & 15.379 & 39.546 & 2.197 \\
\hline 184.548 & 98.865 & 241.67 & 8.788 \\
\hline 155.987 & 46.137 & 43.94 & 15.379 \\
\hline 2.197 & 2.197 & 2.197 & 10.985 \\
\hline 2.197 & 6.591 & 131.82 & 2.197 \\
\hline 2.197 & 2.197 & 812.89 & 6.591 \\
\hline 2.197 & 2.197 & 206.518 & 4.394 \\
\hline 2.197 & 32.955 & 1001.83 & 193.336 \\
\hline 13.182 & 8.788 & 13.182 & 2.197 \\
\hline 61.516 & 2.197 & 19.773 & 90.077 \\
\hline 4.394 & 2.197 & 2.197 & 2399.12 \\
\hline 17.576 & 46.137 & 2.197 & 2.197 \\
\hline 59.319 & 21.97 & 2.197 & 2.197 \\
\hline 2.197 & 248.261 & 8.788 & 8.788 \\
\hline 28.561 & 2.197 & 46.137 & 2.197 \\
\hline 37.349 & 17.576 & 2.197 & 283.413 \\
\hline 96.668 & 6.591 & 8.788 & 68.107 \\
\hline 92.274 & 2.197 & 2.197 & 10.985 \\
\hline 979.862 & 2.197 & 2.197 & 138.411 \\
\hline 4.394 & 2.197 & 2.197 & 4.394 \\
\hline 2.197 & 6.591 & 28.561 & 19.773 \\
\hline 2.197 & 2.197 & 15.379 & 24.167 \\
\hline 8.788 & 195.533 & 169.169 & 558.038 \\
\hline 2.197 & 4.394 & 41.743 & 2.197 \\
\hline 79.092 & 2.197 & 2.197 & 2.197 \\
\hline 4.394 & 21.97 & 46.137 & 54.925 \\
\hline 2.197 & 2.197 & 2.197 & 101.062 \\
\hline
\end{tabular}




\begin{tabular}{|c|c|c|c|}
\hline 2.197 & 2.197 & 2.197 & 4.394 \\
\hline 6.591 & 103.259 & 19.773 & 2.197 \\
\hline 2.197 & 2.197 & 76.895 & 19.773 \\
\hline 6290.01 & 68.107 & 2.197 & 24.167 \\
\hline 6.591 & 8.788 & 59.319 & 2.197 \\
\hline 15.379 & 15.379 & 116.441 & 8385.95 \\
\hline 2.197 & 4.394 & 68.107 & 32.955 \\
\hline 2.197 & 13.182 & 7515.94 & 2.197 \\
\hline 2.197 & 92.274 & 26.364 & 4.394 \\
\hline 2.197 & 2.197 & 2.197 & 15.379 \\
\hline 2.197 & 17.576 & 2.197 & 2.197 \\
\hline 4.394 & 61.516 & 2.197 & 149.396 \\
\hline 145.002 & 50.531 & 4.394 & 17.576 \\
\hline 10.985 & 59.319 & 4.394 & 17.576 \\
\hline 10.985 & 8.788 & 2.197 & 15.379 \\
\hline 2.197 & 2.197 & 6.591 & 10.985 \\
\hline 4.394 & 2.197 & 4.394 & 127.426 \\
\hline 2.197 & 2.197 & 2.197 & 17.576 \\
\hline 17.576 & 6.591 & 2.197 & 2.197 \\
\hline 8.788 & 28.561 & 173.563 & 4.394 \\
\hline 10.985 & 2.197 & 15.379 & 2.197 \\
\hline 54.925 & 68.107 & 13.182 & 2.197 \\
\hline 24.167 & 8.788 & 4.394 & 8.788 \\
\hline 57.122 & 4.394 & 2.197 & 2.197 \\
\hline 37.349 & 43.94 & 2.197 & 2.197 \\
\hline 6.591 & 2.197 & 6.591 & 221.897 \\
\hline 74.698 & 2.197 & 4.394 & 2.197 \\
\hline 37.349 & 2.197 & 2.197 & 4.394 \\
\hline 232.882 & 32.955 & 2.197 & 2.197 \\
\hline 8.788 & 2.197 & 72.501 & 21.97 \\
\hline 15.379 & 85.683 & 2.197 & 10.985 \\
\hline 30.758 & 10.985 & 2.197 & 6.591 \\
\hline 2.197 & 4.394 & 2.197 & 6.591 \\
\hline 2.197 & 2.197 & 2.197 & 30.758 \\
\hline 298.792 & 59.319 & 2.197 & 8.788 \\
\hline 6.591 & 2.197 & 2.197 & 6.591 \\
\hline 2.197 & 17.576 & 2.197 & 4.394 \\
\hline 4.394 & 2.197 & 2.197 & 2.197 \\
\hline 32.955 & 6.591 & 2.197 & 30.758 \\
\hline 4.394 & 8.788 & 24.167 & 81.289 \\
\hline 4.394 & 4.394 & 2.197 & 19.773 \\
\hline 4.394 & 109.85 & 2.197 & 2.197 \\
\hline 2.197 & 2.197 & 2.197 & 15.379 \\
\hline 2.197 & 30.758 & 76.895 & 2.197 \\
\hline 2.197 & 24.167 & 43.94 & 8.788 \\
\hline 39.546 & 4.394 & 4.394 & 46.137 \\
\hline 8.788 & 4.394 & 6.591 & 6.591 \\
\hline 8.788 & 6.591 & 331.747 & 4.394 \\
\hline 8.788 & 6.591 & 83.486 & 4.394 \\
\hline 68.107 & 8.788 & 2.197 & 2.197 \\
\hline
\end{tabular}




\begin{tabular}{|c|c|c|c|}
\hline 8.788 & 6.591 & 30.758 & 19.773 \\
\hline 173.563 & 160.381 & 24.167 & 4.394 \\
\hline 2.197 & 2.197 & 2.197 & 6.591 \\
\hline 2.197 & 4.394 & 2.197 & 8.788 \\
\hline 26.364 & 6.591 & 2.197 & 15.379 \\
\hline 4.394 & 2.197 & 2.197 & 10.985 \\
\hline 41.743 & 2.197 & 1212.74 & 2.197 \\
\hline 26.364 & 6.591 & 2.197 & 2.197 \\
\hline 6.591 & 2.197 & 13.182 & 32.955 \\
\hline 2.197 & 8.788 & 2.197 & 6.591 \\
\hline 6.591 & 103.259 & 2.197 & 8.788 \\
\hline 4.394 & 2.197 & 2.197 & 4.394 \\
\hline 4.394 & 2.197 & 35.152 & 2.197 \\
\hline 4.394 & 2.197 & 6.591 & 32.955 \\
\hline 2.197 & 28.561 & 138.411 & 13.182 \\
\hline 4.394 & 202.124 & 2.197 & 2.197 \\
\hline 90.077 & 50.531 & 65.91 & 63.713 \\
\hline 193.336 & 2.197 & 2.197 & 481.143 \\
\hline 8.788 & 26.364 & 2.197 & 26.364 \\
\hline 2.197 & 2.197 & 136.214 & 8.788 \\
\hline 6.591 & 24.167 & 50.531 & 63.713 \\
\hline 4.394 & 2.197 & 2.197 & 2.197 \\
\hline 6.591 & 2.197 & 2.197 & 2.197 \\
\hline 13.182 & 26.364 & 30.758 & 182.351 \\
\hline 13.182 & 6.591 & 8.788 & 4.394 \\
\hline 10.985 & 72.501 & 4.394 & 28.561 \\
\hline 2.197 & 191.139 & 43.94 & 160.381 \\
\hline 2.197 & 50.531 & 98.865 & 17.576 \\
\hline 2.197 & 2.197 & 6.591 & 4.394 \\
\hline 8.788 & 46.137 & 2.197 & 2.197 \\
\hline 2.197 & 2.197 & 2.197 & 4.394 \\
\hline 2.197 & 164.775 & 2.197 & 2.197 \\
\hline 10.985 & 48.334 & 2.197 & 4.394 \\
\hline 13.182 & 2.197 & 17.576 & 2.197 \\
\hline 21.97 & 26.364 & 2.197 & 8.788 \\
\hline 28.561 & 123.032 & 2.197 & 6.591 \\
\hline 2.197 & 21.97 & 2.197 & 35.152 \\
\hline 28.561 & 4.394 & 2.197 & 63.713 \\
\hline 2.197 & 54.925 & 59.319 & 26.364 \\
\hline 6.591 & 10.985 & 2.197 & 8.788 \\
\hline 2.197 & 4.394 & 2.197 & 52.728 \\
\hline 2.197 & 290.004 & 2.197 & 103.259 \\
\hline 2.197 & 4.394 & 2.197 & 191.139 \\
\hline 19.773 & 4.394 & 2.197 & 206.518 \\
\hline 10.985 & 8.788 & 2.197 & 28.561 \\
\hline 43.94 & 17.576 & 10.985 & 6.591 \\
\hline 4.394 & 2.197 & 4.394 & 72.501 \\
\hline 15.379 & 2.197 & 2.197 & 2.197 \\
\hline 30.758 & 740.389 & 2.197 & 2.197 \\
\hline 2.197 & 6.591 & 2.197 & 2.197 \\
\hline
\end{tabular}




\begin{tabular}{|c|c|c|c|}
\hline 15.379 & 59.319 & 4.394 & 28.561 \\
\hline 4.394 & 4.394 & 2.197 & 232.882 \\
\hline 4.394 & 8.788 & 21.97 & 18562.5 \\
\hline 2.197 & 2.197 & 2.197 & 13.182 \\
\hline 4.394 & 101.062 & 13.182 & 85.683 \\
\hline 2.197 & 19.773 & 10.985 & 2.197 \\
\hline 35.152 & 41.743 & 4.394 & 6.591 \\
\hline 13.182 & 15.379 & 2.197 & 30.758 \\
\hline 41.743 & 4.394 & 2.197 & 19.773 \\
\hline 4.394 & 2.197 & 2.197 & 52.728 \\
\hline 8.788 & 37.349 & 2.197 & 24.167 \\
\hline 24.167 & 2.197 & 393.263 & 8.788 \\
\hline 2.197 & 4.394 & 15.379 & 116.441 \\
\hline 4.394 & 4.394 & 6.591 & 2.197 \\
\hline 2.197 & 2.197 & 114.244 & 2.197 \\
\hline 10.985 & 70.304 & 6.591 & 6.591 \\
\hline 10.985 & 2.197 & 68.107 & 2.197 \\
\hline 2.197 & 2.197 & 2.197 & 41.743 \\
\hline 4.394 & 2.197 & 2.197 & 4.394 \\
\hline 2.197 & 19.773 & 2.197 & 3776.64 \\
\hline 17.576 & 6.591 & 2.197 & 2137.68 \\
\hline 15.379 & 37.349 & 2.197 & 4.394 \\
\hline 4.394 & 52.728 & 2.197 & 6940.32 \\
\hline 2.197 & 98.865 & 2.197 & 2.197 \\
\hline 26.364 & 41.743 & 2.197 & 2.197 \\
\hline 4.394 & 17.576 & 184.548 & 32.955 \\
\hline 17.576 & 2.197 & 10.985 & 10.985 \\
\hline 17.576 & 13.182 & 63.713 & 162.578 \\
\hline 4.394 & 6.591 & 4.394 & 443.794 \\
\hline 300.989 & 94.471 & 19.773 & 2.197 \\
\hline 2.197 & 127.426 & 8.788 & 10.985 \\
\hline 2.197 & 4.394 & 10.985 & 432.809 \\
\hline 2.197 & 2.197 & 6.591 & 6.591 \\
\hline 2.197 & 2.197 & 59.319 & 32.955 \\
\hline 522.886 & 32.955 & 221.897 & 13.182 \\
\hline 15.379 & 10.985 & 35.152 & 208.715 \\
\hline 4.394 & 15.379 & 8.788 & 15.379 \\
\hline 10.985 & 4.394 & 2.197 & 2.197 \\
\hline 39.546 & 30.758 & 2.197 & 4.394 \\
\hline 103.259 & 2.197 & 2.197 & 2.197 \\
\hline 2.197 & 6.591 & 8.788 & 30.758 \\
\hline 35.152 & 79.092 & 2.197 & 35.152 \\
\hline 2.197 & 15.379 & 2.197 & 28.561 \\
\hline 2.197 & 2.197 & 2.197 & 4.394 \\
\hline 355.914 & 2.197 & 2.197 & 10.985 \\
\hline 48.334 & 28.561 & 2.197 & 26.364 \\
\hline 43.94 & 32.955 & 19.773 & 2.197 \\
\hline 74.698 & 32.955 & 252.655 & 2.197 \\
\hline 2.197 & 17.576 & 2.197 & 171.366 \\
\hline 2.197 & 65.91 & 2.197 & 2.197 \\
\hline
\end{tabular}




\begin{tabular}{|c|c|c|c|}
\hline 2.197 & 2.197 & 2.197 & 24.167 \\
\hline 182.351 & 2.197 & 4.394 & 26.364 \\
\hline 4.394 & 30.758 & 2750.64 & 2.197 \\
\hline 2.197 & 68.107 & 19.773 & 79.092 \\
\hline 2.197 & 68.107 & 8.788 & 2.197 \\
\hline 2.197 & 13.182 & 4.394 & 2.197 \\
\hline 2.197 & 2.197 & 10.985 & 2.19 \\
\hline 8.788 & 4.394 & 4.394 & 4.394 \\
\hline 2.197 & 4.394 & 4.394 & 76.895 \\
\hline 2.197 & 6.591 & 6.591 & 221.897 \\
\hline 2.197 & 2.197 & 279.019 & 30.758 \\
\hline 4.394 & 6.591 & 145.002 & 4.394 \\
\hline 2.197 & 2.197 & 274.625 & 114.244 \\
\hline 4.394 & 10.985 & 2.197 & 21.97 \\
\hline 10.985 & 28.561 & 15.379 & 4.394 \\
\hline 17.576 & 8.788 & 2.197 & 19.773 \\
\hline 4.394 & 54.925 & 10.985 & 26.364 \\
\hline 13.182 & 13.182 & 2.197 & 1373.12 \\
\hline 43.94 & 15.379 & 15.379 & 68.107 \\
\hline 2.197 & 2.197 & 15.379 & 2.197 \\
\hline 32.955 & 81.289 & 65.91 & 2.197 \\
\hline 4024.9 & 2.197 & 2.197 & 13.182 \\
\hline 2.197 & 15.379 & 10.985 & 26.364 \\
\hline 4.394 & 21.97 & 2.197 & 32.95 \\
\hline 13.182 & 863.421 & 4.394 & 6.591 \\
\hline 13.182 & 4.394 & 2.197 & 48.33 \\
\hline 37.349 & 61.516 & 2.197 & 8.788 \\
\hline 2.197 & 61.516 & 43.94 & 21.97 \\
\hline 35.152 & 6.591 & 4.394 & 2.197 \\
\hline 199.927 & 142.805 & 43.94 & 2.197 \\
\hline 2.197 & 68.107 & 8.788 & 2.197 \\
\hline 320.762 & 2.197 & 85.683 & 2.197 \\
\hline 2.197 & 85.683 & 39.546 & 2.197 \\
\hline 15.379 & 4.394 & 2.197 & 30.758 \\
\hline 2.197 & 2.197 & 153.79 & 366.899 \\
\hline 87.88 & 2.197 & 4.394 & 4.39 \\
\hline 2.197 & 4.394 & 4.394 & 4.394 \\
\hline 2.197 & 4.394 & 15.379 & 10.98 \\
\hline 43.94 & 6.591 & 2.197 & 171.366 \\
\hline 24.167 & 4.394 & 2.197 & 6.591 \\
\hline 8.788 & 8.788 & 2.197 & 2.197 \\
\hline 358.111 & 6.591 & 2.197 & 902.967 \\
\hline 24.167 & 28.561 & 103.259 & 177.957 \\
\hline 2.197 & 4.394 & 2.197 & 32.955 \\
\hline 2.197 & 8.788 & 6.591 & 32.955 \\
\hline 2.197 & 134.017 & 4.394 & 101.062 \\
\hline 281.216 & 6.591 & 6.591 & 72.501 \\
\hline 8.788 & 94.471 & 61.516 & 4.39 \\
\hline 17.576 & 2.197 & 2.197 & 46.137 \\
\hline 2.197 & 6.591 & 2.197 & 8.788 \\
\hline
\end{tabular}




\begin{tabular}{|c|c|c|c|}
\hline 26.364 & 6.591 & 70.304 & 6.591 \\
\hline 2.197 & 10.985 & 24.167 & 309.777 \\
\hline 4.394 & 24.167 & 8.788 & 8.788 \\
\hline 4.394 & 2.197 & 2.197 & 70.304 \\
\hline 4.394 & 17.576 & 2.197 & 10.985 \\
\hline 10.985 & 4.394 & 2.197 & 4.394 \\
\hline 2.197 & 10.985 & 2.197 & 4.394 \\
\hline 276.822 & 6.591 & 65.91 & 2.197 \\
\hline 2133.29 & 8.788 & 10.985 & 2.197 \\
\hline 2.197 & 32.955 & 59.319 & 4.394 \\
\hline 48.334 & 17.576 & 2.197 & 2.197 \\
\hline 35.152 & 8.788 & 103.259 & 2.197 \\
\hline 8.788 & 8.788 & 2.197 & 4.394 \\
\hline 232.882 & 2.197 & 19032.6 & 4.394 \\
\hline 2.197 & 6.591 & 8.788 & 101.062 \\
\hline 68.107 & 8.788 & 2.197 & 79.092 \\
\hline 10.985 & 2.197 & 2.197 & 175.76 \\
\hline 4.394 & 17.576 & 6.591 & 10.985 \\
\hline 37.349 & 118.638 & 8.788 & 2.197 \\
\hline 270.231 & 13.182 & 4.394 & 1298.43 \\
\hline 142.805 & 202.124 & 76.895 & 2.197 \\
\hline 2.197 & 37.349 & 2.197 & 153.79 \\
\hline 2.197 & 37.349 & 10.985 & 26.364 \\
\hline 8.788 & 30.758 & 2.197 & 4.394 \\
\hline 8.788 & 19.773 & 4.394 & 261.443 \\
\hline 2.197 & 57.122 & 13.182 & 19.773 \\
\hline 8.788 & 2.197 & 4.394 & 4.394 \\
\hline 2.197 & 15.379 & 4.394 & 6.591 \\
\hline 2.197 & 2.197 & 17.576 & 2.197 \\
\hline 4.394 & 15.379 & 2.197 & 37.349 \\
\hline 21.97 & 2.197 & 3853.54 & 114.244 \\
\hline 2.197 & 2.197 & 344.929 & 57.122 \\
\hline 24.167 & 171.366 & 2.197 & 2.197 \\
\hline 2.197 & 2.197 & 35.152 & 2.197 \\
\hline 41.743 & 50.531 & 2.197 & 21.97 \\
\hline 37.349 & 105.456 & 2.197 & 2.197 \\
\hline 5365.07 & 2.197 & 2.197 & 21.97 \\
\hline 2.197 & 39.546 & 19.773 & 2.197 \\
\hline 8.788 & 2.197 & 6.591 & 1008.42 \\
\hline 21.97 & 39.546 & 2.197 & 1682.9 \\
\hline 4.394 & 4.394 & 30.758 & 21.97 \\
\hline 52.728 & 2.197 & 142.805 & 2.197 \\
\hline 4.394 & 2.197 & 4.394 & 2.197 \\
\hline 2.197 & 2.197 & 15.379 & 6.591 \\
\hline 4.394 & 123.032 & 347.126 & 2.197 \\
\hline 366.899 & 10.985 & 2.197 & 41.743 \\
\hline 2.197 & 17.576 & 13.182 & 98.865 \\
\hline 2.197 & 109.85 & 2.197 & 2.197 \\
\hline 17.576 & 2.197 & 8.788 & 2.197 \\
\hline 4.394 & 2.197 & 6.591 & 63.713 \\
\hline
\end{tabular}




\begin{tabular}{|c|c|c|c|}
\hline 32.955 & 2.197 & 2.197 & 6.591 \\
\hline 4.394 & 2.197 & 6.591 & 90.077 \\
\hline 2.197 & 6.591 & 2.197 & 2.197 \\
\hline 2.197 & 202.124 & 2.197 & 8.788 \\
\hline 2.197 & 74.698 & 2.197 & 50.531 \\
\hline 10.985 & 274.625 & 2.197 & 21.97 \\
\hline 131.82 & 15.379 & 2.197 & 41.743 \\
\hline 19.773 & 2.197 & 4.394 & 103.259 \\
\hline 21.97 & 6.591 & 2.197 & 17.576 \\
\hline 70.304 & 10.985 & 10.985 & 39.546 \\
\hline 59.319 & 2.197 & 68.107 & 863.421 \\
\hline 21.97 & 8.788 & 15.379 & 19.773 \\
\hline 6.591 & 21.97 & 26.364 & 10.985 \\
\hline 136.214 & 4.394 & 2.197 & 4.394 \\
\hline 37.349 & 39.546 & 2.197 & 2.197 \\
\hline 127.426 & 30.758 & 26.364 & 2.197 \\
\hline 2.197 & 8.788 & 2.197 & 46.137 \\
\hline 15.379 & 4.394 & 10.985 & 21.97 \\
\hline 2.197 & 2.197 & 114.244 & 114.244 \\
\hline 164.775 & 2.197 & 50.531 & 8.788 \\
\hline 2.197 & 10.985 & 37.349 & 10.985 \\
\hline 37.349 & 90.077 & 134.017 & 4.394 \\
\hline 2.197 & 24.167 & 4.394 & 8.788 \\
\hline 35.152 & 2.197 & 8.788 & 8.788 \\
\hline 2.197 & 37.349 & 2.197 & 2.197 \\
\hline 2.197 & 2.197 & 7904.81 & 2.197 \\
\hline 2.197 & 4.394 & 4.394 & 6.591 \\
\hline 63.713 & 10.985 & 24.167 & 6.591 \\
\hline 2.197 & 13.182 & 2.197 & 153.79 \\
\hline 52.728 & 2.197 & 10.985 & 79.092 \\
\hline 2.197 & 6.591 & 28.561 & 43.94 \\
\hline 2.197 & 19.773 & 2.197 & 2.197 \\
\hline 4.394 & 2.197 & 2.197 & 61.516 \\
\hline 6.591 & 13.182 & 43.94 & 6.591 \\
\hline 164.775 & 4.394 & 28.561 & 6.591 \\
\hline 2.197 & 2.197 & 2.197 & 4.394 \\
\hline 2.197 & 2.197 & 450.385 & 2.197 \\
\hline 8.788 & 2.197 & 4.394 & 28.561 \\
\hline 2.197 & 2.197 & 2.197 & 26.364 \\
\hline 2.197 & 2.197 & 2.197 & 241.67 \\
\hline 2.197 & 6.591 & 2.197 & 27106.6 \\
\hline 8.788 & 54.925 & 46.137 & 8.788 \\
\hline 2.197 & 4.394 & 17.576 & 6.591 \\
\hline 13.182 & 2.197 & 13.182 & 28.561 \\
\hline 125.229 & 32.955 & 4.394 & 19.773 \\
\hline 2.197 & 15.379 & 6.591 & 19.773 \\
\hline 2.197 & 15.379 & 4.394 & 4.394 \\
\hline 140.608 & 24.167 & 2.197 & 112.047 \\
\hline 2172.83 & 10.985 & 2.197 & 57.122 \\
\hline 2.197 & 2.197 & 6.591 & 8.788 \\
\hline
\end{tabular}




\begin{tabular}{|c|c|c|c|}
\hline 37.349 & 32.955 & 13.182 & 6.591 \\
\hline 2.197 & 83.486 & 2.197 & 32.955 \\
\hline 250.458 & 134.017 & 13.182 & 4.394 \\
\hline 39.546 & 2.197 & 2.197 & 2.197 \\
\hline 57.122 & 314.171 & 4.394 & 2.197 \\
\hline 13.182 & 4.394 & 2.197 & 4.394 \\
\hline 2.197 & 2.197 & 2.197 & 4.394 \\
\hline 2.197 & 19.773 & 52.728 & 2.197 \\
\hline 2.197 & 37.349 & 2.197 & 2.197 \\
\hline 2.197 & 61.516 & 327.353 & 6.591 \\
\hline 32.955 & 6.591 & 2.197 & 2.197 \\
\hline 4.394 & 74.698 & 4.394 & 217.503 \\
\hline 6.591 & 24.167 & 2.197 & 32.955 \\
\hline 63.713 & 2.197 & 10.985 & 2.197 \\
\hline 26.364 & 48.334 & 2.197 & 52.728 \\
\hline 43.94 & 72.501 & 2.197 & 6.591 \\
\hline 30.758 & 107.653 & 2.197 & 52.728 \\
\hline 2.197 & 2.197 & 2.197 & 96.668 \\
\hline 2.197 & 8.788 & 2.197 & 37.349 \\
\hline 65.91 & 2.197 & 32.955 & 6.591 \\
\hline 3058.22 & 48.334 & 2.197 & 6.591 \\
\hline 21.97 & 344.929 & 17.576 & 15.379 \\
\hline 6.591 & 13.182 & 6.591 & 35.152 \\
\hline 8.788 & 6.591 & 6.591 & 54.925 \\
\hline 4.394 & 4.394 & 30.758 & 2.197 \\
\hline 2.197 & 2.197 & 2.197 & 2.197 \\
\hline 230.685 & 15.379 & 4.394 & 61.516 \\
\hline 4.394 & 28.561 & 2.197 & 4.394 \\
\hline 30.758 & 2.197 & 2.197 & 4.394 \\
\hline 4.394 & 4.394 & 10.985 & 2.197 \\
\hline 8.788 & 32.955 & 2.197 & 10.985 \\
\hline 52.728 & 2.197 & 2.197 & 2.197 \\
\hline 13.182 & 2.197 & 2.197 & 2.197 \\
\hline 6.591 & 2.197 & 173.563 & 28.561 \\
\hline 6.591 & 30.758 & 6.591 & 61.516 \\
\hline 2.197 & 276.822 & 2.197 & 8.788 \\
\hline 4.394 & 70.304 & 26.364 & 8.788 \\
\hline 6.591 & 2.197 & 2.197 & 8.788 \\
\hline 4.394 & 8.788 & 2.197 & 6.591 \\
\hline 15.379 & 2.197 & 4.394 & 6.591 \\
\hline 2.197 & 30.758 & 4.394 & 92.274 \\
\hline 2.197 & 4.394 & 2.197 & 24.167 \\
\hline 4.394 & 4.394 & 4.394 & 4.394 \\
\hline 81.289 & 70.304 & 43.94 & 193.336 \\
\hline 2.197 & 59.319 & 2.197 & 32.955 \\
\hline 2.197 & 28.561 & 2.197 & 32.955 \\
\hline 70.304 & 13.182 & 2.197 & 10.985 \\
\hline 37.349 & 59.319 & 2.197 & 41.743 \\
\hline 68.107 & 8.788 & 8.788 & 4.394 \\
\hline 98.865 & 4.394 & 2.197 & 112.047 \\
\hline
\end{tabular}




\begin{tabular}{|c|c|c|c|}
\hline 37.349 & 35.152 & 2.197 & 2.197 \\
\hline 28.561 & 6.591 & 30.758 & 2.197 \\
\hline 37.349 & 6.591 & 52.728 & 30.758 \\
\hline 94.471 & 8.788 & 2.197 & 10.985 \\
\hline 112.047 & 10.985 & 4.394 & 2.197 \\
\hline 2.197 & 8.788 & 2.197 & 186.745 \\
\hline 28.561 & 2.197 & 2.197 & 4.394 \\
\hline 2.197 & 4.394 & 2.197 & 2.197 \\
\hline 2.197 & 8.788 & 63.713 & 37.349 \\
\hline 17.576 & 10.985 & 4.394 & 6.591 \\
\hline 32.955 & 2.197 & 4.394 & 39.546 \\
\hline 30.758 & 2.197 & 2.197 & 8.788 \\
\hline 2.197 & 6.591 & 6.591 & 48.334 \\
\hline 4.394 & 30.758 & 6.591 & 17.576 \\
\hline 19.773 & 30.758 & 15.379 & 123.032 \\
\hline 6.591 & 4.394 & 72.501 & 21.97 \\
\hline 5123.4 & 4.394 & 81.289 & 15.379 \\
\hline 68.107 & 2.197 & 10.985 & 13.182 \\
\hline 32.955 & 105.456 & 10.985 & 32.955 \\
\hline 17.576 & 4.394 & 147.199 & 4.394 \\
\hline 19.773 & 2.197 & 4.394 & 41.743 \\
\hline 158.184 & 37.349 & 87.88 & 13.182 \\
\hline 2.197 & 8.788 & 17.576 & 30.758 \\
\hline 26.364 & 68.107 & 6.591 & 30.758 \\
\hline 2.197 & 17.576 & 2.197 & 96.668 \\
\hline 2.197 & 19.773 & 2.197 & 2.197 \\
\hline 2.197 & 2.197 & 15.379 & 19.773 \\
\hline 6.591 & 2.197 & 2.197 & 6.591 \\
\hline 4.394 & 2.197 & 169.169 & 2.197 \\
\hline 4.394 & 2.197 & 2.197 & 164.775 \\
\hline 2.197 & 81.289 & 2.197 & 6.591 \\
\hline 19.773 & 50.531 & 2.197 & 2.197 \\
\hline 2.197 & 6.591 & 26.364 & 2.197 \\
\hline 4.394 & 8.788 & 10.985 & 2.197 \\
\hline 2.197 & 6.591 & 2.197 & 13.182 \\
\hline 388.869 & 6.591 & 4.394 & 2.197 \\
\hline 2.197 & 2.197 & 17.576 & 17.576 \\
\hline 19.773 & 2.197 & 6.591 & 2.197 \\
\hline 72.501 & 39.546 & 8.788 & 2.197 \\
\hline 127.426 & 243.867 & 76.895 & 155.987 \\
\hline 2.197 & 15.379 & 145.002 & 21.97 \\
\hline 8.788 & 13.182 & 3207.62 & 4.394 \\
\hline 2.197 & 2.197 & 2.197 & 13.182 \\
\hline 32.955 & 2.197 & 6.591 & 10.985 \\
\hline 2.197 & 6.591 & 2.197 & 8.788 \\
\hline 2.197 & 68.107 & 43.94 & 39.546 \\
\hline 314.171 & 4.394 & 2.197 & 41.743 \\
\hline 4.394 & 43.94 & 2.197 & 4.394 \\
\hline 13.182 & 13.182 & 2.197 & 123.032 \\
\hline 28.561 & 10.985 & 2.197 & 39.546 \\
\hline
\end{tabular}




\begin{tabular}{|c|c|c|c|}
\hline 2.197 & 43.94 & 19.773 & 43.94 \\
\hline 3539.37 & 2.197 & 10.985 & 6.591 \\
\hline 2.197 & 10.985 & 2.197 & 79.092 \\
\hline 39.546 & 4.394 & 2.197 & 4.394 \\
\hline 2.197 & 19.773 & 2.197 & 39.546 \\
\hline 2.197 & 2.197 & 2.197 & 8.788 \\
\hline 74.698 & 4.394 & 63.713 & 2.197 \\
\hline 43.94 & 2.197 & 6.591 & 30.758 \\
\hline 2.197 & 37.349 & 2.197 & 8.788 \\
\hline 21.97 & 270.231 & 4.394 & 4.394 \\
\hline 2.197 & 147.199 & 4.394 & 2.197 \\
\hline 13.182 & 10.985 & 13.182 & 1289.64 \\
\hline 2.197 & 4.394 & 19.773 & 2.197 \\
\hline 19.773 & 2.197 & 2.197 & 2.197 \\
\hline 85.683 & 4.394 & 8.788 & 4.394 \\
\hline 2.197 & 4.394 & 4.394 & 6.591 \\
\hline 4.394 & 4.394 & 8.788 & 10.985 \\
\hline 6.591 & 30.758 & 4.394 & 21.97 \\
\hline 4.394 & 2.197 & 50.531 & 2.197 \\
\hline 2.197 & 10.985 & 2.197 & 19.773 \\
\hline 8.788 & 2.197 & 4.394 & 10.985 \\
\hline 2.197 & 2.197 & 2.197 & 10.985 \\
\hline 2.197 & 2.197 & 2.197 & 4.394 \\
\hline 160.381 & 2.197 & 2.197 & 4.394 \\
\hline 13.182 & 76.895 & 48.334 & 2.197 \\
\hline 15.379 & 70.304 & 85.683 & 112.047 \\
\hline 54.925 & 8.788 & 46.137 & 2.197 \\
\hline 13.182 & 4.394 & 26.364 & 50.531 \\
\hline 4.394 & 43.94 & 2.197 & 2.197 \\
\hline 2.197 & 30.758 & 2.197 & 10.985 \\
\hline 125.229 & 8.788 & 6.591 & 59.319 \\
\hline 13.182 & 4.394 & 2.197 & 4.394 \\
\hline 8.788 & 2.197 & 6.591 & 322.959 \\
\hline 6.591 & 13.182 & 13.182 & 2.197 \\
\hline 103.259 & 8.788 & 6.591 & 2.197 \\
\hline 2.197 & 105.456 & 24.167 & 2.197 \\
\hline 1731.24 & 120.835 & 4.394 & 2.197 \\
\hline 2.197 & 2.197 & 270.231 & 15.379 \\
\hline 2.197 & 6.591 & 2.197 & 2.197 \\
\hline 2.197 & 2.197 & 6.591 & 4.394 \\
\hline 8.788 & 28.561 & 2.197 & 17.576 \\
\hline 2.197 & 4.394 & 2.197 & 8.788 \\
\hline 129.623 & 2.197 & 17.576 & 43.94 \\
\hline 2.197 & 28.561 & 10.985 & 1507.14 \\
\hline 30.758 & 4.394 & 15.379 & 2.197 \\
\hline 35.152 & 6.591 & 35.152 & 147.199 \\
\hline 2.197 & 13.182 & 2.197 & 10.985 \\
\hline 4.394 & 37.349 & 2.197 & 43.94 \\
\hline 2.197 & 41.743 & 2.197 & 8.788 \\
\hline 2.197 & 43.94 & 2.197 & 4.394 \\
\hline
\end{tabular}




\begin{tabular}{|c|c|c|c|}
\hline 15.379 & 2.197 & 4.394 & 6.591 \\
\hline 37.349 & 538.265 & 2.197 & 2.197 \\
\hline 2.197 & 2.197 & 48.334 & 2.197 \\
\hline 2.197 & 32.955 & 21.97 & 4861.96 \\
\hline 2.197 & 15.379 & 2.197 & 109.85 \\
\hline 30.758 & 13.182 & 17.576 & 2.197 \\
\hline 2.197 & 17.576 & 2.197 & 2.197 \\
\hline 2.197 & 6.591 & 2.197 & 2.197 \\
\hline 13.182 & 4.394 & 2.197 & 30.758 \\
\hline 52.728 & 2.197 & 6.591 & 1559.87 \\
\hline 4.394 & 4.394 & 184.548 & 17.576 \\
\hline 13.182 & 8.788 & 2.197 & 6.591 \\
\hline 114.244 & 6.591 & 2.197 & 4.394 \\
\hline 24.167 & 26.364 & 19.773 & 4.394 \\
\hline 2.197 & 6.591 & 2.197 & 76.895 \\
\hline 2.197 & 4.394 & 2.197 & 26.364 \\
\hline 129.623 & 2.197 & 2.197 & 39.546 \\
\hline 24.167 & 2.197 & 37.349 & 2.197 \\
\hline 2.197 & 2.197 & 4.394 & 2.197 \\
\hline 193.336 & 6.591 & 4.394 & 4.394 \\
\hline 6.591 & 52.728 & 8.788 & 59.319 \\
\hline 2.197 & 8.788 & 2.197 & 2.197 \\
\hline 4.394 & 41.743 & 2.197 & 8.788 \\
\hline 4.394 & 2.197 & 2.197 & 2.197 \\
\hline 11778.1 & 2.197 & 2.197 & 162.578 \\
\hline 41.743 & 4.394 & 2.197 & 2.197 \\
\hline 4.394 & 24.167 & 2.197 & 41.743 \\
\hline 4.394 & 2.197 & 1236.91 & 6.591 \\
\hline 15.379 & 21.97 & 2.197 & 35.152 \\
\hline 1487.37 & 2.197 & 2.197 & 2.197 \\
\hline 2.197 & 79.092 & 2.197 & 46.137 \\
\hline 4.394 & 79.092 & 2.197 & 19.773 \\
\hline 149.396 & 94.471 & 15.379 & 4.394 \\
\hline 149.396 & 17.576 & 6.591 & 4.394 \\
\hline 2.197 & 37.349 & 4.394 & 140.608 \\
\hline 68.107 & 10.985 & 2.197 & 8.788 \\
\hline 17.576 & 2.197 & 2.197 & 2.197 \\
\hline 2.197 & 4.394 & 19.773 & 2.197 \\
\hline 4.394 & 2.197 & 2.197 & 15.379 \\
\hline 4.394 & 4.394 & 6.591 & 26.364 \\
\hline 120.835 & 13.182 & 46.137 & 32.955 \\
\hline 6.591 & 43.94 & 72.501 & 2.197 \\
\hline 10.985 & 406.445 & 8.788 & 2.197 \\
\hline 32.955 & 2.197 & 4.394 & 4.394 \\
\hline 10.985 & 13.182 & 37.349 & 2.197 \\
\hline 4.394 & 2.197 & 43.94 & 397.657 \\
\hline 8.788 & 4.394 & 13.182 & 2.197 \\
\hline 1195.17 & 4.394 & 17.576 & 10.985 \\
\hline 2.197 & 4.394 & 2.197 & 2.197 \\
\hline 4.394 & 35.152 & 6.591 & 2.197 \\
\hline
\end{tabular}




\begin{tabular}{|c|c|c|c|}
\hline 2.197 & 10.985 & 2.197 & 4.394 \\
\hline 2.197 & 4.394 & 17.576 & 63.713 \\
\hline 1788.36 & 1188.58 & 2.197 & 2.197 \\
\hline 2.197 & 26.364 & 2.197 & 560.235 \\
\hline 4.394 & 70.304 & 85.683 & 2.197 \\
\hline 2.197 & 2.197 & 2.197 & 4.394 \\
\hline 50.531 & 4.394 & 26.364 & 2.197 \\
\hline 13.182 & 28.561 & 39.546 & 8.788 \\
\hline 2.197 & 4.394 & 4.394 & 8.788 \\
\hline 37.349 & 208.715 & 8.788 & 2.197 \\
\hline 15.379 & 2.197 & 2.197 & 4.394 \\
\hline 529.477 & 8.788 & 2.197 & 24.167 \\
\hline 136.214 & 10.985 & 8.788 & 32.955 \\
\hline 50.531 & 48.334 & 2.197 & 24.167 \\
\hline 85.683 & 21.97 & 2.197 & 50.531 \\
\hline 2.197 & 6.591 & 10.985 & 50.531 \\
\hline 2.197 & 2.197 & 43.94 & 8.788 \\
\hline 2.197 & 103.259 & 24.167 & 92.274 \\
\hline 8.788 & 17.576 & 8.788 & 2.197 \\
\hline 2.197 & 2.197 & 17.576 & 8.788 \\
\hline 2.197 & 13.182 & 21.97 & 24.167 \\
\hline 19.773 & 30.758 & 2.197 & 8.788 \\
\hline 4.394 & 6.591 & 4.394 & 2.197 \\
\hline 630.539 & 2.197 & 4.394 & 48.334 \\
\hline 2.197 & 8.788 & 2.197 & 2.197 \\
\hline 151.593 & 30.758 & 2.197 & 140.608 \\
\hline 39.546 & 2.197 & 2.197 & 6.591 \\
\hline 10.985 & 2.197 & 2.197 & 54.925 \\
\hline 6.591 & 287.807 & 2.197 & 6.591 \\
\hline 2.197 & 171.366 & 4.394 & 2.197 \\
\hline 2.197 & 50.531 & 2.197 & 17.576 \\
\hline 4.394 & 2.197 & 4.394 & 10.985 \\
\hline 4.394 & 37.349 & 4.394 & 2.197 \\
\hline 2.197 & 2.197 & 208.715 & 2.197 \\
\hline 4.394 & 35.152 & 2.197 & 2.197 \\
\hline 6.591 & 333.944 & 2.197 & 2.197 \\
\hline 8.788 & 120.835 & 4.394 & 149.396 \\
\hline 4.394 & 2.197 & 2.197 & 2.197 \\
\hline 4.394 & 10.985 & 2.197 & 2.197 \\
\hline 8.788 & 226.291 & 17.576 & 8.788 \\
\hline 2.197 & 4.394 & 8.788 & 19.773 \\
\hline 6.591 & 4.394 & 2.197 & 2.197 \\
\hline 13.182 & 848.042 & 2.197 & 2.197 \\
\hline 2.197 & 4.394 & 2.197 & 37.349 \\
\hline 2.197 & 87.88 & 4.394 & 13.182 \\
\hline 1036.98 & 2.197 & 2.197 & 30.758 \\
\hline 35.152 & 10.985 & 6.591 & 10.985 \\
\hline 21.97 & 261.443 & 2.197 & 79.092 \\
\hline 6.591 & 2.197 & 10.985 & 59.319 \\
\hline 28.561 & 13.182 & 4.394 & 19.773 \\
\hline
\end{tabular}




\begin{tabular}{|c|c|c|c|}
\hline 2.197 & 4.394 & 4.394 & 4.394 \\
\hline 2.197 & 68.107 & 2.197 & 6.591 \\
\hline 2.197 & 15.379 & 26.364 & 15.379 \\
\hline 208.715 & 4.394 & 2.197 & 2.197 \\
\hline 4.394 & 8.788 & 2.197 & 4.394 \\
\hline 2.197 & 2.197 & 2.197 & 386.672 \\
\hline 6.591 & 21.97 & 8.788 & 2.197 \\
\hline 98.865 & 83.486 & 21.97 & 2.197 \\
\hline 61.516 & 43.94 & 35.152 & 4.394 \\
\hline 2.197 & 35.152 & 2.197 & 4.394 \\
\hline 10.985 & 147.199 & 8.788 & 2.197 \\
\hline 2.197 & 10.985 & 21.97 & 2.197 \\
\hline 26.364 & 13.182 & 92.274 & 2.197 \\
\hline 21.97 & 103.259 & 19.773 & 1149.03 \\
\hline 35.152 & 24.167 & 2.197 & 2.197 \\
\hline 13.182 & 2.197 & 2.197 & 173.563 \\
\hline 162.578 & 15.379 & 2.197 & 281.216 \\
\hline 2.197 & 202.124 & 4.394 & 2.197 \\
\hline 2.197 & 43.94 & 2.197 & 2.197 \\
\hline 2.197 & 4.394 & 237.276 & 2.197 \\
\hline 2.197 & 81.289 & 2.197 & 2.197 \\
\hline 6.591 & 2.197 & 13.182 & 6.591 \\
\hline 4.394 & 2.197 & 63.713 & 112.047 \\
\hline 4.394 & 6.591 & 2.197 & 8.788 \\
\hline 4.394 & 8.788 & 162.578 & 76.895 \\
\hline 4.394 & 13.182 & 61.516 & 4.394 \\
\hline 410.839 & 2.197 & 120.835 & 24.167 \\
\hline 2.197 & 68.107 & 105.456 & 2.197 \\
\hline 2.197 & 30.758 & 10.985 & 4.394 \\
\hline 8.788 & 2.197 & 39.546 & 4.394 \\
\hline 13.182 & 32.955 & 2.197 & 65.91 \\
\hline 8.788 & 4.394 & 8.788 & 4.394 \\
\hline 6.591 & 21.97 & 2.197 & 4.394 \\
\hline 188.942 & 8.788 & 2.197 & 215.306 \\
\hline 41.743 & 8.788 & 2.197 & 32.955 \\
\hline 35.152 & 2.197 & 2.197 & 24.167 \\
\hline 2.197 & 2.197 & 2.197 & 8.788 \\
\hline 15.379 & 17.576 & 65.91 & 2.197 \\
\hline 19.773 & 4.394 & 2.197 & 17.576 \\
\hline 2.197 & 17.576 & 2.197 & 2.197 \\
\hline 19.773 & 8.788 & 2.197 & 4.394 \\
\hline 15.379 & 2.197 & 2.197 & 50.531 \\
\hline 54.925 & 661.297 & 15.379 & 8.788 \\
\hline 2.197 & 134.017 & 2.197 & 8.788 \\
\hline 28.561 & 13.182 & 6.591 & 13.182 \\
\hline 6.591 & 26.364 & 4.394 & 2.197 \\
\hline 24.167 & 21.97 & 26.364 & 107.653 \\
\hline 2.197 & 2.197 & 2.197 & 72.501 \\
\hline 13.182 & 4.394 & 63.713 & 2.197 \\
\hline 15.379 & 2.197 & 2.197 & 17.576 \\
\hline
\end{tabular}




\begin{tabular}{|c|c|c|c|}
\hline 2.197 & 2.197 & 2.197 & 50.531 \\
\hline 4.394 & 2.197 & 6.591 & 4.394 \\
\hline 2.197 & 215.306 & 52.728 & 28.561 \\
\hline 24.167 & 10.985 & 13.182 & 4.394 \\
\hline 2.197 & 8.788 & 4.394 & 28.561 \\
\hline 74.698 & 48.334 & 2.197 & 65.91 \\
\hline 15.379 & 2.197 & 46.137 & 4.394 \\
\hline 771.147 & 2.197 & 63.713 & 4.394 \\
\hline 2.197 & 30.758 & 6.591 & 19.773 \\
\hline 2.197 & 61.516 & 4.394 & 8.788 \\
\hline 4.394 & 2.197 & 426.218 & 15.379 \\
\hline 2.197 & 30.758 & 2.197 & 8.788 \\
\hline 208.715 & 15.379 & 2.197 & 2.197 \\
\hline 2.197 & 4.394 & 30.758 & 6.591 \\
\hline 2.197 & 2.197 & 4.394 & 2.197 \\
\hline 39.546 & 6.591 & 2.197 & 2.197 \\
\hline 2.197 & 2.197 & 2.197 & 2.197 \\
\hline 48.334 & 8.788 & 2.197 & 32.955 \\
\hline 4.394 & 13.182 & 2.197 & 17.576 \\
\hline 15.379 & 2.197 & 8.788 & 19.773 \\
\hline 72.501 & 70.304 & 32.955 & 2.197 \\
\hline 81.289 & 32.955 & 10.985 & 217.503 \\
\hline 21.97 & 283.413 & 2.197 & 2.197 \\
\hline 6.591 & 17.576 & 94.471 & 63.713 \\
\hline 35.152 & 2.197 & 2.197 & 2.197 \\
\hline 48.334 & 2.197 & 109.85 & 19.773 \\
\hline 10.985 & 2.197 & 4.394 & 4.394 \\
\hline 2.197 & 2.197 & 2.197 & 4.394 \\
\hline 87.88 & 6.591 & 2.197 & 145.002 \\
\hline 28.561 & 10.985 & 2.197 & 8.788 \\
\hline 17.576 & 2.197 & 2.197 & 10.985 \\
\hline 41.743 & 10.985 & 2.197 & 2.197 \\
\hline 2.197 & 404.248 & 2.197 & 4.394 \\
\hline 19.773 & 226.291 & 10.985 & 366.899 \\
\hline 15.379 & 410.839 & 2.197 & 2.197 \\
\hline 114.244 & 237.276 & 2.197 & 21.97 \\
\hline 2.197 & 19.773 & 2.197 & 718.419 \\
\hline 2.197 & 2.197 & 8.788 & 4.394 \\
\hline 17.576 & 2.197 & 2.197 & 193.336 \\
\hline 2.197 & 4.394 & 2.197 & 4.394 \\
\hline 17.576 & 15.379 & 2.197 & 2.197 \\
\hline 2.197 & 2.197 & 2.197 & 8.788 \\
\hline 2.197 & 2.197 & 2.197 & 2.197 \\
\hline 2.197 & 6.591 & 2.197 & 79.092 \\
\hline 8.788 & 6.591 & 2.197 & 19.773 \\
\hline 107.653 & 98.865 & 4.394 & 243.867 \\
\hline 43.94 & 52.728 & 2.197 & 120.835 \\
\hline 4.394 & 19.773 & 2.197 & 4.394 \\
\hline 2.197 & 41.743 & 118.638 & 8.788 \\
\hline 52.728 & 2.197 & 6.591 & 120.835 \\
\hline
\end{tabular}




\begin{tabular}{|c|c|c|c|}
\hline 68.107 & 4.394 & 114.244 & 57.122 \\
\hline 52.728 & 208.715 & 41.743 & 94.471 \\
\hline 57.122 & 13.182 & 2.197 & 10.985 \\
\hline 19.773 & 487.734 & 2.197 & 2.197 \\
\hline 355.914 & 2.197 & 2.197 & 4.394 \\
\hline 24.167 & 2.197 & 2.197 & 6.591 \\
\hline 2.197 & 2.197 & 6.591 & 21.97 \\
\hline 8.788 & 6.591 & 2.197 & 232.882 \\
\hline 26.364 & 48.334 & 2.197 & 8.788 \\
\hline 13.182 & 2.197 & 1131.45 & 41.743 \\
\hline 2.197 & 340.535 & 35.152 & 21.97 \\
\hline 28.561 & 13.182 & 8.788 & 145.002 \\
\hline 2.197 & 4.394 & 72.501 & 2.197 \\
\hline 2.197 & 8.788 & 2.197 & 2.197 \\
\hline 8.788 & 13.182 & 30.758 & 377.884 \\
\hline 116.441 & 6.591 & 6.591 & 2.197 \\
\hline 2.197 & 21.97 & 10.985 & 2.197 \\
\hline 2.197 & 30.758 & 162.578 & 74.698 \\
\hline 8.788 & 6.591 & 19.773 & 17.576 \\
\hline 720.616 & 2.197 & 8.788 & 28.561 \\
\hline 2.197 & 2.197 & 4.394 & 19.773 \\
\hline 2.197 & 2.197 & 13.182 & 2.197 \\
\hline 21.97 & 15.379 & 37.349 & 4.394 \\
\hline 26.364 & 35.152 & 2.197 & 2.197 \\
\hline 2.197 & 6.591 & 6.591 & 2.197 \\
\hline 15.379 & 2.197 & 21.97 & 2.197 \\
\hline 10.985 & 336.141 & 6.591 & 26.364 \\
\hline 206.518 & 2.197 & 15.379 & 8.788 \\
\hline 32.955 & 2.197 & 54.925 & 6.591 \\
\hline 6.591 & 52.728 & 13.182 & 2.197 \\
\hline 32.955 & 13.182 & 43.94 & 2.197 \\
\hline 142.805 & 2.197 & 2.197 & 21.97 \\
\hline 35.152 & 32.955 & 43.94 & 6.591 \\
\hline 138.411 & 2.197 & 2.197 & 8.788 \\
\hline 4.394 & 26.364 & 6.591 & 8.788 \\
\hline 2.197 & 2.197 & 4.394 & 2.197 \\
\hline 2.197 & 6.591 & 13.182 & 114.244 \\
\hline 123.032 & 8.788 & 94.471 & 37.349 \\
\hline 6.591 & 6.591 & 8.788 & 2.197 \\
\hline 2.197 & 8.788 & 2.197 & 4.394 \\
\hline 2.197 & 4.394 & 8.788 & 2.197 \\
\hline 4.394 & 2.197 & 4.394 & 4.394 \\
\hline 4.394 & 6.591 & 4.394 & 2.197 \\
\hline 2.197 & 2645.19 & 4.394 & 2.197 \\
\hline 32.955 & 228.488 & 10.985 & 13.182 \\
\hline 2.197 & 4.394 & 35.152 & 6.591 \\
\hline 2.197 & 94.471 & 2.197 & 2.197 \\
\hline 30.758 & 2.197 & 2.197 & 4.394 \\
\hline 2.197 & 8.788 & 432.809 & 2451.85 \\
\hline 13.182 & 41.743 & 15.379 & 17.576 \\
\hline
\end{tabular}




\begin{tabular}{|c|c|c|c|}
\hline 2.197 & 68.107 & 19.773 & 43.94 \\
\hline 101.062 & 2.197 & 4.394 & 2.197 \\
\hline 4.394 & 2.197 & 2.197 & 8.788 \\
\hline 24.167 & 17.576 & 2.197 & 2.197 \\
\hline 155.987 & 2.197 & 15.379 & 1757.6 \\
\hline 2.197 & 2.197 & 30.758 & 2.197 \\
\hline 4.394 & 2.197 & 2.197 & 2.197 \\
\hline 32.955 & 13.182 & 522.886 & 2.197 \\
\hline 17.576 & 276.822 & 43.94 & 21.97 \\
\hline 2.197 & 65.91 & 353.717 & 85.683 \\
\hline 2.197 & 8.788 & 21.97 & 2.197 \\
\hline 10.985 & 202.124 & 142.805 & 35.152 \\
\hline 2.197 & 4.394 & 2.197 & 8.788 \\
\hline 2.197 & 4.394 & 197.73 & 15.379 \\
\hline 4.394 & 59.319 & 2.197 & 15.379 \\
\hline 30.758 & 52.728 & 4.394 & 46.137 \\
\hline 2.197 & 26.364 & 2.197 & 6.591 \\
\hline 2.197 & 10.985 & 2.197 & 6.591 \\
\hline 402.051 & 155.987 & 2.197 & 98.865 \\
\hline 4.394 & 13.182 & 2.197 & 8.788 \\
\hline 2.197 & 2.197 & 2.197 & 2.197 \\
\hline 15.379 & 2.197 & 2.197 & 2.197 \\
\hline 2.197 & 8.788 & 2.197 & 6.591 \\
\hline 13.182 & 50.531 & 2.197 & 37.349 \\
\hline 2.197 & 4.394 & 17.576 & 8.788 \\
\hline 2.197 & 2.197 & 2.197 & 6.591 \\
\hline 2.197 & 13.182 & 4.394 & 21.97 \\
\hline 4.394 & 105.456 & 2.197 & 2.197 \\
\hline 4.394 & 96.668 & 6.591 & 4.394 \\
\hline 19.773 & 4.394 & 15.379 & 54.925 \\
\hline 8.788 & 6.591 & 2.197 & 4.394 \\
\hline 4.394 & 6.591 & 2.197 & 582.205 \\
\hline 8.788 & 2.197 & 2.197 & 2.197 \\
\hline 138.411 & 19.773 & 2.197 & 2.197 \\
\hline 13.182 & 2.197 & 8.788 & 2.197 \\
\hline 10.985 & 349.323 & 2.197 & 2.197 \\
\hline 6.591 & 21.97 & 2.197 & 333.944 \\
\hline 59.319 & 13.182 & 4.394 & 2.197 \\
\hline 6.591 & 2.197 & 4.394 & 10.985 \\
\hline 2.197 & 2.197 & 48.334 & 17.576 \\
\hline 2.197 & 48.334 & 4.394 & 2.197 \\
\hline 21.97 & 17.576 & 2.197 & 10.985 \\
\hline 134.017 & 57.122 & 2.197 & 26.364 \\
\hline 2.197 & 2.197 & 2.197 & 2.197 \\
\hline 24.167 & 4.394 & 8.788 & 2.197 \\
\hline 13.182 & 2.197 & 2.197 & 6.591 \\
\hline 2.197 & 101.062 & 30.758 & 2.197 \\
\hline 2.197 & 2.197 & 17.576 & 2.197 \\
\hline 2.197 & 6.591 & 2.197 & 13.182 \\
\hline 2.197 & 2.197 & 21.97 & 57.122 \\
\hline
\end{tabular}




\begin{tabular}{|c|c|c|c|}
\hline 2.197 & 2.197 & 21.97 & 10.985 \\
\hline 4.394 & 134.017 & 134.017 & 41.743 \\
\hline 94.471 & 6.591 & 6.591 & 6.591 \\
\hline 2.197 & 4.394 & 4.394 & 10.985 \\
\hline 2.197 & 235.079 & 2.197 & 2.197 \\
\hline 13.182 & 2.197 & 2.197 & 2.197 \\
\hline 4.394 & 2.197 & 4.394 & 2.197 \\
\hline 812.89 & 59.319 & 2.197 & 39.546 \\
\hline 4.394 & 13.182 & 2.197 & 4.394 \\
\hline 10.985 & 8.788 & 17.576 & 4.394 \\
\hline 43.94 & 195.533 & 6.591 & 52.728 \\
\hline 32.955 & 6.591 & 28.561 & 2.197 \\
\hline 63.713 & 28.561 & 2.197 & 4.394 \\
\hline 4.394 & 52.728 & 13.182 & 2.197 \\
\hline 2.197 & 2.197 & 90.077 & 2.197 \\
\hline 2.197 & 26.364 & 6.591 & 13.182 \\
\hline 2.197 & 2.197 & 2.197 & 2.197 \\
\hline 2.197 & 4.394 & 4.394 & 8.788 \\
\hline 2.197 & 2.197 & 6.591 & 8.788 \\
\hline 26.364 & 70.304 & 8.788 & 2.197 \\
\hline 65.91 & 2.197 & 8.788 & 10.985 \\
\hline 6.591 & 2.197 & 13.182 & 28.561 \\
\hline 59.319 & 2.197 & 2.197 & 2.197 \\
\hline 15.379 & 54.925 & 17.576 & 8.788 \\
\hline 2.197 & 17.576 & 2.197 & 13.182 \\
\hline 59.319 & 6.591 & 2.197 & 39.546 \\
\hline 2.197 & 4.394 & 2.197 & 2.197 \\
\hline 2.197 & 518.492 & 2.197 & 2.197 \\
\hline 13.182 & 21.97 & 13.182 & 2.197 \\
\hline 6.591 & 4.394 & 2.197 & 6.591 \\
\hline 841.451 & 186.745 & 1272.06 & 10.985 \\
\hline 30.758 & 2.197 & 2.197 & 101.062 \\
\hline 48.334 & 2.197 & 2.197 & 6.591 \\
\hline 8.788 & 19.773 & 13.182 & 2.197 \\
\hline 4.394 & 54.925 & 8.788 & 2.197 \\
\hline 63.713 & 13.182 & 2.197 & 4.394 \\
\hline 10.985 & 6.591 & 2.197 & 81.289 \\
\hline 2.197 & 17.576 & 21.97 & 8.788 \\
\hline 4.394 & 173.563 & 2.197 & 2.197 \\
\hline 24.167 & 21.97 & 123.032 & 138.411 \\
\hline 149.396 & 21.97 & 2.197 & 4.394 \\
\hline 6.591 & 2.197 & 4.394 & 28.561 \\
\hline 8.788 & 10.985 & 6.591 & 2.197 \\
\hline 6.591 & 8.788 & 2.197 & 24.167 \\
\hline 2.197 & 4.394 & 17.576 & 558.038 \\
\hline 2.197 & 2.197 & 15.379 & 2.197 \\
\hline 727.207 & 37.349 & 2.197 & 188.942 \\
\hline 342.732 & 4.394 & 4.394 & 2.197 \\
\hline 83.486 & 2.197 & 2.197 & 2.197 \\
\hline 2.197 & 4.394 & 2.197 & 13.182 \\
\hline
\end{tabular}




\begin{tabular}{|c|c|c|c|}
\hline 35.152 & 2.197 & 2.197 & 2.197 \\
\hline 28.561 & 2.197 & 2.197 & 19.773 \\
\hline 2.197 & 2.197 & 2.197 & 2.197 \\
\hline 2.197 & 74.698 & 259.246 & 2.197 \\
\hline 8.788 & 30.758 & 2.197 & 2.197 \\
\hline 8.788 & 2.197 & 2.197 & 2.197 \\
\hline 155.987 & 2.197 & 26.364 & 6.591 \\
\hline 2.197 & 175.76 & 2.197 & 2.197 \\
\hline 2.197 & 48.334 & 8.788 & 2.197 \\
\hline 235.079 & 13.182 & 2.197 & 21.97 \\
\hline 54.925 & 37.349 & 2.197 & 120.835 \\
\hline 4.394 & 2.197 & 2.197 & 43.94 \\
\hline 4.394 & 2.197 & 1355.55 & 2.197 \\
\hline 2.197 & 59.319 & 2.197 & 4.394 \\
\hline 70.304 & 50.531 & 184.548 & 15.379 \\
\hline 2.197 & 21.97 & 140.608 & 37.349 \\
\hline 43.94 & 4.394 & 2.197 & 52.728 \\
\hline 48.334 & 48.334 & 2.197 & 2.197 \\
\hline 2.197 & 6.591 & 2.197 & 421.824 \\
\hline 15.379 & 8.788 & 46.137 & 177.957 \\
\hline 15.379 & 6.591 & 4.394 & 10.985 \\
\hline 230.685 & 52.728 & 15.379 & 74.698 \\
\hline 32.955 & 2.197 & 94.471 & 13.182 \\
\hline 26.364 & 15.379 & 43.94 & 41.743 \\
\hline 4.394 & 4.394 & 2.197 & 28.561 \\
\hline 13.182 & 19.773 & 2.197 & 2.197 \\
\hline 2.197 & 4.394 & 2.197 & 8.788 \\
\hline 1656.54 & 4.394 & 13.182 & 46.137 \\
\hline 2.197 & 327.353 & 2.197 & 2.197 \\
\hline 4.394 & 2.197 & 6.591 & 2.197 \\
\hline 173.563 & 13.182 & 24.167 & 8.788 \\
\hline 2.197 & 2.197 & 2.197 & 4.394 \\
\hline 48.334 & 28.561 & 107.653 & 57.122 \\
\hline 81.289 & 28.561 & 4.394 & 81.289 \\
\hline 116.441 & 15.379 & 2.197 & 2.197 \\
\hline 2.197 & 13.182 & 2.197 & 50.531 \\
\hline 2.197 & 4.394 & 26.364 & 15.379 \\
\hline 92.274 & 327.353 & 4.394 & 2.197 \\
\hline 2.197 & 2.197 & 2.197 & 2.197 \\
\hline 2.197 & 2.197 & 2.197 & 26.364 \\
\hline 397.657 & 2.197 & 2.197 & 2.197 \\
\hline 81.289 & 65.91 & 140.608 & 8.788 \\
\hline 8.788 & 79.092 & 24.167 & 2.197 \\
\hline 4.394 & 15.379 & 2.197 & 2.197 \\
\hline 4.394 & 21.97 & 8.788 & 59.319 \\
\hline 2.197 & 4.394 & 4.394 & 28.561 \\
\hline 4.394 & 37.349 & 2.197 & 338.338 \\
\hline 226.291 & 4.394 & 2.197 & 13.182 \\
\hline 2.197 & 4.394 & 4.394 & 19.773 \\
\hline 184.548 & 2.197 & 2.197 & 184.548 \\
\hline
\end{tabular}




\begin{tabular}{|c|c|c|c|}
\hline 2.197 & 2.197 & 2.197 & 15.379 \\
\hline 2.197 & 4.394 & 21.97 & 2.197 \\
\hline 2.197 & 2.197 & 2.197 & 37.349 \\
\hline 2.197 & 848.042 & 4.394 & 2.197 \\
\hline 2.197 & 6.591 & 4.394 & 37.349 \\
\hline 13.182 & 4.394 & 2.197 & 19.773 \\
\hline 2.197 & 8.788 & 50.531 & 2.197 \\
\hline 162.578 & 39.546 & 2.197 & 2.197 \\
\hline 4.394 & 6.591 & 2.197 & 1359.94 \\
\hline 28.561 & 2.197 & 2.197 & 263.64 \\
\hline 2.197 & 76.895 & 2.197 & 19.773 \\
\hline 4.394 & 6.591 & 68.107 & 41.743 \\
\hline 2.197 & 2.197 & 2.197 & 59.319 \\
\hline 30.758 & 6.591 & 8.788 & 101.062 \\
\hline 59.319 & 30.758 & 4.394 & 74.698 \\
\hline 35.152 & 8.788 & 13.182 & 4.394 \\
\hline 6.591 & 19.773 & 2.197 & 4.394 \\
\hline 2.197 & 10.985 & 2.197 & 8.788 \\
\hline 59.319 & 2.197 & 2.197 & 72.501 \\
\hline 6.591 & 536.068 & 24.167 & 35.152 \\
\hline 85.683 & 68.107 & 2.197 & 1326.99 \\
\hline 39.546 & 2.197 & 10.985 & 4.394 \\
\hline 2.197 & 6.591 & 116.441 & 8.788 \\
\hline 28.561 & 2.197 & 4.394 & 26.364 \\
\hline 4.394 & 2.197 & 2.197 & 6.591 \\
\hline 2.197 & 4.394 & 2.197 & 43.94 \\
\hline 4.394 & 2.197 & 16165.5 & 8247.54 \\
\hline 4.394 & 2.197 & 2.197 & 366.899 \\
\hline 4.394 & 2.197 & 43.94 & 6.591 \\
\hline 2.197 & 8.788 & 2812.16 & 70.304 \\
\hline 2.197 & 52.728 & 28.561 & 4.394 \\
\hline 2.197 & 2.197 & 2.197 & 2.197 \\
\hline 2.197 & 8.788 & 2.197 & 4.394 \\
\hline 2.197 & 2.197 & 10.985 & 2.197 \\
\hline 2.197 & 2.197 & 2.197 & 6.591 \\
\hline 19.773 & 2.197 & 2.197 & 4.394 \\
\hline 17.576 & 43.94 & 6.591 & 32.955 \\
\hline 85.683 & 4.394 & 13.182 & 21.97 \\
\hline 39.546 & 19.773 & 54.925 & 6.591 \\
\hline 26.364 & 68.107 & 13.182 & 2.197 \\
\hline 2.197 & 118.638 & 10.985 & 2.197 \\
\hline 149.396 & 10.985 & 13.182 & 2.197 \\
\hline 6.591 & 13.182 & 37.349 & 2.197 \\
\hline 2.197 & 2.197 & 2.197 & 2.197 \\
\hline 70.304 & 8.788 & 2.197 & 2.197 \\
\hline 10.985 & 626.145 & 2.197 & 2.197 \\
\hline 2.197 & 2.197 & 13.182 & 59.319 \\
\hline 2.197 & 2.197 & 162.578 & 24.167 \\
\hline 2.197 & 32.955 & 107.653 & 10.985 \\
\hline 4.394 & 21.97 & 2.197 & 70.304 \\
\hline
\end{tabular}




\begin{tabular}{|c|c|c|c|}
\hline 2.197 & 19.773 & 2.197 & 2.197 \\
\hline 2.197 & 19.773 & 2.197 & 4.394 \\
\hline 149.396 & 87.88 & 2.197 & 6.591 \\
\hline 6.591 & 127.426 & 6.591 & 10.985 \\
\hline 6.591 & 28.561 & 85.683 & 632.736 \\
\hline 6.591 & 195.533 & 37.349 & 76.895 \\
\hline 2.197 & 2.197 & 90.077 & 15.379 \\
\hline 6.591 & 2.197 & 2.197 & 87.88 \\
\hline 50.531 & 4.394 & 8.788 & 147.199 \\
\hline 4.394 & 10.985 & 2.197 & 8.788 \\
\hline 19.773 & 8.788 & 230.685 & 10.985 \\
\hline 4.394 & 19.773 & 70.304 & 8.788 \\
\hline 533.871 & 37.349 & 2.197 & 2.197 \\
\hline 8.788 & 10.985 & 2.197 & 103.259 \\
\hline 2.197 & 2.197 & 4.394 & 13.182 \\
\hline 2.197 & 2.197 & 2.197 & 4.394 \\
\hline 2.197 & 2.197 & 4.394 & 15.379 \\
\hline 48.334 & 10.985 & 2.197 & 6.591 \\
\hline 7779.58 & 21.97 & 2.197 & 10.985 \\
\hline 259.246 & 4.394 & 2.197 & 4.394 \\
\hline 24.167 & 21.97 & 2.197 & 43.94 \\
\hline 1346.76 & 760.162 & 2.197 & 61.516 \\
\hline 6.591 & 2.197 & 2.197 & 105.456 \\
\hline 8.788 & 2.197 & 2.197 & 2.197 \\
\hline 68.107 & 57.122 & 2.197 & 8.788 \\
\hline 2.197 & 30.758 & 59.319 & 30.758 \\
\hline 70.304 & 322.959 & 2.197 & 85.683 \\
\hline 32.955 & 26.364 & 2.197 & 8.788 \\
\hline 2.197 & 6.591 & 2.197 & 2.197 \\
\hline 13.182 & 2.197 & 2.197 & 206.518 \\
\hline 123.032 & 2.197 & 6.591 & 169.169 \\
\hline 4.394 & 4.394 & 2.197 & 10.985 \\
\hline 2.197 & 116.441 & 2.197 & 57.122 \\
\hline 4.394 & 2.197 & 2.197 & 39.546 \\
\hline 6.591 & 2.197 & 85.683 & 10.985 \\
\hline 2.197 & 13.182 & 43.94 & 2.197 \\
\hline 54.925 & 17.576 & 2.197 & 2.197 \\
\hline 6.591 & 19.773 & 129.623 & 4.394 \\
\hline 4.394 & 2.197 & 2.197 & 2.197 \\
\hline 30.758 & 17.576 & 2.197 & 17.576 \\
\hline 2.197 & 957.892 & 1364.34 & 59.319 \\
\hline 41.743 & 32.955 & 76.895 & 4.394 \\
\hline 329.55 & 4.394 & 24.167 & 2.197 \\
\hline 2.197 & 2.197 & 26.364 & 37.349 \\
\hline 79.092 & 8.788 & 142.805 & 39.546 \\
\hline 26.364 & 131.82 & 13.182 & 8.788 \\
\hline 116.441 & 2.197 & 4.394 & 63.713 \\
\hline 24.167 & 35.152 & 2.197 & 140.608 \\
\hline 8.788 & 2.197 & 2.197 & 96.668 \\
\hline 2.197 & 10.985 & 2.197 & 70.304 \\
\hline
\end{tabular}




\begin{tabular}{|c|c|c|c|}
\hline 2.197 & 2.197 & 2.197 & 2.197 \\
\hline 39.546 & 4.394 & 19.773 & 2.197 \\
\hline 2.197 & 13.182 & 2.197 & 2.197 \\
\hline 2.197 & 4.394 & 2.197 & 4.394 \\
\hline 24.167 & 46.137 & 2.197 & 296.595 \\
\hline 50.531 & 4.394 & 2.197 & 2.197 \\
\hline 2.197 & 2.197 & 24.167 & 316.368 \\
\hline 4.394 & 151.593 & 10.985 & 8.788 \\
\hline 6.591 & 83.486 & 4.394 & 2.197 \\
\hline 2.197 & 8.788 & 4.394 & 4.394 \\
\hline 492.128 & 2.197 & 65.91 & 4.394 \\
\hline 2.197 & 4.394 & 52.728 & 2.197 \\
\hline 68.107 & 2.197 & 30.758 & 15.379 \\
\hline 2.197 & 2.197 & 4.394 & 8.788 \\
\hline 68.107 & 8.788 & 28.561 & 2.197 \\
\hline 2.197 & 2.197 & 395.46 & 241.67 \\
\hline 39.546 & 8.788 & 32.955 & 6.591 \\
\hline 8.788 & 21.97 & 30.758 & 336.141 \\
\hline 26.364 & 13.182 & 52.728 & 65.91 \\
\hline 6.591 & 24.167 & 558.038 & 911.755 \\
\hline 15.379 & 6.591 & 4.394 & 30.758 \\
\hline 2.197 & 59.319 & 2.197 & 13.182 \\
\hline 4.394 & 6.591 & 1674.11 & 2.197 \\
\hline 2.197 & 21.97 & 35.152 & 21.97 \\
\hline 2.197 & 50.531 & 54.925 & 26.364 \\
\hline 19.773 & 24.167 & 30.758 & 421.824 \\
\hline 70.304 & 50.531 & 2.197 & 63.713 \\
\hline 57.122 & 6.591 & 2.197 & 4.394 \\
\hline 21.97 & 46.137 & 2.197 & 15.379 \\
\hline 72.501 & 13.182 & 4.394 & 61.516 \\
\hline 2.197 & 2.197 & 4.394 & 2.197 \\
\hline 4.394 & 6.591 & 4.394 & 1469.79 \\
\hline 24.167 & 228.488 & 4.394 & 4.394 \\
\hline 8.788 & 13.182 & 4.394 & 114.244 \\
\hline 2.197 & 2.197 & 2.197 & 2.197 \\
\hline 162.578 & 2.197 & 2.197 & 15.379 \\
\hline 70.304 & 2.197 & 4.394 & 4.394 \\
\hline 35.152 & 2.197 & 2.197 & 292.201 \\
\hline 2.197 & 10.985 & 17.576 & 26.364 \\
\hline 4.394 & 4.394 & 164.775 & 101.062 \\
\hline 52.728 & 26.364 & 4.394 & 57.122 \\
\hline 2.197 & 4.394 & 4.394 & 79.092 \\
\hline 2.197 & 57.122 & 2.197 & 21.97 \\
\hline 2.197 & 21.97 & 2.197 & 2.197 \\
\hline 2.197 & 13.182 & 15.379 & 210.912 \\
\hline 2.197 & 24.167 & 79.092 & 2.197 \\
\hline 26.364 & 15.379 & 4.394 & 2.197 \\
\hline 2.197 & 65.91 & 21.97 & 17.576 \\
\hline 2.197 & 2.197 & 2.197 & 15.379 \\
\hline 105.456 & 70.304 & 2.197 & 59.319 \\
\hline
\end{tabular}




\begin{tabular}{|c|c|c|c|}
\hline 221.897 & 43.94 & 2.197 & 15.379 \\
\hline 59.319 & 2.197 & 13.182 & 6.591 \\
\hline 2.197 & 2.197 & 2.197 & 48.334 \\
\hline 2.197 & 2.197 & 15.379 & 81.289 \\
\hline 13.182 & 2.197 & 2.197 & 6.591 \\
\hline 13.182 & 32.955 & 2.197 & 6.591 \\
\hline 151.593 & 8.788 & 2.197 & 8.788 \\
\hline 2.197 & 57.122 & 2.197 & 26.364 \\
\hline 2.197 & 391.066 & 2.197 & 4.394 \\
\hline 2.197 & 6.591 & 4.394 & 17.576 \\
\hline 24.167 & 10.985 & 13.182 & 19.773 \\
\hline 2.197 & 2.197 & 41.743 & 26.364 \\
\hline 4.394 & 6.591 & 17.576 & 30.758 \\
\hline 4.394 & 92.274 & 6.591 & 6.591 \\
\hline 749.177 & 4.394 & 2.197 & 2.197 \\
\hline 2.197 & 8.788 & 322.959 & 2.197 \\
\hline 8.788 & 2.197 & 2.197 & 8.788 \\
\hline 129.623 & 17.576 & 6.591 & 2.197 \\
\hline 114.244 & 8.788 & 35.152 & 2.197 \\
\hline 26.364 & 2.197 & 2.197 & 274.625 \\
\hline 30.758 & 54.925 & 2.197 & 2.197 \\
\hline 6.591 & 90.077 & 2.197 & 50.531 \\
\hline 4.394 & 6.591 & 8.788 & 10.985 \\
\hline 13.182 & 15.379 & 50.531 & 107.653 \\
\hline 4.394 & 50.531 & 107.653 & 2.197 \\
\hline 2.197 & 105.456 & 2.197 & 2.197 \\
\hline 17.576 & 2.197 & 24.167 & 17.576 \\
\hline 4.394 & 2.197 & 70.304 & 2.197 \\
\hline 8.788 & 2.197 & 6.591 & 1131.45 \\
\hline 50.531 & 43.94 & 2.197 & 2.197 \\
\hline 4.394 & 2.197 & 6.591 & 191.139 \\
\hline 2.197 & 103.259 & 215.306 & 74.698 \\
\hline 2.197 & 24.167 & 2.197 & 177.957 \\
\hline 199.927 & 112.047 & 28.561 & 13.182 \\
\hline 217.503 & 4.394 & 4.394 & 2.197 \\
\hline 15.379 & 46.137 & 17.576 & 4.394 \\
\hline 6.591 & 13.182 & 2.197 & 2.197 \\
\hline 10.985 & 6.591 & 61.516 & 2.197 \\
\hline 72.501 & 2.197 & 566.826 & 8.788 \\
\hline 10.985 & 2.197 & 6.591 & 28.561 \\
\hline 24.167 & 70.304 & 2.197 & 145.002 \\
\hline 2.197 & 2.197 & 2.197 & 6.591 \\
\hline 4.394 & 10.985 & 39.546 & 17.576 \\
\hline 6.591 & 46.137 & 2.197 & 37.349 \\
\hline 4.394 & 15.379 & 72.501 & 2.197 \\
\hline 13.182 & 4.394 & 21.97 & 19.773 \\
\hline 2.197 & 2.197 & 2.197 & 116.441 \\
\hline 2.197 & 10.985 & 2.197 & 24.167 \\
\hline 2.197 & 8.788 & 2.197 & 72.501 \\
\hline 10.985 & 61.516 & 160.381 & 109.85 \\
\hline
\end{tabular}




\begin{tabular}{|c|c|c|c|}
\hline 509.704 & 6.591 & 13.182 & 4.394 \\
\hline 4.394 & 4.394 & 21.97 & 2.197 \\
\hline 4.394 & 125.229 & 2.197 & 2.197 \\
\hline 2.197 & 57.122 & 6.591 & 129.623 \\
\hline 41.743 & 2.197 & 13.182 & 15.379 \\
\hline 6.591 & 2.197 & 46.137 & 2.197 \\
\hline 2.197 & 2.197 & 204.321 & 10.985 \\
\hline 2.197 & 21.97 & 2.197 & 8.788 \\
\hline 24.167 & 6.591 & 13.182 & 6.591 \\
\hline 6.591 & 138.411 & 76.895 & 8.788 \\
\hline 54.925 & 46.137 & 2.197 & 43.94 \\
\hline 2.197 & 4.394 & 2.197 & 4.394 \\
\hline 8.788 & 2.197 & 2.197 & 13.182 \\
\hline 4.394 & 2.197 & 2.197 & 6.591 \\
\hline 2.197 & 87.88 & 2.197 & 2.197 \\
\hline 13.182 & 252.655 & 2.197 & 74.698 \\
\hline 8.788 & 2.197 & 13.182 & 2.197 \\
\hline 2.197 & 2.197 & 2.197 & 2.197 \\
\hline 10.985 & 402.051 & 2.197 & 32.955 \\
\hline 6.591 & 2.197 & 2.197 & 2.197 \\
\hline 2.197 & 72.501 & 2.197 & 6.591 \\
\hline 8.788 & 13.182 & 4.394 & 37.349 \\
\hline 195.533 & 349.323 & 2.197 & 87.88 \\
\hline 15.379 & 4.394 & 6.591 & 2647.38 \\
\hline 8.788 & 2.197 & 81.289 & 13.182 \\
\hline 430.612 & 2.197 & 318.565 & 6.591 \\
\hline 2.197 & 65.91 & 2.197 & 2.197 \\
\hline 8.788 & 2.197 & 2.197 & 2.197 \\
\hline 2.197 & 4.394 & 10.985 & 197.73 \\
\hline 2.197 & 8.788 & 2.197 & 426.218 \\
\hline 4.394 & 268.034 & 2.197 & 2.197 \\
\hline 57.122 & 28.561 & 2.197 & 8.788 \\
\hline 4.394 & 28.561 & 2.197 & 2.197 \\
\hline 2.197 & 2.197 & 2.197 & 4.394 \\
\hline 147.199 & 43.94 & 4.394 & 37.349 \\
\hline 2.197 & 2.197 & 4.394 & 8.788 \\
\hline 2.197 & 41.743 & 8.788 & 2.197 \\
\hline 2.197 & 26.364 & 2.197 & 4.394 \\
\hline 2.197 & 6.591 & 2.197 & 2.197 \\
\hline 2.197 & 118.638 & 10.985 & 26.364 \\
\hline 13.182 & 26.364 & 2.197 & 6.591 \\
\hline 4.394 & 26.364 & 2.197 & 2700.11 \\
\hline 10.985 & 2.197 & 10.985 & 2.197 \\
\hline 4.394 & 2.197 & 4.394 & 10.985 \\
\hline 43.94 & 2.197 & 4.394 & 8.788 \\
\hline 10.985 & 2.197 & 15.379 & 30.758 \\
\hline 37.349 & 43.94 & 17.576 & 13.182 \\
\hline 6.591 & 4.394 & 4.394 & 2.197 \\
\hline 6.591 & 2.197 & 2.197 & 10.985 \\
\hline 26.364 & 2.197 & 2.197 & 2.197 \\
\hline
\end{tabular}




\begin{tabular}{|c|c|c|c|}
\hline 142.805 & 10.985 & 6.591 & 41.743 \\
\hline 6.591 & 2.197 & 6.591 & 13.182 \\
\hline 257.049 & 2.197 & 2.197 & 2.197 \\
\hline 35.152 & 2.197 & 2.197 & 6.591 \\
\hline 2.197 & 2.197 & 21.97 & 2.197 \\
\hline 2.197 & 2.197 & 786.526 & 219.7 \\
\hline 2.197 & 6.591 & 129.623 & 59.319 \\
\hline 2.197 & 4.394 & 2.197 & 30.758 \\
\hline 13.182 & 2.197 & 2.197 & 325.156 \\
\hline 2.197 & 2.197 & 2.197 & 101.062 \\
\hline 2.197 & 17.576 & 41.743 & 39.546 \\
\hline 10.985 & 8.788 & 13.182 & 13.182 \\
\hline 2.197 & 241.67 & 6.591 & 8.788 \\
\hline 8.788 & 6.591 & 32.955 & 15.379 \\
\hline 120.835 & 6.591 & 13.182 & 4.394 \\
\hline 15.379 & 2.197 & 2.197 & 6.591 \\
\hline 2.197 & 2.197 & 46.137 & 17.576 \\
\hline 2.197 & 19.773 & 2.197 & 891.982 \\
\hline 4.394 & 13.182 & 15.379 & 239.473 \\
\hline 2.197 & 2.197 & 2.197 & 19.773 \\
\hline 26.364 & 8.788 & 2.197 & 166.972 \\
\hline 2.197 & 6.591 & 213.109 & 2.197 \\
\hline 83.486 & 4.394 & 26.364 & 2.197 \\
\hline 30.758 & 199.927 & 2.197 & 2159.65 \\
\hline 4.394 & 13.182 & 195.533 & 90.077 \\
\hline 17.576 & 39.546 & 6.591 & 2.197 \\
\hline 79.092 & 4.394 & 2.197 & 4.394 \\
\hline 2.197 & 15.379 & 2.197 & 2.197 \\
\hline 8.788 & 131.82 & 2.197 & 2.197 \\
\hline 6.591 & 2.197 & 13.182 & 4.394 \\
\hline 2.197 & 6.591 & 2.197 & 52.728 \\
\hline 2.197 & 8.788 & 2.197 & 2.197 \\
\hline 2.197 & 6.591 & 2.197 & 8.788 \\
\hline 57.122 & 2.197 & 2.197 & 2.197 \\
\hline 2.197 & 98.865 & 2.197 & 2.197 \\
\hline 2.197 & 50.531 & 2.197 & 2.197 \\
\hline 2.197 & 4.394 & 2.197 & 109.85 \\
\hline 2.197 & 136.214 & 24.167 & 2.197 \\
\hline 6.591 & 4.394 & 2.197 & 8.788 \\
\hline 8.788 & 2.197 & 2.197 & 8517.77 \\
\hline 2.197 & 26.364 & 2.197 & 2.197 \\
\hline 17.576 & 39.546 & 2.197 & 2.197 \\
\hline 17.576 & 37.349 & 2.197 & 2511.17 \\
\hline 4.394 & 8.788 & 2.197 & 2.197 \\
\hline 35.152 & 19.773 & 2.197 & 305.383 \\
\hline 2.197 & 21.97 & 81.289 & 2.197 \\
\hline 2.197 & 21.97 & 9893.09 & 4.394 \\
\hline 13.182 & 79.092 & 10.985 & 2.197 \\
\hline 272.428 & 164.775 & 2.197 & 2.197 \\
\hline 6.591 & 79.092 & 8.788 & 2.197 \\
\hline
\end{tabular}




\begin{tabular}{|c|c|c|c|}
\hline 103.259 & 4.394 & 2.197 & 52.728 \\
\hline 10.985 & 54.925 & 127.426 & 2.197 \\
\hline 2.197 & 6.591 & 2.197 & 28.561 \\
\hline 2.197 & 4.394 & 24.167 & 2.197 \\
\hline 2.197 & 19.773 & 2.197 & 32.955 \\
\hline 4.394 & 70.304 & 2.197 & 35.152 \\
\hline 46.137 & 4.394 & 2.197 & 17.576 \\
\hline 364.702 & 2.197 & 2.197 & 2.197 \\
\hline 48.334 & 2.197 & 2.197 & 43.94 \\
\hline 6.591 & 52.728 & 2.197 & 136.214 \\
\hline 32.955 & 30.758 & 962.286 & 15.379 \\
\hline 24.167 & 8.788 & 2.197 & 28.561 \\
\hline 6.591 & 2.197 & 50.531 & 32.955 \\
\hline 136.214 & 61.516 & 2.197 & 2.197 \\
\hline 2.197 & 4.394 & 6.591 & 6.591 \\
\hline 19.773 & 8.788 & 35.152 & 456.976 \\
\hline 10.985 & 50.531 & 15.379 & 13.182 \\
\hline 2.197 & 2.197 & 2.197 & 15.379 \\
\hline 2.197 & 2.197 & 4.394 & 4.394 \\
\hline 28.561 & 19.773 & 61.516 & 2497.99 \\
\hline 2.197 & 2.197 & 68.107 & 2.197 \\
\hline 2.197 & 2.197 & 2.197 & 8.788 \\
\hline 8.788 & 26.364 & 2.197 & 15.379 \\
\hline 2.197 & 37.349 & 6.591 & 2.197 \\
\hline 63.713 & 10.985 & 8.788 & 2.197 \\
\hline 2.197 & 2.197 & 4.394 & 2.197 \\
\hline 13.182 & 2.197 & 2.197 & 2.197 \\
\hline 2.197 & 2.197 & 92.274 & 4.394 \\
\hline 4.394 & 32.955 & 35.152 & 2.197 \\
\hline 338.338 & 30.758 & 2.197 & 105.456 \\
\hline 32.955 & 13.182 & 105.456 & 2.197 \\
\hline 15.379 & 13.182 & 28.561 & 2.197 \\
\hline 4.394 & 76.895 & 2.197 & 2.197 \\
\hline 2003.66 & 2.197 & 6.591 & 2.197 \\
\hline 112.047 & 4.394 & 2.197 & 8.788 \\
\hline 43.94 & 2.197 & 43.94 & 2.197 \\
\hline 65.91 & 2.197 & 4.394 & 2.197 \\
\hline 4.394 & 8.788 & 30.758 & 344.929 \\
\hline 50.531 & 2.197 & 338.338 & 4.394 \\
\hline 2.197 & 37.349 & 21.97 & 2.197 \\
\hline 15.379 & 2.197 & 184.548 & 2.197 \\
\hline 8.788 & 13.182 & 30.758 & 2.197 \\
\hline 2.197 & 10.985 & 17.576 & 2.197 \\
\hline 48.334 & 26.364 & 19.773 & 2.197 \\
\hline 4.394 & 2.197 & 24.167 & 1353.35 \\
\hline 61.516 & 2.197 & 2.197 & 15.379 \\
\hline 10.985 & 4.394 & 4.394 & 2.197 \\
\hline 26.364 & 13.182 & 101.062 & 2.197 \\
\hline 2.197 & 162.578 & 2.197 & 2.197 \\
\hline 2.197 & 2.197 & 21.97 & 30.758 \\
\hline
\end{tabular}




\begin{tabular}{|c|c|c|c|}
\hline 2.197 & 2.197 & 6.591 & 2.197 \\
\hline 2.197 & 17.576 & 57.122 & 2.197 \\
\hline 21.97 & 2.197 & 2.197 & 28.561 \\
\hline 4.394 & 2.197 & 2.197 & 2.197 \\
\hline 17.576 & 4.394 & 314.171 & 4.394 \\
\hline 2.197 & 35.152 & 15.379 & 2.197 \\
\hline 4.394 & 4.394 & 2.197 & 50.531 \\
\hline 2.197 & 19.773 & 2.197 & 10.985 \\
\hline 6.591 & 2.197 & 2.197 & 164.775 \\
\hline 8.788 & 2.197 & 2.197 & 4.394 \\
\hline 10.985 & 13.182 & 2.197 & 2.197 \\
\hline 2.197 & 2.197 & 2.197 & 2.197 \\
\hline 2.197 & 2.197 & 2.197 & 105.456 \\
\hline 2.197 & 2.197 & 2.197 & 15.379 \\
\hline 4.394 & 2.197 & 2.197 & 4.394 \\
\hline 21.97 & 2.197 & 10.985 & 4.394 \\
\hline 2.197 & 2.197 & 2.197 & 1109.48 \\
\hline 8.788 & 10.985 & 8.788 & 4.394 \\
\hline 2.197 & 6.591 & 2.197 & 26.364 \\
\hline 6.591 & 2.197 & 2.197 & 4.394 \\
\hline 35.152 & 13.182 & 63.713 & 48.334 \\
\hline 46.137 & 2.197 & 2.197 & 8.788 \\
\hline 208.715 & 4.394 & 2.197 & 2.197 \\
\hline 6.591 & 219.7 & 2.197 & 155.987 \\
\hline 4.394 & 10.985 & 2.197 & 4.394 \\
\hline 2.197 & 15.379 & 4.394 & 127.426 \\
\hline 21.97 & 8.788 & 2.197 & 13.182 \\
\hline 162.578 & 2.197 & 2.197 & 10.985 \\
\hline 2.197 & 2.197 & 2.197 & 120.835 \\
\hline 37.349 & 17.576 & 2.197 & 10.985 \\
\hline 2.197 & 32.955 & 329.55 & 13.182 \\
\hline 2.197 & 2.197 & 4.394 & 573.417 \\
\hline 81.289 & 83.486 & 2.197 & 79.092 \\
\hline 8.788 & 177.957 & 2.197 & 13.182 \\
\hline 138.411 & 19.773 & 8.788 & 8.788 \\
\hline 2.197 & 2.197 & 2.197 & 8.788 \\
\hline 4.394 & 2.197 & 61.516 & 57.122 \\
\hline 4.394 & 4.394 & 2.197 & 8.788 \\
\hline 2.197 & 2.197 & 101.062 & 15.379 \\
\hline 6.591 & 4.394 & 6.591 & 85.683 \\
\hline 2.197 & 15.379 & 2.197 & 2.197 \\
\hline 2.197 & 8.788 & 2.197 & 2.197 \\
\hline 79.092 & 4.394 & 2.197 & 6.591 \\
\hline 10.985 & 35.152 & 8.788 & 2.197 \\
\hline 2.197 & 19.773 & 79.092 & 2.197 \\
\hline 2.197 & 232.882 & 6.591 & 6.591 \\
\hline 15.379 & 30.758 & 13.182 & 10.985 \\
\hline 43.94 & 1320.4 & 4.394 & 2.197 \\
\hline 8.788 & 6.591 & 8.788 & 4.394 \\
\hline 2.197 & 441.597 & 120.835 & 76.895 \\
\hline
\end{tabular}




\begin{tabular}{|c|c|c|c|}
\hline 2.197 & 131.82 & 43.94 & 24.167 \\
\hline 2.197 & 2.197 & 10.985 & 30.758 \\
\hline 24.167 & 4.394 & 19.773 & 2.197 \\
\hline 2.197 & 2.197 & 2.197 & 21.97 \\
\hline 3361.41 & 17.576 & 41.743 & 17.576 \\
\hline 4.394 & 1689.49 & 19.773 & 81.289 \\
\hline 2.197 & 30.758 & 450.385 & 90.077 \\
\hline 4.394 & 76.895 & 2.197 & 46.137 \\
\hline 6.591 & 17.576 & 13.182 & 538.265 \\
\hline 2.197 & 15.379 & 4.394 & 20735.3 \\
\hline 2.197 & 26.364 & 4.394 & 13.182 \\
\hline 4.394 & 6.591 & 2.197 & 52.728 \\
\hline 2.197 & 221.897 & 2.197 & 6.591 \\
\hline 32.955 & 72.501 & 43.94 & 30.758 \\
\hline 6.591 & 6.591 & 15.379 & 21.97 \\
\hline 2.197 & 8.788 & 2.197 & 4.394 \\
\hline 2.197 & 61.516 & 4.394 & 8.788 \\
\hline 2.197 & 15.379 & 4.394 & 195.533 \\
\hline 2.197 & 2.197 & 65.91 & 19.773 \\
\hline 6.591 & 87.88 & 8.788 & 6.591 \\
\hline 202.124 & 4.394 & 2.197 & 2.197 \\
\hline 4.394 & 6.591 & 182.351 & 2.197 \\
\hline 39.546 & 2.197 & 2.197 & 50.531 \\
\hline 8.788 & 2.197 & 2.197 & 8.788 \\
\hline 52.728 & 2.197 & 2.197 & 2.197 \\
\hline 2.197 & 15.379 & 2.197 & 338.338 \\
\hline 4.394 & 54.925 & 2.197 & 2.197 \\
\hline 4.394 & 2.197 & 2.197 & 2.197 \\
\hline 2.197 & 28.561 & 2.197 & 2.197 \\
\hline 101.062 & 114.244 & 2.197 & 52.728 \\
\hline 2.197 & 76.895 & 72.501 & 4.394 \\
\hline 2.197 & 2.197 & 2.197 & 21.97 \\
\hline 2.197 & 10.985 & 2.197 & 52.728 \\
\hline 10.985 & 24.167 & 2.197 & 4.394 \\
\hline 2.197 & 10.985 & 2.197 & 50.531 \\
\hline 2.197 & 35.152 & 10.985 & 10.985 \\
\hline 142.805 & 48.334 & 6.591 & 61.516 \\
\hline 81.289 & 21.97 & 15.379 & 15.379 \\
\hline 2.197 & 6.591 & 2.197 & 2.197 \\
\hline 4.394 & 17.576 & 239.473 & 2.197 \\
\hline 21.97 & 50.531 & 654.706 & 13.182 \\
\hline 15.379 & 59.319 & 182.351 & 292.201 \\
\hline 2.197 & 6.591 & 384.475 & 68.107 \\
\hline 248.261 & 4.394 & 15.379 & 63.713 \\
\hline 24.167 & 13.182 & 3064.81 & 19.773 \\
\hline 4.394 & 48.334 & 26.364 & 2.197 \\
\hline 13.182 & 107.653 & 2.197 & 4.394 \\
\hline 2.197 & 28.561 & 142.805 & 41.743 \\
\hline 2.197 & 4.394 & 26.364 & 6.591 \\
\hline 4.394 & 2.197 & 2.197 & 4.394 \\
\hline
\end{tabular}




\begin{tabular}{|c|c|c|c|}
\hline 35.152 & 37.349 & 226.291 & 221.897 \\
\hline 6.591 & 21.97 & 2.197 & 35.152 \\
\hline 13.182 & 8.788 & 109.85 & 2.197 \\
\hline 24.167 & 19.773 & 10.985 & 6.591 \\
\hline 2.197 & 10.985 & 2.197 & 28.561 \\
\hline 2.197 & 2.197 & 8.788 & 2.197 \\
\hline 2.197 & 2.197 & 4.394 & 8.788 \\
\hline 8.788 & 13.182 & 2.197 & 2.197 \\
\hline 2.197 & 2.197 & 388.869 & 116.441 \\
\hline 4.394 & 4.394 & 87.88 & 10.985 \\
\hline 2.197 & 72.501 & 4.394 & 10.985 \\
\hline 6.591 & 2.197 & 4.394 & 30.758 \\
\hline 4.394 & 72.501 & 2.197 & 8.788 \\
\hline 6.591 & 2.197 & 2.197 & 2.197 \\
\hline 30.758 & 94.471 & 68.107 & 421.824 \\
\hline 6.591 & 2.197 & 17.576 & 57.122 \\
\hline 4.394 & 10.985 & 79.092 & 125.229 \\
\hline 2.197 & 48.334 & 6.591 & 32.955 \\
\hline 4.394 & 2.197 & 17.576 & 2.197 \\
\hline 2.197 & 4.394 & 28.561 & 90.077 \\
\hline 8.788 & 2.197 & 13.182 & 191.139 \\
\hline 2.197 & 4.394 & 2.197 & 114.244 \\
\hline 92.274 & 4.394 & 2.197 & 4.394 \\
\hline 13.182 & 4.394 & 48.334 & 8.788 \\
\hline 4.394 & 76.895 & 2.197 & 26.364 \\
\hline 2.197 & 19.773 & 2.197 & 4.394 \\
\hline 221.897 & 243.867 & 8.788 & 8.788 \\
\hline 2.197 & 2.197 & 2.197 & 2.197 \\
\hline 2.197 & 259.246 & 118.638 & 19.773 \\
\hline 2.197 & 896.376 & 2.197 & 2.197 \\
\hline 2.197 & 15.379 & 2.197 & 2.197 \\
\hline 4.394 & 145.002 & 2.197 & 70.304 \\
\hline 4.394 & 2.197 & 2.197 & 4.394 \\
\hline 2.197 & 4.394 & 2.197 & 2.197 \\
\hline 6.591 & 63.713 & 2.197 & 602817 \\
\hline 70.304 & 63.713 & 2.197 & 4.394 \\
\hline 10.985 & 10.985 & 2.197 & 10.985 \\
\hline 2.197 & 68.107 & 2.197 & 125.229 \\
\hline 10.985 & 136.214 & 2.197 & 2.197 \\
\hline 8.788 & 8.788 & 2.197 & 4.394 \\
\hline 24.167 & 6.591 & 2.197 & 6.591 \\
\hline 19.773 & 6.591 & 17.576 & 6.591 \\
\hline 2.197 & 160.381 & 2.197 & 2.197 \\
\hline 4.394 & 30.758 & 2.197 & 13.182 \\
\hline 19.773 & 127.426 & 21.97 & 6.591 \\
\hline 4.394 & 2.197 & 2.197 & 2.197 \\
\hline 4.394 & 8.788 & 4.394 & 39.546 \\
\hline 2.197 & 2.197 & 10.985 & 2.197 \\
\hline 2.197 & 164.775 & 68.107 & 2.197 \\
\hline 8.788 & 8.788 & 4.394 & 72.501 \\
\hline
\end{tabular}




\begin{tabular}{|c|c|c|c|}
\hline 188.942 & 8.788 & 2.197 & 6.591 \\
\hline 8.788 & 2.197 & 8.788 & 24.167 \\
\hline 6.591 & 8.788 & 4.394 & 364.702 \\
\hline 6.591 & 2.197 & 2.197 & 10.985 \\
\hline 2.197 & 43.94 & 35.152 & 10.985 \\
\hline 2.197 & 2.197 & 4.394 & 2.197 \\
\hline 2.197 & 15.379 & 15.379 & 2.197 \\
\hline 2.197 & 2.197 & 2080.56 & 17.576 \\
\hline 2.197 & 2.197 & 10.985 & 61.516 \\
\hline 2.197 & 2.197 & 4.394 & 15.379 \\
\hline 2.197 & 2.197 & 2.197 & 872.209 \\
\hline 665.691 & 2.197 & 72.501 & 2.197 \\
\hline 32.955 & 2.197 & 2.197 & 43.94 \\
\hline 2142.07 & 2.197 & 2.197 & 300.989 \\
\hline 63.713 & 4.394 & 4.394 & 10.985 \\
\hline 8.788 & 2.197 & 138.411 & 2.197 \\
\hline 2.197 & 2.197 & 65.91 & 28.561 \\
\hline 2.197 & 35.152 & 2.197 & 445.991 \\
\hline 2.197 & 8.788 & 2080.56 & 2.197 \\
\hline 43.94 & 43.94 & 21.97 & 8.788 \\
\hline 8.788 & 10.985 & 2.197 & 4.394 \\
\hline 206.518 & 6.591 & 59.319 & 13.182 \\
\hline 2.197 & 8.788 & 17.576 & 19.773 \\
\hline 2.197 & 57.122 & 15.379 & 4.394 \\
\hline 10.985 & 4.394 & 30.758 & 4.394 \\
\hline 2.197 & 15.379 & 109.85 & 17.576 \\
\hline 10.985 & 13.182 & 4.394 & 2.197 \\
\hline 6.591 & 24.167 & 2.197 & 30.758 \\
\hline 2.197 & 19.773 & 2.197 & 2.197 \\
\hline 39.546 & 8.788 & 2.197 & 15.379 \\
\hline 1770.78 & 2.197 & 13.182 & 2.197 \\
\hline 2.197 & 41.743 & 17.576 & 2.197 \\
\hline 2.197 & 65.91 & 2.197 & 2.197 \\
\hline 24.167 & 8.788 & 8.788 & 2.197 \\
\hline 10.985 & 21.97 & 241.67 & 4.394 \\
\hline 81.289 & 17.576 & 19.773 & 8.788 \\
\hline 35.152 & 50.531 & 6.591 & 149.396 \\
\hline 21.97 & 1087.51 & 2.197 & 4.394 \\
\hline 8.788 & 32.955 & 2.197 & 2.197 \\
\hline 125.229 & 2.197 & 2.197 & 2.197 \\
\hline 2.197 & 2.197 & 43.94 & 2.197 \\
\hline 21.97 & 41.743 & 4.394 & 2.197 \\
\hline 19.773 & 15.379 & 32.955 & 2.197 \\
\hline 2.197 & 2.197 & 6.591 & 13.182 \\
\hline 35.152 & 4.394 & 2.197 & 6.591 \\
\hline 136.214 & 43.94 & 2.197 & 30.758 \\
\hline 2.197 & 2.197 & 6.591 & 8.788 \\
\hline 8.788 & 2.197 & 2.197 & 8.788 \\
\hline 4.394 & 8.788 & 2.197 & 52.728 \\
\hline 2.197 & 2.197 & 2.197 & 50.531 \\
\hline
\end{tabular}




\begin{tabular}{|c|c|c|c|}
\hline 10.985 & 15.379 & 2.197 & 6.591 \\
\hline 2.197 & 1043.57 & 10.985 & 10.985 \\
\hline 8.788 & 2.197 & 6.591 & 2.197 \\
\hline 2.197 & 6.591 & 2.197 & 26.364 \\
\hline 4.394 & 2.197 & 2.197 & 50.531 \\
\hline 8.788 & 2372.76 & 2.197 & 2.197 \\
\hline 4.394 & 6.591 & 2.197 & 2.197 \\
\hline 4.394 & 4.394 & 2.197 & 4.394 \\
\hline 2.197 & 6.591 & 2.197 & 6.591 \\
\hline 4.394 & 41.743 & 2.197 & 24.167 \\
\hline 2.197 & 2.197 & 2.197 & 32.955 \\
\hline 13.182 & 4.394 & 2.197 & 19.773 \\
\hline 2.197 & 10.985 & 4.394 & 48.334 \\
\hline 19.773 & 39.546 & 520.689 & 19.773 \\
\hline 2.197 & 21.97 & 2.197 & 28.561 \\
\hline 2.197 & 28.561 & 4.394 & 6.591 \\
\hline 2.197 & 2.197 & 454.779 & 17.576 \\
\hline 13.182 & 15.379 & 103.259 & 24.167 \\
\hline 4.394 & 21.97 & 2.197 & 4.394 \\
\hline 6.591 & 10.985 & 2.197 & 92.274 \\
\hline 3216.41 & 50.531 & 6.591 & 87.88 \\
\hline 4.394 & 2.197 & 2.197 & 2.197 \\
\hline 21.97 & 37.349 & 81.289 & 17.576 \\
\hline 10.985 & 2.197 & 4.394 & 6.591 \\
\hline 2.197 & 4.394 & 2.197 & 6.591 \\
\hline 147.199 & 57.122 & 2.197 & 21.97 \\
\hline 96.668 & 8.788 & 59.319 & 4.394 \\
\hline 74.698 & 21.97 & 2.197 & 37.349 \\
\hline 10.985 & 92.274 & 147.199 & 443.794 \\
\hline 28.561 & 65.91 & 2.197 & 90.077 \\
\hline 2.197 & 2.197 & 2.197 & 467.961 \\
\hline 2280.49 & 37.349 & 13300.6 & 83.486 \\
\hline 4.394 & 2.197 & 2.197 & 41.743 \\
\hline 2.197 & 50.531 & 2.197 & 2.197 \\
\hline 26.364 & 43.94 & 10.985 & 2.197 \\
\hline 2.197 & 2.197 & 39.546 & 2.197 \\
\hline 2.197 & 2.197 & 10.985 & 2.197 \\
\hline 70.304 & 21.97 & 4.394 & 17.576 \\
\hline 10.985 & 4.394 & 2.197 & 186.745 \\
\hline 2.197 & 2.197 & 19.773 & 729.404 \\
\hline 2.197 & 2.197 & 15.379 & 140.608 \\
\hline 2.197 & 259.246 & 17.576 & 180.154 \\
\hline 30.758 & 287.807 & 59.319 & 83.486 \\
\hline 2.197 & 663.494 & 2.197 & 2.197 \\
\hline 2.197 & 15.379 & 2.197 & 41.743 \\
\hline 4.394 & 4.394 & 46.137 & 46.137 \\
\hline 4.394 & 13.182 & 2.197 & 4.394 \\
\hline 4.394 & 4.394 & 2.197 & 4.394 \\
\hline 52.728 & 2.197 & 4.394 & 10.985 \\
\hline 2.197 & 4.394 & 68.107 & 2.197 \\
\hline
\end{tabular}




\begin{tabular}{|c|c|c|c|}
\hline 21.97 & 2.197 & 2.197 & 2.197 \\
\hline 2.197 & 6.591 & 2.197 & 2.197 \\
\hline 50.531 & 2.197 & 4.394 & 4.394 \\
\hline 4.394 & 2.197 & 32.955 & 2.197 \\
\hline 81.289 & 21.97 & 8.788 & 8.788 \\
\hline 76.895 & 4.394 & 6.591 & 2.197 \\
\hline 8.788 & 81.289 & 61.516 & 2.197 \\
\hline 59.319 & 10.985 & 4.394 & 2.197 \\
\hline 2.197 & 182.351 & 39.546 & 41.743 \\
\hline 15.379 & 30.758 & 17.576 & 68.107 \\
\hline 2.197 & 10.985 & 2.197 & 2.197 \\
\hline 4.394 & 26.364 & 10.985 & 4.394 \\
\hline 2.197 & 8.788 & 10.985 & 2.197 \\
\hline 2.197 & 52.728 & 28.561 & 15.379 \\
\hline 2.197 & 79.092 & 2.197 & 4.394 \\
\hline 6.591 & 127.426 & 2.197 & 2.197 \\
\hline 2.197 & 2.197 & 8.788 & 2.197 \\
\hline 2.197 & 17.576 & 4.394 & 8.788 \\
\hline 35.152 & 37.349 & 2.197 & 2.197 \\
\hline 2.197 & 8.788 & 2.197 & 35.152 \\
\hline 37.349 & 46.137 & 92.274 & 28.561 \\
\hline 4.394 & 6.591 & 8.788 & 101.062 \\
\hline 4.394 & 26.364 & 6.591 & 24.167 \\
\hline 8.788 & 4.394 & 2.197 & 2.197 \\
\hline 13.182 & 30.758 & 13.182 & 13.182 \\
\hline 87.88 & 2.197 & 50.531 & 54.925 \\
\hline 6.591 & 19.773 & 17.576 & 15.379 \\
\hline 17.576 & 37.349 & 4.394 & 4.394 \\
\hline 2.197 & 21.97 & 2.197 & 2.197 \\
\hline 41.743 & 83.486 & 1085.32 & 4.394 \\
\hline 2.197 & 6.591 & 2.197 & 4.394 \\
\hline 2.197 & 8.788 & 2.197 & 6.591 \\
\hline 2.197 & 4.394 & 2.197 & 2.197 \\
\hline 6.591 & 123.032 & 2.197 & 8.788 \\
\hline 17.576 & 79.092 & 2.197 & 103.259 \\
\hline 2.197 & 57.122 & 2.197 & 2.197 \\
\hline 2.197 & 2.197 & 2.197 & 2.197 \\
\hline 15.379 & 24.167 & 2.197 & 2.197 \\
\hline 2.197 & 50.531 & 2.197 & 2.197 \\
\hline 17.576 & 296.595 & 2.197 & 6.591 \\
\hline 10.985 & 2.197 & 4.394 & 2.197 \\
\hline 2.197 & 4.394 & 2.197 & 6.591 \\
\hline 2.197 & 15.379 & 2.197 & 2.197 \\
\hline 4.394 & 2.197 & 4.394 & 39.546 \\
\hline 2.197 & 129.623 & 17.576 & 21.97 \\
\hline 19.773 & 2.197 & 160.381 & 13.182 \\
\hline 81.289 & 41.743 & 6.591 & 2.197 \\
\hline 2.197 & 30.758 & 2.197 & 4.394 \\
\hline 4.394 & 2.197 & 2.197 & 6.591 \\
\hline 2.197 & 19.773 & 2.197 & 57.122 \\
\hline
\end{tabular}




\begin{tabular}{|c|c|c|c|}
\hline 4.394 & 2.197 & 2.197 & 2.197 \\
\hline 4.394 & 6.591 & 32.955 & 46.137 \\
\hline 2.197 & 10.985 & 2.197 & 15.379 \\
\hline 2.197 & 1080.92 & 239.473 & 50.531 \\
\hline 2.197 & 2.197 & 2.197 & 450.385 \\
\hline 127.426 & 2.197 & 2.197 & 17.576 \\
\hline 26.364 & 6.591 & 10.985 & 2.197 \\
\hline 21.97 & 8.788 & 41.743 & 123.032 \\
\hline 50.531 & 116.441 & 2.197 & 17.576 \\
\hline 4.394 & 96.668 & 52.728 & 129.623 \\
\hline 4.394 & 2.197 & 2.197 & 2.197 \\
\hline 2.197 & 6.591 & 6.591 & 2.197 \\
\hline 50.531 & 2.197 & 2.197 & 4.394 \\
\hline 4.394 & 2.197 & 2.197 & 2.197 \\
\hline 2.197 & 2.197 & 2.197 & 65.91 \\
\hline 2.197 & 30.758 & 48.334 & 195.533 \\
\hline 15.379 & 6.591 & 10.985 & 19.773 \\
\hline 2.197 & 2.197 & 6.591 & 72.501 \\
\hline 206.518 & 4.394 & 10.985 & 2.197 \\
\hline 2.197 & 4.394 & 2.197 & 2.197 \\
\hline 2.197 & 2.197 & 8.788 & 17.576 \\
\hline 11582.6 & 4.394 & 4.394 & 17.576 \\
\hline 4.394 & 4.394 & 19.773 & 4.394 \\
\hline 2.197 & 10.985 & 28.561 & 15.379 \\
\hline 15.379 & 4.394 & 35.152 & 19.773 \\
\hline 4.394 & 4.394 & 2.197 & 1450.02 \\
\hline 2.197 & 4.394 & 17.576 & 4.394 \\
\hline 8.788 & 8.788 & 30.758 & 2.197 \\
\hline 2.197 & 2.197 & 57.122 & 2.197 \\
\hline 2.197 & 21.97 & 4.394 & 43.94 \\
\hline 19.773 & 2.197 & 2.197 & 2.197 \\
\hline 2.197 & 2.197 & 2.197 & 6.591 \\
\hline 13.182 & 8.788 & 2.197 & 202.124 \\
\hline 2.197 & 10.985 & 17.576 & 2.197 \\
\hline 13.182 & 43.94 & 1860.86 & 2.197 \\
\hline 2.197 & 92.274 & 19.773 & 54.925 \\
\hline 35.152 & 2.197 & 15.379 & 4.394 \\
\hline 10.985 & 2.197 & 4.394 & 35.152 \\
\hline 2.197 & 2.197 & 4.394 & 2.197 \\
\hline 28.561 & 54.925 & 2.197 & 83.486 \\
\hline 2.197 & 13.182 & 2.197 & 4.394 \\
\hline 54.925 & 24.167 & 2.197 & 4.394 \\
\hline 52.728 & 13.182 & 2.197 & 2.197 \\
\hline 4.394 & 8.788 & 2.197 & 52.728 \\
\hline 2.197 & 8.788 & 2.197 & 19.773 \\
\hline 2.197 & 10.985 & 2.197 & 13.182 \\
\hline 15.379 & 6.591 & 2.197 & 4.394 \\
\hline 2.197 & 52.728 & 2.197 & 10.985 \\
\hline 2.197 & 70.304 & 2.197 & 4.394 \\
\hline 6.591 & 2.197 & 4.394 & 2.197 \\
\hline
\end{tabular}




\begin{tabular}{|c|c|c|c|}
\hline 4.394 & 2.197 & 2.197 & 6.591 \\
\hline 26.364 & 15.379 & 215.306 & 32.955 \\
\hline 50.531 & 213.109 & 2.197 & 30.758 \\
\hline 17.576 & 2.197 & 2.197 & 6.591 \\
\hline 8.788 & 8.788 & 2.197 & 17.576 \\
\hline 2.197 & 2.197 & 2.197 & 4.394 \\
\hline 10.985 & 21.97 & 2.197 & 24.167 \\
\hline 2.197 & 21.97 & 21.97 & 2904.43 \\
\hline 155.987 & 15.379 & 2.197 & 588.796 \\
\hline 2.197 & 21.97 & 123.032 & 4.394 \\
\hline 32.955 & 224.094 & 2.197 & 74.698 \\
\hline 2.197 & 6.591 & 2.197 & 48.334 \\
\hline 10.985 & 2.197 & 2.197 & 364.702 \\
\hline 2.197 & 2.197 & 4.394 & 10.985 \\
\hline 32.955 & 35.152 & 2.197 & 35.152 \\
\hline 13.182 & 37.349 & 2.197 & 2.197 \\
\hline 4.394 & 4.394 & 2.197 & 2.197 \\
\hline 10.985 & 4.394 & 2.197 & 26.364 \\
\hline 2.197 & 2.197 & 4.394 & 8.788 \\
\hline 2.197 & 2.197 & 8.788 & 43.94 \\
\hline 83.486 & 15.379 & 2.197 & 4.394 \\
\hline 2.197 & 2.197 & 2.197 & 2.197 \\
\hline 2.197 & 221.897 & 19.773 & 8.788 \\
\hline 2.197 & 10.985 & 24.167 & 2.197 \\
\hline 10.985 & 109.85 & 6.591 & 2.197 \\
\hline 61.516 & 10.985 & 59.319 & 17.576 \\
\hline 2.197 & 83.486 & 2.197 & 193.336 \\
\hline 4.394 & 142.805 & 101.062 & 10.985 \\
\hline 287.807 & 6.591 & 70.304 & 61.516 \\
\hline 4.394 & 24.167 & 39.546 & 10.985 \\
\hline 2.197 & 26.364 & 10.985 & 2.197 \\
\hline 6.591 & 28.561 & 21.97 & 2.197 \\
\hline 2.197 & 6.591 & 8.788 & 2.197 \\
\hline 2.197 & 2.197 & 4.394 & 971.074 \\
\hline 2.197 & 59.319 & 4.394 & 15.379 \\
\hline 2.197 & 15.379 & 35.152 & 92.274 \\
\hline 2.197 & 21.97 & 109.85 & 2.197 \\
\hline 4.394 & 8.788 & 2.197 & 26.364 \\
\hline 2.197 & 50.531 & 2.197 & 8.788 \\
\hline 70.304 & 26.364 & 2.197 & 6.591 \\
\hline 107.653 & 4.394 & 2.197 & 6.591 \\
\hline 2.197 & 162.578 & 2.197 & 8.788 \\
\hline 2.197 & 6.591 & 2.197 & 68.107 \\
\hline 2.197 & 2.197 & 19.773 & 92.274 \\
\hline 2.197 & 2.197 & 13.182 & 2.197 \\
\hline 92.274 & 195.533 & 2.197 & 2.197 \\
\hline 76.895 & 129.623 & 2.197 & 10.985 \\
\hline 26.364 & 15.379 & 10.985 & 50.531 \\
\hline 2.197 & 19.773 & 2.197 & 4.394 \\
\hline 129.623 & 85.683 & 19.773 & 4.394 \\
\hline
\end{tabular}




\begin{tabular}{|c|c|c|c|}
\hline 6.591 & 43.94 & 48.334 & 30.758 \\
\hline 43.94 & 85.683 & 20100.4 & 2.197 \\
\hline 2.197 & 21.97 & 2.197 & 2.197 \\
\hline 26.364 & 10.985 & 8.788 & 15.379 \\
\hline 2.197 & 43.94 & 2.197 & 2.197 \\
\hline 2.197 & 388.869 & 2.197 & 333.944 \\
\hline 2.197 & 2.197 & 2.197 & 28.561 \\
\hline 6.591 & 2.197 & 68.107 & 4.394 \\
\hline 2.197 & 4.394 & 13.182 & 2.197 \\
\hline 2.197 & 4.394 & 806.299 & 237.276 \\
\hline 30.758 & 8.788 & 2.197 & 17.576 \\
\hline 2.197 & 13.182 & 2.197 & 46.137 \\
\hline 2.197 & 2.197 & 13.182 & 10.985 \\
\hline 2.197 & 186.745 & 147.199 & 21.97 \\
\hline 4.394 & 4.394 & 2.197 & 2.197 \\
\hline 4.394 & 13.182 & 70.304 & 6.591 \\
\hline 4.394 & 10.985 & 4.394 & 94.471 \\
\hline 2.197 & 32.955 & 19.773 & 4.394 \\
\hline 15.379 & 92.274 & 2.197 & 147.199 \\
\hline 6.591 & 406.445 & 2.197 & 112.047 \\
\hline 15.379 & 147.199 & 2.197 & 65.91 \\
\hline 6.591 & 92.274 & 2.197 & 72.501 \\
\hline 2.197 & 2.197 & 2.197 & 35.152 \\
\hline 2.197 & 2.197 & 2.197 & 4.394 \\
\hline 2.197 & 61.516 & 8.788 & 87.88 \\
\hline 2.197 & 2.197 & 15.379 & 10.985 \\
\hline 13.182 & 2.197 & 6.591 & 2.197 \\
\hline 3372.39 & 26.364 & 2.197 & 15.379 \\
\hline 19.773 & 6.591 & 2.197 & 19.773 \\
\hline 30.758 & 35.152 & 2.197 & 4.394 \\
\hline 2.197 & 2.197 & 2.197 & 10.985 \\
\hline 19.773 & 2.197 & 2.197 & 2.197 \\
\hline 2.197 & 2.197 & 2.197 & 2.197 \\
\hline 15.379 & 6.591 & 2.197 & 4.394 \\
\hline 59.319 & 4.394 & 4.394 & 30.758 \\
\hline 52.728 & 2.197 & 2.197 & 2.197 \\
\hline 4.394 & 17.576 & 2.197 & 37.349 \\
\hline 2.197 & 6.591 & 90.077 & 39.546 \\
\hline 2.197 & 1298.43 & 402.051 & 39.546 \\
\hline 2.197 & 147.199 & 4.394 & 2.197 \\
\hline 32.955 & 30.758 & 125.229 & 2.197 \\
\hline 2.197 & 10.985 & 2.197 & 169.169 \\
\hline 2.197 & 2.197 & 2.197 & 2.197 \\
\hline 4.394 & 6.591 & 2.197 & 8.788 \\
\hline 15.379 & 41.743 & 70.304 & 2.197 \\
\hline 4.394 & 59.319 & 17.576 & 2.197 \\
\hline 10.985 & 41.743 & 2.197 & 4.394 \\
\hline 15.379 & 61.516 & 4.394 & 276.822 \\
\hline 2.197 & 13.182 & 4.394 & 2.197 \\
\hline 4.394 & 13.182 & 2.197 & 26.364 \\
\hline
\end{tabular}




\begin{tabular}{|c|c|c|c|}
\hline 50.531 & 2.197 & 2.197 & 118.638 \\
\hline 355.914 & 2.197 & 2.197 & 54.925 \\
\hline 2.197 & 28.561 & 2.197 & 65.91 \\
\hline 10.985 & 43.94 & 2.197 & 2.197 \\
\hline 4.394 & 2.197 & 4.394 & 34185.3 \\
\hline 6.591 & 120.835 & 109.85 & 2.197 \\
\hline 10.985 & 134.017 & 2.197 & 2.197 \\
\hline 8.788 & 2.197 & 2.197 & 8.788 \\
\hline 2.197 & 2.197 & 2.197 & 2.197 \\
\hline 43.94 & 28.561 & 2.197 & 37.349 \\
\hline 2.197 & 290.004 & 2.197 & 2.197 \\
\hline 2.197 & 103.259 & 6.591 & 2.197 \\
\hline 2.197 & 4.394 & 17.576 & 2.197 \\
\hline 2.197 & 191.139 & 2.197 & 456.976 \\
\hline 37.349 & 35.152 & 2.197 & 28.561 \\
\hline 2.197 & 24.167 & 2.197 & 8.788 \\
\hline 81.289 & 2.197 & 2.197 & 4.394 \\
\hline 6.591 & 2.197 & 8.788 & 2.197 \\
\hline 2.197 & 2.197 & 46.137 & 2.197 \\
\hline 2.197 & 13.182 & 2.197 & 173.563 \\
\hline 10.985 & 8.788 & 2.197 & 6.591 \\
\hline 116.441 & 17.576 & 2.197 & 8.788 \\
\hline 8.788 & 2.197 & 4.394 & 30.758 \\
\hline 8.788 & 8.788 & 2.197 & 6.591 \\
\hline 2.197 & 2.197 & 2.197 & 57.122 \\
\hline 4.394 & 2.197 & 2.197 & 17.576 \\
\hline 37.349 & 10.985 & 2.197 & 63.713 \\
\hline 2.197 & 4.394 & 37.349 & 8.788 \\
\hline 8.788 & 24.167 & 2.197 & 2.197 \\
\hline 4.394 & 85.683 & 2.197 & 48.334 \\
\hline 26.364 & 2.197 & 4.394 & 6.591 \\
\hline 17.576 & 2.197 & 4.394 & 4.394 \\
\hline 6.591 & 59.319 & 138.411 & 10.985 \\
\hline 2.197 & 4.394 & 2.197 & 15.379 \\
\hline 2.197 & 4.394 & 15.379 & 2.197 \\
\hline 2.197 & 2.197 & 2.197 & 2.197 \\
\hline 2.197 & 314.171 & 17.576 & 98.865 \\
\hline 32.955 & 4.394 & 2.197 & 2.197 \\
\hline 2.197 & 217.503 & 2.197 & 2.197 \\
\hline 2.197 & 2.197 & 6.591 & 123.032 \\
\hline 147.199 & 2.197 & 37.349 & 4.394 \\
\hline 2.197 & 153.79 & 116.441 & 10.985 \\
\hline 8.788 & 8.788 & 10.985 & 2.197 \\
\hline 15.379 & 13.182 & 2.197 & 13.182 \\
\hline 15.379 & 435.006 & 248.261 & 2.197 \\
\hline 2.197 & 314.171 & 2.197 & 4.394 \\
\hline 50.531 & 160.381 & 63.713 & 15.379 \\
\hline 4.394 & 15.379 & 50.531 & 2.197 \\
\hline 2.197 & 35.152 & 2.197 & 2.197 \\
\hline 2.197 & 15.379 & 2.197 & 136.214 \\
\hline
\end{tabular}




\begin{tabular}{|c|c|c|c|}
\hline 10.985 & 2.197 & 6.591 & 50.531 \\
\hline 8.788 & 6.591 & 52.728 & 6.591 \\
\hline 6.591 & 10.985 & 2.197 & 10.985 \\
\hline 2.197 & 10.985 & 2.197 & 26.364 \\
\hline 2.197 & 2.197 & 2.197 & 8.788 \\
\hline 8.788 & 257.049 & 2.197 & 8.788 \\
\hline 13.182 & 21.97 & 2.197 & 13.182 \\
\hline 2.197 & 2.197 & 37.349 & 246.064 \\
\hline 4.394 & 2.197 & 582.205 & 8.788 \\
\hline 19.773 & 13.182 & 2.197 & 24.167 \\
\hline 6.591 & 2.197 & 10.985 & 353.717 \\
\hline 68.107 & 32.955 & 13.182 & 54.925 \\
\hline 2.197 & 360.308 & 2.197 & 2.197 \\
\hline 17.576 & 2.197 & 2.197 & 2.197 \\
\hline 2.197 & 39.546 & 4.394 & 8.788 \\
\hline 8.788 & 39.546 & 2.197 & 2.197 \\
\hline 182.351 & 2.197 & 4.394 & 164.775 \\
\hline 19.773 & 2.197 & 2.197 & 61.516 \\
\hline 6.591 & 24.167 & 6.591 & 4.394 \\
\hline 13.182 & 10.985 & 59.319 & 2.197 \\
\hline 65.91 & 15.379 & 63.713 & 2.197 \\
\hline 2.197 & 19.773 & 8.788 & 2.197 \\
\hline 2.197 & 4.394 & 79.092 & 2.197 \\
\hline 2.197 & 41.743 & 2.197 & 17.576 \\
\hline 76.895 & 2.197 & 2.197 & 48.334 \\
\hline 4.394 & 2.197 & 65.91 & 127.426 \\
\hline 6.591 & 2.197 & 4.394 & 8.788 \\
\hline 6.591 & 2.197 & 2.197 & 692.055 \\
\hline 8.788 & 6.591 & 2.197 & 2.197 \\
\hline 4.394 & 2.197 & 2.197 & 35.152 \\
\hline 2.197 & 2.197 & 35.152 & 753.571 \\
\hline 2.197 & 101.062 & 4.394 & 81.289 \\
\hline 6.591 & 4.394 & 173.563 & 123.032 \\
\hline 127.426 & 35.152 & 2.197 & 2.197 \\
\hline 171.366 & 10.985 & 2.197 & 24.167 \\
\hline 599.781 & 2.197 & 13.182 & 127.426 \\
\hline 76.895 & 445.991 & 10.985 & 3288.91 \\
\hline 2.197 & 79.092 & 2.197 & 4.394 \\
\hline 70.304 & 24.167 & 2.197 & 26.364 \\
\hline 32.955 & 96.668 & 2.197 & 41.743 \\
\hline 2.197 & 101.062 & 26.364 & 114.244 \\
\hline 2.197 & 15.379 & 2.197 & 171.366 \\
\hline 4.394 & 74.698 & 2.197 & 24.167 \\
\hline 2.197 & 2.197 & 4.394 & 83.486 \\
\hline 8.788 & 2.197 & 4.394 & 2.197 \\
\hline 2.197 & 2.197 & 2.197 & 2.197 \\
\hline 184.548 & 17.576 & 4.394 & 2.197 \\
\hline 2.197 & 2.197 & 4.394 & 2.197 \\
\hline 13.182 & 87.88 & 32.955 & 2.197 \\
\hline 4.394 & 2.197 & 191.139 & 13.182 \\
\hline
\end{tabular}




\begin{tabular}{|c|c|c|c|}
\hline 2.197 & 2.197 & 2.197 & 17.576 \\
\hline 4.394 & 2.197 & 2.197 & 21.97 \\
\hline 393.263 & 65.91 & 2.197 & 4.394 \\
\hline 2.197 & 125.229 & 4.394 & 2.197 \\
\hline 8.788 & 19.773 & 101.062 & 15.379 \\
\hline 6.591 & 4.394 & 2.197 & 37.349 \\
\hline 6.591 & 2.197 & 2.197 & 8.788 \\
\hline 10.985 & 2.197 & 2.197 & 2.197 \\
\hline 32.955 & 213.109 & 4.394 & 2.197 \\
\hline 8.788 & 57.122 & 19.773 & 2.197 \\
\hline 19.773 & 39.546 & 92.274 & 85.683 \\
\hline 2.197 & 4.394 & 15.379 & 2.197 \\
\hline 85.683 & 6.591 & 96.668 & 8.788 \\
\hline 2.197 & 68.107 & 6.591 & 2.197 \\
\hline 2.197 & 46.137 & 2.197 & 4.394 \\
\hline 2.197 & 8.788 & 68.107 & 2.197 \\
\hline 2.197 & 2.197 & 2.197 & 48.334 \\
\hline 2.197 & 13.182 & 2.197 & 799.708 \\
\hline 70.304 & 74.698 & 2.197 & 17.576 \\
\hline 4.394 & 8.788 & 74.698 & 8.788 \\
\hline 215.306 & 6.591 & 2.197 & 4.394 \\
\hline 4.394 & 2.197 & 68.107 & 6.591 \\
\hline 2.197 & 6.591 & 2.197 & 2.197 \\
\hline 2.197 & 46.137 & 30.758 & 21.97 \\
\hline 2.197 & 109.85 & 4.394 & 2.197 \\
\hline 92.274 & 327.353 & 6.591 & 2.197 \\
\hline 2.197 & 166.972 & 26.364 & 41.743 \\
\hline 2.197 & 2.197 & 2.197 & 8.788 \\
\hline 2.197 & 15.379 & 2.197 & 37.349 \\
\hline 2.197 & 6.591 & 2.197 & 2.197 \\
\hline 48.334 & 2.197 & 10.985 & 8.788 \\
\hline 2.197 & 2.197 & 2.197 & 4.394 \\
\hline 4.394 & 10.985 & 15.379 & 226.291 \\
\hline 4.394 & 94.471 & 2.197 & 8.788 \\
\hline 4.394 & 28.561 & 15.379 & 2.197 \\
\hline 8.788 & 2.197 & 2.197 & 8.788 \\
\hline 2.197 & 28.561 & 6.591 & 87.88 \\
\hline 4.394 & 2.197 & 270.231 & 1223.73 \\
\hline 2.197 & 19.773 & 21.97 & 6.591 \\
\hline 2.197 & 8.788 & 2.197 & 145.002 \\
\hline 2.197 & 6.591 & 8.788 & 15.379 \\
\hline 2.197 & 43.94 & 4.394 & 6.591 \\
\hline 2.197 & 43.94 & 2.197 & 109.85 \\
\hline 2.197 & 2.197 & 2.197 & 17.576 \\
\hline 2.197 & 21.97 & 4.394 & 8216.78 \\
\hline 35.152 & 2.197 & 94.471 & 782.132 \\
\hline 17.576 & 2.197 & 2.197 & 2.197 \\
\hline 2.197 & 57.122 & 35.152 & 8.788 \\
\hline 2.197 & 333.944 & 19.773 & 127.426 \\
\hline 2.197 & 21.97 & 35.152 & 19.773 \\
\hline
\end{tabular}




\begin{tabular}{|c|c|c|c|}
\hline 2.197 & 2.197 & 2.197 & 4.394 \\
\hline 4.394 & 2.197 & 41.743 & 4.394 \\
\hline 2.197 & 2.197 & 2.197 & 2.197 \\
\hline 2.197 & 21.97 & 2.197 & 2.197 \\
\hline 4.394 & 2.197 & 2.197 & 4.394 \\
\hline 8.788 & 8.788 & 4.394 & 443.794 \\
\hline 13.182 & 4.394 & 2.197 & 279.019 \\
\hline 21.97 & 2.197 & 94.471 & 204.321 \\
\hline 24.167 & 2.197 & 4.394 & 2.197 \\
\hline 2.197 & 50.531 & 48.334 & 2.197 \\
\hline 2.197 & 19.773 & 15.379 & 8.788 \\
\hline 2.197 & 8.788 & 6.591 & 4.394 \\
\hline 2.197 & 2.197 & 4.394 & 6.591 \\
\hline 4.394 & 4.394 & 2.197 & 41.743 \\
\hline 131.82 & 2.197 & 125.229 & 2.197 \\
\hline 281.216 & 4.394 & 2.197 & 4.394 \\
\hline 8.788 & 654.706 & 2.197 & 2.197 \\
\hline 2.197 & 2.197 & 2.197 & 107.653 \\
\hline 120.835 & 219.7 & 17.576 & 6.591 \\
\hline 17.576 & 2.197 & 4.394 & 2.197 \\
\hline 50.531 & 2.197 & 8.788 & 4.394 \\
\hline 4.394 & 2.197 & 2.197 & 35.152 \\
\hline 2.197 & 24.167 & 2.197 & 15.379 \\
\hline 2.197 & 39.546 & 41.743 & 2.197 \\
\hline 6.591 & 10.985 & 2.197 & 2.197 \\
\hline 4.394 & 2.197 & 2.197 & 4.394 \\
\hline 4.394 & 98.865 & 57.122 & 2.197 \\
\hline 107.653 & 35.152 & 2.197 & 41.743 \\
\hline 2.197 & 2.197 & 2.197 & 2.197 \\
\hline 2.197 & 2.197 & 2.197 & 2.197 \\
\hline 17.576 & 52.728 & 2.197 & 61.516 \\
\hline 4.394 & 2.197 & 2.197 & 2.197 \\
\hline 2.197 & 2.197 & 2.197 & 4.394 \\
\hline 6.591 & 39.546 & 2.197 & 8.788 \\
\hline 6.591 & 32.955 & 2.197 & 72.501 \\
\hline 21.97 & 8.788 & 6.591 & 2.197 \\
\hline 52.728 & 2.197 & 2.197 & 2.197 \\
\hline 10.985 & 2.197 & 2.197 & 140.608 \\
\hline 17.576 & 6.591 & 4.394 & 48.334 \\
\hline 4.394 & 43.94 & 30.758 & 2.197 \\
\hline 465.764 & 2.197 & 4.394 & 158.184 \\
\hline 2.197 & 6.591 & 26.364 & 2.197 \\
\hline 2.197 & 2.197 & 2.197 & 21.97 \\
\hline 2.197 & 17.576 & 10.985 & 54.925 \\
\hline 2.197 & 8.788 & 2.197 & 2.197 \\
\hline 2.197 & 2.197 & 2.197 & 13.182 \\
\hline 13.182 & 10.985 & 2.197 & 94.471 \\
\hline 2.197 & 8.788 & 2.197 & 13.182 \\
\hline 2.197 & 24.167 & 136.214 & 2.197 \\
\hline 4.394 & 13.182 & 13.182 & 2.197 \\
\hline
\end{tabular}




\begin{tabular}{|c|c|c|c|}
\hline 397.657 & 19.773 & 219.7 & 2.197 \\
\hline 13.182 & 70.304 & 4.394 & 2.197 \\
\hline 35.152 & 54.925 & 2.197 & 2.197 \\
\hline 37.349 & 2.197 & 2.197 & 2.197 \\
\hline 2.197 & 2.197 & 4.394 & 2.197 \\
\hline 17.576 & 109.85 & 24.167 & 2.197 \\
\hline 6.591 & 4.394 & 2.197 & 50.531 \\
\hline 19.773 & 32.955 & 2.197 & 2.197 \\
\hline 96.668 & 87.88 & 2.197 & 2.197 \\
\hline 15.379 & 4.394 & 15.379 & 13.182 \\
\hline 2.197 & 59.319 & 2.197 & 160.381 \\
\hline 2.197 & 15.379 & 2.197 & 37.349 \\
\hline 4.394 & 6.591 & 2.197 & 30.758 \\
\hline 96.668 & 26.364 & 2.197 & 30.758 \\
\hline 48.334 & 213.109 & 4.394 & 8.788 \\
\hline 10.985 & 46.137 & 6.591 & 6.591 \\
\hline 2.197 & 8.788 & 13.182 & 2.197 \\
\hline 116.441 & 4.394 & 2.197 & 10.985 \\
\hline 4.394 & 120.835 & 2.197 & 63.713 \\
\hline 4.394 & 241.67 & 28.561 & 101.062 \\
\hline 2.197 & 46.137 & 2.197 & 125.229 \\
\hline 19.773 & 17.576 & 4.394 & 127.426 \\
\hline 2.197 & 2.197 & 74.698 & 2.197 \\
\hline 2.197 & 10.985 & 46.137 & 24.167 \\
\hline 2.197 & 24.167 & 81.289 & 2.197 \\
\hline 2.197 & 2.197 & 94.471 & 2.197 \\
\hline 103.259 & 136.214 & 2.197 & 2.197 \\
\hline 4.394 & 107.653 & 2.197 & 2.197 \\
\hline 2.197 & 204.321 & 2.197 & 2.197 \\
\hline 21.97 & 4.394 & 4.394 & 2.197 \\
\hline 2.197 & 50.531 & 19.773 & 63.713 \\
\hline 10.985 & 254.852 & 43.94 & 10.985 \\
\hline 4.394 & 2.197 & 2.197 & 2.197 \\
\hline 2.197 & 52.728 & 2.197 & 2.197 \\
\hline 2.197 & 2.197 & 2.197 & 2.197 \\
\hline 118.638 & 10.985 & 2.197 & 4.394 \\
\hline 2.197 & 26.364 & 8.788 & 2.197 \\
\hline 2.197 & 2.197 & 30.758 & 8.788 \\
\hline 2.197 & 59.319 & 10.985 & 24.167 \\
\hline 2.197 & 4.394 & 8.788 & 6.591 \\
\hline 1986.09 & 2.197 & 377.884 & 2.197 \\
\hline 169.169 & 2.197 & 74.698 & 4.394 \\
\hline 4.394 & 6.591 & 8.788 & 2.197 \\
\hline 2.197 & 520.689 & 2.197 & 46.137 \\
\hline 13.182 & 4.394 & 24.167 & 30.758 \\
\hline 129.623 & 83.486 & 2.197 & 90.077 \\
\hline 8.788 & 641.524 & 2.197 & 2.197 \\
\hline 2.197 & 79.092 & 4.394 & 8.788 \\
\hline 15.379 & 1584.04 & 4.394 & 85.683 \\
\hline 6.591 & 8.788 & 2.197 & 4.394 \\
\hline
\end{tabular}




\begin{tabular}{|c|c|c|c|}
\hline 2.197 & 221.897 & 2.197 & 197.73 \\
\hline 2.197 & 4.394 & 2.197 & 2.197 \\
\hline 2.197 & 2.197 & 2.197 & 46.137 \\
\hline 2.197 & 10.985 & 52.728 & 8.788 \\
\hline 2.197 & 2.197 & 13.182 & 2.197 \\
\hline 2.197 & 19.773 & 2.197 & 4.394 \\
\hline 24.167 & 2.197 & 4.394 & 4.394 \\
\hline 83.486 & 8.788 & 32.955 & 2.197 \\
\hline 85.683 & 72.501 & 314.171 & 2.197 \\
\hline 6.591 & 129.623 & 6.591 & 65.91 \\
\hline 2.197 & 4.394 & 19.773 & 2.197 \\
\hline 19.773 & 65.91 & 41.743 & 63.713 \\
\hline 24.167 & 85.683 & 2.197 & 61.516 \\
\hline 13.182 & 68.107 & 6637.14 & 26.364 \\
\hline 13.182 & 21.97 & 2.197 & 68.107 \\
\hline 2.197 & 2.197 & 41.743 & 35.152 \\
\hline 2.197 & 4.394 & 13.182 & 4.394 \\
\hline 2.197 & 19.773 & 61.516 & 8.788 \\
\hline 13.182 & 81.289 & 2.197 & 52.728 \\
\hline 2.197 & 35.152 & 4.394 & 2.197 \\
\hline 15.379 & 6.591 & 17.576 & 35.152 \\
\hline 338.338 & 10.985 & 15.379 & 19.773 \\
\hline 17.576 & 28.561 & 32.955 & 32.955 \\
\hline 2.197 & 4.394 & 2.197 & 182.351 \\
\hline 39.546 & 10.985 & 2.197 & 96.668 \\
\hline 17.576 & 129.623 & 2.197 & 24.167 \\
\hline 4.394 & 2.197 & 39.546 & 8.788 \\
\hline 6.591 & 311.974 & 4.394 & 19.773 \\
\hline 4.394 & 4.394 & 2.197 & 30.758 \\
\hline 2.197 & 4.394 & 2.197 & 13.182 \\
\hline 2.197 & 136.214 & 2.197 & 61.516 \\
\hline 35.152 & 26.364 & 2.197 & 935.922 \\
\hline 2.197 & 2.197 & 2.197 & 421.824 \\
\hline 26.364 & 2.197 & 2.197 & 28.561 \\
\hline 2.197 & 2.197 & 2.197 & 2.197 \\
\hline 13.182 & 2.197 & 28.561 & 6.591 \\
\hline 15.379 & 2.197 & 8.788 & 2.197 \\
\hline 2.197 & 2.197 & 2.197 & 79.092 \\
\hline 2.197 & 590.993 & 2.197 & 19.773 \\
\hline 8.788 & 197.73 & 265.837 & 4.394 \\
\hline 35.152 & 8.788 & 268.034 & 39.546 \\
\hline 4.394 & 109.85 & 4.394 & 32.955 \\
\hline 2.197 & 2.197 & 57.122 & 4.394 \\
\hline 2.197 & 17.576 & 8.788 & 70.304 \\
\hline 2.197 & 382.278 & 2.197 & 116.441 \\
\hline 10.985 & 6.591 & 2.197 & 2.197 \\
\hline 2.197 & 4.394 & 155.987 & 96.668 \\
\hline 37.349 & 4.394 & 103.259 & 79.092 \\
\hline 8.788 & 2.197 & 17.576 & 19.773 \\
\hline 2.197 & 4.394 & 39.546 & 2.197 \\
\hline
\end{tabular}




\begin{tabular}{|c|c|c|c|}
\hline 6.591 & 13.182 & 8.788 & 2.197 \\
\hline 4.394 & 107.653 & 35.152 & 6.591 \\
\hline 28.561 & 8.788 & 28.561 & 2.197 \\
\hline 120.835 & 2.197 & 2.197 & 2.197 \\
\hline 2.197 & 50.531 & 268.034 & 28.561 \\
\hline 2.197 & 2.197 & 17.576 & 21.97 \\
\hline 2.197 & 15.379 & 4.394 & 8.788 \\
\hline 4.394 & 2.197 & 2.197 & 230.685 \\
\hline 4.394 & 13.182 & 6.591 & 72.501 \\
\hline 17.576 & 15.379 & 4.394 & 13.182 \\
\hline 6.591 & 6.591 & 2.197 & 828.269 \\
\hline 21.97 & 2.197 & 2.197 & 2.197 \\
\hline 162.578 & 235.079 & 2.197 & 19.773 \\
\hline 2.197 & 4.394 & 21.97 & 8.788 \\
\hline 2.197 & 6.591 & 2.197 & 13.182 \\
\hline 4.394 & 2.197 & 90.077 & 8.788 \\
\hline 10.985 & 2.197 & 32.955 & 424.021 \\
\hline 410.839 & 50.531 & 6.591 & 138.411 \\
\hline 10.985 & 6.591 & 2.197 & 2.197 \\
\hline 4.394 & 26.364 & 2.197 & 2.197 \\
\hline 61.516 & 6.591 & 2.197 & 8.788 \\
\hline 32.955 & 2.197 & 2.197 & 6.591 \\
\hline 4.394 & 2.197 & 24.167 & 2.197 \\
\hline 70.304 & 26.364 & 19.773 & 2.197 \\
\hline 2.197 & 2.197 & 2.197 & 21.97 \\
\hline 15.379 & 74.698 & 37.349 & 2.197 \\
\hline 2.197 & 15.379 & 21.97 & 90.077 \\
\hline 2.197 & 30.758 & 290.004 & 83.486 \\
\hline 4.394 & 4.394 & 4.394 & 8.788 \\
\hline 6.591 & 32.955 & 37.349 & 13.182 \\
\hline 2.197 & 10.985 & 21.97 & 13.182 \\
\hline 24.167 & 26.364 & 2.197 & 2.197 \\
\hline 2.197 & 2.197 & 2.197 & 13.182 \\
\hline 2.197 & 50.531 & 6.591 & 2.197 \\
\hline 2.197 & 127.426 & 4.394 & 61.516 \\
\hline 2.197 & 2.197 & 15.379 & 4.394 \\
\hline 2.197 & 2.197 & 2.197 & 30.758 \\
\hline 63.713 & 76.895 & 48.334 & 2.197 \\
\hline 6.591 & 2.197 & 801.905 & 19.773 \\
\hline 15.379 & 239.473 & 24.167 & 13.182 \\
\hline 2.197 & 17.576 & 21.97 & 2.197 \\
\hline 2.197 & 24.167 & 6.591 & 4.394 \\
\hline 2.197 & 2.197 & 28.561 & 2.197 \\
\hline 32.955 & 2.197 & 1197.36 & 8.788 \\
\hline 2.197 & 10.985 & 4.394 & 2.197 \\
\hline 127.426 & 30.758 & 2.197 & 6.591 \\
\hline 2139.88 & 2.197 & 2.197 & 597.584 \\
\hline 6.591 & 26.364 & 2.197 & 61.516 \\
\hline 2.197 & 2.197 & 15.379 & 17.576 \\
\hline 19.773 & 30.758 & 2.197 & 41.743 \\
\hline
\end{tabular}




\begin{tabular}{|c|c|c|c|}
\hline 2.197 & 2.197 & 2.197 & 15.379 \\
\hline 4.394 & 30.758 & 2.197 & 147.199 \\
\hline 4.394 & 24.167 & 2.197 & 6.591 \\
\hline 24.167 & 52.728 & 2.197 & 103.259 \\
\hline 2.197 & 2.197 & 6.591 & 2.197 \\
\hline 26.364 & 2.197 & 158.184 & 2.197 \\
\hline 15.379 & 24.167 & 2.197 & 2.197 \\
\hline 8.788 & 2.197 & 2.197 & 8.788 \\
\hline 6.591 & 4.394 & 8.788 & 118.638 \\
\hline 612.963 & 17.576 & 50.531 & 72.501 \\
\hline 10.985 & 10.985 & 57.122 & 210.912 \\
\hline 13.182 & 6.591 & 2.197 & 4.394 \\
\hline 2.197 & 17.576 & 2.197 & 2.197 \\
\hline 2.197 & 8.788 & 59.319 & 30.758 \\
\hline 2.197 & 13.182 & 303.186 & 2.197 \\
\hline 2.197 & 4.394 & 17.576 & 81.289 \\
\hline 72.501 & 2.197 & 8.788 & 164.775 \\
\hline 26.364 & 219.7 & 2.197 & 2.197 \\
\hline 2.197 & 2.197 & 2.197 & 15.379 \\
\hline 2.197 & 10.985 & 2.197 & 2.197 \\
\hline 2.197 & 28.561 & 109.85 & 2.197 \\
\hline 2.197 & 2.197 & 4.394 & 2.197 \\
\hline 2.197 & 494.325 & 8.788 & 2.197 \\
\hline 439.4 & 32.955 & 2.197 & 2.197 \\
\hline 2.197 & 76.895 & 52.728 & 2.197 \\
\hline 10.985 & 54.925 & 26.364 & 2.197 \\
\hline 2.197 & 2.197 & 2.197 & 4.394 \\
\hline 6.591 & 2.197 & 4.394 & 6.591 \\
\hline 6.591 & 2.197 & 456.976 & 2.197 \\
\hline 6.591 & 2.197 & 107.653 & 10.985 \\
\hline 2.197 & 4.394 & 2.197 & 8.788 \\
\hline 6.591 & 6.591 & 4.394 & 21.97 \\
\hline 13.182 & 4.394 & 28.561 & 2.197 \\
\hline 30.758 & 166.972 & 107.653 & 2.197 \\
\hline 6.591 & 4.394 & 32.955 & 30.758 \\
\hline 48.334 & 15.379 & 2.197 & 4.394 \\
\hline 4.394 & 43.94 & 74.698 & 4.394 \\
\hline 96.668 & 2.197 & 2.197 & 2.197 \\
\hline 955.695 & 10.985 & 112.047 & 17.576 \\
\hline 2.197 & 437.203 & 2.197 & 41.743 \\
\hline 2.197 & 10.985 & 17.576 & 2.197 \\
\hline 4.394 & 2.197 & 2.197 & 2.197 \\
\hline 4.394 & 30.758 & 19.773 & 2.197 \\
\hline 26.364 & 2.197 & 8.788 & 2.197 \\
\hline 17.576 & 2.197 & 2.197 & 21.97 \\
\hline 2.197 & 6.591 & 6.591 & 2.197 \\
\hline 2.197 & 2.197 & 4.394 & 41.743 \\
\hline 2.197 & 21.97 & 217.503 & 2.197 \\
\hline 4.394 & 4.394 & 2.197 & 90.077 \\
\hline 2.197 & 287.807 & 2.197 & 15.379 \\
\hline
\end{tabular}




\begin{tabular}{|c|c|c|c|}
\hline 2.197 & 8.788 & 4.394 & 175.76 \\
\hline 2.197 & 160.381 & 39.546 & 35.152 \\
\hline 2.197 & 19.773 & 48.334 & 123.032 \\
\hline 6.591 & 13.182 & 250.458 & 2.197 \\
\hline 4.394 & 177.957 & 2.197 & 134.017 \\
\hline 608.569 & 193.336 & 15.379 & 918.346 \\
\hline 24.167 & 17.576 & 13.182 & 32.955 \\
\hline 8.788 & 217.503 & 68.107 & 6.591 \\
\hline 2.197 & 15.379 & 2.197 & 61.516 \\
\hline 4.394 & 2.197 & 13.182 & 17.576 \\
\hline 6.591 & 37.349 & 2.197 & 241.67 \\
\hline 2.197 & 94.471 & 2.197 & 6.591 \\
\hline 4.394 & 81.289 & 4.394 & 149.396 \\
\hline 6.591 & 2.197 & 2.197 & 162.578 \\
\hline 2.197 & 6.591 & 2.197 & 70.304 \\
\hline 2.197 & 226.291 & 2.197 & 39.546 \\
\hline 2.197 & 413.036 & 13.182 & 140.608 \\
\hline 98.865 & 10.985 & 4.394 & 19.773 \\
\hline 10.985 & 4.394 & 2.197 & 10.985 \\
\hline 63.713 & 2.197 & 2.197 & 37.349 \\
\hline 79.092 & 2.197 & 2.197 & 6.591 \\
\hline 4.394 & 21.97 & 2.197 & 4.394 \\
\hline 571.22 & 344.929 & 239.473 & 2.197 \\
\hline 17.576 & 30.758 & 2.197 & 2.197 \\
\hline 1889.42 & 2.197 & 4.394 & 2.197 \\
\hline 8.788 & 10.985 & 2.197 & 2.197 \\
\hline 21.97 & 8.788 & 2.197 & 2.197 \\
\hline 4.394 & 37.349 & 10.985 & 6.591 \\
\hline 4.394 & 4.394 & 57.122 & 2.197 \\
\hline 10.985 & 4.394 & 26.364 & 8.788 \\
\hline 2.197 & 30.758 & 17.576 & 37.349 \\
\hline 2.197 & 15.379 & 2.197 & 2.197 \\
\hline 2.197 & 6.591 & 6.591 & 4.394 \\
\hline 248.261 & 4.394 & 32.955 & 10.985 \\
\hline 59.319 & 2.197 & 4.394 & 10.985 \\
\hline 2.197 & 241.67 & 2.197 & 8.788 \\
\hline 4.394 & 15.379 & 30.758 & 26.364 \\
\hline 2.197 & 10.985 & 2.197 & 92.274 \\
\hline 83.486 & 21.97 & 2.197 & 19.773 \\
\hline 270.231 & 54.925 & 8.788 & 949.104 \\
\hline 4.394 & 19.773 & 213.109 & 270.231 \\
\hline 562.432 & 6.591 & 2.197 & 6.591 \\
\hline 10.985 & 2.197 & 52.728 & 2.197 \\
\hline 2.197 & 2.197 & 2.197 & 6.591 \\
\hline 2.197 & 2.197 & 2.197 & 17.576 \\
\hline 6.591 & 2014.65 & 2.197 & 2.197 \\
\hline 2.197 & 39.546 & 2.197 & 6.591 \\
\hline 21.97 & 114.244 & 4.394 & 2.197 \\
\hline 15.379 & 2.197 & 4.394 & 2.197 \\
\hline 2.197 & 2.197 & 2.197 & 2.197 \\
\hline
\end{tabular}




\begin{tabular}{|c|c|c|c|}
\hline 81.289 & 4.394 & 2.197 & 4.394 \\
\hline 109.85 & 8.788 & 786.526 & 26.364 \\
\hline 13.182 & 70.304 & 2.197 & 2.197 \\
\hline 90.077 & 2.197 & 2.197 & 2.197 \\
\hline 8.788 & 17.576 & 2.197 & 2.197 \\
\hline 8.788 & 13.182 & 28.561 & 2.197 \\
\hline 2.197 & 8.788 & 2.197 & 2.197 \\
\hline 4.394 & 48.334 & 2.197 & 2.197 \\
\hline 83.486 & 2.197 & 21.97 & 136.214 \\
\hline 8.788 & 6.591 & 2.197 & 129.623 \\
\hline 4.394 & 4.394 & 101.062 & 48.334 \\
\hline 4.394 & 10.985 & 2.197 & 2.197 \\
\hline 24.167 & 41.743 & 2.197 & 784.329 \\
\hline 2.197 & 24.167 & 2.197 & 4.394 \\
\hline 2.197 & 10.985 & 2.197 & 120.835 \\
\hline 131.82 & 2.197 & 2.197 & 10.985 \\
\hline 147.199 & 39.546 & 10.985 & 2.197 \\
\hline 2.197 & 180.154 & 8.788 & 21.97 \\
\hline 6.591 & 13.182 & 74.698 & 41.743 \\
\hline 208.715 & 43.94 & 2.197 & 4.394 \\
\hline 15.379 & 19.773 & 39.546 & 79.092 \\
\hline 149.396 & 2.197 & 2.197 & 17.576 \\
\hline 714.025 & 85.683 & 2.197 & 8.788 \\
\hline 24.167 & 6.591 & 2.197 & 39.546 \\
\hline 2.197 & 2.197 & 48.334 & 118.638 \\
\hline 2.197 & 15.379 & 4.394 & 4.394 \\
\hline 2.197 & 26.364 & 2.197 & 24.167 \\
\hline 2.197 & 10.985 & 2.197 & 2.197 \\
\hline 2.197 & 177.957 & 2.197 & 4.394 \\
\hline 2.197 & 59.319 & 2.197 & 2.197 \\
\hline 105.456 & 63.713 & 2.197 & 28.561 \\
\hline 52.728 & 8.788 & 2.197 & 13.182 \\
\hline 17.576 & 2.197 & 2.197 & 24.167 \\
\hline 2.197 & 6.591 & 76.895 & 395.46 \\
\hline 4.394 & 19.773 & 2.197 & 467.961 \\
\hline 8.788 & 1100.7 & 2.197 & 30.758 \\
\hline 59.319 & 2.197 & 8.788 & 8.788 \\
\hline 57.122 & 10.985 & 2.197 & 2.197 \\
\hline 2.197 & 17.576 & 2.197 & 127.426 \\
\hline 2.197 & 19.773 & 15.379 & 10.985 \\
\hline 2.197 & 6.591 & 2.197 & 10.985 \\
\hline 24.167 & 2.197 & 164.775 & 59.319 \\
\hline 2.197 & 13.182 & 19.773 & 39.546 \\
\hline 2.197 & 21.97 & 2.197 & 2.197 \\
\hline 4.394 & 4.394 & 30.758 & 28.561 \\
\hline 6.591 & 274.625 & 21.97 & 2.197 \\
\hline 8.788 & 8.788 & 2.197 & 10.985 \\
\hline 2.197 & 2.197 & 43.94 & 2.197 \\
\hline 10.985 & 72.501 & 2.197 & 72.501 \\
\hline 197.73 & 61.516 & 30.758 & 13.182 \\
\hline
\end{tabular}




\begin{tabular}{|c|c|c|c|}
\hline 2.197 & 21.97 & 4.394 & 19.773 \\
\hline 2.197 & 4.394 & 4.394 & 151.593 \\
\hline 8.788 & 13.182 & 700.843 & 265.837 \\
\hline 134.017 & 10.985 & 2.197 & 4.394 \\
\hline 17.576 & 303.186 & 8.788 & 2.197 \\
\hline 10.985 & 2.197 & 6.591 & 285.61 \\
\hline 21.97 & 2.197 & 10.985 & 103.259 \\
\hline 50.531 & 2.197 & 2.197 & 21.97 \\
\hline 59.319 & 2.197 & 2.197 & 8.788 \\
\hline 2.197 & 50.531 & 2.197 & 10.985 \\
\hline 2.197 & 6.591 & 4.394 & 6.591 \\
\hline 4.394 & 2.197 & 2.197 & 4.394 \\
\hline 48.334 & 35.152 & 2.197 & 2.197 \\
\hline 6.591 & 4.394 & 2.197 & 184.548 \\
\hline 26.364 & 48.334 & 6.591 & 2.197 \\
\hline 2.197 & 10.985 & 2.197 & 13.182 \\
\hline 2.197 & 48.334 & 2.197 & 131.82 \\
\hline 24.167 & 32.955 & 2.197 & 158.184 \\
\hline 24.167 & 2.197 & 2.197 & 6.591 \\
\hline 123.032 & 202.124 & 2.197 & 24.167 \\
\hline 19.773 & 17.576 & 2.197 & 46.137 \\
\hline 4.394 & 70.304 & 2.197 & 228.488 \\
\hline 6.591 & 8.788 & 2.197 & 2.197 \\
\hline 15.379 & 28.561 & 2.197 & 175.76 \\
\hline 4.394 & 4.394 & 2.197 & 228.488 \\
\hline 8.788 & 10.985 & 2.197 & 4.394 \\
\hline 26.364 & 15.379 & 2.197 & 6.591 \\
\hline 2.197 & 2.197 & 2612.23 & 37.349 \\
\hline 2.197 & 2.197 & 76.895 & 61.516 \\
\hline 13.182 & 2.197 & 2.197 & 10.985 \\
\hline 13.182 & 30.758 & 2.197 & 70.304 \\
\hline 15.379 & 43.94 & 2.197 & 15.379 \\
\hline 4.394 & 8.788 & 149.396 & 8.788 \\
\hline 32.955 & 37.349 & 13.182 & 8.788 \\
\hline 2.197 & 147.199 & 4.394 & 8.788 \\
\hline 37.349 & 13.182 & 6.591 & 32.955 \\
\hline 59.319 & 4.394 & 2.197 & 6.591 \\
\hline 59.319 & 2.197 & 4.394 & 252.655 \\
\hline 15.379 & 4.394 & 6.591 & 26.364 \\
\hline 2.197 & 10.985 & 54.925 & 2.197 \\
\hline 140.608 & 2.197 & 4.394 & 57.122 \\
\hline 24.167 & 2.197 & 2.197 & 48.334 \\
\hline 2.197 & 30.758 & 8.788 & 107.653 \\
\hline 213.109 & 6.591 & 2.197 & 4.394 \\
\hline 2.197 & 61.516 & 588.796 & 109.85 \\
\hline 4.394 & 4.394 & 13.182 & 2.197 \\
\hline 4.394 & 54.925 & 8.788 & 2.197 \\
\hline 287.807 & 2.197 & 107.653 & 68.107 \\
\hline 2.197 & 103.259 & 4.394 & 2.197 \\
\hline 2.197 & 4.394 & 2.197 & 2.197 \\
\hline
\end{tabular}




\begin{tabular}{|c|c|c|c|}
\hline 2.197 & 13.182 & 2.197 & 8.788 \\
\hline 26.364 & 6.591 & 2.197 & 15.379 \\
\hline 2.197 & 4.394 & 37.349 & 4.394 \\
\hline 2.197 & 21.97 & 2.197 & 4.394 \\
\hline 2.197 & 4.394 & 4.394 & 39.546 \\
\hline 2.197 & 10.985 & 2.197 & 136.214 \\
\hline 2.197 & 114.244 & 580.008 & 13.182 \\
\hline 4.394 & 2.197 & 2.197 & 2.197 \\
\hline 50.531 & 26.364 & 2.197 & 2.197 \\
\hline 2.197 & 177.957 & 2.197 & 21.97 \\
\hline 2.197 & 6.591 & 2.197 & 6.591 \\
\hline 2.197 & 21.97 & 2.197 & 153.79 \\
\hline 2.197 & 4.394 & 2.197 & 17.576 \\
\hline 2.197 & 4.394 & 2.197 & 127.426 \\
\hline 28.561 & 199.927 & 160.381 & 4.394 \\
\hline 4.394 & 2.197 & 4.394 & 43.94 \\
\hline 13.182 & 10.985 & 2.197 & 540.462 \\
\hline 24.167 & 4.394 & 8.788 & 10.985 \\
\hline 2.197 & 19.773 & 6.591 & 2.197 \\
\hline 2.197 & 76.895 & 46.137 & 37.349 \\
\hline 13.182 & 48.334 & 2.197 & 13.182 \\
\hline 2.197 & 32.955 & 6.591 & 6.591 \\
\hline 120.835 & 13.182 & 15.379 & 6.591 \\
\hline 13.182 & 2.197 & 6.591 & 101.062 \\
\hline 10.985 & 10.985 & 41.743 & 10.985 \\
\hline 171.366 & 32.955 & 2.197 & 13.182 \\
\hline 17.576 & 13.182 & 2.197 & 39.546 \\
\hline 151.593 & 204.321 & 2.197 & 10.985 \\
\hline 35.152 & 15.379 & 13.182 & 10.985 \\
\hline 13.182 & 8.788 & 98.865 & 4.394 \\
\hline 13.182 & 2.197 & 6.591 & 8.788 \\
\hline 10.985 & 2.197 & 2.197 & 2.197 \\
\hline 2.197 & 2.197 & 4.394 & 4.394 \\
\hline 2.197 & 2.197 & 2.197 & 134.017 \\
\hline 57.122 & 4.394 & 10.985 & 10.985 \\
\hline 81.289 & 17.576 & 2.197 & 2.197 \\
\hline 2.197 & 32.955 & 2.197 & 4.394 \\
\hline 2.197 & 2.197 & 2.197 & 8.788 \\
\hline 4.394 & 2.197 & 281.216 & 17.576 \\
\hline 6.591 & 6.591 & 4.394 & 4.394 \\
\hline 2.197 & 2.197 & 8.788 & 37.349 \\
\hline 2.197 & 61.516 & 8.788 & 43.94 \\
\hline 2.197 & 6.591 & 2.197 & 19.773 \\
\hline 2.197 & 19.773 & 10.985 & 2.197 \\
\hline 2.197 & 46.137 & 2.197 & 10.985 \\
\hline 13.182 & 30.758 & 6.591 & 4.394 \\
\hline 4.394 & 13.182 & 72.501 & 129.623 \\
\hline 6.591 & 114.244 & 2.197 & 4.394 \\
\hline 2.197 & 4.394 & 41.743 & 41.743 \\
\hline 2.197 & 6.591 & 2.197 & 10.985 \\
\hline
\end{tabular}




\begin{tabular}{|c|c|c|c|}
\hline 2.197 & 2.197 & 2.197 & 68.107 \\
\hline 2.197 & 74.698 & 2.197 & 10.985 \\
\hline 19.773 & 35.152 & 2.197 & 2.197 \\
\hline 4.394 & 6.591 & 2.197 & 2.197 \\
\hline 65.91 & 6.591 & 2.197 & 8.788 \\
\hline 17.576 & 2.197 & 2.197 & 13.182 \\
\hline 2.197 & 2.197 & 81.289 & 52.728 \\
\hline 4.394 & 79.092 & 4.394 & 2.197 \\
\hline 2.197 & 6.591 & 2.197 & 2.197 \\
\hline 30.758 & 28.561 & 10.985 & 26.364 \\
\hline 52.728 & 2.197 & 2.197 & 257.049 \\
\hline 43.94 & 10.985 & 854.633 & 2.197 \\
\hline 2.197 & 6.591 & 103.259 & 2.197 \\
\hline 2.197 & 2.197 & 2.197 & 597.584 \\
\hline 2.197 & 28.561 & 118.638 & 797.511 \\
\hline 15.379 & 30.758 & 2.197 & 98.865 \\
\hline 2.197 & 19.773 & 46.137 & 10.985 \\
\hline 2.197 & 32.955 & 2.197 & 1432.44 \\
\hline 2.197 & 2.197 & 2.197 & 30.758 \\
\hline 2.197 & 15.379 & 2.197 & 10.985 \\
\hline 10.985 & 2.197 & 4.394 & 2.197 \\
\hline 2.197 & 6.591 & 173.563 & 2.197 \\
\hline 15.379 & 2.197 & 195.533 & 15.379 \\
\hline 2.197 & 8.788 & 2.197 & 2.197 \\
\hline 39.546 & 4.394 & 10.985 & 109.85 \\
\hline 10.985 & 4.394 & 2.197 & 116.441 \\
\hline 320.762 & 142.805 & 202.124 & 6.591 \\
\hline 2.197 & 28.561 & 2.197 & 2.197 \\
\hline 68.107 & 285.61 & 4.394 & 21.97 \\
\hline 15.379 & 2.197 & 2.197 & 2.197 \\
\hline 4.394 & 2.197 & 2.197 & 6.591 \\
\hline 4.394 & 13.182 & 4.394 & 4.394 \\
\hline 2.197 & 2.197 & 760.162 & 85.683 \\
\hline 4.394 & 2.197 & 15.379 & 52.728 \\
\hline 2.197 & 2.197 & 268.034 & 182.351 \\
\hline 2.197 & 17.576 & 2.197 & 2.197 \\
\hline 4.394 & 87.88 & 32.955 & 19.773 \\
\hline 2.197 & 10.985 & 10.985 & 35.152 \\
\hline 4.394 & 2.197 & 2.197 & 35.152 \\
\hline 2.197 & 261.443 & 2.197 & 2.197 \\
\hline 6.591 & 6.591 & 17.576 & 17.576 \\
\hline 6.591 & 6.591 & 188.942 & 50.531 \\
\hline 4.394 & 4.394 & 2.197 & 4.394 \\
\hline 6.591 & 10.985 & 2.197 & 4.394 \\
\hline 1783.96 & 10.985 & 2.197 & 8.788 \\
\hline 2.197 & 2.197 & 2.197 & 13.182 \\
\hline 8.788 & 8.788 & 2.197 & 10.985 \\
\hline 41.743 & 24.167 & 2.197 & 2.197 \\
\hline 2.197 & 54.925 & 87.88 & 2.197 \\
\hline 4.394 & 21.97 & 4.394 & 26.364 \\
\hline
\end{tabular}




\begin{tabular}{|c|c|c|c|}
\hline 2.197 & 24.167 & 4.394 & 6.591 \\
\hline 21.97 & 17.576 & 15.379 & 26.364 \\
\hline 1333.58 & 6.591 & 48.334 & 19.773 \\
\hline 2.197 & 2.197 & 2.197 & 8.788 \\
\hline 8.788 & 6.591 & 6.591 & 19.773 \\
\hline 70.304 & 2.197 & 13.182 & 87.88 \\
\hline 37.349 & 85.683 & 8.788 & 61.516 \\
\hline 21.97 & 41.743 & 4.394 & 57.122 \\
\hline 2.197 & 94.471 & 4.394 & 8.788 \\
\hline 2.197 & 52.728 & 2.197 & 26.364 \\
\hline 4.394 & 6.591 & 2.197 & 2.197 \\
\hline 15.379 & 2.197 & 6.591 & 2.197 \\
\hline 10.985 & 8.788 & 68.107 & 2.197 \\
\hline 5826.44 & 2.197 & 30.758 & 6.591 \\
\hline 17.576 & 68.107 & 10.985 & 2.197 \\
\hline 13.182 & 120.835 & 8.788 & 26.364 \\
\hline 2.197 & 13.182 & 48.334 & 4.394 \\
\hline 2.197 & 311.974 & 2.197 & 169.169 \\
\hline 215.306 & 109.85 & 2.197 & 114.244 \\
\hline 81.289 & 2.197 & 2.197 & 37.349 \\
\hline 2.197 & 6.591 & 21.97 & 83.486 \\
\hline 2.197 & 70.304 & 2.197 & 2.197 \\
\hline 2.197 & 21.97 & 24.167 & 2.197 \\
\hline 2.197 & 21.97 & 2.197 & 142.805 \\
\hline 28.561 & 4.394 & 2.197 & 6.591 \\
\hline 2.197 & 2.197 & 2.197 & 2.197 \\
\hline 2.197 & 2.197 & 4.394 & 52.728 \\
\hline 2.197 & 46.137 & 2.197 & 10.985 \\
\hline 32.955 & 206.518 & 2.197 & 2.197 \\
\hline 2.197 & 6.591 & 6.591 & 2.197 \\
\hline 2.197 & 19.773 & 41.743 & 325.156 \\
\hline 21.97 & 74.698 & 4.394 & 24.167 \\
\hline 17.576 & 4.394 & 740.389 & 4363.24 \\
\hline 48.334 & 2.197 & 2.197 & 8.788 \\
\hline 2.197 & 4.394 & 6.591 & 39.546 \\
\hline 2.197 & 2.197 & 30.758 & 10.985 \\
\hline 4.394 & 30.758 & 2.197 & 15.379 \\
\hline 17.576 & 50.531 & 2.197 & 13.182 \\
\hline 4.394 & 4.394 & 2.197 & 971.074 \\
\hline 59.319 & 199.927 & 35.152 & 13.182 \\
\hline 2.197 & 48.334 & 2.197 & 2.197 \\
\hline 2.197 & 41.743 & 2.197 & 30.758 \\
\hline 24.167 & 252.655 & 2.197 & 454.779 \\
\hline 2.197 & 59.319 & 243.867 & 52.728 \\
\hline 4.394 & 2.197 & 2.197 & 2.197 \\
\hline 2.197 & 6.591 & 2.197 & 2.197 \\
\hline 2.197 & 54.925 & 2.197 & 2.197 \\
\hline 2.197 & 373.49 & 28.561 & 26.364 \\
\hline 65.91 & 6.591 & 6.591 & 2.197 \\
\hline 6.591 & 2.197 & 39.546 & 2.197 \\
\hline
\end{tabular}




\begin{tabular}{|c|c|c|c|}
\hline 2.197 & 4.394 & 10.985 & 2.197 \\
\hline 2.197 & 17.576 & 8.788 & 2.197 \\
\hline 4.394 & 2.197 & 2.197 & 2.197 \\
\hline 6.591 & 169.169 & 65.91 & 2.197 \\
\hline 2.197 & 65.91 & 2.197 & 59.319 \\
\hline 19.773 & 2.197 & 2.197 & 142.805 \\
\hline 2.197 & 24.167 & 402.051 & 116.441 \\
\hline 188.942 & 2.197 & 177.957 & 32.955 \\
\hline 4.394 & 2.197 & 4.394 & 65.91 \\
\hline 105.456 & 2.197 & 8.788 & 2.197 \\
\hline 4.394 & 114.244 & 10.985 & 52.728 \\
\hline 2.197 & 2.197 & 109.85 & 23833.1 \\
\hline 2.197 & 2.197 & 15.379 & 35.152 \\
\hline 2.197 & 2.197 & 21.97 & 2.197 \\
\hline 2.197 & 215.306 & 8.788 & 2.197 \\
\hline 4.394 & 30.758 & 2.197 & 8.788 \\
\hline 10.985 & 24.167 & 2.197 & 4.394 \\
\hline 1146.83 & 17.576 & 2.197 & 72.501 \\
\hline 2.197 & 10.985 & 2.197 & 131.82 \\
\hline 17.576 & 160.381 & 2.197 & 2.197 \\
\hline 4.394 & 2.197 & 2.197 & 4.394 \\
\hline 2.197 & 155.987 & 2.197 & 2.197 \\
\hline 2.197 & 30.758 & 2.197 & 87.88 \\
\hline 28.561 & 307.58 & 4.394 & 8.788 \\
\hline 2.197 & 17.576 & 21.97 & 57568 \\
\hline 8.788 & 19.773 & 2.197 & 4.394 \\
\hline 19.773 & 4.394 & 28.561 & 13.182 \\
\hline 4.394 & 19.773 & 101.062 & 131.82 \\
\hline 10.985 & 2.197 & 15.379 & 2.197 \\
\hline 2.197 & 15.379 & 10.985 & 4.394 \\
\hline 2.197 & 10.985 & 676.676 & 2.197 \\
\hline 224.094 & 2.197 & 37.349 & 116.441 \\
\hline 2038.82 & 8.788 & 235.079 & 2.197 \\
\hline 2.197 & 164.775 & 2.197 & 2.197 \\
\hline 2.197 & 2.197 & 6.591 & 2.197 \\
\hline 2.197 & 4.394 & 10.985 & 4.394 \\
\hline 2.197 & 72.501 & 46.137 & 26.364 \\
\hline 2.197 & 2.197 & 61.516 & 21.97 \\
\hline 2.197 & 2.197 & 2.197 & 24.167 \\
\hline 2.197 & 13.182 & 26.364 & 2.197 \\
\hline 13.182 & 4.394 & 2.197 & 2.197 \\
\hline 21.97 & 4.394 & 2.197 & 24.167 \\
\hline 8.788 & 15.379 & 50.531 & 2.197 \\
\hline 50.531 & 4.394 & 8.788 & 2.197 \\
\hline 15.379 & 19.773 & 2.197 & 2.197 \\
\hline 2.197 & 2.197 & 61.516 & 4.394 \\
\hline 17.576 & 2.197 & 109.85 & 6.591 \\
\hline 351.52 & 30.758 & 13.182 & 26.364 \\
\hline 24.167 & 2.197 & 35.152 & 2.197 \\
\hline 2.197 & 2.197 & 19.773 & 2.197 \\
\hline
\end{tabular}




\begin{tabular}{|c|c|c|c|}
\hline 2.197 & 2.197 & 2.197 & 6.591 \\
\hline 2.197 & 109.85 & 171.366 & 30.758 \\
\hline 17.576 & 118.638 & 10.985 & 2.197 \\
\hline 17.576 & 8.788 & 221.897 & 6.591 \\
\hline 2.197 & 8.788 & 2.197 & 13.182 \\
\hline 4.394 & 85.683 & 4.394 & 4.394 \\
\hline 35.152 & 6.591 & 2.197 & 30.758 \\
\hline 134.017 & 68.107 & 2.197 & 2752.84 \\
\hline 4.394 & 2.197 & 26.364 & 2.197 \\
\hline 2.197 & 2.197 & 2.197 & 2.197 \\
\hline 2.197 & 32.955 & 2.197 & 120.835 \\
\hline 4.394 & 10.985 & 2.197 & 10.985 \\
\hline 2.197 & 10.985 & 63.713 & 131.82 \\
\hline 2.197 & 6.591 & 2.197 & 4.394 \\
\hline 2.197 & 17.576 & 2.197 & 8.788 \\
\hline 8.788 & 4.394 & 32.955 & 2.197 \\
\hline 2.197 & 32.955 & 43.94 & 2.197 \\
\hline 2.197 & 6.591 & 6.591 & 2.197 \\
\hline 4.394 & 32.955 & 28.561 & 2.197 \\
\hline 2.197 & 4.394 & 2.197 & 2.197 \\
\hline 15.379 & 2.197 & 15.379 & 4.394 \\
\hline 19.773 & 13.182 & 8.788 & 2.197 \\
\hline 8.788 & 2.197 & 2.197 & 50.531 \\
\hline 2.197 & 10.985 & 2.197 & 2.197 \\
\hline 2.197 & 13.182 & 10.985 & 26.364 \\
\hline 8.788 & 2.197 & 2.197 & 8.788 \\
\hline 59.319 & 13.182 & 13.182 & 26.364 \\
\hline 54.925 & 175.76 & 2.197 & 2.197 \\
\hline 30.758 & 10.985 & 2.197 & 13.182 \\
\hline 441.597 & 612.963 & 15.379 & 52.728 \\
\hline 26.364 & 10.985 & 2.197 & 90.077 \\
\hline 10.985 & 6.591 & 4.394 & 4.394 \\
\hline 13.182 & 8.788 & 2.197 & 2.197 \\
\hline 6.591 & 175.76 & 17.576 & 4.394 \\
\hline 28.561 & 13.182 & 2.197 & 13.182 \\
\hline 2.197 & 54.925 & 15.379 & 2954.96 \\
\hline 2.197 & 41.743 & 4.394 & 2.197 \\
\hline 10.985 & 6.591 & 366.899 & 32.955 \\
\hline 8.788 & 21.97 & 2.197 & 173.563 \\
\hline 4.394 & 6.591 & 2.197 & 37.349 \\
\hline 4.394 & 2.197 & 2.197 & 13.182 \\
\hline 81.289 & 96.668 & 4.394 & 259.246 \\
\hline 50.531 & 4.394 & 21.97 & 3152.69 \\
\hline 2.197 & 2.197 & 6.591 & 180.154 \\
\hline 13.182 & 2.197 & 4.394 & 63.713 \\
\hline 118.638 & 2.197 & 4.394 & 2.197 \\
\hline 10.985 & 4.394 & 10.985 & 19.773 \\
\hline 2.197 & 24.167 & 28.561 & 320.762 \\
\hline 2.197 & 4.394 & 2.197 & 112.047 \\
\hline 2.197 & 4.394 & 13.182 & 8.788 \\
\hline
\end{tabular}




\begin{tabular}{|c|c|c|c|}
\hline 2.197 & 2.197 & 4.394 & 10.985 \\
\hline 2.197 & 4.394 & 2.197 & 10.985 \\
\hline 2.197 & 2.197 & 2.197 & 2.197 \\
\hline 2.197 & 26.364 & 72.501 & 63.713 \\
\hline 2.197 & 2.197 & 2.197 & 19.773 \\
\hline 2.197 & 6.591 & 2.197 & 186.745 \\
\hline 4.394 & 2.197 & 19.773 & 13.182 \\
\hline 2.197 & 6.591 & 2.197 & 19.773 \\
\hline 2.197 & 17.576 & 2.197 & 21.97 \\
\hline 4.394 & 2.197 & 6.591 & 10.985 \\
\hline 2.197 & 2.197 & 2.197 & 28.561 \\
\hline 10.985 & 39.546 & 50.531 & 6.591 \\
\hline 2.197 & 2.197 & 24.167 & 2.197 \\
\hline 2.197 & 21.97 & 6.591 & 2.197 \\
\hline 2.197 & 6.591 & 2.197 & 2.197 \\
\hline 351.52 & 2.197 & 13.182 & 2.197 \\
\hline 2.197 & 54.925 & 4.394 & 96.668 \\
\hline 13.182 & 68.107 & 2.197 & 6.591 \\
\hline 2.197 & 15.379 & 2.197 & 140.608 \\
\hline 4.394 & 166.972 & 2.197 & 8.788 \\
\hline 2.197 & 2.197 & 4.394 & 21.97 \\
\hline 13.182 & 118.638 & 10.985 & 2.197 \\
\hline 50.531 & 2.197 & 2.197 & 2.197 \\
\hline 2.197 & 2.197 & 2.197 & 15.379 \\
\hline 2.197 & 2.197 & 2.197 & 4.394 \\
\hline 6.591 & 2.197 & 2.197 & 2.197 \\
\hline 6.591 & 2.197 & 21.97 & 10.985 \\
\hline 131.82 & 8.788 & 2.197 & 2.197 \\
\hline 2.197 & 8.788 & 2.197 & 4.394 \\
\hline 4.394 & 2.197 & 2.197 & 2.197 \\
\hline 2.197 & 2.197 & 6.591 & 17.576 \\
\hline 4.394 & 2.197 & 24.167 & 2.197 \\
\hline 15.379 & 13.182 & 2.197 & 15.379 \\
\hline 2.197 & 50.531 & 2.197 & 140.608 \\
\hline 2.197 & 6.591 & 2.197 & 2.197 \\
\hline 10.985 & 4.394 & 6.591 & 2.197 \\
\hline 4.394 & 4.394 & 10.985 & 17.576 \\
\hline 19.773 & 2.197 & 17.576 & 2.197 \\
\hline 149.396 & 4.394 & 2.197 & 2.197 \\
\hline 8.788 & 6.591 & 2.197 & 2.197 \\
\hline 8.788 & 21.97 & 19.773 & 2.197 \\
\hline 10.985 & 32.955 & 8.788 & 2.197 \\
\hline 2.197 & 2.197 & 2.197 & 6.591 \\
\hline 188.942 & 2.197 & 28.561 & 4.394 \\
\hline 19.773 & 118.638 & 6.591 & 435.006 \\
\hline 2.197 & 10.985 & 2.197 & 52.728 \\
\hline 371.293 & 2.197 & 2.197 & 39.546 \\
\hline 8.788 & 2.197 & 2.197 & 2.197 \\
\hline 6.591 & 2.197 & 4.394 & 32.955 \\
\hline 204.321 & 4.394 & 21.97 & 15.379 \\
\hline
\end{tabular}




\begin{tabular}{|c|c|c|c|}
\hline 2.197 & 213.109 & 4.394 & 2.197 \\
\hline 2.197 & 2.197 & 145.002 & 689.858 \\
\hline 35.152 & 46.137 & 2.197 & 6.591 \\
\hline 21.97 & 2.197 & 2.197 & 35.152 \\
\hline 2.197 & 4.394 & 2.197 & 2.197 \\
\hline 2.197 & 57.122 & 2.197 & 182.351 \\
\hline 116.441 & 32.955 & 13.182 & 114.244 \\
\hline 6.591 & 2.197 & 2.197 & 28.561 \\
\hline 28.561 & 6.591 & 8.788 & 10.985 \\
\hline 2.197 & 252.655 & 2.197 & 10.985 \\
\hline 6.591 & 109.85 & 2.197 & 59.319 \\
\hline 2.197 & 2.197 & 2.197 & 35.152 \\
\hline 28.561 & 4.394 & 4.394 & 2.197 \\
\hline 2.197 & 24.167 & 37.349 & 2.197 \\
\hline 2.197 & 2.197 & 83.486 & 257.049 \\
\hline 4.394 & 2.197 & 2.197 & 10.985 \\
\hline 10.985 & 8.788 & 2.197 & 114.244 \\
\hline 4.394 & 4.394 & 32.955 & 8.788 \\
\hline 2.197 & 87.88 & 2.197 & 4.394 \\
\hline 4.394 & 474.552 & 24.167 & 4.394 \\
\hline 4.394 & 2.197 & 2.197 & 50.531 \\
\hline 2.197 & 2.197 & 136.214 & 155.987 \\
\hline 17.576 & 2.197 & 19.773 & 8.788 \\
\hline 4.394 & 2.197 & 2.197 & 13.182 \\
\hline 2.197 & 2.197 & 2.197 & 43.94 \\
\hline 17.576 & 2.197 & 2.197 & 8.788 \\
\hline 81.289 & 32.955 & 2.197 & 52.728 \\
\hline 2.197 & 65.91 & 24.167 & 32.955 \\
\hline 17.576 & 6.591 & 2.197 & 1067.74 \\
\hline 10.985 & 195.533 & 4.394 & 6.591 \\
\hline 4.394 & 4.394 & 30.758 & 2.197 \\
\hline 2.197 & 239.473 & 4.394 & 8.788 \\
\hline 2.197 & 13.182 & 6.591 & 101.062 \\
\hline 15.379 & 15.379 & 2.197 & 2.197 \\
\hline 46.137 & 265.837 & 37.349 & 2.197 \\
\hline 96.668 & 2.197 & 15.379 & 2.197 \\
\hline 2.197 & 652.509 & 21.97 & 2.197 \\
\hline 10.985 & 35.152 & 210.912 & 2.197 \\
\hline 705.237 & 13.182 & 15.379 & 2.197 \\
\hline 4.394 & 2.197 & 32.955 & 2.197 \\
\hline 15.379 & 57.122 & 285.61 & 2.197 \\
\hline 2.197 & 10.985 & 2.197 & 2.197 \\
\hline 13.182 & 15.379 & 4.394 & 2.197 \\
\hline 13.182 & 213.109 & 19.773 & 2.197 \\
\hline 15.379 & 2.197 & 2.197 & 496.522 \\
\hline 4.394 & 4.394 & 116.441 & 2.197 \\
\hline 2.197 & 8.788 & 2.197 & 239.473 \\
\hline 2.197 & 166.972 & 2.197 & 4.394 \\
\hline 2.197 & 2.197 & 2.197 & 37.349 \\
\hline 2.197 & 52.728 & 6.591 & 46.137 \\
\hline
\end{tabular}




\begin{tabular}{|c|c|c|c|}
\hline 946.907 & 35.152 & 32.955 & 2.197 \\
\hline 10.985 & 114.244 & 4.394 & 109.85 \\
\hline 61.516 & 103.259 & 2.197 & 2.197 \\
\hline 8.788 & 103.259 & 2.197 & 2.197 \\
\hline 10.985 & 39.546 & 43.94 & 4.394 \\
\hline 10.985 & 4.394 & 2.197 & 17.576 \\
\hline 2.197 & 2.197 & 2.197 & 2.197 \\
\hline 6.591 & 76.895 & 24.167 & 17.576 \\
\hline 2.197 & 30.758 & 2.197 & 4.394 \\
\hline 2.197 & 17.576 & 2.197 & 285.61 \\
\hline 160.381 & 19.773 & 2.197 & 2.197 \\
\hline 21.97 & 6.591 & 4.394 & 8.788 \\
\hline 136.214 & 4.394 & 2.197 & 63.713 \\
\hline 4.394 & 37.349 & 24.167 & 10.985 \\
\hline 8.788 & 10.985 & 109.85 & 4.394 \\
\hline 2895.65 & 2.197 & 17.576 & 2.197 \\
\hline 76.895 & 164.775 & 26.364 & 32.955 \\
\hline 26.364 & 4.394 & 2.197 & 10.985 \\
\hline 21.97 & 8.788 & 4.394 & 8.788 \\
\hline 2.197 & 2.197 & 2355.18 & 2.197 \\
\hline 2.197 & 10.985 & 4.394 & 125.229 \\
\hline 2.197 & 13.182 & 2.197 & 35.152 \\
\hline 2.197 & 48.334 & 13.182 & 8.788 \\
\hline 6.591 & 30.758 & 13.182 & 41.743 \\
\hline 2.197 & 54.925 & 6.591 & 13.182 \\
\hline 6.591 & 4.394 & 4.394 & 6.591 \\
\hline 2.197 & 2.197 & 2.197 & 30.758 \\
\hline 129.623 & 6.591 & 2.197 & 35.152 \\
\hline 2.197 & 4.394 & 2.197 & 4.394 \\
\hline 2.197 & 26.364 & 2.197 & 4.394 \\
\hline 4.394 & 4.394 & 2.197 & 327.353 \\
\hline 2.197 & 8.788 & 70.304 & 81.289 \\
\hline 4.394 & 57.122 & 2.197 & 4.394 \\
\hline 35.152 & 2.197 & 2.197 & 4.394 \\
\hline 120.835 & 54.925 & 263.64 & 17.576 \\
\hline 432.809 & 4.394 & 2.197 & 4.394 \\
\hline 46.137 & 2.197 & 2.197 & 2.197 \\
\hline 19.773 & 17.576 & 391.066 & 2.197 \\
\hline 4.394 & 30.758 & 2.197 & 43.94 \\
\hline 2.197 & 13.182 & 318.565 & 6.591 \\
\hline 26.364 & 19.773 & 24.167 & 2.197 \\
\hline 2.197 & 2.197 & 13.182 & 112.047 \\
\hline 2.197 & 17.576 & 2.197 & 6.591 \\
\hline 2.197 & 48.334 & 35.152 & 221.897 \\
\hline 6.591 & 4.394 & 2.197 & 17.576 \\
\hline 259.246 & 8.788 & 2.197 & 2.197 \\
\hline 1283.05 & 4.394 & 2.197 & 202.124 \\
\hline 2.197 & 19.773 & 6.591 & 4.394 \\
\hline 2.197 & 21.97 & 6.591 & 48.334 \\
\hline 17.576 & 21.97 & 138.411 & 65.91 \\
\hline
\end{tabular}




\begin{tabular}{|c|c|c|c|}
\hline 8.788 & 68.107 & 2.197 & 6.591 \\
\hline 2.197 & 21.97 & 2.197 & 839.254 \\
\hline 2.197 & 107.653 & 8.788 & 8.788 \\
\hline 6.591 & 103.259 & 2.197 & 19.773 \\
\hline 19.773 & 10.985 & 109.85 & 2.197 \\
\hline 59.319 & 105.456 & 10.985 & 138.411 \\
\hline 195.533 & 90.077 & 4.394 & 6.591 \\
\hline 17.576 & 6.591 & 17.576 & 2.197 \\
\hline 4.394 & 2.197 & 13.182 & 43.94 \\
\hline 2.197 & 10.985 & 13.182 & 19.773 \\
\hline 81.289 & 8.788 & 35.152 & 8.788 \\
\hline 2.197 & 118.638 & 8.788 & 2.197 \\
\hline 13.182 & 2.197 & 2.197 & 6.591 \\
\hline 8.788 & 6.591 & 8.788 & 79.092 \\
\hline 59.319 & 19.773 & 10.985 & 15.379 \\
\hline 19.773 & 46.137 & 279.019 & 2.197 \\
\hline 2.197 & 153.79 & 6.591 & 2.197 \\
\hline 2.197 & 68.107 & 15.379 & 35.152 \\
\hline 2.197 & 8.788 & 90.077 & 2.197 \\
\hline 2.197 & 2.197 & 87.88 & 2.197 \\
\hline 2.197 & 10.985 & 8.788 & 2.197 \\
\hline 4.394 & 241.67 & 2.197 & 2.197 \\
\hline 2.197 & 26.364 & 2.197 & 101.062 \\
\hline 2.197 & 76.895 & 2.197 & 4.394 \\
\hline 26.364 & 15.379 & 46.137 & 15.379 \\
\hline 48.334 & 8.788 & 2.197 & 2.197 \\
\hline 235.079 & 2.197 & 4.394 & 21.97 \\
\hline 13.182 & 1015.01 & 4.394 & 2.197 \\
\hline 92.274 & 72.501 & 6.591 & 24.167 \\
\hline 32.955 & 94.471 & 2.197 & 2.197 \\
\hline 2.197 & 2.197 & 2.197 & 2.197 \\
\hline 2.197 & 2.197 & 2.197 & 2.197 \\
\hline 2.197 & 4.394 & 2.197 & 2.197 \\
\hline 15.379 & 10.985 & 2.197 & 2.197 \\
\hline 6.591 & 131.82 & 2.197 & 2.197 \\
\hline 17.576 & 10.985 & 2.197 & 30.758 \\
\hline 48.334 & 2.197 & 61.516 & 8.788 \\
\hline 10.985 & 70.304 & 2.197 & 39.546 \\
\hline 70.304 & 2.197 & 2.197 & 10.985 \\
\hline 8.788 & 4.394 & 2.197 & 2.197 \\
\hline 2.197 & 4.394 & 4.394 & 2.197 \\
\hline 2.197 & 4.394 & 2.197 & 32.955 \\
\hline 15.379 & 15.379 & 15.379 & 2.197 \\
\hline 19.773 & 13.182 & 2.197 & 4.394 \\
\hline 19.773 & 4.394 & 4.394 & 4.394 \\
\hline 26.364 & 41.743 & 120.835 & 72.501 \\
\hline 98.865 & 380.081 & 2.197 & 6.591 \\
\hline 450.385 & 2.197 & 2.197 & 8.788 \\
\hline 21.97 & 4.394 & 4.394 & 2.197 \\
\hline 311.974 & 2.197 & 19.773 & 8.788 \\
\hline
\end{tabular}




\begin{tabular}{|c|c|c|c|}
\hline 15.379 & 2.197 & 8.788 & 28.561 \\
\hline 13.182 & 50.531 & 160.381 & 2.197 \\
\hline 2.197 & 2.197 & 4.394 & 6.591 \\
\hline 8.788 & 65.91 & 153.79 & 2.197 \\
\hline 17.576 & 4.394 & 105.456 & 94.471 \\
\hline 13.182 & 2.197 & 2.197 & 8.788 \\
\hline 2.197 & 68.107 & 8.788 & 17.576 \\
\hline 2.197 & 2.197 & 4.394 & 6.591 \\
\hline 2.197 & 8.788 & 6.591 & 39.546 \\
\hline 2.197 & 2.197 & 2.197 & 94.471 \\
\hline 2.197 & 320.762 & 2.197 & 13.182 \\
\hline 15.379 & 2.197 & 221.897 & 6.591 \\
\hline 2.197 & 17.576 & 2.197 & 2.197 \\
\hline 2.197 & 2.197 & 2.197 & 37.349 \\
\hline 6.591 & 30.758 & 10883.9 & 21.97 \\
\hline 39.546 & 2.197 & 2.197 & 31034.8 \\
\hline 13.182 & 2.197 & 2.197 & 2.197 \\
\hline 6.591 & 13.182 & 39.546 & 2.197 \\
\hline 2.197 & 35.152 & 2.197 & 19.773 \\
\hline 26.364 & 4.394 & 4.394 & 24.167 \\
\hline 2.197 & 21.97 & 4.394 & 164.775 \\
\hline 2.197 & 2.197 & 2.197 & 30.758 \\
\hline 15.379 & 2.197 & 4.394 & 8.788 \\
\hline 13.182 & 13.182 & 21.97 & 8.788 \\
\hline 24.167 & 2.197 & 46.137 & 81.289 \\
\hline 268.034 & 10.985 & 8.788 & 28.561 \\
\hline 21.97 & 195.533 & 24.167 & 228.488 \\
\hline 2.197 & 2.197 & 2.197 & 2.197 \\
\hline 311.974 & 10.985 & 2.197 & 6.591 \\
\hline 30.758 & 4.394 & 2.197 & 8.788 \\
\hline 136.214 & 19.773 & 6.591 & 4.394 \\
\hline 28.561 & 15.379 & 540.462 & 4.394 \\
\hline 10.985 & 2781.4 & 28.561 & 21.97 \\
\hline 37.349 & 61.516 & 2.197 & 2.197 \\
\hline 19.773 & 2.197 & 4.394 & 2.197 \\
\hline 2.197 & 17.576 & 2.197 & 2.197 \\
\hline 2.197 & 160.381 & 57.122 & 2.197 \\
\hline 4.394 & 76.895 & 2.197 & 2.197 \\
\hline 10.985 & 63.713 & 4.394 & 4.394 \\
\hline 50.531 & 39.546 & 2.197 & 6.591 \\
\hline 4.394 & 4.394 & 85.683 & 1239.11 \\
\hline 2.197 & 81.289 & 6.591 & 2.197 \\
\hline 2.197 & 2.197 & 10.985 & 13.182 \\
\hline 6.591 & 39.546 & 17.576 & 4.394 \\
\hline 2.197 & 158.184 & 2.197 & 2.197 \\
\hline 2790.19 & 6.591 & 19.773 & 188.942 \\
\hline 6.591 & 2.197 & 24.167 & 2.197 \\
\hline 2.197 & 8.788 & 2.197 & 2.197 \\
\hline 13.182 & 2.197 & 2.197 & 24.167 \\
\hline 2.197 & 2.197 & 10.985 & 41.743 \\
\hline
\end{tabular}




\begin{tabular}{|c|c|c|c|}
\hline 2.197 & 4.394 & 87.88 & 6.591 \\
\hline 24.167 & 2.197 & 15.379 & 26.364 \\
\hline 2.197 & 8.788 & 8.788 & 43.94 \\
\hline 2.197 & 24.167 & 79.092 & 39.546 \\
\hline 2.197 & 24.167 & 2.197 & 2.197 \\
\hline 8.788 & 2.197 & 2.197 & 2.197 \\
\hline 8.788 & 6.591 & 2.197 & 2.197 \\
\hline 15.379 & 4.394 & 2.197 & 2.197 \\
\hline 2.197 & 28.561 & 2.197 & 2.197 \\
\hline 2.197 & 2.197 & 6.591 & 101.062 \\
\hline 1162.21 & 43.94 & 2.197 & 2.197 \\
\hline 186.745 & 123.032 & 2.197 & 2.197 \\
\hline 13.182 & 2.197 & 804.102 & 2.197 \\
\hline 46.137 & 4.394 & 15.379 & 2.197 \\
\hline 173.563 & 4.394 & 136.214 & 2.197 \\
\hline 2.197 & 226.291 & 2.197 & 2.197 \\
\hline 4.394 & 564.629 & 4.394 & 37.349 \\
\hline 48.334 & 87.88 & 32.955 & 2.197 \\
\hline 2.197 & 2.197 & 48.334 & 4.394 \\
\hline 10.985 & 2.197 & 35.152 & 2.197 \\
\hline 10.985 & 2.197 & 2.197 & 2.197 \\
\hline 2.197 & 2.197 & 15.379 & 30.758 \\
\hline 10.985 & 15.379 & 6.591 & 4.394 \\
\hline 74.698 & 474.552 & 333.944 & 2.197 \\
\hline 46.137 & 17.576 & 2.197 & 2.197 \\
\hline 43.94 & 8.788 & 13.182 & 98.865 \\
\hline 2.197 & 4.394 & 6.591 & 30.758 \\
\hline 26.364 & 32.955 & 2.197 & 4.394 \\
\hline 8.788 & 15.379 & 15.379 & 6.591 \\
\hline 2.197 & 2.197 & 4.394 & 28.561 \\
\hline 52.728 & 46.137 & 21.97 & 2.197 \\
\hline 10.985 & 2.197 & 2.197 & 10.985 \\
\hline 2.197 & 2.197 & 13.182 & 6.591 \\
\hline 26.364 & 96.668 & 109.85 & 2.197 \\
\hline 50.531 & 30.758 & 2.197 & 120.835 \\
\hline 50.531 & 81.289 & 15.379 & 15.379 \\
\hline 2.197 & 114.244 & 2.197 & 103.259 \\
\hline 683.267 & 158.184 & 15.379 & 50.531 \\
\hline 4.394 & 41.743 & 2.197 & 13.182 \\
\hline 2.197 & 50.531 & 72.501 & 50.531 \\
\hline 21.97 & 134.017 & 2.197 & 6.591 \\
\hline 26.364 & 2.197 & 2.197 & 4.394 \\
\hline 1151.23 & 2.197 & 24.167 & 2.197 \\
\hline 6.591 & 8.788 & 2.197 & 90.077 \\
\hline 114.244 & 2.197 & 103.259 & 120.835 \\
\hline 6.591 & 8.788 & 43.94 & 2.197 \\
\hline 2.197 & 2.197 & 17.576 & 2.197 \\
\hline 2.197 & 2.197 & 2.197 & 10.985 \\
\hline 142.805 & 109.85 & 10.985 & 4.394 \\
\hline 10.985 & 74.698 & 2.197 & 2.197 \\
\hline
\end{tabular}




\begin{tabular}{|c|c|c|c|}
\hline 26.364 & 13.182 & 2.197 & 6.591 \\
\hline 37.349 & 4.394 & 2.197 & 6.591 \\
\hline 15.379 & 10.985 & 19.773 & 4.394 \\
\hline 24.167 & 4.394 & 8.788 & 39.546 \\
\hline 13.182 & 83.486 & 2.197 & 2.197 \\
\hline 28.561 & 2.197 & 2.197 & 2.197 \\
\hline 2.197 & 24.167 & 2.197 & 26.364 \\
\hline 2.197 & 125.229 & 28.561 & 101.062 \\
\hline 2.197 & 8.788 & 283.413 & 2.197 \\
\hline 6.591 & 52.728 & 92.274 & 10.985 \\
\hline 6.591 & 164.775 & 59.319 & 2.197 \\
\hline 2.197 & 351.52 & 2.197 & 2.197 \\
\hline 46.137 & 4.394 & 4.394 & 19.773 \\
\hline 2.197 & 96.668 & 2.197 & 79.092 \\
\hline 2.197 & 4.394 & 2.197 & 2.197 \\
\hline 2.197 & 2.197 & 41.743 & 4.394 \\
\hline 2.197 & 4.394 & 2.197 & 4.394 \\
\hline 2.197 & 15.379 & 10.985 & 1546.69 \\
\hline 13.182 & 39.546 & 19.773 & 8.788 \\
\hline 4.394 & 30.758 & 4.394 & 4.394 \\
\hline 41.743 & 21.97 & 10.985 & 17.576 \\
\hline 63.713 & 4.394 & 2.197 & 6.591 \\
\hline 2.197 & 138.411 & 2.197 & 599.781 \\
\hline 6.591 & 518.492 & 2.197 & 17.576 \\
\hline 15.379 & 13.182 & 4.394 & 63.713 \\
\hline 2.197 & 6.591 & 2.197 & 2.197 \\
\hline 13.182 & 6.591 & 4.394 & 268.034 \\
\hline 70.304 & 2.197 & 4.394 & 41.743 \\
\hline 6.591 & 2.197 & 2.197 & 2.197 \\
\hline 4.394 & 10.985 & 13.182 & 2.197 \\
\hline 17.576 & 4.394 & 50.531 & 2.197 \\
\hline 37.349 & 15.379 & 2.197 & 10.985 \\
\hline 2.197 & 6.591 & 4.394 & 4.394 \\
\hline 2.197 & 2.197 & 13.182 & 17.576 \\
\hline 2.197 & 26.364 & 2.197 & 2.197 \\
\hline 6.591 & 10.985 & 8.788 & 21.97 \\
\hline 24.167 & 2.197 & 166.972 & 4.394 \\
\hline 63.713 & 26.364 & 2.197 & 30.758 \\
\hline 4.394 & 2.197 & 17.576 & 210.912 \\
\hline 4.394 & 65.91 & 2.197 & 10.985 \\
\hline 8.788 & 2.197 & 140.608 & 13.182 \\
\hline 2.197 & 2.197 & 21.97 & 15.379 \\
\hline 2.197 & 2.197 & 2.197 & 28.561 \\
\hline 2.197 & 28.561 & 2.197 & 15.379 \\
\hline 6.591 & 2.197 & 685.464 & 4.394 \\
\hline 2.197 & 10.985 & 333.944 & 2.197 \\
\hline 2.197 & 142.805 & 6.591 & 2.197 \\
\hline 2.197 & 4.394 & 15.379 & 10.985 \\
\hline 2.197 & 8.788 & 2.197 & 76.895 \\
\hline 6.591 & 8.788 & 2.197 & 10.985 \\
\hline
\end{tabular}




\begin{tabular}{|c|c|c|c|}
\hline 4.394 & 13.182 & 2.197 & 15.379 \\
\hline 37.349 & 17.576 & 10.985 & 1036.98 \\
\hline 4.394 & 6.591 & 2.197 & 151.593 \\
\hline 28.561 & 10.985 & 4.394 & 406.445 \\
\hline 4.394 & 90.077 & 2.197 & 39.546 \\
\hline 2.197 & 4.394 & 2.197 & 681.07 \\
\hline 731.601 & 4.394 & 2.197 & 2.197 \\
\hline 8.788 & 10.985 & 15.379 & 15.379 \\
\hline 41.743 & 37.349 & 2.197 & 2.197 \\
\hline 287.807 & 4.394 & 15.379 & 6.591 \\
\hline 131.82 & 2.197 & 2.197 & 2.197 \\
\hline 37.349 & 8.788 & 2.197 & 2.197 \\
\hline 15.379 & 4.394 & 4.394 & 57.122 \\
\hline 2.197 & 94.471 & 2.197 & 2.197 \\
\hline 2.197 & 48.334 & 2.197 & 41.743 \\
\hline 112.047 & 13.182 & 2.197 & 46.137 \\
\hline 10.985 & 19.773 & 8.788 & 41.743 \\
\hline 101.062 & 2.197 & 43.94 & 30.758 \\
\hline 35.152 & 6.591 & 250.458 & 94.471 \\
\hline 577.811 & 6.591 & 83.486 & 39.546 \\
\hline 2.197 & 10.985 & 94.471 & 10.985 \\
\hline 8.788 & 2.197 & 48.334 & 17.576 \\
\hline 46.137 & 2.197 & 21.97 & 4.394 \\
\hline 2.197 & 169.169 & 4.394 & 43.94 \\
\hline 26.364 & 48.334 & 4.394 & 81.289 \\
\hline 4.394 & 4.394 & 6.591 & 2.197 \\
\hline 6.591 & 32.955 & 10.985 & 59.319 \\
\hline 2.197 & 15.379 & 2.197 & 2.197 \\
\hline 17.576 & 17.576 & 32.955 & 19.773 \\
\hline 6.591 & 2.197 & 65.91 & 2.197 \\
\hline 2.197 & 8.788 & 2.197 & 2.197 \\
\hline 30.758 & 127.426 & 2.197 & 2.197 \\
\hline 70.304 & 19.773 & 10.985 & 4.394 \\
\hline 8.788 & 2.197 & 19.773 & 57.122 \\
\hline 2.197 & 2.197 & 4.394 & 61.516 \\
\hline 2.197 & 13.182 & 415.233 & 19.773 \\
\hline 24.167 & 68.107 & 21.97 & 4.394 \\
\hline 2.197 & 8.788 & 6.591 & 2.197 \\
\hline 2.197 & 28.561 & 4.394 & 13.182 \\
\hline 2.197 & 2.197 & 2.197 & 2.197 \\
\hline 2.197 & 10.985 & 21.97 & 8.788 \\
\hline 2.197 & 4.394 & 4.394 & 17.576 \\
\hline 2.197 & 2.197 & 4.394 & 2.197 \\
\hline 70.304 & 17.576 & 2.197 & 6.591 \\
\hline 2.197 & 6.591 & 2.197 & 35.152 \\
\hline 2.197 & 2.197 & 2.197 & 39.546 \\
\hline 13.182 & 15.379 & 2.197 & 8.788 \\
\hline 2.197 & 8.788 & 32.955 & 4.394 \\
\hline 2.197 & 6.591 & 2.197 & 2.197 \\
\hline 2.197 & 15.379 & 2.197 & 41.743 \\
\hline
\end{tabular}




\begin{tabular}{|c|c|c|c|}
\hline 4.394 & 4.394 & 10.985 & 4.394 \\
\hline 4.394 & 2.197 & 6.591 & 32.955 \\
\hline 96.668 & 2.197 & 10.985 & 52.728 \\
\hline 8.788 & 61.516 & 46.137 & 2.197 \\
\hline 6.591 & 32.955 & 32.955 & 8.788 \\
\hline 2.197 & 63.713 & 30.758 & 2.197 \\
\hline 13.182 & 195.533 & 4.394 & 8.788 \\
\hline 72.501 & 4.394 & 2.197 & 2.197 \\
\hline 918.346 & 4.394 & 2.197 & 13.182 \\
\hline 6.591 & 164.775 & 15.379 & 4.394 \\
\hline 4.394 & 21.97 & 50.531 & 21.97 \\
\hline 2.197 & 138.411 & 30.758 & 10.985 \\
\hline 37.349 & 4.394 & 52.728 & 4.394 \\
\hline 19.773 & 8.788 & 2.197 & 10.985 \\
\hline 41.743 & 4.394 & 19.773 & 19.773 \\
\hline 24.167 & 28.561 & 59.319 & 8.788 \\
\hline 21.97 & 17.576 & 2.197 & 10.985 \\
\hline 10.985 & 4.394 & 10.985 & 2.197 \\
\hline 19.773 & 13.182 & 615.16 & 6.591 \\
\hline 101.062 & 13.182 & 2.197 & 2.197 \\
\hline 219.7 & 96.668 & 2.197 & 4.394 \\
\hline 2.197 & 2.197 & 2.197 & 15.379 \\
\hline 6.591 & 35.152 & 366.899 & 6.591 \\
\hline 6.591 & 221.897 & 2.197 & 120.835 \\
\hline 2.197 & 48.334 & 2.197 & 19.773 \\
\hline 4.394 & 180.154 & 2.197 & 2.197 \\
\hline 2.197 & 30.758 & 4.394 & 1006.23 \\
\hline 4.394 & 26.364 & 2.197 & 114.244 \\
\hline 6.591 & 2.197 & 2.197 & 2.197 \\
\hline 2.197 & 2.197 & 63.713 & 2.197 \\
\hline 13.182 & 259.246 & 2.197 & 120.835 \\
\hline 52.728 & 2.197 & 2.197 & 2.197 \\
\hline 103.259 & 2.197 & 2.197 & 2.197 \\
\hline 1195.17 & 15.379 & 8.788 & 24.167 \\
\hline 4.394 & 10.985 & 272.428 & 4.394 \\
\hline 10.985 & 24.167 & 2.197 & 351.52 \\
\hline 6.591 & 39.546 & 2.197 & 94.471 \\
\hline 127.426 & 2.197 & 2.197 & 2.197 \\
\hline 2.197 & 4.394 & 129.623 & 10.985 \\
\hline 26.364 & 10.985 & 4.394 & 2.197 \\
\hline 2.197 & 43.94 & 129.623 & 2.197 \\
\hline 4.394 & 63.713 & 50.531 & 2.197 \\
\hline 4.394 & 432.809 & 2.197 & 2.197 \\
\hline 10.985 & 114.244 & 1015.01 & 8.788 \\
\hline 8.788 & 4.394 & 8.788 & 832.663 \\
\hline 4.394 & 8.788 & 28.561 & 173.563 \\
\hline 6.591 & 13.182 & 8.788 & 8.788 \\
\hline 2.197 & 59.319 & 70.304 & 2.197 \\
\hline 2.197 & 83.486 & 35.152 & 116.441 \\
\hline 4.394 & 2.197 & 6.591 & 2.197 \\
\hline
\end{tabular}




\begin{tabular}{|c|c|c|c|}
\hline 2.197 & 19.773 & 2.197 & 2.197 \\
\hline 2.197 & 109.85 & 2.197 & 6.591 \\
\hline 6.591 & 2.197 & 13.182 & 19.773 \\
\hline 4.394 & 2.197 & 4.394 & 59.319 \\
\hline 10.985 & 63.713 & 6.591 & 127.426 \\
\hline 2.197 & 4.394 & 2.197 & 21.97 \\
\hline 2.197 & 2.197 & 8.788 & 4.394 \\
\hline 4.394 & 15.379 & 2.197 & 28.561 \\
\hline 2.197 & 4.394 & 8.788 & 65.91 \\
\hline 4.394 & 4.394 & 2.197 & 50.531 \\
\hline 63.713 & 4.394 & 2.197 & 19.773 \\
\hline 109.85 & 87.88 & 2.197 & 2.197 \\
\hline 202.124 & 17.576 & 3295.5 & 10.985 \\
\hline 24.167 & 2.197 & 2.197 & 4.394 \\
\hline 15.379 & 4.394 & 138.411 & 4.394 \\
\hline 13.182 & 8.788 & 2.197 & 24.167 \\
\hline 2.197 & 109.85 & 2.197 & 250.458 \\
\hline 2.197 & 6.591 & 2.197 & 2.197 \\
\hline 2.197 & 32.955 & 2.197 & 2.197 \\
\hline 37.349 & 24.167 & 164.775 & 6.591 \\
\hline 2.197 & 63.713 & 2.197 & 2.197 \\
\hline 6.591 & 10.985 & 10.985 & 19.773 \\
\hline 2.197 & 109.85 & 120.835 & 2.197 \\
\hline 2.197 & 2.197 & 6.591 & 4.394 \\
\hline 2.197 & 2.197 & 59.319 & 4.394 \\
\hline 2.197 & 10.985 & 17.576 & 8.788 \\
\hline 2.197 & 13.182 & 10.985 & 30.758 \\
\hline 2.197 & 4.394 & 21.97 & 388.869 \\
\hline 65.91 & 10.985 & 57.122 & 15.379 \\
\hline 4.394 & 322.959 & 467.961 & 6.591 \\
\hline 237.276 & 57.122 & 28.561 & 1676.31 \\
\hline 153.79 & 10.985 & 2.197 & 15.379 \\
\hline 2.197 & 2.197 & 2.197 & 2.197 \\
\hline 2.197 & 19.773 & 2.197 & 2.197 \\
\hline 2.197 & 15.379 & 2.197 & 2.197 \\
\hline 4.394 & 8.788 & 2.197 & 656.903 \\
\hline 10.985 & 92.274 & 2.197 & 4.394 \\
\hline 8.788 & 52.728 & 4.394 & 8.788 \\
\hline 10.985 & 2.197 & 2.197 & 28.561 \\
\hline 17.576 & 123.032 & 4.394 & 6.591 \\
\hline 140.608 & 46.137 & 4.394 & 6.591 \\
\hline 13.182 & 10.985 & 2.197 & 63.713 \\
\hline 2.197 & 26.364 & 2.197 & 6.591 \\
\hline 4.394 & 13.182 & 2.197 & 4.394 \\
\hline 6.591 & 2.197 & 2.197 & 2.197 \\
\hline 79.092 & 2.197 & 41.743 & 2.197 \\
\hline 4.394 & 2.197 & 2.197 & 8.788 \\
\hline 2.197 & 2.197 & 2.197 & 30.758 \\
\hline 2.197 & 4.394 & 2.197 & 239.473 \\
\hline 17.576 & 2.197 & 43.94 & 171.366 \\
\hline
\end{tabular}




\begin{tabular}{|c|c|c|c|}
\hline 19.773 & 8.788 & 2.197 & 4.394 \\
\hline 4.394 & 120.835 & 4.394 & 98.865 \\
\hline 8.788 & 6.591 & 2.197 & 24.167 \\
\hline 13.182 & 10.985 & 2.197 & 2.197 \\
\hline 46.137 & 4.394 & 2.197 & 2.197 \\
\hline 4.394 & 153.79 & 4.394 & 39.546 \\
\hline 2.197 & 105.456 & 2.197 & 101.062 \\
\hline 2.197 & 4.394 & 74.698 & 2.197 \\
\hline 2.197 & 10.985 & 13.182 & 74.698 \\
\hline 1076.53 & 2.197 & 26.364 & 4.394 \\
\hline 2.197 & 166.972 & 169.169 & 6.591 \\
\hline 2.197 & 81.289 & 2.197 & 13.182 \\
\hline 395.46 & 2.197 & 43.94 & 13.182 \\
\hline 4.394 & 2.197 & 2.197 & 17.576 \\
\hline 2.197 & 4.394 & 8.788 & 2.197 \\
\hline 2.197 & 61.516 & 2.197 & 6.591 \\
\hline 10.985 & 2.197 & 2.197 & 8.788 \\
\hline 2.197 & 2.197 & 4.394 & 6.591 \\
\hline 28.561 & 2.197 & 221.897 & 61.516 \\
\hline 2.197 & 2.197 & 2.197 & 744.783 \\
\hline 2.197 & 2.197 & 8.788 & 72.501 \\
\hline 2.197 & 4.394 & 2.197 & 19.773 \\
\hline 17.576 & 4.394 & 4.394 & 1083.12 \\
\hline 2.197 & 2.197 & 101.062 & 57.122 \\
\hline 103.259 & 15.379 & 7467.6 & 8.788 \\
\hline 4.394 & 28.561 & 2.197 & 63.713 \\
\hline 32.955 & 140.608 & 4.394 & 54.925 \\
\hline 24.167 & 54.925 & 2.197 & 8.788 \\
\hline 8.788 & 2.197 & 79.092 & 254.852 \\
\hline 2.197 & 2.197 & 26.364 & 15.379 \\
\hline 2.197 & 4.394 & 8.788 & 142.805 \\
\hline 19.773 & 4.394 & 2.197 & 24.167 \\
\hline 4.394 & 6.591 & 2.197 & 2.197 \\
\hline 2.197 & 8.788 & 2.197 & 28.561 \\
\hline 19.773 & 10.985 & 19.773 & 17.576 \\
\hline 129.623 & 4.394 & 2.197 & 4.394 \\
\hline 2.197 & 52.728 & 30.758 & 50.531 \\
\hline 2.197 & 39.546 & 4.394 & 2.197 \\
\hline 4.394 & 30.758 & 35.152 & 4.394 \\
\hline 30.758 & 17.576 & 94.471 & 50.531 \\
\hline 50.531 & 6.591 & 8.788 & 2.197 \\
\hline 59.319 & 30.758 & 2.197 & 2.197 \\
\hline 13.182 & 13.182 & 2.197 & 15.379 \\
\hline 30.758 & 24.167 & 6.591 & 2.197 \\
\hline 2.197 & 114.244 & 2.197 & 8.788 \\
\hline 4.394 & 107.653 & 4.394 & 2.197 \\
\hline 2.197 & 4.394 & 2.197 & 4.394 \\
\hline 4.394 & 65.91 & 445.991 & 92.274 \\
\hline 35.152 & 85.683 & 4.394 & 2.197 \\
\hline 13.182 & 2.197 & 2.197 & 2.197 \\
\hline
\end{tabular}




\begin{tabular}{|c|c|c|c|}
\hline 2.197 & 2.197 & 35.152 & 2.197 \\
\hline 39.546 & 10.985 & 6.591 & 2.197 \\
\hline 4.394 & 4.394 & 13.182 & 2.197 \\
\hline 118.638 & 2.197 & 2.197 & 333.944 \\
\hline 6.591 & 175.76 & 4.394 & 63.713 \\
\hline 15.379 & 28.561 & 6.591 & 2.197 \\
\hline 2.197 & 17.576 & 19.773 & 43.94 \\
\hline 10.985 & 15.379 & 4.394 & 4.394 \\
\hline 8.788 & 149.396 & 2.197 & 46.137 \\
\hline 8.788 & 123.032 & 2.197 & 35.152 \\
\hline 17.576 & 28.561 & 415.233 & 2.197 \\
\hline 2.197 & 2.197 & 2.197 & 2.197 \\
\hline 2.197 & 2.197 & 17.576 & 2.197 \\
\hline 4.394 & 4.394 & 2.197 & 188.942 \\
\hline 32.955 & 2.197 & 4.394 & 10.985 \\
\hline 2.197 & 2.197 & 175.76 & 2.197 \\
\hline 123.032 & 2.197 & 2.197 & 59.319 \\
\hline 2.197 & 4.394 & 2.197 & 654.706 \\
\hline 87.88 & 13.182 & 2.197 & 13.182 \\
\hline 2.197 & 120.835 & 2.197 & 32.955 \\
\hline 2.197 & 50.531 & 2.197 & 59.319 \\
\hline 2.197 & 4.394 & 6.591 & 10.985 \\
\hline 2.197 & 1421.46 & 2.197 & 2.197 \\
\hline 2.197 & 213.109 & 19.773 & 92.274 \\
\hline 322.959 & 2.197 & 322.959 & 2.197 \\
\hline 2.197 & 2.197 & 744.783 & 63.713 \\
\hline 2.197 & 13.182 & 127.426 & 19.773 \\
\hline 2.197 & 8.788 & 2.197 & 4.394 \\
\hline 6.591 & 2.197 & 540.462 & 28.561 \\
\hline 30.758 & 17.576 & 2.197 & 13.182 \\
\hline 48.334 & 41.743 & 2.197 & 8.788 \\
\hline 2.197 & 13.182 & 2.197 & 2.197 \\
\hline 61.516 & 2.197 & 76.895 & 2.197 \\
\hline 19.773 & 2.197 & 30.758 & 272.428 \\
\hline 210.912 & 140.608 & 2.197 & 2.197 \\
\hline 8.788 & 19.773 & 2.197 & 21.97 \\
\hline 6.591 & 43.94 & 17.576 & 4.394 \\
\hline 153.79 & 4.394 & 2.197 & 48.334 \\
\hline 8.788 & 57.122 & 2.197 & 43.94 \\
\hline 15.379 & 39.546 & 6.591 & 2.197 \\
\hline 10.985 & 6.591 & 2.197 & 26.364 \\
\hline 4.394 & 2.197 & 6.591 & 2.197 \\
\hline 2.197 & 15.379 & 6.591 & 4.394 \\
\hline 4.394 & 2.197 & 2.197 & 2.197 \\
\hline 2.197 & 2.197 & 2.197 & 24.167 \\
\hline 2.197 & 276.822 & 4.394 & 2.197 \\
\hline 8.788 & 2.197 & 2.197 & 760.162 \\
\hline 50.531 & 2.197 & 2.197 & 10.985 \\
\hline 6.591 & 6.591 & 149.396 & 10.985 \\
\hline 8.788 & 4.394 & 2.197 & 109.85 \\
\hline
\end{tabular}




\begin{tabular}{|c|c|c|c|}
\hline 39.546 & 4.394 & 48.334 & 17.576 \\
\hline 6.591 & 2.197 & 8.788 & 155.987 \\
\hline 2.197 & 4.394 & 2.197 & 4.394 \\
\hline 21.97 & 103.259 & 2.197 & 2.197 \\
\hline 426.218 & 21.97 & 4.394 & 13.182 \\
\hline 8.788 & 8.788 & 2.197 & 2.197 \\
\hline 4.394 & 50.531 & 4.394 & 6.591 \\
\hline 2.197 & 2.197 & 8.788 & 10.985 \\
\hline 54.925 & 4.394 & 6.591 & 2.197 \\
\hline 8.788 & 8.788 & 2.197 & 112.047 \\
\hline 98.865 & 6.591 & 15.379 & 39.546 \\
\hline 2.197 & 57.122 & 13.182 & 17.576 \\
\hline 46.137 & 85.683 & 136.214 & 64301.8 \\
\hline 4.394 & 90.077 & 2.197 & 2.197 \\
\hline 6.591 & 74.698 & 41.743 & 2.197 \\
\hline 4.394 & 2.197 & 2.197 & 2.197 \\
\hline 19.773 & 4.394 & 2.197 & 580.008 \\
\hline 2.197 & 50.531 & 2.197 & 8.788 \\
\hline 2.197 & 65.91 & 2.197 & 4.394 \\
\hline 2.197 & 13.182 & 2.197 & 32.955 \\
\hline 287.807 & 8.788 & 19.773 & 4.394 \\
\hline 2.197 & 13.182 & 13.182 & 4.394 \\
\hline 2.197 & 2.197 & 1428.05 & 41.743 \\
\hline 2.197 & 8.788 & 2.197 & 2.197 \\
\hline 2.197 & 2.197 & 21.97 & 21.97 \\
\hline 2.197 & 15.379 & 1056.76 & 96.668 \\
\hline 430.612 & 138.411 & 6.591 & 2.197 \\
\hline 586.599 & 46.137 & 4.394 & 2.197 \\
\hline 667.888 & 6.591 & 4.394 & 2.197 \\
\hline 4.394 & 2.197 & 2.197 & 2.197 \\
\hline 6.591 & 2.197 & 79.092 & 28.561 \\
\hline 17.576 & 83.486 & 4.394 & 10.985 \\
\hline 2.197 & 4.394 & 6.591 & 2.197 \\
\hline 2.197 & 15.379 & 2.197 & 4.394 \\
\hline 17.576 & 52.728 & 2.197 & 2.197 \\
\hline 28.561 & 2.197 & 4.394 & 2.197 \\
\hline 15.379 & 2.197 & 8.788 & 28.561 \\
\hline 116.441 & 6.591 & 2.197 & 4.394 \\
\hline 2.197 & 419.627 & 6.591 & 4.394 \\
\hline 41.743 & 10.985 & 2.197 & 4.394 \\
\hline 6.591 & 2.197 & 186.745 & 6.591 \\
\hline 2.197 & 10.985 & 2.197 & 6.591 \\
\hline 48.334 & 26.364 & 4.394 & 6.591 \\
\hline 2.197 & 2.197 & 46.137 & 92.274 \\
\hline 2.197 & 6.591 & 2.197 & 39.546 \\
\hline 6.591 & 4.394 & 2.197 & 10.985 \\
\hline 2.197 & 6.591 & 26.364 & 39.546 \\
\hline 2.197 & 2.197 & 2.197 & 4.394 \\
\hline 10.985 & 4.394 & 2.197 & 2.197 \\
\hline 2.197 & 90.077 & 109.85 & 24.167 \\
\hline
\end{tabular}




\begin{tabular}{|c|c|c|c|}
\hline 15.379 & 2.197 & 2.197 & 676.676 \\
\hline 76.895 & 13.182 & 10.985 & 35.152 \\
\hline 15.379 & 4.394 & 32.955 & 391.066 \\
\hline 2.197 & 4.394 & 2935.19 & 8.788 \\
\hline 2.197 & 68.107 & 2.197 & 8.788 \\
\hline 2.197 & 24.167 & 2.197 & 21.97 \\
\hline 2.197 & 391.066 & 54.925 & 2.197 \\
\hline 15.379 & 134.017 & 4.394 & 10.985 \\
\hline 50.531 & 43.94 & 2.197 & 4.394 \\
\hline 2.197 & 160.381 & 2.197 & 2.197 \\
\hline 2.197 & 2.197 & 2.197 & 2.197 \\
\hline 2.197 & 94.471 & 1540.1 & 21.97 \\
\hline 1026 & 8.788 & 6.591 & 935.922 \\
\hline 70.304 & 87.88 & 2.197 & 15.379 \\
\hline 24.167 & 10.985 & 32.955 & 6.591 \\
\hline 2.197 & 8.788 & 17.576 & 2.197 \\
\hline 17.576 & 2.197 & 8.788 & 125.229 \\
\hline 30.758 & 26.364 & 8.788 & 151.593 \\
\hline 2.197 & 10.985 & 2.197 & 19.773 \\
\hline 37.349 & 2.197 & 456.976 & 30.758 \\
\hline 48.334 & 4.394 & 17.576 & 6.591 \\
\hline 15.379 & 8.788 & 4.394 & 13.182 \\
\hline 351.52 & 4.394 & 2.197 & 6.591 \\
\hline 65.91 & 2.197 & 2.197 & 6.591 \\
\hline 19.773 & 24.167 & 26.364 & 2.197 \\
\hline 15.379 & 65.91 & 52.728 & 2.197 \\
\hline 13.182 & 8.788 & 6.591 & 4.394 \\
\hline 4.394 & 2.197 & 2.197 & 26.364 \\
\hline 4.394 & 39.546 & 6.591 & 125.229 \\
\hline 742.586 & 358.111 & 52.728 & 13.182 \\
\hline 24.167 & 4.394 & 606.372 & 15.379 \\
\hline 2.197 & 2.197 & 6.591 & 6.591 \\
\hline 2.197 & 10.985 & 2.197 & 2.197 \\
\hline 138.411 & 2.197 & 2.197 & 2.197 \\
\hline 15.379 & 2.197 & 4.394 & 2.197 \\
\hline 107.653 & 13.182 & 2.197 & 2.197 \\
\hline 197.73 & 19.773 & 8.788 & 6.591 \\
\hline 4.394 & 2.197 & 4.394 & 19.773 \\
\hline 4.394 & 10.985 & 4.394 & 2.197 \\
\hline 30.758 & 6.591 & 10.985 & 32.955 \\
\hline 2.197 & 37.349 & 13.182 & 2.197 \\
\hline 2.197 & 6.591 & 21.97 & 41.743 \\
\hline 2.197 & 6.591 & 2.197 & 2.197 \\
\hline 2.197 & 2.197 & 2.197 & 65.91 \\
\hline 2.197 & 21.97 & 2.197 & 2.197 \\
\hline 39.546 & 318.565 & 2.197 & 2.197 \\
\hline 15.379 & 21.97 & 2.197 & 2.197 \\
\hline 15.379 & 6.591 & 2.197 & 2.197 \\
\hline 10.985 & 37.349 & 2.197 & 10.985 \\
\hline 15.379 & 8.788 & 68.107 & 2.197 \\
\hline
\end{tabular}




\begin{tabular}{|c|c|c|c|}
\hline 13.182 & 382.278 & 2.197 & 17.576 \\
\hline 68.107 & 6.591 & 2.197 & 4.394 \\
\hline 13.182 & 2.197 & 2.197 & 8.788 \\
\hline 2.197 & 61.516 & 79.092 & 2.197 \\
\hline 2.197 & 15.379 & 2.197 & 15.379 \\
\hline 2.197 & 727.207 & 19.773 & 127.426 \\
\hline 2.197 & 2.197 & 8.788 & 6.591 \\
\hline 4.394 & 2.197 & 590.993 & 4.394 \\
\hline 24.167 & 17.576 & 2.197 & 2.197 \\
\hline 13.182 & 37.349 & 28.561 & 19.773 \\
\hline 2.197 & 2.197 & 2.197 & 8.788 \\
\hline 4.394 & 2.197 & 8.788 & 2.197 \\
\hline 30.758 & 2.197 & 6.591 & 8.788 \\
\hline 2.197 & 160.381 & 70.304 & 98.865 \\
\hline 2.197 & 8.788 & 2.197 & 19.773 \\
\hline 48.334 & 2.197 & 2.197 & 2.197 \\
\hline 85.683 & 15.379 & 39.546 & 239.473 \\
\hline 6.591 & 6.591 & 4.394 & 32.955 \\
\hline 8.788 & 228.488 & 355.914 & 188.942 \\
\hline 2.197 & 28.561 & 65.91 & 6.591 \\
\hline 2.197 & 13.182 & 2.197 & 2.197 \\
\hline 439.4 & 2.197 & 2.197 & 4.394 \\
\hline 4.394 & 369.096 & 2.197 & 153.79 \\
\hline 21.97 & 2.197 & 21.97 & 2.197 \\
\hline 2.197 & 13.182 & 2.197 & 2.197 \\
\hline 2.197 & 4.394 & 8.788 & 2.197 \\
\hline 2.197 & 39.546 & 2.197 & 2.197 \\
\hline 4.394 & 24.167 & 2.197 & 21.97 \\
\hline 17.576 & 2.197 & 4.394 & 10.985 \\
\hline 2.197 & 6.591 & 2.197 & 54.925 \\
\hline 2.197 & 2.197 & 8.788 & 15.379 \\
\hline 749.177 & 17.576 & 6.591 & 6.591 \\
\hline 10.985 & 2.197 & 4.394 & 21.97 \\
\hline 19.773 & 226.291 & 364.702 & 2.197 \\
\hline 10.985 & 2.197 & 2.197 & 19.773 \\
\hline 4.394 & 4.394 & 8.788 & 127.426 \\
\hline 57.122 & 24.167 & 4.394 & 4.394 \\
\hline 6.591 & 19.773 & 4.394 & 13.182 \\
\hline 2.197 & 76.895 & 4.394 & 2.197 \\
\hline 17.576 & 17.576 & 2.197 & 2.197 \\
\hline 26.364 & 8.788 & 2.197 & 4.394 \\
\hline 28.561 & 149.396 & 101.062 & 57.122 \\
\hline 2.197 & 6.591 & 151.593 & 2.197 \\
\hline 6.591 & 19.773 & 21.97 & 13.182 \\
\hline 17.576 & 48.334 & 2.197 & 2.197 \\
\hline 2.197 & 19.773 & 4.394 & 13.182 \\
\hline 2.197 & 6.591 & 2.197 & 8.788 \\
\hline 65.91 & 2.197 & 6.591 & 6.591 \\
\hline 2.197 & 15.379 & 149.396 & 2.197 \\
\hline 28.561 & 4.394 & 2.197 & 68.107 \\
\hline
\end{tabular}




\begin{tabular}{|c|c|c|c|}
\hline 2.197 & 4.394 & 2.197 & 2.197 \\
\hline 13.182 & 39.546 & 2.197 & 2.197 \\
\hline 10.985 & 28.561 & 193.336 & 456.976 \\
\hline 2.197 & 30.758 & 2.197 & 2.197 \\
\hline 2.197 & 4.394 & 8.788 & 48.334 \\
\hline 147.199 & 65.91 & 4.394 & 39.546 \\
\hline 2.197 & 2.197 & 2.197 & 120.835 \\
\hline 21.97 & 2.197 & 4.394 & 10.985 \\
\hline 2.197 & 43.94 & 4.394 & 79.092 \\
\hline 2.197 & 2.197 & 17.576 & 8.788 \\
\hline 10.985 & 2.197 & 6.591 & 2.197 \\
\hline 8.788 & 134.017 & 4.394 & 2.197 \\
\hline 2.197 & 202.124 & 2.197 & 2.197 \\
\hline 2.197 & 4.394 & 2.197 & 2.197 \\
\hline 2.197 & 2.197 & 2.197 & 19.773 \\
\hline 2.197 & 24.167 & 2.197 & 1146.83 \\
\hline 4.394 & 193.336 & 13.182 & 2.197 \\
\hline 2.197 & 4.394 & 2.197 & 478.946 \\
\hline 2.197 & 15.379 & 2.197 & 74.698 \\
\hline 2.197 & 6.591 & 2.197 & 4.394 \\
\hline 4.394 & 79.092 & 28.561 & 8.788 \\
\hline 2.197 & 2.197 & 17.576 & 13.182 \\
\hline 4.394 & 2.197 & 2.197 & 65.91 \\
\hline 65.91 & 50.531 & 15.379 & 24.167 \\
\hline 2.197 & 35.152 & 10.985 & 320.762 \\
\hline 2.197 & 4.394 & 4.394 & 28.561 \\
\hline 48.334 & 2.197 & 2.197 & 4.394 \\
\hline 10.985 & 4.394 & 37.349 & 10.985 \\
\hline 230.685 & 32.955 & 6.591 & 15.379 \\
\hline 2.197 & 48.334 & 4.394 & 10.985 \\
\hline 142.805 & 4.394 & 2.197 & 2.197 \\
\hline 2.197 & 2.197 & 413.036 & 2.197 \\
\hline 2.197 & 2.197 & 4.394 & 109.85 \\
\hline 10.985 & 4.394 & 13.182 & 30.758 \\
\hline 107.653 & 8.788 & 59.319 & 2.197 \\
\hline 2.197 & 10.985 & 4.394 & 4.394 \\
\hline 19.773 & 2.197 & 4.394 & 63.713 \\
\hline 134.017 & 28.561 & 28.561 & 59.319 \\
\hline 2.197 & 2.197 & 8.788 & 107.653 \\
\hline 17.576 & 2.197 & 2.197 & 6.591 \\
\hline 4.394 & 4.394 & 41.743 & 35.152 \\
\hline 2.197 & 237.276 & 2.197 & 8.788 \\
\hline 2.197 & 4.394 & 17.576 & 10.985 \\
\hline 4.394 & 94.471 & 2.197 & 6.591 \\
\hline 52.728 & 8.788 & 6.591 & 2.197 \\
\hline 2.197 & 30.758 & 101.062 & 103.259 \\
\hline 8.788 & 10.985 & 2.197 & 105.456 \\
\hline 4.394 & 4.394 & 68.107 & 2.197 \\
\hline 10.985 & 17.576 & 2.197 & 65.91 \\
\hline 39.546 & 6.591 & 8.788 & 26.364 \\
\hline
\end{tabular}




\begin{tabular}{|c|c|c|c|}
\hline 35.152 & 4.394 & 6.591 & 2.197 \\
\hline 4.394 & 391.066 & 54.925 & 4.394 \\
\hline 2.197 & 4.394 & 4.394 & 30.758 \\
\hline 327.353 & 2.197 & 4.394 & 2.197 \\
\hline 59.319 & 4.394 & 2.197 & 2.197 \\
\hline 2.197 & 24.167 & 2.197 & 2.197 \\
\hline 2.197 & 24.167 & 2.197 & 37.349 \\
\hline 2.197 & 21.97 & 2.197 & 2.197 \\
\hline 2.197 & 15.379 & 61.516 & 2.197 \\
\hline 274.625 & 24.167 & 2.197 & 2.197 \\
\hline 8.788 & 1247.9 & 6.591 & 6.591 \\
\hline 283.413 & 850.239 & 2.197 & 2.197 \\
\hline 35.152 & 2.197 & 2.197 & 6.591 \\
\hline 2.197 & 8.788 & 26.364 & 90.077 \\
\hline 13.182 & 4.394 & 74.698 & 2.197 \\
\hline 32.955 & 13.182 & 6.591 & 96.668 \\
\hline 2.197 & 2.197 & 50.531 & 8.788 \\
\hline 2550.72 & 2.197 & 2.197 & 2.197 \\
\hline 13.182 & 13.182 & 68.107 & 6.591 \\
\hline 2.197 & 8.788 & 2.197 & 138.411 \\
\hline 2.197 & 24.167 & 2.197 & 210.912 \\
\hline 10.985 & 4.394 & 2.197 & 32.955 \\
\hline 2.197 & 79.092 & 2.197 & 6.591 \\
\hline 2.197 & 4.394 & 2.197 & 19.773 \\
\hline 2.197 & 61.516 & 2.197 & 2.197 \\
\hline 4.394 & 30.758 & 2.197 & 2.197 \\
\hline 6.591 & 17.576 & 2.197 & 2.197 \\
\hline 2.197 & 127.426 & 4.394 & 2.197 \\
\hline 2.197 & 166.972 & 2.197 & 2.197 \\
\hline 2.197 & 6.591 & 15.379 & 2.197 \\
\hline 2.197 & 8.788 & 2.197 & 2.197 \\
\hline 6.591 & 2.197 & 15.379 & 2.197 \\
\hline 2.197 & 566.826 & 39.546 & 15.379 \\
\hline 2.197 & 4.394 & 4.394 & 158.184 \\
\hline 8.788 & 2.197 & 6.591 & 2.197 \\
\hline 4.394 & 158.184 & 10.985 & 6.591 \\
\hline 19.773 & 2.197 & 4.394 & 191.139 \\
\hline 2.197 & 15.379 & 6.591 & 2.197 \\
\hline 10985 & 191.139 & 127.426 & 2.197 \\
\hline 13.182 & 2.197 & 2.197 & 2.197 \\
\hline 15.379 & 4.394 & 81.289 & 6.591 \\
\hline 37.349 & 50.531 & 2.197 & 4.394 \\
\hline 151.593 & 8.788 & 6.591 & 4.394 \\
\hline 103.259 & 6.591 & 4.394 & 107.653 \\
\hline 48.334 & 164.775 & 2.197 & 8.788 \\
\hline 8.788 & 2.197 & 2.197 & 4.394 \\
\hline 6.591 & 2.197 & 10.985 & 2.197 \\
\hline 2.197 & 39.546 & 4.394 & 8.788 \\
\hline 173.563 & 129.623 & 4.394 & 2.197 \\
\hline 46.137 & 6.591 & 2.197 & 4.394 \\
\hline
\end{tabular}




\begin{tabular}{|c|c|c|c|}
\hline 10.985 & 2.197 & 4.394 & 109.85 \\
\hline 13.182 & 4.394 & 21.97 & 485.537 \\
\hline 4.394 & 6.591 & 2.197 & 10.985 \\
\hline 48.334 & 2.197 & 2.197 & 8935.2 \\
\hline 17.576 & 2.197 & 2.197 & 8.788 \\
\hline 2.197 & 2.197 & 2.197 & 105.456 \\
\hline 2.197 & 24.167 & 2.197 & 30.758 \\
\hline 2.197 & 2.197 & 24.167 & 30.758 \\
\hline 2.197 & 2.197 & 21.97 & 2.197 \\
\hline 15.379 & 380.081 & 10.985 & 2.197 \\
\hline 39.546 & 8.788 & 2.197 & 2.197 \\
\hline 6.591 & 202.124 & 2.197 & 2.197 \\
\hline 2.197 & 2.197 & 2.197 & 1783.96 \\
\hline 2.197 & 2.197 & 4.394 & 26.364 \\
\hline 63.713 & 2.197 & 2.197 & 134.017 \\
\hline 8.788 & 2.197 & 19.773 & 6.591 \\
\hline 6.591 & 2.197 & 2.197 & 120.835 \\
\hline 4.394 & 8.788 & 6.591 & 43.94 \\
\hline 2.197 & 2.197 & 48.334 & 2.197 \\
\hline 4.394 & 24.167 & 4.394 & 43.94 \\
\hline 10.985 & 13.182 & 2.197 & 43.94 \\
\hline 4.394 & 4.394 & 2.197 & 70.304 \\
\hline 32.955 & 2.197 & 4.394 & 8.788 \\
\hline 6.591 & 2.197 & 4.394 & 21.97 \\
\hline 57.122 & 90.077 & 6.591 & 39.546 \\
\hline 8.788 & 32.955 & 2.197 & 6.591 \\
\hline 13.182 & 2.197 & 2.197 & 63.713 \\
\hline 138.411 & 10.985 & 13.182 & 10.985 \\
\hline 4.394 & 46.137 & 6.591 & 8.788 \\
\hline 4.394 & 57.122 & 4.394 & 2.197 \\
\hline 2.197 & 4.394 & 2.197 & 2.197 \\
\hline 2.197 & 17.576 & 30.758 & 2.197 \\
\hline 57.122 & 4.394 & 4.394 & 2.197 \\
\hline 13.182 & 4.394 & 19.773 & 19.773 \\
\hline 2.197 & 366.899 & 32.955 & 30.758 \\
\hline 2.197 & 217.503 & 13.182 & 8.788 \\
\hline 2.197 & 37.349 & 2.197 & 57.122 \\
\hline 2.197 & 2.197 & 2.197 & 10.985 \\
\hline 35.152 & 2.197 & 2.197 & 4.394 \\
\hline 322.959 & 2.197 & 630.539 & 4.394 \\
\hline 2.197 & 2.197 & 2.197 & 2.197 \\
\hline 21.97 & 2.197 & 8.788 & 94.471 \\
\hline 2.197 & 15.379 & 6.591 & 72.501 \\
\hline 4.394 & 2.197 & 32.955 & 2.197 \\
\hline 2.197 & 2.197 & 2.197 & 17.576 \\
\hline 2.197 & 2.197 & 2.197 & 4.394 \\
\hline 13.182 & 35.152 & 37.349 & 28.561 \\
\hline 13.182 & 2.197 & 4.394 & 76.895 \\
\hline 24.167 & 28.561 & 41.743 & 120.835 \\
\hline 2.197 & 13.182 & 2.197 & 2.197 \\
\hline
\end{tabular}




\begin{tabular}{|c|c|c|c|}
\hline 4.394 & 2.197 & 2.197 & 4.394 \\
\hline 41.743 & 21.97 & 8.788 & 8.788 \\
\hline 10.985 & 70.304 & 21.97 & 24.167 \\
\hline 4.394 & 19.773 & 28.561 & 37.349 \\
\hline 46.137 & 48.334 & 2.197 & 131.82 \\
\hline 4.394 & 57.122 & 13.182 & 2.197 \\
\hline 10.985 & 10.985 & 48.334 & 57.122 \\
\hline 48.334 & 2.197 & 6.591 & 2.197 \\
\hline 4.394 & 43.94 & 8.788 & 10.985 \\
\hline 15.379 & 21.97 & 15.379 & 2.197 \\
\hline 2.197 & 2.197 & 2.197 & 2.197 \\
\hline 2.197 & 24.167 & 2.197 & 8.788 \\
\hline 648.115 & 158.184 & 10.985 & 4.394 \\
\hline 30.758 & 2.197 & 17.576 & 13.182 \\
\hline 10.985 & 2.197 & 26.364 & 2.197 \\
\hline 4.394 & 2.197 & 2.197 & 2.197 \\
\hline 2.197 & 6.591 & 4.394 & 37.349 \\
\hline 24.167 & 6.591 & 2.197 & 2.197 \\
\hline 10.985 & 103.259 & 2.197 & 4.394 \\
\hline 4.394 & 6.591 & 2.197 & 4.394 \\
\hline 162.578 & 4.394 & 101.062 & 366.899 \\
\hline 37.349 & 6.591 & 6.591 & 79.092 \\
\hline 2.197 & 2.197 & 35.152 & 320.762 \\
\hline 6.591 & 15.379 & 2.197 & 287.807 \\
\hline 11024.5 & 21.97 & 92.274 & 76.895 \\
\hline 2.197 & 2.197 & 2.197 & 4.394 \\
\hline 50.531 & 10.985 & 2.197 & 2.197 \\
\hline 19.773 & 35.152 & 2.197 & 138.411 \\
\hline 37.349 & 2.197 & 184.548 & 15.379 \\
\hline 13.182 & 2.197 & 28.561 & 76.895 \\
\hline 8.788 & 57.122 & 2.197 & 1493.96 \\
\hline 6.591 & 10.985 & 2.197 & 24.167 \\
\hline 24.167 & 8.788 & 2.197 & 2.197 \\
\hline 8.788 & 2.197 & 232.882 & 24.167 \\
\hline 2.197 & 21.97 & 2.197 & 2.197 \\
\hline 2.197 & 131.82 & 2.197 & 26.364 \\
\hline 2.197 & 4.394 & 13.182 & 2.197 \\
\hline 4.394 & 24.167 & 6.591 & 8.788 \\
\hline 2.197 & 50.531 & 41.743 & 87.88 \\
\hline 13.182 & 10.985 & 2.197 & 17.576 \\
\hline 2.197 & 2.197 & 4.394 & 6.591 \\
\hline 48.334 & 2.197 & 8.788 & 6.591 \\
\hline 4.394 & 6.591 & 2.197 & 39.546 \\
\hline 2.197 & 17.576 & 6.591 & 2.197 \\
\hline 28.561 & 10.985 & 2.197 & 2.197 \\
\hline 4.394 & 85.683 & 2.197 & 2.197 \\
\hline 2.197 & 32.955 & 2.197 & 8.788 \\
\hline 4.394 & 4.394 & 2.197 & 4.394 \\
\hline 4.394 & 217.503 & 2.197 & 4.394 \\
\hline 6.591 & 48.334 & 112.047 & 24.167 \\
\hline
\end{tabular}




\begin{tabular}{|c|c|c|c|}
\hline 8.788 & 8.788 & 2.197 & 2.197 \\
\hline 54.925 & 8.788 & 2.197 & 32.955 \\
\hline 1801.54 & 19.773 & 4.394 & 2.197 \\
\hline 2.197 & 107.653 & 4.394 & 2.197 \\
\hline 21.97 & 32.955 & 2.197 & 2.197 \\
\hline 4.394 & 19.773 & 30.758 & 26.364 \\
\hline 2.197 & 32.955 & 4.394 & 61.516 \\
\hline 19.773 & 1610.4 & 6.591 & 50.531 \\
\hline 2.197 & 48.334 & 2.197 & 17.576 \\
\hline 8.788 & 2.197 & 2.197 & 8.788 \\
\hline 6.591 & 2.197 & 10.985 & 48.334 \\
\hline 2.197 & 2.197 & 4.394 & 2.197 \\
\hline 2.197 & 15.379 & 149.396 & 35.152 \\
\hline 4.394 & 15.379 & 2.197 & 2.197 \\
\hline 2.197 & 2.197 & 2.197 & 149.396 \\
\hline 2.197 & 2.197 & 46.137 & 41.743 \\
\hline 2.197 & 2.197 & 8.788 & 10.985 \\
\hline 8.788 & 2.197 & 2.197 & 2.197 \\
\hline 4.394 & 61.516 & 68.107 & 2.197 \\
\hline 43.94 & 2.197 & 4.394 & 2.197 \\
\hline 142.805 & 153.79 & 57.122 & 2.197 \\
\hline 21.97 & 19.773 & 2.197 & 4.394 \\
\hline 193.336 & 2.197 & 17.576 & 70.304 \\
\hline 4.394 & 2.197 & 4.394 & 2.197 \\
\hline 2.197 & 70.304 & 8.788 & 4.394 \\
\hline 4.394 & 43.94 & 4.394 & 4.394 \\
\hline 10.985 & 6.591 & 2.197 & 61.516 \\
\hline 2.197 & 2.197 & 2.197 & 2.197 \\
\hline 2.197 & 329.55 & 26.364 & 24.167 \\
\hline 2.197 & 39.546 & 2.197 & 2.197 \\
\hline 4.394 & 4.394 & 2.197 & 8.788 \\
\hline 17.576 & 2.197 & 19.773 & 2.197 \\
\hline 83.486 & 2.197 & 41.743 & 2.197 \\
\hline 17.576 & 8.788 & 2.197 & 14203.6 \\
\hline 13.182 & 4.394 & 4.394 & 54.925 \\
\hline 6.591 & 2.197 & 6.591 & 6.591 \\
\hline 2.197 & 2.197 & 21.97 & 24.167 \\
\hline 527.28 & 6.591 & 39.546 & 10.985 \\
\hline 13.182 & 2.197 & 808.496 & 109.85 \\
\hline 4.394 & 4.394 & 6.591 & 96.668 \\
\hline 35.152 & 2.197 & 6.591 & 2.197 \\
\hline 2.197 & 10.985 & 4.394 & 927.134 \\
\hline 13.182 & 26.364 & 2.197 & 37.349 \\
\hline 2.197 & 208.715 & 76.895 & 4.394 \\
\hline 8.788 & 6.591 & 2.197 & 2.197 \\
\hline 68.107 & 2.197 & 2.197 & 65.91 \\
\hline 6.591 & 2.197 & 10.985 & 8.788 \\
\hline 4.394 & 50.531 & 4.394 & 87.88 \\
\hline 10.985 & 292.201 & 28.561 & 76.895 \\
\hline 4.394 & 2.197 & 4.394 & 17.576 \\
\hline
\end{tabular}




\begin{tabular}{|c|c|c|c|}
\hline 342.732 & 10.985 & 17.576 & 177.957 \\
\hline 2.197 & 6.591 & 2.197 & 30.758 \\
\hline 8.788 & 21.97 & 2.197 & 2.197 \\
\hline 4.394 & 4.394 & 162.578 & 6.591 \\
\hline 4.394 & 4.394 & 8.788 & 265.837 \\
\hline 896.376 & 37.349 & 2.197 & 52.728 \\
\hline 61.516 & 2.197 & 4.394 & 10.985 \\
\hline 41.743 & 4.394 & 35.152 & 10.985 \\
\hline 2.197 & 21.97 & 2.197 & 1298.43 \\
\hline 8.788 & 43.94 & 2.197 & 1878.43 \\
\hline 2.197 & 92.274 & 2.197 & 107.653 \\
\hline 2.197 & 1129.26 & 8.788 & 4.394 \\
\hline 2.197 & 268.034 & 85.683 & 2.197 \\
\hline 6.591 & 4.394 & 2.197 & 674.479 \\
\hline 2.197 & 164.775 & 68.107 & 81.289 \\
\hline 2.197 & 17.576 & 28.561 & 41.743 \\
\hline 13.182 & 2.197 & 8.788 & 8.788 \\
\hline 2.197 & 8.788 & 2.197 & 2.197 \\
\hline 10.985 & 54.925 & 2.197 & 4.394 \\
\hline 4.394 & 4.394 & 2.197 & 155.987 \\
\hline 8.788 & 2.197 & 6.591 & 2.197 \\
\hline 4.394 & 46.137 & 2.197 & 1155.62 \\
\hline 2.197 & 116.441 & 2.197 & 105.456 \\
\hline 17.576 & 10.985 & 2.197 & 2.197 \\
\hline 4.394 & 10.985 & 2.197 & 2.197 \\
\hline 2.197 & 32.955 & 6.591 & 2.197 \\
\hline 226.291 & 6.591 & 8.788 & 2.197 \\
\hline 2.197 & 127.426 & 2.197 & 41.743 \\
\hline 2.197 & 50.531 & 32.955 & 437.203 \\
\hline 4.394 & 39.546 & 2.197 & 81.289 \\
\hline 2.197 & 2.197 & 6.591 & 8.788 \\
\hline 4.394 & 4.394 & 10.985 & 4.394 \\
\hline 4.394 & 2.197 & 2.197 & 41.743 \\
\hline 2.197 & 26.364 & 2.197 & 10.985 \\
\hline 112.047 & 41.743 & 4.394 & 8.788 \\
\hline 6.591 & 109.85 & 2.197 & 1667.52 \\
\hline 101.062 & 61.516 & 4.394 & 26.364 \\
\hline 4.394 & 2.197 & 4.394 & 525.083 \\
\hline 4.394 & 8.788 & 2.197 & 68.107 \\
\hline 2.197 & 228.488 & 2.197 & 8.788 \\
\hline 2.197 & 39.546 & 37.349 & 4.394 \\
\hline 2.197 & 8.788 & 2.197 & 2.197 \\
\hline 2.197 & 2.197 & 6.591 & 2.197 \\
\hline 4.394 & 2.197 & 8.788 & 2.197 \\
\hline 10.985 & 6.591 & 24.167 & 8.788 \\
\hline 6.591 & 2192.61 & 8.788 & 2.197 \\
\hline 4.394 & 10.985 & 30.758 & 24.167 \\
\hline 2.197 & 15.379 & 30.758 & 4.394 \\
\hline 72.501 & 4.394 & 6.591 & 2.197 \\
\hline 4.394 & 4.394 & 107.653 & 13.182 \\
\hline
\end{tabular}




\begin{tabular}{|c|c|c|c|}
\hline 166.972 & 52.728 & 2.197 & 2.197 \\
\hline 87.88 & 2.197 & 2.197 & 32.955 \\
\hline 839.254 & 2.197 & 2.197 & 1254.49 \\
\hline 46.137 & 2.197 & 13.182 & 4.394 \\
\hline 24.167 & 26.364 & 4.394 & 28.561 \\
\hline 731.601 & 13.182 & 13.182 & 30.758 \\
\hline 145.002 & 2.197 & 2.197 & 4466.5 \\
\hline 70.304 & 17.576 & 4.394 & 21.97 \\
\hline 37.349 & 173.563 & 90.077 & 2.197 \\
\hline 3987.55 & 2.197 & 2.197 & 10.985 \\
\hline 2.197 & 2.197 & 6.591 & 87.88 \\
\hline 4.394 & 2.197 & 4.394 & 4.394 \\
\hline 2.197 & 2.197 & 2.197 & 32.955 \\
\hline 2.197 & 2.197 & 6.591 & 10.985 \\
\hline 2.197 & 2.197 & 2.197 & 17.576 \\
\hline 349.323 & 2.197 & 2.197 & 292.201 \\
\hline 28.561 & 4.394 & 6.591 & 32.955 \\
\hline 13.182 & 50.531 & 2.197 & 76.895 \\
\hline 2.197 & 26.364 & 2.197 & 24.167 \\
\hline 2.197 & 6.591 & 2.197 & 661.297 \\
\hline 70.304 & 2.197 & 57.122 & 2.197 \\
\hline 2.197 & 6.591 & 6.591 & 43.94 \\
\hline 129.623 & 4.394 & 8.788 & 6.591 \\
\hline 15.379 & 105.456 & 2.197 & 24.167 \\
\hline 13.182 & 2.197 & 2.197 & 13.182 \\
\hline 48.334 & 2.197 & 4.394 & 8.788 \\
\hline 8.788 & 10.985 & 10.985 & 241.67 \\
\hline 4.394 & 6.591 & 2.197 & 129.623 \\
\hline 37.349 & 35.152 & 19.773 & 26.364 \\
\hline 2.197 & 2.197 & 4.394 & 30.758 \\
\hline 76.895 & 8.788 & 43.94 & 19.773 \\
\hline 63.713 & 24.167 & 2.197 & 4.394 \\
\hline 32.955 & 19.773 & 219.7 & 65.91 \\
\hline 180.154 & 2.197 & 52.728 & 15.379 \\
\hline 116.441 & 6.591 & 2.197 & 15.379 \\
\hline 2.197 & 2.197 & 17.576 & 6.591 \\
\hline 2.197 & 2.197 & 4.394 & 404.248 \\
\hline 4.394 & 4.394 & 46.137 & 6.591 \\
\hline 13.182 & 230.685 & 8.788 & 6.591 \\
\hline 2.197 & 90.077 & 2.197 & 338.338 \\
\hline 24.167 & 2.197 & 2.197 & 2.197 \\
\hline 13.182 & 147.199 & 24.167 & 6.591 \\
\hline 957.892 & 2.197 & 10.985 & 2.197 \\
\hline 2.197 & 2.197 & 2.197 & 2.197 \\
\hline 138.411 & 26.364 & 65.91 & 15.379 \\
\hline 10.985 & 2.197 & 6.591 & 2.197 \\
\hline 13.182 & 2.197 & 158.184 & 19.773 \\
\hline 2.197 & 4.394 & 2.197 & 2.197 \\
\hline 8.788 & 13.182 & 10.985 & 2.197 \\
\hline 28.561 & 2.197 & 32.955 & 2.197 \\
\hline
\end{tabular}




\begin{tabular}{|c|c|c|c|}
\hline 2.197 & 32.955 & 2.197 & 50.531 \\
\hline 2.197 & 2.197 & 2.197 & 2.197 \\
\hline 2.197 & 2.197 & 24.167 & 2.197 \\
\hline 2.197 & 2.197 & 2.197 & 79.092 \\
\hline 92.274 & 4.394 & 10.985 & 2.197 \\
\hline 2.197 & 17.576 & 8.788 & 30.758 \\
\hline 2.197 & 127.426 & 2.197 & 2.197 \\
\hline 2.197 & 13.182 & 2.197 & 13.182 \\
\hline 15.379 & 2.197 & 37.349 & 4.394 \\
\hline 59.319 & 13.182 & 6.591 & 127.426 \\
\hline 8.788 & 15.379 & 52.728 & 48.334 \\
\hline 2.197 & 98.865 & 6.591 & 4.394 \\
\hline 1287.44 & 13.182 & 2.197 & 6.591 \\
\hline 2.197 & 6.591 & 4.394 & 6.591 \\
\hline 6.591 & 2.197 & 21.97 & 15.379 \\
\hline 2.197 & 4.394 & 10.985 & 13.182 \\
\hline 6.591 & 4.394 & 2911.02 & 76.895 \\
\hline 63.713 & 15.379 & 8.788 & 160.381 \\
\hline 8.788 & 8.788 & 43.94 & 15.379 \\
\hline 2.197 & 4.394 & 6.591 & 2.197 \\
\hline 2.197 & 8.788 & 270.231 & 16545.6 \\
\hline 2.197 & 142.805 & 210.912 & 6.591 \\
\hline 2.197 & 15.379 & 2.197 & 134.017 \\
\hline 8.788 & 101.062 & 2.197 & 15.379 \\
\hline 2.197 & 48.334 & 10.985 & 21.97 \\
\hline 48.334 & 2.197 & 2.197 & 30.758 \\
\hline 136.214 & 4.394 & 4.394 & 2.197 \\
\hline 4.394 & 57.122 & 13.182 & 26.364 \\
\hline 2.197 & 2.197 & 32.955 & 6.591 \\
\hline 125.229 & 65.91 & 21.97 & 2.197 \\
\hline 4.394 & 6.591 & 4.394 & 10.985 \\
\hline 21.97 & 2.197 & 2.197 & 2.197 \\
\hline 10.985 & 456.976 & 2.197 & 2.197 \\
\hline 4.394 & 26.364 & 2.197 & 17.576 \\
\hline 4.394 & 2.197 & 2.197 & 2.197 \\
\hline 2.197 & 32.955 & 6.591 & 4.394 \\
\hline 6.591 & 4.394 & 8.788 & 6.591 \\
\hline 2.197 & 2.197 & 4.394 & 15.379 \\
\hline 17.576 & 13.182 & 2.197 & 74.698 \\
\hline 2.197 & 103.259 & 2.197 & 2.197 \\
\hline 127.426 & 252.655 & 15.379 & 13.182 \\
\hline 41.743 & 21.97 & 2.197 & 151.593 \\
\hline 6235.09 & 15.379 & 6.591 & 85.683 \\
\hline 2.197 & 2.197 & 2.197 & 54.925 \\
\hline 2.197 & 6.591 & 2.197 & 15.379 \\
\hline 2.197 & 46.137 & 127.426 & 15.379 \\
\hline 6.591 & 2.197 & 17.576 & 15.379 \\
\hline 2.197 & 26.364 & 4.394 & 6.591 \\
\hline 17.576 & 26.364 & 2.197 & 48.334 \\
\hline 2.197 & 169.169 & 2.197 & 145.002 \\
\hline
\end{tabular}




\begin{tabular}{|c|c|c|c|}
\hline 6.591 & 2.197 & 1078.73 & 127.426 \\
\hline 41.743 & 2.197 & 15.379 & 24.167 \\
\hline 37.349 & 6.591 & 54.925 & 131.82 \\
\hline 2.197 & 4.394 & 61.516 & 8.788 \\
\hline 2.197 & 28.561 & 10.985 & 15.379 \\
\hline 4.394 & 147.199 & 993.044 & 13.182 \\
\hline 74.698 & 24.167 & 4.394 & 6.591 \\
\hline 8.788 & 2.197 & 4.394 & 6.591 \\
\hline 10.985 & 26.364 & 2.197 & 41.743 \\
\hline 6.591 & 17.576 & 4.394 & 4.394 \\
\hline 17.576 & 17.576 & 4.394 & 24.167 \\
\hline 2.197 & 4.394 & 8.788 & 10.985 \\
\hline 2.197 & 13.182 & 8.788 & 6.591 \\
\hline 2.197 & 46.137 & 13.182 & 32.955 \\
\hline 2.197 & 10.985 & 2.197 & 8.788 \\
\hline 2.197 & 21.97 & 65.91 & 2.197 \\
\hline 2.197 & 6.591 & 37.349 & 2.197 \\
\hline 26.364 & 2.197 & 153.79 & 2.197 \\
\hline 2.197 & 4.394 & 2.197 & 2.197 \\
\hline 2.197 & 4.394 & 2.197 & 4.394 \\
\hline 46.137 & 48.334 & 13.182 & 149.396 \\
\hline 2.197 & 6.591 & 4.394 & 13.182 \\
\hline 2.197 & 4.394 & 13.182 & 2.197 \\
\hline 296.595 & 8.788 & 2.197 & 2.197 \\
\hline 15.379 & 6.591 & 2.197 & 2.197 \\
\hline 26.364 & 26.364 & 6.591 & 2.197 \\
\hline 2.197 & 2.197 & 254.852 & 76.895 \\
\hline 13.182 & 13.182 & 2.197 & 4.394 \\
\hline 13.182 & 2.197 & 19.773 & 2.197 \\
\hline 2.197 & 2.197 & 26.364 & 2.197 \\
\hline 2.197 & 70.304 & 8.788 & 28.561 \\
\hline 2.197 & 57.122 & 4.394 & 15.379 \\
\hline 344.929 & 8.788 & 4.394 & 4.394 \\
\hline 2.197 & 4.394 & 13.182 & 37.349 \\
\hline 2.197 & 371.293 & 2.197 & 6.591 \\
\hline 2.197 & 107.653 & 2.197 & 6.591 \\
\hline 2.197 & 32.955 & 72.501 & 2.197 \\
\hline 26.364 & 83.486 & 4.394 & 696.449 \\
\hline 8.788 & 21.97 & 2.197 & 4.394 \\
\hline 28.561 & 32.955 & 2.197 & 1794.95 \\
\hline 2.197 & 2.197 & 2.197 & 6.591 \\
\hline 2.197 & 2.197 & 2.197 & 2.197 \\
\hline 2.197 & 2.197 & 17.576 & 2.197 \\
\hline 8.788 & 4.394 & 2.197 & 2.197 \\
\hline 2.197 & 4.394 & 76.895 & 1074.33 \\
\hline 2.197 & 92.274 & 4.394 & 2.197 \\
\hline 2.197 & 8.788 & 85.683 & 2.197 \\
\hline 2.197 & 28.561 & 13.182 & 2.197 \\
\hline 2.197 & 147.199 & 30.758 & 309.777 \\
\hline 2.197 & 182.351 & 59.319 & 57.122 \\
\hline
\end{tabular}




\begin{tabular}{|c|c|c|c|}
\hline 4.394 & 50.531 & 2.197 & 2.197 \\
\hline 8.788 & 4.394 & 2.197 & 21.97 \\
\hline 2.197 & 221.897 & 2.197 & 8.788 \\
\hline 24.167 & 13.182 & 2.197 & 2.197 \\
\hline 2.197 & 2.197 & 2.197 & 24.167 \\
\hline 2.197 & 35.152 & 2.197 & 17.576 \\
\hline 2.197 & 2.197 & 6.591 & 17.576 \\
\hline 2.197 & 17.576 & 2.197 & 17.576 \\
\hline 4.394 & 8.788 & 28.561 & 17.576 \\
\hline 8.788 & 120.835 & 8.788 & 17.576 \\
\hline 4.394 & 46.137 & 2.197 & 10.985 \\
\hline 24.167 & 221.897 & 2.197 & 2.197 \\
\hline 2.197 & 2.197 & 333.944 & 6.591 \\
\hline 2.197 & 13.182 & 101.062 & 8.788 \\
\hline 4.394 & 41.743 & 15.379 & 21.97 \\
\hline 10.985 & 2.197 & 2.197 & 2.197 \\
\hline 6.591 & 217.503 & 52.728 & 118.638 \\
\hline 344.929 & 573.417 & 6.591 & 6.591 \\
\hline 2.197 & 28.561 & 26.364 & 8.788 \\
\hline 8.788 & 257.049 & 2.197 & 6.591 \\
\hline 65.91 & 19.773 & 2.197 & 76.895 \\
\hline 26.364 & 32.955 & 8.788 & 2438.67 \\
\hline 2.197 & 48.334 & 2.197 & 28.561 \\
\hline 4.394 & 2.197 & 2.197 & 28.561 \\
\hline 4.394 & 10.985 & 4.394 & 32.955 \\
\hline 13.182 & 101.062 & 6.591 & 28.561 \\
\hline 74.698 & 2.197 & 79.092 & 26.364 \\
\hline 94.471 & 17.576 & 8.788 & 208.715 \\
\hline 15.379 & 70.304 & 8.788 & 169.169 \\
\hline 4.394 & 19.773 & 2.197 & 516.295 \\
\hline 2.197 & 37.349 & 6.591 & 17.576 \\
\hline 2.197 & 2.197 & 2.197 & 30.758 \\
\hline 17.576 & 15.379 & 2.197 & 35.152 \\
\hline 2.197 & 48.334 & 2.197 & 83.486 \\
\hline 199.927 & 8.788 & 6.591 & 6.591 \\
\hline 2.197 & 180.154 & 2.197 & 6.591 \\
\hline 8.788 & 21.97 & 2.197 & 24.167 \\
\hline 2.197 & 6.591 & 2.197 & 2.197 \\
\hline 2.197 & 2.197 & 2.197 & 2.197 \\
\hline 61.516 & 4.394 & 4.394 & 63.713 \\
\hline 2.197 & 39.546 & 2.197 & 2.197 \\
\hline 6.591 & 4.394 & 4.394 & 2.197 \\
\hline 2.197 & 4.394 & 13.182 & 208.715 \\
\hline 2.197 & 2.197 & 2.197 & 37.349 \\
\hline 2.197 & 2.197 & 2.197 & 2.197 \\
\hline 30.758 & 2.197 & 2.197 & 2.197 \\
\hline 125.229 & 24.167 & 2.197 & 21.97 \\
\hline 63.713 & 6.591 & 2.197 & 6.591 \\
\hline 26.364 & 2.197 & 2.197 & 4.394 \\
\hline 6.591 & 8.788 & 109.85 & 4.394 \\
\hline
\end{tabular}




\begin{tabular}{|c|c|c|c|}
\hline 2.197 & 4.394 & 2.197 & 63.713 \\
\hline 2.197 & 2.197 & 4.394 & 50.531 \\
\hline 2.197 & 13.182 & 2.197 & 2.197 \\
\hline 8.788 & 2.197 & 19.773 & 2.197 \\
\hline 2.197 & 21.97 & 2.197 & 8.788 \\
\hline 2.197 & 2.197 & 32.955 & 2.197 \\
\hline 4.394 & 2.197 & 1685.1 & 2.197 \\
\hline 15.379 & 2.197 & 61.516 & 2.197 \\
\hline 2.197 & 2.197 & 4.394 & 2.197 \\
\hline 4.394 & 6.591 & 2.197 & 2.197 \\
\hline 6.591 & 28.561 & 4.394 & 2.197 \\
\hline 4.394 & 19.773 & 507.507 & 2.197 \\
\hline 4.394 & 24.167 & 2.197 & 371.293 \\
\hline 21.97 & 4.394 & 13.182 & 4.394 \\
\hline 1696.08 & 8.788 & 2.197 & 13.182 \\
\hline 2.197 & 2.197 & 2.197 & 4.394 \\
\hline 103.259 & 79.092 & 28.561 & 70.304 \\
\hline 94.471 & 2.197 & 2.197 & 4446.73 \\
\hline 10.985 & 2.197 & 8.788 & 74.698 \\
\hline 2.197 & 101.062 & 2.197 & 2.197 \\
\hline 2.197 & 2.197 & 2.197 & 2.197 \\
\hline 2.197 & 2.197 & 2.197 & 2.197 \\
\hline 2.197 & 2.197 & 6.591 & 384.475 \\
\hline 28.561 & 52.728 & 13.182 & 13.182 \\
\hline 2.197 & 57.122 & 843.648 & 2.197 \\
\hline 292.201 & 2.197 & 2.197 & 4.394 \\
\hline 2.197 & 2.197 & 26.364 & 2.197 \\
\hline 61.516 & 2.197 & 2.197 & 37.349 \\
\hline 131.82 & 2.197 & 37.349 & 2.197 \\
\hline 2.197 & 6.591 & 2.197 & 37.349 \\
\hline 2.197 & 2.197 & 26.364 & 13.182 \\
\hline 74.698 & 2.197 & 19.773 & 15.379 \\
\hline 4.394 & 4.394 & 6.591 & 37.349 \\
\hline 2.197 & 103.259 & 21.97 & 2.197 \\
\hline 4.394 & 8.788 & 17.576 & 2.197 \\
\hline 6.591 & 2.197 & 46.137 & 8.788 \\
\hline 4.394 & 8.788 & 3686.57 & 15.379 \\
\hline 30.758 & 2.197 & 4.394 & 15.379 \\
\hline 2.197 & 4.394 & 2.197 & 37.349 \\
\hline 28.561 & 4.394 & 24.167 & 281.216 \\
\hline 8.788 & 6.591 & 15.379 & 43.94 \\
\hline 2.197 & 6.591 & 2.197 & 2.197 \\
\hline 15.379 & 199.927 & 4.394 & 2.197 \\
\hline 1588.43 & 2.197 & 4.394 & 2.197 \\
\hline 2.197 & 2.197 & 105.456 & 57.122 \\
\hline 2.197 & 17.576 & 2.197 & 65.91 \\
\hline 2.197 & 8.788 & 92.274 & 8.788 \\
\hline 746.98 & 37.349 & 2.197 & 48.334 \\
\hline 19.773 & 15.379 & 61.516 & 94.471 \\
\hline 4.394 & 4.394 & 68.107 & 41.743 \\
\hline
\end{tabular}




\begin{tabular}{|c|c|c|c|}
\hline 2.197 & 43.94 & 2.197 & 2.197 \\
\hline 26.364 & 2.197 & 2.197 & 2.197 \\
\hline 2.197 & 32.955 & 2.197 & 2.197 \\
\hline 10.985 & 24.167 & 4.394 & 4.394 \\
\hline 4.394 & 2.197 & 2.197 & 61.516 \\
\hline 6.591 & 24.167 & 70.304 & 37.349 \\
\hline 4.394 & 138.411 & 2.197 & 2.197 \\
\hline 6.591 & 39.546 & 28.561 & 2.197 \\
\hline 2.197 & 2.197 & 15.379 & 13.182 \\
\hline 2.197 & 4.394 & 6.591 & 15.379 \\
\hline 8.788 & 30.758 & 41.743 & 92.274 \\
\hline 626.145 & 2.197 & 6.591 & 4.394 \\
\hline 2.197 & 4.394 & 2.197 & 10.985 \\
\hline 4.394 & 59.319 & 4.394 & 123.032 \\
\hline 4.394 & 129.623 & 10.985 & 2.197 \\
\hline 13.182 & 2.197 & 4.394 & 2.197 \\
\hline 81.289 & 68.107 & 2.197 & 4.394 \\
\hline 2.197 & 2.197 & 2.197 & 81.289 \\
\hline 2.197 & 28.561 & 410.839 & 24.167 \\
\hline 2.197 & 8.788 & 13.182 & 15.379 \\
\hline 8.788 & 19.773 & 2.197 & 6.591 \\
\hline 4.394 & 26.364 & 799.708 & 17.576 \\
\hline 6.591 & 4.394 & 2.197 & 2.197 \\
\hline 10.985 & 6.591 & 28.561 & 6.591 \\
\hline 2.197 & 10.985 & 1663.13 & 2.197 \\
\hline 8.788 & 2.197 & 2.197 & 4.394 \\
\hline 24.167 & 2.197 & 307.58 & 37.349 \\
\hline 24.167 & 2.197 & 8.788 & 50.531 \\
\hline 21.97 & 2.197 & 270.231 & 6.591 \\
\hline 6.591 & 274.625 & 57.122 & 2.197 \\
\hline 191.139 & 52.728 & 94.471 & 2.197 \\
\hline 2.197 & 21.97 & 6.591 & 116.441 \\
\hline 2.197 & 2.197 & 35.152 & 4.394 \\
\hline 2.197 & 10.985 & 6.591 & 4.394 \\
\hline 2.197 & 2.197 & 145.002 & 6.591 \\
\hline 2.197 & 19.773 & 2.197 & 2.197 \\
\hline 15.379 & 2.197 & 61.516 & 2.197 \\
\hline 6.591 & 8.788 & 10.985 & 10.985 \\
\hline 15.379 & 79.092 & 26.364 & 98.865 \\
\hline 2.197 & 28.561 & 46.137 & 39.546 \\
\hline 6.591 & 54.925 & 2.197 & 2.197 \\
\hline 19.773 & 8.788 & 6.591 & 17.576 \\
\hline 6.591 & 30.758 & 2.197 & 2.197 \\
\hline 6.591 & 26.364 & 13.182 & 26.364 \\
\hline 2.197 & 17.576 & 26.364 & 57.122 \\
\hline 8.788 & 8.788 & 2.197 & 2.197 \\
\hline 2.197 & 158.184 & 2.197 & 2.197 \\
\hline 296.595 & 17.576 & 21.97 & 10.985 \\
\hline 90.077 & 8.788 & 320.762 & 145.002 \\
\hline 17.576 & 13.182 & 35.152 & 35.152 \\
\hline
\end{tabular}




\begin{tabular}{|c|c|c|c|}
\hline 6.591 & 1395.09 & 83.486 & 17.576 \\
\hline 2.197 & 43.94 & 235.079 & 2.197 \\
\hline 8.788 & 243.867 & 2.197 & 2.197 \\
\hline 24.167 & 15.379 & 8.788 & 17.576 \\
\hline 2.197 & 4.394 & 2.197 & 17.576 \\
\hline 13.182 & 4.394 & 46.137 & 32.955 \\
\hline 2.197 & 2.197 & 57.122 & 162.578 \\
\hline 4.394 & 2.197 & 4.394 & 54.925 \\
\hline 2.197 & 10.985 & 6.591 & 8.788 \\
\hline 138.411 & 4.394 & 2.197 & 70.304 \\
\hline 2.197 & 15.379 & 2.197 & 4.394 \\
\hline 2.197 & 2.197 & 2.197 & 4.394 \\
\hline 2.197 & 6.591 & 41.743 & 21.97 \\
\hline 4.394 & 19.773 & 46.137 & 10.985 \\
\hline 21.97 & 32.955 & 19.773 & 2.197 \\
\hline 10.985 & 10.985 & 10.985 & 54.925 \\
\hline 8.788 & 2.197 & 2.197 & 79.092 \\
\hline 4.394 & 6.591 & 307.58 & 2.197 \\
\hline 8.788 & 10.985 & 2.197 & 6.591 \\
\hline 2.197 & 2.197 & 2.197 & 2.197 \\
\hline 6.591 & 1004.03 & 2.197 & 32.955 \\
\hline 2.197 & 8.788 & 2.197 & 21.97 \\
\hline 2.197 & 4.394 & 105.456 & 6.591 \\
\hline 1019.41 & 35.152 & 2.197 & 2.197 \\
\hline 8.788 & 8.788 & 2.197 & 57.122 \\
\hline 2.197 & 17.576 & 2.197 & 24.167 \\
\hline 4.394 & 30.758 & 2.197 & 307.58 \\
\hline 4.394 & 2.197 & 2.197 & 4.394 \\
\hline 2.197 & 2.197 & 2.197 & 2.197 \\
\hline 2.197 & 549.25 & 8.788 & 2.197 \\
\hline 2.197 & 2.197 & 2.197 & 13.182 \\
\hline 2.197 & 107.653 & 2.197 & 13.182 \\
\hline 2.197 & 28.561 & 213.109 & 10.985 \\
\hline 13.182 & 8.788 & 28.561 & 8.788 \\
\hline 8.788 & 2.197 & 2.197 & 2.197 \\
\hline 4.394 & 2.197 & 2.197 & 43.94 \\
\hline 8.788 & 13.182 & 2.197 & 8.788 \\
\hline 19.773 & 6.591 & 2.197 & 2.197 \\
\hline 8.788 & 6.591 & 2.197 & 35.152 \\
\hline 362.505 & 4.394 & 15.379 & 2.197 \\
\hline 2.197 & 35.152 & 2.197 & 4314.91 \\
\hline 2.197 & 4.394 & 59.319 & 19.773 \\
\hline 2.197 & 52.728 & 15.379 & 8.788 \\
\hline 4.394 & 140.608 & 353.717 & 10.985 \\
\hline 4.394 & 395.46 & 6.591 & 15.379 \\
\hline 2.197 & 37.349 & 2.197 & 4.394 \\
\hline 2.197 & 2.197 & 2.197 & 30.758 \\
\hline 2.197 & 2.197 & 4.394 & 4.394 \\
\hline 2.197 & 4.394 & 2.197 & 21.97 \\
\hline 112.047 & 4.394 & 4.394 & 8.788 \\
\hline
\end{tabular}




\begin{tabular}{|c|c|c|c|}
\hline 2.197 & 4.394 & 43.94 & 364.702 \\
\hline 123.032 & 2.197 & 4.394 & 13.182 \\
\hline 2.197 & 94.471 & 6.591 & 6.591 \\
\hline 4.394 & 8.788 & 2.197 & 4.394 \\
\hline 43.94 & 17.576 & 2.197 & 4.394 \\
\hline 39.546 & 28.561 & 2.197 & 63.713 \\
\hline 654.706 & 50.531 & 2.197 & 2.197 \\
\hline 149.396 & 1186.38 & 2.197 & 2.197 \\
\hline 74.698 & 2.197 & 2.197 & 2.197 \\
\hline 17.576 & 74.698 & 6.591 & 2.197 \\
\hline 4.394 & 6.591 & 2.197 & 30.758 \\
\hline 2.197 & 19.773 & 2.197 & 8.788 \\
\hline 2.197 & 35.152 & 2.197 & 10.985 \\
\hline 15.379 & 41.743 & 230.685 & 30.758 \\
\hline 2.197 & 10.985 & 2.197 & 4.394 \\
\hline 17.576 & 24.167 & 8.788 & 4.394 \\
\hline 17.576 & 4.394 & 39.546 & 507.507 \\
\hline 17.576 & 6.591 & 4.394 & 2.197 \\
\hline 2.197 & 2.197 & 2.197 & 35.152 \\
\hline 13.182 & 2.197 & 2.197 & 131.82 \\
\hline 41.743 & 26.364 & 6.591 & 4.394 \\
\hline 15.379 & 2.197 & 478.946 & 17.576 \\
\hline 2.197 & 2.197 & 2.197 & 373.49 \\
\hline 2.197 & 2.197 & 2.197 & 63.713 \\
\hline 129.623 & 21.97 & 2.197 & 2.197 \\
\hline 4.394 & 8.788 & 127.426 & 2.197 \\
\hline 35.152 & 2.197 & 32.955 & 155.987 \\
\hline 235.079 & 83.486 & 17.576 & 28.561 \\
\hline 35.152 & 65.91 & 8.788 & 2.197 \\
\hline 2.197 & 114.244 & 4.394 & 35.152 \\
\hline 2.197 & 28.561 & 2.197 & 8.788 \\
\hline 43.94 & 43.94 & 83.486 & 70.304 \\
\hline 10.985 & 4.394 & 15.379 & 10.985 \\
\hline 10.985 & 32.955 & 2.197 & 26.364 \\
\hline 41.743 & 61.516 & 4.394 & 6.591 \\
\hline 17.576 & 560.235 & 61.516 & 15.379 \\
\hline 6.591 & 2.197 & 8.788 & 4.394 \\
\hline 4.394 & 6.591 & 2.197 & 2.197 \\
\hline 162.578 & 72.501 & 4.394 & 19.773 \\
\hline 294.398 & 63.713 & 81.289 & 13.182 \\
\hline 2.197 & 6.591 & 2.197 & 72.501 \\
\hline 2.197 & 8.788 & 2.197 & 2.197 \\
\hline 2.197 & 43.94 & 2.197 & 19.773 \\
\hline 2.197 & 8.788 & 2.197 & 2.197 \\
\hline 6.591 & 2.197 & 2.197 & 10.985 \\
\hline 2.197 & 6.591 & 1722.45 & 6.591 \\
\hline 2.197 & 2.197 & 6.591 & 10.985 \\
\hline 2.197 & 2.197 & 2.197 & 26.364 \\
\hline 10.985 & 2.197 & 2.197 & 70.304 \\
\hline 35.152 & 2.197 & 2.197 & 2.197 \\
\hline
\end{tabular}




\begin{tabular}{|c|c|c|c|}
\hline 8.788 & 2.197 & 2.197 & 98.865 \\
\hline 10.985 & 2.197 & 19.773 & 70.304 \\
\hline 2.197 & 15.379 & 2.197 & 35.152 \\
\hline 2.197 & 6.591 & 2.197 & 120.835 \\
\hline 8.788 & 19.773 & 2.197 & 21.97 \\
\hline 10.985 & 8.788 & 2.197 & 13.182 \\
\hline 8.788 & 79.092 & 2.197 & 4.394 \\
\hline 10.985 & 4.394 & 2.197 & 28.561 \\
\hline 6.591 & 2.197 & 2.197 & 21.97 \\
\hline 8.788 & 4.394 & 2.197 & 37.349 \\
\hline 6.591 & 2.197 & 2.197 & 17.576 \\
\hline 2.197 & 2.197 & 10.985 & 109.85 \\
\hline 15.379 & 92.274 & 6.591 & 28.561 \\
\hline 2.197 & 28.561 & 2.197 & 2.197 \\
\hline 6.591 & 17.576 & 63.713 & 2.197 \\
\hline 2.197 & 37.349 & 4.394 & 15.379 \\
\hline 2.197 & 13.182 & 6.591 & 2.197 \\
\hline 2.197 & 2.197 & 2.197 & 2.197 \\
\hline 17.576 & 4.394 & 4.394 & 8.788 \\
\hline 2.197 & 2.197 & 103.259 & 6.591 \\
\hline 4.394 & 26.364 & 2.197 & 4.394 \\
\hline 8.788 & 6.591 & 2.197 & 70.304 \\
\hline 17.576 & 4.394 & 17.576 & 10.985 \\
\hline 37.349 & 59.319 & 54.925 & 4.394 \\
\hline 232.882 & 2.197 & 2.197 & 24.167 \\
\hline 6.591 & 2.197 & 2.197 & 4.394 \\
\hline 4.394 & 2.197 & 123.032 & 52.728 \\
\hline 131.82 & 13.182 & 226.291 & 24.167 \\
\hline 8.788 & 10.985 & 2.197 & 2.197 \\
\hline 2.197 & 35.152 & 127.426 & 2.197 \\
\hline 10.985 & 6.591 & 24.167 & 2.197 \\
\hline 17.576 & 6.591 & 2.197 & 10.985 \\
\hline 6.591 & 28.561 & 2.197 & 4.394 \\
\hline 472.355 & 2.197 & 8.788 & 2.197 \\
\hline 2.197 & 8.788 & 325.156 & 2.197 \\
\hline 4.394 & 52.728 & 2.197 & 8.788 \\
\hline 8.788 & 2.197 & 2.197 & 2.197 \\
\hline 19.773 & 26.364 & 17.576 & 30.758 \\
\hline 6.591 & 6.591 & 6.591 & 621.751 \\
\hline 2.197 & 21.97 & 2.197 & 39.546 \\
\hline 2.197 & 17.576 & 4.394 & 30.758 \\
\hline 2.197 & 2.197 & 6.591 & 90.077 \\
\hline 39.546 & 41.743 & 2.197 & 15.379 \\
\hline 4.394 & 19.773 & 9831.57 & 26.364 \\
\hline 2.197 & 30.758 & 32.955 & 39.546 \\
\hline 2.197 & 4.394 & 2.197 & 2.197 \\
\hline 2.197 & 32.955 & 13.182 & 4.394 \\
\hline 4.394 & 2.197 & 6.591 & 24.167 \\
\hline 4.394 & 2.197 & 2.197 & 4.394 \\
\hline 2.197 & 15.379 & 4.394 & 2.197 \\
\hline
\end{tabular}




\begin{tabular}{|c|c|c|c|}
\hline 8.788 & 48.334 & 6.591 & 30.758 \\
\hline 19.773 & 52.728 & 37.349 & 59.319 \\
\hline 52.728 & 79.092 & 2.197 & 21.97 \\
\hline 37.349 & 8.788 & 2.197 & 4.394 \\
\hline 116.441 & 13.182 & 74.698 & 2.197 \\
\hline 54.925 & 243.867 & 2.197 & 4.394 \\
\hline 17.576 & 4.394 & 2.197 & 2.197 \\
\hline 70.304 & 2.197 & 2.197 & 57.122 \\
\hline 217.503 & 2.197 & 4.394 & 101.062 \\
\hline 101.062 & 10.985 & 4.394 & 2.197 \\
\hline 79.092 & 79.092 & 4.394 & 41.743 \\
\hline 32.955 & 13.182 & 2.197 & 2.197 \\
\hline 6.591 & 17.576 & 2.197 & 19.773 \\
\hline 114.244 & 41.743 & 2.197 & 6.591 \\
\hline 6.591 & 2.197 & 2.197 & 2.197 \\
\hline 8.788 & 2.197 & 292.201 & 46.137 \\
\hline 4.394 & 184.548 & 2.197 & 6.591 \\
\hline 21.97 & 13.182 & 13.182 & 2.197 \\
\hline 2.197 & 13.182 & 6.591 & 21.97 \\
\hline 39.546 & 4.394 & 13.182 & 21.97 \\
\hline 2.197 & 8.788 & 10.985 & 90.077 \\
\hline 2.197 & 15.379 & 4.394 & 349.323 \\
\hline 4.394 & 4.394 & 4.394 & 2.197 \\
\hline 955.695 & 13.182 & 6.591 & 15.379 \\
\hline 2.197 & 2.197 & 2.197 & 2.197 \\
\hline 4.394 & 10.985 & 52.728 & 41.743 \\
\hline 4.394 & 2.197 & 2.197 & 4.394 \\
\hline 4.394 & 2.197 & 4.394 & 10.985 \\
\hline 10.985 & 6.591 & 10.985 & 8.788 \\
\hline 2.197 & 2.197 & 70.304 & 19.773 \\
\hline 19.773 & 2.197 & 4.394 & 2.197 \\
\hline 2.197 & 10.985 & 4.394 & 17.576 \\
\hline 21.97 & 140.608 & 2.197 & 10.985 \\
\hline 221.897 & 4.394 & 21.97 & 10.985 \\
\hline 2.197 & 17.576 & 2.197 & 6.591 \\
\hline 2.197 & 2.197 & 2.197 & 35.152 \\
\hline 2.197 & 221.897 & 10.985 & 10.985 \\
\hline 8.788 & 2.197 & 2.197 & 2.197 \\
\hline 4.394 & 2.197 & 8.788 & 2.197 \\
\hline 32.955 & 2.197 & 8.788 & 6.591 \\
\hline 4.394 & 13.182 & 2.197 & 10.985 \\
\hline 2.197 & 2.197 & 6.591 & 4.394 \\
\hline 232.882 & 2.197 & 15.379 & 4.394 \\
\hline 2.197 & 8.788 & 2.197 & 92.274 \\
\hline 2.197 & 2.197 & 2.197 & 8.788 \\
\hline 4.394 & 4.394 & 2.197 & 107.653 \\
\hline 6.591 & 2.197 & 13.182 & 2.197 \\
\hline 6.591 & 21.97 & 2.197 & 2.197 \\
\hline 2.197 & 2.197 & 182.351 & 2.197 \\
\hline 17.576 & 6.591 & 2.197 & 2.197 \\
\hline
\end{tabular}




\begin{tabular}{|c|c|c|c|}
\hline 2.197 & 4.394 & 59.319 & 2.197 \\
\hline 2.197 & 35.152 & 4.394 & 103.259 \\
\hline 10.985 & 26.364 & 4.394 & 2.197 \\
\hline 4.394 & 43.94 & 2.197 & 2.197 \\
\hline 70.304 & 6.591 & 2.197 & 2.197 \\
\hline 61.516 & 8.788 & 8.788 & 2.197 \\
\hline 4.394 & 6.591 & 52.728 & 61.516 \\
\hline 17.576 & 52.728 & 2.197 & 61.516 \\
\hline 2.197 & 180.154 & 210.912 & 4.394 \\
\hline 2.197 & 8.788 & 26.364 & 61.516 \\
\hline 4.394 & 2.197 & 4.394 & 13.182 \\
\hline 10.985 & 2.197 & 8.788 & 8.788 \\
\hline 52.728 & 6.591 & 17.576 & 2.197 \\
\hline 6.591 & 4.394 & 4.394 & 4.394 \\
\hline 2.197 & 37.349 & 17.576 & 5782.5 \\
\hline 2.197 & 19.773 & 19.773 & 13.182 \\
\hline 2.197 & 10.985 & 98.865 & 129.623 \\
\hline 10.985 & 369.096 & 641.524 & 10.985 \\
\hline 17.576 & 43.94 & 4.394 & 3187.85 \\
\hline 2.197 & 10.985 & 39.546 & 26.364 \\
\hline 2.197 & 28.561 & 6.591 & 127.426 \\
\hline 2.197 & 4.394 & 24.167 & 19.773 \\
\hline 292.201 & 10.985 & 2.197 & 2.197 \\
\hline 2.197 & 17.576 & 2.197 & 272.428 \\
\hline 4.394 & 2.197 & 2.197 & 4.394 \\
\hline 2.197 & 4.394 & 2.197 & 15.379 \\
\hline 30.758 & 160.381 & 2.197 & 2.197 \\
\hline 6.591 & 13.182 & 10.985 & 13.182 \\
\hline 68.107 & 28.561 & 19.773 & 428.415 \\
\hline 2.197 & 24.167 & 4.394 & 94.471 \\
\hline 902.967 & 41.743 & 2.197 & 162.578 \\
\hline 81.289 & 2.197 & 2.197 & 2.197 \\
\hline 158.184 & 8.788 & 4.394 & 24.167 \\
\hline 19.773 & 13.182 & 4.394 & 81.289 \\
\hline 19.773 & 4.394 & 24.167 & 24.167 \\
\hline 2.197 & 54.925 & 2.197 & 19.773 \\
\hline 2.197 & 2.197 & 13.182 & 85.683 \\
\hline 13.182 & 2.197 & 2.197 & 2.197 \\
\hline 2.197 & 2.197 & 4.394 & 2.197 \\
\hline 2.197 & 2.197 & 6.591 & 2.197 \\
\hline 6.591 & 8.788 & 4.394 & 2.197 \\
\hline 2.197 & 41.743 & 2.197 & 2.197 \\
\hline 2.197 & 24.167 & 2.197 & 46.137 \\
\hline 2.197 & 118.638 & 13.182 & 10.985 \\
\hline 8.788 & 35.152 & 2.197 & 48.334 \\
\hline 4.394 & 43.94 & 79.092 & 8.788 \\
\hline 2.197 & 160.381 & 2.197 & 2.197 \\
\hline 8.788 & 2.197 & 2.197 & 10.985 \\
\hline 4.394 & 70.304 & 2.197 & 30.758 \\
\hline 4.394 & 2.197 & 2.197 & 8.788 \\
\hline
\end{tabular}




\begin{tabular}{|c|c|c|c|}
\hline 15.379 & 10.985 & 2.197 & 37.349 \\
\hline 6.591 & 2.197 & 13.182 & 26.364 \\
\hline 4.394 & 2.197 & 2.197 & 2.197 \\
\hline 145.002 & 2.197 & 2.197 & 46.137 \\
\hline 15.379 & 26.364 & 8.788 & 2.197 \\
\hline 6.591 & 2.197 & 2.197 & 57.122 \\
\hline 8.788 & 129.623 & 32.955 & 2.197 \\
\hline 2.197 & 17.576 & 184.548 & 158.184 \\
\hline 35.152 & 4.394 & 2.197 & 8.788 \\
\hline 2.197 & 2.197 & 2.197 & 2.197 \\
\hline 68.107 & 2.197 & 2.197 & 39.546 \\
\hline 24.167 & 2.197 & 8.788 & 2.197 \\
\hline 2.197 & 15.379 & 6.591 & 4.394 \\
\hline 2.197 & 15.379 & 2.197 & 2.197 \\
\hline 2.197 & 2.197 & 4.394 & 2.197 \\
\hline 2.197 & 10.985 & 2.197 & 6.591 \\
\hline 13.182 & 50.531 & 8.788 & 2.197 \\
\hline 17.576 & 2.197 & 6.591 & 65.91 \\
\hline 13.182 & 2.197 & 2.197 & 13.182 \\
\hline 19.773 & 21.97 & 43.94 & 41.743 \\
\hline 2.197 & 2.197 & 4.394 & 2.197 \\
\hline 2.197 & 2.197 & 46.137 & 10.985 \\
\hline 2.197 & 4.394 & 2.197 & 15.379 \\
\hline 4.394 & 4.394 & 4.394 & 6.591 \\
\hline 2.197 & 2.197 & 2.197 & 48.334 \\
\hline 4.394 & 2.197 & 2.197 & 4.394 \\
\hline 2.197 & 28.561 & 358.111 & 54.925 \\
\hline 4.394 & 24.167 & 50.531 & 15.379 \\
\hline 2.197 & 21.97 & 2.197 & 173.563 \\
\hline 226.291 & 4.394 & 4.394 & 4.394 \\
\hline 2.197 & 4.394 & 15.379 & 125.229 \\
\hline 2.197 & 4.394 & 353.717 & 26.364 \\
\hline 4.394 & 17.576 & 50.531 & 41.743 \\
\hline 26.364 & 57.122 & 24.167 & 2.197 \\
\hline 35.152 & 19.773 & 13.182 & 74.698 \\
\hline 4.394 & 10.985 & 2.197 & 17.576 \\
\hline 13.182 & 6.591 & 2.197 & 19.773 \\
\hline 538.265 & 2.197 & 2.197 & 1436.84 \\
\hline 8.788 & 4.394 & 2.197 & 2.197 \\
\hline 2.197 & 8.788 & 4.394 & 10.985 \\
\hline 8.788 & 17.576 & 4.394 & 2.197 \\
\hline 4.394 & 74.698 & 2.197 & 2.197 \\
\hline 37.349 & 24.167 & 68.107 & 2.197 \\
\hline 2.197 & 13.182 & 37.349 & 48.334 \\
\hline 2.197 & 41.743 & 2.197 & 72.501 \\
\hline 2.197 & 13.182 & 10.985 & 4.394 \\
\hline 4.394 & 4.394 & 177.957 & 2.197 \\
\hline 2.197 & 2.197 & 228.488 & 2.197 \\
\hline 2.197 & 4.394 & 2.197 & 35.152 \\
\hline 2.197 & 727.207 & 2.197 & 4.394 \\
\hline
\end{tabular}




\begin{tabular}{|c|c|c|c|}
\hline 30.758 & 19.773 & 2.197 & 8.788 \\
\hline 8.788 & 2.197 & 2.197 & 35.152 \\
\hline 1056.76 & 6.591 & 2.197 & 13.182 \\
\hline 6.591 & 30.758 & 4.394 & 6.591 \\
\hline 107.653 & 2.197 & 2.197 & 2.197 \\
\hline 2.197 & 2.197 & 8.788 & 4.394 \\
\hline 6.591 & 4.394 & 2.197 & 6.591 \\
\hline 21.97 & 4.394 & 6.591 & 61.516 \\
\hline 2.197 & 8.788 & 10.985 & 2.197 \\
\hline 2.197 & 4.394 & 1175.39 & 2.197 \\
\hline 37.349 & 2.197 & 50.531 & 2.197 \\
\hline 4.394 & 10.985 & 2.197 & 2.197 \\
\hline 2.197 & 10.985 & 215.306 & 10.985 \\
\hline 30.758 & 43.94 & 6.591 & 19.773 \\
\hline 26.364 & 10.985 & 4.394 & 2.197 \\
\hline 1652.14 & 17.576 & 2.197 & 24.167 \\
\hline 2.197 & 4.394 & 85.683 & 2.197 \\
\hline 2.197 & 2.197 & 2.197 & 2.197 \\
\hline 4.394 & 8.788 & 2.197 & 2.197 \\
\hline 2.197 & 6.591 & 2.197 & 171.366 \\
\hline 17.576 & 8.788 & 37.349 & 2.197 \\
\hline 6.591 & 2.197 & 960.089 & 26.364 \\
\hline 131.82 & 116.441 & 160.381 & 59.319 \\
\hline 2.197 & 41.743 & 63.713 & 26.364 \\
\hline 2.197 & 21.97 & 2.197 & 26.364 \\
\hline 2.197 & 6.591 & 6.591 & 54.925 \\
\hline 2.197 & 43.94 & 4.394 & 28.561 \\
\hline 2.197 & 39.546 & 309.777 & 6.591 \\
\hline 4.394 & 6.591 & 2752.84 & 15.379 \\
\hline 4.394 & 59.319 & 19.773 & 6.591 \\
\hline 2.197 & 2.197 & 13.182 & 2.197 \\
\hline 2.197 & 2.197 & 4.394 & 83.486 \\
\hline 24.167 & 2.197 & 2.197 & 2.197 \\
\hline 8.788 & 134.017 & 2.197 & 6.591 \\
\hline 19.773 & 6.591 & 2.197 & 15.379 \\
\hline 13.182 & 4.394 & 4.394 & 4.394 \\
\hline 2.197 & 4.394 & 4.394 & 90.077 \\
\hline 41.743 & 81.289 & 10.985 & 2.197 \\
\hline 6.591 & 4.394 & 2.197 & 2.197 \\
\hline 52.728 & 320.762 & 103.259 & 6.591 \\
\hline 94.471 & 2.197 & 206.518 & 2.197 \\
\hline 2.197 & 4.394 & 6.591 & 2.197 \\
\hline 2.197 & 2.197 & 2.197 & 2.197 \\
\hline 2.197 & 19.773 & 2.197 & 2.197 \\
\hline 2.197 & 32.955 & 17.576 & 2.197 \\
\hline 8.788 & 43.94 & 4.394 & 2.197 \\
\hline 2.197 & 4.394 & 2.197 & 2.197 \\
\hline 674.479 & 314.171 & 2.197 & 15.379 \\
\hline 2.197 & 6.591 & 10.985 & 70.304 \\
\hline 2.197 & 26.364 & 213.109 & 17.576 \\
\hline
\end{tabular}




\begin{tabular}{|c|c|c|c|}
\hline 2.197 & 224.094 & 2.197 & 2.197 \\
\hline 2.197 & 46.137 & 17.576 & 57.122 \\
\hline 4.394 & 37.349 & 4.394 & 4.394 \\
\hline 74.698 & 48.334 & 107.653 & 169.169 \\
\hline 6336.15 & 17.576 & 4.394 & 8.788 \\
\hline 6.591 & 2.197 & 2.197 & 4.394 \\
\hline 2.197 & 30.758 & 17.576 & 8.788 \\
\hline 2.197 & 2.197 & 2.197 & 13.182 \\
\hline 2.197 & 39.546 & 257.049 & 8.788 \\
\hline 1397.29 & 52.728 & 2.197 & 13.182 \\
\hline 2.197 & 193.336 & 2.197 & 54.925 \\
\hline 2.197 & 2.197 & 120.835 & 4.394 \\
\hline 2.197 & 2.197 & 46.137 & 17.576 \\
\hline 8.788 & 2.197 & 46.137 & 2.197 \\
\hline 2.197 & 109.85 & 6.591 & 13.182 \\
\hline 17.576 & 19.773 & 8.788 & 252.655 \\
\hline 2.197 & 2.197 & 2.197 & 8.788 \\
\hline 19.773 & 30.758 & 6.591 & 2.197 \\
\hline 2.197 & 2.197 & 4.394 & 4.394 \\
\hline 2.197 & 4.394 & 2.197 & 13.182 \\
\hline 2.197 & 2.197 & 2.197 & 2.197 \\
\hline 2.197 & 142.805 & 2093.74 & 6.591 \\
\hline 35.152 & 2.197 & 8.788 & 39.546 \\
\hline 2.197 & 4.394 & 32.955 & 107.653 \\
\hline 2.197 & 4.394 & 8.788 & 6.591 \\
\hline 1030.39 & 4.394 & 19.773 & 2.197 \\
\hline 13.182 & 70.304 & 6.591 & 2.197 \\
\hline 2.197 & 2.197 & 54.925 & 72.501 \\
\hline 19.773 & 37.349 & 83.486 & 2.197 \\
\hline 28.561 & 83.486 & 81.289 & 2.197 \\
\hline 35.152 & 4.394 & 2.197 & 2.197 \\
\hline 13.182 & 395.46 & 17.576 & 4.394 \\
\hline 8.788 & 125.229 & 2.197 & 24.167 \\
\hline 24.167 & 768.95 & 2.197 & 4.394 \\
\hline 2.197 & 8.788 & 4.394 & 2.197 \\
\hline 24.167 & 17.576 & 6.591 & 59.319 \\
\hline 6.591 & 6.591 & 17.576 & 37.349 \\
\hline 41.743 & 6.591 & 6.591 & 32.955 \\
\hline 213.109 & 24.167 & 112.047 & 87.88 \\
\hline 2.197 & 15.379 & 2.197 & 6.591 \\
\hline 4.394 & 39.546 & 10.985 & 6.591 \\
\hline 2.197 & 6.591 & 10.985 & 41.743 \\
\hline 6.591 & 17.576 & 8.788 & 6.591 \\
\hline 17.576 & 32.955 & 2.197 & 547.053 \\
\hline 6.591 & 32.955 & 35.152 & 46.137 \\
\hline 239.473 & 61.516 & 2.197 & 81.289 \\
\hline 2.197 & 4.394 & 41.743 & 35.152 \\
\hline 6.591 & 2.197 & 2.197 & 125.229 \\
\hline 2.197 & 230.685 & 2.197 & 8.788 \\
\hline 2.197 & 13.182 & 2.197 & 35.152 \\
\hline
\end{tabular}




\begin{tabular}{|c|c|c|c|}
\hline 26.364 & 32.955 & 2.197 & 17.576 \\
\hline 4.394 & 37.349 & 2.197 & 32.955 \\
\hline 24.167 & 8.788 & 26.364 & 8.788 \\
\hline 17.576 & 10.985 & 2.197 & 59.319 \\
\hline 59.319 & 116.441 & 166.972 & 4.394 \\
\hline 2.197 & 2.197 & 74.698 & 54.925 \\
\hline 2.197 & 8.788 & 10.985 & 17.576 \\
\hline 2.197 & 28.561 & 2.197 & 116.441 \\
\hline 2.197 & 26.364 & 26.364 & 21.97 \\
\hline 10.985 & 6.591 & 2.197 & 2.197 \\
\hline 4.394 & 41.743 & 2.197 & 4.394 \\
\hline 2.197 & 46.137 & 2.197 & 2.197 \\
\hline 26.364 & 2.197 & 46.137 & 21.97 \\
\hline 6.591 & 30.758 & 2.197 & 32.955 \\
\hline 6.591 & 10.985 & 6.591 & 98.865 \\
\hline 4.394 & 4.394 & 2.197 & 6.591 \\
\hline 2.197 & 322.959 & 120.835 & 2.197 \\
\hline 2.197 & 35.152 & 2.197 & 4.394 \\
\hline 37.349 & 72.501 & 4.394 & 4.394 \\
\hline 2.197 & 162.578 & 2.197 & 13.182 \\
\hline 2.197 & 2.197 & 2.197 & 4.394 \\
\hline 39.546 & 37.349 & 72.501 & 50.531 \\
\hline 8.788 & 246.064 & 2.197 & 2.197 \\
\hline 21.97 & 2.197 & 2.197 & 85.683 \\
\hline 153.79 & 2.197 & 180.154 & 4.394 \\
\hline 2.197 & 85.683 & 6.591 & 48.334 \\
\hline 2.197 & 2.197 & 2.197 & 28.561 \\
\hline 2.197 & 17.576 & 8.788 & 4.394 \\
\hline 4.394 & 307.58 & 28.561 & 2.197 \\
\hline 46.137 & 2.197 & 2.197 & 2.197 \\
\hline 26.364 & 6.591 & 2.197 & 2.197 \\
\hline 2.197 & 32.955 & 15.379 & 2.197 \\
\hline 92.274 & 410.839 & 19.773 & 2.197 \\
\hline 2.197 & 32.955 & 52.728 & 37.349 \\
\hline 2.197 & 8.788 & 2.197 & 2.197 \\
\hline 4.394 & 6.591 & 43.94 & 2.197 \\
\hline 2.197 & 8.788 & 2.197 & 10.985 \\
\hline 10.985 & 28.561 & 8.788 & 406.445 \\
\hline 19.773 & 2.197 & 4.394 & 2.197 \\
\hline 2.197 & 2.197 & 6.591 & 17.576 \\
\hline 68.107 & 2.197 & 2.197 & 13.182 \\
\hline 35.152 & 160.381 & 2.197 & 10.985 \\
\hline 10.985 & 4.394 & 13.182 & 204.321 \\
\hline 6.591 & 70.304 & 6.591 & 39.546 \\
\hline 6.591 & 15.379 & 43.94 & 2.197 \\
\hline 2.197 & 522.886 & 2.197 & 2.197 \\
\hline 2.197 & 83.486 & 2.197 & 2.197 \\
\hline 24.167 & 21.97 & 2.197 & 8.788 \\
\hline 30.758 & 2.197 & 6.591 & 6.591 \\
\hline 15.379 & 136.214 & 2.197 & 35.152 \\
\hline
\end{tabular}




\begin{tabular}{|c|c|c|c|}
\hline 21.97 & 17.576 & 2.197 & 2.197 \\
\hline 4.394 & 2.197 & 2.197 & 13.182 \\
\hline 4.394 & 120.835 & 4.394 & 2.197 \\
\hline 2.197 & 15.379 & 2.197 & 2.197 \\
\hline 2.197 & 4.394 & 2.197 & 2.197 \\
\hline 24.167 & 46.137 & 2.197 & 79.092 \\
\hline 28.561 & 19.773 & 4.394 & 28.561 \\
\hline 4.394 & 4.394 & 2.197 & 52.728 \\
\hline 10.985 & 43.94 & 26.364 & 6.591 \\
\hline 2.197 & 2.197 & 2.197 & 21.97 \\
\hline 4.394 & 6.591 & 2.197 & 13.182 \\
\hline 4.394 & 17.576 & 4.394 & 2.197 \\
\hline 4.394 & 15.379 & 70.304 & 10.985 \\
\hline 13.182 & 2.197 & 2.197 & 8.788 \\
\hline 26.364 & 63.713 & 8.788 & 2.197 \\
\hline 6.591 & 2.197 & 2.197 & 19.773 \\
\hline 2.197 & 19.773 & 2.197 & 4.394 \\
\hline 13.182 & 24.167 & 2.197 & 2.197 \\
\hline 10.985 & 10.985 & 17.576 & 2.197 \\
\hline 10.985 & 6.591 & 2.197 & 10.985 \\
\hline 6.591 & 8.788 & 2.197 & 10.985 \\
\hline 6.591 & 4.394 & 10.985 & 6.591 \\
\hline 4.394 & 19.773 & 2.197 & 96.668 \\
\hline 2.197 & 37.349 & 28.561 & 24.167 \\
\hline 4.394 & 2.197 & 2.197 & 74.698 \\
\hline 2.197 & 661.297 & 2.197 & 94.471 \\
\hline 8.788 & 4.394 & 13.182 & 184.548 \\
\hline 8.788 & 13.182 & 2.197 & 43.94 \\
\hline 19.773 & 4.394 & 2.197 & 131.82 \\
\hline 6.591 & 2.197 & 2.197 & 19.773 \\
\hline 2.197 & 2.197 & 10.985 & 43.94 \\
\hline 2.197 & 2.197 & 2.197 & 2.197 \\
\hline 2.197 & 2.197 & 2.197 & 6.591 \\
\hline 2.197 & 2.197 & 4.394 & 219.7 \\
\hline 26.364 & 61.516 & 2.197 & 90.077 \\
\hline 195.533 & 4.394 & 303.186 & 2.197 \\
\hline 19.773 & 4.394 & 2.197 & 169.169 \\
\hline 39.546 & 204.321 & 15.379 & 2.197 \\
\hline 8.788 & 2.197 & 4.394 & 2.197 \\
\hline 2.197 & 4.394 & 13.182 & 17.576 \\
\hline 2.197 & 6.591 & 4.394 & 70.304 \\
\hline 19.773 & 15.379 & 4.394 & 2.197 \\
\hline 8.788 & 15.379 & 2.197 & 2.197 \\
\hline 237.276 & 6.591 & 4.394 & 2.197 \\
\hline 2.197 & 17.576 & 10.985 & 2.197 \\
\hline 2.197 & 2.197 & 6.591 & 2.197 \\
\hline 2.197 & 13.182 & 8.788 & 2.197 \\
\hline 17.576 & 2.197 & 10.985 & 10.985 \\
\hline 2.197 & 59.319 & 6.591 & 2.197 \\
\hline 116.441 & 65.91 & 243.867 & 199.927 \\
\hline
\end{tabular}




\begin{tabular}{|c|c|c|c|}
\hline 2.197 & 24.167 & 4.394 & 13.182 \\
\hline 2.197 & 17.576 & 4.394 & 17.576 \\
\hline 2.197 & 10.985 & 15.379 & 57.122 \\
\hline 2.197 & 10.985 & 2.197 & 87.88 \\
\hline 57.122 & 2.197 & 2.197 & 21.97 \\
\hline 61.516 & 2.197 & 92.274 & 2.197 \\
\hline 6.591 & 2.197 & 30.758 & 2.197 \\
\hline 2.197 & 4.394 & 2.197 & 2.197 \\
\hline 2.197 & 4.394 & 6.591 & 2.197 \\
\hline 2.197 & 2.197 & 26.364 & 2.197 \\
\hline 2.197 & 4.394 & 92.274 & 8.788 \\
\hline 8.788 & 6.591 & 4.394 & 13.182 \\
\hline 6.591 & 4.394 & 2.197 & 2.197 \\
\hline 57.122 & 4.394 & 65.91 & 153.79 \\
\hline 43.94 & 4.394 & 2.197 & 10.985 \\
\hline 2.197 & 48.334 & 2.197 & 43.94 \\
\hline 70.304 & 2.197 & 2.197 & 19.773 \\
\hline 2.197 & 39.546 & 21.97 & 2.197 \\
\hline 2.197 & 4.394 & 2.197 & 2.197 \\
\hline 4.394 & 61.516 & 2.197 & 2.197 \\
\hline 2.197 & 2.197 & 2.197 & 2.197 \\
\hline 2.197 & 2.197 & 63.713 & 6.591 \\
\hline 6.591 & 30.758 & 4.394 & 138.411 \\
\hline 4.394 & 896.376 & 2.197 & 107.653 \\
\hline 4.394 & 2.197 & 1917.98 & 6.591 \\
\hline 6.591 & 79.092 & 2.197 & 13.182 \\
\hline 17.576 & 2.197 & 2.197 & 2.197 \\
\hline 8.788 & 2.197 & 2.197 & 19.773 \\
\hline 2.197 & 404.248 & 263.64 & 142.805 \\
\hline 30.758 & 4.394 & 21.97 & 2.197 \\
\hline 136.214 & 6.591 & 2.197 & 2.197 \\
\hline 2.197 & 4.394 & 8.788 & 105.456 \\
\hline 2.197 & 28.561 & 2.197 & 43.94 \\
\hline 2.197 & 2.197 & 158.184 & 2.197 \\
\hline 28.561 & 2.197 & 2.197 & 2.197 \\
\hline 2.197 & 2.197 & 188.942 & 2.197 \\
\hline 19.773 & 2.197 & 32.955 & 162.578 \\
\hline 2.197 & 2.197 & 21.97 & 8.788 \\
\hline 4.394 & 21.97 & 2.197 & 6.591 \\
\hline 2.197 & 2.197 & 2.197 & 13.182 \\
\hline 2.197 & 2.197 & 94.471 & 13.182 \\
\hline 2.197 & 19.773 & 85.683 & 8.788 \\
\hline 2.197 & 2.197 & 10.985 & 2.197 \\
\hline 10.985 & 10.985 & 72.501 & 2.197 \\
\hline 15.379 & 37.349 & 4.394 & 39.546 \\
\hline 2.197 & 65.91 & 2.197 & 10.985 \\
\hline 6.591 & 8.788 & 2.197 & 186.745 \\
\hline 17.576 & 17.576 & 2.197 & 2.197 \\
\hline 4.394 & 28.561 & 17.576 & 24.167 \\
\hline 6.591 & 243.867 & 2.197 & 52.728 \\
\hline
\end{tabular}




\begin{tabular}{|c|c|c|c|}
\hline 2.197 & 41.743 & 2.197 & 353.717 \\
\hline 2.197 & 8.788 & 4.394 & 2.197 \\
\hline 2.197 & 4.394 & 2.197 & 10.985 \\
\hline 2.197 & 81.289 & 103.259 & 2.197 \\
\hline 6.591 & 8.788 & 2.197 & 2.197 \\
\hline 6.591 & 26.364 & 6.591 & 13.182 \\
\hline 2.197 & 2.197 & 2.197 & 59.319 \\
\hline 2.197 & 37.349 & 2.197 & 46.137 \\
\hline 54.925 & 232.882 & 6.591 & 19.773 \\
\hline 2.197 & 35.152 & 6.591 & 2.197 \\
\hline 2.197 & 250.458 & 105.456 & 4.394 \\
\hline 2.197 & 2.197 & 2.197 & 2.197 \\
\hline 6.591 & 32.955 & 2.197 & 8.788 \\
\hline 10.985 & 441.597 & 92.274 & 48.334 \\
\hline 4.394 & 13.182 & 2.197 & 21.97 \\
\hline 2.197 & 15.379 & 13.182 & 2.197 \\
\hline 4.394 & 580.008 & 2.197 & 17.576 \\
\hline 15.379 & 2.197 & 419.627 & 24.167 \\
\hline 4.394 & 2.197 & 2.197 & 8.788 \\
\hline 2.197 & 2.197 & 2.197 & 2.197 \\
\hline 283.413 & 4.394 & 26.364 & 4.394 \\
\hline 4.394 & 8.788 & 4.394 & 4.394 \\
\hline 2.197 & 2.197 & 2.197 & 4.394 \\
\hline 1423.66 & 10.985 & 125.229 & 2.197 \\
\hline 48.334 & 15.379 & 10.985 & 4.394 \\
\hline 4.394 & 2.197 & 70.304 & 175.76 \\
\hline 2.197 & 41.743 & 13.182 & 19.773 \\
\hline 4.394 & 2.197 & 4.394 & 26.364 \\
\hline 19.773 & 74.698 & 4.394 & 65.91 \\
\hline 17.576 & 76.895 & 4.394 & 118.638 \\
\hline 1759.8 & 2.197 & 127.426 & 2.197 \\
\hline 10.985 & 76.895 & 2.197 & 2.197 \\
\hline 4.394 & 2.197 & 2.197 & 32.955 \\
\hline 2.197 & 4.394 & 268.034 & 246.064 \\
\hline 2.197 & 76.895 & 2.197 & 173.563 \\
\hline 2.197 & 2.197 & 24.167 & 3879.9 \\
\hline 2.197 & 2.197 & 492.128 & 24.167 \\
\hline 2.197 & 2.197 & 26.364 & 2.197 \\
\hline 6.591 & 2.197 & 2.197 & 2.197 \\
\hline 10.985 & 2.197 & 72.501 & 8.788 \\
\hline 2.197 & 6.591 & 2.197 & 4.394 \\
\hline 2.197 & 2.197 & 2.197 & 52.728 \\
\hline 4.394 & 48.334 & 4.394 & 48.334 \\
\hline 15.379 & 2.197 & 26.364 & 13.182 \\
\hline 2.197 & 6.591 & 4.394 & 21.97 \\
\hline 2.197 & 125.229 & 2.197 & 21.97 \\
\hline 4.394 & 17.576 & 2.197 & 2.197 \\
\hline 17.576 & 15.379 & 2.197 & 8.788 \\
\hline 2.197 & 140.608 & 6.591 & 8.788 \\
\hline 8.788 & 8.788 & 2.197 & 2.197 \\
\hline
\end{tabular}




\begin{tabular}{|c|c|c|c|}
\hline 15.379 & 2.197 & 4.394 & 26.364 \\
\hline 6.591 & 4.394 & 6.591 & 50.531 \\
\hline 358.111 & 59.319 & 4.394 & 2.197 \\
\hline 15.379 & 21.97 & 2.197 & 4.394 \\
\hline 6.591 & 28.561 & 81.289 & 30.758 \\
\hline 15.379 & 26.364 & 2.197 & 4.394 \\
\hline 290.004 & 2.197 & 2.197 & 2.197 \\
\hline 8.788 & 46.137 & 26.364 & 8.788 \\
\hline 4.394 & 2.197 & 2.197 & 2.197 \\
\hline 15.379 & 2.197 & 15.379 & 39.546 \\
\hline 2.197 & 10.985 & 6.591 & 10.985 \\
\hline 19.773 & 6.591 & 2.197 & 26.364 \\
\hline 4.394 & 149.396 & 4.394 & 37.349 \\
\hline 2.197 & 35.152 & 39.546 & 2.197 \\
\hline 2.197 & 239.473 & 2.197 & 15.379 \\
\hline 30.758 & 50.531 & 2.197 & 4.394 \\
\hline 351.52 & 10.985 & 4.394 & 8.788 \\
\hline 4.394 & 2.197 & 2.197 & 2.197 \\
\hline 28.561 & 74.698 & 134.017 & 1149.03 \\
\hline 24.167 & 19.773 & 4.394 & 26.364 \\
\hline 2.197 & 52.728 & 2.197 & 15.379 \\
\hline 61.516 & 2.197 & 8.788 & 6.591 \\
\hline 2.197 & 8.788 & 10.985 & 2.197 \\
\hline 4.394 & 2.197 & 2.197 & 333.944 \\
\hline 17.576 & 2.197 & 2.197 & 8.788 \\
\hline 4.394 & 2.197 & 2.197 & 13.182 \\
\hline 10.985 & 408.642 & 177.957 & 13.182 \\
\hline 2.197 & 37.349 & 2.197 & 430.612 \\
\hline 13.182 & 2.197 & 4.394 & 4.394 \\
\hline 2.197 & 6.591 & 2.197 & 19.773 \\
\hline 8.788 & 13.182 & 2.197 & 305.383 \\
\hline 30.758 & 13.182 & 2.197 & 2.197 \\
\hline 2.197 & 2.197 & 2.197 & 15.379 \\
\hline 4.394 & 2.197 & 19.773 & 2.197 \\
\hline 10.985 & 87.88 & 4.394 & 8.788 \\
\hline 2.197 & 145.002 & 2.197 & 145.002 \\
\hline 2.197 & 4.394 & 19.773 & 4.394 \\
\hline 2.197 & 48.334 & 2.197 & 2.197 \\
\hline 2.197 & 28.561 & 2.197 & 30.758 \\
\hline 2.197 & 2.197 & 4.394 & 2.197 \\
\hline 2.197 & 2.197 & 8.788 & 4.394 \\
\hline 30.758 & 2.197 & 8.788 & 28.561 \\
\hline 4.394 & 902.967 & 2.197 & 2.197 \\
\hline 2.197 & 15.379 & 2.197 & 2.197 \\
\hline 2.197 & 6.591 & 2.197 & 4.394 \\
\hline 2.197 & 24.167 & 2.197 & 2.197 \\
\hline 21.97 & 21.97 & 6.591 & 4.394 \\
\hline 4.394 & 2.197 & 2.197 & 15.379 \\
\hline 6.591 & 2.197 & 2.197 & 4.394 \\
\hline 15.379 & 30.758 & 2.197 & 2.197 \\
\hline
\end{tabular}




\begin{tabular}{|c|c|c|c|}
\hline 6.591 & 294.398 & 2.197 & 2.197 \\
\hline 2.197 & 13.182 & 4.394 & 2.197 \\
\hline 4.394 & 4.394 & 509.704 & 4.394 \\
\hline 2.197 & 6.591 & 37.349 & 2.197 \\
\hline 2.197 & 48.334 & 10.985 & 4.394 \\
\hline 4.394 & 4.394 & 2.197 & 2.197 \\
\hline 4.394 & 2.197 & 2.197 & 8.788 \\
\hline 2.197 & 4.394 & 48.334 & 57.122 \\
\hline 4.394 & 4.394 & 2.197 & 10.985 \\
\hline 4.394 & 448.188 & 8.788 & 6.591 \\
\hline 10.985 & 32.955 & 8.788 & 19.773 \\
\hline 2.197 & 2.197 & 19.773 & 6.591 \\
\hline 96.668 & 2.197 & 43.94 & 4.394 \\
\hline 2.197 & 4.394 & 160.381 & 2.197 \\
\hline 2.197 & 17.576 & 2.197 & 2.197 \\
\hline 43.94 & 48.334 & 6.591 & 15.379 \\
\hline 2.197 & 17.576 & 6.591 & 28.561 \\
\hline 4.394 & 6.591 & 2.197 & 2.197 \\
\hline 13.182 & 4.394 & 2.197 & 30.758 \\
\hline 4.394 & 81.289 & 4.394 & 8.788 \\
\hline 2.197 & 129.623 & 2.197 & 19.773 \\
\hline 8.788 & 37.349 & 2.197 & 8.788 \\
\hline 26.364 & 2.197 & 48.334 & 48.334 \\
\hline 70.304 & 26.364 & 6.591 & 52.728 \\
\hline 2.197 & 76.895 & 4.394 & 8.788 \\
\hline 2.197 & 6.591 & 17.576 & 171.366 \\
\hline 2.197 & 134.017 & 2.197 & 26.364 \\
\hline 8.788 & 2.197 & 4.394 & 118.638 \\
\hline 2.197 & 50.531 & 2.197 & 17.576 \\
\hline 68.107 & 2.197 & 2.197 & 4.394 \\
\hline 4.394 & 6.591 & 410.839 & 2.197 \\
\hline 2.197 & 2.197 & 2.197 & 35.152 \\
\hline 2.197 & 2.197 & 2.197 & 112.047 \\
\hline 2.197 & 15.379 & 2.197 & 2.197 \\
\hline 10.985 & 48.334 & 4.394 & 28.561 \\
\hline 2.197 & 2.197 & 2.197 & 2.197 \\
\hline 2.197 & 2.197 & 4.394 & 46.137 \\
\hline 2.197 & 21.97 & 2.197 & 19.773 \\
\hline 10.985 & 547.053 & 2.197 & 4.394 \\
\hline 2.197 & 24.167 & 2.197 & 4.394 \\
\hline 2.197 & 6.591 & 2.197 & 4.394 \\
\hline 2.197 & 15.379 & 2.197 & 8.788 \\
\hline 2.197 & 294.398 & 2.197 & 4.394 \\
\hline 2.197 & 43.94 & 2.197 & 4.394 \\
\hline 2.197 & 54.925 & 2.197 & 30.758 \\
\hline 8.788 & 292.201 & 2.197 & 6.591 \\
\hline 4.394 & 2.197 & 10.985 & 2.197 \\
\hline 61.516 & 116.441 & 15.379 & 26.364 \\
\hline 4.394 & 13.182 & 13.182 & 421.824 \\
\hline 2.197 & 2.197 & 2.197 & 105.456 \\
\hline
\end{tabular}




\begin{tabular}{|c|c|c|c|}
\hline 2.197 & 2.197 & 2.197 & 10.985 \\
\hline 6.591 & 107.653 & 2.197 & 2.197 \\
\hline 2.197 & 6.591 & 4.394 & 182.351 \\
\hline 4.394 & 2.197 & 2.197 & 2.197 \\
\hline 4.394 & 85.683 & 24.167 & 2.197 \\
\hline 59.319 & 1535.7 & 2.197 & 2.197 \\
\hline 4.394 & 17.576 & 2.197 & 2.197 \\
\hline 63.713 & 4.394 & 2.197 & 843.648 \\
\hline 2.197 & 4.394 & 2.197 & 4.394 \\
\hline 13.182 & 2.197 & 15.379 & 21.97 \\
\hline 2.197 & 2.197 & 2.197 & 101.062 \\
\hline 8.788 & 21.97 & 4.394 & 39.546 \\
\hline 2.197 & 2.197 & 533.871 & 43.94 \\
\hline 4.394 & 39.546 & 4.394 & 70.304 \\
\hline 17.576 & 2.197 & 2.197 & 2.197 \\
\hline 2.197 & 2.197 & 50.531 & 1142.44 \\
\hline 35.152 & 2.197 & 28.561 & 4.394 \\
\hline 63.713 & 2.197 & 2.197 & 2.197 \\
\hline 2.197 & 13.182 & 2.197 & 2.197 \\
\hline 2.197 & 4.394 & 2.197 & 2.197 \\
\hline 15.379 & 2.197 & 74.698 & 2.197 \\
\hline 2.197 & 2.197 & 2.197 & 10.985 \\
\hline 6.591 & 8.788 & 2.197 & 96.668 \\
\hline 2.197 & 2.197 & 54.925 & 37.349 \\
\hline 2.197 & 2.197 & 2.197 & 2.197 \\
\hline 2.197 & 8.788 & 2.197 & 17.576 \\
\hline 2.197 & 72.501 & 4.394 & 2.197 \\
\hline 2.197 & 4.394 & 215.306 & 4.394 \\
\hline 2.197 & 10.985 & 74.698 & 2.197 \\
\hline 2.197 & 107.653 & 8.788 & 30.758 \\
\hline 2.197 & 6.591 & 2.197 & 186.745 \\
\hline 2.197 & 160.381 & 39.546 & 129.623 \\
\hline 2.197 & 30.758 & 24.167 & 61.516 \\
\hline 2.197 & 8.788 & 162.578 & 50.531 \\
\hline 2.197 & 6.591 & 2.197 & 35.152 \\
\hline 2.197 & 8.788 & 6.591 & 30.758 \\
\hline 35.152 & 79.092 & 4.394 & 26.364 \\
\hline 2.197 & 2.197 & 2.197 & 2.197 \\
\hline 4.394 & 2.197 & 2.197 & 2.197 \\
\hline 92.274 & 35.152 & 6.591 & 4.394 \\
\hline 26.364 & 2.197 & 94.471 & 26.364 \\
\hline 17.576 & 26.364 & 2.197 & 2.197 \\
\hline 28.561 & 4.394 & 63.713 & 8.788 \\
\hline 2.197 & 2.197 & 6.591 & 2.197 \\
\hline 2.197 & 59.319 & 61.516 & 4.394 \\
\hline 4.394 & 2.197 & 41.743 & 4.394 \\
\hline 2.197 & 131.82 & 4.394 & 28.561 \\
\hline 4.394 & 2.197 & 2.197 & 26.364 \\
\hline 59.319 & 6.591 & 6.591 & 10.985 \\
\hline 19.773 & 2.197 & 2.197 & 37.349 \\
\hline
\end{tabular}




\begin{tabular}{|c|c|c|c|}
\hline 235.079 & 13.182 & 2.197 & 2.197 \\
\hline 8.788 & 28.561 & 8.788 & 2.197 \\
\hline 19.773 & 10.985 & 199.927 & 48.334 \\
\hline 50.531 & 8.788 & 17.576 & 30.758 \\
\hline 2.197 & 2.197 & 4.394 & 30.758 \\
\hline 17.576 & 46.137 & 1228.12 & 2.197 \\
\hline 109.85 & 19.773 & 2.197 & 382.278 \\
\hline 21.97 & 2.197 & 2.197 & 2.197 \\
\hline 8.788 & 26.364 & 6.591 & 37.349 \\
\hline 2.197 & 2.197 & 4.394 & 15.379 \\
\hline 24.167 & 19.773 & 10.985 & 8.788 \\
\hline 2.197 & 10.985 & 445.991 & 37.349 \\
\hline 2.197 & 65.91 & 2.197 & 10.985 \\
\hline 2.197 & 2.197 & 105.456 & 2.197 \\
\hline 2.197 & 2.197 & 8.788 & 2.197 \\
\hline 2.197 & 15.379 & 4.394 & 6.591 \\
\hline 21.97 & 24.167 & 6.591 & 10.985 \\
\hline 52.728 & 6.591 & 81.289 & 650.312 \\
\hline 2.197 & 4.394 & 50.531 & 19.773 \\
\hline 10.985 & 6.591 & 2.197 & 2.197 \\
\hline 15.379 & 2.197 & 2.197 & 215.306 \\
\hline 2.197 & 21.97 & 19.773 & 13.182 \\
\hline 2.197 & 30.758 & 2.197 & 6.591 \\
\hline 15.379 & 17.576 & 2.197 & 4.394 \\
\hline 6.591 & 2.197 & 6.591 & 2.197 \\
\hline 6.591 & 32.955 & 4.394 & 2.197 \\
\hline 4.394 & 32.955 & 2.197 & 15.379 \\
\hline 21.97 & 4.394 & 2.197 & 2.197 \\
\hline 7755.41 & 24.167 & 2.197 & 6.591 \\
\hline 2.197 & 103.259 & 2.197 & 8.788 \\
\hline 4.394 & 4.394 & 2.197 & 10.985 \\
\hline 4.394 & 10.985 & 2.197 & 2.197 \\
\hline 4.394 & 2.197 & 10.985 & 4.394 \\
\hline 10.985 & 2.197 & 797.511 & 6.591 \\
\hline 98.865 & 518.492 & 2.197 & 46.137 \\
\hline 2.197 & 8.788 & 10.985 & 10.985 \\
\hline 8.788 & 2.197 & 2.197 & 2.197 \\
\hline 118.638 & 26.364 & 2.197 & 186.745 \\
\hline 30.758 & 6.591 & 2.197 & 8.788 \\
\hline 26.364 & 8.788 & 2.197 & 4.394 \\
\hline 2.197 & 54.925 & 2.197 & 41.743 \\
\hline 2.197 & 2.197 & 26.364 & 4.394 \\
\hline 2.197 & 4.394 & 4.394 & 2.197 \\
\hline 2.197 & 4.394 & 26.364 & 2.197 \\
\hline 15.379 & 12933.7 & 13.182 & 37.349 \\
\hline 2.197 & 28.561 & 2.197 & 81.289 \\
\hline 2.197 & 153.79 & 2.197 & 10.985 \\
\hline 424.021 & 4.394 & 2.197 & 61.516 \\
\hline 2.197 & 10.985 & 21.97 & 59.319 \\
\hline 2.197 & 39.546 & 4.394 & 68.107 \\
\hline
\end{tabular}




\begin{tabular}{|c|c|c|c|}
\hline 2.197 & 4.394 & 2.197 & 26.364 \\
\hline 15.379 & 68.107 & 8.788 & 6.591 \\
\hline 4.394 & 39.546 & 2.197 & 15.379 \\
\hline 410.839 & 4.394 & 2.197 & 177.957 \\
\hline 760.162 & 652.509 & 35.152 & 195.533 \\
\hline 19.773 & 4.394 & 24.167 & 10.985 \\
\hline 2.197 & 2.197 & 13.182 & 15.379 \\
\hline 19.773 & 19.773 & 4.394 & 35.152 \\
\hline 50.531 & 265.837 & 2.197 & 10.985 \\
\hline 76.895 & 59.319 & 944.71 & 37.349 \\
\hline 2.197 & 26.364 & 2.197 & 105.456 \\
\hline 2.197 & 6.591 & 2.197 & 208.715 \\
\hline 2.197 & 17.576 & 2.197 & 4.394 \\
\hline 243.867 & 182.351 & 2.197 & 224.094 \\
\hline 4.394 & 21.97 & 2.197 & 6.591 \\
\hline 4.394 & 35.152 & 63.713 & 171.366 \\
\hline 2.197 & 39.546 & 2.197 & 2.197 \\
\hline 43.94 & 21.97 & 2.197 & 4.394 \\
\hline 2.197 & 19.773 & 17.576 & 2.197 \\
\hline 8.788 & 4.394 & 4.394 & 37.349 \\
\hline 48.334 & 136.214 & 43.94 & 26.364 \\
\hline 2.197 & 336.141 & 2.197 & 6.591 \\
\hline 164.775 & 17.576 & 4.394 & 43.94 \\
\hline 70.304 & 2.197 & 2.197 & 8.788 \\
\hline 32.955 & 57.122 & 2.197 & 70.304 \\
\hline 2.197 & 21.97 & 2.197 & 24.167 \\
\hline 6.591 & 2.197 & 4.394 & 4.394 \\
\hline 15.379 & 8.788 & 1935.56 & 15.379 \\
\hline 114.244 & 4.394 & 19.773 & 4.394 \\
\hline 4.394 & 2.197 & 26.364 & 298.792 \\
\hline 6.591 & 4.394 & 2.197 & 24.167 \\
\hline 2.197 & 37.349 & 2.197 & 2.197 \\
\hline 2.197 & 10.985 & 13.182 & 4.394 \\
\hline 2.197 & 518.492 & 24.167 & 19.773 \\
\hline 2.197 & 10.985 & 140.608 & 2.197 \\
\hline 10.985 & 6.591 & 4.394 & 17.576 \\
\hline 2.197 & 2.197 & 4.394 & 2.197 \\
\hline 2.197 & 35.152 & 79.092 & 10.985 \\
\hline 2.197 & 59.319 & 608.569 & 24.167 \\
\hline 2.197 & 2.197 & 2.197 & 10.985 \\
\hline 15.379 & 10.985 & 8.788 & 204.321 \\
\hline 2.197 & 2.197 & 28.561 & 37.349 \\
\hline 13.182 & 274.625 & 2.197 & 6.591 \\
\hline 4.394 & 85.683 & 15.379 & 158.184 \\
\hline 68.107 & 4.394 & 142.805 & 6.591 \\
\hline 2.197 & 35.152 & 52.728 & 6.591 \\
\hline 138.411 & 274.625 & 2.197 & 2.197 \\
\hline 208.715 & 123.032 & 28.561 & 2.197 \\
\hline 109.85 & 2.197 & 4.394 & 107.653 \\
\hline 250.458 & 4.394 & 2.197 & 24.167 \\
\hline
\end{tabular}




\begin{tabular}{|c|c|c|c|}
\hline 2.197 & 13.182 & 259.246 & 4.394 \\
\hline 2.197 & 2.197 & 10.985 & 17.576 \\
\hline 4.394 & 17.576 & 26.364 & 13.182 \\
\hline 2.197 & 4.394 & 83.486 & 103.259 \\
\hline 2.197 & 2.197 & 656.903 & 2.197 \\
\hline 2737.46 & 4.394 & 8.788 & 8.788 \\
\hline 96.668 & 2.197 & 2.197 & 2.197 \\
\hline 26.364 & 2.197 & 24.167 & 37.349 \\
\hline 8.788 & 2.197 & 2.197 & 204.321 \\
\hline 4.394 & 158.184 & 2.197 & 8.788 \\
\hline 2.197 & 4.394 & 2.197 & 641.524 \\
\hline 2.197 & 72.501 & 21.97 & 21.97 \\
\hline 2.197 & 694.252 & 90.077 & 26.364 \\
\hline 15.379 & 2.197 & 2.197 & 2.197 \\
\hline 2.197 & 28.561 & 2.197 & 10.985 \\
\hline 2.197 & 4.394 & 2.197 & 43.94 \\
\hline 94.471 & 6.591 & 8.788 & 2.197 \\
\hline 15.379 & 2.197 & 59.319 & 21.97 \\
\hline 17.576 & 2.197 & 46.137 & 889.785 \\
\hline 4.394 & 10.985 & 8.788 & 21.97 \\
\hline 2.197 & 13.182 & 8.788 & 17.576 \\
\hline 19.773 & 2.197 & 692.055 & 2.197 \\
\hline 2.197 & 21.97 & 4.394 & 2.197 \\
\hline 2.197 & 17.576 & 4.394 & 26.364 \\
\hline 2.197 & 6.591 & 2.197 & 538.265 \\
\hline 2.197 & 17.576 & 2.197 & 24.167 \\
\hline 2.197 & 2.197 & 83.486 & 2.197 \\
\hline 28.561 & 116.441 & 4.394 & 2.197 \\
\hline 8.788 & 57.122 & 2.197 & 28.561 \\
\hline 6.591 & 142.805 & 2.197 & 296.595 \\
\hline 2.197 & 39.546 & 2.197 & 2.197 \\
\hline 2.197 & 10.985 & 10.985 & 28.561 \\
\hline 2.197 & 151.593 & 6.591 & 2.197 \\
\hline 2.197 & 2.197 & 2.197 & 8.788 \\
\hline 28.561 & 21.97 & 2.197 & 2.197 \\
\hline 4.394 & 4.394 & 2.197 & 2.197 \\
\hline 13.182 & 2.197 & 24.167 & 4.394 \\
\hline 4.394 & 2.197 & 2.197 & 134.017 \\
\hline 19.773 & 39.546 & 6.591 & 2.197 \\
\hline 2.197 & 4.394 & 10.985 & 4.394 \\
\hline 15.379 & 4.394 & 2.197 & 35.152 \\
\hline 2.197 & 4.394 & 2.197 & 6.591 \\
\hline 4.394 & 8.788 & 21.97 & 57.122 \\
\hline 39.546 & 13.182 & 6.591 & 13.182 \\
\hline 4.394 & 10.985 & 8.788 & 2.197 \\
\hline 43.94 & 26.364 & 4.394 & 2.197 \\
\hline 241.67 & 2.197 & 2.197 & 10.985 \\
\hline 2.197 & 2.197 & 61.516 & 2.197 \\
\hline 8.788 & 125.229 & 2.197 & 2.197 \\
\hline 8.788 & 2.197 & 2.197 & 65.91 \\
\hline
\end{tabular}




\begin{tabular}{|c|c|c|c|}
\hline 672.282 & 112.047 & 2.197 & 2.197 \\
\hline 10.985 & 50.531 & 2.197 & 114.244 \\
\hline 2.197 & 50.531 & 2.197 & 28.561 \\
\hline 2.197 & 6.591 & 8.788 & 2.197 \\
\hline 2.197 & 2.197 & 2.197 & 105.456 \\
\hline 2.197 & 30.758 & 24.167 & 6.591 \\
\hline 65.91 & 32.955 & 2.197 & 6.591 \\
\hline 4.394 & 129.623 & 50.531 & 10.985 \\
\hline 2.197 & 4.394 & 2.197 & 28.561 \\
\hline 2.197 & 72.501 & 514.098 & 4.394 \\
\hline 2.197 & 151.593 & 2.197 & 8.788 \\
\hline 8.788 & 8.788 & 2.197 & 15.379 \\
\hline 2.197 & 70.304 & 2.197 & 461.37 \\
\hline 26.364 & 50.531 & 2.197 & 894.179 \\
\hline 2.197 & 24.167 & 24.167 & 10.985 \\
\hline 393.263 & 4.394 & 2.197 & 138.411 \\
\hline 24.167 & 118.638 & 2.197 & 448.188 \\
\hline 10.985 & 2.197 & 79.092 & 26.364 \\
\hline 32.955 & 2.197 & 531.674 & 57.122 \\
\hline 2.197 & 8.788 & 2.197 & 21.97 \\
\hline 2.197 & 2.197 & 24.167 & 2.197 \\
\hline 1258.88 & 54.925 & 6.591 & 2.197 \\
\hline 30.758 & 24.167 & 1794.95 & 72.501 \\
\hline 13.182 & 8.788 & 2.197 & 2.197 \\
\hline 4.394 & 8.788 & 17.576 & 15.379 \\
\hline 35.152 & 26.364 & 2.197 & 8.788 \\
\hline 6.591 & 32.955 & 6.591 & 46.137 \\
\hline 4.394 & 103.259 & 2.197 & 39.546 \\
\hline 4.394 & 164.775 & 2.197 & 4.394 \\
\hline 46.137 & 6.591 & 4.394 & 39.546 \\
\hline 61.516 & 2.197 & 30.758 & 81.289 \\
\hline 76.895 & 35.152 & 19625.8 & 2.197 \\
\hline 76.895 & 2.197 & 2.197 & 19.773 \\
\hline 2.197 & 6.591 & 2.197 & 79.092 \\
\hline 17.576 & 39.546 & 2.197 & 35.152 \\
\hline 4.394 & 32.955 & 48.334 & 2.197 \\
\hline 30.758 & 21.97 & 8.788 & 215.306 \\
\hline 8.788 & 6.591 & 8.788 & 2.197 \\
\hline 2.197 & 4.394 & 13896 & 2.197 \\
\hline 2.197 & 2.197 & 2.197 & 41.743 \\
\hline 2.197 & 2.197 & 21.97 & 309.777 \\
\hline 2.197 & 2.197 & 10.985 & 35.152 \\
\hline 2.197 & 2.197 & 85.683 & 54.925 \\
\hline 19.773 & 35.152 & 4042.48 & 6.591 \\
\hline 2.197 & 4.394 & 6.591 & 32.955 \\
\hline 105.456 & 6.591 & 13.182 & 6.591 \\
\hline 2.197 & 4.394 & 2.197 & 2.197 \\
\hline 2.197 & 4.394 & 2.197 & 39.546 \\
\hline 2.197 & 2.197 & 2.197 & 2.197 \\
\hline 2.197 & 76.895 & 30.758 & 2317.83 \\
\hline
\end{tabular}




\begin{tabular}{|c|c|c|c|}
\hline 2.197 & 15.379 & 4.394 & 2.197 \\
\hline 2.197 & 13.182 & 2.197 & 2.197 \\
\hline 4.394 & 6.591 & 41.743 & 2.197 \\
\hline 2.197 & 96.668 & 162.578 & 2.197 \\
\hline 4.394 & 4.394 & 2.197 & 8.788 \\
\hline 17.576 & 81.289 & 10209.5 & 24.167 \\
\hline 19.773 & 4.394 & 8.788 & 2.197 \\
\hline 15.379 & 2.197 & 4.394 & 2.197 \\
\hline 6.591 & 2.197 & 4.394 & 2.197 \\
\hline 37.349 & 24.167 & 76.895 & 136.214 \\
\hline 395.46 & 4.394 & 11670.5 & 17.576 \\
\hline 2.197 & 87.88 & 2.197 & 2.197 \\
\hline 2.197 & 2.197 & 4.394 & 2.197 \\
\hline 2.197 & 2.197 & 4.394 & 6498.73 \\
\hline 2.197 & 2.197 & 4.394 & 21.97 \\
\hline 2.197 & 2.197 & 8.788 & 2.197 \\
\hline 2.197 & 2.197 & 2.197 & 147.199 \\
\hline 10.985 & 109.85 & 2.197 & 57.122 \\
\hline 10.985 & 30.758 & 37.349 & 4.394 \\
\hline 10.985 & 2.197 & 2.197 & 2.197 \\
\hline 2.197 & 6.591 & 2.197 & 87.88 \\
\hline 43.94 & 50.531 & 94.471 & 8.788 \\
\hline 4.394 & 35.152 & 2.197 & 2.197 \\
\hline 35.152 & 94.471 & 1326.99 & 2.197 \\
\hline 26.364 & 6.591 & 39.546 & 72.501 \\
\hline 257.049 & 4.394 & 2.197 & 10.985 \\
\hline 848.042 & 4.394 & 6.591 & 2.197 \\
\hline 85.683 & 2.197 & 2.197 & 2.197 \\
\hline 37.349 & 2.197 & 2.197 & 13.182 \\
\hline 232.882 & 15.379 & 4.394 & 8.788 \\
\hline 2.197 & 4.394 & 46.137 & 35.152 \\
\hline 4.394 & 2.197 & 116.441 & 4.394 \\
\hline 70.304 & 32.955 & 2.197 & 17852.8 \\
\hline 8.788 & 6.591 & 21.97 & 37.349 \\
\hline 19.773 & 4.394 & 2.197 & 2.197 \\
\hline 50.531 & 10.985 & 2.197 & 6.591 \\
\hline 4.394 & 8.788 & 13.182 & 8.788 \\
\hline 123.032 & 2.197 & 6.591 & 8.788 \\
\hline 2.197 & 4.394 & 2.197 & 50.531 \\
\hline 2.197 & 2.197 & 6.591 & 8.788 \\
\hline 6.591 & 10.985 & 21.97 & 19.773 \\
\hline 2.197 & 8.788 & 2.197 & 26.364 \\
\hline 4.394 & 6.591 & 24.167 & 57.122 \\
\hline 2.197 & 6.591 & 4.394 & 54.925 \\
\hline 8.788 & 101.062 & 54.925 & 2.197 \\
\hline 2.197 & 10.985 & 8.788 & 72.501 \\
\hline 4.394 & 72.501 & 2.197 & 35.152 \\
\hline 2.197 & 4.394 & 2.197 & 24.167 \\
\hline 2.197 & 6.591 & 2.197 & 4.394 \\
\hline 2.197 & 4.394 & 2.197 & 54.925 \\
\hline
\end{tabular}




\begin{tabular}{|c|c|c|c|}
\hline 2.197 & 6.591 & 46.137 & 15.379 \\
\hline 2.197 & 2.197 & 2.197 & 4.394 \\
\hline 2.197 & 17.576 & 2.197 & 59.319 \\
\hline 2.197 & 4.394 & 2.197 & 8.788 \\
\hline 6.591 & 204.321 & 2.197 & 2.197 \\
\hline 2.197 & 15.379 & 15.379 & 13.182 \\
\hline 15.379 & 2.197 & 4.394 & 2.197 \\
\hline 8.788 & 1296.23 & 13.182 & 4.394 \\
\hline 8.788 & 101.062 & 32.955 & 1975.1 \\
\hline 2.197 & 837.057 & 2.197 & 17.576 \\
\hline 4.394 & 2.197 & 2.197 & 24.167 \\
\hline 10.985 & 6.591 & 2.197 & 10.985 \\
\hline 35.152 & 26.364 & 8.788 & 13.182 \\
\hline 21.97 & 4.394 & 2.197 & 2.197 \\
\hline 4.394 & 2.197 & 2.197 & 2.197 \\
\hline 13.182 & 30.758 & 2.197 & 2.197 \\
\hline 6.591 & 17.576 & 19.773 & 70.304 \\
\hline 13.182 & 37.349 & 21.97 & 4.394 \\
\hline 2.197 & 2.197 & 127.426 & 6.591 \\
\hline 4.394 & 206.518 & 8.788 & 2.197 \\
\hline 4.394 & 4.394 & 2.197 & 15.379 \\
\hline 2.197 & 39.546 & 6.591 & 2.197 \\
\hline 2.197 & 4.394 & 2.197 & 4.394 \\
\hline 15.379 & 2.197 & 8.788 & 2.197 \\
\hline 8.788 & 2.197 & 15.379 & 2.197 \\
\hline 2.197 & 10.985 & 162.578 & 17.576 \\
\hline 2.197 & 206.518 & 4.394 & 2.197 \\
\hline 2.197 & 15.379 & 10.985 & 28.561 \\
\hline 2.197 & 6.591 & 76.895 & 13.182 \\
\hline 2.197 & 577.811 & 24.167 & 2.197 \\
\hline 4.394 & 322.959 & 4.394 & 57.122 \\
\hline 2.197 & 43.94 & 13.182 & 2.197 \\
\hline 2.197 & 4.394 & 2.197 & 2.197 \\
\hline 59.319 & 4.394 & 2.197 & 2.197 \\
\hline 481.143 & 2.197 & 2.197 & 2.197 \\
\hline 19.773 & 61.516 & 4.394 & 2.197 \\
\hline 2.197 & 2.197 & 2.197 & 2.197 \\
\hline 2.197 & 28.561 & 2.197 & 2.197 \\
\hline 2.197 & 8.788 & 4.394 & 6.591 \\
\hline 2.197 & 8.788 & 4.394 & 57.122 \\
\hline 2.197 & 2.197 & 4.394 & 2.197 \\
\hline 4.394 & 2.197 & 2.197 & 21.97 \\
\hline 2.197 & 2.197 & 2.197 & 2.197 \\
\hline 32.955 & 2.197 & 2.197 & 4.394 \\
\hline 19.773 & 15.379 & 2.197 & 26.364 \\
\hline 30.758 & 2.197 & 8.788 & 6.591 \\
\hline 8.788 & 2.197 & 4.394 & 2.197 \\
\hline 195.533 & 2.197 & 4.394 & 21.97 \\
\hline 15.379 & 2.197 & 4.394 & 6.591 \\
\hline 290.004 & 2.197 & 2.197 & 5092.65 \\
\hline
\end{tabular}




\begin{tabular}{|c|c|c|c|}
\hline 37.349 & 2.197 & 1671.92 & 2.197 \\
\hline 166.972 & 2.197 & 2.197 & 2.197 \\
\hline 4.394 & 4.394 & 2.197 & 2.197 \\
\hline 65.91 & 2.197 & 4.394 & 2.197 \\
\hline 59.319 & 2.197 & 4.394 & 4.394 \\
\hline 35.152 & 17.576 & 2.197 & 4.394 \\
\hline 2.197 & 4.394 & 2.197 & 2.197 \\
\hline 202.124 & 26.364 & 6.591 & 8.788 \\
\hline 2.197 & 2.197 & 4.394 & 19.773 \\
\hline 10.985 & 6.591 & 2.197 & 28.561 \\
\hline 85.683 & 61.516 & 4.394 & 26.364 \\
\hline 83.486 & 2.197 & 116.441 & 83.486 \\
\hline 13.182 & 531.674 & 8.788 & 2.197 \\
\hline 30.758 & 15.379 & 8.788 & 118.638 \\
\hline 2.197 & 648.115 & 10.985 & 8.788 \\
\hline 2.197 & 24.167 & 2.197 & 21.97 \\
\hline 21.97 & 160.381 & 2.197 & 28.561 \\
\hline 65.91 & 4.394 & 4.394 & 35.152 \\
\hline 4.394 & 10.985 & 4.394 & 250.458 \\
\hline 15.379 & 2.197 & 2.197 & 81.289 \\
\hline 15.379 & 63.713 & 2.197 & 2.197 \\
\hline 4.394 & 4.394 & 35.152 & 10.985 \\
\hline 2.197 & 6.591 & 243.867 & 4.394 \\
\hline 8.788 & 54.925 & 4.394 & 83.486 \\
\hline 2.197 & 2.197 & 2.197 & 2.197 \\
\hline 2.197 & 2.197 & 136.214 & 32.955 \\
\hline 17.576 & 261.443 & 2.197 & 2.197 \\
\hline 10.985 & 239.473 & 2.197 & 6.591 \\
\hline 52.728 & 39.546 & 2.197 & 364.702 \\
\hline 2.197 & 2.197 & 8.788 & 70.304 \\
\hline 2.197 & 2.197 & 2.197 & 6.591 \\
\hline 4.394 & 17.576 & 24.167 & 4.394 \\
\hline 6.591 & 208.715 & 2.197 & 6.591 \\
\hline 72.501 & 15.379 & 2.197 & 37.349 \\
\hline 241.67 & 37.349 & 2.197 & 259.246 \\
\hline 2.197 & 4.394 & 4.394 & 24.167 \\
\hline 28.561 & 2.197 & 30.758 & 85.683 \\
\hline 349.323 & 2.197 & 2.197 & 13.182 \\
\hline 8.788 & 147.199 & 2.197 & 17.576 \\
\hline 26.364 & 2.197 & 2.197 & 4.394 \\
\hline 2.197 & 2.197 & 65.91 & 4.394 \\
\hline 107.653 & 61.516 & 10.985 & 151.593 \\
\hline 19.773 & 54.925 & 4.394 & 6.591 \\
\hline 26.364 & 2.197 & 2.197 & 30.758 \\
\hline 8.788 & 17.576 & 4.394 & 13.182 \\
\hline 2.197 & 4.394 & 2.197 & 2.197 \\
\hline 24.167 & 10.985 & 2.197 & 10.985 \\
\hline 2.197 & 195.533 & 2.197 & 2.197 \\
\hline 2.197 & 24.167 & 4.394 & 10.985 \\
\hline 2.197 & 4.394 & 2.197 & 50.531 \\
\hline
\end{tabular}




\begin{tabular}{|c|c|c|c|}
\hline 2.197 & 129.623 & 4.394 & 28.561 \\
\hline 2.197 & 2.197 & 90.077 & 21.97 \\
\hline 4.394 & 140.608 & 2.197 & 2.197 \\
\hline 2.197 & 2.197 & 13085.3 & 4.394 \\
\hline 2.197 & 2.197 & 4.394 & 4.394 \\
\hline 19.773 & 109.85 & 2.197 & 386.672 \\
\hline 2.197 & 52.728 & 2.197 & 6.591 \\
\hline 6.591 & 65.91 & 2.197 & 10.985 \\
\hline 2.197 & 2.197 & 6.591 & 17.576 \\
\hline 2.197 & 162.578 & 292.201 & 2948.37 \\
\hline 2.197 & 2.197 & 10.985 & 17.576 \\
\hline 285.61 & 166.972 & 630.539 & 4.394 \\
\hline 6.591 & 417.43 & 87.88 & 10.985 \\
\hline 8.788 & 19.773 & 43.94 & 177.957 \\
\hline 35.152 & 4.394 & 2.197 & 30.758 \\
\hline 777.738 & 19.773 & 4.394 & 8.788 \\
\hline 2.197 & 558.038 & 4.394 & 2.197 \\
\hline 15.379 & 6.591 & 2.197 & 2.197 \\
\hline 15.379 & 32.955 & 118.638 & 52.728 \\
\hline 2.197 & 8.788 & 10.985 & 8.788 \\
\hline 590.993 & 2.197 & 4.394 & 127.426 \\
\hline 6.591 & 2.197 & 4040.28 & 41.743 \\
\hline 2.197 & 2.197 & 2.197 & 10.985 \\
\hline 17.576 & 2.197 & 2.197 & 19.773 \\
\hline 37.349 & 6.591 & 2.197 & 204.321 \\
\hline 35.152 & 4.394 & 2.197 & 17.576 \\
\hline 8.788 & 37.349 & 19.773 & 81.289 \\
\hline 2.197 & 10.985 & 2.197 & 2.197 \\
\hline 2.197 & 15.379 & 39.546 & 8.788 \\
\hline 4.394 & 39.546 & 6.591 & 13.182 \\
\hline 17.576 & 2.197 & 6.591 & 57.122 \\
\hline 2.197 & 4.394 & 2.197 & 15.379 \\
\hline 15.379 & 81.289 & 6.591 & 107.653 \\
\hline 2.197 & 101.062 & 2.197 & 4.394 \\
\hline 19.773 & 46.137 & 107.653 & 4.394 \\
\hline 21.97 & 4.394 & 2.197 & 8.788 \\
\hline 2.197 & 151.593 & 2.197 & 85.683 \\
\hline 2.197 & 13.182 & 6.591 & 17.576 \\
\hline 127.426 & 6.591 & 2.197 & 21.97 \\
\hline 2.197 & 46.137 & 30.758 & 4.394 \\
\hline 2.197 & 6.591 & 35.152 & 4.394 \\
\hline 2.197 & 19.773 & 46.137 & 24.167 \\
\hline 4.394 & 2.197 & 15.379 & 15.379 \\
\hline 2.197 & 8.788 & 30.758 & 2.197 \\
\hline 13.182 & 2.197 & 4.394 & 4.394 \\
\hline 4.394 & 2.197 & 2.197 & 327.353 \\
\hline 4.394 & 6.591 & 2.197 & 46.137 \\
\hline 4.394 & 24.167 & 2.197 & 2.197 \\
\hline 2.197 & 24.167 & 4.394 & 24.167 \\
\hline 8.788 & 4.394 & 81.289 & 2.197 \\
\hline
\end{tabular}




\begin{tabular}{|c|c|c|c|}
\hline 8.788 & 21.97 & 8.788 & 2.197 \\
\hline 2.197 & 2.197 & 2.197 & 716.222 \\
\hline 4.394 & 6.591 & 2.197 & 2.197 \\
\hline 2.197 & 79.092 & 2.197 & 2.197 \\
\hline 24.167 & 6.591 & 13.182 & 2.197 \\
\hline 2.197 & 4.394 & 6.591 & 164.775 \\
\hline 2.197 & 2.197 & 2.197 & 2.197 \\
\hline 6.591 & 24.167 & 10.985 & 4.394 \\
\hline 2.197 & 28.561 & 2.197 & 13.182 \\
\hline 68.107 & 2.197 & 2.197 & 13.182 \\
\hline 2.197 & 61.516 & 351.52 & 17.576 \\
\hline 30.758 & 2.197 & 2.197 & 2.197 \\
\hline 2.197 & 4.394 & 169.169 & 2.197 \\
\hline 465.764 & 239.473 & 2.197 & 21.97 \\
\hline 10.985 & 13.182 & 175.76 & 2.197 \\
\hline 4.394 & 2.197 & 2.197 & 2.197 \\
\hline 2.197 & 10.985 & 2.197 & 8.788 \\
\hline 2.197 & 2.197 & 2.197 & 68.107 \\
\hline 2.197 & 54.925 & 8.788 & 4.394 \\
\hline 2.197 & 6.591 & 21.97 & 2.197 \\
\hline 2.197 & 24.167 & 37.349 & 8.788 \\
\hline 2.197 & 4.394 & 2.197 & 10.985 \\
\hline 41.743 & 2.197 & 2.197 & 8.788 \\
\hline 6.591 & 4.394 & 4.394 & 26.364 \\
\hline 2.197 & 48.334 & 2.197 & 2.197 \\
\hline 2.197 & 21.97 & 2.197 & 2.197 \\
\hline 35.152 & 2.197 & 72.501 & 15.379 \\
\hline 2.197 & 21.97 & 94.471 & 26.364 \\
\hline 13.182 & 30.758 & 2.197 & 8.788 \\
\hline 13.182 & 2.197 & 19.773 & 30.758 \\
\hline 4.394 & 48.334 & 8.788 & 21.97 \\
\hline 2.197 & 6.591 & 87.88 & 79.092 \\
\hline 4.394 & 39.546 & 8.788 & 4.394 \\
\hline 4.394 & 2.197 & 2.197 & 2818.75 \\
\hline 2.197 & 4.394 & 127.426 & 13.182 \\
\hline 4.394 & 131.82 & 2.197 & 2.197 \\
\hline 4.394 & 6.591 & 6.591 & 2.197 \\
\hline 8.788 & 153.79 & 2.197 & 362.505 \\
\hline 4.394 & 2.197 & 10.985 & 13.182 \\
\hline 4.394 & 142.805 & 4.394 & 292.201 \\
\hline 2.197 & 6.591 & 4.394 & 2.197 \\
\hline 2.197 & 2.197 & 2.197 & 15.379 \\
\hline 4.394 & 2.197 & 2.197 & 6.591 \\
\hline 8.788 & 30.758 & 6.591 & 8.788 \\
\hline 28.561 & 8.788 & 2.197 & 188.942 \\
\hline 15.379 & 2.197 & 2.197 & 48.334 \\
\hline 4.394 & 32.955 & 2.197 & 6.591 \\
\hline 2.197 & 24.167 & 4.394 & 10.985 \\
\hline 2.197 & 109.85 & 2.197 & 6.591 \\
\hline 2.197 & 162.578 & 4.394 & 19.773 \\
\hline
\end{tabular}




\begin{tabular}{|c|c|c|c|}
\hline 2.197 & 17.576 & 4.394 & 2.197 \\
\hline 2.197 & 28.561 & 13.182 & 4.394 \\
\hline 21.97 & 15.379 & 48.334 & 13.182 \\
\hline 8.788 & 13.182 & 37.349 & 166.972 \\
\hline 13.182 & 2.197 & 2.197 & 160.381 \\
\hline 19.773 & 15.379 & 336.141 & 21.97 \\
\hline 2.197 & 2.197 & 4.394 & 19.773 \\
\hline 6.591 & 4.394 & 79.092 & 13.182 \\
\hline 2.197 & 2.197 & 79.092 & 2.197 \\
\hline 2.197 & 114.244 & 2.197 & 2.197 \\
\hline 2.197 & 83.486 & 2.197 & 2.197 \\
\hline 26.364 & 103.259 & 94.471 & 13.182 \\
\hline 124893 & 15.379 & 10.985 & 21.97 \\
\hline 2.197 & 2.197 & 10.985 & 2.197 \\
\hline 4.394 & 43.94 & 37.349 & 2.197 \\
\hline 2.197 & 48.334 & 131.82 & 2210.18 \\
\hline 4.394 & 6.591 & 232.882 & 52.728 \\
\hline 2.197 & 6.591 & 4.394 & 114.244 \\
\hline 8.788 & 4.394 & 4.394 & 140.608 \\
\hline 4.394 & 13.182 & 2.197 & 4.394 \\
\hline 2.197 & 8.788 & 2.197 & 6.591 \\
\hline 26.364 & 17.576 & 30.758 & 21.97 \\
\hline 8.788 & 50.531 & 2.197 & 8.788 \\
\hline 193.336 & 505.31 & 2.197 & 265.837 \\
\hline 50.531 & 85.683 & 2.197 & 2.197 \\
\hline 19.773 & 283.413 & 2.197 & 4.394 \\
\hline 2.197 & 4.394 & 2.197 & 21.97 \\
\hline 2.197 & 21.97 & 2.197 & 6.591 \\
\hline 2.197 & 74.698 & 2.197 & 21.97 \\
\hline 4.394 & 2.197 & 2.197 & 35.152 \\
\hline 10.985 & 2.197 & 17.576 & 1280.85 \\
\hline 15.379 & 114.244 & 2.197 & 2.197 \\
\hline 2.197 & 325.156 & 63.713 & 2.197 \\
\hline 2.197 & 50.531 & 35.152 & 2.197 \\
\hline 6.591 & 2.197 & 2.197 & 8.788 \\
\hline 2.197 & 2.197 & 2.197 & 2790.19 \\
\hline 24.167 & 19.773 & 2.197 & 57.122 \\
\hline 2.197 & 2.197 & 4.394 & 30.758 \\
\hline 2.197 & 4.394 & 26.364 & 13.182 \\
\hline 272.428 & 57.122 & 2.197 & 316.368 \\
\hline 2.197 & 37.349 & 4.394 & 8.788 \\
\hline 13.182 & 2.197 & 8.788 & 933.725 \\
\hline 13.182 & 2.197 & 2.197 & 166.972 \\
\hline 2.197 & 19.773 & 2.197 & 6.591 \\
\hline 2.197 & 54.925 & 2.197 & 4.394 \\
\hline 2.197 & 4.394 & 4.394 & 4.394 \\
\hline 8.788 & 79.092 & 4.394 & 2.197 \\
\hline 6.591 & 4.394 & 2.197 & 4.394 \\
\hline 2.197 & 2.197 & 8.788 & 105.456 \\
\hline 2.197 & 2.197 & 10.985 & 21.97 \\
\hline
\end{tabular}




\begin{tabular}{|c|c|c|c|}
\hline 4.394 & 10.985 & 8.788 & 4.394 \\
\hline 32.955 & 2.197 & 19.773 & 10.985 \\
\hline 2.197 & 2.197 & 101.062 & 37.349 \\
\hline 153.79 & 2.197 & 2.197 & 6.591 \\
\hline 6.591 & 26.364 & 8.788 & 39.546 \\
\hline 2.197 & 2.197 & 21.97 & 48.334 \\
\hline 30.758 & 19.773 & 8.788 & 2.197 \\
\hline 2.197 & 2.197 & 8.788 & 2.197 \\
\hline 2.197 & 2.197 & 10.985 & 4.394 \\
\hline 2.197 & 2.197 & 6.591 & 140.608 \\
\hline 10.985 & 2.197 & 68.107 & 6.591 \\
\hline 2.197 & 4.394 & 2.197 & 13.182 \\
\hline 2.197 & 15.379 & 17.576 & 57.122 \\
\hline 21.97 & 4.394 & 17.576 & 4.394 \\
\hline 52.728 & 30.758 & 694.252 & 10.985 \\
\hline 2.197 & 21.97 & 6.591 & 4.394 \\
\hline 13.182 & 10.985 & 2.197 & 2.197 \\
\hline 63.713 & 4.394 & 2.197 & 6.591 \\
\hline 4.394 & 2.197 & 4.394 & 294.398 \\
\hline 2.197 & 61.516 & 2.197 & 8.788 \\
\hline 2.197 & 2.197 & 24.167 & 87.88 \\
\hline 2.197 & 17.576 & 2.197 & 4.394 \\
\hline 2.197 & 4.394 & 2.197 & 2.197 \\
\hline 2.197 & 6.591 & 8.788 & 4.394 \\
\hline 2.197 & 2.197 & 32.955 & 333.944 \\
\hline 2.197 & 10.985 & 57.122 & 87.88 \\
\hline 8.788 & 6.591 & 4.394 & 6.591 \\
\hline 2.197 & 2.197 & 50.531 & 2.197 \\
\hline 2.197 & 15.379 & 10.985 & 2.197 \\
\hline 4.394 & 24.167 & 2.197 & 26.364 \\
\hline 4.394 & 4.394 & 2.197 & 2.197 \\
\hline 17.576 & 48.334 & 2.197 & 8.788 \\
\hline 15.379 & 70.304 & 4.394 & 6.591 \\
\hline 2.197 & 6.591 & 4.394 & 39.546 \\
\hline 4.394 & 19.773 & 2.197 & 2.197 \\
\hline 4.394 & 2.197 & 4.394 & 24.167 \\
\hline 13.182 & 41.743 & 17.576 & 2.197 \\
\hline 2.197 & 2.197 & 4.394 & 6.591 \\
\hline 2.197 & 2.197 & 2.197 & 6.591 \\
\hline 2.197 & 19.773 & 2.197 & 4.394 \\
\hline 2.197 & 4.394 & 2.197 & 28.561 \\
\hline 2.197 & 8.788 & 52.728 & 2.197 \\
\hline 2.197 & 19.773 & 98.865 & 81.289 \\
\hline 4.394 & 2.197 & 35.152 & 6.591 \\
\hline 2.197 & 15.379 & 43.94 & 6.591 \\
\hline 6.591 & 13.182 & 90.077 & 2.197 \\
\hline 2.197 & 6.591 & 8.788 & 118.638 \\
\hline 2.197 & 65.91 & 2.197 & 30.758 \\
\hline 39.546 & 2.197 & 24.167 & 92.274 \\
\hline 290.004 & 6.591 & 2.197 & 2.197 \\
\hline
\end{tabular}




\begin{tabular}{|c|c|c|c|}
\hline 2.197 & 10.985 & 13.182 & 32.955 \\
\hline 116.441 & 4.394 & 15.379 & 76.895 \\
\hline 10.985 & 2.197 & 6.591 & 586.599 \\
\hline 32.955 & 140.608 & 26.364 & 2.197 \\
\hline 17.576 & 8.788 & 2.197 & 63.713 \\
\hline 2.197 & 83.486 & 2.197 & 35.152 \\
\hline 2.197 & 21.97 & 2.197 & 10.985 \\
\hline 2.197 & 4.394 & 2.197 & 15.379 \\
\hline 2.197 & 30.758 & 10.985 & 59.319 \\
\hline 4.394 & 26.364 & 17.576 & 19.773 \\
\hline 4.394 & 4.394 & 2.197 & 4.394 \\
\hline 2.197 & 10.985 & 2.197 & 2.197 \\
\hline 663.494 & 70.304 & 2.197 & 19.773 \\
\hline 19.773 & 186.745 & 4.394 & 17.576 \\
\hline 8.788 & 2.197 & 24.167 & 2.197 \\
\hline 8.788 & 2.197 & 4.394 & 2.197 \\
\hline 140.608 & 26.364 & 2.197 & 72.501 \\
\hline 4.394 & 138.411 & 21.97 & 28.561 \\
\hline 2.197 & 35.152 & 17.576 & 17.576 \\
\hline 2.197 & 13.182 & 6.591 & 2.197 \\
\hline 6.591 & 2.197 & 65.91 & 4.394 \\
\hline 2.197 & 2.197 & 54.925 & 2.197 \\
\hline 2.197 & 2.197 & 2.197 & 2.197 \\
\hline 4.394 & 2.197 & 2.197 & 2.197 \\
\hline 8.788 & 149.396 & 2.197 & 15.379 \\
\hline 2.197 & 4.394 & 2.197 & 24.167 \\
\hline 2.197 & 4.394 & 2.197 & 17.576 \\
\hline 13.182 & 70.304 & 8.788 & 8.788 \\
\hline 10.985 & 2.197 & 50.531 & 2.197 \\
\hline 103.259 & 2.197 & 2.197 & 4.394 \\
\hline 4.394 & 6.591 & 2.197 & 2.197 \\
\hline 2.197 & 19.773 & 2.197 & 46.137 \\
\hline 4.394 & 15.379 & 13.182 & 54.925 \\
\hline 6.591 & 4.394 & 1291.84 & 48.334 \\
\hline 2.197 & 158.184 & 13.182 & 169.169 \\
\hline 2.197 & 2.197 & 2.197 & 10.985 \\
\hline 30.758 & 2.197 & 2.197 & 41.743 \\
\hline 891.982 & 114.244 & 4.394 & 6.591 \\
\hline 15.379 & 19.773 & 2.197 & 2.197 \\
\hline 725.01 & 2.197 & 428.415 & 2.197 \\
\hline 26.364 & 72.501 & 2.197 & 2.197 \\
\hline 26.364 & 10.985 & 13.182 & 13.182 \\
\hline 1733.43 & 10.985 & 15.379 & 182.351 \\
\hline 4.394 & 30.758 & 2.197 & 2.197 \\
\hline 6.591 & 13.182 & 2.197 & 13.182 \\
\hline 166.972 & 2.197 & 2.197 & 24.167 \\
\hline 2.197 & 4125.97 & 8.788 & 10.985 \\
\hline 2.197 & 26.364 & 37.349 & 43.94 \\
\hline 4.394 & 2.197 & 2.197 & 2.197 \\
\hline 8.788 & 2.197 & 15.379 & 2.197 \\
\hline
\end{tabular}




\begin{tabular}{|c|c|c|c|}
\hline 4.394 & 2.197 & 4.394 & 8.788 \\
\hline 2.197 & 35.152 & 6.591 & 101.062 \\
\hline 8.788 & 13.182 & 2.197 & 549.25 \\
\hline 2.197 & 2.197 & 10.985 & 109.85 \\
\hline 2.197 & 21.97 & 8.788 & 41.743 \\
\hline 2.197 & 2.197 & 2.197 & 2.197 \\
\hline 2.197 & 10.985 & 2.197 & 2.197 \\
\hline 2.197 & 2.197 & 74.698 & 4.394 \\
\hline 83.486 & 4.394 & 2.197 & 19.773 \\
\hline 2.197 & 6.591 & 261.443 & 63.713 \\
\hline 4.394 & 41.743 & 10.985 & 6.591 \\
\hline 799.708 & 2.197 & 68.107 & 6.591 \\
\hline 8.788 & 68.107 & 4.394 & 2.197 \\
\hline 6.591 & 19.773 & 2.197 & 2.197 \\
\hline 10.985 & 2.197 & 2.197 & 4.394 \\
\hline 2.197 & 4.394 & 2.197 & 2.197 \\
\hline 2.197 & 316.368 & 63.713 & 10.985 \\
\hline 2.197 & 30.758 & 125.229 & 28.561 \\
\hline 13.182 & 109.85 & 2.197 & 81.289 \\
\hline 2.197 & 15.379 & 2.197 & 24.167 \\
\hline 13.182 & 13.182 & 2.197 & 6.591 \\
\hline 4.394 & 2.197 & 4.394 & 26.364 \\
\hline 2.197 & 6.591 & 2.197 & 92.274 \\
\hline 4.394 & 35.152 & 2.197 & 19.773 \\
\hline 285.61 & 19.773 & 57.122 & 4.394 \\
\hline 13.182 & 72.501 & 2.197 & 206.518 \\
\hline 2.197 & 10.985 & 26.364 & 90.077 \\
\hline 2.197 & 57.122 & 35.152 & 4.394 \\
\hline 76.895 & 6.591 & 2.197 & 2.197 \\
\hline 142.805 & 43.94 & 15.379 & 2.197 \\
\hline 2.197 & 4.394 & 120.835 & 15.379 \\
\hline 2.197 & 4.394 & 2.197 & 4.394 \\
\hline 2.197 & 2.197 & 6.591 & 35.152 \\
\hline 4.394 & 13.182 & 4.394 & 19.773 \\
\hline 2.197 & 232.882 & 2.197 & 6.591 \\
\hline 17.576 & 8.788 & 8.788 & 208.715 \\
\hline 6.591 & 28.561 & 4.394 & 70.304 \\
\hline 6.591 & 8.788 & 79.092 & 2.197 \\
\hline 72.501 & 8.788 & 2.197 & 35.152 \\
\hline 2.197 & 15.379 & 2.197 & 70.304 \\
\hline 4.394 & 41.743 & 4.394 & 2.197 \\
\hline 656.903 & 4.394 & 2.197 & 2.197 \\
\hline 2.197 & 2.197 & 162.578 & 6.591 \\
\hline 4.394 & 4.394 & 2.197 & 13.182 \\
\hline 105.456 & 13.182 & 2.197 & 26.364 \\
\hline 2.197 & 21.97 & 2.197 & 10.985 \\
\hline 2.197 & 65.91 & 2.197 & 52.728 \\
\hline 26.364 & 15.379 & 2.197 & 4.394 \\
\hline 2.197 & 28.561 & 2.197 & 2.197 \\
\hline 6.591 & 28.561 & 2.197 & $184.54 \varepsilon$ \\
\hline
\end{tabular}




\begin{tabular}{|c|c|c|c|}
\hline 2.197 & 6.591 & 1001.83 & 4.394 \\
\hline 2.197 & 997.438 & 43.94 & 10.985 \\
\hline 2.197 & 8.788 & 4.394 & 81.289 \\
\hline 10.985 & 136.214 & 2.197 & 68.107 \\
\hline 15.379 & 10.985 & 48.334 & 2.197 \\
\hline 2.197 & 2.197 & 2.197 & 2.197 \\
\hline 4.394 & 6.591 & 28.561 & 4.394 \\
\hline 2.197 & 2.197 & 4.394 & 10.985 \\
\hline 2.197 & 24.167 & 300.989 & 8.788 \\
\hline 4.394 & 13.182 & 2.197 & 30.758 \\
\hline 114.244 & 6.591 & 2.197 & 50.531 \\
\hline 4.394 & 4.394 & 2.197 & 35.152 \\
\hline 4.394 & 39.546 & 2.197 & 2.197 \\
\hline 2632.01 & 48.334 & 2.197 & 6.591 \\
\hline 2.197 & 6.591 & 2.197 & 94.471 \\
\hline 2.197 & 2.197 & 652.509 & 8.788 \\
\hline 37.349 & 2.197 & 2.197 & 10.985 \\
\hline 17.576 & 10.985 & 4.394 & 2.197 \\
\hline 118.638 & 52.728 & 2.197 & 10.985 \\
\hline 4.394 & 2.197 & 2.197 & 2.197 \\
\hline 4.394 & 112.047 & 2.197 & 2.197 \\
\hline 8.788 & 114.244 & 13.182 & 8.788 \\
\hline 2.197 & 4.394 & 2.197 & 2.197 \\
\hline 2.197 & 4.394 & 2.197 & 2.197 \\
\hline 13.182 & 336.141 & 230.685 & 17.576 \\
\hline 8.788 & 2.197 & 2.197 & 4.394 \\
\hline 43.94 & 50.531 & 15.379 & 145.002 \\
\hline 2.197 & 2.197 & 2.197 & 217.503 \\
\hline 2.197 & 2.197 & 4.394 & 72.501 \\
\hline 2.197 & 685.464 & 2.197 & 32.955 \\
\hline 2.197 & 6.591 & 2.197 & 2.197 \\
\hline 2.197 & 175.76 & 2.197 & 13.182 \\
\hline 6.591 & 32.955 & 4.394 & 2.197 \\
\hline 10.985 & 300.989 & 2.197 & 2.197 \\
\hline 37.349 & 4.394 & 2.197 & 59.319 \\
\hline 329.55 & 39.546 & 2.197 & 2.197 \\
\hline 46.137 & 891.982 & 2.197 & 2.197 \\
\hline 19.773 & 72.501 & 2.197 & 2.197 \\
\hline 2.197 & 246.064 & 6.591 & 21.97 \\
\hline 10.985 & 24.167 & 6.591 & 52.728 \\
\hline 147.199 & 13.182 & 2.197 & 173.563 \\
\hline 2.197 & 2.197 & 2.197 & 2.197 \\
\hline 15.379 & 118.638 & 2.197 & 26.364 \\
\hline 61.516 & 8.788 & 2.197 & 2.197 \\
\hline 35.152 & 28.561 & 2.197 & 13.182 \\
\hline 1015.01 & 2.197 & 2.197 & 6.591 \\
\hline 2.197 & 26.364 & 2.197 & 2.197 \\
\hline 6.591 & 17.576 & 28.561 & 2.197 \\
\hline 2.197 & 485.537 & 2.197 & 19.773 \\
\hline 2.197 & 2.197 & 2.197 & 8.788 \\
\hline
\end{tabular}




\begin{tabular}{|c|c|c|c|}
\hline 4.394 & 15.379 & 2.197 & 4.394 \\
\hline 2.197 & 6.591 & 4.394 & 13.182 \\
\hline 2.197 & 13.182 & 120.835 & 219.7 \\
\hline 2.197 & 2.197 & 2.197 & 59.319 \\
\hline 138.411 & 160.381 & 28.561 & 50.531 \\
\hline 2.197 & 4.394 & 2.197 & 6.591 \\
\hline 2.197 & 4.394 & 8.788 & 50.531 \\
\hline 2.197 & 13.182 & 2.197 & 13.182 \\
\hline 4.394 & 8.788 & 6.591 & 2.197 \\
\hline 4.394 & 2.197 & 175.76 & 118.638 \\
\hline 6.591 & 6.591 & 4.394 & 13.182 \\
\hline 2.197 & 76.895 & 2.197 & 2.197 \\
\hline 2.197 & 4.394 & 4.394 & 2.197 \\
\hline 2.197 & 43.94 & 6.591 & 149.396 \\
\hline 4.394 & 32.955 & 13.182 & 2.197 \\
\hline 4.394 & 81.289 & 6.591 & 4.394 \\
\hline 10.985 & 120.835 & 2.197 & 59.319 \\
\hline 6.591 & 6.591 & 6.591 & 230.685 \\
\hline 2.197 & 109.85 & 107.653 & 13.182 \\
\hline 10.985 & 2.197 & 10.985 & 39.546 \\
\hline 24.167 & 4.394 & 2.197 & 4.394 \\
\hline 2.197 & 41.743 & 4.394 & 13.182 \\
\hline 2.197 & 617.357 & 4.394 & 2.197 \\
\hline 2.197 & 2.197 & 6.591 & 2.197 \\
\hline 6.591 & 2.197 & 112.047 & 2.197 \\
\hline 2.197 & 6.591 & 2.197 & 2.197 \\
\hline 648.115 & 68.107 & 2.197 & 13.182 \\
\hline 10.985 & 6.591 & 17.576 & 50.531 \\
\hline 24.167 & 4.394 & 21.97 & 24.167 \\
\hline 15.379 & 171.366 & 4.394 & 10.985 \\
\hline 10.985 & 41.743 & 41.743 & 17.576 \\
\hline 4.394 & 6.591 & 2.197 & 2.197 \\
\hline 6.591 & 2.197 & 8.788 & 2.197 \\
\hline 4.394 & 2.197 & 2.197 & 2.197 \\
\hline 17.576 & 17.576 & 4.394 & 142.805 \\
\hline 4.394 & 96.668 & 2.197 & 4.394 \\
\hline 10.985 & 509.704 & 1036.98 & 4.394 \\
\hline 2.197 & 166.972 & 2.197 & 2.197 \\
\hline 2.197 & 6.591 & 2.197 & 8.788 \\
\hline 228.488 & 4.394 & 13.182 & 2.197 \\
\hline 118.638 & 17.576 & 2.197 & 2.197 \\
\hline 15.379 & 10.985 & 2.197 & 4.394 \\
\hline 4.394 & 250.458 & 2.197 & 4.394 \\
\hline 15.379 & 2.197 & 2.197 & 8.788 \\
\hline 4.394 & 588.796 & 4.394 & 41.743 \\
\hline 4.394 & 32.955 & 2.197 & 83.486 \\
\hline 6.591 & 2.197 & 4.394 & 2.197 \\
\hline 10.985 & 4.394 & 127.426 & 1138.05 \\
\hline 4.394 & 4.394 & 72.501 & 2.197 \\
\hline 4.394 & 2.197 & 72.501 & 57.122 \\
\hline
\end{tabular}




\begin{tabular}{|c|c|c|c|}
\hline 8.788 & 13.182 & 2.197 & 24.167 \\
\hline 628.342 & 4.394 & 10.985 & 2.197 \\
\hline 8.788 & 2.197 & 13.182 & 19.773 \\
\hline 2.197 & 41.743 & 8.788 & 30.758 \\
\hline 6.591 & 2.197 & 4.394 & 4.394 \\
\hline 6.591 & 2.197 & 4.394 & 35.152 \\
\hline 2.197 & 10.985 & 191.139 & 50.531 \\
\hline 35.152 & 2.197 & 4.394 & 8.788 \\
\hline 2.197 & 2.197 & 2.197 & 4.394 \\
\hline 24.167 & 4.394 & 24.167 & 13.182 \\
\hline 2.197 & 105.456 & 30.758 & 6.591 \\
\hline 4.394 & 2.197 & 30.758 & 4.394 \\
\hline 2.197 & 131.82 & 6.591 & 61.516 \\
\hline 4.394 & 76.895 & 2.197 & 2.197 \\
\hline 2.197 & 2.197 & 61.516 & 26.364 \\
\hline 2.197 & 4.394 & 46.137 & 65.91 \\
\hline 61.516 & 41.743 & 123.032 & 10.985 \\
\hline 2.197 & 4.394 & 50.531 & 114.244 \\
\hline 2.197 & 4.394 & 30.758 & 8.788 \\
\hline 107.653 & 2.197 & 2.197 & 17.576 \\
\hline 15.379 & 2.197 & 103.259 & 68.107 \\
\hline 35.152 & 13.182 & 2.197 & 2.197 \\
\hline 4.394 & 2.197 & 547.053 & 24.167 \\
\hline 8.788 & 2.197 & 2.197 & 8.788 \\
\hline 4.394 & 4.394 & 2.197 & 2.197 \\
\hline 6.591 & 2.197 & 2.197 & 13.182 \\
\hline 6.591 & 2.197 & 2.197 & 6.591 \\
\hline 2.197 & 10.985 & 2.197 & 15.379 \\
\hline 6.591 & 59.319 & 13.182 & 4.394 \\
\hline 4.394 & 2.197 & 2.197 & 32.955 \\
\hline 2.197 & 4.394 & 39.546 & 48.334 \\
\hline 2.197 & 70.304 & 2.197 & 21.97 \\
\hline 276.822 & 147.199 & 2.197 & 2.197 \\
\hline 13.182 & 2.197 & 2.197 & 2.197 \\
\hline 35.152 & 24.167 & 2.197 & 2.197 \\
\hline 2.197 & 125.229 & 10.985 & 2.197 \\
\hline 2.197 & 8.788 & 19.773 & 2.197 \\
\hline 2.197 & 70.304 & 2.197 & 2.197 \\
\hline 4.394 & 538.265 & 2.197 & 2.197 \\
\hline 2.197 & 186.745 & 4.394 & 2.197 \\
\hline 68.107 & 21.97 & 2.197 & 2.197 \\
\hline 10.985 & 608.569 & 2.197 & 101.062 \\
\hline 2.197 & 6.591 & 59.319 & 6.591 \\
\hline 4.394 & 15.379 & 8.788 & 4.394 \\
\hline 15.379 & 2.197 & 4.394 & 2.197 \\
\hline 68.107 & 4.394 & 2.197 & 2.197 \\
\hline 26.364 & 15.379 & 2.197 & 136.214 \\
\hline 101.062 & 4.394 & 2.197 & 2.197 \\
\hline 413.036 & 2.197 & 2.197 & 2.197 \\
\hline 2.197 & 10.985 & 21.97 & 26.364 \\
\hline
\end{tabular}




\begin{tabular}{|c|c|c|c|}
\hline 118.638 & 98.865 & 32.955 & 2.197 \\
\hline 2.197 & 2.197 & 2.197 & 19.773 \\
\hline 2.197 & 39.546 & 1146.83 & 4.394 \\
\hline 2.197 & 112.047 & 13.182 & 2.197 \\
\hline 2.197 & 90.077 & 2.197 & 2.197 \\
\hline 13.182 & 8.788 & 81.289 & 2.197 \\
\hline 2.197 & 39.546 & 2.197 & 2.197 \\
\hline 2.197 & 2.197 & 2.197 & 2.197 \\
\hline 2.197 & 2.197 & 2.197 & 72.501 \\
\hline 2.197 & 28.561 & 63.713 & 2.197 \\
\hline 6.591 & 6.591 & 6.591 & 2.197 \\
\hline 2.197 & 4.394 & 10.985 & 28.561 \\
\hline 68.107 & 2.197 & 342.732 & 50.531 \\
\hline 32.955 & 2.197 & 160.381 & 90.077 \\
\hline 2.197 & 2.197 & 2.197 & 2.197 \\
\hline 2.197 & 8.788 & 2.197 & 13.182 \\
\hline 4.394 & 96.668 & 52.728 & 63.713 \\
\hline 2.197 & 720.616 & 6.591 & 2.197 \\
\hline 2.197 & 98.865 & 215.306 & 10.985 \\
\hline 483.34 & 8.788 & 2.197 & 8.788 \\
\hline 6.591 & 2.197 & 2.197 & 4.394 \\
\hline 10.985 & 2.197 & 2.197 & 28.561 \\
\hline 35.152 & 8.788 & 2.197 & 4.394 \\
\hline 81.289 & 129.623 & 24.167 & 4.394 \\
\hline 6.591 & 59.319 & 2.197 & 2.197 \\
\hline 6.591 & 15.379 & 2.197 & 6905.17 \\
\hline 2.197 & 30.758 & 41.743 & 37.349 \\
\hline 37.349 & 145.002 & 28.561 & 4.394 \\
\hline 2.197 & 4.394 & 19.773 & 489.931 \\
\hline 4.394 & 32.955 & 28.561 & 6.591 \\
\hline 2.197 & 2.197 & 2.197 & 74.698 \\
\hline 2.197 & 35.152 & 2.197 & 6.591 \\
\hline 6.591 & 6.591 & 15.379 & 4.394 \\
\hline 30.758 & 10.985 & 2.197 & 4.394 \\
\hline 8.788 & 4.394 & 4.394 & 15.379 \\
\hline 4.394 & 2.197 & 109.85 & 13.182 \\
\hline 6.591 & 2.197 & 2.197 & 2.197 \\
\hline 10.985 & 2.197 & 79.092 & 250.458 \\
\hline 17.576 & 2.197 & 59.319 & 2.197 \\
\hline 74.698 & 15.379 & 30.758 & 2.197 \\
\hline 2.197 & 52.728 & 2.197 & 2.197 \\
\hline 4.394 & 173.563 & 2.197 & 4.394 \\
\hline 2.197 & 10.985 & 2.197 & 4.394 \\
\hline 4.394 & 13.182 & 2.197 & 13.182 \\
\hline 4.394 & 2.197 & 235.079 & 13.182 \\
\hline 4.394 & 6.591 & 65.91 & 15.379 \\
\hline 2.197 & 87.88 & 2.197 & 15.379 \\
\hline 10.985 & 2.197 & 3682.17 & 2.197 \\
\hline 10.985 & 15.379 & 24.167 & 4.394 \\
\hline 2.197 & 4.394 & 17.576 & 2.197 \\
\hline
\end{tabular}




\begin{tabular}{|c|c|c|c|}
\hline 17.576 & 76.895 & 2.197 & 2.197 \\
\hline 2.197 & 2.197 & 8.788 & 10.985 \\
\hline 738.192 & 4.394 & 4.394 & 2.197 \\
\hline 2.197 & 17.576 & 2.197 & 79.092 \\
\hline 4.394 & 2.197 & 2.197 & 2.197 \\
\hline 13.182 & 8.788 & 15.379 & 2.197 \\
\hline 2.197 & 8.788 & 32.955 & 24.167 \\
\hline 8.788 & 2.197 & 21.97 & 2.197 \\
\hline 43.94 & 15.379 & 2.197 & 4.394 \\
\hline 4.394 & 322.959 & 353.717 & 254.852 \\
\hline 2.197 & 30.758 & 2.197 & 87.88 \\
\hline 2.197 & 2.197 & 4.394 & 839.254 \\
\hline 4.394 & 32.955 & 2.197 & 2.197 \\
\hline 4.394 & 61.516 & 2.197 & 48.334 \\
\hline 2.197 & 2.197 & 2.197 & 2.197 \\
\hline 4.394 & 4.394 & 2.197 & 21.97 \\
\hline 19.773 & 57.122 & 2.197 & 30.758 \\
\hline 6.591 & 2.197 & 2.197 & 2.197 \\
\hline 2.197 & 8.788 & 145.002 & 2.197 \\
\hline 2.197 & 39.546 & 87.88 & 2.197 \\
\hline 4.394 & 182.351 & 8.788 & 32.955 \\
\hline 32.955 & 109.85 & 2.197 & 28.561 \\
\hline 2.197 & 2.197 & 8.788 & 41.743 \\
\hline 4.394 & 177.957 & 2.197 & 15.379 \\
\hline 8.788 & 232.882 & 2.197 & 153.79 \\
\hline 2.197 & 2.197 & 2.197 & 2.197 \\
\hline 46.137 & 153.79 & 10.985 & 4.394 \\
\hline 15.379 & 4.394 & 4.394 & 274.625 \\
\hline 26.364 & 8.788 & 4.394 & 32.955 \\
\hline 2.197 & 2.197 & 2.197 & 6.591 \\
\hline 2.197 & 50.531 & 2.197 & 10.985 \\
\hline 2.197 & 4.394 & 2.197 & 35.152 \\
\hline 41.743 & 8.788 & 2.197 & 15.379 \\
\hline 37.349 & 2.197 & 92.274 & 10.985 \\
\hline 46.137 & 2.197 & 2.197 & 8.788 \\
\hline 2.197 & 2.197 & 32.955 & 10.985 \\
\hline 15.379 & 2.197 & 2.197 & 85.683 \\
\hline 2.197 & 4.394 & 2.197 & 8.788 \\
\hline 39.546 & 6.591 & 2.197 & 4.394 \\
\hline 21.97 & 2.197 & 2.197 & 15.379 \\
\hline 4.394 & 10.985 & 4.394 & 2.197 \\
\hline 50.531 & 41.743 & 4.394 & 2.197 \\
\hline 2.197 & 10.985 & 2.197 & 8.788 \\
\hline 8.788 & 4.394 & 2.197 & 83.486 \\
\hline 1821.31 & 8.788 & 2.197 & 15.379 \\
\hline 2.197 & 4.394 & 4.394 & 4.394 \\
\hline 2.197 & 10.985 & 2.197 & 2.197 \\
\hline 10.985 & 8.788 & 2.197 & 8.788 \\
\hline 2.197 & 2.197 & 19.773 & 2.197 \\
\hline 4.394 & 24.167 & 377.884 & 722.813 \\
\hline
\end{tabular}




\begin{tabular}{|c|c|c|c|}
\hline 4.394 & 65.91 & 2.197 & 2.197 \\
\hline 6.591 & 24.167 & 2.197 & 8.788 \\
\hline 329.55 & 10.985 & 2.197 & 6.591 \\
\hline 2.197 & 191.139 & 6.591 & 114.244 \\
\hline 2.197 & 8.788 & 2.197 & 2.197 \\
\hline 43.94 & 4.394 & 2.197 & 6.591 \\
\hline 70.304 & 17.576 & 2.197 & 243.867 \\
\hline 448.188 & 26.364 & 2.197 & 4.394 \\
\hline 57.122 & 72.501 & 32.955 & 19.773 \\
\hline 2.197 & 15.379 & 13.182 & 2.197 \\
\hline 2.197 & 8.788 & 92.274 & 15.379 \\
\hline 4.394 & 10.985 & 26.364 & 39.546 \\
\hline 2.197 & 13.182 & 2.197 & 2.197 \\
\hline 2.197 & 68.107 & 35.152 & 2.197 \\
\hline 2.197 & 19.773 & 13.182 & 6.591 \\
\hline 2.197 & 30.758 & 6.591 & 2.197 \\
\hline 15.379 & 2.197 & 2.197 & 2.197 \\
\hline 2.197 & 39.546 & 2.197 & 10.985 \\
\hline 17.576 & 2.197 & 2.197 & 2.197 \\
\hline 2.197 & 15.379 & 2.197 & 39.546 \\
\hline 17.576 & 637.13 & 2.197 & 2.197 \\
\hline 2.197 & 6.591 & 2.197 & 2.197 \\
\hline 2.197 & 4.394 & 2.197 & 2.197 \\
\hline 43.94 & 215.306 & 254.852 & 2.197 \\
\hline 52.728 & 2.197 & 2.197 & 2.197 \\
\hline 2.197 & 2.197 & 4.394 & 4.394 \\
\hline 2.197 & 8.788 & 2.197 & 720.616 \\
\hline 13.182 & 85.683 & 74.698 & 34024.9 \\
\hline 13.182 & 37.349 & 4.394 & 1036.98 \\
\hline 28.561 & 4.394 & 26.364 & 74.698 \\
\hline 109.85 & 2.197 & 621.751 & 197.73 \\
\hline 50.531 & 4.394 & 13.182 & 26.364 \\
\hline 10.985 & 10.985 & 2.197 & 2.197 \\
\hline 13.182 & 81.289 & 2.197 & 2.197 \\
\hline 2.197 & 2.197 & 2.197 & 32.955 \\
\hline 2.197 & 61.516 & 160.381 & 1199.56 \\
\hline 4.394 & 6.591 & 39.546 & 4.394 \\
\hline 2.197 & 8.788 & 2.197 & 28.561 \\
\hline 8.788 & 281.216 & 10.985 & 52.728 \\
\hline 2.197 & 26.364 & 2.197 & 24.167 \\
\hline 4.394 & 2.197 & 8.788 & 30.758 \\
\hline 10.985 & 13.182 & 188.942 & 2.197 \\
\hline 2.197 & 2.197 & 2.197 & 2.197 \\
\hline 4.394 & 21.97 & 32.955 & 17.576 \\
\hline 4.394 & 8.788 & 68.107 & 2.197 \\
\hline 4.394 & 35.152 & 2.197 & 21.97 \\
\hline 4.394 & 2.197 & 2.197 & 37.349 \\
\hline 4.394 & 601.978 & 2.197 & 70.304 \\
\hline 2.197 & 8.788 & 6.591 & 21.97 \\
\hline 70.304 & 10.985 & 180.154 & 4.394 \\
\hline
\end{tabular}




\begin{tabular}{|c|c|c|c|}
\hline 2.197 & 48.334 & 2.197 & 6.591 \\
\hline 13.182 & 43.94 & 17.576 & 96.668 \\
\hline 2.197 & 2.197 & 2.197 & 10.985 \\
\hline 2.197 & 4.394 & 129.623 & 195.533 \\
\hline 2.197 & 125.229 & 2.197 & 4.394 \\
\hline 2.197 & 2.197 & 2.197 & 17.576 \\
\hline 8.788 & 6.591 & 13.182 & 125.229 \\
\hline 2.197 & 21.97 & 2.197 & 15.379 \\
\hline 2.197 & 4.394 & 2.197 & 4.394 \\
\hline 2.197 & 35.152 & 2.197 & 6.591 \\
\hline 2.197 & 37.349 & 2.197 & 15.379 \\
\hline 2.197 & 21.97 & 2.197 & 57.122 \\
\hline 17.576 & 74.698 & 2.197 & 13.182 \\
\hline 43.94 & 582.205 & 2.197 & 2.197 \\
\hline 4.394 & 43.94 & 48.334 & 26.364 \\
\hline 2.197 & 46.137 & 2.197 & 107.653 \\
\hline 4.394 & 6.591 & 169.169 & 32.955 \\
\hline 13.182 & 21.97 & 17.576 & 13.182 \\
\hline 72.501 & 4.394 & 2.197 & 2.197 \\
\hline 85.683 & 21.97 & 4.394 & 2.197 \\
\hline 2.197 & 24.167 & 2.197 & 2.197 \\
\hline 6.591 & 2.197 & 123.032 & 13.182 \\
\hline 4.394 & 59.319 & 140.608 & 6.591 \\
\hline 4.394 & 70.304 & 13.182 & 2.197 \\
\hline 4.394 & 8.788 & 2.197 & 2.197 \\
\hline 8.788 & 2.197 & 2.197 & 8.788 \\
\hline 6.591 & 2.197 & 26.364 & 8.788 \\
\hline 4.394 & 4.394 & 496.522 & 4.394 \\
\hline 2.197 & 2.197 & 8.788 & 2.197 \\
\hline 2.197 & 46.137 & 74.698 & 15.379 \\
\hline 2.197 & 10.985 & 2.197 & 39.546 \\
\hline 2.197 & 2.197 & 2.197 & 37.349 \\
\hline 15.379 & 21.97 & 2.197 & 10.985 \\
\hline 2.197 & 153.79 & 2.197 & 41.743 \\
\hline 4.394 & 94.471 & 4.394 & 6.591 \\
\hline 4.394 & 10.985 & 13.182 & 2.197 \\
\hline 4.394 & 2.197 & 2.197 & 2.197 \\
\hline 15.379 & 2.197 & 4.394 & 2.197 \\
\hline 2.197 & 2.197 & 4.394 & 2.197 \\
\hline 2.197 & 158.184 & 1021.6 & 46.137 \\
\hline 35.152 & 2.197 & 2.197 & 2.197 \\
\hline 15.379 & 2.197 & 2.197 & 43.94 \\
\hline 2.197 & 21.97 & 4.394 & 17.576 \\
\hline 2.197 & 52.728 & 6.591 & 2.197 \\
\hline 4.394 & 13.182 & 2.197 & 2.197 \\
\hline 2.197 & 2.197 & 28.561 & 2.197 \\
\hline 4.394 & 2.197 & 272.428 & 2.197 \\
\hline 2.197 & 17.576 & 2.197 & 6.591 \\
\hline 39.546 & 2.197 & 2.197 & 8.788 \\
\hline 2.197 & 2.197 & 13.182 & 2.197 \\
\hline
\end{tabular}




\begin{tabular}{|c|c|c|c|}
\hline 2.197 & 6.591 & 57.122 & 2.197 \\
\hline 15.379 & 8.788 & 4.394 & 2.197 \\
\hline 2.197 & 10.985 & 4.394 & 15.379 \\
\hline 2.197 & 4.394 & 6.591 & 50.531 \\
\hline 2.197 & 76.895 & 2.197 & 15.379 \\
\hline 8.788 & 2.197 & 2.197 & 15.379 \\
\hline 63.713 & 6.591 & 2.197 & 96.668 \\
\hline 30.758 & 2.197 & 83.486 & 2.197 \\
\hline 2.197 & 28.561 & 39.546 & 72.501 \\
\hline 8.788 & 13.182 & 116.441 & 4.394 \\
\hline 17.576 & 54.925 & 32.955 & 6.591 \\
\hline 76.895 & 32.955 & 4.394 & 4.394 \\
\hline 134.017 & 2.197 & 2.197 & 50.531 \\
\hline 32.955 & 6.591 & 2.197 & 2.197 \\
\hline 2.197 & 15.379 & 10.985 & 4.394 \\
\hline 4.394 & 6.591 & 10.985 & 2.197 \\
\hline 2.197 & 10.985 & 2.197 & 10.985 \\
\hline 6.591 & 276.822 & 2.197 & 2.197 \\
\hline 17.576 & 2.197 & 2.197 & 13.182 \\
\hline 2.197 & 2.197 & 2.197 & 81.289 \\
\hline 2.197 & 123.032 & 2.197 & 17.576 \\
\hline 2.197 & 19.773 & 54.925 & 2.197 \\
\hline 15.379 & 4.394 & 155.987 & 6.591 \\
\hline 79.092 & 15.379 & 2.197 & 26.364 \\
\hline 35.152 & 15.379 & 2.197 & 6.591 \\
\hline 21.97 & 24.167 & 6.591 & 6.591 \\
\hline 15.379 & 41.743 & 57.122 & 4.394 \\
\hline 2.197 & 4.394 & 10.985 & 8.788 \\
\hline 17.576 & 30.758 & 17.576 & 2.197 \\
\hline 37.349 & 127.426 & 26.364 & 366.899 \\
\hline 2.197 & 2.197 & 2.197 & 2.197 \\
\hline 8.788 & 8.788 & 19.773 & 733.798 \\
\hline 2.197 & 8.788 & 2.197 & 26.364 \\
\hline 13.182 & 2.197 & 2.197 & 1406.08 \\
\hline 8.788 & 10.985 & 13.182 & 50.531 \\
\hline 17.576 & 10.985 & 2.197 & 2.197 \\
\hline 2.197 & 13.182 & 2.197 & 8.788 \\
\hline 2.197 & 59.319 & 2.197 & 13.182 \\
\hline 32.955 & 15.379 & 2.197 & 292.201 \\
\hline 6.591 & 6.591 & 2366.17 & 4207.25 \\
\hline 2.197 & 26.364 & 2.197 & 26.364 \\
\hline 4.394 & 79.092 & 116.441 & 714.025 \\
\hline 2.197 & 109.85 & 2.197 & 8.788 \\
\hline 2.197 & 13.182 & 4.394 & 109.85 \\
\hline 2.197 & 6.591 & 2.197 & 2.197 \\
\hline 2.197 & 2.197 & 2.197 & 274.625 \\
\hline 2.197 & 2.197 & 2.197 & 252.655 \\
\hline 2.197 & 70.304 & 2.197 & 2.197 \\
\hline 2.197 & 105.456 & 2.197 & 101.062 \\
\hline 2.197 & 46.137 & 24.167 & 32.955 \\
\hline
\end{tabular}




\begin{tabular}{|c|c|c|c|}
\hline 177.957 & 4.394 & 180.154 & 2.197 \\
\hline 4.394 & 68.107 & 6.591 & 105.456 \\
\hline 2.197 & 6.591 & 10.985 & 15.379 \\
\hline 1441.23 & 2.197 & 6.591 & 8.788 \\
\hline 2.197 & 26.364 & 26.364 & 2.197 \\
\hline 2.197 & 2.197 & 2.197 & 4.394 \\
\hline 2.197 & 8.788 & 219.7 & 6.591 \\
\hline 2.197 & 2.197 & 4.394 & 43.94 \\
\hline 2.197 & 37.349 & 94.471 & 2.197 \\
\hline 2.197 & 2.197 & 2.197 & 2.197 \\
\hline 2.197 & 37.349 & 17.576 & 2.197 \\
\hline 13.182 & 90.077 & 2.197 & 2.197 \\
\hline 2.197 & 4.394 & 4.394 & 4.394 \\
\hline 28.561 & 46.137 & 2.197 & 2.197 \\
\hline 158.184 & 70.304 & 1085.32 & 2.197 \\
\hline 17.576 & 107.653 & 2.197 & 2.197 \\
\hline 17.576 & 10.985 & 2.197 & 4.394 \\
\hline 2.197 & 2.197 & 2.197 & 10.985 \\
\hline 294.398 & 166.972 & 2.197 & 2.197 \\
\hline 166.972 & 50.531 & 52.728 & 21.97 \\
\hline 2.197 & 2.197 & 2.197 & 2.197 \\
\hline 8.788 & 15.379 & 2.197 & 4.394 \\
\hline 2.197 & 4.394 & 2.197 & 68.107 \\
\hline 8.788 & 17.576 & 13.182 & 2.197 \\
\hline 8.788 & 4.394 & 24.167 & 2.197 \\
\hline 136.214 & 8.788 & 8.788 & 2.197 \\
\hline 2.197 & 6.591 & 6.591 & 28.561 \\
\hline 4.394 & 4.394 & 4.394 & 37.349 \\
\hline 4.394 & 2.197 & 2.197 & 79.092 \\
\hline 2.197 & 2.197 & 8.788 & 169.169 \\
\hline 2.197 & 2.197 & 6.591 & 149.396 \\
\hline 2.197 & 4.394 & 4.394 & 2.197 \\
\hline 24.167 & 2.197 & 2.197 & 2.197 \\
\hline 2.197 & 41.743 & 15.379 & 6.591 \\
\hline 2.197 & 2.197 & 494.325 & 103.259 \\
\hline 48.334 & 2.197 & 2.197 & 26.364 \\
\hline 2.197 & 21.97 & 72.501 & 131.82 \\
\hline 2.197 & 107.653 & 2.197 & 147.199 \\
\hline 2.197 & 52.728 & 2.197 & 369.096 \\
\hline 2.197 & 2.197 & 2.197 & 4.394 \\
\hline 4.394 & 2.197 & 8.788 & 2.197 \\
\hline 65.91 & 4.394 & 70.304 & 6.591 \\
\hline 43.94 & 13.182 & 8.788 & 2.197 \\
\hline 2.197 & 79.092 & 83.486 & 10.985 \\
\hline 54.925 & 2.197 & 6.591 & 2.197 \\
\hline 17903.4 & 6.591 & 10.985 & 10.985 \\
\hline 24.167 & 15.379 & 37.349 & 72.501 \\
\hline 59.319 & 37.349 & 35.152 & 4.394 \\
\hline 15.379 & 4.394 & 8.788 & 32.955 \\
\hline 4.394 & 4.394 & 2.197 & 6.591 \\
\hline
\end{tabular}




\begin{tabular}{|c|c|c|c|}
\hline 2.197 & 2.197 & 8.788 & 2.197 \\
\hline 6.591 & 2.197 & 13.182 & 8.788 \\
\hline 8.788 & 127.426 & 52.728 & 2.197 \\
\hline 26.364 & 43.94 & 4.394 & 2.197 \\
\hline 2.197 & 6.591 & 6.591 & 54.925 \\
\hline 2.197 & 46.137 & 6.591 & 61.516 \\
\hline 4.394 & 241.67 & 2.197 & 24.167 \\
\hline 4.394 & 2.197 & 2.197 & 37.349 \\
\hline 4.394 & 6.591 & 2.197 & 15.379 \\
\hline 2.197 & 21.97 & 2.197 & 8.788 \\
\hline 371.293 & 6.591 & 4.394 & 28.561 \\
\hline 2.197 & 8.788 & 2.197 & 92.274 \\
\hline 2.197 & 10.985 & 8.788 & 8.788 \\
\hline 2.197 & 59.319 & 2.197 & 41.743 \\
\hline 65.91 & 268.034 & 2.197 & 15.379 \\
\hline 6.591 & 2.197 & 24.167 & 10.985 \\
\hline 2.197 & 41.743 & 4.394 & 46.137 \\
\hline 2.197 & 13.182 & 4.394 & 2.197 \\
\hline 2.197 & 8.788 & 4.394 & 4.394 \\
\hline 4.394 & 35.152 & 2.197 & 182.351 \\
\hline 2.197 & 46.137 & 26.364 & 37.349 \\
\hline 202.124 & 2.197 & 2.197 & 24.167 \\
\hline 41.743 & 2.197 & 248.261 & 2.197 \\
\hline 2.197 & 2.197 & 166.972 & 2.197 \\
\hline 4.394 & 8.788 & 2.197 & 2.197 \\
\hline 13.182 & 13.182 & 28.561 & 2.197 \\
\hline 30.758 & 28.561 & 15.379 & 2.197 \\
\hline 4.394 & 2.197 & 2.197 & 4.394 \\
\hline 2.197 & 2.197 & 2.197 & 4.394 \\
\hline 6.591 & 573.417 & 2.197 & 2.197 \\
\hline 4.394 & 4.394 & 2.197 & 2.197 \\
\hline 2.197 & 2.197 & 2.197 & 2.197 \\
\hline 2.197 & 175.76 & 2.197 & 2.197 \\
\hline 2.197 & 2.197 & 65.91 & 30.758 \\
\hline 2.197 & 13.182 & 8.788 & 4.394 \\
\hline 8.788 & 4.394 & 4.394 & 17.576 \\
\hline 4.394 & 92.274 & 2.197 & 107.653 \\
\hline 2.197 & 4.394 & 10.985 & 4.394 \\
\hline 28.561 & 193.336 & 6.591 & 8.788 \\
\hline 17.576 & 2.197 & 19.773 & 35.152 \\
\hline 2.197 & 21.97 & 6.591 & 8.788 \\
\hline 4.394 & 2.197 & 90.077 & 8.788 \\
\hline 4.394 & 2.197 & 2.197 & 8.788 \\
\hline 2.197 & 4.394 & 4.394 & 17.576 \\
\hline 4.394 & 65.91 & 2.197 & 15.379 \\
\hline 2.197 & 153.79 & 2.197 & 19.773 \\
\hline 37.349 & 6.591 & 2.197 & 48.334 \\
\hline 2.197 & 8.788 & 8.788 & 6.591 \\
\hline 10.985 & 15.379 & 2.197 & 92.274 \\
\hline 52.728 & 19.773 & 2.197 & 2.197 \\
\hline
\end{tabular}




\begin{tabular}{|c|c|c|c|}
\hline 2.197 & 13.182 & 13.182 & 37.349 \\
\hline 2.197 & 4.394 & 2.197 & 4.394 \\
\hline 2.197 & 15.379 & 30.758 & 24.167 \\
\hline 2.197 & 2.197 & 2.197 & 17.576 \\
\hline 15.379 & 21.97 & 2.197 & 10.985 \\
\hline 8.788 & 28.561 & 2.197 & 17.576 \\
\hline 2.197 & 57.122 & 399.854 & 2.197 \\
\hline 4.394 & 2.197 & 2.197 & 2.197 \\
\hline 2.197 & 6.591 & 46.137 & 2.197 \\
\hline 2.197 & 6.591 & 2.197 & 10.985 \\
\hline 15.379 & 123.032 & 2.197 & 4.394 \\
\hline 2.197 & 57.122 & 131.82 & 1520.32 \\
\hline 8.788 & 6.591 & 26.364 & 6.591 \\
\hline 2.197 & 2.197 & 21.97 & 17.576 \\
\hline 2.197 & 2.197 & 13.182 & 10.985 \\
\hline 2.197 & 94.471 & 6.591 & 2.197 \\
\hline 2.197 & 28.561 & 2.197 & 2.197 \\
\hline 21.97 & 2.197 & 123.032 & 94.471 \\
\hline 667.888 & 8.788 & 34264.4 & 2.197 \\
\hline 8.788 & 6.591 & 13.182 & 4.394 \\
\hline 2.197 & 10.985 & 2.197 & 235.079 \\
\hline 2.197 & 30.758 & 151.593 & 87.88 \\
\hline 6.591 & 2.197 & 10.985 & 4.394 \\
\hline 24.167 & 241.67 & 30.758 & 107.653 \\
\hline 8.788 & 2.197 & 2.197 & 19.773 \\
\hline 4.394 & 4.394 & 24.167 & 4.394 \\
\hline 2.197 & 2.197 & 54.925 & 2.197 \\
\hline 2.197 & 6.591 & 41.743 & 13.182 \\
\hline 4.394 & 61.516 & 2.197 & 13.182 \\
\hline 13.182 & 35.152 & 2.197 & 4.394 \\
\hline 10.985 & 28.561 & 4.394 & 136.214 \\
\hline 8.788 & 35.152 & 8.788 & 388.869 \\
\hline 6.591 & 2.197 & 15.379 & 2.197 \\
\hline 21.97 & 2.197 & 4.394 & 2.197 \\
\hline 2.197 & 292.201 & 15.379 & 30.758 \\
\hline 8.788 & 2.197 & 856.83 & 6.591 \\
\hline 2.197 & 90.077 & 2.197 & 2.197 \\
\hline 2.197 & 54.925 & 6.591 & 98.865 \\
\hline 2.197 & 4.394 & 2.197 & 4.394 \\
\hline 4.394 & 6.591 & 59.319 & 6.591 \\
\hline 2.197 & 28.561 & 6.591 & 4.394 \\
\hline 4.394 & 4.394 & 2.197 & 2.197 \\
\hline 2.197 & 210.912 & 10.985 & 6.591 \\
\hline 2.197 & 87.88 & 21.97 & 92.274 \\
\hline 2.197 & 79.092 & 37.349 & 10.985 \\
\hline 2.197 & 10.985 & 6.591 & 4.394 \\
\hline 2.197 & 39.546 & 246.064 & 597.584 \\
\hline 2.197 & 2.197 & 2.197 & 2.197 \\
\hline 4.394 & 162.578 & 2.197 & 35.152 \\
\hline 2.197 & 8.788 & 2.197 & 15.379 \\
\hline
\end{tabular}




\begin{tabular}{|c|c|c|c|}
\hline 2.197 & 2.197 & 2.197 & 2.197 \\
\hline 2.197 & 13.182 & 19.773 & 2.197 \\
\hline 2.197 & 28.561 & 2.197 & 19.773 \\
\hline 2.197 & 46.137 & 4.394 & 21.97 \\
\hline 6.591 & 373.49 & 2.197 & 2.197 \\
\hline 2166.24 & 8.788 & 35.152 & 74.698 \\
\hline 4.394 & 2.197 & 6.591 & 10.985 \\
\hline 17.576 & 26.364 & 2.197 & 169.169 \\
\hline 2.197 & 2.197 & 2.197 & 13.182 \\
\hline 8.788 & 41.743 & 2.197 & 13.182 \\
\hline 8.788 & 68.107 & 823.875 & 2.197 \\
\hline 90.077 & 2.197 & 15.379 & 333.944 \\
\hline 4.394 & 175.76 & 54.925 & 147.199 \\
\hline 2.197 & 8.788 & 2.197 & 6.591 \\
\hline 4.394 & 2.197 & 43.94 & 2.197 \\
\hline 13.182 & 6.591 & 2.197 & 8.788 \\
\hline 443.794 & 17.576 & 2.197 & 19.773 \\
\hline 6.591 & 160.381 & 4.394 & 2.197 \\
\hline 2.197 & 61.516 & 4.394 & 2.197 \\
\hline 65.91 & 2.197 & 4.394 & 26.364 \\
\hline 6.591 & 21.97 & 2.197 & 83.486 \\
\hline 2.197 & 8.788 & 2.197 & 6.591 \\
\hline 2.197 & 8.788 & 21.97 & 2.197 \\
\hline 2.197 & 2.197 & 2.197 & 2.197 \\
\hline 4.394 & 8.788 & 63.713 & 4.394 \\
\hline 2.197 & 8.788 & 6.591 & 13.182 \\
\hline 2.197 & 2.197 & 28.561 & 17.576 \\
\hline 2.197 & 10.985 & 8.788 & 37.349 \\
\hline 2.197 & 41.743 & 43.94 & 10.985 \\
\hline 2.197 & 8.788 & 2.197 & 6.591 \\
\hline 2.197 & 2.197 & 37.349 & 15.379 \\
\hline 8.788 & 10.985 & 2.197 & 15.379 \\
\hline 4.394 & 127.426 & 63.713 & 2.197 \\
\hline 2.197 & 2.197 & 37.349 & 46.137 \\
\hline 13.182 & 2.197 & 10.985 & 41.743 \\
\hline 2.197 & 2.197 & 2.197 & 24.167 \\
\hline 28.561 & 17.576 & 2.197 & 13.182 \\
\hline 2.197 & 2.197 & 26.364 & 24.167 \\
\hline 2.197 & 2.197 & 2.197 & 17.576 \\
\hline 39.546 & 6.591 & 6.591 & 28.561 \\
\hline 2.197 & 35.152 & 10.985 & 2.197 \\
\hline 2.197 & 2.197 & 2.197 & 2.197 \\
\hline 2.197 & 2.197 & 2.197 & 2.197 \\
\hline 13.182 & 13.182 & 2.197 & 10.985 \\
\hline 4.394 & 6.591 & 2.197 & 2.197 \\
\hline 2.197 & 2.197 & 8.788 & 74.698 \\
\hline 2.197 & 85.683 & 2.197 & 4.394 \\
\hline 2.197 & 166.972 & 2.197 & 52.728 \\
\hline 2.197 & 118.638 & 2.197 & 2.197 \\
\hline 2.197 & 70.304 & 208.715 & 2.197 \\
\hline
\end{tabular}




\begin{tabular}{|c|c|c|c|}
\hline 4.394 & 13.182 & 6.591 & 2.197 \\
\hline 6.591 & 114.244 & 4.394 & 13.182 \\
\hline 2.197 & 92.274 & 2.197 & 2.197 \\
\hline 57.122 & 134.017 & 2.197 & 2.197 \\
\hline 2.197 & 28.561 & 2.197 & 180.154 \\
\hline 30.758 & 118.638 & 4.394 & 2.197 \\
\hline 2.197 & 1019.41 & 10.985 & 4.394 \\
\hline 10.985 & 81.289 & 2.197 & 2.197 \\
\hline 24.167 & 13.182 & 32.955 & 136.214 \\
\hline 87.88 & 4.394 & 2.197 & 26.364 \\
\hline 98.865 & 2.197 & 32.955 & 10.985 \\
\hline 74.698 & 8.788 & 13.182 & 4.394 \\
\hline 160.381 & 2.197 & 2.197 & 4.394 \\
\hline 2.197 & 19.773 & 2.197 & 52.728 \\
\hline 180.154 & 4.394 & 114.244 & 4.394 \\
\hline 19.773 & 35.152 & 2.197 & 2.197 \\
\hline 4.394 & 52.728 & 2.197 & 17.576 \\
\hline 21.97 & 4.394 & 210.912 & 96.668 \\
\hline 83.486 & 103.259 & 6.591 & 50.531 \\
\hline 2.197 & 2.197 & 6.591 & 10.985 \\
\hline 17.576 & 96.668 & 2.197 & 26.364 \\
\hline 2.197 & 57.122 & 4.394 & 6.591 \\
\hline 2.197 & 10.985 & 26.364 & 30.758 \\
\hline 2.197 & 52.728 & 107.653 & 2.197 \\
\hline 30.758 & 2.197 & 8.788 & 2.197 \\
\hline 19.773 & 2.197 & 35.152 & 2.197 \\
\hline 8.788 & 17.576 & 2.197 & 8.788 \\
\hline 28.561 & 2.197 & 2.197 & 21.97 \\
\hline 2.197 & 17.576 & 2.197 & 4.394 \\
\hline 2.197 & 15.379 & 2.197 & 63.713 \\
\hline 13.182 & 8.788 & 68.107 & 61.516 \\
\hline 2.197 & 2.197 & 26.364 & 6.591 \\
\hline 2.197 & 2.197 & 2.197 & 2.197 \\
\hline 2.197 & 2.197 & 2.197 & 39.546 \\
\hline 2.197 & 15.379 & 61.516 & 21.97 \\
\hline 8.788 & 74.698 & 2.197 & 2.197 \\
\hline 6.591 & 8.788 & 4.394 & 10.985 \\
\hline 2.197 & 2.197 & 4.394 & 2.197 \\
\hline 81.289 & 4.394 & 4.394 & 21.97 \\
\hline 10.985 & 17.576 & 694.252 & 76.895 \\
\hline 26.364 & 101.062 & 10.985 & 2.197 \\
\hline 6.591 & 325.156 & 2.197 & 4.394 \\
\hline 6.591 & 39.546 & 105.456 & 2.197 \\
\hline 2.197 & 2.197 & 2.197 & 21.97 \\
\hline 2.197 & 19.773 & 2.197 & 2.197 \\
\hline 2.197 & 79.092 & 6.591 & 305.383 \\
\hline 2.197 & 4.394 & 6.591 & 96.668 \\
\hline 2.197 & 10.985 & 19.773 & 17.576 \\
\hline 2.197 & 101.062 & 2.197 & 4.394 \\
\hline 2.197 & 63.713 & 15.379 & 90.077 \\
\hline
\end{tabular}




\begin{tabular}{|c|c|c|c|}
\hline 43.94 & 4.394 & 2.197 & 2.197 \\
\hline 2.197 & 37.349 & 8.788 & 2.197 \\
\hline 8.788 & 6.591 & 6.591 & 13.182 \\
\hline 2.197 & 13.182 & 2.197 & 4.394 \\
\hline 8.788 & 4.394 & 2.197 & 10.985 \\
\hline 4.394 & 59.319 & 2.197 & 13.182 \\
\hline 2.197 & 6.591 & 2.197 & 6.591 \\
\hline 2.197 & 15.379 & 2.197 & 107.653 \\
\hline 10.985 & 2.197 & 6.591 & 4.394 \\
\hline 2.197 & 13.182 & 6.591 & 28.561 \\
\hline 4.394 & 2.197 & 15.379 & 28.561 \\
\hline 2.197 & 17.576 & 79.092 & 15.379 \\
\hline 2.197 & 35.152 & 4.394 & 4.394 \\
\hline 2.197 & 103.259 & 8.788 & 2.197 \\
\hline 6.591 & 13.182 & 8.788 & 123.032 \\
\hline 2.197 & 24.167 & 4.394 & 59.319 \\
\hline 2.197 & 54.925 & 171.366 & 15.379 \\
\hline 2.197 & 107.653 & 50.531 & 2.197 \\
\hline 2.197 & 26.364 & 2.197 & 87.88 \\
\hline 2.197 & 2.197 & 2.197 & 43.94 \\
\hline 4.394 & 10.985 & 6.591 & 6.591 \\
\hline 2.197 & 10.985 & 2.197 & 4.394 \\
\hline 4.394 & 4.394 & 13.182 & 261.443 \\
\hline 2.197 & 21.97 & 186.745 & 8.788 \\
\hline 2.197 & 773.344 & 6.591 & 13.182 \\
\hline 2.197 & 4.394 & 10.985 & 2.197 \\
\hline 2.197 & 59.319 & 2.197 & 65.91 \\
\hline 2.197 & 4.394 & 2.197 & 4.394 \\
\hline 2.197 & 8.788 & 6.591 & 61.516 \\
\hline 2.197 & 19.773 & 2.197 & 6.591 \\
\hline 2.197 & 4.394 & 2036.62 & 4.394 \\
\hline 2.197 & 35.152 & 2.197 & 94.471 \\
\hline 65.91 & 2.197 & 1788.36 & 32.955 \\
\hline 10.985 & 37.349 & 2.197 & 37.349 \\
\hline 50.531 & 6.591 & 2.197 & 6.591 \\
\hline 1509.34 & 61.516 & 2.197 & 10.985 \\
\hline 2.197 & 2.197 & 26.364 & 8.788 \\
\hline 2.197 & 13.182 & 2.197 & 2.197 \\
\hline 8.788 & 2.197 & 2.197 & 2.197 \\
\hline 6.591 & 6.591 & 2.197 & 2.197 \\
\hline 4.394 & 219.7 & 2.197 & 2.197 \\
\hline 2.197 & 13.182 & 41.743 & 193.336 \\
\hline 2.197 & 28.561 & 2.197 & 4.394 \\
\hline 2.197 & 2.197 & 6.591 & 2.197 \\
\hline 8.788 & 6.591 & 10.985 & 4.394 \\
\hline 4.394 & 2.197 & 13.182 & 4.394 \\
\hline 2.197 & 843.648 & 6.591 & 384.475 \\
\hline 90.077 & 17.576 & 2.197 & 6.591 \\
\hline 4.394 & 13.182 & 26.364 & 4.394 \\
\hline 32.955 & 4.394 & 2.197 & 2.197 \\
\hline
\end{tabular}




\begin{tabular}{|c|c|c|c|}
\hline 2.197 & 968.877 & 2.197 & 13.182 \\
\hline 2.197 & 243.867 & 4.394 & 4.394 \\
\hline 8.788 & 2.197 & 2.197 & 28.561 \\
\hline 19.773 & 2.197 & 10.985 & 2.197 \\
\hline 8.788 & 6.591 & 103.259 & 2.197 \\
\hline 2.197 & 4.394 & 4.394 & 41.743 \\
\hline 2.197 & 2.197 & 4.394 & 17.576 \\
\hline 2.197 & 24.167 & 26.364 & 46.137 \\
\hline 24.167 & 155.987 & 2.197 & 39.546 \\
\hline 13.182 & 61.516 & 17.576 & 8.788 \\
\hline 344.929 & 65.91 & 2.197 & 35.152 \\
\hline 15.379 & 59.319 & 6.591 & 2.197 \\
\hline 17.576 & 2.197 & 26.364 & 8.788 \\
\hline 8.788 & 2.197 & 2.197 & 41.743 \\
\hline 6.591 & 4.394 & 2.197 & 2.197 \\
\hline 13.182 & 2.197 & 2.197 & 6.591 \\
\hline 6.591 & 17.576 & 4.394 & 173.563 \\
\hline 24.167 & 2.197 & 2.197 & 46.137 \\
\hline 4.394 & 2.197 & 2.197 & 6.591 \\
\hline 2.197 & 8.788 & 2.197 & 13.182 \\
\hline 2.197 & 10.985 & 17.576 & 28.561 \\
\hline 2.197 & 2.197 & 2.197 & 2.197 \\
\hline 2.197 & 59.319 & 4.394 & 2.197 \\
\hline 2.197 & 2.197 & 2.197 & 24.167 \\
\hline 4.394 & 6.591 & 4.394 & 2.197 \\
\hline 2.197 & 2.197 & 19.773 & 4.394 \\
\hline 2.197 & 2.197 & 17.576 & 2.197 \\
\hline 57.122 & 13.182 & 4.394 & 2.197 \\
\hline 4.394 & 8.788 & 2.197 & 30.758 \\
\hline 2.197 & 2.197 & 4.394 & 4.394 \\
\hline 2.197 & 4.394 & 2.197 & 59.319 \\
\hline 2.197 & 2548.52 & 6.591 & 2.197 \\
\hline 2.197 & 37.349 & 2.197 & 30.758 \\
\hline 4.394 & 4.394 & 2.197 & 24.167 \\
\hline 2.197 & 6.591 & 296.595 & 252.655 \\
\hline 8.788 & 2.197 & 2.197 & 116.441 \\
\hline 15.379 & 8.788 & 6.591 & 8.788 \\
\hline 2.197 & 59.319 & 17.576 & 8.788 \\
\hline 6.591 & 2.197 & 15.379 & 6.591 \\
\hline 2.197 & 8.788 & 2.197 & 6.591 \\
\hline 98.865 & 2.197 & 2.197 & 79.092 \\
\hline 4.394 & 8.788 & 2.197 & 2.197 \\
\hline 2.197 & 2.197 & 26.364 & 15886.5 \\
\hline 8.788 & 13.182 & 6.591 & 30.758 \\
\hline 3310.88 & 74.698 & 101.062 & 10.985 \\
\hline 39.546 & 2.197 & 17.576 & 17.576 \\
\hline 2.197 & 2.197 & 2.197 & 2.197 \\
\hline 2.197 & 2.197 & 2.197 & 65.91 \\
\hline 2.197 & 2.197 & 10.985 & 83.486 \\
\hline 65.91 & 2.197 & 6.591 & 2.197 \\
\hline
\end{tabular}




\begin{tabular}{|c|c|c|c|}
\hline 13.182 & 17.576 & 2.197 & 2.197 \\
\hline 140.608 & 4.394 & 2.197 & 742.586 \\
\hline 70.304 & 30.758 & 2.197 & 4.394 \\
\hline 2.197 & 10.985 & 2.197 & 24.167 \\
\hline 4.394 & 2.197 & 2.197 & 6.591 \\
\hline 32.955 & 2.197 & 2.197 & 191.139 \\
\hline 4.394 & 171.366 & 2.197 & 8.788 \\
\hline 17.576 & 17.576 & 26.364 & 2.197 \\
\hline 19.773 & 19.773 & 2.197 & 21.97 \\
\hline 85.683 & 13.182 & 549.25 & 2.197 \\
\hline 2.197 & 2.197 & 26.364 & 6.591 \\
\hline 13.182 & 92.274 & 2.197 & 50.531 \\
\hline 4.394 & 2.197 & 4.394 & 2.197 \\
\hline 4.394 & 676.676 & 2.197 & 8.788 \\
\hline 13.182 & 13.182 & 2.197 & 87.88 \\
\hline 10.985 & 4.394 & 2.197 & 37.349 \\
\hline 2.197 & 2.197 & 2.197 & 17.576 \\
\hline 2.197 & 217.503 & 2.197 & 2.197 \\
\hline 4.394 & 24.167 & 2.197 & 2.197 \\
\hline 4.394 & 6.591 & 6.591 & 2.197 \\
\hline 2.197 & 10.985 & 2.197 & 2.197 \\
\hline 4.394 & 15.379 & 4.394 & 65.91 \\
\hline 2.197 & 52.728 & 17.576 & 17.576 \\
\hline 2.197 & 6.591 & 21.97 & 39.546 \\
\hline 2.197 & 50.531 & 2.197 & 4.394 \\
\hline 46.137 & 39.546 & 15.379 & 8.788 \\
\hline 2.197 & 4.394 & 24.167 & 938.119 \\
\hline 8.788 & 79.092 & 48.334 & 2.197 \\
\hline 2.197 & 6.591 & 6.591 & 28.561 \\
\hline 2.197 & 2.197 & 2.197 & 2.197 \\
\hline 15.379 & 24.167 & 21.97 & 2.197 \\
\hline 2.197 & 68.107 & 39.546 & 63.713 \\
\hline 68.107 & 8.788 & 2.197 & 553.644 \\
\hline 4.394 & 2.197 & 2.197 & 8.788 \\
\hline 8.788 & 68.107 & 2.197 & 4.394 \\
\hline 21.97 & 54.925 & 2.197 & 2.197 \\
\hline 32.955 & 8.788 & 4.394 & 8.788 \\
\hline 2.197 & 30.758 & 13.182 & 26.364 \\
\hline 10.985 & 4.394 & 4.394 & 103.259 \\
\hline 2.197 & 19.773 & 15.379 & 19.773 \\
\hline 4.394 & 123.032 & 2.197 & 4.394 \\
\hline 2.197 & 94.471 & 949.104 & 4.394 \\
\hline 2.197 & 59.319 & 2.197 & 8.788 \\
\hline 2.197 & 4.394 & 2.197 & 21.97 \\
\hline 2.197 & 35.152 & 35.152 & 15.379 \\
\hline 48.334 & 15.379 & 10.985 & 2.197 \\
\hline 6.591 & 2.197 & 426.218 & 336.141 \\
\hline 15.379 & 13.182 & 2.197 & 2.197 \\
\hline 2.197 & 10.985 & 17.576 & 30.758 \\
\hline 2.197 & 24.167 & 2.197 & 46.137 \\
\hline
\end{tabular}




\begin{tabular}{|c|c|c|c|}
\hline 2.197 & 2.197 & 2.197 & 6.591 \\
\hline 4.394 & 15.379 & 2.197 & 35.152 \\
\hline 2.197 & 182.351 & 2.197 & 2.197 \\
\hline 2.197 & 28.561 & 83.486 & 2.197 \\
\hline 4.394 & 8.788 & 2.197 & 10.985 \\
\hline 6.591 & 2.197 & 2.197 & 116.441 \\
\hline 4.394 & 59.319 & 4.394 & 6.591 \\
\hline 584.402 & 2.197 & 2.197 & 120.835 \\
\hline 15.379 & 50.531 & 2.197 & 81.289 \\
\hline 19.773 & 105.456 & 4.394 & 21.97 \\
\hline 2372.76 & 2.197 & 46.137 & 8.788 \\
\hline 50.531 & 39.546 & 2.197 & 4.394 \\
\hline 19.773 & 68.107 & 2.197 & 2.197 \\
\hline 2.197 & 4.394 & 39.546 & 2.197 \\
\hline 4.394 & 30.758 & 17.576 & 2.197 \\
\hline 2.197 & 30.758 & 2.197 & 2.197 \\
\hline 2.197 & 48.334 & 15.379 & 6.591 \\
\hline 2.197 & 8.788 & 28.561 & 50.531 \\
\hline 32.955 & 6.591 & 85.683 & 54.925 \\
\hline 2.197 & 6.591 & 46.137 & 28.561 \\
\hline 2.197 & 197.73 & 6.591 & 39.546 \\
\hline 2.197 & 2.197 & 13.182 & 424.021 \\
\hline 2.197 & 162.578 & 79.092 & 2.197 \\
\hline 8.788 & 2.197 & 6.591 & 2.197 \\
\hline 2.197 & 21.97 & 10.985 & 83.486 \\
\hline 2.197 & 21.97 & 2.197 & 125.229 \\
\hline 4.394 & 2.197 & 15.379 & 17.576 \\
\hline 2.197 & 28.561 & 1160.02 & 59.319 \\
\hline 2.197 & 6.591 & 2.197 & 2.197 \\
\hline 4.394 & 2.197 & 2.197 & 17.576 \\
\hline 21.97 & 17.576 & 2.197 & 2.197 \\
\hline 169.169 & 4.394 & 8.788 & 2.197 \\
\hline 68.107 & 2.197 & 8.788 & 2.197 \\
\hline 35.152 & 24.167 & 2.197 & 6.591 \\
\hline 8.788 & 35.152 & 6.591 & 2.197 \\
\hline 2.197 & 2.197 & 6.591 & 2.197 \\
\hline 2.197 & 85.683 & 2.197 & 4.394 \\
\hline 698.646 & 6.591 & 2.197 & 112.047 \\
\hline 8.788 & 142.805 & 13.182 & 24.167 \\
\hline 2.197 & 8.788 & 2.197 & 2.197 \\
\hline 2.197 & 43.94 & 2.197 & 4.394 \\
\hline 4.394 & 79.092 & 2.197 & 2.197 \\
\hline 883.194 & 87.88 & 2.197 & 208.715 \\
\hline 4.394 & 2.197 & 4.394 & 283.413 \\
\hline 4.394 & 2.197 & 2.197 & 8.788 \\
\hline 19.773 & 4.394 & 4.394 & 79.092 \\
\hline 2.197 & 15.379 & 2.197 & 8.788 \\
\hline 4.394 & 2.197 & 2.197 & 32.955 \\
\hline 70.304 & 63.713 & 2.197 & 94.471 \\
\hline 2.197 & 2.197 & 2.197 & 17.576 \\
\hline
\end{tabular}




\begin{tabular}{|c|c|c|c|}
\hline 2.197 & 461.37 & 4.394 & 96.668 \\
\hline 13.182 & 384.475 & 70.304 & 6.591 \\
\hline 2.197 & 4.394 & 2.197 & 2.197 \\
\hline 8.788 & 81.289 & 2.197 & 4.394 \\
\hline 8.788 & 2.197 & 2.197 & 2.197 \\
\hline 2.197 & 6.591 & 2.197 & 8.788 \\
\hline 2.197 & 4.394 & 2.197 & 6.591 \\
\hline 6.591 & 4.394 & 2.197 & 13.182 \\
\hline 2.197 & 2.197 & 2.197 & 259.246 \\
\hline 4.394 & 52.728 & 2.197 & 8.788 \\
\hline 2.197 & 4.394 & 10.985 & 2.197 \\
\hline 2.197 & 4.394 & 4.394 & 24.167 \\
\hline 41.743 & 57.122 & 2.197 & 15.379 \\
\hline 2.197 & 2.197 & 4.394 & 2.197 \\
\hline 204.321 & 4.394 & 2.197 & 6.591 \\
\hline 98.865 & 101.062 & 2.197 & 17.576 \\
\hline 2052 & 4.394 & 2.197 & 8.788 \\
\hline 32.955 & 2.197 & 2.197 & 13.182 \\
\hline 2.197 & 294.398 & 8.788 & 2.197 \\
\hline 19.773 & 54.925 & 50.531 & 2.197 \\
\hline 2.197 & 2.197 & 2.197 & 74.698 \\
\hline 2.197 & 8.788 & 2.197 & 2.197 \\
\hline 2.197 & 2.197 & 2.197 & 279.019 \\
\hline 410.839 & 21.97 & 2.197 & 101.062 \\
\hline 24.167 & 4.394 & 24.167 & 158.184 \\
\hline 382.278 & 15.379 & 10.985 & 39.546 \\
\hline 35.152 & 118.638 & 6.591 & 61.516 \\
\hline 2.197 & 2.197 & 8.788 & 2.197 \\
\hline 2.197 & 2.197 & 2.197 & 2.197 \\
\hline 37.349 & 2.197 & 85.683 & 2.197 \\
\hline 24.167 & 21.97 & 2.197 & 4.394 \\
\hline 503.113 & 10.985 & 6.591 & 2.197 \\
\hline 4.394 & 6.591 & 15.379 & 2.197 \\
\hline 39.546 & 17.576 & 8.788 & 6.591 \\
\hline 10.985 & 2.197 & 2.197 & 2.197 \\
\hline 2.197 & 19.773 & 2.197 & 193.336 \\
\hline 6.591 & 112.047 & 19.773 & 2.197 \\
\hline 10.985 & 2.197 & 2.197 & 2.197 \\
\hline 24.167 & 2.197 & 180.154 & 10.985 \\
\hline 169.169 & 2.197 & 2.197 & 19.773 \\
\hline 120.835 & 180.154 & 2.197 & 30.758 \\
\hline 2.197 & 155.987 & 74.698 & 2.197 \\
\hline 4.394 & 2.197 & 15.379 & 17.576 \\
\hline 2.197 & 112.047 & 8.788 & 17.576 \\
\hline 136.214 & 8.788 & 2.197 & 6.591 \\
\hline 2.197 & 17.576 & 76.895 & 15.379 \\
\hline 17.576 & 10.985 & 13.182 & 2.197 \\
\hline 19.773 & 19.773 & 15.379 & 2.197 \\
\hline 2.197 & 2.197 & 8.788 & 2.197 \\
\hline 2.197 & 10.985 & 2.197 & 2.197 \\
\hline
\end{tabular}




\begin{tabular}{|c|c|c|c|}
\hline 2.197 & 4.394 & 2.197 & 15.379 \\
\hline 2.197 & 2.197 & 2.197 & 2.197 \\
\hline 15.379 & 57.122 & 4.394 & 4.394 \\
\hline 6.591 & 2.197 & 43.94 & 17.576 \\
\hline 8.788 & 4.394 & 2.197 & 4.394 \\
\hline 2.197 & 15.379 & 2.197 & 2.197 \\
\hline 300.989 & 219.7 & 8.788 & 4.394 \\
\hline 140.608 & 68.107 & 2.197 & 21.97 \\
\hline 2.197 & 2.197 & 2.197 & 4.394 \\
\hline 13.182 & 2.197 & 107.653 & 4.394 \\
\hline 6.591 & 874.406 & 96.668 & 21.97 \\
\hline 4.394 & 50.531 & 103.259 & 2.197 \\
\hline 35.152 & 2.197 & 4.394 & 10.985 \\
\hline 24.167 & 10.985 & 2.197 & 24.167 \\
\hline 4.394 & 81.289 & 2.197 & 4.394 \\
\hline 4.394 & 39.546 & 2.197 & 2.197 \\
\hline 134.017 & 26.364 & 2.197 & 4.394 \\
\hline 6.591 & 195.533 & 2.197 & 2.197 \\
\hline 2.197 & 30.758 & 8.788 & 2.197 \\
\hline 4.394 & 24.167 & 26.364 & 13.182 \\
\hline 2.197 & 21.97 & 2.197 & 59.319 \\
\hline 6.591 & 2.197 & 15.379 & 13.182 \\
\hline 19.773 & 2.197 & 4.394 & 2.197 \\
\hline 4.394 & 4.394 & 2.197 & 13.182 \\
\hline 4.394 & 68.107 & 2.197 & 2.197 \\
\hline 10.985 & 2.197 & 2.197 & 2.197 \\
\hline 6.591 & 8.788 & 2.197 & 10.985 \\
\hline 2.197 & 259.246 & 2.197 & 13.182 \\
\hline 2.197 & 2.197 & 2.197 & 30.758 \\
\hline 4.394 & 2.197 & 2.197 & 4.394 \\
\hline 39.546 & 6.591 & 2.197 & 8.788 \\
\hline 2.197 & 228.488 & 140.608 & 252.655 \\
\hline 4.394 & 4.394 & 2.197 & 2.197 \\
\hline 2.197 & 760.162 & 2.197 & 8.788 \\
\hline 2.197 & 10.985 & 4.394 & 52.728 \\
\hline 10.985 & 4.394 & 13.182 & 2.197 \\
\hline 4.394 & 30.758 & 4.394 & 2.197 \\
\hline 839.254 & 61.516 & 2.197 & 2.197 \\
\hline 2.197 & 8.788 & 4.394 & 2.197 \\
\hline 4521.43 & 2.197 & 164.775 & 2.197 \\
\hline 4.394 & 2.197 & 21.97 & 41.743 \\
\hline 4.394 & 2.197 & 19.773 & 2.197 \\
\hline 10.985 & 2.197 & 4.394 & 1261.08 \\
\hline 2.197 & 10.985 & 4.394 & 8.788 \\
\hline 43.94 & 4.394 & 28.561 & 10.985 \\
\hline 2.197 & 2.197 & 2.197 & 2.197 \\
\hline 2.197 & 26.364 & 85.683 & 6.591 \\
\hline 65.91 & 32.955 & 2.197 & 19.773 \\
\hline 37.349 & 2.197 & 2.197 & 19.773 \\
\hline 13.182 & 8.788 & 37.349 & 2.197 \\
\hline
\end{tabular}




\begin{tabular}{|c|c|c|c|}
\hline 48.334 & 26.364 & 19.773 & 2.197 \\
\hline 30.758 & 138.411 & 2.197 & 254.852 \\
\hline 2.197 & 19.773 & 4.394 & 13.182 \\
\hline 2.197 & 2.197 & 2.197 & 24.167 \\
\hline 2.197 & 30.758 & 2.197 & 10.985 \\
\hline 4.394 & 2.197 & 10.985 & 19.773 \\
\hline 2.197 & 65.91 & 2.197 & 17.576 \\
\hline 54.925 & 50.531 & 2.197 & 2.197 \\
\hline 265.837 & 2.197 & 4.394 & 19.773 \\
\hline 4.394 & 4.394 & 2.197 & 2.197 \\
\hline 2.197 & 76.895 & 2.197 & 10.985 \\
\hline 419.627 & 13.182 & 2.197 & 224.094 \\
\hline 2.197 & 54.925 & 2.197 & 4.394 \\
\hline 59.319 & 57.122 & 4.394 & 10.985 \\
\hline 17.576 & 63.713 & 4.394 & 107.653 \\
\hline 2.197 & 17.576 & 2.197 & 54.925 \\
\hline 2.197 & 2.197 & 2.197 & 6.591 \\
\hline 15.379 & 2.197 & 2.197 & 4.394 \\
\hline 4.394 & 4.394 & 2.197 & 26.364 \\
\hline 2.197 & 6.591 & 2.197 & 8.788 \\
\hline 4.394 & 21.97 & 2.197 & 188.942 \\
\hline 2.197 & 127.426 & 2.197 & 8.788 \\
\hline 4.394 & 2.197 & 2.197 & 208.715 \\
\hline 120.835 & 4.394 & 21.97 & 35.152 \\
\hline 13.182 & 15.379 & 2.197 & 2.197 \\
\hline 2.197 & 4.394 & 2.197 & 4.394 \\
\hline 57.122 & 2.197 & 8.788 & 6.591 \\
\hline 2.197 & 2.197 & 8.788 & 8.788 \\
\hline 2.197 & 2.197 & 2.197 & 2.197 \\
\hline 2533.14 & 8.788 & 24.167 & 30.758 \\
\hline 2.197 & 6.591 & 37.349 & 2.197 \\
\hline 85.683 & 24.167 & 2.197 & 6.591 \\
\hline 15.379 & 2.197 & 85.683 & 8.788 \\
\hline 4.394 & 50.531 & 2.197 & 2.197 \\
\hline 35.152 & 26.364 & 2.197 & 4.394 \\
\hline 4.394 & 2.197 & 226.291 & 37.349 \\
\hline 15.379 & 39.546 & 4.394 & 8.788 \\
\hline 2.197 & 61.516 & 10.985 & 2.197 \\
\hline 2.197 & 4.394 & 2.197 & 4.394 \\
\hline 2.197 & 2.197 & 2.197 & 6.591 \\
\hline 2.197 & 2.197 & 318.565 & 37.349 \\
\hline 35.152 & 17.576 & 2.197 & 2.197 \\
\hline 4.394 & 43.94 & 57.122 & 39.546 \\
\hline 4.394 & 2.197 & 4.394 & 13.182 \\
\hline 4.394 & 19.773 & 17.576 & 4.394 \\
\hline 8.788 & 6.591 & 2.197 & 48.334 \\
\hline 17.576 & 24.167 & 4.394 & 2.197 \\
\hline 28.561 & 2.197 & 2.197 & 90.077 \\
\hline 10.985 & 4.394 & 2.197 & 2.197 \\
\hline 48.334 & 24.167 & 8.788 & 2.197 \\
\hline
\end{tabular}




\begin{tabular}{|c|c|c|c|}
\hline 6.591 & 155.987 & 8.788 & 2.197 \\
\hline 4.394 & 2.197 & 83.486 & 2.197 \\
\hline 2.197 & 2.197 & 6.591 & 39.546 \\
\hline 2.197 & 4.394 & 24615.2 & 4.394 \\
\hline 2.197 & 6.591 & 2.197 & 259.246 \\
\hline 15.379 & 107.653 & 2.197 & 17.576 \\
\hline 8.788 & 2.197 & 76.895 & 4.394 \\
\hline 81.289 & 30.758 & 8.788 & 129.623 \\
\hline 6.591 & 2.197 & 2.197 & 6.591 \\
\hline 2.197 & 70.304 & 2.197 & 6.591 \\
\hline 4.394 & 4.394 & 8.788 & 19.773 \\
\hline 4.394 & 2.197 & 6.591 & 37.349 \\
\hline 13.182 & 17.576 & 2.197 & 70.304 \\
\hline 4.394 & 8.788 & 2.197 & 15.379 \\
\hline 142.805 & 15.379 & 153.79 & 2.197 \\
\hline 2.197 & 19.773 & 61.516 & 4.394 \\
\hline 2.197 & 10.985 & 2.197 & 384.475 \\
\hline 8.788 & 4.394 & 2.197 & 98.865 \\
\hline 10.985 & 2.197 & 6.591 & 10.985 \\
\hline 2.197 & 2.197 & 13.182 & 81.289 \\
\hline 39.546 & 10.985 & 4.394 & 5907.73 \\
\hline 6.591 & 2.197 & 2.197 & 26.364 \\
\hline 6.591 & 402.051 & 221.897 & 2.197 \\
\hline 4.394 & 2.197 & 19.773 & 68.107 \\
\hline 68.107 & 2.197 & 6.591 & 2.197 \\
\hline 2.197 & 10.985 & 4.394 & 96.668 \\
\hline 2.197 & 2.197 & 13.182 & 4.394 \\
\hline 8.788 & 6.591 & 2.197 & 41.743 \\
\hline 2.197 & 2.197 & 2.197 & 13.182 \\
\hline 74.698 & 2.197 & 2.197 & 2.197 \\
\hline 2.197 & 2.197 & 2.197 & 10.985 \\
\hline 2.197 & 52.728 & 2.197 & 13.182 \\
\hline 2.197 & 259.246 & 2.197 & 24.167 \\
\hline 4.394 & 13.182 & 8.788 & 2.197 \\
\hline 4.394 & 2.197 & 8.788 & 4.394 \\
\hline 28.561 & 2.197 & 24.167 & 17.576 \\
\hline 2.197 & 28.561 & 2.197 & 2.197 \\
\hline 2.197 & 2.197 & 6.591 & 4.394 \\
\hline 2.197 & 2.197 & 4.394 & 10.985 \\
\hline 26.364 & 125.229 & 2.197 & 151.593 \\
\hline 15.379 & 10.985 & 6.591 & 15.379 \\
\hline 8.788 & 4.394 & 2.197 & 10.985 \\
\hline 4.394 & 10.985 & 2.197 & 2.197 \\
\hline 13.182 & 2.197 & 2.197 & 41.743 \\
\hline 6.591 & 8.788 & 48.334 & 2.197 \\
\hline 39.546 & 2.197 & 2.197 & 2.197 \\
\hline 2.197 & 2.197 & 2.197 & 2612.23 \\
\hline 4.394 & 140.608 & 2.197 & 281.216 \\
\hline 2.197 & 48.334 & 2.197 & 4.394 \\
\hline 2.197 & 555.841 & 2.197 & 50.531 \\
\hline
\end{tabular}




\begin{tabular}{|c|c|c|c|}
\hline 2.197 & 109.85 & 2.197 & 2.197 \\
\hline 2.197 & 15.379 & 30.758 & 2.197 \\
\hline 8.788 & 10.985 & 2.197 & 2.197 \\
\hline 4.394 & 2.197 & 10.985 & 2.197 \\
\hline 28.561 & 8.788 & 2.197 & 26.364 \\
\hline 17.576 & 2.197 & 10.985 & 4.394 \\
\hline 2.197 & 10.985 & 59.319 & 101.062 \\
\hline 8.788 & 155.987 & 2.197 & 14937.4 \\
\hline 2.197 & 2.197 & 21.97 & 116.441 \\
\hline 13.182 & 85.683 & 2.197 & 2.197 \\
\hline 19.773 & 17.576 & 2.197 & 8.788 \\
\hline 2.197 & 46.137 & 2.197 & 2.197 \\
\hline 4.394 & 8.788 & 6.591 & 32.955 \\
\hline 4.394 & 2.197 & 32.955 & 8.788 \\
\hline 2.197 & 6.591 & 8.788 & 158.184 \\
\hline 2.197 & 43.94 & 2.197 & 17.576 \\
\hline 30.758 & 61.516 & 4.394 & 2.197 \\
\hline 2.197 & 2.197 & 8.788 & 109.85 \\
\hline 2.197 & 2.197 & 2.197 & 2.197 \\
\hline 2.197 & 2.197 & 2.197 & 32.955 \\
\hline 520.689 & 2.197 & 2.197 & 13.182 \\
\hline 21.97 & 2.197 & 35.152 & 2.197 \\
\hline 8.788 & 6.591 & 2.197 & 6.591 \\
\hline 17.576 & 4.394 & 2.197 & 2.197 \\
\hline 15.379 & 127.426 & 2.197 & 8.788 \\
\hline 19.773 & 2.197 & 15.379 & 2.197 \\
\hline 13.182 & 4.394 & 2.197 & 85.683 \\
\hline 4.394 & 8.788 & 2.197 & 74.698 \\
\hline 2.197 & 2.197 & 2.197 & 4.394 \\
\hline 8.788 & 57.122 & 6.591 & 46.137 \\
\hline 10.985 & 4.394 & 2.197 & 2.197 \\
\hline 21.97 & 2.197 & 109.85 & 478.946 \\
\hline 8.788 & 153.79 & 4.394 & 8.788 \\
\hline 2.197 & 2.197 & 2.197 & 2.197 \\
\hline 2.197 & 151.593 & 4.394 & 79.092 \\
\hline 2.197 & 37.349 & 4.394 & 15.379 \\
\hline 4.394 & 2.197 & 2.197 & 13.182 \\
\hline 13.182 & 2.197 & 4.394 & 4.394 \\
\hline 28.561 & 2.197 & 19.773 & 8.788 \\
\hline 15.379 & 2.197 & 50.531 & 4.394 \\
\hline 6.591 & 4.394 & 15.379 & 79.092 \\
\hline 2.197 & 2.197 & 2.197 & 70.304 \\
\hline 129.623 & 6.591 & 2.197 & 2.197 \\
\hline 4.394 & 59.319 & 2.197 & 2.197 \\
\hline 15.379 & 68.107 & 2.197 & 2.197 \\
\hline 10.985 & 50.531 & 4.394 & 98.865 \\
\hline 32.955 & 2.197 & 79.092 & 13.182 \\
\hline 173.563 & 50.531 & 2.197 & 61.516 \\
\hline 15.379 & 2.197 & 2.197 & 43.94 \\
\hline 4.394 & 2.197 & 2.197 & 4.394 \\
\hline
\end{tabular}




\begin{tabular}{|c|c|c|c|}
\hline 6.591 & 24.167 & 670.085 & 41.743 \\
\hline 2.197 & 17.576 & 13.182 & 43.94 \\
\hline 4.394 & 6.591 & 2.197 & 8.788 \\
\hline 2.197 & 4.394 & 4.394 & 173.563 \\
\hline 2.197 & 81.289 & 2.197 & 8.788 \\
\hline 2.197 & 2.197 & 2.197 & 19.773 \\
\hline 2.197 & 2.197 & 72.501 & 2.197 \\
\hline 2.197 & 2.197 & 24.167 & 10.985 \\
\hline 2.197 & 10.985 & 2.197 & 30.758 \\
\hline 13.182 & 2.197 & 2.197 & 24.167 \\
\hline 2.197 & 17.576 & 2.197 & 590.993 \\
\hline 70.304 & 6.591 & 17.576 & 125.229 \\
\hline 13.182 & 219.7 & 6.591 & 2.197 \\
\hline 2.197 & 10.985 & 2.197 & 4.394 \\
\hline 46.137 & 4.394 & 2.197 & 15.379 \\
\hline 13.182 & 2.197 & 4.394 & 50.531 \\
\hline 120.835 & 13.182 & 2.197 & 8.788 \\
\hline 6.591 & 6.591 & 4.394 & 2.197 \\
\hline 4.394 & 2.197 & 2.197 & 2.197 \\
\hline 2.197 & 2.197 & 2.197 & 204.321 \\
\hline 6.591 & 2.197 & 35.152 & 10.985 \\
\hline 4.394 & 79.092 & 2.197 & 2.197 \\
\hline 6.591 & 57.122 & 35.152 & 2.197 \\
\hline 2.197 & 2.197 & 35.152 & 4.394 \\
\hline 26.364 & 15.379 & 2.197 & 2.197 \\
\hline 4.394 & 37.349 & 98.865 & 2.197 \\
\hline 2.197 & 8.788 & 4.394 & 2.197 \\
\hline 2.197 & 28.561 & 70.304 & 2.197 \\
\hline 2.197 & 24.167 & 4.394 & 35.152 \\
\hline 8.788 & 2.197 & 37.349 & 92.274 \\
\hline 2.197 & 547.053 & 162.578 & 5782.5 \\
\hline 2.197 & 48.334 & 4.394 & 10.985 \\
\hline 2.197 & 2.197 & 13.182 & 2.197 \\
\hline 2.197 & 15.379 & 6.591 & 2.197 \\
\hline 2.197 & 13.182 & 2.197 & 26.364 \\
\hline 2.197 & 105.456 & 2.197 & 4.394 \\
\hline 4.394 & 30.758 & 32.955 & 10.985 \\
\hline 542.659 & 26.364 & 76.895 & 32.955 \\
\hline 753.571 & 24.167 & 2.197 & 3168.07 \\
\hline 6.591 & 2.197 & 41.743 & 103.259 \\
\hline 2.197 & 13.182 & 406.445 & 8.788 \\
\hline 2.197 & 87.88 & 68.107 & 2.197 \\
\hline 28.561 & 6.591 & 19.773 & 4.394 \\
\hline 2.197 & 489.931 & 8.788 & 10.985 \\
\hline 37.349 & 32.955 & 6.591 & 2.197 \\
\hline 4.394 & 1267.67 & 19.773 & 52.728 \\
\hline 2.197 & 2.197 & 6.591 & 15.379 \\
\hline 2.197 & 2.197 & 17.576 & 4.394 \\
\hline 2.197 & 2.197 & 8.788 & 6.591 \\
\hline 24.167 & 120.835 & 101.062 & 21.97 \\
\hline
\end{tabular}




\begin{tabular}{|c|c|c|c|}
\hline 19.773 & 32.955 & 8.788 & 19.773 \\
\hline 21.97 & 782.132 & 2.197 & 15.379 \\
\hline 37.349 & 26.364 & 10.985 & 37.349 \\
\hline 2.197 & 10.985 & 4.394 & 2.197 \\
\hline 4.394 & 8.788 & 6.591 & 2.197 \\
\hline 87.88 & 2.197 & 2.197 & 2.197 \\
\hline 13.182 & 6.591 & 72.501 & 8.788 \\
\hline 2.197 & 21.97 & 6.591 & 2.197 \\
\hline 24.167 & 2.197 & 17.576 & 4.394 \\
\hline 4.394 & 2.197 & 24.167 & 2.197 \\
\hline 4.394 & 13.182 & 37.349 & 57.122 \\
\hline 6.591 & 2.197 & 2.197 & 173.563 \\
\hline 2.197 & 2.197 & 2.197 & 68.107 \\
\hline 4.394 & 2.197 & 4.394 & 39.546 \\
\hline 424.021 & 72.501 & 15.379 & 6.591 \\
\hline 15.379 & 17.576 & 83.486 & 8.788 \\
\hline 10.985 & 764.556 & 2.197 & 28.561 \\
\hline 2.197 & 28.561 & 2.197 & 4.394 \\
\hline 13.182 & 2.197 & 4.394 & 19.773 \\
\hline 39.546 & 290.004 & 2.197 & 52.728 \\
\hline 4.394 & 46.137 & 8.788 & 208.715 \\
\hline 2.197 & 6.591 & 2.197 & 10.985 \\
\hline 4.394 & 70.304 & 2.197 & 2.197 \\
\hline 92.274 & 8.788 & 2.197 & 26.364 \\
\hline 2.197 & 145.002 & 4.394 & 2.197 \\
\hline 24.167 & 2.197 & 8.788 & 4.394 \\
\hline 41.743 & 1454.41 & 2.197 & 8.788 \\
\hline 4.394 & 85.683 & 2.197 & 28.561 \\
\hline 834.86 & 2.197 & 2.197 & 2.197 \\
\hline 46.137 & 6.591 & 2.197 & 4.394 \\
\hline 2.197 & 10.985 & 2.197 & 2.197 \\
\hline 4.394 & 4.394 & 193.336 & 21.97 \\
\hline 13.182 & 2.197 & 6.591 & 50.531 \\
\hline 553.644 & 2.197 & 2.197 & 2.197 \\
\hline 2.197 & 6.591 & 59.319 & 4.394 \\
\hline 6.591 & 39.546 & 531.674 & 13.182 \\
\hline 2.197 & 13.182 & 41.743 & 2.197 \\
\hline 2.197 & 15.379 & 59.319 & 4.394 \\
\hline 2.197 & 10.985 & 865.618 & 2.197 \\
\hline 6.591 & 103.259 & 4.394 & 2.197 \\
\hline 4.394 & 28.561 & 35.152 & 2.197 \\
\hline 15.379 & 2.197 & 4.394 & 2.197 \\
\hline 6.591 & 2.197 & 134.017 & 13.182 \\
\hline 13.182 & 4.394 & 26.364 & 32.955 \\
\hline 2.197 & 17.576 & 2.197 & 4.394 \\
\hline 127.426 & 2.197 & 21.97 & 2.197 \\
\hline 2.197 & 2.197 & 2.197 & 8.788 \\
\hline 250.458 & 57.122 & 4.394 & 4.394 \\
\hline 2.197 & 2.197 & 2.197 & 2.197 \\
\hline 4.394 & 2.197 & 2.197 & 30.758 \\
\hline
\end{tabular}




\begin{tabular}{|c|c|c|c|}
\hline 2.197 & 41.743 & 2.197 & 10.985 \\
\hline 26.364 & 10.985 & 2.197 & 21.97 \\
\hline 325.156 & 8.788 & 2.197 & 13.182 \\
\hline 19.773 & 26.364 & 2.197 & 8.788 \\
\hline 2.197 & 2.197 & 2.197 & 13.182 \\
\hline 8.788 & 74.698 & 2.197 & 2.197 \\
\hline 2.197 & 2.197 & 2.197 & 41.743 \\
\hline 32.955 & 158.184 & 63.713 & 2.197 \\
\hline 2.197 & 4.394 & 6.591 & 6.591 \\
\hline 2.197 & 210.912 & 63.713 & 164.775 \\
\hline 15.379 & 87.88 & 1296.23 & 8.788 \\
\hline 6.591 & 180.154 & 6.591 & 6.591 \\
\hline 9110.96 & 39.546 & 2.197 & 6.591 \\
\hline 26.364 & 10.985 & 2.197 & 2.197 \\
\hline 2.197 & 24.167 & 94.471 & 10.985 \\
\hline 6.591 & 15.379 & 8.788 & 21.97 \\
\hline 2.197 & 28.561 & 13.182 & 2.197 \\
\hline 10.985 & 2.197 & 6.591 & 24.167 \\
\hline 2.197 & 15.379 & 2.197 & 2.197 \\
\hline 2.197 & 2.197 & 21.97 & 6.591 \\
\hline 2.197 & 19.773 & 2.197 & 4.394 \\
\hline 19.773 & 217.503 & 529.477 & 19.773 \\
\hline 6.591 & 2.197 & 17.576 & 4.394 \\
\hline 4.394 & 17.576 & 15.379 & 21.97 \\
\hline 116.441 & 8.788 & 4.394 & 6.591 \\
\hline 94.471 & 24.167 & 408.642 & 41.743 \\
\hline 4.394 & 52.728 & 2.197 & 4.394 \\
\hline 2.197 & 2.197 & 2.197 & 2.197 \\
\hline 2.197 & 285.61 & 8.788 & 19.773 \\
\hline 2.197 & 41.743 & 4.394 & 76.895 \\
\hline 2.197 & 2.197 & 2.197 & 15.379 \\
\hline 226.291 & 92.274 & 505.31 & 138.411 \\
\hline 4.394 & 32.955 & 2.197 & 2.197 \\
\hline 2.197 & 107.653 & 2.197 & 4.394 \\
\hline 70.304 & 68.107 & 2.197 & 270.231 \\
\hline 13.182 & 2.197 & 10.985 & 2.197 \\
\hline 4.394 & 13.182 & 21.97 & 28.561 \\
\hline 114.244 & 228.488 & 2.197 & 6.591 \\
\hline 2.197 & 65.91 & 2.197 & 2.197 \\
\hline 2.197 & 195.533 & 2.197 & 2.197 \\
\hline 2.197 & 2.197 & 15.379 & 28.561 \\
\hline 4.394 & 2.197 & 2.197 & 8.788 \\
\hline 2.197 & 2.197 & 2.197 & 2.197 \\
\hline 90.077 & 76.895 & 15.379 & 81.289 \\
\hline 15.379 & 87.88 & 2.197 & 127.426 \\
\hline 17.576 & 4.394 & 2.197 & 90.077 \\
\hline 8.788 & 103.259 & 6.591 & 4.394 \\
\hline 2.197 & 4.394 & 2.197 & 2.197 \\
\hline 2407.91 & 2.197 & 4.394 & 6.591 \\
\hline 2.197 & 48.334 & 2.197 & 2.197 \\
\hline
\end{tabular}




\begin{tabular}{|c|c|c|c|}
\hline 2.197 & 166.972 & 8.788 & 4.394 \\
\hline 6.591 & 2.197 & 6.591 & 977.665 \\
\hline 6.591 & 52.728 & 2.197 & 17.576 \\
\hline 4.394 & 8.788 & 13.182 & 4.394 \\
\hline 2.197 & 17.576 & 4.394 & 83.486 \\
\hline 4.394 & 48.334 & 54.925 & 6.591 \\
\hline 2.197 & 327.353 & 2.197 & 4.394 \\
\hline 6.591 & 2.197 & 32.955 & 2.197 \\
\hline 2.197 & 13.182 & 63.713 & 21.97 \\
\hline 4.394 & 197.73 & 74.698 & 6.591 \\
\hline 6.591 & 2.197 & 2.197 & 8.788 \\
\hline 454.779 & 26.364 & 204.321 & 2.197 \\
\hline 2.197 & 8.788 & 4.394 & 8.788 \\
\hline 2.197 & 2.197 & 2.197 & 215.306 \\
\hline 2.197 & 2.197 & 2.197 & 4.394 \\
\hline 4.394 & 2.197 & 114.244 & 430.612 \\
\hline 4.394 & 4.394 & 2.197 & 2.197 \\
\hline 922.74 & 8.788 & 8.788 & 2.197 \\
\hline 79.092 & 131.82 & 30.758 & 158.184 \\
\hline 2.197 & 2.197 & 50.531 & 35.152 \\
\hline 4.394 & 4.394 & 4.394 & 2.197 \\
\hline 2.197 & 2.197 & 1854.27 & 17.576 \\
\hline 4.394 & 15.379 & 239.473 & 4.394 \\
\hline 2.197 & 120.835 & 2.197 & 4.394 \\
\hline 37.349 & 48.334 & 32.955 & 13.182 \\
\hline 72.501 & 52.728 & 6.591 & 10.985 \\
\hline 2.197 & 2.197 & 4747.72 & 4.394 \\
\hline 2.197 & 2.197 & 10.985 & 4.394 \\
\hline 4.394 & 6.591 & 2.197 & 4.394 \\
\hline 39.546 & 43.94 & 2.197 & 2.197 \\
\hline 63.713 & 2.197 & 4.394 & 24.167 \\
\hline 2.197 & 19.773 & 4.394 & 10.985 \\
\hline 2.197 & 4.394 & 2.197 & 340.535 \\
\hline 2.197 & 2.197 & 21.97 & 2.197 \\
\hline 8.788 & 2.197 & 4.394 & 48.334 \\
\hline 2.197 & 8.788 & 13.182 & 2.197 \\
\hline 30.758 & 2.197 & 57.122 & 2.197 \\
\hline 8.788 & 50.531 & 4.394 & 2.197 \\
\hline 2.197 & 4.394 & 4.394 & 2.197 \\
\hline 4.394 & 13.182 & 2.197 & 6.591 \\
\hline 4.394 & 8.788 & 2.197 & 8.788 \\
\hline 8.788 & 35.152 & 19.773 & 15.379 \\
\hline 17.576 & 10.985 & 90.077 & 10.985 \\
\hline 8.788 & 30.758 & 2.197 & 26.364 \\
\hline 10.985 & 125.229 & 2.197 & 2.197 \\
\hline 4.394 & 2.197 & 2.197 & 41.743 \\
\hline 2.197 & 2.197 & 4.394 & 8.788 \\
\hline 2.197 & 8.788 & 24.167 & 83.486 \\
\hline 2.197 & 21.97 & 2.197 & 65.91 \\
\hline 2.197 & 43.94 & 2.197 & 57.122 \\
\hline
\end{tabular}




\begin{tabular}{|c|c|c|c|}
\hline 2.197 & 15.379 & 2.197 & 15.379 \\
\hline 35.152 & 79.092 & 2.197 & 37.349 \\
\hline 4.394 & 4.394 & 2.197 & 32.955 \\
\hline 4.394 & 2.197 & 2.197 & 254.852 \\
\hline 6.591 & 4.394 & 26.364 & 26.364 \\
\hline 4.394 & 1004.03 & 46.137 & 2.197 \\
\hline 4.394 & 259.246 & 4.394 & 2.197 \\
\hline 24.167 & 46.137 & 2.197 & 4.394 \\
\hline 39.546 & 2.197 & 4.394 & 2.197 \\
\hline 81.289 & 8.788 & 114.244 & 659.1 \\
\hline 2.197 & 4.394 & 4.394 & 90.077 \\
\hline 2.197 & 4.394 & 2.197 & 2.197 \\
\hline 2.197 & 4.394 & 125.229 & 2.197 \\
\hline 6.591 & 85.683 & 8.788 & 2.197 \\
\hline 87.88 & 6.591 & 2.197 & 4.394 \\
\hline 13.182 & 28.561 & 4.394 & 59.319 \\
\hline 6.591 & 153.79 & 10.985 & 4.394 \\
\hline 2.197 & 19.773 & 2574.88 & 4.394 \\
\hline 2.197 & 48.334 & 6.591 & 2.197 \\
\hline 2.197 & 305.383 & 2.197 & 4.394 \\
\hline 4.394 & 6.591 & 2.197 & 2.197 \\
\hline 8.788 & 2.197 & 2.197 & 6.591 \\
\hline 39.546 & 2.197 & 2.197 & 2.197 \\
\hline 65.91 & 15.379 & 4.394 & 17.576 \\
\hline 4.394 & 8.788 & 10.985 & 19.773 \\
\hline 2.197 & 41.743 & 1463.2 & 2.197 \\
\hline 2.197 & 2.197 & 2.197 & 2.197 \\
\hline 2.197 & 107.653 & 2.197 & 4.394 \\
\hline 2.197 & 4.394 & 15.379 & 17.576 \\
\hline 52.728 & 72.501 & 2.197 & 4.394 \\
\hline 4.394 & 175.76 & 2.197 & 138.411 \\
\hline 6.591 & 329.55 & 32.955 & 4.394 \\
\hline 8.788 & 26.364 & 2.197 & 39.546 \\
\hline 4.394 & 19.773 & 2.197 & 2.197 \\
\hline 43.94 & 13.182 & 4.394 & 4.394 \\
\hline 2.197 & 2.197 & 2.197 & 10.985 \\
\hline 4.394 & 6.591 & 2.197 & 17.576 \\
\hline 10.985 & 41.743 & 4.394 & 52.728 \\
\hline 37.349 & 2.197 & 8.788 & 6.591 \\
\hline 112.047 & 2.197 & 2.197 & 15.379 \\
\hline 6.591 & 2.197 & 6.591 & 4.394 \\
\hline 2.197 & 2.197 & 129.623 & 4.394 \\
\hline 4.394 & 120.835 & 6.591 & 2.197 \\
\hline 13.182 & 619.554 & 2.197 & 101.062 \\
\hline 8.788 & 24.167 & 1546.69 & 4.394 \\
\hline 39.546 & 2.197 & 8.788 & 10.985 \\
\hline 30.758 & 8.788 & 4.394 & 2.197 \\
\hline 10.985 & 4.394 & 35.152 & 2.197 \\
\hline 4.394 & 2.197 & 4.394 & 2.197 \\
\hline 6.591 & 43.94 & 2.197 & 32.955 \\
\hline
\end{tabular}




\begin{tabular}{|c|c|c|c|}
\hline 21.97 & 15.379 & 4.394 & 30.758 \\
\hline 6.591 & 59.319 & 2.197 & 10.985 \\
\hline 2.197 & 72.501 & 52.728 & 2.197 \\
\hline 2.197 & 46.137 & 37.349 & 2.197 \\
\hline 8.788 & 327.353 & 2.197 & 76.895 \\
\hline 37.349 & 10.985 & 2.197 & 35.152 \\
\hline 74.698 & 54.925 & 2.197 & 24.167 \\
\hline 21.97 & 2.197 & 417.43 & 4927.87 \\
\hline 2.197 & 13.182 & 2.197 & 2.197 \\
\hline 101.062 & 4.394 & 2.197 & 2.197 \\
\hline 70.304 & 8.788 & 2.197 & 4.394 \\
\hline 6.591 & 37.349 & 4.394 & 2.197 \\
\hline 4.394 & 37.349 & 2.197 & 2.197 \\
\hline 2.197 & 50.531 & 4.394 & 2.197 \\
\hline 46.137 & 10.985 & 10.985 & 4.394 \\
\hline 2.197 & 10.985 & 6.591 & 2.197 \\
\hline 2.197 & 4.394 & 2.197 & 2.197 \\
\hline 2.197 & 2.197 & 8.788 & 206.518 \\
\hline 2.197 & 2.197 & 13.182 & 2.197 \\
\hline 4.394 & 2.197 & 2.197 & 2.197 \\
\hline 2.197 & 6.591 & 2.197 & 2.197 \\
\hline 2.197 & 37.349 & 4.394 & 2.197 \\
\hline 24.167 & 8.788 & 6.591 & 37.349 \\
\hline 4.394 & 15.379 & 2.197 & 4.394 \\
\hline 17.576 & 2.197 & 2.197 & 2.197 \\
\hline 4.394 & 70.304 & 13.182 & 2.197 \\
\hline 2.197 & 13.182 & 173.563 & 2.197 \\
\hline 2.197 & 13.182 & 8.788 & 46.137 \\
\hline 54.925 & 8.788 & 2.197 & 2.197 \\
\hline \multirow[t]{21}{*}{13.182} & 10.985 & 2.197 & 2.197 \\
\hline & 13.182 & 32.955 & 4.394 \\
\hline & 4.394 & 2.197 & 2.197 \\
\hline & 4.394 & 70.304 & 2.197 \\
\hline & 2.197 & 2.197 & 2.197 \\
\hline & 8.788 & 52.728 & 6.591 \\
\hline & 2.197 & 2.197 & 2.197 \\
\hline & 15.379 & 4.394 & 39.546 \\
\hline & 6.591 & 8.788 & 15.379 \\
\hline & 13.182 & 10.985 & 21.97 \\
\hline & 21.97 & 10.985 & 145.002 \\
\hline & 4.394 & 26.364 & 8.788 \\
\hline & 2.197 & 2.197 & 98.865 \\
\hline & 2.197 & 2.197 & 2.197 \\
\hline & 37.349 & 43.94 & 138.411 \\
\hline & 35.152 & 4.394 & 46.137 \\
\hline & 2.197 & 6.591 & 4.394 \\
\hline & 6.591 & 4.394 & 265.837 \\
\hline & 268.034 & 4.394 & 17.576 \\
\hline & 4.394 & 24.167 & 8.788 \\
\hline & 2.197 & 2.197 & 48.334 \\
\hline
\end{tabular}




\begin{tabular}{|c|c|c|}
\hline 17.576 & 2.197 & 8.788 \\
\hline 63.713 & 6.591 & 2.197 \\
\hline 46.137 & 2.197 & 8.788 \\
\hline 2.197 & 57.122 & 2.197 \\
\hline 2.197 & 30.758 & 2.197 \\
\hline 2.197 & 2.197 & 4.394 \\
\hline 2.197 & 59.319 & 6.591 \\
\hline 2.197 & 118.638 & 4.394 \\
\hline 21.97 & 166.972 & 2.197 \\
\hline 331.747 & 46.137 & 2.197 \\
\hline 2.197 & 195.533 & 65.91 \\
\hline 217.503 & 2.197 & 6.591 \\
\hline 298.792 & 68.107 & 10.985 \\
\hline 24.167 & 2.197 & 2.197 \\
\hline 17.576 & 2.197 & 81.289 \\
\hline 2.197 & 131.82 & 2.197 \\
\hline 24.167 & 2.197 & 70.304 \\
\hline 10.985 & 2.197 & 2.197 \\
\hline 6.591 & 2.197 & 4.394 \\
\hline 6.591 & 2.197 & 2.197 \\
\hline 37.349 & 2.197 & 10.985 \\
\hline 155.987 & 81.289 & 6.591 \\
\hline 48.334 & 2.197 & 8.788 \\
\hline 4.394 & 4.394 & 2.197 \\
\hline 8.788 & 6.591 & 2.197 \\
\hline 142.805 & 4.394 & 6.591 \\
\hline 17.576 & 4.394 & 26.364 \\
\hline 13.182 & 30.758 & 70.304 \\
\hline 2.197 & 241.67 & 6.591 \\
\hline 21.97 & 10.985 & 4.394 \\
\hline 2.197 & 15.379 & 43.94 \\
\hline 6.591 & 2.197 & 2.197 \\
\hline 2.197 & 21.97 & 2.197 \\
\hline 4.394 & 2.197 & 4.394 \\
\hline 2.197 & 2.197 & 6.591 \\
\hline 30.758 & 2.197 & 2.197 \\
\hline 32.955 & 8.788 & 2.197 \\
\hline 2.197 & 41.743 & 15.379 \\
\hline 79.092 & 10.985 & 2.197 \\
\hline 134.017 & 136.214 & 57.122 \\
\hline 26.364 & 54.925 & 261.443 \\
\hline 6.591 & 13.182 & 30.758 \\
\hline 24.167 & 19.773 & 2.197 \\
\hline 94.471 & 98.865 & 30.758 \\
\hline 4.394 & 19.773 & 8.788 \\
\hline 300.989 & 10.985 & 105.456 \\
\hline 13.182 & 13.182 & 17.576 \\
\hline 2.197 & 2.197 & 2.197 \\
\hline 2.197 & 17.576 & 2.197 \\
\hline 4.394 & 94.471 & 17.576 \\
\hline
\end{tabular}




\begin{tabular}{|c|c|c|}
\hline 2.197 & 2.197 & 2.197 \\
\hline 13.182 & 2.197 & 6.591 \\
\hline 4.394 & 2.197 & 4.394 \\
\hline 21.97 & 6.591 & 2.197 \\
\hline 4.394 & 195.533 & 2.197 \\
\hline 162.578 & 2471.62 & 4.394 \\
\hline 21.97 & 3036.25 & 4.394 \\
\hline 70.304 & 4.394 & 2.197 \\
\hline 6.591 & 2.197 & 28.561 \\
\hline 6.591 & 35.152 & 4.394 \\
\hline 13.182 & 2.197 & 2.197 \\
\hline 4.394 & 6.591 & 2.197 \\
\hline 50.531 & 2.197 & 2.197 \\
\hline 6.591 & 2.197 & 19.773 \\
\hline 39.546 & 2.197 & 28.561 \\
\hline 63.713 & 672.282 & 2.197 \\
\hline 322.959 & 109.85 & 6.591 \\
\hline 24.167 & 2.197 & 276.822 \\
\hline 63.713 & 2.197 & 13.182 \\
\hline 123.032 & 2.197 & 2.197 \\
\hline 6.591 & 4.394 & 2.197 \\
\hline 2.197 & 4.394 & 4.394 \\
\hline 2.197 & 2.197 & 2.197 \\
\hline 35.152 & 4.394 & 32.955 \\
\hline 37.349 & 37.349 & 2.197 \\
\hline 2.197 & 2.197 & 26.364 \\
\hline 61.516 & 151.593 & 2.197 \\
\hline 28.561 & 26.364 & 8.788 \\
\hline 26.364 & 46.137 & 37.349 \\
\hline 10.985 & 2.197 & 2.197 \\
\hline 2.197 & 8.788 & 123.032 \\
\hline 8.788 & 2.197 & 2.197 \\
\hline 17.576 & 32.955 & 8.788 \\
\hline 4.394 & 8.788 & 2.197 \\
\hline 8.788 & 4.394 & 2.197 \\
\hline 2.197 & 2.197 & 37.349 \\
\hline 2.197 & 2.197 & 90.077 \\
\hline 30.758 & 2.197 & 186.745 \\
\hline 4.394 & 103.259 & 2.197 \\
\hline 2.197 & 2.197 & 15.379 \\
\hline 2.197 & 2.197 & 177.957 \\
\hline 336.141 & 2.197 & 4.394 \\
\hline 39.546 & 2.197 & 13.182 \\
\hline 8.788 & 17.576 & 2.197 \\
\hline 28.561 & 28.561 & 28.561 \\
\hline 2.197 & 10.985 & 35.152 \\
\hline 127.426 & 8.788 & 2.197 \\
\hline 4.394 & 2.197 & 2.197 \\
\hline 2.197 & 2.197 & 2.197 \\
\hline 2.197 & 6.591 & 15.379 \\
\hline
\end{tabular}




\begin{tabular}{|c|c|c|}
\hline 61.516 & 54.925 & 15.379 \\
\hline 68.107 & 8.788 & 6.591 \\
\hline 28.561 & 17.576 & 123.032 \\
\hline 2.197 & 10.985 & 39.546 \\
\hline 2.197 & 4.394 & 2.197 \\
\hline 2.197 & 4.394 & 70.304 \\
\hline 4.394 & 2.197 & 120.835 \\
\hline 48.334 & 2.197 & 525.083 \\
\hline 6.591 & 4.394 & 19.773 \\
\hline 48.334 & 2.197 & 8.788 \\
\hline 2.197 & 2.197 & 4.394 \\
\hline 8.788 & 81.289 & 4.394 \\
\hline 2.197 & 4.394 & 246.064 \\
\hline 2.197 & 2.197 & 19.773 \\
\hline 2.197 & 2.197 & 2.197 \\
\hline 2.197 & 6.591 & 2.197 \\
\hline 4.394 & 4.394 & 2.197 \\
\hline 6.591 & 4.394 & 10.985 \\
\hline 15.379 & 2.197 & 2.197 \\
\hline 4.394 & 2.197 & 41.743 \\
\hline 4.394 & 2.197 & 2.197 \\
\hline 2.197 & 4.394 & 17.576 \\
\hline 2.197 & 26.364 & 13.182 \\
\hline 35.152 & 2.197 & 50.531 \\
\hline 2.197 & 4.394 & 13.182 \\
\hline 50.531 & 2.197 & 2.197 \\
\hline 63.713 & 4.394 & 2.197 \\
\hline 61.516 & 2.197 & 4.394 \\
\hline 10.985 & 35.152 & 6.591 \\
\hline 26.364 & 2.197 & 72.501 \\
\hline 13.182 & 6.591 & 2.197 \\
\hline 2.197 & 2.197 & 4.394 \\
\hline 6.591 & 2.197 & 6.591 \\
\hline 2.197 & 4.394 & 2.197 \\
\hline 6.591 & 142.805 & 48.334 \\
\hline 32.955 & 10.985 & 2.197 \\
\hline 30.758 & 4.394 & 68.107 \\
\hline 10.985 & 2.197 & 114.244 \\
\hline 6.591 & 37.349 & 105.456 \\
\hline 4.394 & 2.197 & 125.229 \\
\hline 10.985 & 28.561 & 37.349 \\
\hline 17.576 & 2.197 & 4.394 \\
\hline 8.788 & 2.197 & 37.349 \\
\hline 30.758 & 134.017 & 2.197 \\
\hline 15.379 & 68.107 & 59.319 \\
\hline 136.214 & 2.197 & 70.304 \\
\hline 10.985 & 21.97 & 2.197 \\
\hline 19.773 & 2.197 & 15.379 \\
\hline 149.396 & 514.098 & 37.349 \\
\hline 92.274 & 2.197 & 3513 \\
\hline
\end{tabular}




\begin{tabular}{|c|c|c|}
\hline 2.197 & 21.97 & 2.197 \\
\hline 166.972 & 30.758 & 2.197 \\
\hline 17.576 & 2.197 & 131.82 \\
\hline 2.197 & 65.91 & 13.182 \\
\hline 2.197 & 2.197 & 6.591 \\
\hline 2.197 & 4.394 & 2.197 \\
\hline 2.197 & 2.197 & 180.154 \\
\hline 977.665 & 4.394 & 164.775 \\
\hline 2.197 & 195.533 & 114.244 \\
\hline 2.197 & 59.319 & 2.197 \\
\hline 13.182 & 6.591 & 2227.76 \\
\hline 4.394 & 2.197 & 182.351 \\
\hline 2.197 & 8.788 & 46.137 \\
\hline 2.197 & 35.152 & 54.925 \\
\hline 2.197 & 4.394 & 15.379 \\
\hline 2.197 & 4.394 & 2.197 \\
\hline 13.182 & 2.197 & 4.394 \\
\hline 279.019 & 17.576 & 39.546 \\
\hline 21.97 & 2.197 & 2.197 \\
\hline 8.788 & 4.394 & 70011.8 \\
\hline 2.197 & 21.97 & 6.591 \\
\hline 2.197 & 6.591 & 52.728 \\
\hline 8.788 & 19.773 & 2.197 \\
\hline 2.197 & 2.197 & 2.197 \\
\hline 2.197 & 4.394 & 8.788 \\
\hline 2.197 & 2.197 & 6.591 \\
\hline 2.197 & 28.561 & 4.394 \\
\hline 83.486 & 13.182 & 8.788 \\
\hline 6.591 & 2.197 & 43.94 \\
\hline 2.197 & 2.197 & 13.182 \\
\hline 2.197 & 6.591 & 6.591 \\
\hline 41.743 & 2.197 & 4.394 \\
\hline 30.758 & 2.197 & 13.182 \\
\hline 87.88 & 2.197 & 39.546 \\
\hline 6.591 & 39.546 & 2.197 \\
\hline 2.197 & 4.394 & 2.197 \\
\hline 2.197 & 2.197 & 52.728 \\
\hline 4.394 & 142.805 & 2.197 \\
\hline 2.197 & 2.197 & 2.197 \\
\hline 2.197 & 8.788 & 2.197 \\
\hline 2.197 & 2.197 & 2.197 \\
\hline 2.197 & 10.985 & 2.197 \\
\hline 21.97 & 2.197 & 6.591 \\
\hline 2.197 & 4.394 & 2.197 \\
\hline 4.394 & 24.167 & 2.197 \\
\hline 2.197 & 15.379 & 6.591 \\
\hline 4.394 & 171.366 & 2.197 \\
\hline 17.576 & 2.197 & 6.591 \\
\hline 43.94 & 4.394 & 10.985 \\
\hline 32.955 & 17.576 & 17.576 \\
\hline
\end{tabular}




\begin{tabular}{|c|c|c|}
\hline 96.668 & 15.379 & 65.91 \\
\hline 4.394 & 6.591 & 193.336 \\
\hline 2.197 & 90.077 & 2.197 \\
\hline 2.197 & 4.394 & 46.137 \\
\hline 17.576 & 136.214 & 24.167 \\
\hline 2.197 & 2.197 & 2.197 \\
\hline 2.197 & 2.197 & 10.985 \\
\hline 2.197 & 2.197 & 4.394 \\
\hline 6.591 & 4.394 & 17.576 \\
\hline 94.471 & 880.997 & 2.197 \\
\hline 4.394 & 4.394 & 17.576 \\
\hline 2.197 & 10.985 & 2.197 \\
\hline 37.349 & 2.197 & 2.197 \\
\hline 2.197 & 32.955 & 21.97 \\
\hline 83.486 & 21.97 & 13.182 \\
\hline 13.182 & 2.197 & 8.788 \\
\hline 4.394 & 2.197 & 2.197 \\
\hline 298.792 & 6.591 & 4.394 \\
\hline 4.394 & 2.197 & 186.745 \\
\hline 19.773 & 127.426 & 2.197 \\
\hline 2.197 & 13.182 & 8.788 \\
\hline 32.955 & 2.197 & 4.394 \\
\hline 98.865 & 2.197 & 41.743 \\
\hline 52.728 & 2.197 & 10.985 \\
\hline 197.73 & 2.197 & 15.379 \\
\hline 63.713 & 2.197 & 10.985 \\
\hline 24.167 & 2.197 & 48.334 \\
\hline 43.94 & 74.698 & 2.197 \\
\hline 21.97 & 63.713 & 48.334 \\
\hline 30.758 & 8.788 & 6.591 \\
\hline 4.394 & 2.197 & 10.985 \\
\hline 109.85 & 351.52 & 37.349 \\
\hline 68.107 & 2.197 & 6.591 \\
\hline 4.394 & 30.758 & 131.82 \\
\hline 2.197 & 8.788 & 10.985 \\
\hline 4.394 & 353.717 & 65.91 \\
\hline 2.197 & 57.122 & 19.773 \\
\hline 8.788 & 50.531 & 2.197 \\
\hline 26.364 & 6.591 & 2.197 \\
\hline 39.546 & 46.137 & 26.364 \\
\hline 13.182 & 8.788 & 37.349 \\
\hline 30.758 & 15.379 & 48.334 \\
\hline 39.546 & 2.197 & 4971.81 \\
\hline 2.197 & 4.394 & 4.394 \\
\hline 8.788 & 17.576 & 13.182 \\
\hline 2.197 & 123.032 & 10.985 \\
\hline 2.197 & 8.788 & 8.788 \\
\hline 4.394 & 10.985 & 48.334 \\
\hline 2.197 & 21.97 & 13.182 \\
\hline 21.97 & 107.653 & 252.655 \\
\hline
\end{tabular}




\begin{tabular}{|c|c|c|}
\hline 61.516 & 2.197 & 145.002 \\
\hline 30.758 & 2.197 & 2.197 \\
\hline 6.591 & 28.561 & 10.985 \\
\hline 145.002 & 2.197 & 2.197 \\
\hline 441.597 & 15.379 & 81.289 \\
\hline 2.197 & 2.197 & 544.856 \\
\hline 2.197 & 2.197 & 70.304 \\
\hline 2.197 & 180.154 & 4.394 \\
\hline 13.182 & 15.379 & 19.773 \\
\hline 2.197 & 2.197 & 2.197 \\
\hline 4.394 & 2.197 & 2.197 \\
\hline 8.788 & 28.561 & 2.197 \\
\hline 26.364 & 2.197 & 2.197 \\
\hline 13.182 & 10.985 & 90.077 \\
\hline 21.97 & 2.197 & 136.214 \\
\hline 2.197 & 6.591 & 21.97 \\
\hline 24.167 & 2.197 & 4.394 \\
\hline 2.197 & 10.985 & 2.197 \\
\hline 6.591 & 2.197 & 10.985 \\
\hline 19.773 & 2.197 & 2.197 \\
\hline 2.197 & 41.743 & 2.197 \\
\hline 21.97 & 2.197 & 15.379 \\
\hline 4.394 & 2.197 & 2.197 \\
\hline 6.591 & 4.394 & 4949.84 \\
\hline 2.197 & 41.743 & 10.985 \\
\hline 8.788 & 2.197 & 4.394 \\
\hline 87.88 & 2.197 & 2.197 \\
\hline 4.394 & 6.591 & 10.985 \\
\hline 2.197 & 2.197 & 8.788 \\
\hline 2.197 & 4.394 & 43.94 \\
\hline 59.319 & 4.394 & 2.197 \\
\hline 4.394 & 26.364 & 19.773 \\
\hline 6.591 & 2.197 & 61.516 \\
\hline 2.197 & 4.394 & 13.182 \\
\hline 2.197 & 164.775 & 6.591 \\
\hline 96.668 & 101.062 & 4.394 \\
\hline 2.197 & 37.349 & 4.394 \\
\hline 2.197 & 2.197 & 2.197 \\
\hline 2.197 & 8.788 & 13.182 \\
\hline 28.561 & 2.197 & 37.349 \\
\hline 15.379 & 17.576 & 28.561 \\
\hline 2.197 & 8.788 & 2.197 \\
\hline 57.122 & 26.364 & 6.591 \\
\hline 4.394 & 37.349 & 68.107 \\
\hline 54.925 & 2.197 & 59.319 \\
\hline 17.576 & 2.197 & 13.182 \\
\hline 2.197 & 4.394 & 32.955 \\
\hline 24.167 & 4.394 & 72.501 \\
\hline 10.985 & 199.927 & 10.985 \\
\hline 50.531 & 4.394 & 4.394 \\
\hline
\end{tabular}




\begin{tabular}{|c|c|c|}
\hline 2.197 & 2.197 & 4.394 \\
\hline 118.638 & 13.182 & 52.728 \\
\hline 8.788 & 15.379 & 2.197 \\
\hline 4.394 & 35.152 & 6.591 \\
\hline 2.197 & 54.925 & 2.197 \\
\hline 48.334 & 2.197 & 6.591 \\
\hline 70.304 & 151.593 & 8.788 \\
\hline 61.516 & 2.197 & 2.197 \\
\hline 10.985 & 2.197 & 2.197 \\
\hline 6.591 & 8.788 & 120.835 \\
\hline 30.758 & 2.197 & 235.079 \\
\hline 2.197 & 2.197 & 8.788 \\
\hline 2.197 & 2.197 & 13.182 \\
\hline 21.97 & 2.197 & 2.197 \\
\hline 2.197 & 6.591 & 2.197 \\
\hline 10.985 & 2.197 & 230.685 \\
\hline 37.349 & 4.394 & 4.394 \\
\hline 63.713 & 10.985 & 28.561 \\
\hline 10.985 & 2.197 & 228.488 \\
\hline 580.008 & 2.197 & 4.394 \\
\hline 182.351 & 654.706 & 2.197 \\
\hline 24.167 & 21.97 & 35.152 \\
\hline 4.394 & 4.394 & 24.167 \\
\hline 19.773 & 8.788 & 10.985 \\
\hline 2.197 & 10.985 & 6.591 \\
\hline 2.197 & 90.077 & 2.197 \\
\hline 2.197 & 2.197 & 2.197 \\
\hline 2.197 & 2.197 & 226.291 \\
\hline 32.955 & 2.197 & 105.456 \\
\hline 61.516 & 10.985 & 17.576 \\
\hline 65.91 & 2.197 & 17.576 \\
\hline 8.788 & 4.394 & 26.364 \\
\hline 623.948 & 2.197 & 6.591 \\
\hline 19.773 & 2.197 & 4.394 \\
\hline 4.394 & 2.197 & 72.501 \\
\hline 8.788 & 10.985 & 8.788 \\
\hline 6.591 & 19.773 & 4.394 \\
\hline 19.773 & 2.197 & 57.122 \\
\hline 13.182 & 15.379 & 483.34 \\
\hline 8.788 & 2.197 & 2.197 \\
\hline 204.321 & 98.865 & 39.546 \\
\hline 50.531 & 96.668 & 2.197 \\
\hline 4.394 & 2.197 & 2.197 \\
\hline 2.197 & 50.531 & 4.394 \\
\hline 2.197 & 2.197 & 2.197 \\
\hline 2.197 & 2.197 & 8.788 \\
\hline 2.197 & 4.394 & 4.394 \\
\hline 4.394 & 2.197 & 17.576 \\
\hline 32.955 & 2.197 & 2.197 \\
\hline 19.773 & 2.197 & 2.197 \\
\hline
\end{tabular}




\begin{tabular}{|c|c|c|}
\hline 24.167 & 41.743 & 4.394 \\
\hline 59.319 & 4.394 & 2.197 \\
\hline 10.985 & 2.197 & 46.137 \\
\hline 13.182 & 48.334 & 26.364 \\
\hline 4.394 & 4.394 & 2.197 \\
\hline 8.788 & 8.788 & 2.197 \\
\hline 46.137 & 94.471 & 4.394 \\
\hline 4.394 & 2.197 & 169.169 \\
\hline 456.976 & 2.197 & 15.379 \\
\hline 76.895 & 2.197 & 2.197 \\
\hline 21.97 & 4.394 & 8.788 \\
\hline 193.336 & 2.197 & 10.985 \\
\hline 2.197 & 2.197 & 8.788 \\
\hline 41.743 & 127.426 & 6.591 \\
\hline 4.394 & 2.197 & 90.077 \\
\hline 8.788 & 2.197 & 21.97 \\
\hline 4.394 & 2.197 & 50.531 \\
\hline 4.394 & 2.197 & 2.197 \\
\hline 2.197 & 2.197 & 76.895 \\
\hline 2.197 & 74.698 & 10.985 \\
\hline 2.197 & 2.197 & 15.379 \\
\hline 26.364 & 4.394 & 2.197 \\
\hline 391.066 & 21.97 & 2.197 \\
\hline 208.715 & 2.197 & 17.576 \\
\hline 8.788 & 4.394 & 39.546 \\
\hline 4.394 & 2.197 & 10.985 \\
\hline 10.985 & 4.394 & 2.197 \\
\hline 2.197 & 2.197 & 19.773 \\
\hline 26.364 & 4.394 & 28.561 \\
\hline 21.97 & 57.122 & 19.773 \\
\hline 2.197 & 2.197 & 4.394 \\
\hline 37.349 & 2.197 & 26.364 \\
\hline 83.486 & 15.379 & 2.197 \\
\hline 2.197 & 17.576 & 26.364 \\
\hline 166.972 & 57.122 & 8.788 \\
\hline 287.807 & 30.758 & 2.197 \\
\hline 8.788 & 2.197 & 430.612 \\
\hline 8.788 & 63.713 & 2.197 \\
\hline 105.456 & 138.411 & 2.197 \\
\hline 28.561 & 158.184 & 8.788 \\
\hline 68.107 & 52.728 & 63.713 \\
\hline 2.197 & 2.197 & 15.379 \\
\hline 6.591 & 63.713 & 2.197 \\
\hline 2.197 & 4.394 & 2.197 \\
\hline 2.197 & 13.182 & 4.394 \\
\hline 2.197 & 17.576 & 21.97 \\
\hline 2.197 & 35.152 & 2.197 \\
\hline 4.394 & 509.704 & 4.394 \\
\hline 81.289 & 19.773 & 331.747 \\
\hline 2.197 & 118.638 & 4.394 \\
\hline
\end{tabular}




\begin{tabular}{|c|c|c|}
\hline 10.985 & 43.94 & 6.591 \\
\hline 2.197 & 2.197 & 6.591 \\
\hline 28.561 & 2.197 & 107.653 \\
\hline 8.788 & 6.591 & 81.289 \\
\hline 17.576 & 4.394 & 8.788 \\
\hline 112.047 & 6.591 & 10.985 \\
\hline 28.561 & 2.197 & 155.987 \\
\hline 4.394 & 8.788 & 2.197 \\
\hline 2.197 & 10.985 & 4.394 \\
\hline 2.197 & 17.576 & 2.197 \\
\hline 10.985 & 2.197 & 15.379 \\
\hline 2.197 & 4.394 & 564.629 \\
\hline 2.197 & 21.97 & 24.167 \\
\hline 2.197 & 39.546 & 37.349 \\
\hline 54.925 & 2.197 & 2.197 \\
\hline 2.197 & 125.229 & 19.773 \\
\hline 4.394 & 10.985 & 2.197 \\
\hline 10.985 & 74.698 & 2.197 \\
\hline 10.985 & 8.788 & 24.167 \\
\hline 4.394 & 2.197 & 98.865 \\
\hline 4.394 & 21.97 & 2.197 \\
\hline 2.197 & 6.591 & 8.788 \\
\hline 2.197 & 8.788 & 4.394 \\
\hline 19.773 & 2.197 & 8.788 \\
\hline 28.561 & 19.773 & 21.97 \\
\hline 2.197 & 8.788 & 2.197 \\
\hline 13.182 & 2.197 & 17.576 \\
\hline 52.728 & 2.197 & 4.394 \\
\hline 87.88 & 57.122 & 8.788 \\
\hline 70.304 & 2.197 & 2.197 \\
\hline 2.197 & 2.197 & 169.169 \\
\hline 2.197 & 4.394 & 2.197 \\
\hline 90.077 & 6.591 & 43.94 \\
\hline 39.546 & 2.197 & 149.396 \\
\hline 13.182 & 197.73 & 2.197 \\
\hline 2.197 & 6.591 & 57.122 \\
\hline 2.197 & 2.197 & 2.197 \\
\hline 2.197 & 2.197 & 2.197 \\
\hline 6.591 & 2.197 & 10.985 \\
\hline 2.197 & 243.867 & 2.197 \\
\hline 4.394 & 2.197 & 2.197 \\
\hline 2.197 & 6.591 & 4.394 \\
\hline 2.197 & 6.591 & 2.197 \\
\hline 2.197 & 2.197 & 2.197 \\
\hline 74.698 & 4.394 & 2.197 \\
\hline 72.501 & 4.394 & 30.758 \\
\hline 2.197 & 2.197 & 665.691 \\
\hline 6.591 & 2.197 & 2.197 \\
\hline 2.197 & 30.758 & 43.94 \\
\hline 35.152 & 2.197 & 6.591 \\
\hline
\end{tabular}




\begin{tabular}{|c|c|c|}
\hline 15.379 & 2.197 & 2.197 \\
\hline 37.349 & 43.94 & 2.197 \\
\hline 4.394 & 74.698 & 2.197 \\
\hline 2.197 & 8.788 & 2.197 \\
\hline 8.788 & 4.394 & 30.758 \\
\hline 2.197 & 4.394 & 4.394 \\
\hline 2.197 & 15.379 & 2.197 \\
\hline 6.591 & 4.394 & 2.197 \\
\hline 59.319 & 4.394 & 2.197 \\
\hline 109.85 & 4.394 & 13.182 \\
\hline 15.379 & 13.182 & 103.259 \\
\hline 8.788 & 15.379 & 43.94 \\
\hline 21.97 & 37.349 & 2.197 \\
\hline 13.182 & 2.197 & 39.546 \\
\hline 2.197 & 2.197 & 2.197 \\
\hline 2.197 & 2.197 & 41.743 \\
\hline 8.788 & 2.197 & 13.182 \\
\hline 4.394 & 197.73 & 4.394 \\
\hline 57.122 & 276.822 & 13.182 \\
\hline 2.197 & 26.364 & 21.97 \\
\hline 3954.6 & 2.197 & 65.91 \\
\hline 52.728 & 10.985 & 2.197 \\
\hline 4.394 & 41.743 & 10.985 \\
\hline 2.197 & 6.591 & 24.167 \\
\hline 13.182 & 155.987 & 2.197 \\
\hline 2.197 & 39.546 & 4.394 \\
\hline 2.197 & 72.501 & 243.867 \\
\hline 2.197 & 2.197 & 4.394 \\
\hline 21.97 & 21.97 & 83.486 \\
\hline 2.197 & 2.197 & 15.379 \\
\hline 4.394 & 169.169 & 298.792 \\
\hline 2.197 & 2.197 & 4.394 \\
\hline 204.321 & 2.197 & 147.199 \\
\hline 4.394 & 2.197 & 331.747 \\
\hline 10.985 & 32.955 & 57.122 \\
\hline 4.394 & 1533.51 & 8.788 \\
\hline 2.197 & 6.591 & 4.394 \\
\hline 70.304 & 6.591 & 32.955 \\
\hline 2.197 & 2.197 & 127.426 \\
\hline 6.591 & 19.773 & 2.197 \\
\hline 4.394 & 35.152 & 2.197 \\
\hline 10.985 & 184.548 & 10.985 \\
\hline 2.197 & 43.94 & 5991.22 \\
\hline 2.197 & 2.197 & 4.394 \\
\hline 13.182 & 6.591 & 8.788 \\
\hline 13.182 & 2.197 & 21.97 \\
\hline 21.97 & 4.394 & 5017.95 \\
\hline 2.197 & 4.394 & 2.197 \\
\hline 19.773 & 4.394 & 24.167 \\
\hline 15.379 & 2.197 & 24.167 \\
\hline
\end{tabular}




\begin{tabular}{|c|c|c|}
\hline 4.394 & 15.379 & 35.152 \\
\hline 2.197 & 2.197 & 94.471 \\
\hline 2.197 & 4.394 & 2.197 \\
\hline 2.197 & 2.197 & 61.516 \\
\hline 6.591 & 4.394 & 28.561 \\
\hline 2.197 & 2.197 & 2.197 \\
\hline 2.197 & 4.394 & 2.197 \\
\hline 2.197 & 2.197 & 4.394 \\
\hline 2.197 & 2.197 & 164.775 \\
\hline 15.379 & 4.394 & 92.274 \\
\hline 35.152 & 10.985 & 2126.7 \\
\hline 13.182 & 4.394 & 138.411 \\
\hline 4.394 & 2.197 & 2.197 \\
\hline 43.94 & 19.773 & 30.758 \\
\hline 6.591 & 1056.76 & 76.895 \\
\hline 19.773 & 4.394 & 81.289 \\
\hline 188.942 & 59.319 & 2.197 \\
\hline 13.182 & 2.197 & 8.788 \\
\hline 2.197 & 15.379 & 2.197 \\
\hline 19.773 & 2.197 & 8.788 \\
\hline 15.379 & 2.197 & 2.197 \\
\hline 2.197 & 6.591 & 35.152 \\
\hline 15.379 & 2.197 & 94.471 \\
\hline 4.394 & 2.197 & 4.394 \\
\hline 32.955 & 2.197 & 4.394 \\
\hline 68.107 & 50.531 & 1726.84 \\
\hline 4.394 & 4.394 & 2.197 \\
\hline 13.182 & 21.97 & 4.394 \\
\hline 24.167 & 85.683 & 8.788 \\
\hline 2.197 & 2.197 & 2.197 \\
\hline 15.379 & 2.197 & 8.788 \\
\hline 13.182 & 15.379 & 8.788 \\
\hline 2.197 & 17.576 & 8.788 \\
\hline 6.591 & 26.364 & 6.591 \\
\hline 13.182 & 2.197 & 147.199 \\
\hline 2.197 & 2.197 & 90.077 \\
\hline 4.394 & 13.182 & 10.985 \\
\hline 2.197 & 26.364 & 2.197 \\
\hline 4.394 & 127.426 & 2.197 \\
\hline 232.882 & 2.197 & 2.197 \\
\hline 13.182 & 2.197 & 215.306 \\
\hline 2.197 & 8.788 & 250.458 \\
\hline 2.197 & 2.197 & 68.107 \\
\hline 226.291 & 4.394 & 72.501 \\
\hline 2.197 & 30.758 & 4.394 \\
\hline 2.197 & 2.197 & 2.197 \\
\hline 2.197 & 2.197 & 8.788 \\
\hline 2.197 & 2.197 & 112.047 \\
\hline 2.197 & 8.788 & 2.197 \\
\hline 2.197 & 2.197 & 188.942 \\
\hline
\end{tabular}




\begin{tabular}{|c|c|c|}
\hline 2.197 & 4.394 & 6.591 \\
\hline 2.197 & 2.197 & 2.197 \\
\hline 2.197 & 19.773 & 2.197 \\
\hline 4.394 & 2.197 & 2.197 \\
\hline 10.985 & 2.197 & 6.591 \\
\hline 134.017 & 2.197 & 52.728 \\
\hline 303.186 & 6.591 & 6.591 \\
\hline 8.788 & 90.077 & 2.197 \\
\hline 61.516 & 43.94 & 13.182 \\
\hline 125.229 & 4.394 & 13.182 \\
\hline 213.109 & 6.591 & 6.591 \\
\hline 428.415 & 4.394 & 28.561 \\
\hline 65.91 & 15.379 & 2.197 \\
\hline 76.895 & 85.683 & 63.713 \\
\hline 30.758 & 8.788 & 48.334 \\
\hline 173.563 & 2.197 & 2.197 \\
\hline 6.591 & 8.788 & 2.197 \\
\hline 6.591 & 17.576 & 2.197 \\
\hline 4.394 & 15.379 & 92.274 \\
\hline 2.197 & 15.379 & 217.503 \\
\hline 19.773 & 2.197 & 26.364 \\
\hline 4.394 & 4.394 & 65.91 \\
\hline 13.182 & 24.167 & 6.591 \\
\hline 2.197 & 2.197 & 2.197 \\
\hline 54.925 & 41.743 & 8.788 \\
\hline 2.197 & 32.955 & 21.97 \\
\hline 28.561 & 4.394 & 90.077 \\
\hline 8.788 & 4.394 & 2.197 \\
\hline 57.122 & 6.591 & 2.197 \\
\hline 2.197 & 26.364 & 2.197 \\
\hline 30.758 & 4.394 & 2.197 \\
\hline 2.197 & 4.394 & 160.381 \\
\hline 2.197 & 6.591 & 15.379 \\
\hline 54.925 & 4.394 & 41.743 \\
\hline 2.197 & 2.197 & 8.788 \\
\hline 2.197 & 2.197 & 4.394 \\
\hline 2188.21 & 13.182 & 13.182 \\
\hline 8.788 & 59.319 & 153.79 \\
\hline 2.197 & 2.197 & 246.064 \\
\hline 2.197 & 2.197 & 2.197 \\
\hline 70.304 & 2.197 & 2.197 \\
\hline 10.985 & 166.972 & 13.182 \\
\hline 463.567 & 4.394 & 41.743 \\
\hline 10.985 & 13.182 & 2.197 \\
\hline 19.773 & 2.197 & 13.182 \\
\hline 4.394 & 582.205 & 19.773 \\
\hline 4.394 & 13.182 & 63.713 \\
\hline 2.197 & 13.182 & 6.591 \\
\hline 197.73 & 2.197 & 2.197 \\
\hline 13.182 & 2.197 & 127.426 \\
\hline
\end{tabular}




\begin{tabular}{|c|c|c|}
\hline 8.788 & 2.197 & 7926.78 \\
\hline 13.182 & 6.591 & 10.985 \\
\hline 32.955 & 17.576 & 24.167 \\
\hline 6.591 & 6.591 & 2.197 \\
\hline 4.394 & 2.197 & 2.197 \\
\hline 8.788 & 145.002 & 2.197 \\
\hline 2.197 & 8.788 & 2.197 \\
\hline 52.728 & 35.152 & 2.197 \\
\hline 8.788 & 4.394 & 2.197 \\
\hline 17.576 & 2.197 & 2.197 \\
\hline 6.591 & 388.869 & 39.546 \\
\hline 4.394 & 2.197 & 41.743 \\
\hline 24.167 & 4.394 & 2.197 \\
\hline 8.788 & 65.91 & 32.955 \\
\hline 2.197 & 2.197 & 6039.55 \\
\hline 2.197 & 2.197 & 4.394 \\
\hline 2.197 & 2.197 & 8.788 \\
\hline 30.758 & 13.182 & 87.88 \\
\hline 39.546 & 4.394 & 6.591 \\
\hline 114.244 & 2.197 & 59.319 \\
\hline 13.182 & 4.394 & 2.197 \\
\hline 107.653 & 2.197 & 2.197 \\
\hline 37.349 & 155.987 & 28.561 \\
\hline 4.394 & 2.197 & 4.394 \\
\hline 2.197 & 4.394 & 37.349 \\
\hline 327.353 & 2.197 & 2.197 \\
\hline 10.985 & 6.591 & 28.561 \\
\hline 24.167 & 50.531 & 17.576 \\
\hline 4.394 & 2.197 & 19.773 \\
\hline 134.017 & 2.197 & 2.197 \\
\hline 94.471 & 478.946 & 2.197 \\
\hline 8.788 & 17.576 & 103.259 \\
\hline 52.728 & 37.349 & 10.985 \\
\hline 65.91 & 6.591 & 3455.88 \\
\hline 17.576 & 57.122 & 24.167 \\
\hline 13.182 & 2.197 & 4.394 \\
\hline 2.197 & 19.773 & 2.197 \\
\hline 118.638 & 59.319 & 8.788 \\
\hline 2.197 & 13.182 & 21.97 \\
\hline 68.107 & 10.985 & 15.379 \\
\hline 52.728 & 4.394 & 28.561 \\
\hline 28.561 & 2.197 & 4.394 \\
\hline 10.985 & 6.591 & 1816.92 \\
\hline 2.197 & 2.197 & 13.182 \\
\hline 2.197 & 166.972 & 46.137 \\
\hline 26.364 & 83.486 & 21.97 \\
\hline 26.364 & 2.197 & 2.197 \\
\hline 76.895 & 2.197 & 24.167 \\
\hline 54.925 & 35.152 & 4.394 \\
\hline 4.394 & 2.197 & 2.197 \\
\hline
\end{tabular}




\begin{tabular}{|c|c|c|}
\hline 2.197 & 4.394 & 28.561 \\
\hline 6.591 & 4.394 & 2.197 \\
\hline 208.715 & 4.394 & 35.152 \\
\hline 24.167 & 4.394 & 6.591 \\
\hline 188.942 & 13.182 & 6.591 \\
\hline 2.197 & 2.197 & 8.788 \\
\hline 8.788 & 2.197 & 2.197 \\
\hline 2.197 & 37.349 & 2.197 \\
\hline 10.985 & 2.197 & 13.182 \\
\hline 2.197 & 2.197 & 2.197 \\
\hline 83.486 & 4.394 & 59.319 \\
\hline 4.394 & 4.394 & 30.758 \\
\hline 15.379 & 2.197 & 120.835 \\
\hline 2.197 & 4.394 & 30.758 \\
\hline 43.94 & 2.197 & 10.985 \\
\hline 15.379 & 13.182 & 2.197 \\
\hline 2.197 & 120.835 & 2.197 \\
\hline 8.788 & 4.394 & 2.197 \\
\hline 10.985 & 2.197 & 2.197 \\
\hline 15.379 & 4.394 & 2.197 \\
\hline 63.713 & 4.394 & 81.289 \\
\hline 21.97 & 6.591 & 4.394 \\
\hline 2.197 & 6.591 & 2.197 \\
\hline 10.985 & 8.788 & 193.336 \\
\hline 142.805 & 21.97 & 2.197 \\
\hline 4.394 & 98.865 & 6.591 \\
\hline 8.788 & 6.591 & 2.197 \\
\hline 8.788 & 2.197 & 2.197 \\
\hline 21.97 & 17.576 & 2.197 \\
\hline 4.394 & 2.197 & 2.197 \\
\hline 28.561 & 2.197 & 2.197 \\
\hline 2.197 & 8.788 & 30.758 \\
\hline 8.788 & 10.985 & 2.197 \\
\hline 8.788 & 6.591 & 2.197 \\
\hline 2.197 & 8.788 & 2.197 \\
\hline 6.591 & 2.197 & 2.197 \\
\hline 68.107 & 2.197 & 2.197 \\
\hline 700.843 & 116.441 & 2.197 \\
\hline 2.197 & 2.197 & 2.197 \\
\hline 21.97 & 2.197 & 37.349 \\
\hline 15.379 & 2.197 & 13.182 \\
\hline 81.289 & 17.576 & 182.351 \\
\hline 2.197 & 2.197 & 98.865 \\
\hline 35.152 & 13.182 & 2.197 \\
\hline 6.591 & 4.394 & 21.97 \\
\hline 6.591 & 8.788 & 6.591 \\
\hline 68.107 & 83.486 & 4.394 \\
\hline 15.379 & 2.197 & 2.197 \\
\hline 140.608 & 6.591 & 261.443 \\
\hline 2.197 & 6.591 & 15.379 \\
\hline
\end{tabular}




\begin{tabular}{|c|c|c|}
\hline 6.591 & 107.653 & 13.182 \\
\hline 2.197 & 8.788 & 4.394 \\
\hline 2.197 & 4.394 & 37.349 \\
\hline 2.197 & 6.591 & 43.94 \\
\hline 76.895 & 15.379 & 2.197 \\
\hline 76.895 & 2.197 & 2.197 \\
\hline 2.197 & 13.182 & 2.197 \\
\hline 344.929 & 26.364 & 35.152 \\
\hline 10.985 & 2.197 & 129.623 \\
\hline 2.197 & 2.197 & 2.197 \\
\hline 32.955 & 2.197 & 8.788 \\
\hline 2.197 & 2.197 & 13.182 \\
\hline 19.773 & 52.728 & 4.394 \\
\hline 17.576 & 17.576 & 6.591 \\
\hline 2.197 & 1221.53 & 4.394 \\
\hline 182.351 & 1329.18 & 28.561 \\
\hline 2.197 & 2.197 & 4.394 \\
\hline 2.197 & 4.394 & 6.591 \\
\hline 79.092 & 2.197 & 2.197 \\
\hline 13.182 & 2.197 & 13.182 \\
\hline 2.197 & 2.197 & 2.197 \\
\hline 61.516 & 4.394 & 2.197 \\
\hline 41.743 & 6.591 & 24.167 \\
\hline 13.182 & 2.197 & 4.394 \\
\hline 199.927 & 2.197 & 21.97 \\
\hline 118.638 & 2.197 & 6.591 \\
\hline 2.197 & 2.197 & 10.985 \\
\hline 13.182 & 834.86 & 41.743 \\
\hline 120.835 & 2.197 & 6.591 \\
\hline 15.379 & 4.394 & 4.394 \\
\hline 15.379 & 2.197 & 2.197 \\
\hline 61.516 & 37.349 & 2.197 \\
\hline 4.394 & 2.197 & 8.788 \\
\hline 173.563 & 54.925 & 10.985 \\
\hline 298.792 & 15.379 & 26.364 \\
\hline 15.379 & 2.197 & 2.197 \\
\hline 2.197 & 72.501 & 2.197 \\
\hline 2.197 & 2.197 & 2.197 \\
\hline 4.394 & 905.164 & 17.576 \\
\hline 39.546 & 2.197 & 2.197 \\
\hline 57.122 & 17.576 & 21.97 \\
\hline 8.788 & 2.197 & 35.152 \\
\hline 2.197 & 21.97 & 2.197 \\
\hline 37.349 & 6.591 & 2.197 \\
\hline 2.197 & 2.197 & 2.197 \\
\hline 2.197 & 2.197 & 10.985 \\
\hline 2.197 & 37.349 & 2.197 \\
\hline 6.591 & 35.152 & 4.394 \\
\hline 2.197 & 140.608 & 2.197 \\
\hline 8.788 & 2.197 & 6.591 \\
\hline
\end{tabular}




\begin{tabular}{|c|c|c|}
\hline 24.167 & 2.197 & 2.197 \\
\hline 2.197 & 2.197 & 2.197 \\
\hline 2.197 & 2.197 & 26.364 \\
\hline 15.379 & 6.591 & 4.394 \\
\hline 120.835 & 2.197 & 13.182 \\
\hline 15.379 & 17.576 & 2.197 \\
\hline 13.182 & 2.197 & 2.197 \\
\hline 2.197 & 2.197 & 153.79 \\
\hline 63.713 & 2.197 & 2.197 \\
\hline 59.319 & 4.394 & 2.197 \\
\hline 59.319 & 4.394 & 2.197 \\
\hline 105.456 & 26.364 & 296.595 \\
\hline 2.197 & 8.788 & 4.394 \\
\hline 2.197 & 87.88 & 4.394 \\
\hline 140.608 & 2.197 & 2.197 \\
\hline 4.394 & 2.197 & 26.364 \\
\hline 52.728 & 2.197 & 26.364 \\
\hline 32.955 & 13.182 & 2.197 \\
\hline 46.137 & 2.197 & 39.546 \\
\hline 2.197 & 30.758 & 8.788 \\
\hline 4.394 & 4.394 & 2.197 \\
\hline 13.182 & 2.197 & 57.122 \\
\hline 2.197 & 2.197 & 8.788 \\
\hline 4.394 & 2.197 & 676.676 \\
\hline 74.698 & 2.197 & 4.394 \\
\hline 21.97 & 8.788 & 28.561 \\
\hline 10.985 & 28.561 & 2.197 \\
\hline 15.379 & 8.788 & 4.394 \\
\hline 10.985 & 2.197 & 2.197 \\
\hline 8.788 & 2.197 & 296.595 \\
\hline 4.394 & 2.197 & 2.197 \\
\hline 2.197 & 17.576 & 10.985 \\
\hline 4.394 & 6.591 & 76.895 \\
\hline 8.788 & 2.197 & 10.985 \\
\hline 24.167 & 52.728 & 72.501 \\
\hline 37.349 & 28.561 & 8.788 \\
\hline 6.591 & 2.197 & 28.561 \\
\hline 50.531 & 13.182 & 6.591 \\
\hline 13.182 & 15.379 & 134.017 \\
\hline 4.394 & 48.334 & 13.182 \\
\hline 39.546 & 26.364 & 65.91 \\
\hline 2.197 & 8.788 & 158.184 \\
\hline 2.197 & 2.197 & 46.137 \\
\hline 57.122 & 252.655 & 2.197 \\
\hline 13.182 & 26.364 & 4.394 \\
\hline 4.394 & 46.137 & 2.197 \\
\hline 24.167 & 10.985 & 15.379 \\
\hline 54.925 & 2.197 & 15.379 \\
\hline 13.182 & 2.197 & 6.591 \\
\hline 2.197 & 2.197 & 4.394 \\
\hline
\end{tabular}




\begin{tabular}{|c|c|c|}
\hline 19.773 & 41.743 & 2.197 \\
\hline 2.197 & 2.197 & 4.394 \\
\hline 10.985 & 6.591 & 2.197 \\
\hline 21.97 & 4.394 & 19.773 \\
\hline 39.546 & 10.985 & 2.197 \\
\hline 2.197 & 2.197 & 6.591 \\
\hline 6.591 & 2.197 & 2.197 \\
\hline 21.97 & 2.197 & 2.197 \\
\hline 46.137 & 35.152 & 81.289 \\
\hline 2.197 & 15.379 & 2.197 \\
\hline 10.985 & 129.623 & 26.364 \\
\hline 13.182 & 37.349 & 41.743 \\
\hline 39.546 & 19.773 & 2.197 \\
\hline 92.274 & 2.197 & 30.758 \\
\hline 24.167 & 6.591 & 15.379 \\
\hline 2.197 & 24.167 & 2.197 \\
\hline 2.197 & 28.561 & 2.197 \\
\hline 90.077 & 2.197 & 4.394 \\
\hline 615.16 & 39.546 & 10.985 \\
\hline 2.197 & 32.955 & 6.591 \\
\hline 463.567 & 10.985 & 19.773 \\
\hline 2.197 & 2.197 & 50.531 \\
\hline 79.092 & 48.334 & 24.167 \\
\hline 15.379 & 2.197 & 2.197 \\
\hline 134.017 & 112.047 & 6.591 \\
\hline 4.394 & 2.197 & 8.788 \\
\hline 4.394 & 2.197 & 2.197 \\
\hline 13.182 & 2.197 & 2.197 \\
\hline 6.591 & 19.773 & 4.394 \\
\hline 4.394 & 17.576 & 2.197 \\
\hline 13.182 & 15.379 & 35.152 \\
\hline 2.197 & 65.91 & 13.182 \\
\hline 70.304 & 4.394 & 4.394 \\
\hline 28.561 & 8.788 & 72.501 \\
\hline 4.394 & 2.197 & 2.197 \\
\hline 188.942 & 17.576 & 226.291 \\
\hline 342.732 & 4.394 & 37.349 \\
\hline 4.394 & 2.197 & 2.197 \\
\hline 26.364 & 4.394 & 2.197 \\
\hline 15.379 & 2.197 & 4.394 \\
\hline 153.79 & 4.394 & 28.561 \\
\hline 2.197 & 6.591 & 26.364 \\
\hline 2.197 & 2.197 & 6.591 \\
\hline 2.197 & 4.394 & 171.366 \\
\hline 177.957 & 32.955 & 4.394 \\
\hline 2.197 & 35.152 & 24.167 \\
\hline 8.788 & 15.379 & 6.591 \\
\hline 2.197 & 4.394 & 127.426 \\
\hline 2.197 & 4.394 & 4.394 \\
\hline 26.364 & 2.197 & 50.531 \\
\hline
\end{tabular}




\begin{tabular}{|c|c|c|}
\hline 26.364 & 2.197 & 151.593 \\
\hline 4.394 & 8.788 & 8.788 \\
\hline 10.985 & 2.197 & 2.197 \\
\hline 2.197 & 2.197 & 2.197 \\
\hline 4.394 & 6.591 & 48.334 \\
\hline 2.197 & 2.197 & 21.97 \\
\hline 8.788 & 2.197 & 2.197 \\
\hline 2.197 & 2.197 & 8.788 \\
\hline 4.394 & 2.197 & 2.197 \\
\hline 13.182 & 63.713 & 10.985 \\
\hline 13.182 & 2.197 & 4.394 \\
\hline 6.591 & 15.379 & 26.364 \\
\hline 8.788 & 2.197 & 127.426 \\
\hline 4.394 & 2.197 & 2.197 \\
\hline 24.167 & 4.394 & 2.197 \\
\hline 4.394 & 4.394 & 2.197 \\
\hline 2.197 & 10.985 & 2.197 \\
\hline 19.773 & 2.197 & 4.394 \\
\hline 52.728 & 10.985 & 265.837 \\
\hline 8.788 & 81.289 & 52.728 \\
\hline 6.591 & 24.167 & 2.197 \\
\hline 13.182 & 8.788 & 30.758 \\
\hline 129.623 & 2.197 & 4.394 \\
\hline 50.531 & 6.591 & 26.364 \\
\hline 74.698 & 13.182 & 2.197 \\
\hline 2.197 & 15.379 & 21.97 \\
\hline 4.394 & 2.197 & 2.197 \\
\hline 2.197 & 2.197 & 13.182 \\
\hline 2.197 & 2.197 & 6.591 \\
\hline 10.985 & 2.197 & 2.197 \\
\hline 219.7 & 2.197 & 74.698 \\
\hline 32.955 & 2.197 & 2.197 \\
\hline 10.985 & 2.197 & 4.394 \\
\hline 2.197 & 13.182 & 4.394 \\
\hline 43.94 & 2.197 & 10.985 \\
\hline 4.394 & 2.197 & 28.561 \\
\hline 2.197 & 68.107 & 39.546 \\
\hline 2.197 & 21.97 & 13.182 \\
\hline 4.394 & 13.182 & 2.197 \\
\hline 8.788 & 2.197 & 8.788 \\
\hline 4.394 & 19.773 & 57.122 \\
\hline 28.561 & 2.197 & 2.197 \\
\hline 2.197 & 10.985 & 2.197 \\
\hline 8.788 & 72.501 & 28.561 \\
\hline 6.591 & 2.197 & 2.197 \\
\hline 268.034 & 4.394 & 8.788 \\
\hline 59.319 & 8.788 & 2.197 \\
\hline 2.197 & 362.505 & 28.561 \\
\hline 63.713 & 13.182 & 2.197 \\
\hline 2.197 & 103.259 & 17.576 \\
\hline
\end{tabular}




\begin{tabular}{|c|c|c|}
\hline 2.197 & 70.304 & 6.591 \\
\hline 6.591 & 4.394 & 48.334 \\
\hline 2.197 & 2.197 & 32.955 \\
\hline 4.394 & 4.394 & 10.985 \\
\hline 2.197 & 2.197 & 6.591 \\
\hline 8.788 & 10.985 & 2.197 \\
\hline 35.152 & 19.773 & 355.914 \\
\hline 2.197 & 2.197 & 2.197 \\
\hline 127.426 & 35.152 & 43.94 \\
\hline 4.394 & 15.379 & 2.197 \\
\hline 2.197 & 17.576 & 2.197 \\
\hline 13.182 & 4.394 & 2.197 \\
\hline 10.985 & 4.394 & 2.197 \\
\hline 87.88 & 2.197 & 13.182 \\
\hline 17.576 & 6.591 & 2.197 \\
\hline 2.197 & 17.576 & 39.546 \\
\hline 4.394 & 13.182 & 4.394 \\
\hline 2.197 & 4.394 & 2.197 \\
\hline 39.546 & 6.591 & 2.197 \\
\hline 13.182 & 6.591 & 101.062 \\
\hline 32.955 & 4.394 & 4.394 \\
\hline 15.379 & 59.319 & 4.394 \\
\hline 10.985 & 4.394 & 2.197 \\
\hline 68.107 & 2.197 & 2.197 \\
\hline 21.97 & 4.394 & 2.197 \\
\hline 2.197 & 35.152 & 2.197 \\
\hline 52.728 & 2.197 & 2.197 \\
\hline 8.788 & 6.591 & 24.167 \\
\hline 17.576 & 15.379 & 90.077 \\
\hline 43.94 & 6.591 & 52.728 \\
\hline 8.788 & 2.197 & 2.197 \\
\hline 10.985 & 4.394 & 70.304 \\
\hline 24.167 & 30.758 & 2.197 \\
\hline 4.394 & 4.394 & 15.379 \\
\hline 4.394 & 4.394 & 2.197 \\
\hline 2.197 & 2.197 & 13.182 \\
\hline 8.788 & 4.394 & 2.197 \\
\hline 1179.79 & 41.743 & 13.182 \\
\hline 2.197 & 85.683 & 43.94 \\
\hline 21.97 & 4.394 & 30.758 \\
\hline 2.197 & 4.394 & 4.394 \\
\hline 4.394 & 2.197 & 2.197 \\
\hline 48.334 & 4.394 & 4.394 \\
\hline 2.197 & 2.197 & 2.197 \\
\hline 52.728 & 74.698 & 4.394 \\
\hline 1590.63 & 43.94 & 120.835 \\
\hline 90.077 & 13.182 & 19.773 \\
\hline 173.563 & 2.197 & 8.788 \\
\hline 96.668 & 2.197 & 2.197 \\
\hline 10.985 & 2.197 & 46.137 \\
\hline
\end{tabular}




\begin{tabular}{|c|c|c|}
\hline 63.713 & 61.516 & 15.379 \\
\hline 2.197 & 2.197 & 41.743 \\
\hline 43.94 & 21.97 & 30.758 \\
\hline 263.64 & 6.591 & 15.379 \\
\hline 19.773 & 4.394 & 4.394 \\
\hline 2.197 & 2.197 & 4.394 \\
\hline 13.182 & 8.788 & 24.167 \\
\hline 2.197 & 2.197 & 52.728 \\
\hline 10.985 & 2.197 & 37.349 \\
\hline 4.394 & 6.591 & 63.713 \\
\hline 92.274 & 2.197 & 1357.75 \\
\hline 4.394 & 13.182 & 112.047 \\
\hline 8.788 & 8.788 & 15.379 \\
\hline 2.197 & 6.591 & 281.216 \\
\hline 2.197 & 2.197 & 30.758 \\
\hline 2.197 & 19.773 & 4.394 \\
\hline 2.197 & 2.197 & 4.394 \\
\hline 2.197 & 61.516 & 2.197 \\
\hline 6.591 & 2.197 & 2.197 \\
\hline 21.97 & 2.197 & 239.473 \\
\hline 8.788 & 13.182 & 6.591 \\
\hline 4.394 & 2.197 & 6.591 \\
\hline 2.197 & 2.197 & 2.197 \\
\hline 10.985 & 2.197 & 6.591 \\
\hline 46.137 & 4.394 & 4.394 \\
\hline 164.775 & 63.713 & 32.955 \\
\hline 13.182 & 41.743 & 109.85 \\
\hline 2.197 & 2.197 & 2.197 \\
\hline 13.182 & 6.591 & 2.197 \\
\hline 4.394 & 4.394 & 2.197 \\
\hline 28.561 & 8.788 & 70.304 \\
\hline 6.591 & 208.715 & 2.197 \\
\hline 10.985 & 70.304 & 4.394 \\
\hline 19.773 & 21.97 & 50.531 \\
\hline 2.197 & 32.955 & 2.197 \\
\hline 331.747 & 4.394 & 2.197 \\
\hline 4.394 & 8.788 & 4.394 \\
\hline 4.394 & 4.394 & 85.683 \\
\hline 2.197 & 2.197 & 94.471 \\
\hline 8.788 & 4.394 & 70.304 \\
\hline 4.394 & 21.97 & 10.985 \\
\hline 41.743 & 4.394 & 191.139 \\
\hline 39.546 & 4.394 & 32.955 \\
\hline 2.197 & 2.197 & 65.91 \\
\hline 2.197 & 2.197 & 6.591 \\
\hline 2.197 & 2.197 & 2.197 \\
\hline 2.197 & 2.197 & 19.773 \\
\hline 136.214 & 28.561 & 13.182 \\
\hline 8.788 & 10.985 & 48.334 \\
\hline 6.591 & 2.197 & 186.745 \\
\hline
\end{tabular}




\begin{tabular}{|c|c|c|}
\hline 2.197 & 4.394 & 70.304 \\
\hline 2.197 & 48.334 & 54.925 \\
\hline 408.642 & 4.394 & 39.546 \\
\hline 2.197 & 57.122 & 17.576 \\
\hline 35.152 & 2.197 & 30.758 \\
\hline 28.561 & 2.197 & 2.197 \\
\hline 2.197 & 2.197 & 37.349 \\
\hline 2.197 & 48.334 & 28.561 \\
\hline 6.591 & 15.379 & 2.197 \\
\hline 2.197 & 8.788 & 10.985 \\
\hline 26.364 & 28.561 & 10.985 \\
\hline 2.197 & 2.197 & 10.985 \\
\hline 17.576 & 6.591 & 2.197 \\
\hline 2.197 & 2.197 & 17.576 \\
\hline 2.197 & 2.197 & 15.379 \\
\hline 107.653 & 2.197 & 6.591 \\
\hline 46.137 & 6.591 & 8.788 \\
\hline 2.197 & 13.182 & 2.197 \\
\hline 2.197 & 32.955 & 74.698 \\
\hline 6.591 & 4.394 & 2.197 \\
\hline 2.197 & 2.197 & 2.197 \\
\hline 68.107 & 2.197 & 26.364 \\
\hline 4.394 & 15.379 & 15.379 \\
\hline 2.197 & 2.197 & 10.985 \\
\hline 2.197 & 4.394 & 2.197 \\
\hline 2.197 & 2.197 & 2.197 \\
\hline 292.201 & 2.197 & 19.773 \\
\hline 2.197 & 2.197 & 2.197 \\
\hline 6.591 & 4.394 & 6.591 \\
\hline 10.985 & 98.865 & 6.591 \\
\hline 30.758 & 30.758 & 41.743 \\
\hline 85.683 & 10.985 & 39.546 \\
\hline 366.899 & 59.319 & 6.591 \\
\hline 17.576 & 30.758 & 8.788 \\
\hline 28.561 & 2.197 & 2.197 \\
\hline 46.137 & 270.231 & 188.942 \\
\hline 118.638 & 2.197 & 6.591 \\
\hline 2.197 & 2.197 & 2.197 \\
\hline 13.182 & 39.546 & 48.334 \\
\hline 52.728 & 4.394 & 226.291 \\
\hline 15.379 & 41.743 & 57.122 \\
\hline 54.925 & 2.197 & 57.122 \\
\hline 2.197 & 48.334 & 4.394 \\
\hline 2.197 & 4.394 & 2.197 \\
\hline 6.591 & 145.002 & 15.379 \\
\hline 19.773 & 10.985 & 21.97 \\
\hline 63.713 & 2.197 & 2.197 \\
\hline 6.591 & 2.197 & 2.197 \\
\hline 2.197 & 8.788 & 2.197 \\
\hline 2.197 & 8.788 & 46.137 \\
\hline
\end{tabular}




\begin{tabular}{|c|c|c|}
\hline 19.773 & 8.788 & 8.788 \\
\hline 2.197 & 4.394 & 35.152 \\
\hline 112.047 & 6.591 & 24.167 \\
\hline 204.321 & 13.182 & 24.167 \\
\hline 30.758 & 4.394 & 10.985 \\
\hline 254.852 & 8.788 & 2.197 \\
\hline 637.13 & 2.197 & 2.197 \\
\hline 4.394 & 2.197 & 48.334 \\
\hline 41.743 & 2.197 & 83.486 \\
\hline 112.047 & 2.197 & 2.197 \\
\hline 54.925 & 41.743 & 24.167 \\
\hline 2.197 & 2.197 & 358.111 \\
\hline 2.197 & 4.394 & 26.364 \\
\hline 2.197 & 4.394 & 2.197 \\
\hline 2.197 & 26.364 & 63.713 \\
\hline 4.394 & 30.758 & 15.379 \\
\hline 2.197 & 70.304 & 2.197 \\
\hline 4.394 & 2.197 & 2.197 \\
\hline 2.197 & 4.394 & 2.197 \\
\hline 41.743 & 37.349 & 15.379 \\
\hline 265.837 & 191.139 & 10.985 \\
\hline 4.394 & 19.773 & 2.197 \\
\hline 94.471 & 2.197 & 4.394 \\
\hline 4.394 & 2.197 & 2.197 \\
\hline 6.591 & 2.197 & 4.394 \\
\hline 4.394 & 8.788 & 68.107 \\
\hline 2.197 & 2.197 & 184.548 \\
\hline 6.591 & 4.394 & 2.197 \\
\hline 4.394 & 17.576 & 2.197 \\
\hline 4.394 & 6.591 & 2.197 \\
\hline 2.197 & 2.197 & 2.197 \\
\hline 8.788 & 2.197 & 28.561 \\
\hline 2.197 & 24.167 & 2.197 \\
\hline 4.394 & 2.197 & 2.197 \\
\hline 4.394 & 2.197 & 2.197 \\
\hline 48.334 & 6.591 & 487.734 \\
\hline 2.197 & 2.197 & 2.197 \\
\hline 2.197 & 4.394 & 652.509 \\
\hline 6.591 & 6.591 & 2.197 \\
\hline 2.197 & 52.728 & 155.987 \\
\hline 4.394 & 8.788 & 8.788 \\
\hline 13.182 & 2.197 & 39.546 \\
\hline 15.379 & 2.197 & 26.364 \\
\hline 2.197 & 32.955 & 15.379 \\
\hline 4.394 & 263.64 & 2.197 \\
\hline 17.576 & 98.865 & 24.167 \\
\hline 2.197 & 54.925 & 19.773 \\
\hline 2.197 & 4.394 & 50.531 \\
\hline 8.788 & 2.197 & 13.182 \\
\hline 41.743 & 2.197 & 54.925 \\
\hline
\end{tabular}




\begin{tabular}{|c|c|c|}
\hline 4.394 & 2.197 & 692.055 \\
\hline 586.599 & 21.97 & 498.719 \\
\hline 41.743 & 4.394 & 59.319 \\
\hline 43.94 & 2.197 & 2.197 \\
\hline 13.182 & 52.728 & 2.197 \\
\hline 15.379 & 2.197 & 35.152 \\
\hline 6.591 & 10.985 & 46.137 \\
\hline 8.788 & 13.182 & 79.092 \\
\hline 48.334 & 4.394 & 98.865 \\
\hline 2.197 & 2.197 & 50.531 \\
\hline 6.591 & 2.197 & 8.788 \\
\hline 2.197 & 2.197 & 2.197 \\
\hline 413.036 & 2.197 & 4.394 \\
\hline 10.985 & 6.591 & 98.865 \\
\hline 10.985 & 2.197 & 2.197 \\
\hline 138.411 & 30.758 & 8.788 \\
\hline 4.394 & 24.167 & 52.728 \\
\hline 2.197 & 2.197 & 2.197 \\
\hline 2.197 & 2.197 & 123.032 \\
\hline 2.197 & 4.394 & 21.97 \\
\hline 107.653 & 4.394 & 2.197 \\
\hline 2.197 & 4.394 & 21.97 \\
\hline 2.197 & 2.197 & 43.94 \\
\hline 10.985 & 2.197 & 10.985 \\
\hline 15.379 & 6.591 & 30.758 \\
\hline 35.152 & 2.197 & 140.608 \\
\hline 2.197 & 13.182 & 10.985 \\
\hline 2.197 & 2.197 & 10.985 \\
\hline 6.591 & 2.197 & 2.197 \\
\hline 2.197 & 8.788 & 2.197 \\
\hline 10.985 & 4.394 & 2.197 \\
\hline 32.955 & 54.925 & 4.394 \\
\hline 4.394 & 101.062 & 177.957 \\
\hline 145.002 & 10.985 & 2.197 \\
\hline 30.758 & 2.197 & 19.773 \\
\hline 24.167 & 17.576 & 32.955 \\
\hline 4.394 & 2.197 & 4.394 \\
\hline 6.591 & 68.107 & 2.197 \\
\hline 2.197 & 17.576 & 2.197 \\
\hline 2.197 & 2.197 & 10.985 \\
\hline 2.197 & 6.591 & 2.197 \\
\hline 279.019 & 2.197 & 2.197 \\
\hline 2.197 & 13.182 & 2.197 \\
\hline 2.197 & 4.394 & 2.197 \\
\hline 2.197 & 30.758 & 2.197 \\
\hline 4.394 & 2.197 & 17.576 \\
\hline 24.167 & 6.591 & 96.668 \\
\hline 48.334 & 65.91 & 17.576 \\
\hline 17.576 & 2.197 & 2.197 \\
\hline 2.197 & 21.97 & 24.167 \\
\hline
\end{tabular}




\begin{tabular}{|c|c|c|}
\hline 15.379 & 48.334 & 46.137 \\
\hline 6.591 & 2.197 & 8.788 \\
\hline 2.197 & 30.758 & 13.182 \\
\hline 4.394 & 10.985 & 4.394 \\
\hline 61.516 & 6.591 & 2.197 \\
\hline 2.197 & 2.197 & 4.394 \\
\hline 459.173 & 4.394 & 164.775 \\
\hline 98.865 & 2.197 & 26.364 \\
\hline 57.122 & 250.458 & 26.364 \\
\hline 2.197 & 8.788 & 129.623 \\
\hline 8.788 & 41.743 & 2.197 \\
\hline 2.197 & 1305.02 & 2.197 \\
\hline 6.591 & 48.334 & 2.197 \\
\hline 4.394 & 10.985 & 235.079 \\
\hline 10.985 & 2.197 & 4.394 \\
\hline 522.886 & 2.197 & 2.197 \\
\hline 37.349 & 2.197 & 65.91 \\
\hline 61.516 & 2.197 & 4.394 \\
\hline 26.364 & 4.394 & 15.379 \\
\hline 13.182 & 4.394 & 2.197 \\
\hline 8.788 & 421.824 & 4.394 \\
\hline 2.197 & 2.197 & 2816.55 \\
\hline 17.576 & 2.197 & 4.394 \\
\hline 41.743 & 2.197 & 26.364 \\
\hline 4.394 & 2.197 & 155.987 \\
\hline 2.197 & 10.985 & 2.197 \\
\hline 2.197 & 107.653 & 35.152 \\
\hline 2.197 & 19.773 & 19.773 \\
\hline 2.197 & 386.672 & 15.379 \\
\hline 4.394 & 15.379 & 30.758 \\
\hline 13.182 & 2.197 & 6.591 \\
\hline 37.349 & 13617 & 63.713 \\
\hline 2.197 & 6.591 & 4.394 \\
\hline 2.197 & 61.516 & 4.394 \\
\hline 28.561 & 175.76 & 2.197 \\
\hline 2.197 & 4.394 & 4.394 \\
\hline 760.162 & 74.698 & 50.531 \\
\hline 6.591 & 85.683 & 87.88 \\
\hline 136.214 & 2.197 & 2.197 \\
\hline 2.197 & 4.394 & 43.94 \\
\hline 4.394 & 2.197 & 2.197 \\
\hline 28.561 & 2.197 & 21.97 \\
\hline 2.197 & 2.197 & 4.394 \\
\hline 2.197 & 2.197 & 2.197 \\
\hline 4.394 & 85.683 & 2.197 \\
\hline 4.394 & 10.985 & 2.197 \\
\hline 85.683 & 26.364 & 2.197 \\
\hline 6.591 & 2.197 & 177.957 \\
\hline 21.97 & 17083.9 & 35.152 \\
\hline 17.576 & 2.197 & 2.197 \\
\hline
\end{tabular}




\begin{tabular}{|c|c|c|}
\hline 6.591 & 4.394 & 197.73 \\
\hline 305.383 & 2.197 & 28.561 \\
\hline 2.197 & 24.167 & 30.758 \\
\hline 48.334 & 39.546 & 2.197 \\
\hline 2.197 & 6.591 & 151.593 \\
\hline 2.197 & 544.856 & 26.364 \\
\hline 13.182 & 28.561 & 2.197 \\
\hline 109.85 & 10.985 & 2.197 \\
\hline 2.197 & 2.197 & 46.137 \\
\hline 37.349 & 2.197 & 2.197 \\
\hline 48.334 & 2.197 & 6.591 \\
\hline 10.985 & 4.394 & 26.364 \\
\hline 6.591 & 6.591 & 2.197 \\
\hline 2.197 & 30.758 & 2.197 \\
\hline 6.591 & 83.486 & 39.546 \\
\hline 32.955 & 2.197 & 13.182 \\
\hline 54.925 & 2.197 & 338.338 \\
\hline 6.591 & 4.394 & 4.394 \\
\hline 2.197 & 2.197 & 13.182 \\
\hline 2.197 & 13.182 & 4.394 \\
\hline 246.064 & 15.379 & 8.788 \\
\hline 2.197 & 2.197 & 32.955 \\
\hline 6.591 & 8.788 & 2.197 \\
\hline 26.364 & 215.306 & 46.137 \\
\hline 4.394 & 28.561 & 68.107 \\
\hline 4.394 & 2.197 & 129.623 \\
\hline 17.576 & 2.197 & 329.55 \\
\hline 6.591 & 415.233 & 48.334 \\
\hline 8.788 & 2.197 & 116.441 \\
\hline 2.197 & 2.197 & 2.197 \\
\hline 2.197 & 13.182 & 4.394 \\
\hline 316.368 & 2326.62 & 15.379 \\
\hline 8.788 & 2.197 & 6.591 \\
\hline 2.197 & 2.197 & 19.773 \\
\hline 145.002 & 68.107 & 210.912 \\
\hline 2.197 & 19.773 & 17.576 \\
\hline 2.197 & 2.197 & 2.197 \\
\hline 6.591 & 4.394 & 2.197 \\
\hline 2.197 & 2.197 & 10.985 \\
\hline 4.394 & 826.072 & 358.111 \\
\hline 2.197 & 2.197 & 76.895 \\
\hline 13.182 & 26.364 & 10.985 \\
\hline 6.591 & 2.197 & 2.197 \\
\hline 8.788 & 2.197 & 290.004 \\
\hline 10.985 & 252.655 & 4.394 \\
\hline 63.713 & 2.197 & 241.67 \\
\hline 2.197 & 2.197 & 57.122 \\
\hline 35.152 & 2.197 & 68.107 \\
\hline 6.591 & 4.394 & 13.182 \\
\hline 28.561 & 10.985 & 6.591 \\
\hline
\end{tabular}




\begin{tabular}{|c|c|c|}
\hline 2.197 & 2.197 & 24.167 \\
\hline 2.197 & 4.394 & 2.197 \\
\hline 21.97 & 4.394 & 4.394 \\
\hline 6.591 & 2.197 & 2.197 \\
\hline 6.591 & 13.182 & 2.197 \\
\hline 48.334 & 74.698 & 6.591 \\
\hline 6.591 & 4.394 & 21.97 \\
\hline 2.197 & 4.394 & 21.97 \\
\hline 2.197 & 2.197 & 2.197 \\
\hline 26.364 & 2.197 & 2.197 \\
\hline 35.152 & 48.334 & 2.197 \\
\hline 8.788 & 4.394 & 129.623 \\
\hline 41.743 & 2.197 & 2.197 \\
\hline 6.591 & 57.122 & 61.516 \\
\hline 39.546 & 2.197 & 24.167 \\
\hline 2.197 & 2.197 & 4.394 \\
\hline 147.199 & 4.394 & 2.197 \\
\hline 8.788 & 10.985 & 35.152 \\
\hline 6.591 & 6.591 & 15.379 \\
\hline 123.032 & 10.985 & 4.394 \\
\hline 2.197 & 2.197 & 15.379 \\
\hline 4.394 & 4.394 & 28.561 \\
\hline 6.591 & 2.197 & 2.197 \\
\hline 2.197 & 6.591 & 6.591 \\
\hline 4.394 & 4.394 & 2.197 \\
\hline 2.197 & 2.197 & 21.97 \\
\hline 2.197 & 2.197 & 2.197 \\
\hline 158.184 & 2.197 & 6.591 \\
\hline 2.197 & 4.394 & 129.623 \\
\hline 74.698 & 474.552 & 4.394 \\
\hline 68.107 & 10.985 & 2.197 \\
\hline 15.379 & 2.197 & 1195.17 \\
\hline 74.698 & 10.985 & 6.591 \\
\hline 68.107 & 30.758 & 19.773 \\
\hline 4.394 & 215.306 & 41.743 \\
\hline 8.788 & 24.167 & 6.591 \\
\hline 24.167 & 4.394 & 15.379 \\
\hline 4.394 & 2.197 & 3491.03 \\
\hline 6.591 & 92.274 & 2.197 \\
\hline 4.394 & 2.197 & 57.122 \\
\hline 2.197 & 10.985 & 2.197 \\
\hline 8.788 & 90.077 & 2.197 \\
\hline 63.713 & 8.788 & 307.58 \\
\hline 153.79 & 4.394 & 2.197 \\
\hline 6.591 & 2.197 & 2.197 \\
\hline 10.985 & 6.591 & 4.394 \\
\hline 6.591 & 2.197 & 30.758 \\
\hline 13.182 & 4.394 & 17.576 \\
\hline 2.197 & 2.197 & 17.576 \\
\hline 43.94 & 2.197 & 2.197 \\
\hline
\end{tabular}




$\begin{array}{rrr}4.394 & 4.394 & 10.985 \\ 4.394 & 30.758 & 2.197 \\ 43.94 & 2.197 & 15.379 \\ 107.653 & 2.197 & 35.152 \\ 61.516 & 2.197 & 8.788 \\ 4.394 & 4.394 & 19.773 \\ 2.197 & 2.197 & 4.394 \\ 98.865 & 180.154 & 2.197 \\ 4.394 & 366.899 & 1711.46 \\ 90.077 & 68.107 & 2.197 \\ 2.197 & 1138.05 & 4.394 \\ 48.334 & 17.576 & 2.197 \\ 2.197 & 4.394 & 4.394 \\ 68.107 & 17.576 & 24.167 \\ 32.955 & 17.576 & 6.591 \\ 2.197 & 2.197 & 6.591 \\ 2.197 & 2.197 & 4.394 \\ 2.197 & 4.394 & 15.379 \\ 6.591 & 4.394 & 6.591 \\ 74.698 & 8.788 & 292.201 \\ 13.182 & 2.197 & 10.985 \\ 4.394 & 2.197 & \end{array}$




\begin{tabular}{|c|c|c|}
\hline 8.788 & 4.394 & 24.167 \\
\hline 2.197 & 4.394 & 21.97 \\
\hline 21.97 & 2.197 & 24.167 \\
\hline 37.349 & 169.169 & 32.955 \\
\hline 2.197 & 127.426 & 2.197 \\
\hline 112.047 & 6.591 & 850.239 \\
\hline 65.91 & 2.197 & 856.83 \\
\hline 74.698 & 74.698 & 8.788 \\
\hline 28.561 & 10.985 & 226.291 \\
\hline 48.334 & 10.985 & 8.788 \\
\hline 344.929 & 4.394 & 63.713 \\
\hline 4.394 & 8.788 & 1500.55 \\
\hline 105.456 & 2.197 & 112.047 \\
\hline 2.197 & 17.576 & 3042.84 \\
\hline 2.197 & 21.97 & 17.576 \\
\hline 182.351 & 257.049 & 2.197 \\
\hline 2.197 & 17.576 & 2.197 \\
\hline 41.743 & 2.197 & 52.728 \\
\hline 112.047 & 2.197 & 15.379 \\
\hline 4.394 & 6.591 & 2.197 \\
\hline 4.394 & 37.349 & 2.197 \\
\hline 4.394 & 2.197 & 2.197 \\
\hline 4.394 & 21.97 & 235.079 \\
\hline 4.394 & 43.94 & 4.394 \\
\hline 193.336 & 2.197 & 753.571 \\
\hline 2.197 & 2.197 & 10.985 \\
\hline 2.197 & 2.197 & 2.197 \\
\hline 43.94 & 19.773 & 2.197 \\
\hline 28.561 & 2.197 & 2.197 \\
\hline 68.107 & 19.773 & 10.985 \\
\hline 2.197 & 26.364 & 2.197 \\
\hline 4.394 & 21.97 & 19.773 \\
\hline 2.197 & 26.364 & 35.152 \\
\hline 19.773 & 17.576 & 17.576 \\
\hline 8.788 & 2.197 & 2.197 \\
\hline 2.197 & 24.167 & 15.379 \\
\hline 6.591 & 2.197 & 15.379 \\
\hline 2.197 & 2.197 & 8.788 \\
\hline 2.197 & 2.197 & 2.197 \\
\hline 2.197 & 2.197 & 2.197 \\
\hline 4.394 & 103.259 & 8.788 \\
\hline 6.591 & 336.141 & 2.197 \\
\hline 2.197 & 2.197 & 26.364 \\
\hline 35.152 & 4.394 & 90.077 \\
\hline 134.017 & 19.773 & 2.197 \\
\hline 2.197 & 2.197 & 4.394 \\
\hline 6.591 & 4.394 & 19.773 \\
\hline 2.197 & 175.76 & 17.576 \\
\hline 37.349 & 2.197 & 81.289 \\
\hline 4.394 & 6.591 & 2.197 \\
\hline
\end{tabular}




\begin{tabular}{|c|c|c|}
\hline 129.623 & 2.197 & 24.167 \\
\hline 37.349 & 2.197 & 2.197 \\
\hline 2.197 & 2.197 & 4.394 \\
\hline 52.728 & 2.197 & 4.394 \\
\hline 127.426 & 28.561 & 46.137 \\
\hline 2.197 & 6.591 & 68.107 \\
\hline 90.077 & 15.379 & 6.591 \\
\hline 2.197 & 2.197 & 4.394 \\
\hline 4.394 & 2.197 & 8.788 \\
\hline 2.197 & 4.394 & 8.788 \\
\hline 68.107 & 2.197 & 145.002 \\
\hline 10.985 & 2.197 & 386.672 \\
\hline 6.591 & 2.197 & 2.197 \\
\hline 8.788 & 2.197 & 43.94 \\
\hline 516.295 & 2.197 & 39.546 \\
\hline 28.561 & 50.531 & 2.197 \\
\hline 4.394 & 39.546 & 65.91 \\
\hline 41.743 & 4.394 & 2.197 \\
\hline 32.955 & 15.379 & 13.182 \\
\hline 8.788 & 6.591 & 162.578 \\
\hline 32.955 & 48.334 & 4.394 \\
\hline 4.394 & 2.197 & 2.197 \\
\hline 2.197 & 2.197 & 54.925 \\
\hline 35.152 & 2.197 & 2.197 \\
\hline 15.379 & 6.591 & 4.394 \\
\hline 2.197 & 21.97 & 4.394 \\
\hline 24.167 & 10.985 & 6.591 \\
\hline 138.411 & 19.773 & 35.152 \\
\hline 4.394 & 6.591 & 48.334 \\
\hline 2.197 & 2.197 & 6.591 \\
\hline 4.394 & 2.197 & 2.197 \\
\hline 4.394 & 2.197 & 4.394 \\
\hline 8.788 & 4.394 & 30.758 \\
\hline 8.788 & 6.591 & 268.034 \\
\hline 70.304 & 2.197 & 4.394 \\
\hline 2.197 & 2.197 & 32.955 \\
\hline 2.197 & 13.182 & 24.167 \\
\hline 4.394 & 2.197 & 10.985 \\
\hline 15.379 & 2.197 & 4.394 \\
\hline 2.197 & 2.197 & 2.197 \\
\hline 8.788 & 6.591 & 17.576 \\
\hline 4.394 & 17.576 & 13.182 \\
\hline 2.197 & 4.394 & 46.137 \\
\hline 276.822 & 2.197 & 2.197 \\
\hline 4.394 & 2.197 & 2.197 \\
\hline 76.895 & 2.197 & 10.985 \\
\hline 21.97 & 4.394 & 10.985 \\
\hline 4.394 & 68.107 & 6.591 \\
\hline 1904.8 & 2.197 & 4.394 \\
\hline 30.758 & 35.152 & 15.379 \\
\hline
\end{tabular}




\begin{tabular}{|c|c|c|}
\hline 2.197 & 61.516 & 175.76 \\
\hline 500.916 & 50.531 & 2.197 \\
\hline 2.197 & 2.197 & 2.197 \\
\hline 21.97 & 4.394 & 2.197 \\
\hline 70.304 & 4.394 & 8.788 \\
\hline 4.394 & 2.197 & 149.396 \\
\hline 4.394 & 2.197 & 4.394 \\
\hline 10.985 & 2.197 & 2.197 \\
\hline 72.501 & 2.197 & 4.394 \\
\hline 386.672 & 19.773 & 30.758 \\
\hline 39.546 & 2.197 & 4.394 \\
\hline 4.394 & 2.197 & 195.533 \\
\hline 30.758 & 2.197 & 2.197 \\
\hline 2.197 & 4.394 & 580.008 \\
\hline 19.773 & 4.394 & 8.788 \\
\hline 909.558 & 21.97 & 4.394 \\
\hline 4.394 & 15.379 & 10.985 \\
\hline 13.182 & 325.156 & 2.197 \\
\hline 17.576 & 2.197 & 8.788 \\
\hline 10.985 & 4.394 & 608.569 \\
\hline 180.154 & 70.304 & 5187.12 \\
\hline 226.291 & 3363.61 & 10.985 \\
\hline 19.773 & 2.197 & 26.364 \\
\hline 166.972 & 21.97 & 4101.8 \\
\hline 2.197 & 2.197 & 4.394 \\
\hline 6.591 & 151.593 & 35.152 \\
\hline 13.182 & 6.591 & 65.91 \\
\hline 2.197 & 4.394 & 19.773 \\
\hline 37.349 & 2.197 & 17.576 \\
\hline 2.197 & 2.197 & 32.955 \\
\hline 107.653 & 2.197 & 21.97 \\
\hline 10.985 & 2.197 & 61.516 \\
\hline 37.349 & 2.197 & 57.122 \\
\hline 4.394 & 17.576 & 85.683 \\
\hline 28.561 & 2.197 & 2.197 \\
\hline 4.394 & 470.158 & 208.715 \\
\hline 26.364 & 85.683 & 2.197 \\
\hline 65.91 & 6.591 & 10.985 \\
\hline 21.97 & 4.394 & 79.092 \\
\hline 6.591 & 2.197 & 6.591 \\
\hline 6.591 & 2.197 & 6.591 \\
\hline 46.137 & 2.197 & 13.182 \\
\hline 30.758 & 402.051 & 54.925 \\
\hline 2.197 & 2.197 & 6.591 \\
\hline 125.229 & 2.197 & 2.197 \\
\hline 4.394 & 37.349 & 2.197 \\
\hline 2.197 & 2.197 & 26.364 \\
\hline 210.912 & 13.182 & 10.985 \\
\hline 2.197 & 810.693 & 81.289 \\
\hline 2.197 & 30.758 & 2.197 \\
\hline
\end{tabular}




\begin{tabular}{|c|c|c|}
\hline 4.394 & 8.788 & 6.591 \\
\hline 10.985 & 21.97 & 15.379 \\
\hline 2.197 & 2.197 & 4.394 \\
\hline 4.394 & 259.246 & 39.546 \\
\hline 6.591 & 13.182 & 4.394 \\
\hline 2.197 & 13.182 & 21.97 \\
\hline 2.197 & 6.591 & 2.197 \\
\hline 10.985 & 2.197 & 13.182 \\
\hline 4.394 & 79.092 & 4.394 \\
\hline 17.576 & 6.591 & 322.959 \\
\hline 28.561 & 17.576 & 2.197 \\
\hline 39.546 & 2.197 & 2.197 \\
\hline 19.773 & 2.197 & 2.197 \\
\hline 4.394 & 2.197 & 2.197 \\
\hline 13.182 & 19.773 & 17.576 \\
\hline 46.137 & 2.197 & 4.394 \\
\hline 15.379 & 96.668 & 2.197 \\
\hline 8.788 & 2.197 & 6.591 \\
\hline 8.788 & 2.197 & 2.197 \\
\hline 2.197 & 2.197 & 2.197 \\
\hline 2.197 & 4.394 & 26.364 \\
\hline 15.379 & 4.394 & 50.531 \\
\hline 61.516 & 105.456 & 2188.21 \\
\hline 13.182 & 2.197 & 24.167 \\
\hline 4.394 & 2.197 & 4.394 \\
\hline 101.062 & 32.955 & 4.394 \\
\hline 13.182 & 10.985 & 2.197 \\
\hline 252.655 & 2.197 & 2.197 \\
\hline 4.394 & 287.807 & 15.379 \\
\hline 4.394 & 2.197 & 4.394 \\
\hline 197.73 & 2.197 & 10.985 \\
\hline 4.394 & 2.197 & 28.561 \\
\hline 4.394 & 2.197 & 52.728 \\
\hline 2.197 & 98.865 & 8.788 \\
\hline 26.364 & 13.182 & 2.197 \\
\hline 8.788 & 21.97 & 6.591 \\
\hline 6.591 & 39.546 & 10.985 \\
\hline 37.349 & 4.394 & 735.995 \\
\hline 4.394 & 2.197 & 79.092 \\
\hline 4.394 & 2.197 & 4.394 \\
\hline 208.715 & 2.197 & 2.197 \\
\hline 35.152 & 8.788 & 6.591 \\
\hline 4.394 & 15.379 & 505.31 \\
\hline 8.788 & 2.197 & 48.334 \\
\hline 224.094 & 2.197 & 2.197 \\
\hline 6.591 & 21.97 & 13.182 \\
\hline 13.182 & 72.501 & 2.197 \\
\hline 8.788 & 2.197 & 4.394 \\
\hline 79.092 & 76.895 & 2.197 \\
\hline 19.773 & 21.97 & 270.231 \\
\hline
\end{tabular}




\begin{tabular}{|c|c|c|}
\hline 2.197 & 26.364 & 2.197 \\
\hline 17.576 & 2.197 & 10.985 \\
\hline 2.197 & 37.349 & 2.197 \\
\hline 995.241 & 2.197 & 2.197 \\
\hline 2.197 & 2.197 & 19.773 \\
\hline 50.531 & 8.788 & 6.591 \\
\hline 10.985 & 4.394 & 6.591 \\
\hline 6.591 & 19.773 & 83.486 \\
\hline 2.197 & 2.197 & 2.197 \\
\hline 24.167 & 8.788 & 4.394 \\
\hline 2.197 & 10.985 & 2.197 \\
\hline 188.942 & 127.426 & 184.548 \\
\hline 2.197 & 2.197 & 61.516 \\
\hline 17.576 & 4.394 & 125.229 \\
\hline 2.197 & 10.985 & 35.152 \\
\hline 2.197 & 4.394 & 2.197 \\
\hline 4.394 & 6.591 & 2.197 \\
\hline 2.197 & 2.197 & 369.096 \\
\hline 4.394 & 70.304 & 283.413 \\
\hline 70.304 & 4.394 & 2.197 \\
\hline 13.182 & 54.925 & 366.899 \\
\hline 116.441 & 2.197 & 2.197 \\
\hline 2.197 & 311.974 & 26.364 \\
\hline 8.788 & 2.197 & 2.197 \\
\hline 2.197 & 4.394 & 2.197 \\
\hline 2.197 & 4.394 & 2.197 \\
\hline 6.591 & 2.197 & 2.197 \\
\hline 2.197 & 6.591 & 8.788 \\
\hline 2.197 & 2.197 & 4.394 \\
\hline 19.773 & 2.197 & 10.985 \\
\hline 2.197 & 4.394 & 2.197 \\
\hline 2.197 & 2.197 & 4.394 \\
\hline 35.152 & 63.713 & 28.561 \\
\hline 2.197 & 4.394 & 35.152 \\
\hline 17.576 & 4.394 & 39.546 \\
\hline 105.456 & 2.197 & 6.591 \\
\hline 10.985 & 35.152 & 4.394 \\
\hline 4.394 & 8.788 & 2.197 \\
\hline 79.092 & 35.152 & 309.777 \\
\hline 2.197 & 54.925 & 21.97 \\
\hline 10.985 & 2.197 & 2.197 \\
\hline 17.576 & 2.197 & 46.137 \\
\hline 15.379 & 76.895 & 1098.5 \\
\hline 116.441 & 52.728 & 13.182 \\
\hline 24.167 & 15.379 & 8.788 \\
\hline 13.182 & 70.304 & 54.925 \\
\hline 48.334 & 35.152 & 10.985 \\
\hline 37.349 & 2.197 & 4.394 \\
\hline 2.197 & 2.197 & 2.197 \\
\hline 2.197 & 2.197 & 2.197 \\
\hline
\end{tabular}




\begin{tabular}{|c|c|c|}
\hline 116.441 & 8.788 & 4.394 \\
\hline 37.349 & 2.197 & 10.985 \\
\hline 2.197 & 2.197 & 10.985 \\
\hline 250.458 & 4.394 & 98.865 \\
\hline 54.925 & 6.591 & 30.758 \\
\hline 13.182 & 2.197 & 54.925 \\
\hline 4.394 & 4.394 & 6.591 \\
\hline 2.197 & 15.379 & 6.591 \\
\hline 4.394 & 2.197 & 57.122 \\
\hline 180.154 & 2.197 & 2.197 \\
\hline 2.197 & 2.197 & 4.394 \\
\hline 28.561 & 8.788 & 70.304 \\
\hline 2.197 & 48.334 & 17.576 \\
\hline 2.197 & 2.197 & 2.197 \\
\hline 2.197 & 8.788 & 15.379 \\
\hline 6.591 & 10.985 & 10.985 \\
\hline 2.197 & 2.197 & 2.197 \\
\hline 4.394 & 2.197 & 35.152 \\
\hline 268.034 & 3400.96 & 2.197 \\
\hline 52.728 & 2.197 & 2.197 \\
\hline 2.197 & 4.394 & 10.985 \\
\hline 8.788 & 4.394 & 54.925 \\
\hline 175.76 & 2.197 & 13.182 \\
\hline 2.197 & 2.197 & 2.197 \\
\hline 6.591 & 37.349 & 2.197 \\
\hline 32.955 & 4.394 & 2.197 \\
\hline 2.197 & 4.394 & 10.985 \\
\hline 28.561 & 2.197 & 28.561 \\
\hline 4.394 & 8.788 & 6.591 \\
\hline 26.364 & 2.197 & 2.197 \\
\hline 83.486 & 6.591 & 2.197 \\
\hline 2.197 & 2.197 & 10.985 \\
\hline 39.546 & 10.985 & 2.197 \\
\hline 90.077 & 2.197 & 79.092 \\
\hline 10.985 & 37.349 & 2.197 \\
\hline 72.501 & 2.197 & 2.197 \\
\hline 4.394 & 10.985 & 13.182 \\
\hline 2.197 & 15.379 & 24.167 \\
\hline 204.321 & 8.788 & 285.61 \\
\hline 10.985 & 4.394 & 76.895 \\
\hline 68.107 & 2.197 & 2.197 \\
\hline 68.107 & 2.197 & 2.197 \\
\hline 8.788 & 2.197 & 4.394 \\
\hline 35.152 & 15.379 & 2.197 \\
\hline 2.197 & 4.394 & 26.364 \\
\hline 85.683 & 2.197 & 2.197 \\
\hline 6.591 & 41.743 & 37.349 \\
\hline 2.197 & 2.197 & 142.805 \\
\hline 19.773 & 2.197 & 76.895 \\
\hline 19.773 & 4.394 & 15.379 \\
\hline
\end{tabular}




\begin{tabular}{|c|c|c|}
\hline 63.713 & 191.139 & 19.773 \\
\hline 8.788 & 2.197 & 8.788 \\
\hline 8.788 & 32.955 & 8.788 \\
\hline 2.197 & 2.197 & 435.006 \\
\hline 21.97 & 6.591 & 43.94 \\
\hline 2.197 & 17.576 & 13.182 \\
\hline 2.197 & 292.201 & 2.197 \\
\hline 37.349 & 6.591 & 21.97 \\
\hline 17.576 & 371.293 & 13.182 \\
\hline 43.94 & 2.197 & 8.788 \\
\hline 6.591 & 2.197 & 6.591 \\
\hline 4.394 & 2.197 & 2.197 \\
\hline 13.182 & 10.985 & 4.394 \\
\hline 4.394 & 129.623 & 21.97 \\
\hline 4.394 & 8.788 & 10.985 \\
\hline 4.394 & 8.788 & 6.591 \\
\hline 10.985 & 19.773 & 83.486 \\
\hline 4.394 & 2.197 & 4.394 \\
\hline 4.394 & 4.394 & 4.394 \\
\hline 8.788 & 2.197 & 6.591 \\
\hline 4.394 & 6.591 & 8.788 \\
\hline 6.591 & 26.364 & 2.197 \\
\hline 24.167 & 10.985 & 109.85 \\
\hline 13.182 & 2.197 & 4.394 \\
\hline 4.394 & 2.197 & 2.197 \\
\hline 2.197 & 26.364 & 2.197 \\
\hline 4.394 & 6.591 & 2.197 \\
\hline 4.394 & 1863.06 & 2.197 \\
\hline 2.197 & 2.197 & 2.197 \\
\hline 6.591 & 230.685 & 2.197 \\
\hline 2.197 & 2.197 & 54.925 \\
\hline 4.394 & 2.197 & 85.683 \\
\hline 103.259 & 2.197 & 2.197 \\
\hline 2.197 & 2.197 & 70.304 \\
\hline 107.653 & 2.197 & 2.197 \\
\hline 15.379 & 17.576 & 6.591 \\
\hline 2.197 & 2.197 & 30.758 \\
\hline 96.668 & 2.197 & 46.137 \\
\hline 37.349 & 6.591 & 24.167 \\
\hline 17.576 & 4.394 & 4.394 \\
\hline 8.788 & 2.197 & 2.197 \\
\hline 450.385 & 10.985 & 118.638 \\
\hline 65.91 & 2.197 & 2.197 \\
\hline 2.197 & 2.197 & 2.197 \\
\hline 2.197 & 175.76 & 6.591 \\
\hline 197.73 & 21.97 & 39.546 \\
\hline 32.955 & 2.197 & 37.349 \\
\hline 2.197 & 2.197 & 2.197 \\
\hline 13.182 & 2.197 & 10.985 \\
\hline 13.182 & 369.096 & 8.788 \\
\hline
\end{tabular}




\begin{tabular}{|c|c|c|}
\hline 2.197 & 408.642 & 2.197 \\
\hline 8.788 & 2.197 & 2.197 \\
\hline 105.456 & 52.728 & 505.31 \\
\hline 10.985 & 654.706 & 4.394 \\
\hline 2.197 & 522.886 & 79.092 \\
\hline 79.092 & 41.743 & 26.364 \\
\hline 13.182 & 6.591 & 17.576 \\
\hline 13.182 & 2.197 & 85.683 \\
\hline 15.379 & 2.197 & 4.394 \\
\hline 112.047 & 21.97 & 138.411 \\
\hline 2.197 & 2.197 & 127.426 \\
\hline 17.576 & 4.394 & 2.197 \\
\hline 8.788 & 37.349 & 101.062 \\
\hline 2.197 & 26.364 & 2.197 \\
\hline 52.728 & 17.576 & 24.167 \\
\hline 2.197 & 125.229 & 13.182 \\
\hline 68.107 & 32.955 & 10.985 \\
\hline 57.122 & 30.758 & 2.197 \\
\hline 2.197 & 2.197 & 918.346 \\
\hline 4.394 & 6.591 & 2.197 \\
\hline 2.197 & 6.591 & 6.591 \\
\hline 125.229 & 8.788 & 41.743 \\
\hline 2.197 & 61.516 & 21.97 \\
\hline 4.394 & 26.364 & 228.488 \\
\hline 57.122 & 76.895 & 2.197 \\
\hline 6.591 & 4.394 & 39.546 \\
\hline 6.591 & 2.197 & 2.197 \\
\hline 30.758 & 2.197 & 2.197 \\
\hline 6.591 & 2.197 & 123.032 \\
\hline 39.546 & 92.274 & 2506.78 \\
\hline 4.394 & 2.197 & 2.197 \\
\hline 10.985 & 4.394 & 8.788 \\
\hline 32.955 & 13.182 & 2.197 \\
\hline 76.895 & 8.788 & 6.591 \\
\hline 61.516 & 2.197 & 4.394 \\
\hline 2.197 & 2.197 & 10.985 \\
\hline 162.578 & 19.773 & 2.197 \\
\hline 101.062 & 6.591 & 8.788 \\
\hline 142.805 & 4.394 & 8.788 \\
\hline 2.197 & 26.364 & 8.788 \\
\hline 2.197 & 2.197 & 4.394 \\
\hline 2.197 & 2.197 & 2.197 \\
\hline 300.989 & 123.032 & 24.167 \\
\hline 4.394 & 19.773 & 6.591 \\
\hline 17.576 & 21.97 & 48.334 \\
\hline 4.394 & 2.197 & 4.394 \\
\hline 4.394 & 2.197 & 2.197 \\
\hline 6.591 & 10.985 & 30.758 \\
\hline 2.197 & 15.379 & 2.197 \\
\hline 54.925 & 2.197 & 2.197 \\
\hline
\end{tabular}




\begin{tabular}{|c|c|c|}
\hline 2.197 & 68.107 & 2.197 \\
\hline 2.197 & 13.182 & 2.197 \\
\hline 26.364 & 2.197 & 4.394 \\
\hline 2.197 & 2.197 & 17.576 \\
\hline 54.925 & 2.197 & 252.655 \\
\hline 4.394 & 4.394 & 21.97 \\
\hline 17.576 & 4.394 & 24.167 \\
\hline 76.895 & 6.591 & 79.092 \\
\hline 72.501 & 4.394 & 4.394 \\
\hline 57.122 & 2.197 & 4.394 \\
\hline 13.182 & 17.576 & 4.394 \\
\hline 4.394 & 10.985 & 8.788 \\
\hline 153.79 & 6.591 & 2.197 \\
\hline 6.591 & 37.349 & 4.394 \\
\hline 2.197 & 2.197 & 17.576 \\
\hline 2.197 & 13.182 & 8.788 \\
\hline 10.985 & 2.197 & 8.788 \\
\hline 79.092 & 4.394 & 314.171 \\
\hline 2.197 & 2.197 & 160.381 \\
\hline 2.197 & 120.835 & 2.197 \\
\hline 6.591 & 2.197 & 2.197 \\
\hline 46.137 & 35.152 & 68.107 \\
\hline 2.197 & 17.576 & 2.197 \\
\hline 30.758 & 13.182 & 4.394 \\
\hline 2.197 & 8.788 & 355.914 \\
\hline 48.334 & 6.591 & 35.152 \\
\hline 63.713 & 10.985 & 4.394 \\
\hline 4.394 & 2.197 & 4.394 \\
\hline 26.364 & 2.197 & 54.925 \\
\hline 8.788 & 28.561 & 2.197 \\
\hline 2.197 & 2.197 & 112.047 \\
\hline 2.197 & 2.197 & 2695.72 \\
\hline 4.394 & 2.197 & 13.182 \\
\hline 325.156 & 52.728 & 28.561 \\
\hline 8.788 & 41.743 & 59.319 \\
\hline 123.032 & 61.516 & 6.591 \\
\hline 46.137 & 6.591 & 2.197 \\
\hline 65.91 & 2.197 & 2.197 \\
\hline 2.197 & 8.788 & 2.197 \\
\hline 2.197 & 13.182 & 4.394 \\
\hline 30.758 & 4.394 & 15.379 \\
\hline 26.364 & 2.197 & 101.062 \\
\hline 419.627 & 393.263 & 2.197 \\
\hline 59.319 & 13.182 & 39.546 \\
\hline 2.197 & 2.197 & 2.197 \\
\hline 10.985 & 81.289 & 2.197 \\
\hline 24.167 & 2.197 & 6.591 \\
\hline 4.394 & 76.895 & 6.591 \\
\hline 26.364 & 26.364 & 2.197 \\
\hline 142.805 & 2.197 & 4.394 \\
\hline
\end{tabular}




\begin{tabular}{|c|c|c|}
\hline 2.197 & 6.591 & 2.197 \\
\hline 68.107 & 2.197 & 2.197 \\
\hline 2.197 & 4.394 & 2.197 \\
\hline 13.182 & 4.394 & 2.197 \\
\hline 4.394 & 15.379 & 65.91 \\
\hline 2.197 & 202.124 & 92.274 \\
\hline 32.955 & 19.773 & 2.197 \\
\hline 28.561 & 46.137 & 4.394 \\
\hline 30.758 & 8.788 & 2.197 \\
\hline 26.364 & 2.197 & 151.593 \\
\hline 2.197 & 2.197 & 4.394 \\
\hline 968.877 & 10.985 & 696.449 \\
\hline 162.578 & 2.197 & 2.197 \\
\hline 2.197 & 2.197 & 2.197 \\
\hline 2.197 & 2.197 & 2.197 \\
\hline 17.576 & 6.591 & 2.197 \\
\hline 4.394 & 17.576 & 4.394 \\
\hline 6.591 & 228.488 & 2.197 \\
\hline 13.182 & 6.591 & 8.788 \\
\hline 17.576 & 2.197 & 235.079 \\
\hline 8.788 & 24.167 & 2.197 \\
\hline 32.955 & 2.197 & 2.197 \\
\hline 2.197 & 17.576 & 54.925 \\
\hline 2.197 & 2.197 & 2.197 \\
\hline 8.788 & 2.197 & 145.002 \\
\hline 112.047 & 114.244 & 6.591 \\
\hline 2.197 & 2.197 & 8.788 \\
\hline 2.197 & 2.197 & 19.773 \\
\hline 10.985 & 2.197 & 2.197 \\
\hline 2.197 & 103.259 & 52.728 \\
\hline 217.503 & 2.197 & 21.97 \\
\hline 2.197 & 2.197 & 2.197 \\
\hline 43.94 & 2.197 & 24.167 \\
\hline 2.197 & 2.197 & 4.394 \\
\hline 2.197 & 4.394 & 2.197 \\
\hline 13.182 & 32.955 & 4.394 \\
\hline 2.197 & 61.516 & 4.394 \\
\hline 21.97 & 2.197 & 2.197 \\
\hline 125.229 & 37.349 & 4.394 \\
\hline 57.122 & 4.394 & 2.197 \\
\hline 57.122 & 2.197 & 6.591 \\
\hline 8.788 & 4.394 & 2.197 \\
\hline 4.394 & 2.197 & 4.394 \\
\hline 4.394 & 4.394 & 46.137 \\
\hline 2.197 & 2.197 & 2.197 \\
\hline 6.591 & 2.197 & 6.591 \\
\hline 4.394 & 6.591 & 52.728 \\
\hline 2.197 & 2.197 & 250.458 \\
\hline 2.197 & 158.184 & 19.773 \\
\hline 2.197 & 2.197 & 6.591 \\
\hline
\end{tabular}




\begin{tabular}{|c|c|c|}
\hline 63.713 & 17.576 & 19.773 \\
\hline 2.197 & 2.197 & 795.314 \\
\hline 10.985 & 2.197 & 39.546 \\
\hline 2.197 & 2.197 & 347.126 \\
\hline 4.394 & 8.788 & 2.197 \\
\hline 28.561 & 2.197 & 2.197 \\
\hline 6.591 & 2.197 & 4.394 \\
\hline 30.758 & 63.713 & 65.91 \\
\hline 6.591 & 2.197 & 8.788 \\
\hline 48.334 & 2.197 & 61.516 \\
\hline 96.668 & 4.394 & 6.591 \\
\hline 171.366 & 6.591 & 32.955 \\
\hline 2.197 & 4.394 & 4.394 \\
\hline 43.94 & 2.197 & 8.788 \\
\hline 4.394 & 2.197 & 10.985 \\
\hline 2.197 & 6.591 & 2.197 \\
\hline 52.728 & 439.4 & 2.197 \\
\hline 28.561 & 92.274 & 4497.26 \\
\hline 8.788 & 8.788 & 2.197 \\
\hline 4.394 & 8.788 & 553.644 \\
\hline 2.197 & 30.758 & 32.955 \\
\hline 2.197 & 6.591 & 2.197 \\
\hline 2.197 & 15.379 & 109.85 \\
\hline 39.546 & 15.379 & 6.591 \\
\hline 8.788 & 87.88 & 15.379 \\
\hline 2.197 & 24.167 & 19.773 \\
\hline 6.591 & 4.394 & 160.381 \\
\hline 19.773 & 2.197 & 17.576 \\
\hline 2181.62 & 2.197 & 4.394 \\
\hline 19.773 & 461.37 & 19.773 \\
\hline 184.548 & 2.197 & 79.092 \\
\hline 41.743 & 48.334 & 2.197 \\
\hline 2.197 & 2.197 & 2.197 \\
\hline 35.152 & 2.197 & 2.197 \\
\hline 2.197 & 114.244 & 2.197 \\
\hline 98.865 & 48.334 & 2.197 \\
\hline 2.197 & 61.516 & 2.197 \\
\hline 2.197 & 43.94 & 26.364 \\
\hline 8.788 & 2.197 & 210.912 \\
\hline 68.107 & 2.197 & 13.182 \\
\hline 4.394 & 2.197 & 65.91 \\
\hline 2.197 & 2.197 & 2.197 \\
\hline 2.197 & 15.379 & 13.182 \\
\hline 26.364 & 8.788 & 59.319 \\
\hline 68.107 & 2.197 & 94.471 \\
\hline 21.97 & 2.197 & 2.197 \\
\hline 4.394 & 30.758 & 6.591 \\
\hline 4.394 & 39.546 & 2.197 \\
\hline 2.197 & 2.197 & 87.88 \\
\hline 4.394 & 601.978 & 17.576 \\
\hline
\end{tabular}




\begin{tabular}{|c|c|c|}
\hline 81.289 & 2.197 & 59.319 \\
\hline 15.379 & 4.394 & 4.394 \\
\hline 10.985 & 35.152 & 6.591 \\
\hline 17.576 & 2.197 & 2.197 \\
\hline 2.197 & 4.394 & 2.197 \\
\hline 4.394 & 2.197 & 2.197 \\
\hline 15.379 & 2.197 & 2.197 \\
\hline 48.334 & 2.197 & 21.97 \\
\hline 2.197 & 2.197 & 24.167 \\
\hline 92.274 & 32.955 & 237.276 \\
\hline 254.852 & 2.197 & 4.394 \\
\hline 6.591 & 2.197 & 261.443 \\
\hline 37.349 & 17.576 & 19.773 \\
\hline 2.197 & 8.788 & 4.394 \\
\hline 4.394 & 26.364 & 4.394 \\
\hline 4.394 & 17.576 & 2.197 \\
\hline 6.591 & 26.364 & 21.97 \\
\hline 2.197 & 4.394 & 4.394 \\
\hline 35.152 & 4.394 & 2.197 \\
\hline 24.167 & 10.985 & 8.788 \\
\hline 57.122 & 2.197 & 15.379 \\
\hline 68.107 & 2.197 & 2.197 \\
\hline 4.394 & 2.197 & 2.197 \\
\hline 2.197 & 41.743 & 101.062 \\
\hline 2.197 & 28.561 & 4.394 \\
\hline 2.197 & 2.197 & 4.394 \\
\hline 13.182 & 2.197 & 8.788 \\
\hline 134.017 & 2.197 & 50.531 \\
\hline 4.394 & 13.182 & 6.591 \\
\hline 2.197 & 46.137 & 6.591 \\
\hline 2.197 & 8.788 & 4.394 \\
\hline 54.925 & 2.197 & 57.122 \\
\hline 4.394 & 2.197 & 4.394 \\
\hline 2.197 & 4.394 & 4.394 \\
\hline 17.576 & 2.197 & 63.713 \\
\hline 2.197 & 2.197 & 98.865 \\
\hline 2.197 & 4.394 & 2.197 \\
\hline 46.137 & 4.394 & 32.955 \\
\hline 76.895 & 13.182 & 2.197 \\
\hline 2.197 & 2.197 & 129.623 \\
\hline 134.017 & 28.561 & 4.394 \\
\hline 2.197 & 2.197 & 43.94 \\
\hline 2.197 & 2.197 & 10.985 \\
\hline 19.773 & 24.167 & 17.576 \\
\hline 46.137 & 2.197 & 35.152 \\
\hline 2.197 & 2.197 & 59.319 \\
\hline 74.698 & 8.788 & 68.107 \\
\hline 2.197 & 155.987 & 15.379 \\
\hline 2.197 & 2.197 & 30.758 \\
\hline 6.591 & 4.394 & 13.182 \\
\hline
\end{tabular}




\begin{tabular}{|c|c|c|}
\hline 10.985 & 10.985 & 4.394 \\
\hline 2.197 & 15910.7 & 432.809 \\
\hline 54.925 & 10.985 & 46.137 \\
\hline 6.591 & 2.197 & 230.685 \\
\hline 2.197 & 2.197 & 21.97 \\
\hline 21.97 & 87.88 & 70.304 \\
\hline 50.531 & 10.985 & 30.758 \\
\hline 105.456 & 4.394 & 2.197 \\
\hline 32.955 & 37.349 & 4.394 \\
\hline 15.379 & 10.985 & 94.471 \\
\hline 2.197 & 4.394 & 112.047 \\
\hline 8.788 & 37.349 & 2.197 \\
\hline 4.394 & 17.576 & 2.197 \\
\hline 2.197 & 39.546 & 8.788 \\
\hline 169.169 & 8.788 & 2.197 \\
\hline 41.743 & 28.561 & 19.773 \\
\hline 268.034 & 41.743 & 4.394 \\
\hline 4.394 & 2.197 & 2.197 \\
\hline 19.773 & 2.197 & 4.394 \\
\hline 79.092 & 17.576 & 52.728 \\
\hline 2.197 & 2.197 & 24.167 \\
\hline 4.394 & 2.197 & 8.788 \\
\hline 17.576 & 2.197 & 2.197 \\
\hline 4.394 & 2.197 & 6.591 \\
\hline 2.197 & 8.788 & 4.394 \\
\hline 4.394 & 72.501 & 6.591 \\
\hline 2.197 & 2.197 & 103.259 \\
\hline 4.394 & 6.591 & 4.394 \\
\hline 39.546 & 2.197 & 4.394 \\
\hline 4.394 & 2.197 & 4.394 \\
\hline 2.197 & 2.197 & 2.197 \\
\hline 2.197 & 10.985 & 13.182 \\
\hline 2.197 & 6.591 & 2.197 \\
\hline 197.73 & 2.197 & 13.182 \\
\hline 26.364 & 2.197 & 2.197 \\
\hline 8.788 & 2.197 & 2.197 \\
\hline 4.394 & 2.197 & 10.985 \\
\hline 4.394 & 8.788 & 151.593 \\
\hline 4.394 & 2.197 & 2.197 \\
\hline 2.197 & 6.591 & 4.394 \\
\hline 2.197 & 2.197 & 8.788 \\
\hline 4.394 & 10.985 & 72.501 \\
\hline 6.591 & 8.788 & 4.394 \\
\hline 112.047 & 41.743 & 2.197 \\
\hline 6.591 & 2.197 & 2.197 \\
\hline 4.394 & 17.576 & 24.167 \\
\hline 2.197 & 8.788 & 8.788 \\
\hline 57.122 & 2.197 & 4.394 \\
\hline 2.197 & 2.197 & 30.758 \\
\hline 188.942 & 2.197 & 6.591 \\
\hline
\end{tabular}




\begin{tabular}{|c|c|c|}
\hline 103.259 & 2.197 & 17.576 \\
\hline 13.182 & 2.197 & 74.698 \\
\hline 2.197 & 24.167 & 54.925 \\
\hline 153.79 & 8.788 & 13.182 \\
\hline 65.91 & 166.972 & 4.394 \\
\hline 13.182 & 41.743 & 6.591 \\
\hline 4.394 & 13.182 & 2.197 \\
\hline 6.591 & 13.182 & 24.167 \\
\hline 2.197 & 39.546 & 735.995 \\
\hline 2.197 & 15.379 & 4.394 \\
\hline 54.925 & 4.394 & 2.197 \\
\hline 41.743 & 4.394 & 8.788 \\
\hline 2.197 & 2.197 & 103.259 \\
\hline 52.728 & 2.197 & 59.319 \\
\hline 21.97 & 428.415 & 68.107 \\
\hline 107.653 & 2.197 & 13.182 \\
\hline 19.773 & 2.197 & 4.394 \\
\hline 4.394 & 2.197 & 21.97 \\
\hline 39.546 & 10.985 & 107.653 \\
\hline 287.807 & 32.955 & 2.197 \\
\hline 19.773 & 17.576 & 2.197 \\
\hline 6.591 & 2.197 & 85.683 \\
\hline 4.394 & 2.197 & 2.197 \\
\hline 24.167 & 4.394 & 37.349 \\
\hline 54.925 & 96.668 & 15.379 \\
\hline 4.394 & 4.394 & 28.561 \\
\hline 2.197 & 2.197 & 10.985 \\
\hline 4.394 & 4.394 & 26.364 \\
\hline 2.197 & 2.197 & 4.394 \\
\hline 2.197 & 4.394 & 2.197 \\
\hline 2.197 & 2.197 & 2.197 \\
\hline 2.197 & 2.197 & 2.197 \\
\hline 6.591 & 74.698 & 94.471 \\
\hline 19.773 & 2.197 & 494.325 \\
\hline 17.576 & 19.773 & 15.379 \\
\hline 2.197 & 4.394 & 21.97 \\
\hline 19.773 & 13.182 & 2.197 \\
\hline 17.576 & 6.591 & 2.197 \\
\hline 2.197 & 2.197 & 2.197 \\
\hline 2.197 & 15.379 & 2.197 \\
\hline 6.591 & 136.214 & 2.197 \\
\hline 10.985 & 4.394 & 2.197 \\
\hline 21.97 & 10.985 & 2.197 \\
\hline 21.97 & 2.197 & 867.815 \\
\hline 19.773 & 28.561 & 2.197 \\
\hline 2.197 & 2.197 & 15.379 \\
\hline 10.985 & 8.788 & 2.197 \\
\hline 4.394 & 4.394 & 2.197 \\
\hline 13.182 & 2.197 & 4.394 \\
\hline 8.788 & 4.394 & 13.182 \\
\hline
\end{tabular}




\begin{tabular}{|c|c|c|}
\hline 145.002 & 24.167 & 2.197 \\
\hline 17.576 & 2.197 & 10.985 \\
\hline 4.394 & 4.394 & 39.546 \\
\hline 70.304 & 4.394 & 127.426 \\
\hline 68.107 & 2.197 & 10.985 \\
\hline 8.788 & 4.394 & 2.197 \\
\hline 26.364 & 2.197 & 149.396 \\
\hline 2.197 & 28.561 & 4.394 \\
\hline 2.197 & 4.394 & 8.788 \\
\hline 123.032 & 2.197 & 8.788 \\
\hline 10.985 & 2.197 & 10.985 \\
\hline 96.668 & 2.197 & 10.985 \\
\hline 57.122 & 2.197 & 15.379 \\
\hline 8.788 & 2.197 & 2.197 \\
\hline 15.379 & 112.047 & 46.137 \\
\hline 4.394 & 2.197 & 215.306 \\
\hline 2.197 & 2.197 & 4.394 \\
\hline 13.182 & 2.197 & 10.985 \\
\hline 17.576 & 2.197 & 2.197 \\
\hline 10.985 & 6.591 & 37.349 \\
\hline 2.197 & 30.758 & 13.182 \\
\hline 4.394 & 17.576 & 28.561 \\
\hline 4.394 & 2.197 & 4.394 \\
\hline 2.197 & 6.591 & 4.394 \\
\hline 50.531 & 19.773 & 6.591 \\
\hline 10.985 & 6.591 & 17.576 \\
\hline 109.85 & 127.426 & 13.182 \\
\hline 81.289 & 219.7 & 41.743 \\
\hline 2.197 & 659.1 & 8.788 \\
\hline 15.379 & 2.197 & 2.197 \\
\hline 19.773 & 7454.42 & 261.443 \\
\hline 4.394 & 19.773 & 10.985 \\
\hline 28.561 & 21.97 & 57.122 \\
\hline 96.668 & 2.197 & 2.197 \\
\hline 4.394 & 2.197 & 6.591 \\
\hline 4.394 & 8.788 & 2.197 \\
\hline 4.394 & 8.788 & 2.197 \\
\hline 2.197 & 4.394 & 2.197 \\
\hline 32.955 & 2.197 & 191.139 \\
\hline 349.323 & 2.197 & 46.137 \\
\hline 103.259 & 2.197 & 2.197 \\
\hline 19.773 & 2.197 & 83.486 \\
\hline 35.152 & 2.197 & 98.865 \\
\hline 4.394 & 4.394 & 32.955 \\
\hline 26.364 & 2.197 & 2.197 \\
\hline 2.197 & 48.334 & 41.743 \\
\hline 2.197 & 114.244 & 6.591 \\
\hline 30.758 & 41.743 & 2.197 \\
\hline 54.925 & 2.197 & 2.197 \\
\hline 79.092 & 13.182 & 8.788 \\
\hline
\end{tabular}




\begin{tabular}{|c|c|c|}
\hline 24.167 & 2.197 & 2.197 \\
\hline 13.182 & 4.394 & 738.192 \\
\hline 149.396 & 6.591 & 595.387 \\
\hline 2.197 & 2.197 & 2.197 \\
\hline 4.394 & 13.182 & 4.394 \\
\hline 30.758 & 8.788 & 24.167 \\
\hline 6.591 & 4.394 & 2.197 \\
\hline 41.743 & 32.955 & 17.576 \\
\hline 4.394 & 4.394 & 59.319 \\
\hline 50.531 & 268.034 & 17.576 \\
\hline 15.379 & 2.197 & 2.197 \\
\hline 21.97 & 2.197 & 35.152 \\
\hline 2.197 & 2.197 & 6.591 \\
\hline 6.591 & 48.334 & 4.394 \\
\hline 134.017 & 252.655 & 8.788 \\
\hline 2.197 & 43.94 & 76.895 \\
\hline 8.788 & 2.197 & 15.379 \\
\hline 26.364 & 37.349 & 28.561 \\
\hline 430.612 & 2.197 & 76.895 \\
\hline 2.197 & 4.394 & 43.94 \\
\hline 13.182 & 19.773 & 54.925 \\
\hline 2.197 & 2.197 & 4.394 \\
\hline 8.788 & 2.197 & 63.713 \\
\hline 90.077 & 2.197 & 6.591 \\
\hline 13.182 & 123.032 & 10.985 \\
\hline 2.197 & 21.97 & 149.396 \\
\hline 2.197 & 30.758 & 114.244 \\
\hline 4.394 & 8.788 & 8.788 \\
\hline 52.728 & 59.319 & 2.197 \\
\hline 52.728 & 90.077 & 2.197 \\
\hline 19.773 & 4.394 & 10.985 \\
\hline 2.197 & 929.331 & 2.197 \\
\hline 8.788 & 10.985 & 4.394 \\
\hline 48.334 & 6.591 & 8.788 \\
\hline 50.531 & 8.788 & 17.576 \\
\hline 2.197 & 6.591 & 6.591 \\
\hline 2.197 & 582.205 & 32.955 \\
\hline 43.94 & 2.197 & 4.394 \\
\hline 19.773 & 46.137 & 50.531 \\
\hline 2.197 & 28.561 & 30.758 \\
\hline 61.516 & 2.197 & 4.394 \\
\hline 2.197 & 2.197 & 6.591 \\
\hline 2.197 & 2.197 & 15.379 \\
\hline 2.197 & 13.182 & 39.546 \\
\hline 68.107 & 4.394 & 4.394 \\
\hline 65.91 & 2.197 & 15009.9 \\
\hline 4.394 & 2.197 & 2.197 \\
\hline 10.985 & 2.197 & 2.197 \\
\hline 15.379 & 2.197 & 208.715 \\
\hline 6.591 & 30.758 & 2.197 \\
\hline
\end{tabular}




\begin{tabular}{|c|c|c|}
\hline 10.985 & 17.576 & 2.197 \\
\hline 4.394 & 15.379 & 57.122 \\
\hline 2.197 & 6.591 & 28.561 \\
\hline 63.713 & 21.97 & 2.197 \\
\hline 116.441 & 10.985 & 6.591 \\
\hline 13.182 & 749.177 & 105.456 \\
\hline 15.379 & 2.197 & 2.197 \\
\hline 39.546 & 2.197 & 2.197 \\
\hline 6.591 & 2.197 & 26.364 \\
\hline 43.94 & 17.576 & 15.379 \\
\hline 39.546 & 2.197 & 2.197 \\
\hline 2.197 & 2.197 & 26.364 \\
\hline 4.394 & 43.94 & 2.197 \\
\hline 6.591 & 2.197 & 358.111 \\
\hline 4.394 & 65.91 & 41.743 \\
\hline 21.97 & 2.197 & 2.197 \\
\hline 17.576 & 2.197 & 15.379 \\
\hline 46.137 & 6.591 & 199.927 \\
\hline 2.197 & 6.591 & 2.197 \\
\hline 21.97 & 13.182 & 6.591 \\
\hline 70.304 & 10.985 & 140.608 \\
\hline 76.895 & 30.758 & 4.394 \\
\hline 6.591 & 241.67 & 8.788 \\
\hline 2.197 & 4.394 & 2.197 \\
\hline 59.319 & 2.197 & 6.591 \\
\hline 8.788 & 30.758 & 2.197 \\
\hline 68.107 & 8.788 & 2.197 \\
\hline 6.591 & 6.591 & 76.895 \\
\hline 120.835 & 2.197 & 2.197 \\
\hline 6.591 & 2.197 & 2.197 \\
\hline 8.788 & 373.49 & 26.364 \\
\hline 72.501 & 10.985 & 145.002 \\
\hline 2.197 & 2.197 & 13.182 \\
\hline 39.546 & 2.197 & 4.394 \\
\hline 35.152 & 2.197 & 39.546 \\
\hline 24.167 & 21.97 & 13.182 \\
\hline 28.561 & 41.743 & 2.197 \\
\hline 6.591 & 2.197 & 164.775 \\
\hline 2.197 & 2.197 & 57.122 \\
\hline 2.197 & 2.197 & 129.623 \\
\hline 6.591 & 8.788 & 8.788 \\
\hline 2.197 & 2.197 & 4.394 \\
\hline 19.773 & 1318.2 & 15.379 \\
\hline 70.304 & 193.336 & 57.122 \\
\hline 17.576 & 4.394 & 127.426 \\
\hline 8.788 & 155.987 & 208.715 \\
\hline 4.394 & 2.197 & 30.758 \\
\hline 2.197 & 2.197 & 50.531 \\
\hline 35.152 & 17.576 & 15.379 \\
\hline 37.349 & 2.197 & 30.758 \\
\hline
\end{tabular}




\begin{tabular}{|c|c|c|}
\hline 478.946 & 30.758 & 19.773 \\
\hline 46.137 & 2.197 & 6.591 \\
\hline 125.229 & 2.197 & 4.394 \\
\hline 2.197 & 43.94 & 4.394 \\
\hline 2.197 & 2.197 & 2.197 \\
\hline 2.197 & 8.788 & 68.107 \\
\hline 70.304 & 2.197 & 6.591 \\
\hline 8.788 & 300.989 & 6.591 \\
\hline 17.576 & 2.197 & 2.197 \\
\hline 15.379 & 4.394 & 487.734 \\
\hline 32.955 & 155.987 & 8.788 \\
\hline 30.758 & 50.531 & 72.501 \\
\hline 21.97 & 175.76 & 4.394 \\
\hline 8.788 & 35.152 & 6.591 \\
\hline 13.182 & 6.591 & 21.97 \\
\hline 13.182 & 6.591 & 6.591 \\
\hline 2.197 & 41.743 & 4.394 \\
\hline 54.925 & 145.002 & 4.394 \\
\hline 30.758 & 2.197 & 10.985 \\
\hline 6.591 & 160.381 & 10.985 \\
\hline 2.197 & 63.713 & 21.97 \\
\hline 177.957 & 2.197 & 4.394 \\
\hline 90.077 & 43.94 & 10.985 \\
\hline 68.107 & 162.578 & 2.197 \\
\hline 2.197 & 4.394 & 2.197 \\
\hline 4.394 & 4.394 & 2.197 \\
\hline 17.576 & 43.94 & 2.197 \\
\hline 35.152 & 2.197 & 2.197 \\
\hline 2.197 & 4.394 & 6.591 \\
\hline 2.197 & 2.197 & 2.197 \\
\hline 10.985 & 21.97 & 2.197 \\
\hline 19.773 & 114.244 & 4.394 \\
\hline 2.197 & 6.591 & 24.167 \\
\hline 2.197 & 241.67 & 61.516 \\
\hline 2.197 & 2.197 & 28.561 \\
\hline 70.304 & 2.197 & 70.304 \\
\hline 2.197 & 17.576 & 4.394 \\
\hline 2.197 & 2.197 & 35.152 \\
\hline 2.197 & 2.197 & 10.985 \\
\hline 19.773 & 10.985 & 2.197 \\
\hline 4.394 & 2.197 & 6.591 \\
\hline 13.182 & 6.591 & 4.394 \\
\hline 32.955 & 15.379 & 2.197 \\
\hline 32.955 & 4.394 & 2.197 \\
\hline 41.743 & 2.197 & 2.197 \\
\hline 4.394 & 94.471 & 2.197 \\
\hline 8.788 & 204.321 & 2.197 \\
\hline 79.092 & 4.394 & 2.197 \\
\hline 4.394 & 10.985 & 2.197 \\
\hline 17.576 & 21.97 & 2.197 \\
\hline
\end{tabular}




$\begin{array}{rrr}136.214 & 85.683 & 2.197 \\ 2.197 & 2.197 & 41.743 \\ 2.197 & 8.788 & 50.531 \\ 4.394 & 2.197 & 59.319 \\ 2.197 & 2.197 & 134.017 \\ 4.394 & 6.591 & 43.94 \\ 6.591 & 2.197 & 101.062 \\ 4.394 & 28.561 & 2.197 \\ 15.379 & 35.152 & 26.364 \\ 13.182 & 39.546 & 30.758 \\ 54.925 & 6.591 & 2.197 \\ 10.985 & 2.197 & 2.197 \\ 2.197 & 35.152 & 48.334 \\ 6.591 & 4.394 & 4.394 \\ 10.985 & 2.197 & 2.197 \\ 2.197 & 17.576 & 26.364 \\ 17.576 & 2.197 & 221.897 \\ 30.758 & 3686.57 & 13.182 \\ 2.197 & 2.197 & 2.197 \\ 8.788 & 3.197 \\ 17.576 & 2.394 & 2.1957 \\ 2.197 & 2.197 & 50.531 \\ 17.576 & 2.394 & 83.486 \\ 4.394 & 2.197 & \end{array}$




\begin{tabular}{|c|c|c|}
\hline 252.655 & 2.197 & 13.182 \\
\hline 6.591 & 4.394 & 8.788 \\
\hline 2.197 & 4.394 & 114.244 \\
\hline 4.394 & 4.394 & 24.167 \\
\hline 2.197 & 19.773 & 68.107 \\
\hline 2.197 & 2.197 & 2.197 \\
\hline 2.197 & 43.94 & 13.182 \\
\hline 57.122 & 4.394 & 2.197 \\
\hline 2.197 & 2.197 & 2.197 \\
\hline 2.197 & 24.167 & 21.97 \\
\hline 70.304 & 8.788 & 10.985 \\
\hline 2.197 & 8.788 & 13.182 \\
\hline 90.077 & 2.197 & 4.394 \\
\hline 72.501 & 17.576 & 2.197 \\
\hline 8.788 & 59.319 & 32.955 \\
\hline 419.627 & 15.379 & 206.518 \\
\hline 4.394 & 6.591 & 2.197 \\
\hline 13.182 & 32.955 & 2.197 \\
\hline 6.591 & 2.197 & 4.394 \\
\hline 21.97 & 2.197 & 237.276 \\
\hline 10.985 & 59.319 & 4.394 \\
\hline 10.985 & 2.197 & 101.062 \\
\hline 87.88 & 2.197 & 21.97 \\
\hline 32.955 & 2.197 & 2.197 \\
\hline 87.88 & 2.197 & 8.788 \\
\hline 26.364 & 2.197 & 2.197 \\
\hline 2.197 & 2.197 & 2.197 \\
\hline 4.394 & 2.197 & 4.394 \\
\hline 2.197 & 2.197 & 2.197 \\
\hline 2.197 & 8.788 & 4.394 \\
\hline 2.197 & 8.788 & 39.546 \\
\hline 10.985 & 37.349 & 8.788 \\
\hline 50.531 & 4.394 & 2.197 \\
\hline 276.822 & 2.197 & 13.182 \\
\hline 26.364 & 333.944 & 15.379 \\
\hline 4.394 & 10.985 & 43.94 \\
\hline 2.197 & 15.379 & 39.546 \\
\hline 2.197 & 4.394 & 2.197 \\
\hline 8.788 & 57.122 & 37.349 \\
\hline 21.97 & 2.197 & 10.985 \\
\hline 103.259 & 2.197 & 90.077 \\
\hline 2.197 & 4.394 & 35.152 \\
\hline 136.214 & 652.509 & 19.773 \\
\hline 2.197 & 2.197 & 24.167 \\
\hline 46.137 & 2.197 & 6.591 \\
\hline 8.788 & 140.608 & 98.865 \\
\hline 46.137 & 2.197 & 15.379 \\
\hline 17.576 & 2.197 & 169.169 \\
\hline 6.591 & 2.197 & 98.865 \\
\hline 4.394 & 21.97 & 8.788 \\
\hline
\end{tabular}




\begin{tabular}{|c|c|c|}
\hline 2.197 & 116.441 & 2.197 \\
\hline 8.788 & 2.197 & 57.122 \\
\hline 13.182 & 26.364 & 96.668 \\
\hline 177.957 & 4.394 & 17.576 \\
\hline 52.728 & 6.591 & 6.591 \\
\hline 10.985 & 2.197 & 13.182 \\
\hline 17.576 & 4.394 & 4.394 \\
\hline 162.578 & 17.576 & 15.379 \\
\hline 8.788 & 74.698 & 50.531 \\
\hline 39.546 & 13.182 & 4.394 \\
\hline 2.197 & 6.591 & 2.197 \\
\hline 2.197 & 290.004 & 155.987 \\
\hline 32.955 & 2.197 & 4.394 \\
\hline 2.197 & 2.197 & 6.591 \\
\hline 19.773 & 2.197 & 10.985 \\
\hline 224.094 & 2.197 & 2.197 \\
\hline 138.411 & 8.788 & 2.197 \\
\hline 2.197 & 70.304 & 4.394 \\
\hline 182.351 & 10.985 & 2.197 \\
\hline 2.197 & 8.788 & 8.788 \\
\hline 4.394 & 103.259 & 98.865 \\
\hline 2.197 & 2.197 & 39.546 \\
\hline 26.364 & 2.197 & 2.197 \\
\hline 13.182 & 94.471 & 6.591 \\
\hline 2.197 & 2.197 & 2.197 \\
\hline 4.394 & 13.182 & 24.167 \\
\hline 21.97 & 2.197 & 2.197 \\
\hline 2.197 & 103.259 & 4.394 \\
\hline 2.197 & 2.197 & 10.985 \\
\hline 4.394 & 4.394 & 8.788 \\
\hline 2.197 & 2.197 & 54.925 \\
\hline 2.197 & 4.394 & 8.788 \\
\hline 68.107 & 59.319 & 32.955 \\
\hline 2.197 & 2.197 & 2.197 \\
\hline 4.394 & 4.394 & 2.197 \\
\hline 98.865 & 10.985 & 2.197 \\
\hline 285.61 & 2.197 & 4.394 \\
\hline 19.773 & 37.349 & 8.788 \\
\hline 61.516 & 10.985 & 4.394 \\
\hline 2.197 & 2.197 & 6.591 \\
\hline 17.576 & 2.197 & 39.546 \\
\hline 54.925 & 2.197 & 72.501 \\
\hline 2.197 & 2.197 & 2.197 \\
\hline 6.591 & 2.197 & 6.591 \\
\hline 26.364 & 2.197 & 2.197 \\
\hline 2.197 & 2.197 & 6.591 \\
\hline 2.197 & 2.197 & 85.683 \\
\hline 248.261 & 4.394 & 2.197 \\
\hline 101.062 & 30.758 & 6.591 \\
\hline 4.394 & 2.197 & 2.197 \\
\hline
\end{tabular}




\begin{tabular}{|c|c|c|}
\hline 17.576 & 32.955 & 2.197 \\
\hline 32.955 & 48.334 & 21.97 \\
\hline 2.197 & 13.182 & 21.97 \\
\hline 2.197 & 13.182 & 30.758 \\
\hline 2.197 & 13.182 & 2.197 \\
\hline 6.591 & 2.197 & 2.197 \\
\hline 6.591 & 2.197 & 2.197 \\
\hline 241.67 & 2.197 & 2.197 \\
\hline 98.865 & 2.197 & 138.411 \\
\hline 13.182 & 30.758 & 2.197 \\
\hline 41.743 & 2.197 & 2.197 \\
\hline 30.758 & 2.197 & 15.379 \\
\hline 4.394 & 2.197 & 2.197 \\
\hline 6.591 & 2.197 & 32.955 \\
\hline 2.197 & 4.394 & 8.788 \\
\hline 2.197 & 6.591 & 4.394 \\
\hline 28.561 & 4.394 & 169.169 \\
\hline 2.197 & 2.197 & 2.197 \\
\hline 2.197 & 6.591 & 35.152 \\
\hline 243.867 & 98.865 & 8.788 \\
\hline 2.197 & 52.728 & 2.197 \\
\hline 70.304 & 4.394 & 10.985 \\
\hline 41.743 & 68.107 & 2.197 \\
\hline 2.197 & 2.197 & 8.788 \\
\hline 2.197 & 2.197 & 2.197 \\
\hline 50.531 & 15.379 & 6.591 \\
\hline 41.743 & 2.197 & 4.394 \\
\hline 10.985 & 2.197 & 4.394 \\
\hline 21.97 & 105.456 & 125.229 \\
\hline 292.201 & 2.197 & 26.364 \\
\hline 2.197 & 2.197 & 2.197 \\
\hline 6.591 & 6.591 & 98.865 \\
\hline 2.197 & 15.379 & 17.576 \\
\hline 21.97 & 48.334 & 61.516 \\
\hline 41.743 & 8.788 & 2.197 \\
\hline 10.985 & 2.197 & 19.773 \\
\hline 15.379 & 4.394 & 6.591 \\
\hline 268.034 & 4.394 & 2.197 \\
\hline 2.197 & 2.197 & 6.591 \\
\hline 52.728 & 2.197 & 2.197 \\
\hline 2.197 & 239.473 & 10.985 \\
\hline 26.364 & 2.197 & 101.062 \\
\hline 2.197 & 13.182 & 2.197 \\
\hline 2.197 & 2.197 & 2.197 \\
\hline 6.591 & 13.182 & 2.197 \\
\hline 15.379 & 2.197 & 2.197 \\
\hline 48.334 & 2.197 & 24.167 \\
\hline 2.197 & 63.713 & 4.394 \\
\hline 26.364 & 2.197 & 2.197 \\
\hline 2.197 & 6.591 & 175.76 \\
\hline
\end{tabular}




\begin{tabular}{|c|c|c|}
\hline 2.197 & 2.197 & 103.259 \\
\hline 17.576 & 2.197 & 10.985 \\
\hline 8.788 & 8.788 & 2.197 \\
\hline 74.698 & 2.197 & 61.516 \\
\hline 4.394 & 10.985 & 19.773 \\
\hline 430.612 & 300.989 & 118.638 \\
\hline 6.591 & 2.197 & 2.197 \\
\hline 13.182 & 13.182 & 46.137 \\
\hline 2.197 & 2.197 & 2.197 \\
\hline 4.394 & 2.197 & 2.197 \\
\hline 43.94 & 32.955 & 4.394 \\
\hline 4.394 & 8.788 & 2.197 \\
\hline 17.576 & 6.591 & 2.197 \\
\hline 4.394 & 2.197 & 2.197 \\
\hline 32.955 & 10.985 & 2.197 \\
\hline 4.394 & 2.197 & 2.197 \\
\hline 2.197 & 32.955 & 4.394 \\
\hline 4.394 & 2.197 & 2.197 \\
\hline 2.197 & 65.91 & 35.152 \\
\hline 17.576 & 891.982 & 2.197 \\
\hline 107.653 & 4.394 & 2.197 \\
\hline 4.394 & 4.394 & 2.197 \\
\hline 2.197 & 17.576 & 2.197 \\
\hline 26.364 & 2.197 & 123.032 \\
\hline 6.591 & 4.394 & 125.229 \\
\hline 2.197 & 107.653 & 2.197 \\
\hline 2.197 & 54.925 & 85.683 \\
\hline 6.591 & 15.379 & 2.197 \\
\hline 182.351 & 13.182 & 24.167 \\
\hline 76.895 & 30.758 & 2.197 \\
\hline 2.197 & 221.897 & 2.197 \\
\hline 439.4 & 32.955 & 2.197 \\
\hline 2.197 & 2.197 & 6.591 \\
\hline 2.197 & 467.961 & 2.197 \\
\hline 10.985 & 19.773 & 2.197 \\
\hline 6.591 & 2.197 & 15.379 \\
\hline 4.394 & 2.197 & 54.925 \\
\hline 8.788 & 4.394 & 140.608 \\
\hline 1403.88 & 2.197 & 6.591 \\
\hline 2.197 & 13.182 & 4.394 \\
\hline 4.394 & 10.985 & 32.955 \\
\hline 2.197 & 2.197 & 296.595 \\
\hline 4.394 & 2.197 & 4.394 \\
\hline 37.349 & 136.214 & 57.122 \\
\hline 6.591 & 2.197 & 81.289 \\
\hline 57.122 & 4.394 & 4.394 \\
\hline 2.197 & 8.788 & 61.516 \\
\hline 68.107 & 8.788 & 2.197 \\
\hline 6.591 & 6.591 & 10.985 \\
\hline 2.197 & 6.591 & 19.773 \\
\hline
\end{tabular}




\begin{tabular}{|c|c|c|}
\hline 98.865 & 4.394 & 8.788 \\
\hline 10.985 & 4.394 & 2.197 \\
\hline 17.576 & 90.077 & 19.773 \\
\hline 32.955 & 28.561 & 2.197 \\
\hline 4.394 & 8.788 & 15.379 \\
\hline 2.197 & 21.97 & 107.653 \\
\hline 2.197 & 2.197 & 6.591 \\
\hline 6.591 & 4.394 & 10.985 \\
\hline 46.137 & 2.197 & 2.197 \\
\hline 72.501 & 4.394 & 90.077 \\
\hline 32.955 & 177.957 & 63.713 \\
\hline 2.197 & 15.379 & 2.197 \\
\hline 2.197 & 105.456 & 6.591 \\
\hline 48.334 & 2.197 & 21.97 \\
\hline 4.394 & 6.591 & 24.167 \\
\hline 2.197 & 2.197 & 10.985 \\
\hline 32.955 & 2137.68 & 2.197 \\
\hline 2.197 & 2.197 & 10.985 \\
\hline 70.304 & 7544.5 & 531.674 \\
\hline 4.394 & 1045.77 & 35.152 \\
\hline 57.122 & 2.197 & 2.197 \\
\hline 169.169 & 2.197 & 8.788 \\
\hline 13.182 & 2.197 & 2.197 \\
\hline 6.591 & 79.092 & 2.197 \\
\hline 2.197 & 2.197 & 2.197 \\
\hline 10.985 & 4.394 & 17.576 \\
\hline 6.591 & 2.197 & 24.167 \\
\hline 2.197 & 4.394 & 8.788 \\
\hline 2.197 & 2.197 & 2.197 \\
\hline 136.214 & 4.394 & 4.394 \\
\hline 2.197 & 2.197 & 112.047 \\
\hline 6.591 & 2.197 & 4.394 \\
\hline 2.197 & 2.197 & 2.197 \\
\hline 94.471 & 2.197 & 2.197 \\
\hline 518.492 & 50.531 & 180.154 \\
\hline 215.306 & 1814.72 & 26.364 \\
\hline 4.394 & 21.97 & 4.394 \\
\hline 2.197 & 2.197 & 388.869 \\
\hline 2.197 & 15.379 & 116.441 \\
\hline 21.97 & 10.985 & 2.197 \\
\hline 41.743 & 15.379 & 2.197 \\
\hline 21.97 & 10.985 & 6.591 \\
\hline 4.394 & 4.394 & 98.865 \\
\hline 2.197 & 2.197 & 2.197 \\
\hline 2.197 & 4.394 & 26.364 \\
\hline 30.758 & 4.394 & 2.197 \\
\hline 4.394 & 2.197 & 70.304 \\
\hline 2.197 & 226.291 & 6.591 \\
\hline 2.197 & 6.591 & 46.137 \\
\hline 10.985 & 6.591 & 1157.82 \\
\hline
\end{tabular}




\begin{tabular}{|c|c|c|}
\hline 4.394 & 19.773 & 4.394 \\
\hline 39.546 & 4.394 & 50.531 \\
\hline 72.501 & 19.773 & 4.394 \\
\hline 118.638 & 54.925 & 4.394 \\
\hline 4.394 & 6.591 & 8.788 \\
\hline 707.434 & 8.788 & 13.182 \\
\hline 2.197 & 2.197 & 2.197 \\
\hline 26.364 & 8.788 & 32.955 \\
\hline 8.788 & 8.788 & 2.197 \\
\hline 2.197 & 92.274 & 136.214 \\
\hline 6.591 & 10.985 & 2.197 \\
\hline 30.758 & 2.197 & 2.197 \\
\hline 19.773 & 2.197 & 2.197 \\
\hline 2.197 & 4.394 & 4.394 \\
\hline 13.182 & 6.591 & 54.925 \\
\hline 8.788 & 10.985 & 4.394 \\
\hline 15.379 & 4.394 & 6.591 \\
\hline 50.531 & 845.845 & 254.852 \\
\hline 4.394 & 4.394 & 4.394 \\
\hline 24.167 & 90.077 & 257.049 \\
\hline 54.925 & 399.854 & 28.561 \\
\hline 2.197 & 2.197 & 32.955 \\
\hline 2.197 & 2.197 & 2.197 \\
\hline 4.394 & 184.548 & 2.197 \\
\hline 10.985 & 10.985 & 30.758 \\
\hline 19.773 & 2.197 & 68.107 \\
\hline 4.394 & 4.394 & 8.788 \\
\hline 10.985 & 2.197 & 85.683 \\
\hline 2.197 & 2.197 & 4.394 \\
\hline 43.94 & 4.394 & 417.43 \\
\hline 37.349 & 2.197 & 2.197 \\
\hline 21.97 & 2.197 & 2089.35 \\
\hline 15.379 & 6.591 & 593.19 \\
\hline 2.197 & 2.197 & 15.379 \\
\hline 30.758 & 2.197 & 2.197 \\
\hline 2.197 & 2.197 & 6.591 \\
\hline 81.289 & 120.835 & 13.182 \\
\hline 2.197 & 2.197 & 21.97 \\
\hline 43.94 & 10.985 & 15.379 \\
\hline 2.197 & 43.94 & 8.788 \\
\hline 2.197 & 15.379 & 4.394 \\
\hline 184.548 & 619.554 & 6.591 \\
\hline 43.94 & 10.985 & 6.591 \\
\hline 10.985 & 349.323 & 10.985 \\
\hline 2.197 & 26.364 & 6.591 \\
\hline 2.197 & 50.531 & 17.576 \\
\hline 2.197 & 4.394 & 28.561 \\
\hline 48.334 & 4.394 & 2.197 \\
\hline 17.576 & 2.197 & 87.88 \\
\hline 17.576 & 52.728 & 98.865 \\
\hline
\end{tabular}




\begin{tabular}{|c|c|c|}
\hline 2.197 & 4.394 & 24.167 \\
\hline 6.591 & 15.379 & 8.788 \\
\hline 112.047 & 24.167 & 2.197 \\
\hline 76.895 & 4.394 & 8.788 \\
\hline 2.197 & 81.289 & 118.638 \\
\hline 2.197 & 2.197 & 2.197 \\
\hline 287.807 & 2.197 & 4.394 \\
\hline 24.167 & 4.394 & 2.197 \\
\hline 15.379 & 215.306 & 2.197 \\
\hline 4.394 & 24.167 & 2.197 \\
\hline 4.394 & 404.248 & 8.788 \\
\hline 2.197 & 2.197 & 87.88 \\
\hline 2.197 & 2.197 & 4.394 \\
\hline 30.758 & 2.197 & 2.197 \\
\hline 2.197 & 24.167 & 30.758 \\
\hline 39.546 & 2.197 & 2.197 \\
\hline 2.197 & 15.379 & 2.197 \\
\hline 2.197 & 8.788 & 8.788 \\
\hline 13.182 & 6.591 & 30.758 \\
\hline 4.394 & 24.167 & 210.912 \\
\hline 70.304 & 2.197 & 10.985 \\
\hline 6.591 & 13.182 & 6.591 \\
\hline 140.608 & 2.197 & 2.197 \\
\hline 4.394 & 2.197 & 2.197 \\
\hline 10.985 & 10.985 & 4.394 \\
\hline 10.985 & 2.197 & 85.683 \\
\hline 46.137 & 2.197 & 39.546 \\
\hline 6.591 & 2.197 & 17.576 \\
\hline 4.394 & 2.197 & 19.773 \\
\hline 2.197 & 17.576 & 13.182 \\
\hline 6.591 & 2.197 & 30.758 \\
\hline 2.197 & 2.197 & 2.197 \\
\hline 2.197 & 2.197 & 2.197 \\
\hline 2.197 & 2.197 & 2.197 \\
\hline 2.197 & 2.197 & 2069.57 \\
\hline 8.788 & 2.197 & 118.638 \\
\hline 307.58 & 2.197 & 6.591 \\
\hline 2.197 & 2.197 & 17.576 \\
\hline 70.304 & 10.985 & 347.126 \\
\hline 153.79 & 2.197 & 43913.6 \\
\hline 92.274 & 2.197 & 37.349 \\
\hline 2.197 & 2.197 & 15.379 \\
\hline 2.197 & 2.197 & 2.197 \\
\hline 6.591 & 2.197 & 2.197 \\
\hline 2.197 & 6.591 & 26.364 \\
\hline 39.546 & 4.394 & 120.835 \\
\hline 17.576 & 28.561 & 15.379 \\
\hline 24.167 & 1606.01 & 17.576 \\
\hline 127.426 & 2.197 & 24.167 \\
\hline 2.197 & 2.197 & 92.274 \\
\hline
\end{tabular}




\begin{tabular}{|c|c|c|}
\hline 35.152 & 2.197 & 26.364 \\
\hline 2.197 & 9117.55 & 41.743 \\
\hline 2.197 & 123.032 & 169.169 \\
\hline 46.137 & 2.197 & 4.394 \\
\hline 19.773 & 2.197 & 46.137 \\
\hline 94.471 & 2.197 & 10.985 \\
\hline 158.184 & 19.773 & 656.903 \\
\hline 138.411 & 6.591 & 2.197 \\
\hline 311.974 & 4.394 & 123.032 \\
\hline 204.321 & 6.591 & 6.591 \\
\hline 4.394 & 2.197 & 2.197 \\
\hline 13.182 & 4.394 & 2.197 \\
\hline 28.561 & 4.394 & 10.985 \\
\hline 87.88 & 13.182 & 26.364 \\
\hline 2.197 & 4.394 & 76.895 \\
\hline 4.394 & 4.394 & 37.349 \\
\hline 50.531 & 2.197 & 28.561 \\
\hline 28.561 & 2.197 & 1944.34 \\
\hline 41.743 & 4.394 & 24.167 \\
\hline 4.394 & 2.197 & 28.561 \\
\hline 21.97 & 8.788 & 2.197 \\
\hline 6.591 & 2.197 & 6.591 \\
\hline 6.591 & 19.773 & 13.182 \\
\hline 4.394 & 303.186 & 134.017 \\
\hline 15.379 & 8.788 & 6.591 \\
\hline 2.197 & 2.197 & 54.925 \\
\hline 19.773 & 2.197 & 6.591 \\
\hline 8.788 & 101.062 & 10.985 \\
\hline 46.137 & 2.197 & 94.471 \\
\hline 4.394 & 4.394 & 37.349 \\
\hline 2.197 & 10.985 & 4.394 \\
\hline 79.092 & 13.182 & 6.591 \\
\hline 2.197 & 8.788 & 4.394 \\
\hline 2.197 & 2.197 & 17.576 \\
\hline 2.197 & 2.197 & 259.246 \\
\hline 2.197 & 254.852 & 30.758 \\
\hline 6.591 & 24.167 & 344.929 \\
\hline 2.197 & 2.197 & 2.197 \\
\hline 94.471 & 2.197 & 6.591 \\
\hline 17.576 & 2.197 & 4.394 \\
\hline 17.576 & 13.182 & 15.379 \\
\hline 2.197 & 8.788 & 13.182 \\
\hline 19.773 & 4.394 & 413.036 \\
\hline 52.728 & 2.197 & 43.94 \\
\hline 4.394 & 2.197 & 2.197 \\
\hline 6.591 & 54.925 & 810.693 \\
\hline 13.182 & 2.197 & 118.638 \\
\hline 2.197 & 2.197 & 2.197 \\
\hline 50.531 & 2.197 & 32.955 \\
\hline 10.985 & 21.97 & 2.197 \\
\hline
\end{tabular}




\begin{tabular}{|c|c|c|}
\hline 43.94 & 2.197 & 123.032 \\
\hline 79.092 & 2.197 & 4.394 \\
\hline 8.788 & 2.197 & 17.576 \\
\hline 79.092 & 2.197 & 8.788 \\
\hline 87.88 & 116.441 & 112.047 \\
\hline 4.394 & 43.94 & 28.561 \\
\hline 8.788 & 2.197 & 6.591 \\
\hline 17.576 & 2.197 & 2.197 \\
\hline 2.197 & 2.197 & 4.394 \\
\hline 21.97 & 24.167 & 41.743 \\
\hline 2.197 & 13.182 & 555.841 \\
\hline 6.591 & 2.197 & 26.364 \\
\hline 37.349 & 2.197 & 15.379 \\
\hline 2.197 & 2.197 & 4.394 \\
\hline 2.197 & 2.197 & 30.758 \\
\hline 52.728 & 2.197 & 2.197 \\
\hline 48.334 & 2.197 & 32.955 \\
\hline 6.591 & 6.591 & 17.576 \\
\hline 4.394 & 4.394 & 6.591 \\
\hline 2.197 & 21552.6 & 938.119 \\
\hline 2.197 & 2.197 & 2.197 \\
\hline 52.728 & 4.394 & 4.394 \\
\hline 116.441 & 2.197 & 8.788 \\
\hline 2.197 & 4.394 & 4.394 \\
\hline 364.702 & 2.197 & 59.319 \\
\hline 46.137 & 8.788 & 35.152 \\
\hline 2.197 & 57.122 & 6.591 \\
\hline 4.394 & 13.182 & 320.762 \\
\hline 2.197 & 24.167 & 2.197 \\
\hline 184.548 & 2.197 & 43.94 \\
\hline 101.062 & 35.152 & 2.197 \\
\hline 2.197 & 2.197 & 4.394 \\
\hline 65.91 & 101.062 & 2.197 \\
\hline 21.97 & 2.197 & 2.197 \\
\hline 2.197 & 2.197 & 2.197 \\
\hline 19.773 & 6.591 & 342.732 \\
\hline 2.197 & 2.197 & 4.394 \\
\hline 2.197 & 6.591 & 4.394 \\
\hline 4.394 & 4.394 & 2.197 \\
\hline 4.394 & 2.197 & 15.379 \\
\hline 43.94 & 37.349 & 48.334 \\
\hline 270.231 & 76.895 & 10.985 \\
\hline 103.259 & 6.591 & 551.447 \\
\hline 2.197 & 6.591 & 13.182 \\
\hline 17.576 & 2.197 & 2.197 \\
\hline 2.197 & 43.94 & 15.379 \\
\hline 13.182 & 158.184 & 59.319 \\
\hline 43.94 & 2.197 & 4.394 \\
\hline 2.197 & 2.197 & 39.546 \\
\hline 2.197 & 2.197 & 177.957 \\
\hline
\end{tabular}




\begin{tabular}{|c|c|c|}
\hline 2.197 & 2.197 & 15.379 \\
\hline 6.591 & 2.197 & 15.379 \\
\hline 10.985 & 2.197 & 2.197 \\
\hline 8.788 & 35.152 & 17.576 \\
\hline 4.394 & 43.94 & 155.987 \\
\hline 63.713 & 2.197 & 116.441 \\
\hline 85.683 & 2.197 & 26.364 \\
\hline 2.197 & 4.394 & 3644.82 \\
\hline 2.197 & 2.197 & 17.576 \\
\hline 2.197 & 2.197 & 2.197 \\
\hline 2.197 & 2.197 & 4.394 \\
\hline 2.197 & 21.97 & 43.94 \\
\hline 2.197 & 2.197 & 57.122 \\
\hline 4.394 & 8.788 & 2.197 \\
\hline 8.788 & 2.197 & 2.197 \\
\hline 4.394 & 2.197 & 2.197 \\
\hline 8.788 & 2.197 & 2.197 \\
\hline 4.394 & 2.197 & 30.758 \\
\hline 2.197 & 2.197 & 13.182 \\
\hline 2.197 & 4.394 & 4.394 \\
\hline 19.773 & 46.137 & 39.546 \\
\hline 6.591 & 10.985 & 2.197 \\
\hline 17.576 & 2.197 & 6.591 \\
\hline 10.985 & 2.197 & 10.985 \\
\hline 2.197 & 4.394 & 2.197 \\
\hline 112.047 & 6.591 & 2.197 \\
\hline 37.349 & 13.182 & 4.394 \\
\hline 19.773 & 8.788 & 8.788 \\
\hline 2.197 & 2.197 & 63.713 \\
\hline 6.591 & 134.017 & 6.591 \\
\hline 28.561 & 2.197 & 19.773 \\
\hline 4.394 & 2.197 & 2.197 \\
\hline 2.197 & 2.197 & 28.561 \\
\hline 76.895 & 10.985 & 4.394 \\
\hline 4.394 & 72.501 & 21.97 \\
\hline 4.394 & 6.591 & 6.591 \\
\hline 17.576 & 2.197 & 6.591 \\
\hline 15.379 & 2.197 & 6.591 \\
\hline 6.591 & 2.197 & 17.576 \\
\hline 2.197 & 347.126 & 43.94 \\
\hline 6.591 & 30.758 & 116.441 \\
\hline 17.576 & 2.197 & 2.197 \\
\hline 2.197 & 17.576 & 2.197 \\
\hline 2.197 & 2.197 & 57.122 \\
\hline 2.197 & 8.788 & 15.379 \\
\hline 2.197 & 2.197 & 68.107 \\
\hline 2.197 & 74.698 & 2.197 \\
\hline 2.197 & 4.394 & 4.394 \\
\hline 116.441 & 70.304 & 10.985 \\
\hline 15.379 & 4.394 & 19.773 \\
\hline
\end{tabular}




\begin{tabular}{|c|c|c|}
\hline 10.985 & 2.197 & 2.197 \\
\hline 2.197 & 8.788 & 2.197 \\
\hline 4.394 & 2.197 & 114.244 \\
\hline 184.548 & 13.182 & 37.349 \\
\hline 270.231 & 4.394 & 8.788 \\
\hline 125.229 & 19.773 & 19.773 \\
\hline 2.197 & 2.197 & 2.197 \\
\hline 4.394 & 15.379 & 382.278 \\
\hline 2.197 & 6.591 & 6.591 \\
\hline 6.591 & 4.394 & 2.197 \\
\hline 6.591 & 4.394 & 2.197 \\
\hline 2.197 & 6355.92 & 2.197 \\
\hline 4.394 & 1026 & 26.364 \\
\hline 19.773 & 8.788 & 134.017 \\
\hline 13.182 & 24.167 & 13.182 \\
\hline 2.197 & 4.394 & 13.182 \\
\hline 8.788 & 162.578 & 15.379 \\
\hline 28.561 & 4.394 & 142.805 \\
\hline 26.364 & 2.197 & 32.955 \\
\hline 221.897 & 8.788 & 13.182 \\
\hline 24.167 & 13.182 & 8.788 \\
\hline 15.379 & 2.197 & 54.925 \\
\hline 19.773 & 54.925 & 21.97 \\
\hline 2.197 & 4.394 & 15.379 \\
\hline 114.244 & 70.304 & 2.197 \\
\hline 123.032 & 2.197 & 15.379 \\
\hline 2.197 & 59.319 & 4.394 \\
\hline 2.197 & 2.197 & 2.197 \\
\hline 2.197 & 6.591 & 2.197 \\
\hline 2.197 & 2.197 & 2.197 \\
\hline 2.197 & 8.788 & 257.049 \\
\hline 2.197 & 39.546 & 4.394 \\
\hline 158.184 & 10.985 & 698.646 \\
\hline 4.394 & 2.197 & 19.773 \\
\hline 6.591 & 2.197 & 6.591 \\
\hline 17.576 & 2.197 & 13.182 \\
\hline 138.411 & 8.788 & 2.197 \\
\hline 4.394 & 48.334 & 6.591 \\
\hline 10.985 & 13.182 & 2.197 \\
\hline 2.197 & 61.516 & 2.197 \\
\hline 8.788 & 15.379 & 2.197 \\
\hline 2.197 & 72.501 & 65.91 \\
\hline 6.591 & 30.758 & 6.591 \\
\hline 26.364 & 13.182 & 2.197 \\
\hline 112.047 & 4.394 & 19.773 \\
\hline 4.394 & 2.197 & 2.197 \\
\hline 6.591 & 2.197 & 2.197 \\
\hline 4.394 & 2.197 & 37.349 \\
\hline 32.955 & 8.788 & 24.167 \\
\hline 68.107 & 2.197 & 57.122 \\
\hline
\end{tabular}




\begin{tabular}{|c|c|c|}
\hline 28.561 & 2.197 & 2.197 \\
\hline 2.197 & 8.788 & 10.985 \\
\hline 8.788 & 2.197 & 6.591 \\
\hline 52.728 & 2.197 & 4.394 \\
\hline 4.394 & 2.197 & 15.379 \\
\hline 15.379 & 2.197 & 19.773 \\
\hline 129.623 & 2.197 & 8.788 \\
\hline 166.972 & 8.788 & 2.197 \\
\hline 21.97 & 2.197 & 13.182 \\
\hline 4.394 & 24.167 & 24.167 \\
\hline 39.546 & 2.197 & 2.197 \\
\hline 63.713 & 24.167 & 158.184 \\
\hline 21.97 & 2.197 & 6.591 \\
\hline 4.394 & 8.788 & 2.197 \\
\hline 19.773 & 4.394 & 2.197 \\
\hline 4.394 & 68.107 & 2.197 \\
\hline 2.197 & 4.394 & 6.591 \\
\hline 6.591 & 2.197 & 2.197 \\
\hline 8.788 & 2.197 & 17.576 \\
\hline 68.107 & 2.197 & 13.182 \\
\hline 4.394 & 193.336 & 30.758 \\
\hline 215.306 & 6.591 & 2.197 \\
\hline 8.788 & 24.167 & 2.197 \\
\hline 10.985 & 21.97 & 6.591 \\
\hline 61.516 & 10.985 & 136.214 \\
\hline 43.94 & 4.394 & 28.561 \\
\hline 2.197 & 4.394 & 2.197 \\
\hline 2.197 & 4.394 & 2.197 \\
\hline 10.985 & 10.985 & 2.197 \\
\hline 2.197 & 6.591 & 2.197 \\
\hline 2.197 & 39.546 & 21.97 \\
\hline 21.97 & 2.197 & 26.364 \\
\hline 35.152 & 4.394 & 112.047 \\
\hline 30.758 & 74.698 & 15.379 \\
\hline 2.197 & 4.394 & 21.97 \\
\hline 10.985 & 2.197 & 30.758 \\
\hline 19.773 & 105.456 & 134.017 \\
\hline 68.107 & 241.67 & 13.182 \\
\hline 13.182 & 17.576 & 32.955 \\
\hline 2.197 & 2.197 & 2.197 \\
\hline 41.743 & 19.773 & 39.546 \\
\hline 15.379 & 6.591 & 6.591 \\
\hline 105.456 & 2.197 & 2.197 \\
\hline 2.197 & 2.197 & 2.197 \\
\hline 2.197 & 2.197 & 4.394 \\
\hline 2.197 & 2.197 & 2.197 \\
\hline 6.591 & 2.197 & 880.997 \\
\hline 116.441 & 13.182 & 52.728 \\
\hline 59.319 & 2.197 & 2.197 \\
\hline 6.591 & 2.197 & 4.394 \\
\hline
\end{tabular}




\begin{tabular}{|c|c|c|}
\hline 46.137 & 2.197 & 2.197 \\
\hline 26.364 & 4.394 & 10.985 \\
\hline 8.788 & 2.197 & 8.788 \\
\hline 6.591 & 4.394 & 28.561 \\
\hline 8.788 & 4.394 & 4.394 \\
\hline 8.788 & 2.197 & 35.152 \\
\hline 13.182 & 2.197 & 79.092 \\
\hline 2.197 & 2.197 & 2.197 \\
\hline 2.197 & 61.516 & 15.379 \\
\hline 2.197 & 2.197 & 2.197 \\
\hline 2.197 & 2.197 & 125.229 \\
\hline 8.788 & 2.197 & 4.394 \\
\hline 6.591 & 17.576 & 2.197 \\
\hline 4.394 & 2.197 & 37.349 \\
\hline 8.788 & 2.197 & 15.379 \\
\hline 2.197 & 6.591 & 15.379 \\
\hline 6.591 & 24.167 & 65.91 \\
\hline 46.137 & 8.788 & 2.197 \\
\hline 153.79 & 2.197 & 2.197 \\
\hline 4.394 & 6.591 & 307.58 \\
\hline 61.516 & 6.591 & 30.758 \\
\hline 43.94 & 123.032 & 2.197 \\
\hline 13.182 & 2.197 & 4.394 \\
\hline 186.745 & 2.197 & 2.197 \\
\hline 2.197 & 2.197 & 2.197 \\
\hline 2.197 & 2.197 & 6.591 \\
\hline 68.107 & 10.985 & 35.152 \\
\hline 4.394 & 2.197 & 17.576 \\
\hline 15.379 & 142.805 & 21.97 \\
\hline 4.394 & 4.394 & 50.531 \\
\hline 21.97 & 2.197 & 2.197 \\
\hline 2.197 & 2.197 & 4.394 \\
\hline 105.456 & 6.591 & 6.591 \\
\hline 4.394 & 2.197 & 4.394 \\
\hline 48.334 & 2.197 & 41.743 \\
\hline 6.591 & 4.394 & 6.591 \\
\hline 10.985 & 2.197 & 4.394 \\
\hline 4.394 & 4.394 & 32.955 \\
\hline 10.985 & 2.197 & 37.349 \\
\hline 10.985 & 19.773 & 2.197 \\
\hline 48.334 & 19.773 & 6.591 \\
\hline 2.197 & 15.379 & 478.946 \\
\hline 13.182 & 2.197 & 2.197 \\
\hline 4.394 & 2.197 & 2.197 \\
\hline 6.591 & 8.788 & 15.379 \\
\hline 2.197 & 6.591 & 10.985 \\
\hline 101.062 & 17.576 & 2.197 \\
\hline 2.197 & 142.805 & 2.197 \\
\hline 6.591 & 6.591 & 8.788 \\
\hline 2.197 & 2.197 & 2.197 \\
\hline
\end{tabular}




\begin{tabular}{|c|c|c|}
\hline 2.197 & 19.773 & 8.788 \\
\hline 2.197 & 24.167 & 4.394 \\
\hline 2.197 & 2.197 & 6.591 \\
\hline 17.576 & 2.197 & 2.197 \\
\hline 46.137 & 2.197 & 2.197 \\
\hline 32.955 & 2.197 & 4.394 \\
\hline 28.561 & 2.197 & 2.197 \\
\hline 4.394 & 6.591 & 6.591 \\
\hline 2.197 & 2.197 & 10.985 \\
\hline 2.197 & 2.197 & 4.394 \\
\hline 2.197 & 2.197 & 15.379 \\
\hline 4.394 & 2.197 & 123.032 \\
\hline 61.516 & 10.985 & 59.319 \\
\hline 252.655 & 2.197 & 32.955 \\
\hline 32.955 & 2.197 & 2.197 \\
\hline 4.394 & 2.197 & 6.591 \\
\hline 4.394 & 26.364 & 48.334 \\
\hline 28.561 & 35.152 & 173.563 \\
\hline 24.167 & 4.394 & 4.394 \\
\hline 94.471 & 2.197 & 26.364 \\
\hline 2.197 & 2.197 & 2.197 \\
\hline 68.107 & 4.394 & 28.561 \\
\hline 19.773 & 15.379 & 26.364 \\
\hline 4.394 & 2.197 & 30.758 \\
\hline 6.591 & 17.576 & 2.197 \\
\hline 10.985 & 2.197 & 74.698 \\
\hline 2.197 & 2.197 & 544.856 \\
\hline 2.197 & 70.304 & 2.197 \\
\hline 15.379 & 2.197 & 149.396 \\
\hline 2.197 & 6.591 & 2.197 \\
\hline 26.364 & 2.197 & 2.197 \\
\hline 52.728 & 2.197 & 2.197 \\
\hline 13.182 & 2.197 & 8.788 \\
\hline 21.97 & 10.985 & 1113.88 \\
\hline 2.197 & 4.394 & 2.197 \\
\hline 24.167 & 2.197 & 6.591 \\
\hline 2.197 & 4.394 & 2.197 \\
\hline 50.531 & 4.394 & 2.197 \\
\hline 24.167 & 2.197 & 2.197 \\
\hline 24.167 & 2.197 & 13.182 \\
\hline 4.394 & 4.394 & 35.152 \\
\hline 193.336 & 136.214 & 8.788 \\
\hline 2.197 & 371.293 & 2.197 \\
\hline 2.197 & 52.728 & 2.197 \\
\hline 13.182 & 8.788 & 21.97 \\
\hline 26.364 & 8.788 & 8.788 \\
\hline 2.197 & 10.985 & 21.97 \\
\hline 4.394 & 6.591 & 61.516 \\
\hline 28.561 & 4.394 & 2.197 \\
\hline 19.773 & 2.197 & 2.197 \\
\hline
\end{tabular}




\begin{tabular}{|c|c|c|}
\hline 175.76 & 74.698 & 13.182 \\
\hline 8.788 & 4.394 & 15.379 \\
\hline 52.728 & 2.197 & 10.985 \\
\hline 2.197 & 13.182 & 92.274 \\
\hline 90.077 & 17.576 & 10.985 \\
\hline 57.122 & 10.985 & 35.152 \\
\hline 43.94 & 4.394 & 57.122 \\
\hline 13.182 & 1133.65 & 90.077 \\
\hline 4.394 & 28.561 & 8.788 \\
\hline 2.197 & 2.197 & 15.379 \\
\hline 21.97 & 6.591 & 13.182 \\
\hline 15.379 & 2.197 & 35.152 \\
\hline 2.197 & 347.126 & 184.548 \\
\hline 2.197 & 21.97 & 13.182 \\
\hline 13.182 & 15.379 & 13.182 \\
\hline 2.197 & 13.182 & 564.629 \\
\hline 2.197 & 8.788 & 2.197 \\
\hline 48.334 & 4.394 & 4.394 \\
\hline 94.471 & 13.182 & 120.835 \\
\hline 10.985 & 2.197 & 6.591 \\
\hline 4.394 & 2.197 & 4.394 \\
\hline 68.107 & 2.197 & 2.197 \\
\hline 13.182 & 13.182 & 2.197 \\
\hline 24.167 & 2.197 & 19.773 \\
\hline 2.197 & 4.394 & 2.197 \\
\hline 116.441 & 32.955 & 4.394 \\
\hline 52.728 & 6.591 & 17.576 \\
\hline 2.197 & 2.197 & 30.758 \\
\hline 2.197 & 4.394 & 21.97 \\
\hline 72.501 & 6.591 & 15.379 \\
\hline 2.197 & 30.758 & 922.74 \\
\hline 2.197 & 19.773 & 8.788 \\
\hline 61.516 & 2.197 & 15.379 \\
\hline 13.182 & 72.501 & 63.713 \\
\hline 2.197 & 28.561 & 2.197 \\
\hline 46.137 & 2.197 & 4.394 \\
\hline 2.197 & 4.394 & 4.394 \\
\hline 21.97 & 6.591 & 2.197 \\
\hline 10.985 & 2.197 & 32.955 \\
\hline 15.379 & 703.04 & 54.925 \\
\hline 2.197 & 30.758 & 571.22 \\
\hline 2.197 & 19.773 & 57.122 \\
\hline 6.591 & 2.197 & 2.197 \\
\hline 4.394 & 2.197 & 39.546 \\
\hline 17.576 & 26.364 & 553.644 \\
\hline 8.788 & 10.985 & 26.364 \\
\hline 105.456 & 21.97 & 2.197 \\
\hline 83.486 & 24.167 & 2.197 \\
\hline 15.379 & 2.197 & 4.394 \\
\hline 6.591 & 15.379 & 6.591 \\
\hline
\end{tabular}




\begin{tabular}{|c|c|c|}
\hline 10.985 & 210.912 & 2.197 \\
\hline 17.576 & 50.531 & 81.289 \\
\hline 68.107 & 2.197 & 15.379 \\
\hline 21.97 & 2.197 & 114.244 \\
\hline 2.197 & 87.88 & 17.576 \\
\hline 254.852 & 17.576 & 17.576 \\
\hline 4.394 & 8.788 & 8.788 \\
\hline 54.925 & 17.576 & 8.788 \\
\hline 48.334 & 4.394 & 2.197 \\
\hline 6.591 & 2.197 & 162.578 \\
\hline 6.591 & 6.591 & 13.182 \\
\hline 13.182 & 2.197 & 2.197 \\
\hline 65.91 & 6.591 & 13.182 \\
\hline 26.364 & 15.379 & 6.591 \\
\hline 4.394 & 8.788 & 50.531 \\
\hline 2.197 & 24.167 & 28.561 \\
\hline 10.985 & 15.379 & 2.197 \\
\hline 4.394 & 57.122 & 562.432 \\
\hline 2.197 & 6.591 & 8.788 \\
\hline 2.197 & 300.989 & 28.561 \\
\hline 2.197 & 2.197 & 180.154 \\
\hline 13.182 & 2.197 & 21.97 \\
\hline 2.197 & 35.152 & 6.591 \\
\hline 43.94 & 13.182 & 6.591 \\
\hline 2.197 & 21.97 & 2.197 \\
\hline 4.394 & 2.197 & 588.796 \\
\hline 2.197 & 8.788 & 4.394 \\
\hline 4.394 & 15.379 & 4.394 \\
\hline 2.197 & 74.698 & 2.197 \\
\hline 2.197 & 15.379 & 10.985 \\
\hline 2.197 & 11444.2 & 2.197 \\
\hline 2.197 & 24.167 & 2.197 \\
\hline 2.197 & 17.576 & 30.758 \\
\hline 68.107 & 63.713 & 4.394 \\
\hline 10.985 & 26.364 & 2.197 \\
\hline 4.394 & 2.197 & 52.728 \\
\hline 2.197 & 2.197 & 6.591 \\
\hline 2.197 & 2.197 & 116.441 \\
\hline 6.591 & 26.364 & 184.548 \\
\hline 35.152 & 57.122 & 15.379 \\
\hline 467.961 & 10.985 & 162.578 \\
\hline 17.576 & 2.197 & 101.062 \\
\hline 17.576 & 32.955 & 4.394 \\
\hline 6.591 & 2.197 & 32.955 \\
\hline 10.985 & 92.274 & 2.197 \\
\hline 83.486 & 4.394 & 17.576 \\
\hline 217.503 & 6.591 & 2.197 \\
\hline 13.182 & 4.394 & 2.197 \\
\hline 28.561 & 4.394 & 2.197 \\
\hline 92.274 & 2.197 & 2.197 \\
\hline
\end{tabular}




\begin{tabular}{|c|c|c|}
\hline 4.394 & 2.197 & 2.197 \\
\hline 2.197 & 2.197 & 32.955 \\
\hline 13.182 & 2.197 & 81.289 \\
\hline 4.394 & 2.197 & 19.773 \\
\hline 254.852 & 2.197 & 6.591 \\
\hline 43.94 & 2.197 & 4.394 \\
\hline 2.197 & 8.788 & 17.576 \\
\hline 2.197 & 52.728 & 331.747 \\
\hline 41.743 & 13.182 & 2.197 \\
\hline 2.197 & 2.197 & 2.197 \\
\hline 2.197 & 2.197 & 4.394 \\
\hline 4.394 & 4.394 & 2.197 \\
\hline 26.364 & 2.197 & 2.197 \\
\hline 63.713 & 2.197 & 4.394 \\
\hline 6.591 & 15.379 & 43.94 \\
\hline 2.197 & 2.197 & 142.805 \\
\hline 2.197 & 2.197 & 10.985 \\
\hline 268.034 & 2.197 & 2.197 \\
\hline 4.394 & 2.197 & 2.197 \\
\hline 4.394 & 2.197 & 2.197 \\
\hline 4.394 & 2.197 & 15.379 \\
\hline 2.197 & 2.197 & 2.197 \\
\hline 8.788 & 17.576 & 2.197 \\
\hline 208.715 & 37.349 & 151.593 \\
\hline 4.394 & 13.182 & 63.713 \\
\hline 105.456 & 19.773 & 2.197 \\
\hline 63.713 & 15.379 & 155.987 \\
\hline 6.591 & 6.591 & 4.394 \\
\hline 10.985 & 17.576 & 48.334 \\
\hline 2.197 & 98.865 & 2.197 \\
\hline 4.394 & 65.91 & 59.319 \\
\hline 96.668 & 76.895 & 4.394 \\
\hline 2.197 & 6.591 & 19.773 \\
\hline 4.394 & 2.197 & 24.167 \\
\hline 4.394 & 2.197 & 46.137 \\
\hline 85.683 & 2.197 & 177.957 \\
\hline 4.394 & 92.274 & 3941.42 \\
\hline 92.274 & 10.985 & 6.591 \\
\hline 21.97 & 19.773 & 4.394 \\
\hline 4.394 & 2.197 & 213.109 \\
\hline 112.047 & 37.349 & 386.672 \\
\hline 2.197 & 325.156 & 799.708 \\
\hline 37.349 & 19.773 & 127.426 \\
\hline 4.394 & 2.197 & 70.304 \\
\hline 4.394 & 6.591 & 17.576 \\
\hline 10.985 & 35.152 & 63.713 \\
\hline 10.985 & 2.197 & 107.653 \\
\hline 8.788 & 8.788 & 2.197 \\
\hline 85.683 & 2.197 & 8.788 \\
\hline 136.214 & 2.197 & 8.788 \\
\hline
\end{tabular}




\begin{tabular}{|c|c|c|}
\hline 13.182 & 283.413 & 26.364 \\
\hline 2.197 & 4.394 & 8.788 \\
\hline 2.197 & 254.852 & 6.591 \\
\hline 72.501 & 48.334 & 8.788 \\
\hline 2.197 & 46.137 & 2.197 \\
\hline 2.197 & 243.867 & 26.364 \\
\hline 4.394 & 788.723 & 274.625 \\
\hline 10.985 & 4.394 & 15.379 \\
\hline 4.394 & 2.197 & 4.394 \\
\hline 2.197 & 6.591 & 13.182 \\
\hline 26.364 & 4.394 & 2.197 \\
\hline 21.97 & 2.197 & 8.788 \\
\hline 2.197 & 70.304 & 32.955 \\
\hline 79.092 & 28.561 & 8.788 \\
\hline 2.197 & 19.773 & 6.591 \\
\hline 2.197 & 2.197 & 15.379 \\
\hline 10.985 & 2.197 & 2.197 \\
\hline 4.394 & 4.394 & 19.773 \\
\hline 2.197 & 2.197 & 2.197 \\
\hline 70.304 & 6.591 & 48.334 \\
\hline 2.197 & 2.197 & 2.197 \\
\hline 15.379 & 2.197 & 8.788 \\
\hline 24.167 & 294.398 & 21.97 \\
\hline 2.197 & 2.197 & 2.197 \\
\hline 2.197 & 2.197 & 8.788 \\
\hline 2.197 & 54.925 & 17.576 \\
\hline 149.396 & 4.394 & 676.676 \\
\hline 2.197 & 4.394 & 2.197 \\
\hline 19.773 & 2.197 & 50.531 \\
\hline 377.884 & 24.167 & 30.758 \\
\hline 41.743 & 13.182 & 65.91 \\
\hline 4.394 & 32.955 & 28.561 \\
\hline 4.394 & 2.197 & 4.394 \\
\hline 6.591 & 2.197 & 261.443 \\
\hline 32.955 & 2.197 & 21.97 \\
\hline 6.591 & 2.197 & 1597.22 \\
\hline 54.925 & 2.197 & 2.197 \\
\hline 13.182 & 2.197 & 2.197 \\
\hline 2.197 & 4.394 & 28.561 \\
\hline 70.304 & 4.394 & 134.017 \\
\hline 32.955 & 19.773 & 72.501 \\
\hline 2.197 & 6.591 & 1083.12 \\
\hline 2.197 & 19.773 & 2.197 \\
\hline 37.349 & 2.197 & 2.197 \\
\hline 15.379 & 2.197 & 21.97 \\
\hline 6.591 & 2.197 & 2.197 \\
\hline 2.197 & 2.197 & 6.591 \\
\hline 13.182 & 2.197 & 72.501 \\
\hline 2.197 & 2.197 & 2.197 \\
\hline 15.379 & 4.394 & 2.197 \\
\hline
\end{tabular}




\begin{tabular}{|c|c|c|}
\hline 6.591 & 166.972 & 6.591 \\
\hline 68.107 & 2.197 & 46.137 \\
\hline 2.197 & 2.197 & 2.197 \\
\hline 2.197 & 6.591 & 8.788 \\
\hline 8.788 & 6.591 & 87.88 \\
\hline 10.985 & 50.531 & 2.197 \\
\hline 65.91 & 2.197 & 72.501 \\
\hline 6.591 & 37.349 & 2.197 \\
\hline 15.379 & 116.441 & 2.197 \\
\hline 2.197 & 85.683 & 8.788 \\
\hline 21.97 & 2.197 & 4.394 \\
\hline 4.394 & 4.394 & 68.107 \\
\hline 10.985 & 4.394 & 6.591 \\
\hline 4.394 & 2.197 & 6.591 \\
\hline 94.471 & 83.486 & 2.197 \\
\hline 2.197 & 2.197 & 2.197 \\
\hline 2.197 & 30.758 & 2.197 \\
\hline 24.167 & 2.197 & 2.197 \\
\hline 4.394 & 2.197 & 6.591 \\
\hline 481.143 & 2.197 & 217.503 \\
\hline 2.197 & 2.197 & 6.591 \\
\hline 39.546 & 8.788 & 13.182 \\
\hline 6.591 & 57.122 & 30.758 \\
\hline 8.788 & 6.591 & 173.563 \\
\hline 2.197 & 6.591 & 1254.49 \\
\hline 2.197 & 2.197 & 2.197 \\
\hline 10.985 & 419.627 & 4.394 \\
\hline 2.197 & 2.197 & 6.591 \\
\hline 588.796 & 2.197 & 2.197 \\
\hline 2.197 & 558.038 & 10.985 \\
\hline 28.561 & 648.115 & 30.758 \\
\hline 2.197 & 2.197 & 17.576 \\
\hline 2.197 & 4.394 & 4.394 \\
\hline 8.788 & 2.197 & 24.167 \\
\hline 2.197 & 2.197 & 13.182 \\
\hline 2.197 & 13.182 & 46.137 \\
\hline 4.394 & 2.197 & 107.653 \\
\hline 2.197 & 6.591 & 15.379 \\
\hline 6.591 & 6.591 & 147.199 \\
\hline 8.788 & 4.394 & 217.503 \\
\hline 4.394 & 2.197 & 4.394 \\
\hline 2.197 & 6.591 & 6.591 \\
\hline 4.394 & 37.349 & 4.394 \\
\hline 6.591 & 28.561 & 30.758 \\
\hline 4.394 & 8.788 & 59.319 \\
\hline 4.394 & 415.233 & 94.471 \\
\hline 2.197 & 79.092 & 4.394 \\
\hline 2.197 & 2.197 & 4.394 \\
\hline 6.591 & 8.788 & 13.182 \\
\hline 2.197 & 8.788 & 41.743 \\
\hline
\end{tabular}




\begin{tabular}{|c|c|c|}
\hline 2.197 & 4.394 & 2.197 \\
\hline 2.197 & 2.197 & 2.197 \\
\hline 2.197 & 2.197 & 2.197 \\
\hline 8.788 & 4.394 & 35.152 \\
\hline 8.788 & 404.248 & 90.077 \\
\hline 2.197 & 4.394 & 10.985 \\
\hline 24.167 & 2.197 & 2.197 \\
\hline 4.394 & 15.379 & 17.576 \\
\hline 19.773 & 61.516 & 6.591 \\
\hline 15.379 & 24.167 & 678.873 \\
\hline 21.97 & 2.197 & 35.152 \\
\hline 2.197 & 1043.57 & 4.394 \\
\hline 2.197 & 2.197 & 6.591 \\
\hline 39.546 & 4.394 & 4.394 \\
\hline 6.591 & 2.197 & 2.197 \\
\hline 2.197 & 15.379 & 6.591 \\
\hline 8.788 & 4.394 & 2.197 \\
\hline 2.197 & 2.197 & 2.197 \\
\hline 149.396 & 193.336 & 26.364 \\
\hline 10.985 & 2.197 & 2.197 \\
\hline 13.182 & 2.197 & 6.591 \\
\hline 276.822 & 17.576 & 28.561 \\
\hline 2.197 & 4.394 & 2.197 \\
\hline 2.197 & 2.197 & 6.591 \\
\hline 15.379 & 4.394 & 2.197 \\
\hline 4.394 & 2.197 & 2.197 \\
\hline 21.97 & 63.713 & 2.197 \\
\hline 4.394 & 2.197 & 2.197 \\
\hline 41.743 & 39.546 & 1674.11 \\
\hline 19.773 & 2.197 & 195.533 \\
\hline 171.366 & 35.152 & 81.289 \\
\hline 90.077 & 10.985 & 4.394 \\
\hline 8.788 & 21.97 & 2.197 \\
\hline 2.197 & 2.197 & 4.394 \\
\hline 21.97 & 2.197 & 41.743 \\
\hline 81.289 & 6.591 & 10.985 \\
\hline 2.197 & 4.394 & 30.758 \\
\hline 2.197 & 4.394 & 4.394 \\
\hline 2.197 & 2.197 & 4.394 \\
\hline 4.394 & 72.501 & 61.516 \\
\hline 2.197 & 2.197 & 2.197 \\
\hline 2.197 & 2.197 & 2109.12 \\
\hline 8.788 & 2.197 & 4.394 \\
\hline 2.197 & 2.197 & 87.88 \\
\hline 2.197 & 2.197 & 8.788 \\
\hline 101.062 & 2.197 & 15.379 \\
\hline 195.533 & 2.197 & 4.394 \\
\hline 4.394 & 2.197 & 6.591 \\
\hline 2.197 & 2.197 & 1790.55 \\
\hline 24.167 & 2.197 & 48.334 \\
\hline
\end{tabular}




\begin{tabular}{|c|c|c|}
\hline 2.197 & 8.788 & 4.394 \\
\hline 37.349 & 2.197 & 2.197 \\
\hline 2.197 & 70.304 & 2.197 \\
\hline 43.94 & 2.197 & 4.394 \\
\hline 28.561 & 169.169 & 2.197 \\
\hline 17.576 & 153.79 & 8.788 \\
\hline 2.197 & 2.197 & 98.865 \\
\hline 4.394 & 6.591 & 21.97 \\
\hline 621.751 & 57.122 & 2.197 \\
\hline 4.394 & 2.197 & 2.197 \\
\hline 2.197 & 4.394 & 6.591 \\
\hline 30.758 & 4.394 & 2.197 \\
\hline 32.955 & 6.591 & 2.197 \\
\hline 4.394 & 4.394 & 2.197 \\
\hline 30.758 & 2.197 & 13.182 \\
\hline 272.428 & 13.182 & 155.987 \\
\hline 32.955 & 2.197 & 39.546 \\
\hline 13.182 & 2.197 & 6.591 \\
\hline 41.743 & 10.985 & 4.394 \\
\hline 4.394 & 4.394 & 4.394 \\
\hline 39.546 & 39.546 & 21.97 \\
\hline 2.197 & 6.591 & 2.197 \\
\hline 21.97 & 4.394 & 17.576 \\
\hline 8.788 & 4.394 & 2.197 \\
\hline 2.197 & 4.394 & 50.531 \\
\hline 19.773 & 26.364 & 2.197 \\
\hline 2.197 & 4.394 & 2.197 \\
\hline 2.197 & 2.197 & 2.197 \\
\hline 136.214 & 2.197 & 2.197 \\
\hline 2.197 & 32.955 & 101.062 \\
\hline 50.531 & 166.972 & 2.197 \\
\hline 30.758 & 94.471 & 6.591 \\
\hline 10.985 & 2.197 & 107.653 \\
\hline 771.147 & 50.531 & 6.591 \\
\hline 2.197 & 2.197 & 2.197 \\
\hline 2.197 & 32.955 & 8.788 \\
\hline 4.394 & 41.743 & 2.197 \\
\hline 2.197 & 2.197 & 59.319 \\
\hline 4.394 & 2.197 & 24.167 \\
\hline 4.394 & 2.197 & 13.182 \\
\hline 21.97 & 4.394 & 4.394 \\
\hline 24.167 & 50.531 & 2.197 \\
\hline 2.197 & 2.197 & 2.197 \\
\hline 98.865 & 2.197 & 2.197 \\
\hline 8.788 & 17.576 & 10.985 \\
\hline 6.591 & 2.197 & 39.546 \\
\hline 19.773 & 2.197 & 2.197 \\
\hline 15.379 & 2.197 & 41.743 \\
\hline 2.197 & 261.443 & 6.591 \\
\hline 30.758 & 2.197 & 35.152 \\
\hline
\end{tabular}




\begin{tabular}{|c|c|c|}
\hline 10.985 & 39.546 & 70.304 \\
\hline 41.743 & 19.773 & 320.762 \\
\hline 8.788 & 30.758 & 8.788 \\
\hline 52.728 & 2.197 & 533.871 \\
\hline 131.82 & 2.197 & 8.788 \\
\hline 13.182 & 96.668 & 10.985 \\
\hline 6.591 & 241.67 & 26.364 \\
\hline 2.197 & 10.985 & 4.394 \\
\hline 2.197 & 8.788 & 32.955 \\
\hline 28.561 & 2.197 & 8.788 \\
\hline 2.197 & 2.197 & 2.197 \\
\hline 28.561 & 531.674 & 8.788 \\
\hline 2.197 & 228.488 & 17.576 \\
\hline 2.197 & 2.197 & 2.197 \\
\hline 32.955 & 8.788 & 2.197 \\
\hline 2.197 & 2.197 & 628.342 \\
\hline 6.591 & 268.034 & 4.394 \\
\hline 4.394 & 2.197 & 4.394 \\
\hline 15.379 & 4.394 & 10.985 \\
\hline 268.034 & 13.182 & 3915.05 \\
\hline 21.97 & 4.394 & 4.394 \\
\hline 81.289 & 2.197 & 92.274 \\
\hline 2.197 & 2.197 & 2.197 \\
\hline 632.736 & 6.591 & 4.394 \\
\hline 4.394 & 2.197 & 2.197 \\
\hline 4.394 & 2.197 & 48.334 \\
\hline 2.197 & 19.773 & 8.788 \\
\hline 2.197 & 4.394 & 17.576 \\
\hline 10.985 & 2.197 & 6.591 \\
\hline 10.985 & 2.197 & 2.197 \\
\hline 81.289 & 48.334 & 131.82 \\
\hline 43.94 & 2.197 & 4.394 \\
\hline 2.197 & 1006.23 & 2.197 \\
\hline 226.291 & 9405.36 & 10.985 \\
\hline 2.197 & 4.394 & 17.576 \\
\hline 19.773 & 6.591 & 2.197 \\
\hline 2.197 & 2.197 & 2.197 \\
\hline 2.197 & 2.197 & 96.668 \\
\hline 6.591 & 2.197 & 138.411 \\
\hline 10.985 & 8.788 & 8.788 \\
\hline 2.197 & 2.197 & 4.394 \\
\hline 2.197 & 2.197 & 2.197 \\
\hline 15.379 & 2.197 & 13.182 \\
\hline 140.608 & 8.788 & 15.379 \\
\hline 8.788 & 8.788 & 28.561 \\
\hline 2.197 & 2.197 & 15.379 \\
\hline 8.788 & 8.788 & 2.197 \\
\hline 13.182 & 2.197 & 2.197 \\
\hline 19.773 & 151.593 & 4.394 \\
\hline 15.379 & 2.197 & 6.591 \\
\hline
\end{tabular}




\begin{tabular}{|c|c|c|}
\hline 72.501 & 10.985 & 26.364 \\
\hline 4.394 & 35.152 & 4.394 \\
\hline 924.937 & 2.197 & 8.788 \\
\hline 87.88 & 4.394 & 4.394 \\
\hline 39.546 & 2.197 & 13.182 \\
\hline 314.171 & 2.197 & 15.379 \\
\hline 30.758 & 21.97 & 6.591 \\
\hline 6.591 & 2.197 & 2.197 \\
\hline 65.91 & 2.197 & 1122.67 \\
\hline 59.319 & 2.197 & 46.137 \\
\hline 2.197 & 4.394 & 6.591 \\
\hline 2.197 & 2.197 & 6.591 \\
\hline 2.197 & 2.197 & 52.728 \\
\hline 2.197 & 2.197 & 37.349 \\
\hline 4.394 & 2.197 & 217.503 \\
\hline 17.576 & 145.002 & 54.925 \\
\hline 41.743 & 4.394 & 13.182 \\
\hline 10.985 & 46.137 & 2.197 \\
\hline 140.608 & 2.197 & 4.394 \\
\hline 39.546 & 6.591 & 2.197 \\
\hline 2.197 & 257.049 & 48.334 \\
\hline 4.394 & 30.758 & 70.304 \\
\hline 2.197 & 4.394 & 2.197 \\
\hline 2.197 & 2.197 & 24.167 \\
\hline 239.473 & 2.197 & 83.486 \\
\hline 30.758 & 2.197 & 2.197 \\
\hline 24.167 & 2.197 & 4.394 \\
\hline 52.728 & 6.591 & 300.989 \\
\hline 4.394 & 2.197 & 4.394 \\
\hline 162.578 & 6.591 & 2.197 \\
\hline 2.197 & 6.591 & 16679.6 \\
\hline 2.197 & 13.182 & 232.882 \\
\hline 32.955 & 2.197 & 15.379 \\
\hline 15.379 & 252.655 & 13.182 \\
\hline 83.486 & 32.955 & 19.773 \\
\hline 4.394 & 24.167 & 2.197 \\
\hline 37.349 & 4.394 & 8.788 \\
\hline 4.394 & 21.97 & 2.197 \\
\hline 10.985 & 85.683 & 17.576 \\
\hline 2.197 & 2.197 & 2.197 \\
\hline 24.167 & 2.197 & 112.047 \\
\hline 70.304 & 2.197 & 81.289 \\
\hline 2.197 & 2.197 & 837.057 \\
\hline 6.591 & 30.758 & 4.394 \\
\hline 2.197 & 2.197 & 10.985 \\
\hline 6.591 & 114.244 & 6.591 \\
\hline 2.197 & 4.394 & 2.197 \\
\hline 8.788 & 72.501 & 65.91 \\
\hline 6.591 & 15.379 & 28.561 \\
\hline 2.197 & 30.758 & 15.379 \\
\hline
\end{tabular}




\begin{tabular}{|c|c|c|}
\hline 8.788 & 57.122 & 4.394 \\
\hline 147.199 & 2.197 & 32.955 \\
\hline 4.394 & 48.334 & 2.197 \\
\hline 2.197 & 2.197 & 2.197 \\
\hline 4.394 & 4.394 & 290.004 \\
\hline 2.197 & 4.394 & 4.394 \\
\hline 149.396 & 4.394 & 30.758 \\
\hline 17.576 & 8.788 & 2.197 \\
\hline 2.197 & 4.394 & 19.773 \\
\hline 74.698 & 4.394 & 2.197 \\
\hline 4.394 & 50.531 & 21.97 \\
\hline 2.197 & 4.394 & 2.197 \\
\hline 2.197 & 6.591 & 358.111 \\
\hline 103.259 & 8.788 & 166.972 \\
\hline 2.197 & 2.197 & 2.197 \\
\hline 4.394 & 2.197 & 2.197 \\
\hline 8.788 & 2.197 & 54.925 \\
\hline 2.197 & 8.788 & 6.591 \\
\hline 2.197 & 1388.5 & 4.394 \\
\hline 17.576 & 19.773 & 87.88 \\
\hline 8.788 & 2.197 & 26.364 \\
\hline 2.197 & 6.591 & 52.728 \\
\hline 4.394 & 760.162 & 160.381 \\
\hline 193.336 & 21.97 & 129.623 \\
\hline 2.197 & 2.197 & 10.985 \\
\hline 127.426 & 41.743 & 52.728 \\
\hline 4.394 & 4.394 & 2.197 \\
\hline 35.152 & 2.197 & 4.394 \\
\hline 10.985 & 2.197 & 65.91 \\
\hline 2.197 & 4.394 & 2.197 \\
\hline 10.985 & 239.473 & 19.773 \\
\hline 2.197 & 8.788 & 114.244 \\
\hline 26.364 & 8.788 & 8.788 \\
\hline 4.394 & 6.591 & 151.593 \\
\hline 2.197 & 4.394 & 2.197 \\
\hline 2.197 & 6.591 & 2.197 \\
\hline 43.94 & 2.197 & 2.197 \\
\hline 46.137 & 8.788 & 2.197 \\
\hline 4.394 & 4.394 & 10.985 \\
\hline 4.394 & 2.197 & 74.698 \\
\hline 2.197 & 17.576 & 8.788 \\
\hline 103.259 & 6.591 & 28.561 \\
\hline 30.758 & 250.458 & 2.197 \\
\hline 2.197 & 13.182 & 74.698 \\
\hline 26.364 & 26.364 & 21.97 \\
\hline 4.394 & 2.197 & 2.197 \\
\hline 13.182 & 8.788 & 30.758 \\
\hline 4.394 & 98.865 & 2.197 \\
\hline 6.591 & 2.197 & 13.182 \\
\hline 6.591 & 2.197 & 4.394 \\
\hline
\end{tabular}




\begin{tabular}{|c|c|c|}
\hline 331.747 & 37.349 & 6.591 \\
\hline 6.591 & 6.591 & 2.197 \\
\hline 2.197 & 96.668 & 2.197 \\
\hline 43.94 & 2.197 & 2.197 \\
\hline 17.576 & 2.197 & 4.394 \\
\hline 10.985 & 24.167 & 21.97 \\
\hline 6.591 & 37.349 & 2.197 \\
\hline 4.394 & 4.394 & 30.758 \\
\hline 50.531 & 8.788 & 2.197 \\
\hline 30.758 & 142.805 & 10.985 \\
\hline 2.197 & 13.182 & 2.197 \\
\hline 2.197 & 4.394 & 26.364 \\
\hline 13.182 & 153.79 & 68.107 \\
\hline 17.576 & 2.197 & 8.788 \\
\hline 8.788 & 24.167 & 10.985 \\
\hline 6.591 & 2.197 & 2.197 \\
\hline 17.576 & 2.197 & 19.773 \\
\hline 76.895 & 2.197 & 4.394 \\
\hline 2.197 & 2.197 & 6.591 \\
\hline 2.197 & 2.197 & 17.576 \\
\hline 2.197 & 8.788 & 24.167 \\
\hline 2.197 & 10.985 & 35.152 \\
\hline 26.364 & 2.197 & 2.197 \\
\hline 4.394 & 2.197 & 2.197 \\
\hline 4.394 & 4.394 & 6.591 \\
\hline 2.197 & 37.349 & 2.197 \\
\hline 37.349 & 8.788 & 2.197 \\
\hline 15.379 & 2.197 & 2.197 \\
\hline 8.788 & 2.197 & 8.788 \\
\hline 408.642 & 2.197 & 8.788 \\
\hline 85.683 & 2.197 & 6.591 \\
\hline 63.713 & 6.591 & 2.197 \\
\hline 6.591 & 8.788 & 4.394 \\
\hline 2.197 & 4.394 & 1333.58 \\
\hline 19.773 & 4.394 & 21.97 \\
\hline 2.197 & 21.97 & 155.987 \\
\hline 2.197 & 311.974 & 2.197 \\
\hline 8.788 & 15.379 & 2.197 \\
\hline 17.576 & 2.197 & 26.364 \\
\hline 8.788 & 37.349 & 17.576 \\
\hline 41.743 & 2.197 & 2.197 \\
\hline 15.379 & 2.197 & 4.394 \\
\hline 8.788 & 24.167 & 4.394 \\
\hline 1298.43 & 2.197 & 2.197 \\
\hline 6.591 & 2.197 & 2.197 \\
\hline 290.004 & 2.197 & 2.197 \\
\hline 2.197 & 2.197 & 4.394 \\
\hline 2.197 & 2.197 & 101.062 \\
\hline 15.379 & 13.182 & 41.743 \\
\hline 147.199 & 2.197 & 120.835 \\
\hline
\end{tabular}




\begin{tabular}{|c|c|c|}
\hline 13.182 & 21.97 & 4.394 \\
\hline 2.197 & 2.197 & 15.379 \\
\hline 15.379 & 4.394 & 19.773 \\
\hline 21.97 & 2.197 & 386.672 \\
\hline 8.788 & 2.197 & 92.274 \\
\hline 26.364 & 4.394 & 35.152 \\
\hline 2.197 & 24.167 & 6.591 \\
\hline 4.394 & 2.197 & 6.591 \\
\hline 2.197 & 4.394 & 2.197 \\
\hline 2.197 & 4.394 & 94.471 \\
\hline 24.167 & 2.197 & 2.197 \\
\hline 19.773 & 2.197 & 13.182 \\
\hline 2.197 & 2.197 & 37.349 \\
\hline 8.788 & 2.197 & 2.197 \\
\hline 4598.32 & 2.197 & 307.58 \\
\hline 13.182 & 17.576 & 28.561 \\
\hline 21.97 & 2.197 & 2.197 \\
\hline 83.486 & 116.441 & 2.197 \\
\hline 76.895 & 10.985 & 4.394 \\
\hline 37.349 & 1649.95 & 6.591 \\
\hline 2.197 & 15.379 & 2.197 \\
\hline 182.351 & 2.197 & 4.394 \\
\hline 21.97 & 83.486 & 59.319 \\
\hline 4.394 & 63.713 & 21.97 \\
\hline 6.591 & 2.197 & 125.229 \\
\hline 10.985 & 8.788 & 4.394 \\
\hline 8.788 & 2.197 & 224.094 \\
\hline 4.394 & 4.394 & 8.788 \\
\hline 2.197 & 4.394 & 2.197 \\
\hline 21.97 & 6.591 & 19.773 \\
\hline 4.394 & 4.394 & 10.985 \\
\hline 2.197 & 4.394 & 24.167 \\
\hline 17.576 & 2.197 & 37.349 \\
\hline 24.167 & 2.197 & 41.743 \\
\hline 2.197 & 4.394 & 48.334 \\
\hline 158.184 & 2809.96 & 13.182 \\
\hline 2.197 & 2.197 & 2.197 \\
\hline 2.197 & 2.197 & 4.394 \\
\hline 2.197 & 4.394 & 2.197 \\
\hline 6.591 & 10.985 & 10.985 \\
\hline 8.788 & 23351.9 & 1733.43 \\
\hline 57.122 & 13.182 & 2.197 \\
\hline 4.394 & 10.985 & 15.379 \\
\hline 107.653 & 10.985 & 2.197 \\
\hline 140.608 & 4.394 & 2.197 \\
\hline 19.773 & 2.197 & 2.197 \\
\hline 30.758 & 4.394 & 4.394 \\
\hline 2.197 & 2.197 & 6.591 \\
\hline 2.197 & 2.197 & 125.229 \\
\hline 2.197 & 61.516 & 4.394 \\
\hline
\end{tabular}




\begin{tabular}{|c|c|c|}
\hline 4.394 & 2.197 & 2.197 \\
\hline 2.197 & 2.197 & 10.985 \\
\hline 259.246 & 17.576 & 10.985 \\
\hline 19.773 & 2.197 & 98.865 \\
\hline 85.683 & 2.197 & 13.182 \\
\hline 39.546 & 2.197 & 8.788 \\
\hline 8.788 & 43.94 & 41.743 \\
\hline 4.394 & 4.394 & 2.197 \\
\hline 112.047 & 63.713 & 2.197 \\
\hline 8.788 & 2.197 & 2.197 \\
\hline 2.197 & 4.394 & 28.561 \\
\hline 6.591 & 2.197 & 2.197 \\
\hline 15.379 & 4.394 & 19.773 \\
\hline 65.91 & 2.197 & 74.698 \\
\hline 4.394 & 41.743 & 219.7 \\
\hline 2.197 & 2.197 & 3262.54 \\
\hline 4.394 & 333.944 & 10.985 \\
\hline 2.197 & 4.394 & 70.304 \\
\hline 4.394 & 2.197 & 2.197 \\
\hline 21.97 & 6.591 & 2.197 \\
\hline 153.79 & 2.197 & 8.788 \\
\hline 32.955 & 6.591 & 52.728 \\
\hline 2.197 & 6.591 & 2.197 \\
\hline 4.394 & 10.985 & 8.788 \\
\hline 250.458 & 184.548 & 10.985 \\
\hline 2.197 & 6.591 & 13.182 \\
\hline 10.985 & 32.955 & 8.788 \\
\hline 24.167 & 2.197 & 76.895 \\
\hline 10.985 & 2.197 & 74.698 \\
\hline 8.788 & 8.788 & 8.788 \\
\hline 4.394 & 2.197 & 2.197 \\
\hline 4.394 & 2.197 & 2.197 \\
\hline 6.591 & 2.197 & 6.591 \\
\hline 28.561 & 2.197 & 2.197 \\
\hline 70.304 & 4.394 & 136.214 \\
\hline 10.985 & 2.197 & 8.788 \\
\hline 10.985 & 4.394 & 4.394 \\
\hline 72.501 & 2.197 & 2.197 \\
\hline 2.197 & 10.985 & 2.197 \\
\hline 4.394 & 15.379 & 2.197 \\
\hline 2.197 & 85.683 & 13.182 \\
\hline 61.516 & 2.197 & 15.379 \\
\hline 4.394 & 2.197 & 21.97 \\
\hline 2.197 & 2.197 & 10.985 \\
\hline 2.197 & 2.197 & 4.394 \\
\hline 162.578 & 2.197 & 2.197 \\
\hline 98.865 & 127.426 & 15.379 \\
\hline 184.548 & 2.197 & 2.197 \\
\hline 6.591 & 30.758 & 441.597 \\
\hline 26.364 & 17.576 & 70.304 \\
\hline
\end{tabular}




\begin{tabular}{|c|c|c|}
\hline 13.182 & 8388.15 & 41.743 \\
\hline 199.927 & 2.197 & 4.394 \\
\hline 13.182 & 61.516 & 10.985 \\
\hline 4.394 & 6.591 & 138.411 \\
\hline 2.197 & 2.197 & 450.385 \\
\hline 46.137 & 2.197 & 8.788 \\
\hline 2.197 & 2.197 & 2.197 \\
\hline 6.591 & 2.197 & 4.394 \\
\hline 10.985 & 263.64 & 2.197 \\
\hline 2.197 & 8.788 & 50.531 \\
\hline 4.394 & 8.788 & 37.349 \\
\hline 94.471 & 4.394 & 153.79 \\
\hline 6.591 & 2.197 & 344.929 \\
\hline 39.546 & 8.788 & 2.197 \\
\hline 195.533 & 2.197 & 39.546 \\
\hline 4.394 & 6.591 & 6.591 \\
\hline 4.394 & 35.152 & 13.182 \\
\hline 101.062 & 210.912 & 10.985 \\
\hline 19.773 & 193.336 & 2.197 \\
\hline 8.788 & 2.197 & 2.197 \\
\hline 10.985 & 160.381 & 13.182 \\
\hline 15.379 & 4.394 & 6.591 \\
\hline 43.94 & 2.197 & 2.197 \\
\hline 13.182 & 13.182 & 13.182 \\
\hline 17.576 & 24.167 & 10.985 \\
\hline 8.788 & 118.638 & 68.107 \\
\hline 24.167 & 2.197 & 4.394 \\
\hline 4.394 & 48.334 & 2.197 \\
\hline 4.394 & 2.197 & 2.197 \\
\hline 4.394 & 8.788 & 28.561 \\
\hline 17.576 & 2.197 & 241.67 \\
\hline 2.197 & 2.197 & 13.182 \\
\hline 8.788 & 2.197 & 2.197 \\
\hline 26.364 & 2.197 & 4.394 \\
\hline 26.364 & 2.197 & 2.197 \\
\hline 430.612 & 2.197 & 6.591 \\
\hline 21.97 & 2.197 & 129.623 \\
\hline 105.456 & 2.197 & 2.197 \\
\hline 2.197 & 2.197 & 13.182 \\
\hline 2.197 & 2.197 & 4.394 \\
\hline 4.394 & 2.197 & 2.197 \\
\hline 4.394 & 6.591 & 114.244 \\
\hline 4.394 & 2.197 & 24.167 \\
\hline 298.792 & 21.97 & 2.197 \\
\hline 155.987 & 2.197 & 2.197 \\
\hline 10.985 & 4.394 & 2.197 \\
\hline 6.591 & 39.546 & 30.758 \\
\hline 2.197 & 4.394 & 19.773 \\
\hline 2.197 & 2.197 & 2.197 \\
\hline 19.773 & 4.394 & 13.182 \\
\hline
\end{tabular}




\begin{tabular}{|c|c|c|}
\hline 4.394 & 10.985 & 6.591 \\
\hline 197.73 & 2.197 & 46.137 \\
\hline 35.152 & 94.471 & 10.985 \\
\hline 28.561 & 4.394 & 2.197 \\
\hline 6.591 & 4.394 & 28.561 \\
\hline 41.743 & 8.788 & 2.197 \\
\hline 279.019 & 2.197 & 109.85 \\
\hline 21.97 & 17.576 & 10.985 \\
\hline 166.972 & 17.576 & 2.197 \\
\hline 8.788 & 2.197 & 2.197 \\
\hline 4.394 & 70.304 & 4.394 \\
\hline 2.197 & 2.197 & 30.758 \\
\hline 140.608 & 2.197 & 123.032 \\
\hline 250.458 & 129.623 & 13.182 \\
\hline 13.182 & 46.137 & 2.197 \\
\hline 2.197 & 4.394 & 50.531 \\
\hline 2.197 & 2.197 & 2.197 \\
\hline 17.576 & 6.591 & 81.289 \\
\hline 17.576 & 2.197 & 4.394 \\
\hline 6.591 & 2.197 & 2.197 \\
\hline 26.364 & 35.152 & 70.304 \\
\hline 17.576 & 4.394 & 2.197 \\
\hline 35.152 & 2.197 & 4.394 \\
\hline 74.698 & 10.985 & 41.743 \\
\hline 43.94 & 2.197 & 2.197 \\
\hline 4.394 & 2.197 & 37.349 \\
\hline 6.591 & 4.394 & 2.197 \\
\hline 2.197 & 4.394 & 2.197 \\
\hline 6.591 & 4.394 & 4.394 \\
\hline 2.197 & 2.197 & 6.591 \\
\hline 41.743 & 4.394 & 2.197 \\
\hline 173.563 & 2.197 & 26.364 \\
\hline 2.197 & 2.197 & 2.197 \\
\hline 57.122 & 2.197 & 6.591 \\
\hline 4.394 & 2.197 & 4.394 \\
\hline 17.576 & 241.67 & 2.197 \\
\hline 4.394 & 10.985 & 24.167 \\
\hline 4.394 & 2.197 & 4752.11 \\
\hline 2.197 & 2.197 & 6.591 \\
\hline 61.516 & 4.394 & 2.197 \\
\hline 2.197 & 8.788 & 94.471 \\
\hline 2.197 & 48.334 & 46.137 \\
\hline 2.197 & 2.197 & 2.197 \\
\hline 4.394 & 4.394 & 46.137 \\
\hline 4.394 & 2.197 & 2.197 \\
\hline 349.323 & 2.197 & 6.591 \\
\hline 4.394 & 26.364 & 2.197 \\
\hline 450.385 & 125.229 & 8.788 \\
\hline 8.788 & 2.197 & 4.394 \\
\hline 2.197 & 4.394 & 4.394 \\
\hline
\end{tabular}




\begin{tabular}{|c|c|c|}
\hline 43.94 & 138.411 & 439.4 \\
\hline 4.394 & 2.197 & 2.197 \\
\hline 127.426 & 243.867 & 4.394 \\
\hline 140.608 & 2.197 & 52.728 \\
\hline 2.197 & 2.197 & 4.394 \\
\hline 2.197 & 2.197 & 6.591 \\
\hline 8.788 & 2.197 & 2.197 \\
\hline 2.197 & 2.197 & 169.169 \\
\hline 4.394 & 2.197 & 2.197 \\
\hline 43.94 & 6.591 & 6.591 \\
\hline 6.591 & 2.197 & 32.955 \\
\hline 4.394 & 340.535 & 2.197 \\
\hline 101.062 & 10.985 & 2.197 \\
\hline 13.182 & 4.394 & 153.79 \\
\hline 10.985 & 2.197 & 2.197 \\
\hline 2.197 & 48.334 & 8.788 \\
\hline 15.379 & 2.197 & 39.546 \\
\hline 26.364 & 2.197 & 1050.17 \\
\hline 2.197 & 90.077 & 2.197 \\
\hline 2.197 & 4.394 & 2.197 \\
\hline 85.683 & 2.197 & 10.985 \\
\hline 13.182 & 2.197 & 15.379 \\
\hline 2.197 & 37.349 & 2.197 \\
\hline 15.379 & 15.379 & 2.197 \\
\hline 13.182 & 2.197 & 6.591 \\
\hline 10.985 & 2.197 & 2.197 \\
\hline 17.576 & 90.077 & 2.197 \\
\hline 37.349 & 2.197 & 2.197 \\
\hline 24.167 & 30.758 & 4.394 \\
\hline 2.197 & 4.394 & 4.394 \\
\hline 4.394 & 24.167 & 21.97 \\
\hline 2.197 & 2.197 & 155.987 \\
\hline 4.394 & 2.197 & 4.394 \\
\hline 204.321 & 24.167 & 2.197 \\
\hline 8.788 & 8.788 & 30.758 \\
\hline 2.197 & 24.167 & 30.758 \\
\hline 30.758 & 90.077 & 153.79 \\
\hline 8.788 & 2.197 & 2.197 \\
\hline 118.638 & 94.471 & 2.197 \\
\hline 2.197 & 37.349 & 6.591 \\
\hline 2.197 & 15.379 & 2.197 \\
\hline 6.591 & 17.576 & 2.197 \\
\hline 4.394 & 24.167 & 4.394 \\
\hline 2.197 & 3866.72 & 19.773 \\
\hline 8.788 & 2.197 & 8.788 \\
\hline 4.394 & 52.728 & 4.394 \\
\hline 2.197 & 4.394 & 19.773 \\
\hline 4.394 & 2.197 & 19.773 \\
\hline 54.925 & 2.197 & 6.591 \\
\hline 17.576 & 2.197 & 8.788 \\
\hline
\end{tabular}




\begin{tabular}{|c|c|c|}
\hline 8.788 & 2.197 & 2.197 \\
\hline 2.197 & 24.167 & 880.997 \\
\hline 28.561 & 4.394 & 30.758 \\
\hline 2.197 & 2.197 & 96.668 \\
\hline 68.107 & 2.197 & 79.092 \\
\hline 2.197 & 19.773 & 81.289 \\
\hline 74.698 & 2.197 & 6.591 \\
\hline 2.197 & 2.197 & 41.743 \\
\hline 4.394 & 57.122 & 17.576 \\
\hline 21.97 & 2.197 & 90.077 \\
\hline 2.197 & 2.197 & 2.197 \\
\hline 8.788 & 96.668 & 2.197 \\
\hline 15.379 & 85.683 & 2.197 \\
\hline 87.88 & 2.197 & 2.197 \\
\hline 10.985 & 2.197 & 2.197 \\
\hline 4.394 & 2.197 & 6.591 \\
\hline 4.394 & 6.591 & 2.197 \\
\hline 2.197 & 228.488 & 4.394 \\
\hline 2.197 & 2.197 & 37.349 \\
\hline 46.137 & 2.197 & 6.591 \\
\hline 30.758 & 10.985 & 2.197 \\
\hline 4.394 & 17.576 & 10.985 \\
\hline 48.334 & 6.591 & 10.985 \\
\hline 17.576 & 199.927 & 50.531 \\
\hline 76.895 & 4.394 & 322.959 \\
\hline 74.698 & 2.197 & 2.197 \\
\hline 35.152 & 2.197 & 6.591 \\
\hline 19.773 & 10.985 & 6.591 \\
\hline 2.197 & 2.197 & 17.576 \\
\hline 41.743 & 2.197 & 15.379 \\
\hline 145.002 & 2.197 & 494.325 \\
\hline 15.379 & 2.197 & 2.197 \\
\hline 10.985 & 2.197 & 32.955 \\
\hline 129.623 & 2.197 & 24.167 \\
\hline 15.379 & 10.985 & 2.197 \\
\hline 26.364 & 8.788 & 6.591 \\
\hline 2.197 & 2.197 & 2.197 \\
\hline 2.197 & 2.197 & 15.379 \\
\hline 2.197 & 4.394 & 81.289 \\
\hline 19.773 & 2.197 & 186.745 \\
\hline 4.394 & 13.182 & 30.758 \\
\hline 4.394 & 2.197 & 54.925 \\
\hline 147.199 & 8.788 & 28.561 \\
\hline 2.197 & 2.197 & 6.591 \\
\hline 17.576 & 21.97 & 54.925 \\
\hline 4.394 & 15.379 & 17.576 \\
\hline 52.728 & 28.561 & 6.591 \\
\hline 210.912 & 4.394 & 17.576 \\
\hline 2.197 & 19.773 & 37.349 \\
\hline 2.197 & 2.197 & 4.394 \\
\hline
\end{tabular}




\begin{tabular}{|c|c|c|}
\hline 4.394 & 2.197 & 10.985 \\
\hline 19.773 & 2.197 & 2.197 \\
\hline 28.561 & 4.394 & 24.167 \\
\hline 2.197 & 4.394 & 28.561 \\
\hline 4.394 & 35.152 & 28.561 \\
\hline 52.728 & 26.364 & 4.394 \\
\hline 48.334 & 10.985 & 2.197 \\
\hline 2.197 & 2.197 & 2.197 \\
\hline 2.197 & 2.197 & 2.197 \\
\hline 2.197 & 6.591 & 586.599 \\
\hline 2.197 & 8.788 & 37.349 \\
\hline 2.197 & 8.788 & 8.788 \\
\hline 2.197 & 228.488 & 39.546 \\
\hline 32.955 & 8.788 & 6.591 \\
\hline 65.91 & 4.394 & 65.91 \\
\hline 2.197 & 61.516 & 2.197 \\
\hline 2.197 & 4.394 & 2.197 \\
\hline 24.167 & 4.394 & 2.197 \\
\hline 48.334 & 4.394 & 4.394 \\
\hline 2.197 & 92.274 & 4.394 \\
\hline 4.394 & 2.197 & 48.334 \\
\hline 43.94 & 2.197 & 35.152 \\
\hline 65.91 & 2.197 & 19.773 \\
\hline 8.788 & 101.062 & 4.394 \\
\hline 10.985 & 8.788 & 2.197 \\
\hline 37.349 & 2.197 & 10.985 \\
\hline 28.561 & 2.197 & 3447.09 \\
\hline 39.546 & 46.137 & 13.182 \\
\hline 6.591 & 2.197 & 98.865 \\
\hline 17.576 & 2.197 & 204.321 \\
\hline 2.197 & 8.788 & 94.471 \\
\hline 103.259 & 2.197 & 4.394 \\
\hline 15.379 & 2.197 & 21.97 \\
\hline 15.379 & 13.182 & 8.788 \\
\hline 476.749 & 2.197 & 2.197 \\
\hline 2.197 & 10.985 & 193.336 \\
\hline 2.197 & 2.197 & 437.203 \\
\hline 81.289 & 76.895 & 52.728 \\
\hline 10.985 & 4.394 & 4.394 \\
\hline 21.97 & 2.197 & 123.032 \\
\hline 4.394 & 2.197 & 21.97 \\
\hline 6.591 & 2.197 & 13.182 \\
\hline 24.167 & 2.197 & 50.531 \\
\hline 65.91 & 10.985 & 3587.7 \\
\hline 2.197 & 2.197 & 2.197 \\
\hline 2.197 & 2.197 & 173.563 \\
\hline 6.591 & 6.591 & 4.394 \\
\hline 197.73 & 2.197 & 48.334 \\
\hline 4.394 & 2.197 & 87.88 \\
\hline 8.788 & 21.97 & 4.394 \\
\hline
\end{tabular}




\begin{tabular}{|c|c|c|}
\hline 17.576 & 13.182 & 6.591 \\
\hline 2.197 & 1961.92 & 10.985 \\
\hline 19.773 & 2.197 & 13.182 \\
\hline 4.394 & 8.788 & 52.728 \\
\hline 2.197 & 2.197 & 2.197 \\
\hline 13.182 & 2.197 & 4.394 \\
\hline 6.591 & 10.985 & 2.197 \\
\hline 2.197 & 2.197 & 10.985 \\
\hline 2.197 & 32.955 & 4.394 \\
\hline 2.197 & 2.197 & 2.197 \\
\hline 6.591 & 2.197 & 6.591 \\
\hline 6.591 & 35.152 & 2.197 \\
\hline 57.122 & 8.788 & 4.394 \\
\hline 39.546 & 2.197 & 8.788 \\
\hline 6.591 & 43.94 & 1643.36 \\
\hline 10.985 & 21.97 & 2.197 \\
\hline 2.197 & 35.152 & 4.394 \\
\hline 2.197 & 15.379 & 13.182 \\
\hline 6.591 & 13.182 & 2.197 \\
\hline 10.985 & 2.197 & 2.197 \\
\hline 1612.6 & 2.197 & 21.97 \\
\hline 2.197 & 2.197 & 105.456 \\
\hline 2.197 & 2.197 & 8.788 \\
\hline 2.197 & 2.197 & 2.197 \\
\hline 217.503 & 4.394 & 2.197 \\
\hline 2.197 & 2.197 & 54.925 \\
\hline 32.955 & 2.197 & 63.713 \\
\hline 94.471 & 569.023 & 4.394 \\
\hline 4.394 & 2.197 & 13.182 \\
\hline 17.576 & 4.394 & 19.773 \\
\hline 19.773 & 6.591 & 2.197 \\
\hline 2.197 & 182.351 & 2.197 \\
\hline 4.394 & 2.197 & 2.197 \\
\hline 2.197 & 13.182 & 2.197 \\
\hline 2.197 & 4.394 & 46.137 \\
\hline 6.591 & 217.503 & 2.197 \\
\hline 2.197 & 74.698 & 6.591 \\
\hline 4.394 & 2.197 & 120.835 \\
\hline 15.379 & 2.197 & 2.197 \\
\hline 2.197 & 2.197 & 2.197 \\
\hline 63.713 & 17.576 & 4.394 \\
\hline 83.486 & 6.591 & 254.852 \\
\hline 30.758 & 2.197 & 10.985 \\
\hline 92.274 & 2.197 & 6.591 \\
\hline 2.197 & 10.985 & 8.788 \\
\hline 4.394 & 202.124 & 26.364 \\
\hline 52.728 & 4.394 & 8.788 \\
\hline 2.197 & 59.319 & 975.468 \\
\hline 26.364 & 4.394 & 19.773 \\
\hline 6.591 & 74.698 & 2.197 \\
\hline
\end{tabular}




\begin{tabular}{|c|c|c|}
\hline 4.394 & 364.702 & 19.773 \\
\hline 2.197 & 4.394 & 2.197 \\
\hline 96.668 & 2.197 & 2.197 \\
\hline 290.004 & 2.197 & 6.591 \\
\hline 2.197 & 2.197 & 24.167 \\
\hline 2.197 & 65.91 & 19.773 \\
\hline 2.197 & 6.591 & 6.591 \\
\hline 24.167 & 39.546 & 32.955 \\
\hline 2.197 & 28.561 & 10.985 \\
\hline 21.97 & 6.591 & 8.788 \\
\hline 4.394 & 30.758 & 4.394 \\
\hline 21.97 & 2.197 & 6.591 \\
\hline 2.197 & 2.197 & 2.197 \\
\hline 114.244 & 131.82 & 15.379 \\
\hline 4.394 & 17.576 & 6.591 \\
\hline 2.197 & 2.197 & 6.591 \\
\hline 4.394 & 2.197 & 2.197 \\
\hline 85.683 & 2.197 & 8.788 \\
\hline 1353.35 & 2.197 & 426.218 \\
\hline 2.197 & 8.788 & 19.773 \\
\hline 28.561 & 2.197 & 8.788 \\
\hline 6.591 & 87.88 & 13.182 \\
\hline 6.591 & 2.197 & 13.182 \\
\hline 228.488 & 10.985 & 17.576 \\
\hline 32.955 & 30.758 & 6.591 \\
\hline 10.985 & 10.985 & 19.773 \\
\hline 15.379 & 2.197 & 268.034 \\
\hline 81.289 & 2.197 & 8.788 \\
\hline 4.394 & 2.197 & 4.394 \\
\hline 580.008 & 131.82 & 50.531 \\
\hline 2.197 & 4.394 & 4.394 \\
\hline 59.319 & 24.167 & 2.197 \\
\hline 2.197 & 4.394 & 13.182 \\
\hline 2.197 & 6.591 & 13.182 \\
\hline 4.394 & 13.182 & 19.773 \\
\hline 87.88 & 2.197 & 17.576 \\
\hline 103.259 & 2.197 & 8.788 \\
\hline 2.197 & 134.017 & 35.152 \\
\hline 2.197 & 4.394 & 50.531 \\
\hline 4.394 & 6.591 & 347.126 \\
\hline 4.394 & 2.197 & 268.034 \\
\hline 19.773 & 26.364 & 2.197 \\
\hline 48.334 & 43.94 & 2.197 \\
\hline 2.197 & 2.197 & 2.197 \\
\hline 4.394 & 2.197 & 15.379 \\
\hline 43.94 & 15.379 & 15.379 \\
\hline 2.197 & 230.685 & 268.034 \\
\hline 2.197 & 81.289 & 4.394 \\
\hline 24.167 & 17.576 & 46.137 \\
\hline 4.394 & 10.985 & 2.197 \\
\hline
\end{tabular}




\begin{tabular}{|c|c|c|}
\hline 2.197 & 6.591 & 41.743 \\
\hline 2.197 & 246.064 & 15.379 \\
\hline 103.259 & 2.197 & 2.197 \\
\hline 10.985 & 30.758 & 902.967 \\
\hline 2.197 & 2.197 & 17.576 \\
\hline 217.503 & 4.394 & 52.728 \\
\hline 118.638 & 2.197 & 4.394 \\
\hline 2.197 & 2.197 & 634.933 \\
\hline 76.895 & 72.501 & 116.441 \\
\hline 221.897 & 70.304 & 101.062 \\
\hline 8.788 & 4.394 & 32.955 \\
\hline 123.032 & 2.197 & 2.197 \\
\hline 28.561 & 10.985 & 2.197 \\
\hline 2.197 & 30.758 & 2.197 \\
\hline 107.653 & 1074.33 & 2.197 \\
\hline 46.137 & 15.379 & 57.122 \\
\hline 21.97 & 21.97 & 2.197 \\
\hline 28.561 & 2.197 & 32.955 \\
\hline 24.167 & 6.591 & 4.394 \\
\hline 2.197 & 32.955 & 15.379 \\
\hline 224.094 & 2.197 & 2.197 \\
\hline 2.197 & 720.616 & 4.394 \\
\hline 46.137 & 6.591 & 52.728 \\
\hline 116.441 & 2.197 & 2.197 \\
\hline 2.197 & 331.747 & 4679.61 \\
\hline 2.197 & 6.591 & 8.788 \\
\hline 8.788 & 505.31 & 2.197 \\
\hline 15.379 & 2.197 & 37.349 \\
\hline 2.197 & 2.197 & 4.394 \\
\hline 79.092 & 2.197 & 28.561 \\
\hline 408.642 & 4.394 & 32.955 \\
\hline 2.197 & 2.197 & 6.591 \\
\hline 2.197 & 2.197 & 26.364 \\
\hline 4.394 & 32.955 & 32.955 \\
\hline 68.107 & 6.591 & 32.955 \\
\hline 19.773 & 10.985 & 2.197 \\
\hline 4.394 & 2.197 & 30.758 \\
\hline 155.987 & 4.394 & 8.788 \\
\hline 13.182 & 2.197 & 6.591 \\
\hline 10.985 & 2.197 & 2.197 \\
\hline 74.698 & 8.788 & 76.895 \\
\hline 4.394 & 2.197 & 129.623 \\
\hline 2.197 & 4.394 & 6.591 \\
\hline 2.197 & 2.197 & 17.576 \\
\hline 4.394 & 8.788 & 13.182 \\
\hline 35.152 & 4.394 & 1469.79 \\
\hline 6.591 & 6.591 & 90.077 \\
\hline 6.591 & 2.197 & 46.137 \\
\hline 6.591 & 4.394 & 2.197 \\
\hline 2.197 & 173.563 & 4.394 \\
\hline
\end{tabular}




\begin{tabular}{|c|c|c|}
\hline 6.591 & 2.197 & 123.032 \\
\hline 2.197 & 2.197 & 2.197 \\
\hline 61.516 & 24.167 & 2.197 \\
\hline 79.092 & 2.197 & 4.394 \\
\hline 21.97 & 2.197 & 109.85 \\
\hline 4.394 & 2.197 & 24.167 \\
\hline 92.274 & 2.197 & 2.197 \\
\hline 15.379 & 2.197 & 10.985 \\
\hline 46.137 & 8.788 & 6.591 \\
\hline 6.591 & 6.591 & 2.197 \\
\hline 206.518 & 6.591 & 210.912 \\
\hline 21.97 & 19.773 & 32.955 \\
\hline 6.591 & 37.349 & 2.197 \\
\hline 2.197 & 2.197 & 10.985 \\
\hline 10.985 & 17.576 & 13.182 \\
\hline 19.773 & 2.197 & 21.97 \\
\hline 8.788 & 26.364 & 2.197 \\
\hline 17.576 & 2.197 & 10.985 \\
\hline 6.591 & 4.394 & 2.197 \\
\hline 4.394 & 2.197 & 10.985 \\
\hline 15.379 & 4.394 & 4.394 \\
\hline 4.394 & 2.197 & 24.167 \\
\hline 2.197 & 2.197 & 70.304 \\
\hline 32.955 & 2.197 & 21.97 \\
\hline 2.197 & 10.985 & 2.197 \\
\hline 19.773 & 1346.76 & 590.993 \\
\hline 19.773 & 101.062 & 940.316 \\
\hline 24.167 & 28.561 & 30.758 \\
\hline 4.394 & 2.197 & 2.197 \\
\hline 2.197 & 166.972 & 2.197 \\
\hline 2.197 & 17.576 & 13.182 \\
\hline 2.197 & 39.546 & 2.197 \\
\hline 2.197 & 2.197 & 2.197 \\
\hline 17.576 & 50.531 & 1935.56 \\
\hline 98.865 & 2.197 & 4.394 \\
\hline 17.576 & 105.456 & 8.788 \\
\hline 13.182 & 4.394 & 338.338 \\
\hline 2.197 & 2.197 & 46.137 \\
\hline 6.591 & 2.197 & 15.379 \\
\hline 59.319 & 4.394 & 6.591 \\
\hline 2.197 & 8.788 & 2.197 \\
\hline 2.197 & 13.182 & 68.107 \\
\hline 4.394 & 6.591 & 2.197 \\
\hline 39.546 & 4.394 & 2.197 \\
\hline 13.182 & 8.788 & 70.304 \\
\hline 2.197 & 8.788 & 24.167 \\
\hline 2.197 & 2.197 & 70.304 \\
\hline 2.197 & 2.197 & 4.394 \\
\hline 4.394 & 26.364 & 2.197 \\
\hline 19.773 & 2.197 & 2.197 \\
\hline
\end{tabular}




\begin{tabular}{|c|c|c|}
\hline 4.394 & 4.394 & 15.379 \\
\hline 10.985 & 2.197 & 2.197 \\
\hline 15.379 & 2.197 & 4.394 \\
\hline 166.972 & 2.197 & 4.394 \\
\hline 76.895 & 4.394 & 2.197 \\
\hline 15.379 & 158.184 & 15.379 \\
\hline 2.197 & 8.788 & 10.985 \\
\hline 61.516 & 4.394 & 298.792 \\
\hline 2.197 & 32.955 & 2.197 \\
\hline 2.197 & 6.591 & 2.197 \\
\hline 2.197 & 21.97 & 24.167 \\
\hline 2.197 & 6.591 & 13.182 \\
\hline 24.167 & 2.197 & 2.197 \\
\hline 2.197 & 4.394 & 28.561 \\
\hline 26.364 & 2.197 & 37.349 \\
\hline 13.182 & 2.197 & 39.546 \\
\hline 10.985 & 79.092 & 6.591 \\
\hline 6.591 & 4.394 & 13.182 \\
\hline 2.197 & 37.349 & 19.773 \\
\hline 83.486 & 2.197 & 8.788 \\
\hline 2.197 & 2.197 & 13.182 \\
\hline 2.197 & 4.394 & 2.197 \\
\hline 2.197 & 2.197 & 2.197 \\
\hline 15.379 & 2.197 & 261.443 \\
\hline 61.516 & 4.394 & 114.244 \\
\hline 228.488 & 2.197 & 41.743 \\
\hline 4.394 & 2.197 & 81.289 \\
\hline 2.197 & 37.349 & 8.788 \\
\hline 28.561 & 6.591 & 3502.02 \\
\hline 8.788 & 2.197 & 6.591 \\
\hline 2.197 & 4.394 & 6.591 \\
\hline 15.379 & 15.379 & 6.591 \\
\hline 2.197 & 6.591 & 37.349 \\
\hline 2.197 & 2.197 & 171.366 \\
\hline 26.364 & 153.79 & 24.167 \\
\hline 2.197 & 10.985 & 35.152 \\
\hline 125.229 & 2.197 & 35.152 \\
\hline 2.197 & 2.197 & 2539.73 \\
\hline 13.182 & 2.197 & 4.394 \\
\hline 4.394 & 8.788 & 8.788 \\
\hline 2.197 & 30.758 & 294.398 \\
\hline 46.137 & 4.394 & 6.591 \\
\hline 2.197 & 6.591 & 4.394 \\
\hline 21.97 & 2.197 & 2.197 \\
\hline 28.561 & 2.197 & 6.591 \\
\hline 2.197 & 4.394 & 2.197 \\
\hline 2.197 & 206.518 & 10.985 \\
\hline 4.394 & 2.197 & 2.197 \\
\hline 15.379 & 254.852 & 39.546 \\
\hline 30.758 & 2.197 & 10.985 \\
\hline
\end{tabular}




\begin{tabular}{|c|c|c|}
\hline 2.197 & 6.591 & 4.394 \\
\hline 39.546 & 2.197 & 48.334 \\
\hline 2.197 & 15.379 & 8.788 \\
\hline 30.758 & 2.197 & 13.182 \\
\hline 129.623 & 74.698 & 2.197 \\
\hline 41.743 & 2.197 & 340.535 \\
\hline 17.576 & 4.394 & 61.516 \\
\hline 136.214 & 2.197 & 74.698 \\
\hline 19.773 & 50.531 & 15.379 \\
\hline 2.197 & 4.394 & 619.554 \\
\hline 2.197 & 2.197 & 2.197 \\
\hline 46.137 & 2.197 & 2.197 \\
\hline 4.394 & 2.197 & 54.925 \\
\hline 208.715 & 2.197 & 46.137 \\
\hline 4.394 & 10.985 & 6.591 \\
\hline 4.394 & 2.197 & 2.197 \\
\hline 2.197 & 2.197 & 2.197 \\
\hline 2.197 & 90.077 & 17.576 \\
\hline 8.788 & 90.077 & 2.197 \\
\hline 13.182 & 28.561 & 2.197 \\
\hline 2.197 & 2.197 & 2.197 \\
\hline 30.758 & 21.97 & 32.955 \\
\hline 2.197 & 8.788 & 26.364 \\
\hline 17.576 & 41.743 & 817.284 \\
\hline 46.137 & 2.197 & 4.394 \\
\hline 158.184 & 927.134 & 6.591 \\
\hline 6.591 & 59.319 & 8.788 \\
\hline 32.955 & 2.197 & 15.379 \\
\hline 57.122 & 41.743 & 2.197 \\
\hline 2.197 & 2.197 & 13.182 \\
\hline 2.197 & 4.394 & 13.182 \\
\hline 8.788 & 13.182 & 2.197 \\
\hline 28.561 & 2.197 & 10.985 \\
\hline 8.788 & 125.229 & 13.182 \\
\hline 17.576 & 197.73 & 467.961 \\
\hline 6.591 & 19.773 & 112.047 \\
\hline 2.197 & 2.197 & 2.197 \\
\hline 17.576 & 2.197 & 2.197 \\
\hline 2.197 & 28.561 & 2.197 \\
\hline 72.501 & 145.002 & 13.182 \\
\hline 101.062 & 362.505 & 107.653 \\
\hline 2.197 & 2.197 & 402.051 \\
\hline 4.394 & 2.197 & 57.122 \\
\hline 2.197 & 2.197 & 17.576 \\
\hline 4.394 & 131.82 & 90.077 \\
\hline 2.197 & 120.835 & 76.895 \\
\hline 15.379 & 2.197 & 2.197 \\
\hline 81.289 & 24.167 & 8.788 \\
\hline 50.531 & 10.985 & 2.197 \\
\hline 8.788 & 2.197 & 6.591 \\
\hline
\end{tabular}




\begin{tabular}{|c|c|c|}
\hline 13.182 & 24.167 & 2.197 \\
\hline 4.394 & 10.985 & 2.197 \\
\hline 2.197 & 61.516 & 2.197 \\
\hline 2.197 & 96.668 & 92.274 \\
\hline 26.364 & 76.895 & 6.591 \\
\hline 41.743 & 2.197 & 2.197 \\
\hline 17.576 & 112.047 & 2.197 \\
\hline 28.561 & 2.197 & 4.394 \\
\hline 2.197 & 2.197 & 2.197 \\
\hline 79.092 & 2.197 & 6.591 \\
\hline 8.788 & 2.197 & 2.197 \\
\hline 2.197 & 4.394 & 2.197 \\
\hline 4.394 & 41.743 & 298.792 \\
\hline 43.94 & 17.576 & 2.197 \\
\hline 2.197 & 6.591 & 39.546 \\
\hline 2.197 & 8.788 & 79.092 \\
\hline 2.197 & 2.197 & 164.775 \\
\hline 2.197 & 2.197 & 15.379 \\
\hline 136.214 & 2.197 & 6.591 \\
\hline 24.167 & 2.197 & 103.259 \\
\hline 4.394 & 13.182 & 2.197 \\
\hline 8.788 & 4.394 & 138.411 \\
\hline 6.591 & 8.788 & 26.364 \\
\hline 4.394 & 2.197 & 26.364 \\
\hline 742.586 & 10.985 & 6.591 \\
\hline 2.197 & 2.197 & 2.197 \\
\hline 4.394 & 4.394 & 21.97 \\
\hline 46.137 & 107.653 & 155.987 \\
\hline 8.788 & 2.197 & 4.394 \\
\hline 21.97 & 26.364 & 184.548 \\
\hline 2.197 & 19.773 & 2.197 \\
\hline 2.197 & 2.197 & 35.152 \\
\hline 4.394 & 2.197 & 24.167 \\
\hline 2.197 & 10.985 & 173.563 \\
\hline 96.668 & 210.912 & 243.867 \\
\hline 2.197 & 2.197 & 2.197 \\
\hline 2.197 & 2.197 & 294.398 \\
\hline 2.197 & 2.197 & 10.985 \\
\hline 35.152 & 4.394 & 76.895 \\
\hline 2.197 & 2.197 & 2.197 \\
\hline 43.94 & 65.91 & 4.394 \\
\hline 4.394 & 2.197 & 41.743 \\
\hline 15.379 & 2.197 & 4.394 \\
\hline 4.394 & 76.895 & 10.985 \\
\hline 17.576 & 4.394 & 24.167 \\
\hline 6.591 & 74.698 & 8.788 \\
\hline 10.985 & 46.137 & 10.985 \\
\hline 4.394 & 8.788 & 4.394 \\
\hline 6.591 & 2.197 & 39.546 \\
\hline 4.394 & 2.197 & 32.955 \\
\hline
\end{tabular}




\begin{tabular}{|c|c|c|}
\hline 63.713 & 2.197 & 8.788 \\
\hline 72.501 & 2.197 & 2.197 \\
\hline 2.197 & 2.197 & 19.773 \\
\hline 2.197 & 2.197 & 37.349 \\
\hline 2.197 & 8.788 & 1010.62 \\
\hline 2.197 & 2.197 & 54.925 \\
\hline 6.591 & 21.97 & 65.91 \\
\hline 2.197 & 2.197 & 17.576 \\
\hline 2.197 & 2258.52 & 2.197 \\
\hline 21.97 & 112.047 & 2.197 \\
\hline 13.182 & 2.197 & 4.394 \\
\hline 26.364 & 2.197 & 151.593 \\
\hline 775.541 & 19.773 & 4.394 \\
\hline 24.167 & 57.122 & 13.182 \\
\hline 2.197 & 4.394 & 13.182 \\
\hline 6.591 & 2.197 & 4.394 \\
\hline 15.379 & 2.197 & 243.867 \\
\hline 4.394 & 4.394 & 4.394 \\
\hline 63.713 & 2.197 & 76.895 \\
\hline 1827.9 & 2.197 & 8.788 \\
\hline 2.197 & 2.197 & 2.197 \\
\hline 6.591 & 2.197 & 2.197 \\
\hline 204.321 & 2.197 & 257.049 \\
\hline 2.197 & 24.167 & 2.197 \\
\hline 10.985 & 6.591 & 10.985 \\
\hline 2.197 & 2.197 & 114.244 \\
\hline 4.394 & 4.394 & 2.197 \\
\hline 8.788 & 2.197 & 17.576 \\
\hline 2.197 & 15.379 & 2.197 \\
\hline 4.394 & 8.788 & 15.379 \\
\hline 15.379 & 531.674 & 19.773 \\
\hline 54.925 & 145.002 & 17.576 \\
\hline 2.197 & 4.394 & 57.122 \\
\hline 6.591 & 2.197 & 147.199 \\
\hline 24.167 & 90.077 & 83.486 \\
\hline 32.955 & 4.394 & 4.394 \\
\hline 54.925 & 120.835 & 46.137 \\
\hline 2.197 & 2.197 & 24.167 \\
\hline 2.197 & 2.197 & 2.197 \\
\hline 54.925 & 8.788 & 4.394 \\
\hline 26.364 & 76.895 & 2.197 \\
\hline 76.895 & 782.132 & 4.394 \\
\hline 6.591 & 10.985 & 54.925 \\
\hline 83.486 & 32.955 & 8.788 \\
\hline 10.985 & 2.197 & 32.955 \\
\hline 15.379 & 2.197 & 59.319 \\
\hline 15.379 & 59.319 & 26.364 \\
\hline 4.394 & 2.197 & 2.197 \\
\hline 6.591 & 2.197 & 68.107 \\
\hline 19.773 & 2.197 & 74.698 \\
\hline
\end{tabular}




\begin{tabular}{|c|c|c|}
\hline 2.197 & 8.788 & 24.167 \\
\hline 2.197 & 2.197 & 215.306 \\
\hline 57.122 & 41.743 & 32.955 \\
\hline 19.773 & 21.97 & 2.197 \\
\hline 15.379 & 2.197 & 2.197 \\
\hline 107.653 & 2.197 & 2.197 \\
\hline 125.229 & 35.152 & 10.985 \\
\hline 74.698 & 17.576 & 4.394 \\
\hline 2.197 & 30.758 & 2.197 \\
\hline 2.197 & 4.394 & 2.197 \\
\hline 6.591 & 2.197 & 2.197 \\
\hline 46.137 & 2.197 & 8.788 \\
\hline 4.394 & 2.197 & 13.182 \\
\hline 2.197 & 2.197 & 2.197 \\
\hline 46.137 & 83.486 & 13.182 \\
\hline 54.925 & 4.394 & 6.591 \\
\hline 2.197 & 10.985 & 6.591 \\
\hline 2.197 & 2.197 & 2.197 \\
\hline 131.82 & 2.197 & 59.319 \\
\hline 41.743 & 26.364 & 2.197 \\
\hline 43.94 & 15.379 & 2.197 \\
\hline 24.167 & 4.394 & 48.334 \\
\hline 15.379 & 2.197 & 8.788 \\
\hline 208.715 & 391.066 & 13.182 \\
\hline 8.788 & 2.197 & 48.334 \\
\hline 52.728 & 340.535 & 13.182 \\
\hline 2.197 & 39.546 & 4.394 \\
\hline 32.955 & 424.021 & 2.197 \\
\hline 224.094 & 2.197 & 24.167 \\
\hline 391.066 & 2.197 & 2.197 \\
\hline 6.591 & 2.197 & 2.197 \\
\hline 2.197 & 112.047 & 8.788 \\
\hline 2.197 & 2.197 & 663.494 \\
\hline 4.394 & 10.985 & 13.182 \\
\hline 8.788 & 2.197 & 10.985 \\
\hline 2.197 & 2.197 & 48.334 \\
\hline 147.199 & 2.197 & 13.182 \\
\hline 37.349 & 2.197 & 15.379 \\
\hline 6.591 & 2.197 & 4.394 \\
\hline 39.546 & 2.197 & 8.788 \\
\hline 2.197 & 21.97 & 898.573 \\
\hline 43.94 & 494.325 & 13.182 \\
\hline 4.394 & 57.122 & 52.728 \\
\hline 6.591 & 2.197 & 8.788 \\
\hline 79.092 & 10.985 & 17.576 \\
\hline 32.955 & 2.197 & 15.379 \\
\hline 17.576 & 2.197 & 4.394 \\
\hline 2.197 & 2.197 & 2.197 \\
\hline 4.394 & 2.197 & 2.197 \\
\hline 4.394 & 2.197 & 2.197 \\
\hline
\end{tabular}




\begin{tabular}{|c|c|c|}
\hline 2.197 & 10.985 & 2.197 \\
\hline 6.591 & 2.197 & 4.394 \\
\hline 6.591 & 2.197 & 151.593 \\
\hline 2.197 & 59.319 & 188.942 \\
\hline 21.97 & 8.788 & 52.728 \\
\hline 4.394 & 2.197 & 28.561 \\
\hline 2.197 & 2.197 & 2.197 \\
\hline 37.349 & 26.364 & 2.197 \\
\hline 30.758 & 432.809 & 92.274 \\
\hline 145.002 & 2.197 & 4.394 \\
\hline 41.743 & 6.591 & 2.197 \\
\hline 2.197 & 215.306 & 2.197 \\
\hline 2.197 & 19.773 & 2.197 \\
\hline 4.394 & 32.955 & 2.197 \\
\hline 37.349 & 138.411 & 26.364 \\
\hline 15.379 & 32.955 & 2.197 \\
\hline 92.274 & 50.531 & 39.546 \\
\hline 2.197 & 4.394 & 39.546 \\
\hline 2.197 & 2.197 & 2.197 \\
\hline 2.197 & 2.197 & 180.154 \\
\hline 74.698 & 10.985 & 30.758 \\
\hline 2.197 & 15.379 & 15.379 \\
\hline 13.182 & 4.394 & 17.576 \\
\hline 48.334 & 4.394 & 2.197 \\
\hline 13.182 & 2.197 & 41.743 \\
\hline 4.394 & 169.169 & 2.197 \\
\hline 4.394 & 17.576 & 10.985 \\
\hline 28.561 & 15.379 & 4.394 \\
\hline 4.394 & 2.197 & 213.109 \\
\hline 2.197 & 2.197 & 83.486 \\
\hline 17.576 & 24.167 & 4.394 \\
\hline 24.167 & 94.471 & 2.197 \\
\hline 54.925 & 8.788 & 6.591 \\
\hline 17.576 & 2.197 & 10.985 \\
\hline 41.743 & 8.788 & 19.773 \\
\hline 8.788 & 4.394 & 107.653 \\
\hline 6.591 & 26.364 & 52.728 \\
\hline 21.97 & 2.197 & 19.773 \\
\hline 101.062 & 13.182 & 2.197 \\
\hline 6.591 & 2.197 & 19.773 \\
\hline 459.173 & 2.197 & 59.319 \\
\hline 2.197 & 2.197 & 13.182 \\
\hline 61.516 & 2.197 & 2.197 \\
\hline 2.197 & 2.197 & 46.137 \\
\hline 6.591 & 2.197 & 15.379 \\
\hline 54.925 & 2.197 & 35.152 \\
\hline 2.197 & 2.197 & 2.197 \\
\hline 17.576 & 2.197 & 54.925 \\
\hline 8.788 & 4.394 & 35.152 \\
\hline 21.97 & 2.197 & 13.182 \\
\hline
\end{tabular}




\begin{tabular}{|c|c|c|}
\hline 2.197 & 166.972 & 15.379 \\
\hline 4.394 & 30.758 & 4.394 \\
\hline 6.591 & 26.364 & 6.591 \\
\hline 2.197 & 4.394 & 24.167 \\
\hline 2.197 & 24.167 & 2.197 \\
\hline 94.471 & 120.835 & 4.394 \\
\hline 4.394 & 2.197 & 2.197 \\
\hline 37.349 & 19.773 & 2.197 \\
\hline 21.97 & 4.394 & 2.197 \\
\hline 6.591 & 4.394 & 105.456 \\
\hline 2.197 & 15.379 & 4.394 \\
\hline 32.955 & 2.197 & 24.167 \\
\hline 26.364 & 4.394 & 63.713 \\
\hline 26.364 & 2.197 & 90.077 \\
\hline 46.137 & 46.137 & 109.85 \\
\hline 94.471 & 6.591 & 8.788 \\
\hline 2.197 & 13.182 & 10.985 \\
\hline 2.197 & 191.139 & 17.576 \\
\hline 6.591 & 6.591 & 35.152 \\
\hline 114.244 & 4.394 & 98.865 \\
\hline 2.197 & 6.591 & 94.471 \\
\hline 19.773 & 2.197 & 129.623 \\
\hline 79.092 & 142.805 & 1135.85 \\
\hline 2.197 & 50.531 & 105.456 \\
\hline 136.214 & 2.197 & 19.773 \\
\hline 21.97 & 2.197 & 2.197 \\
\hline 274.625 & 30.758 & 2.197 \\
\hline 6.591 & 2.197 & 8.788 \\
\hline 2.197 & 2.197 & 2.197 \\
\hline 4.394 & 10.985 & 32.955 \\
\hline 6.591 & 2.197 & 160.381 \\
\hline 6.591 & 30.758 & 15.379 \\
\hline 10.985 & 6.591 & 10.985 \\
\hline 21.97 & 2.197 & 470.158 \\
\hline 239.473 & 13.182 & 2.197 \\
\hline 4.394 & 2.197 & 2.197 \\
\hline 4.394 & 2.197 & 2.197 \\
\hline 57.122 & 10.985 & 2.197 \\
\hline 50.531 & 2.197 & 2.197 \\
\hline 39.546 & 2.197 & 54.925 \\
\hline 2.197 & 13.182 & 6.591 \\
\hline 2.197 & 39.546 & 2.197 \\
\hline 50.531 & 6.591 & 219.7 \\
\hline 8.788 & 2.197 & 2.197 \\
\hline 24.167 & 13.182 & 13.182 \\
\hline 50.531 & 37.349 & 6.591 \\
\hline 6.591 & 67067.8 & 2.197 \\
\hline 2.197 & 2.197 & 8.788 \\
\hline 386.672 & 2.197 & 43.94 \\
\hline 10.985 & 2.197 & 4.394 \\
\hline
\end{tabular}




\begin{tabular}{|c|c|c|}
\hline 79.092 & 8.788 & 87.88 \\
\hline 4.394 & 2.197 & 4.394 \\
\hline 142.805 & 2.197 & 46.137 \\
\hline 4.394 & 2.197 & 2.197 \\
\hline 6.591 & 2.197 & 2.197 \\
\hline 2.197 & 191.139 & 2.197 \\
\hline 6.591 & 219.7 & 19.773 \\
\hline 2.197 & 131.82 & 52.728 \\
\hline 6.591 & 105.456 & 24.167 \\
\hline 83.486 & 15.379 & 4.394 \\
\hline 4.394 & 2.197 & 2.197 \\
\hline 8.788 & 2.197 & 2.197 \\
\hline 8.788 & 17.576 & 21.97 \\
\hline 30.758 & 6.591 & 4.394 \\
\hline 8.788 & 2.197 & 2.197 \\
\hline 118.638 & 2.197 & 41.743 \\
\hline 4.394 & 21.97 & 2.197 \\
\hline 4.394 & 8.788 & 92.274 \\
\hline 4.394 & 24.167 & 610.766 \\
\hline 6.591 & 2.197 & 76.895 \\
\hline 52.728 & 2.197 & 24.167 \\
\hline 2.197 & 2.197 & 32.955 \\
\hline 6.591 & 17.576 & 4.394 \\
\hline 2.197 & 15.379 & 4.394 \\
\hline 2.197 & 2.197 & 2.197 \\
\hline 2.197 & 4.394 & 43.94 \\
\hline 2.197 & 2.197 & 10.985 \\
\hline 17.576 & 32.955 & 2.197 \\
\hline 4.394 & 2.197 & 2.197 \\
\hline 2.197 & 2.197 & 2524.35 \\
\hline 2.197 & 2.197 & 15.379 \\
\hline 4.394 & 30.758 & 32.955 \\
\hline 2.197 & 2.197 & 2.197 \\
\hline 2.197 & 296.595 & 2.197 \\
\hline 4.394 & 2.197 & 2.197 \\
\hline 46.137 & 32.955 & 35.152 \\
\hline 2.197 & 2.197 & 4.394 \\
\hline 2.197 & 6.591 & 6.591 \\
\hline 26.364 & 50.531 & 476.749 \\
\hline 4.394 & 2.197 & 4.394 \\
\hline 8.788 & 8.788 & 2.197 \\
\hline 2.197 & 19.773 & 127.426 \\
\hline 4.394 & 8.788 & 2.197 \\
\hline 37.349 & 10.985 & 116.441 \\
\hline 4.394 & 57.122 & 2.197 \\
\hline 131.82 & 2.197 & 28.561 \\
\hline 26.364 & 4.394 & 109.85 \\
\hline 4.394 & 2.197 & 2.197 \\
\hline 83.486 & 2.197 & 2.197 \\
\hline 2.197 & 6.591 & 37.349 \\
\hline
\end{tabular}




\begin{tabular}{|c|c|c|}
\hline 239.473 & 2.197 & 735.995 \\
\hline 19.773 & 2.197 & 46.137 \\
\hline 15.379 & 15.379 & 169.169 \\
\hline 727.207 & 6.591 & 13.182 \\
\hline 46.137 & 2.197 & 37.349 \\
\hline 4.394 & 2.197 & 2.197 \\
\hline 4.394 & 6.591 & 136.214 \\
\hline 2.197 & 2.197 & 2.197 \\
\hline 2.197 & 19.773 & 4.394 \\
\hline 2.197 & 6.591 & 10.985 \\
\hline 83.486 & 8.788 & 4.394 \\
\hline 17.576 & 19.773 & 4.394 \\
\hline 2.197 & 2.197 & 13.182 \\
\hline 2.197 & 19.773 & 2.197 \\
\hline 4.394 & 6.591 & 861.224 \\
\hline 2.197 & 2.197 & 1102.89 \\
\hline 15.379 & 2.197 & 151.593 \\
\hline 2.197 & 2.197 & 2.197 \\
\hline 8.788 & 2.197 & 8.788 \\
\hline 199.927 & 4.394 & 4.394 \\
\hline 41.743 & 83.486 & 2.197 \\
\hline 10.985 & 4.394 & 8.788 \\
\hline 30.758 & 101.062 & 2.197 \\
\hline 26.364 & 10.985 & 17.576 \\
\hline 24.167 & 90.077 & 94.471 \\
\hline 6.591 & 2.197 & 30.758 \\
\hline 6.591 & 17.576 & 96.668 \\
\hline 6.591 & 2.197 & 195.533 \\
\hline 10.985 & 24.167 & 2.197 \\
\hline 76.895 & 215.306 & 2157.45 \\
\hline 24.167 & 8.788 & 15.379 \\
\hline 70.304 & 4.394 & 26.364 \\
\hline 17.576 & 2.197 & 17.576 \\
\hline 21.97 & 2.197 & 15.379 \\
\hline 164.775 & 2.197 & 2.197 \\
\hline 8.788 & 2.197 & 116.441 \\
\hline 4.394 & 2.197 & 2.197 \\
\hline 2.197 & 8.788 & 2.197 \\
\hline 15.379 & 96.668 & 2.197 \\
\hline 41.743 & 2.197 & 2.197 \\
\hline 8.788 & 4.394 & 17.576 \\
\hline 28.561 & 2.197 & 254.852 \\
\hline 81.289 & 2.197 & 2.197 \\
\hline 4.394 & 2.197 & 2.197 \\
\hline 2.197 & 4.394 & 8.788 \\
\hline 8.788 & 2.197 & 2.197 \\
\hline 19.773 & 8.788 & 8.788 \\
\hline 2.197 & 2.197 & 6.591 \\
\hline 68.107 & 2.197 & 4.394 \\
\hline 52.728 & 2.197 & 2.197 \\
\hline
\end{tabular}




\begin{tabular}{|c|c|c|}
\hline 39.546 & 2.197 & 46.137 \\
\hline 1491.76 & 6.591 & 6.591 \\
\hline 8.788 & 6.591 & 2.197 \\
\hline 39.546 & 70.304 & 35.152 \\
\hline 13.182 & 17.576 & 6.591 \\
\hline 2.197 & 81.289 & 13.182 \\
\hline 2.197 & 2.197 & 8.788 \\
\hline 2.197 & 2.197 & 87.88 \\
\hline 4.394 & 2.197 & 39.546 \\
\hline 2.197 & 6.591 & 2.197 \\
\hline 10.985 & 2.197 & 6.591 \\
\hline 8.788 & 382.278 & 10.985 \\
\hline 6.591 & 19.773 & 153.79 \\
\hline 10.985 & 6.591 & 2.197 \\
\hline 116.441 & 2.197 & 54.925 \\
\hline 2.197 & 2.197 & 2.197 \\
\hline 2.197 & 68.107 & 13.182 \\
\hline 4.394 & 6.591 & 10.985 \\
\hline 2.197 & 6.591 & 2.197 \\
\hline 50.531 & 79.092 & 2.197 \\
\hline 2.197 & 59.319 & 2.197 \\
\hline 4.394 & 2.197 & 325.156 \\
\hline 28.561 & 2.197 & 19.773 \\
\hline 46.137 & 112.047 & 2.197 \\
\hline 46.137 & 2.197 & 2.197 \\
\hline 24.167 & 764.556 & 6.591 \\
\hline 8.788 & 942.513 & 46.137 \\
\hline 6.591 & 26.364 & 2.197 \\
\hline 52.728 & 26.364 & 17.576 \\
\hline 8.788 & 6.591 & 287.807 \\
\hline 24.167 & 4.394 & 17.576 \\
\hline 2.197 & 2.197 & 21.97 \\
\hline 8.788 & 24.167 & 2.197 \\
\hline 15.379 & 4.394 & 2.197 \\
\hline 54.925 & 2.197 & 2.197 \\
\hline 8.788 & 15.379 & 32.955 \\
\hline 8.788 & 32.955 & 32.955 \\
\hline 8.788 & 10.985 & 4.394 \\
\hline 2.197 & 2.197 & 13.182 \\
\hline 265.837 & 46.137 & 8.788 \\
\hline 6.591 & 2.197 & 48.334 \\
\hline 217.503 & 2.197 & 28.561 \\
\hline 41.743 & 496.522 & 6.591 \\
\hline 13.182 & 70.304 & 70.304 \\
\hline 24.167 & 1674.11 & 274.625 \\
\hline 2.197 & 8.788 & 87.88 \\
\hline 257.049 & 17.576 & 4.394 \\
\hline 6.591 & 2.197 & 68.107 \\
\hline 24.167 & 2.197 & 10.985 \\
\hline 2.197 & 2.197 & 4.394 \\
\hline
\end{tabular}




\begin{tabular}{|c|c|c|}
\hline 6.591 & 4.394 & 2.197 \\
\hline 2.197 & 2.197 & 2.197 \\
\hline 6.591 & 4.394 & 2.197 \\
\hline 4.394 & 2.197 & 2.197 \\
\hline 13.182 & 19.773 & 2.197 \\
\hline 61.516 & 4.394 & 2.197 \\
\hline 92.274 & 4.394 & 8.788 \\
\hline 2.197 & 2.197 & 32.955 \\
\hline 4.394 & 4.394 & 46.137 \\
\hline 4.394 & 2.197 & 2.197 \\
\hline 2.197 & 522.886 & 2.197 \\
\hline 24.167 & 2.197 & 24.167 \\
\hline 2.197 & 2.197 & 2.197 \\
\hline 54.925 & 2.197 & 2.197 \\
\hline 61.516 & 24.167 & 4.394 \\
\hline 13.182 & 4.394 & 4.394 \\
\hline 10.985 & 4.394 & 213.109 \\
\hline 4.394 & 2.197 & 50.531 \\
\hline 10.985 & 46.137 & 2.197 \\
\hline 6.591 & 2.197 & 2.197 \\
\hline 125.229 & 17.576 & 13.182 \\
\hline 8.788 & 15.379 & 4.394 \\
\hline 4.394 & 2.197 & 597.584 \\
\hline 13.182 & 2.197 & 17.576 \\
\hline 8.788 & 13.182 & 2.197 \\
\hline 2.197 & 4.394 & 2.197 \\
\hline 2.197 & 41.743 & 4.394 \\
\hline 50.531 & 2.197 & 15.379 \\
\hline 19.773 & 4.394 & 2.197 \\
\hline 4.394 & 2.197 & 2.197 \\
\hline 2.197 & 17.576 & 173.563 \\
\hline 4.394 & 2.197 & 2.197 \\
\hline 4.394 & 8.788 & 8.788 \\
\hline 6.591 & 2.197 & 94.471 \\
\hline 8.788 & 2.197 & 2.197 \\
\hline 4.394 & 79.092 & 10.985 \\
\hline 6.591 & 2.197 & 2.197 \\
\hline 105.456 & 92.274 & 6.591 \\
\hline 6.591 & 2.197 & 2.197 \\
\hline 94.471 & 2.197 & 61.516 \\
\hline 2.197 & 393.263 & 59.319 \\
\hline 28.561 & 2.197 & 4.394 \\
\hline 19.773 & 2.197 & 2.197 \\
\hline 4.394 & 2.197 & 6.591 \\
\hline 4.394 & 2.197 & 2.197 \\
\hline 2.197 & 2.197 & 13.182 \\
\hline 4.394 & 2.197 & 4.394 \\
\hline 61.516 & 4.394 & 2.197 \\
\hline 4.394 & 2.197 & 2.197 \\
\hline 4.394 & 10.985 & 19.773 \\
\hline
\end{tabular}




\begin{tabular}{|c|c|c|}
\hline 13.182 & 2.197 & 10.985 \\
\hline 2.197 & 2.197 & 70.304 \\
\hline 4.394 & 4.394 & 4.394 \\
\hline 4.394 & 2.197 & 8.788 \\
\hline 2.197 & 127.426 & 50.531 \\
\hline 10.985 & 2.197 & 6.591 \\
\hline 39.546 & 8.788 & 195.533 \\
\hline 30.758 & 10.985 & 215.306 \\
\hline 2.197 & 2.197 & 94.471 \\
\hline 24.167 & 10.985 & 61.516 \\
\hline 74.698 & 4.394 & 6.591 \\
\hline 8.788 & 4.394 & 24.167 \\
\hline 13.182 & 2.197 & 2.197 \\
\hline 173.563 & 7026.01 & 2.197 \\
\hline 65.91 & 2.197 & 6.591 \\
\hline 68.107 & 2.197 & 21.97 \\
\hline 6.591 & 4.394 & 46.137 \\
\hline 24.167 & 2.197 & 24.167 \\
\hline 2.197 & 24.167 & 2.197 \\
\hline 4.394 & 19.773 & 13.182 \\
\hline 2.197 & 6.591 & 26.364 \\
\hline 43.94 & 6.591 & 8.788 \\
\hline 15.379 & 2.197 & 2.197 \\
\hline 166.972 & 2.197 & 4.394 \\
\hline 4.394 & 4.394 & 118.638 \\
\hline 2.197 & 10.985 & 129.623 \\
\hline 2.197 & 2.197 & 285.61 \\
\hline 6.591 & 2.197 & 4.394 \\
\hline 188.942 & 6.591 & 28.561 \\
\hline 2.197 & 4.394 & 59.319 \\
\hline 26.364 & 2.197 & 39.546 \\
\hline 175.76 & 19.773 & 6.591 \\
\hline 13.182 & 2.197 & 37.349 \\
\hline 35.152 & 2.197 & 10.985 \\
\hline 2.197 & 6.591 & 32.955 \\
\hline 59.319 & 2.197 & 17.576 \\
\hline 2.197 & 2.197 & 320.762 \\
\hline 4.394 & 224.094 & 333.944 \\
\hline 300.989 & 13.182 & 83.486 \\
\hline 15.379 & 2.197 & 98.865 \\
\hline 13.182 & 2.197 & 166.972 \\
\hline 4.394 & 2.197 & 13.182 \\
\hline 8.788 & 6.591 & 2.197 \\
\hline 10.985 & 4.394 & 37.349 \\
\hline 76.895 & 4.394 & 2.197 \\
\hline 21.97 & 8.788 & 13.182 \\
\hline 2.197 & 650.312 & 70.304 \\
\hline 96.668 & 50.531 & 2.197 \\
\hline 4.394 & 6.591 & 8.788 \\
\hline 2.197 & 6.591 & 2.197 \\
\hline
\end{tabular}




\begin{tabular}{|c|c|c|}
\hline 2.197 & 2.197 & 6.591 \\
\hline 120.835 & 4.394 & 94.471 \\
\hline 2.197 & 4.394 & 8.788 \\
\hline 8.788 & 2.197 & 353.717 \\
\hline 74.698 & 2.197 & 2.197 \\
\hline 2.197 & 59.319 & 8.788 \\
\hline 10.985 & 2.197 & 2.197 \\
\hline 2.197 & 4.394 & 375.687 \\
\hline 48.334 & 4.394 & 32.955 \\
\hline 26.364 & 6.591 & 37.349 \\
\hline 21.97 & 2.197 & 50.531 \\
\hline 4.394 & 4.394 & 199.927 \\
\hline 17.576 & 2.197 & 39.546 \\
\hline 2.197 & 4.394 & 2.197 \\
\hline 2.197 & 6.591 & 8.788 \\
\hline 153.79 & 4.394 & 2.197 \\
\hline 52.728 & 228.488 & 19.773 \\
\hline 2.197 & 6.591 & 6.591 \\
\hline 2.197 & 15.379 & 459.173 \\
\hline 197.73 & 10.985 & 2.197 \\
\hline 24.167 & 61.516 & 4.394 \\
\hline 6.591 & 2.197 & 2.197 \\
\hline 138.411 & 2.197 & 85.683 \\
\hline 340.535 & 6.591 & 2.197 \\
\hline 2.197 & 2.197 & 8.788 \\
\hline 28.561 & 4.394 & 41.743 \\
\hline 2.197 & 4.394 & 24.167 \\
\hline 6.591 & 43.94 & 380.081 \\
\hline 10.985 & 87.88 & 8.788 \\
\hline 4.394 & 4.394 & 8.788 \\
\hline 4.394 & 2.197 & 2.197 \\
\hline 2.197 & 2.197 & 4.394 \\
\hline 8.788 & 74.698 & 6.591 \\
\hline 6.591 & 2.197 & 2.197 \\
\hline 10.985 & 6.591 & 13.182 \\
\hline 26.364 & 6.591 & 2.197 \\
\hline 8.788 & 140.608 & 2.197 \\
\hline 17.576 & 17.576 & 2.197 \\
\hline 2.197 & 2.197 & 19.773 \\
\hline 8.788 & 13.182 & 2.197 \\
\hline 4.394 & 41.743 & 2.197 \\
\hline 2.197 & 4.394 & 24.167 \\
\hline 2.197 & 4.394 & 2.197 \\
\hline 21.97 & 2.197 & 2.197 \\
\hline 32.955 & 689.858 & 57.122 \\
\hline 155.987 & 4.394 & 13.182 \\
\hline 2.197 & 2.197 & 54.925 \\
\hline 2.197 & 2.197 & 6.591 \\
\hline 19.773 & 15.379 & 8.788 \\
\hline 180.154 & 35.152 & 48.334 \\
\hline
\end{tabular}




\begin{tabular}{|c|c|c|}
\hline 63.713 & 2.197 & 57.122 \\
\hline 32.955 & 2.197 & 13.182 \\
\hline 21.97 & 68.107 & 2.197 \\
\hline 393.263 & 2.197 & 140.608 \\
\hline 41.743 & 48.334 & 81.289 \\
\hline 48.334 & 2.197 & 85.683 \\
\hline 2.197 & 13.182 & 268.034 \\
\hline 32.955 & 2.197 & 4.394 \\
\hline 2.197 & 15.379 & 500.916 \\
\hline 4.394 & 6.591 & 4.394 \\
\hline 6.591 & 39.546 & 21.97 \\
\hline 4.394 & 2.197 & 37.349 \\
\hline 2.197 & 2.197 & 2.197 \\
\hline 2.197 & 35.152 & 2.197 \\
\hline 8.788 & 35.152 & 2.197 \\
\hline 2.197 & 2.197 & 999.635 \\
\hline 2.197 & 35.152 & 2.197 \\
\hline 19.773 & 28.561 & 2.197 \\
\hline 4.394 & 2.197 & 2.197 \\
\hline 15.379 & 2.197 & 2.197 \\
\hline 21.97 & 2.197 & 406.445 \\
\hline 252.655 & 2.197 & 2.197 \\
\hline 8.788 & 26.364 & 2.197 \\
\hline 4.394 & 2.197 & 6.591 \\
\hline 173.563 & 2.197 & 6.591 \\
\hline 61.516 & 4.394 & 32.955 \\
\hline 10.985 & 2.197 & 2.197 \\
\hline 2.197 & 2.197 & 30.758 \\
\hline 26.364 & 2.197 & 2.197 \\
\hline 2.197 & 2.197 & 2.197 \\
\hline 2.197 & 2.197 & 10.985 \\
\hline 2.197 & 59.319 & 68.107 \\
\hline 15.379 & 2.197 & 26.364 \\
\hline 10.985 & 65.91 & 338.338 \\
\hline 85.683 & 125.229 & 17.576 \\
\hline 10.985 & 30.758 & 257.049 \\
\hline 2.197 & 2.197 & 32.955 \\
\hline 2.197 & 8.788 & 270.231 \\
\hline 10.985 & 2.197 & 2.197 \\
\hline 2.197 & 19.773 & 37.349 \\
\hline 8.788 & 2.197 & 2.197 \\
\hline 8.788 & 68.107 & 17.576 \\
\hline 8.788 & 6.591 & 90.077 \\
\hline 2.197 & 4.394 & 1047.97 \\
\hline 2.197 & 2.197 & 37.349 \\
\hline 19.773 & 4.394 & 35.152 \\
\hline 35.152 & 8.788 & 2.197 \\
\hline 13.182 & 24.167 & 8.788 \\
\hline 28.561 & 2.197 & 24.167 \\
\hline 2.197 & 6.591 & 13.182 \\
\hline
\end{tabular}




\begin{tabular}{|c|c|c|}
\hline 2.197 & 2.197 & 57.122 \\
\hline 13.182 & 4.394 & 2.197 \\
\hline 46.137 & 17.576 & 2.197 \\
\hline 15.379 & 32.955 & 2.197 \\
\hline 2.197 & 4.394 & 6.591 \\
\hline 2.197 & 4.394 & 4.394 \\
\hline 37.349 & 153.79 & 48.334 \\
\hline 96.668 & 57.122 & 2.197 \\
\hline 30.758 & 2.197 & 2.197 \\
\hline 81.289 & 4.394 & 6.591 \\
\hline 24.167 & 2.197 & 10.985 \\
\hline 160.381 & 2.197 & 8.788 \\
\hline 68.107 & 6.591 & 98.865 \\
\hline 6.591 & 2.197 & 17.576 \\
\hline 21.97 & 2.197 & 92.274 \\
\hline 2.197 & 2.197 & 10.985 \\
\hline 2.197 & 39.546 & 6.591 \\
\hline 900.77 & 2.197 & 17.576 \\
\hline 4.394 & 2.197 & 24.167 \\
\hline 2.197 & 4.394 & 17.576 \\
\hline 2.197 & 15.379 & 4.394 \\
\hline 125.229 & 2.197 & 4.394 \\
\hline 19.773 & 72.501 & 4.394 \\
\hline 2.197 & 4.394 & 26.364 \\
\hline 6.591 & 911.755 & 15.379 \\
\hline 19.773 & 24.167 & 125.229 \\
\hline 10.985 & 32.955 & 43.94 \\
\hline 2.197 & 8.788 & 48.334 \\
\hline 15.379 & 90.077 & 10.985 \\
\hline 4.394 & 35.152 & 68.107 \\
\hline 8.788 & 4.394 & 6.591 \\
\hline 6.591 & 6.591 & 17.576 \\
\hline 61.516 & 4.394 & 4.394 \\
\hline 4.394 & 435.006 & 26.364 \\
\hline 8.788 & 19.773 & 2.197 \\
\hline 30.758 & 2.197 & 13.182 \\
\hline 180.154 & 2.197 & 2.197 \\
\hline 6.591 & 15.379 & 142.805 \\
\hline 4.394 & 10.985 & 96.668 \\
\hline 24.167 & 24.167 & 2.197 \\
\hline 59.319 & 68.107 & 2.197 \\
\hline 2.197 & 10.985 & 26.364 \\
\hline 2.197 & 39.546 & 2.197 \\
\hline 6.591 & 151.593 & 8.788 \\
\hline 10.985 & 2.197 & 2.197 \\
\hline 4.394 & 351.52 & 37.349 \\
\hline 116.441 & 4.394 & 2.197 \\
\hline 2.197 & 15.379 & 2.197 \\
\hline 35.152 & 6.591 & 76.895 \\
\hline 10.985 & 2.197 & 17.576 \\
\hline
\end{tabular}




\begin{tabular}{|c|c|c|}
\hline 2.197 & 6.591 & 94.471 \\
\hline 39.546 & 2.197 & 2.197 \\
\hline 4.394 & 2.197 & 817.284 \\
\hline 2.197 & 2.197 & 8.788 \\
\hline 72.501 & 2.197 & 4.394 \\
\hline 8.788 & 2.197 & 8.788 \\
\hline 2.197 & 254.852 & 2.197 \\
\hline 2.197 & 2.197 & 1863.06 \\
\hline 287.807 & 4.394 & 52.728 \\
\hline 4.394 & 2.197 & 74.698 \\
\hline 41.743 & 39.546 & 309.777 \\
\hline 2.197 & 4.394 & 26.364 \\
\hline 8.788 & 2.197 & 28.561 \\
\hline 2.197 & 4.394 & 8.788 \\
\hline 2.197 & 17.576 & 8.788 \\
\hline 2.197 & 30.758 & 10.985 \\
\hline 2.197 & 2.197 & 10.985 \\
\hline 30.758 & 2.197 & 21.97 \\
\hline 8.788 & 2.197 & 98.865 \\
\hline 294.398 & 6.591 & 54.925 \\
\hline 10.985 & 13.182 & 823.875 \\
\hline 35.152 & 604.175 & 87.88 \\
\hline 2.197 & 125.229 & 2.197 \\
\hline 186.745 & 26.364 & 6.591 \\
\hline 81.289 & 24.167 & 54.925 \\
\hline 4.394 & 2.197 & 28.561 \\
\hline 2.197 & 2.197 & 2.197 \\
\hline 2.197 & 4.394 & 2.197 \\
\hline 4.394 & 2.197 & 4.394 \\
\hline 65.91 & 61.516 & 10.985 \\
\hline 6.591 & 2.197 & 492.128 \\
\hline 15.379 & 2.197 & 41.743 \\
\hline 32.955 & 17.576 & 2.197 \\
\hline 4.394 & 21.97 & 2.197 \\
\hline 52.728 & 2.197 & 232.882 \\
\hline 2.197 & 17.576 & 19.773 \\
\hline 8.788 & 2.197 & 4.394 \\
\hline 393.263 & 2.197 & 10.985 \\
\hline 2.197 & 2.197 & 437.203 \\
\hline 2.197 & 74.698 & 8.788 \\
\hline 35.152 & 74.698 & 4.394 \\
\hline 30.758 & 15.379 & 2.197 \\
\hline 85.683 & 2.197 & 35.152 \\
\hline 10.985 & 17.576 & 10.985 \\
\hline 4.394 & 10.985 & 2.197 \\
\hline 46.137 & 103.259 & 2.197 \\
\hline 4.394 & 10.985 & 4.394 \\
\hline 4.394 & 2.197 & 4.394 \\
\hline 4.394 & 2.197 & 21.97 \\
\hline 4.394 & 6.591 & 120.835 \\
\hline
\end{tabular}




\begin{tabular}{|c|c|c|}
\hline 2.197 & 30.758 & 15.379 \\
\hline 30.758 & 2.197 & 155.987 \\
\hline 8.788 & 17.576 & 24.167 \\
\hline 147.199 & 2.197 & 127.426 \\
\hline 6.591 & 4.394 & 2.197 \\
\hline 4.394 & 2.197 & 311.974 \\
\hline 4.394 & 2.197 & 2.197 \\
\hline 4.394 & 61.516 & 246.064 \\
\hline 2.197 & 2.197 & 116.441 \\
\hline 8.788 & 8.788 & 8.788 \\
\hline 72.501 & 2.197 & 30.758 \\
\hline 10.985 & 6.591 & 2.197 \\
\hline 2.197 & 17.576 & 2.197 \\
\hline 160.381 & 547.053 & 48.334 \\
\hline 32.955 & 2.197 & 2.197 \\
\hline 2.197 & 6.591 & 52.728 \\
\hline 797.511 & 4.394 & 6.591 \\
\hline 2.197 & 24.167 & 2.197 \\
\hline 2.197 & 2.197 & 26.364 \\
\hline 114.244 & 6.591 & 6.591 \\
\hline 35.152 & 15.379 & 30.758 \\
\hline 30.758 & 8.788 & 13.182 \\
\hline 4.394 & 8.788 & 6.591 \\
\hline 39.546 & 2.197 & 2.197 \\
\hline 15.379 & 37.349 & 2.197 \\
\hline 21.97 & 452.582 & 24.167 \\
\hline 39.546 & 2.197 & 35.152 \\
\hline 4.394 & 2.197 & 13.182 \\
\hline 101.062 & 2.197 & 118.638 \\
\hline 59.319 & 151.593 & 59.319 \\
\hline 2.197 & 2.197 & 2.197 \\
\hline 4.394 & 2.197 & 2.197 \\
\hline 59.319 & 24.167 & 26.364 \\
\hline 2.197 & 2.197 & 15.379 \\
\hline 10.985 & 68.107 & 35.152 \\
\hline 2.197 & 2.197 & 10.985 \\
\hline 41.743 & 13.182 & 8.788 \\
\hline 2.197 & 2.197 & 10.985 \\
\hline 2.197 & 2.197 & 4.394 \\
\hline 4.394 & 2.197 & 8.788 \\
\hline 2.197 & 2.197 & 19.773 \\
\hline 4.394 & 2.197 & 566.826 \\
\hline 138.411 & 2.197 & 6.591 \\
\hline 13.182 & 114.244 & 105.456 \\
\hline 2.197 & 6.591 & 254.852 \\
\hline 2.197 & 2.197 & 32.955 \\
\hline 85.683 & 2.197 & 2.197 \\
\hline 28.561 & 2.197 & 13.182 \\
\hline 145.002 & 2.197 & 6.591 \\
\hline 2.197 & 19.773 & 8.788 \\
\hline
\end{tabular}




\begin{tabular}{|c|c|c|}
\hline 2.197 & 459.173 & 2.197 \\
\hline 90.077 & 28.561 & 6.591 \\
\hline 164.775 & 2.197 & 46.137 \\
\hline 2.197 & 48.334 & 186.745 \\
\hline 32.955 & 15.379 & 6.591 \\
\hline 8.788 & 52.728 & 4.394 \\
\hline 147.199 & 15.379 & 15.379 \\
\hline 6.591 & 21.97 & 21.97 \\
\hline 358.111 & 2.197 & 184.548 \\
\hline 8.788 & 667.888 & 32.955 \\
\hline 10.985 & 2.197 & 2.197 \\
\hline 2.197 & 2.197 & 2.197 \\
\hline 2.197 & 6.591 & 191.139 \\
\hline 4.394 & 2.197 & 48.334 \\
\hline 4.394 & 52.728 & 4.394 \\
\hline 43.94 & 6.591 & 6.591 \\
\hline 10.985 & 10.985 & 35.152 \\
\hline 2.197 & 243.867 & 2.197 \\
\hline 4.394 & 2.197 & 2.197 \\
\hline 21.97 & 4.394 & 2.197 \\
\hline 2.197 & 4.394 & 4.394 \\
\hline 24.167 & 2.197 & 13.182 \\
\hline 32.955 & 6.591 & 2.197 \\
\hline 2.197 & 15.379 & 4.394 \\
\hline 4.394 & 2.197 & 28.561 \\
\hline 2.197 & 90.077 & 2.197 \\
\hline 41.743 & 2.197 & 8.788 \\
\hline 2.197 & 2.197 & 4.394 \\
\hline 43.94 & 2.197 & 2.197 \\
\hline 24.167 & 2.197 & 4.394 \\
\hline 10.985 & 2.197 & 37.349 \\
\hline 2.197 & 2.197 & 92.274 \\
\hline 2.197 & 2.197 & 2.197 \\
\hline 2.197 & 2.197 & 2.197 \\
\hline 2.197 & 6.591 & 21.97 \\
\hline 13.182 & 4.394 & 696.449 \\
\hline 2.197 & 4.394 & 221.897 \\
\hline 26.364 & 2.197 & 39.546 \\
\hline 2.197 & 2.197 & 797.511 \\
\hline 13.182 & 351.52 & 2.197 \\
\hline 2.197 & 17.576 & 43.94 \\
\hline 2.197 & 2.197 & 2.197 \\
\hline 2.197 & 4.394 & 6.591 \\
\hline 30.758 & 4.394 & 81.289 \\
\hline 13.182 & 13.182 & 224.094 \\
\hline 6.591 & 6.591 & 2.197 \\
\hline 10.985 & 2.197 & 2.197 \\
\hline 32.955 & 2.197 & 2.197 \\
\hline 215.306 & 2.197 & 24.167 \\
\hline 4.394 & 6.591 & 8.788 \\
\hline
\end{tabular}




\begin{tabular}{|c|c|c|}
\hline 19.773 & 46.137 & 15.379 \\
\hline 70.304 & 13.182 & 61.516 \\
\hline 243.867 & 2.197 & 43.94 \\
\hline 4.394 & 4.394 & 61.516 \\
\hline 15.379 & 13.182 & 2.197 \\
\hline 13.182 & 2.197 & 2.197 \\
\hline 2.197 & 2.197 & 17.576 \\
\hline 2.197 & 2.197 & 61.516 \\
\hline 4.394 & 10.985 & 52.728 \\
\hline 4.394 & 4.394 & 96.668 \\
\hline 2.197 & 1318.2 & 30.758 \\
\hline 2.197 & 2.197 & 2.197 \\
\hline 2.197 & 2.197 & 10.985 \\
\hline 4.394 & 4.394 & 2.197 \\
\hline 50.531 & 2.197 & 72.501 \\
\hline 380.081 & 2.197 & 6.591 \\
\hline 2.197 & 52.728 & 32.955 \\
\hline 2.197 & 6.591 & 129.623 \\
\hline 24.167 & 2.197 & 8.788 \\
\hline 4.394 & 19.773 & 4.394 \\
\hline 2.197 & 8.788 & 52.728 \\
\hline 13.182 & 35.152 & 2.197 \\
\hline 2.197 & 43.94 & 54.925 \\
\hline 2.197 & 4.394 & 2.197 \\
\hline 2.197 & 30.758 & 19.773 \\
\hline 4.394 & 4.394 & 2.197 \\
\hline 6.591 & 37.349 & 63.713 \\
\hline 21.97 & 1388.5 & 2.197 \\
\hline 13.182 & 340.535 & 4.394 \\
\hline 125.229 & 21.97 & 2.197 \\
\hline 50.531 & 6.591 & 10.985 \\
\hline 2.197 & 2.197 & 2.197 \\
\hline 65.91 & 2.197 & 4.394 \\
\hline 13.182 & 4.394 & 17.576 \\
\hline 13.182 & 2.197 & 8.788 \\
\hline 2.197 & 2.197 & 4.394 \\
\hline 2.197 & 15.379 & 1801.54 \\
\hline 17.576 & 30.758 & 2.197 \\
\hline 50.531 & 6.591 & 10.985 \\
\hline 15.379 & 2.197 & 24.167 \\
\hline 26.364 & 4.394 & 8.788 \\
\hline 54.925 & 2.197 & 19.773 \\
\hline 140.608 & 2.197 & 2.197 \\
\hline 4.394 & 10.985 & 10.985 \\
\hline 17.576 & 2.197 & 17.576 \\
\hline 2.197 & 6.591 & 26695.7 \\
\hline 2.197 & 6.591 & 2.197 \\
\hline 195.533 & 2.197 & 8.788 \\
\hline 13.182 & 2.197 & 30.758 \\
\hline 19.773 & 4.394 & 298.792 \\
\hline
\end{tabular}




\begin{tabular}{|c|c|c|}
\hline 28.561 & 4.394 & 2.197 \\
\hline 2.197 & 2.197 & 4.394 \\
\hline 6.591 & 4.394 & 4.394 \\
\hline 2.197 & 17.576 & 4.394 \\
\hline 46.137 & 2.197 & 10.985 \\
\hline 2.197 & 2.197 & 30.758 \\
\hline 107.653 & 2.197 & 28.561 \\
\hline 4.394 & 28.561 & 37.349 \\
\hline 50.531 & 2.197 & 13.182 \\
\hline 83.486 & 2.197 & 2.197 \\
\hline 6.591 & 254.852 & 2.197 \\
\hline 6.591 & 2.197 & 6.591 \\
\hline 24.167 & 2.197 & 2.197 \\
\hline 57.122 & 8.788 & 2.197 \\
\hline 4.394 & 2.197 & 4.394 \\
\hline 87.88 & 15.379 & 259.246 \\
\hline 32.955 & 15.379 & 2.197 \\
\hline 41.743 & 2.197 & 2.197 \\
\hline 13.182 & 10.985 & 28.561 \\
\hline 230.685 & 2.197 & 4.394 \\
\hline 2.197 & 4.394 & 8.788 \\
\hline 2.197 & 2.197 & 252.655 \\
\hline 19.773 & 2058.59 & 4.394 \\
\hline 182.351 & 2.197 & 10.985 \\
\hline 160.381 & 118.638 & 2.197 \\
\hline 37.349 & 10.985 & 8.788 \\
\hline 43.94 & 2.197 & 2.197 \\
\hline 4.394 & 8.788 & 8.788 \\
\hline 114.244 & 6.591 & 4.394 \\
\hline 8.788 & 63.713 & 2.197 \\
\hline 24.167 & 4.394 & 8.788 \\
\hline 4.394 & 8021.25 & 4.394 \\
\hline 10.985 & 2.197 & 10.985 \\
\hline 15.379 & 2.197 & 2.197 \\
\hline 4.394 & 21.97 & 6.591 \\
\hline 4.394 & 10.985 & 10.985 \\
\hline 2.197 & 8.788 & 17.576 \\
\hline 2.197 & 2.197 & 72.501 \\
\hline 8.788 & 112.047 & 6.591 \\
\hline 2.197 & 6.591 & 2.197 \\
\hline 2.197 & 2.197 & 2.197 \\
\hline 37.349 & 2.197 & 92.274 \\
\hline 4.394 & 2.197 & 6.591 \\
\hline 4.394 & 2.197 & 8.788 \\
\hline 32.955 & 2.197 & 2.197 \\
\hline 98.865 & 2.197 & 2.197 \\
\hline 26.364 & 21.97 & 2.197 \\
\hline 153.79 & 32.955 & 2.197 \\
\hline 13.182 & 2.197 & 2.197 \\
\hline 37.349 & 2.197 & 2.197 \\
\hline
\end{tabular}




\begin{tabular}{|c|c|c|}
\hline 8.788 & 2.197 & 21.97 \\
\hline 17.576 & 2.197 & 2.197 \\
\hline 199.927 & 2.197 & 2.197 \\
\hline 6.591 & 2.197 & 2.197 \\
\hline 59.319 & 8.788 & 21.97 \\
\hline 2.197 & 92.274 & 15.379 \\
\hline 2.197 & 2.197 & 4.394 \\
\hline 2.197 & 180.154 & 1792.75 \\
\hline 13.182 & 8.788 & 79.092 \\
\hline 2.197 & 254.852 & 43.94 \\
\hline 4.394 & 6.591 & 24.167 \\
\hline 6.591 & 28.561 & 4.394 \\
\hline 30.758 & 2.197 & 2.197 \\
\hline 8.788 & 70.304 & 6.591 \\
\hline 2.197 & 2.197 & 15.379 \\
\hline 2.197 & 8.788 & 8.788 \\
\hline 8.788 & 26.364 & 120.835 \\
\hline 21.97 & 114.244 & 285.61 \\
\hline 24.167 & 2.197 & 2.197 \\
\hline 6.591 & 2.197 & 13.182 \\
\hline 8.788 & 4.394 & 35.152 \\
\hline 10.985 & 2.197 & 4.394 \\
\hline 2.197 & 4.394 & 35.152 \\
\hline 966.68 & 2.197 & 120.835 \\
\hline 24.167 & 2.197 & 10.985 \\
\hline 2.197 & 2.197 & 10.985 \\
\hline 19.773 & 8.788 & 21.97 \\
\hline 57.122 & 15.379 & 19.773 \\
\hline 153.79 & 68.107 & 61.516 \\
\hline 221.897 & 28.561 & 2.197 \\
\hline 101.062 & 2.197 & 17.576 \\
\hline 2.197 & 6.591 & 17.576 \\
\hline 15.379 & 65.91 & 4.394 \\
\hline 2.197 & 6.591 & 13.182 \\
\hline 166.972 & 48.334 & 65.91 \\
\hline 46.137 & 19.773 & 2.197 \\
\hline 2.197 & 2.197 & 2.197 \\
\hline 101.062 & 19.773 & 30.758 \\
\hline 13.182 & 50.531 & 17.576 \\
\hline 26.364 & 2.197 & 4.394 \\
\hline 41.743 & 61.516 & 28.561 \\
\hline 2.197 & 2.197 & 4.394 \\
\hline 2.197 & 4.394 & 19.773 \\
\hline 2.197 & 2.197 & 13.182 \\
\hline 4.394 & 2.197 & 15.379 \\
\hline 46.137 & 6.591 & 4.394 \\
\hline 2.197 & 4.394 & 54.925 \\
\hline 142.805 & 74.698 & 2.197 \\
\hline 4.394 & 2.197 & 13.182 \\
\hline 4.394 & 35.152 & 2.197 \\
\hline
\end{tabular}




\begin{tabular}{|c|c|c|}
\hline 30.758 & 2.197 & 24.167 \\
\hline 2.197 & 2.197 & 2.197 \\
\hline 2.197 & 2.197 & 2.197 \\
\hline 2.197 & 2.197 & 252.655 \\
\hline 180.154 & 4.394 & 8.788 \\
\hline 2.197 & 960.089 & 22866.4 \\
\hline 2.197 & 30.758 & 24.167 \\
\hline 13.182 & 2.197 & 4.394 \\
\hline 39.546 & 19.773 & 2.197 \\
\hline 2.197 & 72.501 & 15.379 \\
\hline 4.394 & 2.197 & 8.788 \\
\hline 2.197 & 2.197 & 4.394 \\
\hline 4.394 & 74.698 & 2.197 \\
\hline 193.336 & 13.182 & 349.323 \\
\hline 52.728 & 8.788 & 13.182 \\
\hline 24.167 & 2.197 & 19.773 \\
\hline 28.561 & 6.591 & 2.197 \\
\hline 8.788 & 2.197 & 2.197 \\
\hline 13.182 & 197.73 & 3422.93 \\
\hline 123.032 & 2.197 & 4.394 \\
\hline 24.167 & 2.197 & 6.591 \\
\hline 274.625 & 2.197 & 2.197 \\
\hline 87.88 & 2.197 & 17.576 \\
\hline 6.591 & 2.197 & 32.955 \\
\hline 6.591 & 4.394 & 2.197 \\
\hline 10.985 & 4.394 & 2.197 \\
\hline 6.591 & 2.197 & 2.197 \\
\hline 2.197 & 28.561 & 2.197 \\
\hline 2.197 & 109.85 & 2.197 \\
\hline 8.788 & 6.591 & 2.197 \\
\hline 2.197 & 134.017 & 2.197 \\
\hline 4.394 & 2.197 & 8.788 \\
\hline 8.788 & 2.197 & 2.197 \\
\hline 2.197 & 50.531 & 160.381 \\
\hline 48.334 & 10.985 & 15.379 \\
\hline 65.91 & 2.197 & 54.925 \\
\hline 149.396 & 2.197 & 15.379 \\
\hline 15.379 & 2.197 & 15.379 \\
\hline 13.182 & 4.394 & 2.197 \\
\hline 50.531 & 15.379 & 15.379 \\
\hline 50.531 & 2.197 & 2.197 \\
\hline 52.728 & 6.591 & 13.182 \\
\hline 35.152 & 2.197 & 19.773 \\
\hline 2.197 & 2.197 & 39.546 \\
\hline 2.197 & 2.197 & 30.758 \\
\hline 21.97 & 8.788 & 4.394 \\
\hline 4.394 & 2.197 & 2.197 \\
\hline 96.668 & 8.788 & 15.379 \\
\hline 8.788 & 2.197 & 4.394 \\
\hline 2.197 & 6.591 & 41.743 \\
\hline
\end{tabular}




\begin{tabular}{|c|c|c|}
\hline 54.925 & 10.985 & 6.591 \\
\hline 6.591 & 8.788 & 28.561 \\
\hline 2.197 & 4.394 & 4.394 \\
\hline 30.758 & 2.197 & 2.197 \\
\hline 30.758 & 10.985 & 21.97 \\
\hline 85.683 & 17.576 & 57.122 \\
\hline 15.379 & 6.591 & 2.197 \\
\hline 59.319 & 2.197 & 213.109 \\
\hline 4.394 & 4.394 & 6.591 \\
\hline 17.576 & 26.364 & 2.197 \\
\hline 65.91 & 2.197 & 2.197 \\
\hline 4.394 & 2.197 & 2.197 \\
\hline 8.788 & 2.197 & 50.531 \\
\hline 41.743 & 13.182 & 4.394 \\
\hline 2.197 & 228.488 & 26.364 \\
\hline 63.713 & 6.591 & 300.989 \\
\hline 8.788 & 59.319 & 145.002 \\
\hline 6.591 & 8.788 & 4.394 \\
\hline 2.197 & 2.197 & 87.88 \\
\hline 6.591 & 2.197 & 65.91 \\
\hline 2.197 & 2.197 & 13.182 \\
\hline 26.364 & 2.197 & 10.985 \\
\hline 566.826 & 90.077 & 2.197 \\
\hline 46.137 & 30.758 & 28.561 \\
\hline 4.394 & 2.197 & 96.668 \\
\hline 32.955 & 6.591 & 2.197 \\
\hline 30.758 & 17.576 & 81.289 \\
\hline 72.501 & 2.197 & 4.394 \\
\hline 2.197 & 98.865 & 2.197 \\
\hline 169.169 & 83.486 & 48.334 \\
\hline 32.955 & 2.197 & 30.758 \\
\hline 215.306 & 823.875 & 2.197 \\
\hline 224.094 & 17.576 & 2.197 \\
\hline 4.394 & 8.788 & 2.197 \\
\hline 19.773 & 13.182 & 120.835 \\
\hline 17.576 & 2.197 & 4.394 \\
\hline 15.379 & 2.197 & 32.955 \\
\hline 6.591 & 6.591 & 15.379 \\
\hline 2.197 & 722.813 & 26.364 \\
\hline 2.197 & 13.182 & 2.197 \\
\hline 2.197 & 2.197 & 4.394 \\
\hline 2.197 & 2.197 & 4.394 \\
\hline 2.197 & 52.728 & 2.197 \\
\hline 43.94 & 43.94 & 26.364 \\
\hline 32.955 & 10.985 & 2.197 \\
\hline 74.698 & 6.591 & 2.197 \\
\hline 145.002 & 2.197 & 13.182 \\
\hline 2.197 & 30.758 & 118.638 \\
\hline 8.788 & 37.349 & 2.197 \\
\hline 41.743 & 2.197 & 2.197 \\
\hline
\end{tabular}




\begin{tabular}{|c|c|c|}
\hline 2.197 & 17.576 & 48.334 \\
\hline 2.197 & 6.591 & 2.197 \\
\hline 57.122 & 8.788 & 2.197 \\
\hline 17.576 & 3717.32 & 2.197 \\
\hline 2.197 & 2.197 & 19.773 \\
\hline 10.985 & 68.107 & 2.197 \\
\hline 8.788 & 2.197 & 2.197 \\
\hline 112.047 & 4.394 & 8.788 \\
\hline 68.107 & 8.788 & 123.032 \\
\hline 41.743 & 2.197 & 4.394 \\
\hline 52.728 & 2.197 & 21.97 \\
\hline 2.197 & 2.197 & 13.182 \\
\hline 2.197 & 2.197 & 13.182 \\
\hline 6.591 & 37.349 & 250.458 \\
\hline 2.197 & 6.591 & 109.85 \\
\hline 2.197 & 2.197 & 136.214 \\
\hline 13.182 & 6.591 & 6.591 \\
\hline 28.561 & 4.394 & 2.197 \\
\hline 511.901 & 15.379 & 2.197 \\
\hline 2.197 & 2.197 & 24.167 \\
\hline 2.197 & 4.394 & 2.197 \\
\hline 2.197 & 4.394 & 74.698 \\
\hline 41.743 & 4.394 & 41.743 \\
\hline 79.092 & 2.197 & 239.473 \\
\hline 92.274 & 2.197 & 775.541 \\
\hline 2.197 & 6.591 & 2.197 \\
\hline 2.197 & 35.152 & 2.197 \\
\hline 527.28 & 2.197 & 11242 \\
\hline 118.638 & 2.197 & 2.197 \\
\hline 70.304 & 2.197 & 2.197 \\
\hline 10.985 & 15.379 & 373.49 \\
\hline 4.394 & 19.773 & 8.788 \\
\hline 2.197 & 2.197 & 8.788 \\
\hline 4.394 & 2.197 & 28.561 \\
\hline 48.334 & 17.576 & 13.182 \\
\hline 2.197 & 6.591 & 177.957 \\
\hline 2.197 & 2.197 & 8.788 \\
\hline 101.062 & 21.97 & 15.379 \\
\hline 153.79 & 15.379 & 6.591 \\
\hline 8.788 & 2.197 & 32.955 \\
\hline 54.925 & 6.591 & 28.561 \\
\hline 2.197 & 2.197 & 2.197 \\
\hline 2.197 & 2.197 & 2.197 \\
\hline 2.197 & 2.197 & 6.591 \\
\hline 1700.48 & 6.591 & 59.319 \\
\hline 10.985 & 13.182 & 151.593 \\
\hline 32.955 & 2.197 & 37.349 \\
\hline 15.379 & 65.91 & 2.197 \\
\hline 2.197 & 6.591 & 81.289 \\
\hline 4.394 & 6.591 & 10.985 \\
\hline
\end{tabular}




\begin{tabular}{|c|c|c|}
\hline 4.394 & 2.197 & 2.197 \\
\hline 85.683 & 318.565 & 30.758 \\
\hline 65.91 & 24.167 & 2.197 \\
\hline 63.713 & 74.698 & 2.197 \\
\hline 2.197 & 2.197 & 4.394 \\
\hline 63.713 & 10.985 & 2.197 \\
\hline 2.197 & 6.591 & 85.683 \\
\hline 19.773 & 2.197 & 2.197 \\
\hline 2.197 & 21.97 & 147.199 \\
\hline 10.985 & 2.197 & 4.394 \\
\hline 4.394 & 6.591 & 6.591 \\
\hline 13.182 & 4.394 & 8.788 \\
\hline 4.394 & 2.197 & 19.773 \\
\hline 90.077 & 21.97 & 2.197 \\
\hline 13.182 & 2.197 & 96.668 \\
\hline 19.773 & 19.773 & 10.985 \\
\hline 4.394 & 8.788 & 10.985 \\
\hline 6.591 & 6.591 & 2.197 \\
\hline 85.683 & 6.591 & 2.197 \\
\hline 10.985 & 6.591 & 134.017 \\
\hline 13.182 & 2.197 & 2.197 \\
\hline 131.82 & 2.197 & 2.197 \\
\hline 2.197 & 2.197 & 6.591 \\
\hline 2.197 & 6.591 & 8.788 \\
\hline 707.434 & 8.788 & 28.561 \\
\hline 162.578 & 74.698 & 10.985 \\
\hline 2.197 & 21.97 & 24.167 \\
\hline 2.197 & 2.197 & 186.745 \\
\hline 2.197 & 2.197 & 2.197 \\
\hline 4.394 & 24.167 & 10.985 \\
\hline 43.94 & 4.394 & 2.197 \\
\hline 21.97 & 2.197 & 6.591 \\
\hline 6.591 & 4.394 & 8.788 \\
\hline 21.97 & 4.394 & 2.197 \\
\hline 4.394 & 48.334 & 2.197 \\
\hline 30.758 & 2.197 & 2.197 \\
\hline 112.047 & 4.394 & 2.197 \\
\hline 52.728 & 2.197 & 4.394 \\
\hline 4.394 & 35.152 & 30.758 \\
\hline 230.685 & 2.197 & 2.197 \\
\hline 4.394 & 4.394 & 61.516 \\
\hline 6.591 & 2.197 & 2.197 \\
\hline 15.379 & 4.394 & 8.788 \\
\hline 4.394 & 2.197 & 2.197 \\
\hline 6.591 & 2.197 & 8.788 \\
\hline 6.591 & 74.698 & 35.152 \\
\hline 4.394 & 4.394 & 19.773 \\
\hline 360.308 & 24.167 & 2.197 \\
\hline 54.925 & 15.379 & 35.152 \\
\hline 4.394 & 439.4 & 28.561 \\
\hline
\end{tabular}




\begin{tabular}{|c|c|c|}
\hline 15.379 & 4.394 & 2.197 \\
\hline 8.788 & 8.788 & 437.203 \\
\hline 4.394 & 32.955 & 8.788 \\
\hline 358.111 & 4.394 & 15.379 \\
\hline 24.167 & 2.197 & 2.197 \\
\hline 2.197 & 2.197 & 4.394 \\
\hline 24.167 & 4.394 & 2.197 \\
\hline 35.152 & 2.197 & 79.092 \\
\hline 13.182 & 6.591 & 26.364 \\
\hline 4.394 & 2.197 & 6.591 \\
\hline 4.394 & 2.197 & 4.394 \\
\hline 32.955 & 4.394 & 2.197 \\
\hline 2.197 & 4.394 & 21.97 \\
\hline 4.394 & 2.197 & 2.197 \\
\hline 232.882 & 2.197 & 2.197 \\
\hline 107.653 & 318.565 & 6.591 \\
\hline 342.732 & 4.394 & 2.197 \\
\hline 35.152 & 2.197 & 2.197 \\
\hline 896.376 & 2.197 & 52.728 \\
\hline 17.576 & 518.492 & 6.591 \\
\hline 28.561 & 19.773 & 21.97 \\
\hline 2.197 & 2.197 & 397.657 \\
\hline 8.788 & 2.197 & 17.576 \\
\hline 2.197 & 2.197 & 17.576 \\
\hline 2.197 & 2.197 & 2.197 \\
\hline 24.167 & 2.197 & 2.197 \\
\hline 738.192 & 15.379 & 2.197 \\
\hline 127.426 & 8.788 & 4.394 \\
\hline 61.516 & 151.593 & 2.197 \\
\hline 4.394 & 4.394 & 30.758 \\
\hline 221.897 & 2.197 & 2.197 \\
\hline 8.788 & 2.197 & 92.274 \\
\hline 2.197 & 30.758 & 966.68 \\
\hline 4.394 & 2.197 & 15.379 \\
\hline 48.334 & 2.197 & 19.773 \\
\hline 26.364 & 2.197 & 8.788 \\
\hline 4.394 & 2.197 & 61.516 \\
\hline 15.379 & 2.197 & 2.197 \\
\hline 2.197 & 2.197 & 4.394 \\
\hline 2.197 & 4.394 & 2.197 \\
\hline 2.197 & 13.182 & 2.197 \\
\hline 41.743 & 2.197 & 2.197 \\
\hline 21.97 & 2.197 & 63.713 \\
\hline 6.591 & 2.197 & 4.394 \\
\hline 300.989 & 151.593 & 2.197 \\
\hline 2.197 & 2.197 & 2.197 \\
\hline 26.364 & 2.197 & 2.197 \\
\hline 2.197 & 4.394 & 2.197 \\
\hline 4.394 & 92.274 & 10.985 \\
\hline 46.137 & 6.591 & 8.788 \\
\hline
\end{tabular}




\begin{tabular}{|c|c|c|}
\hline 37.349 & 4.394 & 2.197 \\
\hline 101.062 & 24.167 & 26.364 \\
\hline 10.985 & 39.546 & 4.394 \\
\hline 41.743 & 17.576 & 2.197 \\
\hline 10.985 & 63.713 & 90.077 \\
\hline 10.985 & 604.175 & 24.167 \\
\hline 2.197 & 2.197 & 6.591 \\
\hline 13.182 & 17.576 & 2.197 \\
\hline 4.394 & 2.197 & 2.197 \\
\hline 109.85 & 4.394 & 661.297 \\
\hline 13.182 & 109.85 & 2.197 \\
\hline 2.197 & 2.197 & 2.197 \\
\hline 2.197 & 2.197 & 590.993 \\
\hline 136.214 & 2.197 & 2.197 \\
\hline 2.197 & 918.346 & 2.197 \\
\hline 2.197 & 8.788 & 6.591 \\
\hline 4.394 & 4.394 & 2.197 \\
\hline 70.304 & 4.394 & 15.379 \\
\hline 6.591 & 6.591 & 74.698 \\
\hline 83.486 & 173.563 & 83.486 \\
\hline 2.197 & 32.955 & 2.197 \\
\hline 2.197 & 17.576 & 164.775 \\
\hline 19.773 & 2.197 & 2.197 \\
\hline 6.591 & 2.197 & 575.614 \\
\hline 26.364 & 4.394 & 24.167 \\
\hline 24.167 & 26.364 & 13.182 \\
\hline 35.152 & 2.197 & 13.182 \\
\hline 21.97 & 2.197 & 8.788 \\
\hline 6.591 & 70.304 & 478.946 \\
\hline 2.197 & 2.197 & 6.591 \\
\hline 2.197 & 623.948 & 6.591 \\
\hline 2.197 & 6.591 & 15.379 \\
\hline 2.197 & 2.197 & 65.91 \\
\hline 8.788 & 2.197 & 125.229 \\
\hline 285.61 & 2.197 & 2.197 \\
\hline 2.197 & 79.092 & 2.197 \\
\hline 2.197 & 2.197 & 107.653 \\
\hline 13.182 & 2.197 & 4.394 \\
\hline 61.516 & 4.394 & 4.394 \\
\hline 19.773 & 2.197 & 57.122 \\
\hline 13.182 & 2.197 & 4.394 \\
\hline 331.747 & 10.985 & 2.197 \\
\hline 19.773 & 208.715 & 2.197 \\
\hline 8.788 & 6.591 & 6.591 \\
\hline 1362.14 & 8.788 & 8.788 \\
\hline 162.578 & 10.985 & 4.394 \\
\hline 6.591 & 6.591 & 15.379 \\
\hline 21.97 & 4.394 & 2.197 \\
\hline 32.955 & 2.197 & 2.197 \\
\hline 43.94 & 2.197 & 2.197 \\
\hline
\end{tabular}




\begin{tabular}{|c|c|c|}
\hline 2.197 & 2.197 & 4.394 \\
\hline 1140.24 & 21.97 & 2.197 \\
\hline 2.197 & 2.197 & 2.197 \\
\hline 112.047 & 65.91 & 2.197 \\
\hline 8.788 & 52.728 & 8.788 \\
\hline 24.167 & 6.591 & 2.197 \\
\hline 17.576 & 2.197 & 6.591 \\
\hline 6.591 & 13.182 & 81.289 \\
\hline 8.788 & 39.546 & 6.591 \\
\hline 2.197 & 2.197 & 4.394 \\
\hline 2.197 & 4.394 & 21.97 \\
\hline 63.713 & 2.197 & 250.458 \\
\hline 10.985 & 19.773 & 6.591 \\
\hline 593.19 & 4.394 & 19.773 \\
\hline 13.182 & 2.197 & 2.197 \\
\hline 2.197 & 4.394 & 15.379 \\
\hline 2.197 & 109.85 & 186.745 \\
\hline 2.197 & 184.548 & 125.229 \\
\hline 24.167 & 2.197 & 2.197 \\
\hline 4.394 & 2.197 & 2.197 \\
\hline 19.773 & 2.197 & 4.394 \\
\hline 10.985 & 2.197 & 6.591 \\
\hline 13.182 & 4.394 & 46.137 \\
\hline 17.576 & 30.758 & 2.197 \\
\hline 2.197 & 114.244 & 2.197 \\
\hline 2.197 & 94.471 & 8.788 \\
\hline 2.197 & 2.197 & 8.788 \\
\hline 10.985 & 10.985 & 2.197 \\
\hline 2.197 & 261.443 & 15.379 \\
\hline 30.758 & 307.58 & 8.788 \\
\hline 2.197 & 8.788 & 101.062 \\
\hline 24.167 & 2.197 & 26.364 \\
\hline 2.197 & 4.394 & 2.197 \\
\hline 2.197 & 8.788 & 92.274 \\
\hline 4.394 & 2.197 & 4.394 \\
\hline 10.985 & 2.197 & 24.167 \\
\hline 2.197 & 2.197 & 4.394 \\
\hline 6.591 & 35.152 & 2.197 \\
\hline 21.97 & 2.197 & 2.197 \\
\hline 2.197 & 4.394 & 2.197 \\
\hline 2.197 & 2.197 & 4.394 \\
\hline 2.197 & 79.092 & 13.182 \\
\hline 2.197 & 2.197 & 65.91 \\
\hline 76.895 & 2.197 & 17.576 \\
\hline 17.576 & 72.501 & 2.197 \\
\hline 184.548 & 272.428 & 26.364 \\
\hline 6.591 & 2.197 & 4.394 \\
\hline 2.197 & 10.985 & 109.85 \\
\hline 70.304 & 4.394 & 17.576 \\
\hline 6.591 & 2.197 & 32.955 \\
\hline
\end{tabular}




\begin{tabular}{|c|c|c|}
\hline 4.394 & 2.197 & 2038.82 \\
\hline 8.788 & 2.197 & 6.591 \\
\hline 134.017 & 2.197 & 6.591 \\
\hline 48.334 & 2.197 & 41.743 \\
\hline 4.394 & 2.197 & 2.197 \\
\hline 43.94 & 2.197 & 4.394 \\
\hline 21.97 & 2.197 & 6.591 \\
\hline 94.471 & 2.197 & 83.486 \\
\hline 6.591 & 2.197 & 6.591 \\
\hline 81.289 & 13.182 & 4.394 \\
\hline 28.561 & 6.591 & 2.197 \\
\hline 83.486 & 2.197 & 2.197 \\
\hline 2.197 & 2.197 & 2.197 \\
\hline 2.197 & 8.788 & 2.197 \\
\hline 2.197 & 4.394 & 6.591 \\
\hline 2.197 & 2.197 & 19.773 \\
\hline 2.197 & 43.94 & 37.349 \\
\hline 2.197 & 125.229 & 4.394 \\
\hline 2.197 & 2.197 & 39.546 \\
\hline 50.531 & 2.197 & 50.531 \\
\hline 94.471 & 2.197 & 2.197 \\
\hline 10.985 & 2.197 & 35.152 \\
\hline 21.97 & 37.349 & 4.394 \\
\hline 10.985 & 254.852 & 61.516 \\
\hline 70.304 & 4.394 & 13.182 \\
\hline 4.394 & 15.379 & 4.394 \\
\hline 2.197 & 15.379 & 134.017 \\
\hline 2.197 & 2.197 & 24.167 \\
\hline 164.775 & 2.197 & 6.591 \\
\hline 4.394 & 127.426 & 17.576 \\
\hline 6.591 & 2.197 & 21.97 \\
\hline 4.394 & 2.197 & 2.197 \\
\hline 19.773 & 2.197 & 32.955 \\
\hline 4.394 & 2.197 & 17.576 \\
\hline 125.229 & 8.788 & 2.197 \\
\hline 6.591 & 2.197 & 10.985 \\
\hline 2.197 & 2.197 & 898.573 \\
\hline 4.394 & 162.578 & 2.197 \\
\hline 4.394 & 123.032 & 2.197 \\
\hline 2.197 & 2.197 & 13.182 \\
\hline 37.349 & 2.197 & 2.197 \\
\hline 6.591 & 2.197 & 39.546 \\
\hline 2.197 & 4.394 & 2.197 \\
\hline 28.561 & 2.197 & 13.182 \\
\hline 4.394 & 4.394 & 2.197 \\
\hline 4.394 & 2.197 & 39.546 \\
\hline 147.199 & 2.197 & 6.591 \\
\hline 2.197 & 2.197 & 2.197 \\
\hline 35.152 & 17.576 & 103.259 \\
\hline 6.591 & 2.197 & 10.985 \\
\hline
\end{tabular}




\begin{tabular}{|c|c|c|}
\hline 2.197 & 2.197 & 8.788 \\
\hline 39.546 & 6.591 & 59.319 \\
\hline 17.576 & 70.304 & 206.518 \\
\hline 10.985 & 15.379 & 1579.64 \\
\hline 2.197 & 2.197 & 21.97 \\
\hline 2.197 & 2.197 & 4.394 \\
\hline 2.197 & 2.197 & 26.364 \\
\hline 15.379 & 2.197 & 17.576 \\
\hline 6.591 & 2.197 & 30.758 \\
\hline 2.197 & 2.197 & 4.394 \\
\hline 10.985 & 296.595 & 83.486 \\
\hline 65.91 & 57.122 & 50.531 \\
\hline 94.471 & 17.576 & 4.394 \\
\hline 10.985 & 2.197 & 4.394 \\
\hline 8.788 & 2.197 & 13.182 \\
\hline 15.379 & 2.197 & 358.111 \\
\hline 4.394 & 4.394 & 4.394 \\
\hline 2.197 & 193.336 & 96.668 \\
\hline 2.197 & 8.788 & 2.197 \\
\hline 2.197 & 15.379 & 2.197 \\
\hline 2.197 & 15.379 & 13.182 \\
\hline 32.955 & 2.197 & 3860.13 \\
\hline 2.197 & 151.593 & 4.394 \\
\hline 32.955 & 70.304 & 4.394 \\
\hline 2.197 & 4.394 & 4.394 \\
\hline 76.895 & 4.394 & 10.985 \\
\hline 2.197 & 2.197 & 4.394 \\
\hline 2.197 & 3247.17 & 13.182 \\
\hline 175.76 & 4.394 & 2.197 \\
\hline 2.197 & 15.379 & 30.758 \\
\hline 2.197 & 19.773 & 2.197 \\
\hline 26.364 & 2.197 & 8.788 \\
\hline 2.197 & 2.197 & 2.197 \\
\hline 4.394 & 10.985 & 2.197 \\
\hline 2.197 & 48.334 & 2.197 \\
\hline 4.394 & 17.576 & 2.197 \\
\hline 2.197 & 2.197 & 4.394 \\
\hline 13.182 & 2.197 & 4.394 \\
\hline 4.394 & 59.319 & 272.428 \\
\hline 10.985 & 2.197 & 2.197 \\
\hline 8.788 & 6.591 & 311.974 \\
\hline 19.773 & 32.955 & 2.197 \\
\hline 4.394 & 13.182 & 8.788 \\
\hline 2.197 & 2.197 & 59.319 \\
\hline 10.985 & 2.197 & 215.306 \\
\hline 8.788 & 2.197 & 4.394 \\
\hline 6.591 & 10.985 & 3161.48 \\
\hline 37.349 & 15963.4 & 24.167 \\
\hline 2.197 & 6.591 & 6.591 \\
\hline 13.182 & 59.319 & 21.97 \\
\hline
\end{tabular}




\begin{tabular}{|c|c|c|}
\hline 4.394 & 2.197 & 19.773 \\
\hline 4.394 & 2.197 & 4.394 \\
\hline 13.182 & 21.97 & 6.591 \\
\hline 8.788 & 50.531 & 15.379 \\
\hline 2.197 & 13.182 & 2.197 \\
\hline 4.394 & 2.197 & 19.773 \\
\hline 210.912 & 10.985 & 28.561 \\
\hline 90.077 & 2.197 & 2.197 \\
\hline 26.364 & 76.895 & 13.182 \\
\hline 15.379 & 2.197 & 35.152 \\
\hline 300.989 & 2.197 & 57.122 \\
\hline 4.394 & 4.394 & 92.274 \\
\hline 4.394 & 63.713 & 15.379 \\
\hline 2.197 & 4.394 & 13.182 \\
\hline 4.394 & 8.788 & 4.394 \\
\hline 6588.8 & 2.197 & 239.473 \\
\hline 15.379 & 2.197 & 4.394 \\
\hline 21.97 & 2.197 & 2.197 \\
\hline 2.197 & 2.197 & 112.047 \\
\hline 35.152 & 4.394 & 15.379 \\
\hline 2.197 & 2.197 & 15.379 \\
\hline 4.394 & 6.591 & 15.379 \\
\hline 4.394 & 2.197 & 703.04 \\
\hline 2.197 & 13.182 & 2.197 \\
\hline 2.197 & 402.051 & 28.561 \\
\hline 2.197 & 4.394 & 149.396 \\
\hline 2.197 & 4.394 & 2.197 \\
\hline 13.182 & 208.715 & 8.788 \\
\hline 4.394 & 19.773 & 4.394 \\
\hline 2.197 & 2.197 & 50.531 \\
\hline 26.364 & 4.394 & 4.394 \\
\hline 2.197 & 4.394 & 2.197 \\
\hline 8.788 & 8.788 & 15.379 \\
\hline 2.197 & 4.394 & 2.197 \\
\hline 21.97 & 6.591 & 336.141 \\
\hline 349.323 & 254.852 & 2.197 \\
\hline 2.197 & 8.788 & 6.591 \\
\hline 103.259 & 4.394 & 46.137 \\
\hline 2.197 & 4.394 & 10.985 \\
\hline 6.591 & 2.197 & 2.197 \\
\hline 35.152 & 2.197 & 4.394 \\
\hline 2.197 & 4.394 & 4.394 \\
\hline 10.985 & 2.197 & 2.197 \\
\hline 410.839 & 19.773 & 2.197 \\
\hline 19.773 & 2.197 & 6.591 \\
\hline 90.077 & 2.197 & 177.957 \\
\hline 46.137 & 8.788 & 6.591 \\
\hline 30.758 & 2.197 & 2.197 \\
\hline 729.404 & 17.576 & 2.197 \\
\hline 17.576 & 4.394 & 2.197 \\
\hline
\end{tabular}




\begin{tabular}{|c|c|c|}
\hline 6.591 & 26.364 & 10.985 \\
\hline 15.379 & 65.91 & 309.777 \\
\hline 8.788 & 2.197 & 4.394 \\
\hline 2.197 & 21.97 & 10.985 \\
\hline 94.471 & 37.349 & 4.394 \\
\hline 2.197 & 432.809 & 10.985 \\
\hline 2.197 & 54.925 & 6.591 \\
\hline 13.182 & 30.758 & 4.394 \\
\hline 30.758 & 17.576 & 4.394 \\
\hline 50.531 & 61.516 & 32.955 \\
\hline 2.197 & 2.197 & 48.334 \\
\hline 4.394 & 2.197 & 6.591 \\
\hline 52.728 & 2.197 & 2.197 \\
\hline 26.364 & 2.197 & 4.394 \\
\hline 2.197 & 2.197 & 2.197 \\
\hline 2.197 & 2.197 & 28.561 \\
\hline 70.304 & 2.197 & 28.561 \\
\hline 2.197 & 2.197 & 28.561 \\
\hline 28.561 & 2.197 & 2.197 \\
\hline 10.985 & 2.197 & 48.334 \\
\hline 48.334 & 8.788 & 6.591 \\
\hline 138.411 & 15.379 & 61.516 \\
\hline 103.259 & 2.197 & 28.561 \\
\hline 94.471 & 13.182 & 8.788 \\
\hline 6.591 & 8.788 & 4.394 \\
\hline 35.152 & 4.394 & 162.578 \\
\hline 4.394 & 6.591 & 6.591 \\
\hline 6.591 & 2.197 & 2.197 \\
\hline 2.197 & 6.591 & 8.788 \\
\hline 173.563 & 13.182 & 41.743 \\
\hline 2.197 & 2.197 & 17.576 \\
\hline 4.394 & 2.197 & 94.471 \\
\hline 2.197 & 74.698 & 35.152 \\
\hline 48.334 & 2.197 & 43.94 \\
\hline 79.092 & 2.197 & 109.85 \\
\hline 162.578 & 2.197 & 32.955 \\
\hline 8.788 & 4.394 & 41.743 \\
\hline 2.197 & 4.394 & 2.197 \\
\hline 1792.75 & 2.197 & 2.197 \\
\hline 4.394 & 6.591 & 2.197 \\
\hline 19.773 & 30.758 & 46.137 \\
\hline 131.82 & 24.167 & 6.591 \\
\hline 4.394 & 2.197 & 8.788 \\
\hline 790.92 & 2.197 & 41.743 \\
\hline 30.758 & 2.197 & 8.788 \\
\hline 19.773 & 96.668 & 17.576 \\
\hline 2.197 & 2.197 & 4.394 \\
\hline 182.351 & 395.46 & 17.576 \\
\hline 26.364 & 2.197 & 2.197 \\
\hline 8.788 & 2.197 & 39.546 \\
\hline
\end{tabular}




\begin{tabular}{|c|c|c|}
\hline 8.788 & 2.197 & 6.591 \\
\hline 13.182 & 2.197 & 21.97 \\
\hline 6.591 & 2.197 & 37.349 \\
\hline 54.925 & 4.394 & 17.576 \\
\hline 50.531 & 2.197 & 85.683 \\
\hline 8.788 & 2.197 & 15.379 \\
\hline 57.122 & 2.197 & 10.985 \\
\hline 19.773 & 2.197 & 197.73 \\
\hline 2.197 & 2.197 & 1140.24 \\
\hline 2.197 & 4.394 & 10.985 \\
\hline 74.698 & 4.394 & 2.197 \\
\hline 4.394 & 2.197 & 10.985 \\
\hline 10.985 & 70.304 & 112.047 \\
\hline 257.049 & 2.197 & 2.197 \\
\hline 19.773 & 127.426 & 555.841 \\
\hline 2.197 & 2.197 & 2.197 \\
\hline 65.91 & 2.197 & 281.216 \\
\hline 676.676 & 2.197 & 2.197 \\
\hline 2.197 & 13.182 & 2.197 \\
\hline 2.197 & 50.531 & 4.394 \\
\hline 8.788 & 32.955 & 2.197 \\
\hline 2.197 & 415.233 & 4.394 \\
\hline 103.259 & 4.394 & 19.773 \\
\hline 2.197 & 35.152 & 2.197 \\
\hline 6.591 & 2.197 & 4.394 \\
\hline 2.197 & 2.197 & 153.79 \\
\hline 35.152 & 8.788 & 28.561 \\
\hline 63.713 & 2.197 & 204.321 \\
\hline 2.197 & 41.743 & 83.486 \\
\hline 426.218 & 10.985 & 15.379 \\
\hline 32.955 & 85.683 & 87.88 \\
\hline 8.788 & 2.197 & 10.985 \\
\hline 129.623 & 2.197 & 957.892 \\
\hline 2.197 & 24.167 & 13.182 \\
\hline 2.197 & 2.197 & 8.788 \\
\hline 6.591 & 2.197 & 13.182 \\
\hline 217.503 & 32.955 & 68.107 \\
\hline 239.473 & 2.197 & 81.289 \\
\hline 4.394 & 6.591 & 17.576 \\
\hline 4.394 & 2.197 & 81.289 \\
\hline 35.152 & 59.319 & 24.167 \\
\hline 2.197 & 48.334 & 24.167 \\
\hline 8.788 & 175.76 & 2.197 \\
\hline 30.758 & 35.152 & 8.788 \\
\hline 645.918 & 2.197 & 584.402 \\
\hline 2.197 & 4.394 & 43.94 \\
\hline 24.167 & 2.197 & 2.197 \\
\hline 2.197 & 4.394 & 46.137 \\
\hline 2.197 & 2.197 & 26.364 \\
\hline 13.182 & 2.197 & 2.197 \\
\hline
\end{tabular}




\begin{tabular}{|c|c|c|}
\hline 4.394 & 2.197 & 19.773 \\
\hline 472.355 & 15.379 & 4.394 \\
\hline 8.788 & 2.197 & 2.197 \\
\hline 2.197 & 2.197 & 4.394 \\
\hline 2.197 & 4.394 & 10.985 \\
\hline 2.197 & 2.197 & 28.561 \\
\hline 2.197 & 2.197 & 4.394 \\
\hline 6.591 & 2.197 & 2.197 \\
\hline 6.591 & 2.197 & 140.608 \\
\hline 41.743 & 2.197 & 226.291 \\
\hline 21.97 & 30.758 & 15.379 \\
\hline 35.152 & 24.167 & 2.197 \\
\hline 4.394 & 2.197 & 2.197 \\
\hline 10.985 & 2.197 & 4.394 \\
\hline 52.728 & 2.197 & 606.372 \\
\hline 13.182 & 2.197 & 4.394 \\
\hline 2.197 & 59.319 & 210.912 \\
\hline 8.788 & 2.197 & 90.077 \\
\hline 26.364 & 6.591 & 663.494 \\
\hline 2.197 & 2.197 & 24.167 \\
\hline 2.197 & 2.197 & 101.062 \\
\hline 6.591 & 2.197 & 2.197 \\
\hline 2.197 & 4.394 & 4.394 \\
\hline 4.394 & 2.197 & 4.394 \\
\hline 17.576 & 2.197 & 2.197 \\
\hline 17.576 & 6.591 & 6.591 \\
\hline 39.546 & 2.197 & 50.531 \\
\hline 15.379 & 2.197 & 26.364 \\
\hline 10.985 & 21.97 & 2.197 \\
\hline 61.516 & 19.773 & 2.197 \\
\hline 6.591 & 39.546 & 2.197 \\
\hline 4.394 & 13.182 & 2.197 \\
\hline 32.955 & 2.197 & 2.197 \\
\hline 2.197 & 2.197 & 4.394 \\
\hline 2.197 & 2.197 & 6.591 \\
\hline 21.97 & 2.197 & 68.107 \\
\hline 2.197 & 10.985 & 15.379 \\
\hline 2.197 & 26.364 & 8.788 \\
\hline 2.197 & 2.197 & 2.197 \\
\hline 2.197 & 103.259 & 8.788 \\
\hline 19.773 & 136.214 & 28.561 \\
\hline 2.197 & 2.197 & 4.394 \\
\hline 4.394 & 24.167 & 2.197 \\
\hline 2.197 & 2.197 & 6.591 \\
\hline 4.394 & 24.167 & 208.715 \\
\hline 4.394 & 21.97 & 48.334 \\
\hline 2.197 & 4.394 & 2.197 \\
\hline 2.197 & 59.319 & 57.122 \\
\hline 2.197 & 314.171 & 19.773 \\
\hline 2.197 & 2.197 & 191.139 \\
\hline
\end{tabular}




\begin{tabular}{|c|c|c|}
\hline 57.122 & 2.197 & 74.698 \\
\hline 259.246 & 709.631 & 4.394 \\
\hline 8.788 & 2.197 & 65.91 \\
\hline 48.334 & 2.197 & 2.197 \\
\hline 182.351 & 2.197 & 4.394 \\
\hline 4.394 & 2.197 & 2.197 \\
\hline 85.683 & 6.591 & 2.197 \\
\hline 28.561 & 197.73 & 2.197 \\
\hline 380.081 & 2.197 & 8.788 \\
\hline 2.197 & 4.394 & 2.197 \\
\hline 248.261 & 2.197 & 6.591 \\
\hline 259.246 & 2.197 & 13.182 \\
\hline 4.394 & 6.591 & 169.169 \\
\hline 4.394 & 2.197 & 4.394 \\
\hline 2.197 & 28.561 & 52.728 \\
\hline 43.94 & 2.197 & 2.197 \\
\hline 8.788 & 435.006 & 26.364 \\
\hline 17.576 & 48.334 & 37.349 \\
\hline 10.985 & 2.197 & 30.758 \\
\hline 10.985 & 4.394 & 26.364 \\
\hline 2.197 & 87.88 & 26.364 \\
\hline 4.394 & 8.788 & 46.137 \\
\hline 6.591 & 4.394 & 2.197 \\
\hline 4.394 & 13.182 & 2.197 \\
\hline 4.394 & 2.197 & 2.197 \\
\hline 2.197 & 8.788 & 2.197 \\
\hline 90.077 & 4.394 & 2.197 \\
\hline 8.788 & 147.199 & 575.614 \\
\hline 140.608 & 2.197 & 6.591 \\
\hline 155.987 & 17.576 & 2.197 \\
\hline 101.062 & 1537.9 & 24.167 \\
\hline 2.197 & 13.182 & 4.394 \\
\hline 72.501 & 21.97 & 21.97 \\
\hline 8.788 & 402.051 & 15.379 \\
\hline 37.349 & 2.197 & 2.197 \\
\hline 28.561 & 2.197 & 50.531 \\
\hline 4.394 & 2.197 & 10.985 \\
\hline 39.546 & 68.107 & 52.728 \\
\hline 39.546 & 13.182 & 15.379 \\
\hline 6.591 & 4.394 & 639.327 \\
\hline 2.197 & 2.197 & 2.197 \\
\hline 13.182 & 2.197 & 2.197 \\
\hline 8.788 & 98.865 & 81.289 \\
\hline 68.107 & 2.197 & 311.974 \\
\hline 2.197 & 90.077 & 4.394 \\
\hline 32.955 & 4.394 & 2.197 \\
\hline 235.079 & 13.182 & 2.197 \\
\hline 6.591 & 2.197 & 944.71 \\
\hline 92.274 & 837.057 & 43.94 \\
\hline 147.199 & 32.955 & 4.394 \\
\hline
\end{tabular}




\begin{tabular}{|c|c|c|}
\hline 2.197 & 8.788 & 2.197 \\
\hline 2.197 & 2.197 & 6.591 \\
\hline 2.197 & 2.197 & 2.197 \\
\hline 2.197 & 6.591 & 4.394 \\
\hline 26.364 & 2.197 & 4.394 \\
\hline 24.167 & 4.394 & 83.486 \\
\hline 4.394 & 2.197 & 59.319 \\
\hline 4.394 & 2.197 & 173.563 \\
\hline 4.394 & 2.197 & 2.197 \\
\hline 2.197 & 2.197 & 8.788 \\
\hline 2.197 & 2.197 & 2.197 \\
\hline 68.107 & 6.591 & 68.107 \\
\hline 4.394 & 4.394 & 28.561 \\
\hline 2.197 & 10.985 & 2.197 \\
\hline 2.197 & 2.197 & 2.197 \\
\hline 2.197 & 2.197 & 6.591 \\
\hline 13.182 & 37.349 & 2.197 \\
\hline 90.077 & 2.197 & 2.197 \\
\hline 147.199 & 2.197 & 118.638 \\
\hline 2.197 & 2.197 & 6.591 \\
\hline 10.985 & 2.197 & 17.576 \\
\hline 48.334 & 37.349 & 50.531 \\
\hline 2.197 & 2.197 & 4.394 \\
\hline 74.698 & 15.379 & 2.197 \\
\hline 2.197 & 2.197 & 2.197 \\
\hline 2.197 & 4.394 & 2.197 \\
\hline 8.788 & 35.152 & 237.276 \\
\hline 311.974 & 4.394 & 2.197 \\
\hline 70.304 & 2.197 & 4.394 \\
\hline 28.561 & 2.197 & 2.197 \\
\hline 35.152 & 10.985 & 4.394 \\
\hline 21.97 & 4.394 & 2.197 \\
\hline 4.394 & 24.167 & 19.773 \\
\hline 24.167 & 6.591 & 4.394 \\
\hline 8.788 & 13.182 & 2.197 \\
\hline 6.591 & 6.591 & 10.985 \\
\hline 6.591 & 4.394 & 35.152 \\
\hline 2.197 & 13.182 & 2.197 \\
\hline 81.289 & 10.985 & 2.197 \\
\hline 2.197 & 4.394 & 35.152 \\
\hline 8.788 & 2.197 & 107.653 \\
\hline 50.531 & 4.394 & 2.197 \\
\hline 4.394 & 2.197 & 13.182 \\
\hline 2.197 & 15.379 & 2.197 \\
\hline 4.394 & 17.576 & 2.197 \\
\hline 4.394 & 2.197 & 72.501 \\
\hline 65.91 & 2.197 & 259.246 \\
\hline 13.182 & 2.197 & 2.197 \\
\hline 2.197 & 8.788 & 17.576 \\
\hline 2.197 & 10.985 & 10.985 \\
\hline
\end{tabular}




\begin{tabular}{|c|c|c|}
\hline 2.197 & 4.394 & 2.197 \\
\hline 28.561 & 2.197 & 6.591 \\
\hline 2.197 & 2.197 & 10.985 \\
\hline 2.197 & 8.788 & 13.182 \\
\hline 70.304 & 10.985 & 2.197 \\
\hline 2.197 & 2.197 & 6.591 \\
\hline 193.336 & 24.167 & 4.394 \\
\hline 30.758 & 2.197 & 13.182 \\
\hline 2.197 & 120.835 & 4.394 \\
\hline 87.88 & 2.197 & 17.576 \\
\hline 68.107 & 4.394 & 30.758 \\
\hline 13.182 & 13.182 & 10.985 \\
\hline 10.985 & 8.788 & 13.182 \\
\hline 4.394 & 24.167 & 4.394 \\
\hline 6.591 & 2.197 & 2.197 \\
\hline 2.197 & 2.197 & 2.197 \\
\hline 4.394 & 4.394 & 259.246 \\
\hline 21.97 & 2.197 & 19.773 \\
\hline 2.197 & 6.591 & 59.319 \\
\hline 2.197 & 4.394 & 39.546 \\
\hline 6.591 & 2.197 & 4.394 \\
\hline 1019.41 & 4.394 & 10.985 \\
\hline 2.197 & 4.394 & 155.987 \\
\hline 162.578 & 2.197 & 8.788 \\
\hline 68.107 & 8.788 & 173.563 \\
\hline 2.197 & 4.394 & 46.137 \\
\hline 68.107 & 8.788 & 2.197 \\
\hline 39.546 & 2.197 & 2.197 \\
\hline 6.591 & 2.197 & 4.394 \\
\hline 4.394 & 2.197 & 2.197 \\
\hline 6.591 & 2.197 & 10.985 \\
\hline 2.197 & 2.197 & 2.197 \\
\hline 2.197 & 35.152 & 8.788 \\
\hline 4.394 & 2.197 & 2.197 \\
\hline 6.591 & 2.197 & 4.394 \\
\hline 241.67 & 4.394 & 6.591 \\
\hline 2.197 & 41391.5 & 2.197 \\
\hline 83.486 & 4.394 & 32.955 \\
\hline 2.197 & 2.197 & 21.97 \\
\hline 2.197 & 2.197 & 103.259 \\
\hline 6.591 & 2.197 & 2.197 \\
\hline 4.394 & 2.197 & 2.197 \\
\hline 15.379 & 2.197 & 2.197 \\
\hline 160.381 & 2.197 & 85.683 \\
\hline 13.182 & 224.094 & 24.167 \\
\hline 114.244 & 120.835 & 336.141 \\
\hline 13.182 & 10.985 & 21.97 \\
\hline 65.91 & 2.197 & 2.197 \\
\hline 6.591 & 4.394 & 2.197 \\
\hline 35.152 & 70.304 & 17.576 \\
\hline
\end{tabular}




\begin{tabular}{|c|c|c|}
\hline 134.017 & 2.197 & 2.197 \\
\hline 10.985 & 2.197 & 48.334 \\
\hline 151.593 & 2.197 & 2.197 \\
\hline 6.591 & 2.197 & 4.394 \\
\hline 4.394 & 2.197 & 101.062 \\
\hline 4.394 & 2.197 & 48.334 \\
\hline 149.396 & 2.197 & 4.394 \\
\hline 2.197 & 2.197 & 10.985 \\
\hline 30.758 & 4.394 & 48.334 \\
\hline 61.516 & 52.728 & 2.197 \\
\hline 10.985 & 6.591 & 6.591 \\
\hline 6.591 & 2.197 & 63.713 \\
\hline 10.985 & 2.197 & 54.925 \\
\hline 2.197 & 8.788 & 46.137 \\
\hline 2.197 & 54.925 & 13.182 \\
\hline 21.97 & 13.182 & 2.197 \\
\hline 65.91 & 4.394 & 6.591 \\
\hline 19.773 & 39.546 & 15.379 \\
\hline 13.182 & 4.394 & 4.394 \\
\hline 13.182 & 2.197 & 4.394 \\
\hline 76.895 & 15.379 & 4.394 \\
\hline 4.394 & 6.591 & 8.788 \\
\hline 15.379 & 15.379 & 2.197 \\
\hline 2.197 & 2.197 & 8.788 \\
\hline 13.182 & 2.197 & 129.623 \\
\hline 4.394 & 1713.66 & 15.379 \\
\hline 2.197 & 2.197 & 63.713 \\
\hline 2.197 & 2.197 & 1889.42 \\
\hline 43.94 & 2.197 & 4.394 \\
\hline 213.109 & 8.788 & 503.113 \\
\hline 4.394 & 4.394 & 4.394 \\
\hline 76.895 & 54.925 & 2.197 \\
\hline 57.122 & 2.197 & 2.197 \\
\hline 2.197 & 72.501 & 10.985 \\
\hline 90.077 & 4.394 & 15.379 \\
\hline 2.197 & 2.197 & 2.197 \\
\hline 2.197 & 41.743 & 24.167 \\
\hline 6.591 & 6.591 & 32.955 \\
\hline 2.197 & 2.197 & 8.788 \\
\hline 1353.35 & 2.197 & 2.197 \\
\hline 28.561 & 2.197 & 4.394 \\
\hline 32.955 & 2.197 & 2.197 \\
\hline 2.197 & 2.197 & 39.546 \\
\hline 4.394 & 2.197 & 39.546 \\
\hline 39.546 & 2.197 & 4.394 \\
\hline 32.955 & 2.197 & 19.773 \\
\hline 8.788 & 4.394 & 2.197 \\
\hline 32.955 & 2.197 & 4.394 \\
\hline 4.394 & 347.126 & 46.137 \\
\hline 4.394 & 15.379 & 19.773 \\
\hline
\end{tabular}




\begin{tabular}{|c|c|c|}
\hline 28.561 & 4.394 & 1421.46 \\
\hline 10.985 & 6.591 & 4.394 \\
\hline 8.788 & 8.788 & 17.576 \\
\hline 21.97 & 90.077 & 973.271 \\
\hline 96.668 & 32.955 & 2.197 \\
\hline 2.197 & 21.97 & 28.561 \\
\hline 2.197 & 2.197 & 4.394 \\
\hline 2.197 & 48.334 & 21.97 \\
\hline 2.197 & 2.197 & 4.394 \\
\hline 2.197 & 13.182 & 120.835 \\
\hline 2.197 & 43.94 & 98.865 \\
\hline 2.197 & 6.591 & 19.773 \\
\hline 24.167 & 4.394 & 79.092 \\
\hline 6.591 & 15.379 & 39.546 \\
\hline 4.394 & 158.184 & 24.167 \\
\hline 4.394 & 2.197 & 17.576 \\
\hline 46.137 & 17.576 & 2.197 \\
\hline 8.788 & 2.197 & 15.379 \\
\hline 476.749 & 2.197 & 8.788 \\
\hline 74.698 & 2.197 & 74.698 \\
\hline 2.197 & 2.197 & 48.334 \\
\hline 32.955 & 4.394 & 4.394 \\
\hline 68.107 & 2.197 & 2.197 \\
\hline 15.379 & 28.561 & 28.561 \\
\hline 19.773 & 4.394 & 2.197 \\
\hline 2.197 & 28.561 & 4.394 \\
\hline 8.788 & 2.197 & 32.955 \\
\hline 13.182 & 2.197 & 6.591 \\
\hline 2.197 & 2.197 & 2.197 \\
\hline 131.82 & 43.94 & 35.152 \\
\hline 21.97 & 39.546 & 2.197 \\
\hline 2.197 & 37.349 & 2.197 \\
\hline 2.197 & 6.591 & 21.97 \\
\hline 6.591 & 6.591 & 2.197 \\
\hline 158.184 & 10.985 & 2.197 \\
\hline 26.364 & 39.546 & 371.293 \\
\hline 8.788 & 8.788 & 32.955 \\
\hline 8.788 & 2.197 & 4.394 \\
\hline 2.197 & 2.197 & 21.97 \\
\hline 30.758 & 2.197 & 6.591 \\
\hline 59.319 & 87.88 & 2.197 \\
\hline 2.197 & 2.197 & 430.612 \\
\hline 4.394 & 39.546 & 2.197 \\
\hline 2.197 & 24.167 & 2.197 \\
\hline 10.985 & 13.182 & 92.274 \\
\hline 26.364 & 48.334 & 15.379 \\
\hline 2.197 & 2.197 & 2.197 \\
\hline 2.197 & 2.197 & 74.698 \\
\hline 15.379 & 2.197 & 2.197 \\
\hline 39.546 & 2.197 & 4.394 \\
\hline
\end{tabular}




\begin{tabular}{|c|c|c|}
\hline 300.989 & 2.197 & 140.608 \\
\hline 15.379 & 19.773 & 83.486 \\
\hline 15.379 & 15.379 & 2.197 \\
\hline 259.246 & 2.197 & 2.197 \\
\hline 10.985 & 2.197 & 2.197 \\
\hline 140.608 & 63.713 & 15.379 \\
\hline 17.576 & 6.591 & 57.122 \\
\hline 10.985 & 6.591 & 57.122 \\
\hline 83.486 & 4.394 & 136.214 \\
\hline 70.304 & 4.394 & 26.364 \\
\hline 24.167 & 2.197 & 2.197 \\
\hline 13.182 & 2.197 & 2.197 \\
\hline 50.531 & 6.591 & 8.788 \\
\hline 35.152 & 2.197 & 2.197 \\
\hline 2.197 & 6.591 & 96.668 \\
\hline 1808.13 & 2.197 & 4.394 \\
\hline 2.197 & 177.957 & 8.788 \\
\hline 2.197 & 21.97 & 15.379 \\
\hline 2.197 & 2.197 & 123.032 \\
\hline 85.683 & 2.197 & 4.394 \\
\hline 2.197 & 2.197 & 46.137 \\
\hline 2.197 & 6.591 & 2.197 \\
\hline 41.743 & 195.533 & 87.88 \\
\hline 6.591 & 2.197 & 15.379 \\
\hline 4.394 & 63.713 & 15.379 \\
\hline 6.591 & 2.197 & 46.137 \\
\hline 72.501 & 2.197 & 43.94 \\
\hline 59.319 & 59.319 & 8.788 \\
\hline 24.167 & 2.197 & 107.653 \\
\hline 35.152 & 8.788 & 4.394 \\
\hline 32.955 & 4.394 & 4.394 \\
\hline 52.728 & 2.197 & 8.788 \\
\hline 24.167 & 4.394 & 136.214 \\
\hline 2.197 & 340.535 & 30769 \\
\hline 4.394 & 2.197 & 270.231 \\
\hline 2.197 & 2.197 & 2.197 \\
\hline 2.197 & 2.197 & 300.989 \\
\hline 54.925 & 2.197 & 10.985 \\
\hline 4.394 & 19.773 & 83.486 \\
\hline 15.379 & 125.229 & 4.394 \\
\hline 35.152 & 17.576 & 2.197 \\
\hline 2.197 & 6.591 & 10.985 \\
\hline 87.88 & 4.394 & 8.788 \\
\hline 109.85 & 2.197 & 2.197 \\
\hline 24.167 & 2.197 & 13.182 \\
\hline 8.788 & 2.197 & 17.576 \\
\hline 6.591 & 8.788 & 2.197 \\
\hline 2.197 & 15.379 & 2.197 \\
\hline 219.7 & 68.107 & 48.334 \\
\hline 2.197 & 15.379 & 2.197 \\
\hline
\end{tabular}




\begin{tabular}{|c|c|c|}
\hline 4.394 & 21.97 & 35.152 \\
\hline 72.501 & 6.591 & 2.197 \\
\hline 2.197 & 96.668 & 10.985 \\
\hline 2.197 & 2.197 & 30.758 \\
\hline 2.197 & 26.364 & 8.788 \\
\hline 13.182 & 4.394 & 54.925 \\
\hline 35.152 & 30.758 & 2.197 \\
\hline 4.394 & 4.394 & 221.897 \\
\hline 127.426 & 10.985 & 13.182 \\
\hline 2.197 & 35.152 & 2.197 \\
\hline 10.985 & 2.197 & 10.985 \\
\hline 8.788 & 103.259 & 4.394 \\
\hline 8.788 & 112.047 & 10.985 \\
\hline 10.985 & 2.197 & 52.728 \\
\hline 2.197 & 6.591 & 68.107 \\
\hline 15.379 & 331.747 & 2.197 \\
\hline 26.364 & 2.197 & 2.197 \\
\hline 63.713 & 2.197 & 4.394 \\
\hline 8.788 & 6.591 & 2.197 \\
\hline 2.197 & 2.197 & 2.197 \\
\hline 4.394 & 2.197 & 28.561 \\
\hline 4.394 & 26.364 & 8.788 \\
\hline 199.927 & 8.788 & 41.743 \\
\hline 13.182 & 2.197 & 136.214 \\
\hline 52.728 & 17.576 & 4.394 \\
\hline 30.758 & 54.925 & 94.471 \\
\hline 4.394 & 4.394 & 17.576 \\
\hline 147.199 & 2.197 & 274.625 \\
\hline 2.197 & 4.394 & 4.394 \\
\hline 2.197 & 21.97 & 10.985 \\
\hline 4.394 & 2328.82 & 6.591 \\
\hline 98.865 & 2.197 & 2.197 \\
\hline 4.394 & 2.197 & 26.364 \\
\hline 8.788 & 2.197 & 2.197 \\
\hline 17.576 & 37.349 & 2.197 \\
\hline 50.531 & 322.959 & 24.167 \\
\hline 10.985 & 28.561 & 2.197 \\
\hline 57.122 & 2.197 & 2.197 \\
\hline 43.94 & 2.197 & 4.394 \\
\hline 21.97 & 246.064 & 118.638 \\
\hline 13.182 & 2.197 & 15.379 \\
\hline 2.197 & 4.394 & 4.394 \\
\hline 59.319 & 6.591 & 15.379 \\
\hline 2.197 & 8.788 & 1823.51 \\
\hline 10.985 & 10.985 & 41.743 \\
\hline 2.197 & 2.197 & 2.197 \\
\hline 2.197 & 19.773 & 4.394 \\
\hline 4.394 & 2.197 & 2.197 \\
\hline 48.334 & 21.97 & 2.197 \\
\hline 10.985 & 2.197 & 8.788 \\
\hline
\end{tabular}




\begin{tabular}{|c|c|c|}
\hline 2.197 & 2.197 & 35.152 \\
\hline 10.985 & 26.364 & 6.591 \\
\hline 61.516 & 4.394 & 10.985 \\
\hline 2.197 & 24.167 & 24.167 \\
\hline 4.394 & 39.546 & 2.197 \\
\hline 6.591 & 2.197 & 10.985 \\
\hline 2.197 & 2.197 & 26.364 \\
\hline 10.985 & 4.394 & 2.197 \\
\hline 2.197 & 2.197 & 37.349 \\
\hline 19.773 & 39.546 & 8.788 \\
\hline 21.97 & 4.394 & 4.394 \\
\hline 30.758 & 590.993 & 43.94 \\
\hline 15.379 & 254.852 & 2.197 \\
\hline 2.197 & 13.182 & 2.197 \\
\hline 134.017 & 4.394 & 250.458 \\
\hline 35.152 & 612.963 & 2.197 \\
\hline 17.576 & 2.197 & 37.349 \\
\hline 160.381 & 4.394 & 79.092 \\
\hline 8.788 & 4.394 & 6.591 \\
\hline 39.546 & 2.197 & 4.394 \\
\hline 2.197 & 8.788 & 2.197 \\
\hline 2.197 & 8.788 & 15.379 \\
\hline 2.197 & 162.578 & 10.985 \\
\hline 13.182 & 2.197 & 63.713 \\
\hline 10.985 & 2.197 & 4.394 \\
\hline 314.171 & 8.788 & 2.197 \\
\hline 15.379 & 4.394 & 2.197 \\
\hline 6.591 & 24.167 & 2.197 \\
\hline 4.394 & 443.794 & 4.394 \\
\hline 2.197 & 2.197 & 13.182 \\
\hline 10.985 & 2.197 & 4.394 \\
\hline 2.197 & 180.154 & 573.417 \\
\hline 2.197 & 2.197 & 4.394 \\
\hline 8.788 & 4.394 & 2.197 \\
\hline 26.364 & 2.197 & 19.773 \\
\hline 6.591 & 2.197 & 2.197 \\
\hline 13.182 & 43.94 & 2.197 \\
\hline 19.773 & 8.788 & 24.167 \\
\hline 173.563 & 2.197 & 17.576 \\
\hline 2.197 & 2.197 & 2.197 \\
\hline 2.197 & 467.961 & 2.197 \\
\hline 2.197 & 32.955 & 2.197 \\
\hline 96.668 & 2.197 & 2.197 \\
\hline 2.197 & 2.197 & 43.94 \\
\hline 28.561 & 2.197 & 48.334 \\
\hline 2.197 & 2.197 & 2.197 \\
\hline 70.304 & 39.546 & 2.197 \\
\hline 4.394 & 2.197 & 2.197 \\
\hline 2.197 & 8.788 & 57.122 \\
\hline 6.591 & 2.197 & 2.197 \\
\hline
\end{tabular}




\begin{tabular}{|c|c|c|}
\hline 410.839 & 2.197 & 4.394 \\
\hline 19.773 & 2.197 & 15.379 \\
\hline 17.576 & 10.985 & 2.197 \\
\hline 2.197 & 2.197 & 30.758 \\
\hline 4.394 & 15.379 & 8.788 \\
\hline 2.197 & 718.419 & 13.182 \\
\hline 39.546 & 2.197 & 94.471 \\
\hline 452.582 & 6.591 & 402.051 \\
\hline 26.364 & 2.197 & 2.197 \\
\hline 37.349 & 10.985 & 2.197 \\
\hline 4.394 & 28.561 & 2.197 \\
\hline 6.591 & 2.197 & 2.197 \\
\hline 35.152 & 2.197 & 2.197 \\
\hline 8.788 & 2.197 & 63.713 \\
\hline 303.186 & 2.197 & 6.591 \\
\hline 4.394 & 2.197 & 8.788 \\
\hline 10.985 & 4.394 & 24.167 \\
\hline 6.591 & 140.608 & 2.197 \\
\hline 13.182 & 8.788 & 10.985 \\
\hline 28.561 & 2.197 & 6.591 \\
\hline 39.546 & 2.197 & 103.259 \\
\hline 2.197 & 6.591 & 489.931 \\
\hline 2.197 & 63.713 & 10.985 \\
\hline 116.441 & 8.788 & 261.443 \\
\hline 2.197 & 2.197 & 13.182 \\
\hline 2.197 & 2.197 & 21.97 \\
\hline 6.591 & 10.985 & 28.561 \\
\hline 13.182 & 2.197 & 265.837 \\
\hline 4.394 & 24.167 & 8.788 \\
\hline 61.516 & 8.788 & 6.591 \\
\hline 2.197 & 4.394 & 17.576 \\
\hline 2.197 & 4.394 & 2.197 \\
\hline 2.197 & 160.381 & 41.743 \\
\hline 2.197 & 92.274 & 10.985 \\
\hline 19.773 & 147.199 & 8.788 \\
\hline 632.736 & 30.758 & 9809.6 \\
\hline 4.394 & 2.197 & 72.501 \\
\hline 2.197 & 2.197 & 10.985 \\
\hline 2.197 & 50.531 & 41.743 \\
\hline 2.197 & 123.032 & 2.197 \\
\hline 21.97 & 48.334 & 2.197 \\
\hline 6.591 & 2.197 & 10.985 \\
\hline 406.445 & 2.197 & 2.197 \\
\hline 87.88 & 2.197 & 2.197 \\
\hline 8.788 & 21.97 & 4.394 \\
\hline 26.364 & 2.197 & 1485.17 \\
\hline 2.197 & 48.334 & 709.631 \\
\hline 2.197 & 13.182 & 13.182 \\
\hline 2.197 & 2.197 & 6.591 \\
\hline 2.197 & 39.546 & 2.197 \\
\hline
\end{tabular}




\begin{tabular}{|c|c|c|}
\hline 35.152 & 28.561 & 2.197 \\
\hline 2.197 & 4.394 & 21.97 \\
\hline 4.394 & 4.394 & 28.561 \\
\hline 6.591 & 4.394 & 92.274 \\
\hline 19.773 & 21.97 & 8.788 \\
\hline 94.471 & 2.197 & 2.197 \\
\hline 46.137 & 32.955 & 123.032 \\
\hline 96.668 & 213.109 & 4.394 \\
\hline 41.743 & 6.591 & 6.591 \\
\hline 15.379 & 2.197 & 551.447 \\
\hline 854.633 & 2.197 & 30.758 \\
\hline 4.394 & 4.394 & 294.398 \\
\hline 24.167 & 481.143 & 336.141 \\
\hline 2.197 & 6.591 & 2.197 \\
\hline 2.197 & 32.955 & 68.107 \\
\hline 2.197 & 54.925 & 2.197 \\
\hline 2.197 & 2.197 & 8.788 \\
\hline 26.364 & 35.152 & 81.289 \\
\hline 13.182 & 2.197 & 59.319 \\
\hline 26.364 & 2.197 & 2.197 \\
\hline 287.807 & 2.197 & 19.773 \\
\hline 2.197 & 4.394 & 15.379 \\
\hline 2.197 & 2.197 & 59.319 \\
\hline 2.197 & 2.197 & 92.274 \\
\hline 2.197 & 4.394 & 8.788 \\
\hline 118.638 & 173.563 & 68.107 \\
\hline 2.197 & 2.197 & 10.985 \\
\hline 30.758 & 4.394 & 118.638 \\
\hline 41.743 & 10.985 & 4.394 \\
\hline 21.97 & 4.394 & 2.197 \\
\hline 2.197 & 28.561 & 371.293 \\
\hline 149.396 & 4.394 & 2.197 \\
\hline 511.901 & 2.197 & 2.197 \\
\hline 24.167 & 8.788 & 39.546 \\
\hline 83.486 & 15.379 & 13.182 \\
\hline 13.182 & 2.197 & 6.591 \\
\hline 8.788 & 107.653 & 65.91 \\
\hline 4.394 & 2.197 & 6.591 \\
\hline 2.197 & 2.197 & 2.197 \\
\hline 2.197 & 2.197 & 2.197 \\
\hline 61.516 & 2.197 & 26.364 \\
\hline 4.394 & 2.197 & 2.197 \\
\hline 10.985 & 10.985 & 17.576 \\
\hline 26.364 & 2.197 & 2.197 \\
\hline 149.396 & 2.197 & 2.197 \\
\hline 2.197 & 8.788 & 15.379 \\
\hline 8.788 & 2.197 & 10.985 \\
\hline 26.364 & 4.394 & 8.788 \\
\hline 2.197 & 21.97 & 19.773 \\
\hline 63.713 & 15.379 & 8.788 \\
\hline
\end{tabular}




\begin{tabular}{|c|c|c|}
\hline 46.137 & 2.197 & 17.576 \\
\hline 2.197 & 2.197 & 26.364 \\
\hline 32.955 & 2.197 & 2.197 \\
\hline 10.985 & 17.576 & 21.97 \\
\hline 4.394 & 2.197 & 6.591 \\
\hline 39.546 & 2.197 & 953.498 \\
\hline 85.683 & 2.197 & 6.591 \\
\hline 4.394 & 2.197 & 96.668 \\
\hline 26.364 & 237.276 & 2.197 \\
\hline 39.546 & 2.197 & 15.379 \\
\hline 2.197 & 28.561 & 2.197 \\
\hline 2.197 & 338.338 & 8.788 \\
\hline 65.91 & 52.728 & 43.94 \\
\hline 32.955 & 2.197 & 2.197 \\
\hline 2.197 & 2.197 & 6.591 \\
\hline 4.394 & 8.788 & 4.394 \\
\hline 41.743 & 19.773 & 292.201 \\
\hline 2.197 & 314.171 & 52.728 \\
\hline 2.197 & 6.591 & 977.665 \\
\hline 4.394 & 4.394 & 2.197 \\
\hline 4.394 & 1017.21 & 4.394 \\
\hline 24.167 & 4.394 & 2.197 \\
\hline 151.593 & 6.591 & 4.394 \\
\hline 112.047 & 6.591 & 2.197 \\
\hline 166.972 & 2.197 & 13.182 \\
\hline 26.364 & 2.197 & 15.379 \\
\hline 6.591 & 8.788 & 8.788 \\
\hline 4.394 & 28.561 & 13.182 \\
\hline 10.985 & 2.197 & 19.773 \\
\hline 182.351 & 21.97 & 169.169 \\
\hline 41.743 & 134.017 & 300.989 \\
\hline 2.197 & 30.758 & 2.197 \\
\hline 2.197 & 2.197 & 6.591 \\
\hline 243.867 & 2.197 & 261.443 \\
\hline 41.743 & 8.788 & 43.94 \\
\hline 2.197 & 2.197 & 2.197 \\
\hline 166.972 & 30.758 & 81.289 \\
\hline 37.349 & 2.197 & 2.197 \\
\hline 4.394 & 54.925 & 2.197 \\
\hline 30.758 & 2.197 & 2.197 \\
\hline 2.197 & 37.349 & 4365.44 \\
\hline 4.394 & 6.591 & 4.394 \\
\hline 30.758 & 26.364 & 2.197 \\
\hline 2.197 & 2.197 & 2.197 \\
\hline 10.985 & 17.576 & 2.197 \\
\hline 24.167 & 30.758 & 116.441 \\
\hline 17.576 & 4.394 & 8.788 \\
\hline 85.683 & 2.197 & 13.182 \\
\hline 10.985 & 2.197 & 10.985 \\
\hline 2.197 & 2.197 & 8.788 \\
\hline
\end{tabular}




\begin{tabular}{|c|c|c|}
\hline 28.561 & 2.197 & 2.197 \\
\hline 26.364 & 8.788 & 13.182 \\
\hline 120.835 & 2.197 & 4.394 \\
\hline 2.197 & 61.516 & 2.197 \\
\hline 2.197 & 4.394 & 6.591 \\
\hline 2.197 & 15.379 & 37.349 \\
\hline 6.591 & 2.197 & 19.773 \\
\hline 8.788 & 6.591 & 186.745 \\
\hline 28.561 & 2.197 & 2.197 \\
\hline 2.197 & 2.197 & 2.197 \\
\hline 6.591 & 922.74 & 6.591 \\
\hline 2.197 & 17.576 & 26.364 \\
\hline 19.773 & 2.197 & 4.394 \\
\hline 4.394 & 2.197 & 2.197 \\
\hline 2.197 & 2.197 & 2.197 \\
\hline 10.985 & 2.197 & 4.394 \\
\hline 81.289 & 8.788 & 39.546 \\
\hline 37.349 & 2.197 & 65.91 \\
\hline 4.394 & 2.197 & 2.197 \\
\hline 2.197 & 2.197 & 2.197 \\
\hline 52.728 & 2.197 & 10.985 \\
\hline 4.394 & 118.638 & 2.197 \\
\hline 2.197 & 2.197 & 2.197 \\
\hline 2.197 & 4.394 & 120.835 \\
\hline 2.197 & 2.197 & 85.683 \\
\hline 54.925 & 10.985 & 2.197 \\
\hline 90.077 & 2.197 & 962.286 \\
\hline 2.197 & 4.394 & 17.576 \\
\hline 8.788 & 2.197 & 52.728 \\
\hline 265.837 & 4.394 & 103.259 \\
\hline 2.197 & 79.092 & 6.591 \\
\hline 13.182 & 2.197 & 2.197 \\
\hline 182.351 & 26.364 & 6.591 \\
\hline 303.186 & 2.197 & 2.197 \\
\hline 30.758 & 2.197 & 4.394 \\
\hline 17.576 & 2.197 & 2.197 \\
\hline 142.805 & 2.197 & 2.197 \\
\hline 4.394 & 2.197 & 2.197 \\
\hline 15.379 & 2.197 & 68.107 \\
\hline 2.197 & 70.304 & 8.788 \\
\hline 17.576 & 2.197 & 2.197 \\
\hline 32.955 & 4.394 & 195.533 \\
\hline 8.788 & 2.197 & 692.055 \\
\hline 8.788 & 2.197 & 4.394 \\
\hline 2.197 & 41.743 & 15.379 \\
\hline 2.197 & 6.591 & 2.197 \\
\hline 8.788 & 81.289 & 2.197 \\
\hline 2.197 & 21.97 & 52.728 \\
\hline 2.197 & 2.197 & 2.197 \\
\hline 6.591 & 2.197 & 4.394 \\
\hline
\end{tabular}




\begin{tabular}{|c|c|c|}
\hline 6.591 & 2.197 & 147.199 \\
\hline 2.197 & 4.394 & 4.394 \\
\hline 6.591 & 2.197 & 131.82 \\
\hline 4.394 & 2.197 & 6.591 \\
\hline 6.591 & 2.197 & 8.788 \\
\hline 35.152 & 127.426 & 2.197 \\
\hline 90.077 & 28.561 & 2.197 \\
\hline 52.728 & 15.379 & 6.591 \\
\hline 4.394 & 2.197 & 39.546 \\
\hline 30.758 & 26.364 & 2.197 \\
\hline 32.955 & 4.394 & 474.552 \\
\hline 8.788 & 2.197 & 6.591 \\
\hline 43.94 & 4.394 & 4.394 \\
\hline 2.197 & 2.197 & 2.197 \\
\hline 351.52 & 2.197 & 8.788 \\
\hline 322.959 & 2.197 & 887.588 \\
\hline 2.197 & 2.197 & 2.197 \\
\hline 37.349 & 2.197 & 2.197 \\
\hline 4.394 & 329.55 & 171.366 \\
\hline 219.7 & 2.197 & 68.107 \\
\hline 21.97 & 41.743 & 10.985 \\
\hline 2.197 & 32.955 & 21.97 \\
\hline 2.197 & 2.197 & 2.197 \\
\hline 83.486 & 68.107 & 6.591 \\
\hline 41.743 & 2.197 & 2.197 \\
\hline 17.576 & 2.197 & 2.197 \\
\hline 63.713 & 6.591 & 8.788 \\
\hline 4.394 & 2.197 & 6.591 \\
\hline 6.591 & 2.197 & 2.197 \\
\hline 2.197 & 39.546 & 94.471 \\
\hline 2.197 & 6.591 & 2.197 \\
\hline 50.531 & 2.197 & 2.197 \\
\hline 4.394 & 13.182 & 4.394 \\
\hline 4.394 & 90.077 & 4.394 \\
\hline 17.576 & 109.85 & 2.197 \\
\hline 59.319 & 15.379 & 155.987 \\
\hline 6.591 & 26.364 & 2.197 \\
\hline 8.788 & 6.591 & 4.394 \\
\hline 65.91 & 2.197 & 2.197 \\
\hline 120.835 & 19.773 & 13.182 \\
\hline 68.107 & 2.197 & 2.197 \\
\hline 131.82 & 79.092 & 6.591 \\
\hline 13.182 & 30.758 & 2.197 \\
\hline 8.788 & 2.197 & 2.197 \\
\hline 2.197 & 6.591 & 2.197 \\
\hline 10.985 & 4.394 & 129.623 \\
\hline 52.728 & 24.167 & 4.394 \\
\hline 8.788 & 2.197 & 4.394 \\
\hline 4.394 & 4.394 & 52.728 \\
\hline 19.773 & 191.139 & 10.985 \\
\hline
\end{tabular}




\begin{tabular}{|c|c|c|}
\hline 129.623 & 2.197 & 10.985 \\
\hline 13.182 & 2.197 & 2.197 \\
\hline 57.122 & 6.591 & 76.895 \\
\hline 17.576 & 15.379 & 2.197 \\
\hline 6.591 & 2.197 & 30.758 \\
\hline 4.394 & 2.197 & 2.197 \\
\hline 109.85 & 2.197 & 13.182 \\
\hline 30.758 & 61.516 & 10.985 \\
\hline 32.955 & 2.197 & 6.591 \\
\hline 6.591 & 2.197 & 2.197 \\
\hline 4.394 & 2.197 & 10.985 \\
\hline 6.591 & 10.985 & 6.591 \\
\hline 28.561 & 4.394 & 32.955 \\
\hline 788.723 & 2.197 & 2216.77 \\
\hline 32.955 & 2.197 & 217.503 \\
\hline 35.152 & 6.591 & 6.591 \\
\hline 118.638 & 8.788 & 4.394 \\
\hline 8.788 & 2.197 & 30.758 \\
\hline 41.743 & 2.197 & 114.244 \\
\hline 162.578 & 2.197 & 8.788 \\
\hline 4.394 & 15.379 & 2.197 \\
\hline 2.197 & 4.394 & 4.394 \\
\hline 4.394 & 2.197 & 2.197 \\
\hline 15.379 & 52.728 & 2.197 \\
\hline 13.182 & 4.394 & 4.394 \\
\hline 19.773 & 2.197 & 4.394 \\
\hline 155.987 & 85.683 & 19.773 \\
\hline 30.758 & 2.197 & 26.364 \\
\hline 19.773 & 2.197 & 15.379 \\
\hline 2.197 & 4.394 & 2.197 \\
\hline 2.197 & 6.591 & 2.197 \\
\hline 2.197 & 30.758 & 2.197 \\
\hline 21.97 & 15.379 & 8.788 \\
\hline 70.304 & 8.788 & 4.394 \\
\hline 72.501 & 2.197 & 6.591 \\
\hline 15.379 & 2.197 & 26.364 \\
\hline 8.788 & 2.197 & 2.197 \\
\hline 10.985 & 2.197 & 4.394 \\
\hline 6.591 & 8.788 & 43.94 \\
\hline 2.197 & 26.364 & 2.197 \\
\hline 114.244 & 2.197 & 26.364 \\
\hline 2.197 & 2.197 & 4.394 \\
\hline 4.394 & 13.182 & 24.167 \\
\hline 109.85 & 2.197 & 4.394 \\
\hline 6.591 & 13.182 & 4.394 \\
\hline 8.788 & 188.942 & 13.182 \\
\hline 32.955 & 17.576 & 8.788 \\
\hline 50.531 & 2.197 & 2.197 \\
\hline 10.985 & 15.379 & 467.961 \\
\hline 2.197 & 2.197 & 2.197 \\
\hline
\end{tabular}




\begin{tabular}{|c|c|c|}
\hline 338.338 & 2.197 & 441.597 \\
\hline 57.122 & 4.394 & 17.576 \\
\hline 21.97 & 2.197 & 4.394 \\
\hline 2.197 & 2.197 & 6.591 \\
\hline 74.698 & 48.334 & 241.67 \\
\hline 43.94 & 4.394 & 2.197 \\
\hline 2.197 & 13.182 & 4.394 \\
\hline 123.032 & 19.773 & 48.334 \\
\hline 4.394 & 2.197 & 13.182 \\
\hline 8.788 & 39.546 & 19.773 \\
\hline 4.394 & 8.788 & 68.107 \\
\hline 4.394 & 2153.06 & 8.788 \\
\hline 2.197 & 2.197 & 162.578 \\
\hline 342.732 & 4.394 & 8.788 \\
\hline 2.197 & 37.349 & 85.683 \\
\hline 4.394 & 4.394 & 2.197 \\
\hline 6.591 & 85.683 & 8.788 \\
\hline 8.788 & 2.197 & 127.426 \\
\hline 21.97 & 4.394 & 6.591 \\
\hline 83.486 & 8.788 & 2.197 \\
\hline 2.197 & 10.985 & 26.364 \\
\hline 2.197 & 8.788 & 2.197 \\
\hline 2.197 & 61.516 & 2.197 \\
\hline 1272.06 & 2.197 & 37.349 \\
\hline 162.578 & 13.182 & 6.591 \\
\hline 4.394 & 2.197 & 15.379 \\
\hline 2.197 & 2.197 & 26.364 \\
\hline 114.244 & 103.259 & 2221.17 \\
\hline 8.788 & 2.197 & 13.182 \\
\hline 17.576 & 2.197 & 2.197 \\
\hline 6.591 & 2.197 & 2.197 \\
\hline 103.259 & 10.985 & 4.394 \\
\hline 43.94 & 2.197 & 4.394 \\
\hline 140.608 & 2.197 & 24.167 \\
\hline 26.364 & 2.197 & 39.546 \\
\hline 2.197 & 2.197 & 13.182 \\
\hline 2.197 & 2.197 & 975.468 \\
\hline 6.591 & 2.197 & 4.394 \\
\hline 131.82 & 171.366 & 92.274 \\
\hline 30.758 & 2.197 & 4.394 \\
\hline 10.985 & 2.197 & 2.197 \\
\hline 2.197 & 2.197 & 2.197 \\
\hline 160.381 & 4.394 & 2.197 \\
\hline 6.591 & 2.197 & 547.053 \\
\hline 6.591 & 2.197 & 2.197 \\
\hline 2.197 & 8.788 & 4.394 \\
\hline 39.546 & 2.197 & 54.925 \\
\hline 10.985 & 6.591 & 63.713 \\
\hline 134.017 & 4.394 & 2.197 \\
\hline 2.197 & 2.197 & 21.97 \\
\hline
\end{tabular}




\begin{tabular}{|c|c|c|}
\hline 41.743 & 8.788 & 6.591 \\
\hline 17.576 & 2.197 & 2.197 \\
\hline 2.197 & 2.197 & 235.079 \\
\hline 2.197 & 1447.82 & 393.263 \\
\hline 6.591 & 10.985 & 2.197 \\
\hline 6.591 & 2.197 & 13.182 \\
\hline 2.197 & 2.197 & 4.394 \\
\hline 2.197 & 2.197 & 2.197 \\
\hline 4.394 & 15.379 & 241.67 \\
\hline 8.788 & 4.394 & 107.653 \\
\hline 2.197 & 296.595 & 4.394 \\
\hline 237.276 & 2.197 & 17.576 \\
\hline 2.197 & 6.591 & 4.394 \\
\hline 2.197 & 10.985 & 274.625 \\
\hline 68.107 & 24.167 & 21.97 \\
\hline 2.197 & 4.394 & 2.197 \\
\hline 13.182 & 2.197 & 21.97 \\
\hline 6.591 & 6.591 & 2.197 \\
\hline 21.97 & 6.591 & 94.471 \\
\hline 2.197 & 2.197 & 4.394 \\
\hline 87.88 & 279.019 & 39.546 \\
\hline 13.182 & 2.197 & 46.137 \\
\hline 2.197 & 2.197 & 17.576 \\
\hline 2.197 & 2.197 & 2.197 \\
\hline 4.394 & 2.197 & 74.698 \\
\hline 10.985 & 13.182 & 4.394 \\
\hline 41.743 & 648.115 & 26.364 \\
\hline 175.76 & 2.197 & 41.743 \\
\hline 4.394 & 30.758 & 4.394 \\
\hline 4.394 & 2.197 & 50.531 \\
\hline 2.197 & 24.167 & 26.364 \\
\hline 2.197 & 74.698 & 4.394 \\
\hline 2.197 & 2.197 & 4.394 \\
\hline 2.197 & 32.955 & 8.788 \\
\hline 4.394 & 2.197 & 41.743 \\
\hline 2.197 & 46.137 & 6.591 \\
\hline 6.591 & 4.394 & 13.182 \\
\hline 2.197 & 28.561 & 2.197 \\
\hline 2.197 & 2.197 & 4.394 \\
\hline 4.394 & 2.197 & 843.648 \\
\hline 2.197 & 2.197 & 2.197 \\
\hline 21.97 & 43.94 & 6.591 \\
\hline 4.394 & 2.197 & 722.813 \\
\hline 15.379 & 13.182 & 4.394 \\
\hline 6.591 & 2073.97 & 2.197 \\
\hline 2.197 & 15.379 & 2.197 \\
\hline 4.394 & 59.319 & 13.182 \\
\hline 2.197 & 37.349 & 138.411 \\
\hline 81.289 & 2.197 & 2.197 \\
\hline 4.394 & 4.394 & 26.364 \\
\hline
\end{tabular}




\begin{tabular}{|c|c|c|}
\hline 46.137 & 4.394 & 2.197 \\
\hline 2.197 & 8.788 & 2.197 \\
\hline 4.394 & 2.197 & 2.197 \\
\hline 59.319 & 4.394 & 2.197 \\
\hline 2.197 & 2.197 & 30.758 \\
\hline 6.591 & 4.394 & 54.925 \\
\hline 101.062 & 4.394 & 4.394 \\
\hline 57.122 & 2.197 & 333.944 \\
\hline 2.197 & 2.197 & 13.182 \\
\hline 92.274 & 2.197 & 4.394 \\
\hline 21.97 & 2.197 & 6.591 \\
\hline 24.167 & 6.591 & 10.985 \\
\hline 2.197 & 6.591 & 15.379 \\
\hline 19.773 & 2.197 & 10.985 \\
\hline 39.546 & 2.197 & 30.758 \\
\hline 6.591 & 2.197 & 2.197 \\
\hline 4.394 & 4.394 & 4.394 \\
\hline 39.546 & 4.394 & 2.197 \\
\hline 2.197 & 2.197 & 2.197 \\
\hline 2.197 & 19.773 & 2.197 \\
\hline 2.197 & 2.197 & 6.591 \\
\hline 197.73 & 2.197 & 10.985 \\
\hline 138.411 & 90.077 & 8.788 \\
\hline 85.683 & 41.743 & 17.576 \\
\hline 57.122 & 15.379 & 6.591 \\
\hline 4.394 & 127.426 & 2.197 \\
\hline 10.985 & 79.092 & 37.349 \\
\hline 6.591 & 1214.94 & 182.351 \\
\hline 2.197 & 4.394 & 39.546 \\
\hline 96.668 & 10.985 & 8.788 \\
\hline 8.788 & 13.182 & 2.197 \\
\hline 2.197 & 2.197 & 39.546 \\
\hline 26.364 & 2.197 & 39.546 \\
\hline 123.032 & 2.197 & 13.182 \\
\hline 305.383 & 2.197 & 79.092 \\
\hline 6.591 & 4.394 & 61.516 \\
\hline 87.88 & 2.197 & 65.91 \\
\hline 2.197 & 2.197 & 4.394 \\
\hline 65.91 & 2.197 & 13.182 \\
\hline 21.97 & 2.197 & 13.182 \\
\hline 13.182 & 4.394 & 2.197 \\
\hline 2.197 & 2.197 & 61.516 \\
\hline 2.197 & 217.503 & 2.197 \\
\hline 8.788 & 2.197 & 4.394 \\
\hline 4.394 & 21.97 & 2.197 \\
\hline 6.591 & 2.197 & 2.197 \\
\hline 2.197 & 32.955 & 2.197 \\
\hline 8.788 & 13.182 & 8.788 \\
\hline 68.107 & 2.197 & 2.197 \\
\hline 8.788 & 2.197 & 2.197 \\
\hline
\end{tabular}




\begin{tabular}{|c|c|c|}
\hline 79.092 & 8.788 & 2.197 \\
\hline 4.394 & 4.394 & 2.197 \\
\hline 162.578 & 2.197 & 8.788 \\
\hline 2.197 & 2.197 & 2.197 \\
\hline 4.394 & 2.197 & 37.349 \\
\hline 2.197 & 21.97 & 4.394 \\
\hline 46.137 & 15.379 & 10.985 \\
\hline 24.167 & 6.591 & 2.197 \\
\hline 74.698 & 6.591 & 2.197 \\
\hline 2.197 & 6.591 & 2.197 \\
\hline 13.182 & 8.788 & 26.364 \\
\hline 13.182 & 17.576 & 2.197 \\
\hline 136.214 & 2.197 & 8.788 \\
\hline 46.137 & 2.197 & 536.068 \\
\hline 2.197 & 32.955 & 4.394 \\
\hline 2.197 & 2.197 & 2.197 \\
\hline 158.184 & 30.758 & 2.197 \\
\hline 13.182 & 19.773 & 5356.29 \\
\hline 8.788 & 2.197 & 4.394 \\
\hline 2.197 & 2.197 & 48.334 \\
\hline 2.197 & 92.274 & 191.139 \\
\hline 10.985 & 2.197 & 10.985 \\
\hline 10.985 & 2.197 & 10.985 \\
\hline 2.197 & 13.182 & 2.197 \\
\hline 2.197 & 4.394 & 19.773 \\
\hline 2.197 & 2.197 & 2.197 \\
\hline 101.062 & 28.561 & 2.197 \\
\hline 4.394 & 2.197 & 2.197 \\
\hline 4.394 & 4.394 & 32.955 \\
\hline 2.197 & 15.379 & 17.576 \\
\hline 13.182 & 6.591 & 41.743 \\
\hline 2.197 & 15.379 & 79.092 \\
\hline 8.788 & 39.546 & 39.546 \\
\hline 2.197 & 6.591 & 10.985 \\
\hline 15.379 & 15.379 & 13.182 \\
\hline 2.197 & 2.197 & 72.501 \\
\hline 184.548 & 26.364 & 8.788 \\
\hline 13.182 & 2.197 & 46.137 \\
\hline 459.173 & 43.94 & 13.182 \\
\hline 177.957 & 13.182 & 109.85 \\
\hline 19.773 & 116.441 & 138.411 \\
\hline 13.182 & 15.379 & 2.197 \\
\hline 2.197 & 8.788 & 4.394 \\
\hline 2.197 & 2.197 & 4.394 \\
\hline 4.394 & 6.591 & 4.394 \\
\hline 8.788 & 2.197 & 2.197 \\
\hline 96.668 & 17.576 & 265.837 \\
\hline 81.289 & 90.077 & 13.182 \\
\hline 4.394 & 2.197 & 6.591 \\
\hline 2.197 & 2.197 & 8.788 \\
\hline
\end{tabular}




\begin{tabular}{|c|c|c|}
\hline 6.591 & 2.197 & 30.758 \\
\hline 2.197 & 2.197 & 15.379 \\
\hline 6.591 & 10.985 & 2.197 \\
\hline 2.197 & 2.197 & 90.077 \\
\hline 2.197 & 4.394 & 15.379 \\
\hline 17.576 & 6.591 & 107.653 \\
\hline 4.394 & 10.985 & 32.955 \\
\hline 2.197 & 4.394 & 28.561 \\
\hline 4.394 & 2.197 & 13.182 \\
\hline 964.483 & 43.94 & 2.197 \\
\hline 83.486 & 10.985 & 15.379 \\
\hline 8.788 & 35.152 & 2.197 \\
\hline 32.955 & 2.197 & 35.152 \\
\hline 90.077 & 2.197 & 4.394 \\
\hline 8.788 & 17.576 & 32.955 \\
\hline 10.985 & 1500.55 & 149.396 \\
\hline 149.396 & 81.289 & 28.561 \\
\hline 19.773 & 21.97 & 2.197 \\
\hline 4.394 & 19.773 & 2.197 \\
\hline 83.486 & 2.197 & 8.788 \\
\hline 2.197 & 13.182 & 2.197 \\
\hline 4.394 & 4.394 & 2.197 \\
\hline 19.773 & 2.197 & 43.94 \\
\hline 6.591 & 2.197 & 50.531 \\
\hline 173.563 & 125.229 & 15.379 \\
\hline 2.197 & 296.595 & 342.732 \\
\hline 2.197 & 2.197 & 28.561 \\
\hline 94.471 & 15.379 & 13.182 \\
\hline 39.546 & 65.91 & 2.197 \\
\hline 123.032 & 61.516 & 10.985 \\
\hline 8.788 & 10.985 & 17.576 \\
\hline 13.182 & 32.955 & 41.743 \\
\hline 2.197 & 24.167 & 1285.24 \\
\hline 2.197 & 63.713 & 8.788 \\
\hline 21.97 & 2.197 & 57.122 \\
\hline 35.152 & 24.167 & 32.955 \\
\hline 21.97 & 8.788 & 2.197 \\
\hline 415.233 & 37.349 & 13.182 \\
\hline 208.715 & 2.197 & 2.197 \\
\hline 8.788 & 147.199 & 287.807 \\
\hline 48.334 & 2.197 & 2.197 \\
\hline 24.167 & 6.591 & 641.524 \\
\hline 83.486 & 2.197 & 4.394 \\
\hline 13.182 & 17.576 & 37.349 \\
\hline 6.591 & 2.197 & 2.197 \\
\hline 15.379 & 481.143 & 39.546 \\
\hline 19.773 & 8.788 & 735.995 \\
\hline 13.182 & 2.197 & 8.788 \\
\hline 6.591 & 48.334 & 46.137 \\
\hline 8.788 & 8.788 & 4.394 \\
\hline
\end{tabular}




\begin{tabular}{|c|c|c|}
\hline 28.561 & 6.591 & 10.985 \\
\hline 92.274 & 2.197 & 4.394 \\
\hline 8.788 & 2.197 & 2.197 \\
\hline 2.197 & 79.092 & 2.197 \\
\hline 2.197 & 17.576 & 2.197 \\
\hline 21.97 & 4.394 & 2.197 \\
\hline 2.197 & 6.591 & 2.197 \\
\hline 17.576 & 4.394 & 120.835 \\
\hline 17.576 & 2.197 & 15.379 \\
\hline 48.334 & 2.197 & 2.197 \\
\hline 2.197 & 2.197 & 2.197 \\
\hline 4.394 & 6.591 & 103.259 \\
\hline 10.985 & 43.94 & 6.591 \\
\hline 2.197 & 8.788 & 26.364 \\
\hline 2.197 & 10.985 & 79.092 \\
\hline 4.394 & 2.197 & 4.394 \\
\hline 32.955 & 50.531 & 142.805 \\
\hline 13.182 & 2.197 & 15.379 \\
\hline 4.394 & 2.197 & 8.788 \\
\hline 10.985 & 10.985 & 527.28 \\
\hline 2.197 & 2.197 & 2.197 \\
\hline 4.394 & 103.259 & 2.197 \\
\hline 2.197 & 2.197 & 2.197 \\
\hline 2.197 & 2.197 & 114.244 \\
\hline 2.197 & 2.197 & 2.197 \\
\hline 2.197 & 83.486 & 4.394 \\
\hline 4.394 & 2.197 & 6.591 \\
\hline 2.197 & 4.394 & 24.167 \\
\hline 2.197 & 2.197 & 2.197 \\
\hline 2.197 & 4.394 & 177.957 \\
\hline 32.955 & 2.197 & 10.985 \\
\hline 10.985 & 2.197 & 2.197 \\
\hline 164.775 & 10.985 & 6.591 \\
\hline 2.197 & 2.197 & 366.899 \\
\hline 50.531 & 81.289 & 2.197 \\
\hline 32.955 & 15.379 & 90.077 \\
\hline 24.167 & 2.197 & 10.985 \\
\hline 4.394 & 2.197 & 2.197 \\
\hline 52.728 & 4.394 & 2.197 \\
\hline 83.486 & 4.394 & 2.197 \\
\hline 136.214 & 2.197 & 149.396 \\
\hline 81.289 & 237.276 & 4.394 \\
\hline 4.394 & 35.152 & 2.197 \\
\hline 228.488 & 68.107 & 15.379 \\
\hline 6.591 & 15.379 & 82488.6 \\
\hline 153.79 & 2.197 & 13.182 \\
\hline 112.047 & 6.591 & 8.788 \\
\hline 6.591 & 63.713 & 57.122 \\
\hline 50.531 & 2.197 & 10.985 \\
\hline 26.364 & 8.788 & 4.394 \\
\hline
\end{tabular}




\begin{tabular}{|c|c|c|}
\hline 26.364 & 32.955 & 6.591 \\
\hline 2.197 & 109.85 & 8.788 \\
\hline 2.197 & 2.197 & 4.394 \\
\hline 46.137 & 4.394 & 96.668 \\
\hline 4.394 & 2.197 & 52.728 \\
\hline 2.197 & 2.197 & 30.758 \\
\hline 37.349 & 2.197 & 92.274 \\
\hline 2.197 & 2.197 & 39.546 \\
\hline 4.394 & 2.197 & 2.197 \\
\hline 4.394 & 13.182 & 4.394 \\
\hline 26.364 & 4.394 & 2.197 \\
\hline 232.882 & 17.576 & 96.668 \\
\hline 28.561 & 24.167 & 43.94 \\
\hline 2.197 & 4.394 & 92.274 \\
\hline 6.591 & 26.364 & 155.987 \\
\hline 2.197 & 4.394 & 2.197 \\
\hline 2.197 & 136.214 & 2.197 \\
\hline 8.788 & 4.394 & 41.743 \\
\hline 6.591 & 15.379 & 37.349 \\
\hline 32.955 & 15.379 & 272.428 \\
\hline 129.623 & 4.394 & 61.516 \\
\hline 21.97 & 364.702 & 4.394 \\
\hline 4.394 & 15.379 & 94.471 \\
\hline 15.379 & 2.197 & 2.197 \\
\hline 37.349 & 10.985 & 6.591 \\
\hline 13.182 & 2.197 & 15.379 \\
\hline 2.197 & 28.561 & 83.486 \\
\hline 4.394 & 8.788 & 13.182 \\
\hline 4.394 & 2.197 & 4330.29 \\
\hline 213.109 & 2.197 & 28.561 \\
\hline 13.182 & 114.244 & 1067.74 \\
\hline 24.167 & 4.394 & 8.788 \\
\hline 2.197 & 6.591 & 15.379 \\
\hline 169.169 & 15.379 & 109.85 \\
\hline 2.197 & 2.197 & 4.394 \\
\hline 799.708 & 13.182 & 4.394 \\
\hline 2.197 & 2.197 & 101.062 \\
\hline 15.379 & 17.576 & 59.319 \\
\hline 19.773 & 2.197 & 21.97 \\
\hline 2.197 & 114.244 & 17.576 \\
\hline 2.197 & 6.591 & 2.197 \\
\hline 41.743 & 118.638 & 6.591 \\
\hline 15.379 & 2.197 & 2172.83 \\
\hline 4.394 & 6.591 & 2.197 \\
\hline 10.985 & 2.197 & 4.394 \\
\hline 30.758 & 30.758 & 35.152 \\
\hline 24.167 & 10.985 & 13.182 \\
\hline 21.97 & 43.94 & 186.745 \\
\hline 81.289 & 4.394 & 2.197 \\
\hline 6.591 & 17.576 & 10.985 \\
\hline
\end{tabular}




\begin{tabular}{|c|c|c|}
\hline 2.197 & 6.591 & 4.394 \\
\hline 28.561 & 2.197 & 13.182 \\
\hline 30.758 & 2.197 & 527.28 \\
\hline 63.713 & 2.197 & 35.152 \\
\hline 2.197 & 2.197 & 129.623 \\
\hline 4.394 & 2.197 & 72.501 \\
\hline 2.197 & 2.197 & 10.985 \\
\hline 94.471 & 35.152 & 4.394 \\
\hline 26.364 & 2.197 & 4.394 \\
\hline 10.985 & 4.394 & 48.334 \\
\hline 8.788 & 2.197 & 10.985 \\
\hline 21.97 & 4.394 & 182.351 \\
\hline 19.773 & 32.955 & 219.7 \\
\hline 2.197 & 2.197 & 13.182 \\
\hline 6.591 & 2.197 & 2.197 \\
\hline 17.576 & 2.197 & 8.788 \\
\hline 28.561 & 13.182 & 26.364 \\
\hline 17.576 & 2.197 & 26.364 \\
\hline 2.197 & 30.758 & 6.591 \\
\hline 2.197 & 2.197 & 8.788 \\
\hline 2.197 & 6.591 & 96.668 \\
\hline 4.394 & 2.197 & 72.501 \\
\hline 10.985 & 2.197 & 2.197 \\
\hline 4.394 & 8.788 & 13.182 \\
\hline 2.197 & 10.985 & 2.197 \\
\hline 2.197 & 2.197 & 32.955 \\
\hline 63.713 & 4.394 & 6.591 \\
\hline 15.379 & 4.394 & 2.197 \\
\hline 4.394 & 8.788 & 26.364 \\
\hline 6.591 & 46.137 & 8.788 \\
\hline 39.546 & 30.758 & 6.591 \\
\hline 39.546 & 292.201 & 15.379 \\
\hline 109.85 & 8.788 & 940.316 \\
\hline 63.713 & 2.197 & 2.197 \\
\hline 17.576 & 4.394 & 2.197 \\
\hline 2.197 & 2.197 & 2.197 \\
\hline 2.197 & 2.197 & 4.394 \\
\hline 2.197 & 2.197 & 2.197 \\
\hline 8.788 & 52.728 & 13.182 \\
\hline 2.197 & 2.197 & 6.591 \\
\hline 2.197 & 10.985 & 41.743 \\
\hline 2.197 & 8.788 & 48.334 \\
\hline 2.197 & 2.197 & 2.197 \\
\hline 2.197 & 2.197 & 17.576 \\
\hline 1410.47 & 8.788 & 6.591 \\
\hline 24.167 & 2.197 & 2.197 \\
\hline 48.334 & 2.197 & 4.394 \\
\hline 32.955 & 2.197 & 4.394 \\
\hline 37.349 & 32.955 & 2.197 \\
\hline 41.743 & 2.197 & 2.197 \\
\hline
\end{tabular}




\begin{tabular}{|c|c|c|}
\hline 125.229 & 270.231 & 2.197 \\
\hline 4.394 & 239.473 & 158.184 \\
\hline 2.197 & 2.197 & 10.985 \\
\hline 8.788 & 13.182 & 17.576 \\
\hline 2.197 & 2.197 & 2.197 \\
\hline 2.197 & 674.479 & 4.394 \\
\hline 46.137 & 2.197 & 2.197 \\
\hline 6.591 & 131.82 & 2.197 \\
\hline 2.197 & 19.773 & 19.773 \\
\hline 2.197 & 17.576 & 2357.38 \\
\hline 2.197 & 10.985 & 19.773 \\
\hline 6.591 & 61.516 & 10.985 \\
\hline 48.334 & 2.197 & 74.698 \\
\hline 41.743 & 2.197 & 32.955 \\
\hline 13.182 & 39.546 & 8.788 \\
\hline 2.197 & 6.591 & 2.197 \\
\hline 6.591 & 2.197 & 8.788 \\
\hline 6.591 & 2.197 & 4.394 \\
\hline 19.773 & 2.197 & 2.197 \\
\hline 2.197 & 4.394 & 2.197 \\
\hline 24.167 & 2.197 & 21.97 \\
\hline 6.591 & 21.97 & 134.017 \\
\hline 37.349 & 347.126 & 4.394 \\
\hline 98.865 & 32.955 & 37.349 \\
\hline 10.985 & 2.197 & 2.197 \\
\hline 48.334 & 61.516 & 8.788 \\
\hline 4.394 & 6.591 & 26.364 \\
\hline 35.152 & 30.758 & 2.197 \\
\hline 6.591 & 10.985 & 131.82 \\
\hline 13.182 & 2.197 & 2.197 \\
\hline 2.197 & 6.591 & 28.561 \\
\hline 2.197 & 2.197 & 8.788 \\
\hline 2.197 & 4.394 & 4.394 \\
\hline 2.197 & 437.203 & 4.394 \\
\hline 81.289 & 4.394 & 17.576 \\
\hline 4.394 & 17.576 & 19.773 \\
\hline 2.197 & 10.985 & 4.394 \\
\hline 2.197 & 285.61 & 32.955 \\
\hline 4.394 & 4.394 & 4.394 \\
\hline 10.985 & 8.788 & 136.214 \\
\hline 4.394 & 2.197 & 8.788 \\
\hline 4.394 & 4.394 & 4.394 \\
\hline 6.591 & 21.97 & 8.788 \\
\hline 2.197 & 35.152 & 8.788 \\
\hline 94.471 & 2.197 & 4.394 \\
\hline 70.304 & 17.576 & 2.197 \\
\hline 2.197 & 2.197 & 54.925 \\
\hline 2.197 & 6.591 & 21.97 \\
\hline 2.197 & 2.197 & 2.197 \\
\hline 8.788 & 4.394 & 19.773 \\
\hline
\end{tabular}




\begin{tabular}{|c|c|c|}
\hline 15.379 & 10.985 & 19.773 \\
\hline 184.548 & 2.197 & 8.788 \\
\hline 24.167 & 2.197 & 2.197 \\
\hline 15.379 & 15.379 & 4.394 \\
\hline 4.394 & 2.197 & 4.394 \\
\hline 4.394 & 8.788 & 2.197 \\
\hline 364.702 & 4.394 & 19.773 \\
\hline 2.197 & 21.97 & 6.591 \\
\hline 6.591 & 2.197 & 2.197 \\
\hline 39.546 & 2.197 & 2.197 \\
\hline 2.197 & 15.379 & 2.197 \\
\hline 2.197 & 15.379 & 46.137 \\
\hline 6.591 & 8.788 & 2.197 \\
\hline 24.167 & 17.576 & 43.94 \\
\hline 2.197 & 1794.95 & 125.229 \\
\hline 6.591 & 2.197 & 8.788 \\
\hline 15.379 & 17.576 & 2.197 \\
\hline 98.865 & 2.197 & 10.985 \\
\hline 24.167 & 13.182 & 50.531 \\
\hline 118.638 & 406.445 & 129.623 \\
\hline 4.394 & 41.743 & 24.167 \\
\hline 2.197 & 2.197 & 4.394 \\
\hline 2.197 & 8.788 & 6.591 \\
\hline 2.197 & 2.197 & 166.972 \\
\hline 2.197 & 4.394 & 4.394 \\
\hline 2.197 & 4.394 & 4.394 \\
\hline 4.394 & 21.97 & 4.394 \\
\hline 4.394 & 48.334 & 2.197 \\
\hline 2.197 & 2.197 & 6.591 \\
\hline 17.576 & 694.252 & 13.182 \\
\hline 35.152 & 4.394 & 4.394 \\
\hline 101.062 & 2.197 & 4.394 \\
\hline 74.698 & 8.788 & 21.97 \\
\hline 2.197 & 92.274 & 2.197 \\
\hline 35.152 & 2.197 & 2.197 \\
\hline 94.471 & 68.107 & 26.364 \\
\hline 4.394 & 4.394 & 2.197 \\
\hline 54.925 & 87.88 & 184.548 \\
\hline 15.379 & 2.197 & 48.334 \\
\hline 24.167 & 2.197 & 274.625 \\
\hline 4.394 & 8.788 & 4.394 \\
\hline 70.304 & 2.197 & 61.516 \\
\hline 2.197 & 2.197 & 2.197 \\
\hline 17.576 & 2.197 & 6.591 \\
\hline 79.092 & 2.197 & 32.955 \\
\hline 30.758 & 4.394 & 8.788 \\
\hline 2.197 & 597.584 & 2.197 \\
\hline 46.137 & 2.197 & 17.576 \\
\hline 169.169 & 2.197 & 19.773 \\
\hline 4.394 & 2.197 & 2.197 \\
\hline
\end{tabular}




\begin{tabular}{|c|c|c|}
\hline 81.289 & 41.743 & 2.197 \\
\hline 129.623 & 57.122 & 2.197 \\
\hline 2.197 & 61.516 & 2.197 \\
\hline 103.259 & 430.612 & 8.788 \\
\hline 13.182 & 2.197 & 32.955 \\
\hline 164.775 & 15.379 & 421.824 \\
\hline 8.788 & 2.197 & 2.197 \\
\hline 2.197 & 39.546 & 30.758 \\
\hline 6.591 & 2.197 & 50.531 \\
\hline 768.95 & 6.591 & 13.182 \\
\hline 10.985 & 8.788 & 43.94 \\
\hline 4.394 & 8.788 & 6.591 \\
\hline 2.197 & 17.576 & 17.576 \\
\hline 4.394 & 136.214 & 68.107 \\
\hline 2.197 & 2.197 & 8.788 \\
\hline 2.197 & 4.394 & 19.773 \\
\hline 6.591 & 155.987 & 2.197 \\
\hline 2.197 & 199.927 & 4.394 \\
\hline 30.758 & 101.062 & 19.773 \\
\hline 32.955 & 2.197 & 15.379 \\
\hline 2.197 & 4.394 & 4.394 \\
\hline 2.197 & 2.197 & 72.501 \\
\hline 17.576 & 2.197 & 26.364 \\
\hline 43.94 & 388.869 & 6.591 \\
\hline 2.197 & 2.197 & 39.546 \\
\hline 4.394 & 2.197 & 107.653 \\
\hline 173.563 & 2.197 & 4.394 \\
\hline 4.394 & 6.591 & 17.576 \\
\hline 15.379 & 4.394 & 2.197 \\
\hline 17.576 & 2.197 & 540.462 \\
\hline 61.516 & 2.197 & 2.197 \\
\hline 4.394 & 2.197 & 10.985 \\
\hline 10.985 & 2.197 & 6.591 \\
\hline 17.576 & 2.197 & 13.182 \\
\hline 2.197 & 17.576 & 13.182 \\
\hline 28.561 & 21.97 & 107.653 \\
\hline 2.197 & 2.197 & 2.197 \\
\hline 6.591 & 2.197 & 2.197 \\
\hline 59.319 & 210.912 & 92.274 \\
\hline 2.197 & 8.788 & 13.182 \\
\hline 4.394 & 2.197 & 2.197 \\
\hline 21.97 & 155.987 & 13.182 \\
\hline 28.561 & 489.931 & 19.773 \\
\hline 8.788 & 2.197 & 2.197 \\
\hline 26.364 & 28.561 & 37.349 \\
\hline 8.788 & 17.576 & 2.197 \\
\hline 2.197 & 2.197 & 2.197 \\
\hline 6.591 & 6.591 & 2.197 \\
\hline 10.985 & 6.591 & 2.197 \\
\hline 6.591 & 6.591 & 2.197 \\
\hline
\end{tabular}




\begin{tabular}{|c|c|c|}
\hline 2.197 & 2.197 & 48.334 \\
\hline 52.728 & 4.394 & 2.197 \\
\hline 2.197 & 2.197 & 48.334 \\
\hline 19.773 & 70.304 & 217.503 \\
\hline 43.94 & 39.546 & 2.197 \\
\hline 2.197 & 1900.4 & 24.167 \\
\hline 8.788 & 2.197 & 43.94 \\
\hline 17.576 & 17.576 & 39.546 \\
\hline 2.197 & 2.197 & 175.76 \\
\hline 4.394 & 2.197 & 2.197 \\
\hline 46.137 & 224.094 & 79.092 \\
\hline 195.533 & 21.97 & 224.094 \\
\hline 26.364 & 4.394 & 10.985 \\
\hline 2.197 & 2.197 & 8.788 \\
\hline 2.197 & 4.394 & 65.91 \\
\hline 2.197 & 94.471 & 2.197 \\
\hline 2.197 & 2.197 & 35.152 \\
\hline 2.197 & 4.394 & 2.197 \\
\hline 2.197 & 2.197 & 21.97 \\
\hline 4.394 & 17.576 & 50.531 \\
\hline 39.546 & 15.379 & 28.561 \\
\hline 2.197 & 123.032 & 8.788 \\
\hline 2.197 & 26.364 & 57.122 \\
\hline 4.394 & 10.985 & 15.379 \\
\hline 142.805 & 8.788 & 4.394 \\
\hline 4.394 & 46.137 & 15.379 \\
\hline 186.745 & 2.197 & 6.591 \\
\hline 709.631 & 4.394 & 145.002 \\
\hline 96.668 & 4.394 & 6.591 \\
\hline 4.394 & 13.182 & 2.197 \\
\hline 15.379 & 76.895 & 8.788 \\
\hline 4.394 & 10.985 & 4.394 \\
\hline 98.865 & 32.955 & 2.197 \\
\hline 19.773 & 96.668 & 28.561 \\
\hline 465.764 & 6.591 & 4.394 \\
\hline 43.94 & 2.197 & 226.291 \\
\hline 4.394 & 2.197 & 105.456 \\
\hline 4.394 & 2.197 & 2.197 \\
\hline 6.591 & 2.197 & 79.092 \\
\hline 2.197 & 2.197 & 307.58 \\
\hline 39.546 & 2.197 & 2.197 \\
\hline 2.197 & 2.197 & 2.197 \\
\hline 2.197 & 2.197 & 2.197 \\
\hline 2.197 & 2.197 & 219.7 \\
\hline 30.758 & 101.062 & 17.576 \\
\hline 35.152 & 2.197 & 2.197 \\
\hline 2.197 & 2.197 & 4.394 \\
\hline 2.197 & 2.197 & 10.985 \\
\hline 2.197 & 2.197 & 2.197 \\
\hline 2.197 & 21.97 & 830.466 \\
\hline
\end{tabular}




\begin{tabular}{|c|c|c|}
\hline 2.197 & 2.197 & 41.743 \\
\hline 2.197 & 2.197 & 2.197 \\
\hline 2.197 & 2.197 & 63.713 \\
\hline 6.591 & 26.364 & 6.591 \\
\hline 41.743 & 6.591 & 46.137 \\
\hline 498.719 & 4.394 & 2.197 \\
\hline 184.548 & 35.152 & 2.197 \\
\hline 2.197 & 2.197 & 2.197 \\
\hline 19.773 & 19.773 & 2.197 \\
\hline 2.197 & 37.349 & 2.197 \\
\hline 15.379 & 19.773 & 65.91 \\
\hline 81.289 & 6.591 & 4.394 \\
\hline 134.017 & 2.197 & 46.137 \\
\hline 24.167 & 2.197 & 54.925 \\
\hline 2.197 & 2.197 & 35.152 \\
\hline 2.197 & 2.197 & 8.788 \\
\hline 2.197 & 24.167 & 2.197 \\
\hline 2.197 & 2.197 & 4.394 \\
\hline 114.244 & 863.421 & 28.561 \\
\hline 4.394 & 21.97 & 8.788 \\
\hline 41.743 & 15.379 & 17.576 \\
\hline 6.591 & 4.394 & 2.197 \\
\hline 52.728 & 17.576 & 10.985 \\
\hline 76.895 & 54.925 & 4.394 \\
\hline 6.591 & 94.471 & 237.276 \\
\hline 2.197 & 6.591 & 37.349 \\
\hline 2.197 & 52.728 & 90.077 \\
\hline 35.152 & 112.047 & 10.985 \\
\hline 2.197 & 2.197 & 24.167 \\
\hline 30.758 & 13.182 & 4.394 \\
\hline 2.197 & 2.197 & 48.334 \\
\hline 224.094 & 2.197 & 2.197 \\
\hline 6.591 & 28.561 & 2.197 \\
\hline 30.758 & 19.773 & 21.97 \\
\hline 2.197 & 2.197 & 19.773 \\
\hline 8.788 & 2.197 & 92.274 \\
\hline 529.477 & 4.394 & 6.591 \\
\hline 6.591 & 30.758 & 2.197 \\
\hline 2.197 & 4.394 & 35.152 \\
\hline 6.591 & 2.197 & 79.092 \\
\hline 2.197 & 2.197 & 386.672 \\
\hline 114.244 & 6.591 & 2.197 \\
\hline 6.591 & 8.788 & 2.197 \\
\hline 57.122 & 4.394 & 8.788 \\
\hline 41.743 & 153.79 & 19.773 \\
\hline 41.743 & 30.758 & 19.773 \\
\hline 2.197 & 10.985 & 4.394 \\
\hline 2.197 & 8.788 & 2.197 \\
\hline 2.197 & 2.197 & 2.197 \\
\hline 2.197 & 2.197 & 6.591 \\
\hline
\end{tabular}




\begin{tabular}{|c|c|c|}
\hline 2.197 & 2.197 & 24.167 \\
\hline 296.595 & 2.197 & 4.394 \\
\hline 28.561 & 2.197 & 15.379 \\
\hline 2.197 & 2.197 & 2.197 \\
\hline 50.531 & 2.197 & 6.591 \\
\hline 2.197 & 65.91 & 239.473 \\
\hline 2.197 & 2.197 & 10.985 \\
\hline 2.197 & 4.394 & 2.197 \\
\hline 21.97 & 2.197 & 68.107 \\
\hline 8.788 & 19.773 & 10.985 \\
\hline 32.955 & 8.788 & 2.197 \\
\hline 2.197 & 28.561 & 17.576 \\
\hline 2.197 & 13.182 & 4.394 \\
\hline 63.713 & 32.955 & 6.591 \\
\hline 10.985 & 101.062 & 2.197 \\
\hline 13.182 & 52.728 & 2.197 \\
\hline 4.394 & 15.379 & 2.197 \\
\hline 24.167 & 65.91 & 26.364 \\
\hline 4.394 & 2.197 & 2.197 \\
\hline 2.197 & 2.197 & 2.197 \\
\hline 6.591 & 10.985 & 17.576 \\
\hline 4.394 & 2.197 & 2.197 \\
\hline 2.197 & 463.567 & 2.197 \\
\hline 19.773 & 2.197 & 15.379 \\
\hline 2.197 & 123.032 & 2.197 \\
\hline 90.077 & 4.394 & 2.197 \\
\hline 28.561 & 15.379 & 2.197 \\
\hline 41.743 & 8.788 & 2581.47 \\
\hline 4.394 & 6.591 & 19.773 \\
\hline 15.379 & 2.197 & 131.82 \\
\hline 2.197 & 26.364 & 13.182 \\
\hline 2.197 & 4.394 & 441.597 \\
\hline 6.591 & 8.788 & 2.197 \\
\hline 2.197 & 2.197 & 2.197 \\
\hline 2.197 & 2.197 & 2.197 \\
\hline 8.788 & 43.94 & 26.364 \\
\hline 2.197 & 54.925 & 2.197 \\
\hline 13.182 & 2.197 & 2.197 \\
\hline 2.197 & 2.197 & 17.576 \\
\hline 2.197 & 2.197 & 28.561 \\
\hline 39.546 & 2.197 & 131.82 \\
\hline 15.379 & 2.197 & 15.379 \\
\hline 10.985 & 204.321 & 2.197 \\
\hline 15.379 & 6.591 & 43.94 \\
\hline 10.985 & 4.394 & 2.197 \\
\hline 158.184 & 4.394 & 395.46 \\
\hline 59.319 & 2.197 & 8.788 \\
\hline 24.167 & 70.304 & 2.197 \\
\hline 2.197 & 2.197 & 8.788 \\
\hline 24.167 & 2.197 & 8.788 \\
\hline
\end{tabular}




\begin{tabular}{|c|c|c|}
\hline 2.197 & 2.197 & 4319.3 \\
\hline 2.197 & 6.591 & 140.608 \\
\hline 6.591 & 2.197 & 4.394 \\
\hline 2.197 & 2.197 & 6.591 \\
\hline 935.922 & 2.197 & 395.46 \\
\hline 24.167 & 2.197 & 549.25 \\
\hline 2.197 & 2.197 & 2.197 \\
\hline 2.197 & 138.411 & 4.394 \\
\hline 2.197 & 50.531 & 35.152 \\
\hline 2.197 & 2.197 & 50.531 \\
\hline 10.985 & 2.197 & 206.518 \\
\hline 221.897 & 2.197 & 4.394 \\
\hline 10.985 & 6.591 & 8.788 \\
\hline 19.773 & 24.167 & 2.197 \\
\hline 10.985 & 26.364 & 2.197 \\
\hline 8.788 & 28.561 & 369.096 \\
\hline 2.197 & 2.197 & 54.925 \\
\hline 138.411 & 2.197 & 6.591 \\
\hline 28.561 & 4.394 & 4.394 \\
\hline 2.197 & 6.591 & 2.197 \\
\hline 13.182 & 2.197 & 13.182 \\
\hline 2.197 & 2.197 & 2.197 \\
\hline 6.591 & 6.591 & 4.394 \\
\hline 50.531 & 28.561 & 2.197 \\
\hline 2.197 & 43.94 & 4.394 \\
\hline 17.576 & 4.394 & 4.394 \\
\hline 2.197 & 8.788 & 92.274 \\
\hline 2.197 & 4.394 & 19.773 \\
\hline 30.758 & 13.182 & 2.197 \\
\hline 116.441 & 753.571 & 32.955 \\
\hline 52.728 & 4.394 & 2.197 \\
\hline 10.985 & 2.197 & 35.152 \\
\hline 74.698 & 4.394 & 30.758 \\
\hline 8.788 & 6.591 & 6.591 \\
\hline 13.182 & 2.197 & 28.561 \\
\hline 4.394 & 81.289 & 2.197 \\
\hline 2.197 & 35.152 & 4.394 \\
\hline 15.379 & 4.394 & 19.773 \\
\hline 19.773 & 4.394 & 2.197 \\
\hline 105.456 & 2.197 & 8.788 \\
\hline 2.197 & 2.197 & 39.546 \\
\hline 4.394 & 59.319 & 125.229 \\
\hline 10.985 & 2.197 & 35.152 \\
\hline 2.197 & 2.197 & 2.197 \\
\hline 151.593 & 8.788 & 4.394 \\
\hline 6.591 & 2.197 & 41.743 \\
\hline 120.835 & 4.394 & 6.591 \\
\hline 21.97 & 4.394 & 6.591 \\
\hline 4.394 & 6.591 & 2.197 \\
\hline 4.394 & 13.182 & 2.197 \\
\hline
\end{tabular}




\begin{tabular}{|c|c|c|}
\hline 2.197 & 2.197 & 19.773 \\
\hline 4.394 & 2.197 & 2.197 \\
\hline 21.97 & 2.197 & 63.713 \\
\hline 4.394 & 4.394 & 85.683 \\
\hline 39.546 & 6.591 & 10.985 \\
\hline 10.985 & 2.197 & 2.197 \\
\hline 15.379 & 4.394 & 39.546 \\
\hline 10.985 & 2.197 & 2.197 \\
\hline 8.788 & 2.197 & 1843.28 \\
\hline 30.758 & 21.97 & 4.394 \\
\hline 26.364 & 8.788 & 10.985 \\
\hline 17.576 & 6.591 & 63.713 \\
\hline 2.197 & 4.394 & 281.216 \\
\hline 2.197 & 96.668 & 173.563 \\
\hline 26.364 & 72.501 & 35.152 \\
\hline 2.197 & 107.653 & 8.788 \\
\hline 2.197 & 2.197 & 2.197 \\
\hline 2.197 & 26.364 & 4.394 \\
\hline 50.531 & 21.97 & 2.197 \\
\hline 10.985 & 26.364 & 17.576 \\
\hline 342.732 & 4.394 & 4.394 \\
\hline 57.122 & 4.394 & 2.197 \\
\hline 296.595 & 13.182 & 13.182 \\
\hline 4.394 & 8.788 & 10.985 \\
\hline 4.394 & 2.197 & 13.182 \\
\hline 10.985 & 32.955 & 59.319 \\
\hline 2.197 & 2.197 & 19.773 \\
\hline 26.364 & 13.182 & 2.197 \\
\hline 2.197 & 6.591 & 65.91 \\
\hline 8.788 & 2.197 & 2.197 \\
\hline 28.561 & 4.394 & 2.197 \\
\hline 2.197 & 8.788 & 2.197 \\
\hline 10.985 & 79.092 & 4.394 \\
\hline 13.182 & 6.591 & 2.197 \\
\hline 123.032 & 2.197 & 2.197 \\
\hline 21.97 & 4.394 & 28.561 \\
\hline 136.214 & 21.97 & 252.655 \\
\hline 2.197 & 239.473 & 142.805 \\
\hline 46.137 & 72.501 & 15.379 \\
\hline 2.197 & 2.197 & 134.017 \\
\hline 4.394 & 8.788 & 6.591 \\
\hline 294.398 & 8.788 & 2.197 \\
\hline 26.364 & 13.182 & 15.379 \\
\hline 68.107 & 2.197 & 90.077 \\
\hline 13.182 & 24.167 & 52.728 \\
\hline 13.182 & 26.364 & 2.197 \\
\hline 8.788 & 116.441 & 19.773 \\
\hline 28.561 & 2.197 & 2.197 \\
\hline 32.955 & 2.197 & 24.167 \\
\hline 15.379 & 294.398 & 28.561 \\
\hline
\end{tabular}




\begin{tabular}{|c|c|c|}
\hline 2.197 & 24.167 & 8.788 \\
\hline 13.182 & 4.394 & 87.88 \\
\hline 87.88 & 151.593 & 10.985 \\
\hline 4.394 & 2.197 & 17.576 \\
\hline 4.394 & 2.197 & 2.197 \\
\hline 2.197 & 2.197 & 131.82 \\
\hline 65.91 & 4.394 & 2.197 \\
\hline 2.197 & 2.197 & 4.394 \\
\hline 19.773 & 32.955 & 452.582 \\
\hline 4.394 & 30.758 & 10.985 \\
\hline 68.107 & 2.197 & 4.394 \\
\hline 6.591 & 2.197 & 8.788 \\
\hline 87.88 & 2.197 & 19.773 \\
\hline 15.379 & 2.197 & 4.394 \\
\hline 4.394 & 2.197 & 2.197 \\
\hline 6.591 & 26.364 & 2.197 \\
\hline 19.773 & 35.152 & 2.197 \\
\hline 17.576 & 2.197 & 2.197 \\
\hline 4.394 & 13.182 & 2.197 \\
\hline 4.394 & 21.97 & 6.591 \\
\hline 2.197 & 4.394 & 46.137 \\
\hline 63.713 & 4.394 & 4.394 \\
\hline 2.197 & 43.94 & 6.591 \\
\hline 2.197 & 26.364 & 6.591 \\
\hline 83.486 & 48.334 & 2.197 \\
\hline 2.197 & 72.501 & 4.394 \\
\hline 2.197 & 2.197 & 98.865 \\
\hline 87.88 & 10.985 & 54.925 \\
\hline 57.122 & 4.394 & 2.197 \\
\hline 13.182 & 4.394 & 2.197 \\
\hline 24.167 & 85.683 & 6.591 \\
\hline 6.591 & 19.773 & 19.773 \\
\hline 2.197 & 4.394 & 15.379 \\
\hline 4.394 & 2.197 & 43.94 \\
\hline 4.394 & 8.788 & 164.775 \\
\hline 26.364 & 6.591 & 4.394 \\
\hline 4.394 & 2.197 & 327.353 \\
\hline 96.668 & 4.394 & 6.591 \\
\hline 10.985 & 2.197 & 21.97 \\
\hline 32.955 & 4.394 & 1069.94 \\
\hline 6.591 & 103.259 & 4.394 \\
\hline 10.985 & 2.197 & 4.394 \\
\hline 101.062 & 2.197 & 2.197 \\
\hline 4.394 & 4.394 & 35.152 \\
\hline 2.197 & 6.591 & 26.364 \\
\hline 39.546 & 2.197 & 24.167 \\
\hline 2.197 & 2.197 & 52.728 \\
\hline 112.047 & 2.197 & 6.591 \\
\hline 4.394 & 2.197 & 24.167 \\
\hline 35.152 & 26.364 & 79.092 \\
\hline
\end{tabular}




\begin{tabular}{|c|c|c|}
\hline 4.394 & 6.591 & 63.713 \\
\hline 19.773 & 2.197 & 10.985 \\
\hline 19.773 & 41.743 & 35.152 \\
\hline 2.197 & 2.197 & 483.34 \\
\hline 2.197 & 17.576 & 6.591 \\
\hline 2.197 & 4.394 & 2.197 \\
\hline 10.985 & 2.197 & 239.473 \\
\hline 13.182 & 10.985 & 6.591 \\
\hline 4.394 & 2.197 & 147.199 \\
\hline 360.308 & 4.394 & 21.97 \\
\hline 26.364 & 46.137 & 10.985 \\
\hline 2.197 & 2.197 & 2.197 \\
\hline 2.197 & 6.591 & 2.197 \\
\hline 2.197 & 6.591 & 2.197 \\
\hline 59.319 & 65.91 & 21.97 \\
\hline 32.955 & 4.394 & 4.394 \\
\hline 4.394 & 2.197 & 2.197 \\
\hline 134.017 & 39.546 & 4.394 \\
\hline 4.394 & 15.379 & 2.197 \\
\hline 2.197 & 19.773 & 2.197 \\
\hline 2.197 & 17.576 & 6.591 \\
\hline 74.698 & 2.197 & 2.197 \\
\hline 26.364 & 2.197 & 2.197 \\
\hline 98.865 & 2.197 & 2.197 \\
\hline 380.081 & 2.197 & 2.197 \\
\hline 50.531 & 74.698 & 2.197 \\
\hline 4.394 & 2.197 & 19.773 \\
\hline 4.394 & 2.197 & 2.197 \\
\hline 4.394 & 248.261 & 2.197 \\
\hline 24.167 & 17.576 & 13.182 \\
\hline 6.591 & 8.788 & 2.197 \\
\hline 8.788 & 15.379 & 2.197 \\
\hline 199.927 & 4.394 & 4.394 \\
\hline 2.197 & 15.379 & 35.152 \\
\hline 386.672 & 28.561 & 19.773 \\
\hline 149.396 & 2.197 & 2.197 \\
\hline 8.788 & 2.197 & 37.349 \\
\hline 10.985 & 19.773 & 57.122 \\
\hline 2.197 & 61.516 & 4.394 \\
\hline 43.94 & 41.743 & 90.077 \\
\hline 90.077 & 2.197 & 85.683 \\
\hline 2.197 & 2.197 & 10.985 \\
\hline 2.197 & 8.788 & 6.591 \\
\hline 2.197 & 2.197 & 6.591 \\
\hline 2.197 & 2.197 & 59.319 \\
\hline 41.743 & 2.197 & 10.985 \\
\hline 59.319 & 6.591 & 268.034 \\
\hline 74.698 & 59.319 & 4.394 \\
\hline 2.197 & 13.182 & 147.199 \\
\hline 87.88 & 59.319 & 2.197 \\
\hline
\end{tabular}




\begin{tabular}{|c|c|c|}
\hline 61.516 & 2.197 & 2.197 \\
\hline 2.197 & 8.788 & 37.349 \\
\hline 30.758 & 4.394 & 17.576 \\
\hline 4.394 & 24.167 & 17.576 \\
\hline 4.394 & 21.97 & 2.197 \\
\hline 24.167 & 35.152 & 32.955 \\
\hline 158.184 & 2.197 & 13.182 \\
\hline 4.394 & 17.576 & 303.186 \\
\hline 4.394 & 6.591 & 2.197 \\
\hline 32.955 & 2.197 & 160.381 \\
\hline 2.197 & 4.394 & 13.182 \\
\hline 2.197 & 4.394 & 26.364 \\
\hline 15.379 & 4.394 & 155.987 \\
\hline 2.197 & 6.591 & 2.197 \\
\hline 30.758 & 2.197 & 2.197 \\
\hline 2.197 & 2.197 & 10.985 \\
\hline 26.364 & 76.895 & 43.94 \\
\hline 2.197 & 8.788 & 2.197 \\
\hline 202.124 & 2.197 & 2.197 \\
\hline 2.197 & 4.394 & 2.197 \\
\hline 6.591 & 2.197 & 2.197 \\
\hline 6.591 & 24.167 & 4.394 \\
\hline 4.394 & 6.591 & 2.197 \\
\hline 10.985 & 7109.49 & 21.97 \\
\hline 2.197 & 2.197 & 28.561 \\
\hline 59.319 & 2.197 & 61.516 \\
\hline 2.197 & 2.197 & 2.197 \\
\hline 61.516 & 8.788 & 2.197 \\
\hline 79.092 & 92.274 & 10.985 \\
\hline 2.197 & 2.197 & 35.152 \\
\hline 127.426 & 2.197 & 2.197 \\
\hline 4.394 & 10.985 & 2.197 \\
\hline 4.394 & 68.107 & 19.773 \\
\hline 63.713 & 10.985 & 32.955 \\
\hline 17.576 & 28.561 & 6.591 \\
\hline 4.394 & 2.197 & 87.88 \\
\hline 2.197 & 435.006 & 120.835 \\
\hline 2.197 & 46.137 & 162.578 \\
\hline 8.788 & 54.925 & 4.394 \\
\hline 15.379 & 2.197 & 4.394 \\
\hline 54.925 & 10.985 & 2.197 \\
\hline 79.092 & 2.197 & 2.197 \\
\hline 39.546 & 432.809 & 61.516 \\
\hline 2.197 & 39.546 & 30.758 \\
\hline 2.197 & 15.379 & 57.122 \\
\hline 4.394 & 39.546 & 160.381 \\
\hline 2.197 & 2.197 & 2.197 \\
\hline 4.394 & 2.197 & 6.591 \\
\hline 2.197 & 217.503 & 28.561 \\
\hline 61.516 & 15.379 & 2.197 \\
\hline
\end{tabular}




\begin{tabular}{|c|c|c|}
\hline 21.97 & 6.591 & 4.394 \\
\hline 8.788 & 10.985 & 30.758 \\
\hline 43.94 & 2.197 & 993.044 \\
\hline 2.197 & 4.394 & 408.642 \\
\hline 4.394 & 428.415 & 6.591 \\
\hline 147.199 & 10.985 & 788.723 \\
\hline 2.197 & 30.758 & 19.773 \\
\hline 6.591 & 2.197 & 2.197 \\
\hline 109.85 & 2.197 & 2.197 \\
\hline 2.197 & 2.197 & 4.394 \\
\hline 17.576 & 764.556 & 54.925 \\
\hline 15.379 & 21.97 & 1348.96 \\
\hline 2.197 & 4.394 & 54.925 \\
\hline 2.197 & 15.379 & 2.197 \\
\hline 28.561 & 6.591 & 13.182 \\
\hline 2.197 & 243.867 & 24.167 \\
\hline 19.773 & 2.197 & 10.985 \\
\hline 2.197 & 17.576 & 2.197 \\
\hline 2.197 & 10.985 & 15.379 \\
\hline 2.197 & 26.364 & 2.197 \\
\hline 19.773 & 4.394 & 4.394 \\
\hline 24.167 & 2.197 & 2.197 \\
\hline 17.576 & 2.197 & 351.52 \\
\hline 8.788 & 19.773 & 4.394 \\
\hline 19.773 & 2.197 & 2.197 \\
\hline 19.773 & 4.394 & 90.077 \\
\hline 2.197 & 2.197 & 2.197 \\
\hline 107.653 & 4.394 & 8.788 \\
\hline 149.396 & 4.394 & 140.608 \\
\hline 101.062 & 2.197 & 2.197 \\
\hline 6.591 & 4.394 & 2.197 \\
\hline 186.745 & 4.394 & 2.197 \\
\hline 4.394 & 129.623 & 10.985 \\
\hline 35.152 & 6.591 & 2.197 \\
\hline 28.561 & 26.364 & 2.197 \\
\hline 2.197 & 4.394 & 48.334 \\
\hline 722.813 & 2.197 & 46.137 \\
\hline 2.197 & 2.197 & 2.197 \\
\hline 10.985 & 48.334 & 145.002 \\
\hline 248.261 & 123.032 & 127.426 \\
\hline 4.394 & 4.394 & 2.197 \\
\hline 8.788 & 30.758 & 2.197 \\
\hline 13.182 & 2.197 & 30.758 \\
\hline 8.788 & 2.197 & 2.197 \\
\hline 2.197 & 2.197 & 30.758 \\
\hline 4.394 & 6.591 & 4.394 \\
\hline 15.379 & 2.197 & 2.197 \\
\hline 2.197 & 1370.93 & 2.197 \\
\hline 26.364 & 4.394 & 32.955 \\
\hline 61.516 & 6.591 & 4.394 \\
\hline
\end{tabular}




\begin{tabular}{|c|c|c|}
\hline 2.197 & 6.591 & 15.379 \\
\hline 15.379 & 2.197 & 52.728 \\
\hline 17.576 & 2.197 & 4.394 \\
\hline 19.773 & 6.591 & 6.591 \\
\hline 127.426 & 6.591 & 4.394 \\
\hline 6.591 & 10.985 & 4.394 \\
\hline 2.197 & 4.394 & 2.197 \\
\hline 193.336 & 8.788 & 2.197 \\
\hline 2.197 & 2.197 & 2.197 \\
\hline 76.895 & 4.394 & 15.379 \\
\hline 19.773 & 15.379 & 39.546 \\
\hline 94.471 & 2.197 & 46.137 \\
\hline 19.773 & 4.394 & 219.7 \\
\hline 6.591 & 2.197 & 85.683 \\
\hline 6.591 & 2.197 & 946.907 \\
\hline 24.167 & 2.197 & 15.379 \\
\hline 10.985 & 2.197 & 26.364 \\
\hline 2.197 & 2.197 & 2.197 \\
\hline 6.591 & 2.197 & 109.85 \\
\hline 13.182 & 10.985 & 2.197 \\
\hline 24.167 & 6.591 & 13.182 \\
\hline 2.197 & 2.197 & 50.531 \\
\hline 2.197 & 101.062 & 26.364 \\
\hline 32.955 & 17.576 & 41.743 \\
\hline 6.591 & 2.197 & 202.124 \\
\hline 94.471 & 2.197 & 30.758 \\
\hline 8.788 & 2.197 & 24.167 \\
\hline 13.182 & 2.197 & 2.197 \\
\hline 83.486 & 19.773 & 2.197 \\
\hline 6.591 & 8.788 & 2.197 \\
\hline 180.154 & 2.197 & 4.394 \\
\hline 2.197 & 116.441 & 24.167 \\
\hline 8.788 & 13.182 & 4.394 \\
\hline 2.197 & 2.197 & 2.197 \\
\hline 30.758 & 531.674 & 24.167 \\
\hline 17.576 & 2.197 & 6.591 \\
\hline 4.394 & 4.394 & 109.85 \\
\hline 21.97 & 2.197 & 2.197 \\
\hline 4.394 & 2.197 & 21.97 \\
\hline 2.197 & 2.197 & 2.197 \\
\hline 15.379 & 2.197 & 208.715 \\
\hline 6.591 & 2.197 & 2.197 \\
\hline 101.062 & 2.197 & 2.197 \\
\hline 43.94 & 487.734 & 2.197 \\
\hline 17.576 & 2.197 & 15.379 \\
\hline 15.379 & 4.394 & 558.038 \\
\hline 145.002 & 195.533 & 6.591 \\
\hline 2.197 & 4.394 & 4.394 \\
\hline 10.985 & 10.985 & 17.576 \\
\hline 2.197 & 48.334 & 8.788 \\
\hline
\end{tabular}




\begin{tabular}{|c|c|c|}
\hline 2.197 & 2.197 & 439.4 \\
\hline 107.653 & 2.197 & 4.394 \\
\hline 6.591 & 2.197 & 2.197 \\
\hline 4.394 & 2.197 & 127.426 \\
\hline 19.773 & 2.197 & 10.985 \\
\hline 19.773 & 10.985 & 4.394 \\
\hline 19.773 & 2.197 & 24.167 \\
\hline 13.182 & 4.394 & 2.197 \\
\hline 2.197 & 177.957 & 13.182 \\
\hline 2.197 & 19.773 & 48.334 \\
\hline 37.349 & 19.773 & 4.394 \\
\hline 2.197 & 4.394 & 46.137 \\
\hline 2.197 & 15.379 & 2.197 \\
\hline 43.94 & 30.758 & 2.197 \\
\hline 4.394 & 2.197 & 10.985 \\
\hline 153.79 & 2.197 & 13.182 \\
\hline 4.394 & 129.623 & 2.197 \\
\hline 87.88 & 2.197 & 63.713 \\
\hline 2.197 & 2.197 & 6.591 \\
\hline 4.394 & 6.591 & 21.97 \\
\hline 2.197 & 2.197 & 237.276 \\
\hline 2.197 & 13.182 & 26.364 \\
\hline 2.197 & 10.985 & 2.197 \\
\hline 2.197 & 8.788 & 2.197 \\
\hline 4.394 & 6.591 & 6.591 \\
\hline 8.788 & 10.985 & 8.788 \\
\hline 41.743 & 8.788 & 2.197 \\
\hline 166.972 & 4.394 & 2.197 \\
\hline 43.94 & 2.197 & 50.531 \\
\hline 10.985 & 19.773 & 551.447 \\
\hline 4.394 & 793.117 & 6.591 \\
\hline 26.364 & 8.788 & 2.197 \\
\hline 90.077 & 28.561 & 6.591 \\
\hline 2.197 & 6.591 & 13.182 \\
\hline 6.591 & 2.197 & 8.788 \\
\hline 59.319 & 2.197 & 6.591 \\
\hline 2.197 & 6.591 & 10.985 \\
\hline 8.788 & 46.137 & 2.197 \\
\hline 2.197 & 2.197 & 32.955 \\
\hline 2.197 & 6.591 & 2.197 \\
\hline 19.773 & 2.197 & 4.394 \\
\hline 2.197 & 4.394 & 2.197 \\
\hline 15.379 & 309.777 & 8.788 \\
\hline 13.182 & 2.197 & 166.972 \\
\hline 39.546 & 2.197 & 46.137 \\
\hline 4.394 & 26.364 & 2.197 \\
\hline 2.197 & 2.197 & 2.197 \\
\hline 92.274 & 2.197 & 2.197 \\
\hline 107.653 & 2.197 & 35.152 \\
\hline 21.97 & 2.197 & 52.728 \\
\hline
\end{tabular}




\begin{tabular}{|c|c|c|}
\hline 2.197 & 72.501 & 6.591 \\
\hline 10.985 & 4.394 & 54.925 \\
\hline 2.197 & 2.197 & 8.788 \\
\hline 59.319 & 90.077 & 6.591 \\
\hline 43.94 & 4.394 & 15.379 \\
\hline 87.88 & 2.197 & 10.985 \\
\hline 2.197 & 90.077 & 13.182 \\
\hline 4.394 & 10.985 & 17.576 \\
\hline 8.788 & 17.576 & 2.197 \\
\hline 19.773 & 6.591 & 4.394 \\
\hline 8.788 & 2.197 & 2.197 \\
\hline 37.349 & 4.394 & 6.591 \\
\hline 333.944 & 6.591 & 162.578 \\
\hline 8.788 & 4.394 & 2.197 \\
\hline 4.394 & 4.394 & 6.591 \\
\hline 6.591 & 2.197 & 13.182 \\
\hline 2.197 & 65.91 & 6.591 \\
\hline 19.773 & 2.197 & 8.788 \\
\hline 37.349 & 10.985 & 15.379 \\
\hline 4.394 & 2.197 & 32.955 \\
\hline 57.122 & 24.167 & 8.788 \\
\hline 634.933 & 2.197 & 13.182 \\
\hline 39.546 & 395.46 & 2.197 \\
\hline 8.788 & 2.197 & 8.788 \\
\hline 61.516 & 2.197 & 2.197 \\
\hline 94.471 & 28.561 & 19.773 \\
\hline 48.334 & 2.197 & 2.197 \\
\hline 2.197 & 2.197 & 28.561 \\
\hline 2.197 & 2.197 & 2.197 \\
\hline 4.394 & 2.197 & 6.591 \\
\hline 17.576 & 4.394 & 123.032 \\
\hline 17.576 & 6.591 & 2.197 \\
\hline 35.152 & 2.197 & 6.591 \\
\hline 8.788 & 8.788 & 2.197 \\
\hline 15.379 & 13.182 & 8.788 \\
\hline 399.854 & 2.197 & 2.197 \\
\hline 2.197 & 4.394 & 4.394 \\
\hline 2.197 & 2.197 & 2.197 \\
\hline 17.576 & 24.167 & 160.381 \\
\hline 2.197 & 2.197 & 2.197 \\
\hline 136.214 & 2.197 & 28.561 \\
\hline 13.182 & 10.985 & 17.576 \\
\hline 43.94 & 2.197 & 8.788 \\
\hline 2.197 & 2.197 & 101.062 \\
\hline 2.197 & 6.591 & 79.092 \\
\hline 4.394 & 2.197 & 4.394 \\
\hline 70.304 & 32.955 & 19.773 \\
\hline 125.229 & 54.925 & 26.364 \\
\hline 6.591 & 2.197 & 2.197 \\
\hline 2.197 & 2.197 & 2.197 \\
\hline
\end{tabular}




\begin{tabular}{|c|c|c|}
\hline 15.379 & 2.197 & 13.182 \\
\hline 2.197 & 6.591 & 4.394 \\
\hline 2.197 & 2.197 & 2.197 \\
\hline 2.197 & 57.122 & 123.032 \\
\hline 15.379 & 8.788 & 2.197 \\
\hline 8.788 & 4.394 & 142.805 \\
\hline 2.197 & 2.197 & 57.122 \\
\hline 217.503 & 2.197 & 46.137 \\
\hline 2.197 & 2.197 & 2.197 \\
\hline 221.897 & 4.394 & 94.471 \\
\hline 15.379 & 41.743 & 2.197 \\
\hline 2.197 & 2.197 & 2.197 \\
\hline 81.289 & 6.591 & 2.197 \\
\hline 2.197 & 4.394 & 2.197 \\
\hline 2.197 & 2.197 & 2.197 \\
\hline 6.591 & 2.197 & 2.197 \\
\hline 15.379 & 48.334 & 4.394 \\
\hline 72.501 & 13.182 & 4.394 \\
\hline 331.747 & 2.197 & 28.561 \\
\hline 6.591 & 1502.75 & 2.197 \\
\hline 28.561 & 2.197 & 6.591 \\
\hline 8.788 & 2.197 & 2.197 \\
\hline 2.197 & 24.167 & 2.197 \\
\hline 2.197 & 35.152 & 2.197 \\
\hline 35.152 & 2.197 & 4.394 \\
\hline 94.471 & 4.394 & 169.169 \\
\hline 6.591 & 32.955 & 79.092 \\
\hline 72.501 & 19.773 & 103.259 \\
\hline 2.197 & 17.576 & 4.394 \\
\hline 30.758 & 17.576 & 87.88 \\
\hline 101.062 & 39.546 & 2.197 \\
\hline 4.394 & 2.197 & 2.197 \\
\hline 6.591 & 2.197 & 2.197 \\
\hline 59.319 & 2.197 & 32.955 \\
\hline 2.197 & 8.788 & 28.561 \\
\hline 4.394 & 2.197 & 15.379 \\
\hline 65.91 & 19.773 & 4.394 \\
\hline 41.743 & 6.591 & 10.985 \\
\hline 17.576 & 26.364 & 10.985 \\
\hline 10.985 & 2.197 & 2.197 \\
\hline 4.394 & 2.197 & 2.197 \\
\hline 28.561 & 13.182 & 2.197 \\
\hline 37.349 & 2.197 & 90.077 \\
\hline 101.062 & 85.683 & 2.197 \\
\hline 52.728 & 10.985 & 136.214 \\
\hline 10.985 & 37.349 & 2.197 \\
\hline 674.479 & 21.97 & 382.278 \\
\hline 68.107 & 19.773 & 180.154 \\
\hline 2.197 & 2.197 & 21.97 \\
\hline 2.197 & 4.394 & 2.197 \\
\hline
\end{tabular}




\begin{tabular}{|c|c|c|}
\hline 2.197 & 10.985 & 6.591 \\
\hline 28.561 & 2.197 & 2.197 \\
\hline 10.985 & 2.197 & 6.591 \\
\hline 21.97 & 2.197 & 2.197 \\
\hline 90.077 & 13.182 & 13.182 \\
\hline 4.394 & 2.197 & 6.591 \\
\hline 2.197 & 10.985 & 19.773 \\
\hline 2.197 & 85.683 & 2.197 \\
\hline 46.137 & 2.197 & 50.531 \\
\hline 52.728 & 48.334 & 24.167 \\
\hline 15.379 & 37.349 & 2.197 \\
\hline 8.788 & 4.394 & 6.591 \\
\hline 4.394 & 32.955 & 103.259 \\
\hline 195.533 & 4.394 & 2.197 \\
\hline 4.394 & 48.334 & 4.394 \\
\hline 46.137 & 2.197 & 2.197 \\
\hline 445.991 & 65.91 & 6.591 \\
\hline 61.516 & 79.092 & 43.94 \\
\hline 57.122 & 263.64 & 68.107 \\
\hline 19.773 & 24.167 & 21.97 \\
\hline 43.94 & 2.197 & 2.197 \\
\hline 10.985 & 248.261 & 43.94 \\
\hline 37.349 & 19.773 & 24.167 \\
\hline 4.394 & 2.197 & 10.985 \\
\hline 2.197 & 52.728 & 74.698 \\
\hline 8.788 & 261.443 & 120.835 \\
\hline 8.788 & 10.985 & 13.182 \\
\hline 8.788 & 15.379 & 8.788 \\
\hline 103.259 & 2.197 & 8.788 \\
\hline 2.197 & 17.576 & 10.985 \\
\hline 13.182 & 2.197 & 2.197 \\
\hline 153.79 & 2.197 & 265.837 \\
\hline 10.985 & 2.197 & 13.182 \\
\hline 32.955 & 2.197 & 2.197 \\
\hline 2.197 & 17.576 & 48.334 \\
\hline 2.197 & 2.197 & 17.576 \\
\hline 149.396 & 4.394 & 4.394 \\
\hline 1658.73 & 1340.17 & 2.197 \\
\hline 17.576 & 2161.85 & 13.182 \\
\hline 2.197 & 2.197 & 8.788 \\
\hline 4.394 & 2.197 & 4.394 \\
\hline 17.576 & 26.364 & 2.197 \\
\hline 76.895 & 4.394 & 32.955 \\
\hline 13.182 & 2.197 & 4.394 \\
\hline 2.197 & 2.197 & 2.197 \\
\hline 2.197 & 15.379 & 2.197 \\
\hline 4.394 & 17.576 & 2.197 \\
\hline 30.758 & 15.379 & 32.955 \\
\hline 6.591 & 57.122 & 8.788 \\
\hline 191.139 & 140.608 & 24.167 \\
\hline
\end{tabular}




\begin{tabular}{|c|c|c|}
\hline 4.394 & 239.473 & 461.37 \\
\hline 2.197 & 43.94 & 6.591 \\
\hline 2.197 & 4.394 & 21.97 \\
\hline 6.591 & 2.197 & 226.291 \\
\hline 2.197 & 2.197 & 2.197 \\
\hline 2.197 & 2.197 & 26.364 \\
\hline 21.97 & 21.97 & 10.985 \\
\hline 221.897 & 2.197 & 46.137 \\
\hline 59.319 & 2.197 & 8.788 \\
\hline 6.591 & 2.197 & 79.092 \\
\hline 4.394 & 2.197 & 8.788 \\
\hline 112.047 & 184.548 & 8.788 \\
\hline 17.576 & 35.152 & 8.788 \\
\hline 2.197 & 408.642 & 57.122 \\
\hline 4.394 & 4.394 & 4.394 \\
\hline 2.197 & 331.747 & 35.152 \\
\hline 43.94 & 43.94 & 4.394 \\
\hline 4.394 & 15.379 & 2.197 \\
\hline 2.197 & 19.773 & 57.122 \\
\hline 10.985 & 13.182 & 6.591 \\
\hline 96.668 & 6.591 & 90.077 \\
\hline 8.788 & 2.197 & 2.197 \\
\hline 2.197 & 83.486 & 142.805 \\
\hline 6.591 & 4.394 & 2.197 \\
\hline 2.197 & 4.394 & 1041.38 \\
\hline 2.197 & 2.197 & 4.394 \\
\hline 2.197 & 2.197 & 21.97 \\
\hline 24.167 & 2.197 & 30.758 \\
\hline 252.655 & 2.197 & 4.394 \\
\hline 2.197 & 2.197 & 2.197 \\
\hline 4.394 & 2.197 & 127.426 \\
\hline 2.197 & 155.987 & 6.591 \\
\hline 41.743 & 35.152 & 6.591 \\
\hline 2.197 & 2.197 & 57.122 \\
\hline 15.379 & 8.788 & 15.379 \\
\hline 10.985 & 276.822 & 2.197 \\
\hline 43.94 & 2.197 & 2.197 \\
\hline 26.364 & 2.197 & 17.576 \\
\hline 8.788 & 41.743 & 2.197 \\
\hline 2.197 & 2.197 & 10.985 \\
\hline 4.394 & 6.591 & 4.394 \\
\hline 24.167 & 2.197 & 57.122 \\
\hline 2.197 & 2.197 & 37.349 \\
\hline 2.197 & 2.197 & 2.197 \\
\hline 2.197 & 101.062 & 4.394 \\
\hline 2.197 & 2.197 & 83.486 \\
\hline 2.197 & 6.591 & 10.985 \\
\hline 2.197 & 2.197 & 2.197 \\
\hline 6.591 & 2.197 & 4.394 \\
\hline 6.591 & 8.788 & 2.197 \\
\hline
\end{tabular}




\begin{tabular}{|c|c|c|}
\hline 4.394 & 15.379 & 239.473 \\
\hline 2.197 & 6.591 & 70.304 \\
\hline 41.743 & 2.197 & 162.578 \\
\hline 13.182 & 160.381 & 83.486 \\
\hline 8.788 & 63.713 & 4.394 \\
\hline 173.563 & 2.197 & 314.171 \\
\hline 2.197 & 17.576 & 8.788 \\
\hline 2.197 & 2.197 & 8.788 \\
\hline 228.488 & 2.197 & 8.788 \\
\hline 30.758 & 2.197 & 338.338 \\
\hline 2.197 & 2.197 & 129.623 \\
\hline 2.197 & 2.197 & 2.197 \\
\hline 4.394 & 10.985 & 4.394 \\
\hline 2.197 & 2.197 & 30.758 \\
\hline 10.985 & 2.197 & 2.197 \\
\hline 2.197 & 26.364 & 6.591 \\
\hline 39.546 & 50.531 & 3460.27 \\
\hline 4.394 & 2.197 & 815.087 \\
\hline 2.197 & 24.167 & 2.197 \\
\hline 6.591 & 81.289 & 4.394 \\
\hline 2.197 & 2.197 & 6.591 \\
\hline 2.197 & 2.197 & 43.94 \\
\hline 63.713 & 2.197 & 4.394 \\
\hline 2.197 & 1181.99 & 15.379 \\
\hline 2.197 & 2.197 & 52.728 \\
\hline 10.985 & 46.137 & 48.334 \\
\hline 17.576 & 32.955 & 8.788 \\
\hline 2.197 & 76.895 & 8.788 \\
\hline 193.336 & 59.319 & 2.197 \\
\hline 2.197 & 2.197 & 39.546 \\
\hline 30.758 & 2.197 & 2.197 \\
\hline 2.197 & 2.197 & 19.773 \\
\hline 6.591 & 8.788 & 2.197 \\
\hline 13.182 & 2.197 & 19.773 \\
\hline 76.895 & 2.197 & 17.576 \\
\hline 19.773 & 2.197 & 2.197 \\
\hline 8.788 & 70.304 & 35.152 \\
\hline 85.683 & 39.546 & 2.197 \\
\hline 2.197 & 92.274 & 377.884 \\
\hline 2.197 & 57.122 & 15.379 \\
\hline 2.197 & 2.197 & 39.546 \\
\hline 2.197 & 2.197 & 360.308 \\
\hline 109.85 & 2.197 & 2.197 \\
\hline 2.197 & 2.197 & 2.197 \\
\hline 15.379 & 2.197 & 2.197 \\
\hline 57.122 & 2.197 & 54.925 \\
\hline 4.394 & 2.197 & 83.486 \\
\hline 96.668 & 2.197 & 2.197 \\
\hline 17.576 & 8.788 & 2.197 \\
\hline 2.197 & 2.197 & 118.638 \\
\hline
\end{tabular}




\begin{tabular}{|c|c|c|}
\hline 2.197 & 2.197 & 19.773 \\
\hline 2.197 & 4.394 & 8.788 \\
\hline 4.394 & 2.197 & 6.591 \\
\hline 2.197 & 8.788 & 2.197 \\
\hline 6.591 & 2.197 & 2.197 \\
\hline 10.985 & 26.364 & 35.152 \\
\hline 87.88 & 4.394 & 10.985 \\
\hline 24.167 & 2115.71 & 4.394 \\
\hline 2.197 & 1737.83 & 213.109 \\
\hline 8.788 & 85.683 & 26.364 \\
\hline 2.197 & 32.955 & 8.788 \\
\hline 4.394 & 2.197 & 52.728 \\
\hline 63.713 & 28.561 & 2.197 \\
\hline 160.381 & 8.788 & 4.394 \\
\hline 87.88 & 2.197 & 13.182 \\
\hline 138.411 & 221.897 & 74.698 \\
\hline 2.197 & 2.197 & 15.379 \\
\hline 8.788 & 2.197 & 10.985 \\
\hline 32.955 & 2.197 & 6.591 \\
\hline 2.197 & 1865.25 & 3341.64 \\
\hline 2.197 & 6.591 & 6.591 \\
\hline 37.349 & 6.591 & 667.888 \\
\hline 2.197 & 6.591 & 2.197 \\
\hline 8.788 & 61.516 & 2.197 \\
\hline 8.788 & 2.197 & 17.576 \\
\hline 6.591 & 4.394 & 2.197 \\
\hline 15.379 & 2.197 & 19.773 \\
\hline 46.137 & 19.773 & 2.197 \\
\hline 4.394 & 4.394 & 738.192 \\
\hline 68.107 & 19.773 & 2.197 \\
\hline 2.197 & 2.197 & 21.97 \\
\hline 52.728 & 32.955 & 46.137 \\
\hline 28.561 & 2.197 & 6.591 \\
\hline 13.182 & 2.197 & 10.985 \\
\hline 10.985 & 19.773 & 6.591 \\
\hline 17.576 & 2.197 & 4.394 \\
\hline 8.788 & 347.126 & 171.366 \\
\hline 6.591 & 4.394 & 13.182 \\
\hline 287.807 & 2.197 & 57.122 \\
\hline 2.197 & 2.197 & 57.122 \\
\hline 2.197 & 2.197 & 2.197 \\
\hline 2.197 & 24.167 & 2.197 \\
\hline 2.197 & 2.197 & 19.773 \\
\hline 2.197 & 2.197 & 10.985 \\
\hline 32.955 & 8.788 & 2.197 \\
\hline 2.197 & 2.197 & 4.394 \\
\hline 13.182 & 4.394 & 4.394 \\
\hline 19.773 & 4.394 & 2.197 \\
\hline 2.197 & 8.788 & 2.197 \\
\hline 46.137 & 4.394 & 6.591 \\
\hline
\end{tabular}




\begin{tabular}{|c|c|c|}
\hline 2.197 & 35.152 & 4.394 \\
\hline 202.124 & 4.394 & 92.274 \\
\hline 19.773 & 4.394 & 68.107 \\
\hline 30.758 & 2.197 & 134.017 \\
\hline 15.379 & 4.394 & 2.197 \\
\hline 2.197 & 2.197 & 2.197 \\
\hline 2.197 & 26.364 & 2.197 \\
\hline 2.197 & 2.197 & 4.394 \\
\hline 2.197 & 8.788 & 32.955 \\
\hline 4.394 & 8.788 & 2.197 \\
\hline 41.743 & 6.591 & 15.379 \\
\hline 13.182 & 10.985 & 2.197 \\
\hline 30.758 & 2.197 & 15.379 \\
\hline 6.591 & 2.197 & 123.032 \\
\hline 32.955 & 900.77 & 2.197 \\
\hline 2.197 & 217.503 & 39.546 \\
\hline 162.578 & 90.077 & 52.728 \\
\hline 39.546 & 2.197 & 4.394 \\
\hline 41.743 & 10.985 & 2.197 \\
\hline 10.985 & 4.394 & 21.97 \\
\hline 230.685 & 4.394 & 13.182 \\
\hline 4.394 & 74.698 & 8.788 \\
\hline 59.319 & 2.197 & 4.394 \\
\hline 6.591 & 8.788 & 2.197 \\
\hline 2.197 & 2.197 & 2.197 \\
\hline 39.546 & 8.788 & 15.379 \\
\hline 4.394 & 26.364 & 2.197 \\
\hline 2.197 & 13.182 & 90.077 \\
\hline 2.197 & 4.394 & 2.197 \\
\hline 2.197 & 164.775 & 2.197 \\
\hline 2.197 & 2.197 & 8.788 \\
\hline 2.197 & 2.197 & 17.576 \\
\hline 158.184 & 2.197 & 2.197 \\
\hline 2.197 & 2.197 & 35.152 \\
\hline 52.728 & 2.197 & 13.182 \\
\hline 4.394 & 443.794 & 186.745 \\
\hline 37.349 & 197.73 & 2.197 \\
\hline 32.955 & 24.167 & 2.197 \\
\hline 4.394 & 26.364 & 8.788 \\
\hline 6.591 & 19.773 & 2.197 \\
\hline 4.394 & 2.197 & 2.197 \\
\hline 28.561 & 125.229 & 21.97 \\
\hline 105.456 & 39.546 & 17.576 \\
\hline 72.501 & 305.383 & 59.319 \\
\hline 54.925 & 10.985 & 4.394 \\
\hline 4.394 & 2.197 & 2.197 \\
\hline 10.985 & 10.985 & 123.032 \\
\hline 59.319 & 2.197 & 1504.94 \\
\hline 2.197 & 2.197 & 2.197 \\
\hline 10.985 & 6.591 & 41.743 \\
\hline
\end{tabular}




\begin{tabular}{|c|c|c|}
\hline 2.197 & 2.197 & 2.197 \\
\hline 6.591 & 15.379 & 2.197 \\
\hline 30.758 & 2.197 & 140.608 \\
\hline 4.394 & 2.197 & 52.728 \\
\hline 2.197 & 8.788 & 2.197 \\
\hline 2.197 & 6.591 & 2.197 \\
\hline 2.197 & 2.197 & 2.197 \\
\hline 61.516 & 2.197 & 81.289 \\
\hline 21.97 & 2.197 & 648.115 \\
\hline 4.394 & 10.985 & 2.197 \\
\hline 15.379 & 2.197 & 24.167 \\
\hline 39.546 & 2.197 & 15.379 \\
\hline 2.197 & 2.197 & 50.531 \\
\hline 10.985 & 6.591 & 61.516 \\
\hline 6.591 & 2.197 & 120.835 \\
\hline 10.985 & 13.182 & 8.788 \\
\hline 305.383 & 4.394 & 2.197 \\
\hline 2.197 & 6.591 & 59.319 \\
\hline 6.591 & 4.394 & 10.985 \\
\hline 8.788 & 1164.41 & 13.182 \\
\hline 37.349 & 2.197 & 2.197 \\
\hline 8.788 & 46.137 & 19.773 \\
\hline 26.364 & 2.197 & 28.561 \\
\hline 2.197 & 4.394 & 6.591 \\
\hline 4.394 & 2.197 & 2.197 \\
\hline 59.319 & 68.107 & 37.349 \\
\hline 43.94 & 17.576 & 81.289 \\
\hline 2.197 & 48.334 & 2.197 \\
\hline 26.364 & 17.576 & 386.672 \\
\hline 2.197 & 140.608 & 41.743 \\
\hline 13.182 & 2.197 & 2.197 \\
\hline 8.788 & 2.197 & 2.197 \\
\hline 72.501 & 4.394 & 8.788 \\
\hline 4.394 & 6.591 & 2.197 \\
\hline 2.197 & 2.197 & 4.394 \\
\hline 2.197 & 2.197 & 2.197 \\
\hline 24.167 & 2.197 & 2.197 \\
\hline 2.197 & 2.197 & 8.788 \\
\hline 76.895 & 2.197 & 24.167 \\
\hline 109.85 & 4.394 & 17.576 \\
\hline 10.985 & 2.197 & 28.561 \\
\hline 2.197 & 2.197 & 1751.01 \\
\hline 279.019 & 28.561 & 17.576 \\
\hline 4.394 & 39.546 & 74.698 \\
\hline 13.182 & 2.197 & 6.591 \\
\hline 2.197 & 6.591 & 26.364 \\
\hline 2.197 & 2.197 & 114.244 \\
\hline 28.561 & 2.197 & 6.591 \\
\hline 2.197 & 21.97 & 32.955 \\
\hline 4.394 & 2.197 & 2.197 \\
\hline
\end{tabular}




\begin{tabular}{|c|c|c|}
\hline 15.379 & 2.197 & 8.788 \\
\hline 2.197 & 8.788 & 19.773 \\
\hline 2.197 & 202.124 & 2.197 \\
\hline 26.364 & 2.197 & 10.985 \\
\hline 6.591 & 32.955 & 2.197 \\
\hline 70.304 & 15.379 & 28.561 \\
\hline 15.379 & 2.197 & 527.28 \\
\hline 2.197 & 555.841 & 43.94 \\
\hline 8.788 & 2.197 & 208.715 \\
\hline 10.985 & 4.394 & 4.394 \\
\hline 155.987 & 2.197 & 4.394 \\
\hline 6.591 & 39.546 & 26.364 \\
\hline 61.516 & 85.683 & 2.197 \\
\hline 217.503 & 4.394 & 2326.62 \\
\hline 2.197 & 2.197 & 8.788 \\
\hline 81.289 & 2.197 & 83.486 \\
\hline 8.788 & 2.197 & 812.89 \\
\hline 10.985 & 4.394 & 4.394 \\
\hline 70.304 & 2.197 & 8.788 \\
\hline 4.394 & 24.167 & 2.197 \\
\hline 4.394 & 4.394 & 2.197 \\
\hline 210.912 & 290.004 & 15.379 \\
\hline 35.152 & 2.197 & 2.197 \\
\hline 13.182 & 15.379 & 2.197 \\
\hline 59.319 & 17.576 & 21.97 \\
\hline 2.197 & 2.197 & 6.591 \\
\hline 35.152 & 59.319 & 2.197 \\
\hline 4.394 & 347.126 & 129.623 \\
\hline 6.591 & 87.88 & 2.197 \\
\hline 4.394 & 52.728 & 6.591 \\
\hline 6.591 & 48.334 & 8838.53 \\
\hline 13.182 & 8.788 & 4.394 \\
\hline 151.593 & 30.758 & 41.743 \\
\hline 2.197 & 2.197 & 59.319 \\
\hline 2.197 & 134.017 & 15.379 \\
\hline 8.788 & 4.394 & 15.379 \\
\hline 6.591 & 6.591 & 2.197 \\
\hline 2.197 & 21.97 & 349.323 \\
\hline 2.197 & 4.394 & 10.985 \\
\hline 333.944 & 2.197 & 6.591 \\
\hline 2.197 & 21693.2 & 17.576 \\
\hline 68.107 & 17.576 & 2.197 \\
\hline 63.713 & 4.394 & 57.122 \\
\hline 8.788 & 2.197 & 19.773 \\
\hline 26.364 & 2.197 & 43.94 \\
\hline 8.788 & 2.197 & 6.591 \\
\hline 15.379 & 340.535 & 834.86 \\
\hline 2.197 & 2.197 & 158.184 \\
\hline 4.394 & 2.197 & 30.758 \\
\hline 2.197 & 2.197 & 65.91 \\
\hline
\end{tabular}




\begin{tabular}{|c|c|c|}
\hline 6.591 & 2.197 & 8.788 \\
\hline 4.394 & 46.137 & 32.955 \\
\hline 4.394 & 2.197 & 26.364 \\
\hline 8.788 & 2.197 & 2.197 \\
\hline 2.197 & 4.394 & 4.394 \\
\hline 224.094 & 2.197 & 2.197 \\
\hline 32.955 & 39.546 & 17.576 \\
\hline 10.985 & 2.197 & 2.197 \\
\hline 2.197 & 15.379 & 21.97 \\
\hline 10.985 & 2.197 & 83.486 \\
\hline 10.985 & 35.152 & 4.394 \\
\hline 41.743 & 4.394 & 2.197 \\
\hline 2.197 & 48.334 & 2432.08 \\
\hline 26.364 & 2.197 & 2.197 \\
\hline 21.97 & 296.595 & 39.546 \\
\hline 116.441 & 2.197 & 4.394 \\
\hline 8.788 & 2.197 & 4.394 \\
\hline 4.394 & 2.197 & 48.334 \\
\hline 4.394 & 2.197 & 210.912 \\
\hline 39.546 & 2.197 & 4.394 \\
\hline 46.137 & 2.197 & 21.97 \\
\hline 15.379 & 2.197 & 26.364 \\
\hline 164.775 & 2.197 & 37.349 \\
\hline 30.758 & 6.591 & 8.788 \\
\hline 4.394 & 6.591 & 226.291 \\
\hline 2.197 & 10.985 & 37.349 \\
\hline 28.561 & 2.197 & 136.214 \\
\hline 2.197 & 2.197 & 2.197 \\
\hline 4.394 & 63.713 & 2.197 \\
\hline 2.197 & 2.197 & 2.197 \\
\hline 13.182 & 2.197 & 10.985 \\
\hline 4.394 & 4.394 & 17.576 \\
\hline 2.197 & 8.788 & 57.122 \\
\hline 28.561 & 2.197 & 63.713 \\
\hline 17.576 & 2.197 & 580.008 \\
\hline 6.591 & 2.197 & 2.197 \\
\hline 6.591 & 2.197 & 2.197 \\
\hline 2.197 & 15.379 & 105.456 \\
\hline 19.773 & 2.197 & 2.197 \\
\hline 17.576 & 15.379 & 8.788 \\
\hline 2.197 & 2.197 & 173.563 \\
\hline 4.394 & 32.955 & 21.97 \\
\hline 2.197 & 2.197 & 1368.73 \\
\hline 35.152 & 2.197 & 50.531 \\
\hline 32.955 & 2.197 & 26.364 \\
\hline 2.197 & 2.197 & 162.578 \\
\hline 10.985 & 43.94 & 50.531 \\
\hline 15.379 & 2.197 & 2.197 \\
\hline 65.91 & 2.197 & 15.379 \\
\hline 6.591 & 17.576 & 125.229 \\
\hline
\end{tabular}




\begin{tabular}{|c|c|c|}
\hline 61.516 & 2.197 & 2.197 \\
\hline 4.394 & 2.197 & 15.379 \\
\hline 2.197 & 153.79 & 39.546 \\
\hline 2.197 & 26.364 & 10.985 \\
\hline 103.259 & 28.561 & 2.197 \\
\hline 4.394 & 2.197 & 2.197 \\
\hline 4.394 & 2.197 & 19.773 \\
\hline 2.197 & 2.197 & 2.197 \\
\hline 24.167 & 2.197 & 35.152 \\
\hline 2.197 & 2.197 & 61.516 \\
\hline 2.197 & 4.394 & 2.197 \\
\hline 2.197 & 2.197 & 8.788 \\
\hline 4.394 & 2.197 & 2.197 \\
\hline 373.49 & 52.728 & 4.394 \\
\hline 4.394 & 2.197 & 6.591 \\
\hline 13.182 & 2.197 & 2.197 \\
\hline 4.394 & 2.197 & 72.501 \\
\hline 37.349 & 735.995 & 2.197 \\
\hline 6.591 & 15.379 & 138.411 \\
\hline 2.197 & 68.107 & 10.985 \\
\hline 2.197 & 6.591 & 6.591 \\
\hline 24.167 & 2.197 & 54.925 \\
\hline 13.182 & 8.788 & 131.82 \\
\hline 149.396 & 2.197 & 863.421 \\
\hline 8.788 & 43.94 & 8.788 \\
\hline 101.062 & 2.197 & 2.197 \\
\hline 52.728 & 2.197 & 21.97 \\
\hline 2.197 & 8.788 & 8.788 \\
\hline 19.773 & 24.167 & 2.197 \\
\hline 2.197 & 10.985 & 43.94 \\
\hline 136.214 & 10.985 & 57.122 \\
\hline 17.576 & 2.197 & 2.197 \\
\hline 2.197 & 15.379 & 6.591 \\
\hline 2.197 & 303.186 & 57.122 \\
\hline 19.773 & 37.349 & 21.97 \\
\hline 2.197 & 4.394 & 4.394 \\
\hline 30.758 & 2.197 & 17.576 \\
\hline 52.728 & 2.197 & 76.895 \\
\hline 2.197 & 127.426 & 46.137 \\
\hline 13.182 & 2.197 & 2.197 \\
\hline 2.197 & 131.82 & 90.077 \\
\hline 4.394 & 6.591 & 191.139 \\
\hline 17.576 & 65.91 & 2.197 \\
\hline 149.396 & 10.985 & 2.197 \\
\hline 28.561 & 17.576 & 2.197 \\
\hline 2.197 & 2.197 & 6.591 \\
\hline 2.197 & 6.591 & 2.197 \\
\hline 2.197 & 6.591 & 59.319 \\
\hline 2.197 & 6.591 & 74.698 \\
\hline 2.197 & 6.591 & 43.94 \\
\hline
\end{tabular}




\begin{tabular}{|c|c|c|}
\hline 531.674 & 2.197 & 6.591 \\
\hline 2.197 & 2.197 & 2.197 \\
\hline 28.561 & 2.197 & 2.197 \\
\hline 153.79 & 2.197 & 417.43 \\
\hline 2.197 & 17.576 & 182.351 \\
\hline 10.985 & 435.006 & 2.197 \\
\hline 70.304 & 26.364 & 204.321 \\
\hline 41.743 & 19.773 & 2355.18 \\
\hline 19.773 & 263.64 & 24.167 \\
\hline 2.197 & 2.197 & 6.591 \\
\hline 6.591 & 21.97 & 83.486 \\
\hline 57.122 & 2.197 & 2.197 \\
\hline 68.107 & 13.182 & 35.152 \\
\hline 8.788 & 26.364 & 32.955 \\
\hline 125.229 & 2.197 & 26.364 \\
\hline 2.197 & 8.788 & 50.531 \\
\hline 4.394 & 2.197 & 2.197 \\
\hline 2.197 & 4.394 & 2.197 \\
\hline 2.197 & 2.197 & 6.591 \\
\hline 6.591 & 2.197 & 2.197 \\
\hline 13.182 & 2.197 & 2.197 \\
\hline 52.728 & 41.743 & 2.197 \\
\hline 98.865 & 19.773 & 2.197 \\
\hline 32.955 & 2.197 & 15.379 \\
\hline 158.184 & 2.197 & 13.182 \\
\hline 2.197 & 138.411 & 28.561 \\
\hline 54.925 & 2.197 & 2568.29 \\
\hline 2.197 & 2.197 & 15.379 \\
\hline 6.591 & 63.713 & 2.197 \\
\hline 96.668 & 15.379 & 52.728 \\
\hline 90.077 & 2.197 & 2.197 \\
\hline 6.591 & 2.197 & 8.788 \\
\hline 28.561 & 4.394 & 10.985 \\
\hline 4.394 & 4.394 & 4.394 \\
\hline 8.788 & 13.182 & 2.197 \\
\hline 17.576 & 24.167 & 41.743 \\
\hline 2.197 & 2.197 & 4.394 \\
\hline 2.197 & 48.334 & 72.501 \\
\hline 52.728 & 2.197 & 10.985 \\
\hline 268.034 & 4.394 & 4.394 \\
\hline 6.591 & 2.197 & 46.137 \\
\hline 19.773 & 4.394 & 37.349 \\
\hline 4.394 & 6.591 & 2.197 \\
\hline 112.047 & 2.197 & 57.122 \\
\hline 96.668 & 197.73 & 19.773 \\
\hline 2.197 & 81.289 & 2.197 \\
\hline 15.379 & 2.197 & 2.197 \\
\hline 21.97 & 2.197 & 39.546 \\
\hline 13.182 & 136.214 & 37.349 \\
\hline 10.985 & 2.197 & 8.788 \\
\hline
\end{tabular}




\begin{tabular}{|c|c|c|}
\hline 19.773 & 2.197 & 4.394 \\
\hline 6.591 & 4.394 & 6.591 \\
\hline 1006.23 & 8.788 & 2.197 \\
\hline 35.152 & 4.394 & 2.197 \\
\hline 6.591 & 2.197 & 19.773 \\
\hline 61.516 & 2.197 & 2.197 \\
\hline 10.985 & 4.394 & 158.184 \\
\hline 87.88 & 2.197 & 298.792 \\
\hline 131.82 & 2.197 & 151.593 \\
\hline 90.077 & 2.197 & 2.197 \\
\hline 15.379 & 2.197 & 52.728 \\
\hline 199.927 & 259.246 & 2.197 \\
\hline 270.231 & 2.197 & 59.319 \\
\hline 52.728 & 6.591 & 52.728 \\
\hline 28.561 & 4.394 & 6.591 \\
\hline 63.713 & 8.788 & 41.743 \\
\hline 2.197 & 4.394 & 4.394 \\
\hline 15.379 & 4.394 & 4.394 \\
\hline 4.394 & 2.197 & 23532.1 \\
\hline 285.61 & 239.473 & 87.88 \\
\hline 2.197 & 30.758 & 109.85 \\
\hline 70.304 & 46.137 & 26.364 \\
\hline 2.197 & 4.394 & 2.197 \\
\hline 2.197 & 21.97 & 2.197 \\
\hline 8.788 & 30.758 & 2.197 \\
\hline 2.197 & 2.197 & 2.197 \\
\hline 10.985 & 2.197 & 8.788 \\
\hline 6.591 & 2.197 & 19.773 \\
\hline 28.561 & 2.197 & 263.64 \\
\hline 4.394 & 4.394 & 17.576 \\
\hline 136.214 & 2.197 & 4.394 \\
\hline 6.591 & 2.197 & 2.197 \\
\hline 6.591 & 10.985 & 35.152 \\
\hline 145.002 & 37.349 & 87.88 \\
\hline 8.788 & 4.394 & 6.591 \\
\hline 2.197 & 4.394 & 1017.21 \\
\hline 8.788 & 54.925 & 4.394 \\
\hline 2.197 & 2.197 & 19.773 \\
\hline 8.788 & 8.788 & 15.379 \\
\hline 10.985 & 2.197 & 2.197 \\
\hline 10.985 & 2.197 & 24.167 \\
\hline 4.394 & 59.319 & 10.985 \\
\hline 4.394 & 2.197 & 13.182 \\
\hline 26.364 & 2.197 & 6.591 \\
\hline 2.197 & 19.773 & 10.985 \\
\hline 274.625 & 2.197 & 35.152 \\
\hline 2.197 & 103.259 & 8.788 \\
\hline 10.985 & 2.197 & 15.379 \\
\hline 2.197 & 52.728 & 8.788 \\
\hline 2.197 & 4.394 & 50.531 \\
\hline
\end{tabular}




\begin{tabular}{|c|c|c|}
\hline 2.197 & 17.576 & 1698.28 \\
\hline 6.591 & 10.985 & 2.197 \\
\hline 4.394 & 2.197 & 46.137 \\
\hline 10.985 & 2.197 & 4.394 \\
\hline 2.197 & 6.591 & 8.788 \\
\hline 59.319 & 21.97 & 8.788 \\
\hline 4.394 & 8.788 & 46.137 \\
\hline 17.576 & 338.338 & 28.561 \\
\hline 48.334 & 32.955 & 6.591 \\
\hline 32.955 & 2.197 & 147.199 \\
\hline 41.743 & 35.152 & 2.197 \\
\hline 32.955 & 134.017 & 2.197 \\
\hline 6.591 & 2.197 & 39.546 \\
\hline 37.349 & 2.197 & 8.788 \\
\hline 24.167 & 2.197 & 2.197 \\
\hline 8.788 & 2.197 & 2.197 \\
\hline 2.197 & 26.364 & 10.985 \\
\hline 2.197 & 4.394 & 2.197 \\
\hline 1129.26 & 2.197 & 28.561 \\
\hline 57.122 & 24.167 & 2.197 \\
\hline 2.197 & 2.197 & 32.955 \\
\hline 2.197 & 6.591 & 63.713 \\
\hline 4.394 & 4.394 & 10.985 \\
\hline 59.319 & 81.289 & 2.197 \\
\hline 30.758 & 2.197 & 2.197 \\
\hline 360.308 & 2.197 & 4.394 \\
\hline 2.197 & 2.197 & 54.925 \\
\hline 4.394 & 6.591 & 10.985 \\
\hline 2.197 & 94.471 & 10.985 \\
\hline 2.197 & 2.197 & 17.576 \\
\hline 37.349 & 2.197 & 4.394 \\
\hline 32.955 & 2.197 & 15.379 \\
\hline 4.394 & 2.197 & 8.788 \\
\hline 2.197 & 72.501 & 2.197 \\
\hline 2.197 & 2.197 & 4.394 \\
\hline 2.197 & 32.955 & 2.197 \\
\hline 4.394 & 5323.33 & 43.94 \\
\hline 37.349 & 4.394 & 2.197 \\
\hline 24.167 & 2.197 & 70.304 \\
\hline 19.773 & 2.197 & 4.394 \\
\hline 17.576 & 4.394 & 2.197 \\
\hline 2.197 & 8.788 & 6.591 \\
\hline 8.788 & 138.411 & 15.379 \\
\hline 15.379 & 2.197 & 79.092 \\
\hline 2.197 & 2.197 & 19.773 \\
\hline 8.788 & 2.197 & 2.197 \\
\hline 26.364 & 2.197 & 8.788 \\
\hline 4.394 & 4.394 & 4.394 \\
\hline 35.152 & 17.576 & 65.91 \\
\hline 134.017 & 63.713 & 4.394 \\
\hline
\end{tabular}




\begin{tabular}{|c|c|c|}
\hline 92.274 & 2.197 & 21.97 \\
\hline 19.773 & 2.197 & 169.169 \\
\hline 6.591 & 4.394 & 2.197 \\
\hline 2.197 & 2.197 & 6.591 \\
\hline 2.197 & 155.987 & 19.773 \\
\hline 4.394 & 2.197 & 2.197 \\
\hline 54.925 & 4.394 & 83.486 \\
\hline 13.182 & 2.197 & 6.591 \\
\hline 4.394 & 28.561 & 2.197 \\
\hline 2.197 & 8.788 & 8.788 \\
\hline 2.197 & 10.985 & 8.788 \\
\hline 2.197 & 6.591 & 2.197 \\
\hline 2.197 & 2.197 & 2.197 \\
\hline 21.97 & 6.591 & 24.167 \\
\hline 15.379 & 10.985 & 208.715 \\
\hline 4.394 & 6.591 & 19.773 \\
\hline 2.197 & 4.394 & 4.394 \\
\hline 19.773 & 2.197 & 41.743 \\
\hline 6.591 & 6.591 & 72.501 \\
\hline 17.576 & 395.46 & 2.197 \\
\hline 6.591 & 4.394 & 26.364 \\
\hline 2.197 & 4.394 & 147.199 \\
\hline 8.788 & 2.197 & 4.394 \\
\hline 287.807 & 6.591 & 2.197 \\
\hline 19.773 & 2.197 & 13.182 \\
\hline 2.197 & 2.197 & 2.197 \\
\hline 8.788 & 90.077 & 2.197 \\
\hline 4.394 & 2.197 & 4.394 \\
\hline 2.197 & 2.197 & 19.773 \\
\hline 2.197 & 76.895 & 2.197 \\
\hline 2.197 & 4.394 & 7871.85 \\
\hline 10.985 & 70.304 & 32.955 \\
\hline 39.546 & 4.394 & 2.197 \\
\hline 2.197 & 4.394 & 19.773 \\
\hline 6.591 & 4.394 & 37.349 \\
\hline 8.788 & 21.97 & 21.97 \\
\hline 2.197 & 2.197 & 2.197 \\
\hline 19.773 & 2.197 & 35.152 \\
\hline 10.985 & 2.197 & 2.197 \\
\hline 41.743 & 2.197 & 30.758 \\
\hline 2.197 & 6.591 & 4.394 \\
\hline 17.576 & 13.182 & 2.197 \\
\hline 26.364 & 13.182 & 2.197 \\
\hline 2.197 & 2.197 & 2.197 \\
\hline 2.197 & 2.197 & 2.197 \\
\hline 2.197 & 6.591 & 15.379 \\
\hline 4.394 & 4.394 & 28.561 \\
\hline 81.289 & 2.197 & 540.462 \\
\hline 4.394 & 2.197 & 90.077 \\
\hline 10.985 & 24.167 & 8.788 \\
\hline
\end{tabular}




\begin{tabular}{|c|c|c|}
\hline 17.576 & 2.197 & 13.182 \\
\hline 2.197 & 8.788 & 4.394 \\
\hline 4.394 & 116.441 & 2.197 \\
\hline 19.773 & 2.197 & 6.591 \\
\hline 70.304 & 15.379 & 4.394 \\
\hline 41.743 & 13.182 & 43.94 \\
\hline 2.197 & 2.197 & 2.197 \\
\hline 2.197 & 35.152 & 123.032 \\
\hline 8.788 & 2.197 & 10.985 \\
\hline 46.137 & 35.152 & 7316.01 \\
\hline 30.758 & 2.197 & 13.182 \\
\hline 2.197 & 13.182 & 50.531 \\
\hline 2.197 & 171.366 & 43.94 \\
\hline 17.576 & 2.197 & 577.811 \\
\hline 17.576 & 123.032 & 39.546 \\
\hline 6.591 & 4692.79 & 4.394 \\
\hline 2.197 & 452.582 & 1157.82 \\
\hline 2.197 & 2.197 & 206.518 \\
\hline 6.591 & 13.182 & 6.591 \\
\hline 8.788 & 2.197 & 2.197 \\
\hline 26.364 & 8.788 & 2.197 \\
\hline 2.197 & 6.591 & 81.289 \\
\hline 79.092 & 57.122 & 4.394 \\
\hline 39.546 & 2.197 & 6.591 \\
\hline 268.034 & 2.197 & 32.955 \\
\hline 2.197 & 2.197 & 13.182 \\
\hline 21.97 & 8.788 & 6.591 \\
\hline 4.394 & 21.97 & 2.197 \\
\hline 2.197 & 2.197 & 46.137 \\
\hline 2.197 & 2.197 & 15.379 \\
\hline 46.137 & 2.197 & 2.197 \\
\hline 46.137 & 2.197 & 2.197 \\
\hline 2.197 & 173.563 & 2.197 \\
\hline 4.394 & 81.289 & 4.394 \\
\hline 2.197 & 50.531 & 4.394 \\
\hline 15.379 & 24.167 & 2.197 \\
\hline 87.88 & 2.197 & 28.561 \\
\hline 8.788 & 270.231 & 2.197 \\
\hline 6.591 & 2.197 & 15.379 \\
\hline 28.561 & 2.197 & 4.394 \\
\hline 8.788 & 2.197 & 4.394 \\
\hline 8.788 & 4.394 & 2.197 \\
\hline 85.683 & 2.197 & 2.197 \\
\hline 217.503 & 4.394 & 2.197 \\
\hline 26.364 & 2.197 & 2.197 \\
\hline 2.197 & 21.97 & 2.197 \\
\hline 2.197 & 17.576 & 8.788 \\
\hline 21.97 & 101.062 & 2.197 \\
\hline 4.394 & 6.591 & 94.471 \\
\hline 15.379 & 2.197 & 13.182 \\
\hline
\end{tabular}




\begin{tabular}{|c|c|c|}
\hline 8.788 & 2.197 & 37.349 \\
\hline 6.591 & 8.788 & 1915.78 \\
\hline 2.197 & 8.788 & 8.788 \\
\hline 101.062 & 2.197 & 10.985 \\
\hline 2.197 & 4.394 & 10.985 \\
\hline 10.985 & 2.197 & 6.591 \\
\hline 39.546 & 2.197 & 15.379 \\
\hline 2.197 & 4.394 & 4.394 \\
\hline 8.788 & 4.394 & 2.197 \\
\hline 19.773 & 2.197 & 2.197 \\
\hline 10.985 & 6.591 & 6.591 \\
\hline 2.197 & 2.197 & 6.591 \\
\hline 10.985 & 4.394 & 32.955 \\
\hline 714.025 & 2.197 & 2.197 \\
\hline 10.985 & 2.197 & 2.197 \\
\hline 21.97 & 21.97 & 2.197 \\
\hline 15.379 & 2.197 & 2.197 \\
\hline 41.743 & 24.167 & 26.364 \\
\hline 6.591 & 2.197 & 2.197 \\
\hline 15.379 & 4.394 & 63.713 \\
\hline 118.638 & 63.713 & 4.394 \\
\hline 4.394 & 54.925 & 228.488 \\
\hline 4.394 & 13.182 & 61.516 \\
\hline 193.336 & 72.501 & 6.591 \\
\hline 2.197 & 2.197 & 10.985 \\
\hline 37.349 & 61.516 & 2.197 \\
\hline 17.576 & 4.394 & 24.167 \\
\hline 32.955 & 6.591 & 32.955 \\
\hline 28.561 & 4.394 & 608.569 \\
\hline 2.197 & 41.743 & 61.516 \\
\hline 175.76 & 30.758 & 6.591 \\
\hline 2.197 & 63.713 & 171.366 \\
\hline 8.788 & 17.576 & 6.591 \\
\hline 8.788 & 6.591 & 6.591 \\
\hline 2.197 & 17.576 & 6.591 \\
\hline 2.197 & 24.167 & 57.122 \\
\hline 4.394 & 10.985 & 57.122 \\
\hline 2.197 & 6.591 & 10.985 \\
\hline 85.683 & 2.197 & 4.394 \\
\hline 4.394 & 83.486 & 4.394 \\
\hline 39.546 & 8.788 & 2.197 \\
\hline 8.788 & 2.197 & 2.197 \\
\hline 21.97 & 2.197 & 28.561 \\
\hline 37.349 & 21.97 & 2.197 \\
\hline 26.364 & 101.062 & 2.197 \\
\hline 2.197 & 59.319 & 17.576 \\
\hline 72.501 & 2.197 & 125.229 \\
\hline 92.274 & 226.291 & 63.713 \\
\hline 2.197 & 2.197 & 17.576 \\
\hline 19.773 & 2.197 & 2.197 \\
\hline
\end{tabular}




\begin{tabular}{|c|c|c|}
\hline 2.197 & 2.197 & 2.197 \\
\hline 8.788 & 10.985 & 17.576 \\
\hline 2.197 & 2.197 & 30.758 \\
\hline 4.394 & 2.197 & 8.788 \\
\hline 2.197 & 2.197 & 21.97 \\
\hline 46.137 & 2.197 & 2.197 \\
\hline 160.381 & 26.364 & 6.591 \\
\hline 263.64 & 35.152 & 2.197 \\
\hline 2.197 & 4.394 & 28.561 \\
\hline 2.197 & 173.563 & 2.197 \\
\hline 59.319 & 4.394 & 4.394 \\
\hline 2.197 & 37.349 & 2.197 \\
\hline 19.773 & 6.591 & 2.197 \\
\hline 46.137 & 2.197 & 37.349 \\
\hline 35.152 & 2.197 & 41.743 \\
\hline 17.576 & 2.197 & 8.788 \\
\hline 105.456 & 13.182 & 28.561 \\
\hline 4.394 & 2.197 & 428.415 \\
\hline 2.197 & 21.97 & 8.788 \\
\hline 2.197 & 4.394 & 2.197 \\
\hline 6.591 & 4.394 & 6.591 \\
\hline 2.197 & 2.197 & 4.394 \\
\hline 2.197 & 4.394 & 2.197 \\
\hline 101.062 & 2.197 & 2.197 \\
\hline 4.394 & 2.197 & 6.591 \\
\hline 4.394 & 6.591 & 123.032 \\
\hline 2.197 & 8.788 & 37.349 \\
\hline 2.197 & 15.379 & 2.197 \\
\hline 21.97 & 90.077 & 2.197 \\
\hline 4.394 & 2.197 & 63.713 \\
\hline 112.047 & 8.788 & 26.364 \\
\hline 17.576 & 920.543 & 17.576 \\
\hline 2.197 & 4.394 & 30.758 \\
\hline 8.788 & 2.197 & 26.364 \\
\hline 46.137 & 2.197 & 2.197 \\
\hline 32.955 & 2.197 & 2.197 \\
\hline 166.972 & 2.197 & 2.197 \\
\hline 21.97 & 2.197 & 10.985 \\
\hline 6.591 & 28.561 & 2.197 \\
\hline 8.788 & 57.122 & 15.379 \\
\hline 2.197 & 4.394 & 43.94 \\
\hline 2.197 & 114.244 & 8.788 \\
\hline 2.197 & 2.197 & 2.197 \\
\hline 26.364 & 2.197 & 4.394 \\
\hline 13.182 & 10.985 & 4.394 \\
\hline 15.379 & 6.591 & 4.394 \\
\hline 6.591 & 17.576 & 287.807 \\
\hline 2.197 & 8.788 & 6.591 \\
\hline 30.758 & 2.197 & 6.591 \\
\hline 8.788 & 8.788 & 8.788 \\
\hline
\end{tabular}




\begin{tabular}{|c|c|c|}
\hline 4.394 & 35.152 & 61.516 \\
\hline 54.925 & 4.394 & 24.167 \\
\hline 2.197 & 2.197 & 2.197 \\
\hline 43.94 & 15.379 & 2.197 \\
\hline 10.985 & 2.197 & 81.289 \\
\hline 2.197 & 285.61 & 10.985 \\
\hline 2135.48 & 41.743 & 2.197 \\
\hline 2.197 & 2.197 & 2.197 \\
\hline 2.197 & 2.197 & 10.985 \\
\hline 2.197 & 6.591 & 2.197 \\
\hline 2.197 & 6.591 & 13.182 \\
\hline 59.319 & 8.788 & 2.197 \\
\hline 13.182 & 2.197 & 2.197 \\
\hline 4.394 & 10.985 & 1017.21 \\
\hline 39.546 & 2.197 & 2.197 \\
\hline 10.985 & 4.394 & 8.788 \\
\hline 41.743 & 2.197 & 6.591 \\
\hline 24.167 & 15.379 & 2.197 \\
\hline 274.625 & 8.788 & 2.197 \\
\hline 2.197 & 8.788 & 6.591 \\
\hline 59.319 & 61.516 & 1867.45 \\
\hline 276.822 & 2.197 & 136.214 \\
\hline 85.683 & 2.197 & 103.259 \\
\hline 8.788 & 2.197 & 4.394 \\
\hline 10.985 & 19.773 & 83.486 \\
\hline 2.197 & 35.152 & 32.955 \\
\hline 2.197 & 70.304 & 17.576 \\
\hline 28.561 & 4.394 & 123.032 \\
\hline 2.197 & 8.788 & 2.197 \\
\hline 37.349 & 32.955 & 6.591 \\
\hline 2.197 & 2.197 & 112.047 \\
\hline 4.394 & 4.394 & 180.154 \\
\hline 2.197 & 6.591 & 6.591 \\
\hline 2.197 & 2.197 & 2.197 \\
\hline 10.985 & 2.197 & 13.182 \\
\hline 10.985 & 2.197 & 4.394 \\
\hline 199.927 & 2.197 & 4.394 \\
\hline 4.394 & 2.197 & 4.394 \\
\hline 8.788 & 50.531 & 10.985 \\
\hline 4.394 & 2.197 & 24.167 \\
\hline 2.197 & 2.197 & 13.182 \\
\hline 2.197 & 2.197 & 57.122 \\
\hline 2.197 & 37.349 & 28.561 \\
\hline 17.576 & 92.274 & 2.197 \\
\hline 2.197 & 24.167 & 114.244 \\
\hline 13.182 & 19.773 & 43.94 \\
\hline 30.758 & 2.197 & 35.152 \\
\hline 2.197 & 2.197 & 43.94 \\
\hline 15.379 & 96.668 & 2.197 \\
\hline 10.985 & 63.713 & 6.591 \\
\hline
\end{tabular}




\begin{tabular}{|c|c|c|}
\hline 2.197 & 24.167 & 6.591 \\
\hline 8.788 & 6.591 & 2.197 \\
\hline 2.197 & 24.167 & 2.197 \\
\hline 32.955 & 2.197 & 2.197 \\
\hline 4.394 & 2.197 & 21.97 \\
\hline 19.773 & 4.394 & 43.94 \\
\hline 2.197 & 2.197 & 2.197 \\
\hline 85.683 & 2.197 & 65.91 \\
\hline 13.182 & 2.197 & 413.036 \\
\hline 4.394 & 2.197 & 37.349 \\
\hline 4.394 & 35.152 & 2.197 \\
\hline 160.381 & 2.197 & 79.092 \\
\hline 2.197 & 2.197 & 2.197 \\
\hline 2.197 & 6812.9 & 2.197 \\
\hline 32.955 & 191.139 & 39.546 \\
\hline 35.152 & 2.197 & 43.94 \\
\hline 39.546 & 4.394 & 2.197 \\
\hline 4.394 & 57.122 & 26.364 \\
\hline 17.576 & 2.197 & 2.197 \\
\hline 2.197 & 10.985 & 2.197 \\
\hline 2.197 & 52.728 & 4.394 \\
\hline 10.985 & 26.364 & 8.788 \\
\hline 8.788 & 10.985 & 30.758 \\
\hline 13.182 & 15.379 & 2.197 \\
\hline 6.591 & 276.822 & 41.743 \\
\hline 41.743 & 1667.52 & 39.546 \\
\hline 4.394 & 30.758 & 182.351 \\
\hline 63.713 & 2.197 & 2.197 \\
\hline 4.394 & 43.94 & 2.197 \\
\hline 6.591 & 2.197 & 2.197 \\
\hline 17.576 & 4.394 & 2.197 \\
\hline 32.955 & 4.394 & 19.773 \\
\hline 54.925 & 4.394 & 32.955 \\
\hline 87.88 & 24.167 & 10.985 \\
\hline 228.488 & 2.197 & 4.394 \\
\hline 2.197 & 2.197 & 6.591 \\
\hline 2.197 & 83.486 & 4.394 \\
\hline 2.197 & 2.197 & 2.197 \\
\hline 6.591 & 2.197 & 30.758 \\
\hline 2.197 & 2.197 & 17.576 \\
\hline 2.197 & 8.788 & 6.591 \\
\hline 2.197 & 2.197 & 28.561 \\
\hline 13.182 & 2.197 & 72.501 \\
\hline 28.561 & 10.985 & 37.349 \\
\hline 26.364 & 149.396 & 43.94 \\
\hline 8.788 & 2.197 & 10.985 \\
\hline 59.319 & 2.197 & 13.182 \\
\hline 2.197 & 6.591 & 28.561 \\
\hline 2.197 & 2.197 & 2.197 \\
\hline 21.97 & 4.394 & 13.182 \\
\hline
\end{tabular}




\begin{tabular}{|c|c|c|}
\hline 8.788 & 2.197 & 6.591 \\
\hline 32.955 & 2.197 & 2.197 \\
\hline 39.546 & 17.576 & 114.244 \\
\hline 158.184 & 65.91 & 2.197 \\
\hline 2.197 & 2.197 & 2.197 \\
\hline 28.561 & 26.364 & 6.591 \\
\hline 17.576 & 2.197 & 19.773 \\
\hline 32.955 & 2.197 & 2.197 \\
\hline 54.925 & 4.394 & 10.985 \\
\hline 28.561 & 13.182 & 15.379 \\
\hline 4.394 & 19.773 & 10.985 \\
\hline 2.197 & 17.576 & 10.985 \\
\hline 28.561 & 26.364 & 2.197 \\
\hline 2.197 & 2.197 & 6.591 \\
\hline 300.989 & 2.197 & 37.349 \\
\hline 28.561 & 8.788 & 103.259 \\
\hline 2.197 & 4.394 & 21.97 \\
\hline 6.591 & 2.197 & 2.197 \\
\hline 96.668 & 4.394 & 2.197 \\
\hline 2.197 & 13.182 & 2.197 \\
\hline 2.197 & 2.197 & 15.379 \\
\hline 4.394 & 2.197 & 4.394 \\
\hline 26.364 & 4.394 & 15.379 \\
\hline 13.182 & 4.394 & 19.773 \\
\hline 2.197 & 265.837 & 59.319 \\
\hline 4.394 & 2.197 & 2.197 \\
\hline 32.955 & 32.955 & 8.788 \\
\hline 4.394 & 59.319 & 81.289 \\
\hline 2.197 & 586.599 & 30.758 \\
\hline 4.394 & 4.394 & 219.7 \\
\hline 2.197 & 4.394 & 2.197 \\
\hline 68.107 & 21.97 & 35.152 \\
\hline 10.985 & 19.773 & 13.182 \\
\hline 2.197 & 21.97 & 6.591 \\
\hline 4.394 & 2.197 & 17.576 \\
\hline 2.197 & 2.197 & 2.197 \\
\hline 10.985 & 2.197 & 19.773 \\
\hline 48.334 & 4.394 & 50.531 \\
\hline 50.531 & 2.197 & 2.197 \\
\hline 160.381 & 2.197 & 4.394 \\
\hline 17.576 & 2.197 & 50.531 \\
\hline 235.079 & 2.197 & 15.379 \\
\hline 17.576 & 43.94 & 4.394 \\
\hline 131.82 & 8.788 & 26.364 \\
\hline 41.743 & 10.985 & 2.197 \\
\hline 98.865 & 160.381 & 4.394 \\
\hline 15.379 & 2.197 & 2.197 \\
\hline 347.126 & 21.97 & 85.683 \\
\hline 13.182 & 13.182 & 8.788 \\
\hline 8.788 & 4.394 & 2.197 \\
\hline
\end{tabular}




\begin{tabular}{|c|c|c|}
\hline 705.237 & 547.053 & 32.955 \\
\hline 2.197 & 2.197 & 2.197 \\
\hline 155.987 & 2.197 & 63.713 \\
\hline 2.197 & 4.394 & 43.94 \\
\hline 61.516 & 3023.07 & 19.773 \\
\hline 15.379 & 4.394 & 48.334 \\
\hline 2.197 & 2.197 & 26.364 \\
\hline 13.182 & 6.591 & 138.411 \\
\hline 2.197 & 4.394 & 865.618 \\
\hline 6.591 & 32.955 & 17.576 \\
\hline 2.197 & 83.486 & 2.197 \\
\hline 8.788 & 8.788 & 4.394 \\
\hline 8.788 & 296.595 & 2.197 \\
\hline 24.167 & 28.561 & 17.576 \\
\hline 57.122 & 2.197 & 6.591 \\
\hline 26.364 & 880.997 & 8.788 \\
\hline 26.364 & 39.546 & 59.319 \\
\hline 4.394 & 173.563 & 6.591 \\
\hline 10.985 & 4.394 & 32.955 \\
\hline 2.197 & 4.394 & 39.546 \\
\hline 8.788 & 4.394 & 21.97 \\
\hline 107.653 & 2.197 & 43.94 \\
\hline 35.152 & 2.197 & 630.539 \\
\hline 4.394 & 109.85 & 2.197 \\
\hline 101.062 & 2.197 & 2.197 \\
\hline 4.394 & 4.394 & 15.379 \\
\hline 4.394 & 19.773 & 6.591 \\
\hline 4.394 & 52.728 & 4.394 \\
\hline 2.197 & 43.94 & 2.197 \\
\hline 2.197 & 10.985 & 6.591 \\
\hline 193.336 & 2.197 & 4.394 \\
\hline 8.788 & 101.062 & 8.788 \\
\hline 13.182 & 2.197 & 17.576 \\
\hline 2.197 & 6.591 & 2.197 \\
\hline 43.94 & 4.394 & 241.67 \\
\hline 4.394 & 2.197 & 17.576 \\
\hline 37.349 & 2.197 & 175.76 \\
\hline 30.758 & 2.197 & 4.394 \\
\hline 4.394 & 2.197 & 4.394 \\
\hline 2.197 & 28.561 & 94.471 \\
\hline 41.743 & 2.197 & 470.158 \\
\hline 65.91 & 2.197 & 4.394 \\
\hline 8.788 & 384.475 & 98.865 \\
\hline 10.985 & 8.788 & 116.441 \\
\hline 6.591 & 4.394 & 6.591 \\
\hline 15.379 & 4.394 & 92.274 \\
\hline 65.91 & 2.197 & 30.758 \\
\hline 2.197 & 2.197 & 21.97 \\
\hline 2.197 & 6.591 & 19.773 \\
\hline 57.122 & 26.364 & 10.985 \\
\hline
\end{tabular}




\begin{tabular}{|c|c|c|}
\hline 4.394 & 2.197 & 13.182 \\
\hline 15.379 & 2.197 & 151.593 \\
\hline 2.197 & 15.379 & 30.758 \\
\hline 6.591 & 2.197 & 149.396 \\
\hline 2.197 & 6.591 & 10.985 \\
\hline 4.394 & 2.197 & 2.197 \\
\hline 17.576 & 26.364 & 2.197 \\
\hline 173.563 & 2.197 & 24.167 \\
\hline 2.197 & 26.364 & 4.394 \\
\hline 2.197 & 15.379 & 10.985 \\
\hline 2.197 & 2.197 & 13.182 \\
\hline 46.137 & 8.788 & 10.985 \\
\hline 8.788 & 6.591 & 21.97 \\
\hline 24.167 & 2.197 & 2.197 \\
\hline 2.197 & 123.032 & 182.351 \\
\hline 441.597 & 2.197 & 134.017 \\
\hline 35.152 & 10.985 & 68.107 \\
\hline 6.591 & 2.197 & 2.197 \\
\hline 15.379 & 30.758 & 2.197 \\
\hline 2.197 & 10.985 & 10.985 \\
\hline 30.758 & 6.591 & 149.396 \\
\hline 2.197 & 2.197 & 224.094 \\
\hline 140.608 & 15.379 & 450.385 \\
\hline 6.591 & 2.197 & 4.394 \\
\hline 2.197 & 2.197 & 2.197 \\
\hline 358.111 & 2.197 & 48.334 \\
\hline 6.591 & 2.197 & 35.152 \\
\hline 2.197 & 2.197 & 19.773 \\
\hline 6.591 & 2.197 & 8.788 \\
\hline 186.745 & 6.591 & 28.561 \\
\hline 8.788 & 28.561 & 8.788 \\
\hline 2.197 & 46.137 & 2.197 \\
\hline 4.394 & 17.576 & 2.197 \\
\hline 210.912 & 116.441 & 2.197 \\
\hline 2.197 & 2.197 & 2.197 \\
\hline 8.788 & 2.197 & 17.576 \\
\hline 2.197 & 24.167 & 35.152 \\
\hline 4.394 & 2.197 & 6.591 \\
\hline 2.197 & 2.197 & 4.394 \\
\hline 10.985 & 597.584 & 74.698 \\
\hline 2.197 & 4.394 & 65.91 \\
\hline 13.182 & 2.197 & 65.91 \\
\hline 2.197 & 10.985 & 4.394 \\
\hline 2.197 & 6.591 & 10.985 \\
\hline 10.985 & 13.182 & 28.561 \\
\hline 21.97 & 41.743 & 24.167 \\
\hline 8.788 & 39.546 & 2.197 \\
\hline 6.591 & 2.197 & 28.561 \\
\hline 2.197 & 2.197 & 83.486 \\
\hline 2.197 & 2.197 & 6.591 \\
\hline
\end{tabular}




\begin{tabular}{|c|c|c|}
\hline 2.197 & 2.197 & 197.73 \\
\hline 26.364 & 4.394 & 76.895 \\
\hline 2.197 & 123.032 & 17.576 \\
\hline 4.394 & 4.394 & 604.175 \\
\hline 2.197 & 2.197 & 19.773 \\
\hline 4.394 & 2.197 & 10.985 \\
\hline 116.441 & 6.591 & 2.197 \\
\hline 19.773 & 4.394 & 48.334 \\
\hline 41.743 & 2.197 & 15.379 \\
\hline 4.394 & 2.197 & 87.88 \\
\hline 10.985 & 10.985 & 2.197 \\
\hline 2.197 & 6.591 & 13.182 \\
\hline 2.197 & 2.197 & 138.411 \\
\hline 2.197 & 2.197 & 109.85 \\
\hline 2.197 & 2.197 & 13.182 \\
\hline 87.88 & 2.197 & 4.394 \\
\hline 26.364 & 2.197 & 41.743 \\
\hline 6.591 & 2.197 & 2.197 \\
\hline 2.197 & 50.531 & 19.773 \\
\hline 2.197 & 424.021 & 2.197 \\
\hline 129.623 & 8.788 & 10.985 \\
\hline 94.471 & 48.334 & 2.197 \\
\hline 2.197 & 10.985 & 2.197 \\
\hline 17.576 & 129.623 & 8.788 \\
\hline 2.197 & 4.394 & 2.197 \\
\hline 303.186 & 48.334 & 8.788 \\
\hline 2.197 & 63.713 & 131.82 \\
\hline 2.197 & 68.107 & 109.85 \\
\hline 281.216 & 2.197 & 17.576 \\
\hline 19.773 & 2.197 & 6.591 \\
\hline 6.591 & 2.197 & 2.197 \\
\hline 63.713 & 2.197 & 2.197 \\
\hline 24.167 & 24.167 & 2.197 \\
\hline 2.197 & 4.394 & 24.167 \\
\hline 2.197 & 19.773 & 2.197 \\
\hline 6.591 & 2.197 & 43.94 \\
\hline 2.197 & 2.197 & 2091.54 \\
\hline 2.197 & 2.197 & 2.197 \\
\hline 17.576 & 2.197 & 472.355 \\
\hline 2.197 & 2.197 & 2.197 \\
\hline 2.197 & 2.197 & 13.182 \\
\hline 8.788 & 2.197 & 41.743 \\
\hline 35.152 & 8.788 & 6.591 \\
\hline 2.197 & 2.197 & 54.925 \\
\hline 149.396 & 41.743 & 37.349 \\
\hline 6.591 & 2.197 & 6.591 \\
\hline 46.137 & 2.197 & 2724.28 \\
\hline 13.182 & 4.394 & 6.591 \\
\hline 8.788 & 4963.02 & 24.167 \\
\hline 4.394 & 2.197 & 17.576 \\
\hline
\end{tabular}




\begin{tabular}{|c|c|c|}
\hline 182.351 & 4.394 & 2.197 \\
\hline 160.381 & 2.197 & 2891.25 \\
\hline 151.593 & 10.985 & 975.468 \\
\hline 2.197 & 37.349 & 21.97 \\
\hline 2.197 & 4.394 & 270.231 \\
\hline 8.788 & 2.197 & 15.379 \\
\hline 4.394 & 2.197 & 164.775 \\
\hline 4.394 & 2.197 & 57.122 \\
\hline 4.394 & 90.077 & 81.289 \\
\hline 17.576 & 2.197 & 19.773 \\
\hline 6.591 & 2.197 & 6.591 \\
\hline 8.788 & 270.231 & 17.576 \\
\hline 26.364 & 2.197 & 296.595 \\
\hline 426.218 & 2.197 & 28.561 \\
\hline 4.394 & 2.197 & 28.561 \\
\hline 285.61 & 54.925 & 13.182 \\
\hline 4.394 & 2.197 & 123.032 \\
\hline 2.197 & 2.197 & 30.758 \\
\hline 4.394 & 17.576 & 237.276 \\
\hline 10.985 & 43.94 & 35.152 \\
\hline 13.182 & 28.561 & 21.97 \\
\hline 2.197 & 2.197 & 2.197 \\
\hline 2.197 & 2.197 & 4.394 \\
\hline 15.379 & 26.364 & 4.394 \\
\hline 4.394 & 2.197 & 39.546 \\
\hline 4.394 & 2.197 & 17.576 \\
\hline 46.137 & 41.743 & 4.394 \\
\hline 15.379 & 8.788 & 2.197 \\
\hline 26.364 & 2.197 & 2.197 \\
\hline 2.197 & 2.197 & 4.394 \\
\hline 4.394 & 2.197 & 6.591 \\
\hline 6.591 & 2.197 & 109.85 \\
\hline 8.788 & 8.788 & 4.394 \\
\hline 57.122 & 2.197 & 26.364 \\
\hline 6.591 & 43.94 & 2.197 \\
\hline 21.97 & 4.394 & 6.591 \\
\hline 2.197 & 320.762 & 2.197 \\
\hline 4.394 & 63.713 & 4.394 \\
\hline 6.591 & 2.197 & 2.197 \\
\hline 6.591 & 10.985 & 63.713 \\
\hline 2.197 & 2.197 & 21.97 \\
\hline 2.197 & 2.197 & 4.394 \\
\hline 4.394 & 8.788 & 6.591 \\
\hline 6.591 & 2.197 & 2.197 \\
\hline 39.546 & 2.197 & 424.021 \\
\hline 4.394 & 2.197 & 6.591 \\
\hline 26.364 & 2.197 & 13.182 \\
\hline 13.182 & 151.593 & 158.184 \\
\hline 6.591 & 4.394 & 103.259 \\
\hline 171.366 & 2.197 & 147.199 \\
\hline
\end{tabular}




\begin{tabular}{|c|c|c|}
\hline 4.394 & 4.394 & 50.531 \\
\hline 6.591 & 19.773 & 41.743 \\
\hline 28.561 & 54.925 & 4.394 \\
\hline 19.773 & 6.591 & 24.167 \\
\hline 6.591 & 28.561 & 2.197 \\
\hline 193.336 & 6.591 & 4.394 \\
\hline 4.394 & 4.394 & 13.182 \\
\hline 180.154 & 2.197 & 2.197 \\
\hline 6.591 & 2.197 & 2.197 \\
\hline 15.379 & 2.197 & 2.197 \\
\hline 15.379 & 2.197 & 26.364 \\
\hline 8.788 & 28.561 & 24.167 \\
\hline 35.152 & 52.728 & 68.107 \\
\hline 24.167 & 4.394 & 2.197 \\
\hline 15.379 & 2.197 & 107.653 \\
\hline 4.394 & 6.591 & 432.809 \\
\hline 4.394 & 2.197 & 2.197 \\
\hline 2.197 & 2.197 & 10.985 \\
\hline 52.728 & 2.197 & 2.197 \\
\hline 76.895 & 134.017 & 2.197 \\
\hline 2.197 & 309.777 & 59.319 \\
\hline 28.561 & 261.443 & 195.533 \\
\hline 2.197 & 2.197 & 26.364 \\
\hline 24.167 & 6.591 & 221.897 \\
\hline 2.197 & 2.197 & 6.591 \\
\hline 2.197 & 2.197 & 10.985 \\
\hline 2.197 & 2.197 & 2.197 \\
\hline 2.197 & 2.197 & 8.788 \\
\hline 2.197 & 6.591 & 19.773 \\
\hline 8.788 & 15.379 & 2.197 \\
\hline 1373.12 & 129.623 & 6.591 \\
\hline 13.182 & 24.167 & 276.822 \\
\hline 68.107 & 15.379 & 503.113 \\
\hline 191.139 & 6.591 & 786.526 \\
\hline 2.197 & 2.197 & 32.955 \\
\hline 15.379 & 145.002 & 2.197 \\
\hline 17.576 & 118.638 & 320.762 \\
\hline 26.364 & 21.97 & 2.197 \\
\hline 533.871 & 8.788 & 6.591 \\
\hline 79.092 & 6.591 & 4.394 \\
\hline 6.591 & 6.591 & 2.197 \\
\hline 6.591 & 35.152 & 145.002 \\
\hline 15.379 & 213.109 & 10.985 \\
\hline 4.394 & 17.576 & 8.788 \\
\hline 2.197 & 2.197 & 65.91 \\
\hline 4.394 & 6.591 & 79.092 \\
\hline 2.197 & 4.394 & 35.152 \\
\hline 74.698 & 6.591 & 2.197 \\
\hline 4.394 & 164.775 & 6.591 \\
\hline 26.364 & 2.197 & 2.197 \\
\hline
\end{tabular}




\begin{tabular}{|c|c|c|}
\hline 6.591 & 26.364 & 2.197 \\
\hline 26.364 & 2.197 & 76.895 \\
\hline 410.839 & 19.773 & 4.394 \\
\hline 30.758 & 4.394 & 8.788 \\
\hline 507.507 & 10.985 & 10.985 \\
\hline 15.379 & 2.197 & 4.394 \\
\hline 4.394 & 2.197 & 4.394 \\
\hline 224.094 & 13.182 & 2.197 \\
\hline 186.745 & 2.197 & 81.289 \\
\hline 8.788 & 2.197 & 2.197 \\
\hline 35.152 & 103.259 & 6.591 \\
\hline 28.561 & 4.394 & 63.713 \\
\hline 2.197 & 6.591 & 6.591 \\
\hline 2.197 & 24.167 & 13.182 \\
\hline 61.516 & 6.591 & 24.167 \\
\hline 2.197 & 246.064 & 4.394 \\
\hline 4.394 & 2.197 & 26.364 \\
\hline 2.197 & 4.394 & 79.092 \\
\hline 2.197 & 4.394 & 13.182 \\
\hline 4.394 & 6.591 & 13.182 \\
\hline 17.576 & 2.197 & 116.441 \\
\hline 2.197 & 2.197 & 63.713 \\
\hline 4.394 & 4.394 & 13.182 \\
\hline 4.394 & 2.197 & 54.925 \\
\hline 8.788 & 6.591 & 10.985 \\
\hline 2.197 & 186.745 & 13.182 \\
\hline 2.197 & 2.197 & 70.304 \\
\hline 4.394 & 131.82 & 8.788 \\
\hline 15.379 & 2.197 & 15.379 \\
\hline 6.591 & 37.349 & 2.197 \\
\hline 19.773 & 4.394 & 573.417 \\
\hline 8.788 & 13.182 & 79.092 \\
\hline 2.197 & 13.182 & 2.197 \\
\hline 8.788 & 685.464 & 2.197 \\
\hline 4.394 & 4.394 & 19.773 \\
\hline 4.394 & 4.394 & 309.777 \\
\hline 37.349 & 2.197 & 136.214 \\
\hline 26.364 & 6.591 & 2.197 \\
\hline 408.642 & 120.835 & 2.197 \\
\hline 1370.93 & 2.197 & 2.197 \\
\hline 6.591 & 8.788 & 4.394 \\
\hline 8.788 & 2.197 & 2.197 \\
\hline 2.197 & 8.788 & 61.516 \\
\hline 2.197 & 46.137 & 48.334 \\
\hline 37.349 & 2.197 & 4.394 \\
\hline 101.062 & 2.197 & 8.788 \\
\hline 4.394 & 72.501 & 2.197 \\
\hline 2.197 & 8.788 & 15.379 \\
\hline 2.197 & 87.88 & 2.197 \\
\hline 15.379 & 2.197 & 2.197 \\
\hline
\end{tabular}




\begin{tabular}{|c|c|c|}
\hline 2.197 & 10.985 & 2.197 \\
\hline 2.197 & 8.788 & 2.197 \\
\hline 28.561 & 2.197 & 30.758 \\
\hline 4.394 & 6.591 & 10.985 \\
\hline 21.97 & 21.97 & 8.788 \\
\hline 15.379 & 4.394 & 28.561 \\
\hline 13.182 & 6.591 & 5918.72 \\
\hline 109.85 & 4.394 & 13.182 \\
\hline 35.152 & 2.197 & 15.379 \\
\hline 2.197 & 4.394 & 2.197 \\
\hline 252.655 & 250.458 & 6.591 \\
\hline 2.197 & 6.591 & 17.576 \\
\hline 155.987 & 32.955 & 8.788 \\
\hline 32.955 & 2.197 & 15.379 \\
\hline 13.182 & 8.788 & 15.379 \\
\hline 37.349 & 13.182 & 28.561 \\
\hline 28.561 & 8.788 & 35.152 \\
\hline 6.591 & 175.76 & 2.197 \\
\hline 116.441 & 8.788 & 127.426 \\
\hline 2.197 & 15.379 & 35.152 \\
\hline 6.591 & 2.197 & 2.197 \\
\hline 2.197 & 2.197 & 46.137 \\
\hline 2.197 & 2.197 & 2.197 \\
\hline 35.152 & 2.197 & 41.743 \\
\hline 4.394 & 2.197 & 48.334 \\
\hline 6.591 & 2.197 & 2.197 \\
\hline 4.394 & 2.197 & 2.197 \\
\hline 50.531 & 2.197 & 2.197 \\
\hline 6.591 & 2.197 & 4.394 \\
\hline 4.394 & 48.334 & 10.985 \\
\hline 8.788 & 54.925 & 98.865 \\
\hline 2.197 & 3420.73 & 94.471 \\
\hline 54.925 & 43.94 & 21.97 \\
\hline 2.197 & 2.197 & 2.197 \\
\hline 6.591 & 2.197 & 2.197 \\
\hline 28.561 & 1983.89 & 2.197 \\
\hline 2.197 & 13.182 & 2.197 \\
\hline 155.987 & 2.197 & 15.379 \\
\hline 65.91 & 30.758 & 21.97 \\
\hline 6.591 & 4.394 & 13.182 \\
\hline 46.137 & 2.197 & 2.197 \\
\hline 2.197 & 2.197 & 81.289 \\
\hline 2.197 & 2.197 & 2.197 \\
\hline 43.94 & 10.985 & 43.94 \\
\hline 2.197 & 10.985 & 8.788 \\
\hline 2.197 & 2.197 & 6.591 \\
\hline 6.591 & 120.835 & 21.97 \\
\hline 4.394 & 2.197 & 37.349 \\
\hline 35.152 & 61.516 & 19.773 \\
\hline 2.197 & 13.182 & 4.394 \\
\hline
\end{tabular}




\begin{tabular}{|c|c|c|}
\hline 2.197 & 112.047 & 2.197 \\
\hline 52.728 & 15.379 & 2.197 \\
\hline 4.394 & 2.197 & 6.591 \\
\hline 10.985 & 2.197 & 28.561 \\
\hline 19.773 & 2.197 & 79.092 \\
\hline 37.349 & 2.197 & 4.394 \\
\hline 76.895 & 35.152 & 24.167 \\
\hline 2.197 & 24.167 & 2.197 \\
\hline 2.197 & 43.94 & 2.197 \\
\hline 2.197 & 2.197 & 2.197 \\
\hline 10.985 & 2.197 & 4.394 \\
\hline 28.561 & 2.197 & 92.274 \\
\hline 116.441 & 6.591 & 13.182 \\
\hline 221.897 & 2.197 & 43.94 \\
\hline 4.394 & 125.229 & 2.197 \\
\hline 50.531 & 8.788 & 54.925 \\
\hline 21.97 & 4.394 & 243.867 \\
\hline 46.137 & 2.197 & 6.591 \\
\hline 13.182 & 28.561 & 13.182 \\
\hline 2.197 & 26.364 & 4.394 \\
\hline 43.94 & 4.394 & 59.319 \\
\hline 63.713 & 2.197 & 2.197 \\
\hline 4.394 & 4.394 & 17.576 \\
\hline 41.743 & 2.197 & 2.197 \\
\hline 103.259 & 8.788 & 37.349 \\
\hline 10.985 & 15.379 & 15.379 \\
\hline 6.591 & 26.364 & 13.182 \\
\hline 8.788 & 221.897 & 2.197 \\
\hline 2.197 & 17.576 & 4.394 \\
\hline 21.97 & 2.197 & 103.259 \\
\hline 13.182 & 52.728 & 6.591 \\
\hline 41.743 & 8.788 & 28.561 \\
\hline 4.394 & 652.509 & 48.334 \\
\hline 228.488 & 6.591 & 2.197 \\
\hline 19.773 & 2.197 & 72.501 \\
\hline 4.394 & 2.197 & 35.152 \\
\hline 43.94 & 28.561 & 2.197 \\
\hline 2.197 & 15.379 & 2.197 \\
\hline 2.197 & 415.233 & 15.379 \\
\hline 15.379 & 15.379 & 15.379 \\
\hline 6.591 & 37.349 & 10.985 \\
\hline 10.985 & 2.197 & 26.364 \\
\hline 54.925 & 2.197 & 2.197 \\
\hline 26.364 & 4.394 & 195.533 \\
\hline 57.122 & 4.394 & 6.591 \\
\hline 123.032 & 4.394 & 2.197 \\
\hline 6.591 & 6.591 & 19.773 \\
\hline 210.912 & 2.197 & 8.788 \\
\hline 24.167 & 2.197 & 26.364 \\
\hline 147.199 & 2.197 & 290.004 \\
\hline
\end{tabular}




\begin{tabular}{|c|c|c|}
\hline 4.394 & 35.152 & 6.591 \\
\hline 2.197 & 2.197 & 2.197 \\
\hline 8.788 & 6.591 & 10.985 \\
\hline 15.379 & 2.197 & 50.531 \\
\hline 4.394 & 13.182 & 17.576 \\
\hline 2.197 & 68.107 & 24582.2 \\
\hline 208.715 & 2.197 & 2.197 \\
\hline 13.182 & 2.197 & 103.259 \\
\hline 4.394 & 61.516 & 182.351 \\
\hline 26.364 & 19.773 & 2.197 \\
\hline 2.197 & 2.197 & 6.591 \\
\hline 2.197 & 307.58 & 2.197 \\
\hline 2.197 & 6.591 & 2.197 \\
\hline 19.773 & 21.97 & 2.197 \\
\hline 2.197 & 4.394 & 2.197 \\
\hline 19.773 & 6.591 & 2.197 \\
\hline 2.197 & 50.531 & 24.167 \\
\hline 2.197 & 6.591 & 2.197 \\
\hline 2.197 & 2.197 & 8.788 \\
\hline 68.107 & 2.197 & 2.197 \\
\hline 8.788 & 2.197 & 39.546 \\
\hline 300.989 & 2.197 & 13127.1 \\
\hline 2.197 & 2.197 & 641.524 \\
\hline 4.394 & 4.394 & 2.197 \\
\hline 94.471 & 2.197 & 2.197 \\
\hline 19.773 & 4.394 & 24.167 \\
\hline 1102.89 & 4.394 & 10.985 \\
\hline 10.985 & 4.394 & 2.197 \\
\hline 13.182 & 2.197 & 50.531 \\
\hline 4.394 & 180.154 & 74.698 \\
\hline 2.197 & 206.518 & 2.197 \\
\hline 8.788 & 2.197 & 2.197 \\
\hline 4.394 & 140.608 & 8.788 \\
\hline 340.535 & 4.394 & 8.788 \\
\hline 8.788 & 32.955 & 2.197 \\
\hline 2.197 & 2.197 & 10.985 \\
\hline 399.854 & 158.184 & 4.394 \\
\hline 13.182 & 2.197 & 30.758 \\
\hline 2.197 & 2.197 & 2.197 \\
\hline 46.137 & 2.197 & 8.788 \\
\hline 28.561 & 2.197 & 2.197 \\
\hline 4.394 & 8.788 & 4.394 \\
\hline 19.773 & 8.788 & 10.985 \\
\hline 6.591 & 276.822 & 43.94 \\
\hline 314.171 & 26.364 & 2.197 \\
\hline 2.197 & 4.394 & 10.985 \\
\hline 287.807 & 2.197 & 8.788 \\
\hline 2.197 & 2.197 & 50.531 \\
\hline 2.197 & 2.197 & 4.394 \\
\hline 30.758 & 147.199 & 2.197 \\
\hline
\end{tabular}




\begin{tabular}{|c|c|c|}
\hline 65.91 & 54.925 & 17.576 \\
\hline 153.79 & 2.197 & 10.985 \\
\hline 26.364 & 2.197 & 10.985 \\
\hline 2.197 & 83.486 & 70.304 \\
\hline 2.197 & 861.224 & 2.197 \\
\hline 13.182 & 6.591 & 4.394 \\
\hline 13.182 & 4.394 & 217.503 \\
\hline 81.289 & 2.197 & 2.197 \\
\hline 52.728 & 24.167 & 2.197 \\
\hline 13.182 & 8.788 & 138.411 \\
\hline 74.698 & 2.197 & 241.67 \\
\hline 131.82 & 2.197 & 10.985 \\
\hline 4.394 & 4.394 & 6.591 \\
\hline 79.092 & 26.364 & 2.197 \\
\hline 10.985 & 2.197 & 10.985 \\
\hline 48.334 & 2.197 & 2.197 \\
\hline 63.713 & 2.197 & 112.047 \\
\hline 191.139 & 8.788 & 15.379 \\
\hline 6.591 & 24.167 & 24.167 \\
\hline 63.713 & 4.394 & 766.753 \\
\hline 24.167 & 4.394 & 2.197 \\
\hline 70.304 & 87.88 & 26.364 \\
\hline 2.197 & 331.747 & 2.197 \\
\hline 8.788 & 35.152 & 2.197 \\
\hline 2.197 & 2.197 & 87.88 \\
\hline 2.197 & 4.394 & 13.182 \\
\hline 10.985 & 37.349 & 37.349 \\
\hline 2.197 & 4.394 & 2.197 \\
\hline 4.394 & 2.197 & 79.092 \\
\hline 2.197 & 2.197 & 19.773 \\
\hline 28.561 & 2.197 & 2.197 \\
\hline 6.591 & 24.167 & 6.591 \\
\hline 79.092 & 4.394 & 4.394 \\
\hline 10.985 & 2.197 & 2.197 \\
\hline 13.182 & 2.197 & 8.788 \\
\hline 2.197 & 17.576 & 13.182 \\
\hline 21.97 & 2.197 & 2.197 \\
\hline 2.197 & 2.197 & 4.394 \\
\hline 4.394 & 19.773 & 2.197 \\
\hline 4.394 & 2.197 & 698.646 \\
\hline 4.394 & 4.394 & 4.394 \\
\hline 2.197 & 35.152 & 2.197 \\
\hline 17.576 & 2.197 & 48.334 \\
\hline 6.591 & 210.912 & 8.788 \\
\hline 24.167 & 2.197 & 13.182 \\
\hline 6.591 & 2.197 & 37.349 \\
\hline 2.197 & 61.516 & 57.122 \\
\hline 4.394 & 2.197 & 15.379 \\
\hline 8.788 & 2.197 & 26.364 \\
\hline 6.591 & 8.788 & 8.788 \\
\hline
\end{tabular}




\begin{tabular}{|c|c|c|}
\hline 2.197 & 2.197 & 39.546 \\
\hline 4.394 & 4.394 & 2.197 \\
\hline 4.394 & 35.152 & 2.197 \\
\hline 85.683 & 13.182 & 6.591 \\
\hline 2.197 & 103.259 & 731.601 \\
\hline 26.364 & 566.826 & 6.591 \\
\hline 10.985 & 13.182 & 2.197 \\
\hline 2.197 & 1592.82 & 2.197 \\
\hline 10.985 & 4.394 & 4.394 \\
\hline 13.182 & 19.773 & 6.591 \\
\hline 2.197 & 4.394 & 145.002 \\
\hline 2.197 & 155.987 & 4.394 \\
\hline 6.591 & 2.197 & 2.197 \\
\hline 6.591 & 10.985 & 2.197 \\
\hline 15.379 & 2.197 & 8.788 \\
\hline 15.379 & 364.702 & 349.323 \\
\hline 101.062 & 8.788 & 15.379 \\
\hline 32.955 & 2.197 & 2.197 \\
\hline 13.182 & 2.197 & 2.197 \\
\hline 6.591 & 35.152 & 4.394 \\
\hline 2.197 & 17.576 & 2.197 \\
\hline 2.197 & 210.912 & 30.758 \\
\hline 10.985 & 4.394 & 15.379 \\
\hline 265.837 & 766.753 & 10.985 \\
\hline 94.471 & 2.197 & 10.985 \\
\hline 505.31 & 2.197 & 10.985 \\
\hline 4.394 & 28.561 & 15.379 \\
\hline 83.486 & 2.197 & 2.197 \\
\hline 17.576 & 2.197 & 4.394 \\
\hline 17.576 & 13.182 & 2.197 \\
\hline 50.531 & 6.591 & 8.788 \\
\hline 2.197 & 2.197 & 10.985 \\
\hline 19.773 & 2.197 & 2.197 \\
\hline 26.364 & 2.197 & 32.955 \\
\hline 2.197 & 19.773 & 2.197 \\
\hline 2.197 & 2.197 & 31557.7 \\
\hline 10.985 & 2.197 & 134.017 \\
\hline 35.152 & 160.381 & 50.531 \\
\hline 65.91 & 2.197 & 65.91 \\
\hline 6.591 & 30.758 & 43.94 \\
\hline 46.137 & 13.182 & 32.955 \\
\hline 2.197 & 32.955 & 79.092 \\
\hline 153.79 & 4.394 & 21.97 \\
\hline 2.197 & 4.394 & 437.203 \\
\hline 140.608 & 13.182 & 59.319 \\
\hline 2.197 & 2.197 & 263.64 \\
\hline 2.197 & 4.394 & 30.758 \\
\hline 2.197 & 28.561 & 2.197 \\
\hline 54.925 & 4.394 & 2.197 \\
\hline 26.364 & 41.743 & 24.167 \\
\hline
\end{tabular}




\begin{tabular}{|c|c|c|}
\hline 13.182 & 10.985 & 2.197 \\
\hline 4.394 & 2.197 & 2.197 \\
\hline 4.394 & 2.197 & 2836.33 \\
\hline 2.197 & 2.197 & 2.197 \\
\hline 8.788 & 4.394 & 39.546 \\
\hline 2.197 & 2.197 & 630.539 \\
\hline 6.591 & 6.591 & 13.182 \\
\hline 2.197 & 2.197 & 8.788 \\
\hline 2.197 & 2.197 & 21.97 \\
\hline 202.124 & 2.197 & 37.349 \\
\hline 2.197 & 371.293 & 2.197 \\
\hline 10.985 & 13.182 & 2.197 \\
\hline 15.379 & 145.002 & 2.197 \\
\hline 57.122 & 13.182 & 4.394 \\
\hline 19.773 & 2.197 & 19.773 \\
\hline 2.197 & 8.788 & 2.197 \\
\hline 2.197 & 8.788 & 2.197 \\
\hline 39.546 & 83.486 & 43.94 \\
\hline 2.197 & 2.197 & 30.758 \\
\hline 2.197 & 2.197 & 2.197 \\
\hline 8.788 & 8.788 & 4.394 \\
\hline 28.561 & 21.97 & 4.394 \\
\hline 2.197 & 30.758 & 10.985 \\
\hline 2.197 & 465.764 & 2.197 \\
\hline 4.394 & 2.197 & 2.197 \\
\hline 6.591 & 164.775 & 2.197 \\
\hline 114.244 & 169.169 & 2.197 \\
\hline 4.394 & 2.197 & 6.591 \\
\hline 2.197 & 2.197 & 2.197 \\
\hline 285.61 & 6.591 & 4.394 \\
\hline 4.394 & 68.107 & 30.758 \\
\hline 4.394 & 4.394 & 6.591 \\
\hline 6.591 & 4.394 & 4.394 \\
\hline 123.032 & 4.394 & 2.197 \\
\hline 351.52 & 19.773 & 151.593 \\
\hline 15.379 & 2.197 & 2.197 \\
\hline 4.394 & 2.197 & 2.197 \\
\hline 68.107 & 2.197 & 2.197 \\
\hline 6.591 & 15.379 & 46.137 \\
\hline 6.591 & 81.289 & 314.171 \\
\hline 13.182 & 4.394 & 24.167 \\
\hline 6.591 & 2.197 & 8.788 \\
\hline 8.788 & 169.169 & 6.591 \\
\hline 114.244 & 17.576 & 46.137 \\
\hline 28.561 & 2.197 & 2.197 \\
\hline 2.197 & 98.865 & 8.788 \\
\hline 13.182 & 8295.87 & 28.561 \\
\hline 4.394 & 2.197 & 2.197 \\
\hline 2.197 & 615.16 & 52.728 \\
\hline 94.471 & 8.788 & 360.308 \\
\hline
\end{tabular}




\begin{tabular}{|c|c|c|}
\hline 43.94 & 2.197 & 676.676 \\
\hline 24.167 & 2095.94 & 2.197 \\
\hline 28.561 & 8.788 & 21.97 \\
\hline 101.062 & 6.591 & 2.197 \\
\hline 4.394 & 2.197 & 46.137 \\
\hline 70.304 & 2.197 & 48.334 \\
\hline 2.197 & 2.197 & 28.561 \\
\hline 2.197 & 8.788 & 35.152 \\
\hline 94.471 & 4.394 & 2.197 \\
\hline 37.349 & 4.394 & 6.591 \\
\hline 4.394 & 2.197 & 28.561 \\
\hline 8.788 & 2.197 & 166.972 \\
\hline 8.788 & 2.197 & 63.713 \\
\hline 2.197 & 2.197 & 10.985 \\
\hline 17.576 & 8.788 & 279.019 \\
\hline 94.471 & 2.197 & 2.197 \\
\hline 4.394 & 15.379 & 30.758 \\
\hline 2.197 & 4.394 & 4.394 \\
\hline 17.576 & 8.788 & 13.182 \\
\hline 6.591 & 2.197 & 2.197 \\
\hline 13.182 & 2.197 & 13.182 \\
\hline 4.394 & 586.599 & 30.758 \\
\hline 21.97 & 4.394 & 4.394 \\
\hline 19.773 & 2.197 & 8.788 \\
\hline 2.197 & 213.109 & 48.334 \\
\hline 54.925 & 2.197 & 210.912 \\
\hline 116.441 & 52.728 & 21.97 \\
\hline 2.197 & 4.394 & 2.197 \\
\hline 4.394 & 10.985 & 4.394 \\
\hline 2.197 & 2.197 & 26.364 \\
\hline 46.137 & 2.197 & 41.743 \\
\hline 10.985 & 151.593 & 2.197 \\
\hline 4.394 & 6.591 & 54.925 \\
\hline 4.394 & 2.197 & 10.985 \\
\hline 2.197 & 2.197 & 24.167 \\
\hline 32.955 & 24.167 & 2.197 \\
\hline 28.561 & 65.91 & 2.197 \\
\hline 131.82 & 2.197 & 2.197 \\
\hline 13.182 & 2.197 & 17.576 \\
\hline 30.758 & 2.197 & 2.197 \\
\hline 74.698 & 72.501 & 123.032 \\
\hline 2.197 & 4.394 & 50.531 \\
\hline 2.197 & 2.197 & 54.925 \\
\hline 2.197 & 19.773 & 54.925 \\
\hline 6.591 & 37.349 & 37.349 \\
\hline 61.516 & 2.197 & 2.197 \\
\hline 177.957 & 26.364 & 48.334 \\
\hline 10.985 & 68.107 & 37.349 \\
\hline 4.394 & 610.766 & 2.197 \\
\hline 1454.41 & 2.197 & 395.46 \\
\hline
\end{tabular}




\begin{tabular}{|c|c|c|}
\hline 8.788 & 2.197 & 388.869 \\
\hline 1412.67 & 2.197 & 8.788 \\
\hline 366.899 & 19.773 & 79.092 \\
\hline 57.122 & 81.289 & 2.197 \\
\hline 24.167 & 2.197 & 2.197 \\
\hline 2.197 & 2.197 & 26.364 \\
\hline 2.197 & 2.197 & 70.304 \\
\hline 6.591 & 15.379 & 15.379 \\
\hline 175.76 & 173.563 & 81.289 \\
\hline 10.985 & 6.591 & 2.197 \\
\hline 2.197 & 2.197 & 6.591 \\
\hline 10.985 & 2.197 & 79.092 \\
\hline 2.197 & 2.197 & 17.576 \\
\hline 21.97 & 4.394 & 8.788 \\
\hline 8.788 & 2.197 & 4.394 \\
\hline 46.137 & 15.379 & 4.394 \\
\hline 2.197 & 241.67 & 57.122 \\
\hline 4.394 & 28.561 & 15.379 \\
\hline 4.394 & 2.197 & 50.531 \\
\hline 2.197 & 848.042 & 4.394 \\
\hline 94.471 & 8.788 & 2.197 \\
\hline 2.197 & 2.197 & 43.94 \\
\hline 2.197 & 41.743 & 6.591 \\
\hline 50.531 & 4.394 & 2.197 \\
\hline 26.364 & 39.546 & 10.985 \\
\hline 21.97 & 6.591 & 2.197 \\
\hline 17.576 & 2.197 & 2.197 \\
\hline 169.169 & 2.197 & 6.591 \\
\hline 160.381 & 52.728 & 85.683 \\
\hline 2.197 & 59.319 & 2.197 \\
\hline 246.064 & 2.197 & 2.197 \\
\hline 4.394 & 131.82 & 441.597 \\
\hline 2.197 & 2.197 & 15.379 \\
\hline 2.197 & 2.197 & 1803.74 \\
\hline 8.788 & 79.092 & 61.516 \\
\hline 43.94 & 2.197 & 2.197 \\
\hline 19.773 & 2.197 & 96.668 \\
\hline 10.985 & 221.897 & 1447.82 \\
\hline 6.591 & 2.197 & 4.394 \\
\hline 2.197 & 2.197 & 136.214 \\
\hline 162.578 & 26.364 & 6.591 \\
\hline 6.591 & 2.197 & 138.411 \\
\hline 6.591 & 2.197 & 2.197 \\
\hline 19.773 & 2.197 & 17.576 \\
\hline 15.379 & 2.197 & 4.394 \\
\hline 32.955 & 4.394 & 2.197 \\
\hline 4.394 & 2.197 & 65.91 \\
\hline 2.197 & 2.197 & 13.182 \\
\hline 4.394 & 2.197 & 204.321 \\
\hline 6.591 & 6.591 & 15.379 \\
\hline
\end{tabular}




\begin{tabular}{|c|c|c|}
\hline 59.319 & 4.394 & 32.955 \\
\hline 2.197 & 74.698 & 872.209 \\
\hline 2.197 & 2.197 & 2.197 \\
\hline 90.077 & 2.197 & 2.197 \\
\hline 4.394 & 6.591 & 10.985 \\
\hline 15.379 & 52.728 & 43.94 \\
\hline 127.426 & 57.122 & 2.197 \\
\hline 2.197 & 2.197 & 37.349 \\
\hline 2.197 & 4.394 & 2.197 \\
\hline 2.197 & 24.167 & 123.032 \\
\hline 15.379 & 2.197 & 6.591 \\
\hline 4.394 & 2.197 & 48.334 \\
\hline 54.925 & 10.985 & 6.591 \\
\hline 4.394 & 15.379 & 24.167 \\
\hline 6.591 & 68.107 & 10.985 \\
\hline 8.788 & 182.351 & 79.092 \\
\hline 2.197 & 6.591 & 28.561 \\
\hline 4.394 & 2.197 & 6.591 \\
\hline 2.197 & 2.197 & 30.758 \\
\hline 2.197 & 63.713 & 2.197 \\
\hline 2.197 & 2.197 & 43.94 \\
\hline 2.197 & 2.197 & 279.019 \\
\hline 32.955 & 2.197 & 439.4 \\
\hline 2.197 & 2.197 & 65.91 \\
\hline 2.197 & 2.197 & 70.304 \\
\hline 2.197 & 105.456 & 54.925 \\
\hline 2.197 & 15.379 & 129.623 \\
\hline 1647.75 & 4.394 & 8.788 \\
\hline 19.773 & 2.197 & 8.788 \\
\hline 311.974 & 50.531 & 2.197 \\
\hline 2.197 & 2.197 & 46.137 \\
\hline 61.516 & 2.197 & 17.576 \\
\hline 2.197 & 2.197 & 2.197 \\
\hline 4.394 & 2.197 & 21.97 \\
\hline 2.197 & 2.197 & 46.137 \\
\hline 4.394 & 94.471 & 2.197 \\
\hline 4.394 & 48.334 & 30.758 \\
\hline 4.394 & 4.394 & 4.394 \\
\hline 2.197 & 37.349 & 28.561 \\
\hline 32.955 & 4.394 & 2.197 \\
\hline 2.197 & 4.394 & 10.985 \\
\hline 10.985 & 1915.78 & 463.567 \\
\hline 19.773 & 2.197 & 19.773 \\
\hline 54.925 & 2.197 & 2.197 \\
\hline 2.197 & 10.985 & 2.197 \\
\hline 4.394 & 4.394 & 2.197 \\
\hline 17.576 & 4.394 & 10.985 \\
\hline 19.773 & 590.993 & 30.758 \\
\hline 39.546 & 2.197 & 2.197 \\
\hline 2.197 & 197.73 & 15.379 \\
\hline
\end{tabular}




\begin{tabular}{|c|c|c|}
\hline 52.728 & 6.591 & 2.197 \\
\hline 72.501 & 2.197 & 74.698 \\
\hline 2.197 & 279.019 & 83.486 \\
\hline 28.561 & 65.91 & 8.788 \\
\hline 35.152 & 2.197 & 17.576 \\
\hline 10.985 & 2.197 & 2.197 \\
\hline 21.97 & 10.985 & 8.788 \\
\hline 48.334 & 2.197 & 2.197 \\
\hline 193.336 & 2.197 & 4.394 \\
\hline 61.516 & 4.394 & 4.394 \\
\hline 19.773 & 6.591 & 28.561 \\
\hline 19.773 & 15.379 & 21.97 \\
\hline 4.394 & 24.167 & 37.349 \\
\hline 68.107 & 138.411 & 2.197 \\
\hline 2.197 & 8.788 & 2.197 \\
\hline 4.394 & 2.197 & 13.182 \\
\hline 2.197 & 386.672 & 81.289 \\
\hline 4.394 & 2.197 & 4.394 \\
\hline 2.197 & 2.197 & 30.758 \\
\hline 50.531 & 2.197 & 4.394 \\
\hline 2.197 & 2.197 & 43.94 \\
\hline 6.591 & 2.197 & 4.394 \\
\hline 2.197 & 30.758 & 13.182 \\
\hline 13.182 & 10.985 & 8.788 \\
\hline 8.788 & 17.576 & 48.334 \\
\hline 13.182 & 2.197 & 26.364 \\
\hline 15.379 & 527.28 & 590.993 \\
\hline 30.758 & 6.591 & 10.985 \\
\hline 6.591 & 2.197 & 107.653 \\
\hline 4.394 & 2.197 & 17.576 \\
\hline 2.197 & 2.197 & 2.197 \\
\hline 2.197 & 2.197 & 2.197 \\
\hline 2.197 & 2.197 & 2.197 \\
\hline 2.197 & 2.197 & 37.349 \\
\hline 4.394 & 2.197 & 6.591 \\
\hline 2.197 & 2.197 & 2.197 \\
\hline 37.349 & 2.197 & 48.334 \\
\hline 2.197 & 2.197 & 2.197 \\
\hline 2.197 & 4.394 & 57.122 \\
\hline 10.985 & 2.197 & 2.197 \\
\hline 2.197 & 2.197 & 2.197 \\
\hline 2.197 & 13.182 & 2.197 \\
\hline 2.197 & 6.591 & 4.394 \\
\hline 2.197 & 123.032 & 72.501 \\
\hline 1135.85 & 2.197 & 331.747 \\
\hline 2.197 & 8.788 & 4.394 \\
\hline 2.197 & 35.152 & 26.364 \\
\hline 19.773 & 2.197 & 101.062 \\
\hline 2.197 & 15.379 & 24.167 \\
\hline 10.985 & 2.197 & 8.788 \\
\hline
\end{tabular}




\begin{tabular}{|c|c|c|}
\hline 2.197 & 4.394 & 98.865 \\
\hline 98.865 & 2.197 & 41.743 \\
\hline 57.122 & 2.197 & 8.788 \\
\hline 37.349 & 24.167 & 2.197 \\
\hline 15.379 & 2.197 & 6.591 \\
\hline 24.167 & 19.773 & 8.788 \\
\hline 32.955 & 2.197 & 2.197 \\
\hline 6.591 & 2.197 & 15.379 \\
\hline 2.197 & 17.576 & 2.197 \\
\hline 2.197 & 37.349 & 4.394 \\
\hline 2.197 & 4.394 & 4.394 \\
\hline 511.901 & 2.197 & 2.197 \\
\hline 103.259 & 2.197 & 6.591 \\
\hline 2.197 & 2.197 & 19.773 \\
\hline 6.591 & 8.788 & 50.531 \\
\hline 2.197 & 15.379 & 26.364 \\
\hline 4.394 & 2.197 & 21.97 \\
\hline 8.788 & 26.364 & 4.394 \\
\hline 2.197 & 10.985 & 8.788 \\
\hline 2.197 & 129.623 & 90.077 \\
\hline 2.197 & 2.197 & 2.197 \\
\hline 46.137 & 2.197 & 2.197 \\
\hline 59.319 & 2.197 & 4.394 \\
\hline 2.197 & 19.773 & 8.788 \\
\hline 24.167 & 2.197 & 13.182 \\
\hline 2.197 & 4.394 & 43.94 \\
\hline 13.182 & 13.182 & 19.773 \\
\hline 37.349 & 4.394 & 8.788 \\
\hline 26.364 & 2.197 & 13.182 \\
\hline 4.394 & 2.197 & 13.182 \\
\hline 6.591 & 6.591 & 8.788 \\
\hline 2.197 & 2.197 & 41.743 \\
\hline 28.561 & 96.668 & 17.576 \\
\hline 35.152 & 2.197 & 2.197 \\
\hline 2.197 & 2.197 & 6.591 \\
\hline 149.396 & 4.394 & 2.197 \\
\hline 6.591 & 175.76 & 2.197 \\
\hline 15.379 & 8.788 & 4.394 \\
\hline 10.985 & 2.197 & 2.197 \\
\hline 2.197 & 2.197 & 30.758 \\
\hline 35.152 & 320.762 & 2.197 \\
\hline 2.197 & 68.107 & 85.683 \\
\hline 65.91 & 1718.05 & 2.197 \\
\hline 4.394 & 2.197 & 8.788 \\
\hline 4.394 & 2.197 & 19.773 \\
\hline 6.591 & 10.985 & 2.197 \\
\hline 4.394 & 248.261 & 10.985 \\
\hline 4.394 & 4.394 & 15.379 \\
\hline 6.591 & 373.49 & 15.379 \\
\hline 10.985 & 4.394 & 8.788 \\
\hline
\end{tabular}




\begin{tabular}{|c|c|c|}
\hline 26.364 & 6.591 & 61.516 \\
\hline 2.197 & 2.197 & 37.349 \\
\hline 303.186 & 46.137 & 46.137 \\
\hline 15.379 & 514.098 & 305.383 \\
\hline 4.394 & 4.394 & 19.773 \\
\hline 21.97 & 4.394 & 8.788 \\
\hline 63.713 & 26.364 & 329.55 \\
\hline 13.182 & 2.197 & 32.955 \\
\hline 8.788 & 4.394 & 243.867 \\
\hline 2.197 & 2.197 & 39.546 \\
\hline 2.197 & 2.197 & 4.394 \\
\hline 2.197 & 639.327 & 232.882 \\
\hline 21.97 & 2.197 & 2.197 \\
\hline 4.394 & 2.197 & 10.985 \\
\hline 72.501 & 57.122 & 2.197 \\
\hline 6.591 & 134.017 & 151.593 \\
\hline 46.137 & 2.197 & 57.122 \\
\hline 43.94 & 4.394 & 6.591 \\
\hline 2.197 & 2.197 & 2.197 \\
\hline 10.985 & 4.394 & 160.381 \\
\hline 2.197 & 4.394 & 4.394 \\
\hline 4.394 & 10.985 & 6.591 \\
\hline 28.561 & 2.197 & 37.349 \\
\hline 4.394 & 32.955 & 32.955 \\
\hline 2.197 & 21.97 & 37.349 \\
\hline 15.379 & 15.379 & 50.531 \\
\hline 19.773 & 2.197 & 112.047 \\
\hline 2.197 & 2.197 & 39.546 \\
\hline 123.032 & 4.394 & 2.197 \\
\hline 2.197 & 50.531 & 6.591 \\
\hline 92.274 & 1458.81 & 4.394 \\
\hline 21.97 & 13.182 & 83.486 \\
\hline 2.197 & 2.197 & 2.197 \\
\hline 10.985 & 2.197 & 2.197 \\
\hline 134.017 & 2.197 & 59.319 \\
\hline 8.788 & 4.394 & 52.728 \\
\hline 17.576 & 2.197 & 188.942 \\
\hline 2.197 & 2.197 & 2.197 \\
\hline 39.546 & 10.985 & 2.197 \\
\hline 8.788 & 2.197 & 116.441 \\
\hline 19.773 & 140.608 & 683.267 \\
\hline 6.591 & 2.197 & 2.197 \\
\hline 2.197 & 10.985 & 2.197 \\
\hline 2.197 & 2.197 & 2.197 \\
\hline 41.743 & 8.788 & 15.379 \\
\hline 8.788 & 2.197 & 4.394 \\
\hline 2.197 & 10.985 & 61.516 \\
\hline 2.197 & 2.197 & 28.561 \\
\hline 2.197 & 10.985 & 4.394 \\
\hline 6.591 & 39.546 & 6.591 \\
\hline
\end{tabular}




\begin{tabular}{|c|c|c|}
\hline 140.608 & 24.167 & 8.788 \\
\hline 15.379 & 2.197 & 6.591 \\
\hline 17.576 & 2.197 & 32.955 \\
\hline 136.214 & 2.197 & 70.304 \\
\hline 30.758 & 2.197 & 19.773 \\
\hline 2.197 & 10.985 & 134.017 \\
\hline 2.197 & 4.394 & 2.197 \\
\hline 120.835 & 6.591 & 15.379 \\
\hline 8.788 & 2.197 & 4.394 \\
\hline 8.788 & 4.394 & 2.197 \\
\hline 8.788 & 155.987 & 136.214 \\
\hline 2.197 & 6.591 & 2.197 \\
\hline 8.788 & 2.197 & 254.852 \\
\hline 4.394 & 2.197 & 2.197 \\
\hline 8.788 & 2.197 & 37.349 \\
\hline 8.788 & 2.197 & 4.394 \\
\hline 8.788 & 2.197 & 2.197 \\
\hline 17.576 & 2.197 & 13.182 \\
\hline 6.591 & 6.591 & 2.197 \\
\hline 2.197 & 26.364 & 2.197 \\
\hline 2.197 & 10.985 & 171.366 \\
\hline 39.546 & 43.94 & 2.197 \\
\hline 6.591 & 46.137 & 15.379 \\
\hline 24.167 & 4.394 & 331.747 \\
\hline 6.591 & 164.775 & 8.788 \\
\hline 6.591 & 4.394 & 6.591 \\
\hline 2.197 & 2.197 & 10.985 \\
\hline 24.167 & 17.576 & 50.531 \\
\hline 2.197 & 32.955 & 2.197 \\
\hline 35.152 & 4.394 & 134.017 \\
\hline 2.197 & 2.197 & 138.411 \\
\hline 2.197 & 6.591 & 4.394 \\
\hline 8.788 & 2.197 & 32.955 \\
\hline 17.576 & 21.97 & 2.197 \\
\hline 46.137 & 4.394 & 94.471 \\
\hline 15.379 & 2.197 & 6.591 \\
\hline 191.139 & 2.197 & 1667.52 \\
\hline 4.394 & 2.197 & 2.197 \\
\hline 24.167 & 4.394 & 52.728 \\
\hline 6.591 & 4.394 & 2.197 \\
\hline 15.379 & 13.182 & 2.197 \\
\hline 472.355 & 6.591 & 13.182 \\
\hline 2.197 & 15.379 & 24.167 \\
\hline 8.788 & 2.197 & 4.394 \\
\hline 2.197 & 6.591 & 307.58 \\
\hline 4.394 & 2.197 & 158.184 \\
\hline 35.152 & 2.197 & 103.259 \\
\hline 6.591 & 52.728 & 4.394 \\
\hline 2.197 & 15.379 & 4.394 \\
\hline 2.197 & 2.197 & 13.182 \\
\hline
\end{tabular}




\begin{tabular}{|c|c|c|}
\hline 4.394 & 2.197 & 15.379 \\
\hline 8.788 & 2.197 & 2.197 \\
\hline 2.197 & 2.197 & 8.788 \\
\hline 2.197 & 2.197 & 6.591 \\
\hline 21.97 & 4.394 & 19.773 \\
\hline 48.334 & 4.394 & 175.76 \\
\hline 4.394 & 2.197 & 68.107 \\
\hline 37.349 & 30.758 & 13.182 \\
\hline 8.788 & 63.713 & 2.197 \\
\hline 2.197 & 975.468 & 26.364 \\
\hline 612.963 & 8.788 & 4.394 \\
\hline 98.865 & 6.591 & 2.197 \\
\hline 4.394 & 15.379 & 30.758 \\
\hline 2.197 & 6.591 & 57.122 \\
\hline 39.546 & 15.379 & 48.334 \\
\hline 2.197 & 2.197 & 2.197 \\
\hline 2.197 & 1465.4 & 35.152 \\
\hline 90.077 & 19.773 & 2.197 \\
\hline 2.197 & 41.743 & 2.197 \\
\hline 2.197 & 2.197 & 2.197 \\
\hline 202.124 & 39.546 & 8.788 \\
\hline 2.197 & 2.197 & 2.197 \\
\hline 81.289 & 2.197 & 21.97 \\
\hline 2.197 & 37.349 & 2.197 \\
\hline 57.122 & 8.788 & 24.167 \\
\hline 21.97 & 2.197 & 17.576 \\
\hline 4.394 & 2.197 & 2.197 \\
\hline 61.516 & 17.576 & 37.349 \\
\hline 8.788 & 2.197 & 79.092 \\
\hline 39.546 & 105.456 & 551.447 \\
\hline 59.319 & 10.985 & 4.394 \\
\hline 2.197 & 2.197 & 8.788 \\
\hline 2.197 & 13.182 & 6.591 \\
\hline 6.591 & 4.394 & 74.698 \\
\hline 2.197 & 21.97 & 2.197 \\
\hline 2.197 & 76.895 & 136.214 \\
\hline 2.197 & 4.394 & 8.788 \\
\hline 2.197 & 46.137 & 125.229 \\
\hline 10.985 & 8.788 & 2.197 \\
\hline 21.97 & 2.197 & 2.197 \\
\hline 28.561 & 4.394 & 4.394 \\
\hline 2.197 & 15.379 & 160.381 \\
\hline 6.591 & 4.394 & 2.197 \\
\hline 24.167 & 103.259 & 2.197 \\
\hline 2.197 & 37.349 & 15.379 \\
\hline 246.064 & 349.323 & 2.197 \\
\hline 17.576 & 8.788 & 30.758 \\
\hline 10.985 & 17.576 & 2.197 \\
\hline 8.788 & 24.167 & 8.788 \\
\hline 32.955 & 2.197 & 59.319 \\
\hline
\end{tabular}




\begin{tabular}{|c|c|c|}
\hline 2.197 & 2.197 & 37.349 \\
\hline 24.167 & 96.668 & 4.394 \\
\hline 21.97 & 17.576 & 252.655 \\
\hline 6.591 & 4.394 & 48.334 \\
\hline 4.394 & 2.197 & 8.788 \\
\hline 2.197 & 2.197 & 151.593 \\
\hline 15.379 & 21.97 & 2.197 \\
\hline 4.394 & 10.985 & 52.728 \\
\hline 2.197 & 10.985 & 2.197 \\
\hline 4.394 & 81.289 & 656.903 \\
\hline 2.197 & 2.197 & 81.289 \\
\hline 4.394 & 2.197 & 4.394 \\
\hline 2.197 & 2.197 & 15.379 \\
\hline 8.788 & 4.394 & 48.334 \\
\hline 4.394 & 2.197 & 28.561 \\
\hline 2.197 & 4.394 & 2.197 \\
\hline 4.394 & 63.713 & 4.394 \\
\hline 415.233 & 2.197 & 98.865 \\
\hline 8.788 & 4.394 & 2.197 \\
\hline 177.957 & 13.182 & 79.092 \\
\hline 10.985 & 4.394 & 2.197 \\
\hline 37.349 & 26.364 & 2.197 \\
\hline 15.379 & 21.97 & 39.546 \\
\hline 8.788 & 2.197 & 28.561 \\
\hline 2.197 & 2.197 & 15.379 \\
\hline 2.197 & 2.197 & 26.364 \\
\hline 35.152 & 329.55 & 252.655 \\
\hline 4.394 & 15.379 & 2.197 \\
\hline 681.07 & 4.394 & 4.394 \\
\hline 17.576 & 4.394 & 2.197 \\
\hline 103.259 & 6.591 & 2.197 \\
\hline 4.394 & 24.167 & 2.197 \\
\hline 6.591 & 17.576 & 10.985 \\
\hline 2.197 & 48.334 & 54.925 \\
\hline 24.167 & 81.289 & 4.394 \\
\hline 35.152 & 859.027 & 2.197 \\
\hline 2.197 & 2.197 & 160.381 \\
\hline 19.773 & 2.197 & 4.394 \\
\hline 2.197 & 8.788 & 24.167 \\
\hline 10.985 & 601.978 & 6.591 \\
\hline 17.576 & 2.197 & 35.152 \\
\hline 59.319 & 8.788 & 386.672 \\
\hline 4.394 & 10.985 & 24.167 \\
\hline 2.197 & 6.591 & 2.197 \\
\hline 257.049 & 10.985 & 35.152 \\
\hline 2.197 & 8.788 & 2.197 \\
\hline 35.152 & 2.197 & 2.197 \\
\hline 10.985 & 2.197 & 83.486 \\
\hline 8.788 & 24.167 & 2.197 \\
\hline 4.394 & 4.394 & 26.364 \\
\hline
\end{tabular}




\begin{tabular}{|c|c|c|}
\hline 2.197 & 6.591 & 50.531 \\
\hline 43.94 & 13.182 & 30.758 \\
\hline 2.197 & 26.364 & 4.394 \\
\hline 10.985 & 8.788 & 2.197 \\
\hline 8.788 & 13.182 & 6.591 \\
\hline 2.197 & 4.394 & 8.788 \\
\hline 2.197 & 2.197 & 6.591 \\
\hline 2.197 & 15.379 & 10.985 \\
\hline 8.788 & 2.197 & 4.394 \\
\hline 19.773 & 6.591 & 8.788 \\
\hline 17.576 & 85.683 & 13.182 \\
\hline 28.561 & 8.788 & 4.394 \\
\hline 6.591 & 35.152 & 72.501 \\
\hline 120.835 & 32.955 & 96.668 \\
\hline 15.379 & 235.079 & 26.364 \\
\hline 30.758 & 6.591 & 2.197 \\
\hline 76.895 & 4.394 & 17.576 \\
\hline 41.743 & 101.062 & 15.379 \\
\hline 19.773 & 4.394 & 2.197 \\
\hline 72.501 & 2.197 & 70.304 \\
\hline 21.97 & 4.394 & 2.197 \\
\hline 4.394 & 6.591 & 32.955 \\
\hline 39.546 & 123.032 & 571.22 \\
\hline 2.197 & 136.214 & 2.197 \\
\hline 21.97 & 1630.17 & 547.053 \\
\hline 112.047 & 8.788 & 68.107 \\
\hline 41.743 & 48.334 & 2.197 \\
\hline 24.167 & 2.197 & 2.197 \\
\hline 21.97 & 2.197 & 37.349 \\
\hline 2.197 & 2.197 & 96.668 \\
\hline 8.788 & 2.197 & 63.713 \\
\hline 19.773 & 4.394 & 8.788 \\
\hline 26.364 & 4.394 & 465.764 \\
\hline 2.197 & 4.394 & 28.561 \\
\hline 70.304 & 98.865 & 6.591 \\
\hline 32.955 & 81.289 & 171.366 \\
\hline 2.197 & 2.197 & 2.197 \\
\hline 21.97 & 21.97 & 2.197 \\
\hline 8.788 & 15.379 & 2.197 \\
\hline 8.788 & 10.985 & 10.985 \\
\hline 13.182 & 8.788 & 24.167 \\
\hline 2.197 & 4.394 & 4.394 \\
\hline 2.197 & 15.379 & 37.349 \\
\hline 173.563 & 46.137 & 2.197 \\
\hline 2.197 & 127.426 & 2.197 \\
\hline 13.182 & 35.152 & 2.197 \\
\hline 17.576 & 2.197 & 35.152 \\
\hline 123.032 & 52.728 & 300.989 \\
\hline 116.441 & 4.394 & 123.032 \\
\hline 6.591 & 2.197 & 6.591 \\
\hline
\end{tabular}




\begin{tabular}{|c|c|c|}
\hline 8.788 & 2.197 & 325.156 \\
\hline 8.788 & 2.197 & 590.993 \\
\hline 98.865 & 2.197 & 1348.96 \\
\hline 6.591 & 2.197 & 105.456 \\
\hline 2.197 & 2.197 & 37.349 \\
\hline 32.955 & 10.985 & 4.394 \\
\hline 24.167 & 2.197 & 13.182 \\
\hline 2.197 & 19.773 & 4.394 \\
\hline 6.591 & 2.197 & 4.394 \\
\hline 2.197 & 2.197 & 2.197 \\
\hline 2.197 & 2.197 & 138.411 \\
\hline 654.706 & 10.985 & 927.134 \\
\hline 4.394 & 2.197 & 4.394 \\
\hline 2.197 & 2.197 & 2.197 \\
\hline 32.955 & 2.197 & 2.197 \\
\hline 19.773 & 4.394 & 859.027 \\
\hline 2.197 & 4.394 & 2.197 \\
\hline 6.591 & 37.349 & 65.91 \\
\hline 4.394 & 8.788 & 15.379 \\
\hline 6.591 & 101.062 & 35.152 \\
\hline 4.394 & 162.578 & 68.107 \\
\hline 30.758 & 4.394 & 705.237 \\
\hline 8.788 & 8.788 & 15.379 \\
\hline 2.197 & 15.379 & 70.304 \\
\hline 48.334 & 13.182 & 149.396 \\
\hline 4.394 & 19.773 & 70.304 \\
\hline 10.985 & 2.197 & 8.788 \\
\hline 8.788 & 2.197 & 226.291 \\
\hline 248.261 & 2.197 & 26.364 \\
\hline 2.197 & 2.197 & 105.456 \\
\hline 28.561 & 101.062 & 224.094 \\
\hline 28.561 & 149.396 & 4.394 \\
\hline 13.182 & 2.197 & 2.197 \\
\hline 32.955 & 2.197 & 2.197 \\
\hline 4.394 & 8.788 & 2.197 \\
\hline 2.197 & 2.197 & 2.197 \\
\hline 2.197 & 6.591 & 8.788 \\
\hline 4.394 & 2.197 & 2.197 \\
\hline 19.773 & 2.197 & 149.396 \\
\hline 4.394 & 92.274 & 2.197 \\
\hline 134.017 & 2.197 & 1685.1 \\
\hline 6.591 & 4.394 & 2.197 \\
\hline 6.591 & 10.985 & 81.289 \\
\hline 2.197 & 2.197 & 4.394 \\
\hline 202.124 & 6.591 & 26.364 \\
\hline 26.364 & 2.197 & 2.197 \\
\hline 15.379 & 15.379 & 2.197 \\
\hline 6.591 & 2.197 & 2.197 \\
\hline 4.394 & 2.197 & 2.197 \\
\hline 30.758 & 2.197 & 39.546 \\
\hline
\end{tabular}




\begin{tabular}{|c|c|c|}
\hline 8.788 & 8.788 & 2293.67 \\
\hline 46.137 & 2.197 & 10.985 \\
\hline 2.197 & 24.167 & 4.394 \\
\hline 2.197 & 26.364 & 2.197 \\
\hline 6.591 & 2.197 & 2.197 \\
\hline 2.197 & 28.561 & 4.394 \\
\hline 19.773 & 2.197 & 2.197 \\
\hline 913.952 & 8.788 & 10.985 \\
\hline 13.182 & 2.197 & 208.715 \\
\hline 2.197 & 6.591 & 30.758 \\
\hline 6.591 & 13.182 & 8.788 \\
\hline 2.197 & 10.985 & 2.197 \\
\hline 6.591 & 17.576 & 4.394 \\
\hline 17.576 & 2.197 & 43.94 \\
\hline 10.985 & 2.197 & 2.197 \\
\hline 48.334 & 17.576 & 35.152 \\
\hline 30.758 & 13.182 & 307.58 \\
\hline 81.289 & 4.394 & 24.167 \\
\hline 169.169 & 6.591 & 94.471 \\
\hline 114.244 & 4.394 & 142.805 \\
\hline 4.394 & 2.197 & 10.985 \\
\hline 13.182 & 4.394 & 6.591 \\
\hline 2.197 & 2.197 & 4.394 \\
\hline 10.985 & 17.576 & 32.955 \\
\hline 8.788 & 13.182 & 2.197 \\
\hline 17.576 & 4.394 & 4.394 \\
\hline 2.197 & 2.197 & 19.773 \\
\hline 10.985 & 305.383 & 15.379 \\
\hline 8.788 & 4.394 & 175.76 \\
\hline 6.591 & 2.197 & 4.394 \\
\hline 17.576 & 2.197 & 85.683 \\
\hline 13.182 & 246.064 & 4.394 \\
\hline 8.788 & 15.379 & 2.197 \\
\hline 81.289 & 2.197 & 21.97 \\
\hline 276.822 & 2.197 & 2.197 \\
\hline 6.591 & 2.197 & 2.197 \\
\hline 54.925 & 4.394 & 2.197 \\
\hline 2.197 & 4.394 & 2.197 \\
\hline 2.197 & 4.394 & 13.182 \\
\hline 4.394 & 10.985 & 2.197 \\
\hline 39.546 & 6.591 & 2.197 \\
\hline 8.788 & 17.576 & 26.364 \\
\hline 19.773 & 6.591 & 6.591 \\
\hline 4.394 & 81.289 & 2.197 \\
\hline 4.394 & 6.591 & 8.788 \\
\hline 2.197 & 2.197 & 21.97 \\
\hline 50.531 & 384.475 & 21.97 \\
\hline 6.591 & 43.94 & 2.197 \\
\hline 4.394 & 2.197 & 6.591 \\
\hline 424.021 & 4.394 & 2.197 \\
\hline
\end{tabular}




\begin{tabular}{|c|c|c|}
\hline 13.182 & 2.197 & 2.197 \\
\hline 41.743 & 300.989 & 2.197 \\
\hline 19.773 & 2.197 & 2.197 \\
\hline 46.137 & 10.985 & 206.518 \\
\hline 15.379 & 24.167 & 19.773 \\
\hline 116.441 & 54.925 & 4.394 \\
\hline 8.788 & 4.394 & 15.379 \\
\hline 24.167 & 57.122 & 8.788 \\
\hline 6.591 & 393.263 & 2.197 \\
\hline 2.197 & 35.152 & 2.197 \\
\hline 32.955 & 2.197 & 2.197 \\
\hline 19.773 & 6.591 & 2.197 \\
\hline 6.591 & 2.197 & 46.137 \\
\hline 2.197 & 8.788 & 4.394 \\
\hline 4.394 & 2.197 & 10.985 \\
\hline 6.591 & 19.773 & 2.197 \\
\hline 129.623 & 2.197 & 2.197 \\
\hline 109.85 & 2.197 & 13.182 \\
\hline 37.349 & 2.197 & 4.394 \\
\hline 298.792 & 2.197 & 10.985 \\
\hline 13.182 & 10.985 & 17.576 \\
\hline 4.394 & 4.394 & 10.985 \\
\hline 46.137 & 43.94 & 10.985 \\
\hline 103.259 & 46.137 & 2.197 \\
\hline 175.76 & 2.197 & 52.728 \\
\hline 39.546 & 2.197 & 13.182 \\
\hline 2.197 & 2.197 & 13.182 \\
\hline 4.394 & 21.97 & 2.197 \\
\hline 2.197 & 46.137 & 19948.8 \\
\hline 2.197 & 2.197 & 4.394 \\
\hline 26.364 & 2.197 & 17.576 \\
\hline 8.788 & 4.394 & 2.197 \\
\hline 2.197 & 2.197 & 103.259 \\
\hline 4.394 & 61.516 & 32.955 \\
\hline 15.379 & 2.197 & 2.197 \\
\hline 52.728 & 2.197 & 173.563 \\
\hline 2.197 & 2.197 & 13.182 \\
\hline 2.197 & 2.197 & 6.591 \\
\hline 2.197 & 2.197 & 2.197 \\
\hline 92.274 & 94.471 & 8.788 \\
\hline 4.394 & 13.182 & 79.092 \\
\hline 4.394 & 24.167 & 204.321 \\
\hline 10.985 & 2.197 & 15.379 \\
\hline 2.197 & 8.788 & 2.197 \\
\hline 188.942 & 19.773 & 2.197 \\
\hline 28.561 & 2.197 & 35.152 \\
\hline 129.623 & 219.7 & 175.76 \\
\hline 4.394 & 30.758 & 8.788 \\
\hline 65.91 & 8.788 & 2.197 \\
\hline 588.796 & 4.394 & 219.7 \\
\hline
\end{tabular}




\begin{tabular}{|c|c|c|}
\hline 10.985 & 4.394 & 6.591 \\
\hline 329.55 & 8.788 & 4.394 \\
\hline 50.531 & 6.591 & 10.985 \\
\hline 4.394 & 13034.8 & 2.197 \\
\hline 2.197 & 15.379 & 2.197 \\
\hline 2.197 & 68.107 & 2.197 \\
\hline 26.364 & 8.788 & 2.197 \\
\hline 13.182 & 120.835 & 41.743 \\
\hline 41.743 & 283.413 & 6.591 \\
\hline 2.197 & 37.349 & 2.197 \\
\hline 35.152 & 17.576 & 10.985 \\
\hline 2.197 & 10.985 & 228.488 \\
\hline 28.561 & 2.197 & 6.591 \\
\hline 28.561 & 395.46 & 500.916 \\
\hline 149.396 & 2.197 & 8.788 \\
\hline 19.773 & 10.985 & 4.394 \\
\hline 2.197 & 83.486 & 13.182 \\
\hline 2.197 & 2.197 & 2.197 \\
\hline 96.668 & 65.91 & 90.077 \\
\hline 17.576 & 70.304 & 21.97 \\
\hline 2.197 & 2.197 & 37.349 \\
\hline 6.591 & 17.576 & 2.197 \\
\hline 2.197 & 4.394 & 6.591 \\
\hline 4.394 & 2.197 & 2.197 \\
\hline 2.197 & 944.71 & 2.197 \\
\hline 26.364 & 2.197 & 21.97 \\
\hline 6.591 & 6.591 & 8.788 \\
\hline 2.197 & 4.394 & 8.788 \\
\hline 2.197 & 15.379 & 13.182 \\
\hline 4.394 & 2.197 & 2.197 \\
\hline 8.788 & 276.822 & 24.167 \\
\hline 6.591 & 50.531 & 2.197 \\
\hline 46.137 & 4.394 & 123.032 \\
\hline 10.985 & 560.235 & 8.788 \\
\hline 26.364 & 118.638 & 232.882 \\
\hline 2.197 & 13.182 & 10.985 \\
\hline 2.197 & 2.197 & 43.94 \\
\hline 2.197 & 6.591 & 24.167 \\
\hline 50.531 & 8.788 & 46.137 \\
\hline 8.788 & 2.197 & 17.576 \\
\hline 6.591 & 129.623 & 4.394 \\
\hline 123.032 & 2.197 & 6.591 \\
\hline 2.197 & 2.197 & 79.092 \\
\hline 2.197 & 4.394 & 6.591 \\
\hline 65.91 & 4.394 & 6.591 \\
\hline 6.591 & 17.576 & 13.182 \\
\hline 2.197 & 6.591 & 4.394 \\
\hline 2.197 & 4.394 & 13.182 \\
\hline 24.167 & 2.197 & 54.925 \\
\hline 2.197 & 2.197 & 2.197 \\
\hline
\end{tabular}




\begin{tabular}{|c|c|c|}
\hline 2.197 & 2.197 & 2.197 \\
\hline 6.591 & 4.394 & 2.197 \\
\hline 197.73 & 6.591 & 4.394 \\
\hline 8.788 & 2.197 & 2.197 \\
\hline 30.758 & 2.197 & 54.925 \\
\hline 43.94 & 10.985 & 463.567 \\
\hline 6.591 & 74.698 & 2.197 \\
\hline 41.743 & 2.197 & 4.394 \\
\hline 6.591 & 37.349 & 15.379 \\
\hline 2.197 & 8.788 & 2.197 \\
\hline 2.197 & 6.591 & 4.394 \\
\hline 8.788 & 2.197 & 4.394 \\
\hline 2.197 & 105.456 & 626.145 \\
\hline 6.591 & 4.394 & 4.394 \\
\hline 4.394 & 2.197 & 32.955 \\
\hline 307.58 & 230.685 & 4.394 \\
\hline 4.394 & 13.182 & 10.985 \\
\hline 129.623 & 19.773 & 59.319 \\
\hline 61.516 & 10.985 & 48.334 \\
\hline 6.591 & 24.167 & 6.591 \\
\hline 8.788 & 21.97 & 10.985 \\
\hline 2.197 & 13.182 & 6.591 \\
\hline 15.379 & 10.985 & 2.197 \\
\hline 2.197 & 6.591 & 2.197 \\
\hline 173.563 & 8.788 & 57.122 \\
\hline 2.197 & 4.394 & 10.985 \\
\hline 2.197 & 2.197 & 4.394 \\
\hline 2.197 & 2.197 & 15.379 \\
\hline 4.394 & 92.274 & 8.788 \\
\hline 2.197 & 50.531 & 37.349 \\
\hline 2.197 & 15.379 & 19.773 \\
\hline 2.197 & 2.197 & 246.064 \\
\hline 8.788 & 10.985 & 142.805 \\
\hline 2.197 & 2.197 & 10.985 \\
\hline 6.591 & 2.197 & 2482.61 \\
\hline 2.197 & 17.576 & 4.394 \\
\hline 421.824 & 2.197 & 10.985 \\
\hline 17.576 & 2.197 & 26.364 \\
\hline 15.379 & 4.394 & 2.197 \\
\hline 17.576 & 52.728 & 30.758 \\
\hline 4.394 & 2.197 & 21.97 \\
\hline 13.182 & 2.197 & 2.197 \\
\hline 4.394 & 2.197 & 6.591 \\
\hline 17.576 & 2.197 & 2.197 \\
\hline 15.379 & 2.197 & 4.394 \\
\hline 6.591 & 6.591 & 4.394 \\
\hline 131.82 & 8.788 & 10.985 \\
\hline 8.788 & 1010.62 & 6.591 \\
\hline 2.197 & 2.197 & 17.576 \\
\hline 109.85 & 2.197 & 57.122 \\
\hline
\end{tabular}




\begin{tabular}{|c|c|c|}
\hline 21.97 & 1036.98 & 2.197 \\
\hline 2.197 & 4.394 & 2.197 \\
\hline 8.788 & 41.743 & 6.591 \\
\hline 2.197 & 2.197 & 2.197 \\
\hline 2.197 & 15.379 & 2.197 \\
\hline 272.428 & 2.197 & 17.576 \\
\hline 2.197 & 197.73 & 10.985 \\
\hline 81.289 & 2.197 & 4.394 \\
\hline 15.379 & 6.591 & 17.576 \\
\hline 4.394 & 248.261 & 4.394 \\
\hline 2.197 & 50.531 & 123.032 \\
\hline 2.197 & 24.167 & 2.197 \\
\hline 103.259 & 17.576 & 17.576 \\
\hline 155.987 & 2.197 & 2.197 \\
\hline 2.197 & 4.394 & 48.334 \\
\hline 35.152 & 2.197 & 10.985 \\
\hline 4.394 & 2.197 & 4.394 \\
\hline 32.955 & 2.197 & 17.576 \\
\hline 120.835 & 2.197 & 4.394 \\
\hline 6.591 & 123.032 & 8.788 \\
\hline 2.197 & 32.955 & 2.197 \\
\hline 10.985 & 2.197 & 4.394 \\
\hline 63.713 & 10.985 & 2.197 \\
\hline 2.197 & 39.546 & 4.394 \\
\hline 103.259 & 6.591 & 74.698 \\
\hline 8.788 & 2.197 & 41.743 \\
\hline 2.197 & 21.97 & 2.197 \\
\hline 15.379 & 30.758 & 4.394 \\
\hline 41.743 & 2.197 & 2.197 \\
\hline 4.394 & 246.064 & 70.304 \\
\hline 61.516 & 8.788 & 6.591 \\
\hline 2.197 & 4.394 & 61.516 \\
\hline 17.576 & 4.394 & 145.002 \\
\hline 2.197 & 406.445 & 28.561 \\
\hline 50.531 & 32.955 & 57.122 \\
\hline 4.394 & 6.591 & 4.394 \\
\hline 10.985 & 4.394 & 87.88 \\
\hline 94.471 & 15.379 & 70.304 \\
\hline 17.576 & 57.122 & 4.394 \\
\hline 2.197 & 37.349 & 123.032 \\
\hline 15.379 & 2.197 & 39.546 \\
\hline 2.197 & 2.197 & 6.591 \\
\hline 2.197 & 4.394 & 456.976 \\
\hline 2.197 & 6.591 & 2528.75 \\
\hline 6.591 & 2.197 & 4.394 \\
\hline 15.379 & 6.591 & 4.394 \\
\hline 85.683 & 136.214 & 2.197 \\
\hline 2.197 & 2.197 & 28.561 \\
\hline 41.743 & 17.576 & 28.561 \\
\hline 13.182 & 2.197 & 13.182 \\
\hline
\end{tabular}




\begin{tabular}{|c|c|c|}
\hline 206.518 & 2.197 & 92.274 \\
\hline 13.182 & 10.985 & 2.197 \\
\hline 6.591 & 316.368 & 32.955 \\
\hline 8.788 & 6.591 & 4.394 \\
\hline 8.788 & 2.197 & 2.197 \\
\hline 2.197 & 6.591 & 4.394 \\
\hline 2.197 & 6.591 & 6.591 \\
\hline 13.182 & 19.773 & 4.394 \\
\hline 125.229 & 4.394 & 35.152 \\
\hline 6.591 & 15.379 & 2.197 \\
\hline 2.197 & 4.394 & 19.773 \\
\hline 4.394 & 2.197 & 151.593 \\
\hline 57.122 & 10.985 & 4.394 \\
\hline 21.97 & 68.107 & 2.197 \\
\hline 2.197 & 35.152 & 2.197 \\
\hline 134.017 & 2190.41 & 358.111 \\
\hline 2.197 & 2.197 & 2.197 \\
\hline 4.394 & 6.591 & 13.182 \\
\hline 48.334 & 2.197 & 4.394 \\
\hline 10.985 & 10.985 & 79.092 \\
\hline 6.591 & 171.366 & 35.152 \\
\hline 8.788 & 2.197 & 41.743 \\
\hline 10.985 & 2.197 & 32.955 \\
\hline 6.591 & 909.558 & 10.985 \\
\hline 2.197 & 76.895 & 59.319 \\
\hline 2.197 & 2.197 & 8.788 \\
\hline 4.394 & 15.379 & 37.349 \\
\hline 138.411 & 3822.78 & 76.895 \\
\hline 2.197 & 2.197 & 24.167 \\
\hline 2.197 & 4.394 & 4.394 \\
\hline 2.197 & 2.197 & 2.197 \\
\hline 28.561 & 2.197 & 4.394 \\
\hline 6.591 & 217.503 & 15.379 \\
\hline 2.197 & 2.197 & 320.762 \\
\hline 900.77 & 2.197 & 35.152 \\
\hline 6.591 & 2.197 & 68.107 \\
\hline 37.349 & 2.197 & 2.197 \\
\hline 2.197 & 2.197 & 2.197 \\
\hline 123.032 & 2.197 & 2.197 \\
\hline 70.304 & 2.197 & 24.167 \\
\hline 4.394 & 2.197 & 2.197 \\
\hline 232.882 & 2.197 & 8.788 \\
\hline 2.197 & 79.092 & 204.321 \\
\hline 17.576 & 2.197 & 96.668 \\
\hline 6.591 & 2.197 & 15.379 \\
\hline 94.471 & 2.197 & 26.364 \\
\hline 17.576 & 2.197 & 204.321 \\
\hline 8.788 & 2.197 & 6.591 \\
\hline 4.394 & 43.94 & 13.182 \\
\hline 2.197 & 254.852 & 8.788 \\
\hline
\end{tabular}




\begin{tabular}{|c|c|c|}
\hline 2.197 & 496.522 & 2.197 \\
\hline 2.197 & 2.197 & 2.197 \\
\hline 10.985 & 2.197 & 2.197 \\
\hline 6.591 & 2.197 & 8.788 \\
\hline 6.591 & 129.623 & 4.394 \\
\hline 2.197 & 2.197 & 1063.35 \\
\hline 125.229 & 2.197 & 19.773 \\
\hline 8.788 & 166.972 & 4.394 \\
\hline 6.591 & 17.576 & 32.955 \\
\hline 2.197 & 37.349 & 384.475 \\
\hline 13.182 & 13.182 & 8.788 \\
\hline 59.319 & 4.394 & 2.197 \\
\hline 2.197 & 6.591 & 39.546 \\
\hline 4.394 & 19.773 & 41.743 \\
\hline 2.197 & 2.197 & 2.197 \\
\hline 2.197 & 2.197 & 30.758 \\
\hline 6.591 & 204.321 & 169.169 \\
\hline 2.197 & 21.97 & 10.985 \\
\hline 2.197 & 10.985 & 63.713 \\
\hline 4.394 & 54.925 & 8.788 \\
\hline 17.576 & 2.197 & 808.496 \\
\hline 65.91 & 6.591 & 193.336 \\
\hline 4.394 & 4.394 & 8.788 \\
\hline 48.334 & 2.197 & 10.985 \\
\hline 94.471 & 4.394 & 13.182 \\
\hline 13.182 & 145.002 & 2.197 \\
\hline 101.062 & 2.197 & 8.788 \\
\hline 2.197 & 2.197 & 8.788 \\
\hline 2.197 & 6.591 & 13.182 \\
\hline 2.197 & 2.197 & 10.985 \\
\hline 30.758 & 13.182 & 46.137 \\
\hline 6.591 & 2.197 & 2.197 \\
\hline 13.182 & 2.197 & 52.728 \\
\hline 10.985 & 2.197 & 4.394 \\
\hline 2.197 & 107.653 & 54.925 \\
\hline 10.985 & 2.197 & 21.97 \\
\hline 32.955 & 4.394 & 6.591 \\
\hline 105.456 & 210.912 & 6.591 \\
\hline 193.336 & 43.94 & 2.197 \\
\hline 6.591 & 246.064 & 2.197 \\
\hline 10.985 & 4.394 & 2.197 \\
\hline 15.379 & 4.394 & 15.379 \\
\hline 24.167 & 120.835 & 155.987 \\
\hline 70.304 & 276.822 & 4.394 \\
\hline 2.197 & 19.773 & 2.197 \\
\hline 2.197 & 32.955 & 2.197 \\
\hline 6.591 & 2.197 & 2.197 \\
\hline 2.197 & 4.394 & 2.197 \\
\hline 4.394 & 2.197 & 19.773 \\
\hline 13.182 & 85.683 & 10.985 \\
\hline
\end{tabular}




\begin{tabular}{|c|c|c|}
\hline 6.591 & 2.197 & 48.334 \\
\hline 8.788 & 118.638 & 2.197 \\
\hline 10.985 & 26.364 & 28.561 \\
\hline 2.197 & 10.985 & 8.788 \\
\hline 2.197 & 83.486 & 2.197 \\
\hline 4.394 & 39.546 & 15.379 \\
\hline 107.653 & 6.591 & 265.837 \\
\hline 2.197 & 76.895 & 2.197 \\
\hline 30.758 & 2.197 & 6.591 \\
\hline 4.394 & 2.197 & 63.713 \\
\hline 17.576 & 2.197 & 19.773 \\
\hline 2.197 & 2.197 & 48.334 \\
\hline 15.379 & 2.197 & 41.743 \\
\hline 6.591 & 2.197 & 2.197 \\
\hline 17.576 & 2.197 & 2.197 \\
\hline 2.197 & 784.329 & 19.773 \\
\hline 2.197 & 15.379 & 39.546 \\
\hline 377.884 & 30.758 & 26.364 \\
\hline 4.394 & 43.94 & 883.194 \\
\hline 13.182 & 6.591 & 72.501 \\
\hline 15.379 & 6.591 & 61.516 \\
\hline 13.182 & 2.197 & 15.379 \\
\hline 2.197 & 10.985 & 10.985 \\
\hline 4.394 & 96.668 & 8.788 \\
\hline 2.197 & 3244.97 & 2043.21 \\
\hline 2.197 & 19.773 & 6.591 \\
\hline 2.197 & 2.197 & 2.197 \\
\hline 19.773 & 1111.68 & 105.456 \\
\hline 2.197 & 2.197 & 4.394 \\
\hline 2.197 & 10.985 & 15466.9 \\
\hline 19.773 & 2.197 & 10.985 \\
\hline 4.394 & 2.197 & 26.364 \\
\hline 15.379 & 4.394 & 13.182 \\
\hline 35.152 & 2.197 & 72.501 \\
\hline 10.985 & 81.289 & 281.216 \\
\hline 6.591 & 2.197 & 2.197 \\
\hline 2.197 & 2.197 & 2.197 \\
\hline 6.591 & 98.865 & 116.441 \\
\hline 17.576 & 24.167 & 2.197 \\
\hline 43.94 & 37.349 & 4.394 \\
\hline 4.394 & 59.319 & 15.379 \\
\hline 59.319 & 2.197 & 485.537 \\
\hline 98.865 & 21.97 & 19.773 \\
\hline 6.591 & 15.379 & 1135.85 \\
\hline 6.591 & 4.394 & 2.197 \\
\hline 4.394 & 52.728 & 70.304 \\
\hline 35.152 & 2.197 & 2.197 \\
\hline 2.197 & 2.197 & 2.197 \\
\hline 26.364 & 6.591 & 2.197 \\
\hline 17.576 & 2.197 & 2.197 \\
\hline
\end{tabular}




\begin{tabular}{|c|c|c|}
\hline 4.394 & 2.197 & 2.197 \\
\hline 2.197 & 2.197 & 6.591 \\
\hline 2.197 & 4.394 & 59.319 \\
\hline 43.94 & 24.167 & 24.167 \\
\hline 6.591 & 35.152 & 2.197 \\
\hline 10.985 & 10.985 & 107.653 \\
\hline 2.197 & 4.394 & 68.107 \\
\hline 32.955 & 52.728 & 2.197 \\
\hline 2.197 & 57.122 & 2.197 \\
\hline 4.394 & 2.197 & 2.197 \\
\hline 2.197 & 24.167 & 8.788 \\
\hline 2.197 & 2.197 & 4.394 \\
\hline 2.197 & 2.197 & 6.591 \\
\hline 103.259 & 2.197 & 83.486 \\
\hline 50.531 & 8.788 & 4.394 \\
\hline 6.591 & 4.394 & 4.394 \\
\hline 6.591 & 2.197 & 2.197 \\
\hline 59.319 & 39.546 & 10.985 \\
\hline 8.788 & 19.773 & 41.743 \\
\hline 2.197 & 6.591 & 2.197 \\
\hline 2.197 & 4.394 & 2.197 \\
\hline 4.394 & 28.561 & 37.349 \\
\hline 17.576 & 19.773 & 48.334 \\
\hline 2.197 & 17.576 & 52.728 \\
\hline 15.379 & 43.94 & 21.97 \\
\hline 8.788 & 6.591 & 145.002 \\
\hline 10.985 & 2.197 & 2.197 \\
\hline 8.788 & 2.197 & 2.197 \\
\hline 145.002 & 2.197 & 2.197 \\
\hline 26.364 & 2.197 & 30.758 \\
\hline 4.394 & 37.349 & 28.561 \\
\hline 151.593 & 184.548 & 4.394 \\
\hline 68.107 & 8.788 & 270.231 \\
\hline 19.773 & 96.668 & 70.304 \\
\hline 2.197 & 2.197 & 50.531 \\
\hline 92.274 & 30.758 & 10.985 \\
\hline 2.197 & 4.394 & 76.895 \\
\hline 2.197 & 6.591 & 59.319 \\
\hline 6.591 & 2.197 & 15.379 \\
\hline 15.379 & 50.531 & 52.728 \\
\hline 32.955 & 2.197 & 4.394 \\
\hline 2.197 & 749.177 & 652.509 \\
\hline 2.197 & 290.004 & 6.591 \\
\hline 2.197 & 2.197 & 19.773 \\
\hline 2.197 & 2.197 & 4.394 \\
\hline 65.91 & 21.97 & 26.364 \\
\hline 2.197 & 17.576 & 39.546 \\
\hline 8.788 & 13.182 & 21.97 \\
\hline 123.032 & 94.471 & 37.349 \\
\hline 2.197 & 4.394 & 4.394 \\
\hline
\end{tabular}




\begin{tabular}{|c|c|c|}
\hline 2.197 & 217.503 & 2.197 \\
\hline 24.167 & 68.107 & 134.017 \\
\hline 2.197 & 2.197 & 158.184 \\
\hline 2.197 & 26.364 & 4.394 \\
\hline 24.167 & 193.336 & 21.97 \\
\hline 8.788 & 2.197 & 43.94 \\
\hline 2.197 & 8.788 & 10.985 \\
\hline 2.197 & 2.197 & 39.546 \\
\hline 2.197 & 2.197 & 26.364 \\
\hline 2.197 & 2.197 & 63.713 \\
\hline 10.985 & 4.394 & 2.197 \\
\hline 15.379 & 2.197 & 309.777 \\
\hline 2.197 & 4.394 & 54.925 \\
\hline 6.591 & 6.591 & 206.518 \\
\hline 2.197 & 257.049 & 103.259 \\
\hline 2.197 & 98.865 & 2.197 \\
\hline 195.533 & 2.197 & 26.364 \\
\hline 26.364 & 2.197 & 2.197 \\
\hline 2.197 & 4.394 & 2.197 \\
\hline 2.197 & 4.394 & 6.591 \\
\hline 2.197 & 21.97 & 6.591 \\
\hline 2.197 & 19.773 & 10.985 \\
\hline 2.197 & 8.788 & 76.895 \\
\hline 24.167 & 2.197 & 114.244 \\
\hline 2.197 & 127.426 & 13.182 \\
\hline 2.197 & 19.773 & 54.925 \\
\hline 2.197 & 2.197 & 140.608 \\
\hline 17.576 & 6.591 & 188.942 \\
\hline 8.788 & 112.047 & 2.197 \\
\hline 15.379 & 2.197 & 2.197 \\
\hline 81.289 & 48.334 & 15.379 \\
\hline 74.698 & 10.985 & 6.591 \\
\hline 63.713 & 4.394 & 24.167 \\
\hline 2.197 & 15.379 & 35.152 \\
\hline 2.197 & 2.197 & 68.107 \\
\hline 6.591 & 4.394 & 6.591 \\
\hline 6.591 & 2.197 & 26.364 \\
\hline 6.591 & 85.683 & 4.394 \\
\hline 65.91 & 2.197 & 68.107 \\
\hline 2.197 & 2.197 & 2.197 \\
\hline 6.591 & 6.591 & 46.137 \\
\hline 2.197 & 2.197 & 2.197 \\
\hline 2.197 & 2.197 & 6.591 \\
\hline 68.107 & 2.197 & 26.364 \\
\hline 19.773 & 6.591 & 8.788 \\
\hline 2.197 & 30.758 & 4.394 \\
\hline 2.197 & 2.197 & 2.197 \\
\hline 2.197 & 2.197 & 13.182 \\
\hline 2.197 & 26.364 & 2.197 \\
\hline 19.773 & 4.394 & 2.197 \\
\hline
\end{tabular}




\begin{tabular}{|c|c|c|}
\hline 8.788 & 2.197 & 2.197 \\
\hline 2.197 & 2.197 & 15.379 \\
\hline 2.197 & 116.441 & 2.197 \\
\hline 2.197 & 2.197 & 10.985 \\
\hline 2.197 & 2.197 & 48.334 \\
\hline 28.561 & 2.197 & 21.97 \\
\hline 6.591 & 4.394 & 2.197 \\
\hline 4.394 & 81.289 & 580.008 \\
\hline 19.773 & 17.576 & 586.599 \\
\hline 6.591 & 2.197 & 109.85 \\
\hline 19.773 & 2.197 & 59.319 \\
\hline 2.197 & 153.79 & 41.743 \\
\hline 21.97 & 2.197 & 10.985 \\
\hline 15.379 & 4.394 & 193.336 \\
\hline 32.955 & 2.197 & 160.381 \\
\hline 2.197 & 92.274 & 17.576 \\
\hline 4.394 & 2.197 & 26.364 \\
\hline 4.394 & 6.591 & 2.197 \\
\hline 4.394 & 2.197 & 2.197 \\
\hline 2.197 & 4.394 & 4.394 \\
\hline 2.197 & 2.197 & 191.139 \\
\hline 21.97 & 8.788 & 4.394 \\
\hline 6.591 & 21.97 & 35.152 \\
\hline 2.197 & 10.985 & 114.244 \\
\hline 19.773 & 2.197 & 30.758 \\
\hline 8.788 & 4.394 & 32.955 \\
\hline 26.364 & 4.394 & 2.197 \\
\hline 15.379 & 2315.64 & 6.591 \\
\hline 2.197 & 2.197 & 35.152 \\
\hline 54.925 & 2.197 & 6.591 \\
\hline 6.591 & 81.289 & 6.591 \\
\hline 4.394 & 2.197 & 202.124 \\
\hline 2.197 & 2.197 & 30.758 \\
\hline 6.591 & 4.394 & 1381.91 \\
\hline 19.773 & 1232.52 & 415.233 \\
\hline 28.561 & 2.197 & 4.394 \\
\hline 4.394 & 2.197 & 85.683 \\
\hline 28.561 & 2.197 & 24.167 \\
\hline 2.197 & 10.985 & 4.394 \\
\hline 2.197 & 6.591 & 63.713 \\
\hline 6.591 & 2.197 & 13.182 \\
\hline 13.182 & 2.197 & 3488.84 \\
\hline 8.788 & 4.394 & 2.197 \\
\hline 8.788 & 21.97 & 2.197 \\
\hline 63.713 & 2.197 & 4.394 \\
\hline 2.197 & 8.788 & 43.94 \\
\hline 41.743 & 41.743 & 54.925 \\
\hline 21.97 & 76.895 & 19.773 \\
\hline 26.364 & 43.94 & 24.167 \\
\hline 17.576 & 4.394 & 15.379 \\
\hline
\end{tabular}




\begin{tabular}{|c|c|c|}
\hline 2.197 & 4.394 & 206.518 \\
\hline 2.197 & 2.197 & 24.167 \\
\hline 2.197 & 54.925 & 13.182 \\
\hline 8.788 & 2.197 & 4.394 \\
\hline 2.197 & 2.197 & 43.94 \\
\hline 2.197 & 19.773 & 4.394 \\
\hline 6.591 & 105.456 & 2.197 \\
\hline 4.394 & 41.743 & 15.379 \\
\hline 13.182 & 70.304 & 19.773 \\
\hline 13.182 & 21.97 & 210.912 \\
\hline 10.985 & 2.197 & 309.777 \\
\hline 6.591 & 4.394 & 2.197 \\
\hline 10.985 & 6.591 & 2.197 \\
\hline 15.379 & 68.107 & 15.379 \\
\hline 6.591 & 2.197 & 2.197 \\
\hline 13.182 & 37.349 & 19.773 \\
\hline 4.394 & 2.197 & 12248.3 \\
\hline 6.591 & 17.576 & 2.197 \\
\hline 4.394 & 2.197 & 15.379 \\
\hline 6.591 & 4.394 & 2.197 \\
\hline 4.394 & 6.591 & 2.197 \\
\hline 2.197 & 26.364 & 2.197 \\
\hline 2.197 & 2.197 & 6.591 \\
\hline 6.591 & 6.591 & 586.599 \\
\hline 2.197 & 6.591 & 107.653 \\
\hline 8.788 & 2.197 & 4.394 \\
\hline 10.985 & 8.788 & 17.576 \\
\hline 2.197 & 4.394 & 37.349 \\
\hline 19.773 & 138.411 & 2.197 \\
\hline 2.197 & 2.197 & 1267.67 \\
\hline 2.197 & 2.197 & 2.197 \\
\hline 2.197 & 2.197 & 74.698 \\
\hline 2.197 & 2.197 & 4.394 \\
\hline 2.197 & 2.197 & 15.379 \\
\hline 13.182 & 4.394 & 2.197 \\
\hline \multirow[t]{15}{*}{4.394} & 2.197 & 8.788 \\
\hline & 87.88 & 2.197 \\
\hline & 4.394 & 2.197 \\
\hline & 26.364 & 2.197 \\
\hline & 1155.62 & 2.197 \\
\hline & 2.197 & 2.197 \\
\hline & 2.197 & 2.197 \\
\hline & 17833 & 21.97 \\
\hline & 6.591 & 13.182 \\
\hline & 4.394 & 6.591 \\
\hline & 254.852 & 32.955 \\
\hline & 131.82 & 2.197 \\
\hline & 2.197 & 57.122 \\
\hline & 136.214 & 17.576 \\
\hline & 4.394 & 120.835 \\
\hline
\end{tabular}




\begin{tabular}{|c|c|}
\hline 8.788 & 90.077 \\
\hline 4.394 & 50.531 \\
\hline 39.546 & 722.813 \\
\hline 4.394 & 140.608 \\
\hline 4.394 & 15.379 \\
\hline 2.197 & 30.758 \\
\hline 10.985 & 26.364 \\
\hline 21.97 & 2.197 \\
\hline 96.668 & 140.608 \\
\hline 26.364 & 6.591 \\
\hline 2.197 & 13.182 \\
\hline 775.541 & 2.197 \\
\hline 15.379 & 13.182 \\
\hline 37.349 & 2.197 \\
\hline 15.379 & 13.182 \\
\hline 2.197 & 26.364 \\
\hline 4.394 & 15.379 \\
\hline 8.788 & 2.197 \\
\hline 263.64 & 4.394 \\
\hline 28.561 & 2.197 \\
\hline 17.576 & 123.032 \\
\hline 2.197 & 10.985 \\
\hline 26.364 & 8.788 \\
\hline 2.197 & 63.713 \\
\hline 43.94 & 2.197 \\
\hline 2.197 & 28.561 \\
\hline 2.197 & 17.576 \\
\hline 6.591 & 8.788 \\
\hline 2.197 & 26.364 \\
\hline 54.925 & 2.197 \\
\hline 2.197 & 2.197 \\
\hline 2.197 & 70.304 \\
\hline 2.197 & 57.122 \\
\hline 2.197 & 4.394 \\
\hline 15.379 & 10.985 \\
\hline 8.788 & 8.788 \\
\hline 2.197 & 19.773 \\
\hline 2.197 & 2.197 \\
\hline 112.047 & 6.591 \\
\hline 13.182 & 13.182 \\
\hline 19.773 & 101.062 \\
\hline 2.197 & 2.197 \\
\hline 2.197 & 24.167 \\
\hline 24.167 & 4.394 \\
\hline 19.773 & 153.79 \\
\hline 2.197 & 281.216 \\
\hline 4.394 & 4.394 \\
\hline 2.197 & 10.985 \\
\hline 2.197 & 87.88 \\
\hline 4.394 & 823.875 \\
\hline
\end{tabular}




\begin{tabular}{|c|c|}
\hline 32.955 & 2.197 \\
\hline 2.197 & 52.728 \\
\hline 90.077 & 2.197 \\
\hline 2.197 & 6.591 \\
\hline 30.758 & 8.788 \\
\hline 4.394 & 37.349 \\
\hline 24.167 & 2.197 \\
\hline 6.591 & 149.396 \\
\hline 10.985 & 48.334 \\
\hline 6.591 & 50.531 \\
\hline 10.985 & 15.379 \\
\hline 2.197 & 4.394 \\
\hline 13.182 & 4.394 \\
\hline 4.394 & 26.364 \\
\hline 6.591 & 577.811 \\
\hline 6.591 & 134.017 \\
\hline 15.379 & 81.289 \\
\hline 43.94 & 2.197 \\
\hline 43.94 & 2.197 \\
\hline 30.758 & 2.197 \\
\hline 118.638 & 10.985 \\
\hline 15.379 & 17.576 \\
\hline 8.788 & 43.94 \\
\hline 2.197 & 28.561 \\
\hline 10.985 & 6.591 \\
\hline 43.94 & 2062.98 \\
\hline 37.349 & 19.773 \\
\hline 10.985 & 4.394 \\
\hline 2.197 & 2.197 \\
\hline 2.197 & 85.683 \\
\hline 21.97 & 344.929 \\
\hline 2.197 & 26.364 \\
\hline 2.197 & 4268.77 \\
\hline 8.788 & 8.788 \\
\hline 2.197 & 35.152 \\
\hline 46.137 & 13.182 \\
\hline 2.197 & 1045.77 \\
\hline 2.197 & 2.197 \\
\hline 107.653 & 26.364 \\
\hline 2.197 & 46.137 \\
\hline 72.501 & 2.197 \\
\hline 4.394 & 2.197 \\
\hline 4.394 & 4.394 \\
\hline 19.773 & 37.349 \\
\hline 17.576 & 10.985 \\
\hline 43.94 & 4.394 \\
\hline 30.758 & 8.788 \\
\hline 2.197 & 4.394 \\
\hline 2.197 & 2.197 \\
\hline 17.576 & 26.364 \\
\hline
\end{tabular}




\begin{tabular}{|c|c|}
\hline 134.017 & 257.049 \\
\hline 151.593 & 2.197 \\
\hline 2.197 & 10.985 \\
\hline 2.197 & 4.394 \\
\hline 2.197 & 10.985 \\
\hline 2.197 & 2.197 \\
\hline 2.197 & 2.197 \\
\hline 2.197 & 96.668 \\
\hline 2.197 & 41.743 \\
\hline 17.576 & 525.083 \\
\hline 17.576 & 2.197 \\
\hline 2.197 & 8.788 \\
\hline 4.394 & 142.805 \\
\hline 6.591 & 15.379 \\
\hline 59.319 & 50.531 \\
\hline 2.197 & 83.486 \\
\hline 4.394 & 32.955 \\
\hline 2.197 & 30.758 \\
\hline 2.197 & 61.516 \\
\hline 19.773 & 2.197 \\
\hline 2.197 & 353.717 \\
\hline 2.197 & 92.274 \\
\hline 6.591 & 8.788 \\
\hline 2.197 & 2.197 \\
\hline 30.758 & 175.76 \\
\hline 2.197 & 6.591 \\
\hline 74.698 & 2.197 \\
\hline 10.985 & 17.576 \\
\hline 2.197 & 46.137 \\
\hline 2.197 & 28.561 \\
\hline 2.197 & 8.788 \\
\hline 2.197 & 21.97 \\
\hline 2.197 & 26.364 \\
\hline 2.197 & 32.955 \\
\hline 1160.02 & 2.197 \\
\hline 2.197 & 15.379 \\
\hline 2.197 & 32.955 \\
\hline 2.197 & 2.197 \\
\hline 17.576 & 2.197 \\
\hline 2.197 & 4.394 \\
\hline 2.197 & 2.197 \\
\hline 2.197 & 4.394 \\
\hline 10.985 & 13333.6 \\
\hline 4.394 & 2.197 \\
\hline 4.394 & 6.591 \\
\hline 2.197 & 2.197 \\
\hline 149.396 & 601.978 \\
\hline 39.546 & 4.394 \\
\hline 46.137 & 4.394 \\
\hline 15.379 & 4.394 \\
\hline
\end{tabular}




\begin{tabular}{|c|c|}
\hline 35.152 & 70.304 \\
\hline 2.197 & 19.773 \\
\hline 2.197 & 2.197 \\
\hline 2.197 & 6.591 \\
\hline 2.197 & 4.394 \\
\hline 2.197 & 21.97 \\
\hline 2.197 & 37.349 \\
\hline 81.289 & 2.197 \\
\hline 8.788 & 270.231 \\
\hline 17.576 & 2.197 \\
\hline 2.197 & 4.394 \\
\hline 114.244 & 2.197 \\
\hline 19.773 & 2.197 \\
\hline 2.197 & 15.379 \\
\hline 26.364 & 19.773 \\
\hline 74.698 & 2.197 \\
\hline 17.576 & 129.623 \\
\hline 2.197 & 92.274 \\
\hline 2.197 & 6.591 \\
\hline 360.308 & 6.591 \\
\hline 2.197 & 4.394 \\
\hline 46.137 & 101.062 \\
\hline 101.062 & 2.197 \\
\hline 320.762 & 15.379 \\
\hline 2.197 & 118.638 \\
\hline 6.591 & 2.197 \\
\hline 6.591 & 87.88 \\
\hline 2.197 & 17.576 \\
\hline 19.773 & 2.197 \\
\hline 2.197 & 13.182 \\
\hline 2.197 & 2.197 \\
\hline 8.788 & 235.079 \\
\hline 15.379 & 15.379 \\
\hline 2.197 & 74.698 \\
\hline 107.653 & 215.306 \\
\hline 2.197 & 19.773 \\
\hline 2.197 & 21.97 \\
\hline 2.197 & 204.321 \\
\hline 2.197 & 26.364 \\
\hline 21.97 & 4.394 \\
\hline 26.364 & 68.107 \\
\hline 4.394 & 2.197 \\
\hline 8.788 & 15.379 \\
\hline 6.591 & 2.197 \\
\hline 2.197 & 4.394 \\
\hline 270.231 & 606.372 \\
\hline 6.591 & 72.501 \\
\hline 13.182 & 2.19 \\
\hline 105.456 & 2.197 \\
\hline 2.197 & 177.957 \\
\hline
\end{tabular}




\begin{tabular}{|c|c|}
\hline 2.197 & 296.595 \\
\hline 37.349 & 2.197 \\
\hline 149.396 & 2.197 \\
\hline 2.197 & 2.197 \\
\hline 2.197 & 2.197 \\
\hline 4.394 & 13.182 \\
\hline 2.197 & 54.925 \\
\hline 17.576 & 13.182 \\
\hline 13.182 & 2.197 \\
\hline 10.985 & 2.197 \\
\hline 13.182 & 2.197 \\
\hline 162.578 & 4.394 \\
\hline 2.197 & 4.394 \\
\hline 2.197 & 13.182 \\
\hline 2.197 & 8.788 \\
\hline 2.197 & 10.985 \\
\hline 65.91 & 46.137 \\
\hline 2.197 & 2.197 \\
\hline 2.197 & 2.197 \\
\hline 470.158 & 52.728 \\
\hline 4.394 & 1786.16 \\
\hline 169.169 & 134.017 \\
\hline 10.985 & 134.017 \\
\hline 15.379 & 8.788 \\
\hline 2.197 & 6.591 \\
\hline 294.398 & 2.197 \\
\hline 15.379 & 57.122 \\
\hline 17.576 & 2.197 \\
\hline 54.925 & 13.182 \\
\hline 2.197 & 21.97 \\
\hline 2.197 & 2.197 \\
\hline 2.197 & 2.197 \\
\hline 2.197 & 59.319 \\
\hline 2.197 & 2.197 \\
\hline 13.182 & 6.591 \\
\hline 2.197 & 17.576 \\
\hline 24.167 & 2.197 \\
\hline 6.591 & 28.561 \\
\hline 2.197 & 2.197 \\
\hline 4.394 & 6.591 \\
\hline 2.197 & 13.182 \\
\hline 8.788 & 6.591 \\
\hline 48.334 & 2.197 \\
\hline 17.576 & 2.197 \\
\hline 8.788 & 4.394 \\
\hline 30.758 & 81.289 \\
\hline 26.364 & 8.788 \\
\hline 4.394 & 46.137 \\
\hline 6.591 & 65.91 \\
\hline 2.197 & 21.97 \\
\hline
\end{tabular}




\begin{tabular}{|c|c|}
\hline 2.197 & 120.835 \\
\hline 2.197 & 15.379 \\
\hline 4.394 & 4.394 \\
\hline 2.197 & 2.197 \\
\hline 6.591 & 2.197 \\
\hline 4290.74 & 4.394 \\
\hline 30.758 & 21.97 \\
\hline 85.683 & 46.137 \\
\hline 281.216 & 4.394 \\
\hline 2.197 & 2.197 \\
\hline 2.197 & 35.152 \\
\hline 2.197 & 15.379 \\
\hline 2.197 & 21.97 \\
\hline 2.197 & 2.197 \\
\hline 19.773 & 13.182 \\
\hline 2.197 & 4.394 \\
\hline 4.394 & 2.197 \\
\hline 6.591 & 292.201 \\
\hline 2.197 & 2.197 \\
\hline 2.197 & 120.835 \\
\hline 2.197 & 35.152 \\
\hline 39.546 & 4.394 \\
\hline 2.197 & 2.197 \\
\hline 2.197 & 2.197 \\
\hline 2.197 & 2.197 \\
\hline 254.852 & 79.092 \\
\hline 2.197 & 2.197 \\
\hline 26.364 & 6.591 \\
\hline 17.576 & 70.304 \\
\hline 2.197 & 70.304 \\
\hline 2.197 & 2.197 \\
\hline 2.197 & 4.394 \\
\hline 2.197 & 2.197 \\
\hline 2.197 & 4.394 \\
\hline 26.364 & 15.379 \\
\hline 129.623 & 290.004 \\
\hline 13.182 & 2.197 \\
\hline 127.426 & 2.197 \\
\hline 4.394 & 2.197 \\
\hline 206.518 & 2.197 \\
\hline 10.985 & 30.758 \\
\hline 819.481 & 28.561 \\
\hline 2.197 & 10.985 \\
\hline 24.167 & 2.197 \\
\hline 2.197 & 92.274 \\
\hline 24.167 & 276.822 \\
\hline 2.197 & 2.197 \\
\hline 2.197 & 39.546 \\
\hline 6.591 & 6.591 \\
\hline 17.576 & 94.471 \\
\hline
\end{tabular}




\begin{tabular}{|c|c|}
\hline 81.289 & 188.942 \\
\hline 4.394 & 17.576 \\
\hline 6.591 & 6.591 \\
\hline 2.197 & 72.501 \\
\hline 2.197 & 562.432 \\
\hline 24.167 & 4.394 \\
\hline 2.197 & 4.394 \\
\hline 4.394 & 101.062 \\
\hline 2.197 & 2.197 \\
\hline 2.197 & 428.415 \\
\hline 2.197 & 8.788 \\
\hline 155.987 & 101.062 \\
\hline 15.379 & 46.137 \\
\hline 2.197 & 10.985 \\
\hline 395.46 & 252.655 \\
\hline 6949.11 & 2.197 \\
\hline 2.197 & 2.197 \\
\hline 880.997 & 2.197 \\
\hline 303.186 & 2.197 \\
\hline 2.197 & 2.197 \\
\hline 2.197 & 2.197 \\
\hline 2.197 & 15.379 \\
\hline 48.334 & 101.062 \\
\hline 46.137 & 19.773 \\
\hline 4.394 & 331.747 \\
\hline 2.197 & 274.625 \\
\hline 68.107 & 10.985 \\
\hline 13.182 & 21.97 \\
\hline 98.865 & 4.394 \\
\hline 2.197 & 2.197 \\
\hline 2.197 & 2.197 \\
\hline 2.197 & 4.394 \\
\hline 43.94 & 448.188 \\
\hline 2.197 & 4.394 \\
\hline 41.743 & 164.775 \\
\hline 10.985 & 2.197 \\
\hline 15.379 & 2.197 \\
\hline 4.394 & 2.197 \\
\hline 391.066 & 2.197 \\
\hline 32.955 & 39.546 \\
\hline 2.197 & 2.197 \\
\hline 2.197 & 43.94 \\
\hline 68.107 & 41.743 \\
\hline 2.197 & 274.625 \\
\hline 2.197 & 17.576 \\
\hline 81.289 & 6.591 \\
\hline 15.379 & 6.591 \\
\hline 2.197 & 15.379 \\
\hline 32.955 & 2.197 \\
\hline 2.197 & 6.591 \\
\hline
\end{tabular}




\begin{tabular}{|c|c|}
\hline 37.349 & 6.591 \\
\hline 2.197 & 10.985 \\
\hline 21.97 & 224.094 \\
\hline 2.197 & 5338.71 \\
\hline 2.197 & 2.197 \\
\hline 2.197 & 19.773 \\
\hline 2.197 & 2.197 \\
\hline 30.758 & 52.728 \\
\hline 74.698 & 8.788 \\
\hline 19.773 & 2.197 \\
\hline 6.591 & 10.985 \\
\hline 4.394 & 32.955 \\
\hline 4.394 & 4.394 \\
\hline 4.394 & 17.576 \\
\hline 2.197 & 15438.3 \\
\hline 15.379 & 10.985 \\
\hline 186.745 & 10.985 \\
\hline 19.773 & 300.989 \\
\hline 15.379 & 24.167 \\
\hline 2.197 & 4.394 \\
\hline 43.94 & 105.456 \\
\hline 65.91 & 52.728 \\
\hline 2.197 & 4.394 \\
\hline 21.97 & 10.985 \\
\hline 4.394 & 147.199 \\
\hline 6.591 & 2.197 \\
\hline 68.107 & 94.471 \\
\hline 6.591 & 2.197 \\
\hline 6.591 & 2.197 \\
\hline 2.197 & 2.197 \\
\hline 41.743 & 123.032 \\
\hline 8.788 & 24.167 \\
\hline 6.591 & 2.197 \\
\hline 72.501 & 63.713 \\
\hline 87.88 & 17.576 \\
\hline 28.561 & 8.788 \\
\hline 6.591 & 13.182 \\
\hline 103.259 & 461.37 \\
\hline 32.955 & 2.197 \\
\hline 76.895 & 4.394 \\
\hline 98.865 & 2.197 \\
\hline 2.197 & 2.197 \\
\hline 54.925 & 2.197 \\
\hline 59.319 & 2.197 \\
\hline 87.88 & 4.394 \\
\hline 248.261 & 2.197 \\
\hline 2.197 & 2.197 \\
\hline 26.364 & 13.182 \\
\hline 2.197 & 2.197 \\
\hline 8.788 & 63.713 \\
\hline
\end{tabular}




\begin{tabular}{|c|c|}
\hline 2.197 & 46.137 \\
\hline 4.394 & 10.985 \\
\hline 2.197 & 17.576 \\
\hline 667.888 & 15.379 \\
\hline 26.364 & 2.197 \\
\hline 2.197 & 2.197 \\
\hline 41.743 & 8.788 \\
\hline 24.167 & 6.591 \\
\hline 92.274 & 8.788 \\
\hline 35.152 & 17.576 \\
\hline 10.985 & 21.97 \\
\hline 72.501 & 96.668 \\
\hline 2.197 & 65.91 \\
\hline 4.394 & 8.788 \\
\hline 428.415 & 13.182 \\
\hline 2.197 & 2.197 \\
\hline 235.079 & 4.394 \\
\hline 30.758 & 4.394 \\
\hline 2.197 & 2.197 \\
\hline 2.197 & 1564.26 \\
\hline 6.591 & 2.197 \\
\hline 2.197 & 2.197 \\
\hline 2.197 & 6.591 \\
\hline 2.197 & 15.379 \\
\hline 39.546 & 2.197 \\
\hline 8.788 & 241.67 \\
\hline 10.985 & 4.394 \\
\hline 6.591 & 2.197 \\
\hline 8.788 & 13.182 \\
\hline 503.113 & 48.334 \\
\hline 8.788 & 32.955 \\
\hline 2.197 & 4.394 \\
\hline 2.197 & 15.379 \\
\hline 4.394 & 24.167 \\
\hline 2.197 & 28.561 \\
\hline 8.788 & 65.91 \\
\hline 4.394 & 2.197 \\
\hline 2.197 & 13.182 \\
\hline 8.788 & 129.623 \\
\hline 193.336 & 1254.49 \\
\hline 2.197 & 10.985 \\
\hline 10.985 & 2.197 \\
\hline 2.197 & 2.197 \\
\hline 4.394 & 4.394 \\
\hline 10.985 & 13.182 \\
\hline 26.364 & 2.197 \\
\hline 4.394 & 8.788 \\
\hline 70.304 & 21.97 \\
\hline 24.167 & 5365.07 \\
\hline 287.807 & 43.9 \\
\hline
\end{tabular}




\begin{tabular}{|c|c|}
\hline 19.773 & 6.591 \\
\hline 2.197 & 2.197 \\
\hline 6.591 & 2.197 \\
\hline 6.591 & 2.197 \\
\hline 4.394 & 2.197 \\
\hline 24.167 & 4.394 \\
\hline 8.788 & 2.197 \\
\hline 21.97 & 2.197 \\
\hline 522.886 & 2.197 \\
\hline 153.79 & 2.197 \\
\hline 10.985 & 2.197 \\
\hline 19.773 & 39.546 \\
\hline 43.94 & 2.197 \\
\hline 15.379 & 79.092 \\
\hline 2.197 & 74.698 \\
\hline 13.182 & 9543.77 \\
\hline 37.349 & 171.366 \\
\hline 2.197 & 19.773 \\
\hline 2.197 & 4.394 \\
\hline 50.531 & 2869.28 \\
\hline 472.355 & 87.88 \\
\hline 6.591 & 15.379 \\
\hline 10.985 & 2.197 \\
\hline 6.591 & 24.167 \\
\hline 19.773 & 41.743 \\
\hline 8.788 & 140.608 \\
\hline 2.197 & 13.182 \\
\hline 2.197 & 4.394 \\
\hline 2.197 & 6.591 \\
\hline 2.197 & 2.197 \\
\hline 2.197 & 4.394 \\
\hline 2.197 & 2.197 \\
\hline 2.197 & 35.152 \\
\hline 4.394 & 2.197 \\
\hline 2.197 & 63.713 \\
\hline 2.197 & 61.516 \\
\hline 6.591 & 182.351 \\
\hline 21.97 & 8.788 \\
\hline 35.152 & 32.955 \\
\hline 2.197 & 2.197 \\
\hline 26.364 & 85.683 \\
\hline 21.97 & 52.728 \\
\hline 13.182 & 35.152 \\
\hline 15.379 & 37.349 \\
\hline 4.394 & 6.591 \\
\hline 2.197 & 50.531 \\
\hline 217.503 & 4.394 \\
\hline 70.304 & 2.197 \\
\hline 2.197 & 4.394 \\
\hline 2.197 & 2.197 \\
\hline
\end{tabular}




\begin{tabular}{|c|c|}
\hline 21.97 & 221.897 \\
\hline 4.394 & 2.197 \\
\hline 2.197 & 2.197 \\
\hline 70.304 & 2.197 \\
\hline 6.591 & 13.182 \\
\hline 30.758 & 4.394 \\
\hline 2.197 & 6.591 \\
\hline 8.788 & 46.137 \\
\hline 4.394 & 8.788 \\
\hline 6.591 & 6.591 \\
\hline 6.591 & 61.516 \\
\hline 162.578 & 105.456 \\
\hline 2.197 & 10.985 \\
\hline 35.152 & 37.349 \\
\hline 13.182 & 8.788 \\
\hline 6.591 & 24.167 \\
\hline 4677.41 & 2.197 \\
\hline 2.197 & 6.591 \\
\hline 8.788 & 8.788 \\
\hline 13.182 & 854.633 \\
\hline 4.394 & 6.591 \\
\hline 8.788 & 459.173 \\
\hline 17.576 & 24.167 \\
\hline 10.985 & 6.591 \\
\hline 2.197 & 6.591 \\
\hline 18373.5 & 24.167 \\
\hline 30.758 & 351.52 \\
\hline 388.869 & 2.197 \\
\hline 13.182 & 61.516 \\
\hline 2.197 & 50.531 \\
\hline 6.591 & 2.197 \\
\hline 6.591 & 10.985 \\
\hline 2.197 & 8.788 \\
\hline 2.197 & 10.985 \\
\hline 2.197 & 63.713 \\
\hline 2.197 & 63.713 \\
\hline 2.197 & 2.197 \\
\hline 26.364 & 4.394 \\
\hline 13.182 & 17.576 \\
\hline 2.197 & 28.561 \\
\hline 2.197 & 2.197 \\
\hline 2.197 & 15.379 \\
\hline 19.773 & 230.685 \\
\hline 2.197 & 13.182 \\
\hline 2.197 & 15.379 \\
\hline 2.197 & 13.182 \\
\hline 2.197 & 4.394 \\
\hline 10.985 & 4.394 \\
\hline 155.987 & 4.394 \\
\hline 48.334 & 37.349 \\
\hline
\end{tabular}




\begin{tabular}{|c|c|}
\hline 248.261 & 241.67 \\
\hline 2.197 & 136.214 \\
\hline 6.591 & 2.197 \\
\hline 2.197 & 28.561 \\
\hline 76.895 & 2.197 \\
\hline 2.197 & 32.955 \\
\hline 17.576 & 2.197 \\
\hline 52.728 & 2.197 \\
\hline 6.591 & 2.197 \\
\hline 6.591 & 6.591 \\
\hline 61.516 & 463.567 \\
\hline 10.985 & 2.197 \\
\hline 8.788 & 21.97 \\
\hline 70.304 & 63.713 \\
\hline 118.638 & 182.351 \\
\hline 35.152 & 105.456 \\
\hline 2.197 & 15.379 \\
\hline 8.788 & 37.349 \\
\hline 4.394 & 15.379 \\
\hline 6.591 & 48.334 \\
\hline 63.713 & 2.197 \\
\hline 13.182 & 2.197 \\
\hline 2.197 & 8.788 \\
\hline 2.197 & 8.788 \\
\hline 2.197 & 19.773 \\
\hline 2.197 & 8.788 \\
\hline 13.182 & 134.017 \\
\hline 76.895 & 2.197 \\
\hline 6.591 & 2.197 \\
\hline 13.182 & 151.593 \\
\hline 2.197 & 10.985 \\
\hline 432.809 & 155.987 \\
\hline 2.197 & 19.773 \\
\hline 39.546 & 2.197 \\
\hline 2.197 & 10.985 \\
\hline 2.197 & 10.985 \\
\hline 28.561 & 61.516 \\
\hline 8.788 & 2.197 \\
\hline 191.139 & 8.788 \\
\hline 15.379 & 19.773 \\
\hline 54.925 & 13.182 \\
\hline 8.788 & 72.501 \\
\hline 406.445 & 28.561 \\
\hline 2.197 & 6.591 \\
\hline 2.197 & 6.591 \\
\hline 43.94 & 17.576 \\
\hline 70.304 & 349.323 \\
\hline 4.394 & 268.034 \\
\hline 2.197 & 2.197 \\
\hline 2.197 & 48.33 \\
\hline
\end{tabular}




\begin{tabular}{|c|c|}
\hline 2.197 & 17.576 \\
\hline 2.197 & 2818.75 \\
\hline 2.197 & 2.197 \\
\hline 28.561 & 21.97 \\
\hline 6.591 & 2.197 \\
\hline 79.092 & 24.167 \\
\hline 6.591 & 4.394 \\
\hline 10.985 & 19.773 \\
\hline 2.197 & 2.197 \\
\hline 2.197 & 2.197 \\
\hline 344.929 & 10.985 \\
\hline 2.197 & 6.591 \\
\hline 30.758 & 2.197 \\
\hline 21.97 & 13.182 \\
\hline 4.394 & 26.364 \\
\hline 4.394 & 30.758 \\
\hline 19.773 & 10.985 \\
\hline 2.197 & 2.197 \\
\hline 6.591 & 81.289 \\
\hline 2.197 & 59.319 \\
\hline 2.197 & 21.97 \\
\hline 52.728 & 24.167 \\
\hline 4.394 & 8.788 \\
\hline 2.197 & 35.152 \\
\hline 2.197 & 10.985 \\
\hline 2.197 & 2027.83 \\
\hline 2.197 & 2.197 \\
\hline 4.394 & 8.788 \\
\hline 2.197 & 8.788 \\
\hline 2.197 & 13.182 \\
\hline 2.197 & 41.743 \\
\hline 2.197 & 28.561 \\
\hline 4.394 & 13.182 \\
\hline 63.713 & 10.985 \\
\hline 94.471 & 10.985 \\
\hline 4.394 & 26.364 \\
\hline 24.167 & 15.379 \\
\hline 10.985 & 30.758 \\
\hline 2.197 & 24.167 \\
\hline 109.85 & 13171 \\
\hline 2.197 & 450.385 \\
\hline 2.197 & 6.591 \\
\hline 4.394 & 26.364 \\
\hline 6.591 & 149.396 \\
\hline 2.197 & 2.197 \\
\hline 2.197 & 2.197 \\
\hline 30.758 & 6.591 \\
\hline 103.259 & 35.152 \\
\hline 43.94 & 13.182 \\
\hline 373.49 & 17.576 \\
\hline
\end{tabular}




\begin{tabular}{|c|c|}
\hline 670.085 & 43.94 \\
\hline 2.197 & 68.107 \\
\hline 13.182 & 72.501 \\
\hline 6.591 & 15.379 \\
\hline 184.548 & 24.167 \\
\hline 57.122 & 19.773 \\
\hline 2.197 & 254.852 \\
\hline 2.197 & 15.379 \\
\hline 81.289 & 6.591 \\
\hline 4.394 & 8900.05 \\
\hline 13.182 & 39.546 \\
\hline 17.576 & 24.167 \\
\hline 6.591 & 254.852 \\
\hline 8.788 & 4.394 \\
\hline 2.197 & 4.394 \\
\hline 2.197 & 35.152 \\
\hline 8.788 & 2.197 \\
\hline 37.349 & 173.563 \\
\hline 2.197 & 8.788 \\
\hline 87.88 & 2.197 \\
\hline 30.758 & 65.91 \\
\hline 2.197 & 4.394 \\
\hline 2.197 & 2.197 \\
\hline 2.197 & 2.197 \\
\hline 2.197 & 8.788 \\
\hline 94.471 & 2.197 \\
\hline 430.612 & 2.197 \\
\hline 39.546 & 2.197 \\
\hline 2.197 & 2.197 \\
\hline 195.533 & 2.197 \\
\hline 85.683 & 2.197 \\
\hline 26.364 & 2.197 \\
\hline 2.197 & 30.758 \\
\hline 4.394 & 4.394 \\
\hline 4.394 & 30.758 \\
\hline 52.728 & 10.985 \\
\hline 459.173 & 4.394 \\
\hline 990.847 & 2.197 \\
\hline 32.955 & 85.683 \\
\hline 10.985 & 32.955 \\
\hline 15.379 & 6.591 \\
\hline 6.591 & 72.501 \\
\hline 4.394 & 6.591 \\
\hline 2.197 & 8.788 \\
\hline 158.184 & 48.334 \\
\hline 2.197 & 2.19 \\
\hline 2.197 & 10.985 \\
\hline 2.197 & 2.19 \\
\hline 2.197 & 2.197 \\
\hline 2.197 & 50.531 \\
\hline
\end{tabular}




\begin{tabular}{|c|c|}
\hline 2.197 & 408.642 \\
\hline 2.197 & 2.197 \\
\hline 2.197 & 48.334 \\
\hline 2.197 & 46.137 \\
\hline 2.197 & 419.627 \\
\hline 8.788 & 2.197 \\
\hline 4.394 & 250.458 \\
\hline 4.394 & 30.758 \\
\hline 173.563 & 74.698 \\
\hline 43.94 & 118.638 \\
\hline 2.197 & 8.788 \\
\hline 2.197 & 13.182 \\
\hline 2.197 & 4.394 \\
\hline 2.197 & 21.97 \\
\hline 6.591 & 10.985 \\
\hline 17.576 & 2.197 \\
\hline 19.773 & 37.349 \\
\hline 197.73 & 48.334 \\
\hline 4.394 & 2.197 \\
\hline 2.197 & 6.591 \\
\hline 59.319 & 180.154 \\
\hline 59.319 & 17.576 \\
\hline 2.197 & 4.394 \\
\hline 4.394 & 786.526 \\
\hline 2.197 & 4.394 \\
\hline 17.576 & 188.942 \\
\hline 2.197 & 2.197 \\
\hline 4.394 & 112.047 \\
\hline 2.197 & 2.197 \\
\hline 2.197 & 72.501 \\
\hline 2.197 & 2.197 \\
\hline 2.197 & 151.593 \\
\hline 4.394 & 17.576 \\
\hline 4.394 & 37.349 \\
\hline 2.197 & 8.788 \\
\hline 19.773 & 2.197 \\
\hline 2.197 & 2.197 \\
\hline 65.91 & 6.591 \\
\hline 10.985 & 10.985 \\
\hline 2.197 & 2.197 \\
\hline 48.334 & 177.957 \\
\hline 4.394 & 2.197 \\
\hline 2.197 & 4.394 \\
\hline 2.197 & 6.591 \\
\hline 8.788 & 37.349 \\
\hline 83.486 & 37.349 \\
\hline 15.379 & 2.197 \\
\hline 2.197 & 8.788 \\
\hline 2.197 & 90.077 \\
\hline 28.561 & 6.591 \\
\hline
\end{tabular}




\begin{tabular}{|c|c|}
\hline 2.197 & 6.591 \\
\hline 2.197 & 57.122 \\
\hline 2.197 & 61.516 \\
\hline 17.576 & 6.591 \\
\hline 2.197 & 2.197 \\
\hline 4.394 & 19.773 \\
\hline 4.394 & 46.137 \\
\hline 4.394 & 2.197 \\
\hline 2.197 & 2.197 \\
\hline 4.394 & 2.197 \\
\hline 4.394 & 2.197 \\
\hline 2.197 & 2.197 \\
\hline 4.394 & 149.396 \\
\hline 2.197 & 2.197 \\
\hline 215.306 & 2.197 \\
\hline 2.197 & 35.152 \\
\hline 4.394 & 8.788 \\
\hline 136.214 & 4.394 \\
\hline 4.394 & 8.788 \\
\hline 19.773 & 13.182 \\
\hline 2.197 & 2.197 \\
\hline 19.773 & 4.394 \\
\hline 145.002 & 24.167 \\
\hline 2.197 & 2.197 \\
\hline 2.197 & 41.743 \\
\hline 8.788 & 79.092 \\
\hline 2.197 & 15.379 \\
\hline 2.197 & 59.319 \\
\hline 2.197 & 50.531 \\
\hline 2.197 & 584.402 \\
\hline 19.773 & 307.58 \\
\hline 81.289 & 2.197 \\
\hline 2.197 & 2.197 \\
\hline 19.773 & 32.955 \\
\hline 109.85 & 21.97 \\
\hline 316.368 & 13.182 \\
\hline 1309.41 & 19.773 \\
\hline 4.394 & 2.197 \\
\hline 72.501 & 70.304 \\
\hline 19.773 & 5756.14 \\
\hline 68.107 & 173.563 \\
\hline 4.394 & 28.561 \\
\hline 2.197 & 2.197 \\
\hline 30.758 & 2.197 \\
\hline 24.167 & 79.092 \\
\hline 2.197 & 35.152 \\
\hline 6.591 & 129.623 \\
\hline 15.379 & 4.394 \\
\hline 13.182 & 6.591 \\
\hline 2.197 & 270.231 \\
\hline
\end{tabular}




\begin{tabular}{|c|c|}
\hline 13.182 & 70.304 \\
\hline 2.197 & 19.773 \\
\hline 2.197 & 63.713 \\
\hline 2.197 & 2.197 \\
\hline 70.304 & 2.197 \\
\hline 435.006 & 4.394 \\
\hline 92.274 & 6.591 \\
\hline 6.591 & 2414.5 \\
\hline 2.197 & 2.197 \\
\hline 2.197 & 4.394 \\
\hline 2.197 & 2.197 \\
\hline 883.194 & 8.788 \\
\hline 2.197 & 28.561 \\
\hline 94.471 & 4.394 \\
\hline 105.456 & 2.197 \\
\hline 142.805 & 41.743 \\
\hline 15.379 & 10.985 \\
\hline 19.773 & 61.516 \\
\hline 109.85 & 885.391 \\
\hline 4.394 & 4.394 \\
\hline 4.394 & 10.985 \\
\hline 2.197 & 35.152 \\
\hline 19.773 & 59.319 \\
\hline 2.197 & 8.788 \\
\hline 2.197 & 186.745 \\
\hline 2.197 & 17.576 \\
\hline 2.197 & 1581.84 \\
\hline 4.394 & 4.394 \\
\hline 4.394 & 87.88 \\
\hline 2.197 & 10.985 \\
\hline 37.349 & 2.197 \\
\hline 37.349 & 4.394 \\
\hline 2.197 & 6.591 \\
\hline 2.197 & 32.955 \\
\hline 13.182 & 762.359 \\
\hline 10.985 & 52.728 \\
\hline 2.197 & 8.788 \\
\hline 6.591 & 123.032 \\
\hline 17.576 & 46.137 \\
\hline 2.197 & 6.591 \\
\hline 129.623 & 4.394 \\
\hline 2.197 & 4.394 \\
\hline 21.97 & 32.955 \\
\hline 2.197 & 2.197 \\
\hline 6.591 & 90.077 \\
\hline 722.813 & 15.379 \\
\hline 2.197 & 10.985 \\
\hline 101.062 & 1942.15 \\
\hline 72.501 & 2.197 \\
\hline 4.394 & 4.394 \\
\hline
\end{tabular}




\begin{tabular}{|c|c|}
\hline 4.394 & 2.197 \\
\hline 87.88 & 6.591 \\
\hline 957.892 & 57.122 \\
\hline 2.197 & 15.379 \\
\hline 116.441 & 13.182 \\
\hline 15.379 & 4.394 \\
\hline 4.394 & 4.394 \\
\hline 2.197 & 4.394 \\
\hline 19.773 & 158.184 \\
\hline 6.591 & 35.152 \\
\hline 2.197 & 6.591 \\
\hline 4.394 & 39.546 \\
\hline 28.561 & 35.152 \\
\hline 4.394 & 2.197 \\
\hline 358.111 & 2.197 \\
\hline 8.788 & 10.985 \\
\hline 4.394 & 28.561 \\
\hline 4.394 & 4.394 \\
\hline 6.591 & 17.576 \\
\hline 4.394 & 19.773 \\
\hline 2.197 & 79.092 \\
\hline 195.533 & 107.653 \\
\hline 8.788 & 2.197 \\
\hline 8.788 & 2.197 \\
\hline 2.197 & 15.379 \\
\hline 54.925 & 142.805 \\
\hline 19.773 & 4.394 \\
\hline 6.591 & 4.394 \\
\hline 87.88 & 129.623 \\
\hline 19.773 & 10.985 \\
\hline 2.197 & 195.533 \\
\hline 2.197 & 50.531 \\
\hline 287.807 & 2.197 \\
\hline 443.794 & 32.955 \\
\hline 13.182 & 15.379 \\
\hline 17.576 & 6.591 \\
\hline 4.394 & 2.197 \\
\hline 2.197 & 81.289 \\
\hline 2.197 & 13.182 \\
\hline 193.336 & 13.182 \\
\hline 4.394 & 175.76 \\
\hline 32.955 & 4.394 \\
\hline 83.486 & 4.394 \\
\hline 6.591 & 65.91 \\
\hline 2.197 & 560.235 \\
\hline 2.197 & 2.197 \\
\hline 2.197 & 4.394 \\
\hline 4.394 & 6.591 \\
\hline 2.197 & 4.394 \\
\hline 2.197 & 217.503 \\
\hline
\end{tabular}




\begin{tabular}{|c|c|}
\hline 8.788 & 2.197 \\
\hline 4.394 & 10.985 \\
\hline 134.017 & 8.788 \\
\hline 147.199 & 21.97 \\
\hline 349.323 & 2.197 \\
\hline 15.379 & 94.471 \\
\hline 2.197 & 2.197 \\
\hline 26.364 & 2.197 \\
\hline 4.394 & 4.394 \\
\hline 2.197 & 2.197 \\
\hline 2.197 & 2.197 \\
\hline 2.197 & 10.985 \\
\hline 10.985 & 2.197 \\
\hline 8.788 & 24.167 \\
\hline 142.805 & 48.334 \\
\hline 17.576 & 34938.9 \\
\hline 2.197 & 6.591 \\
\hline 50.531 & 217.503 \\
\hline 6.591 & 1195.17 \\
\hline 2.197 & 2.197 \\
\hline 2.197 & 57.122 \\
\hline 4.394 & 26.364 \\
\hline 8.788 & 2.197 \\
\hline 2.197 & 118.638 \\
\hline 2.197 & 32.955 \\
\hline 63.713 & 4.394 \\
\hline 32.955 & 217.503 \\
\hline 37.349 & 109.85 \\
\hline 72.501 & 2.197 \\
\hline 10.985 & 35.152 \\
\hline 224.094 & 16993.8 \\
\hline 127.426 & 76.895 \\
\hline 10.985 & 8.788 \\
\hline 2.197 & 15.379 \\
\hline 2.197 & 6.591 \\
\hline 2.197 & 63.713 \\
\hline 13.182 & 2.197 \\
\hline 424.021 & 6.591 \\
\hline 2.197 & 15.379 \\
\hline 52.728 & 2.197 \\
\hline 15.379 & 13.182 \\
\hline 2.197 & 13.182 \\
\hline 2.197 & 98.865 \\
\hline 92.274 & 2.197 \\
\hline 24.167 & 145.002 \\
\hline 4.394 & 2.197 \\
\hline 41.743 & 2.197 \\
\hline 2.197 & 4.394 \\
\hline 6.591 & 2.197 \\
\hline 369.096 & 226.291 \\
\hline
\end{tabular}




\begin{tabular}{|c|c|}
\hline 186.745 & 4.394 \\
\hline 2.197 & 6.591 \\
\hline 2.197 & 2.197 \\
\hline 2.197 & 10.985 \\
\hline 2.197 & 57.122 \\
\hline 2.197 & 10.985 \\
\hline 2.197 & 8.788 \\
\hline 28.561 & 4.394 \\
\hline 13.182 & 6.591 \\
\hline 26.364 & 43.94 \\
\hline 2.197 & 4.394 \\
\hline 4.394 & 21.97 \\
\hline 4.394 & 4.394 \\
\hline 2.197 & 8.788 \\
\hline 136.214 & 8.788 \\
\hline 41.743 & 8.788 \\
\hline 2.197 & 140.608 \\
\hline 19.773 & 4.394 \\
\hline 48.334 & 74.698 \\
\hline 103.259 & 57.122 \\
\hline 2.197 & 21.97 \\
\hline 10.985 & 6.591 \\
\hline 4.394 & 2.197 \\
\hline 4.394 & 2.197 \\
\hline 2.197 & 96.668 \\
\hline 37.349 & 26.364 \\
\hline 13.182 & 2.197 \\
\hline 2.197 & 6.591 \\
\hline 28.561 & 2.197 \\
\hline 4.394 & 6.591 \\
\hline 2.197 & 72.501 \\
\hline 555.841 & 2.197 \\
\hline 26.364 & 8.788 \\
\hline 2.197 & 2.197 \\
\hline 2.197 & 52.728 \\
\hline 2.197 & 98.865 \\
\hline 2.197 & 8.788 \\
\hline 8.788 & 4.394 \\
\hline 81.289 & 8.788 \\
\hline 606.372 & 4.394 \\
\hline 4.394 & 2.197 \\
\hline 6.591 & 2.197 \\
\hline 4.394 & 2.197 \\
\hline 4.394 & 2.197 \\
\hline 4.394 & 21.97 \\
\hline 43.94 & 6.591 \\
\hline 2.197 & 2.197 \\
\hline 2.197 & 2.197 \\
\hline 46.137 & 21.9 \\
\hline 2.197 & 24.16 \\
\hline
\end{tabular}




\begin{tabular}{|c|c|}
\hline 4.394 & 2.197 \\
\hline 2.197 & 2.197 \\
\hline 2.197 & 140.608 \\
\hline 2.197 & 21.97 \\
\hline 2.197 & 2.197 \\
\hline 2.197 & 61.516 \\
\hline 2.197 & 2.197 \\
\hline 2.197 & 4.394 \\
\hline 103.259 & 6.591 \\
\hline 32.955 & 4.394 \\
\hline 13.182 & 10.985 \\
\hline 6.591 & 21.97 \\
\hline 4.394 & 2.197 \\
\hline 213.109 & 19.773 \\
\hline 2.197 & 21.97 \\
\hline 17.576 & 39.546 \\
\hline 4.394 & 15.379 \\
\hline 2.197 & 4.394 \\
\hline 6.591 & 50.531 \\
\hline 6.591 & 8.788 \\
\hline 19.773 & 24.167 \\
\hline 2.197 & 162.578 \\
\hline 4.394 & 123.032 \\
\hline 6.591 & 2.197 \\
\hline 615.16 & 6.591 \\
\hline 861.224 & 2.197 \\
\hline 63.713 & 2.197 \\
\hline 41.743 & 13.182 \\
\hline 2.197 & 28.561 \\
\hline 4.394 & 2.197 \\
\hline 17.576 & 2.197 \\
\hline 2.197 & 4.394 \\
\hline 4.394 & 2.197 \\
\hline 2.197 & 162.578 \\
\hline 8.788 & 6.591 \\
\hline 777.738 & 2.197 \\
\hline 2.197 & 2.197 \\
\hline 118.638 & 318.565 \\
\hline 4.394 & 6.591 \\
\hline 2.197 & 2.197 \\
\hline 2.197 & 50.531 \\
\hline 70.304 & 364.702 \\
\hline 6.591 & 10.985 \\
\hline 4.394 & 2.197 \\
\hline 2.197 & 2.197 \\
\hline 2.197 & 13.182 \\
\hline 39.546 & 17.576 \\
\hline 17.576 & 2.197 \\
\hline 2.197 & 2.197 \\
\hline 4.394 & 817.284 \\
\hline
\end{tabular}




\begin{tabular}{|c|c|}
\hline 2.197 & 57.122 \\
\hline 2.197 & 46.137 \\
\hline 2.197 & 61.516 \\
\hline 2.197 & 4.394 \\
\hline 28.561 & 116.441 \\
\hline 2.197 & 19.773 \\
\hline 2.197 & 13.182 \\
\hline 4.394 & 24.167 \\
\hline 4.394 & 6.591 \\
\hline 70.304 & 39.546 \\
\hline 61.516 & 180.154 \\
\hline 15.379 & 19.773 \\
\hline 26.364 & 57.122 \\
\hline 2.197 & 13.182 \\
\hline 123.032 & 17.576 \\
\hline 30.758 & 8.788 \\
\hline 4.394 & 8.788 \\
\hline 180.154 & 2.197 \\
\hline 2.197 & 8.788 \\
\hline 4.394 & 19.773 \\
\hline 2.197 & 13.182 \\
\hline 2.197 & 98.865 \\
\hline 2.197 & 6.591 \\
\hline 2.197 & 28.561 \\
\hline 2.197 & 37.349 \\
\hline 1199.56 & 136.214 \\
\hline 2.197 & 2.197 \\
\hline 8.788 & 342.732 \\
\hline 35.152 & 2.197 \\
\hline 2.197 & 37.349 \\
\hline 4.394 & 63.713 \\
\hline 2.197 & 13.182 \\
\hline 21.97 & 19.773 \\
\hline 2.197 & 90.077 \\
\hline 43.94 & 2.197 \\
\hline 4.394 & 24.167 \\
\hline 2.197 & 41.743 \\
\hline 2.197 & 19.773 \\
\hline 4.394 & 24.167 \\
\hline 281.216 & 2.197 \\
\hline 21.97 & 13.182 \\
\hline 4.394 & 131.82 \\
\hline 32.955 & 43.94 \\
\hline 125.229 & 19.773 \\
\hline 4.394 & 13.182 \\
\hline 6.591 & 2.197 \\
\hline 10.985 & 131.82 \\
\hline 2.197 & 28.561 \\
\hline 2.197 & 1348.96 \\
\hline 2.197 & 10.985 \\
\hline
\end{tabular}




\begin{tabular}{|c|c|}
\hline 8.788 & 6.591 \\
\hline 65.91 & 2.197 \\
\hline 4.394 & 4.394 \\
\hline 4.394 & 85.683 \\
\hline 2.197 & 6.591 \\
\hline 6.591 & 2.197 \\
\hline 4.394 & 2.197 \\
\hline 2.197 & 2.197 \\
\hline 4.394 & 54.925 \\
\hline 2.197 & 19.773 \\
\hline 4.394 & 2.197 \\
\hline 39.546 & 17.576 \\
\hline 63.713 & 46.137 \\
\hline 98.865 & 41.743 \\
\hline 151.593 & 28.561 \\
\hline 8.788 & 309.777 \\
\hline 2.197 & 6.591 \\
\hline 2.197 & 24.167 \\
\hline 2.197 & 4.394 \\
\hline 50.531 & 2.197 \\
\hline 2.197 & 15.379 \\
\hline 13.182 & 2.197 \\
\hline 43.94 & 37.349 \\
\hline 4.394 & 74.698 \\
\hline 8.788 & 52.728 \\
\hline 2.197 & 13.182 \\
\hline 2.197 & 65.91 \\
\hline 13.182 & 6.591 \\
\hline 2.197 & 8.788 \\
\hline 2.197 & 292.201 \\
\hline 2.197 & 6.591 \\
\hline 2.197 & 2.197 \\
\hline 2.197 & 21.97 \\
\hline 8.788 & 2.197 \\
\hline 2.197 & 8.788 \\
\hline 4.394 & 2.197 \\
\hline 2.197 & 10.985 \\
\hline 2.197 & 8.788 \\
\hline 2.197 & 8.788 \\
\hline 2.197 & 21.97 \\
\hline 2.197 & 8.788 \\
\hline 2.197 & 13.182 \\
\hline 2.197 & 4.394 \\
\hline 4.394 & 2.197 \\
\hline 2.197 & 173.563 \\
\hline 61.516 & 8.788 \\
\hline 2.197 & 19.773 \\
\hline 30.758 & 19.773 \\
\hline 2.197 & 39.546 \\
\hline 6.591 & 4.394 \\
\hline
\end{tabular}




\begin{tabular}{|c|c|}
\hline 61.516 & 2.197 \\
\hline 217.503 & 2.197 \\
\hline 70.304 & 2.197 \\
\hline 48.334 & 2.197 \\
\hline 13.182 & 15.379 \\
\hline 2.197 & 6.591 \\
\hline 2.197 & 10.985 \\
\hline 50.531 & 147.199 \\
\hline 43.94 & 10.985 \\
\hline 4.394 & 1590.63 \\
\hline 10.985 & 26.364 \\
\hline 4.394 & 92.274 \\
\hline 4.394 & 28.561 \\
\hline 70.304 & 96.668 \\
\hline 2.197 & 2.197 \\
\hline 4.394 & 17.576 \\
\hline 10.985 & 2.197 \\
\hline 2.197 & 4.394 \\
\hline 2.197 & 10.985 \\
\hline 10.985 & 4.394 \\
\hline 2.197 & 2.197 \\
\hline 30.758 & 87.88 \\
\hline 131.82 & 35.152 \\
\hline 98.865 & 2.197 \\
\hline 2.197 & 28.561 \\
\hline 2.197 & 76.895 \\
\hline 2.197 & 35.152 \\
\hline 19.773 & 2.197 \\
\hline 59.319 & 2.197 \\
\hline 8.788 & 4.394 \\
\hline 114.244 & 10.985 \\
\hline 13.182 & 96.668 \\
\hline 6.591 & 226.291 \\
\hline 2.197 & 2.197 \\
\hline 4.394 & 125.229 \\
\hline 2.197 & 19.773 \\
\hline 24.167 & 8.788 \\
\hline 6.591 & 4.394 \\
\hline 2.197 & 46.137 \\
\hline 2.197 & 2.197 \\
\hline 10.985 & 13.182 \\
\hline 21.97 & 4.394 \\
\hline 309.777 & 54.925 \\
\hline 8.788 & 21.97 \\
\hline 4.394 & 15.379 \\
\hline 21.97 & 10.985 \\
\hline 292.201 & 6.591 \\
\hline 2.197 & 2.197 \\
\hline 4.394 & 2.197 \\
\hline 2.197 & 10.985 \\
\hline
\end{tabular}




\begin{tabular}{|c|c|}
\hline 2.197 & 1834.49 \\
\hline 17.576 & 8.788 \\
\hline 4.394 & 37.349 \\
\hline 61.516 & 4.394 \\
\hline 6.591 & 8.788 \\
\hline 8.788 & 160.381 \\
\hline 4.394 & 2.197 \\
\hline 35.152 & 17.576 \\
\hline 17.576 & 10.985 \\
\hline 184.548 & 4.394 \\
\hline 72.501 & 6.591 \\
\hline 2.197 & 4.394 \\
\hline 35.152 & 17.576 \\
\hline 4.394 & 2.197 \\
\hline 120.835 & 2.197 \\
\hline 39.546 & 19.773 \\
\hline 2.197 & 4.394 \\
\hline 28.561 & 52.728 \\
\hline 4.394 & 2.197 \\
\hline 2.197 & 2.197 \\
\hline 92.274 & 4.394 \\
\hline 4.394 & 6.591 \\
\hline 2.197 & 2.197 \\
\hline 206.518 & 4.394 \\
\hline 2.197 & 6.591 \\
\hline 2.197 & 52.728 \\
\hline 10.985 & 54.925 \\
\hline 2.197 & 50.531 \\
\hline 2.197 & 2.197 \\
\hline 2.197 & 21.97 \\
\hline 2.197 & 1709.27 \\
\hline 2.197 & 2.197 \\
\hline 2.197 & 4.394 \\
\hline 456.976 & 4.394 \\
\hline 8.788 & 4.394 \\
\hline 74.698 & 417.43 \\
\hline 10.985 & 474.552 \\
\hline 2.197 & 8.788 \\
\hline 2.197 & 101.062 \\
\hline 4.394 & 17.576 \\
\hline 2.197 & 8.788 \\
\hline 2.197 & 72.501 \\
\hline 10.985 & 32.955 \\
\hline 4.394 & 32.955 \\
\hline 6.591 & 2.197 \\
\hline 39.546 & 4266.57 \\
\hline 6.591 & 2.197 \\
\hline 8.788 & 465.764 \\
\hline 2.197 & 2.197 \\
\hline 15.379 & 177.957 \\
\hline
\end{tabular}




\begin{tabular}{|c|c|}
\hline 30.758 & 2.197 \\
\hline 8.788 & 8.788 \\
\hline 1573.05 & 26.364 \\
\hline 413.036 & 2.197 \\
\hline 4.394 & 10.985 \\
\hline 81.289 & 19.773 \\
\hline 8.788 & 26.364 \\
\hline 6.591 & 24.167 \\
\hline 101.062 & 17.576 \\
\hline 8.788 & 81.289 \\
\hline 4.394 & 127.426 \\
\hline 21.97 & 177.957 \\
\hline 221.897 & 2.197 \\
\hline 4.394 & 48.334 \\
\hline 13.182 & 17.576 \\
\hline 2.197 & 17.576 \\
\hline 21.97 & 2.197 \\
\hline 2.197 & 28.561 \\
\hline 15.379 & 6.591 \\
\hline 65.91 & 2.197 \\
\hline 2.197 & 101.062 \\
\hline 2.197 & 10.985 \\
\hline 4.394 & 6.591 \\
\hline 19.773 & 2.197 \\
\hline 2.197 & 2.197 \\
\hline 54.925 & 103.259 \\
\hline 4.394 & 10.985 \\
\hline 6.591 & 153.79 \\
\hline 2.197 & 10.985 \\
\hline 4.394 & 8.788 \\
\hline 4.394 & 193.336 \\
\hline 6.591 & 2.197 \\
\hline 2.197 & 4.394 \\
\hline 516.295 & 13.182 \\
\hline 2.197 & 21.97 \\
\hline 19.773 & 15.379 \\
\hline 17.576 & 10.985 \\
\hline 26.364 & 21.97 \\
\hline 2.197 & 2.197 \\
\hline 2.197 & 2.197 \\
\hline 4.394 & 2.197 \\
\hline 2.197 & 28.561 \\
\hline 2.197 & 101.062 \\
\hline 4.394 & 4.394 \\
\hline 2.197 & 107.653 \\
\hline 2.197 & 52.728 \\
\hline 4.394 & 4.394 \\
\hline 2.197 & 2675.95 \\
\hline 8.788 & 24.167 \\
\hline 10.985 & 2.19 \\
\hline
\end{tabular}




\begin{tabular}{|c|c|}
\hline 4.394 & 59.319 \\
\hline 43.94 & 4.394 \\
\hline 2.197 & 2.197 \\
\hline 4.394 & 2.197 \\
\hline 15.379 & 10.985 \\
\hline 2.197 & 24.167 \\
\hline 235.079 & 48.334 \\
\hline 2.197 & 83.486 \\
\hline 2.197 & 63.713 \\
\hline 8.788 & 92.274 \\
\hline 30.758 & 63.713 \\
\hline 28.561 & 15.379 \\
\hline 15.379 & 68.107 \\
\hline 15.379 & 127.426 \\
\hline 327.353 & 129.623 \\
\hline 2.197 & 2.197 \\
\hline 2.197 & 10.985 \\
\hline 13.182 & 2.197 \\
\hline 2.197 & 2.197 \\
\hline 32.955 & 2.197 \\
\hline 790.92 & 2.197 \\
\hline 2.197 & 21.97 \\
\hline 8.788 & 2.197 \\
\hline 2.197 & 13.182 \\
\hline 6.591 & 15.379 \\
\hline 2.197 & 81.289 \\
\hline 24.167 & 120.835 \\
\hline 2.197 & 276.822 \\
\hline 2.197 & 10.985 \\
\hline 6.591 & 2.197 \\
\hline 19.773 & 4.394 \\
\hline 37.349 & 4.394 \\
\hline 17.576 & 37.349 \\
\hline 674.479 & 2.197 \\
\hline 1313.81 & 24.167 \\
\hline 4.394 & 10.985 \\
\hline 2.197 & 6.591 \\
\hline 8.788 & 586.599 \\
\hline 2.197 & 61.516 \\
\hline 112.047 & 2.197 \\
\hline 2.197 & 276.822 \\
\hline 15.379 & 2.197 \\
\hline 39.546 & 17.576 \\
\hline 2.197 & 28.561 \\
\hline 6.591 & 13.182 \\
\hline 83.486 & 731.601 \\
\hline 2.197 & 188.942 \\
\hline 6.591 & 35.152 \\
\hline 8.788 & 6.591 \\
\hline 2.197 & 2.197 \\
\hline
\end{tabular}




\begin{tabular}{|c|c|}
\hline 2.197 & 4.394 \\
\hline 294.398 & 32.955 \\
\hline 4.394 & 24.167 \\
\hline 8.788 & 92.274 \\
\hline 4.394 & 4.394 \\
\hline 19.773 & 2.197 \\
\hline 26.364 & 48.334 \\
\hline 13.182 & 2.197 \\
\hline 2.197 & 2.197 \\
\hline 2.197 & 2.197 \\
\hline 81.289 & 4.394 \\
\hline 2.197 & 24.167 \\
\hline 2.197 & 2.197 \\
\hline 2.197 & 10.985 \\
\hline 2.197 & 2.197 \\
\hline 4.394 & 2.197 \\
\hline 6.591 & 26.364 \\
\hline 4.394 & 10.985 \\
\hline 57.122 & 4.394 \\
\hline 28.561 & 4.394 \\
\hline 166.972 & 2.197 \\
\hline 4.394 & 74.698 \\
\hline 68.107 & 32.955 \\
\hline 63.713 & 2.197 \\
\hline 2.197 & 500.916 \\
\hline 2.197 & 2.197 \\
\hline 2.197 & 2.197 \\
\hline 2.197 & 4.394 \\
\hline 2.197 & 6.591 \\
\hline 13.182 & 48.334 \\
\hline 13.182 & 4.394 \\
\hline 21.97 & 2.197 \\
\hline 10.985 & 916.149 \\
\hline 43.94 & 2.197 \\
\hline 2.197 & 46.137 \\
\hline 32.955 & 237.276 \\
\hline 949.104 & 37.349 \\
\hline 10.985 & 120.835 \\
\hline 103.259 & 92.274 \\
\hline 30.758 & 250.458 \\
\hline 2.197 & 15.379 \\
\hline 2.197 & 41.743 \\
\hline 43.94 & 24.167 \\
\hline 103.259 & 61.516 \\
\hline 2421.09 & 41.743 \\
\hline 2.197 & 2.197 \\
\hline 32.955 & 2.197 \\
\hline 6.591 & 2.197 \\
\hline 10.985 & 52.728 \\
\hline 4.394 & 4.394 \\
\hline
\end{tabular}




\begin{tabular}{|c|c|}
\hline 112.047 & 4.394 \\
\hline 4.394 & 1520.32 \\
\hline 180.154 & 15.379 \\
\hline 2.197 & 6.591 \\
\hline 2.197 & 24.167 \\
\hline 57.122 & 4.394 \\
\hline 6.591 & 2.197 \\
\hline 2.197 & 6.591 \\
\hline 2.197 & 32.955 \\
\hline 2.197 & 2.197 \\
\hline 10.985 & 15.379 \\
\hline 2.197 & 17.576 \\
\hline 30.758 & 15.379 \\
\hline 2.197 & 35.152 \\
\hline 2.197 & 4.394 \\
\hline 145.002 & 2.197 \\
\hline 2.197 & 43.94 \\
\hline 2.197 & 35.152 \\
\hline 2.197 & 2.197 \\
\hline 2.197 & 2.197 \\
\hline 2.197 & 6.591 \\
\hline 4.394 & 2.197 \\
\hline 4.394 & 8.788 \\
\hline 4.394 & 41.743 \\
\hline 81.289 & 19.773 \\
\hline 15.379 & 2.197 \\
\hline 2.197 & 4.394 \\
\hline 309.777 & 4.394 \\
\hline 6.591 & 24.167 \\
\hline 13.182 & 492.128 \\
\hline 6.591 & 153.79 \\
\hline 2.197 & 2.197 \\
\hline 274.625 & 2.197 \\
\hline 2.197 & 204.321 \\
\hline 2.197 & 43.94 \\
\hline 17.576 & 15.379 \\
\hline 6.591 & 2.197 \\
\hline 24.167 & 4.394 \\
\hline 140.608 & 2.197 \\
\hline 2.197 & 35.152 \\
\hline 131.82 & 300.989 \\
\hline 2.197 & 19.773 \\
\hline 2.197 & 6.591 \\
\hline 4.394 & 2.197 \\
\hline 6.591 & 8.788 \\
\hline 4.394 & 2.197 \\
\hline 48.334 & 8.788 \\
\hline 4.394 & 2.197 \\
\hline 2.197 & 2.197 \\
\hline 2.197 & 2.197 \\
\hline
\end{tabular}




\begin{tabular}{|c|c|}
\hline 52.728 & 2.197 \\
\hline 10.985 & 2.197 \\
\hline 94.471 & 2.197 \\
\hline 6.591 & 96.668 \\
\hline 2.197 & 10.985 \\
\hline 2.197 & 21.97 \\
\hline 4.394 & 4.394 \\
\hline 2.197 & 50.531 \\
\hline 2.197 & 17.576 \\
\hline 2.197 & 10.985 \\
\hline 2.197 & 30.758 \\
\hline 2.197 & 13.182 \\
\hline 2.197 & 15.379 \\
\hline 19.773 & 2.197 \\
\hline 2.197 & 10.985 \\
\hline 2.197 & 8.788 \\
\hline 4.394 & 6.591 \\
\hline 21.97 & 17.576 \\
\hline 26.364 & 2.197 \\
\hline 4.394 & 26.364 \\
\hline 35.152 & 4.394 \\
\hline 13.182 & 2.197 \\
\hline 8.788 & 2.197 \\
\hline 24.167 & 24.167 \\
\hline 2.197 & 19.773 \\
\hline 46.137 & 46.137 \\
\hline 2.197 & 375.687 \\
\hline 21.97 & 15.379 \\
\hline 57.122 & 13.182 \\
\hline 2.197 & 26.364 \\
\hline 4.394 & 2.197 \\
\hline 26.364 & 2.197 \\
\hline 4.394 & 6.591 \\
\hline 6.591 & 2.197 \\
\hline 54.925 & 21.97 \\
\hline 2.197 & 8.788 \\
\hline 4.394 & 4.394 \\
\hline 6.591 & 4.394 \\
\hline 8.788 & 243.867 \\
\hline 145.002 & 4.394 \\
\hline 19.773 & 10.985 \\
\hline 2.197 & 2.197 \\
\hline 4.394 & 4.394 \\
\hline 15.379 & 21.97 \\
\hline 2.197 & 48.334 \\
\hline 30.758 & 13.182 \\
\hline 6.591 & 52.728 \\
\hline 8.788 & 48.334 \\
\hline 2.197 & 19.773 \\
\hline 54.925 & 13.182 \\
\hline
\end{tabular}




\begin{tabular}{|c|c|}
\hline 4.394 & 30.758 \\
\hline 2.197 & 39.546 \\
\hline 15.379 & 43.94 \\
\hline 2.197 & 13.182 \\
\hline 26.364 & 2.197 \\
\hline 30.758 & 4.394 \\
\hline 2.197 & 2.197 \\
\hline 2.197 & 4.394 \\
\hline 19.773 & 2.197 \\
\hline 2.197 & 8.788 \\
\hline 2.197 & 2.197 \\
\hline 4.394 & 2.197 \\
\hline 2.197 & 8.788 \\
\hline 87.88 & 65.91 \\
\hline 26.364 & 10.985 \\
\hline 4.394 & 24.167 \\
\hline 114.244 & 8.788 \\
\hline 8.788 & 10.985 \\
\hline 127.426 & 2.197 \\
\hline 2.197 & 21.97 \\
\hline 2.197 & 2.197 \\
\hline 2.197 & 2.197 \\
\hline 2.197 & 15.379 \\
\hline 17.576 & 41.743 \\
\hline 19.773 & 2.197 \\
\hline 15.379 & 2.197 \\
\hline 46.137 & 98.865 \\
\hline 2.197 & 4.394 \\
\hline 487.734 & 2.197 \\
\hline 15.379 & 349.323 \\
\hline 8.788 & 257.049 \\
\hline 19.773 & 52.728 \\
\hline 19.773 & 6.591 \\
\hline 2.197 & 21.97 \\
\hline 2.197 & 63.713 \\
\hline 2.197 & 61.516 \\
\hline 28.561 & 2.197 \\
\hline 19.773 & 4.394 \\
\hline 35.152 & 6.591 \\
\hline 2.197 & 17.576 \\
\hline 59.319 & 2.197 \\
\hline 2.197 & 13.182 \\
\hline 8.788 & 43.94 \\
\hline 1157.82 & 43.94 \\
\hline 2.197 & 15.379 \\
\hline 1537.9 & 2.197 \\
\hline 2.197 & 2.197 \\
\hline 2.197 & 10.985 \\
\hline 2.197 & 2.197 \\
\hline 13.182 & 28.561 \\
\hline
\end{tabular}




\begin{tabular}{|c|c|}
\hline 65.91 & 15.379 \\
\hline 2.197 & 39.546 \\
\hline 4.394 & 120.835 \\
\hline 19.773 & 26.364 \\
\hline 41.743 & 21.97 \\
\hline 41.743 & 17.576 \\
\hline 13.182 & 57.122 \\
\hline 199.927 & 8.788 \\
\hline 8.788 & 24.167 \\
\hline 54.925 & 21.97 \\
\hline 2.197 & 15.379 \\
\hline 6.591 & 2.197 \\
\hline 19.773 & 21.97 \\
\hline 173.563 & 2.197 \\
\hline 2.197 & 15.379 \\
\hline 2.197 & 2.197 \\
\hline 2.197 & 138.411 \\
\hline 8.788 & 2.197 \\
\hline 4.394 & 2.197 \\
\hline 79.092 & 13.182 \\
\hline 193.336 & 26.364 \\
\hline 2.197 & 305.383 \\
\hline 15.379 & 41.743 \\
\hline 24.167 & 8.788 \\
\hline 151.593 & 2.197 \\
\hline 83.486 & 21.97 \\
\hline 2.197 & 101.062 \\
\hline 50.531 & 52.728 \\
\hline 8.788 & 4.394 \\
\hline 2.197 & 48.334 \\
\hline 2.197 & 59.319 \\
\hline 2.197 & 2.197 \\
\hline 2.197 & 37.349 \\
\hline 2.197 & 4.394 \\
\hline 2.197 & 2.197 \\
\hline 13.182 & 4.394 \\
\hline 2.197 & 2.197 \\
\hline 2.197 & 8.788 \\
\hline 237.276 & 2.197 \\
\hline 2.197 & 15.379 \\
\hline 120.835 & 134.017 \\
\hline 2.197 & 85.683 \\
\hline 235.079 & 2.197 \\
\hline 2.197 & 109.85 \\
\hline 2.197 & 2.197 \\
\hline 43.94 & 70.304 \\
\hline 2.197 & 2.197 \\
\hline 2.197 & 32.955 \\
\hline 2.197 & 15.379 \\
\hline 2.197 & 37.349 \\
\hline
\end{tabular}




\begin{tabular}{|c|c|}
\hline 8.788 & 92.274 \\
\hline 15.379 & 257.049 \\
\hline 10.985 & 6.591 \\
\hline 2.197 & 10.985 \\
\hline 50.531 & 63.713 \\
\hline 2.197 & 24.167 \\
\hline 4.394 & 17.576 \\
\hline 43.94 & 21.97 \\
\hline 2.197 & 4.394 \\
\hline 35.152 & 13.182 \\
\hline 10.985 & 48.334 \\
\hline 19.773 & 13.182 \\
\hline 28.561 & 134.017 \\
\hline 8.788 & 2.197 \\
\hline 13.182 & 2.197 \\
\hline 4.394 & 2.197 \\
\hline 74.698 & 2.197 \\
\hline 2.197 & 2.197 \\
\hline 2.197 & 2.197 \\
\hline 10.985 & 2.197 \\
\hline 2.197 & 2.197 \\
\hline 17.576 & 6.591 \\
\hline 4.394 & 2.197 \\
\hline 2.197 & 4.394 \\
\hline 2.197 & 2.197 \\
\hline 270.231 & 15.379 \\
\hline 63.713 & 4.394 \\
\hline 39.546 & 4.394 \\
\hline 6.591 & 6.591 \\
\hline 2.197 & 8.788 \\
\hline 114.244 & 13.182 \\
\hline 2.197 & 2.197 \\
\hline 2.197 & 2.197 \\
\hline 184.548 & 2.197 \\
\hline 8.788 & 15.379 \\
\hline 2.197 & 30.758 \\
\hline 10.985 & 10.985 \\
\hline 15.379 & 6.591 \\
\hline 2.197 & 13.182 \\
\hline 6.591 & 2.197 \\
\hline 46.137 & 4.394 \\
\hline 46.137 & 4.394 \\
\hline 6.591 & 4.394 \\
\hline 32.955 & 2.197 \\
\hline 2.197 & 57.122 \\
\hline 26.364 & 35.152 \\
\hline 8.788 & 17.576 \\
\hline 26.364 & 6.591 \\
\hline 2.197 & 37.349 \\
\hline 162.578 & 98.865 \\
\hline
\end{tabular}




$\begin{array}{rr}518.492 & 6.591 \\ 35.152 & 17.576 \\ 2.197 & 131.82 \\ 430.612 & 57.122 \\ 2.197 & 17.576 \\ 26.364 & 2.197 \\ 2.197 & 19.773 \\ 37.349 & 127.426 \\ 72.501 & 41.743 \\ 79.092 & 2.197 \\ 41.743 & 21.97 \\ 4.394 & 2.197 \\ 2.197 & 68.107 \\ 13.182 & 28.561 \\ 4.394 & 41.743 \\ 406.445 & 1904.8 \\ 8.788 & 101.062 \\ 68.107 & 10.985 \\ 10.985 & 6.591 \\ 2.197 & 19.773 \\ 63.713 & 28.561 \\ 8.788 & 2.197 \\ 2.197 & \end{array}$




\begin{tabular}{|c|c|}
\hline 2.197 & 2.197 \\
\hline 35.152 & 2.197 \\
\hline 28.561 & 2.197 \\
\hline 10.985 & 2.197 \\
\hline 2.197 & 54.925 \\
\hline 10.985 & 2.197 \\
\hline 4.394 & 46.137 \\
\hline 2.197 & 72.501 \\
\hline 4.394 & 474.552 \\
\hline 8.788 & 8.788 \\
\hline 8.788 & 2.197 \\
\hline 2.197 & 4.394 \\
\hline 54.925 & 2.197 \\
\hline 2.197 & 6.591 \\
\hline 10.985 & 63.713 \\
\hline 8.788 & 215.306 \\
\hline 13.182 & 2.197 \\
\hline 2.197 & 2.197 \\
\hline 107.653 & 2.197 \\
\hline 2.197 & 10.985 \\
\hline 8.788 & 57.122 \\
\hline 4.394 & 2.197 \\
\hline 2.197 & 41.743 \\
\hline 2.197 & 101.062 \\
\hline 4.394 & 2.197 \\
\hline 2.197 & 4.394 \\
\hline 2.197 & 2.197 \\
\hline 2.197 & 6.591 \\
\hline 445.991 & 6.591 \\
\hline 15.379 & 21.97 \\
\hline 404.248 & 2.197 \\
\hline 15.379 & 2.197 \\
\hline 8.788 & 30.758 \\
\hline 2.197 & 2.197 \\
\hline 4.394 & 2.197 \\
\hline 15.379 & 6.591 \\
\hline 138.411 & 2.197 \\
\hline 8.788 & 408.642 \\
\hline 2.197 & 76.895 \\
\hline 2.197 & 24.167 \\
\hline 81.289 & 391.066 \\
\hline 2.197 & 39.546 \\
\hline 2.197 & 4.394 \\
\hline 2.197 & 21.97 \\
\hline 79.092 & 8.788 \\
\hline 2.197 & 26.364 \\
\hline 70.304 & 26.364 \\
\hline 1478.58 & 2.197 \\
\hline 4.394 & 8.788 \\
\hline 6.591 & 2.197 \\
\hline
\end{tabular}




\begin{tabular}{|c|c|}
\hline 15.379 & 6.591 \\
\hline 48.334 & 2.197 \\
\hline 59.319 & 4.394 \\
\hline 2.197 & 4.394 \\
\hline 6.591 & 112.047 \\
\hline 19.773 & 112.047 \\
\hline 32.955 & 2.197 \\
\hline 2.197 & 2.197 \\
\hline 2.197 & 63.713 \\
\hline 2.197 & 6.591 \\
\hline 2.197 & 76.895 \\
\hline 2.197 & 8.788 \\
\hline 4.394 & 2.197 \\
\hline 2.197 & 13.182 \\
\hline 136.214 & 2.197 \\
\hline 2.197 & 15.379 \\
\hline 2.197 & 46.137 \\
\hline 26.364 & 59.319 \\
\hline 2.197 & 48.334 \\
\hline 2.197 & 797.511 \\
\hline 2.197 & 250.458 \\
\hline 2.197 & 962.286 \\
\hline 2.197 & 61.516 \\
\hline 4.394 & 72.501 \\
\hline 2.197 & 21.97 \\
\hline 4.394 & 79.092 \\
\hline 4.394 & 59.319 \\
\hline 4.394 & 2.197 \\
\hline 46.137 & 2.197 \\
\hline 2.197 & 26.364 \\
\hline 573.417 & 19.773 \\
\hline 120.835 & 1010.62 \\
\hline 2.197 & 2.197 \\
\hline 2.197 & 336.141 \\
\hline 21.97 & 8.788 \\
\hline 17.576 & 4.394 \\
\hline 2.197 & 2.197 \\
\hline 32.955 & 10.985 \\
\hline 2.197 & 90.077 \\
\hline 2.197 & 59.319 \\
\hline 37.349 & 2.197 \\
\hline 2.197 & 35.152 \\
\hline 2.197 & 2.197 \\
\hline 2.197 & 19.773 \\
\hline 15.379 & 2.197 \\
\hline 2.197 & 15.379 \\
\hline 2.197 & 76.895 \\
\hline 2.197 & 4.394 \\
\hline 2.197 & 43.94 \\
\hline 2.197 & 4.394 \\
\hline
\end{tabular}




\begin{tabular}{|c|c|}
\hline 65.91 & 8.788 \\
\hline 2.197 & 79.092 \\
\hline 4.394 & 2.197 \\
\hline 162.578 & 26.364 \\
\hline 37.349 & 17.576 \\
\hline 4.394 & 8.788 \\
\hline 15.379 & 6.591 \\
\hline 26.364 & 2.197 \\
\hline 2.197 & 2.197 \\
\hline 10.985 & 19.773 \\
\hline 28.561 & 2.197 \\
\hline 2.197 & 2.197 \\
\hline 2.197 & 8.788 \\
\hline 30.758 & 2.197 \\
\hline 2.197 & 4.394 \\
\hline 2.197 & 120.835 \\
\hline 6.591 & 8.788 \\
\hline 4.394 & 19.773 \\
\hline 2.197 & 2.197 \\
\hline 10.985 & 90.077 \\
\hline 10.985 & 52.728 \\
\hline 10.985 & 48.334 \\
\hline 4.394 & 4.394 \\
\hline 2.197 & 112.047 \\
\hline 2.197 & 13.182 \\
\hline 2.197 & 79.092 \\
\hline 26.364 & 17.576 \\
\hline 2.197 & 151.593 \\
\hline 10.985 & 2.197 \\
\hline 15.379 & 6.591 \\
\hline 30.758 & 2.197 \\
\hline 10.985 & 4.394 \\
\hline 4.394 & 8.788 \\
\hline 4.394 & 131.82 \\
\hline 4.394 & 48.334 \\
\hline 4.394 & 6.591 \\
\hline 4.394 & 4.394 \\
\hline 24.167 & 28.561 \\
\hline 4.394 & 2.197 \\
\hline 15.379 & 2.197 \\
\hline 837.057 & 17.576 \\
\hline 2.197 & 8.788 \\
\hline 26.364 & 37.349 \\
\hline 2.197 & 4.394 \\
\hline 2.197 & 4.394 \\
\hline 4.394 & 10.985 \\
\hline 2.197 & 4.394 \\
\hline 2.197 & 4.394 \\
\hline 2.197 & 19.773 \\
\hline 41.743 & 50.531 \\
\hline
\end{tabular}




\begin{tabular}{|c|c|}
\hline 43.94 & 30.758 \\
\hline 21.97 & 24.167 \\
\hline 10.985 & 109.85 \\
\hline 13.182 & 4.394 \\
\hline 37.349 & 6.591 \\
\hline 2.197 & 13.182 \\
\hline 28.561 & 2.197 \\
\hline 52.728 & 2.197 \\
\hline 2.197 & 17.576 \\
\hline 2.197 & 24.167 \\
\hline 2.197 & 59.319 \\
\hline 4.394 & 4.394 \\
\hline 384.475 & 8.788 \\
\hline 4.394 & 2.197 \\
\hline 2.197 & 755.768 \\
\hline 13.182 & 109.85 \\
\hline 6.591 & 274.625 \\
\hline 4.394 & 59.319 \\
\hline 63.713 & 10.985 \\
\hline 4.394 & 2.197 \\
\hline 4.394 & 24.167 \\
\hline 43.94 & 68.107 \\
\hline 4.394 & 2.197 \\
\hline 2.197 & 2.197 \\
\hline 2.197 & 35.152 \\
\hline 2.197 & 41.743 \\
\hline 2.197 & 221.897 \\
\hline 2.197 & 48.334 \\
\hline 10.985 & 338.338 \\
\hline 4.394 & 8.788 \\
\hline 6.591 & 2.197 \\
\hline 13.182 & 39.546 \\
\hline 50.531 & 303.186 \\
\hline 32.955 & 6.591 \\
\hline 39.546 & 35.152 \\
\hline 70.304 & 2.197 \\
\hline 35.152 & 2.197 \\
\hline 8.788 & 202.124 \\
\hline 24.167 & 145.002 \\
\hline 8.788 & 171.366 \\
\hline 2.197 & 2.197 \\
\hline 65.91 & 57.122 \\
\hline 10.985 & 63.713 \\
\hline 584.402 & 4.394 \\
\hline 2.197 & 103.259 \\
\hline 68.107 & 8.788 \\
\hline 24.167 & 2.197 \\
\hline 52.728 & 19.773 \\
\hline 114.244 & 19.773 \\
\hline 2.197 & 2.19 \\
\hline
\end{tabular}




\begin{tabular}{|c|c|}
\hline 2.197 & 30.758 \\
\hline 13.182 & 54.925 \\
\hline 2.197 & 2.197 \\
\hline 2.197 & 6.591 \\
\hline 191.139 & 109.85 \\
\hline 28.561 & 6.591 \\
\hline 4.394 & 6.591 \\
\hline 8.788 & 2.197 \\
\hline 815.087 & 13.182 \\
\hline 2.197 & 17.576 \\
\hline 17.576 & 2.197 \\
\hline 2.197 & 10.985 \\
\hline 2.197 & 2.197 \\
\hline 19.773 & 21.97 \\
\hline 123.032 & 15.379 \\
\hline 2.197 & 26.364 \\
\hline 2.197 & 2.197 \\
\hline 2.197 & 2.197 \\
\hline 2.197 & 94.471 \\
\hline 6.591 & 8.788 \\
\hline 2.197 & 2.197 \\
\hline 2.197 & 81.289 \\
\hline 6.591 & 2.197 \\
\hline 39.546 & 24.167 \\
\hline 2.197 & 252.655 \\
\hline 2.197 & 606.372 \\
\hline 2.197 & 6.591 \\
\hline 6.591 & 43.94 \\
\hline 2.197 & 109.85 \\
\hline 4.394 & 1076.53 \\
\hline 2.197 & 4.394 \\
\hline 4.394 & 2.197 \\
\hline 2.197 & 2.197 \\
\hline 2.197 & 2.197 \\
\hline 406.445 & 41.743 \\
\hline 32.955 & 2.197 \\
\hline 2.197 & 2.197 \\
\hline 2.197 & 2.197 \\
\hline 2.197 & 2.197 \\
\hline 2.197 & 4.394 \\
\hline 48.334 & 48.334 \\
\hline 2.197 & 2.197 \\
\hline 6.591 & 8.788 \\
\hline 2.197 & 2.197 \\
\hline 101.062 & 19.773 \\
\hline 2.197 & 149.396 \\
\hline 2.197 & 4.394 \\
\hline 8.788 & 217.503 \\
\hline 81.289 & 4.394 \\
\hline 2.197 & 2.197 \\
\hline
\end{tabular}




\begin{tabular}{|c|c|}
\hline 2.197 & 2.197 \\
\hline 24.167 & 6.591 \\
\hline 2.197 & 4.394 \\
\hline 8.788 & 2.197 \\
\hline 2.197 & 21.97 \\
\hline 2.197 & 219.7 \\
\hline 19.773 & 4.394 \\
\hline 37.349 & 2.197 \\
\hline 6.591 & 32.955 \\
\hline 4.394 & 8.788 \\
\hline 4.394 & 6.591 \\
\hline 10.985 & 1997.07 \\
\hline 68.107 & 2.197 \\
\hline 261.443 & 4.394 \\
\hline 2.197 & 4.394 \\
\hline 2.197 & 41.743 \\
\hline 2.197 & 24.167 \\
\hline 37.349 & 2.197 \\
\hline 48.334 & 28.561 \\
\hline 2.197 & 105.456 \\
\hline 2.197 & 6.591 \\
\hline 2.197 & 891.982 \\
\hline 2.197 & 2.197 \\
\hline 2.197 & 17.576 \\
\hline 2.197 & 114.244 \\
\hline 2.197 & 2.197 \\
\hline 2.197 & 10.985 \\
\hline 17.576 & 17.576 \\
\hline 21.97 & 2.197 \\
\hline 4.394 & 2.197 \\
\hline 136.214 & 8.788 \\
\hline 54.925 & 125.229 \\
\hline 4.394 & 10.985 \\
\hline 2.197 & 52.728 \\
\hline 15.379 & 2.197 \\
\hline 21.97 & 10.985 \\
\hline 10.985 & 2.197 \\
\hline 48.334 & 2.197 \\
\hline 2.197 & 362.505 \\
\hline 26.364 & 48.334 \\
\hline 6.591 & 19.773 \\
\hline 2.197 & 10.985 \\
\hline 6.591 & 184.548 \\
\hline 13.182 & 15.379 \\
\hline 2.197 & 81.289 \\
\hline 2.197 & 155.987 \\
\hline 8.788 & 4.394 \\
\hline 2.197 & 2.197 \\
\hline 15.379 & 2.197 \\
\hline 15.379 & 125.229 \\
\hline
\end{tabular}




\begin{tabular}{|c|c|}
\hline 4.394 & 4.394 \\
\hline 2.197 & 26.364 \\
\hline 2.197 & 24.167 \\
\hline 199.927 & 48.334 \\
\hline 4.394 & 96.668 \\
\hline 2.197 & 2.197 \\
\hline 2.197 & 300.989 \\
\hline 2.197 & 26.364 \\
\hline 8.788 & 4.394 \\
\hline 4.394 & 2.197 \\
\hline 10.985 & 1515.93 \\
\hline 2.197 & 74.698 \\
\hline 13.182 & 2.197 \\
\hline 112.047 & 92.274 \\
\hline 2.197 & 10.985 \\
\hline 2.197 & 4.394 \\
\hline 4.394 & 2.197 \\
\hline 4.394 & 2.197 \\
\hline 2.197 & 2.197 \\
\hline 2.197 & 15.379 \\
\hline 2.197 & 10.985 \\
\hline 131.82 & 65.91 \\
\hline 2.197 & 4.394 \\
\hline 2.197 & 90.077 \\
\hline 2.197 & 43.94 \\
\hline 2.197 & 54.925 \\
\hline 4.394 & 4.394 \\
\hline 2.197 & 4.394 \\
\hline 21.97 & 10.985 \\
\hline 68.107 & 4.394 \\
\hline 4.394 & 118.638 \\
\hline 182.351 & 8.788 \\
\hline 50.531 & 8.788 \\
\hline 8.788 & 2.197 \\
\hline 760.162 & 4.394 \\
\hline 2.197 & 114.244 \\
\hline 8.788 & 2.197 \\
\hline 2.197 & 2.197 \\
\hline 13.182 & 87.88 \\
\hline 21.97 & 105.456 \\
\hline 4.394 & 10.985 \\
\hline 2.197 & 2.197 \\
\hline 2.197 & 17.576 \\
\hline 2.197 & 10.985 \\
\hline 2.197 & 586.599 \\
\hline 2.197 & 404.248 \\
\hline 2.197 & 6.591 \\
\hline 94.471 & 2.197 \\
\hline 2.197 & 2.197 \\
\hline 35.152 & 90.07 \\
\hline
\end{tabular}




\begin{tabular}{|c|c|}
\hline 2.197 & 2.197 \\
\hline 2.197 & 472.355 \\
\hline 6.591 & 35.152 \\
\hline 4.394 & 2.197 \\
\hline 4.394 & 37.349 \\
\hline 8.788 & 6.591 \\
\hline 2.197 & 2.197 \\
\hline 2.197 & 2.197 \\
\hline 13.182 & 87.88 \\
\hline 2.197 & 2.197 \\
\hline 2.197 & 127.426 \\
\hline 6.591 & 2.197 \\
\hline 6.591 & 39.546 \\
\hline 2.197 & 28.561 \\
\hline 8.788 & 24.167 \\
\hline 2.197 & 454.779 \\
\hline 2.197 & 6.591 \\
\hline 8.788 & 10.985 \\
\hline 235.079 & 48.334 \\
\hline 2.197 & 10.985 \\
\hline 10.985 & 19.773 \\
\hline 10.985 & 459.173 \\
\hline 2.197 & 1414.87 \\
\hline 136.214 & 171.366 \\
\hline 13.182 & 2.197 \\
\hline 4.394 & 375.687 \\
\hline 13.182 & 41.743 \\
\hline 2.197 & 8.788 \\
\hline 4.394 & 4.394 \\
\hline 4.394 & 21.97 \\
\hline 2.197 & 6.591 \\
\hline 2.197 & 17.576 \\
\hline 2.197 & 4.394 \\
\hline 2.197 & 2.197 \\
\hline 21.97 & 19.773 \\
\hline 2.197 & 13.182 \\
\hline 17.576 & 2.197 \\
\hline 13.182 & 30.758 \\
\hline 4.394 & 518.492 \\
\hline 4.394 & 79.092 \\
\hline 24.167 & 120.835 \\
\hline 4.394 & 30.758 \\
\hline 2.197 & 766.753 \\
\hline 2.197 & 2.197 \\
\hline 2.197 & 43.94 \\
\hline 2.197 & 8.788 \\
\hline 46.137 & 24.167 \\
\hline 24.167 & 975.468 \\
\hline 57.122 & 2.197 \\
\hline 4.394 & 4.394 \\
\hline
\end{tabular}




\begin{tabular}{|c|c|}
\hline 2.197 & 13.182 \\
\hline 2.197 & 2.197 \\
\hline 2.197 & 43.94 \\
\hline 4.394 & 2.197 \\
\hline 2.197 & 2.197 \\
\hline 142.805 & 2.197 \\
\hline 2.197 & 2.197 \\
\hline 2.197 & 6.591 \\
\hline 2.197 & 19.773 \\
\hline 63.713 & 2.197 \\
\hline 8.788 & 2.197 \\
\hline 15.379 & 28.561 \\
\hline 2.197 & 10.985 \\
\hline 4.394 & 4.394 \\
\hline 419.627 & 4.394 \\
\hline 8.788 & 4.394 \\
\hline 2.197 & 6.591 \\
\hline 83.486 & 2.197 \\
\hline 107.653 & 2.197 \\
\hline 142.805 & 21.97 \\
\hline 21.97 & 6.591 \\
\hline 8.788 & 13.182 \\
\hline 2.197 & 76.895 \\
\hline 4.394 & 43.94 \\
\hline 74.698 & 26.364 \\
\hline 2.197 & 2.197 \\
\hline 4.394 & 10.985 \\
\hline 2.197 & 68.107 \\
\hline 10.985 & 4.394 \\
\hline 85.683 & 2.197 \\
\hline 6.591 & 382.278 \\
\hline 4.394 & 230.685 \\
\hline 2.197 & 24.167 \\
\hline 2.197 & 8.788 \\
\hline 2.197 & 6.591 \\
\hline 4.394 & 184.548 \\
\hline 21.97 & 2.197 \\
\hline 2.197 & 2.197 \\
\hline 87.88 & 239.473 \\
\hline 37.349 & 28.561 \\
\hline 61.516 & 2.197 \\
\hline 37.349 & 13.182 \\
\hline 21.97 & 2.197 \\
\hline 4.394 & 26.364 \\
\hline 112.047 & 48.334 \\
\hline 116.441 & 2.197 \\
\hline 2.197 & 2.197 \\
\hline 10.985 & 6.591 \\
\hline 4.394 & 2.197 \\
\hline 4.394 & 52.728 \\
\hline
\end{tabular}




\begin{tabular}{|c|c|}
\hline 2.197 & 43.94 \\
\hline 37.349 & 2.197 \\
\hline 10.985 & 13.182 \\
\hline 35.152 & 48.334 \\
\hline 4.394 & 2.197 \\
\hline 8.788 & 83.486 \\
\hline 13.182 & 2.197 \\
\hline 2.197 & 63.713 \\
\hline 2.197 & 4.394 \\
\hline 2.197 & 28.561 \\
\hline 2.197 & 166.972 \\
\hline 24.167 & 265.837 \\
\hline 8.788 & 2.197 \\
\hline 32.955 & 2.197 \\
\hline 116.441 & 85.683 \\
\hline 32.955 & 2.197 \\
\hline 57.122 & 4.394 \\
\hline 2.197 & 4.394 \\
\hline 6.591 & 2.197 \\
\hline 2.197 & 2.197 \\
\hline 2.197 & 2.197 \\
\hline 2.197 & 46.137 \\
\hline 46.137 & 30.758 \\
\hline 17.576 & 2.197 \\
\hline 48.334 & 2.197 \\
\hline 54.925 & 87.88 \\
\hline 2.197 & 2.197 \\
\hline 4.394 & 32.955 \\
\hline 2.197 & 24.167 \\
\hline 2.197 & 28.561 \\
\hline 10.985 & 2.197 \\
\hline 4.394 & 4.394 \\
\hline 2.197 & 6.591 \\
\hline 155.987 & 2.197 \\
\hline 30.758 & 197.73 \\
\hline 26.364 & 2.197 \\
\hline 2.197 & 52.728 \\
\hline 4.394 & 6.591 \\
\hline 2.197 & 2.197 \\
\hline 1311.61 & 2.197 \\
\hline 4.394 & 2.197 \\
\hline 35.152 & 19.773 \\
\hline 4.394 & 2.197 \\
\hline 2.197 & 8.788 \\
\hline 35.152 & 8.788 \\
\hline 6.591 & 92.274 \\
\hline 6.591 & 19.773 \\
\hline 8.788 & 103.259 \\
\hline 134.017 & 8.788 \\
\hline 96.668 & 2.197 \\
\hline
\end{tabular}




\begin{tabular}{|c|c|}
\hline 10.985 & 2.197 \\
\hline 10.985 & 2.197 \\
\hline 2.197 & 15.379 \\
\hline 2.197 & 4.394 \\
\hline 98.865 & 13.182 \\
\hline 13.182 & 70.304 \\
\hline 10.985 & 8.788 \\
\hline 2.197 & 30.758 \\
\hline 2.197 & 65.91 \\
\hline 8.788 & 4.394 \\
\hline 2.197 & 17.576 \\
\hline 6.591 & 8.788 \\
\hline 2.197 & 525.083 \\
\hline 2.197 & 65.91 \\
\hline 2.197 & 19.773 \\
\hline 4.394 & 13.182 \\
\hline 2.197 & 2.197 \\
\hline 2.197 & 4.394 \\
\hline 2.197 & 4.394 \\
\hline 92.274 & 2.197 \\
\hline 2.197 & 39.546 \\
\hline 2.197 & 76.895 \\
\hline 8.788 & 21.97 \\
\hline 2.197 & 41.743 \\
\hline 129.623 & 4.394 \\
\hline 283.413 & 59.319 \\
\hline 2.197 & 2.197 \\
\hline 2.197 & 24.167 \\
\hline 17.576 & 4.394 \\
\hline 2.197 & 48.334 \\
\hline 4.394 & 2.197 \\
\hline 4.394 & 2.197 \\
\hline 2.197 & 26.364 \\
\hline 2.197 & 2.197 \\
\hline 19.773 & 37.349 \\
\hline 125.229 & 2.197 \\
\hline 43.94 & 2.197 \\
\hline 52.728 & 2.197 \\
\hline 4.394 & 21.97 \\
\hline 6.591 & 26.364 \\
\hline 8.788 & 4.394 \\
\hline 2.197 & 83.486 \\
\hline 4.394 & 19.773 \\
\hline 4.394 & 109.85 \\
\hline 81.289 & 13.182 \\
\hline 6.591 & 59.319 \\
\hline 10.985 & 8.788 \\
\hline 10.985 & 21.97 \\
\hline 2.197 & 6.591 \\
\hline 6.591 & 395.46 \\
\hline
\end{tabular}




\begin{tabular}{|c|c|}
\hline 15.379 & 10.985 \\
\hline 478.946 & 4.394 \\
\hline 4.394 & 54.925 \\
\hline 2.197 & 10.985 \\
\hline 4.394 & 65.91 \\
\hline 2.197 & 39.546 \\
\hline 2.197 & 336.141 \\
\hline 28.561 & 188.942 \\
\hline 4.394 & 15.379 \\
\hline 4.394 & 4.394 \\
\hline 2.197 & 2.197 \\
\hline 2.197 & 15.379 \\
\hline 2.197 & 19.773 \\
\hline 2.197 & 10.985 \\
\hline 2.197 & 8.788 \\
\hline 46.137 & 19.773 \\
\hline 2.197 & 949.104 \\
\hline 2.197 & 125.229 \\
\hline 52.728 & 17.576 \\
\hline 6.591 & 2.197 \\
\hline 13.182 & 4.394 \\
\hline 35.152 & 162.578 \\
\hline 6.591 & 2.197 \\
\hline 2.197 & 2.197 \\
\hline 2.197 & 131.82 \\
\hline 2.197 & 10.985 \\
\hline 4.394 & 2.197 \\
\hline 61.516 & 10.985 \\
\hline 4.394 & 8.788 \\
\hline 2.197 & 46.137 \\
\hline 2.197 & 8.788 \\
\hline 2.197 & 17.576 \\
\hline 2.197 & 41.743 \\
\hline 8.788 & 2.197 \\
\hline 13.182 & 46.137 \\
\hline 191.139 & 2.197 \\
\hline 10.985 & 2.197 \\
\hline 68.107 & 2.197 \\
\hline 2.197 & 2.197 \\
\hline 2.197 & 59.319 \\
\hline 41.743 & 61.516 \\
\hline 2.197 & 10.985 \\
\hline 2.197 & 13.182 \\
\hline 10.985 & 61.516 \\
\hline 2.197 & 32.955 \\
\hline 4.394 & 61.516 \\
\hline 4.394 & 85.683 \\
\hline 4.394 & 4.394 \\
\hline 4.394 & 2.197 \\
\hline 2.197 & 39.546 \\
\hline
\end{tabular}




\begin{tabular}{|c|c|}
\hline 4.394 & 6.591 \\
\hline 52.728 & 230.685 \\
\hline 63.713 & 65.91 \\
\hline 8.788 & 15.379 \\
\hline 28.561 & 24.167 \\
\hline 37.349 & 2.197 \\
\hline 4.394 & 2.197 \\
\hline 2.197 & 13.182 \\
\hline 2.197 & 4.394 \\
\hline 15.379 & 37.349 \\
\hline 2.197 & 15.379 \\
\hline 15.379 & 107.653 \\
\hline 2.197 & 2.197 \\
\hline 4.394 & 15.379 \\
\hline 8.788 & 184.548 \\
\hline 4.394 & 2.197 \\
\hline 48.334 & 98.865 \\
\hline 2.197 & 10.985 \\
\hline 386.672 & 13.182 \\
\hline 19.773 & 26.364 \\
\hline 4.394 & 6.591 \\
\hline 10.985 & 2.197 \\
\hline 26.364 & 8.788 \\
\hline 2.197 & 10.985 \\
\hline 21.97 & 120.835 \\
\hline 2.197 & 4.394 \\
\hline 112.047 & 2.197 \\
\hline 8.788 & 30.758 \\
\hline 177.957 & 8.788 \\
\hline 48.334 & 52.728 \\
\hline 4.394 & 4.394 \\
\hline 4.394 & 17.576 \\
\hline 2.197 & 24.167 \\
\hline 2.197 & 169.169 \\
\hline 2.197 & 2.197 \\
\hline 2.197 & 4.394 \\
\hline 2.197 & 6.591 \\
\hline 24.167 & 2.197 \\
\hline 4.394 & 2.197 \\
\hline 2.197 & 41.743 \\
\hline 4.394 & 4.394 \\
\hline 10.985 & 50.531 \\
\hline 2.197 & 10.985 \\
\hline 2.197 & 48.334 \\
\hline 10.985 & 2.197 \\
\hline 8.788 & 2.197 \\
\hline 4.394 & 2.197 \\
\hline 81.289 & 142.805 \\
\hline 15.379 & 155.987 \\
\hline 193.336 & 2.197 \\
\hline
\end{tabular}




\begin{tabular}{|c|c|}
\hline 8.788 & 2.197 \\
\hline 6.591 & 10.985 \\
\hline 6.591 & 2.197 \\
\hline 10.985 & 153.79 \\
\hline 2.197 & 2.197 \\
\hline 2.197 & 123.032 \\
\hline 101.062 & 10.985 \\
\hline 2.197 & 2.197 \\
\hline 2.197 & 109.85 \\
\hline 2.197 & 8.788 \\
\hline 193.336 & 10.985 \\
\hline 21.97 & 2.197 \\
\hline 8.788 & 15.379 \\
\hline 74.698 & 4.394 \\
\hline 10.985 & 15.379 \\
\hline 4.394 & 50.531 \\
\hline 10.985 & 28.561 \\
\hline 6.591 & 39.546 \\
\hline 2.197 & 19.773 \\
\hline 15.379 & 2.197 \\
\hline 2.197 & 338.338 \\
\hline 8.788 & 87.88 \\
\hline 37.349 & 2.197 \\
\hline 2.197 & 61.516 \\
\hline 2.197 & 2.197 \\
\hline 4.394 & 81.289 \\
\hline 4.394 & 10.985 \\
\hline 76.895 & 13.182 \\
\hline 6.591 & 2.197 \\
\hline 2.197 & 15.379 \\
\hline 30.758 & 8.788 \\
\hline 57.122 & 30.758 \\
\hline 2.197 & 2.197 \\
\hline 2.197 & 2.197 \\
\hline 2.197 & 21.97 \\
\hline 13.182 & 4.394 \\
\hline 4.394 & 4.394 \\
\hline 4.394 & 2.197 \\
\hline 2.197 & 50.531 \\
\hline 46.137 & 4.394 \\
\hline 2.197 & 43.94 \\
\hline 13.182 & 4.394 \\
\hline 10.985 & 2.197 \\
\hline 138.411 & 4.394 \\
\hline 2.197 & 2.197 \\
\hline 4.394 & 2.197 \\
\hline 24.167 & 61.516 \\
\hline 140.608 & 37.349 \\
\hline 2.197 & 109.85 \\
\hline 17.576 & 54.925 \\
\hline
\end{tabular}




\begin{tabular}{|c|c|}
\hline 2.197 & 52.728 \\
\hline 72.501 & 32.955 \\
\hline 26.364 & 43.94 \\
\hline 2.197 & 226.291 \\
\hline 32.955 & 10.985 \\
\hline 4.394 & 17.576 \\
\hline 4.394 & 8.788 \\
\hline 4.394 & 2.197 \\
\hline 4.394 & 6.591 \\
\hline 4.394 & 39.546 \\
\hline 4.394 & 6.591 \\
\hline 2.197 & 15.379 \\
\hline 7278.66 & 158.184 \\
\hline 17.576 & 116.441 \\
\hline 2.197 & 2.197 \\
\hline 4.394 & 120.835 \\
\hline 2.197 & 13.182 \\
\hline 2.197 & 26.364 \\
\hline 2.197 & 105.456 \\
\hline 2.197 & 57.122 \\
\hline 2.197 & 52.728 \\
\hline 98.865 & 8.788 \\
\hline 54.925 & 28.561 \\
\hline 2.197 & 24.167 \\
\hline 663.494 & 175.76 \\
\hline 2.197 & 204.321 \\
\hline 6.591 & 4.394 \\
\hline 158.184 & 6.591 \\
\hline 2.197 & 112.047 \\
\hline 2.197 & 4.394 \\
\hline 6.591 & 15.379 \\
\hline 13.182 & 2.197 \\
\hline 39.546 & 2.197 \\
\hline 2.197 & 2.197 \\
\hline 10.985 & 2.197 \\
\hline 6.591 & 2.197 \\
\hline 123.032 & 32.955 \\
\hline 2.197 & 8.788 \\
\hline 4.394 & 61.516 \\
\hline 2.197 & 4.394 \\
\hline 131.82 & 46.137 \\
\hline 8.788 & 43.94 \\
\hline 4.394 & 90.077 \\
\hline 8.788 & 2.197 \\
\hline 79.092 & 281.216 \\
\hline 182.351 & 103.259 \\
\hline 2.197 & 4.394 \\
\hline 85.683 & 4.394 \\
\hline 13.182 & 114.244 \\
\hline 2.197 & 2.197 \\
\hline
\end{tabular}




\begin{tabular}{|c|c|}
\hline 2.197 & 2.197 \\
\hline 2.197 & 2.197 \\
\hline 2.197 & 4.394 \\
\hline 8.788 & 46.137 \\
\hline 4.394 & 96.668 \\
\hline 15.379 & 8.788 \\
\hline 2.197 & 17.576 \\
\hline 8.788 & 37.349 \\
\hline 50.531 & 8.788 \\
\hline 6.591 & 2.197 \\
\hline 2.197 & 74.698 \\
\hline 10.985 & 94.471 \\
\hline 195.533 & 26.364 \\
\hline 2.197 & 65.91 \\
\hline 2.197 & 114.244 \\
\hline 96.668 & 46.137 \\
\hline 4.394 & 85.683 \\
\hline 4.394 & 41.743 \\
\hline 2.197 & 92.274 \\
\hline 2.197 & 149.396 \\
\hline 13.182 & 6.591 \\
\hline 4.394 & 2.197 \\
\hline 2.197 & 2.197 \\
\hline 4.394 & 54.925 \\
\hline 15.379 & 8.788 \\
\hline 10.985 & 28.561 \\
\hline 43.94 & 2.197 \\
\hline 8.788 & 65.91 \\
\hline 2.197 & 2.197 \\
\hline 2.197 & 50.531 \\
\hline 2.197 & 2.197 \\
\hline 15.379 & 70.304 \\
\hline 2.197 & 35.152 \\
\hline 2.197 & 127.426 \\
\hline 8.788 & 2.197 \\
\hline 28.561 & 2.197 \\
\hline 2.197 & 17.576 \\
\hline 8.788 & 46.137 \\
\hline 92.274 & 74.698 \\
\hline 6.591 & 8.788 \\
\hline 573.417 & 2.197 \\
\hline 8.788 & 79.092 \\
\hline 8.788 & 239.473 \\
\hline 307.58 & 2.197 \\
\hline 4.394 & 30.758 \\
\hline 4.394 & 2.197 \\
\hline 2.197 & 2.197 \\
\hline 2.197 & 10.985 \\
\hline 8.788 & 10.985 \\
\hline 28.561 & 2.197 \\
\hline
\end{tabular}




\begin{tabular}{|c|c|}
\hline 26.364 & 83.486 \\
\hline 2.197 & 10.985 \\
\hline 21.97 & 8.788 \\
\hline 2.197 & 21.97 \\
\hline 228.488 & 26.364 \\
\hline 202.124 & 2.197 \\
\hline 50.531 & 10.985 \\
\hline 8.788 & 2.197 \\
\hline 4.394 & 79.092 \\
\hline 4.394 & 17.576 \\
\hline 2.197 & 4.394 \\
\hline 2.197 & 28.561 \\
\hline 15.379 & 2.197 \\
\hline 26.364 & 57.122 \\
\hline 4.394 & 43.94 \\
\hline 2.197 & 6.591 \\
\hline 65.91 & 6.591 \\
\hline 2.197 & 54.925 \\
\hline 87.88 & 21.97 \\
\hline 4.394 & 21.97 \\
\hline 4.394 & 15.379 \\
\hline 4.394 & 15.379 \\
\hline 2.197 & 2.197 \\
\hline 2.197 & 2.197 \\
\hline 90.077 & 2.197 \\
\hline 24.167 & 39.546 \\
\hline 2.197 & 149.396 \\
\hline 2.197 & 41.743 \\
\hline 2.197 & 6.591 \\
\hline 2.197 & 30.758 \\
\hline 2.197 & 19.773 \\
\hline 2.197 & 48.334 \\
\hline 50.531 & 2.197 \\
\hline 2.197 & 21.97 \\
\hline 2.197 & 15.379 \\
\hline 19.773 & 2.197 \\
\hline 151.593 & 37.349 \\
\hline 13.182 & 2.197 \\
\hline 87.88 & 17.576 \\
\hline 2.197 & 2.197 \\
\hline 2.197 & 39.546 \\
\hline 57.122 & 4.394 \\
\hline 2.197 & 4.394 \\
\hline 2.197 & 2.197 \\
\hline 2.197 & 26.364 \\
\hline 2.197 & 4.394 \\
\hline 2.197 & 2.197 \\
\hline 2.197 & 17.576 \\
\hline 13.182 & 30.758 \\
\hline 15.379 & 24.167 \\
\hline
\end{tabular}




\begin{tabular}{|c|c|}
\hline 26.364 & 2.197 \\
\hline 2.197 & 2.197 \\
\hline 19.773 & 103.259 \\
\hline 828.269 & 13.182 \\
\hline 24.167 & 35.152 \\
\hline 153.79 & 35.152 \\
\hline 2.197 & 19.773 \\
\hline 2.197 & 8.788 \\
\hline 8.788 & 32.955 \\
\hline 6.591 & 2.197 \\
\hline 19.773 & 2.197 \\
\hline 2.197 & 2.197 \\
\hline 24.167 & 2.197 \\
\hline 2.197 & 6.591 \\
\hline 92.274 & 10.985 \\
\hline 4.394 & 30.758 \\
\hline 2.197 & 2.197 \\
\hline 2.197 & 6.591 \\
\hline 8.788 & 15.379 \\
\hline 2.197 & 10.985 \\
\hline 6.591 & 4.394 \\
\hline 8.788 & 17.576 \\
\hline 4.394 & 8.788 \\
\hline 2.197 & 4.394 \\
\hline 2.197 & 8.788 \\
\hline 2.197 & 10.985 \\
\hline 15.379 & 4.394 \\
\hline 13.182 & 2.197 \\
\hline 2.197 & 6.591 \\
\hline 13.182 & 26.364 \\
\hline 2.197 & 2.197 \\
\hline 2.197 & 2.197 \\
\hline 4.394 & 2.197 \\
\hline 2.197 & 2.197 \\
\hline 215.306 & 30.758 \\
\hline 24.167 & 4.394 \\
\hline 2.197 & 2.197 \\
\hline 10.985 & 2.197 \\
\hline 4.394 & 2.197 \\
\hline 2.197 & 4.394 \\
\hline 197.73 & 21.97 \\
\hline 24.167 & 17.576 \\
\hline 2.197 & 26.364 \\
\hline 2.197 & 10.985 \\
\hline 10.985 & 10.985 \\
\hline 4.394 & 4.394 \\
\hline 15.379 & 2.197 \\
\hline 4.394 & 19.773 \\
\hline 2.197 & 2.197 \\
\hline 6.591 & 2.197 \\
\hline
\end{tabular}




\begin{tabular}{|c|c|}
\hline 35.152 & 19.773 \\
\hline 13.182 & 10.985 \\
\hline 10.985 & 2.197 \\
\hline 76.895 & 4.394 \\
\hline 2.197 & 2.197 \\
\hline 46.137 & 4.394 \\
\hline 2.197 & 2.197 \\
\hline 6.591 & 2.197 \\
\hline 2.197 & 4.394 \\
\hline 2.197 & 2.197 \\
\hline 4.394 & 24.167 \\
\hline 4.394 & 24.167 \\
\hline 68.107 & 15.379 \\
\hline 2.197 & 6.591 \\
\hline 46.137 & 13.182 \\
\hline 2.197 & 24.167 \\
\hline 4.394 & 2.197 \\
\hline 2.197 & 2.197 \\
\hline 2.197 & 2.197 \\
\hline 107.653 & 30.758 \\
\hline 65.91 & 8.788 \\
\hline 30.758 & 10.985 \\
\hline 8.788 & 24.167 \\
\hline 8.788 & 52.728 \\
\hline 4.394 & 4.394 \\
\hline 13.182 & 10.985 \\
\hline 1346.76 & 13.182 \\
\hline 2.197 & 2.197 \\
\hline 4.394 & 13.182 \\
\hline 28.561 & 2.197 \\
\hline 87.88 & 2.197 \\
\hline 4.394 & 2.197 \\
\hline 26.364 & 2.197 \\
\hline 2.197 & 2.197 \\
\hline 213.109 & 4.394 \\
\hline 13.182 & 10.985 \\
\hline 2.197 & 2.197 \\
\hline 6.591 & 8.788 \\
\hline 4.394 & 17.576 \\
\hline 2.197 & 19.773 \\
\hline 6.591 & 17.576 \\
\hline 4.394 & 4.394 \\
\hline 15.379 & 2.197 \\
\hline 8.788 & 2.197 \\
\hline 4.394 & 2.197 \\
\hline 8.788 & 10.985 \\
\hline 4.394 & 32.955 \\
\hline \multicolumn{2}{|l|}{61.516} \\
\hline 4.394 & \\
\hline 2.197 & \\
\hline
\end{tabular}


768.95

298.792

35.152

4.394

65.91

2.197

6.591

2.197

2.197

2.197

2.197

2.197

2.197

2.197

2.197

2.197

103.259

2.197

2.197

19.773

2.197

17.576

599.781

50.531

32.955

6.591

61.516

2.197

24.167

2.197

4.394

2.197

8.788

2.197

15.379

76.895

72.501

4.394

10.985

4.394

6.591

17.576 
28.561

90.077

13.182

2.197

6.591

46.137

149.396

30.758

8.788

4.394

2.197

2.197

2.197

2.197

4.394

94.471

13.182

2.197

173.563

2.197

391.066

125.229

35.152

10.985

4.394

8.788

107.653

2.197

10.985

57.122

3686.57

17.576

4.394

2.197

2.197

6.591

4.394

19.773

37.349

37.349

2.197

2.197

39.546

2.197

4.394

2.197

2.197

2.197

2.197

2.197 


4.394
21.97
4.394
4.394
2.197
2.197
103.259
2.197
76.895
6.591
2.197
417.43
6.591
2.197
28.561
6.591
2.197
8.788
2.197
2.197
13.182
8.788
10.985
2049.8
2.197
2.197
145.002
8.788
2.197
2.197
10.985
28.561
21.97
4.394
2.197
6.591
2.197
41.743
2.197
2.197
4.394
6.591
35.152
283.413
2.197
2.197




$\begin{array}{r}751.374 \\ 2.197 \\ 2.197 \\ 2.197 \\ 4.394 \\ 19.773 \\ 4.394 \\ 2.197 \\ 21.97 \\ 2.197 \\ 79.092 \\ 17.576 \\ 17.576 \\ 2.197 \\ 17.576 \\ 2.197 \\ 2.197 \\ 10.985 \\ 2.197 \\ 6.591 \\ 2.197 \\ 4.394 \\ 65.91 \\ 19.773 \\ 4.394 \\ 2.197 \\ 4.394 \\ 41.743 \\ 2.197 \\ 37.349 \\ 272.428 \\ 2.197 \\ 1887.22 \\ 6.591 \\ 48.334 \\ 2.197 \\ 6.591 \\ 2.197 \\ 70.304 \\ 2.197 \\ 2.197 \\ 15.379 \\ 2.197 \\ 2.197 \\ 2.197 \\ 2.197 \\ 4.394 \\ \hline\end{array}$




$$
\begin{array}{r}
2.197 \\
8.788 \\
2.197 \\
4.394 \\
4.394 \\
43.94 \\
15.379 \\
2.197 \\
8.788 \\
2.197 \\
2.197 \\
8.788
\end{array}
$$

281.216

2.197

24.167

2.197

10.985

6.591

268.034

2.197

61.516

109.85

30.758

536.068

4.394

2.197

2.197

26.364

79.092

2.197

2.197

1775.18

10.985

24.167

6.591

81.289

26.364

10.985

21.97

2.197

10.985

2.197

2.197

4.394

2.197

2.197

2.197

8.788

37.349

4.394 


$$
\begin{array}{r}
10.985 \\
41.743 \\
2.197 \\
2.197 \\
10.985 \\
6.591 \\
6.591 \\
2.197 \\
2.197 \\
10.985 \\
90.077 \\
2.197 \\
15.379 \\
2.197 \\
2.197 \\
2.197 \\
4.394 \\
2.197 \\
2.197 \\
2.197 \\
17.576 \\
10.985 \\
15.379 \\
30.758 \\
2.197 \\
72.501 \\
6.591 \\
2.197 \\
4.394 \\
2.197 \\
2.197 \\
4.394 \\
2.197 \\
17.576 \\
8.788 \\
13.182 \\
2.197 \\
4.394 \\
2.197 \\
2.197 \\
2.197 \\
4.394 \\
4.394 \\
2.197 \\
2.197 \\
2.197 \\
4.394 \\
2.197 \\
6.591
\end{array}
$$


2.197

2.197

2.197

2.197

15.379

2.197

2.197

2.197

2.197

2.197

2.197

2.197

8.788

10.985

87.88

2.197

4.394

4.394

4.394

8.788

2.197

2.197

4.394

2.197

2.197

4.394

2.197

15.379

2.197

4.394

2.197

6.591

6.591

4.394

63.713

10.985

2.197

17.576

2.197

4.394

2.197

2.197

2.197

10.985

90.077

37.349

2.197

2.197

8.788

6.591 
57.122

2.197

2.197

19.773

6.591

35.152

2.197

6.591

2.197

2.197

2.197

2.197

2.197

4.394

13.182

2.197

2.197

6.591

10.985

2.197

184.548

2.197

2.197

2.197

2.197

8.788

15.379

164.775

32.955

21.97

2.197

13.182

4.394

2.197

2.197

32.955

2.197

15.379

2.197

2.197

26.364

30.758

2.197

8.788

2.197

6.591

120.835

21.97

30.758

2.197 


$\begin{array}{r}4.394 \\ 2.197 \\ 2.197 \\ 4.394 \\ 237.276 \\ 2.197 \\ 79.092 \\ 21.97 \\ 2.197 \\ 2.197 \\ 10.985 \\ 85.683 \\ 4.394 \\ 24.167 \\ 2.197 \\ 72.501 \\ 8.788 \\ 2.197 \\ 4.394 \\ 2.197 \\ 4.394 \\ 2.197 \\ 2.197 \\ 2.197 \\ \hline 463.567 \\ 4.394 \\ \hline 15.379 \\ 2.197 \\ 2.1967 \\ 2.197 \\ \hline 10.985 \\ \hline\end{array}$




$$
\begin{array}{r}
2.197 \\
83.486 \\
4.394 \\
28.561 \\
17.576 \\
318.565 \\
2.197 \\
2.197 \\
74.698 \\
2.197 \\
2.197 \\
2.197 \\
17.576 \\
2.197 \\
2.197 \\
2.197 \\
28.561 \\
2.197 \\
2.197 \\
2.197 \\
13.182 \\
6.591 \\
6.591 \\
70.304 \\
2.197 \\
107.653 \\
32.955 \\
6.591 \\
2.197 \\
2.197 \\
2.197 \\
2.197 \\
4.394 \\
2.39 \\
2.197 \\
10.989 \\
13.182 \\
4.394 \\
2.197 \\
4.394 \\
13.182 \\
13.182 \\
2.197 \\
21.97 \\
2.197 \\
\hline
\end{array}
$$




$$
\begin{array}{r}
149.396 \\
2.197 \\
2.197 \\
134.017 \\
6.591 \\
50.531 \\
2.197 \\
13.182 \\
2.197 \\
26.364 \\
10.985 \\
4.394 \\
21.97 \\
4.394 \\
13.182 \\
6.591 \\
10.985 \\
6.591 \\
2.197 \\
2.197 \\
2.197 \\
6.591 \\
37.349 \\
54.925 \\
13.182 \\
2.197 \\
276.822 \\
4.394 \\
8.788 \\
76.895 \\
85.683 \\
2.197 \\
3.394 \\
2.197 \\
6.989 \\
6.595 \\
2.197 \\
4.394 \\
4.394 \\
6.591 \\
2.197 \\
2.197 \\
4.394 \\
2.197 \\
2.197 \\
\hline
\end{array}
$$


52.728

2.197

2.197

2.197

2.197

2.197

4.394

4.394

41.743

39.546

6.591

8.788

2.197

6.591

4.394

2.197

2.197

101.062

2.197

6.591

48.334

2.197

2.197

2.197

4.394

2.197

37.349

57.122

2.197

2.197

2.197

4.394

118.638

254.852

21.97

4.394

52.728

2.197

28.561

2.197

2.197

404.248

182.351

17.576

4.394

2.197

24.167

43.94

8.788

4.394 


$$
\begin{array}{r}
2.197 \\
2.197 \\
4.394 \\
2.197 \\
8.788 \\
35.152 \\
4.394 \\
2.197 \\
15.379 \\
2.197 \\
8.788 \\
4.394 \\
2.197 \\
6.591 \\
24.167 \\
28.561 \\
2.197 \\
19.773 \\
2.197 \\
2.197 \\
2.197 \\
2.197 \\
8.788 \\
2.197 \\
2.197 \\
507.507 \\
17.576 \\
2.197 \\
10.985 \\
8.788 \\
30.758 \\
2.197 \\
4.394 \\
10.985 \\
4.394 \\
4.394 \\
4.394 \\
6.591 \\
1333.58 \\
8.788 \\
2.197 \\
2.197 \\
59.319 \\
41.743 \\
2.199 \\
\hline
\end{array}
$$


26.364

10.985

6.591

2.197

2.197

2.197

8.788

2.197

63.713

8.788

4.394

2.197

4.394

4.394

63.713

8.788

2.197

26.364

2.197

2.197

28.561

10.985

2.197

2.197

32.955

2.197

4.394

2.197

13.182

4.394

10.985

2.197

32.955

19.773

2.197

2.197

4.394

4.394

2.197

4.394

2.197

32.955

2.197

2.197

72.501

13.182

13.182

6.591

4.394

191.139 


$$
\begin{array}{r}
6.591 \\
6.591 \\
2.197 \\
2.197 \\
4.394 \\
43.94 \\
61.516 \\
24.167 \\
39.546 \\
6.591 \\
2.197 \\
2.197 \\
2.197 \\
2.197 \\
2.197 \\
28.561 \\
13.182 \\
32.955 \\
2.197 \\
4.394 \\
2.197 \\
24.167 \\
140.608 \\
2.197 \\
2.197 \\
21.97 \\
2.197 \\
30.758 \\
13.182 \\
13.182 \\
224.094 \\
2.197 \\
2.197 \\
2.197 \\
\hline 2.197 \\
2.728 \\
2.197 \\
2.197 \\
2.197 \\
2.197 \\
70.304 \\
\hline 12.963 \\
\hline
\end{array}
$$


28.561

2.197

2.197

2.197

26.364

2.197

2.197

283.413

2.197

4.394

37.349

24.167

6.591

116.441

2.197

6.591

2.197

90.077

8.788

70.304

2.197

6.591

2.197

24.167

2.197

4.394

2.197

2.197

2.197

39.546

105.456

2.197

13.182

2.197

2.197

533.871

10.985

6.591

43.94

180.154

6.591

63.713

542.659

4.394

37.349

2.197

2.197

2.197

2.197

87.88 


$$
\begin{array}{r}
2.197 \\
54.925 \\
2.197 \\
2.197 \\
2.197 \\
2.197 \\
46.137 \\
116.441 \\
4.394 \\
2.197 \\
17.576 \\
4.394 \\
2.197 \\
8.788 \\
4.394 \\
6.591 \\
6.591 \\
4.394 \\
19.773 \\
32.955 \\
24.167 \\
169.169 \\
4.394 \\
2.197 \\
461.37 \\
13.182 \\
13.182 \\
4.394 \\
2.197 \\
32.955 \\
13.182 \\
6.591 \\
2.197 \\
2.197 \\
4.394 \\
2.1989 \\
4.394 \\
\hline 189 \\
10.985 \\
2.197 \\
30.758 \\
13.182 \\
2.197 \\
21.97 \\
\hline
\end{array}
$$




$$
\begin{array}{r}
2.197 \\
2.197 \\
166.972 \\
2.197 \\
2.197 \\
285.61 \\
2.197 \\
2.197 \\
19.773 \\
26.364 \\
4.394 \\
17.576 \\
4.394 \\
2.197 \\
503.113 \\
4.394 \\
2.197 \\
17.576 \\
52.728 \\
24.167 \\
21.97 \\
10.985 \\
24.167 \\
2.197 \\
6.591 \\
2.197 \\
17.576 \\
2.197 \\
13.182 \\
24.167 \\
2.197 \\
2.197 \\
4.394 \\
116.441 \\
13.182 \\
39.546 \\
2.197 \\
96.668 \\
6.591 \\
2.197 \\
2.197 \\
2.197 \\
2.197 \\
2.197 \\
2.197 \\
8.788 \\
4.394 \\
\hline
\end{array}
$$




$$
\begin{array}{r}
2.197 \\
4.394 \\
8.788 \\
2.197 \\
2.197 \\
2.197 \\
2.197 \\
32.955 \\
2.197 \\
2.197 \\
670.085 \\
2.197 \\
4.394 \\
2.197 \\
2.197 \\
8.788 \\
2.197 \\
13.182 \\
2.197 \\
4.394 \\
10.985 \\
2.197 \\
13.182 \\
2.197 \\
2.197 \\
21.97 \\
6.591 \\
4.394 \\
13.182 \\
39.546 \\
2.197 \\
4.394 \\
24.167 \\
2.197 \\
6.591 \\
4.394 \\
2.197 \\
2.197 \\
13.182 \\
10.985 \\
2.197 \\
2.197 \\
\hline
\end{array}
$$


15.379

109.85

6.591

147.199

6.591

54.925

4.394

35.152

4.394

6.591

15.379

24.167

35.152

2.197

61.516

10.985

39.546

2.197

2.197

2.197

2.197

2.197

2.197

74.698

2.197

28.561

4.394

21.97

2.197

13.182

68.107

2.197

2.197

4.394

30.758

2.197

6.591

202.124

30.758

28.561

2142.07

2.197

32.955

2.197

2.197

2.197

127.426

10.985

369.096

30.758 
15.379

35.152

2.197

4.394

48.334

13.182

21.97

10.985

96.668

19.773

2.197

2.197

2.197

2.197

2.197

2.197

2.197

2.197

2.197

4.394

2.197

2.197

6.591

21.97

6.591

39.546

2.197

2.197

6.591

2.197

2.197

2.197

2.197

2.197

17.576

8.788

2.197

2.197

2.197

43.94

32.955

4.394

4.394

107.653

2.197

2.197

2.197

2.197

6.591

13.182 
10.985

24.167

19.773

50.531

13.182

6.591

6.591

4.394

8.788

17.576

2.197

867.815

52.728

28.561

2.197

2.197

371.293

61.516

13.182

164.775

2.197

2.197

103.259

50.531

4.394

2.197

2.197

2.197

6.591

4.394

164.775

243.867

24.167

2.197

39.546

2.197

10.985

6.591

2.197

85.683

2.197

2.197

2.197

8.788

2.197

4.394

4.394

4.394

6.591

2.197 
2.197
2.197
54.925
48.334
125.229
4.394
10.985
4.394
2.197
246.064
21.97
6.591
123.032
63.713
2.197
213.109
13.182
2.197
2.197
8.788

355.914

670.085

10.985

3132.92

2.197

2.197

19.773

2.197

8.788

10.985

2.197

8.788

13.182

4.394

6.591

41.743

2.197

10.985

21.97

6.591

94.471

70.304

90.077

6.591

4.394

4.394

2.197

2.197

30.758

46.137 
2.197

15.379

2.197

4.394

26.364

70.304

4.394

4.394

19.773

2.197

52.728

15.379

50.531

2.197

8.788

2.197

15.379

19.773

2.197

2.197

21.97

2.197

48.334

15.379

41.743

2.197

4.394

2.197

199.927

6.591

19.773

2.197

4.394

81.289

87.88

13.182

2.197

2.197

54.925

13.182

8.788

2.197

4.394

891.982

4.394

2.197

37.349

13.182

2.197

2.197 
10.985

10.985

4.394

6.591

2065.18

2.197

2.197

13.182

19.773

2.197

2.197

2.197

21.97

2.197

35.152

2.197

2.197

4.394

2.197

2.197

241.67

4.394

2.197

2.197

15.379

15.379

2.197

2.197

39.546

83.486

13.182

2.197

1214.94

35.152

10.985

4.394

2.197

2.197

10.985

393.263

145.002

10.985

19.773

10.985

30.758

8.788

4.394

17.576

6.591

2.197 


$$
\begin{array}{r}
4.394 \\
13.182 \\
8.788 \\
2.197 \\
2.197 \\
2.197 \\
2.197 \\
59.319 \\
2.197 \\
6.591 \\
8.788 \\
2.197 \\
4.394 \\
15.379 \\
10.985 \\
2.197 \\
10.985 \\
4.394 \\
112.047 \\
6.591 \\
2.197 \\
24.167 \\
4.394 \\
30.758 \\
2.197 \\
2.197 \\
15.379 \\
109.85 \\
2.197 \\
6.591 \\
6.591 \\
2.197 \\
2.197 \\
2.197 \\
94.471 \\
2.197 \\
2.197 \\
13.197 \\
2.197 \\
2.197 \\
6.591 \\
2.197 \\
2.197 \\
2.197 \\
\hline
\end{array}
$$


331.747

2.197

2.197

2.197

4.394

26.364

19.773

4.394

6.591

43.94

252.655

1590.63

2.197

2.197

4.394

2.197

2.197

2.197

85.683

2.197

13.182

4.394

8.788

17.576

2.197

15.379

24.167 


$\begin{array}{r}6.591 \\ 10.985 \\ 6.591 \\ 2.197 \\ 2.197 \\ 4.394 \\ 68.107 \\ 2.197 \\ 2.197 \\ 2.197 \\ 13.182 \\ 160.381 \\ 140.608 \\ 8.788 \\ 4.394 \\ 2.197 \\ 2.197 \\ 105.456 \\ 24.167 \\ 2.197 \\ 2.197 \\ 4.394 \\ 6.591 \\ 4.394 \\ 4.394 \\ 4.394 \\ 2.197 \\ 21.97 \\ 2.197 \\ 353.717 \\ 26.364 \\ 2.197 \\ 2.197 \\ 4.394 \\ 13.182 \\ 2.197 \\ 6.591 \\ 2.197 \\ 1017.21 \\ 197.73 \\ 28.561 \\ 83.486 \\ 118.638 \\ 24.167 \\ 2.394 \\ \hline \\ \hline\end{array}$


8.788

35.152

2.197

127.426

76.895

4.394

30.758

2.197

2.197

2.197

50.531

2.197

2.197

6.591

74.698

2.197

6.591

2.197

8.788

10.985

15.379

6.591

4.394

8.788

17.576

2.197

2.197

4.394

94.471

2.197

2.197

2.197

2.197

2.197

2.197

147.199

4.394

8.788

8.788

2.197

19.773

2.197

4.394

2.197

21.97

8.788

65.91

2.197

2.197

2.197 


8.788
17.576
105.456
15.379
6.591
39.546
8.788
2.197
4.394
19.773
2.197
2.197
2.197
2.197
39.546
21.97
333.944
2.197
4.394
298.792
21.97
26.364
10.985
2.197
21.97
2.197
8.788
2.197
2.197
43.94
17.576
4.394
8.788
10.985
2.197
13.182
2.197
2.197
26.364
4.394
4.394
4.394
6.591
2.197
2.197
2.197
2.197




$$
\begin{array}{r}
2.197 \\
4.394 \\
76.895 \\
2.197 \\
2.197 \\
2.197 \\
13.182 \\
2.197 \\
4.394 \\
10.985 \\
4.394 \\
19.773 \\
10.985 \\
173.563 \\
32.955 \\
2.197 \\
2.197 \\
21.97 \\
6.591 \\
2.197 \\
2.197 \\
4.394 \\
6.591 \\
15.379 \\
2.197 \\
4.394 \\
17.576 \\
2.197 \\
2.197 \\
2.197 \\
39.546 \\
17.576 \\
26.364 \\
2.197 \\
2.197 \\
2.197 \\
2.197 \\
48.334 \\
415.233 \\
19.773 \\
2.197 \\
41.743 \\
2.197 \\
4.394 \\
\end{array}
$$




$\begin{array}{r}46.137 \\ 2.197 \\ 2.197 \\ 2.197 \\ 2.197 \\ 4.394 \\ 4.394 \\ 2.197 \\ 2.197 \\ 2.197 \\ 2.197 \\ 6.591 \\ 10.985 \\ 68.107 \\ 2.197 \\ 2.197 \\ 2.197 \\ 28.561 \\ 2.197 \\ 4.394 \\ 32.955 \\ 219.7 \\ 195.533 \\ 2.197 \\ 19.773 \\ 2.197 \\ 2.197 \\ 2.197 \\ 2.197 \\ 32.955 \\ 6.591 \\ 4.394 \\ 2.197 \\ 15.379 \\ 2.197 \\ 2.197 \\ 4.394 \\ 4.394 \\ 2.197 \\ 4.394 \\ 2.197 \\ 2.197 \\ 28.561 \\ 30.758 \\ 2.197 \\ 2.197 \\ 6.591 \\ 2.197 \\ \hline\end{array}$




$\begin{array}{r}2.197 \\ 2.197 \\ 30.758 \\ 4.394 \\ 1834.49 \\ 4.394 \\ 8.788 \\ 8.788 \\ 2.197 \\ 90.077 \\ 10.985 \\ 2.197 \\ 17.576 \\ 2.197 \\ 6.591 \\ 2.197 \\ 2.197 \\ 21.97 \\ 8.788 \\ 109.85 \\ 19.773 \\ 369.096 \\ 8.788 \\ 4.394 \\ 17.576 \\ 2.197 \\ 2.197 \\ 2.197 \\ \hline 10.985 \\ \hline 2.788 \\ 8.788 \\ 2.197 \\ \hline 2.394 \\ \hline 10.985 \\ 15.379 \\ 35.152 \\ 6.591 \\ 2.197 \\ 10.985 \\ 145.002 \\ 13.182 \\ 15.379 \\ 21.97 \\ 2.197 \\ \hline 219 \\ \hline\end{array}$


2.197
37.349
2.197
4.394
15.379
59.319
8.788
2.197

59.319

10.985

2.197

76.895

140.608

2.197

6.591

2.197

13.182

2.197

6.591

15.379

2.197

424.021

76.895

2.197

4.394

180.154

35.152

13.182

2.197

6.591

134.017

114.244

10.985

74.698

2.197

199.927

2.197

2.197

2.197

2.197

2.197

4.394

4.394

24.167

28.561

2.197

2.197

2.197

2.197

2.197 


$$
\begin{aligned}
& 2.197 \\
& 2.197 \\
& 2.197 \\
& 2.197 \\
& 2.197 \\
& 2.197 \\
& 15.379 \\
& 2.197 \\
& 4.394 \\
& 588.796 \\
& 2.197 \\
& 2.197 \\
& 2.197 \\
& 21.97 \\
& 8.788 \\
& 24.167 \\
& 4.394 \\
& 4.394 \\
& 19.773 \\
& 8.788 \\
& 13.182 \\
& 26.364 \\
& 2.197 \\
& 19.773 \\
& 2.197 \\
& 6.591 \\
& 4.394 \\
& 2.197 \\
& 252.655 \\
& 2.197 \\
& 13.182 \\
& 2.197 \\
& 2.197 \\
& 43.94 \\
& 160.381 \\
& 8.788 \\
& 2.197 \\
& 4.394 \\
& 59.319 \\
& 8.788 \\
& 30.758 \\
& 676.676 \\
& 2.197 \\
& 2.197 \\
& 17.576 \\
& 4.394 \\
& 6.591 \\
& 10.985 \\
& 8.788 \\
& 15.379
\end{aligned}
$$


169.169

2.197

2.197

2.197

2.197

43.94

92.274

8.788

24.167

2.197

19.773

2.197

202.124

4.394

4.394

6.591

85.683

4.394

10.985

96.668

32.955

17.576

2.197

2.197

13.182

15.379

4323.7

74.698

17.576

467.961

2.197

30.758

2.197

98.865

2.197

2.197

2.197

2.197

2.197

2.197

15.379

52.728

217.503

68.107

2.197

21.97

2.197

8.788

2.197

2.197 
2.197

13.182

2.197

2.197

2.197

28.561

2.197

4.394

2.197

2.197

24.167

2.197

4.394

6.591

6.591

2.197

24.167

2.197

2.197

2.197

2.197

2.197

2.197

4.394

2.197

19.773

28.561

2.197

2.197

2.197

6.591

28.561

94.471

32.955

2.197

26.364

2.197

208.715

2.197

2.197

21.97

2.197

6.591

10.985

8.788

10.985

2.197

39.546

4.394

2.197 


$$
\begin{array}{r}
4.394 \\
2.197 \\
4.394 \\
43.94 \\
2.197 \\
2.197 \\
8.788 \\
2.197 \\
15.379 \\
46.137 \\
4.394 \\
24.167 \\
17.576 \\
13.182 \\
8.788 \\
50.531 \\
13.182 \\
2.197 \\
2.197 \\
4.394 \\
2.197 \\
2.197 \\
61.516 \\
6.591 \\
8.788 \\
2.197 \\
17.576 \\
4.394 \\
366.899 \\
2.197 \\
4.394 \\
35.152 \\
2.197 \\
2.197 \\
2.197 \\
4.394 \\
2.197 \\
2.197 \\
2.197 \\
2.197 \\
2.197 \\
2.197 \\
2.197 \\
4.394 \\
\hline
\end{array}
$$




$$
\begin{array}{r}
2.197 \\
2.197 \\
650.312 \\
2.197 \\
2.197 \\
2.197 \\
2.197 \\
2.197 \\
250.458 \\
6.591 \\
57.122 \\
4.394 \\
2.197 \\
17.576 \\
13.182 \\
8.788 \\
24.167 \\
2.197 \\
6.591 \\
2.197 \\
4.394 \\
6.591 \\
2.197 \\
2.197 \\
127.426 \\
28.561 \\
65.91 \\
32.955 \\
15.379 \\
145.002 \\
195.533 \\
2.197 \\
2.197 \\
2.197 \\
2.197 \\
2.394 \\
2.394 \\
32.955 \\
43.94 \\
24.167 \\
2.197 \\
4.394 \\
2.197 \\
24.167 \\
\hline
\end{array}
$$


19.773

13.182

8.788

4.394

6.591

10.985

6.591

6.591

2.197

8.788

26.364

2.197

2.197

195.533

21.97

6.591

13.182

6.591

4.394

2.197

15.379

32.955

4.394

2.197

2.197

2.197

10.985

6.591

8.788

4.394

10.985

2.197

2.197

8.788

2.197

2.197

6.591

2.197

2.197

21.97

15.379

2.197

43.94

4.394

6.591

2.197

2.197

2.197

2.197

2.197 


$$
\begin{array}{r}
10.985 \\
4.394 \\
30.758 \\
15.379 \\
2.197 \\
4.394 \\
85.683 \\
4.394 \\
101.062 \\
10.985 \\
4.394 \\
2.197 \\
698.646 \\
4.394 \\
30.758 \\
2.197 \\
4.394 \\
2.197 \\
120.835 \\
4.394 \\
2.197 \\
37.349 \\
4.394 \\
2.197 \\
2.197 \\
13.182 \\
2.197 \\
2.197 \\
79.092 \\
2.197 \\
2.197 \\
4.394 \\
2.197 \\
4.394 \\
2.197 \\
17.576 \\
2.197 \\
344.929 \\
2.197 \\
166.972 \\
24.167 \\
6.591 \\
8.788 \\
37.531 \\
\hline 127.06 \\
\hline
\end{array}
$$


10.985

2.197

6.591

4.394

2.197

15.379

13.182

2.197

232.882

4.394

30.758

13.182

2.197

65.91

2.197

6.591

2.197

2.197

32.955

2.197

2.197

2.197

2.197

74.698

10.985

35.152

4.394

2.197

101.062

50.531

8.788

65.91

13.182

37.349

4.394

2.197

4.394

6.591

2.197

2.197

4.394

2.197

2.197

2.197

57.122

6.591

2.197

37.349

4.394

2.197 


$\begin{array}{r}4.394 \\ 2.197 \\ 6.591 \\ 4.394 \\ 37.349 \\ 90.077 \\ 2.197 \\ 2.197 \\ 2.197 \\ 4.394 \\ 4.394 \\ 2.197 \\ 2.197 \\ 4.394 \\ 2.197 \\ 2.197 \\ 41.743 \\ 4.394 \\ 2.197 \\ 2.197 \\ 2.197 \\ 28.561 \\ 17.576 \\ 2.197 \\ 2.197 \\ 2.197 \\ 2.197 \\ 2.197 \\ 2.37 \\ 2.197 \\ 2.364 \\ 2.1989 \\ 2.197 \\ 2.197 \\ 26.364 \\ 57.122 \\ \hline 182.351 \\ 4.394 \\ 2.197 \\ 8.788 \\ 2.197 \\ 2.197 \\ \hline\end{array}$


17.576

57.122

2.197

32.955

2.197

2.197

2.197

8.788

37.349

2.197

171.366

4.394

531.674

26.364

4.394

4.394

10.985

4.394

2.197

4.394

24.167

2.197

2.197

96.668

4.394

4.394

2.197

6.591

17.576

2.197

2.197

4.394

4.394

4.394

2.197

30.758

8.788

246.064

478.946

2.197

4.394

13.182

17.576

26.364

2.197

4.394

4.394

2.197

4.394

26.364 
2.197

6.591

19.773

2.197

2.197

4.394

57.122

10.985

17.576

4.394

13.182

2.197

6.591

19.773

2.197

4.394

4.394

2.197

2.197

2.197

2.197

26.364

2.197

13.182

37.349

4.394

10.985

4.394

2.197

6.591

2.197

15.379

10.985

2.197

2.197

6.591

4.394

8.788

4.394

4.394

2.197

4.394

6.591

81.289

2.197

2.197

2.197

4.394

2.197

83.486 


$$
\begin{array}{r}
839.254 \\
8.788 \\
52.728 \\
2.197 \\
2.197 \\
2.197 \\
2.197 \\
2.197 \\
4.394 \\
4.394 \\
4.394 \\
2.197 \\
2.197 \\
52.728 \\
19.773 \\
74.698 \\
4.394 \\
2.197 \\
13.182 \\
2.197 \\
15.379 \\
57.122 \\
4.394 \\
4.394 \\
26.364 \\
2.197 \\
2.197 \\
2.197 \\
4.394 \\
2.197 \\
17.576 \\
10.985 \\
2.197 \\
13.182 \\
21.97 \\
2.197 \\
101.062 \\
4.394 \\
2.197 \\
65.91 \\
2.197 \\
2.197 \\
2.197 \\
2.197 \\
2.197
\end{array}
$$




$$
\begin{array}{r}
2.197 \\
2.197 \\
2.197 \\
4.394 \\
10.985 \\
10.985 \\
13.182 \\
19.773 \\
8.788 \\
32.955 \\
2.197 \\
2.197 \\
74.698 \\
2.197 \\
6.591 \\
2.197 \\
2.197 \\
2.197 \\
8.788 \\
2.197 \\
2.197 \\
2.197 \\
2.197 \\
39.546 \\
6.591 \\
2.197 \\
2.197 \\
2.197 \\
2.197 \\
\hline 3.394 \\
4.385 \\
2.394 \\
2.197 \\
2.197 \\
\hline
\end{array}
$$




$\begin{array}{r}8.788 \\ 4.394 \\ 241.67 \\ 2339.8 \\ 2.197 \\ 10.985 \\ 103.259 \\ 65.91 \\ 6.591 \\ 4.394 \\ 2.197 \\ 8.788 \\ 6.591 \\ 2.197 \\ 13.182 \\ 2.197 \\ 52.728 \\ 2.197 \\ 2.197 \\ 6.591 \\ 8.788 \\ 8.788 \\ 8.788 \\ 4.394 \\ 17.576 \\ 10.985 \\ 2.197 \\ 2.197 \\ 46.137 \\ 2.197 \\ 2.197 \\ 4.394 \\ 2049.8 \\ 4.394 \\ 617.357 \\ 2.197 \\ 8.788 \\ 4.394 \\ 2.197 \\ 4.394 \\ 8.788 \\ 8.788 \\ 6.591 \\ 2.197 \\ 4.394 \\ 2.197 \\ 4.394 \\ \hline\end{array}$




$$
\begin{array}{r}
2.197 \\
6.591 \\
30.758 \\
2.197 \\
2.197 \\
4.394 \\
2.197 \\
2.197 \\
4.394 \\
2.197 \\
2.197 \\
4.394 \\
15.379 \\
2.197 \\
2.197 \\
6.591 \\
8.788 \\
10.985 \\
109.85 \\
2.197 \\
10.985 \\
2.197 \\
2.197 \\
4.394 \\
2.197 \\
50.531 \\
35.152 \\
30.758 \\
2.197 \\
2.197 \\
4.394 \\
2.197 \\
17.576 \\
48.334 \\
2.197 \\
149.396 \\
2.197 \\
2.197 \\
2.197 \\
10.797 \\
10.985 \\
10.985 \\
8.788 \\
2.197 \\
\hline
\end{array}
$$




$$
\begin{array}{r}
19.773 \\
41.743 \\
2.197 \\
4.394 \\
2.197 \\
2.197 \\
2.197 \\
2.197 \\
4.394 \\
2.197 \\
37.349 \\
2.197 \\
13.182 \\
856.83 \\
2.197 \\
2.197 \\
48.334 \\
35.152 \\
2.197 \\
10.985 \\
8.788 \\
399.854 \\
2.197 \\
4.394 \\
6.591 \\
83.486 \\
2.197 \\
4.394 \\
2.197 \\
2.197 \\
8.788 \\
2.1997 \\
2.197 \\
\hline 197 \\
2.197 \\
2.591 \\
2.197 \\
2.197 \\
24.167 \\
2.197 \\
2.197 \\
2.197 \\
\hline 1.289 \\
\hline
\end{array}
$$




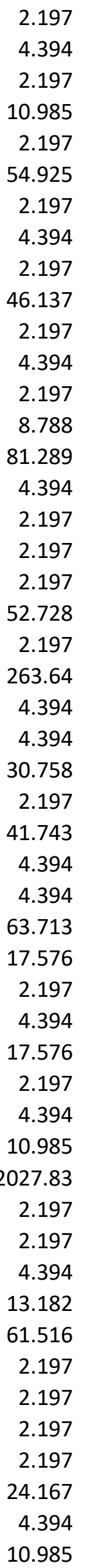


17.576

184.548

2.197

13.182

21.97

2.197

2.197

164.775

6.591

2.197

2.197

21.97

4.394

775.541

17.576

2.197

2.197

2.197

4.394

17.576

4.394

21.97

6.591

2.197

4.394

70.304

15.379

2.197

2.197

2.197

4.394

2.197

4.394

6.591

2.197

21.97

2.197

6.591

2.197

2.197

2.197

2.197

10.985

2.197

243.867

76.895

2.197

2.197

2.197

2.197 


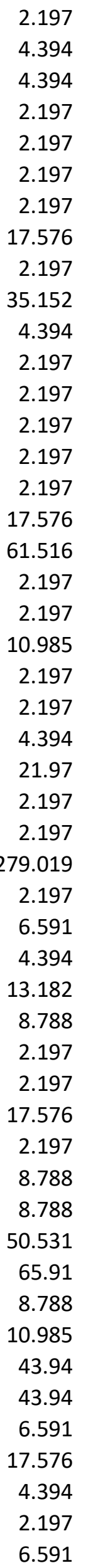


46.137

2.197

2.197

2.197

2.197

2.197

2.197

52.728

35.152

2.197

4.394

2.197

2.197

2.197

2.197

2.197

2.197

2.197

2.197

8.788

4.394

4.394

21.97

4.394

63.713

2.197

2.197

2.197

2.197

2.197

4.394

2.197

35.152

4.394

19.773

15.379

2.197

2.197

2.197

4.394

2.197

2.197

2.197

2.197

74.698

1614.79

32.955

1307.21

4.394

13.182 
52.728

4.394

2.197

10.985

2.197

37.349

342.732

2.197

2.197

2.197

2.197

2.197

2.197

2.197

2.197

2.197

19.773

30.758

2.197

41.743

134.017

17.576

4.394

2.197

2.197

26.364

4.394

4.394

39.546

13.182

37.349

2.197

10.985

6.591

4.394

204.321

2.197

2.197

15.379

68.107

10.985

2.197

2.197

13.182

8.788

35.152

2.197

2.197

46.137

2.197 


$$
\begin{array}{r}
10.985 \\
6.591 \\
2.197 \\
2.197 \\
2.197 \\
59.319 \\
2.197 \\
2.197 \\
2.197 \\
2.197 \\
2.197 \\
8.788 \\
3444.9 \\
2.197 \\
2.197 \\
4.394 \\
2.197 \\
15.379 \\
2.197 \\
28.561 \\
2.197 \\
2.197 \\
149.396 \\
4.394 \\
28.561 \\
2.197 \\
4.394 \\
4.394 \\
2.197 \\
2.197 \\
2.197 \\
10.985 \\
19.773 \\
2.197 \\
37.349 \\
878.8 \\
2.197 \\
2.197 \\
2.197 \\
2.197 \\
2.197 \\
2.197 \\
2.197 \\
2.197 \\
2.197 \\
6.591 \\
\hline \\
\hline
\end{array}
$$


2.197

164.775

4.394

296.595

4.394

6.591

2.197

2.197

2.197

6.591

107.653

2.197

4.394

4.394

6.591

2.197

4.394

17.576

35.152

4.394

48.334

4.394

459.173

8.788

2.197

2.197

2.197

2.197

41.743

17.576

125.229

127.426

2.197

2.197

153.79

61.516

2.197

2.197

2.197

2.197

35.152

24.167

26.364

6.591

2.197

35.152

10.985

2.197

35.152

103.259 
173.563

13.182

6.591

2.197

15.379

10.985

2.197

13.182

2.197

4.394

169.169

2.197

2.197

6.591

8.788

2.197

2.197

30.758

28.561

72.501

10.985

6.591

2.197

2.197

2.197

4.394 


$$
\begin{array}{r}
10.985 \\
10.985 \\
15.379 \\
61.516 \\
6.591 \\
2.197 \\
2.197 \\
127.426 \\
17.576 \\
39.546 \\
41.743 \\
2.197 \\
2.197 \\
2.197 \\
2.197 \\
2.197 \\
13.182 \\
26.364 \\
2.197 \\
388.869 \\
2.197 \\
2.197 \\
2.197 \\
2.197 \\
4.394 \\
23679.3 \\
2.197 \\
4.394 \\
8.788 \\
76.895 \\
4.394 \\
8.788 \\
6.591 \\
21.97 \\
21.97 \\
10.985 \\
28.561 \\
21.97 \\
15.379 \\
4.394 \\
8.1989 \\
2.197 \\
2.197 \\
2.197 \\
\hline
\end{array}
$$




$$
\begin{array}{r}
4.394 \\
2.197 \\
2.197 \\
6.591 \\
4.394 \\
4.394 \\
19.773 \\
6.591 \\
2.197 \\
2.197 \\
26.364 \\
2.197 \\
2.197 \\
57.122 \\
8.788 \\
4.394 \\
2.197 \\
30.758 \\
109.85 \\
15.379 \\
92.274 \\
2.197 \\
4.394 \\
4.394 \\
26.364 \\
2.197 \\
2.197 \\
46.137 \\
2.197 \\
2.197 \\
6.591 \\
8.788 \\
2.379 \\
2.398 \\
2.569 \\
2.197 \\
15.379 \\
4.394 \\
590.993 \\
41.743 \\
2.197 \\
6.591 \\
2.197 \\
2.197 \\
\hline
\end{array}
$$


96.668

26.364

10.985

2.197

32.955

21.97

19.773

28.561

4.394

4.394

182.351

2.197

15.379

6.591

2.197

4.394

4.394

19.773

2.197

4.394

2.197

15.379

109.85

13.182

2.197

6.591

213.109

6.591

6.591

65.91

2.197

85.683

2.197

2.197

136.214

2.197

6.591

19.773

8.788

2.197

4.394

4.394

30.758

21.97

72.501

4.394

4.394

37.349

2.197

2.197 


$\begin{array}{r}2.197 \\ 2.197 \\ 4.394 \\ 2.197 \\ 63.713 \\ 4.394 \\ 4.394 \\ 2.197 \\ 2.197 \\ 30.758 \\ 406.445 \\ 2.197 \\ 39.546 \\ 2.197 \\ 4.394 \\ 746.98 \\ 50.531 \\ 2.197 \\ 4.394 \\ 2.197 \\ 2.197 \\ 2.197 \\ 4.394 \\ 2.197 \\ 2.197 \\ 2.197 \\ 15.379 \\ 6.591 \\ 17.576 \\ 24.167 \\ 2.197 \\ 1179.79 \\ 15.379 \\ 2.199 \\ 2.197 \\ \hline 10.985 \\ 4.394 \\ 103.259 \\ 2.197 \\ 2.197 \\ 41.743 \\ 2.197 \\ 2.197 \\ \hline\end{array}$




$$
\begin{array}{r}
6.591 \\
147.199 \\
2.197 \\
2.197 \\
10.985 \\
10.985 \\
48.334 \\
2.197 \\
19.773 \\
2.197 \\
2.197 \\
2.197 \\
6.591 \\
87.88 \\
4.394 \\
2.197 \\
2.197 \\
285.61 \\
2.197 \\
21.97 \\
2.197 \\
2.197 \\
2.197 \\
2.197 \\
41.743 \\
2.197 \\
2.197 \\
2.197 \\
2.197 \\
2.197 \\
41.743 \\
13.182 \\
8.788 \\
856.83 \\
21.97 \\
68.107 \\
2.197 \\
21.97 \\
2.197 \\
2.197 \\
87.88 \\
2.197 \\
6.591 \\
8.788 \\
17.576 \\
15.379 \\
2.197 \\
\hline .394 \\
\hline
\end{array}
$$




$$
\begin{array}{r}
8.788 \\
10.985 \\
2.197 \\
2.197 \\
21.97 \\
4.394 \\
134.017 \\
129.623 \\
50.531 \\
4.394 \\
2.197 \\
4.394 \\
72.501 \\
2.197 \\
4.394 \\
6.591 \\
2.197 \\
4.394 \\
2.197 \\
6.591 \\
2.197 \\
2.197 \\
2.197 \\
68.107 \\
2.197 \\
2.197 \\
52.728 \\
4.394 \\
13.182 \\
2.197 \\
15.379 \\
2.197 \\
2.197 \\
2.197 \\
17.576 \\
2.197 \\
19.773 \\
35.152 \\
6.591 \\
2.197 \\
6.591 \\
2.197 \\
2.197 \\
\hline 2.197 \\
\hline
\end{array}
$$




$$
\begin{array}{r}
8.788 \\
21.97 \\
4.394 \\
10.985 \\
2.197 \\
2.197 \\
21.97 \\
10.985 \\
6.591 \\
52.728 \\
2.197 \\
2.197 \\
2.197 \\
2.197 \\
8.788 \\
2.197 \\
10.985 \\
2.197 \\
59.319 \\
19.773 \\
41.743 \\
2.197 \\
4.394 \\
2.197 \\
15.379 \\
151.593 \\
10.985 \\
15.379 \\
13.182 \\
2.197 \\
90.077 \\
2.197 \\
2.379 \\
2.82 .193 \\
2.197 \\
\hline \\
\hline 2.758 \\
6.304 \\
6.591 \\
4.394 \\
2.197 \\
15.379 \\
395.46 \\
2.197 \\
\hline
\end{array}
$$




$$
\begin{array}{r}
129.623 \\
32.955 \\
8.788 \\
2.197 \\
19.773 \\
2.197 \\
492.128 \\
2.197 \\
61.516 \\
48.334 \\
87.88 \\
287.807 \\
21.97 \\
94.471 \\
26.364 \\
57.122 \\
10.985 \\
4.394 \\
19.773 \\
19.773 \\
2.197 \\
4.394 \\
35.152 \\
2.197 \\
2.197 \\
30.758 \\
2.197 \\
2.197 \\
2.197 \\
11549.6 \\
2.197 \\
8.788 \\
2.197 \\
19.773 \\
35.152 \\
21.97 \\
4.394 \\
2.197 \\
2.197 \\
2.197 \\
2.197 \\
96.668 \\
2.197 \\
2.1957 \\
8.788
\end{array}
$$




$$
\begin{array}{r}
6.591 \\
79.092 \\
32.955 \\
4.394 \\
92.274 \\
2.197 \\
26.364 \\
4.394 \\
17.576 \\
6.591 \\
8.788 \\
6.591 \\
8.788 \\
65.91 \\
30.758 \\
8.788 \\
10.985 \\
21.97 \\
6.591 \\
4.394 \\
15.379 \\
232.882 \\
134.017 \\
15.379 \\
4.394 \\
4.394 \\
4.394 \\
2.197 \\
2.197 \\
2.197 \\
2.591 \\
2.197 \\
15.379 \\
4.399 \\
2.394 \\
2.197 \\
2.197 \\
79.092 \\
13.182 \\
6.591 \\
6.591 \\
2.197 \\
2.197 \\
2.197 \\
2.197 \\
\hline
\end{array}
$$




$$
\begin{array}{r}
2.197 \\
10.985 \\
4.394 \\
32.955 \\
2.197 \\
4.394 \\
2.197 \\
4.394 \\
4.394 \\
32.955 \\
54.925 \\
2.197 \\
2.197 \\
2.197 \\
2.197 \\
2.197 \\
65.91 \\
50.531 \\
4.394 \\
2.197 \\
4.394 \\
74.698 \\
2.197 \\
13.182 \\
2.197 \\
10.985 \\
2.197 \\
296.595 \\
276.822 \\
32.955 \\
15.379 \\
32.955 \\
2.197 \\
2.197 \\
48.334 \\
2.197 \\
2.197 \\
2.197 \\
2.197 \\
13.187 \\
2.197 \\
8.789 \\
2.197 \\
\end{array}
$$




$$
\begin{array}{r} 
\\
6.591 \\
4.394 \\
10.985 \\
2.197 \\
17.576 \\
2.197 \\
2.197 \\
2.197 \\
13.182 \\
81.289 \\
2.197 \\
2.197 \\
61.516 \\
10.985 \\
2.197 \\
6.591 \\
6.591 \\
6.591 \\
4.394 \\
2.197 \\
21.97 \\
4.394 \\
4.394 \\
2.197 \\
2.197 \\
2.197 \\
2.197 \\
2.197 \\
2.197 \\
2.197 \\
2.197 \\
8.788 \\
4.394 \\
4.394 \\
2.197 \\
6.591 \\
15.379 \\
2.197 \\
2.1979 \\
2.197 \\
2.788 \\
2.197 \\
\hline .394 \\
\hline .197 \\
\hline
\end{array}
$$


63.713

2.197

4.394

4.394

6.591

2.197

6.591

4.394

8.788

10.985

2.197

2.197

8.788

13.182

191.139

74.698

257.049

6.591

28.561

8.788

2.197

2.197

2.197

182.351

92.274

8.788

2.197

21.97

2.197

2.197

2.197

6.591

6.591

26.364

2.197

2.197

2.197

2.197

17.576

4.394

2.197

2.197

10.985

2.197

10.985

6.591

2.197

24.167

2.197

2.197 
2.197

13.182

6.591

2.197

2.197

2.197

19.773

76.895

2.197

2.197

2.197

4.394

2.197

2.197

10.985

2.197

35.152

35.152

35.152

2.197

2.197

15.379

8.788

779.935

2.197

28.561

2.197

10.985

6.591

2.197

6.591

6.591

26.364

2.197

2.197

2.197

4.394

2.197

2.197

2.197

2.197

2.197

4.394

6.591

4.394

2.197

10.985

2.197

19.773

4.394 


$\begin{array}{r}4.394 \\ 2.197 \\ 52.728 \\ 147.199 \\ 138.411 \\ 10.985 \\ 39.546 \\ 2.197 \\ 6.591 \\ 17.576 \\ 2.197 \\ 1076.53 \\ 4.394 \\ 2.197 \\ 26.364 \\ 48.334 \\ 2.197 \\ 2.197 \\ 2.197 \\ 2.197 \\ 2.197 \\ 26.364 \\ 2.197 \\ 2.197 \\ 2.197 \\ 2.197 \\ 4.394 \\ 4.394 \\ 151.593 \\ 21.97 \\ 2.197 \\ 155.987 \\ 162.578 \\ 28.561 \\ 2.197 \\ 2.197 \\ 15.379 \\ 2.197 \\ 2.199 \\ 2.197 \\ 8.788 \\ 6.591 \\ 2.197 \\ \hline 59 \\ \hline\end{array}$


10.985

2.197

2.197

309.777

4.394

485.537

13.182

13.182

186.745

4.394

2.197

4.394

83.486

68.107

6.591

39.546

37.349

2.197

2.197

13.182

15.379

8.788

4.394

85.683

2.197

2.197

2.197

8.788

101.062

8.788

28.561

2.197

2.197

2.197

6.591

2.197

6.591

2.197

4.394

4.394

2.197

26.364

4.394

2.197

2.197

24.167

8.788

4.394

8.788

2.197 


$$
\begin{array}{r}
2.197 \\
2.197 \\
2.197 \\
2.197 \\
2.197 \\
76.895 \\
24.167 \\
6.591 \\
4.394 \\
2.197 \\
8.788 \\
15.379 \\
2.197 \\
10.985 \\
8.788 \\
13.182 \\
2.197 \\
2.197 \\
2.197 \\
2.197 \\
57.122 \\
2.197 \\
15.379 \\
2.197 \\
41.743 \\
2.197 \\
2.197 \\
70.304 \\
2.197 \\
2.197 \\
2.197 \\
2.197 \\
2.197 \\
10.985 \\
13.198 \\
\hline 3.195 \\
\hline 19.773 \\
2.197 \\
2.197 \\
2.197 \\
2.197 \\
30.758 \\
\hline .394 \\
\hline .591 \\
\hline
\end{array}
$$


10.985

4.394

8.788

72.501

2.197

8.788

2.197

2.197

195.533

2.197

2.197

8.788

4.394

8.788

10.985

2.197

2.197

6.591

2.197

2.197

2.197

2.197

43.94

2.197

19.773

8.788

955.695

24.167

24.167

10.985

19.773

2.197

39.546

39.546

32.955

2.197

2.197

21.97

2.197

2.197

2.197

6.591

2.197

10.985

2.197

24.167

21.97

2.197

2.197

52.728 


$$
\begin{array}{r}
6.591 \\
2.197 \\
6.591 \\
2.197 \\
24.167 \\
2.197 \\
2.197 \\
8.788 \\
17.576 \\
52.728 \\
2.197 \\
2.197 \\
8.788 \\
101.062 \\
118.638 \\
2.197 \\
10.985 \\
6.591 \\
2.197 \\
2.197 \\
2.197 \\
19.773 \\
4.394 \\
2.197 \\
125.229 \\
2.197 \\
2.197 \\
2.197 \\
4.394 \\
4.394 \\
4.394 \\
4.394 \\
4.394 \\
8.788 \\
4.394 \\
4.394 \\
2.197 \\
2.197 \\
2.197 \\
6.591 \\
63.713 \\
142.805 \\
4.394 \\
2.197 \\
2.197 \\
10.985 \\
2.197 \\
2.197 \\
2.197
\end{array}
$$


10.985

4.394

2.197

13.182

4.394

2.197

2.197

2.197

57.122

30.758

4.394

17.576

17.576

2.197

347.126

2.197

2.197

2.197

2.197

2.197

4.394

2.197

28.561

4.394

1261.08

4.394

21.97

28.561

10.985

2.197

4.394

8.788

2.197

2.197

2.197

2.197

4.394

935.922

4.394

1841.09

2.197

4.394

2.197

13.182

21.97

50.531

15.379

4.394

79.092

4.394 


$$
\begin{array}{r}
2.197 \\
2.197 \\
4.394 \\
2.197 \\
4.394 \\
131.82 \\
2.197 \\
4.394 \\
2.197 \\
8.788 \\
17.576 \\
2.197 \\
13.182 \\
10.985 \\
2.197 \\
2.197 \\
2.197 \\
50.531 \\
2.197 \\
2.197 \\
35.152 \\
39.546 \\
2.197 \\
24.167 \\
2.197 \\
4.394 \\
19.773 \\
8.788 \\
74.698 \\
6.591 \\
2.197 \\
15.379 \\
10.985 \\
13.188 \\
2.57 \\
2.197 \\
17.576 \\
2.197 \\
2.197 \\
\hline 3.197 \\
35.152 \\
2.197 \\
\hline .788 \\
\hline
\end{array}
$$


6.591
6.591
2.197
2.197
2.197
116.441
2.197
10.985
6.591
2.197
2.197
15.379
8.788
6.591
2.197
2.197
57.122
10.985
28.561

232.882

21.97

2.197

2.197

2.197

2.197

2.197

2.197

4.394

2.197

39.546

2.197

6.591

2.197

287.807

2.197

4.394

37.349

30.758

4.394

30.758

2.197

8.788

8.788

2.197

2.197

2.197

2.197

2.197

120.835

6.591 


$$
\begin{array}{r}
101.062 \\
4.394 \\
2.197 \\
15.379 \\
2.197 \\
2.197 \\
4.394 \\
2.197 \\
2.197 \\
2.197 \\
21.97 \\
24.167 \\
17.576 \\
2.197 \\
8.788 \\
2.197 \\
4.394 \\
2.197 \\
94.471 \\
2.197 \\
15.379 \\
15.379 \\
2.197 \\
2.197 \\
2.197 \\
15.379 \\
2.197 \\
2.197 \\
17.576 \\
2.197 \\
30.758 \\
8722.09 \\
6.591 \\
2.197 \\
2.197 \\
4.394 \\
2.197 \\
2.197 \\
2.197 \\
202.124 \\
8.788 \\
4.394 \\
2.197 \\
2.197 \\
\hline \\
\hline
\end{array}
$$


15.379

10.985

2201.39

2.197

2.197

4.394

6.591

2.197

6.591

2.197

6.591

184.548

4.394

6.591

8.788

98.865

2.197

4.394

2.197

2.197

17.576

72.501

4.394

2.197

17.576

2.197

92.274

2.197

10.985

2.197

2.197

2.197

2.197

17.576

2.197

2.197

2.197

13.182

28.561

2.197

2.197

2.197

2.197

46.137

4.394

4.394

2.197

2.197

13.182

10.985 


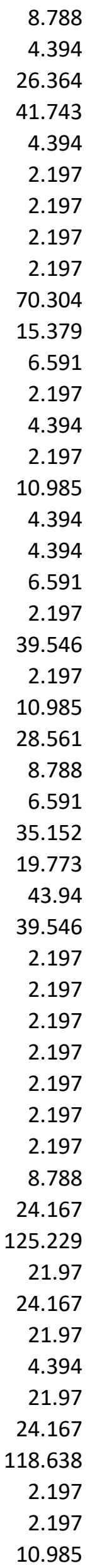




$$
\begin{array}{r}
8.788 \\
6.591 \\
4.394 \\
2.197 \\
15.379 \\
8.788 \\
10.985 \\
2.197 \\
4.394 \\
2.197 \\
2.197 \\
4.394 \\
70.304 \\
26.364 \\
19.773 \\
2.197 \\
2.197 \\
2.197 \\
116.441 \\
26.364 \\
2.197 \\
2.197 \\
4.394 \\
4.394 \\
2.197 \\
2.197 \\
26.364 \\
2.197 \\
76.895 \\
4.394 \\
4.394 \\
4.394 \\
37.349 \\
46.137 \\
6.591 \\
2.197 \\
2.197 \\
311.974 \\
145.002 \\
4.394 \\
2.197 \\
2.197 \\
13.182 \\
2.197 \\
6.591
\end{array}
$$


6.591

19.773

8.788

2.197

2.197

2.197

13.182

6.591

2.197

10.985

2.197

4.394

4.394

26.364

24.167

2.197

272.428

5452.95

2.197

4.394

4.394

41.743

2.197

10.985

4.394

2.197

443.794

46.137

2.197

50.531

4.394

4.394

8.788

21.97

6.591

6.591

63.713

2.197

2.197

2.197

2.197

2.197

2.197

32.955

2.197

2.197

13.182

8.788

166.972

2.197 


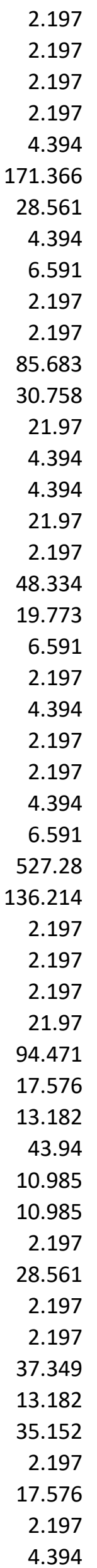




$$
\begin{array}{r}
2.197 \\
2.197 \\
6.591 \\
21.97 \\
32.955 \\
41.743 \\
2.197 \\
4.394 \\
2.197 \\
8.788 \\
120.835 \\
2.197 \\
2.197 \\
15.379 \\
2.197 \\
1094.11 \\
4.394 \\
50.531 \\
2.197 \\
182.351 \\
26.364 \\
6.591 \\
2.197 \\
74.698 \\
35.152 \\
10.985 \\
2.197 \\
6.591 \\
2.197 \\
142.805 \\
2.197 \\
32.955 \\
2.197 \\
2.197 \\
4.939 \\
2.395 \\
2.394 \\
2.394 \\
6.591 \\
2.197 \\
4.394 \\
2.197 \\
90.077 \\
13.182 \\
\hline
\end{array}
$$


15.379

2.197

2.197

2.197

2.197

4.394

8.788

4.394

109.85

2.197

2.197

2.197

2.197

37.349

8.788

19.773

26.364

2.197

2.197

4.394

10.985

2.197

2.197

2.197

4.394

2.197

2.197

13.182

10.985

4.394

2.197

182.351

6.591

2.197

2.197

2.197

2.197

10.985

57.122

10.985

160.381

32.955

24.167

4.394

37.349

81.289

10.985

2.197

6.591

118.638 
17.576

48.334

10.985

43.94

4.394

2.197

15.379

6.591

2.197

52.728

463.567

6.591

2.197

2.197

2.197

2.197

2.197

2.197

2.197

2.197

13.182

109.85

2.197

2.197

6.591

2.197

10.985

2.197

2.197

4.394

74.698

2.197

461.37

8.788

2.197

17.576

2.197

8.788

28.561

2.197

8.788

19.773

2.197

2.197

2.197

2.197

26.364

8.788

90.077

8.788 


$$
\begin{array}{r}
4.394 \\
4.394 \\
4.394 \\
85.683 \\
2.197 \\
2.197 \\
2.197 \\
2.197 \\
21.97 \\
2.197 \\
8.788 \\
4.394 \\
17.576 \\
129.623 \\
21.97 \\
311.974 \\
4.394 \\
2.197 \\
2.197 \\
2.197 \\
2.197 \\
17.576 \\
4.394 \\
2.197 \\
2.197 \\
2.197 \\
2.197 \\
8.788 \\
2.197 \\
13.182 \\
2.197 \\
2.197 \\
2.197 \\
2.187 \\
2.197 \\
127.426
\end{array}
$$


85.683

90.077

6.591

151.593

2.197

2.197

2.197

2.197

2.197

2.197

2.197

2.197

2.197

6.591

6.591

2.197

2.197

2.197

13.182

4.394

8.788

41.743

4.394

4.394

2.197

4.394

8.788

81.289

4.394

4.394

2.197

4.394

2.197

6.591

2.197

4.394

35.152

13.182

13.182

2.197

19.773

2.197

8.788

17.576

4.394

24.167

4.394

10.985

2.197

4.394 
48.334

4.394

2.197

327.353

8.788

2.197

182.351

83.486

6.591

17.576

10.985

2.197

43.94

4.394

15.379

2.197

8.788

2.197

2.197

98.865

13.182

17.576

8.788

2.197

2.197

2.197

15.379

2.197

2.197

2.197

4.394

4.394

147.199

4.394

2.197

2.197

2.197

355.914

2.197

24.167

4.394

2.197

2.197

2.197

6.591

2.197

2.197

24.167

2.197

30.758 
2.197

2.197

2.197

13.182

2.197

4.394

2.197

4.394

2.197

2.197

2.197

2.197

96.668

2.197

2.197

2.197

57.122

15.379

13.182

2.197

10.985

4.394

6.591

2.197

4.394

2.197

4.394

6.591

2.197

4.394

4.394

2.197

2.197

2.197

2.197

24.167

8.788

21.97

15.379

15.379

61.516

2.197

2.197

21.97

2.197

92.274

2.197

2.197

19.773

17.576 


$$
\begin{array}{r}
2.197 \\
2.197 \\
6.591 \\
4.394 \\
2.197 \\
10.985 \\
13.182 \\
2.197 \\
2.197 \\
2.197 \\
2.197 \\
15.379 \\
6.591 \\
2.197 \\
54.925 \\
2.197 \\
2.197 \\
318.565 \\
37.349 \\
4.394 \\
489.931 \\
2.197 \\
2.197 \\
6.591 \\
37.349 \\
4.394 \\
2.197 \\
13.182 \\
2.197 \\
2.197 \\
2.197 \\
10.985 \\
28.561 \\
8.788 \\
2.197 \\
8.788 \\
2.197 \\
2.197 \\
59.319 \\
6.591 \\
2.197 \\
10.985 \\
6.591 \\
2.197 \\
6.591 \\
2.197 \\
\hline
\end{array}
$$




$$
\begin{array}{r}
2.197 \\
8.788 \\
8.788 \\
19.773 \\
2.197 \\
10.985 \\
2.197 \\
2.197 \\
4.394 \\
21.97 \\
4.394 \\
2.197 \\
2.197 \\
2.197 \\
4.394 \\
8.788 \\
83.486 \\
90.077 \\
4.394 \\
2.197 \\
2.197 \\
30.758 \\
2.197 \\
6.591 \\
2.197 \\
2.197 \\
6.591 \\
6.591 \\
2.197 \\
2.197 \\
215.306 \\
19.773 \\
2.197 \\
8.788 \\
4.394 \\
4.394 \\
6.591 \\
24.167 \\
72.501 \\
6.591 \\
15.379 \\
4.394 \\
2.197 \\
2.197 \\
6.591 \\
2.197 \\
2.197
\end{array}
$$




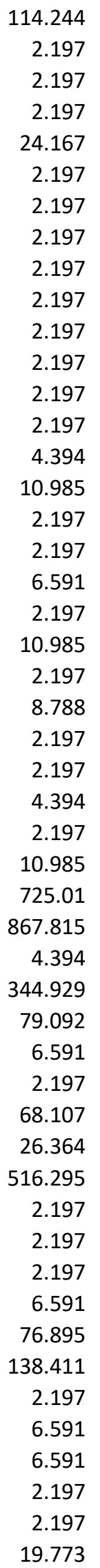


2.197

32.955

41.743

4.394

4.394

2.197

15.379

8.788

85.683

2.197

2.197

8.788

6.591

6.591

2.197

2.197

4.394

2.197

2.197

4.394

2.197

107.653

2.197

87.88

2.197

15.379

21.97

59.319

2.197

2.197

76.895

2.197

2.197

8.788

17.576

28.561

4.394

4.394

24.167

2.197

24.167

59.319

2.197

8.788

4.394

70.304

24.167

4.394

4.394

6.591 


$$
\begin{array}{r} 
\\
2.197 \\
2.197 \\
2.197 \\
2.197 \\
2.197 \\
70.304 \\
4.394 \\
4.394 \\
43.94 \\
8.788 \\
21.97 \\
19.773 \\
2.197 \\
8.788 \\
15.379 \\
13.182 \\
2.197 \\
2.197 \\
2.197 \\
2.197 \\
6.591 \\
4.394 \\
2.197 \\
28.561 \\
2.197 \\
2.197 \\
15.379 \\
2.197 \\
2.197 \\
92.274 \\
2.197 \\
63.713 \\
2.197 \\
4.394 \\
6.591 \\
2.197 \\
2.197 \\
8.788 \\
52.728 \\
2.197 \\
4.394 \\
4.394 \\
2.197 \\
2.1979 \\
\\
2.197
\end{array}
$$




$$
\begin{array}{r}
2.197 \\
2.197 \\
4.394 \\
2.197 \\
2.197 \\
2.197 \\
2.197 \\
2.197 \\
10.985 \\
2.197 \\
6.591 \\
4.394 \\
2.197 \\
48.334 \\
43.94 \\
26.364 \\
2.197 \\
6.591 \\
39.546 \\
2.197 \\
2.197 \\
13.182 \\
6.591 \\
140.608 \\
10.985 \\
204.321 \\
2.197 \\
2.197 \\
2.197 \\
4.394 \\
35.152 \\
13.182 \\
854.633 \\
32.955 \\
2.197 \\
127.426 \\
2.197 \\
4.394 \\
2.197 \\
4.1997 \\
2.197 \\
2.197 \\
\hline
\end{array}
$$




$$
\begin{aligned}
& 2.197 \\
& 2.197 \\
& 2.197 \\
& 2.197 \\
& 4.394 \\
& 28.561 \\
& 2.197 \\
& 94.471 \\
& 2.197 \\
& 2.197 \\
& 2.197 \\
& 30.758 \\
& 13.182 \\
& 13.182 \\
& 2.197 \\
& 2.197 \\
& 2.197 \\
& 2.197 \\
& 4.394 \\
& 6.591 \\
& 2.197 \\
& 4.394 \\
& 4.394 \\
& 2.197 \\
& 2.197 \\
& 46.137 \\
& 52.728 \\
& 342.732 \\
& 24.167 \\
& 26.364 \\
& 6.591 \\
& 61.516 \\
& 2.197 \\
& 6.591 \\
& 2.197 \\
& 2.197 \\
& 2.197 \\
& 2.197 \\
& 2.197 \\
& 13.182 \\
& 2.197 \\
& 6.591 \\
& 10.985 \\
& 19.773 \\
& 2.197 \\
& 2.197 \\
& 19.773 \\
& 83.486 \\
& 19.773 \\
& 4.394
\end{aligned}
$$


2.197
2.197
268.034
54.925
50.531
4.394
54.925
10.985
4.394
2.197
41.743
4.394
4.394
2.197
2.197
118.638
2.197
2.197
15.379
2.197
2.197
112.047
4.394
4.394
15.379
19.773
4.394
41.743
43.94
4.394
762.359

762.359

2.197

4.394

2.197

2.197

2.197

2.197

13.182

2.197

24.167

2.197

2.197

2.197

2.197

2.197

2.197

2.197

2.197

13.182

41.743 


$$
\begin{array}{r}
2.197 \\
2.197 \\
6.591 \\
2.197 \\
10.985 \\
28.561 \\
4.394 \\
2.197 \\
4.394 \\
30.758 \\
2.197 \\
2.197 \\
2.197 \\
2.197 \\
37.349 \\
2.197 \\
142.805 \\
463.567 \\
4.394 \\
4.394 \\
4.394 \\
2.197 \\
24.167 \\
4.394 \\
13.182 \\
2.197 \\
10.985 \\
8.788 \\
8.788 \\
15.379 \\
15.379 \\
2.197 \\
2.197 \\
2.197 \\
6.591 \\
2.197 \\
2.197 \\
10.985 \\
307.58 \\
224.094 \\
79.092 \\
4.394 \\
2.197 \\
2.197 \\
2.197 \\
\hline \\
\hline
\end{array}
$$




$$
\begin{aligned}
& 37.349 \\
& 2.197 \\
& 2.197 \\
& 4.394 \\
& 2.197 \\
& 39.546 \\
& 151.593 \\
& 6.591 \\
& 2.197 \\
& 10.985 \\
& 4.394 \\
& 2.197 \\
& 164.775 \\
& 2.197 \\
& 4.394 \\
& 10.985 \\
& 206.518 \\
& 10.985 \\
& 307.58 \\
& 15.379 \\
& 109.85 \\
& 37.349 \\
& 4.394 \\
& 2.197 \\
& 4.394 \\
& 2.197 \\
& 2.197 \\
& 575.614 \\
& 2.197 \\
& 2.197 \\
& 21.97 \\
& 2.197 \\
& 24.167 \\
& 13.182 \\
& 35.152 \\
& 30.758 \\
& 32.955 \\
& 2.197 \\
& 2.197 \\
& 28.561 \\
& 4.394 \\
& 19.773 \\
& 4.394 \\
& 2.197 \\
& 15.379 \\
& 2.197 \\
& 24.167 \\
& 6.591 \\
& 2.197 \\
& 2.197
\end{aligned}
$$


219.7

973.271

2.197

65.91

2.197

2.197

2.197

13.182

21.97

48.334

2.197

13.182

13.182

13.182

2.197

24.167

4.394

4.394

4.394

2.197

24.167

2.197

2.197

2.197

2.197

2.197

2.197

2.197

15.379

79.092

2.197

243.867

2.197

4.394

2.197

6.591

2.197

2.197

2.197

41.743

13.182

13.182

4.394

2.197

15.379

2.197

52.728

8.788

4.394

19.773 


$\begin{array}{r}2.197 \\ 2.197 \\ 2.197 \\ 2.197 \\ 13.182 \\ 24.167 \\ 2.197 \\ 2.197 \\ 2.197 \\ 4.394 \\ 6.591 \\ 2.197 \\ 2.197 \\ 6.591 \\ 13.182 \\ 2.197 \\ 2.197 \\ 79.092 \\ 2.197 \\ 17.576 \\ 6.591 \\ 2.197 \\ 21.97 \\ 6.591 \\ 2.197 \\ \hline 59.319 \\ 2.197 \\ 21.97 \\ 103.259 \\ 13.182 \\ 6.591 \\ 85.683 \\ 13.773 \\ 26.364 \\ 2.197 \\ 2.197 \\ 2.197 \\ 4.394 \\ 4.394 \\ \hline 4.394 \\ \hline .394 \\ \hline .397 \\ \hline\end{array}$


2.197
13.182
2.197
4.394
4.394
2.197
21.97
2.197
2.197
6.591
112.047
4.394
164.775
10.985
28.561
10.985
208.715
2.197
24.167
8.788
2.197
2.197
2.197
2.197
59.319

59.319

294.398

17.576

39.546

2.197

145.002

4.394

8.788

19.773

6.591

2.197

127.426

28.561

4.394

8.788

2.197

6.591

32.955

4.394

2.197

2.197

2.197

149.396

2.197

2.197

6.591 


$\begin{array}{r}4.394 \\ 59.319 \\ 41.743 \\ 283.413 \\ 112.047 \\ 2.197 \\ 52.728 \\ 2.197 \\ 8.788 \\ 2.197 \\ 2.197 \\ 17.576 \\ 8.788 \\ 2.197 \\ 2.197 \\ 2.197 \\ 2.197 \\ 2.197 \\ 2.197 \\ 17.576 \\ 2.197 \\ 175.76 \\ 114.244 \\ 2.197 \\ 230.685 \\ 2.197 \\ 109.85 \\ 35.152 \\ 2.197 \\ 2.197 \\ 59.319 \\ 2.197 \\ 2.197 \\ 2.197 \\ 2.197 \\ 2.197 \\ 2.197 \\ 4.394 \\ 4.394 \\ 26.364 \\ 103.259 \\ 2.197 \\ 2.197 \\ 4.394 \\ 2.197 \\ 4.99 \\ \hline\end{array}$


2.197

270.231

13.182

2.197

2.197

2.197

4.394

17.576

6.591

4.394

15.379

13.182

485.537

6.591

2.197

2.197

2.197

2.197

17.576

4.394

4.394

35.152

6.591

2.197

13.182

4.394

2.197

57.122

37.349

17.576

52.728

2.197

2.197

28.561

28.561

698.646

2.197

2.197

125.229

17.576

10.985

10.985

8.788

127.426

4.394

217.503

17.576

2.197

6.591

6.591 
459.173
8.788
13.182
13.182
2.197
2.197
37.349
166.972
17.576
13.182
2.197
15.379
96.668
4.394
13.182
2.197
8.788
87.88

13.182

290.004

2.197

8.788

6.591

17.576

2.197

4.394

6.591

2.197

228.488

6.591

8.788

2.197

2.197

6.591

112.047

13.182

2.197

4.394

173.563

4.394

17.576

2.197

2.197

2.197

6.591

2.197

8.788

30.758

145.002

2.197 


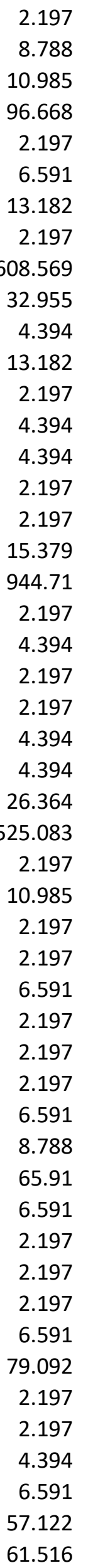


24.167

10.985

21.97

4.394

8.788

2.197

2.197

2.197

19.773

2.197

59.319

10.985

50.531

4.394

10.985

26.364

32.955

4.394

496.522

15.379

35.152

17.576

10.985

26.364

2.197

2.197

120.835

4.394

10.985

24.167

65.91

48.334

30.758

327.353

4.394

6.591

4.394

39.546

2.197

6.591

6.591

2.197

2.197

6.591

4.394

2.197

2.197

13.182

4.394

2.197 
17.576

2.197

24.167

10.985

2.197

2.197

6.591

15.379

26.364

8.788

2.197

2.197

2.197

2.197

10.985

2.197

101.062

2.197

2.197

2.197

2.197

4.394

6.591

37.349

2.197

15.379

2.197

101.062

2.197

6.591

2.197

2.197

4.394

13.182

2.197

10.985

175.76

2.197

2.197

13.182

2.197

4.394

2.197

2.197

2.197

6.591

15.379

10.985

8.788

4.394 
2.197

19.773

2.197

4.394

2.197

6.591

2.197

2.197

246.064

8.788

2.197

41.743

2.197

28.561

2.197

10.985

2.197

2.197

21.97

4.394

2.197

65.91

6.591

2.197

2.197

2.197

2.197

10.985

6.591

15.379

46.137

2.197

35.152

2.197

4.394

2.197

6.591

2.197

4.394

2.197

37.349

21.97

2.197

2.197

4.394

17.576

2.197

4.394

17.576

4.394 
32.955

4.394

125.229

30.758

2.197

39.546

6.591

261.443

32.955

28.561

10.985

17.576

63.713

2.197

13.182

2.197

41.743

2.197

2.197

8.788

4.394

94.471

8.788

28.561

6.591

4.394

13.182

13.182

35.152

65.91

72.501

19.773

2.197

2.197

21.97

188.942

6.591

4.394

8.788

4.394

6.591

2.197

2.197

19.773

4.394

70.304

2.197

2.197

4.394

10.985 


$\begin{array}{r}13.182 \\ 4.394 \\ 4.394 \\ 2.197 \\ 8.788 \\ 158.184 \\ 2.197 \\ 21.97 \\ 129.623 \\ 6.591 \\ 2.197 \\ 43.94 \\ 125.229 \\ 2.197 \\ 2618.82 \\ 2.197 \\ 21.97 \\ 8.788 \\ 8.788 \\ 4.394 \\ 145.002 \\ 599.781 \\ 4.394 \\ 2.197 \\ 6.591 \\ 2.197 \\ 2.197 \\ 2.197 \\ 2.197 \\ 4.394 \\ 6.591 \\ 2.197 \\ 10.985 \\ 2.197 \\ 2.197 \\ 2.197 \\ 6.591 \\ 8.594 \\ 6.598 \\ 8.591 \\ 8.788 \\ \hline\end{array}$




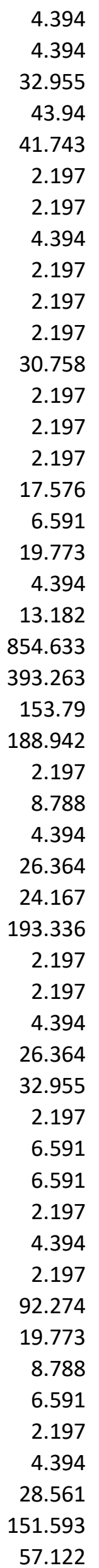




$\begin{array}{r}4.394 \\ 8.788 \\ 24.167 \\ 311.974 \\ 116.441 \\ 2.197 \\ 17.576 \\ 4.394 \\ 6.591 \\ 2.197 \\ 2.197 \\ 19.773 \\ 2.197 \\ 2.197 \\ 13.182 \\ 6.591 \\ 2.197 \\ 2.197 \\ 10.985 \\ 8.788 \\ 50.531 \\ 32.955 \\ 6.591 \\ 2.197 \\ 2.197 \\ 6.591 \\ 4.394 \\ 15.379 \\ 21.97 \\ 10.985 \\ 2.197 \\ 2.197 \\ 6.591 \\ 2.197 \\ 6.591 \\ 125.229 \\ 54.925 \\ 2.197 \\ 4.394 \\ 2.197 \\ 4.394 \\ 4.394 \\ 4.394 \\ 2.197 \\ 2.197 \\ \hline\end{array}$


19.773

2.197

2.197

2.197

2.197

4.394

2.197

2.197

8.788

6.591

138.411

4.394

2.197

2.197

2.197

4.394

2.197

2.197

4.394

6.591

2.197

13.182

2.197

4.394

2.197

8.788

17.576

177.957

8.788

13.182

8.788

4.394

2.197

2.197

2.197

8.788

2.197

57.122

2.197

107.653

2.197

70.304

37.349

107.653

2.197

2.197

8.788

2.197

4.394

2.197 


$$
\begin{array}{r}
19.773 \\
4.394 \\
10.985 \\
24.167 \\
19.773 \\
2.197 \\
8.788 \\
2.197 \\
24.167 \\
10.985 \\
15.379 \\
10.985 \\
2.197 \\
2.197 \\
2.197 \\
4.394 \\
2.197 \\
6.591 \\
4.394 \\
2.197 \\
6.591 \\
4.394 \\
2.197 \\
26.364 \\
8.788 \\
10.985 \\
2.197 \\
2.197 \\
2.197 \\
116.441 \\
4.394 \\
2.197 \\
101.062 \\
50.531 \\
2.197 \\
4.394 \\
4.394 \\
6.591 \\
2.197 \\
2.197 \\
4.394 \\
6.591 \\
2.197 \\
2.197 \\
2.197 \\
2.197 \\
2.197 \\
210.912
\end{array}
$$




$$
\begin{array}{r}
21.97 \\
43.94 \\
131.82 \\
6.591 \\
2.197 \\
85.683 \\
98.865 \\
54.925 \\
2.197 \\
2.197 \\
2.197 \\
2.197 \\
799.708 \\
57.122 \\
112.047 \\
2.197 \\
2.197 \\
2.197 \\
4.394 \\
4.394 \\
52.728 \\
2.197 \\
2.197 \\
4.394 \\
2.197 \\
2.197 \\
4.394 \\
4.394 \\
13.182 \\
2.197 \\
2.197 \\
10.985 \\
48.334 \\
2.197 \\
2.197 \\
\hline 2.197 \\
2.197 \\
2.197 \\
4.394 \\
4.394 \\
2.197 \\
50.531 \\
2.197 \\
\hline
\end{array}
$$




$$
\begin{array}{r}
6.591 \\
8.788 \\
2.197 \\
2.197 \\
2.197 \\
4.394 \\
6.591 \\
10.985 \\
2.197 \\
2.197 \\
2.197 \\
2.197 \\
2.197 \\
8.788 \\
21.97 \\
30.758 \\
2.197 \\
10.985 \\
2.197 \\
6.591 \\
96.668 \\
2.197 \\
8.788 \\
2.197 \\
65.91 \\
2.197 \\
46.137 \\
32.955 \\
30.758 \\
13.182 \\
2.197 \\
2.197 \\
2.197 \\
4.394 \\
6.197 \\
\hline 10.595 \\
2.197 \\
4.394 \\
2.197 \\
2.197 \\
2.197 \\
2.197 \\
8.788 \\
8.788 \\
\hline
\end{array}
$$




$$
\begin{array}{r}
2.197 \\
2.197 \\
10.985 \\
2.197 \\
6.591 \\
13.182 \\
2.197 \\
2.197 \\
4.394 \\
6.591 \\
65.91 \\
2.197 \\
17.576 \\
8.788 \\
4.394 \\
2.197 \\
2.197 \\
2.197 \\
8.788 \\
4.394 \\
2.197 \\
26.364 \\
15.379 \\
2.197 \\
2.197 \\
17.576 \\
8.788 \\
127.426 \\
2.197 \\
4.394 \\
8.788 \\
37.349 \\
4.394 \\
2.197 \\
19.773 \\
50.531 \\
87.88 \\
4.394 \\
4.394 \\
2.394 \\
17.576 \\
17.576 \\
4.394 \\
2.197 \\
\hline
\end{array}
$$


10.985

15.379

2.197

175.76

2.197

10.985

2.197

2.197

2.197

4.394

2.197

8.788

2.197

516.295

4.394

4.394

43.94

4.394

2.197

2.197

2.197

2.197

2.197

2.197

61.516

10.985

2.197

2.197

6.591

112.047

10.985

2.197

2.197

2.197

41.743

57.122

2.197

28.561

48.334

6.591

2.197

37.349

2.197

2.197

2.197

2.197

2.197

2.197

2.197

2.197 


$$
\begin{array}{r}
4.394 \\
37.349 \\
8.788 \\
2.197 \\
17.576 \\
8.788 \\
2.197 \\
17.576 \\
6.591 \\
6.591 \\
134.017 \\
6.591 \\
39.546 \\
2.197 \\
2.197 \\
2.197 \\
15.379 \\
6.591 \\
19.773 \\
4.394 \\
17.576 \\
10.985 \\
722.813 \\
6.591 \\
2.197 \\
2.197 \\
2.197 \\
13.182 \\
43.94 \\
2.197 \\
24.167 \\
52.728 \\
4.394 \\
4.394 \\
8.195 \\
2.197 \\
103.259 \\
2.197 \\
2.197 \\
19.773 \\
155.987 \\
35.159 \\
39.546 \\
26.364 \\
19.773 \\
\hline
\end{array}
$$


2.197

2.197

2.197

4.394

4.394

2.197

2.197

2.197

10.985

10.985

2.197

2.197

2.197

39.546

2.197

17.576

2.197

6.591

2.197

2.197

63.713

15.379

10.985

72.501

4.394

2.197

19.773

109.85

2.197

54.925

2.197

10.985

2.197

4.394

2.197

15.379

50.531

2.197

2.197

2.197

17.576

17.576

2.197

4.394

2.197

68.107

37.349

8.788

10.985

24.167 


$$
\begin{array}{r}
478.946 \\
26.364 \\
2.197 \\
131.82 \\
28.561 \\
2.197 \\
2.197 \\
2.197 \\
4.394 \\
2.197 \\
2.197 \\
309.777 \\
2.197 \\
13.182 \\
2.197 \\
39.546 \\
15.379 \\
4.394 \\
6.591 \\
2.197 \\
2.197 \\
8.788 \\
2.197 \\
6.591 \\
54.925 \\
94.471 \\
2.197 \\
2.197 \\
2.197 \\
6.591 \\
4.19 .773 \\
4.394 \\
2.197 \\
8.788 \\
34.925
\end{array}
$$




$$
\begin{array}{r}
6.591 \\
2.197 \\
2.197 \\
435.006 \\
8.788 \\
28.561 \\
2.197 \\
19.773 \\
2.197 \\
54.925 \\
6.591 \\
32.955 \\
2.197 \\
21.97 \\
2.197 \\
4.394 \\
2.197 \\
2.197 \\
316.368 \\
92.274 \\
2.197 \\
4.394 \\
87.88 \\
4.394 \\
13.182 \\
8.788 \\
105.456 \\
15.379 \\
4.394 \\
2.197 \\
39.546 \\
2.197 \\
2.197 \\
2.197 \\
2.197 \\
2.197 \\
2.197 \\
24.167 \\
4.394 \\
74.698 \\
28.561 \\
2.197 \\
2.197 \\
2.197 \\
\end{array}
$$


28.561

2.197

2.197

2.197

2.197

2.197

65.91

2.197

4.394

8.788

2.197

65.91

52.728

101.062

8.788

2.197

2.197

2.197

2.197

10.985

4.394

2.197

4.394

8.788

21.97

2.197

276.822

19742.2

4.394

2.197

2.197

4.394

30.758

2.197

2.197

2.197

2.197

2.197

2.197

2.197

2.197

2985.72

15.379

2.197

2.197

10.985

2.197

4.394

4.394

6.591 


$\begin{array}{r}8.788 \\ 2.197 \\ 15.379 \\ 4.394 \\ 87.88 \\ 2.197 \\ 2.197 \\ 2.197 \\ 4.394 \\ 2.197 \\ 2.197 \\ 41.743 \\ 303.186 \\ 43.94 \\ 2.197 \\ 2.197 \\ 241.67 \\ 254.852 \\ 2.197 \\ 26.364 \\ 2.197 \\ 127.426 \\ 2.197 \\ 2.197 \\ 4.394 \\ 2.197 \\ 2.197 \\ 24.167 \\ 2.197 \\ 6.591 \\ 28.561 \\ 2.197 \\ 28.561 \\ 2.197 \\ 4.394 \\ 2.197 \\ 2.197 \\ 2.197 \\ 2.319 \\ 2.97 \\ \hline 14.197 \\ 13.189 \\ 10.985 \\ \hline\end{array}$


17.576

2.197

2.197

2.197

2.197

2.197

2.197

2.197

2.197

799.708

2.197

4.394

2.197

2.197

19.773

6.591

2.197

2.197

96.668

32.955

2.197

202.124

21.97

35.152

6.591

2.197

2.197

2.197

17.576

2.197

4.394

4.394

24.167

8.788

10.985

6.591

10.985

4.394

4.394

2.197

8.788

125.229

87.88

2.197

2.197

2.197

2.197

2.197

2.197

2.197 
2.197

2.197

2.197

2.197

2.197

2.197

2.197

2.197

2.197

2.197

4.394

21.97

4.394

87.88

2.197

13.182

4.394

8.788

2.197

85.683

2.197

2.197

4.394

2.197

2.197

2.197

149.396

6.591

48.334

2.197

2.197

2.197

2.197

2.197

2.197

2.197

19.773

8.788

4.394

6.591

2.197

2.197

35.152

6.591

2.197

2.197

10.985

94.471

2.197

70.304 
2.197

13.182

52.728

2.197

8.788

4.394

8.788

2.197

193.336

41.743

2.197

2.197

43.94

21.97

2.197

37.349

52.728

2.197

2.197

32.955

2.197

2.197

101.062

2.197

2.197

21.97

15.379

120.835

24.167

155.987

2.197

2.197

8.788

2.197

2.197

2.197

2.197

2.197

2.197

2.197

123.032

2.197

2.197

2.197

2.197

2.197

81.289

6.591

19.773

230.685 


4.394
4.394
4.394
2.197
4.394
169.169
4.394
79.092
2.197
4.394
19.773
105.456
8.788
2.197
13.182
2.197
10.985
2.197
2.197
4.394
4.394
140.608
30.758
2.197
2.197
689.858
83.486
6.591
166.972
10.985
2.197
2.197
2.197
2.197
2.197
2.197
270.231
48.334
8.788
10.985
2.197
17.576
10.985
50.531
48.334
13.182
2.788




$$
\begin{aligned}
& 4.394 \\
& 17.576 \\
& 4.394 \\
& 4.394 \\
& 2.197 \\
& 37.349 \\
& 19.773 \\
& 15.379 \\
& 72.501 \\
& 4.394 \\
& 2.197 \\
& 2.197 \\
& 2.197 \\
& 21.97 \\
& 4.394 \\
& 2.197 \\
& 2.197 \\
& 2.197 \\
& 2.197 \\
& 2.197 \\
& 19.773 \\
& 2.197 \\
& 2.197 \\
& 232.882 \\
& 2.197 \\
& 6.591 \\
& 2.197 \\
& 2.197 \\
& 8.788 \\
& 2.197 \\
& 46.137 \\
& 52.728 \\
& 2.197 \\
& 21.97 \\
& 8.788 \\
& 8.788 \\
& 21.97 \\
& 4.394 \\
& 4.394 \\
& 4.394 \\
& 2.197 \\
& 2.197 \\
& 48.334 \\
& 10.985 \\
& 24.167 \\
& 70.304 \\
& 426.218 \\
& 246.064 \\
& 4.394 \\
& 4.394
\end{aligned}
$$




$\begin{array}{r}2.197 \\ 2.197 \\ 10.985 \\ 2.197 \\ 19.773 \\ 24.167 \\ 26.364 \\ 2.197 \\ 2.197 \\ 2.197 \\ 2.197 \\ 2.197 \\ 2.197 \\ 2.197 \\ 2.197 \\ 4.394 \\ 4.394 \\ 4.394 \\ 2.197 \\ 2.197 \\ 2.197 \\ 2.197 \\ 2.197 \\ 6.591 \\ 10.985 \\ 8.788 \\ 140.608 \\ 2.197 \\ 70.304 \\ 2.197 \\ 26.364 \\ 6.591 \\ 2.197 \\ 32.955 \\ 2.197 \\ 19.773 \\ 41.743 \\ 2.197 \\ 2.197 \\ 2.197 \\ 4.394 \\ 2.197 \\ 2.197 \\ 4.394 \\ 2.197 \\ \hline .394 \\ \hline\end{array}$




$$
\begin{array}{r}
4.394 \\
1854.27 \\
17.576 \\
39.546 \\
41.743 \\
4.394 \\
15.379 \\
13.182 \\
6.591 \\
32.955 \\
4.394 \\
10.985 \\
4.394 \\
2.197 \\
28.561 \\
4.394 \\
8.788 \\
2.197 \\
224.094 \\
13.182 \\
15.379 \\
107.653 \\
24.167 \\
50.531 \\
6.591 \\
2.197 \\
94.471 \\
2.197 \\
2.197 \\
2.197 \\
10.985 \\
2.197 \\
2.197 \\
10.092 \\
15.379 \\
19.793 \\
8.798 \\
4.398 \\
2.394 \\
2.197 \\
13.182 \\
151.593 \\
2.197 \\
\hline
\end{array}
$$




$$
\begin{array}{r}
2.197 \\
8.788 \\
2.197 \\
2.197 \\
2.197 \\
4.394 \\
2.197 \\
32.955 \\
2.197 \\
19.773 \\
2.197 \\
2.197 \\
2.197 \\
8.788 \\
2.197 \\
2.197 \\
2.197 \\
2.197 \\
2.197 \\
2.197 \\
2.197 \\
2.197 \\
2.197 \\
10.985 \\
4.394 \\
8.788 \\
2.197 \\
17.576 \\
2.197 \\
2.197 \\
942.513 \\
17.576 \\
13.379 \\
4.394 \\
\hline \\
\hline 2.189 \\
2.198 \\
2.97 \\
48.334 \\
4.394 \\
6.591 \\
4.394 \\
2.1985 \\
\hline
\end{array}
$$




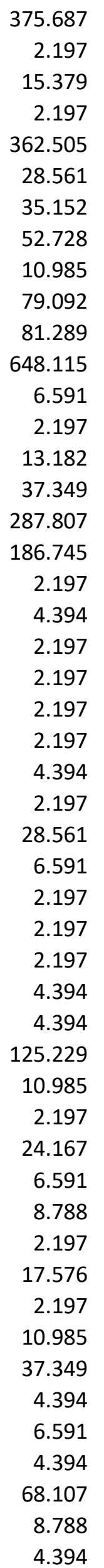




$\begin{array}{r}4.394 \\ 186.745 \\ 2.197 \\ 2.197 \\ 125.229 \\ 2.197 \\ 10.985 \\ 4.394 \\ 10.985 \\ 2.197 \\ 13.182 \\ 6.591 \\ 4.394 \\ 2.197 \\ 2.197 \\ 1955.33 \\ 2.197 \\ 24.167 \\ 2.197 \\ 54.925 \\ 276.822 \\ 2.197 \\ 2.197 \\ 2.197 \\ 39.546 \\ 116.441 \\ 2.197 \\ 2.197 \\ 4.394 \\ 4.394 \\ 6.591 \\ 2.197 \\ 19.773 \\ 10.985 \\ 2.197 \\ 16.197 \\ 16.579 \\ 26.378 \\ 2.379 \\ 2.197 \\ 2.197 \\ 8.788 \\ 4.394 \\ 6.591 \\ \hline\end{array}$


4.394

35.152

39.546

28.561

17.576

2.197

4.394

6.591

10.985

2.197

2.197

4.394

48.334

4.394

8.788

2.197

2.197

24.167

8.788

281.216

35.152

2.197

4.394

81.289

26.364

26.364

19.773

2.197

2.197

2.197

1166.61

59.319

2.197

151.593

17.576

2.197

2.197

8.788

13.182

2.197

13.182

83.486

4.394

2.197

2.197

19.773

4.394

26.364

2.197

2.197 


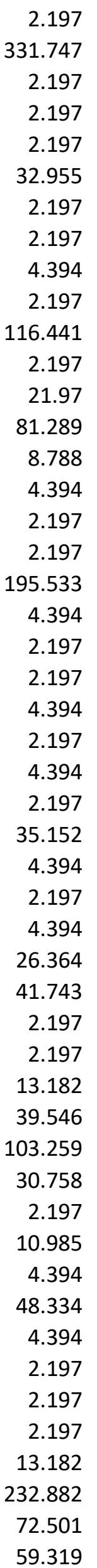




134.017
21.97
6.591
6.591
54.925
2515.56
2.197
2.197
2.197
2.197
2.197
2.197
2.197
2.197
2.197
6.591
2.197
46.137
24.167
2.197
39.546
2.197
2.197
2.197
61.516
2.197
2.197
6.591
2.197
2.197
6.591
26.364
8.788
54.925
4.394
138.411
74.698
250.458
2.197
4.394
54.925
2.197
72.197
10.987
2.197
142.805


13.182

2.197

2.197

2.197

2.197

4.394

8.788

2.197

6.591

70.304

4.394

703.04

2.197

4.394

2.197

8.788

2.197

63.713

24.167

6.591

10.985

2.197

2.197

2.197

52.728

15.379

68.107

26.364

35.152

2.197

52.728

2.197

4.394

2.197

2.197

4.394

2.197

2.197

2.197

2.197

10.985

4.394

2.197

2.197

10.985

202.124

2.197

6.591

13.182

30.758 


$$
\begin{array}{r}
2.197 \\
4.394 \\
43.94 \\
28.561 \\
4.394 \\
2.197 \\
4.394 \\
6.591 \\
2.197 \\
2.197 \\
2.197 \\
2.197 \\
10.985 \\
2.197 \\
13.182 \\
2.197 \\
2.197 \\
50.531 \\
4.394 \\
1192.97 \\
2.197 \\
2.197 \\
48.334 \\
17.576 \\
17.576 \\
2.197 \\
2.197 \\
13.182 \\
13.379 \\
13.197 \\
145.002 \\
2.197 \\
265.837 \\
10.985 \\
72.501 \\
2.197 \\
4.394 \\
17.576 \\
2.197 \\
2.197 \\
2.197 \\
13.182 \\
13.182 \\
145.002 \\
\hline
\end{array}
$$




$$
\begin{array}{r}
8.788 \\
2.197 \\
2.197 \\
6.591 \\
10.985 \\
2.197 \\
2.197 \\
2.197 \\
15.379 \\
6.591 \\
889.785 \\
39.546 \\
342.732 \\
2.197 \\
15.379 \\
39.546 \\
13.182 \\
402.051 \\
4.394 \\
2.197 \\
2.197 \\
26.364 \\
2.197 \\
6.591 \\
2.197 \\
35.152 \\
57.122 \\
2.197 \\
19.773 \\
2.197 \\
4.394 \\
26.364 \\
2.197 \\
2.197 \\
2.197 \\
\hline 3.713 \\
8.788 \\
2.197 \\
336.141 \\
2.197 \\
6.591 \\
30.759 \\
2.197 \\
\hline
\end{array}
$$


2.197

2.197

15.379

13.182

4.394

39.546

4.394

2.197

2.197

17.576

10.985

6.591

83.486

8.788

2.197

2.197

145.002

35.152

2.197

2.197

2.197

2.197

35.152

2.197

2.197

8.788

65.91

21.97

4.394

8.788

24.167

15.379

2.197

2.197

43.94

28.561

10.985

24.167

8.788

4.394

2.197

4.394

8.788

2.197

13.182

8.788

2.197

4.394

61.516

2.197 


$\begin{array}{r}21.97 \\ 8.788 \\ 63.713 \\ 10.985 \\ 2.197 \\ 2.197 \\ 2.197 \\ 10.985 \\ 4.394 \\ 2.197 \\ 4.394 \\ 2.197 \\ 6.591 \\ 4.394 \\ 123.032 \\ 2.197 \\ 2.197 \\ 30.758 \\ 2.197 \\ 2.197 \\ 2.197 \\ 4.394 \\ 4.394 \\ 2.197 \\ 4.394 \\ 2.197 \\ 118.638 \\ 6.591 \\ 2.197 \\ 6.591 \\ 2.197 \\ \hline 123.032 \\ 6.591 \\ 10.985 \\ 4.394 \\ 2.197 \\ 17.576 \\ 8.788 \\ 2.197 \\ 2.197 \\ 19.773 \\ 2.197 \\ 92.274 \\ 10.985 \\ 50.531 \\ \hline\end{array}$




$\begin{array}{r}4.394 \\ 50.531 \\ 17.576 \\ 2.197 \\ 2.197 \\ 2.197 \\ 2.197 \\ 2.197 \\ 21.97 \\ 2.197 \\ 2.197 \\ 2.197 \\ 2.197 \\ 2.197 \\ 10.985 \\ 2.197 \\ 48.334 \\ 13.182 \\ 193.336 \\ 6.591 \\ 2.197 \\ 4.394 \\ \hline 4.394 \\ 2.197 \\ 2.197 \\ 2.197 \\ \hline 10.985 \\ \hline\end{array}$




$\begin{array}{r}4.394 \\ 2.197 \\ 72.501 \\ 4.394 \\ 2.197 \\ 39.546 \\ 2.197 \\ 4.394 \\ 2.197 \\ 6.591 \\ 43.94 \\ 6.591 \\ 2.197 \\ 2.197 \\ 4.394 \\ 4.394 \\ 2.197 \\ 4.394 \\ 2.197 \\ 2.197 \\ 2.197 \\ 2.197 \\ 26.364 \\ 15.379 \\ 2.197 \\ 30.758 \\ 248.261 \\ 26.364 \\ 4.394 \\ 2.197 \\ 6.591 \\ 4.394 \\ 2.197 \\ 4.1939 \\ 2.394 \\ 2.197 \\ 10.985 \\ 8.788 \\ 4.394 \\ 2.197 \\ 2.197 \\ 2.197 \\ 2.197 \\ 4.394 \\ \hline 394 \\ \hline\end{array}$




$$
\begin{array}{r}
61.516 \\
4.394 \\
39.546 \\
32.955 \\
4.394 \\
219.7 \\
2.197 \\
2.197 \\
432.809 \\
210.912 \\
10.985 \\
2.197 \\
2.197 \\
4.394 \\
4.394 \\
19.773 \\
140.608 \\
19.773 \\
2.197 \\
2.197 \\
2.197 \\
4.394 \\
2.197 \\
2.197 \\
6.591 \\
2.197 \\
4.394 \\
4.394 \\
4.394 \\
2.197 \\
37.349 \\
2.197 \\
8.788 \\
13.182 \\
6.591 \\
2.197 \\
10.985 \\
4.394 \\
4.394 \\
4.397 \\
2.197 \\
2.197 \\
15.364 \\
10.985 \\
\hline
\end{array}
$$




$\begin{array}{r}2.197 \\ 2.197 \\ 2.197 \\ 8.788 \\ 2.197 \\ 2.197 \\ 21.97 \\ 456.976 \\ 4.394 \\ 4.394 \\ 37.349 \\ 2.197 \\ 2.197 \\ 6.591 \\ 2.197 \\ 4.394 \\ 13.182 \\ 2.197 \\ 32.955 \\ 41.743 \\ 4.394 \\ 4.394 \\ 4.394 \\ 8.788 \\ 2.197 \\ 32.955 \\ 2.197 \\ 2.197 \\ 37.349 \\ 439.4 \\ 39.546 \\ 2.197 \\ 2.197 \\ 276.822 \\ 4.394 \\ 10.985 \\ 14673.8 \\ 30.758 \\ 155.987 \\ 2.197 \\ 61.516 \\ 48.334 \\ \hline 3.503 \\ \hline 365 \\ \hline\end{array}$


19.773

2.197

2.197

331.747

2.197

24.167

4.394

8.788

2.197

127.426

6.591

4.394

10.985

2.197

35.152

2.197

65.91

8.788

39.546

10.985

2.197

6.591

4.394

2.197

14849.5

1942.15

2.197

2.197

2.197

2.197

2.197

19.773

215.306

19.773

98.865

2.197

6.591

52.728

19.773

50.531

43.94

4.394

4.394

4.394

13.182

6.591

48.334

4.394

6.591

19.773 


$$
\begin{array}{r}
8.788 \\
2.197 \\
4.394 \\
32.955 \\
39.546 \\
2.197 \\
17.576 \\
4.394 \\
4.394 \\
2.197 \\
2.197 \\
57.122 \\
54.925 \\
307.58 \\
107.653 \\
83.486 \\
2.197 \\
4.394 \\
2.197 \\
4.394 \\
2.197 \\
28.561 \\
19.773 \\
4.394 \\
52.728 \\
30.758 \\
15.379 \\
17.576 \\
8.788 \\
48.334 \\
21.97 \\
2.197 \\
10.985 \\
30.758 \\
65.91 \\
2.197 \\
2.197 \\
10.985 \\
10.985 \\
26.364 \\
6.591 \\
2.197 \\
2.197 \\
2.197 \\
37.197 \\
\hline
\end{array}
$$




$$
\begin{array}{r}
10.985 \\
17.576 \\
37.349 \\
15.379 \\
13.182 \\
48.334 \\
10.985 \\
2.197 \\
8.788 \\
2.197 \\
10.985 \\
37.349 \\
8.788 \\
2.197 \\
17.576 \\
17.576 \\
6.591 \\
2.197 \\
2.197 \\
19.773 \\
4.394 \\
4.394 \\
41.743 \\
2.197 \\
2.197 \\
43.94 \\
2.197 \\
2.197 \\
2.197 \\
2.197 \\
2.197 \\
2.197 \\
28.561 \\
4.394 \\
6.591 \\
37.359 \\
4.349 \\
3.394 \\
6.591 \\
2.199 \\
4.397 \\
2.197 \\
\end{array}
$$




$$
\begin{array}{r}
10.985 \\
8.788 \\
2.197 \\
19.773 \\
2.197 \\
2.197 \\
2.197 \\
2.197 \\
2.197 \\
15.379 \\
2.197 \\
4.394 \\
2.197 \\
76.895 \\
2.197 \\
2.197 \\
2.197 \\
2.197 \\
2.197 \\
26.364 \\
43.94 \\
155.987 \\
2.197 \\
24.167 \\
26.364 \\
8.788 \\
2.197 \\
4.394 \\
2.197 \\
2.197 \\
2.197 \\
70.304 \\
4.394 \\
208.715 \\
10.985 \\
4.394 \\
2.197 \\
24.167 \\
30.758 \\
8.788 \\
83.486 \\
17.576 \\
13.197 \\
2.197 \\
\hline \\
\hline
\end{array}
$$




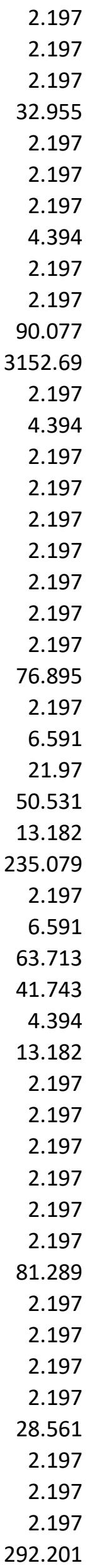




$\begin{array}{r}208.715 \\ 309.777 \\ 2.197 \\ 4.394 \\ 2.197 \\ 2.197 \\ 2.197 \\ 4.394 \\ 2.197 \\ 151.593 \\ 2.197 \\ 2.197 \\ 10.985 \\ 4.394 \\ 13.182 \\ 2.197 \\ 2.197 \\ 8.788 \\ 1203.96 \\ 90.077 \\ 2.197 \\ 393.263 \\ 2.19 \\ 2.197 \\ \hline 3451.49 \\ 2.197 \\ 2.197 \\ 2.197 \\ 15.379 \\ 4.394 \\ 2.197 \\ 4.394 \\ 2.197 \\ 2.197 \\ 4.197 \\ 2.197 \\ 2.197 \\ 2.197 \\ 2.197 \\ 2.197 \\ 2.197 \\ \hline\end{array}$


2.197

2.197

2.197

2.197

4.394

6.591

13.182

17.576

10.985

17.576

4.394

4.394

8.788

2.197

4.394

2.197

2.197

2.197

74.698

2.197

2.197

39.546

2.197

2.197

4.394

50.531

2.197

4.394

2.197

8.788

98.865

10.985

4.394

4.394

2.197

13.182

21.97

63.713

8.788

2.197

13.182

8.788

4.394

50.531

15.379

24.167

2.197

6.591

2.197

2.197 
4.394
2.197
4.394
6.591
305.383
4.394
4.394
2.197
41.743
4.394
59.319
2.197
68.107
2.197
8.788
10.985
13.182
8.788
6.591
8.788
2.197
54.925
2.197
4.394
5628.71

5628.71

533.871

10.985

96.668

19.773

1296.23

2.197

74.698

2.197

46.137

2.197

2.197

8.788

2.197

4.394

26.364

37.349

26.364

2.197

4.394

2.197

2.197

2.197

10.985

48.334

2.197 


$$
\begin{array}{r}
2.197 \\
2.197 \\
28.561 \\
2.197 \\
8.788 \\
50.531 \\
94.471 \\
2.197 \\
6.591 \\
322.959 \\
4.394 \\
50.531 \\
109.85 \\
4.394 \\
6.591 \\
8.788 \\
43.94 \\
9031.87 \\
2.197 \\
2.197 \\
57.122 \\
2.197 \\
8.788 \\
2.197 \\
2.197 \\
2.197 \\
6.591 \\
2.197 \\
26.364 \\
2.197 \\
8.788 \\
26.364 \\
2.197 \\
2.197 \\
26.364 \\
2.197 \\
138.411 \\
2.197 \\
2.197 \\
2.197 \\
41.743 \\
2.397 \\
2.397 \\
2.197
\end{array}
$$




\subsection{7}

2.197

39.546

8.788

15.379

6.591

46.137

2.197

4.394

362.505

2.197

6.591

2.197

35.152

8.788

2.197

2.197

26.364

15.379

199.927

28.561

10.985

15.379

109.85

435.006

6.591

2.197

13.182

21.97

2.197

2.197

4.394

184.548

2.197

2.197

4.394

2.197

2.197

19.773

8.788

140.608

54.925

10.985

2.197

2.197

8.788

2.197

2.197

136.214

50.531 


$$
\begin{array}{r}
6.591 \\
2.197 \\
2.197 \\
2.197 \\
2.197 \\
2.197 \\
2.197 \\
10.985 \\
6.591 \\
17.576 \\
50.531 \\
2.197 \\
500.916 \\
2.197 \\
2.197 \\
147.199 \\
2.197 \\
61.516 \\
2.197 \\
59.319 \\
32.955 \\
2.197 \\
15.379 \\
6.591 \\
4.394 \\
2.197 \\
83.486 \\
2.197 \\
2.197 \\
4.394 \\
1493.96 \\
4.394 \\
2.197 \\
151.593 \\
254.852 \\
4.394 \\
2.197 \\
2.197 \\
15.379 \\
46.137 \\
2.197 \\
2.197 \\
2.197 \\
2.197 \\
\hline
\end{array}
$$


6.591

300.989

13.182

4.394

2.197

2.197

2.197

8.788

28.561

4.394

287.807

2.197

2.197

39.546

17.576

4.394

2.197

10.985

2.197

2.197

2.197

6.591

4.394

10.985

2.197

8.788

2.197

13.182

6.591

2.197

193.336

4.394

2.197

54.925

26.364

4.394

224.094

30.758

6.591

21.97

109.85

2.197

4.394

13.182

8.788

2.197

6.591

2.197

2.197

6.591 


$$
\begin{array}{r}
2.197 \\
2.197 \\
2.197 \\
101.062 \\
6.591 \\
4.394 \\
2.197 \\
166.972 \\
224.094 \\
4.394 \\
213.109 \\
83.486 \\
57.122 \\
2.197 \\
4.394 \\
2.197 \\
4.394 \\
13.182 \\
2.197 \\
158.184 \\
2.197 \\
6.591 \\
46.137 \\
2.197 \\
164.775 \\
8.788 \\
2.197 \\
2.197 \\
6.591 \\
37.349 \\
24.167 \\
8.788 \\
120.835 \\
13.182 \\
2.197 \\
2.197 \\
2.197 \\
2.197 \\
2.197 \\
2.197 \\
2.197 \\
6.591 \\
2.197 \\
2.197 \\
2.197 \\
2.197 \\
2.197 \\
\hline
\end{array}
$$




$$
\begin{array}{r}
2.197 \\
202.124 \\
2.197 \\
41.743 \\
4.394 \\
13.182 \\
19.773 \\
15.379 \\
8.788 \\
2.197 \\
2.197 \\
17.576 \\
19.773 \\
2.197 \\
2.197 \\
2.197 \\
114.244 \\
4.394 \\
2.197 \\
4.394 \\
4.394 \\
2.197 \\
2.197 \\
13.182 \\
8.788 \\
4.394 \\
153.79 \\
94.471 \\
463.567 \\
15.379 \\
4.394 \\
8.788 \\
4.394 \\
2084.95 \\
2.197 \\
8.788 \\
2.197 \\
2.197 \\
2.197 \\
2.197 \\
4.394 \\
2.197 \\
2.197 \\
\hline
\end{array}
$$




$$
\begin{array}{r}
2.197 \\
8.788 \\
8.788 \\
2.197 \\
2.197 \\
8.788 \\
2.197 \\
2.197 \\
65.91 \\
4.394 \\
19.773 \\
2.197 \\
37.349 \\
8.788 \\
87.88 \\
15.379 \\
39.546 \\
208.715 \\
105.456 \\
4.394 \\
155.987 \\
65.91 \\
6.591 \\
2.197 \\
2.197 \\
2.197 \\
17.576 \\
4.394 \\
2.197 \\
2.197 \\
2.197 \\
2.197 \\
10.985 \\
13.182 \\
26.364 \\
8.788 \\
8.788 \\
2.197 \\
6.591 \\
2.197 \\
4.394 \\
4.394 \\
4.394 \\
2.394 \\
2.197 \\
\\
\hline 169 \\
\hline
\end{array}
$$




$$
\begin{array}{r}
19.773 \\
4.394 \\
4.394 \\
2.197 \\
2.197 \\
46.137 \\
8.788 \\
4.394 \\
30.758 \\
57.122 \\
2.197 \\
2.197 \\
2.197 \\
2.197 \\
2.197 \\
4.394 \\
4.394 \\
764.556 \\
4.394 \\
4.394 \\
2.197 \\
2.197 \\
2.197 \\
13.182 \\
2.197 \\
107.653 \\
6.591 \\
6.591 \\
32.955 \\
4.394 \\
2.197 \\
6.591 \\
4.394 \\
114.244 \\
2.197 \\
2.197 \\
13.182 \\
2.379 \\
2.197 \\
2.197 \\
2.683 \\
16.569 \\
48.578 \\
\hline
\end{array}
$$




$$
\begin{array}{r}
30.758 \\
10.985 \\
4.394 \\
15.379 \\
61.516 \\
15.379 \\
70.304 \\
2.197 \\
2.197 \\
6.591 \\
2.197 \\
180.154 \\
2.197 \\
2.197 \\
4.394 \\
2.197 \\
2.197 \\
24.167 \\
2.197 \\
30.758 \\
6.591 \\
2.197 \\
10.985 \\
2.197 \\
13.182 \\
586.599 \\
46.137 \\
43.94 \\
2.197 \\
2.197 \\
2.197 \\
13.182 \\
94.471 \\
15.379 \\
2.197 \\
2.197 \\
2.197 \\
2.197 \\
2.197 \\
2.197 \\
2.197 \\
32.394 \\
63.713 \\
2.197 \\
\hline
\end{array}
$$


54.925

28.561

8.788

516.295

4.394

2.197

2.197

19.773

2.197

2.197

2.197

4.394

2.197

6.591

2.197

90.077

4.394

4.394

4.394

2.197

2.197

6.591

6.591

2.197

6.591

28.561

4.394

123.032

2.197

2.197

2.197

17.576

35.152

4.394

15.379

683.267

4.394

74.698

6.591

2.197

6.591

4.394

32.955

8.788

127.426

21.97

2.197

6.591

4.394

6.591 


$$
\begin{array}{r}
4.394 \\
4.394 \\
4.394 \\
136.214 \\
4.394 \\
76.895 \\
2.197 \\
4.394 \\
4.394 \\
2.197 \\
2.197 \\
28.561 \\
41.743 \\
8.788 \\
8.788 \\
26.364 \\
13.182 \\
560.235 \\
17.576 \\
6.591 \\
24.167 \\
2.197 \\
4800.44 \\
15.379 \\
246.064 \\
4.394 \\
8.788 \\
10.985 \\
2.197 \\
2.197 \\
65.91 \\
19.773 \\
48.334 \\
232.882 \\
24.167 \\
2.197 \\
2.197 \\
2.197 \\
2.197 \\
17.579 \\
\hline 12.57 \\
\hline 12.047 \\
\hline 1.394 \\
\hline
\end{array}
$$




$$
\begin{aligned}
& 2.197 \\
& 70.304 \\
& 30.758 \\
& 2.197 \\
& 2.197 \\
& 8.788 \\
& 2.197 \\
& 17.576 \\
& 2.197 \\
& 314.171 \\
& 2.197 \\
& 2.197 \\
& 2.197 \\
& 2.197 \\
& 2.197 \\
& 6.591 \\
& 2.197 \\
& 2.197 \\
& 2.197 \\
& 74.698 \\
& 13.182 \\
& 2.197 \\
& 13.182 \\
& 17.576 \\
& 136.214 \\
& 2.197 \\
& 4.394 \\
& 4.394 \\
& 10.985 \\
& 4.394 \\
& 2.197 \\
& 24.167 \\
& 92.274 \\
& 2.197 \\
& 8.788 \\
& 6.591 \\
& 79.092 \\
& 8.788 \\
& 24.167 \\
& 2.197 \\
& 17.576 \\
& 2.197 \\
& 21.97 \\
& 2.197 \\
& 138.411 \\
& 2.197 \\
& 28.561 \\
& 120.835 \\
& 2.197 \\
& 2.197
\end{aligned}
$$




$$
\begin{array}{r}
2.197 \\
511.901 \\
24.167 \\
70.304 \\
59.319 \\
2.197 \\
2.197 \\
13.182 \\
2.197 \\
2.197 \\
21.97 \\
2.197 \\
2.197 \\
2.197 \\
2.197 \\
2.197 \\
4.394 \\
2.197 \\
2.197 \\
166.972 \\
105.456 \\
2.197 \\
21.97 \\
19.773 \\
173.563 \\
35.152 \\
10.985 \\
4.394 \\
4.394 \\
24.167 \\
6.591 \\
26.364 \\
2.197 \\
2.197 \\
8.788 \\
2.197 \\
13.182 \\
2.197 \\
2.197 \\
2.197 \\
2.197 \\
2.197 \\
2.197 \\
6.591 \\
2.197 \\
2.197 \\
2.197 \\
2.197
\end{array}
$$




$$
\begin{aligned}
& 2.197 \\
& 2.197 \\
& 2.197 \\
& 4.394 \\
& 6.591 \\
& 6.591 \\
& 2.197 \\
& 2.197 \\
& 2.197 \\
& 30.758 \\
& 2.197 \\
& 43.94 \\
& 19.773 \\
& 250.458 \\
& 4.394 \\
& 17.576 \\
& 2.197 \\
& 19.773 \\
& 19.773 \\
& 2.197 \\
& 2.197 \\
& 2.197 \\
& 13.182 \\
& 2.197 \\
& 8.788 \\
& 59.319 \\
& 54.925 \\
& 118.638 \\
& 28.561 \\
& 166.972 \\
& 21.97 \\
& 2.197 \\
& 4.394 \\
& 8.788 \\
& 2.197 \\
& 2.197 \\
& 28.561 \\
& 2.197 \\
& 8.788 \\
& 4.394 \\
& 37.349 \\
& 2.197 \\
& 149.396 \\
& 2.197 \\
& 10.985 \\
& 19.773 \\
& 2.197 \\
& 10.985 \\
& 2.197 \\
& 2.197
\end{aligned}
$$




$$
\begin{array}{r}
2.197 \\
21.97 \\
37.349 \\
2.197 \\
50.531 \\
265.837 \\
17.576 \\
17.576 \\
4.394 \\
4.394 \\
1656.54 \\
6.591 \\
2.197 \\
4.394 \\
13.182 \\
2.197 \\
2.197 \\
10.985 \\
4.394 \\
2.197 \\
2.197 \\
2.197 \\
2.197 \\
2.197 \\
2.197 \\
6.591 \\
6.591 \\
19.773 \\
10.985 \\
54.925 \\
2.197 \\
2.197 \\
35.152 \\
2.197 \\
2.197 \\
2.197 \\
2.197 \\
10.985 \\
4.394 \\
2.197 \\
26.364 \\
\hline 07.653 \\
\hline .393 \\
\hline
\end{array}
$$




$$
\begin{array}{r}
2.197 \\
2.197 \\
2.197 \\
21.97 \\
4.394 \\
10.985 \\
2.197 \\
1245.7 \\
2.197 \\
17.576 \\
2.197 \\
4.394 \\
2.197 \\
2.197 \\
4.394 \\
2.197 \\
6.591 \\
28.561 \\
6.591 \\
489.931 \\
4.394 \\
2.197 \\
4.394 \\
19.773 \\
2.197 \\
70.304 \\
2.197 \\
4.394 \\
8.788 \\
28.561 \\
4.394 \\
2.197 \\
2.197 \\
6.591 \\
2.1976 \\
2.197 \\
2.197 \\
4194.07 \\
6.591 \\
2.197 \\
21.97 \\
2.197 \\
4.394 \\
2.197 \\
2.197 \\
\hline
\end{array}
$$




$\begin{array}{r}103.259 \\ 19.773 \\ 2.197 \\ 2.197 \\ 19.773 \\ 2.197 \\ 43.94 \\ 81.289 \\ 10.985 \\ 2.197 \\ 54.925 \\ 2.197 \\ 8.788 \\ 2.197 \\ 26.364 \\ 24.167 \\ 2.197 \\ 2.197 \\ 28.561 \\ 13.182 \\ 2.197 \\ 15.379 \\ 112.047 \\ 17.576 \\ 39.546 \\ 2.197 \\ 2.197 \\ 8.788 \\ 15.379 \\ 2.197 \\ 2.197 \\ 2.197 \\ 2.197 \\ 2.197 \\ 2.197 \\ \hline 107.653 \\ 2.197 \\ 2.197 \\ 2.197 \\ 2.197 \\ 6.591 \\ \hline 167 \\ \hline\end{array}$




$$
\begin{array}{r}
30.758 \\
4.394 \\
6.591 \\
1573.05 \\
4.394 \\
30.758 \\
8.788 \\
2.197 \\
889.785 \\
6.591 \\
6.591 \\
2.197 \\
2.197 \\
8.788 \\
17.576 \\
4.394 \\
6.591 \\
10.985 \\
19.773 \\
2.197 \\
50.531 \\
2.197 \\
2.197 \\
276.822 \\
15.379 \\
79.092 \\
32.955 \\
2.197 \\
8.788 \\
2.197 \\
6.591 \\
6.591 \\
10.985 \\
2.197 \\
6.591 \\
19.773 \\
2.1969 \\
2.197 \\
2.197 \\
2.197 \\
2.197 \\
4.394 \\
2.197 \\
\hline
\end{array}
$$




$$
\begin{aligned}
& 2.197 \\
& 2.197 \\
& 26.364 \\
& 4.394 \\
& 2.197 \\
& 2.197 \\
& 2.197 \\
& 2.197 \\
& 2.197 \\
& 2.197 \\
& 35.152 \\
& 19.773 \\
& 2.197 \\
& 61.516 \\
& 2.197 \\
& 4.394 \\
& 26.364 \\
& 4.394 \\
& 6.591 \\
& 35.152 \\
& 57.122 \\
& 2.197 \\
& 4.394 \\
& 4.394 \\
& 21.97 \\
& 318.565 \\
& 17.576 \\
& 4497.26 \\
& 2.197 \\
& 61.516 \\
& 24.167 \\
& 17.576 \\
& 10.985 \\
& 2.197 \\
& 11633.1 \\
& 2.197 \\
& 2.197 \\
& 68.107 \\
& 120.835 \\
& 30.758 \\
& 2.197 \\
& 26.364 \\
& 6.591 \\
& 2.197 \\
& 32.955 \\
& 17.576 \\
& 4.394 \\
& 4.394 \\
& 54.925 \\
& 2.197
\end{aligned}
$$




$$
\begin{array}{r}
4.394 \\
2.197 \\
1348.96 \\
30.758 \\
10.985 \\
2.197 \\
2.197 \\
10.985 \\
4.394 \\
57.122 \\
173.563 \\
4.394 \\
4.394 \\
2.197 \\
2.197 \\
15.379 \\
10.985 \\
4.394 \\
30.758 \\
4.394 \\
6.591 \\
15.379 \\
2.197 \\
2.197 \\
37.349 \\
4.394 \\
4.394 \\
8.788 \\
28.561 \\
2.197 \\
41.743 \\
2.197 \\
4.394 \\
17.576 \\
2.197 \\
2.197 \\
15.379 \\
6.591 \\
8.394 \\
2.197 \\
2.394 \\
30.758 \\
2.197 \\
\hline
\end{array}
$$


10.985

35.152

26.364

17.576

2.197

48.334

4.394

2.197

4.394

28.561

10.985

2.197

4.394

2.197

26.364

2.197

246.064

32.955

24.167

37.349

17.576

2.197

6.591

2.197

2.197

6.591

2.197

112.047

15.379

4.394

2.197

6.591

2.197

6.591

8.788

4.394

2.197

208.715

340.535

2719.89

2.197

59.319

4.394

224.094

219.7

2.197

2.197

2.197

2.197

4.394 


$\begin{array}{r}8.788 \\ 8.788 \\ 112.047 \\ 2.197 \\ 2.197 \\ 46.137 \\ 2.197 \\ 10.985 \\ 4.394 \\ 309.777 \\ 2175.03 \\ 2.197 \\ 8.788 \\ 21.97 \\ 87.88 \\ 4.394 \\ 2.197 \\ 48.334 \\ 39.546 \\ 265.837 \\ 131.82 \\ 68.107 \\ 4.394 \\ 2.197 \\ 2.197 \\ 210.912 \\ 219.7 \\ 2.197 \\ 10.985 \\ 2.197 \\ 250.458 \\ 8.788 \\ 37.349 \\ 74.698 \\ 2.197 \\ 4.394 \\ 8.788 \\ 4.394 \\ 2.197 \\ 2.197 \\ 8.788 \\ 2.197 \\ 2.197 \\ 2.597 \\ \hline\end{array}$




$$
\begin{array}{r}
68.107 \\
6.591 \\
123.032 \\
1436.84 \\
149.396 \\
21.97 \\
2.197 \\
2.197 \\
142.805 \\
2.197 \\
15.379 \\
74.698 \\
54.925 \\
6.591 \\
17.576 \\
2.197 \\
2.197 \\
2.197 \\
2.197 \\
79.092 \\
6.591 \\
32.955 \\
2.197 \\
6.591 \\
151.593 \\
15.379 \\
4.394 \\
2.197 \\
8.788 \\
2.197 \\
2.197 \\
232.882 \\
2.197 \\
4.394 \\
4.394 \\
2.197 \\
19.773 \\
2.197 \\
4.394 \\
2.1997 \\
2.197 \\
8.788 \\
2.197 \\
4.394 \\
\\
\hline
\end{array}
$$




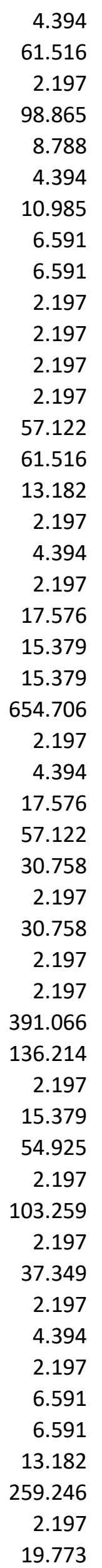


2.197

15.379

2.197

2.197

2.197

4.394

6.591

54.925

43.94

8.788

2.197

35.152

2.197

17.576

21.97

2.197

2.197

17.576

28.561

2.197

2.197

46.137

17.576

21.97

4.394

72.501

54.925

17.576

8.788

162.578

54.925

406.445

2.197

8.788

2.197

2.197

8.788

2.197

4.394

24.167

2.197

4.394

2.197

61.516

4.394

2.197

2.197

4.394

92.274

287.807 
13.182

2.197

2.197

2.197

39.546

37.349

4.394

2.197

2.197

2.197

4.394

4.394

2.197

8.788

145.002

10.985

250.458

155.987

13.182

32.955

4.394

6.591

2.197

10.985

6.591

2.197

6.591

2.197

2.197

2.197

26.364

2.197

13.182

2.197

4.394

46.137

2.197

2.197

19.773

116.441

112.047

79.092

4.394

8.788

571.22

57.122

105.456

8.788

26.364

2.197 


$$
\begin{array}{r}
6.591 \\
6.591
\end{array}
$$

15.379

2.197

13.182

2.197

41.743

30.758

210.912

19.773

6.591

2.197

10.985

6.591

2.197

28.561

2.197

92.274

1619.19

26.364

4.394

4.394

59.319

19.773

217.503

661.297

2.197

72.501

2.197

65.91

2.197

8.788

2.197

57.122

8.788

4.394

35.152

57.122

2.197

2.197

41.743

4.394

43.94

10.985

2.197

6.591

2.197

2.197

320.762

2.197 


$$
\begin{aligned}
& 147.199 \\
& 17.576 \\
& 26.364 \\
& 8.788 \\
& 2.197 \\
& 72.501 \\
& 2.197 \\
& 6.591 \\
& 10.985 \\
& 2.197 \\
& 2.197 \\
& 2.197 \\
& 2.197 \\
& 2.197 \\
& 2.197 \\
& 2.197 \\
& 87.88 \\
& 43.94 \\
& 39.546 \\
& 395.46 \\
& 114.244 \\
& 19.773 \\
& 52.728 \\
& 112.047 \\
& 2.197 \\
& 17.576 \\
& 79.092 \\
& 2.197 \\
& 96.668 \\
& 2.197 \\
& 68.107 \\
& 85.683 \\
& 4.394 \\
& 4.394 \\
& 2.197 \\
& 2.197 \\
& 2.197 \\
& 13.182 \\
& 15.379 \\
& 364.702 \\
& 15.379 \\
& 8.788 \\
& 4.394 \\
& 19.773 \\
& 188.942 \\
& 4.394 \\
& 4.394 \\
& 4.394 \\
& 17.576 \\
& 4.394
\end{aligned}
$$


15.379

17.576

90.077

2.197

8.788

2.197

4.394

2.197

2.197

2.197

21.97

2.197

2.197

15.379

6.591

4.394

57.122

2.197

2.197

399.854

2.197

2.197

2.197

2.197

4.394

2.197

74.698

2.197

2.197

2.197

2.197

6.591

2.197

2.197

413.036

6.591

24.167

2.197

4.394

19.773

250.458

6.591

10.985

37.349

13.182

2.197

10.985

2.197

15.379

2.197 


$$
\begin{array}{r}
4.394 \\
8.788 \\
182.351 \\
6.591 \\
13.182 \\
8.788 \\
2.197 \\
2.197 \\
158.184 \\
2.197 \\
6.591 \\
2.197 \\
4.394 \\
4.394 \\
2.197 \\
4.394 \\
4.394 \\
2.197 \\
4.394 \\
2.197 \\
2.197 \\
2.197 \\
2.197 \\
4.394 \\
2.197 \\
2.197 \\
4.394 \\
2.197 \\
26.364 \\
2.197 \\
2.197 \\
10.985 \\
2.197 \\
2.197 \\
296.595 \\
10.985 \\
13.182 \\
2.197 \\
19.773 \\
6.591 \\
2.197 \\
8.788 \\
2.197 \\
6.591 \\
2.197 \\
4.394 \\
2.197 \\
2.197 \\
2.197 \\
8.788
\end{array}
$$




$$
\begin{array}{r}
61.516 \\
2.197 \\
2.197 \\
24.167 \\
2.197 \\
13.182 \\
26.364 \\
21.97 \\
263.64 \\
26.364 \\
63.713 \\
4.394 \\
4.394 \\
6.591 \\
4.394 \\
13.182 \\
4.394 \\
4.394 \\
2.197 \\
4.394 \\
2.197 \\
61.516 \\
4.394 \\
2.197 \\
57.122 \\
19.773 \\
46.137 \\
2.197 \\
35.152 \\
76.895 \\
375.687 \\
6.591 \\
4.394 \\
4.197 \\
4.394 \\
2.94 \\
2.197 \\
4.394 \\
4.394 \\
4.394 \\
6.591 \\
2.197 \\
17.576 \\
61.516 \\
17.576 \\
\hline
\end{array}
$$




$$
\begin{array}{r}
2.197 \\
30.758 \\
24.167 \\
4.394 \\
2.197 \\
2.197 \\
2.197 \\
15.379 \\
8.788 \\
2.197 \\
2.197 \\
2.197 \\
2.197 \\
41.743 \\
2.197 \\
37.349 \\
105.456 \\
2.197 \\
101.062 \\
2.197 \\
4.394 \\
2.197 \\
2.197 \\
6.591 \\
8.788 \\
13.182 \\
2.197 \\
2.197 \\
2.197 \\
2.197 \\
2.197 \\
2.197 \\
2.197 \\
2.197 \\
2.197 \\
2.197 \\
\hline 13.182 \\
2.197 \\
10.989 \\
2.197 \\
2.197 \\
4.394 \\
32.955 \\
\hline
\end{array}
$$


2.197
2.197
30.758
2.197
26.364
2.197
4.394
4.394
4.394
2.197
4.394
112.047
15.379
2.197
10.985
2.197
4.394
105.456
35.152
21.97
19.773
6.591
8.788
4.394

650.312

2.197

2.197

30.758

2.197

15.379

28.561

2.197

2.197

4.394

43.94

4.394

8.788

65.91

118.638

105.456

2.197

2.197

2.197

2.197

2.197

13.182

19.773

2.197

2.197

8.788 


$$
\begin{array}{r}
2.197 \\
2.197 \\
2.197 \\
8.788 \\
2.197 \\
2.197 \\
24.167 \\
21.97 \\
26.364 \\
2.197 \\
2.197 \\
2.197 \\
116.441 \\
2.197 \\
24.167 \\
32.955 \\
2.197 \\
2.197 \\
4.394 \\
142.805 \\
15.379 \\
13.182 \\
2.197 \\
2.197 \\
2.197 \\
2.197 \\
2.197 \\
10.985 \\
15.379 \\
4.394 \\
8.788 \\
2.197 \\
2.197 \\
2.591 \\
2.197 \\
2.182 \\
2.197 \\
2.197 \\
2.197 \\
2.197 \\
48.334 \\
32.955 \\
2.197 \\
2.197 \\
\hline
\end{array}
$$


2.197

13.182

76.895

6.591

39.546

30.758

2.197

2.197

8.788

6.591

2.197

4.394

2.197

252.655

2.197

65.91

30.758

10.985

2.197

2.197

2.197

2.197

2.197

4.394

2.197

6.591

10.985

2.197

2.197

4.394

2.197

2.197

17.576

2.197

10.985

2.197

2.197

2.197

4.394

2.197

46.137

59.319

4.394

15.379

228.488

2.197

2.197

2.197

2.197

6.591 


$$
\begin{aligned}
& 4.394 \\
& 2.197 \\
& 8.788 \\
& 2.197 \\
& 35.152 \\
& 4.394 \\
& 2.197 \\
& 15.379 \\
& 2.197 \\
& 4.394 \\
& 10.985 \\
& 13.182 \\
& 2.197 \\
& 8.788 \\
& 13.182 \\
& 2.197 \\
& 8.788 \\
& 6.591 \\
& 2.197 \\
& 2.197 \\
& 2.197 \\
& 2.197 \\
& 59.319 \\
& 2.197 \\
& 2.197 \\
& 4.394 \\
& 166.972 \\
& 17.576 \\
& 2.197 \\
& 15.379 \\
& 10.985 \\
& 551.447 \\
& 15.379 \\
& 30.758 \\
& 4.394 \\
& 2.197 \\
& 4.394 \\
& 2.197 \\
& 13.182 \\
& 2.197 \\
& 2.197 \\
& 10.985 \\
& 4.394 \\
& 17.576 \\
& 112.047 \\
& 2.197 \\
& 2.197 \\
& 2.197 \\
& 2.197 \\
& 15.379
\end{aligned}
$$


21.97

35.152

2.197

112.047

2.197

2.197

8.788

2.197

4.394

6.591

2.197

4.394

17.576

138.411

10.985

10.985

2.197

2.197

2.197

2.197

61.516

2.197

2.197

2.197

2.197

4.394

1463.2

13.182

8.788

2.197

50.531

4.394

4.394

8.788

2.197

10.985

8.788

8.788

4.394

2.197

19.773

2.197

2.197

4.394

4.394

46.137

2.197

2.197

2.197

2.197 


$$
\begin{array}{r}
4.394 \\
2.197 \\
4.394 \\
10.985 \\
72.501 \\
2.197 \\
87.88 \\
17.576 \\
32.955 \\
2.197 \\
4.394 \\
21.97 \\
68.107 \\
15.379 \\
30.758 \\
8.788 \\
6.591 \\
2.197 \\
2.197 \\
57.122 \\
10.985 \\
2.197 \\
83.486 \\
19.773 \\
2.197 \\
17.576 \\
6.591 \\
2.197 \\
2.197 \\
2.197 \\
6.591 \\
2.197 \\
261.443 \\
17.576 \\
3743.69 \\
2.197 \\
2.197 \\
329.59 \\
2.197 \\
2.197 \\
2.197 \\
28.596 \\
\hline \\
\hline
\end{array}
$$


13.182

65.91

28.561

4.394

136.214

2.197

32.955

43.94

6.591

2.197

24.167

2.197

10.985

4.394

26.364

8.788

4.394

2.197

24.167

279.019

2.197

6.591

2.197

2.197

2.197

57.122

2.197

4.394

2.197

10.985

8.788

2.197

4.394

74.698

13.182

2.197

30.758

6.591

2128.89

2.197

4.394

15.379

2.197

2.197

2.197

2.197

2.197

158.184

30.758

2.197 
96.668

32.955

19.773

2.197

4.394

13.182

2.197

8.788

489.931

10.985

10.985

4.394

10.985

2.197

2.197

32.955

52.728

19.773

13.182

4.394

6.591

35.152

2.197

784.329

2.197

2.197

2.197

2.197

2.197

17.576

2.197

32.955

24.167

4.394

2.197

6.591

2.197

2.197

2.197

30.758

10.985

6.591

2.197

4.394

10.985

2.197

2.197

17.576

19.773

2.197 


$$
\begin{array}{r}
2.197 \\
2.197 \\
10.985 \\
13.182 \\
10.985 \\
8.788 \\
46.137 \\
19.773 \\
6.591 \\
2.197 \\
2.197 \\
19.773 \\
2.197 \\
2.197 \\
8.788 \\
41.743 \\
17.576 \\
155.987 \\
13.182 \\
59.319 \\
2.197 \\
944.71 \\
26.364 \\
24.167 \\
2.197 \\
17.576 \\
2.197 \\
2.197 \\
4.394 \\
2.197 \\
2.197 \\
4.394 \\
2.197 \\
140.608 \\
2.197 \\
2.197 \\
45.379 \\
41.743 \\
8.788 \\
21.97 \\
6.591 \\
6.591 \\
4.394 \\
8.788 \\
4.394 \\
\hline 408 \\
\hline
\end{array}
$$




$$
\begin{array}{r}
2.197 \\
10.985 \\
2.197 \\
8.788 \\
4.394 \\
28.561 \\
2.197 \\
2.197 \\
4.394 \\
162.578 \\
2.197 \\
6.591 \\
2.197 \\
37.349 \\
17.576 \\
290.004 \\
2.197 \\
2.197 \\
2.197 \\
2.197 \\
8.788 \\
2.197 \\
2.197 \\
43.94 \\
4.394 \\
8.788 \\
8.788 \\
4.394 \\
83.486 \\
4.394 \\
2.197 \\
13.182 \\
4.394 \\
2.197 \\
2.197 \\
2.197 \\
2.197 \\
2.197 \\
13.182 \\
2.197 \\
17.576 \\
10.985 \\
32.353 \\
\hline 3.182 \\
\hline
\end{array}
$$




$$
\begin{array}{r}
2.197 \\
2.197 \\
4.394 \\
32.955 \\
2.197 \\
8.788 \\
43.94 \\
4.394 \\
2.197 \\
2.197 \\
2.197 \\
2.197 \\
2.197 \\
118.638 \\
2.197 \\
4.394 \\
15.379 \\
2.197 \\
142.805 \\
6.591 \\
2.197 \\
37.349 \\
2.197 \\
4.394 \\
2.197 \\
6.591 \\
43.94 \\
13.182 \\
2.197 \\
448.188 \\
2.197 \\
6153.8 \\
46.137 \\
8.788 \\
41.743 \\
2.197 \\
68.107 \\
17.576 \\
4.394 \\
2.197 \\
8.788 \\
2.197 \\
39.546 \\
13.182 \\
250.458 \\
4.394 \\
\\
\hline
\end{array}
$$




$$
\begin{array}{r}
8.788 \\
4.394 \\
4.394 \\
13.182 \\
2.197 \\
2.197 \\
237.276 \\
59.319 \\
6.591 \\
10.985 \\
2.197 \\
2.197 \\
164.775 \\
131.82 \\
17.576 \\
4.394 \\
4.394 \\
105.456 \\
59.319 \\
2.197 \\
2.197 \\
2.197 \\
30.758 \\
4.394 \\
10.985 \\
2.197 \\
35.152 \\
50.531 \\
2.197 \\
6.591 \\
4.394 \\
32.955 \\
4.394 \\
2.197 \\
2.197 \\
358.111 \\
10.985 \\
4.394 \\
15.379 \\
4.394 \\
2.197 \\
15.379 \\
98.865 \\
2.197 \\
2.197 \\
6.591 \\
8.789 \\
\hline
\end{array}
$$




$$
\begin{array}{r}
2.197 \\
2.197 \\
50.531 \\
76.895 \\
2.197 \\
2.197 \\
2.197 \\
79.092 \\
2.197 \\
2.197 \\
4.394 \\
2.197 \\
134.017 \\
2.197 \\
2.197 \\
806.299 \\
26.364 \\
24.167 \\
6.591 \\
17.576 \\
10.985 \\
2.197 \\
2.197 \\
10.985 \\
2.197 \\
61.516 \\
2.197 \\
4.394 \\
6.591 \\
24.167 \\
28.561 \\
2.197 \\
182.351 \\
2.197 \\
10.985 \\
2.197 \\
193.336 \\
37.349 \\
2.197 \\
2.197 \\
2.197 \\
2.197 \\
4.394 \\
2.197 \\
4.394 \\
2.197 \\
2.197 \\
\\
\hline
\end{array}
$$


2.197

2.197

17.576

2.197

2.197

2.197

2.197

6.591

35.152

72.501

19.773

4.394

30.758

6.591

17.576

6.591

2.197

2.197

6.591

2.197

4.394

4.394

68.107

83.486

2.197

2.197

2.197

24.167

17.576

4.394

8.788

6.591

2.197

2.197

35.152

2.197

8.788

2.197

30.758

2.197

2.197

32.955

8.788

6.591

4.394

76.895

28.561

35.152

65.91

6.591 


$$
\begin{array}{r}
17.576 \\
10.985 \\
2.197 \\
6.591 \\
35.152 \\
2.197 \\
4.394 \\
2.197 \\
2.197 \\
6.591 \\
2.197 \\
263.64 \\
2.197 \\
2.197 \\
2.197 \\
1832.3 \\
2.197 \\
2.197 \\
2.197 \\
279.019 \\
2.197 \\
10.985 \\
10.985 \\
6.591 \\
2.197 \\
61.516 \\
6.591 \\
17.576 \\
432.809 \\
2.197 \\
8.788 \\
2.197 \\
4.394 \\
2.197 \\
2.197 \\
2.197 \\
46.137 \\
17.576 \\
2.197 \\
4.394 \\
8.788 \\
2.197 \\
8.788 \\
57.122 \\
13.182 \\
2.197 \\
\hline
\end{array}
$$




$$
\begin{array}{r}
2.197 \\
6.591 \\
2.197 \\
4.394 \\
17.576 \\
2.197 \\
2.197 \\
2.197 \\
2.197 \\
2.197 \\
8.788 \\
15.379 \\
13.182 \\
118.638 \\
2.197 \\
6.591 \\
4.394 \\
2.197 \\
2.197 \\
2.197 \\
10.985 \\
790.92 \\
2.197 \\
17.576 \\
26.364 \\
54.925 \\
2.197 \\
2.197 \\
2.197 \\
52.728 \\
2.197 \\
2.197 \\
2.197 \\
2.398 \\
2.197 \\
2.394 \\
2.394 \\
2.199 \\
2.197 \\
6.591 \\
2.197 \\
112.047 \\
2.197 \\
41.743 \\
\hline
\end{array}
$$




$$
\begin{array}{r}
2.197 \\
2.197 \\
118.638 \\
2.197 \\
2.197 \\
6.591 \\
6.591 \\
2.197 \\
2.197 \\
10.985 \\
2.197 \\
136.214 \\
4.394 \\
30.758 \\
8.788 \\
2.197 \\
2.197 \\
2.197 \\
2.197 \\
19.773 \\
2.197 \\
57.122 \\
2.197 \\
26.364 \\
2.197 \\
311.974 \\
21.97 \\
2.197 \\
4.394 \\
2.197 \\
2.197 \\
2.197 \\
\hline \\
\hline 39.546 \\
8.788 \\
4.394 \\
6.591 \\
2.197 \\
2.197 \\
2.197 \\
2.197 \\
2.197 \\
2.197 \\
2.197 \\
\hline 2.39 \\
\hline
\end{array}
$$




$$
\begin{array}{r}
21.97 \\
2.197 \\
6.591 \\
61.516 \\
158.184 \\
8.788 \\
13.182 \\
19.773 \\
4.394 \\
2.197 \\
2.197 \\
17.576 \\
2.197 \\
6.591 \\
35.152 \\
4.394 \\
2.197 \\
2.197 \\
6.591 \\
2.197 \\
4.394 \\
4.394 \\
85.683 \\
4.394 \\
8.788 \\
8.788 \\
4.394 \\
4.394 \\
2.197 \\
2.197 \\
8.788 \\
2.197 \\
4.394 \\
15.379 \\
13.182 \\
6.591 \\
24.167 \\
10.985 \\
2.197 \\
28.561 \\
109.85 \\
32.955 \\
2.197 \\
\hline 3.152 \\
\hline
\end{array}
$$


2.197
2.197
2.197
2.197
131.82
26.364
2.197
13.182
28.561
257.049
15.379
2.197
2.197
8.788
2.197
26.364
226.291
6.591
32.955
129.623
83.486
120.835
6.591
4.394
24.167
2.197
8.788
164.775

164.775

2.197

2.197

30.758

2.197

15.379

6.591

4.394

17.576

2.197

94.471

6.591

41.743

37.349

41.743

21.97

10.985

65.91

2.197

2.197

1199.56

26.364

2.197 
2.197

2.197

15.379

2.197

2.197

2.197

61.516

15.379

17.576

2.197

32.955

21.97

37.349

2.197

54.925

46.137

19.773

125.229

85.683

2.197

46.137

4.394

2.197

4.394

4.394

2.197

2.197

2.197

92.274

48.334

28.561

4.394

6.591

8.788

116.441

43.94

8.788

8.788

8.788

2.197

2.197

2.197

4.394

4.394

2.197

2.197

68.107

2.197

2.197

103.259 


$$
\begin{array}{r}
2.197 \\
8.788 \\
6.591 \\
17.576 \\
4.394 \\
19.773 \\
10.985 \\
235.079 \\
46.137 \\
397.657 \\
441.597 \\
2.197 \\
2.197 \\
6.591 \\
61.516 \\
2.197 \\
2.197 \\
2.197 \\
2.197 \\
2.197 \\
4.394 \\
215.306 \\
2.197 \\
2.197 \\
2.197 \\
2.197 \\
2.197 \\
388.869 \\
2.197 \\
8.788 \\
2.197 \\
2.197 \\
2.197 \\
2.197 \\
2.197 \\
\hline 05.079 \\
101.062 \\
2.197 \\
8.788 \\
2.197 \\
2.197 \\
19.773 \\
15.379 \\
2.197 \\
\hline
\end{array}
$$




$\begin{array}{r}2.197 \\ 17.576 \\ 2.197 \\ 15.379 \\ 2.197 \\ 63.713 \\ 2.197 \\ 54.925 \\ 13.182 \\ 6.591 \\ 2.197 \\ 8.788 \\ 8.788 \\ 8.788 \\ 2.197 \\ 32.955 \\ 24.167 \\ 6.591 \\ 2.197 \\ 2.197 \\ 8.788 \\ 13.182 \\ 35.152 \\ 35.152 \\ 13.182 \\ 4.394 \\ 573.417 \\ 4.394 \\ 15.379 \\ 8.788 \\ 2.197 \\ 32.955 \\ 6.591 \\ 26.364 \\ 2.728 \\ 24.167 \\ 17.576 \\ 2.197 \\ 50.531 \\ 2.197 \\ 2.197 \\ 2.197 \\ \hline 17.531 \\ 505.31 \\ \hline\end{array}$




$$
\begin{array}{r}
37.349 \\
2.197 \\
4.394 \\
2.197 \\
4.394 \\
101.062 \\
48.334 \\
2.197 \\
19.773 \\
4.394 \\
8.788 \\
2.197 \\
21.97 \\
2.197 \\
2.197 \\
4.394 \\
54.925 \\
4.394 \\
21.97 \\
4.394 \\
15.379 \\
19.773 \\
2.197 \\
2.197 \\
4.394 \\
4.394 \\
17.576 \\
15.379 \\
10.985 \\
2.197 \\
4.394 \\
2.197 \\
70.304 \\
2.197 \\
8.788 \\
2.197 \\
2.197 \\
2.197
\end{array}
$$




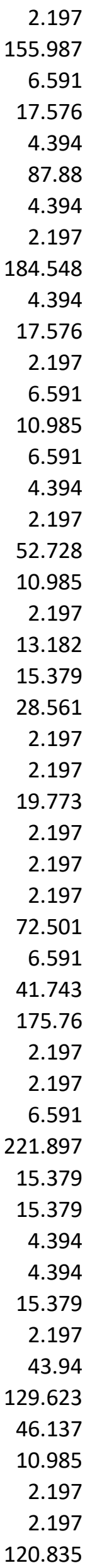




$\begin{array}{r}2.197 \\ 32.955 \\ 2.197 \\ 2.197 \\ 4.394 \\ 8.788 \\ 2.197 \\ 13.182 \\ 186.745 \\ 265.837 \\ 2.197 \\ 13.182 \\ 6.591 \\ 2.197 \\ 4.394 \\ 30.758 \\ 4.394 \\ 2.197 \\ 8.788 \\ 2.197 \\ 2.197 \\ 129.623 \\ 2.197 \\ 2.197 \\ 46.137 \\ 30.758 \\ 15.379 \\ 4.394 \\ 6.591 \\ 2.197 \\ 2.197 \\ 2.197 \\ 6.591 \\ 2.197 \\ 2.197 \\ \hline 2.788 \\ 17.576 \\ 2.197 \\ 2.197 \\ 70.304 \\ 2.197 \\ \hline 3.394 \\ 32.364 \\ 32.955 \\ \hline\end{array}$




\begin{tabular}{|c|}
\hline 4.394 \\
\hline 4.394 \\
\hline 13.182 \\
\hline 4.394 \\
\hline 2.197 \\
\hline 57.122 \\
\hline 4.394 \\
\hline 19.773 \\
\hline 54.925 \\
\hline 13.182 \\
\hline 4.394 \\
\hline 6.591 \\
\hline 30.758 \\
\hline 26.364 \\
\hline 72.501 \\
\hline 15.379 \\
\hline 250.458 \\
\hline 2.197 \\
\hline 103.259 \\
\hline 19.773 \\
\hline 2.197 \\
\hline 4.394 \\
\hline 2.197 \\
\hline 10.985 \\
\hline 2.197 \\
\hline 4.394 \\
\hline 4.394 \\
\hline 6.591 \\
\hline 6.591 \\
\hline 2.197 \\
\hline 2.197 \\
\hline 4.394 \\
\hline 2.197 \\
\hline 4.394 \\
\hline 19.773 \\
\hline 6.591 \\
\hline 2.197 \\
\hline 2.197 \\
\hline 19.773 \\
\hline 19.773 \\
\hline 4.394 \\
\hline 4.394 \\
\hline 2.197 \\
\hline 46.137 \\
\hline 4.394 \\
\hline 10.985 \\
\hline 59.319 \\
\hline 8.788 \\
\hline 2.197 \\
\hline 6.591 \\
\hline
\end{tabular}




$\begin{array}{r}123.032 \\ 2.197 \\ 2.197 \\ 2.197 \\ 4.394 \\ 4.394 \\ 4.394 \\ 2.197 \\ 4.394 \\ 2.197 \\ 2.197 \\ 2.197 \\ 72.501 \\ 4.394 \\ 2.197 \\ 48.334 \\ 2.197 \\ 2.197 \\ 19.773 \\ 2.197 \\ 2.197 \\ 32.955 \\ 6.591 \\ 46.137 \\ 13.182 \\ 13.182 \\ 13.182 \\ 2.197 \\ \hline 92.274 \\ 2.197 \\ 15.379 \\ 4.1997 \\ \hline 4.394 \\ 10.985 \\ 298.792 \\ 52.728 \\ 4.394 \\ 26.364 \\ 57.122 \\ 41.743 \\ 2.197 \\ 26.364 \\ 32.955 \\ 2.197 \\ \hline\end{array}$


92.274

8.788

10.985

2.197

2.197

2.197

61.516

43.94

57.122

10.985

6.591

6.591

41.743

2.197

13.182

10.985

43.94

4.394

6.591

70.304

48.334

2.197

4.394

4.394

4.394

105.456

6.591

2.197

2.197

4.394

8.788

6.591

2.197

17.576

103.259

4.394

4.394

2.197

2.197

188.942

17.576

2.197

2.197

2.197

2.197

79.092

2.197

4.394

2.197

2.197 


$$
\begin{array}{r}
2.197 \\
4.394 \\
2.197 \\
2.197 \\
15.379 \\
30.758 \\
70.304 \\
15.379 \\
2.197 \\
2.197 \\
6.591 \\
52.728 \\
2.197 \\
2.197 \\
2.197 \\
2.197 \\
2.197 \\
109.85 \\
2.197 \\
2.197 \\
17.576 \\
4.394 \\
2.197 \\
2.197 \\
32.955 \\
6.591 \\
4.394 \\
8.788 \\
26.364 \\
8.788 \\
24.167 \\
4.394 \\
2.197 \\
2.197 \\
2.1997 \\
2.197 \\
2.197 \\
6.591 \\
6.591 \\
30.758 \\
2.197 \\
\hline 3.182 \\
\hline .197 \\
\hline
\end{array}
$$


2.197

318.565

26.364

15.379

28.561

37.349

2.197

13.182

74.698

15.379

13.182

4.394

2.197

8.788

2.197

17.576

17.576

2.197

54.925

2.197

21.97

2.197

39.546

15.379

2.197

2.197

39.546

10.985

2.197

2.197

2.197

19.773

8.788

19.773

26.364

30.758

10.985

6.591

32.955

2.197

2.197

46.137

15.379

2.197

2.197

2.197

19.773

2.197

4.394

21.97 
2.197
4.394
24.167
65.91
6.591
2.197
68.107
28.561
2.197
21.97
21.97
10.985
2.197
2.197
2.197
8.788

243.867

21.97

50.531

2.197

2.197

8.788

2.197

6.591

8.788

2.197

57.122

6.591

41.743

4.394

10.985

24.167

2.197

4.394

19.773

13.182

6.591

8.788

153.79

52.728

2.197

30.758

46.137

10.985

2.197

2.197

2.197

13.182

26.364

2.197 
32.955

2.197

2.197

2.197

2.197

2.197

2.197

2.197

6.591

4.394

2.197

2.197

2.197

10.985

2.197

2.197

8.788

2.197

4.394

2.197

4.394

2.197

2.197

13.182

2.197

24.167

2.197

2.197

2.197

19.773

8.788

8.788

6.591

2.197

2.197

15.379

41.743

8.788

4.394

2.197

2.197

15.379

4.394

8.788

2.197

4.394

52.728

2.197

15.379

8.788 
2.197

35.152

4.394

4.394

13.182

6.591

4.394

17.576

6.591

8.788

4.394

6.591

2.197

8.788

8.788

2.197

8.788

2.197

2.197

2.197

21.97

13.182

15.379

10.985

10.985

15.379

15.379

6.591

2.197

2.197

2.197

2.197

2.197

10.985

30.758

2.197

2.197

2.197

2.197

2.197 